\title{
Soil Stabilization/Solidification Grout Mix Design Study for the C-, K-, L-, and P-Reactor Seepage Basins Closure Final Report
}

by

P. Nakagawa

Westinghouse Savannah River Company

Savannah River Site

Aiken, South Carolina 29808

This paper was prepared in connection with work done under the above contract number with the U.S. Department of Energy. By acceptance of this paper, the publisher and/or recipient acknowledges the U. S. Government's right to retain a nonexclusive, royalty-free license in and to any copyright covering this paper, along with the right to reproduce and to authorize others to reproduce all or part of the copyrighted paper. 
This document was prepared in conjunction with work accomplished under Contract No. DE-AC09-96SR18500 with the U. S. Department of Energy.

\section{DISCLAIMER}

This report was prepared as an account of work sponsored by an agency of the United States Government. Neither the United States Government nor any agency thereof, nor any of their employees, makes any warranty, express or implied, or assumes any legal liability or responsibility for the accuracy, completeness, or usefulness of any information, apparatus, product or process disclosed, or represents that its use would not infringe privately owned rights. Reference herein to any specific commercial product, process or service by trade name, trademark, manufacturer, or otherwise does not necessarily constitute or imply its endorsement, recommendation, or favoring by the United States Government or any agency thereof. The views and opinions of authors expressed herein do not necessarily state or reflect those of the United States Government or any agency thereof.

This report has been reproduced directly from the best available copy.

Available for sale to the public, in paper, from: U.S. Department of Commerce, National Technical Information Service, 5285 Port Royal Road, Springfield, VA 22161, phone: (800) 553-6847, fax: (703) 605-6900

email: orders@ntis.fedworld.gov

online ordering: http://www.ntis.gov/help/index.asp

Available electronically at http://www.osti.gov/bridge

Available for a processing fee to U.S. Department of Energy and its contractors, in paper, from: U.S. Department of Energy, Office of Scientific and Technical Information, P.O. Box 62, Oak Ridge, TN 37831-0062,

phone: (865)576-8401,

fax: (865)576-5728

email: $\underline{\text { reports@ adonis.osti.gov }}$ 
United States Department of Energy

Savannah River Site

SOIL STABILIZATION/SOLIDIFICATION

GROUT MIX DESIGN STUDY FOR THE

C-, K-, L-, AND P-REACTOR

SEEPAGE BASINS CLOSURE

FINAL RE BORR

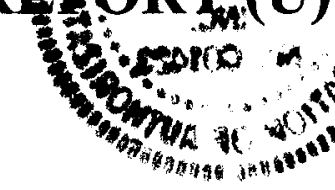

WSRC-RP-99-4075

Revision 1

August 1999

Westinghouse Savannah River Company

Savannah River Site

Aiken, S.C. 29808

Prepared for U.S. Department of Energy Under

Contract DE-AC09-96SR18500 
This report documents the laboratory bench-scale soil stabilization/solidification design study performed for Westinghouse Savannah River Company under Contract C001311N by Science Applications International Corporation (SAIC) Enginccring, Inc. Factors out of SAIC's direct control affect the scalability from bench scale testing to full field applications, including, but not limited to; basin variability, mixing type and application, and experience of the $\mathrm{S} / \mathrm{S}$ contractor. SAIC does not warrant, guarantee, or assume any responsibility for full-scale field application of the recommended mixes.

Larry M. Deschaine, PE
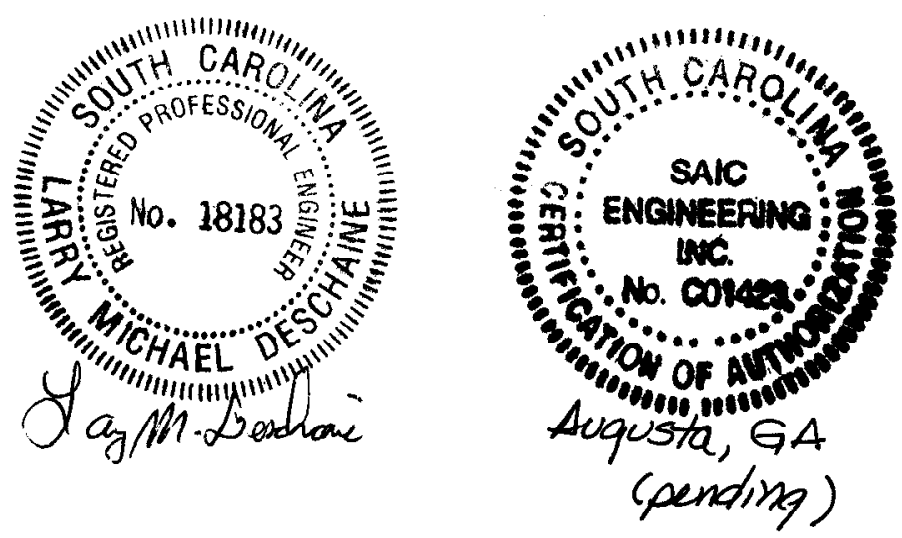


\section{EXECUTIVE SUMMARY}

At the Savannah River Site (SRS), reactor seepage basins received low-level radioactive purge water from the disassembly basins between 1957 and 1991. Almost all of the radioactivity was due to tritium which migrated quickly from the basins via leaching. Most of the remaining radioactivity is associated with cesium-137 (Cs-137), strontium-90 (Sr-90), and plutonium-239/240 (Pu-239/240). These constituents are present in soils immediately beneath each basin.

Although the natural properties of the local soils tend to immobilize these constituents within a few feet of the bottom of the basin, in-situ stabilization/solidification (S/S) treatment has been selected to supplement the natural retention properties of the local soils. This treatment process combines the soil with a grout mixture to form a reduced permeability material which further reduces contaminant mobility. Following stabilization of contaminated soils, each basin is then provided with drainage control, such as a geotextile membrane or other acceptable means, backfilled, and the surrounding area contoured to reduce the infiltration of rain water above the stabilized monolith.

This Revision 1 report documents the results of a bench-scale study conducted by SAIC under Contract No. C001311N to develop a grout mix capable of stabilizing the constituents of concern via the S/S treatment of the soils for reactor seepage basins at four SRS locations: C-Area (CRSB), K-Area (KRSB), L-Area (LRSB), and P-Area (PRSB). It recommends a specific grout mix for each reactor seepage basin and provides recommendations, limitations, and constraints for the full-scale field implementation.

Based on a review of literature and previous stabilization studies conducted at SRS, four grout mixes were selected for study. Portland cement, the basic ingredient, was combined with bentonite alone, silicate alone (four percent by weight), bentonite with silicate (four percent by weight), and silicate alone (two percent by weight).

The general geologic settings of the four seepage basins are very similar. However, a significant degree of variability was observed within each basin, with soils ranging from sands to clays. Therefore, the possible variability in lithology within a reactor seepage basin was a primary consideration in developing the technical approach for the study.

Soils from CRSB and PRSB were divided or segregated to represent the range of lithology within the sample material: coarse (sandy), primarily fine (clayey), and the overall bulk 
combination of coarse and fine soil material (bulk fractions). Soils from KRSB and LRSB were segregated into only coarse and fine fractions. The grout mixes were evaluated on each soil fraction from each reactor seepage basin.

The tests performed under this study fall into three general categories: (1) geotechnical tests, (2) chemical characterization, and (3) S/S performance testing.

With respect to geotechnical testing, the particle size of the soils showed variation in the amounts of silt and clay, as shown in Table ES-1. Although soils at each basin exhibit a range in type, CRSB soils were found to have the higher percentage of sands while PRSB and LRSB have higher clay contents. The smaller sized particles (silts and clays) are important, since particle size distribution appears to be important to the grout set process and may effect the hydraulic conductivity of the treated material.

The chemical and radiological characterization of the soils performed during this study showed that:

- Chlorides, nitrates, nitrites, sulfates, and sulfites are not present at levels which would have an inhibitory effect on the cementitious reactions during the curing of Portland cement.

- Cs-137 and Sr-90 were present at moderate levels in C-, K- and PRSB (174 - 2040 pCi/g of Cs-137 and 5-26 pCi/g of Sr-90)

- Pu-239/240 was present at low levels $(0.2-4.1 \mathrm{pCi} / \mathrm{g})$

- LRSB soils had low levels of radioactivity: $<2 \mathrm{pCi} / \mathrm{g}$ of Cs-137, $<0.4 \mathrm{pCi} / \mathrm{g}$ of Sr-90, and $<0.06 \mathrm{pCi} / \mathrm{g}$ of $\mathrm{Pu} 239 / 240$

Soils from each of the basins were mixed with each of the four grout mixes under evaluation. The treated material was tested to evaluate how effectively each formulation met the performance objectives. Tables ES-2 through ES-5 list the performance objectives and present the results of this testing for each basin by soil fraction. Tables ES-6 through ES-9 list the performance objectives and present the results by grout formulation for each soil fraction. Tables ES-10 and ES-11 show the average hydraulic conductivity values by grout formulation for each reactor seepage basin. Key findings of the performance testing are:

- Unconfined compressive strength (UCS) requirements were met by all mix formulations for all soil fractions. 
- The leach indices for all formulations met requirements and were at least 2 orders of magnitude above the performance requirement. This indicates the COCs are immobilized within the grout mix for all formulations for all soil fractions.

- Shrink/swell, volume increase, $\mathrm{pH}$, temperature rise, false set, and bleed fluid criteria were met by all formulations for all soil fractions at all reactor seepage basins.

- Set time met or exceeded the criterion for all formulations and all soil fractions, except for the LRSB formulations.

- For CRSB (16 soil/mix formulations), the hydraulic conductivity after 28 days of curing ranged from $6 \times 10^{-7}$ to $9 \times 10^{-9}$ centimeters $/$ second $(\mathrm{cm} / \mathrm{sec})$.

- For KRSB (eight soil/mix formulations), the hydraulic conductivity after 28 days of curing ranged from $2 \times 10^{-6}$ to $2 \times 10^{-9} \mathrm{~cm} / \mathrm{sec}$.

- For LRSB (eight soil/mix formulations), the hydraulic conductivity after 28 days of curing ranged from $3 \times 10^{-8}$ to $5 \times 10^{-10} \mathrm{~cm} / \mathrm{sec}$.

- For PRSB (16 soil/mix formulations), the hydraulic conductivity after 28 days of curing ranged from $6 \times 10^{-8}$ to $4 \times 10^{-10} \mathrm{~cm} / \mathrm{sec}$.

- Following water immersion for 90-days, the formulations for experienced a one to two order-of-magnitude increase in hydraulic conductivity:

CRSB ranged from $2 \times 10^{-5}$ to $5 \times 10^{-8} \mathrm{~cm} / \mathrm{sec}$

KRSB ranged from $1 \times 10^{-5}$ to $9 \times 10^{-7} \mathrm{~cm} / \mathrm{sec}$

LRSB ranged from $2 \times 10^{-6}$ to $9 \times 10^{-9} \mathrm{~cm} / \mathrm{sec}$

PRSB ranged from $3 \times 10^{-6}$ to $4 \times 10^{-7} \mathrm{~cm} / \mathrm{sec}$

As highlighted in Tables ES-2 through ES-9, with the exception of the hydraulic conductivity results and the set time for the LRSB, all performance criteria were met by each formulation for each soil fraction for all four reactor seepage basins. While at least one formulation for CRSB and KRSBs met the performance criterion for the 28-day hydraulic conductivity, the observed 28-day values did not consistently achieve a hydraulic conductivity of $1 \times 10^{-8} \mathrm{~cm} / \mathrm{sec}$. 
The hydraulic conductivity performance criterion was met by three of the formulations in every soil fraction for LRSB and PRSB. As shown in Table ES-10, the average hydraulic conductivity of at least one formulation met the performance criterion in KRSB, LRSB, and PRSB. For CRSB, the average hydraulic conductivity for all formulations was an order of magnitude higher than the performance criterion.

The 28-day hydraulic conductivity test results showed a large variability, with no clear pattern related to grout mix or soil fraction. The variability observed in the 28-day tests is attributed to the resolution and repeatability of the technique using these soil types. No additional testing of these grout mixes is recommended for CRSB, since the formulations meet all of the other desired criteria and there is no clear increase or decrease in hydraulic conductivity with any of the mix formulations.

Table ES-11 illustrates that none of the formulations for any soil fraction was able to achieve a hydraulic conductivity of $1 \times 10^{-8} \mathrm{~cm} / \mathrm{sec}$ after immersion in water for 90 days. The increase in hydraulic conductivity occurred in all soil types and all formulations.

Importantly, the 90-day immersion test is not representative of post treatment conditions at any of the reactor seepage basins. The true water table at each basin is well below the contaminated soils, and the perched zones that are now present at some of the basins will not be present after the soils are stabilized and the basins have been backfilled and sloped for proper drainage. Consequently, while the measured 90-day immersion hydraulic conductivity values do not meet the performance objectives, this test does not represent expected field conditions and should not form the basis for modification of formulations that fulfill the requirements of more representative field conditions.

The set time for all formulations for the LRSB soil fractions did not meet the performance criterion. A set retarder may be necessary during full-scale implementation at LRSB, and this should be determined during a pilot test prior to full-scale implementation.

With these qualified exceptions, the performance tests showed that all four mixes met all performance criteria. For those criteria that were not met, no clear correlation was found to suggest that these could be obtained without an adverse impact on other criteria. Therefore, reagent cost was used as the primary basis to select the recommended formulation. Based on the results of the bench scale tests a formation with $15 \%$ Portland cement and $5 \%$ bentonite by weight in the final treated material is recommended for the full-scale treatment of the CRSB, KRSB, LRSB, and PRSB soils. 
While the final detailed methodology will be determined by the successful contractor for the full scale work, the following discussion may provide useful insight into successful $\mathrm{S} / \mathrm{S}$ practices. To be effective, the grout mix must be thoroughly mixed with the contaminated soil. The clay content of the soils will require high speed, high shear mixing. A dual auger mixer would produce the high energy, high shear mixing required to adequate mix the grout and soils. Excavators or rake-like attachments on excavators would not produce the high energy, high shear mixing required. Jet-grouting has been effective in sandy soils, but has had inadequate mixing results in soils with higher clay contents. Therefore, the latter two delivery systems are not recommended. To ensure a complete grout monolith, the construction process should follow specific procedures:

- Multiple auger passes at each column should be made to completely mix the grout throughout the column. Two thirds of the grout volume should be injected during the initial downward pass and the remainder injected during the initial upward pass.

- An overlap between the columns is necessary to ensure the all of the contaminated soil is treated. The overlap volume should be between five and ten percent. Mapping of the column placement on the surface profile of each reactor seepage basin will verify that the overlap volume will be between five and ten percent.

- The auger position for each column should be precisely located by survey equipment and recorded to verify a consistent overlap of columns.

- A batch plant capable of providing an individual grout batch for each column or sets of columns should be used.

- The weights of Portland cement and bentonite and the volume of water should be recorded for each grout batch and controlled to within plus or minus one percent of the desired value as evaluated in the final bench scale study.

- The amount of water can be modified to adjust the viscosity of the grout to match the $\mathrm{S} / \mathrm{S}$ vendor's batching, grout pumping and mixing equipment.

- Each grout batch should be used within a maximum of 2 hours of batching. The results of the bench scale testing suggest that a retarder will not be nceded at CRSB, KRSB, or PRSB, but a retarder may be needed at LRSB. If a set retarder is used at any of the reactor seepage 
basins, the effect of the set retarder on the performance of the treated material should be determined during pilot testing.

- Temperature and soil moisture are both known to affect grout mixes as they set. The following guidance is provided for dealing with changing ambient conditions during fullscale implementation.

Modification of the $\mathrm{S} / \mathrm{S}$ treatment will not be required as long as the moisture content of the soil is greater than $10 \%(\mathrm{dwb})$. If the soil requires the addition of water to prevent drying and cracking of the grout-treated soil, water can be added directly to the soil or by adjusting the volume of water added to the grout. (Variability of soil moisture content within a 11 to $20 \%$ range had little to no effect on the $\mathrm{S} / \mathrm{S}$ testing results.)

The core and surface temperatures of the treated material should be monitored to determine temperature rise during full-scale treatment and compared with predictions. The S/S temperature, temperature gradients, and rate of temperature change should remain within final grout design parameters to prevent cracking of the grouted soil. Common industrial practice is to control the rate of temperature change and keep the surface temperature within final grout design parameters to minimize cracking of the grouted soil. If higher temperatures are encountered, additional water may be added to the grout mix to serve as a heat sink and other temperature control means or methods may be used.

Field operations should cease when temperatures fall below $32^{\circ} \mathrm{F}$.

Ambient temperatures above $100^{\circ} \mathrm{F}$ may accelerate the reaction rates and ruin the final product and may affect the viscosity and pumpability of the grout. Additional water or a set retarder may be added to the grout mix to decrease the viscosity and increase the pumpability of the grout.

- To meet unique vendor requirements, limited field refinement of the grout mix may be done to adapt the grout mix to the batching, grout pumping, and mixing equipment. This should be limited only to the addition of water or the use of a set retarder.

- Additional water can be added only to account for low soil moisture content, to lower the temperature rise of grouted soil, or to decrease the grout viscosity.

- A set retarder may be used to prolong the time between batching and injection of the grout mix or to decrease the viscosity of the grout. If a set retarder is used, field pilot testing 
should be conducted to determine the effect of the set retarder on the performance of the treated material.

The daily system operation cost for the S/S treatment of a cubic yd of soil is estimated to be approximately $\$ 115$, based on daily production rates for a single auger system. The estimated unit cost does not include fixed project costs nor ancillary costs associated with the project, such as air permits for the batch plant, administrative and support costs (site preparation, administrative staff, etc.), mobilization and demobilization, analytical costs for treated material and grout mix samples, nor any contingency for project uncertainties. The estimate was compared to unit costs contained in Environmental Cost Handling Options and Solutions (ECHOS) by R. S. Means. While direct comparison was not possible; units costs for equipment, labor, and material in ECHOS were comparable to those used to develop the above estimate. 
Soil Stabilization/Solidification Grout Mix Design Study WSRC-RP-99-4075. Revision 1 C-, K-, L-, and P-Reactor Seepage Basins Closure (U)

Table ES-1. General Particle Size Distribution

\begin{tabular}{|l|c|c|c|c|}
\hline \multicolumn{1}{|c|}{ Location } & Gravel & Sand & Silt & Clay \\
\hline CRSB & $4 \%$ & $75 \%$ & $6 \%$ & $15 \%$ \\
\hline KRSB & $1 \%$ & $70 \%$ & $11 \%$ & $15 \%$ \\
\hline LRSB & $2 \%$ & $67 \%$ & $10 \%$ & $21 \%$ \\
\hline PRSB & $4 \%$ & $68 \%$ & $8 \%$ & $20 \%$ \\
\hline
\end{tabular}


ES-2. CRSB Data Summary by Soil Fractions

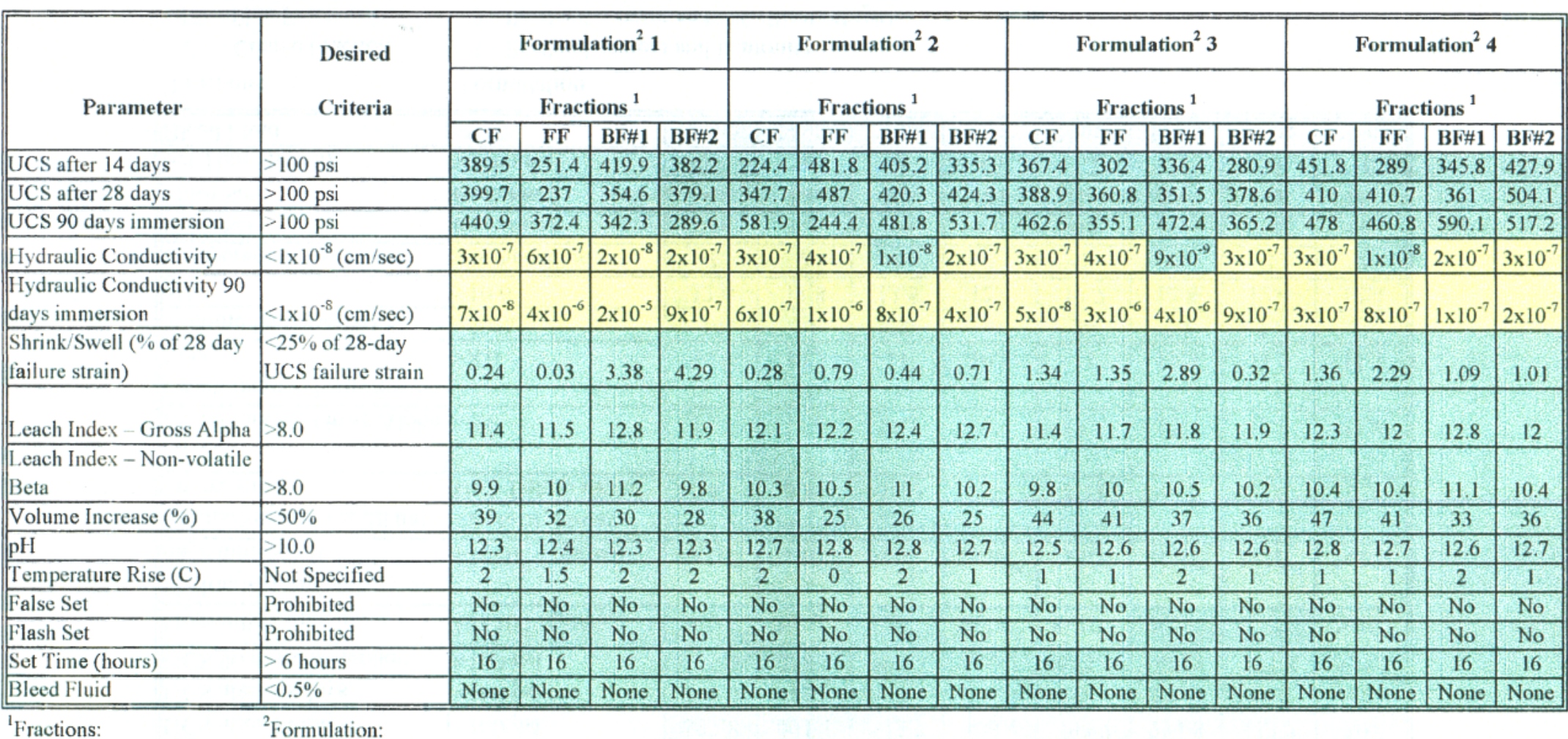

l'Frations:

${ }^{2}$ Formulation:

$\mathrm{CF}$ - Coarse Fraction . 1 - Portland cement and bentonite

FF - Fine Fraction 2 - Portland cement and $4 \%$ silicate (high)

BF\#1 - Bulk Fraction \#1 3 - Portland cement, bentonite, and $2 \%$ silicate (low)

BF\#2 - Bulk Fraction \#2 4 - Portland cement, bentonite, and $4 \%$ silicate (high)

nd - Not determined

Meets the performance criterion

Does not meet the performance criterion 


\section{ES-3. KRSB Data Summary by Soil Fractions}

\begin{tabular}{|c|c|c|c|c|c|c|c|c|c|c|}
\hline \multirow{3}{*}{ Parameter } & \multirow{3}{*}{$\begin{array}{l}\text { Desired } \\
\text { Criteria }\end{array}$} & \multicolumn{2}{|c|}{ Formulation ${ }^{2} 1$} & \multicolumn{2}{|c|}{ Formulation 2} & \multicolumn{2}{|c|}{ Formulation ${ }^{2} 3$} & \multicolumn{2}{|c|}{ Formulation $^{2} 4$} & \\
\hline & & \multicolumn{2}{|c|}{ Fractions $^{1}$} & \multicolumn{2}{|c|}{ Fractions $^{1}$} & \multicolumn{2}{|c|}{ Fractions $^{1}$} & \multicolumn{2}{|c|}{ Fractions $^{1}$} & \\
\hline & & CF & FF & CF & FF & $\mathbf{C F}$ & FF & CF & FF & \\
\hline UCS after 14 days & $>100 \mathrm{psi}$ & 537.8 & 401.1 & 512.7 & 403.4 & 493.7 & 274.8 & 412.2 & 307 & \\
\hline UCS after 28 days & $>100 \mathrm{psi}$ & 500.7 & 412.2 & 619.1 & 372.4 & 532.7 & 318.3 & 541 & 462.2 & \\
\hline UCS 90 days immersion & $>100 \mathrm{psi}$ & 539.5 & 258.1 & 593.3 & 410.2 & 448.4 & 333.5 & 477.8 & 525.3 & \\
\hline \begin{tabular}{|l|} 
Hydraulic Conductivity \\
\end{tabular} & $<1 \times 10^{-8}(\mathrm{~cm} / \mathrm{sec})$ & $1 \times 10^{-8}$ & $2 \times 10^{-8}$ & $3 \times 10^{-9}$ & $9 \times 10^{-7}$ & $8 \times 10^{-7}$ & $2 \times 10^{-9}$ & $3 \times 10^{-9}$ & $2 \times 10^{-6}$ & \\
\hline \begin{tabular}{|l|} 
Hydraulic Conductivity 90 \\
days immersion
\end{tabular} & $<1 \times 10^{-8}(\mathrm{~cm} / \mathrm{sec})$ & $4 \times 10^{-6}$ & $3 \times 10^{-6}$ & $9 \times 10^{-7}$ & $1 \times 10^{-6}$ & $1 \times 10^{-6}$ & $4 \times 10^{-6}$ & $1 \times 10^{-5}$ & $3 \times 10^{-6}$ & \\
\hline $\begin{array}{l}\text { Shrink/Swell (\% of } 28 \text { day } \\
\text { failure strain) }\end{array}$ & $\begin{array}{l}<25 \% \text { of } 28 \text {-day } \\
\text { UCS failure strain } \\
\end{array}$ & 0.64 & 0.39 & $<0.01$ & 0.34 & 4.11 & $<0,01$ & 1.06 & 0.2 & \\
\hline Leach Index - Gross Alpha & $>8.0$ & 11.7 & 11.6 & 12.1 & 12 & 12.6 & 12.3 & 12.9 & 12.4 & \\
\hline $\begin{array}{l}\text { Leach Index - Non-volatile } \\
\text { Beta }\end{array}$ & $>8.0$ & 10.9 & 10.2 & 11 & 10.6 & 11 & 10.5 & 11.5 & 11.1 & \\
\hline \begin{tabular}{|l|} 
Volume Increase (\%) \\
\end{tabular} & $<50 \%$ & 29 & 24 & 28 & 20 & 36 & 30 & 39 & 30 & \\
\hline $\mathrm{pH}$ & $>10.0$ & 12.6 & 12.8 & 12.5 & 12.6 & 12.7 & 12.8 & 12.4 & 12.4 & \\
\hline Temperature Rise (C) & Not Specified & 2 & 2 & 1 & 2 & 2 & 2 & 2 & 2 & \\
\hline False Set & Prohibited & No & No & No & No & No & No & No & No & \\
\hline Flash Set & Prohibited & No & No & No & No & No & No & No & No & \\
\hline Set Time (hours) & $>6$ hours & 16 & 16 & 16 & 16 & 16 & 16 & 16 & 16 & \\
\hline Bleed Fluid & $<0.5 \%$ & None & None & None & None & None & None & None & None & \\
\hline Fractions: & \multicolumn{9}{|l|}{${ }^{2}$ Formulation: } & \\
\hline $\mathrm{CF}$ - Coarse Fraction & & \\
\hline FF - Fine Fraction & & & & & & & & & 2 - Portland cement and $4 \%$ silicate (high) & \\
\hline BF\#1 - Bulk Fraction \#1 & \multicolumn{9}{|c|}{3 - Portland cement, bentonite, and $2 \%$ silicate (low) } & \\
\hline $\begin{array}{l}\text { BF\#2 - Bulk Fraction \#2 } \\
\text { nd - Not determined }\end{array}$ & \multicolumn{9}{|c|}{4 - Portland cement, bentonite, and $4 \%$ silicate (high) } & \\
\hline $\begin{array}{l}\text { Meets the perform } \\
\text { Does not meet the }\end{array}$ & $\begin{array}{l}\text { ance criterion } \\
\text { performance criteri }\end{array}$ & & & & & & & & & : \\
\hline
\end{tabular}


ES-4. PRSB Data Summary by Soil Fractions

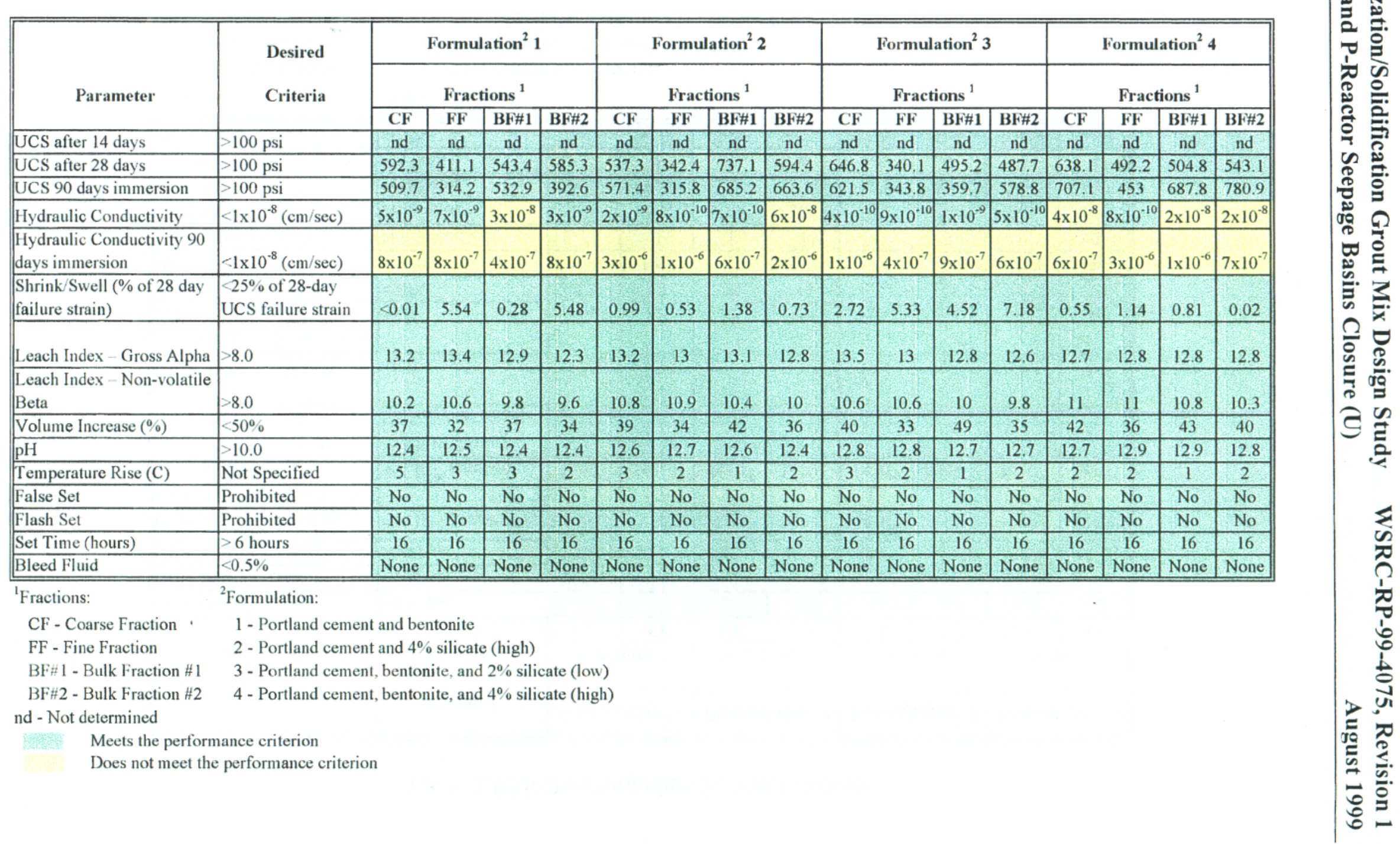




\section{ES-5. LRSB Data Summary by Soil Fractions}

\begin{tabular}{|c|c|c|c|c|c|c|c|c|c|}
\hline \multirow{3}{*}{ Parameter } & \multirow{3}{*}{$\begin{array}{l}\text { Desired } \\
\text { Criteria }\end{array}$} & \multicolumn{2}{|c|}{ Formulation $^{2} 1$} & \multicolumn{2}{|c|}{ Formulation $^{2} 2$} & \multicolumn{2}{|c|}{ Formulation $^{2} 3$} & \multicolumn{2}{|c|}{ Formulation $^{2} 4$} \\
\hline & & \multicolumn{2}{|c|}{ Fractions $^{1}$} & \multicolumn{2}{|c|}{ Fractions $^{1}$} & \multicolumn{2}{|c|}{ Fractions $^{1}$} & \multicolumn{2}{|c|}{ Fractions $^{1}$} \\
\hline & & CF & FF & CF & FF & CF & FF & CF & FF \\
\hline UCS after 14 days & $>100$ psi & nd & nd & nd & nd & nd & nd & nd & nd \\
\hline UCS after 28 days & $>100 \mathrm{psi}$ & 473.1 & 490.6 & 639.6 & 376.6 & 607.6 & 417.5 & 509.6 & 546,6 \\
\hline UCS 90 days immersion & $>100 \mathrm{psi}$ & 613 & 479.9 & 982.5 & 461.6 & 426.9 & 499.3 & 697 & 459.8 \\
\hline Hydraulic Conductivity & $<1 \times 10^{-8}(\mathrm{~cm} / \mathrm{sec})$ & $9 \times 10^{-9}$ & $7 \times 10^{-10}$ & $3 \times 10^{-9}$ & $7 \times 10^{-9}$ & $3 \times 10^{-9}$ & $5 \times 10^{-10}$ & $3 \times 10^{-8}$ & $2 \times 10^{-9}$ \\
\hline $\begin{array}{l}\text { Hydraulic Conductivity } 90 \\
\text { days immersion }\end{array}$ & $<1 \times 10^{-8}(\mathrm{~cm} / \mathrm{sec})$ & $1 \times 10^{-6}$ & $1 \times 10^{-6}$ & $2 \times 10^{-6}$ & $2 \times 10^{-7}$ & $1 \times 10^{-6}$ & $9 \times 10^{-9}$ & $2 \times 10^{-6}$ & $2 \times 10^{-8}$ \\
\hline $\begin{array}{l}\text { Shrink/Swell (\% of } 28 \text { day } \\
\text { failure strain) }\end{array}$ & $\begin{array}{l}<25 \% \text { of } 28 \text {-day } \\
\text { UCS failure strain } \\
\end{array}$ & 0.22 & 2.32 & 0.75 & 0.1 & 4.13 & 1,98 & 1.12 & 1.07 \\
\hline Leach Index - Gross Alpha & $>8.0$ & 12.6 & 12.8 & 13.1 & 13.1 & 12.4 & 13 & 12.4 & 13.1 \\
\hline $\begin{array}{l}\text { Leach Index - Non-volatile } \\
\text { Beta }\end{array}$ & $>8.0$ & nd & nd & nd & nd & nd & nd & nd & nd \\
\hline Volume Increase (\%) & $<50 \%$ & 33 & 33 & 36 & 34 & 34 & 33 & 40 & 37 \\
\hline $\mathrm{pH}$ & $>10.0$ & 12.4 & 12.5 & 12.7 & 12.6 & 12.7 & 12.6 & 12.8 & 12.9 \\
\hline Temperature Rise (C) & Not Specified & 3 & 3 & 1 & 1 & 1 & 2 & 0 & 2 \\
\hline False Set & Prohibited & No & No & No & No & No & No & No & No \\
\hline Flash Set & Prohibited & Yes & Yes & Yes & Yes & Yes & Yes & Yes & Yes \\
\hline Set Time (hours) & $>6$ hours & 4 & 4 & 4 & 4 & 4 & 4 & 4 & 4 \\
\hline Bleed Fluid & $<0.5 \%$ & None & None & None & None & None & None & None & None \\
\hline
\end{tabular}

${ }^{1}$ Fractions:

${ }^{2}$ Formulation:

CF - Coarse Fraction $\quad 1$ - Portland cement and bentonite

FF - Fine Fraction 2 - Portland cement and $4 \%$ silicate (high)

BF\# 1 - Bulk Fraction \# $1 \quad 3$ - Portland cement, bentonite, and 2\% silicate (low)

BF\#2 - Bulk Fraction \#2 4 - Portland cement, bentonite, and 4\% silicate (high)

nd - Not determined

Note: Leach Index for nonvolatile beta not determined since beta activities in LRSB soils were below the detection limit Meets the performance criterion

Does not meet the performance criterion 


\section{ES-6. CRSB Data Summary by Formulations}

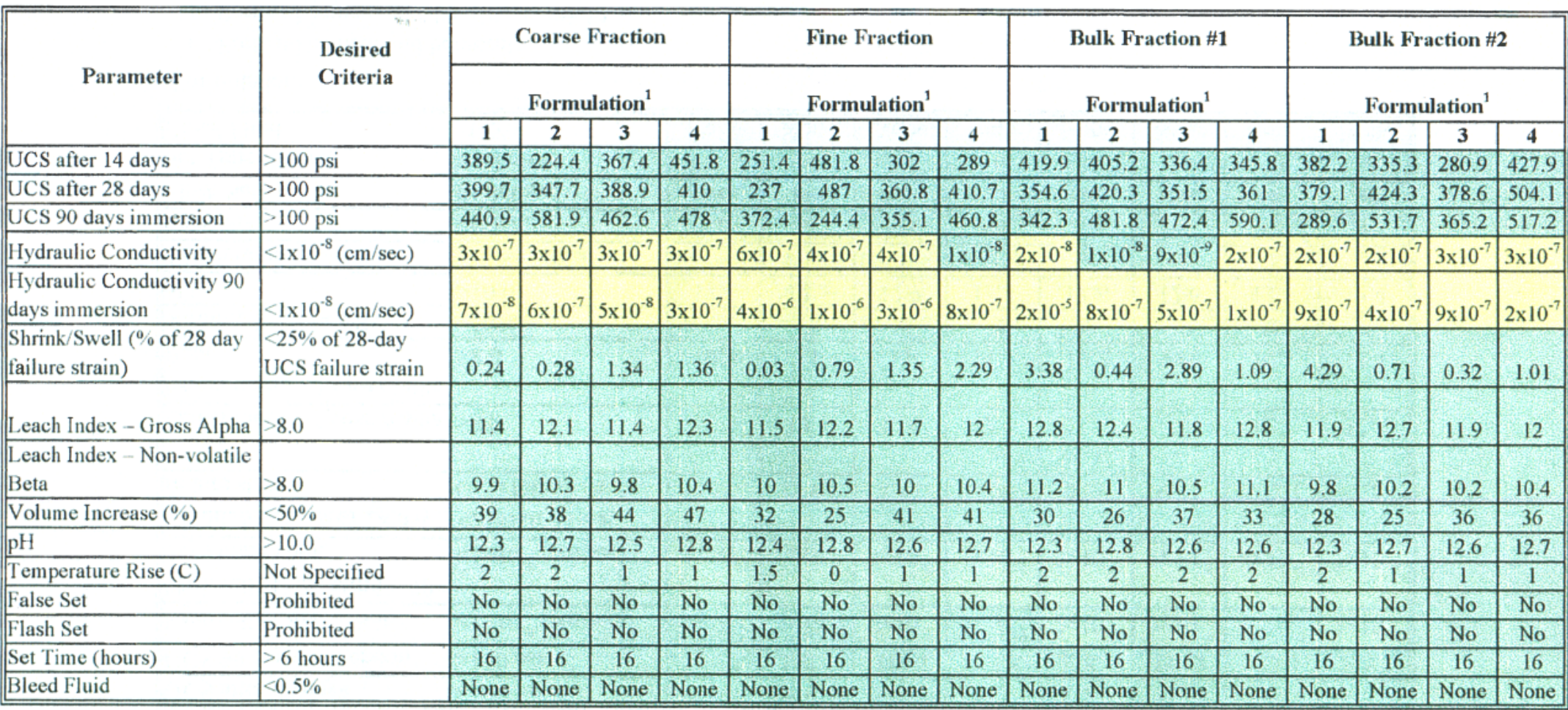

${ }^{1}$ Formulation:

1 - Portland cement and bentonite

2 - Portland cement and $4 \%$ silicate (high)

3 - Portland cement, bentonite, and 2\% silicate (low)

4 - Portland cement, bentonite, and $4 \%$ silicate (high)

nd - Not determined

Meets the performance criterion

Does not meet the performance criterion 


\section{ES-7. KRSB Data Summary by Formulations}

\begin{tabular}{|c|c|c|c|c|c|c|c|c|c|}
\hline \multirow{3}{*}{ Parameter } & \multirow{3}{*}{$\begin{array}{l}\text { Desired } \\
\text { Criteria }\end{array}$} & \multicolumn{4}{|c|}{ Coarse Fraction } & \multicolumn{4}{|c|}{ Fine Fraction } \\
\hline & & \multicolumn{4}{|c|}{ Formulation $^{1}$} & \multicolumn{4}{|c|}{ Formulation $^{1}$} \\
\hline & & 1 & 2 & 3 & 4 & 1 & 2 & 3 & 4 \\
\hline UCS after 14 days & $>100 \mathrm{psi}$ & 537.8 & 512.7 & 493.7 & 412.2 & 401.1 & 403.4 & 274.8 & 307 \\
\hline UCS after 28 days & $>100 \mathrm{psi}$ & 500.7 & 619.1 & 532.7 & 541 & 412.2 & 372.4 & 318.3 & 462.2 \\
\hline UCS 90 days immersion & $>100 \mathrm{psi}$ & 539.5 & 593.3 & 448.4 & 477.8 & 258.1 & 410.2 & 333.5 & 525.3 \\
\hline Hydraulic Conductivity & $<1 \times 10^{-8}(\mathrm{~cm} / \mathrm{sec})$ & $1 \times 10^{-8}$ & $3 \times 10^{-9}$ & $8 \times 10^{-7}$ & $3 \times 10^{-9}$ & $2 \times 10^{-8}$ & $9 \times 10^{-7}$ & $2 \times 10^{-9}$ & $2 \times 10^{-6}$ \\
\hline $\begin{array}{l}\text { Hydraulic Conductivity } 90 \\
\text { days immersion }\end{array}$ & $<1 \times 10^{-8}(\mathrm{~cm} / \mathrm{sec})$ & $4 \times 10^{-6}$ & $9 \times 10^{-7}$ & $1 \times 10^{-6}$ & $1 \times 10^{-5}$ & $3 \times 10^{-6}$ & $1 \times 10^{-6}$ & $4 \times 10^{-6}$ & $3 \times 10^{-6}$ \\
\hline $\begin{array}{l}\text { Shrink/Swell (\% of } 28 \text { day } \\
\text { failure strain) }\end{array}$ & $\begin{array}{l}<25 \% \text { of } 28 \text {-day } \\
\text { UCS failure strain }\end{array}$ & 0.64 & $<0.01$ & 4.11 & 1.06 & 0.39 & 0.34 & $<0.01$ & 0.2 \\
\hline Leach Index - Gross Alpha & $>8.0$ & 11.7 & 12.1 & 12.6 & 12.9 & 11.6 & 12 & 12.3 & 12.4 \\
\hline $\begin{array}{l}\text { Leach Index - Non-volatile } \\
\text { Beta }\end{array}$ & $>8.0$ & 10.9 & 11 & 11 & 11.5 & 10.2 & 10.6 & 10.5 & 11.1 \\
\hline \begin{tabular}{|l} 
Volume Increase (\%) \\
\end{tabular} & $<50 \%$ & 29 & 28 & 36 & 39 & 24 & 20 & 30 & 30 \\
\hline $\mathrm{pH}$ & $>10.0$ & 12.6 & 12.5 & 12.7 & 12.4 & 12.8 & 12.6 & 12.8 & 12.4 \\
\hline Temperature Rise (C) & Not Specified & 2 & 1 & 2 & 2 & 2 & 2 & 2 & 2 \\
\hline False Set & Prohibited & No & No & No & No & No & No & No & No \\
\hline Flash Set & Prohibited & No & No & No & No & No & No & No & No \\
\hline Set Time (hours) & $>6$ hours & 16 & 16 & 16 & 16 & 16 & 16 & 16 & 16 \\
\hline Bleed Fluid & $<0.5 \%$ & None & None & None & None & None & None & None & None \\
\hline
\end{tabular}

${ }^{1}$ Formulation:

1 - Portland cement and bentonite

2 - Portland cement and $4 \%$ silicate (high)

3 - Portland cement, bentonite, and 2\% silicate (low)

4 - Portland cement, bentonite, and 4\% silicate (high) nd - Not determined

Meets the performance criterion

Does not meet the performance criterion 


\section{ES-8. PRSB Data Summary by Formulations}

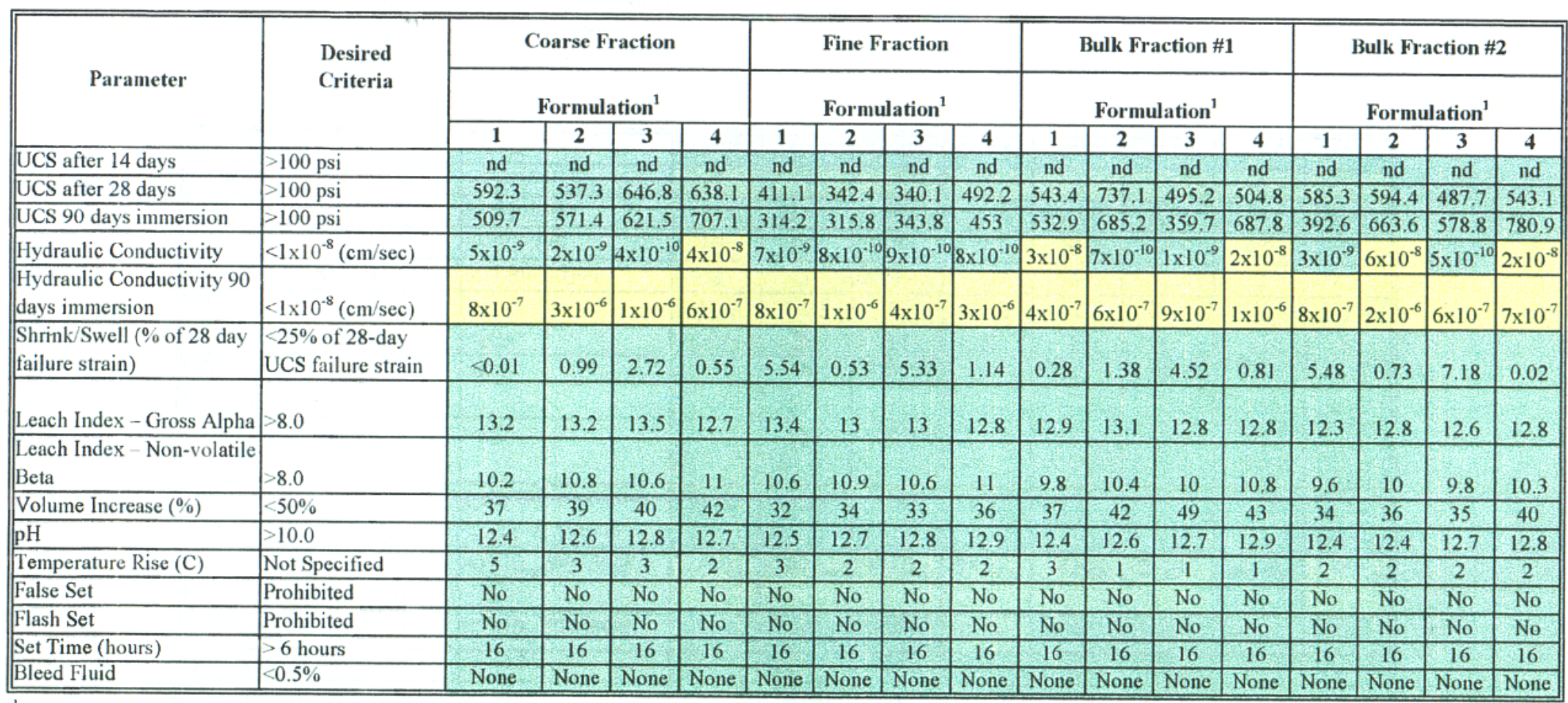

${ }^{1}$ Formulation:

1 - Portland cement and bentonite

2 - Portland cement and $4 \%$ silicate (high)

3 - Portland cement, bentonite, and $2 \%$ silicate (low)

4 - Portland cement, bentonite, and $4 \%$ silicate (high) nd - Not determined

Meets the performance criterion

Does not meet the performance criterion 


\section{ES-9. LRSB Data Summary by Formulations}

\begin{tabular}{|c|c|c|c|c|c|c|c|c|c|}
\hline \multirow{3}{*}{ Parameter } & \multirow{3}{*}{$\begin{array}{l}\text { Desired } \\
\text { Criteria }\end{array}$} & \multicolumn{4}{|c|}{ Coarse Fraction } & \multicolumn{4}{|c|}{ Fine Fraction } \\
\hline & & \multicolumn{4}{|c|}{ Formulation $^{1}$} & \multicolumn{4}{|c|}{ Formulation $^{1}$} \\
\hline & & 1 & 2 & 3 & 4 & 1 & 2 & 3 & 4 \\
\hline UCS after 14 days & $>100 \mathrm{psi}$ & nd & nd & nd & nd & nd & nd & nd & nd \\
\hline UCS after 28 days & $>100 \mathrm{psi}$ & 473.1 & 639.6 & 607.6 & 509.6 & 490.6 & 376.6 & 417.5 & 546.6 \\
\hline UCS 90 days immersion & $>100 \mathrm{psi}$ & 613 & 982.5 & 426.9 & 697 & 479.9 & 461.6 & 499.3 & 459.8 \\
\hline Hydraulic Conductivity & $<1 \times 10^{-8}(\mathrm{~cm} / \mathrm{sec})$ & $9 \times 10^{-9}$ & $3 \times 10^{-9}$ & $3 \times 10^{-9}$ & $3 \times 10^{-8}$ & $7 \times 10^{-10}$ & $7 \times 10^{-9}$ & $5 \times 10^{-10}$ & $2 \times 10^{-9}$ \\
\hline $\begin{array}{l}\text { Hydraulic Conductivity } 90 \\
\text { days immersion }\end{array}$ & $<1 \times 10^{-8}(\mathrm{~cm} / \mathrm{sec})$ & $1 \times 10^{-6}$ & $2 \times 10^{-6}$ & $1 \times 10^{-6}$ & $2 \times 10^{-6}$ & $1 \times 10^{-6}$ & $2 \times 10^{-7}$ & $9 \times 10^{-9}$ & $2 \times 10^{-8}$ \\
\hline $\begin{array}{l}\text { Shrink/Swell ( } \% \text { of } 28 \text { day } \\
\text { failure strain) }\end{array}$ & $\begin{array}{l}<25 \% \text { of } 28 \text {-day } \\
\text { UCS failure strain }\end{array}$ & 0.22 & 0.75 & 4.13 & 1.12 & 2.32 & 0.1 & 1.98 & 1.07 \\
\hline Leach Index - Gross Alpha & $>8.0$ & 12.6 & 13.1 & 12.4 & 12.4 & 12.8 & 13.1 & 13 & 13.1 \\
\hline $\begin{array}{l}\text { Leach Index - Non-volatile } \\
\text { Beta }\end{array}$ & $>8.0$ & nd & nd & nd & nd & nd & nd & nd & nd \\
\hline Volume Increase (\%) & $<50 \%$ & 33 & 36 & 34 & 40 & 33 & 34 & 33 & 37 \\
\hline $\mathrm{pH}$ & $>10.0$ & 12.4 & 12.7 & 12.7 & 12.8 & 12.5 & 12.6 & 12.6 & 12.9 \\
\hline Temperature Rise (C) & Not Specified & 3 & 1 & 1 & 0 & 3 & 1 & 2 & 2 \\
\hline False Set & Prohibited & No & No & No & No & $\mathrm{No}$ & No & No & No \\
\hline Flash Set & Prohibited & Yes & Yes & Yes & Yes & Yes & Yes & Yes & Yes \\
\hline Set Time (hours) & $>6$ hours & 4 & 4 & 4 & 4 & 4 & 4 & 4 & 4 \\
\hline Bleed Fluid & $<0.5 \%$ & None & None & None & None & None & None & None & None \\
\hline
\end{tabular}

'Formulation:

1 - Portland cement and bentonite

2 - Portland cement and $4 \%$ silicate (high)

3 - Portland cement, bentonite, and $2 \%$ silicate (low)

4 - Portland cement, bentonite, and 4\% silicate (high)

nd - Not determined

Note: Leach Index for nonvolatile beta not determined since beta activities in LRSB soils were below the detection limit Meets the performance criterion

Does not meet the performance criterion 
Table ES-10. Average Hydraulic Conductivity by Formulation All Soil Fractions - 28-Day Cure

\begin{tabular}{||c|c|c|c|c||}
\hline \multirow{2}{*}{$\begin{array}{c}\text { Seepage } \\
\text { Basin }\end{array}$} & \multicolumn{4}{|c||}{ Average Hydraulic Conductivity (cm/sec) } \\
\cline { 2 - 5 } & Formulation $^{\mathbf{1}}$ & Formulation $^{2}$ & Formulation $^{2}$ & Formulation $^{2}$ \\
& $\mathbf{1}$ & $\mathbf{2}$ & $\mathbf{3}$ & $\mathbf{4}$ \\
\hline CRSB & $2 \mathrm{E}-07$ & $1 \mathrm{E}-07$ & $1 \mathrm{E}-07$ & $1 \mathrm{E}-07$ \\
\hline KRSB & $1 \mathrm{E}-08$ & $5 \mathrm{E}-08$ & $4 \mathrm{E}-08$ & $8 \mathrm{E}-08$ \\
\hline PRSB & $7 \mathrm{E}-09$ & $3 \mathrm{E}-09$ & $7 \mathrm{E}-10$ & $1 \mathrm{E}-08$ \\
\hline LRSB & $3 \mathrm{E}-09$ & $5 \mathrm{E}-09$ & $1 \mathrm{E}-09$ & $8 \mathrm{E}-09$ \\
\hline
\end{tabular}

${ }^{1}$ Logarithmic average to the base 10

${ }^{2}$ Formulation:

1 - Portland cement and bentonite

2 - Portland cement and $4 \%$ silicate (high)

3 - Portland cement, bentonite, and $2 \%$ silicate (low)

4 - Portland cement, bentonite, and $4 \%$ silicate (high)

Meets the performance criterion

Does not meet the performance criterion

Table ES-11. Average Hydraulic Conductivity by Formulation All Soil Fractions - 28-Day Cure, 90-Day Immersion

\begin{tabular}{||c|c|c|c|c||}
\hline \multirow{2}{*}{$\begin{array}{c}\text { Seepage } \\
\text { Basin }\end{array}$} & \multicolumn{4}{|c||}{ Average Hydraulic Conductivity (cm/sec) } \\
\cline { 2 - 5 } & $\begin{array}{c}\text { Formulation } \\
\text { 1 }\end{array}$ & Formulation $^{\mathbf{1}}$ & Formulation $^{2}$ & Formulation $^{2}$ \\
& $\mathbf{2}$ & $\mathbf{3}$ & $\mathbf{4}$ \\
\hline CRSB & $1 \mathrm{E}-06$ & $7 \mathrm{E}-07$ & $5 \mathrm{E}-07$ & $3 \mathrm{E}-07$ \\
\hline KRSB & $3 \mathrm{E}-06$ & $9 \mathrm{E}-07$ & $2 \mathrm{E}-06$ & $5 \mathrm{E}-06$ \\
\hline PRSB & $7 \mathrm{E}-07$ & $1 \mathrm{E}-06$ & $7 \mathrm{E}-07$ & $1 \mathrm{E}-06$ \\
\hline LRSB & $1 \mathrm{E}-06$ & $6 \mathrm{E}-07$ & $9 \mathrm{E}-08$ & $2 \mathrm{E}-07$ \\
\hline
\end{tabular}

${ }^{1}$ Logarithmic average to the base 10

${ }^{2}$ Formulation:

1 - Portland cement and bentonite

2 - Portland cement and $4 \%$ silicate (high)

3 - Portland cement, bentonite, and $2 \%$ silicate (low)

4 - Portland cement, bentonite, and $4 \%$ silicate (high)

Does not meet the performance criterion 
This page intentionally left blank. 


\section{TABLE OF CONTENTS}

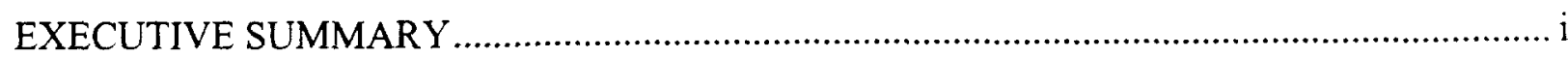

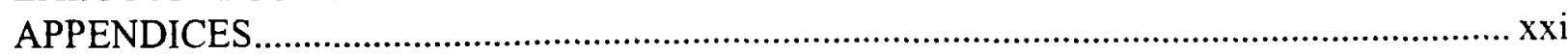

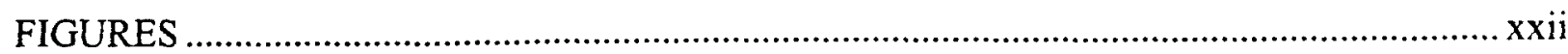

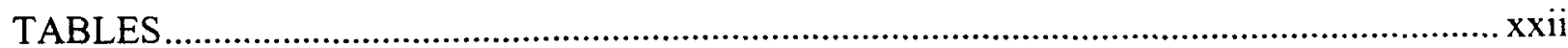

ACRONYMS

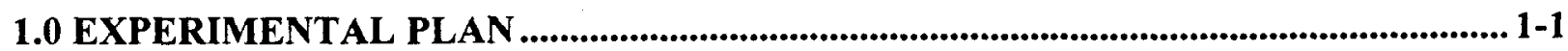

1.1 Project Description........................................................................................... 1-1

1.1.1 Background .............................................................................................. $1-1$

1.1.2 C-Reactor Seepage Basin........................................................................... 1-2

1.1.3 K-Reactor Seepage Basin........................................................................... 1-2

1.1.4 L-Reactor Seepage Basin ........................................................................... 1-3

1.1.5 P-Reactor Seepage Basin .................................................................................. 1-3

1.1.6 Related Immobilization Investigation at L-Area Oil and Chemical Basin .......... 1-3

1.2 Technical Approach .......................................................................................... 1-5

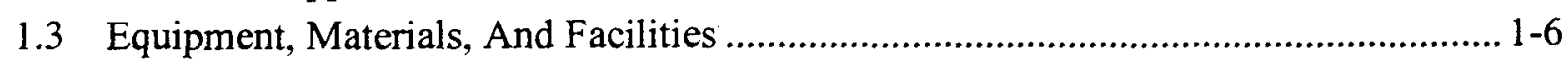

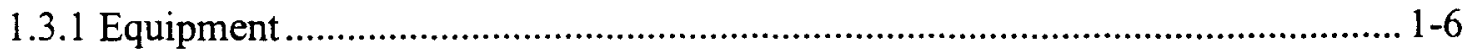

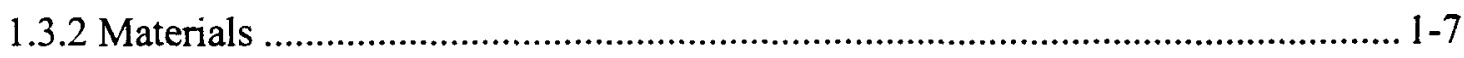

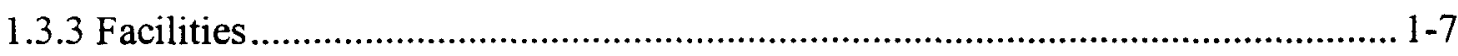

1.4 Sample Transportation And Tracking.................................................................... 1-7

1.4.1 Sample Transportation ................................................................... 1-7

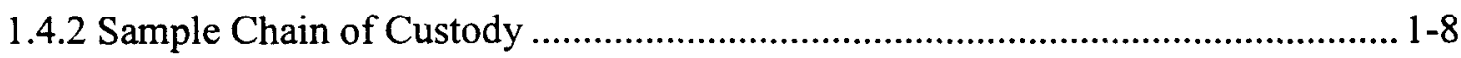

1.4.3 Sample Labeling ........................................................................................ 1-8

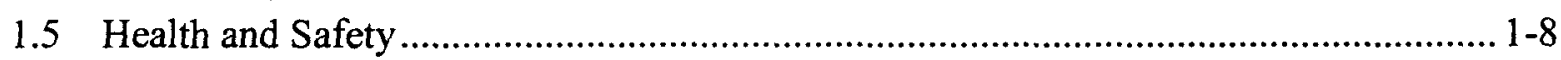

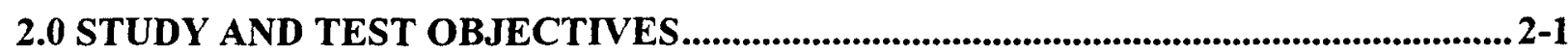

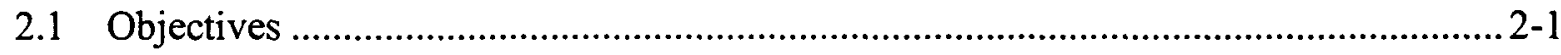

2.2 Study Performance Standards .............................................................................. 2-1

3.0 TEST OBSERVATIONS AND RESULTS ...............................................................

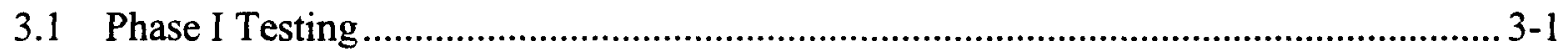

3.1.1 Treatability Testing on CRSB Soils................................................................ 3-1

3.1.2 Treatability Testing on KRSB Soils ......................................................... 3-2

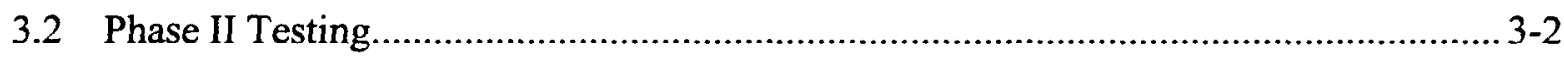

3.2.1 Treatability Testing on PRSB Soils ................................................................ 3-2

3.2.2 Treatability Testing on LRSB Soils ............................................................... 3-3

3.3 Grout Mix Design ........................................................................................ 3-3 


\section{TABLE OF CONTENTS (Continued)}

3.4 Stabilization Formulation Development .................................................................... 3-4

3.5 Testing Results.

3.5.1 Soil Characterization................................................................................. 3-4

3.5.2 Treated Material Performance Testing …………............................................. 3-5

4.0 DATA ANALYSIS AND INTERPRETATION .................................................................. 4-1

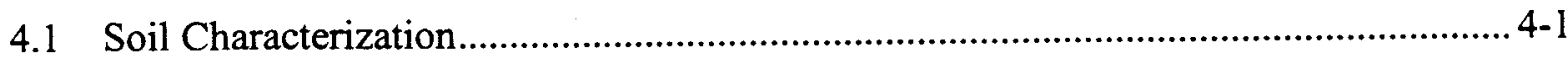

4.1.1 CRSB Soil Fractions.....................................................................................

4.1.2 KRSB Soil Fractions.............................................................................. 4-2

4.1.3 PRSB Soil Fractions .................................................................................. 4-3

4.1.4 LRSB Soil Fractions ................................................................................ 4-4

4.2 Treated Material Performance Testing ……….......................................................... 4-5

4.2.1 Treated Material Performance Testing ................................................................ 4-5

5.0 CONCLUSIONS AND RECOMMENDATIONS............................................................ 5-1

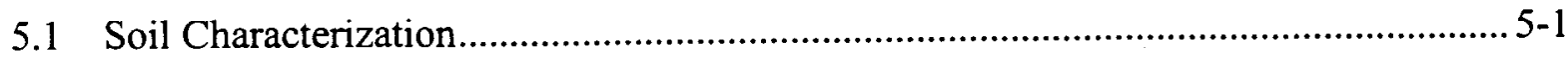

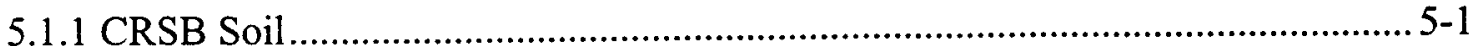

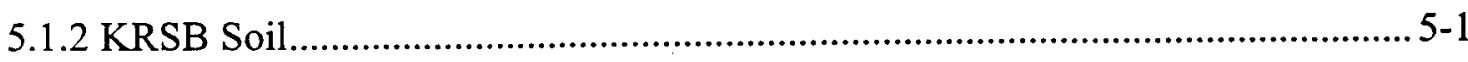

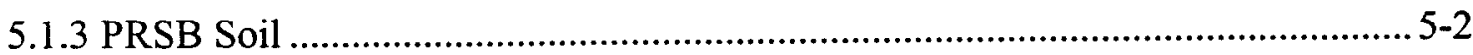

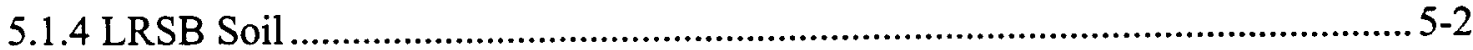

5.2 Stabilization/Solidification Testing ........................................................................ 5-2

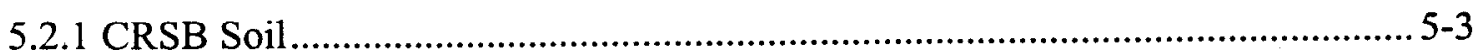

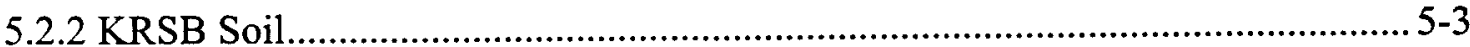

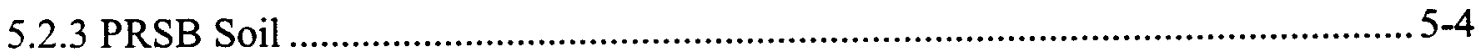

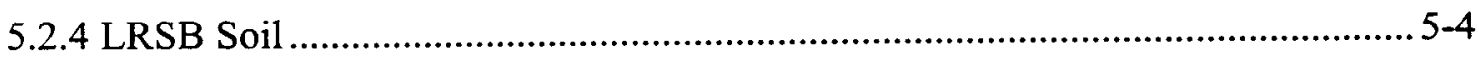

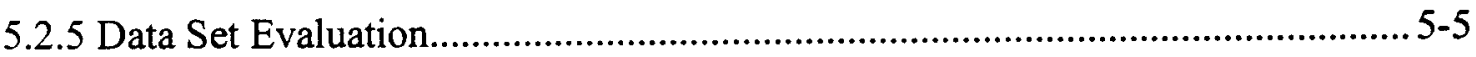

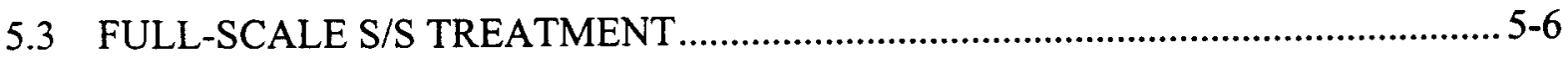

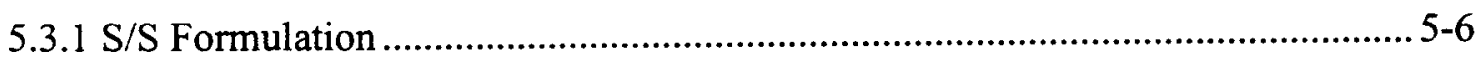

5.3.2 Process Mixing Constraints ......................................................................... 5-10

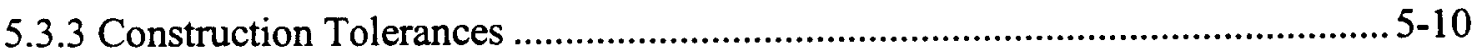

5.4 Estimated Construction Unit Cost .......................................................................... 5-13 


\section{APPENDICES}

Appendix A

Geotechnical Testing Results for the C-, K-, L-, and PRSB

Attachment A

Sample Number Cross-Reference List

Attachment B

Analysis Results

CRSB and KRSB Soil Geotechnical Results

Moisture Content

Atterberg Limits

Specific Gravity

Soil Classification

Particle Size Analysis

14-Day Unconfined Compressive Strength (UCS)

28-Day UCS

28-Day Cure, 90-Day Immersion UCS

28-Day Expansion/Shrinkage

28-Day Permeability

90-Day Immersion Permeability

Leach Index

LRSB and PRSB Soil Geotechnical Results

Moisture Content

Specific Gravity

Atterberg Limits

Soil Classification

Particle Size Analysis

28-Day UCS

28-Day Cure, 90-Day Immersion UCS

28-Day Expansion/Shrinkage

28-Day Permeability

90-Day Immersion Permeability

Leach Index

\section{Attachment $C$}

Chain-of-Custody \& Request-for-Analysis Records Attachment D

Variance Reports

Appendix B

Sampling Notes

Appendix C

CSRB Sampling Map 


\section{APPENDICES (Continued)}

Appendix D

Test Procedures

Appendix E

Analytical Methods

Appendix $F$

Laboratory Health and Safety

Appendix G

Stabilization/Solidification Additives

\section{FIGURES}

Figure 3-1. Flowchart for the Segregation of the Reactor Seepage Basin Soils 3-6

Figure 3-2. Flowchart for Formulation Testing on Reactor Seepage Basin Soils 3-7

\section{TABLES}

Table ES-1. General Particle Size Distribution viii

Table ES-2. CRSB Data Summary by Soil Fractions ix

Table ES-3. KRSB Data Summary by Soil Fractions................................................................

Table ES-4. PRSB Data Summary by Soil Fractions ................................................................. xi

Table ES-5. LRSB Data Summary by Soil Fractions ................................................................. xii

Table ES-6. CRSB Data Summary by Formulations .....................................................................

Table ES-7. KRSB Data Summary by Formulations................................................................ xiv

Table ES-8. PRSB Data Summary by Formulations ................................................................. Xv

Table ES-9. LRSB Data Summary by Formulations ................................................................... xvi

Table ES-10. Average Hydraulic Conductivity by Formulation All Soil Fractions 28-Day Cure ...................................................................................................

Table ES-11. Average Hydraulic Conductivity by Formulation All Soil Fractions - 28-Day Cure, 90-Day Immersion .................................................................................. $\mathrm{xvii}$

Table 1-1. Grout Design Mixes for the Reactor Seepage Basin Soils ........................................ 1-9

Table 1-2. Treatability Testing Sample Numbering Scheme..................................................... 1-10

Table 2-1. Performance Criteria for the S/S Treated Material................................................... 2-3

Table 3-1. Samples Generated During Treatability Testing of CRSB Soil Samples.................... 3-8 


\section{TABLES (Continued)}

Table 3-2. Grout Mix Designs for the CRSB Soil $3-9$

Table 3-3. Samples Generated During Treatability Testing of KRSB Soil Samples 3-10

Table 3-4. Grout Mix Designs for the KRSB Soil. 3-11

Table 3-5. Samples Generated During Treatability Testing of PRSB Soil Samples. 3-12

Table 3-6. Grout Mix Designs for the PRSB Soil 3-13

Table 3-7. Samples Generated During Treatability Testing of LRSB Soil Samples. $3-14$

Table 3-8. Grout Mix Designs for the LRSB Soil 3-15

Table 3-9. Physical Characterization of the CRSB Soil Fractions 3-15

Table 3-10. Particle Size Distribution of the CRSB Soil Fractions. 3-16

Table 3-11. Chemical Characterization of the CRSB Soil Fractions. 3-16

Table 3-12. Radionuclide Characterization of the CRSB Soil Fractions. 3-17

Table 3-13. Physical Characterization of the KRSB Soil Fractions... 3-17

Table 3-14. Particle Size Distribution of the KRSB Soil Fractions.. 3-18

Table 3-15. Chemical Characterization of the KRSB Soil Fractions 3-18

Table 3-16. Radionuclide Characterization of the KRSB Soil Fractions . 3-19

Table 3-17. Physical Characterization of the PRSB Soil Fractions. 3-19

Table 3-18. Particle Size Distribution of the PRSB Soil Fractions . 3-20

Table 3-19. Chemical Characterization of the PRSB Soil Fractions. $3-20$

Table 3-20. Radionuclide Characterization of the PRSB Soil Fractions. $3-21$

Table 3-21. Physical Characterization of the LRSB Soil Fractions. 3-21

Table 3-22. Particle Size Distribution of the LRSB Soil Fractions... 3-22

Table 3-23. Chemical Characterization of the LRSB Soil Fractions. 3-22

Table 3-24. Radionuclide Characterization of the PRSB Soil Fractions ................................. 3-23

Table 3-25. Results for Treated Material from Coarse Fraction of CRSB Soils ...................... 3-24

Table 3-26. Results for Treated Material from Fine Fraction of CRSB Soils ........................... 3-25

Table 3-27. Results for Treated Material from Bulk Fraction \#1 of CRSB Soils ..................... 3-26

Table 3-28. Results for Treated Material from Bulk Fraction \#2 of CRSB Soils ..................... 3-27

Table 3-29. Results for Treated Material from Coarse Fraction of KRSB Soils ...................... 3-28

Table 3-30. Results for Treated Material from Fine Fraction of KRSB Soils .......................... 3-29

Table 3-31. Results for Treated Material from Coarse Fraction of PRSB Soils........................3-30

Table 3-32. Results for Treated Material from Fine Fraction of PRSB Soils........................... 3-31

Table 3-33. Results for Treated Material from Bulk Fraction \#1 of PRSB Soils ...................... 3-32

Table 3-34. Results for Treated Material from Bulk Fraction \#2 of PRSB Soils...................... 3-33

Table 3-35. Results for Treated Material from Coarse Fraction of LRSB Soils .......................3-34

Table 3-36. Results for Treated Material from Fine Fraction of LRSB Soils ...........................3-35 


\section{TABLES (Continued)}

Table 4-1. Average Hydraulic Conductivity by Formulation All Soil Fractions - 28-Day

Cure. 4-12

Table 4-2. Average Hydraulic Conductivity by Formulation All Soil Fractions - 28-Day Cure,

90-Day Immersion 4-12

Table 5-1. Estimated Construction Unit Cost . 5-15 


\section{ACRONYMS}

ACI American Concrete Institute

ANS American Nuclear Society

ASTM American Society for Testing and Materials

${ }^{\circ} \mathrm{C} \quad$ Degrees Celsius

$\mathrm{cm} / \mathrm{sec} \quad$ centimeter/second

Co Cobalt

$\mathrm{COC}$ Contaminant of Concern

CRSB C-Reactor Seepage Basin

Cs Cesium

DOE Department of Energy

dwb dry weight basis

ETDC Environmental Technology Development Center

${ }^{\circ} \mathrm{F} \quad$ Degrees Fahrenheit

$\mathrm{ft} \quad \mathrm{ft}$

g Gram

in inch

IT IT Corporation

$\mathrm{kg} \quad$ Kilogram

KRSB K-Reactor Seepage Basin

LAOCB L-Area Oil and Chemical Basin

LRSB L-Reactor Seepage Basin

$\mathrm{mg} / \mathrm{kg} \quad$ milligrams per kilograms

$\mathrm{mL} \quad$ milliliter

NIST National Institute of Standards and Technology

NRC Nuclear Regulatory Commission

$\mathrm{pCi} / \mathrm{g}$ picoCuries per gram

POR pilot operations room

PRSB P-Reactor Seepage Basin

psi pounds per square inch

PTSM Principal Threat Source Material

$\mathrm{Pu} \quad$ Plutonium

QA/QC Quality Assurance/Quality Control

RMA Radiological Material Area

rpm revolutions per minute

ROD Record of Decision

SAIC Science Applications International Company

SCDHEC South Carolina Department of Health and Environmental Control

Sr Strontium

SRS Savannah River Site

S/S Stabilization/Solidification

TDL Technology Development Laboratory 
ACRONYMS (Continued)

UCS Unconfined Compressive Strength

US EPA United States Environmental Protection Agency

WSRC Westinghouse Savannah River Company 


\subsection{EXPERIMENTAL PLAN}

\subsection{Project Description}

\subsubsection{Background}

Between 1957 and 1991, reactor seepage basins received low-level radioactive purge water from the disassembly basins at operating Savannah River Site (SRS) production reactors. This water purge was necessary to keep the tritium concentration in the disassembly basin water within safe levels. Before the use of mixed-bed deionizers and sand filters began in the 1960s, purge water was pumped directly from the disassembly basins to the seepage basins. From 1970 to 1978, the seepage basins for active reactors were bypassed. In 1978, C-Reactor Seepage Basin (CRSB), L-Reactor Seepage Basin (LRSB), and P-Reactor Seepage Basin (PRSB) were reactivated. The K-Reactor Seepage Basin (KRSB) was used only from 1957 to 1960 . None of the four basins have been active since 1991 .

Although many radionuclides were in the purge water discharged to the basins, almost all of the radioactivity was due to tritium. Tritium is highly mobile and migrated relatively quickly from the basins via leaching. Most of the remaining radioactivity is the result of small amounts of cobalt-60 (Co-60), strontium-90 (Sr-90), cesium-137 (Cs-137), and alpha emitters such as plutonium-239/240 (Pu-239/240). Cobalt-60 has a relatively short half-life; therefore, Cs-137, Sr-90, and Pu-239/240 are of more concern with respect to future leaching to groundwater.

The CRSB, KRSB, LRSB, and PRSBs are scheduled for closure during the next several years. Under the "Plug-In Record of Decision (ROD)" approach, the sclccted remediation technology for CRSB, KRSB, LRSB and PRSB is the in-situ stabilization/solidification (S/S) treatment of soils beneath the basins. Following stabilization of contaminated soils, each basin is then provided with drainage control, such as a geotextile membrane or other acceptable means, backfilled, and the surrounding area contoured to reduce the infiltration of rain water above the stabilized monolith.

A grout mix utilizing various reagents and additives/admixtures is required for the definitive $\mathrm{S} / \mathrm{S}$ remediation design. A grout mix design developed during this study conducted by SAIC under Contract No. C001311N will be recommended for the in-situ S/S of the contaminants of concern (COCs) in each of the reactor seepage basins.

A previous study (Section 1.6) evaluated grout mixes utilizing various reagents (e.g., Portland cement, bentonite, and soluble silicates) for the in-situ $\mathrm{S} / \mathrm{S}$ treatment of soils. The results of that study were used as the basis for selecting formulations evaluated in this study. 
A phased approach was used to identify the grout mix to be recommended for each of the four seepage basins. Phase I, the treatability testing on the CRSB and KRSB soils, involved four grout formulations of Portland cement, bentonite, and soluble silicates in various combinations. Each formulation was tested on soil formulated to encompass the expected variation in lithology and geotechnical properties within each reactor seepage basin (Section 1.2). In Phase II, the same four grout formulations were tested on the LRSB and PRSB soils, again using soil material which encompassed the expected variation in lithology and geotechnical properties for each basin.

\subsubsection{C-Reactor Seepage Basin}

The CRSB is three unlined basins constructed in the late 1950s and operated from 1959 to 1970. Wastewater from the reactor disassembly basin was discharged into the southeastern end of the L-shaped Basin No. 1. A cascade overflow pipe located in the top of the basin wall connects it to Basin No. 2. Basin No. 2 also has a similar cascade overflow into Basin No. 3. Basin No. 3 would have received wastewater if Basins No. 1 and 2 were nearly full.

Radiation surveys and shallow soil sampling indicate that Basin No. 3 was used sparingly. In-situ $\mathrm{S} / \mathrm{S}$ is not included in the remedial action plan for the soil in Basin No. 3. Therefore, Basin No. 3 will not be discussed further in this document.

The Principal Threat Source Materials (PTSMs) are Cs-137, Sr-90, and Pu-239/240. Basin No. 1 contains the highest concentrations of these radioactive materials, with the highest detected concentrations found in the 4 to $6 \mathrm{ft}$ depth. In Basin No. 2 the highest detected concentrations were found in the 1 to $4 \mathrm{ft}$ depth range.

\subsubsection{K-Reactor Seepage Basin}

The KRSB was constructed in 1957 as an unlined basin. The K-Reactor disassembly basin discharged low-level radioactive wastewater by gravity to the eastside of the KRSB.

The PTSMs for KRSB are also Cs-137, Sr-90, and Pu-239/240. Approximately 83 percent of the non-volatile beta activity is in the top $1 \mathrm{ft}$ of soil, 90 percent in the top $3 \mathrm{ft}$ of soil, and at least 95 percent of the activity is above a depth of $7 \mathrm{ft}$. 


\subsubsection{L-Reactor Seepage Basin}

The LRSB is an unlined, L-shaped earthen basin which received low-level radioactive purge water from 1958 until 1968 when L-Reactor was placed on warm standby. In 1978, four soil samples were collected inside the LRSB. These samples contained Co-60 (1052.2 picoCuries per gram $[\mathrm{pCi} / \mathrm{g}]), \mathrm{Cs}-137$ (258 pCi/g) and $\mathrm{Sr}-90(70.6 \mathrm{pCi} / \mathrm{g})$.

\subsubsection{P-Reactor Seepage Basin}

The PRSB consists of three unlined basins connected in series, located to the west of the P-Reactor perimeter fence. Two 400-ft underground process sewer lines extended from the P-Reactor disassembly basin to Basin 1 . The basins have not received wastewater since the P-Reactor was shut down for repairs in 1991.

In 1972, a limited number of soil cores taken to a depth of $1.5 \mathrm{ft}$ were analyzed for radionuclides. Maximum Cs-137 activities of 7,100 pCi/g were noted. In 1976, five surface soil samples (4-in deep) were taken from each basin. The maximum Cs-137 activity was observed to be $4,400 \mathrm{pCi} / \mathrm{g}$ and the maximum Sr-89/90 concentration was $600 \mathrm{pCi} / \mathrm{g}$. In 1978, sixteen borings were advanced to a maximum depth of $20 \mathrm{ft}$. These samples were analyzed for Co-60, Cs-137, and Sr-90. The highest activities were measured in Basins 1 and 2, with elevated activity levels in the top $5 \mathrm{ft}$.

\subsubsection{Related Immobilization Investigation at L-Area Oil and Chemical Basin}

The stabilization of soils and waste materials was previously selected as the remedial action for the L-Area Oil and Chemical Basin (LAOCB). A laboratory-scale immobilization study was conducted to evaluate the effectiveness of various grout formulations in immobilizing the radionuclides present in the $\mathrm{LAOCB}$ soil. The similarity of the soils and COCs present at the $L A O C B$ and the four reactor seepage basins suggest that the immobilization study for the LAOCB is very relevant to addressing the soil stabilization at the C-, K-, L-, P-Area reactor seepage basins.

The radionuclides of concem in the LAOCB included all the PTSMs identified in the C-, K-, L-, and P-Area reactor seepage basins: Cs-137, Sr-90, and Pu-239/240. The LAOCB also contained additional radionuclides not identified as PTSMs in the reactor seepage basins: Americium-241, Co-60, Uranium-234, -235, and -238. 
The LAOCB immobilization study was conducted in three phases: reagent screening, reagent optimization, and extended performance testing. The reagent screening phase evaluated mixing characteristics and performance of six possible mixtures: Portland cement, Portland cement and flyash, Portland cement and bentonite, Portland cement and silicate, Portland cement and hematite, and Portland cement and apatite.

The objective of the second phase was to determine the optimum dosage ratios of three selected mixes. The screening phase showed that mixes having a high ratio of reagents to soil (high mixes) appeared to reduce leachability of COCs more appreciably than mixes with a low ratio. Consequently, three high mixes were retained for further evaluation in extended performance testing: Portland cement, Portland cement and bentonite, and Portland cement and silicate.

The objective of the extended testing was to determine the long-term performance of soils treated with three selected reagent combinations. There were three significant results of this phase of testing:

- The unconfined compressive strength (UCS) of all three mixes exceeded the Nuclear Regulatory Commission (NRC) guidelines for compressive strengths of at least 500 pounds per square inch (psi).

- The compressive strength of all three mixtures were significantly reduced by the 90-day water immersion. The greatest reduction in average compressive strength was seen in the Portland Cement sample which was reduced by 89 percent to 450 psi.

- The three mixtures all effectively reduced the leachability of gross alpha and nonvolatile beta (reduced the leachability to below 20 percent).

The study results suggested that both bentonite and silicate additives significantly increased the effectiveness of Portland cement at reducing the leachability of radionuclides of concern. The LAOCB study recommended additional testing of mixes using a combination of Portland cement, bentonite, and sodium silicate to determine the mixture that would be most effective for the least cost. Dosage ratios of 0.15 Portland cement to 0.05 bentonite to 0.04 sodium silicate (fraction of the total weight), respectively, were recommended for further evaluation in a pilot study. Because of the relatively high cost of the sodium silicate, it was recommended that a lower sodium silicate dosage ratio of 0.02 be evaluated to determine how much benefit is achieved by adding more sodium silicate. 
Based on the results of the LAOCB study, four grout mixes were identified for evaluation in this study: Portland cement and bentonite; Portland cement and sodium silicate; Portland cement. bentonite, and sodium silicate (low); and Portland cement, bentonite, and sodium silicate (high). These four grout mixes are defined in Table 1-1.

\subsection{Technical Approach}

The technical approach to the S/S treatability testing on the soil from the four reactor seepage basins was based on the similarity of the chemical nature of the COCs and the method of contamination for each reactor seepage basin. Therefore, the grout mix for the reactor seepage basins did not have to compensate for variability in the activity of radionuclides, or the concentration of heavy metals, organic, or inorganic constituents. The major variability for which the $\mathrm{S} / \mathrm{S}$ mix design must compensate is the possible variability in lithology within a reactor seepage basin (e.g., clay lenses).

The samples used for the LAOCB treatability testing consisted of reddish and tan sandy soil, reddish and tan sandy soil $(80 \%)$ mixed with small amounts of olive green clay $(20 \%)$, and olive green clay $(80 \%)$ mixed with reddish sandy soil $(20 \%)$. Since the reactor seepage basins are located in areas of similar lithology to the $\angle A O C B$, it was likely that a similar range in soil material would encountered for the CRSB, KRSB, LRSB, and PRSB soils. The S/S grout mix design must be effective within a similar range of lithologic properties.

The technical approach to the treatability testing was to divide, or segregate, the soils from each reactor seepage basin into fractions which represent the range of lithology represented by the sample material. This was accomplished by physically segregating the soil sample material from each reactor seepage basin into fractions which are primarily coarse (sandy), fractions which are primarily fine (clayey), and fractions which represent the overall bulk combination of coarse and fine soil material (bulk fractions). The bulk fraction samples were considered to represent the typical or expected lithological conditions, while the fine and coarse fraction samples would represent lithologic end members.

Soil from the CRSB was collected specifically for this study. Samples were collected from Basin 1 and Basin 2 (Appendix C). Soil from KRSB, LRSB, and PRSB was the residual from previous characterization activities. The precise location within the basins and sample collection interval is not known for KRSB, LRSB, and PRSB. However, review of the sampling plans for these units suggest that these samples are representative of overall basin conditions. 
For the CRSB and LRSB, sufficient soil samples were available to allow for the physical segregation discussed above. For the KRSB and PRSB soils, the quantity of the samples available for performing the tests was limited. Therefore, the KRSB and PRSB soils were segregated into only coarse and fine fraction samples, representing the extremes in lithology for the these reactor seepage basins.

The fractions resulting from the physical segregation of the soil material from each reactor seepage basin were used in the $\mathrm{S} / \mathrm{S}$ grout mix design testing. During this testing, four grout mixes (Table 1-1) were tested on each fraction of each reactor seepage basin. The $S / S$ treated material from each soil/grout mix combination was subjected to physical and chemical testing to determine if it met the specified performance criteria (Section 2.2).

A copy of the Work Plan, which outlines the test procedures and grout mix designs, is included in Appendix D. Section 3.0 discusses how the technical approach was applied to each of the reactor seepage basin soils.

\subsection{Equipment, Materials, And Facilities}

\subsubsection{Equipment}

The equipment used in the laboratory-scale S/S treatability testing included:

- $2 \mathrm{ft}$ by $3 \mathrm{ft}$ plastic trays,

- Stainless steel mixing bowls,

- 5 quart planetary mixer,

- 3 in by 6 in right cylinder plastic molds,

- 2 in by 4 in right cylinder plastic molds,

- Stainless steel spatulas,

- Laboratory balances, and

- Graduated cylinders. 


\subsubsection{Materials}

The reagents used during the laboratory-scale S/S treatability testing included:

- Type I Portland cement,

- Sodium bentonite, and

- Type $\mathrm{N}$ soluble silicates.

Information on these reagents is included in Appendix G.

The personal protective equipment used during the treatability study included:

- Nitrile gloves,

- Tyvek coveralls, and

- Tyvek boot covers.

\subsubsection{Facilities}

The laboratory-scale $\mathrm{S} / \mathrm{S}$ treatability testing was conducted at the IT Corporation (IT) Technology Development Laboratory (TDL) in Knoxville, TN. Geotechnical testing on the CRSB, LRSB, PRSB, and KRSB soils and the S/S treated materials was conducted at IT's Environmental Technology Development Center (ETDC) in Oak Ridge, TN.

The TDL and ETDC each maintain a Radioactive Materials License from the State of Tennessee and operate under the treatability exemption in 40 CFR 261.4. The treatability study and other activities were performed under these relevant permits, licenses, or exemptions.

\subsection{Sample Transportation And Tracking}

\subsubsection{Sample Transportation}

Soil samples from the CRSB, KRSB, LRSB, and PRSB were transported by licensed shipper from the Radiological Material Area (RMA) at Building 772-7B, SRS, to the ETDC. Chain of custody forms accompanied the sample shipment. Upon receipt at the ETDC, the soil samples were each given a unique sample number and logged into the ETDC sample inventory system. 
The unique sample number was used to track materials such as samples, test-residues. test products, and reagents throughout the project.

Upon receipt, the outside of the containers was smeared to measure external radiation contamination, and the dose rate at the surface of the containers was measured. The results were consistent with the initial gross alpha and non-volatile beta radiation data received from WSRC. The containers were then moved to the pilot operations room (POR) at the ETDC. The containers were opened to visibly assess the condition of the contents in the POR.

\subsubsection{Sample Chain of Custody}

A strict Chain-of-Custody Record was maintained for all samples received at the TDL in Knoxville, Tennessee or the ETDC in Oak Ridge, Tennessee. Sample transfers external to the treatability facility were accompanied by an IT combined Analysis Request and Chain-ofCustody Record. Samples transferred to the treatability analytical department or Geotechnical Laboratory were also accompanied by an Analysis Request and Chain-of-Custody Record. Copies of all Analysis Request and Chain-of-Custody Records are maintained in the project file.

\subsubsection{Sample Labeling}

Samples were assigned a unique sample identification which described the reactor seepage basin, the soil fraction or subsample, the formulation, applicable cure period, testing to be conducted on the sample, and any subsample required for the applicable testing. Table 1-2 details the sample numbering categories and their applicable codes.

For example, Sample Number C422F00 was the unconfined compressive strength sample, cured 28 days, from the cement + high silicate formulation from the Fine Fraction of the CRSB soil material. Likewise, Sample L612H02 was the second leachate subsample from the ANS 16.1 leachability test on the treated material, cured 28 days, from the cement + bentonite formulation on the Bulk Fraction $\# 2$ of the LRSB soil material.

\subsection{Health and Safety}

A copy of the Chemical Hygiene Plan for the TDL and ETDC facilities is included in Appendix F. 
Soil Stabilization/Solidification Grout Mix Design Study C-, K-, L-, and P-Reactor Seepage Basins Closure (U)
WSRC-RP-99-4075. Revision 1 August 1999

Table 1-1. Grout Design Mixes for the Reactor Seepage Basin Soils

\begin{tabular}{|c|c|c|c|c|c|c|c|}
\hline \multirow{2}{*}{ Formulation } & Soil & \multicolumn{2}{|c|}{ Portland Cement } & \multicolumn{2}{c|}{ Sodium Bentonite } & \multicolumn{2}{c|}{ Sodium Silicate } \\
\cline { 2 - 8 } & $\mathbf{( g m )}$ & $(\mathbf{g m})$ & $\begin{array}{c}(\% \mathbf{b y} \\
\text { weight })\end{array}$ & $(\mathbf{g m})$ & $\begin{array}{c}(\% \text { by } \\
\text { weight) }\end{array}$ & (gm) & $\begin{array}{c}\text { (\% by } \\
\text { weight) }\end{array}$ \\
\hline 1 & 2,500 & 469 & 15 & 156 & 5 & 0 & 0 \\
\hline 2 & 2,500 & 463 & 15 & 0 & 0 & 124 & 4 \\
\hline 3 & 2,500 & 481 & 15 & 160 & 5 & 65 & 2 \\
\hline 4 & 2,500 & 494 & 15 & 165 & 5 & 132 & 4 \\
\hline
\end{tabular}


Table 1-2. Treatability Testing Sample Numbering Scheme

\begin{tabular}{|c|c|c|c|}
\hline Category & Code & Category & Code \\
\hline \multirow{4}{*}{ Reactor } & $\mathrm{C}=\mathrm{C}$ Reactor Seepage Basin & \multirow[t]{16}{*}{ Test } & $A=C O C s$ \\
\hline & $\mathrm{K}=\mathrm{K}$ Reactor Seepage Basin & & $\mathrm{B}=$ gross alpha. nonvolatile beta \\
\hline & $\mathrm{L}=\mathrm{L}$ Reactor Seepage Basin & & $\mathrm{C}=$ Inorganics \\
\hline & $\mathrm{P}=\mathrm{P}$ Reactor Seepage Basin & & $\mathrm{D}=$ Total carbonation activity \\
\hline \multirow{8}{*}{ Subsample } & $1=$ Grab 1 (Soil before segregation) & & $\mathrm{E}=$ Oxidation/reduction potential \\
\hline & 2 = Grab 2 (Soil before segregation) & & $\mathrm{F}=$ Unconfined Compressive Strength \\
\hline & $3=$ Coarse Fraction & & $\mathrm{G}=$ Hydraulic Conductivity \\
\hline & $4=$ Fine Fraction & & $\mathrm{H}=$ ANS 16.1 Leachability \\
\hline & $5=$ Bulk Fraction $\# 1$ & & $\mathrm{I}=$ Temperature \\
\hline & $6=$ Bulk Fraction $\# 2$ & & $\mathrm{~J}=\mathrm{pH}$ \\
\hline & $\begin{aligned} 7= & \text { Remaining Soil \#1 (After } \\
& \text { Segregation) }\end{aligned}$ & & $\mathrm{K}=$ Shrink/Swell \\
\hline & $\begin{aligned} 8= & \text { Remaining Sample \#2 (After } \\
& \text { Segregation) }\end{aligned}$ & & $\mathrm{L}=$ Bleed Volume \\
\hline \multirow{4}{*}{ Formulation } & $1=$ Cement + bentonite & & $\mathrm{M}=$ Volume Increase \\
\hline & $2=$ Cement + high silicate & & $\mathrm{N}=$ Setting \\
\hline & $3=$ Cement + bentonite + low silicate & & $\mathrm{Q}=$ Geotechnical \\
\hline & $4=$ Cement + bentonite + high silicate & & \\
\hline \multirow{4}{*}{ Cure Period } & $0=$ Not Applicable & \multirow{2}{*}{$\begin{array}{c}\text { Test } \\
\text { Subsample }\end{array}$} & $01-10$, when Test $=\mathrm{H}$ \\
\hline & $1=14$ days & & Otherwise $=00$ \\
\hline & $2=28$ days & & \\
\hline & $3=90$ days immersed & & \\
\hline
\end{tabular}




\subsection{STUDY AND TEST OBJECTIVES}

\section{$2.1 \quad$ Objectives}

The purpose of this study was to perform bench-scale (laboratory-scale) studies to develop grout mix designs which can stabilize and solidify the radiological contaminants in the CRSB, KRSB, LRSB, and PRSB soils. One objective is to determine if a unique grout mix design is required for the in-situ S/S each seepage basin. It is expected that the depth of $\mathrm{S} / \mathrm{S}$ will not exceed $20 \mathrm{ft}$ below the existing basin bottoms, and in most cases will probably be less than $10 \mathrm{ft}$.

The study was implemented in two phases. The first phase of work, designated Phase I, was implemented using the CRSB and KRSB soils. With Phase I substantially complete, Phase II was implemented using the LRSB and PRSB soils.

Initial treatability testing (Phase I) on the CRSB and KRSB soils involved the four formulations defined in Table 1-1. Each formulation was replicated on the soil fractions for each reactor seepage basin. In Phase II, the four formulations were tested on the LRSB and PRSB soils, again on soil fractions from each reactor seepage basin.

The grout mix recommended for each reactor seepage basin will be the one that the test results indicate will produce a stabilized waste monolith (grouted waste) which meets the performance requirements/acceptance criteria (Section 2.2). Information on construction tolerances and mixing process constraints are provided in Section 5.3. Field optimization tests are not expected to be necessary as long as the construction tolerances, PTSMs, and mixing process constraints identified as a result of this study are not exceeded. A pilot test may be necessary to verify the full scale application method provides acceptable mixing or to determine the effect of additives, such as set retarders, if they are found to be needed.

\subsection{Study Performance Standards}

The primary goal of S/S treatment is to minimize the mobility of the PTSMs. Soil S/S treatment is a performance-based remedial approach since it does not reduce the total mass of the PTSMs and cannot in itself achieve concentration-based remedial objectives. The performance standards for the S/S treated soils are given in Table 2-1.

Additional performance parameters such as total carbonation activity, sulfates, sulfides, sulfite, chloride, nitrate, nitrite, nitric compounds, boric compounds, and oxidation/reduction potential had been considered for the $\mathrm{S} / \mathrm{S}$ treated material. Total carbonation activity would be required to 
demonstrate that the stabilized material contains sufficient carbonation to maintain an alkaline $\mathrm{pH}$. The Portland cement (15 percent of the total treated weight) added as part of the $\mathrm{S} / \mathrm{S}$ grout mix will produce a sufficient total carbonation activity level to satisfy this criterion. Therefore, the determination of total carbonation activity on the S/S treated material would not supply any useful information.

When high salt content wastes are stabilized, the sulfate, sulfide, sulfite, nitrate, nitrite, and nitric and boric compound levels are of concern, as the presence of these chemical species in high $(>50,000$ milligram $/$ kilogram $(\mathrm{mg} / \mathrm{kg})$ ) levels can affect the physical properties of the final $\mathrm{S} / \mathrm{S}$ treated material. However, there is no indication that the soils beneath the CRSB, KRSB, LRSB or PRSB contain high levels of these chemical species. Furthermore, previous $\mathrm{S} / \mathrm{S}$ treatability work on the LAOCB soils indicated minimal anion leaching from similar $\mathrm{S} / \mathrm{S}$ treated soils using similar reagents. Therefore, performance criteria for these chemical species would not influence the physical stability of the $\mathrm{S} / \mathrm{S}$ treated material.

Certain contaminants, such as arsenic, chromium, and selenium, can easily exist in a number of valance states. The immobilization of these contaminants can be affected by the oxidation/ reduction potential of the $\mathrm{S} / \mathrm{S}$ treated material. The immobilization of the PTSMs are not affected by their valance state and the oxidation/reduction potential of the $S / S$ treated material will not affect its performance. Therefore, performance criteria for these parameters were not considered further. 
Table 2-1. Performance Criteria for the S/S Treated Material

\begin{tabular}{|c|c|c|}
\hline Parameter & Criteria & Standard Test Method \\
\hline Batching Weight Precision & $\leq 5 \%$ & NIST traceable \\
\hline Batching Mix Ratio Variation & $\leq 0.5 \mathrm{gal} / \mathrm{lb}$ & NIST traceable \\
\hline $\begin{array}{l}\text { Unconfined Compressive Strength at } 14 \\
\text { days Cure }\end{array}$ & $>100 \mathrm{psi}$ & ASTM D 2166 \\
\hline $\begin{array}{l}\text { Unconfined Compressive Strength at } 28 \\
\text { days Cure }\end{array}$ & $>100 \mathrm{psi}$ & ASTM D 2166 \\
\hline $\begin{array}{l}\text { Unconfined Compressive Strength after } \\
\text { Water Immersion for } 90 \text { days }\end{array}$ & $>100 \mathrm{psi}$ & $\begin{array}{l}\text { ASTM D 2166, immersion per } \\
\text { NRC guidelines }\end{array}$ \\
\hline Hydraulic Conductivity at 28 days Cure & $<1 \times 10 \mathrm{E}-08 \mathrm{~cm} / \mathrm{sec}$ & ASTM D 5084 \\
\hline $\begin{array}{l}\text { Hydraulic Conductivity after Water } \\
\text { Immersion for } 90 \text { days }\end{array}$ & $<1 \times 10 \mathrm{E}-08 \mathrm{~cm} / \mathrm{sec}$ & $\begin{array}{l}\text { ASTM D 5084, immersion per } \\
\text { NRC guidelines }\end{array}$ \\
\hline $\begin{array}{l}\text { Leachability Indices for Gross Alpha, } \\
\text { Nonvolatile Beta }\end{array}$ & $>8.0$ & ANS 16.1 \\
\hline Temperature at Core and Surface & $<100^{\circ} \mathrm{F}$ & ASTM C 1064 \\
\hline $\mathrm{pH}$ & $>10.0$ & ASTM D 4972 \\
\hline Shrink/Swell & $\begin{array}{l}<25 \% \text { of } 28 \text {-day UCS } \\
\text { failure strain }\end{array}$ & ASTM D 3877 \\
\hline Bleed Fluid & $<0.5 \%$ & ANS 55.1 or EPA 9095 \\
\hline Volume Increase & $<50 \%$ & None \\
\hline Hazardous dust/vapor release & -- & Per OSHA Standards \\
\hline $\begin{array}{l}\text { Gas generation due to chemical, } \\
\text { biological, organic, thermal, and } \\
\text { radiation sources }\end{array}$ & $\begin{array}{l}\text { Lesser of: } 0.5 \mathrm{moles} / \mathrm{cf} / \mathrm{yr} \\
\text { or } 800 \mathrm{moles} / \mathrm{cf} \text { total }\end{array}$ & $\begin{array}{l}\text { Per } 10 \text { CFR } 60 \text { or Per NUREG- } \\
\text { CR- } 2333\end{array}$ \\
\hline Initial Set Time & $>6$ hours & ASTM C 191 \\
\hline Final Set Time & $<180$ days & ASTM C 191 \\
\hline False Set & Prohibited & ASTM C 191 \\
\hline Flash Set & Prohibited & ASTM C 191 \\
\hline
\end{tabular}


This page intentionally left blank. 


\subsection{TEST OBSERVATIONS AND RESULTS}

The bench-scale treatability testing demonstrated how the treated material from the selected grout mix designs performed with respect to the performance criteria listed in Table 2-1. The technical approach used for selecting the soil fractions for $S / S$ testing was described in Section 1.2. The analytical methods utilized during the testing are included in Appendix E. Appendix A contains the raw data from the testing.

\subsection{Phase I Testing}

The first phase of the S/S treatability study involved the soils from the CRSB and KRSB, the basins scheduled for early closure. The flowcharts in Figures 3-1 and 3-2 demonstrate the progression of the treatability testing.

\subsubsection{Treatability Testing on CRSB Soils}

Step 1. The soil material from the CRSB was placed into two plastic trays, each $2 \mathrm{ft}$ wide by $3 \mathrm{ft}$ long. Two 1-quart grab samples, which appeared representative of the soil material in the tray, were collected for geotechnical and characterization analysis (Table 3-1).

$\underline{\text { Step 2. }}$. Based on visual inspection, the soil material was segregated by lithological properties into samples for formulation mix design testing. The objective of this segregation was to produce samples for testing which bracketed the anticipated range in lithologic properties. This ensured that the performance of the mix designs was evaluated against the potential variability in lithology within the CRSB.

Claycy material was segregated by removing visible "clay balls" from one of the trays. This material was combined to make the Fine Fraction sample. Coarse material remaining in the tray was segregated and combined to make the Coarse Fraction sample. The material in the other tray was homogenized and the two Bulk Fraction samples for mix design testing were prepared. Two 1-quart samples of the remaining material were also collected for geotechnical analysis (Remaining Samples).

Step 3. The four fractions (coarse, fine, and 2 bulk) were used for formulation mix design testing. Four grout mix formulations were made on each fraction (Table 3-2). 


\subsubsection{Treatability Testing on KRSB Soils}

Step 1 . The soil material from the KRSB was placed into a plastic tray, $2 \mathrm{ft}$ wide by $3 \mathrm{ft}$ long. Two 1-quart grab samples, which appeared representative of the soil material in the tray, were collected for geotechnical and characterization analysis (Table 3-3).

Step 2. Based on visual inspection, the soil material was segregated by lithological properties into samples for formulation mix design testing. Clayey material was segregated by removing visible "clay balls". This material was combined to make a fine fraction sample for mix design testing. Coarse material remaining in the tray was segregated and combined to make a coarse fraction sample for mix design testing. The objective of this segregation was to produce samples for mix design testing which bracket the anticipated range in lithologic properties. This ensured that the performance of the mix designs was evaluated against the potential variability in lithology within the KRSB. The remaining material was homogenized and two 1-quart samples were also collected for geotechnical analysis.

Step 3. The two fractions (coarse and fine) segregated above were used for formulation mix design testing. Four formulations were made on each fraction (Table 3-4).

\subsection{Phase II Testing}

The second phase of the $S / S$ treatability study on the reactor seepage basin soils involved the soils from the LRSB and PRSB. This phase began during the latter part of the Phase I testing. The flowcharts in Figures 3-1 and 3-2 demonstrate the progression of the treatability testing.

\subsubsection{Treatability Testing on PRSB Soils}

Step 1 . The soil material from the PRSB was placed into two plastic trays, each $2 \mathrm{ft}$ wide by $3 \mathrm{ft}$ long. Two 1-quart grab samples, which appeared representative of the soil material in the tray, were collected for geotechnical and characterization analysis (Table 3-5).

Step 2. Based on visual inspection, the soil material was segregated by lithological properties into samples for formulation mix design testing. The objective of this segregation was to produce samples for mix design testing which bracket the anticipated range in lithologic properties. This ensured that the performance of the mix designs was evaluated against the potential variability in lithology within the PRSB.

Clayey material was segregated by removing visible "clay balls" from one tray. These were combined to make a Fine Fraction sample. Coarse material remaining in the tray were segregated 
and combined to make a Coarse Fraction sample. The remaining material was homogenized and two Bulk Fraction samples were prepared. Two 1-quart samples were also collected for geotechnical analysis (Remaining Samples).

Step 3. The four fractions (coarse, fine, and 2 bulk) were used for formulation mix design testing. Four formulations were made on each fraction (Table 3-6).

\subsubsection{Treatability Testing on LRSB Soils}

Step 1. The soil material from the LRSB was placed into a plastic tray, $2 \mathrm{ft}$ wide by $3 \mathrm{ft}$ long. Two l-quart grab samples, which appeared representative of the soil material in the tray, were collected for geotechnical and characterization analysis (Table 3-7).

Step 2. Based on visual inspection, the soil material was segregated by lithological properties into samples with the objective of producing samples which bracketed the anticipated range in lithologic properties. This ensured that the performance of the mix designs was evaluated against the potential variability in lithology within the LRSB.

Clayey material was segregated by removing visible "clay balls". These were combined to make a Fine Fraction sample. Coarse material remaining was segregated and combined to make a Coarse Fraction sample. The remaining material was homogenized, and two 1-quart samples were collected for geotechnical analysis (Remaining Samples).

Step 3. The two fractions (coarse and fine) segregated above were used for formulation mix design testing. Four formulations were made on each fraction (Table 3-8).

\subsection{Grout Mix Design}

The $\mathrm{S} / \mathrm{S}$ grout mixes for each basin were prepared using the soil from the appropriate basin, selected additives (admixtures), and other grout mix components. Four mixes were prepared for each reactor seepage basin (see Table 1-1 for specific weights and ratios):

- Portland cement and bentonite

- Portland cement and high silicate

- Portland cement, bentonite, and high silicate

- Portland cement, bentonite, and low silicate. 
In developing the mixes, the components (including cementitious and pozzolanic components and their chemical constituents) were of high quality and of known composition. and commercially available. All cementitious and pozzolanic components were chemically stable and were environmentally nonhazardous before and after their $\mathrm{S} / \mathrm{S}$ applications.

\subsection{Stabilization Formulation Development}

Each formulation was mixed in a planetary mixer. A 2,500 $\mathrm{g}$ aliquot of reactor soil was added to the mixing bowl. The dry reagents were weighed, blended, and mixed with an equal weight of water. Soluble silicate or additional water was added to the reagent slurry. The reagent slurry was then added to the soil in the mixing bowl. The soil and reagent slurry was mixed at 30-40 revolutions per minute (rpm) for 1 to 2 minutes to produce a homogeneous treated material.

The treated material was poured into appropriate molds, vibrated to remove air pockets, and capped to minimize moisture loss. The molded material was allowed to cure at ambient laboratory temperature (approximately $72^{\circ} \mathrm{F}$ ) in sealed coolers.

\subsection{Testing Results}

\subsubsection{Soil Characterization}

The soil samples from Steps 1 and 2 were subjected to the characterization testing listed in Tables 3-1, 3-3, 3-4, and 3-5. Results are presented in Appendix A, Attachment B.

\subsubsection{CRSB Soil Fractions}

The results for the characterization analyses on the CRSB soil fractions are summarized in Tables 3-9 through 3-12.

\subsubsection{KRSB Soil Fractions}

The results for the characterization analyses on the KRSB soil fraction are summarized in Tables 3-13 through 3-16.

\subsubsection{PRSB Soil Fractions}

The results for the characterization analyses on the PRSB soil fraction are summarized in Tables 3-17 through 3-20. 


\subsubsection{LRSB Soil Fractions}

The results for the characterization analyses on the LRSB soil fraction are summarized in Tables 3-21 through 3-24.

\subsubsection{Treated Material Performance Testing}

The cured treated matcrial was subjected to the performance criteria testing listed in Tables 3-1, 3-3, 3-4 and 3-5 for Step 3. The tests were the same as the performance criteria (Table 2-1) for the final stabilized waste material; with the exception of the criteria for the batching, hazardous dust/vapor release, and the gas generation. The results from the performance testing provide information on the leachability, durability, hydraulic conductivity, strength, and long-term durability of the S/S treated soil material. Test results are in Appendix A, Attachment A.

\subsubsection{CRSB Treated Material}

The results of the performance testing on the treated material produced from the treatment of the CRSB soil fractions are summarized in Tables 3-25 through 3-28.

\subsubsection{KRSB Treated Material}

The results of the performance testing on the treated material produced from the treatment of the KRSB soil fractions are summarized in Tables 3-29 and 3-30.

\subsubsection{PRSB Treated Material}

The results of the performance testing on the treated material produced from the treatment of the PRSB soil fractions are summarized in Tables 3-31 through 3-34.

\subsubsection{LRSB Treated Material}

The results of the performance testing on the treated material produced from the treatment of the LRSB soil fractions are summarized in Tables 3-35 and 3-36. 


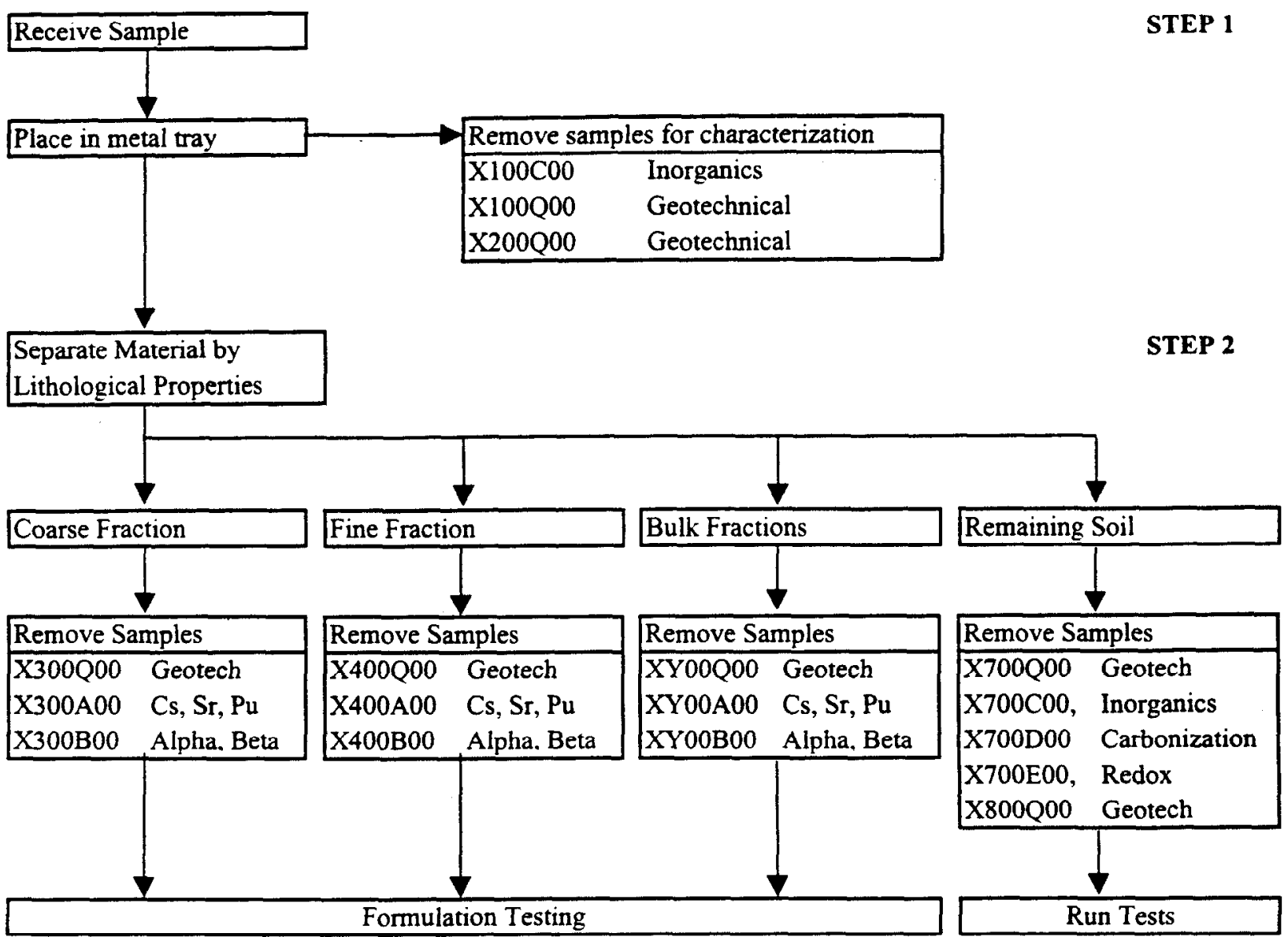

$\mathrm{X}=\mathrm{C}, \mathrm{K}, \mathrm{L}$, or $\mathrm{P}$ (Reactor)

$\mathrm{Y}=5$ or 6 (Subsample)

Figure 3-1. Flowchart for the Segregation of the Reactor Seepage Basin Soils 


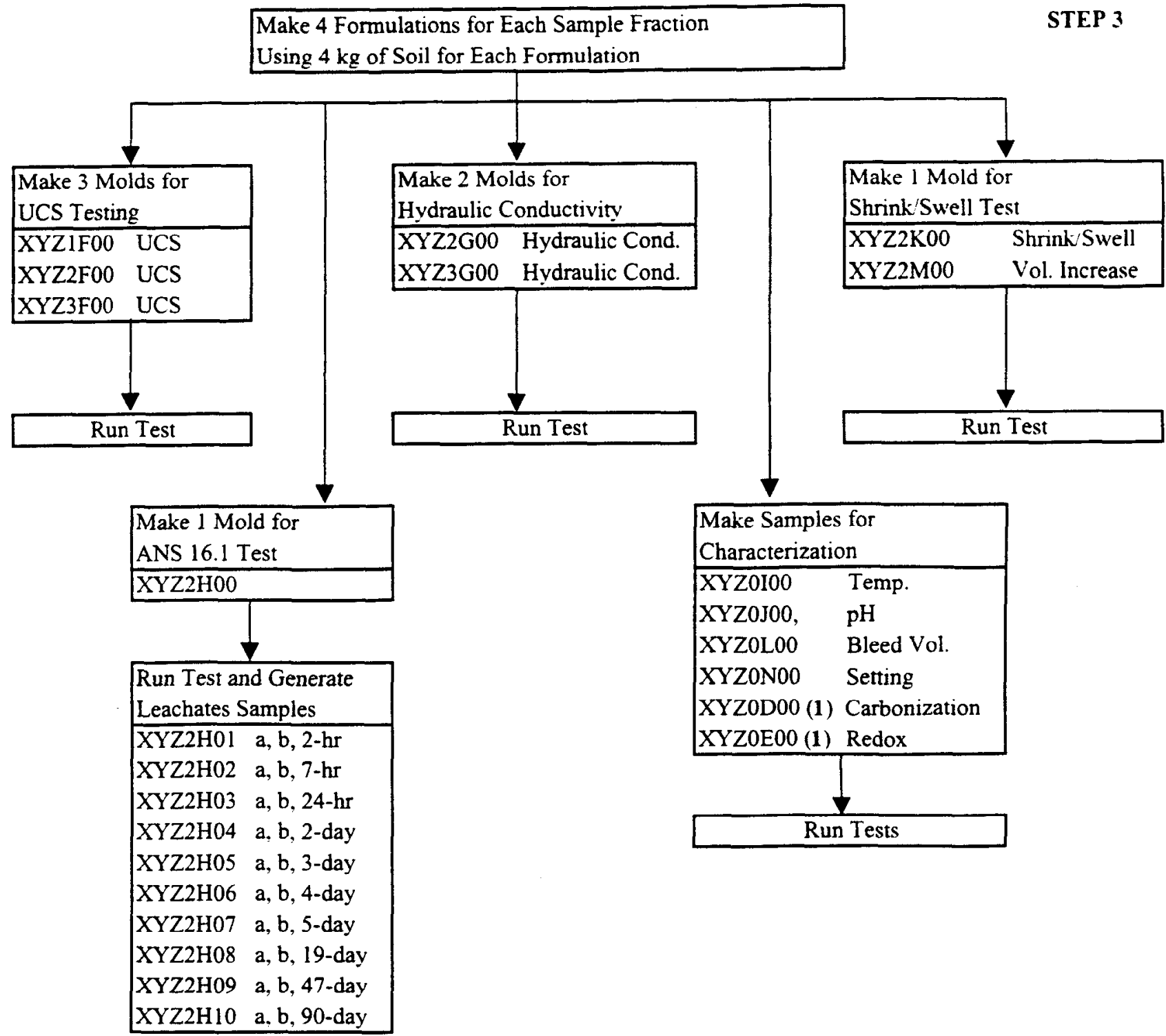

(1) Only on two randomly selected formulations

$\mathrm{X}=\mathrm{C}, \mathrm{K}, \mathrm{L}$, or $\mathrm{P}$ (Reactor)

$\mathrm{Y}=3,4,5$, or 6 (Subsample)

$\mathrm{Z}=1,2,3$, or 4 (Formulation)

UCS $=$ Unconfined Compressive Strength

Figure 3-2. Flowchart for Formulation Testing on Reactor Seepage Basin Soils 
Table 3-1. Samples Generated During Treatability Testing of CRSB Soil Samples

\begin{tabular}{|c|c|c|c|c|c|}
\hline \multirow{2}{*}{ Testing Parameters } & \multirow{2}{*}{ Method } & \multicolumn{4}{|c|}{ Number of Tests } \\
\hline & & Total & Step 1 & Step 2 & Step 3 \\
\hline Atterberg Limits & ASTM D 4318 & 8 & 2 & 0 & \\
\hline Grain Size Distribution & ASTM D422 & 8 & 2 & 6 & \\
\hline Moisture Content & ASTM D2216 & 8 & 2 & 6 & \\
\hline Specific Gravity & ASTM D854 & 8 & 2 & 6 & \\
\hline USCS Soil Classification & ASTM D2487 & 8 & 2 & 6 & \\
\hline Cesium-137 & Gas Flow & 4 & & 4 & \\
\hline Plutonium-239/240 & Gas Flow & 4 & & 4 & \\
\hline Strontium-90 & Gas Flow & 4 & & 4 & \\
\hline Gross Alpha & Gas Flow & 164 & & 4 & 160 \\
\hline Non-volatile Beta & Gas Flow & 164 & & 4 & 160 \\
\hline Chloride Compounds & SW-846 Method 9056 & 1 & 1 & & \\
\hline Nitrate & SW-846 Method 9056 & 1 & 1 & & \\
\hline Nitrite & ASTM D9056 & 1 & 1 & & \\
\hline Oxidation/Reduction State & ASTM D1498 & 1 & 1 & & \\
\hline Sulfate & SW-846 Method 9056 & 1 & 1 & & \\
\hline Sulfide & SW-846 Method 9034 & 1 & 1 & & \\
\hline UCS@14 days cure & ASTM D2166 & 16 & & & 16 \\
\hline UCS@28 days cure & ASTM D2166 & 16 & & & 16 \\
\hline USC @90 days immersion & ASTM D2166 & 16 & & & 16 \\
\hline Hydraulic Conductivity@28 days & ASTM D5084 & 16 & & & 16 \\
\hline Hydraulic Conductivity@90 days immersion & ASTM D5084 & 16 & & & 16 \\
\hline Temperature & ASTM C1064 & 16 & & & 16 \\
\hline $\mathrm{pH}$ & SW-846 Method 9045C & 16 & & & 16 \\
\hline Shrinkage/Swell & ASTM D3877 & 16 & & & 16 \\
\hline Bleed Fluid & SW-846 Method 9095 & 16 & & & 16 \\
\hline Sel Time & ASTM C191 & 16 & & & 16 \\
\hline
\end{tabular}


Soil Stabilization/Solidification Grout Mix Design Study

WSRC-RP-99-4075, Revision 1

C-, K-, L-, and P-Reactor Seepage Basins Closure (U)

August 1999

Table 3-2. Grout Mix Designs for the CRSB Soil

\begin{tabular}{|c|c|c|c|c|c|}
\hline \multirow[t]{2}{*}{ Formulation ${ }^{1}$} & \multirow[t]{2}{*}{$\begin{array}{c}\text { Soil } \\
\text { Fraction }\end{array}$} & $\begin{array}{l}\text { Portland } \\
\text { Cement }\end{array}$ & $\begin{array}{c}\text { Soluble } \\
\text { Bentonite }\end{array}$ & $\begin{array}{l}\text { Sodium } \\
\text { Silicate }\end{array}$ & Water \\
\hline & & \multicolumn{4}{|c|}{ Mix Ratio ${ }^{2}$} \\
\hline l & \multirow{4}{*}{ Coarse } & 0.15 & 0.05 & -- & 0.20 \\
\hline 2 & & 0.15 & -- & 0.04 & 0.19 \\
\hline 3 & & 0.15 & 0.05 & 0.02 & 0.22 \\
\hline 4 & & 0.15 & 0.05 & 0.04 & 0.24 \\
\hline l & \multirow[t]{4}{*}{ Fine } & 0.15 & 0.05 & -- & 0.20 \\
\hline 2 & & 0.15 & -- & 0.04 & 0.19 \\
\hline 3 & & 0.15 & 0.05 & 0.02 & 0.22 \\
\hline 4 & & $\overline{0.15}$ & 0.05 & $\overline{0.04}$ & $\overline{0.24}$ \\
\hline 1 & \multirow{4}{*}{$\begin{array}{c}\text { Bulk } \\
\text { Fraction \# } \\
1\end{array}$} & 0.15 & 0.05 & -- & 0.20 \\
\hline 2 & & 0.15 & -- & 0.04 & 0.19 \\
\hline 3 & & 0.15 & 0.05 & 0.02 & 0.22 \\
\hline 4 & & 0.15 & 0.05 & 0.04 & 0.24 \\
\hline 1 & \multirow{4}{*}{$\begin{array}{c}\text { Bulk } \\
\text { Fraction \# } \\
2\end{array}$} & 0.15 & 0.05 & -- & 0.20 \\
\hline 2 & & 0.15 & -- & 0.04 & 0.19 \\
\hline 3 & & 0.15 & 0.05 & 0.02 & 0.22 \\
\hline 4 & & 0.15 & 0.05 & 0.04 & 0.24 \\
\hline
\end{tabular}

Formulation:

1 - Portland cement and bentonite

2 - Portland cement and 4\% silicate (high)

3 - Portland cement, bentonite, and 2\% silicate (low)

4 - Portland cement, bentonite, and 4\% silicate (high)

2 Fraction of total weight 


\section{Table 3-3. Samples Generated During Treatability Testing of KRSB Soil Samples}

\begin{tabular}{|c|c|c|c|c|c|}
\hline \multirow{2}{*}{ Testing Parameters } & \multirow{2}{*}{ Method } & \multicolumn{4}{|c|}{ Number of Tests } \\
\hline & & Total & Step 1 & Step 2 & Step 3 \\
\hline Atterberg Limits & ASTM D 4318 & 6 & 2 & 4 & \\
\hline Grain Size Distribution & ASTM D422 & 6 & 2 & 4 & \\
\hline Moisture Content & ASTM D2216 & 6 & 2 & 4 & \\
\hline Specific Gravity & ASTM D854 & 6 & 2 & 4 & \\
\hline USCS Soil Classification & ASTM D2487 & 6 & 2 & 4 & \\
\hline Cesium-137 & Gas Flow & 2 & & 2 & \\
\hline Plutonium-239/240 & Gas Flow & 2 & & 2 & \\
\hline Strontium-90 & Gas Flow & 2 & & 2 & \\
\hline Gross Alpha & Gas Flow & 82 & & 2 & 80 \\
\hline Non-volatile Beta & Gas Flow & 82 & & 2 & 80 \\
\hline Chloride Compounds & SW-846 Method 9056 & 1 & 1 & & \\
\hline Nitrate & SW-846 Method 9056 & 1 & 1 & & \\
\hline Nitrite & ASTM D9056 & 1 & 1 & & \\
\hline Oxidation/Reduction State & ASTM D1498 & 1 & 1 & & \\
\hline Sulfate & SW-846 Method 9056 & 1 & 1 & & \\
\hline Sulfide & SW-846 Method 9034 & 1 & 1 & & \\
\hline UCS @ 14 days cure & ASTM D2166 & 8 & & & 8 \\
\hline UCS@ 28 days cure & ASTM D2166 & 8 & & & 8 \\
\hline USC (a) 90 days immersion & ASTM D2166 & 8 & & & 8 \\
\hline Hydraulic Conductivity @ 28 days & ASTM D5084 & 8 & & & 8 \\
\hline Hydraulic Conductivity @90 days immersion & ASTM D5084 & 8 & & & 8 \\
\hline Temperature & ASTM C1064 & 8 & & & 8 \\
\hline $\mathrm{pH}$ & SW-846 Method 9045C & 8 & & & 8 \\
\hline Shrinkage/Swell & ASTM D3877 & 8 & & & 8 \\
\hline Bleed Fluid & SW-846 Method 9095 & 8 & & & 8 \\
\hline Set Time & ASTM C 191 & 8 & & & 8 \\
\hline
\end{tabular}


Table 3-4. Grout Mix Designs for the KRSB Soil

\begin{tabular}{|c|c|c|c|c|c|}
\hline \multirow[t]{2}{*}{ Formulation ${ }^{1}$} & \multirow[t]{2}{*}{$\begin{array}{c}\text { Soil } \\
\text { Fraction }\end{array}$} & $\begin{array}{l}\text { Portland } \\
\text { Cement }\end{array}$ & $\begin{array}{l}\text { Soluble } \\
\text { Bentonite }\end{array}$ & $\begin{array}{l}\text { Sodium } \\
\text { Silicate }\end{array}$ & Water \\
\hline & & \multicolumn{4}{|c|}{ Mix Ratio $^{2}$} \\
\hline 1 & \multirow[t]{4}{*}{ Coarse } & 0.15 & 0.05 & -- & $\overline{0.20}$ \\
\hline 2 & & 0.15 & - & 0.04 & 0.19 \\
\hline 3 & & 0.15 & 0.05 & 0.02 & 0.22 \\
\hline 4 & & 0.15 & 0.05 & 0.04 & 0.24 \\
\hline 1 & \multirow[t]{4}{*}{ Fine } & 0.15 & 0.05 & -- & 0.20 \\
\hline 2 & & 0.15 & -- & 0.04 & 0.19 \\
\hline 3 & & 0.15 & 0.05 & 0.02 & 0.22 \\
\hline 4 & & 0.15 & 0.05 & 0.04 & 0.24 \\
\hline
\end{tabular}

Formulation:

1 - Portland cement and bentonite

2 - Portland cement and 4\% silicate (high)

3 - Portland cement, bentonite, and 2\% silicate (low)

4 - Portland cement, bentonite, and 4\% silicate (high)

2 Fraction of total weight 


\section{Table 3-5. Samples Generated During Treatability Testing of PRSB Soil Samples}

\begin{tabular}{|c|c|c|c|c|c|}
\hline \multirow{2}{*}{ Testing Parameters } & \multirow{2}{*}{ Method } & \multicolumn{4}{|c|}{ Number of Tests } \\
\hline & & Total & Step 1 & Step 2 & Step 3 \\
\hline Atterberg Limits & ASTM D 4318 & 8 & 2 & 6 & \\
\hline Grain Size Distribution & ASTM D422 & 8 & 2 & 6 & \\
\hline Moisture Content & ASTM D2216 & 8 & 2 & 6 & \\
\hline Specific Gravity & ASTM D854 & 8 & 2 & 6 & \\
\hline USCS Soil Classification & ASTM D2487 & 8 & 2 & 6 & \\
\hline Cesium- 137 & Gas Flow & 4 & & 4 & \\
\hline Plutonium-239/240 & Gas Flow & 4 & & 4 & \\
\hline Strontium-90 & Gas Flow & 4 & & 4 & \\
\hline Gross Alpha & Gas Flow & 164 & & 4 & 160 \\
\hline Non-volatile Beta & Gas Flow & 164 & & 4 & 160 \\
\hline Chloride Compounds & SW-846 Method 9056 & 1 & 1 & & \\
\hline Nitrate & SW-846 Method 9056 & 1 & 1 & & \\
\hline Nitrite & ASTM D9056 & 1 & 1 & & \\
\hline Oxidation/Reduction State & ASTM D1498 & 1 & 1 & & \\
\hline Sulfate & SW-846 Method 9056 & 1 & 1 & & \\
\hline Sulfide & SW-846 Method 9034 & 1 & 1 & & \\
\hline UCS@14 days cure & ASTM D2166 & 16 & & & 16 \\
\hline UCS@28 days cure & ASTM D2166 & 16 & & & 16 \\
\hline USC @ 90 days immersion & ASTM D2166 & 16 & & & 16 \\
\hline Hydraulic Conductivity@28 days & ASTM D5084 & 16 & & & 16 \\
\hline Hydraulic Conductivity @ 90 days immersion & ASTM D5084 & 16 & & & 16 \\
\hline Temperature & ASTM C1064 & 16 & & & 16 \\
\hline $\mathrm{pH}$ & SW-846 Method 9045C & 16 & & & 16 \\
\hline Shrinkage/Swell & ASTM D3877 & 16 & & & 16 \\
\hline Bleed Fluid & SW-846 Method 9095 & 16 & & & 16 \\
\hline Set Time & ASTM C191 & 16 & & & 16 \\
\hline
\end{tabular}


Soil Stabilization/Solidification Grout Mix Design Study

C-, K-, L-, and P-Reactor Seepage Basins Closure (U)

Table 3-6. Grout Mix Designs for the PRSB Soil

\begin{tabular}{|c|c|c|c|c|c|}
\hline \multirow[t]{2}{*}{ Formulation ${ }^{1}$} & \multirow[t]{2}{*}{$\begin{array}{c}\text { Soil } \\
\text { Fraction }\end{array}$} & $\begin{array}{c}\text { Portland } \\
\text { Cement }\end{array}$ & $\begin{array}{l}\text { Soluble } \\
\text { Bentonite }\end{array}$ & $\begin{array}{l}\text { Sodium } \\
\text { Silicate }\end{array}$ & Water \\
\hline & & \multicolumn{4}{|c|}{ Mix Ratio $^{2}$} \\
\hline 1 & \multirow[t]{4}{*}{ Coarse } & 0.15 & 0.05 & -- & 0.20 \\
\hline 2 & & 0.15 & -- & 0.04 & 0.19 \\
\hline 3 & & 0.15 & 0.05 & 0.02 & 0.22 \\
\hline 4 & & 0.15 & $\overline{0.05}$ & 0.04 & $\overline{0.24}$ \\
\hline 1 & \multirow[t]{4}{*}{ Fine } & 0.15 & 0.05 & -- & 0.20 \\
\hline 2 & & 0.15 & -- & 0.04 & 0.19 \\
\hline 3 & & 0.15 & 0.05 & 0.02 & 0.22 \\
\hline 4 & & 0.15 & 0.05 & 0.04 & $\overline{0.24}$ \\
\hline 1 & \multirow[t]{4}{*}{ Bulk \#1 } & $\overline{0.15}$ & 0.05 & -- & $\overline{0.20}$ \\
\hline 2 & & 0.15 & -- & 0.04 & 0.19 \\
\hline 3 & & 0.15 & 0.05 & 0.02 & 0.22 \\
\hline 4 & & 0.15 & $\overline{0.05}$ & 0.04 & 0.24 \\
\hline 1 & \multirow[t]{4}{*}{ Bulk \#2 } & 0.15 & 0.05 & -- & 0.20 \\
\hline 2 & & 0.15 & -- & $\overline{0.04}$ & 0.19 \\
\hline 3 & & 0.15 & 0.05 & 0.02 & 0.22 \\
\hline 4 & & 0.15 & 0.05 & 0.04 & 0.24 \\
\hline
\end{tabular}

Formulation:

1 - Portland cement and bentonite

2 - Portland cement and $4 \%$ silicate (high)

3 - Portland cement, bentonite, and $2 \%$ silicate (low)

4 - Portland cement, bentonite, and $4 \%$ silicate (high)

2 Fraction of total weight 
Table 3-7. Samples Generated During Treatability Testing of LRSB Soil Samples

\begin{tabular}{|c|c|c|c|c|c|}
\hline \multirow{2}{*}{ Testing Parameters } & \multirow{2}{*}{ Method } & \multicolumn{4}{|c|}{ Number of Tests } \\
\hline & & Total & Step 1 & Step 2 & Step 3 \\
\hline Afterberg Limits & ASTM D 4318 & 8 & 2 & 6 & \\
\hline Grain Size Distribution & ASTM D422 & 8 & 2 & 6 & \\
\hline Moisture Content & ASTM D2216 & 8 & 2 & 6 & \\
\hline Specific Gravity & ASTM D854 & 8 & 2 & 6 & \\
\hline USCS Soil Classification & ASTM D2487 & 8 & 2 & 6 & \\
\hline Cesium-137 & Gas Flow & 4 & & 4 & \\
\hline Plutonium-239/240 & Gas Flow & 4 & & 4 & \\
\hline Strontium-90 & Gas Flow & 4 & & 4 & \\
\hline Gross Alpha & Gas Flow & 164 & & 4 & 160 \\
\hline Non-volatile Beta & Gas Flow & 164 & & 4 & 160 \\
\hline Chloride Compounds & SW-846 Method 9056 & 1 & 1 & & \\
\hline Nitrate & SW-846 Method 9056 & 1 & 1 & & \\
\hline Nitrite & ASTM D 9056 & $T$ & 1 & & \\
\hline Oxidation/Reduction State & ASTM D1498 & 1 & I & & \\
\hline Sulfate & SW-846 Method 9056 & 1 & 1 & & \\
\hline Sulfide & SW-846 Method 9034 & 1 & 1 & & \\
\hline UCS@14 days cure & ASTM D2166 & 16 & & & 16 \\
\hline UCS@ 28 days cure & ASTM D2166 & 16 & & & 16 \\
\hline USC@90 days immersion & ASTM D2166 & 16 & & & 16 \\
\hline Hydraulic Conductivity@28 days & ASTM D5084 & 16 & & & 16 \\
\hline Hydraulic Conductivity @90 days immersion & ASTM D5084 & 16 & & & 16 \\
\hline Temperature & ASTM C1064 & 16 & & & 16 \\
\hline $\mathrm{pH}$ & SW-846 Method $9045 C$ & 16 & & & 16 \\
\hline Shrinkage/Swell & ASTM D3877 & 16 & & & 16 \\
\hline Bleed Fluid & SW-846 Method 9095 & 16 & & & 16 \\
\hline Set Time & ASTM C191 & 16 & & & 16 \\
\hline
\end{tabular}


Soil Stabilization/Solidification Grout Mix Design Study $\quad$ WSRC-RP-99-4075, Revision 1 C-, K-, L-, and P-Reactor Seepage Basins Closure (U)

August 1999

Table 3-8. Grout Mix Designs for the LRSB Soil

\begin{tabular}{|c|c|c|c|c|c|}
\hline \multirow[t]{2}{*}{ Formulation ${ }^{1}$} & \multirow[t]{2}{*}{$\begin{array}{c}\text { Soil } \\
\text { Fraction }\end{array}$} & $\begin{array}{l}\text { Portland } \\
\text { Cement }\end{array}$ & $\begin{array}{l}\text { Soluble } \\
\text { Bentonite }\end{array}$ & $\begin{array}{l}\text { Sodium } \\
\text { Silicate }\end{array}$ & Water \\
\hline & & \multicolumn{4}{|c|}{ Mix Ratio $^{2}$} \\
\hline 1 & \multirow{4}{*}{ Coarse } & 0.15 & 0.05 & -- & 0.20 \\
\hline 2 & & 0.15 & - & $\overline{0.04}$ & 0.19 \\
\hline 3 & & 0.15 & 0.05 & 0.02 & 0.22 \\
\hline 4 & & $\overline{0.15}$ & 0.05 & 0.04 & $\overline{0.24}$ \\
\hline 1 & \multirow{4}{*}{ Fine } & 0.15 & 0.05 & -- & 0.20 \\
\hline 2 & & 0.15 & - & 0.04 & 0.19 \\
\hline 3 & & 0.15 & 0.05 & 0.02 & 0.22 \\
\hline 4 & & $\overline{0.15}$ & 0.05 & $\overline{0.04}$ & 0.24 \\
\hline
\end{tabular}

Formulation:

1 - Portland cement and bentonite

2 - Portland cement and $4 \%$ silicate (high)

3 - Portland cement, bentonite, and $2 \%$ silicate (low)

4 - Portland cement, bentonite, and $4 \%$ silicate (high)

2 Fraction of total weight

Table 3-9. Physical Characterization of the CRSB Soil Fractions

\begin{tabular}{||c|c|c|c|c|c||}
\hline \multirow{2}{*}{ Soil Fraction } & \multirow{2}{*}{$\begin{array}{c}\text { Moisture } \\
\text { Content } \\
(\% \text { dwb })\end{array}$} & \multicolumn{2}{|c|}{$\begin{array}{c}\text { Liquid } \\
\text { Limit }\end{array}$} & \multicolumn{4}{|c|}{$\begin{array}{c}\text { Plastic } \\
\text { Limit }\end{array}$} & $\begin{array}{c}\text { Plasticity } \\
\text { Index }\end{array}$ & \multirow{2}{*}{$\begin{array}{c}\text { USCS } \\
\text { Class }\end{array}$} \\
\cline { 3 - 6 } & & \multicolumn{3}{|c||}{ Moisture Content (\%) } & \\
\hline Grab Sample\#1 & 14.5 & 26 & 16 & 10 & CL \\
\hline Grab Sample \#2 & 18.5 & 20 & 17 & 3 & ML \\
\hline Coarse Fraction & 12.3 & 21 & 18 & 3 & ML \\
\hline Fine Fraction & 20.0 & 25 & 16 & 9 & CL \\
\hline Bulk Sample \#1 & 14.5 & 24 & 16 & 8 & CL \\
\hline Bulk Sample \#2 & 14.5 & 27 & 15 & 12 & CL \\
\hline Remaining Sample \#1 & 12.6 & 23 & 15 & 8 & CL \\
\hline Remaining Sample \#2 & 13.1 & 21 & 16 & 5 & CL-ML \\
\hline \hline
\end{tabular}

determined on a dry weight basis 
Table 3-10. Particle Size Distribution of the CRSB Soil Fractions

\begin{tabular}{|c|c|c|c|c||}
\hline \multirow{2}{*}{ Particle Size Fraction } & \multicolumn{4}{|c|}{ Percent Solids by Weight } \\
\cline { 2 - 5 } & Gravel & Sand & Silt & Clay \\
\hline Grab Sample \#1 & 2.4 & 71.1 & 7.5 & 19.0 \\
\hline Grab Sample \#2 & 4.7 & 76.9 & 5.7 & 12.7 \\
\hline Coarse Fraction & 2.3 & 79.2 & 5.3 & 13.2 \\
\hline Fine Fraction & 1.9 & 70.5 & 8.4 & 19.2 \\
\hline Bulk Fraction \#1 & 2.0 & 76.8 & 6.5 & 14.7 \\
\hline Bulk Fraction \#2 & 4.5 & 74.8 & 5.5 & 15.2 \\
\hline Remaining Sample \#1 & 9.8 & 71.2 & 5.6 & 13.4 \\
\hline Remaining Sample \#2 & 11.2 & 70.1 & 5.1 & 13.6 \\
\hline \hline
\end{tabular}

Table 3-11. Chemical Characterization of the CRSB Soil Fractions

\begin{tabular}{||l|c|c|}
\hline Sample & Bulk Sample \#1 & Remaining Soil \#1 \\
\hline Parameter & \multicolumn{2}{|c|}{ Result } \\
\hline Chloride $(\mathrm{mg} / \mathrm{kg})$ & $0.629 \mathrm{~J}$ & $1.2 \mathrm{~J}$ \\
\hline Nitrite $(\mathrm{mg} / \mathrm{kg})$ & $<0.551$ & $<0.565$ \\
\hline Nitrate $(\mathrm{mg} / \mathrm{kg})$ & $<0.551$ & $4.1 \mathrm{~J}$ \\
\hline Sulfate $(\mathrm{mg} / \mathrm{kg})$ & $10.1 \mathrm{~J}$ & $11.1 \mathrm{~J}$ \\
\hline Sulfide $(\mathrm{mg} / \mathrm{kg})$ & $<8.99$ & $<8.99$ \\
\hline Relative Oxidation $(\mathrm{mV})$ & nd & $237 \mathrm{~J}$ \\
\hline Reduction Potential $(\mathrm{mV})$ & nd & $441 \mathrm{~J}$ \\
\hline
\end{tabular}

$\mathrm{J}=$ Estimated 
Soil Stabilization/Solidification Grout Mix Design Study $\quad$ WSRC-RP-99-4075. Revision 1 C-, K-, L-, and P-Reactor Seepage Basins Closure (U) August 1999

Table 3-12. Radionuclide Characterization of the CRSB Soil Fractions

\begin{tabular}{|l|c|c|c|c||}
\hline \hline Sample & $\begin{array}{c}\text { Coarse } \\
\text { Fraction }\end{array}$ & $\begin{array}{c}\text { Fine } \\
\text { Fraction }\end{array}$ & $\begin{array}{c}\text { Bulk } \\
\text { Fraction \#1 }\end{array}$ & $\begin{array}{c}\text { Bulk } \\
\text { Fraction \#2 }\end{array}$ \\
\hline Parameter & \multicolumn{4}{|c|}{ Result } \\
\hline Cesium-137 (pCi/g) & 266 & 279 & 683 & 246 \\
\hline Plutonium-239/240 (pCi/g) & $3.47 \mathrm{~J}$ & $3.18 \mathrm{~J}$ & $3.83 \mathrm{~J}$ & $4.12 \mathrm{~J}$ \\
\hline Strontium-90 $(\mathrm{pCi} / \mathrm{g})$ & $18.1 \mathrm{~J}$ & $9.5 \mathrm{~J}$ & $13.6 \mathrm{~J}$ & $11.5 \mathrm{~J}$ \\
\hline Gross Alpha $(\mathrm{pCi} / \mathrm{g})$ & 13.5 & 16.1 & 24.3 & 23.2 \\
\hline Non-volatile Beta $(\mathrm{pCi} / \mathrm{g})$ & 170 & 224 & 284 & 182 \\
\hline
\end{tabular}

$\mathrm{J}=$ Estimated

Table 3-13. Physical Characterization of the KRSB Soil Fractions

\begin{tabular}{|c|c|c|c|c|c|}
\hline \multirow[t]{2}{*}{ Soil Fraction } & \multirow{2}{*}{$\begin{array}{c}\text { Moisture } \\
\text { Content } \\
\text { (\% dwb) }\end{array}$} & $\begin{array}{c}\text { Liquid } \\
\text { Limit }\end{array}$ & $\begin{array}{l}\text { Plastic } \\
\text { Limit }\end{array}$ & $\begin{array}{l}\text { Plasticity } \\
\text { Index }\end{array}$ & \multirow[t]{2}{*}{$\begin{array}{l}\text { USCS } \\
\text { Class }\end{array}$} \\
\hline & & \multicolumn{3}{|c|}{ Moisture Content (\%) } & \\
\hline Grab Sample \#1 & $\overline{9.7}$ & $\overline{\mathrm{NP}}$ & $\overline{\mathrm{NP}}$ & $\overline{\mathrm{NP}}$ & $\overline{\mathrm{NP}}$ \\
\hline Grab Sample \#2 & 18.5 & 30 & 16 & 14 & $\overline{\mathrm{CL}}$ \\
\hline Coarse Fraction & 11.3 & $\mathrm{NP}$ & $\overline{\mathrm{NP}}$ & $\overline{\mathrm{NP}}$ & $\overline{N P}$ \\
\hline Fine Fraction & 22.7 & 33 & 18 & 15 & $\overline{\mathrm{CL}}$ \\
\hline Remaining Sample \#1 & 13.2 & 23 & 16 & 7 & $\overline{C L}-\mathrm{ML}$ \\
\hline Remaining Sample \#2 & 15.1 & 23 & 16 & 7 & CL-ML \\
\hline
\end{tabular}

${ }^{1} \mathrm{NP}=$ nonplastic 
Soil Stabilization/Solidification Grout Mix Design Study

WSRC-RP-99-4075, Revision 1

C-, K-, L-, and P-Reactor Seepage Basins Closure (U)

Table 3-14. Particle Size Distribution of the KRSB Soil Fractions

\begin{tabular}{|c|c|c|c|c|}
\hline \multirow{2}{*}{ Particle Size Fraction } & \multicolumn{4}{|c|}{ Percent Solids by Weight } \\
\cline { 2 - 5 } & Gravel & Sand & Silt & Clay \\
\hline Grab Sample \#1 & 0.3 & 81.6 & 5.9 & 12.2 \\
\hline Grab Sample \#2 & 1.1 & 67.4 & 11.4 & 20.1 \\
\hline Coarse Fraction & 1.0 & 80.4 & 7.5 & 11.1 \\
\hline Fine Fraction & 1.2 & 62.9 & 15.4 & 20.5 \\
\hline Remaining Sample \#1 & 3.6 & 73.1 & 8.0 & 15.3 \\
\hline Remaining Sample \#2 & 0.7 & 74.6 & 9.2 & 15.5 \\
\hline
\end{tabular}

Table 3-15. Chemical Characterization of the KRSB Soil Fractions

\begin{tabular}{||l|c|c||}
\hline Sample & Bulk Sample \#1 & Remaining Soil \\
\hline Parameter & \multicolumn{2}{|c|}{ Result } \\
\hline Chloride $(\mathrm{mg} / \mathrm{kg})$ & $<2.78$ & $0.74 \mathrm{~J}$ \\
\hline Nitrite $(\mathrm{mg} / \mathrm{kg})$ & $<0.556$ & $<0.569$ \\
\hline Nitrate $(\mathrm{mg} / \mathrm{kg})$ & $8.35 \mathrm{~J}$ & $8.64 \mathrm{~J}$ \\
\hline Sulfate $(\mathrm{mg} / \mathrm{kg})$ & $9.09 \mathrm{~J}$ & $6.75 \mathrm{~J}$ \\
\hline Sulfide $(\mathrm{mg} / \mathrm{kg})$ & $<8.7$ & $<8.99$ \\
\hline Relative Oxidation $(\mathrm{mV})$ & nd & $292 \mathrm{~J}$ \\
\hline Reduction Potential $(\mathrm{mV})$ & nd & $496 \mathrm{~J}$ \\
\hline
\end{tabular}

$\mathrm{J}=$-Estimated 
Soil Stabilization/Solidification Grout Mix Design Study

WSRC-RP-99-4075, Revision 1 C-, K-, L-, and P-Reactor Seepage Basins Closure (U)

Table 3-16. Radionuclide Characterization of the KRSB Soil Fractions

\begin{tabular}{||l|c|c||}
\hline Sample & Coarse Fraction & Fine Fraction \\
\hline Parameter & \multicolumn{2}{|c|}{ Result } \\
\hline Cesium-137 (pCi/g) & 1240 & 227 \\
\hline Plutonium-239/240 (pCi/g) & $3.83 \mathrm{~J}$ & $1.93 \mathrm{~J}$ \\
\hline Strontium-90 (pCi/g) & $13.2 \mathrm{~J}$ & $5.02 \mathrm{~J}$ \\
\hline Gross Alpha $(\mathrm{pCi} / \mathrm{g})$ & 21.1 & 12.9 \\
\hline Non-volatile Beta $(\mathrm{pCi} / \mathrm{g})$ & 704 & 293 \\
\hline
\end{tabular}

$\mathrm{J}=$ Estimated

Table 3-17. Physical Characterization of the PRSB Soil Fractions

\begin{tabular}{|c|c|c|c|c|c|}
\hline \multirow[t]{2}{*}{ Soil Fraction } & \multirow{2}{*}{$\begin{array}{c}\text { Moisture } \\
\text { Content } \\
\left(\% \text { dwb }^{1}\right)\end{array}$} & $\begin{array}{l}\text { Liquid } \\
\text { Limit }\end{array}$ & $\begin{array}{l}\text { Plastic } \\
\text { Limit }\end{array}$ & $\begin{array}{l}\text { Plasticity } \\
\text { Index }\end{array}$ & \multirow[t]{2}{*}{$\begin{array}{l}\text { USCS } \\
\text { Class }\end{array}$} \\
\hline & & \multicolumn{3}{|c|}{ Moisture Content (\%) } & \\
\hline Coarse Fraction & 14.9 & 33 & 19 & 14 & $\overline{\mathrm{CL}}$ \\
\hline Fine Fraction & 19.6 & 38 & 20 & 18 & $\overline{\mathrm{CL}}$ \\
\hline Bulk Sample \#1 & 14.9 & 39 & 19 & 20 & $\overline{\mathrm{CL}}$ \\
\hline Bulk Sample \#2 & 13.1 & 45 & 21 & 24 & $\overline{\mathrm{CL}}$ \\
\hline Grab Sample \#1 & 13.1 & 35 & 18 & 17 & $\overline{C L}$ \\
\hline Grab Sample \#2 & 14.9 & 44 & 21 & 23 & $\overline{\mathrm{CL}}$ \\
\hline Remaining Sample \#1 & 14.9 & 46 & 20 & 26 & $\overline{\mathrm{CL}}$ \\
\hline Remaining Sample \#2 & 15.9 & 50 & 23 & 27 & $\overline{\mathrm{CH}}$ \\
\hline
\end{tabular}

Determined on a dry weight basis 
Table 3-18. Particle Size Distribution of the PRSB Soil Fractions

\begin{tabular}{|c|c|c|c|c||}
\hline \multirow{2}{*}{$\begin{array}{c}\text { Particle Size } \\
\text { Fraction }\end{array}$} & \multicolumn{4}{|c|}{ Percent Solids by Weight } \\
\cline { 2 - 5 } & Gravel & Sand & Silt & Clay \\
\hline Coarse Fraction & 3.8 & 67.8 & 9.4 & 19.0 \\
\hline Fine Fraction & 1.2 & 68.8 & 7.7 & 22.3 \\
\hline Bulk Fraction \#1 & 0.9 & 68.6 & 10.9 & 19.6 \\
\hline Bulk Fraction \#2 & 1.6 & 63.6 & 10.7 & 24.1 \\
\hline Grab Sample \#1 & 1.4 & 66.9 & 9.1 & 22.6 \\
\hline Grab Sample \#2 & 5.1 & 69.1 & 7.5 & 18.3 \\
\hline Remaining Sample \#1 & 3.7 & 65.7 & 9.8 & 20.8 \\
\hline Remaining Sample \#2 & 0.9 & 67.3 & 7.8 & 24.0 \\
\hline
\end{tabular}

Table 3-19. Chemical Characterization of the PRSB Soil Fractions

\begin{tabular}{||l|c|c||}
\hline \hline Sample & Bulk Sample \#1 & Remaining Soil \#1 \\
\hline Parameter & \multicolumn{2}{|c|}{ Result } \\
\hline Chloride $(\mathrm{mg} / \mathrm{kg})$ & $1.46 \mathrm{~J}$ & $0.875 \mathrm{~J}$ \\
\hline Nitrite $(\mathrm{mg} / \mathrm{kg})$ & $<0.552$ & $<0.565$ \\
\hline Nitrate $(\mathrm{mg} / \mathrm{kg})$ & $<0.552$ & $0.227 \mathrm{~J}$ \\
\hline Sulfate $(\mathrm{mg} / \mathrm{kg})$ & 14.1 & $11.3 \mathrm{~J}$ \\
\hline Sulfide $(\mathrm{mg} / \mathrm{kg})$ & $<8.99$ & $<9.09$ \\
\hline Relative Oxidation $(\mathrm{mV})$ & nd & $394 \mathrm{~J}$ \\
\hline Reduction Potential $(\mathrm{mV})$ & nd & $598 \mathrm{~J}$ \\
\hline
\end{tabular}


Soil Stabilization/Solidification Grout Mix Design Study

C-, K-, L-, and P-Reactor Seepage Basins Closure (U)

WSRC-RP-99-4075, Revision 1

August 1999

Table 3-20. Radionuclide Characterization of the PRSB Soil Fractions

\begin{tabular}{||l|c|c|c|c||}
\hline \hline Sample & $\begin{array}{c}\text { Coarse } \\
\text { Fraction }\end{array}$ & $\begin{array}{c}\text { Fine } \\
\text { Fraction }\end{array}$ & $\begin{array}{c}\text { Bulk } \\
\text { Fraction \#1 }\end{array}$ & $\begin{array}{c}\text { Bulk } \\
\text { Fraction \#2 }\end{array}$ \\
\hline Parameter & \multicolumn{4}{|c||}{ Result } \\
\hline Cesium-137 (pCi/g) & 273 & 2040 & 226 & 174 \\
\hline Plutonium-239/240 (pCi/g) & 0.182 & 1.62 & 0.289 & 0.292 \\
\hline Strontium-90 (pCi/g) & 9.33 & 14.0 & 19.0 & 25.8 \\
\hline Gross Alpha (pCi/g) & 19.0 & 32.3 & 17.9 & 13.5 \\
\hline Non-volatile Beta (pCi/g) & 239 & 1340 & 338 & 183 \\
\hline
\end{tabular}

Table 3-21. Physical Characterization of the LRSB Soil Fractions

\begin{tabular}{|c|c|c|c|c|c|}
\hline \multirow[t]{2}{*}{ Soil Fraction } & \multirow{2}{*}{$\begin{array}{c}\text { Moisture } \\
\text { Content } \\
(\% \text { dwb) }\end{array}$} & $\begin{array}{l}\text { Liquid } \\
\text { Limit }\end{array}$ & $\begin{array}{c}\text { Plastic } \\
\text { Limit }\end{array}$ & $\begin{array}{l}\text { Plasticity } \\
\text { Index }\end{array}$ & \multirow[t]{2}{*}{$\begin{array}{l}\text { USCS } \\
\text { Class }\end{array}$} \\
\hline & & \multicolumn{3}{|c|}{ Moisture Content (\%) } & \\
\hline Grab Sample \#1 & 14.7 & 34 & 17 & 17 & $\overline{\mathrm{CL}}$ \\
\hline Grab Sample \#2 & 15.1 & 32 & 17 & 15 & $\overline{\mathrm{CL}}$ \\
\hline Coarse Fraction & 15.1 & 36 & 16 & 20 & $\overline{\mathrm{CL}}$ \\
\hline Fine Fraction & 15.9 & 36 & 17 & 19 & $\overline{\mathrm{CL}}$ \\
\hline $\begin{array}{l}\text { Remaining } \\
\text { Sample \#1 }\end{array}$ & 14.6 & 34 & 17 & 17 & $\overline{\mathrm{CL}}$ \\
\hline $\begin{array}{l}\text { Remaining } \\
\text { Sample \#2 }\end{array}$ & 14.9 & 33 & 17 & 16 & $\overline{\mathrm{CL}}$ \\
\hline
\end{tabular}


Table 3-22. Particle Size Distribution of the LRSB Soil Fractions

\begin{tabular}{||c|c|c|c|c||}
\hline \hline \multirow{2}{*}{$\begin{array}{c}\text { Particle Size } \\
\text { Fraction }\end{array}$} & \multicolumn{4}{|c|}{ Percent Solids by Weight } \\
\cline { 2 - 5 } & Gravel & Sand & Silt & Clay \\
\hline Coarse Fraction & 0.8 & 65.0 & 9.9 & 24.3 \\
\hline Fine Fraction & 0.2 & 67.8 & 9.7 & 22.3 \\
\hline Grab Sample \#1 & 0.4 & 72.2 & 7.3 & 20.1 \\
\hline Grab Sample \#2 & 0.4 & 65.8 & 9.8 & 24.0 \\
\hline Remaining Sample \#1 & 0.5 & 65.5 & 10.8 & 23.2 \\
\hline Remaining Sample \#2 & 0.7 & 64.7 & 10.8 & 23.8 \\
\hline
\end{tabular}

Table 3-23. Chemical Characterization of the LRSB Soil Fractions

\begin{tabular}{|l|c|c||}
\hline Sample & Bulk Sample \#1 & Remaining Soil \\
\hline Parameter & \multicolumn{2}{|c|}{ Result } \\
\hline Chloride $(\mathrm{mg} / \mathrm{kg})$ & 3.43 & 3.37 \\
\hline Nitrite $(\mathrm{mg} / \mathrm{kg})$ & $<0.582$ & $<0.568$ \\
\hline Nitrate $(\mathrm{mg} / \mathrm{kg})$ & $0.396 \mathrm{~J}$ & 0.613 \\
\hline Sulfate $(\mathrm{mg} / \mathrm{kg})$ & $4.46 \mathrm{~J}$ & $3.44 \mathrm{~J}$ \\
\hline Sulfide $(\mathrm{mg} / \mathrm{kg})$ & $<9.2$ & $<9.09$ \\
\hline Relative Oxidation $(\mathrm{mV})$ & nd & $368 \mathrm{~J}$ \\
\hline Reduction Potential $(\mathrm{mV})$ & nd & $572 \mathrm{~J}$ \\
\hline
\end{tabular}

$\mathrm{J}=$ Estimated 
Table 3-24. Radionuclide Characterization of the PRSB Soil Fractions

\begin{tabular}{|l|c|c|}
\hline Sample & Coarse Fraction & Fine Fraction \\
\hline Parameter & \multicolumn{2}{|c|}{ Result } \\
\hline Cesium-137 $(\mathrm{pCi} / \mathrm{g})$ & 1.77 & 0.999 \\
\hline Plutonium-239/240 (pCi/g) & $<0.06$ & $<0.06$ \\
\hline Strontium-90 (pCi/g) & $<0.4$ & $<0.4$ \\
\hline Gross Alpha $(\mathrm{pCi} / \mathrm{g})$ & 13.4 & 17.9 \\
\hline Non-volatile Beta $(\mathrm{pCi} / \mathrm{g})$ & $<7.33$ & $<7.58$ \\
\hline
\end{tabular}


Soil Stabilization/Solidification Grout Mix Design Study

WSRC-RP-99-4075, Revision 1

C-, K-, L-, and P-Reactor Seepage Basins Closure (U)

August 1999

Table 3-25. Results for Treated Material from Coarse Fraction of CRSB Soils

\begin{tabular}{||l|c|c|c|c||}
\hline \hline CRSB Soil Fraction & \multicolumn{4}{|c||}{ Coarse Fraction } \\
\hline Formulation ${ }^{1}$ & 1 & 2 & 3 & 4 \\
\hline Parameter & \multicolumn{4}{|c||}{ Result } \\
\hline UCS after 14 days (psi) & 389.5 & 224.4 & 367.4 & 451.8 \\
\hline UCS after 28 days (psi) & 399.7 & 347.7 & 388.9 & 410.0 \\
\hline $\begin{array}{l}\text { UCS after 28 days cure and 90 days } \\
\text { immersion in water (psi) }\end{array}$ & 440.9 & 581.9 & 462.6 & 478.0 \\
\hline $\begin{array}{l}\text { Hydraulic Conductivity after 28 days } \\
\text { (cm/sec) }\end{array}$ & $3 \mathrm{E}-07$ & $3 \mathrm{E}-07$ & $3 \mathrm{E}-07$ & $3 \mathrm{E}-07$ \\
\hline $\begin{array}{l}\text { Hydraulic Conductivity after 28 days cure } \\
\text { and 90 days immersion in water (cm/sec) }\end{array}$ & $7 \mathrm{E}-08$ & $6 \mathrm{E}-07$ & $5 \mathrm{E}-08$ & $3 \mathrm{E}-07$ \\
\hline Shrink/Swell (\% of 28 day failure strain) & 0.24 & 0.28 & 1.34 & 1.36 \\
\hline Leach Index - Gross Alpha & 11.4 & 12.1 & 11.4 & 12.3 \\
\hline Leach Index - Non-volatile Beta & 9.9 & 10.3 & 9.8 & 10.4 \\
\hline Volume Increase (\%) & 39 & 38 & 44 & 47 \\
\hline pH & 12.3 & 12.7 & 12.5 & 12.8 \\
\hline Temperature Rise $\left({ }^{\circ} \mathrm{C}\right)$ & 2 & 2 & 1 & 1 \\
\hline False Set & No & No & No & No \\
\hline Flash Set & No & No & No & No \\
\hline Set Time (hours) & 16 & 16 & 16 & 16 \\
\hline Bleed Fluid & None & None & None & None \\
\hline
\end{tabular}

Formulation:

1 - Portland cement and bentonite

2 - Portland cement and $4 \%$ silicate (high)

3 - Portland cement, bentonite, and $2 \%$ silicate (low)

4 - Portland cement, bentonite, and $4 \%$ silicate (high) 
Table 3-26. Results for Treated Material from Fine Fraction of CRSB Soils

\begin{tabular}{||l|c|c|c|c||}
\hline \hline CRSB Soil Fraction & \multicolumn{4}{|c|}{ Fine Fraction } \\
\hline Formulation ${ }^{1}$ & 1 & 2 & 3 & 4 \\
\hline Parameter & \multicolumn{4}{|c||}{ Result } \\
\hline UCS after 14 days (psi) & 251.4 & 481.8 & 302.0 & 289.0 \\
\hline UCS after 28 days (psi) & 237.0 & 487.0 & 360.8 & 410.7 \\
\hline $\begin{array}{l}\text { UCS after 28 days cure and 90 days } \\
\text { immersion in water (psi) }\end{array}$ & 372.4 & 244.4 & 355.1 & 460.8 \\
\hline $\begin{array}{l}\text { Hydraulic Conductivity after 28 days } \\
\text { (cm/sec) }\end{array}$ & $6 \mathrm{E}-07$ & $4 \mathrm{E}-07$ & $4 \mathrm{E}-07$ & $1 \mathrm{E}-08$ \\
\hline $\begin{array}{l}\text { Hydraulic Conductivity after 28 days cure } \\
\text { and 90 days immersion (cm/sec) }\end{array}$ & $4 \mathrm{E}-06$ & $1 \mathrm{E}-06$ & $3 \mathrm{E}-06$ & $8 \mathrm{E}-07$ \\
\hline Shrink/Swell (\% of 28 day failure strain) & 0.03 & 0.79 & 1.35 & 2.29 \\
\hline Leach Index - Gross Alpha & 11.5 & 12.2 & 11.7 & 12.0 \\
\hline Leach Index - Non-volatile Beta & 10.0 & 10.5 & 10.0 & 10.4 \\
\hline Volume Increase (\%) & 32 & 25 & 41 & 41 \\
\hline pH & 12.4 & 12.8 & 12.6 & 12.7 \\
\hline Temperature Rise ( $\left.{ }^{\circ} \mathrm{C}\right)$ & 1.5 & 0 & 1 & 1 \\
\hline False Set & No & No & No & No \\
\hline Flash Set & No & No & No & No \\
\hline Set Time (hours) & 16 & 16 & 16 & 16 \\
\hline Bleed Fluid & None & None & None & None \\
\hline
\end{tabular}

Formulation:

1 - Portland cement and bentonite

2 - Portland cement and $4 \%$ silicate (high)

3 - Portland cement, bentonite, and $2 \%$ silicate (low)

4 - Portland cement, bentonite, and $4 \%$ silicate (high) 
Table 3-27. Results for Treated Material from Bulk Fraction \#1 of CRSB Soils

\begin{tabular}{||l|c|c|c|c||}
\hline CRSB Soil Fraction & \multicolumn{4}{|c||}{ Bulk Fraction \#1 } \\
\hline Formulation ${ }^{1}$ & 1 & 2 & 3 & 4 \\
\hline Parameter & \multicolumn{4}{|c||}{ Result } \\
\hline UCS after 14 days (psi) & 419.9 & 405.2 & 336.4 & 345.8 \\
\hline UCS after 28 days (psi) & 354.6 & 420.3 & 351.5 & 361.0 \\
\hline $\begin{array}{l}\text { UCS after 28 days cure and 90 days } \\
\text { immersion in water (psi) }\end{array}$ & 342.3 & 481.8 & 472.4 & 590.1 \\
\hline $\begin{array}{l}\text { Hydraulic Conductivity after 28 days } \\
\text { (cm/sec) }\end{array}$ & $2 \mathrm{E}-08$ & $1 \mathrm{E}-08$ & $9 \mathrm{E}-09$ & $2 \mathrm{E}-07$ \\
\hline $\begin{array}{l}\text { Hydraulic Conductivity after 28 days cure } \\
\text { and 90 days immersion in water (cm/sec) }\end{array}$ & $2 \mathrm{E}-05$ & $8 \mathrm{E}-07$ & $5 \mathrm{E}-07$ & $1 \mathrm{E}-07$ \\
\hline Shrink/Swell (\% of 28 day failure strain) & 3.38 & 0.44 & 2.89 & 1.09 \\
\hline Leach Index - Gross Alpha & 12.8 & 12.4 & 11.8 & 12.8 \\
\hline Leach Index - Non-volatile Beta & 11.2 & 11.0 & 10.5 & 11.1 \\
\hline Volume Increase (\%) & 30 & 26 & 37 & 33 \\
\hline pH & 12.3 & 12.8 & 12.6 & 12.6 \\
\hline Temperature Rise $\left({ }^{\circ} \mathrm{C}\right)$ & 2 & 2 & 2 & 2 \\
\hline False Set & No & No & No & No \\
\hline Flash Set & No & No & No & No \\
\hline Set Time (hours) & 16 & 16 & 16 & 16 \\
\hline Bleed Fluid & None & None & None & None \\
\hline
\end{tabular}

Formulation:

1 - Portland cement and bentonite

2 - Portland cement and $4 \%$ silicate (high)

3 - Portland cement, bentonite, and $2 \%$ silicate (low)

4 - Portland cement, bentonite, and $4 \%$ silicate (high) 
Soil Stabilization/Solidification Grout Mix Design Study C-, K-, L-, and P-Reactor Seepage Basins Closure (U)

Table 3-28. Results for Treated Material from Bulk Fraction \#2 of CRSB Soils

\begin{tabular}{|l|c|c|c|c||}
\hline \hline CRSB Soil Fraction & \multicolumn{4}{|c|}{ Bulk Fraction \#2 } \\
\hline Formulation $^{1}$ & $\mathbf{1}$ & $\mathbf{2}$ & $\mathbf{3}$ & $\mathbf{4}$ \\
\hline Parameter & \multicolumn{4}{|c||}{ Result } \\
\hline UCS after 14 days (psi) & 382.2 & 335.3 & 280.9 & 427.9 \\
\hline UCS after 28 days (psi) & 379.1 & 424.3 & 378.6 & 504.1 \\
\hline $\begin{array}{l}\text { UCS after 28 days cure and 90 days } \\
\text { immersion in water (psi) }\end{array}$ & 289.6 & 531.7 & 365.2 & 517.2 \\
\hline $\begin{array}{l}\text { Hydraulic Conductivity after 28 days } \\
\text { (cm/sec) }\end{array}$ & $2 \mathrm{E}-07$ & $2 \mathrm{E}-07$ & $3 \mathrm{E}-07$ & $3 \mathrm{E}-07$ \\
\hline $\begin{array}{l}\text { Hydraulic Conductivity after 28 days cure } \\
\text { and 90 days immersion in water (cm/sec) }\end{array}$ & $9 \mathrm{E}-07$ & $4 \mathrm{E}-07$ & $9 \mathrm{E}-07$ & $2 \mathrm{E}-07$ \\
\hline Shrink/Swell & 4.29 & 0.71 & 0.32 & 1.01 \\
\hline Leach Index - Gross Alpha & 11.9 & 12.7 & 11.9 & 12.0 \\
\hline Leach Index - Non-volatile Beta & 9.8 & 10.2 & 10.2 & 10.4 \\
\hline Volume Increase (\%) & 28 & 25 & 36 & 36 \\
\hline pH & 12.3 & 12.7 & 12.6 & 12.7 \\
\hline Temperature Rise ( $\left.{ }^{\circ} \mathrm{C}\right)$ & 2 & 1 & 1 & 1 \\
\hline False Set & No & No & No & No \\
\hline Flash Set & No & No & No & No \\
\hline Set Time (hours) & 16 & 16 & 16 & 16 \\
\hline Bleed Fluid & None & None & None & None \\
\hline
\end{tabular}

Formulation:

1 - Portland cement and bentonite

2 - Portland cement and 4\% silicate (high)

3 - Portland cement, bentonite, and $2 \%$ silicate (low)

4 - Portland cement, bentonite, and $4 \%$ silicate (high) 
Table 3-29. Results for Treated Material from Coarse Fraction of KRSB Soils

\begin{tabular}{||l|c|c|c|c|c||}
\hline KRSB Soil Fraction & \multicolumn{4}{|c||}{ Coarse Fraction } \\
\hline Formulation & \multicolumn{1}{|c|}{ Result } \\
\hline Parameter & 1 & 2 & $\mathbf{3}$ & $\mathbf{4}$ \\
\hline UCS after 16 days (psi) & 537.8 & 512.7 & 493.7 & 412.2 \\
\hline UCS after 28 days (psi) & 500.7 & 619.1 & 532.7 & 541.0 \\
\hline $\begin{array}{l}\text { UCS after 28 days cure and 90 days } \\
\text { immersion in water (psi) }\end{array}$ & 539.5 & 593.3 & 448.4 & 477.8 \\
\hline $\begin{array}{l}\text { Hydraulic Conductivity after 28 days } \\
\text { (cm/sec) }\end{array}$ & $1 \mathrm{E}-08$ & $3 \mathrm{E}-09$ & $8 \mathrm{E}-07$ & $3 \mathrm{E}-09$ \\
\hline $\begin{array}{l}\text { Hydraulic Conductivity after 28 days cure } \\
\text { and 90 days immersion in water (cm/sec) }\end{array}$ & $4 \mathrm{E}-06$ & $9 \mathrm{E}-07$ & $1 \mathrm{E}-06$ & $1 \mathrm{E}-05$ \\
\hline Shrink/Swell (\% of 28 day failure strain) & 0.64 & $<0.01$ & 4.11 & 1.06 \\
\hline Leach Index - Gross Alpha & 11.7 & 12.1 & 12.6 & 12.9 \\
\hline Leach Index - Non-volatile Beta & 10.9 & 11.0 & 11.0 & 11.5 \\
\hline Volume Increase (\%) & 29 & 28 & 36 & 39 \\
\hline pH & 12.6 & 12.5 & 12.7 & 12.4 \\
\hline Temperature Rise ( $\left.{ }^{\circ} \mathrm{C}\right)$ & 2 & 1 & 2 & 2 \\
\hline False Set & No & No & No & No \\
\hline Flash Set & No & No & No & No \\
\hline Set Time (hours) & 16 & 16 & 16 & 16 \\
\hline Bleed Fluid & None & None & None & None \\
\hline
\end{tabular}

Formulation:

1 - Portland cement and bentonite

2 - Portland cement and $4 \%$ silicate (high)

3 - Portland cement, bentonite, and $2 \%$ silicate (low)

4 - Portland cement, bentonite, and $4 \%$ silicate (high) 
Soil Stabilization/Solidification Grout Mix Design Study

Table 3-30. Results for Treated Material from Fine Fraction of KRSB Soils

\begin{tabular}{||l|c|c|c|c||}
\hline \hline KRSB Soil Fraction & \multicolumn{4}{|c||}{ Fine Fraction } \\
\hline Formulation ' & 1 & 2 & 3 & 4 \\
\hline Parameter & \multicolumn{4}{|c||}{ Result } \\
\hline UCS after 16 days (psi) & 401.1 & 403.4 & 274.8 & 307.0 \\
\hline UCS after 28 days (psi) & 412.2 & 372.4 & 318.3 & 462.2 \\
\hline $\begin{array}{l}\text { UCS after 28 days cure and 90 days } \\
\text { immersion in water (psi) }\end{array}$ & 258.1 & 410.2 & 333.5 & 525.3 \\
\hline $\begin{array}{l}\text { Hydraulic Conductivity after 28 days } \\
\text { (cm/sec) }\end{array}$ & $2 \mathrm{E}-08$ & $9 \mathrm{E}-07$ & $2 \mathrm{E}-09$ & $2 \mathrm{E}-06$ \\
\hline $\begin{array}{l}\text { Hydraulic Conductivity after 28 days cure } \\
\text { and 90 days immersion in water (cm/sec) }\end{array}$ & $3 \mathrm{E}-06$ & $1 \mathrm{E}-06$ & $4 \mathrm{E}-06$ & $3 \mathrm{E}-06$ \\
\hline Shrink/Swell (\% of 28 day failure strain) & 0.39 & 0.34 & $<0.01$ & 0.20 \\
\hline Leach Index - Gross Alpha & 11.6 & 12.0 & 12.3 & 12.4 \\
\hline Leach Index - Non-volatile Beta & 10.2 & 10.6 & 10.5 & 11.1 \\
\hline Volume Increase (\%) & 24 & 20 & 30 & 30 \\
\hline pH & 12.8 & 12.6 & 12.8 & 12.4 \\
\hline Temperature Rise (C) & 2 & 2 & 2 & 2 \\
\hline False Set & No & No & No & No \\
\hline Flash Set & No & No & No & No \\
\hline Set Time (hours) & 16 & 16 & 16 & 16 \\
\hline Bleed Fluid & None & None & None & None \\
\hline
\end{tabular}

Formulation:

1 - Portland cement and bentonite

2 - Portland cement and $4 \%$ silicate (high)

3 - Portland cement, bentonite, and $2 \%$ silicate (low)

4 - Portland cement, bentonite, and $4 \%$ silicate (high) 
Table 3-31. Results for Treated Material from Coarse Fraction of PRSB Soils

\begin{tabular}{||l|c|c|c|c||}
\hline \hline PRSB Soil Fraction & \multicolumn{4}{|c|}{ Coarse Fraction } \\
\hline Formulation ' & 1 & 2 & 3 & 4 \\
\hline Parameter & \multicolumn{4}{|c|}{ Result } \\
\hline UCS after 14 days (psi) & $\mathrm{np}^{2}$ & $\mathrm{np}^{2}$ & $\mathrm{np}^{2}$ & $\mathrm{np}^{2}$ \\
\hline UCS after 28 days (psi) & 592.3 & 537.3 & 646.8 & 638.1 \\
\hline $\begin{array}{l}\text { UCS after 28 days cure and 90 days } \\
\text { immersion in water (psi) }\end{array}$ & 509.7 & 571.4 & 621.5 & 707.1 \\
\hline $\begin{array}{l}\text { Hydraulic Conductivity after 28 days } \\
\text { (cm/sec) }\end{array}$ & $5 \mathrm{E}-09$ & $2 \mathrm{E}-09$ & $4 \mathrm{E}-10$ & $4 \mathrm{E}-08$ \\
\hline $\begin{array}{l}\text { Hydraulic Conductivity after 28 days cure } \\
\text { and 90 days immersion in water (cm/sec) }\end{array}$ & $8 \mathrm{E}-07$ & $3 \mathrm{E}-06$ & $1 \mathrm{E}-06$ & $6 \mathrm{E}-07$ \\
\hline Shrink/Swell (\% of 28 day failure strain) & $<0.01$ & 0.99 & 2.72 & 0.55 \\
\hline Leach Index - Gross Alpha & 13.2 & 13.2 & 13.5 & 12.7 \\
\hline Leach Index - Non-volatile Beta & 10.2 & 10.8 & 10.6 & 11.0 \\
\hline Volume Increase $(\%)$ & 37 & 39 & 40 & 42 \\
\hline pH & 12.4 & 12.6 & 12.8 & 12.7 \\
\hline Temperature Rise ( ${ }^{\circ} \mathrm{C}$ ) & 5 & 3 & 3 & 2 \\
\hline False Set & No & No & No & No \\
\hline Flash Set & No & No & No & No \\
\hline Set Time (hours) & 16 & 16 & 16 & 16 \\
\hline Bleed Fluid & Nonc & None & None & None \\
\hline \hline
\end{tabular}

Formulation:

1 - Portland cement and bentonite

2 - Portland cement and $4 \%$ silicate (high)

3 - Portland cement, bentonite, and $2 \%$ silicate (low)

4 - Portland cement, bentonite, and $4 \%$ silicate (high)

$2 \mathrm{np}$ - this test was not performed 
Soil Stabilization/Solidification Grout Mix Design Study

C-, K-, L-, and P-Reactor Seepage Basins Closure (U)

WSRC-RP-99-4075. Revision 1

August 1999

Table 3-32. Results for Treated Material from Fine Fraction of PRSB Soils

\begin{tabular}{|l|c|c|c|c||}
\hline \hline PRSB Soil Fraction & \multicolumn{4}{|c|}{ Fine Fraction } \\
\hline Formulation ${ }^{1}$ & 1 & 2 & 3 & 4 \\
\hline Parameter & \multicolumn{4}{|c||}{ Result } \\
\hline UCS after 14 days (psi) & $\mathrm{np}^{2}$ & $\mathrm{np}^{2}$ & $\mathrm{np}^{2}$ & $\mathrm{np}^{2}$ \\
\hline UCS after 28 days (psi) & 411.1 & 342.4 & 340.1 & 492.2 \\
\hline $\begin{array}{l}\text { UCS after 28 days cure and 90 days } \\
\text { immersion in water (psi) }\end{array}$ & 314.2 & 315.8 & 343.8 & 453.0 \\
\hline $\begin{array}{l}\text { Hydraulic Conductivity after 28 days } \\
\text { (cm/sec) }\end{array}$ & $7 \mathrm{E}-09$ & $8 \mathrm{E}-10$ & $9 \mathrm{E}-10$ & $8 \mathrm{E}-10$ \\
\hline $\begin{array}{l}\text { Hydraulic Conductivity after 28 days cure } \\
\text { and 90 days immersion (cm/sec) }\end{array}$ & $8 \mathrm{E}-07$ & $1 \mathrm{E}-06$ & $4 \mathrm{E}-07$ & $3 \mathrm{E}-06$ \\
\hline Shrink/Swell (\% of 28 day failure strain) & 5.54 & 0.53 & 5.33 & 1.14 \\
\hline Leach Index - Gross Alpha & 13.4 & 13.0 & 13.0 & 12.8 \\
\hline Leach Index - Non-volatile Beta & 10.6 & 10.9 & 10.6 & 11.0 \\
\hline Volume Increase (\%) & 32 & 34 & 33 & 36 \\
\hline pH & 12.5 & 12.7 & 12.8 & 12.9 \\
\hline Temperature Rise ( ${ }^{\circ} \mathrm{C}$ ) & 3 & 2 & 2 & 2 \\
\hline False Set & No & No & No & No \\
\hline Flash Set & No & No & No & No \\
\hline Set Time (hours) & 16 & 16 & 16 & 16 \\
\hline Bleed Fluid & None & None & None & None \\
\hline \hline
\end{tabular}

Formulation:

1 - Portland cement and bentonite

2 - Portland cement and $4 \%$ silicate (high)

3 - Portland cement, bentonite, and $2 \%$ silicate (low)

4 - Portland cement, bentonite, and $4 \%$ silicate (high)

$2 \mathrm{np}$ - this test was not performed 
Table 3-33. Results for Treated Material from Bulk Fraction \#1 of PRSB Soils

\begin{tabular}{|c|c|c|c|c|}
\hline \multirow{2}{*}{$\begin{array}{l}\text { PRSB Soil Fraction } \\
\text { Formulation }^{1}\end{array}$} & \multicolumn{4}{|c|}{ Bulk Fraction \#1 } \\
\hline & 1 & 2 & 3 & 4 \\
\hline Parameter & \multicolumn{4}{|c|}{ Result } \\
\hline UCS after 14 days (psi) & $n p^{2}$ & $\mathrm{np}^{2}$ & $n p^{2}$ & $n p^{2}$ \\
\hline UCS after 28 days (psi) & 543.4 & 737.1 & 495.2 & 504.8 \\
\hline $\begin{array}{l}\text { UCS after } 28 \text { days cure and } 90 \text { days } \\
\text { immersion in water (psi) }\end{array}$ & 532.9 & 685.2 & 359.7 & 687.8 \\
\hline $\begin{array}{l}\text { Hydraulic Conductivity after } 28 \text { days } \\
(\mathrm{cm} / \mathrm{sec})\end{array}$ & $3 \mathrm{E}-08$ & $7 \mathrm{E}-10$ & $1 \mathrm{E}-09$ & $2 \mathrm{E}-08$ \\
\hline $\begin{array}{l}\text { Hydraulic Conductivity after } 28 \text { days cure } \\
\text { and } 90 \text { days immersion in water }(\mathrm{cm} / \mathrm{sec})\end{array}$ & $4 \mathrm{E}-07$ & $6 \mathrm{E}-07$ & $9 \mathrm{E}-07$ & $1 \mathrm{E}-06$ \\
\hline Shrink/Swell ( $\%$ of 28 day failure strain) & 0.28 & 1.38 & 4.52 & 0.81 \\
\hline Leach Index - Gross Alpha & 12.9 & 13.1 & 12.8 & 12.8 \\
\hline Leach Index - Non-volatile Beta & 9.8 & 10.4 & 10.0 & 10.8 \\
\hline Volume Increase (\%) & 37 & 42 & 49 & 43 \\
\hline $\mathrm{pH}$ & 12.4 & 12.6 & 12.7 & 12.9 \\
\hline Temperature Rise $\left({ }^{\circ} \mathrm{C}\right)$ & 3 & 1 & 1 & 1 \\
\hline False Set & No & No & No & No \\
\hline Flash Set & No & No & No & No \\
\hline Set Time (hours) & 16 & 16 & 16 & 16 \\
\hline Bleed Fluid & None & None & None & None \\
\hline $\begin{array}{l}\text { Formulation: } \\
1 \text { - Portland cement and bentonite } \\
2 \text { - Portland cement and } 4 \% \text { silicate (hig } \\
3 \text { - Portland cement, bentonite, and } 2 \% \\
4 \text { - Portland cement, bentonite, and } 4 \% \\
2 \text { np - this test was not performed }\end{array}$ & $\begin{array}{l}\text { cate (lo } \\
\text { cate (hi }\end{array}$ & & & \\
\hline
\end{tabular}


Soil Stabilization/Solidification Grout Mix Design Study WSRC-RP-99-4075, Revision 1 C-, K-, L-, and P-Reactor Seepage Basins Closure (U)

Table 3-34. Results for Treated Material from Bulk Fraction \#2 of PRSB Soils

\begin{tabular}{|l|c|c|c|c||}
\hline \hline PRSB Soil Fraction & \multicolumn{4}{|c|}{ Bulk Fraction \#2 } \\
\hline Formulation' & 1 & 2 & 3 & 4 \\
\hline Parameter & \multicolumn{4}{|c||}{ Result } \\
\hline UCS after 14 days (psi) & $\mathrm{np}^{2}$ & $\mathrm{np}^{2}$ & $\mathrm{np}^{2}$ & $\mathrm{np}^{2}$ \\
\hline UCS after 28 days (psi) & 585.3 & 594.4 & 487.7 & 543.1 \\
\hline $\begin{array}{l}\text { UCS after 28 days cure and 90 days } \\
\text { immersion in water (psi) }\end{array}$ & 392.6 & 663.6 & 578.8 & 780.9 \\
\hline $\begin{array}{l}\text { Hydraulic Conductivity after 28 days } \\
\text { (cm/sec) }\end{array}$ & $3 \mathrm{E}-09$ & $6 \mathrm{E}-08$ & $5 \mathrm{E}-10$ & $2 \mathrm{E}-08$ \\
\hline $\begin{array}{l}\text { Hydraulic Conductivity after 28 days cure } \\
\text { and 90 days immersion in water (cm/sec) }\end{array}$ & $8 \mathrm{E}-07$ & $2 \mathrm{E}-06$ & $6 \mathrm{E}-07$ & $7 \mathrm{E}-07$ \\
\hline Shrink/Swell (\% of 28 day failure strain) & 5.48 & 0.73 & 7.18 & 0.02 \\
\hline Leach Index - Gross Alpha & 12.3 & 12.8 & 12.6 & 12.8 \\
\hline Leach Index - Non-volatile Beta & 9.6 & 10.0 & 9.8 & 10.3 \\
\hline Volume Increase (\%) & 34 & 36 & 35 & 40 \\
\hline PH & 12.4 & 12.4 & 12.7 & 12.8 \\
\hline Temperature Rise ( $\left.{ }^{\circ} \mathrm{C}\right)$ & 2 & 2 & 2 & 2 \\
\hline False Set & No & No & No & No \\
\hline Flash Set & No & No & No & No \\
\hline Set Time (hours) & 16 & 16 & 16 & 16 \\
\hline Bleed Fluid & None & None & None & None \\
\hline \hline
\end{tabular}

Formulation:

1 - Portland cement and bentonite

2 - Portland cement and $4 \%$ silicate (high)

3 - Portland cement, bentonite, and $2 \%$ silicate (low)

4 - Portland cement, bentonite, and $4 \%$ silicate (high)

$2 \mathrm{np}$ - this test was not performed 
Soil Stabilization/Solidification Grout Mix Design Study

WSRC-RP-99-4075, Revision 1

C-, K-, L-, and P-Reactor Seepage Basins Closure (U)

August 1999

Table 3-35. Results for Treated Material from Coarse Fraction of LRSB Soils

\begin{tabular}{|c|c|c|c|c|}
\hline LRSB Soil Fraction & \multicolumn{4}{|c|}{ Coarse Fraction } \\
\hline Formulation $^{1}$ & 1 & 2 & 3 & 4 \\
\hline Parameter & \multicolumn{4}{|c|}{ Result } \\
\hline UCS after 14 days (psi) & $n p^{2}$ & $n p^{2}$ & $n p^{2}$ & $\mathrm{np}^{2}$ \\
\hline UCS after 28 days (psi) & 473.1 & 639.6 & 607.6 & 509.6 \\
\hline $\begin{array}{l}\text { UCS after } 28 \text { days cure and } 90 \text { days } \\
\text { immersion in water (psi) }\end{array}$ & 613.0 & 982.5 & 426.9 & 697.0 \\
\hline $\begin{array}{l}\text { Hydraulic Conductivity after } 28 \text { days } \\
(\mathrm{cm} / \mathrm{sec})\end{array}$ & $9 \mathrm{E}-09$ & $3 E-09$ & $3 \mathrm{E}-09$ & $3 \mathrm{E}-08$ \\
\hline $\begin{array}{l}\text { Hydraulic Conductivity after } 28 \text { days cure } \\
\text { and } 90 \text { days immersion in water }(\mathrm{cm} / \mathrm{sec})\end{array}$ & $1 \mathrm{E}-06$ & $2 \mathrm{E}-06$ & $1 \mathrm{E}-06$ & $2 \mathrm{E}-06$ \\
\hline Shrink/Swell ( $\%$ of 28 day failure strain) & 0.22 & 0.75 & 4.13 & 1.12 \\
\hline Leach Index - Gross Alpha & 12.6 & 13.1 & 12.4 & 12.4 \\
\hline Leach Index - Non-volatile Beta ${ }^{3}$ & nd & nd & nd & $\overline{\text { nd }}$ \\
\hline Volume Increase (\%) & 33 & 36 & 34 & 40 \\
\hline $\mathrm{PH}$ & 12.4 & 12.7 & 12.7 & 12.8 \\
\hline Temperature Rise $\left({ }^{\circ} \mathrm{C}\right)$ & 3 & 1 & 1 & 0 \\
\hline False Set & No & No & No & No \\
\hline Flash Set & Yes & Yes & Yes & Yes \\
\hline Set Time (hours) & 4 & 4 & 4 & 4 \\
\hline Bleed Fluid & None & None & None & None \\
\hline
\end{tabular}

Formulation:

1 - Portland cement and bentonite

2 - Portland cement and 4\% silicate (high)

3 - Portland cement, bentonite, and $2 \%$ silicate (low)

4 - Portland cement, bentonite, and $4 \%$ silicate (high)

$2 \mathrm{np}$ - this test was not performed

3 Total non-volatile beta value less than detection limit 
Table 3-36. Results for Treated Material from Fine Fraction of LRSB Soils

\begin{tabular}{|c|c|c|c|c|}
\hline \multirow{2}{*}{$\begin{array}{l}\text { LRSB Soil Fraction } \\
\text { Formulation }^{1}\end{array}$} & \multicolumn{4}{|c|}{ Fine Fraction } \\
\hline & 1 & 2 & 3 & 4 \\
\hline Parameter & \multicolumn{4}{|c|}{ Result } \\
\hline UCS after 14 days (psi) & $\overline{n p^{2}}$ & $n \mathrm{p}^{2}$ & $\overline{n p^{2}}$ & $n p^{2}$ \\
\hline UCS after 28 days (psi) & 490.6 & $\overline{376.6}$ & 417.5 & 546.6 \\
\hline $\begin{array}{l}\text { UCS after } 28 \text { days cure and } 90 \text { days } \\
\text { immersion in water ( } p s i)\end{array}$ & 479.9 & 461.6 & 499.3 & 459.8 \\
\hline $\begin{array}{l}\text { Hydraulic Conductivity after } 28 \text { days } \\
(\mathrm{cm} / \mathrm{sec})\end{array}$ & $7 \mathrm{E}-10$ & $7 \mathrm{E}-09$ & $5 \mathrm{E}-10$ & $2 \mathrm{E}-09$ \\
\hline $\begin{array}{l}\text { Hydraulic Conductivity after } 28 \text { days cure } \\
\text { and } 90 \text { days immersion in water }(\mathrm{cm} / \mathrm{sec})\end{array}$ & $1 \mathrm{E}-06$ & 2-E07 & $9 \mathrm{E}-09$ & $2 \mathrm{E}-08$ \\
\hline Shrink/Swell (\% of 28 day failure strain) & 2.32 & 0.10 & 1.98 & 1.07 \\
\hline Leach Index - Gross Alpha & 12.8 & 13.1 & 13.0 & 13.1 \\
\hline Leach Index - Non-volatile Beta ${ }^{3}$ & nd & nd & nd & nd \\
\hline Volume Increase (\%) & 33 & 34 & 33 & 37 \\
\hline $\mathrm{pH}$ & 12.5 & 12.6 & 12.6 & 12.9 \\
\hline Temperature Rise (C) & 3 & 1 & 2 & 2 \\
\hline False Set & No & No & No & No \\
\hline Flash Set & Yes & Yes & Yes & Yes \\
\hline Set Time (hours) & 4 & 4 & 4 & 4 \\
\hline Bleed Fluid & None & None & None & None \\
\hline
\end{tabular}

Formulation:

1 - Portland cement and bentonite

2 - Portland cement and $4 \%$ silicate (high)

3 - Portland cement, bentonite, and $2 \%$ silicate (low)

4 - Portland cement, bentonite, and $4 \%$ silicate (high)

$2 \mathrm{np}$ - this test was not performed

3 Total non-volatile beta value was less than detection limit 
This page intentionally left blank. 


\subsection{DATA ANALYSIS AND INTERPRETATION}

\subsection{Soil Characterization}

\subsubsection{CRSB Soil Fractions}

Visually the CRSB soil, when initially placed in the plastic pans, appeared to be composed of balls of clay-aggregated material ("clay balls") and loamy sand. A sample of each soil type was obtained for characterization (Grab Samples \#1 and \#2). The coarse and fine fractions were prepared as outlined in Section 3.1.1. The remaining material was homogenized and separated into two Bulk Fractions. Two samples (Remaining Soil \#1 and Remaining Soil \#2) were collected from the residual soil for analysis. Samples were also obtained from the bulk fractions (Bulk Fraction \#1 and Bulk Fraction \#2).

The particle size distribution results (Table 3-9) were as expected. The Coarse Fraction contained more sand-sized particles and less clay-sized particles than the Fine Fraction, and the Bulk Fractions were intermediate. The particle size of the grab samples, one from the clay balls and one from the loamy sand, mirrored those of the coarse and fine fraction. Consistent with the material selected for the samples, Grab Sample \#1 had a particle size distribution similar to that of the Fine Fraction, while Grab Sample \#2 had a particle size distribution similar to that of the Coarse Fraction. The Remaining Soil samples had particle size distributions similar to those of the Bulk Fraction samples. The Fine Fraction and Grab Sample \#1 were classified as sandy loam/sandy clay loam soils. The Coarse Fraction and Grab Sample \#2 were classified as loamy sand soils. The Bulk Fraction and Remaining Soil samples were classified as sandy loam soils.

The moisture content and Atterberg limits results (Table 3-10) were in agreement with the particle size distribution results. The Fine Fraction and Grab Sample \#1 had higher moisture contents, higher liquid limits, and higher plasticity indices, as would be expected due to their higher clay content. Conversely, the Coarse Fraction and Grab Sample \#2 had lower moisture contents, lower liquid limits, and low plasticity indices, as expected due to their higher sand content. Overall, the results for the Bulk Fractions and the Remaining Soil samples were intermediate between these two extremes.

The chemical characterization of the Bulk Sample\#1 and the Remaining Soil \#1 samples indicated that the samples contained low levels of chloride, nitrite, nitrate, sulfate, and sulfide (Table 3-11). The presence of these compounds at these low levels suggests that they will have little or no inhibitory effect on the cementitious reactions which occur during the curing of 
Portland cement. The oxidation/reduction data for the Remaining Soil \#1 sample indicated an oxidation/reduction potential well within the typical range for surface soils.

The radionuclide results in Table 3-12 indicate the levels of radionuclides in each sample were similar, although Cs-137 and gross beta levels showed variability.

\subsubsection{KRSB Soil Fractions}

The KRSB soil visually appeared to be composed of balls of clay-aggregated material ("clay balls") and loamy sand. A sample of each was obtained for characterization (Grab Samples \#1 and \#2). The Coarse and Fine Fractions were prepared as outlined in section 3.1.2. The soil remaining after extraction of the Course and Fine Fractions was homogenized prior to obtaining the Remaining Soil samples.

The particle size distribution results (Table 3-13) confirm that the Coarse Fraction contained more sand-sized particles and less clay-sized particles than the Fine Fraction. The particle size of the Grab Samples, one from the clay balls and one from the loamy sand, mirrored those of the Coarse and Fine Fraction. Grab Sample \#2 had a particle size distribution similar to that of the Fine Fraction, while Grab Sample \#1 had a particle size distribution similar to that of the Coarse Fraction. The particle size distributions of the Remaining Soil samples were intermediate of the Coarse and Fine Fractions. The Fine Fraction and Grab Sample \#2 were classified as sandy clay loam soils. The coarse fraction and Grab Sample \#1 were classified as loamy sand soils. The Remaining Soil samples were classified as sandy loam soils.

The moisture content and Atterberg limits results (Table 3-14) were in agreement with the particle size distribution results. The Fine Fraction and Grab Sample \#2 had higher moisture contents, higher liquid limits, and higher plasticity indices, as would be expected due to their higher clay content. Conversely, the Coarse Fraction and Grab Sample \#1 had lower moisture contents and were non-plastic, as would be expected with their higher sand content. The results for the Remaining Soil samples were intermediate.

The chemical characterization of the Bulk Sample \#1 and the Remaining Soil \#1 samples indicated that the samples contained low levels of chloride, nitrite, nitrate, sulfate, and sulfide (Table 3-15). The presence of these compounds at these low levels suggests that these chemical compounds will have little or no inhibitory effect on the cementitious reactions which occur during the curing of Portland cement. The oxidation/reduction data for the Remaining Soil \#1 sample indicated the oxidation/reduction potential of the sample was well within the typical range for surface soils. 
The radionuclide results in Table 3-16 indicate levels of radionuclides in the coarse and fine fractions were similar, although the Coarse Fraction contained higher Cs-137 and gross beta levels.

\subsubsection{PRSB Soil Fractions}

The PRSB soil was composed primarily of balls of clay-aggregated material ("clay balls") with minor amounts of loamy sand. Grab samples were obtained for characterization (Grab Samples \#1 and \#2). The Coarse and Fine Fractions were obtained as outlined in Section 3.2.1. The remaining material was homogenized and split into two bulk fractions and two samples (Remaining Soil \#1 and Remaining Soil \#2). Samples were also obtained from the bulk fractions (Bulk Fraction \#1 and Bulk Fraction \#2).

The particle size distribution results (Table 3-17) indicated that the PRSB Coarse Fraction contained more sand-sized particles and less clay-sized particles than the PRSB Fine Fraction. The particle size distributions of the PRSB Bulk Fractions, the Grab Samples, and the Remaining Soil samples were intermediate to the PRSB Coarse and Fine Fractions. The PRSB Fine Fraction and Remaining Soil \#1 were classified as loam soils. The PRSB Coarse Fraction and the other samples were classified as sandy clay loam soils.

The moisture content and Atterberg limits results (Table 3-18) are in agreement with the particle size distribution results. There were very little differences seen for the moisture contents, liquid limits, plastic limits, and plasticity indices between the PRSB samples. This was probably due to their higher clay contents than the CRSB and KRSB soils.

The chemical characterization of the Bulk Sample\#l and the Remaining Soil \#l samples indicated that the samples contained low levels of chloride, nitrite, nitrate, sulfate, and sulfide (Table 3-19). At these low levels, these chemical compounds will have little to no inhibitory effect on the cementitious reactions which occur during the curing of Portland cement. The oxidation/reduction data for the Remaining Soil \#1 sample indicated the oxidation/reduction potential of the sample was well within the typical range for surface soils.

The radionuclide results in Table 3-20 indicate levels of radionuclides in Coarse and Bulk Fractions were similar, though these was some variability, especially with the Cs-137, Sr-90, and gross beta levels. The Fine Fraction contained significantly higher Cs-137, Pu-239/240, and gross beta levels. 


\subsubsection{LRSB Soil Fractions}

The LRSB soil was composed of balls of clay-aggregated material ("clay balls") and loamy sand. A sample of each was obtained for characterization (Grab Samples \#1 and \#2). The Coarse and Fine Fractions were obtained as outlined in Section 3.2.2. The remaining soil was homogenized prior to obtaining the Remaining Soil samples.

The particle size distribution results (Table 3-21) indicated that the Coarse Fraction contained more sand-sized particles and less clay-sized particles than the Fine Fraction. The particle size of the grab samples, one from the clay balls and one from the loamy sand, mirrored those of the Coarse and Fine Fraction. Grab Sample \#1 had a particle size distribution similar to that of the Fine Fraction, while Grab Sample \#2 had a particle size distribution similar to that of the Coarse Fraction. The particle size distributions of the Remaining Soil samples were intermediate of the Coarse and Fine Fractions. The Fine Fraction and Grab Sample \#2 would be classified as loam soils. The Coarse Fraction and Grab Sample \#1 were classified as sandy clay loam soils. The Remaining Soil samples were classified as sandy loam soils.

The moisture content and Atterberg limits results (Table 3-22) were in agreement with the particle size distribution results. As with the PRSB soils, there was very little differences seen for the moisture contents, liquid limits, plastic limits, and plasticity indices between the LRSB samples. This was probably due to their higher clay contents than the CRSB and KRSB soils.

The chemical characterization of the Bulk Sample\#1 and the Remaining Soil \#1 samples indicated that the samples contained low levels of chloride, nitrite, nitrate, sulfate, and sulfide (Table 3-23). At these low levels, these chemical compounds will have little or no inhibitory cffect on the cementitious reactions which occur during the curing of Portland cement. The oxidation/reduction data for the Remaining Soil \#1 sample indicated an oxidation/reduction potential of the sample well within the typical range for surface soils.

The radionuclide results in Table 3-24 indicate levels of radionuclides in the coarse and fine fractions were similar, though the levels of Cs-137, Pu-239/240, Sr-90, and gross beta in the PRSB soil fractions were much lower than soil fractions from the other three reactor seepage basins. 


\subsection{Treated Material Performance Testing}

\subsubsection{Treated Material Performance Testing}

The cured treated material was subjected to the performance criteria testing listed in Tables 3-1, 3-3, 3-5 and 3-7 for Step 3. The tests were conducted to evaluate the effectiveness of the final stabilized waste material performance criteria (Table 2-1), with the exception of the criteria for the batching, hazardous dust/vapor release and the gas generation which were not performed. The results from the performance testing provide information on the leachability, durability, hydraulic conductivity, strength, and long-term durability of the S/S treated soil material. Test results are in Appendix A, Attachment A.

\subsubsection{CRSB Treated Material}

The results of the performance testing on the treated material produced from the treatment of the CRSB soil fractions are summarized in Tables 3-25 through 3-28.

The UCS results indicated that the treated material from the all soil fractions reached 200 to 400 psi strength within 14 days of curing, exceeding the performance criterion of 100 psi. Overall, little additional strength was gained over the next 14 days (28 days total) of curing. Variation in the UCS was not correlated to the amount of sodium bentonite or sodium silicate in the formulation, indicating that these had little effect on the UCS attained for a given formulation.

The hydraulic conductivity, after 28 days of curing, ranged from $6 \times 10^{-7}$ to $9 \times 10^{-9} \mathrm{~cm} / \mathrm{sec}$ for the treated material from the CRSB soil fractions. The Portland cement and sodium silicate and Portland cement, bentonite, and sodium silicate (low) formulations produced treated material met the performance criterion $\left(<1 \times 10^{-8} \mathrm{~cm} / \mathrm{sec}\right)$ for Bulk Fraction\#1. The average hydraulic conductivity for each formulation considering all soil fractions were essentially identical, approximately $1 \times 10^{-7} \mathrm{~cm} / \mathrm{sec}$ (Table 4-1).

Immersion in water for 90 days had little to no detrimental effect on the UCS values for the CRSB formulations. Most formulations had an increase in UCS during the 90 day water immersion; only the fine soil, Portland cement and silicate (high) and bulk fraction \#2 soil, Portland cement and bentonite had significant reductions in UCS ( $50 \%$ and $24 \%$, respectively).

Immersion in water affected the hydraulic conductivity of the formulations for the CRSB soils. Previous stabilization testing experience has indicated that immersion in water will increase the hydraulic conductivity by 1 to 2 orders of magnitude. Minor increases or decreases within the 
expected range were seen for the hydraulic conductivity after immersion for most CRSB formulations. With the exception of the Fine Fraction, nearly all formulations in all soil fractions had hydraulic conductivity values of less than $1 \times 10^{-6} \mathrm{~cm} / \mathrm{sec}$ even after immersion in water for 90 days. The average hydraulic conductivity for each formulation ranged from $1 \times 10^{-6} \mathrm{~cm} / \mathrm{sec}$ to $3 \times 10^{-7} \mathrm{~cm} / \mathrm{sec}$ (Table 4-2).

The gross alpha and gross nonvolatile beta leach indices for a treated materials from the CRSB soil fractions met the performance criterion ( $>8)$. Generally, the Portland cement and bentonite and Portland cement, bentonite, and silicate (low) formulations had lower leach indices, suggesting that the high addition level of sodium silicate increases the immobilization of the radionuclides.

The shrink/swell results indicated that the treated material from the CRSB did not exhibit significant deformation during such events. The shrink/swell values were well below the performance criterion of less than $25 \%$ of the 28 -day UCS failure strain.

The volume increases for the treated CRSB material were similar to those reported for similar grout stabilization projects and met the performance criterion of $<50 \%$. The volume increases were slightly higher for the CRSB coarse fraction as compared to the other fractions.

The $\mathrm{pH}$ values of the treated material from the CRSB fractions were high (12.2 to 12.8), exceeding the performance criterion $(>10)$. These $\mathrm{pH}$ values may drop slightly over time as the treated material is exposed to carbonate in the environment.

The setting characteristics of the treated material from the CRSB soil fractions were favorable. The temperature rises of the treated material, measured in the cure within 1 hour after treatment, were low $\left(0-2{ }^{\circ} \mathrm{C}\right)$, mostly due to the low Portland cement addition level. During full-scale treatment, it is likely that the temperature rise of the treated material would be much larger. The treated material did not false or flash set, and the treated material set overnight. No bleed fluid was noted for the treated material within 24 hours of treatment.

\subsubsection{KRSB Treated Material}

The results of the performance testing on the treated material produced from the KRSB soil fractions are summarized in Tables 3-29 and 3-30.

The UCS results indicated that the treated material from the KRSB soil fractions reached 270 to $540 \mathrm{psi}$ strength within 16 days of curing. These UCS values exceed the performance criterion of 
100 psi. The UCS measurements were taken on Day 16 instead of Day 14. as Day 14 was a Saturday. Overall, little additional strength was gained over the next 12 days (28 days total) of curing. Variation in the UCS was not correlated to the amount of sodium bentonite or sodium silicate in the formulation, indicating that this parameter had little effect on the UCS attained for a given formulation.

The hydraulic conductivity after 28 days of curing ranged from $2 \times 10^{-6}$ to $2 \times 10^{-9} \mathrm{~cm} / \mathrm{sec}$ for the treated material from the KRSB soil fractions. For the KRSB Coarse Fraction, the treated material from three of the four formulations met the performance criterion $\left(<1 \times 10^{-8} \mathrm{~cm} / \mathrm{sec}\right)$, with only the Portland cement, bentonite, and silicate (low) formulation not meeting it. For the KRSB Fine Fraction, the Portland cement, bentonite, and silicate (low) was the only formulation which met the performance criterion, although the Portland cement and bentonite formulation nearly met it with a hydraulic conductivity of $2 \times 10^{-8} \mathrm{~cm} / \mathrm{sec}$. The average hydraulic conductivity for the Portland cement and bentonite formulation met the performance criterion, while the other three formulations did not meet the performance criterion (Table 4-1).

Immersion in water for 90 days had little to no detrimental effect on the UCS values for the KRSB formulations. Most formulations had an increase in UCS during the 90 day water immersion; only the coarse soil, Portland cement, bentonite and silicate (low) and fine soil, Portland cement and bentonite had significant reductions in UCS (16\% and 37\%, respectively).

The immersion in water did significantly affect the hydraulic conductivity of the all formulations for the KRSB soils. Only one formulation in each soil type met a value of $1 \times 10^{-6} \mathrm{~cm} / \mathrm{sec}$. Previous stabilization testing shows that immersion in water will increase the hydraulic conductivity by 1 to 2 orders of magnitude. Several of the formulations for the KRSB had larger increases in hydraulic conductivity after the 90-day immersion in water, though this may be an artifact of their low initial hydraulic conductivity. None of the formulations met the performance criterion for the average hydraulic conductivity (Table 4-2).

The gross alpha and gross nonvolatile beta leach indices for a treated materials from the KRSB soil fractions met the performance criterion (>8). Generally, the Portland cement and bentonite formulation had the lowest leach indices, suggesting that the addition of sodium silicate increases the immobilization of the radionuclides.

The shrink/swell results indicate that the treated material from the KRSB will not exhibit significant deformation during such events. The shrink/swell values were well below the performance criterion of less than $25 \%$ of the 28 -day UCS failure strain. 
The volume increases for the treated KRSB material was similar to those reported for similar grout stabilization projects. The volume increase performance criterion of less than $50 \%$ was met by all KRSB formulations.

The $\mathrm{pH}$ values of the treated material from the KRSB fractions were high (11.6 to 12.8). exceeding the performance criterion $(>10)$. These $\mathrm{pH}$ values may drop slightly over time as the treated material is exposed to carbonate in the environment.

The setting characteristics of the treated material from the KRSB soil fractions were favorable. The temperature rises of the treated material, measured in the cure within 1 hour after treatment, were low $\left(0-2{ }^{\circ} \mathrm{C}\right)$, mostly due to the low Portland cement addition level. During full-scale treatment, it is likely that the temperature rise of the treated material would be much larger. The treated material did not false or flash set, and the treated material set overnight. No bleed fluid was noted for the treated material within 24 hours of treatment.

\subsubsection{PRSB Treated Material}

The results of the performance testing on the treated material produced from the PRSB soil fractions are summarized in Tables 3-31 through 3-34.

The UCS results indicated that the treated material from the PRSB soil fractions achieve a strength of 340 to $740 \mathrm{psi}$ within 28 days of curing. These UCS values exceed the performance criterion of 100 psi. The 14 day UCS testing was mistakenly omitted during the testing of the PRSB treated material. Variation in the UCS was not correlated to the amount of sodium bentonite or sodium silicate in the formulation, indicating that this parameter had little effect on the UCS attained for a given formulation.

The hydraulic conductivity after 28 days of curing ranged from $6 \times 10^{-8}$ to $4 \times 10^{-10} \mathrm{~cm} / \mathrm{sec}$ for the treated material from the PRSB soil fractions. Overall, the average hydraulic conductivity for the treated materials from all formulations for each of the PRSB soil fractions met or exceeded the performance criterion $\left(<1 \times 10^{-8} \mathrm{~cm} / \mathrm{sec}\right)$. The Portland cement, bentonite, and silicate (low) met the hydraulic conductivity performance criterion for all formulations and for all PRSB soil fractions. The Portland cement and bentonite formulation met the performance criteria for all PRSB soil fractions, with the exception of the Bulk Fraction \#1 where the hydraulic conductivity was $3 \times 10^{-8} \mathrm{~cm} / \mathrm{sec}$. The Portland cement, bentonite, and silicate (high) formulation met the performance criterion for the Fine Fraction and was between $4 \times 10^{-8}$ and $2 \times 10^{-8} \mathrm{~cm} / \mathrm{sec}$ for the other PRSB soil fractions. The Portland cement and silicate (high) met the performance criterion 
in all PRSB soil fractions, except the Bulk Fraction \#2. The average hydraulic conductivity for all formulations met the performance criterion (Table 4-1).

Immersion in water for 90 days had little to no detrimental effect on the UCS values for the PRSB formulations. All formations for all soil fractions exceeded the performance criterion of 100 psi. Only two test formulations had reductions in UCS exceeding $25 \%$. Most formulations had slight a change in UCS during the 90 day water immersion.

Immersion in water affected the hydraulic conductivity of the formulations for the PRSB soils. Previous stabilization testing experience has indicated that immersion in water will increase the hydraulic conductivity by one to two orders of magnitude. The least reduction in the hydraulic conductivity following immersion in water was experienced by the Portland cement with bentonite mix, slightly more than two orders of magnitude. All of the other formulations had reductions of more than three orders of magnitude in at least one soil fraction. The Portland cement, bentonite, and $4 \%$ silicate (high) formulation had a reduction of less than two orders of magnitude in three of the soil fractions. After immersion in water for 90 days, the treated material from PRSB soil had hydraulic conductivity values on the order of $1 \times 10^{-6} \mathrm{~cm} / \mathrm{sec}$, regardless of formulation or soil fraction.

The gross alpha and gross nonvolatile beta leach indices for the treated materials from the PRSB soil fractions met the performance criterion $(>8)$. The leach indices for all formulations were consistent and comparable.

The shrink/swell results indicated that the treated material from the PRSB did not exhibit significant deformation during such events. The shrink/swell values were well below the performance criteria of less than $25 \%$ of the 28 -day UCS failure strain.

The volume increases for the treated PRSB material were similar to those reported for other RSB soils and met the performance criterion of $50 \%$ increase.

The $\mathrm{pH}$ values of the treated material from the PRSB fractions were high (12.4 to 12.9), exceeding the performance criterion $(>10)$. These $\mathrm{pH}$ values may drop slightly over time as the treated material is exposed to carbonate in the environment.

The setting characteristics of the treated material from the PRSB soil fractions were favorable. The temperature rises of the treated material, measured in the cure within one hour after treatment, were low $\left(1-5^{\circ} \mathrm{C}\right)$, mostly attributable to the low percentage of Portland cement added. During full-scale treatment, it is likely that the temperature rise of the treated material 
would be much larger. The treated material did not false or flash set, and the treated material set overnight. No bleed fluid was noted for the treated material within 24 hours of treatment.

\subsubsection{LRSB Treated Material}

The results of the performance testing on the treated material produced from the LRSB soil fractions are summarized in Tables 3-35 and 3-36.

The UCS results indicated that the treated material from the LRSB soil fractions reached 370 to 640 psi within 28 days of curing. These UCS values exceed the performance criterion of 100 psi. The 14 day UCS testing was mistakenly omitted during the testing of the LRSB treated material. Variation in the UCS was not correlated to the amount of sodium bentonite or sodium silicate in the formulation, indicating that this parameter had little effect on the UCS attained for a given formulation.

The average hydraulic conductivity after 28 days of curing for all formulations was less than $1 \times 10^{-8} \mathrm{~cm} / \mathrm{sec}$. The hydraulic conductivity ranged from $3 \times 10^{-8}$ to $5 \times 10^{-10} \mathrm{~cm} / \mathrm{sec}$ for the treated material from the LRSB soil fractions. For the LRSB coarse fraction, the treated material from all formulations met the performance criterion $\left(<1 \times 10^{-8} \mathrm{~cm} / \mathrm{sec}\right)$, with the exception of the Portland cement, bentonite, and silicate (high) formulation $\left(3 \times 10^{-8} \mathrm{~cm} / \mathrm{sec}\right)$. For the LRSB fine fraction, the material from all formulations met the performance criterion.

Immersion in water for 90 days had little to no detrimental effect on the UCS values for the LRSB formulations. Most formulations had an increase in UCS during the 90 day water immersion, while one formulation had a reduction in UCS greater than $25 \%$.

Immersion in water affected the hydraulic conductivity of the formulations for the LRSB soils. Typically, immersion in water will increase the hydraulic conductivity by one to two orders of magnitude. For the LRSB Coarse Fraction, immersion in water reduced the hydraulic conductivity of the treated material to approximately $1 \times 10^{-6} \mathrm{~cm} / \mathrm{sec}$, regardless of the formulation or initial hydraulic conductivity. Decreases within the expected range were seen for the hydraulic conductivity after immersion for all of the formulations on the LRSB Fine Fraction.

The gross alpha leach indices for a treated materials from the LRSB soil fractions met the performance criterion (>8). Generally, the Portland cement and silicate (high) formulation had the highest leach indices. Since the nonvolatile gross beta level in the LRSB soil fractions were below the detection limit, leach indices could not be determined for the nonvolatile gross beta contamination in the LRSB soil fractions. 
The shrink/swell results indicated that the treated material from the CRSB did not exhibit significant deformation during such events. The shrink/swell values were well below the performance criterion of less than $25 \%$ of the 28 -day UCS failure strain.

The volume increases for the treated LRSB material were similar to those reported for the other RSB soils and met the performance criterion of less than $50 \%$ increase in volume.

The $\mathrm{pH}$ values of the treated material from the LRSB fractions were high (12.4 to 12.9), exceeding the performance criterion $(>10)$. These $\mathrm{pH}$ values may drop slightly over time as the treated material is exposed to carbonate in the environment.

The setting characteristics of the treated material from the LRSB soil fractions were not favorable. The temperature rises of the treated material, measured in the cure within one hour after treatment, were low $\left(1-3{ }^{\circ} \mathrm{C}\right)$, mostly attributable to the low percentage of Portland cement added. During full-scale treatment, it is likely that the temperature rise of the treated material would be much larger. The treated material for all formulations appeared to flash set within four hours of mixing. Flash setting may occur during full-scale treatment and produce problems, especially with sampling of the treated material. No bleed fluid was noted for the treated material within 24 hours of treatment. 
Table 4-1. Average Hydraulic Conductivity by Formulation

All Soil Fractions - 28-Day Cure

\begin{tabular}{|c|c|c|c|c|}
\hline \multirow{2}{*}{$\begin{array}{c}\text { Seepage } \\
\text { Basin }\end{array}$} & \multicolumn{4}{|c|}{ Average Hydraulic Conductivity $(\mathrm{cm} / \mathrm{sec})^{1}$} \\
\hline & $\begin{array}{c}\text { Formulation }^{2} \\
1\end{array}$ & $\begin{array}{c}\text { Formulation }^{2} \\
\mathbf{2} \\
\end{array}$ & $\begin{array}{c}\text { Formulation }^{2} \\
3 \\
\end{array}$ & $\begin{array}{c}\text { Formulation }^{2} \\
4\end{array}$ \\
\hline CRSB & $2 \mathrm{E}-07$ & $1 \mathrm{E}-07$ & \begin{tabular}{|l|}
$1 \mathrm{E}-07$ \\
\end{tabular} & $1 \mathrm{E}-07$ \\
\hline KRSB & $1 \mathrm{E}-08$ & $5 \mathrm{E}-08$ & $4 \mathrm{E}-08$ & $8 \mathrm{E}-08$ \\
\hline PRSB & $7 \mathrm{E}-09$ & $3 \mathrm{E}-09$ & $7 \mathrm{E}-10$ & $1 \mathrm{E}-08$ \\
\hline LRSB & $3 \mathrm{E}-09$ & $5 \mathrm{E}-09$ & $1 \mathrm{E}-09$ & $8 \mathrm{E}-09$ \\
\hline $\begin{array}{l}\text { Logarithm } \\
\text { Formulatio } \\
1 \text { - Portlan } \\
2 \text { - Portlan } \\
3 \text { - Portlan } \\
4 \text { - Portlan }\end{array}$ & $\begin{array}{l}\text { ment and bentc } \\
\text { ment and } 4 \% \mathrm{~s} \\
\text { ment, bentonit } \\
\text { ment, bentonit }\end{array}$ & $\begin{array}{l}\text { se } 10 \\
\text { onite } \\
\text { ilicate (high) } \\
\text { e, and } 2 \% \text { silic } \\
e \text {, and } 4 \% \text { silic }\end{array}$ & $\begin{array}{l}\text { (low) } \\
\text { (high) }\end{array}$ & \\
\hline
\end{tabular}

Table 4-2. Average Hydraulic Conductivity by Formulation All Soil Fractions - 28-Day Cure, 90-Day Immersion

\begin{tabular}{|l|c|c|c|c|}
\hline \multirow{3}{*}{$\begin{array}{c}\text { Seepage } \\
\text { Basin }\end{array}$} & \multicolumn{3}{|c|}{ Average Hydraulic Conductivity (cm/sec) } \\
\cline { 2 - 5 } CRSB & $\begin{array}{c}\text { Formulation }^{2} \\
\mathbf{1}\end{array}$ & $\begin{array}{c}\text { Formulation }^{2} \\
\mathbf{2}\end{array}$ & $\begin{array}{c}\text { Formulation }^{2} \\
\mathbf{3}\end{array}$ & $\begin{array}{c}\text { Formulation }^{2} \\
\mathbf{4}\end{array}$ \\
\cline { 2 - 5 } & $1 \mathrm{E}-06$ & $7 \mathrm{E}-07$ & $5 \mathrm{E}-07$ & $3 \mathrm{E}-07$ \\
\hline KRSB & $3 \mathrm{E}-06$ & $9 \mathrm{E}-07$ & $2 \mathrm{E}-06$ & $5 \mathrm{E}-06$ \\
\hline PRSB & $7 \mathrm{E}-07$ & $1 \mathrm{E}-06$ & $7 \mathrm{E}-07$ & $1 \mathrm{E}-06$ \\
\hline LRSB & $1 \mathrm{E}-06$ & $6 \mathrm{E}-07$ & $9 \mathrm{E}-08$ & $2 \mathrm{E}-07$ \\
\hline
\end{tabular}
Logarithmic average to the base 10
Formulation:
1- Portland cement and bentonite
2- Portland cement and 4\% silicate (high)
3- Portland cement, bentonite, and 2\% silicate (low)
4- Portland cement, bentonite, and 4\% silicate (high)




\subsection{CONCLUSIONS AND RECOMMENDATIONS}

\subsection{Soil Characterization}

\subsubsection{CRSB Soil}

Four samples (Grab Sample \#1, Grab Sample \#2, Remaining Soil \#1 and Remaining Soil \#2) of the CRSB soil and samples of the four fractions (fine, coarse, Bulk Fraction \#1, and Bulk Fraction \#2) were subjected to physical characterization. The physical characterization of the CRSB samples indicate that the separation of the CRSB soil into the various fractions for S/S testing produced fractions which vary in their physical properties and reflect the lithologic variability which may occur within the CRSB.

The chemical characterization of the Bulk Sample \#1 and the Remaining Soil \#1 samples indicated that the samples contained low levels of chloride, nitrite, nitrate, sulfate, and sulfide. The presence of these compounds at these low levels suggests that these chemical compounds will have little to no inhibitory effect on the cementitious reactions which occur during the curing of Portland cement. The radionuclide characterization results on the CRSB soil indicated that the CRSB soils contained moderate levels of Cs-137 and Sr-90 and low levels of Pu-239/240.

\subsubsection{KRSB Soil}

Four samples (Grab Sample \#1, Grab Sample \#2, Remaining Soil \#1 and Remaining Soil \#2) of the KRSB soil and samples of the two fractions (fine and coarse) were subjected to physical characterization. The physical characterization of the KRSB samples indicate that the separation of the KRSB soil into fine and coarse fractions for S/S testing produced fractions which vary in their physical properties and reflect the lithologic variability which may occur within the KRSB. The chemical characterization of the Bulk Sample \#1 and the Remaining Soil \#1 samples indicated that the samples contained low levels of chloride, nitrite, nitrate, sulfate, and sulfide. The presence of these compounds at these low levels suggests that these chemical compounds will have little to no inhibitory effect on the cementitious reactions which occur during the curing of Portland cement. The radionuclide characterization results on the KRSB soil fractions indicated that the fine fraction had high levels of Cs-137, moderate levels of Sr-90 and low levels of Pu-239/240 and the coarse fraction contained moderate levels of Cs-137 and Sr-90 and low levels of Pu-239/240. 


\subsubsection{PRSB Soil}

Four samples (Grab Sample \#1, Grab Sample \#2, Remaining Soil \#1 and Remaining Soil \#2) of the PRSB soil and samples of the four fractions (fine, coarse, Bulk Fraction \#1, and Bulk Fraction \#2) were subjected to physical characterization. The physical characterization of the PRSB samples indicate that the separation of the PRSB soil into the various fractions for S/S testing produced fractions which vary in their physical properties and reflect the lithologic variability which may occur within the PRSB.

The chemical characterization of the Bulk Sample \#1 and the Remaining Soil \#1 samples indicated that the samples contained low levels of chloride, nitrite, nitrate, sulfate, and sulfide. The presence of these compounds at these low levels suggests that these chemical compounds will have little to no inhibitory effect on the cementitious reactions which occur during the curing of Portland cement. The radionuclide characterization results on the PRSB soil fractions indicated that the fine fraction contained high levels of Cs-137, moderate levels of Sr-90, and low levels of $\mathrm{Pu}-239 / 240$; while the other fractions contained moderate levels of Cs-137 and Sr-90 and low levels of Pu-239/240.

\subsubsection{LRSB Soil}

Four samples (Grab Sample \#1, Grab Sample \#2, Remaining Soil \#1 and Remaining Soil \#2) of the LRSB soil and samples of the two fractions (fine and coarse) were subjected to physical characterization. The physical characterization of the LRSB samples indicate that the separation of the LRSB soil into the various fractions for S/S testing produced fractions which vary in their physical properties and reflect the lithologic variability which may occur within the LRSB.

The chemical characterization of the Bulk Sample \#1 and the Remaining Soil \#1 samples indicated that the samples contained low levels of chloride, nitrite, nitrate, sulfate, and sulfide. The presence of these compounds at these low levels suggests that these chemical compounds will have little to no inhibitory effect on the cementitious reactions which occur during the curing of Portland cement. The radionuclide characterization results indicated that the LRSB fractions contained low levels of Cs-137, Sr-90, and Pu-239/240.

\subsection{Stabilization/Solidification Testing}

The bench-scale treatability testing was conducted to demonstrate how the treated material from the selected $\mathrm{S} / \mathrm{S}$ grout mix designs performed with respect to the performance criteria for the final stabilized waste material, with the exception of the criteria for the batching, hazardous 
dust/vapor release and the gas generation. The results from the performance testing provide information on the leachability, durability, hydraulic conductivity, strength, and long-term durability of the $\mathrm{S} / \mathrm{S}$ treated soil material.

\subsubsection{CRSB Soil}

With the exception of the hydraulic conductivity, the treated material from all formulations met the performance criteria (Table 2-1). This indicates that the four grout design mixes listed in Table 1-1 could adequately handle the variability in lithology that may be expected within the CRSB.

The hydraulic conductivity after 28 days of curing ranged from $6 \times 10^{-7}$ to $9 \times 10^{-9} \mathrm{~cm} / \mathrm{s}$ for the treated material from the CRSB soil fractions. For the CRSB coarse fraction and bulk fraction \#2, the Portland cement, bentonite, and sodium silicate (high) formulation had the lowest hydraulic conductivity. For the CRSB fine fraction, only the Portland cement, bentonite, and silicate (high) formulation did not meet the performance criterion. This may be an anomaly as all the other formulations for the CRSB fine fraction met the hydraulic conductivity performance criterion.

The immersion in water affected the hydraulic conductivity of the formulations for the CRSB soils, with most formulations experiencing the expected one to two order-of-magnitude increase in hydraulic conductivity.

\subsubsection{KRSB Soil}

With the exception of the hydraulic conductivity, the treated material from all formulations met the performance criteria (Table 2-1). This indicates that the four grout design mixes listed in Table 1-1 could adequately handle the variability in lithology that may be expected within the KRSB.

The hydraulic conductivity after 28 days of curing ranged from $2 \times 10^{-6}$ to $2 \times 10^{-9} \mathrm{~cm} / \mathrm{s}$ for the treated material from the KRSB soil fractions. For the KRSB coarse fraction, the treated material from three formulations met the performance criterion $\left(<1 \times 10^{-8} \mathrm{~cm} / \mathrm{sec}\right)$, with the lowest value for the two formulations with high silicate. For the KRSB fine fraction, the Portland cement, bentonite, and silicate (low) formulation has a hydraulic conductivity of $2 \times 10^{-8} \mathrm{~cm} / \mathrm{sec}$. 
The immersion in water significantly affected the hydraulic conductivity of the all formulations for the KRSB soils, with most formulations experiencing the expected one to two order-ofmagnitude increase in hydraulic conductivity.

\subsubsection{PRSB Soil}

With the exception of the hydraulic conductivity following 90-day immersion in water, the treated material from all formulations met the performance criterion (Table 2-1). This indicates that the four grout design mixes listed in Table 1-1 could adequately handle the variability in lithology that may be expected within the PRSB.

The average hydraulic conductivity after 28 days of curing for the treated material from the PRSB soil fractions met the performance criterion $\left(1 \times 10^{-8} \mathrm{~cm} / \mathrm{sec}\right)$, ranging from $6 \times 10^{-8}$ to $4 \times 10^{-10} \mathrm{~cm} / \mathrm{sec}$. The Portland cement, bentonite, and silicate (low) formulation met the hydraulic conductivity performance criterion for all of the PRSB soil fractions. The other formulations met the performance criterion on most of the PRSB soil fractions.

The immersion in water affected the hydraulic conductivity of the formulations for the PRSB soils, with most formulations experiencing the expected one to two order-of-magnitude increase in hydraulic conductivity. After immersion in water for 90 days, the treated material from PRSB soil had hydraulic conductivity values on the order of $1 \times 10^{-6} \mathrm{~cm} / \mathrm{sec}$, regardless of formulation or soil fraction.

\subsubsection{LRSB Soil}

With the exception of the hydraulic conductivity following 90-day immersion in water, the treated material from all formulations met the performance criterion (Table 2-1). This indicates that the four grout design mixes listed in Table 1-1 could adequately handle the variability in lithology that may be expected within the KRSB.

The average hydraulic conductivity after 28 days of curing for all formulations met the performance criterion $\left(<1 \times 10^{-8} \mathrm{~cm} / \mathrm{sec}\right)$. Individual mixes ranged from $3 \times 10^{-8}$ to $5 \times 10^{-10} \mathrm{~cm} / \mathrm{sec}$ for the treated material from the LRSB soil fractions. For the LRSB coarse fraction, the treated material from all formulations met the performance criterion, with the exception of the Portland cement, bentonite, and silicate (high) formulation $\left(3 \times 10^{-8} \mathrm{~cm} / \mathrm{sec}\right)$. For the LRSB fine fraction, the material from all formulations met the performance criterion. 
The immersion in water significantly affected the hydraulic conductivity of the all formulations for the LRSB soils. For the LRSB Coarse Fraction, immersion in water reduced the hydraulic conductivity of the treated material to approximately $1 \times 10^{-6} \mathrm{~cm} / \mathrm{sec}$, regardless of the formulation or initial hydraulic conductivity. Decreases within the expected range of one to two orders-ofmagnitude were seen for the hydraulic conductivity after immersion for all of the formulations on the LRSB Fine Fraction.

\subsubsection{Data Set Evaluation}

The consistency of the laboratory results was evaluated by building a model that used the grain size and grout mix information to calculate individual key performance parameters. The performance parameters analyzed were hydraulic conductivity (28 and 90-day), unconfined compressive strength $(14,28$, and 90-day), and leachability index. Information from all the four basins were grouped into one data set as not enough data was present at any individual basin for this analysis. A neural network was used in the analysis to identify if outliers were present in the data sets. An outlier would be identificd if given similar input information of grain size and grout mix, one of the outputs was inconsistent with the rest of the information in the data set. The results of the analysis showed:

- Leachability Index - The data (100\%) could be reproduced to within $10 \%$ of the laboratory data.

- UCS (14 and 28 day) - The data (100\%) could be reproduced to within $25 \%$ of the laboratory data;

- UCS (90-day) - The data ( 47 of 48 data points, or $98 \%$ ) could be reproduced to within $25 \%$ of the laboratory data. The sample from KRSB, Portland cement and bentonite, fine fraction had a comparison error of $31 \%$.

- Hydraulic Conductivity (28-day) - The data (44 of 48 data points, or $92 \%$ ) could be reproduced within one order of magnitude. Samples from KRSB (Portland cement and 4\% silicate, fine fraction), KRSB (Portland cement, bentonite, and 4\% silicate, fine fraction), LRSB (Portland cement and 4\% silicate, fine fraction), and LRSB (Portland cement, bentonite, and $4 \%$ silicate, fine fraction) appear to be outliers when evaluating the data set as a whole.

- Hydraulic Conductivity (90-day) - The data (44 of 48 data points, or 92\%) could be reproduced within one order of magnitude. Samples from KRSB in (Portland cement, 
bentonite, and 2\% silicate, fine fraction), KRSB (Portland cement, bentonite, and 4\% silicate, fine fraction), LRSB (Portland cement, bentonite, and $2 \%$ silicate, fine fraction), and LRSB (Portland cement, bentonite, and 4\% silicate, fine fraction) appear to be outliers when evaluating the data set as a whole.

In Summary, the data sets are very consistent and useful for their intended purpose. The outliers may be a result of an inconsistency with the test method or a unique feature of the basin material.

The 14 day unconfined compressive strength tests were similar in value, though slightly stronger on average to the 28-day tests. The 14-day UCS tests averaged $376 \mathrm{psi}$, with a standard deviation of 84 psi. The 28-day UCS test results averaged 416 psi, with a similar standard deviation of 83 psi. Some individual 14-day test results were stronger than 28-day test results, with one being up to $18 \%$ stronger. This apparent anomaly is not unexpected when testing fine-grained soil cements. This was compounded with the fact that only one break was made at each test duration, instead of three as is common, due to lack of available soil to test. All of the results exceed the performance requirement of $100 \mathrm{psi}$.

\subsection{FULL-SCALE S/S TREATMENT}

This bench-scale study has been conducted in accordance with industry practice, and the grout mix recommended for the treatment of soils in the reactor seepage basins (Section 5.3.1) has been demonstrated to be successful at the bench (i.e. laboratory) scale. Scaling this work, and the documentation of successful results, to full scale field applications adds many unknowns. These unknowns include, but are not limited to, variability in the basin chemistry and geology; S/S contractor mixing method, application, and experience; and weather during the mixing operations. While the actual construction activities during full-scale operations are outside of SAIC's control, supplemental information has been provided to deal with some of the potential unknowns which may arise during the actual field activity (Sections 5.3.2 and 5.3.3). These construction practices and lessons learned have enhanced the effectiveness of other successful $\mathrm{S} / \mathrm{S}$ projects.

\subsubsection{S/S Formulation}

\subsubsection{Selected Formulation}

The remediation technology chosen for these basins is soil solidification/stabilization. The purpose of solidification is to produce a strong, structurally stable waste form with a reduced leach potential. The purpose of the stabilization effort is to reduce a waste's solubility and/or 
chemical reactivity using a tailored reagent. Two classifications of soil stabilization techniques are possible for deployment for the basins, ex-situ and in-situ. An ex-situ method would require that the soils in the basins be excavated, mixed with the stabilizing mixture, and returned to the basin. The in-situ techniques treat the soils within the basins by mixing them in-place with augers, grout rakes or other mechanical means. Both techniques have advantages and disadvantages. For example, the ex-situ process requires a significant amount of material handling and transport, while the in-situ approach brings a portable mixing apparatus to the soil material to be stabilized. The ex-situ process provides for more complete mixing and quality assurance/quality control (QA/QC) control, while it can be difficult to control the $\mathrm{QA} / \mathrm{QC}$ when using the in-situ process. BSRI has determined as part of the feasibility process that, all things considered, in-situ soil stabilization is the method of choice. The common methods for in-situ stabilization include a dual auger, a single auger, and a rake-like instrument with grout injection points at the bottom. In terms of the mixing energy imparted to the soil stabilization process, the dual auger technology best emulates the mixing of a twin shaft pug mill, a device commonly used in ex-situ material mixing. One of the keys to successful in-situ $\mathrm{S} / \mathrm{S}$ is getting the grout to the bottom of the target stabilization area. These basins may require excavating areas to loosen, and/or pre-treat, soils in order for the mixing process to occur.

The recommended mixes are starting points for the final grout design, as there was not enough soil to do a complete study or mix design. The laboratory bench scale tests indicate the feasibility of pursuing the use of S/S technology for these basins. Various factors can affect the scalability of the results from the bench scale testing to full field applications, including, but not limited to; basin variability, mixing type and application, and experience of the $\mathrm{S} / \mathrm{S}$ contractor. The selected vendor may modify the final mixture, since new or modifications to the $\mathrm{S} / \mathrm{S}$ technology are continually occurring. It is also prudent to incorporate lessons learned from the L- and F-Area projects currently underway, into the final grout mix design process.

The Portland cement and bentonite grout mix is recommended for the full-scale $\mathrm{S} / \mathrm{S}$ treatment of the CRSB, KRSB, LRSB, and PRSB soils. The S/S testing results (Section 3) for the treated material from the CRSB, KRSB, LRSB, and PRSB soils were similar and showed little variability that could be consistently attributed to the grout mix formulation composition. Therefore, reagent cost was used as the primary basis to select the recommended formulation. The reagent costs used for this comparison are listed in Appendix G.

The inclusion of sodium silicate solution as part of the $\mathrm{S} / \mathrm{S}$ formulation is not recommended, since it did not consistently improve the UCS or hydraulic conductivity of the treated material as compared to the Portland cement and bentonite formulation. Although the addition of sodium 
silicate solution may slightly increase the gross alpha and gross nonvolatile beta leach indices, the leach indices for the Portland cement and bentonite formulations were at least two orders of magnitude above the performance requirement. Further, the Portland cement, bentonite, and silicate formulations appeared to have higher volume increases when compared to Portland cement and bentonite and the Portland cement and silicate formulations.

The recommended formulation would involve mixing sufficient Portland cement and sodium bentonite into the soil so that these reagents account for $15 \%$ and $5 \%$, respectively of the total weight of the soil and the reagents. The amount of water used to slurry the reagents into a grout will depend on the vendor-specific batching, grout pumping, and mixing equipment employed for the full-scale treatment.

\subsubsection{Application of Selected Formulation}

In-situ $\mathrm{S} / \mathrm{S}$ is a complex process that requires significant $\mathrm{QA} / \mathrm{QC}$ emphasis to maximize the probability of success. BSRI has included a significant list of QA/QC checks in the specifications for the L-Area and the F-Area projects which are recommended for incorporation into future bids. In addition, the following are suggestions are made:

- Include an equipment shakeout to see if the grout mix is compatible with equipment and make certain the pilot test works. Expand the pilot test from an equipment shakedown in one area to testing the full process in three areas chosen to represent soils with the finest grain size texture, the average grain size distribution, and the coarsest grain size. This step will assess whether or not the $\mathrm{S} / \mathrm{S}$ process will work for the soil types expected in the basin, and, if failures do occur, will save the contractor from significant possible rework. Conduct the full 28 day test regime, and then make a go or no-go decision for the full-scale project. If a failure of the performance specification does occur, consider mixing the soil or adding components to blend and precondition the soil to a grain size that will work, or adjust the grout mix for individual portions of the basin.

- Consider trade-offs when designing the final grout mix. For example, soil grout matrices typically obtain higher strength and lower permeability if water is not added in excess (i.e., if free soil water could be available for the cementitous reactions) However, the workability of such a grout formulation would be poor.

- Select the time of the year that the in-situ $\mathrm{S} / \mathrm{S}$ work is performed to minimize temperature extremes. Freezing retards the overall reaction rate and may damage the matrix, while 
summer temperatures in excess of 100 degrees $\mathrm{F}$ can accelerate the reaction rate and may also ruin the final product.

- Conduct a geotechnical evaluation of the material below the area to be stabilized and determine if the $\mathrm{S} / \mathrm{S}$ monolith will be subject to adverse settling, differential or otherwise, that may cause the monolith to crack.

- Control the introduction of water to the final S/S monolith via a water management system, such a cap. The design of the cap should include the results of the geotechnical evaluation to assess whether excess settlement could compromise the cap integrity.

For the selected Portland cement and bentonite formulation, the vendor would be required to calculate the volume of material to be treated with each injection or penetration of the mixing equipment. The vendor would then multiply the volume by the bulk density of the soil, as determined by the vendor, to determine the weight of soil to be treated by the injection or penetration. An example of this calculation is given below:

Footprint of mixing tool: $\quad 29$ square $\mathrm{ft}$

Depth of treatment: $\quad 10 \mathrm{ft}$

Volume of soil to be treated: $\quad 29 * 10=290$ cubic $\mathrm{ft}(10.74$ cubic yards $)$

Bulk Density of soil: $\quad 1.4$ tons/cubic yard

Weight of Soil to be Treated: $\quad 10.74 * 1.4=15.04$ tons

The weight of soil to be treated by each injection and the ratios of the reagent can then be used to calculate the amount of reagent required:
Ratio of Portland Cement: $\quad 15 \%$
Ratio of Sodium Bentonite: $\quad 5 \%$
Total Weight of Soil + Reagents: $\quad 15.04 *[1+(15 / 100)+(5 / 100)]=18.04$ tons
Weight Portland Cement: $\quad 18.04 *(15 / 100)=2.71$ tons
Weight Sodium Bentonite: $\quad 18.04 *(5 / 100)=0.90$ tons 
The result of this example is that the vendor would need to supply 2.71 tons of Portland cement and 0.90 tons of sodium bentonite to the $10 \mathrm{ft}$ depth of soil to be treated with one injection or penetration of the mixing equipment. The amount of water needed to slurry the reagents is vendor-specific and depends on the specific batching, grout pumping and mixing equipment. The minimum amount of water used to slurry the reagents would be $1: 1$ by weight. For the above example, the weight of reagents is 3.61 tons $(2.71+0.90)$. Therefore, the minimum water for slurrying reagents in the example is 3.61 tons (or 865 gallons).

\subsubsection{Process Mixing Constraints}

The major process mixing constraint for the Portland cement and bentonite grout mix is that the grout mix must be thoroughly mixed with the contaminated soil. The primary source of failure during full-scale treatment is often the lack of thorough mixing of the reagent grout and the soil. The soil types in the four reactor seepage basins ranged from sandy loams and loams to sandy clay loams. While the sandy nature of the soils make mixing of the grout and soil easier, the presence of soil material with higher clay contents indicate that high speed, high shcar mixing would be required.

Mixing by excavators or rake-likc attachments on excavators would not produce the high energy, high shear mixing required to thoroughly blend the grout and the soil. Jet-grouting has been effective in sandy soils and debris, but has had incomplete or inadequate mixing results in soils with higher clay contents. Auger mixer mounted either on crane bodies or excavators, would be sufficient to produce the high energy, high shear mixing required to adequate mix the grout and the reactor seepage basin soils.

Even with the auger mixing, mixing times for each column must be sufficient to thoroughly mix the injected grout and the soil. Multiple passes up and down at each column should be employed to ensure that the grout is completely mixed throughout the column. Preferably $2 / 3$ of the grout volume for each column should be injected during the initial downward pass and the remaining $1 / 3$ injected during the initial upward pass.

\subsubsection{Construction Tolerances}

\subsubsection{Column Overlap}

The overlap between the columns should be sufficient to ensure that all of the contaminated soil is treated. A minimum total overlap volume of $5 \%$ and a maximum of $10 \%$ should be considered for each of the reactor seepage basins. The minimum typically ensures that no untreated material 
is left in the reactor seepage basin, while the maximum prevents the over-addition of reagents. The over-addition of reagents may lend to higher temperature rises in the treated material. The vendor should determine the positions of each column within each reactor seepage basin prior to beginning field work. Mapping of the column placement on the surface profile of each reactor seepage basin will verify that the overlap volume will be between 5 and $10 \%$.

The position of each column should be surveyed in and recorded to verify that the placement is consistent with the vendor's treatment map. The center position of each column should be within \pm 1.5 inches of the planned position. With most auger mixing equipment, this accuracy will still maintain sufficient overlap to ensure that all of the contaminated soil is treated.

\subsubsection{Grout Batching}

The grout should be produced in a batch plant capable of providing an individual grout batch for each column. The weights of Portland cement and bentonite and the volume of water should be recorded for each grout batch. The weights should be controlled to within $\pm 1 \%$ of the desired value. The metering pump for the water should also control the water addition to within $\pm 1 \%$ of the required value. The desired weights of the Portland cement and bentonite in each grout batch should be calculated from the volume of soil to be treated in the column, the in-situ bulk density of the reactor seepage basin soil, and the recommended S/S formulation. An example of this calculation is given in Section 5.3.1.2. The amount of water should also be determined in a similar fashion, but can be modified to adjust the viscosity of the grout to match the S/S vendor's batching, grout pumping and mixing equipment.

Each grout batch should be used within a maximum of 2 hours of batching. If a set retarder is used to prolong the time between batching and injection of the grout, testing should be conducted to determine the effect of the set retarder on the performance of the treated material.

\subsubsection{Moisture Content}

For the soils used in the grout mix design testing, the moisture content ranged from 11 to $23 \%$ (dwb). Variability of moisture content within this range had little to no effect on the S/S testing results. During full-scale S/S application in the CRSB and KRSB, modification of the S/S treatment will not be required as long as the moisture content of the soil is greater than $10 \%$ (dwb). Soil with lower moisture contents will require the addition of water to prevent drying and cracking of the grout-treated soil. The modification for low moisture content soils can be either by the addition of water directly to the soil to be treated or by the adjustment of the volume of 
water added to the grout. An example calculation to determine the amount of additional water needed is given below for a situation when the soil moisture is less than $10 \%(\mathrm{dwb})$ :

$\begin{array}{ll}\text { Assumed Soil Moisture Content: } & 7 \%(\mathrm{dwb}) \\ \text { Footprint of mixing tool: } & 29 \text { square } \mathrm{ft} \\ \text { Depth of treatment: } & 10 \mathrm{ft} \\ \text { Volume of soil to be treated: } & 29 * 10=290 \text { cubic } \mathrm{ft}(10.74 \text { cubic yards }) \\ \text { Bulk Density of soil: } & 1.4 \text { tons/cubic yard } \\ \text { Weight of Soil to be Treated: } & 10.74 * 1.4=15.04 \text { tons } \\ \text { Weight of Water Present: } & 15.04 * 0.07=1.05 \text { tons } \\ \text { Weight Water at } 15 \% \text { (dwb): } & 15.04 * 0.15=2.26 \text { tons } \\ \text { Weight of Water Needed: } & 2.26-1.05=1.20 \text { tons }\end{array}$

Therefore, the amount of water to apply to the 29 square $\mathrm{ft}$ column treatment area is 1.20 tons (288 gallons). The water could be slowly applied to the surface and allowed to seep into the soil. Alternatively, the water could be added to the grout batch for that column.

\subsubsection{Lithology}

The objective of separating the soil material from the reactor seepage basins by soil texture prior to grout mix design testing was to allow the evaluation of lithological variation on the performance of the grout mixes. The testing results indicated that lithological variability should have little to no variability on the performance of the grout mix during full-scale $\mathrm{S} / \mathrm{S}$ operations. Therefore, the Portland cement and bentonite formulation will be applicable for use over the entire CRSB, KRSB, LRSB, and PRSB with respect to lithology variations.

\subsubsection{Temperature}

Although the bench-scale testing results indicated that small temperature rises would be associated with the Portland cement and bentonite formulation, higher temperature rises are often seen during full-scale S/S operations. The selected S/S vendor should monitor the core and surface temperatures of the treated material to determine temperature rise during full-scale 
treatment. Common industrial practice is to control the rate of temperature change and keep the surface temperature within final grout design parameters to minimize cracking of the grouted soil. If the vendor encounters higher temperatures, the vendor may add additional water to the grout mix. The additional water in the grout will serve as a heat sink to slow the heat rise.

\subsubsection{Ambient Conditions}

The use of the Portland cement and bentonite grout mix should not be limited by ambient conditions, with the exception of ambient temperatures below $32^{\circ} \mathrm{F}$ or above $100^{\circ} \mathrm{F}$.

$\mathrm{S} / \mathrm{S}$ operations should cease when temperatures fall below $32^{\circ} \mathrm{F}$. At this point, all lines should be bled to present freezing of liquid contained in them. S/S operations can resume when warmer temperatures occur.

Ambient temperatures above $100^{\circ} \mathrm{F}$ may accelerate the reaction rates and ruin the final product and affect the viscosity and pumpability of the grout. Based on the vendor-specified batching and grout pumping equipment employed, the vendor may need to add additional water or a set retarder to the grout mix to decrease the viscosity and increase the pumpability of the grout. If a set retarder is used, testing should be conducted to determine the effect of the set retarder on the performance of the treated material.

\subsubsection{Field Refinement}

As required by the $\mathrm{S} / \mathrm{S}$ vendor, limited field refinement of the grout mix may be done to adapt the grout mix to the vendor-specified batching, grout pumping, and mixing equipment. The field refinement can only be the addition of water or the use of a set retarder. Additional water can only be added to the grout mix to account for low soil moisture content, to lower the temperature rise of grouted soil, or to decrease the grout viscosity. A set retarder may be used to prolong the time between batching and injection of the grout mix or to decrease the viscosity of the grout. If a set retarder is used, pilot scale testing should be conducted to determine the effect of the set retarder on the performance of the treated material.

\subsection{Estimated Construction Unit Cost}

The costs of in-situ technology applied to the reactor seepage basins can be estimated on a perunit-of-waste-treated basis. Multiple vendors are capable of providing in-situ S/S services to the depths necessary to treat the reactor seepage basins. The estimated unit cost is provided for the daily operation and does not include fixed project costs. The following estimate is based upon information contained in the report Technical Assessment of In Situ Stabilization/Solidification 
Remedial Technologies for Potential Application to Soils and Sludges at the Petroleum Products Corporation Site in Pembroke Park, Broward County, Florida, EPA Contract No. 68-C5-0001, Science Applications International Corporation, October 1995. The prices and costs contained in this report are considered representative of current costs and prices. It does not consider ancillary costs associated with the project, such as air permits for the batch plant, administrative and support costs (site preparation, administrative staff, etc.), mobilization and dcmobilization, analytical costs for treated material and grout mix samples, nor any contingency for project uncertainties.

The cost for the $\mathrm{S} / \mathrm{S}$ treatment of a cubic yd of soil is estimated to be approximately $\$ 115$, as shown in Table 5-1. The estimate is based on daily production rates for a single auger system. This estimate was compared to unit costs contained in Environmental Cost Handling Options and Solutions (ECHOS) by R. S. Means. A direct comparison of costs was not possible; however units costs for equipment, labor, and material in ECHOS were comparable to those used to develop the above estimate. Costs not considered in this estimate could add substantially to the overall cost. For example, mobilization/demobilization could add an additional $\$ 150,000$ to $\$ 200,000$ to the overall project cost. 
Soil Stabilization/Solidification Grout Mix Design Study

WSRC-RP-99-4075. Revision 1 C-, K-, L-, and P-Reactor Seepage Basins Closure (U)

Table 5-1. Estimated Construction Unit Cost

\begin{tabular}{|c|c|c|c|}
\hline Category & Number & Cost per Day & $\begin{array}{c}\text { Total Cost per } \\
\text { Day }\end{array}$ \\
\hline \multicolumn{4}{|l|}{ Equipment } \\
\hline Auger Mixing Plant & & $\$ 11,500$ & $\$ 11,500$ \\
\hline Batch Plant & & $\$ 2.500$ & $\$ 2,500$ \\
\hline Excavator & & $\$ 500$ & $\$ 500$ \\
\hline Grout Pump & & $\$ 1,000$ & $\$ 1,000$ \\
\hline \multicolumn{3}{|r|}{ SUBTOTAL } & $\$ 15,500$ \\
\hline \multicolumn{4}{|l|}{ Labor } \\
\hline Auger Operator & 1 & $\$ 600$ & $\$ 600$ \\
\hline Batch Plant Operator & 1 & $\$ 475$ & $\$ 475$ \\
\hline Excavator Operator & 1 & $\$ 500$ & $\$ 500$ \\
\hline Health \& Safety Officer & 1 & $\$ 450$ & $\$ 450$ \\
\hline Site Supervisor & 1 & $\$ 600$ & $\$ 600$ \\
\hline Field Engineer & 1 & $\$ 600$ & $\$ 600$ \\
\hline Laborer & 3 & $\$ 350$ & $\$ 1,050$ \\
\hline \multicolumn{3}{|r|}{ SUBTOTAL } & $\$ 4,275$ \\
\hline \multicolumn{4}{|l|}{ Materials $^{1}$} \\
\hline Portland Cement & $\$ 85$ per Ton & $\$ 5,355$ & $\$ 5,355$ \\
\hline Bentonite & $\$ 200$ per Ton & $\$ 4,200$ & $\$ 4,200$ \\
\hline & & SUBTOTAL & $\$ 9,555$ \\
\hline \multicolumn{3}{|c|}{ TOTAL DAILY COST } & $\$ 28,655$ \\
\hline \multicolumn{3}{|l|}{ Average Cost per Cubic Yd } & $\$ 115$ \\
\hline
\end{tabular}

1 Based on treating 250 cubic yards of soil per day, 1.4 tons per cubic yard of soil, and 15\% Portland cement and $5 \%$ bentonite by weight in the final treated material. 
This page intentionally left blank. 


\section{Appendix A}

Geotechnical Testing Results for the C-, K-, L-, and PRSB 
Soil Stabilization/Solidification Grout Mix Design Study WSRC-RP-99-4075, Revision 1 C-, K-, L-, and P-Reactor Seepage Basins Closure (U) August 1999

This page intentionally left blank. 
Paul Lear

May 28, 1999

IT Corporation

304 Directors Drive

Knoxville, Tennessee 37923

This is the Certificate of Analysis for the following samples:

Project ID:

Project Number:

Date Received by Lab:

Number of Samples:

Sample Type:
SAIC Savannah River Site

777304.00010500

December 23, 1998 and January 19, 1999

One hundred thirty-four (134)

Bulk and stabilized soils

\section{Introduction/Case Narrative}

One hundred thirty-four soil samples were received by the IT Geotechnical Laboratory between December 23, 1998 and January 19, 1999. Fourteen bulk samples were submitted for determination of moisture content, particle-size distribution, Atterberg limits, specific gravity, and engineering soil classification (USCS). One hundred twenty stabilized soil cylinder sample were submitted for determination of unconfined compressive strength (USC), coefficient of permeability (hydraulic conductivity), and one-dimensional expansion/shrinkage. UCS tests were performed at 28 day specimen age, and after specimens were immersed in water for 90 days after an initial 28 day cure. Permeability and expansion/shrinkage tests were performed at 28 day specimen age.

Please see Appendix A, Sample Number Cross Reference List; Appendix B, Analysis Results; Appendix C, Chain-of-Custody and Request-for-Analysis Record; and Appendix $D$, Variance Reports. Appendices are divided by blue sheets. Sections within appendices are divided by green sheets.

Reviewed and Approved:

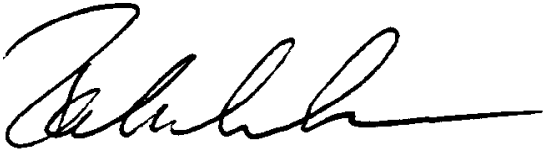

Ralph Cole

Laboratory Manager, Geotechnical Services 


\section{-II. Analytical Results/Methodology}

REFERENCES:

Annual Book of ASTM Standards, Section 4, Construction, Volume 04.08, Soil and Rock (I), and Volume 04.09, Soil and Rock (II), 1998.

United States Army Corps of Engineers, Laboratory Soils Testing. Engineer Manual (EM) 1110-2-1906, Appendix II, 1970.

Requested Methods:

Particle-Size Distribution of Soils

ASTM D 422

Specific Gravity of Soils.

ASTM D 854

Unconfined Compressive Strength of Cohesive Soil

ASTM D 2166

Water (Moisture) Content of Soil and Rock

Classification of Soils for Engineering Purposes (United Soil

ASTM D 2487

Classification System)

ASTM D 3877

Soil-Lime Mixtures. ASTM D 4318

Liquid Limit, Plastic Limit and Plasticity Index of Soils

ASTM D 5084

Flexible-Wall Permeameter ASTM D 422

Particle-Size Distribution of Soils

Additional Method

Unit Weights, Porosity, Void Ratio, and Degree of Saturation USACOE

\section{Quality Control}

Quality control checks such as duplicates and spikes (QC samples), are not normally applicable to geotechnical testing. This is due largely to the inability of obtaining samples with known characteristics, the heterogeneous nature of the samples, and quality control procedures built-in to the analytical method.

QC measures to ensure accuracy and precision of test results include the following:

- $100 \%$ verification of all numerical results - raw data entries, transcriptions and calculations entered by lab technicians are checked, recalculated and verified. Most data calculations are performed by computer programs.

- Data validation through test reasonableness - summaries of all test results for individual reports are reviewed to determine the overall reasonableness of data and to determine the presence of any data that may be considered outliers.

- Quality control procedures are built into most standardized geotechnical procedures. For example, liquid limit and plastic limit analyses call for re-analyses and specify acceptance criteria. 
- Routine instrument calibration - instruments, gauges and equipment used in testing are calibrated on a routine basis. All instrument calibration follows ASTM or manufacturer guidelines.

- Maintenance of all past calibration records - calibration records and certification documents of all instruments, gauges and equipment are updated routinely and maintained in the Quality Control Coordinators Quality/Operations files.

- Certified and trained personnel - all technicians are certified by the National Institute for Certification of Engineering Technicians (NICET) in geotechnical soil testing, and are trained in the application of standard laboratory procedures for geotechnical analyses as well as the quality assurance measures implemented by IT.

\section{IV.Data Qualification}

Unless noted otherwise, moisture content information is determined on the dry sample basis. Asreceived water content data is reported as ancillary information.

\section{Permeability}

A specific gravity value of 2.65 was assumed for use in permeability calculations where applicable.

Steady-state requirement: Section 8.5 .3 (constant head test) requires at least four values of hydraulic conductivity be obtained in which (1) the ratio of outflow to inflow rate is between 0.75 and 1.25 , and (2) the hydraulic conductivity is steady. The test values are considered to be steady when four consecutive tests fall within $\pm 25 \%$ of the mean value for $k \geq 1 \times 10^{-8} \mathrm{~cm} / \mathrm{s}$, or within $\pm 50 \%$ of the mean value for $k<1 \times 10^{-8} \mathrm{~cm} / \mathrm{s}$, and a plot of the hydraulic conductivity versus time shows no significant upward or downward trend. Of the specimens aged 28 days, sample numbers ETDC-, ETDC-7871, ETDC-7874, ETDC-7877, ETDC-7879, ETDC-7970 ETDC-7973 and ETDC-7974 did not meet the steady-state requirements of ASTM D 5084 due to one or more tests falling outside the specification limits. Of the specimens aged 28 days followed by a 90 day immersion period, sample numbers ETDC-7822, ETDC-7827 and ETDC-7833 did not meet the steady-state requirements of ASTM D 5084 due to one or more tests falling outside the specification limits

In addition, no sample was determined to be at least $95 \%$ saturated as estimated by the b-value method outlined in section 8.3.3.1. Incompressible materials such as those tested here often do not yield a high b-value even though saturated, as discussed in Note 8 of the method.

\section{Expansion/Shrinkage}

The sample material did not fit the sample criteria of ASTM D 3877. The method assumes natural bulk soils will be processed and compacted into test rings for testing. Samples were received as solidified cylinders which were placed into rings by cutting to length and forcing the ring over the 
specimen. Most specimens were of such a hard consistency that placement into the rings caused voids around the specimen periphery and/or upper/lower surfaces. For this reason, specimen densities are not reported here.

Expansion test specimens were loaded into consolidation cells and water was added to the cells to saturate the specimens. The initial seating load was placed on each specimen and the degree of deflection recorded after the necessary elapsed time. Most expansion specimens consolidated under the initial seating load. For dial readings indicating expansion, the reading was corrected for swelling of the filter papers used in the cell. Thus, all expansion samples were found to consolidate under the initial load, and/or no expansion was observed. Because no expansion was observed, no further loads were added to the specimens, no expansion curves were generated, and samples were judged to be non-expansive.

No raw sample was available for determination of shrinkage factors by ASTM D 427. This information is required in sections 9.2 (height shrinkage) and 9.3 (volume shrinkage) for determining how much to air-dry the specimens in shrinkage testing. All samples were tested using the height shrinkage method. 
Paul Lear

April 19, 1999

IT Corporation

304 Directors Drive

Knoxville, Tennessee 37923

This is the Certificate of Analysis for the following samples:

Project ID:

Project Number:

Date Received by Lab:

Number of Samples:

Sample Type:
SAIC Savannah River Site

777304.00010500

November 23 through December 15, 1998

One hundred fifty-eight (158)

Bulk and stabilized soils

\section{Introduction/Case Narrative}

One hundred fifty-eight soil samples were received by the IT Geotechnical Laboratory between November 23 and December 15, 1998. Fourteen bulk samples were submitted for determination of moisture content, particle-size distribution, Atterberg limits, specific gravity, and engineering soil classification (USCS). One hundred forty-four stabilized soil cylinder sample were submitted for determination of unconfined compressive strength (USC), coefficient of permeability (hydraulic conductivity), and one-dimensional expansion/shrinkage. UCS tests were performed at 14 and 28 day specimen ages, and after specimens were immersed in water for 90 days after an initial 28 day cure. Permeability and expansion/shrinkage tests were performed at 28 day specimen age, and after specimens were immersed in water for 90 days after the initial 28 day cure.

Please see Appendix A, Sample Number Cross Reference List; Appendix B, Analysis Results; Appendix C, Chain-of-Custody and Request-for-Analysis Record; and Appendix D, Variance Reports. Appendices are divided by blue sheets. Sections within appendices are divided by green sheets.

Reviewed and Approved:

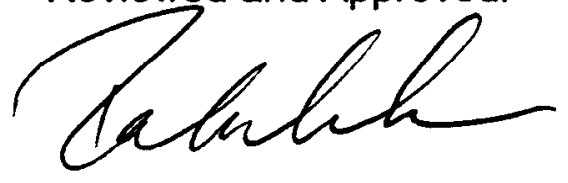

Ralph Cole

Laboratory Manager, Geotechnical Services 


\section{-II. Analytical Results/Methodology}

\section{REFERENCES:}

Annual Book of ASTM Standards, Section 4, Construction, Volume 04.08, Soil and Rock (I), and Volume 04.09, Soil and Rock (II), 1998.

United States Army Corps of Engineers, Laboratory Soils Testing. Engineer Manual (EM) 1110-2-1906, Appendix II, 1970.

\section{Requested Methods:}

Particle-Size Distribution of Soils ASTM D 422

Specific Gravity of Soils. ASTM D 854

Unconfined Compressive Strength of Cohesive Soil ASTM D 2166

Water (Moisture) Content of Soil and Rock. ASTM D 2216

Classification of Soils for Engineering Purposes (United Soil

Classification System). ASTM D 2487

One-Dimensional Expansion, Shrinkage, and Uplift Pressure of

Soil-Lime Mixtures. ASTM D 3877

Liquid Limit, Plastic Limit and Plasticity Index of Soils ASTM D 4318

Hydraulic Conductivity of Saturated Porous Materials Using a

Flexible-Wall Permeameter. ASTM D 5084

Particle-Size Distribution of Soils ASTM D 422

Additional Method

Unit Weights, Porosity, Void Ratio, and Degree of Saturation USACOE

\section{Quality Control}

Quality control checks such as duplicates and spikes (QC samples), are not normally applicable to geotechnical testing. This is due largely to the inability of obtaining samples with known characteristics, the heterogeneous nature of the samples, and quality control procedures built-in to the analytical method.

QC measures to ensure accuracy and precision of test results include the following:

- $100 \%$ verification of all numerical results - raw data entries, transcriptions and calculations entered by lab technicians are checked, recalculated and verified. Most data calculations are performed by computer programs.

- Data validation through test reasonableness - summaries of all test results for individual reports are reviewed to determine the overall reasonableness of data and to determine the presence of any data that may be considered outliers.

- Quality control procedures are built into most standardized geotechnical procedures. For example, liquid limit and plastic limit analyses call for re-analyses and specify acceptance criteria. 
- Routine instrument calibration - instruments, gauges and equipment used in testing are calibrated on a routine basis. All instrument calibration follows ASTM or manufacturer guidelines.

- Maintenance of all past calibration records - calibration records and certification documents of all instruments, gauges and equipment are updated routinely and maintained in the Quality Control Coordinators Quality/Operations files.

- Certified and trained personnel - all technicians are certified by the National Institute for Certification of Engineering Technicians (NICET) in geotechnical soil testing, and are trained in the application of standard laboratory procedures for geotechnical analyses as well as the quality assurance measures implemented by IT.

\section{IV.Data Qualification}

Unless noted othenwise, moisture content information is determined on the dry sample basis. Asreceived water content data is reported as ancillary information.

\section{Permeability}

A specific gravity value of 2.65 was assumed for use in permeability and phase relation calculations where applicable.

Steady-state requirement: Section 8.5.3 (constant head test) requires at least four values of hydraulic conductivity be obtained in which (1) the ratio of outflow to inflow rate is between 0.75 and 1.25 , and (2) the hydraulic conductivity is steady. The test values are considered to be steady when four consecutive tests fall within $\pm 25 \%$ of the mean value for $k \geq 1 \times 10^{-8} \mathrm{~cm} / \mathrm{s}$, or within $\pm 50 \%$ of the mean value for $k<1 \times 10^{-8} \mathrm{~cm} / \mathrm{s}$, and a plot of the hydraulic conductivity versus time shows no significant upward or downward trend. Of the specimens aged 28 days, sample numbers ETDC8178, ETDC-8081, ETDC-8082, ETDC-8085, ETDC-8086, and ETDC-8093 did not meet the steadystate requirements of ASTM D 5084 due to one or more tests falling outside the specification limits. Of the specimens aged 28 days followed by a 90 day immersion period, sample numbers ETDC8124 and ETDC-8242 did not meet the steady-state requirements of ASTM D 5084 due to one or more tests falling outside the specification limits.

In addition, no sample was determined to be at least $95 \%$ saturated as estimated by the b-value method outlined in section 8.3.3.1. Incompressible materials such as those tested here often do not yield a high b-value even though saturated, as discussed in Note 8 of the method.

Sample number ETDC-8080 did not yield positive flow in the effluent burette during any test. One or more tests did not yieid positive flow in the effluent burette for other samples, as well. Negative flow was observed in both influent and effluent in all cases, and this phenomenon was not attributable to leakage in the permeameter/pressure panel system. 


\section{Expansion/Shrinkage}

The sample material did not fit the sample criteria of ASTM D 3877. The method assumes natural bulk soils will be processed and compacted into test rings for testing. Samples were received as solidified cylinders, which were placed into rings by cutting to length and forcing the ring over the specimen. Most specimens were of such a hard consistency that placement into the rings caused voids around the specimen periphery and/or upper/lower surfaces. For this reason, specimen densities are not reported here.

After test specimens were placed into rings, the specimens were mounted in consolidation cells. Water was added to the cells to saturate the specimens. The initial seating load was placed on each specimen and the degree of deflection (as measured by a dial indicator) recorded after the necessary elapsed time. Most expansion specimens consolidated under the initial seating load. For dial readings indicating expansion, the reading was corrected for swelling of the filter papers used in the cell. Thus, all expansion samples were found to consolidate under the initial load, and/or no expansion was observed. Because no expansion was obsenved, no further loads were added to the specimens, no expansion curves were generated, and samples were judged to be non-expansive.

No raw sample was available for determination of shrinkage factors by ASTM D 427. This information is required in sections 9.2 (height shrinkage) and 9.3 (volume shrinkage) for determining how far to air-dry the specimens in shrinkage testing. All samples were tested using the height shrinkage method. 


\section{Attachment A \\ Sample Number Cross-Reference}




\section{SAMPLE NUMBER CROSS-REFERENCE LIST}

LAB SAMPLE NO. CLIENT SAMPLE NO. MATRIX

ETDC-7805

ETDC-7806

ETDC-7807

ETDC-7808

ETDC-7809

ETDC-7810

ETDC-7811

ETDC-7812

ETDC-7813

ETDC-7814

ETDC-7815

ETDC-7816

ETDC-7817

ETDC-7818

ETDC-7819

ETDC-7820

ETDC-7821

ETDC-7822

ETDC-7823

ETDC-7824

ETDC-7825

ETDC-7826

ETDC-7827

ETDC-7828

ETDC-7829

ETDC-7830

ETDC-7831

ETDC-7832

ETDC-7833

ETDC-7834

ETDC-7835

ETDC-7836

ETDC-7837
C311F00

C321F00

C331F00

C341F00

C411F00

C421F00

C431F00

C441F00

C511F00

C521F00

C531F00

C541F00

C611F00

C621F00

C631F00

C641F00

C312KOO

C322K0O

C332K0O

C342K0O

C412K0O

$\mathrm{C} 422 \mathrm{KOO}$

$\mathrm{C} 432 \mathrm{KOO}$

C442K00

C512KOO

C522KOO

C532KOO

C542KOO

C612KOO

C622KOO

C632KOO

C642KOO

C313F00
Stabilized Soil Stabilized Soil Stabilized Soil Stabilized Soil Stabilized Soil Stabilized Soil Stabilized Soil Stabilized Soil Stabilized Soil Stabilized Soil Stabilized Soil Stabilized Soil Stabilized Soil Stabilized Soil Stabilized Soil Stabilized Soil Stabilized Soil Stabilized Soil Stabilized Soil Stabilized Soil Stabilized Soil Stabilized Soil Stabilized Soil Stabilized Soil Stabilized Soil Stabilized Soil Stabilized Soil Stabilized Soil Stabilized Soil Stabilized Soil Stabilized Soil Stabilized Soil Stabilized Soil 


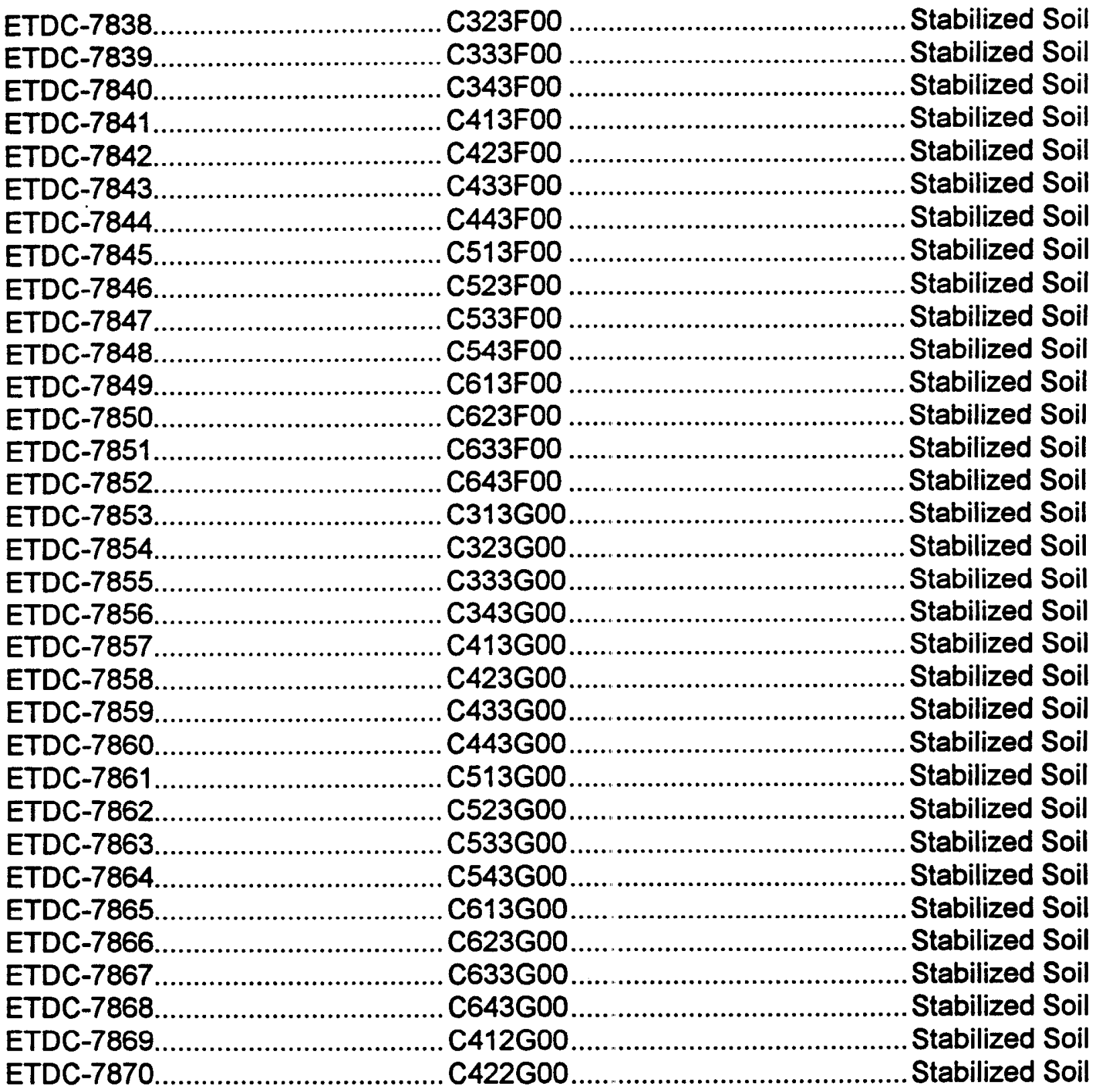




\section{SAMPLE NUMBER CROSS-REFERENCE LIST}

\begin{tabular}{|c|c|c|}
\hline LAB SAMPLE NO. & CLIENT SAMPLE NO. & .......MATRIX \\
\hline 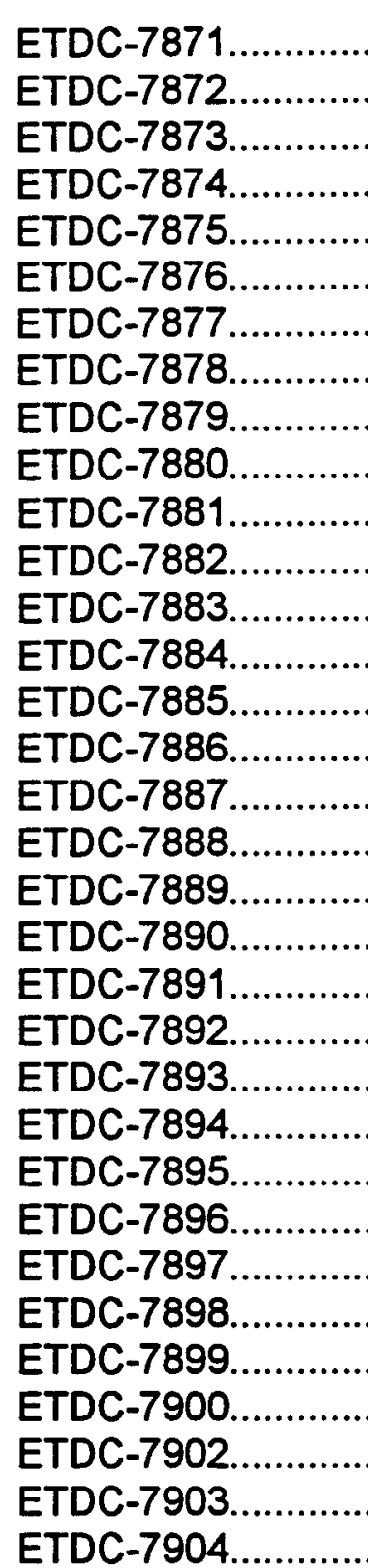 & 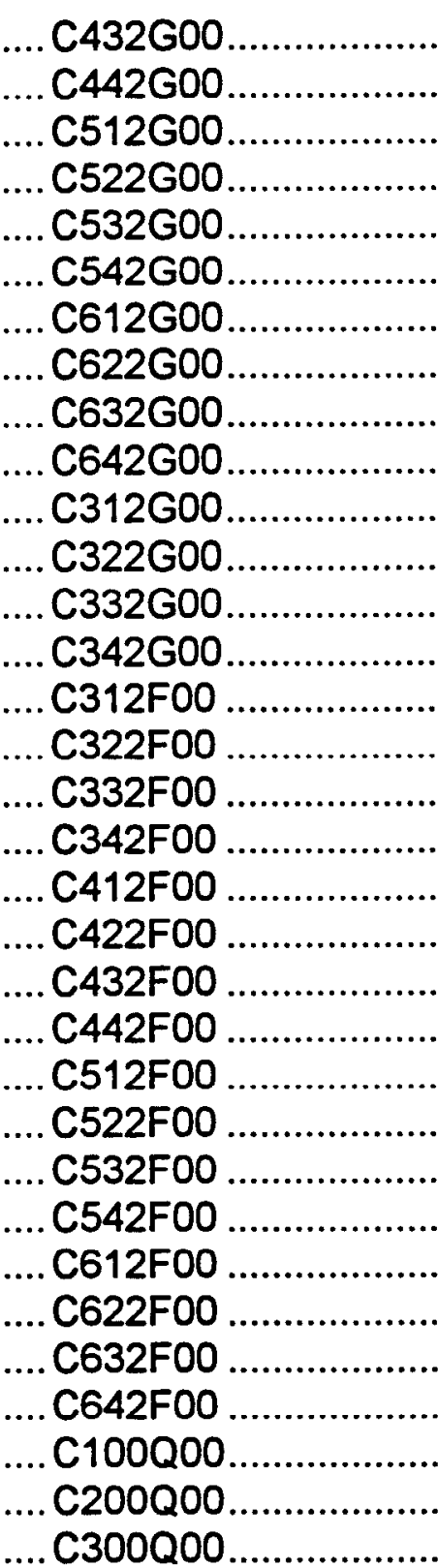 & 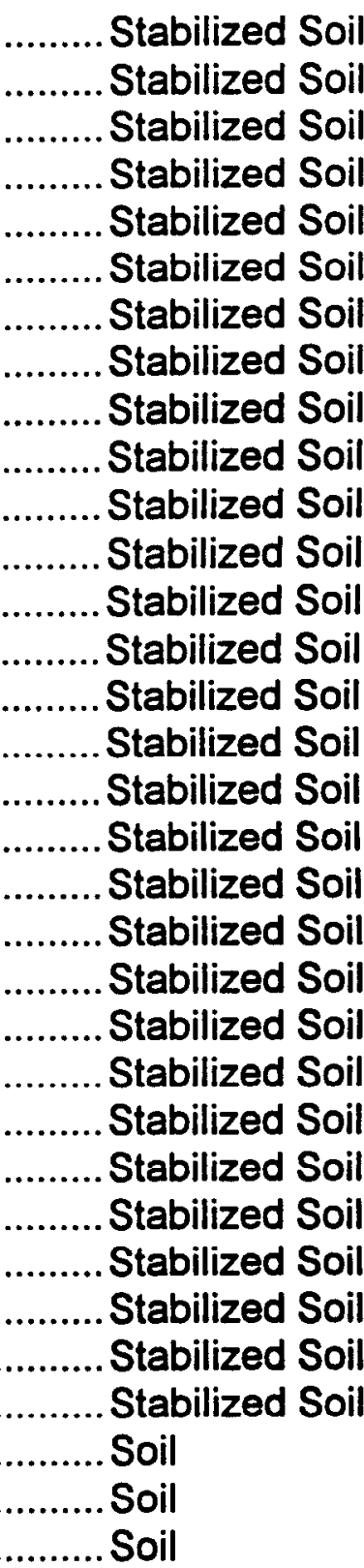 \\
\hline
\end{tabular}




\section{SAMPLE NUMBER CROSS-REFERENCE LIST}

LAB SAMPLE NO.

CLIENT SAMPLE NO.
MATRIX

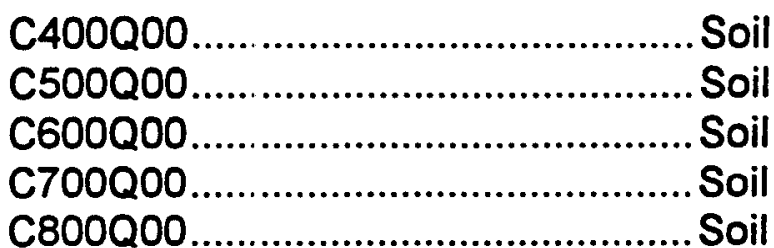

K100Q00

Soil

K200Q00 .................................................

K300Q00 .......................................... Soil

K400Q00 ......................................... Soil

K700Q00 .......................................... Soil

K800Q00 ........................................... Soil

K311F00.......................................... Stabilized Soil

K321F00 Stabilized Soil K331F00

K341F00

K411F00

K421F00

$\mathrm{K} 431 \mathrm{~F} 00$

K441F00

K312K00

$\mathrm{K} 322 \mathrm{KOO}$

K332K00

$\mathrm{K} 342 \mathrm{KOO}$

K412KOO

$\mathrm{K} 422 \mathrm{KOO}$

$\mathrm{K} 432 \mathrm{KOO}$

K442K0O

K312F00

$\mathrm{K} 322 \mathrm{F0O}$

K332F0O.

K342FOO

$\mathrm{K} 412 \mathrm{~F} 00$
Stabilized Soil

Stabilized Soil

Stabilized Soil

Stabilized Soil

Stabilized Soil

Stabilized Soil

Stabilized Soil

Stabilized Soil

Stabilized Soil

Stabilized Soil

Stabilized Soil

Stabilized Soil

Stabilized Soil

Stabilized Soil

Stabilized Soil

Stabilized Soil

Stabilized Soil

Stabilized Soil
Stabilized Soil 


\section{SAMPLE NUMBER CROSS-REFERENCE LIST}

LAB SAMPLE NO. CLIENT SAMPLE NO. $\quad$.......MATRIX

\begin{tabular}{|c|c|c|}
\hline \\
\hline TDC-7981 & K432F00. & Stabilized So \\
\hline TDC-7982 &. $\mathrm{K} 442 \mathrm{~F} 00$. & Stabilized Soi \\
\hline TDC-7983 & K312G00 & Stabilized Soi \\
\hline TDC-7984 & K322G00 & .. Stabilized Soil \\
\hline TDC-7985 & $\mathrm{K} 332 \mathrm{G} 00$ & Stabilized Soil \\
\hline TDC-7986 &.. $\mathrm{K} 342 \mathrm{G} 00$ & Stabilized Soil \\
\hline ETDC-7987 &.. $\mathrm{K} 412 \mathrm{G} 00$ & Stabilized Soil \\
\hline TDC-7988 &.. $\mathrm{K} 422 \mathrm{G} 00$ & Stabilized Soi \\
\hline TDC-7989 &.. $\mathrm{K} 432 \mathrm{G} 00$ & Stabilized Soil \\
\hline TDC-7990 &.. $\mathrm{K} 442 \mathrm{G} 00$ & Stab \\
\hline TDC-7991 & .. K313F00. & Sta \\
\hline TDC-7992 & .. K323F00. & \\
\hline ETDC-7993 & $3 F 00$. & Soil \\
\hline TDC-7994 & .. K343F00. & Stabilized Soi \\
\hline TDC-7995 & K413F00. & Stabilized Soi \\
\hline TDC-7996 & K423F00. & . Stabilized Soi \\
\hline TDC-7997 & K433F00.. & .. Stabilized Soi \\
\hline TDC-7998 & K443F00. & . Stabilized Soi \\
\hline TDC-7999 & K313G00. & . Stabilized Soi \\
\hline TDC-8000 & K323G00. & .. Stabilized Soil \\
\hline TDC-8001 & K333G00. & ..Stabi \\
\hline ETDC-8002 & .. K343G00. & Stabilized \\
\hline TDC-8003 & ... K413G00. & .. Stabilized \\
\hline $\begin{array}{l}\text { TDC-8004 } \\
\text { TDC-8005 }\end{array}$ & ... K423G00 & . \\
\hline $\begin{array}{l}\text { TDC-8005 } \\
\text { TDC-8006 }\end{array}$ & ...K433GG00. & \\
\hline
\end{tabular}




\section{SAMPLE NUMBER CROSS-REFERENCE LIST}

\section{LAB SAMPLE NO.}

CLIENT SAMPLE NO.

\section{MATRIX}

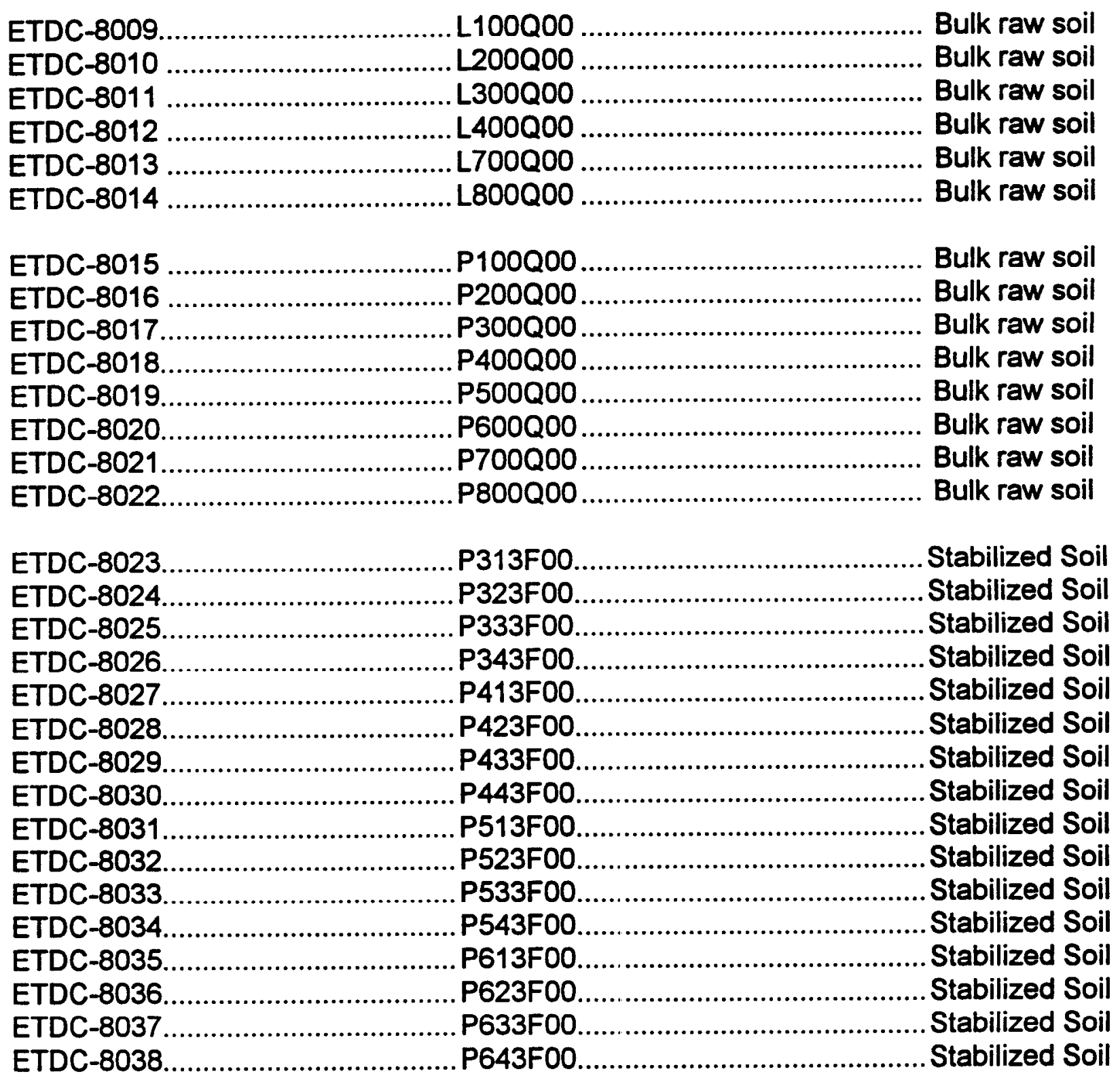




\section{SAMPLE NUMBER CROSS-REFERENCE LIST}

LAB SAMPLE NO. CLIENT SAMPLE NO. MATRIX

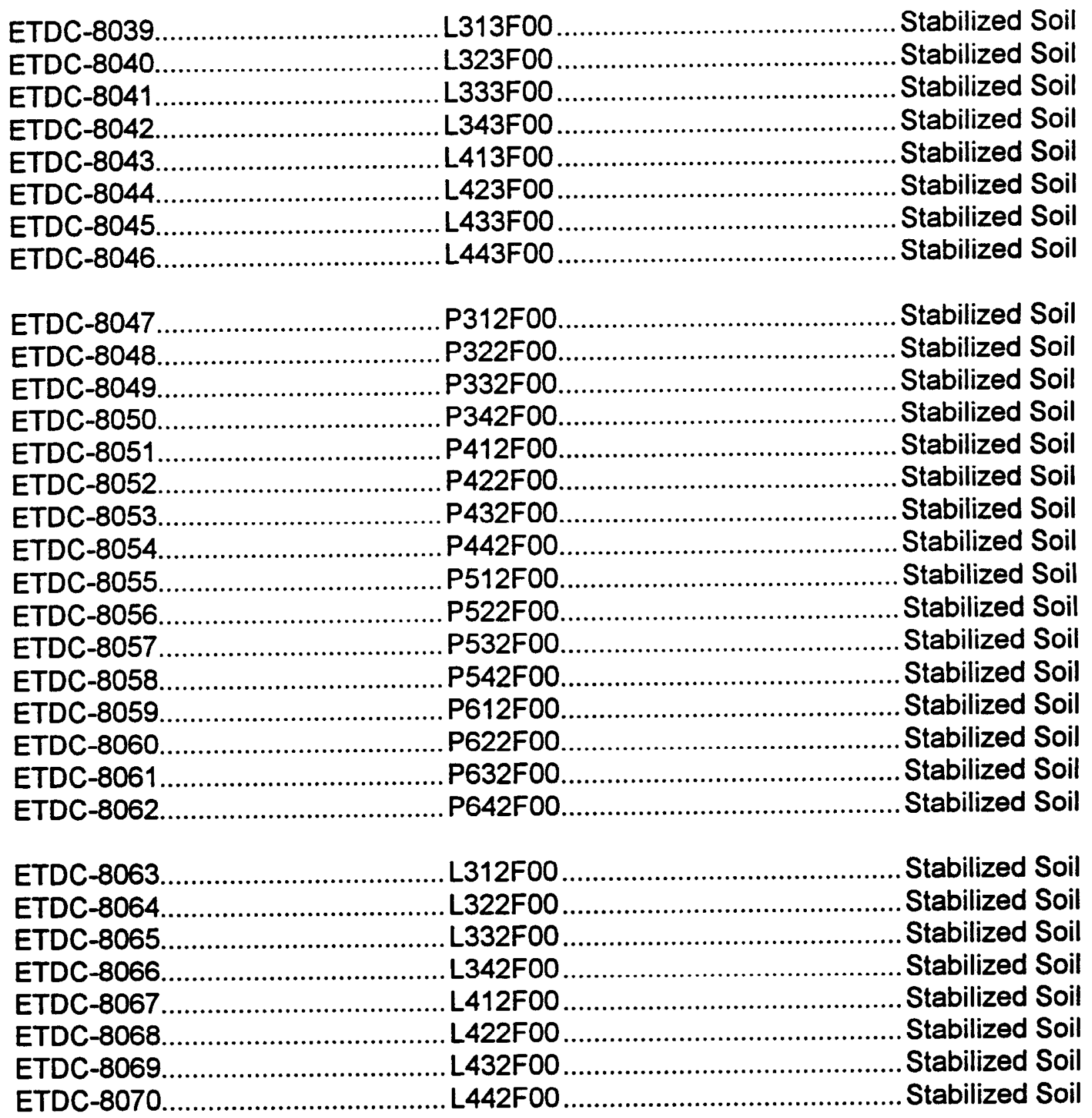




\section{SAMPLE NUMBER CROSS-REFERENCE LIST}

\section{LAB SAMPLE NO.}

ETDC-8071

ETDC-8072

ETDC-8073.

ETDC-8074.

ETDC-8075.

ETDC-8076.

ETDC-8077.

ETDC-8078.

ETDC-8079.

ETDC-8080.

ETDC-8081

ETDC-8082

ETDC-8083.

ETDC-8084.

ETDC-8085

ETDC-8086

ETDC-8087.

ETDC-8088

ETDC-8089.

ETDC-8090

ETDC-8091

ETDC-8092

ETDC-8093

ETDC-8094

ETDC-8095

ETDC-8096

ETDC-8097

ETDC-8098

ETDC-8099

ETDC-8100

ETDC-8101

ETDC-8102
CLIENT SAMPLE NO.

MATRIX
P312K0O

P322K00

P332K00

P342K00

P412K00

$\mathrm{P} 422 \mathrm{KOO}$

$\mathrm{P} 432 \mathrm{KOO}$

$\mathrm{P} 442 \mathrm{KOO}$

P512K00

P522K00

P532K00

$\mathrm{P} 542 \mathrm{~K} 00$

P612K00

P622K0O

P632K0O

P642K00

L312KOO L322K0O. L332KOO

L342KOO

L412KOO

L422KOO

L432KOO

L442KOO

P312G00

P322G00

P332G00

P342G00

P412G00

P422G00

P432G00

P442G00
Stabilized Soil Stabilized Soil Stabilized Soil Stabilized Soil Stabilized Soil Stabilized Soil Stabilized Soil Stabilized Soil Stabilized Soil Stabilized Soil Stabilized Soil Stabilized Soil Stabilized Soil Stabilized Soil Stabilized Soil Stabilized Soil

Stabilized Soil Stabilized Soil Stabilized Soil Stabilized Soil Stabilized Soil Stabilized Soil Stabilized Soil Stabilized Soil

Stabilized Soil Stabilized Soil Stabilized Soil Stabilized Soil Stabilized Soil Stabilized Soil Stabilized Soil Stabilized Soil 


\section{SAMPLE NUMBER CROSS-REFERENCE LIST}

\section{LAB SAMPLE NO.}

ETDC-8103

ETDC-8104

ETDC-8105

ETDC-8106

ETDC-8107.

ETDC-8108.

ETDC-8109

ETDC-8110

ETDC-8111

ETDC-8112

ETDC-8113

ETDC-8114

ETDC-8115

ETDC-8116.

ETDC-8117.

ETDC-8118.

ETDC-8119.

ETDC-8120

ETDC-8121

ETDC-8122

ETDC-8123

ETDC-8124

ETDC-8125

ETDC-8126

ETDC-8127

ETDC-8128

ETDC-8129

ETDC-8130

ETDC-8131

ETDC-8132

ETDC-8133

ETDC-8134
CLIENT SAMPLE NO.

P512G00

P522G00

P532G00

P542G00

P612G00

P622G00

P632G00

P642G00

L312G00

L322G00

L332G00

L342G00

L412G00

L422G00

L432G00

L442G00

P313G00

P323G00

P333G00

P343G00

P413G00

P423G00

P433G00

P443G00

P513G00

P523G00

P533G00

P543G00

P613G00

P623G00

P633G00

P643G00
MATRIX

Stabilized Soil Stabilized Soil Stabilized Soil Stabilized Soil Stabilized Soil Stabilized Soil Stabilized Soil Stabilized Soil

Stabilized Soil Stabilized Soil Stabilized Soil Stabilized Soil Stabilized Soil Stabilized Soil Stabilized Soil Stabilized Soil

Stabilized Soil Stabilized Soil Stabilized Soil Stabilized Soil Stabilized Soil Stabilized Soil Stabilized Soil Stabilized Soil Stabilized Soil Stabilized Soil Stabilized Soil Stabilized Soil Stabilized Soil Stabilized Soil Stabilized Soil Stabilized Soil 


\section{SAMPLE NUMBER CROSS-REFERENCE LIST}

LAB SAMPLE NO.

CLIENT SAMPLE NO.

L313G00

L323G00

ETDC-8136

ETDC-8137

ETDC-8138

ETDC-8139

ETDC-8140

ETDC-8141

ETDC-8142
L333G00

L343G00

L413G00

L423G00

L433G00

L443G00
MATRIX

Stabilized Soil Stabilized Soil Stabilized Soil Stabilized Soil Stabilized Soil Stabilized Soil Stabilized Soil Stabilized Soil 


\section{Attachment B}

\section{Analysis Results}




\section{Series Index Test Results}




\section{MOISTURE CONTENT}

PROJECT NAME

SAIC - Savannah River
PROJECT NUMBER

777304.00010500

\begin{tabular}{|c|c|c|c|c|}
\hline $\begin{array}{c}\text { IT LAB } \\
\text { SAMPLE NO. }\end{array}$ & $\begin{array}{c}\text { CLIENT } \\
\text { SAMPLE NO. }\end{array}$ & $\begin{array}{l}\text { MOISTURE, \% } \\
\text { ASTM D } 2216\end{array}$ & $\begin{array}{c}\text { MOISTURE , \% } \\
\text { SW846 }\end{array}$ & $\begin{array}{l}\text { SOLIDS. \% } \\
\text { SW846 }\end{array}$ \\
\hline ETDC-7902 & $\mathrm{C} 100000$ & 14.5 & 12.7 & 87.3 \\
\hline ETDC-7903 & $\mathrm{C2} 200000$ & 18.5 & 15.6 & 84.4 \\
\hline ETDC-7904 & $\mathrm{C} 300000$ & 12.3 & 10.9 & 89.1 \\
\hline ETDC-7905 & $\mathrm{C400000}$ & 20.0 & 16.7 & 83.3 \\
\hline ETDC-7906 & C500000 & 14.5 & 12.7 & 87.3 \\
\hline ETDC-7907 & 6600000 & 14.5 & 12.7 & 87.3 \\
\hline ETDC-7908 & $\mathrm{C700000}$ & 12.6 & 11.2 & 88.8 \\
\hline ETDC-7909 & $\mathrm{C} 800000$ & 13.1 & 11.6 & 88.4 \\
\hline & & & & \\
\hline & & & & \\
\hline & & & & \\
\hline & & & & \\
\hline & & & & \\
\hline & & & & \\
\hline & & & & \\
\hline & & & & \\
\hline & & & & \\
\hline & & & & \\
\hline & & & & \\
\hline
\end{tabular}

ASTM D 2216 results are based on dry sample weight. SW846 results are based on wet sample weight.

Solids content is determined by subtracting the SW846 moisture (\%) from 100. 


\section{ATTERBERG LIMITS ASTM D 4318}

PROJECT NAME:

SAIC / SAVANNAH RIVER
PROJECT NO.

777304.00010500

\section{ATTERBERG LIMITS RESULTS}

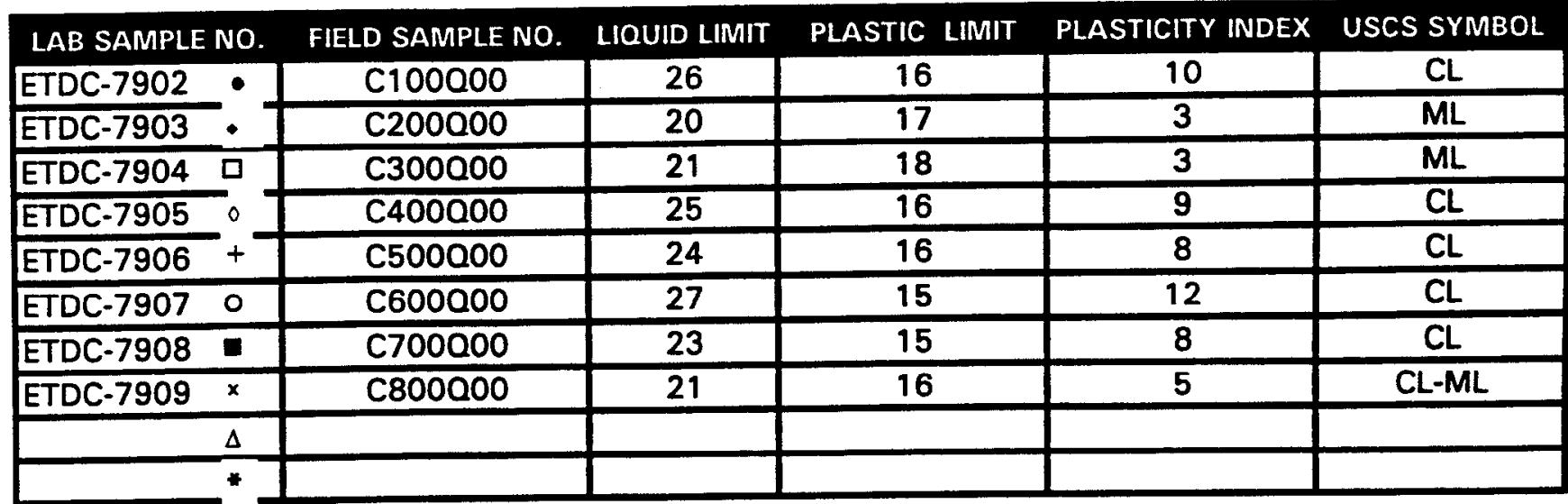

-NP = Nonplastic

\section{PLASTICITY CHART}

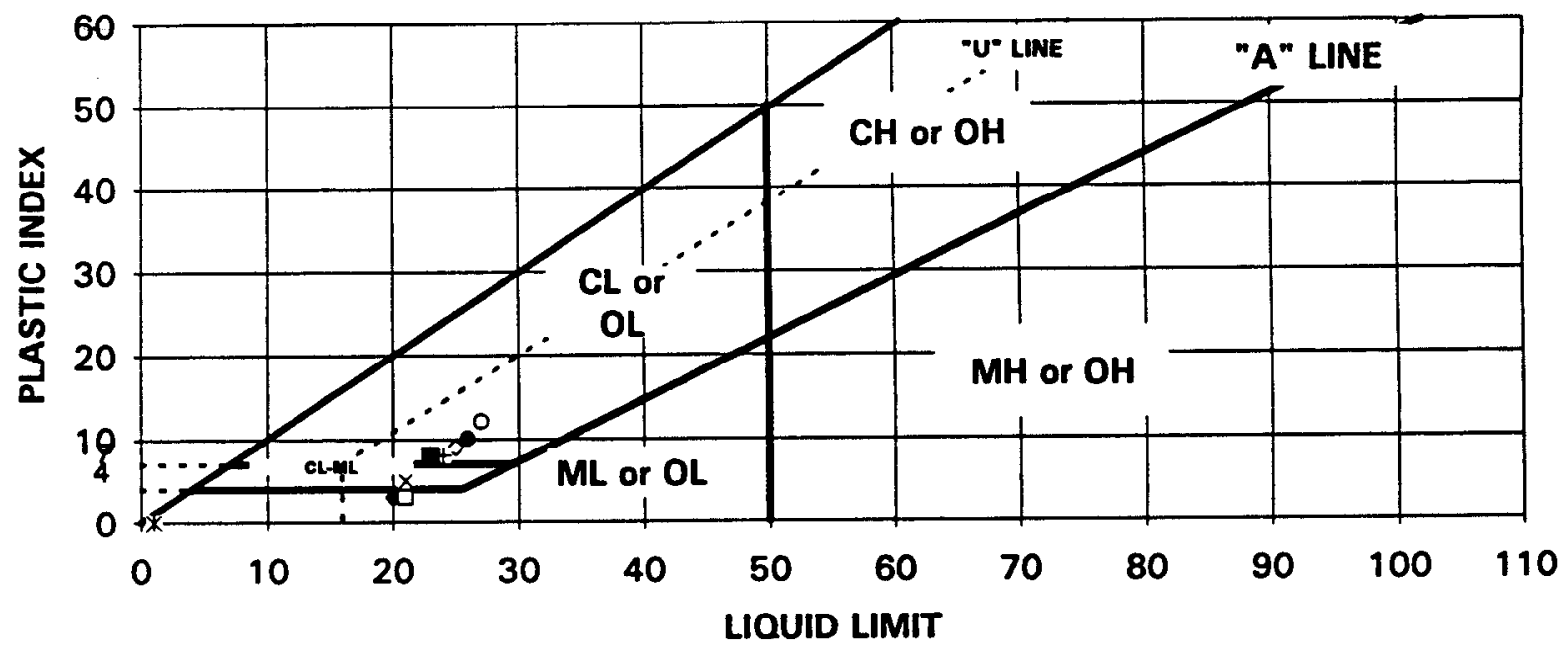




\section{SPECIFIC GRAVITY \\ ASTM D 854}

PROJECT NAME:

PROJECT NUMBER:

SAIC / SAVANNAH RIVER

777304.00010500

IT LAB SAMPLE NO.

CLIENT SAMPLE NO.

SPECIFIC GRAVITY

\begin{tabular}{|c|c|c|}
\hline ETDC-7902 & C100000 & 2.6440 \\
\hline ETDC-7903 & C200000 & 2.6353 \\
\hline ETDC-7904 & C300000 & 2.6593 \\
\hline ETDC-7905 & C400000 & 2.6439 \\
\hline ETDC-7906 & C500000 & 2.6413 \\
\hline ETDC-7907 & C600000 & 2.6352 \\
\hline ETDC-7908 & C700000 & 2.6508 \\
\hline ETDC-7909 & C800000 & 2.6412 \\
\hline & & \\
\hline & & \\
\hline & & \\
\hline & & \\
\hline & & \\
\hline & & \\
\hline & & \\
\hline & & \\
\hline & & \\
\hline & & \\
\hline & & \\
\hline
\end{tabular}




\section{SOIL CLASSIFICATION} ASTM D 2487

\begin{tabular}{|c|c|c|c|c|}
\hline & $\begin{array}{l}\text { ETDC } \\
\text { NUMBER: }\end{array}$ & $\begin{array}{l}\text { CLIENT } \\
\text { NUMBER: }\end{array}$ & $\begin{array}{l}\text { GROUP } \\
\text { SYMBOL: }\end{array}$ & $\begin{array}{l}\text { CLASSIFICATION- } \\
\text { GROUP NAME: }\end{array}$ \\
\hline SAMPLE 1: & ETDC-7902 & $\mathrm{C} 100000$ & sc & CLAYEY SAND \\
\hline SAMPLE 2: & ETDC-7903 & $\mathrm{C} 200000$ & SM & SILTY SAND \\
\hline SAMPLE 3: & ETDC-7904 & C300000 & SM & SILTY SAND \\
\hline SAMPLE 4: & ETDC-7905 & $\mathrm{C} 400000$ & sc & CLAYEY SAND \\
\hline SAMPLE 5: & ETDC-7906 & C500000 & sc & CLAYEY SAND \\
\hline SAMPLE 6: & ETDC-7907 & $\mathrm{C600000}$ & SC & CLAYEY SAND \\
\hline SAMPLE 7: & ETDC-7908 & $c 700000$ & sc & CLAYEY SAND \\
\hline SAMPLE 8: & ETDC-7909 & $\mathrm{C} 800000$ & SC-SM & SILTY, CLAYEY SAND \\
\hline SAMPLE 9: & & & & \\
\hline SAMPLE 10: & & & & \\
\hline
\end{tabular}




\section{PARTICLE-SIZE ANALYSIS \\ ASTM D 422}

Project Name

SAIC / SAVANNAH RIVER

Project No.

777304.00010500

Specific Gravity $=2.6440$
Client Sample No. $\quad \mathrm{C} 100000$

IT Lab Sample No. ETDC-7902
Moisture Content $=14.5 \%$

based on dry sample woight

SIEVE ANALYSIS

\begin{tabular}{|c|c|c|c|}
\hline C & $\begin{array}{c}\text { Sieve } \\
\text { No. }\end{array}$ & $\begin{array}{c}\text { Diameter } \\
\mathrm{mm}\end{array}$ & $\begin{array}{c}\text { Percent } \\
\text { Finer }\end{array}$ \\
\cline { 2 - 4 } O & $3^{\prime \prime}$ & 75.000 & $100.0 \%$ \\
A & $1.5^{n}$ & 37.500 & $100.0 \%$ \\
\hline S & $0.75^{\prime \prime}$ & 19.000 & $100.0 \%$ \\
\cline { 2 - 4 } E & $0.375^{\prime \prime}$ & 9.500 & $98.6 \%$ \\
\cline { 2 - 4 } & $\# 4$ & 4.750 & $97.6 \%$ \\
\hline & $\# 10$ & 2.000 & $95.1 \%$ \\
\hline
\end{tabular}

\begin{tabular}{|c|c|c|c|}
\hline & $\begin{array}{c}\text { Sieve } \\
\text { No. }\end{array}$ & $\begin{array}{c}\text { Diameter } \\
\mathrm{mm}\end{array}$ & $\begin{array}{c}\text { Percent } \\
\text { Finer }\end{array}$ \\
\cline { 2 - 4 } F & $\# 20$ & 0.850 & $80.2 \%$ \\
\hline N & $\# 40$ & 0.425 & $56.7 \%$ \\
\hline E & $\# 60$ & 0.250 & $42.0 \%$ \\
\cline { 2 - 4 } & $\# 100$ & 0.149 & $33.7 \%$ \\
\cline { 2 - 4 } & $\# 140$ & 0.106 & $29.5 \%$ \\
\hline & $\# 200$ & 0.075 & $26.5 \%$ \\
\hline
\end{tabular}

HYDROMETER ANALYSIS

\begin{tabular}{|c|c|c|}
\hline \multirow{4}{*}{$\begin{array}{l}H \\
Y \\
D \\
R\end{array}$} & $\begin{array}{c}\text { Diameter } \\
\mathbf{m m} \\
\end{array}$ & $\begin{array}{c}\text { Percent } \\
\text { Finer } \\
\end{array}$ \\
\hline & & \\
\hline & & \\
\hline & & \\
\hline 0 & 0.02197 & $26.0 \%$ \\
\hline M & 0.01282 & $24.1 \%$ \\
\hline$E$ & 0.00910 & $23.4 \%$ \\
\hline$E$ & 0.00640 & $21.5 \%$ \\
\hline $\mathbf{R}$ & 0.00456 & $20.3 \%$ \\
\hline & 0.00315 & $19.0 \%$ \\
\hline & 0.00132 & $17.7 \%$ \\
\hline
\end{tabular}


Page 15 of 187

Paul Lear

IT Corporation

April 19, 1999

IT Project ID: SAIC Savannah River Site

IT Project No.: 777304.00010500
IT GEOTECHNICAL

LABORATORY

OAK RIDGE, TN

(423) 482-6497

\section{SAIC I SAVANNAH RIVER}

U.S. STANDARD SIEVE SIZES

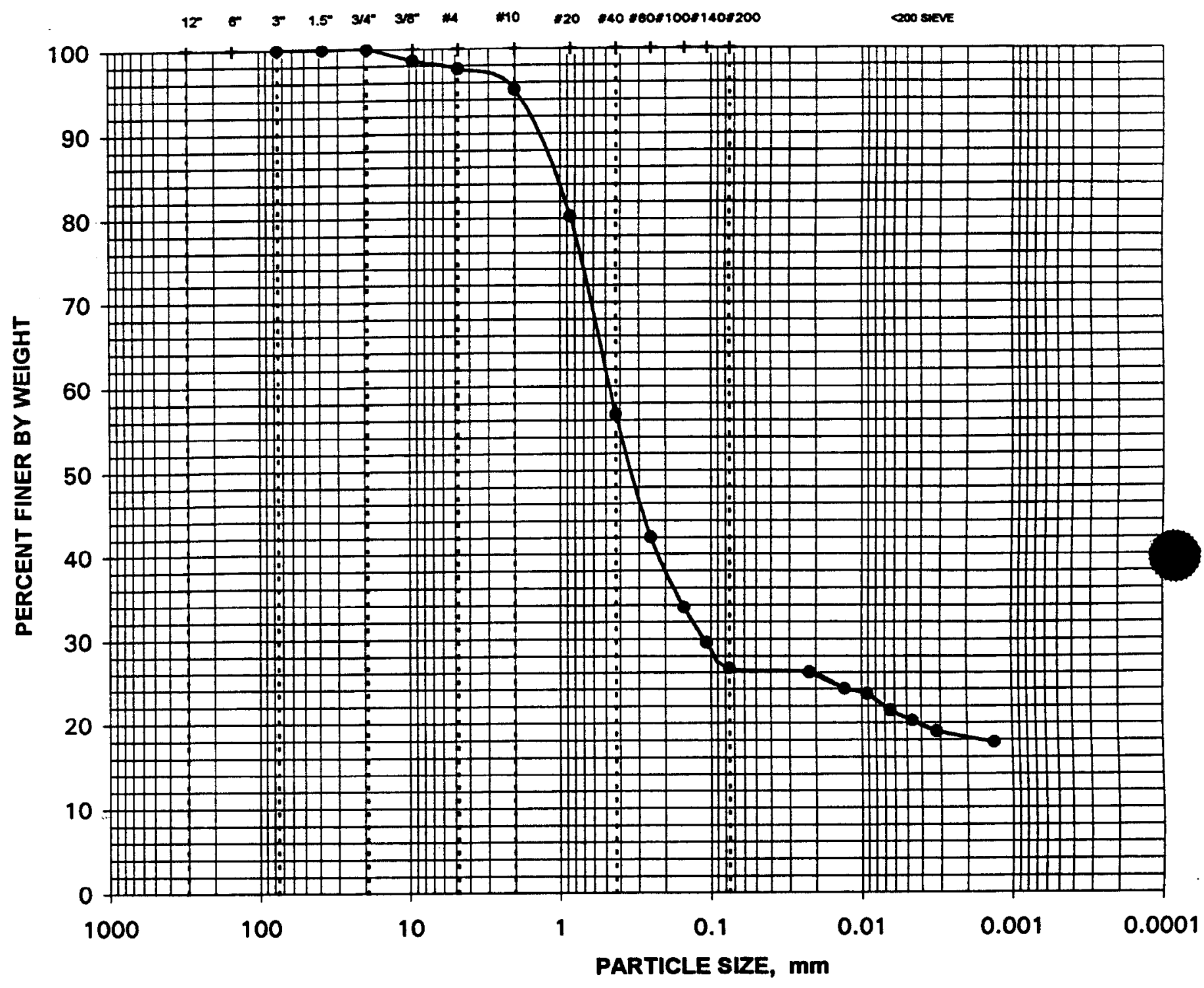

CLIENT SAMPLE NO.: $\quad$ C100Q00

iT LAB SAMPLE NO.:

ETDC-7902

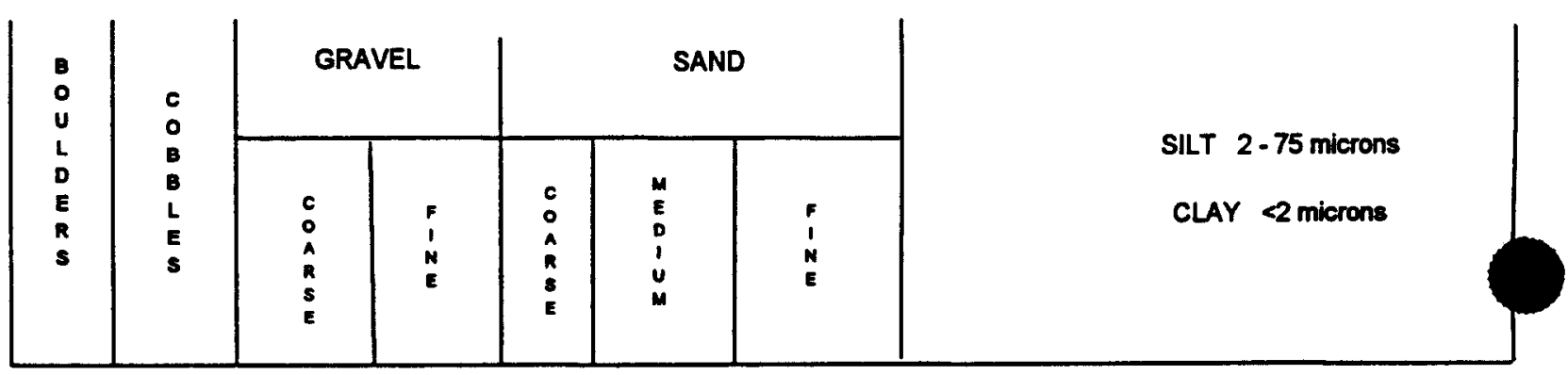




\section{PARTICLE-SIZE ANAL YSIS ASTM D 422}

Project Name

Project No.

Specific Gravity $=2.6353$
Client Sample No. C200000

IT Lab Sample No. ETDC-7903

Moisture Content $=18.5 \%$

besed on dry semple weight

SIEVE ANALYSIS

\begin{tabular}{|c|c|c|c|}
\hline & $\begin{array}{c}\text { Sieve } \\
\text { No. }\end{array}$ & $\begin{array}{c}\text { Diameter } \\
\mathrm{mm}\end{array}$ & $\begin{array}{c}\text { Percent } \\
\text { Finer }\end{array}$ \\
\hline & $3^{\prime \prime}$ & 75.000 & $100.0 \%$ \\
\hline R & $1.5^{\prime \prime}$ & 37.500 & $100.0 \%$ \\
\hline S & $0.75^{n}$ & 19.000 & $100.0 \%$ \\
\hline E & $0.375^{\prime \prime}$ & 9.500 & $98.1 \%$ \\
\hline & $\# 4$ & 4.750 & $95.3 \%$ \\
\hline & $\# 10$ & 2.000 & $91.2 \%$ \\
\hline
\end{tabular}

\begin{tabular}{|c|c|c|c|}
\hline & $\begin{array}{c}\text { Sieve } \\
\text { No. }\end{array}$ & $\begin{array}{c}\text { Diameter } \\
\mathrm{mm}\end{array}$ & $\begin{array}{c}\text { Percent } \\
\text { Finer }\end{array}$ \\
\cline { 2 - 4 } F & $\# 20$ & 0.850 & $82.6 \%$ \\
\cline { 2 - 4 } I & $\# 40$ & 0.425 & $64.4 \%$ \\
\hline E & $\# 60$ & 0.250 & $45.0 \%$ \\
\cline { 2 - 4 } & $\# 100$ & 0.149 & $29.2 \%$ \\
\cline { 2 - 4 } & $\# 140$ & 0.106 & $22.3 \%$ \\
\hline & $\# 200$ & 0.075 & $18.4 \%$ \\
\hline
\end{tabular}

HYDROMETER ANALYSIS

\begin{tabular}{|c|c|c|}
\hline & $\begin{array}{c}\text { Diameter } \\
\mathrm{mm}\end{array}$ & $\begin{array}{c}\text { Percent } \\
\text { Finer }\end{array}$ \\
\hline H & & \\
\cline { 2 - 3 } & & \\
\hline$R$ & & $17.8 \%$ \\
\hline & & $17.2 \%$ \\
\hline$M$ & 0.03623 & $16.5 \%$ \\
\hline T & 0.02300 & $15.3 \%$ \\
\hline E & 0.01333 & $14.0 \%$ \\
\hline$R$ & 0.00946 & $13.4 \%$ \\
\hline & 0.00666 & $12.7 \%$ \\
\hline & 0.00472 & $12.7 \%$ \\
\hline
\end{tabular}


Page 17 of 187

IT GEOTECHNICAL

Paul Lear

IT Corporation

April 19, 1999

IT Project ID: SAIC Savannah River Site

IT Project No.: 777304.00010500

LABORATORY

OAK RIDGE, TN

(423) 482-6497

\section{SAIC I SAVANNAH RIVER}

\section{U.S. STANDARD SIEVE SIZES}

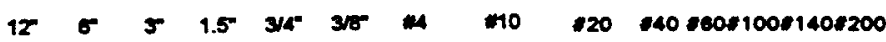

\section{HYDROMETER}

QDo sIeve

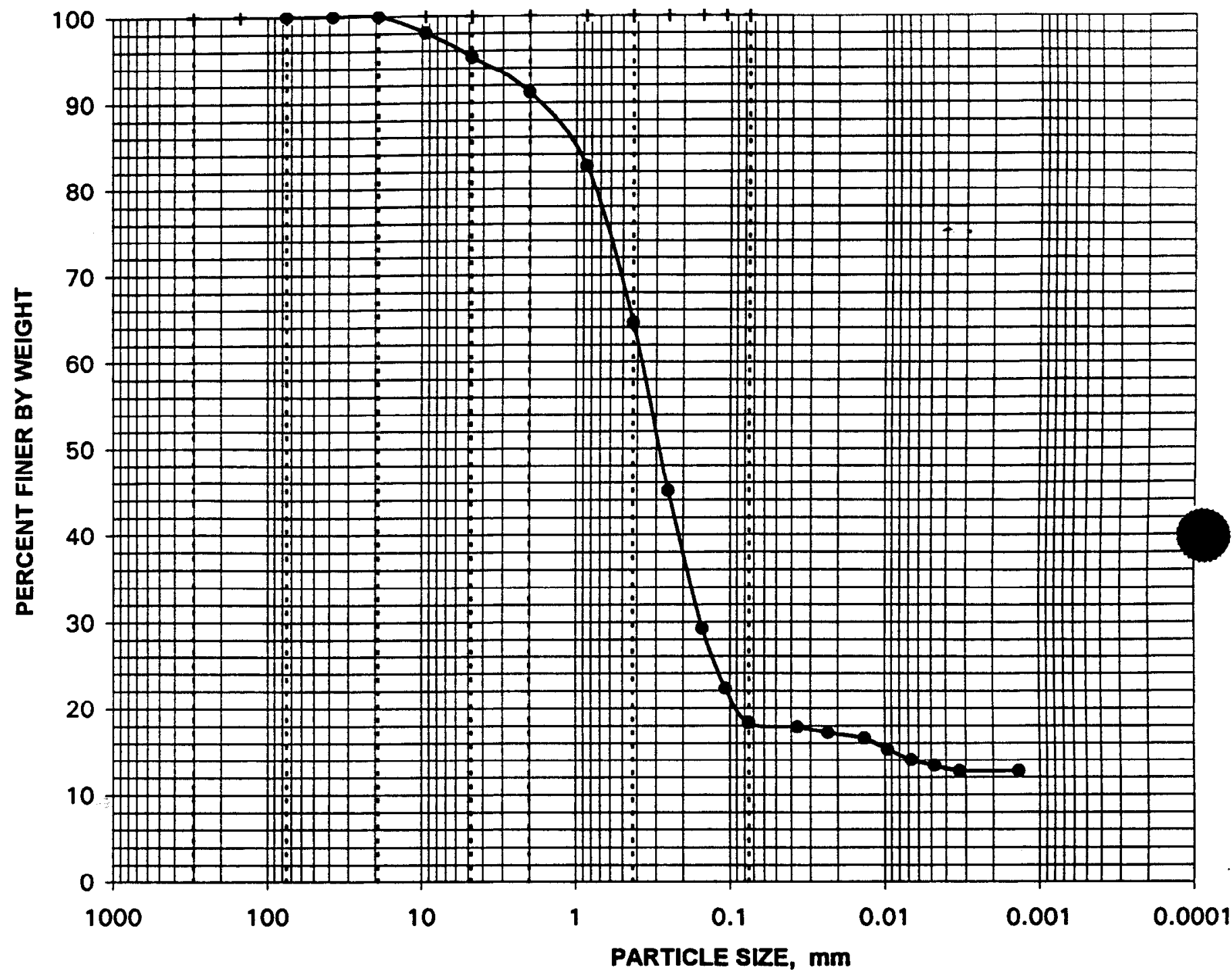

ClUENT SAMPLE NO: $\quad$ C200Q00

iT LAB SAMPLE NO.:

ETDC-7903

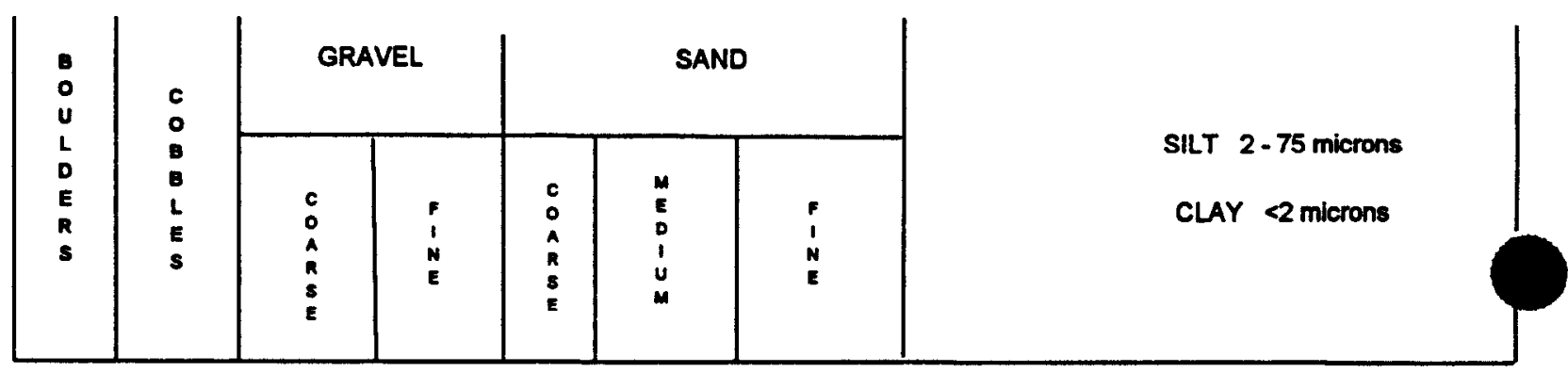




\section{PARTICLE-SIZE ANALYSIS \\ ASTM D 422}

Project Name

Project No.

Specific Gravity $=2.6593$
Client Sample No. $\quad$ C300000

IT Lab Sample No. ETDC-7904

Moisture Content $=12.3 \%$

based on dry sample woight

SIEVE ANALYSIS

\begin{tabular}{|c|c|c|c|}
\hline & $\begin{array}{c}\text { Sieve } \\
\text { No. }\end{array}$ & $\begin{array}{c}\text { Diameter } \\
\mathrm{mm}\end{array}$ & $\begin{array}{c}\text { Percent } \\
\text { Finer }\end{array}$ \\
\hline & $3^{\prime \prime}$ & 75.000 & $100.0 \%$ \\
\hline A & $1.5^{\prime \prime}$ & 37.500 & $100.0 \%$ \\
\hline R & $0.75^{\prime \prime}$ & 19.000 & $100.0 \%$ \\
S & $0.375^{\circ}$ & 9.500 & $98.6 \%$ \\
E & $\# 4$ & 4.750 & $97.7 \%$ \\
\cline { 2 - 4 } & $\# 4$ & 2.000 & $94.9 \%$ \\
\hline
\end{tabular}

\begin{tabular}{|c|c|c|c|}
\hline & $\begin{array}{c}\text { Sieve } \\
\text { No. }\end{array}$ & $\begin{array}{c}\text { Diameter } \\
\mathrm{mm}\end{array}$ & $\begin{array}{c}\text { Percent } \\
\text { Finer }\end{array}$ \\
\cline { 2 - 4 } F & $\# 20$ & 0.850 & $83.6 \%$ \\
\cline { 2 - 4 } N & $\# 40$ & 0.425 & $58.0 \%$ \\
\hline \multirow{1}{*}{ E } & $\# 60$ & 0.250 & $39.6 \%$ \\
\cline { 2 - 4 } & $\# 100$ & 0.149 & $27.8 \%$ \\
\hline & $\# 140$ & 0.106 & $22.2 \%$ \\
\hline & $\# 200$ & 0.075 & $18.5 \%$ \\
\hline
\end{tabular}

HYDROMETER ANALYSIS

\begin{tabular}{|c|c|c|}
\hline & $\begin{array}{c}\text { Diameter } \\
\mathrm{mm}\end{array}$ & $\begin{array}{c}\text { Percent } \\
\text { Finer }\end{array}$ \\
\hline$H$ & & \\
\hline & & \\
\hline$R$ & 0.03560 & $18.2 \%$ \\
\hline & 0.02256 & $17.5 \%$ \\
\hline$M$ & 0.01307 & $16.9 \%$ \\
\hline T & 0.00918 & $15.7 \%$ \\
\hline$E$ & 0.00651 & $15.0 \%$ \\
\hline$R$ & 0.00464 & $13.8 \%$ \\
\hline & 0.00318 & $13.2 \%$ \\
\hline & 0.00133 & $13.2 \%$ \\
\hline
\end{tabular}


Page 19 of 187

Paul Lear

IT GEOTECHNICAL

IT Corporation

April 19, 1999

IT Project ID: SAIC Savannah River Site

LABORATORY

IT Project No.: 777304.00010500

OAK RIDGE, TN

(423) 482-6497

\section{SAIC / SAVANNAH RIVER}

U.S. STANDARD SIEVE SIZES

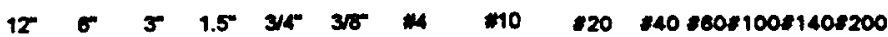

HYDROMETER

CoO save

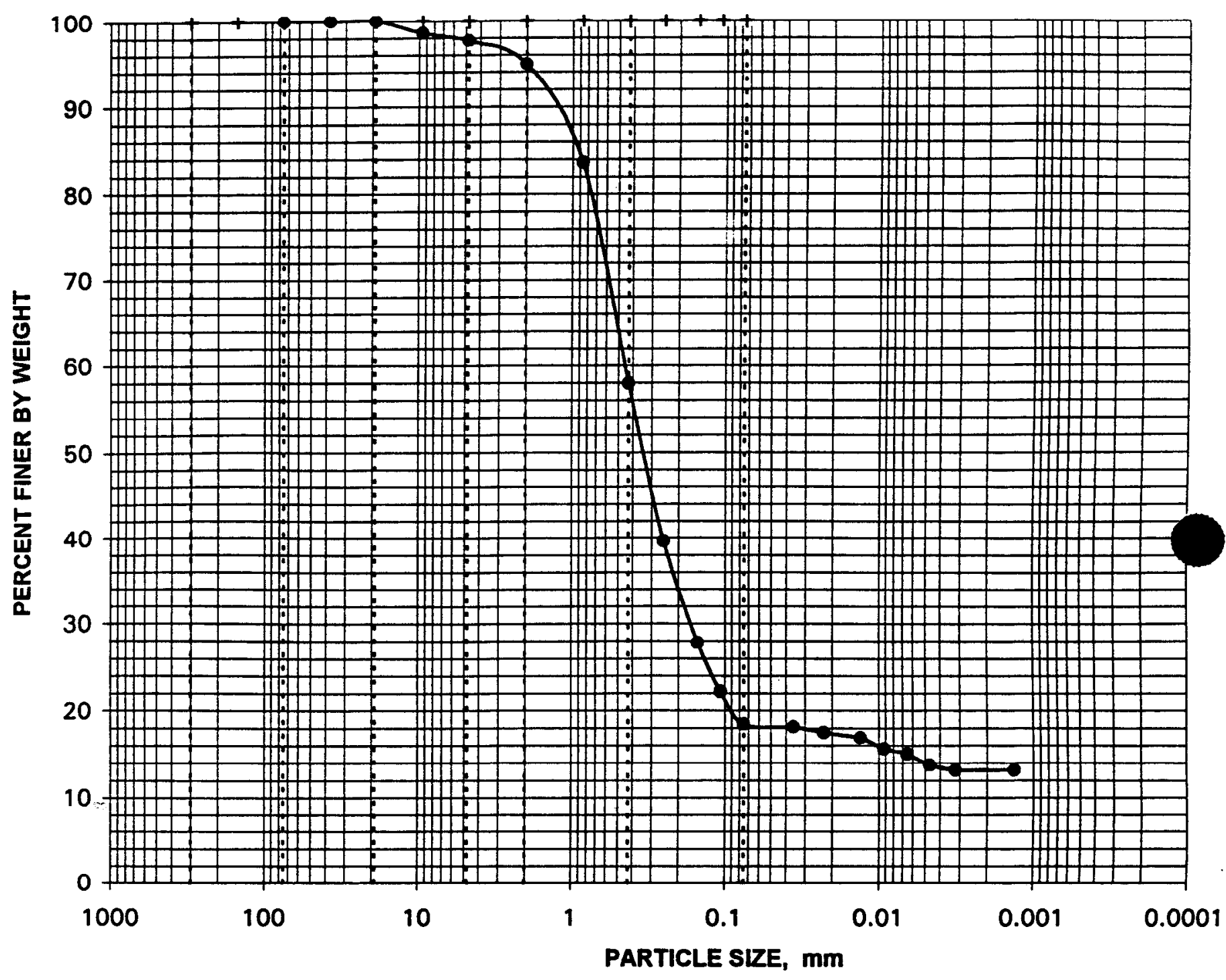

CLIENT SAMPLE NO.: $\quad$ C300Q00

IT LAB SAMPLE NO.:

ETDC-7904

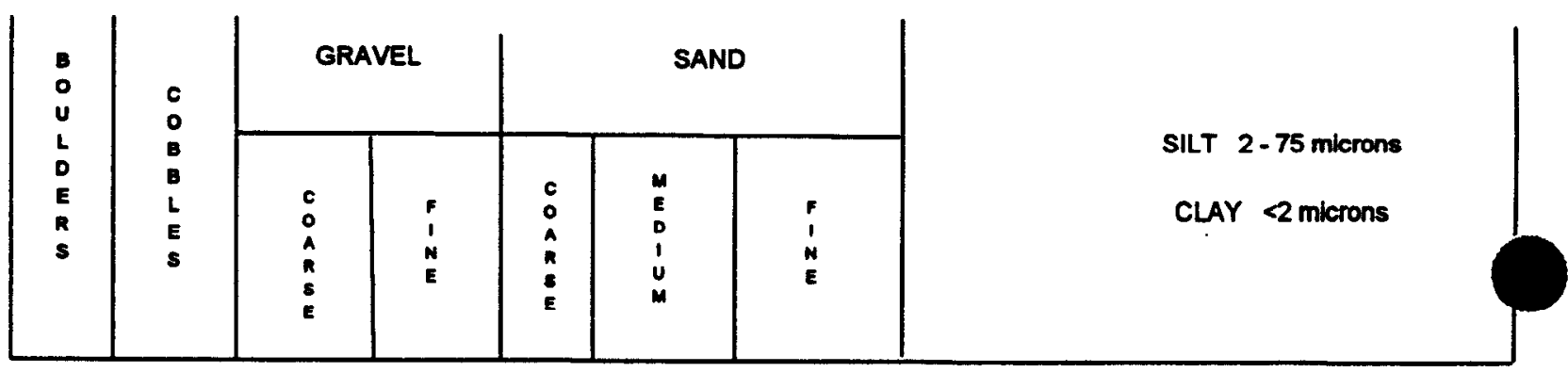


Project Name

SAIC / SAVANNAH RIVER

Project No.

777304.00010500

Specific Gravity $=2.6439$
Client Sample No. $\quad \mathrm{C} 400000$

IT Lab Sample No. ETDC-7905

Moisture Content $=20.0 \%$

besed on dry samplo woight

SIEVE ANALYSIS

\begin{tabular}{|c|c|c|c|}
\hline & $\begin{array}{c}\text { Sieve } \\
\text { No. }\end{array}$ & $\begin{array}{c}\text { Diameter } \\
\mathrm{mm}\end{array}$ & $\begin{array}{c}\text { Percent } \\
\text { Finer }\end{array}$ \\
\cline { 2 - 4 } O & $3^{\prime \prime}$ & 75.000 & $100.0 \%$ \\
\hline R & $1.5^{\prime \prime}$ & 37.500 & $100.0 \%$ \\
\cline { 2 - 4 } S & $0.75^{\prime \prime}$ & 19.000 & $100.0 \%$ \\
\hline E & $0.375^{\prime \prime}$ & 9.500 & $99.3 \%$ \\
\hline & $\# 4$ & 4.750 & $98.1 \%$ \\
\hline & $\# 10$ & 2.000 & $95.2 \%$ \\
\hline
\end{tabular}

\begin{tabular}{|c|c|c|c|}
\hline & $\begin{array}{c}\text { Sieve } \\
\text { No. }\end{array}$ & $\begin{array}{c}\text { Diameter } \\
\mathrm{mm}\end{array}$ & $\begin{array}{c}\text { Percent } \\
\text { Finer }\end{array}$ \\
\cline { 2 - 4 } F & $\# 20$ & 0.850 & $85.9 \%$ \\
\hline N & $\# 40$ & 0.425 & $67.1 \%$ \\
\hline \multirow{4}{*}{ E } & $\# 60$ & 0.250 & $50.9 \%$ \\
\cline { 2 - 4 } & $\# 100$ & 0.149 & $38.3 \%$ \\
\cline { 2 - 4 } & $\# 140$ & 0.106 & $32.0 \%$ \\
\hline & $\# 200$ & 0.075 & $27.6 \%$ \\
\hline
\end{tabular}

HYDROMETER ANALYSIS

\begin{tabular}{|c|c|c|}
\hline \multirow{2}{*}{$\begin{array}{l}H \\
Y\end{array}$} & $\begin{array}{c}\text { Diameter } \\
\mathrm{mm}\end{array}$ & $\begin{array}{c}\text { Percent } \\
\text { Finer }\end{array}$ \\
\hline & & \\
\hline \multirow{2}{*}{$\begin{array}{l}\mathrm{D} \\
\mathrm{R}\end{array}$} & & \\
\hline & 0.03473 & $27.2 \%$ \\
\hline & 0.02201 & $26.5 \%$ \\
\hline & 0.01292 & $23.9 \%$ \\
\hline \multirow{2}{*}{$\begin{array}{l}E \\
T\end{array}$} & 0.00904 & $23.2 \%$ \\
\hline & 0.00645 & $21.2 \%$ \\
\hline \multirow[t]{3}{*}{$\mathbf{R}$} & 0.00460 & $19.9 \%$ \\
\hline & 0.00315 & $19.2 \%$ \\
\hline & 0.00132 & $18.6 \%$ \\
\hline
\end{tabular}




\section{SAIC / SAVANNAH RIVER}

\section{U.S. STANDARD SIEVE SIZES}

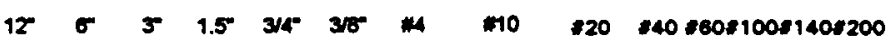

HYDROMETER

Coo seve

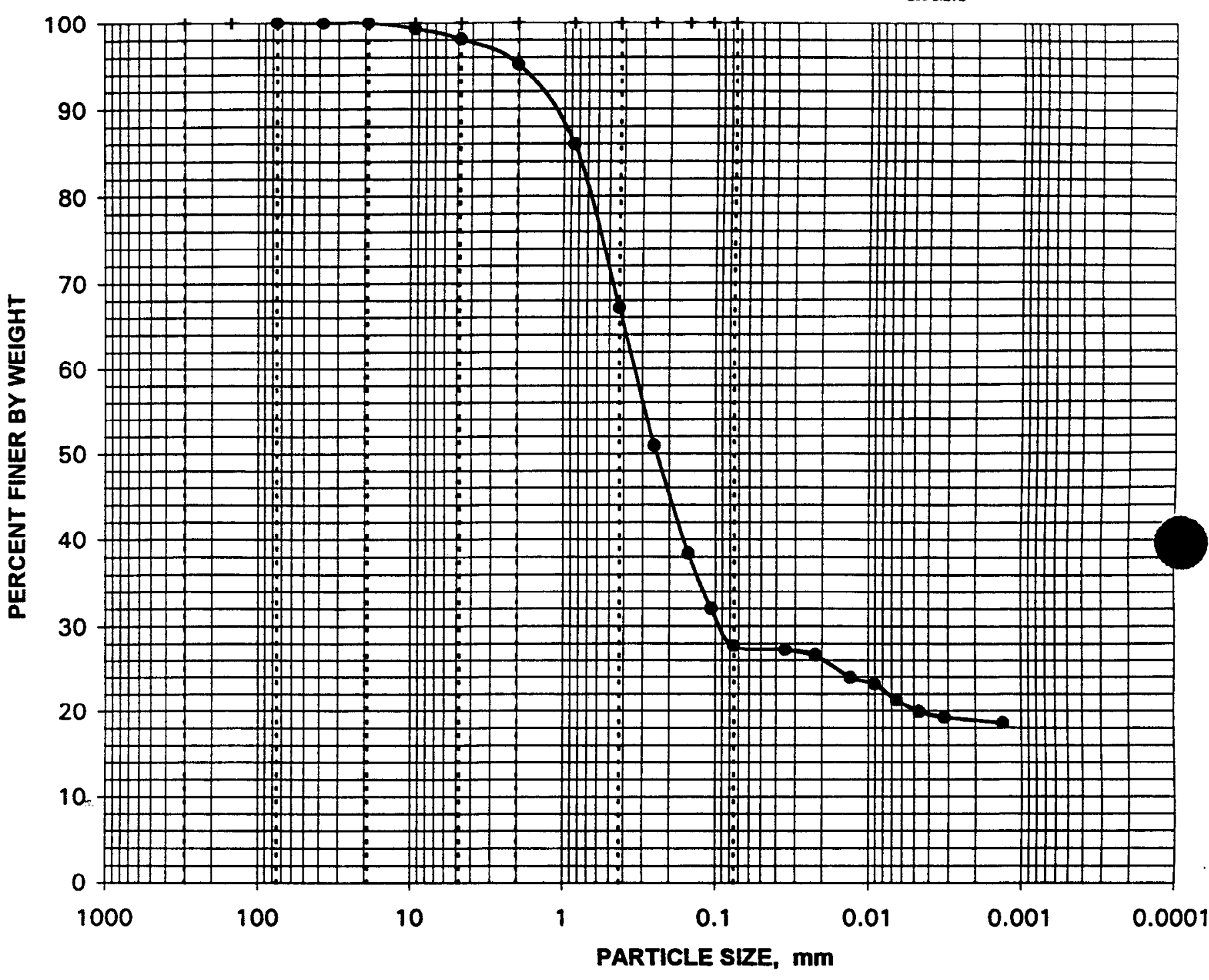

Client sample no.: $\quad$ C400Q00

IT LAB SAMPLE NO.:

ETDC-7905

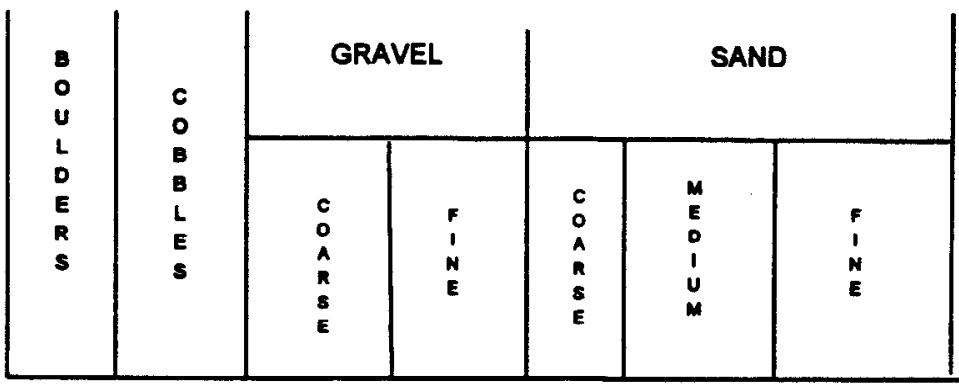

SILT 2 - 75 microns

CLAY $<2$ microns 


\section{PARTICLE-SIZE ANALYSIS}

\section{ASTM D 422}

Project Name

SAIC

C I SAVANNAH RIVER

777304.00010500

Project No.

Specific Gravity $=2.6413$
Client Sample No. $\quad$ C500000

IT Lab Sample No. ETDC-7906

Moisture Content $=\quad 14.5 \%$

based on dry sample woight

SIEVE ANALYSIS

\begin{tabular}{|c|c|c|c|}
\hline & $\begin{array}{c}\text { Sieve } \\
\text { No. }\end{array}$ & $\begin{array}{c}\text { Diameter } \\
\mathrm{mm}\end{array}$ & $\begin{array}{c}\text { Percent } \\
\text { Finer }\end{array}$ \\
\cline { 2 - 4 } $\mathrm{O}$ & $3^{\prime \prime}$ & 75.000 & $100.0 \%$ \\
\hline $\mathrm{A}$ & $1.5^{\prime \prime}$ & 37.500 & $100.0 \%$ \\
$\mathrm{R}$ & $0.75^{\prime \prime}$ & 19.000 & $100.0 \%$ \\
$\mathrm{~S}$ & $0.375^{\prime \prime}$ & 9.500 & $99.3 \%$ \\
$\mathrm{E}$ & $\# 4$ & 4.750 & $98.0 \%$ \\
\cline { 2 - 4 } & $\# 4$ & 2.000 & $95.2 \%$ \\
\hline
\end{tabular}

\begin{tabular}{|c|c|c|c|}
\hline & $\begin{array}{c}\text { Sieve } \\
\text { No. }\end{array}$ & $\begin{array}{c}\text { Diameter } \\
\mathrm{mm}\end{array}$ & $\begin{array}{c}\text { Percent } \\
\text { Finer }\end{array}$ \\
\cline { 2 - 4 } F & $\# 20$ & 0.850 & $84.4 \%$ \\
\hline N & $\# 40$ & 0.425 & $61.8 \%$ \\
\cline { 2 - 4 } E & $\# 60$ & 0.250 & $43.7 \%$ \\
\cline { 2 - 4 } & $\# 100$ & 0.149 & $31.1 \%$ \\
\cline { 2 - 4 } & $\# 140$ & 0.106 & $25.1 \%$ \\
\hline & $\# 200$ & 0.075 & $21.2 \%$ \\
\hline
\end{tabular}

HYDROMETER ANALYSIS

\begin{tabular}{|c|c|c|}
\hline \multirow{3}{*}{$\begin{array}{l}H \\
Y \\
D\end{array}$} & $\begin{array}{c}\text { Diameter } \\
\mathbf{m m}\end{array}$ & $\begin{array}{c}\text { Percent } \\
\text { Finer }\end{array}$ \\
\hline & & \\
\hline & & \\
\hline$R$ & 0.03545 & $20.5 \%$ \\
\hline 0 & 0.02256 & $19.4 \%$ \\
\hline M & 0.01296 & $18.2 \%$ \\
\hline E & 0.00920 & $17.6 \%$ \\
\hline E & 0.00656 & $15.8 \%$ \\
\hline $\mathbf{R}$ & 0.00465 & $15.3 \%$ \\
\hline & 0.00319 & $14.7 \%$ \\
\hline & 0.00133 & $14.7 \%$ \\
\hline
\end{tabular}




\section{SAIC / SAVANNAH RIVER}

\section{U.S. STANDARD SIEVE SIZES}

$12 \quad \sigma \quad 5 \quad 1.5 \%$ 3/4 $3 / 5 \%$
HYDROMETER

coo aneve

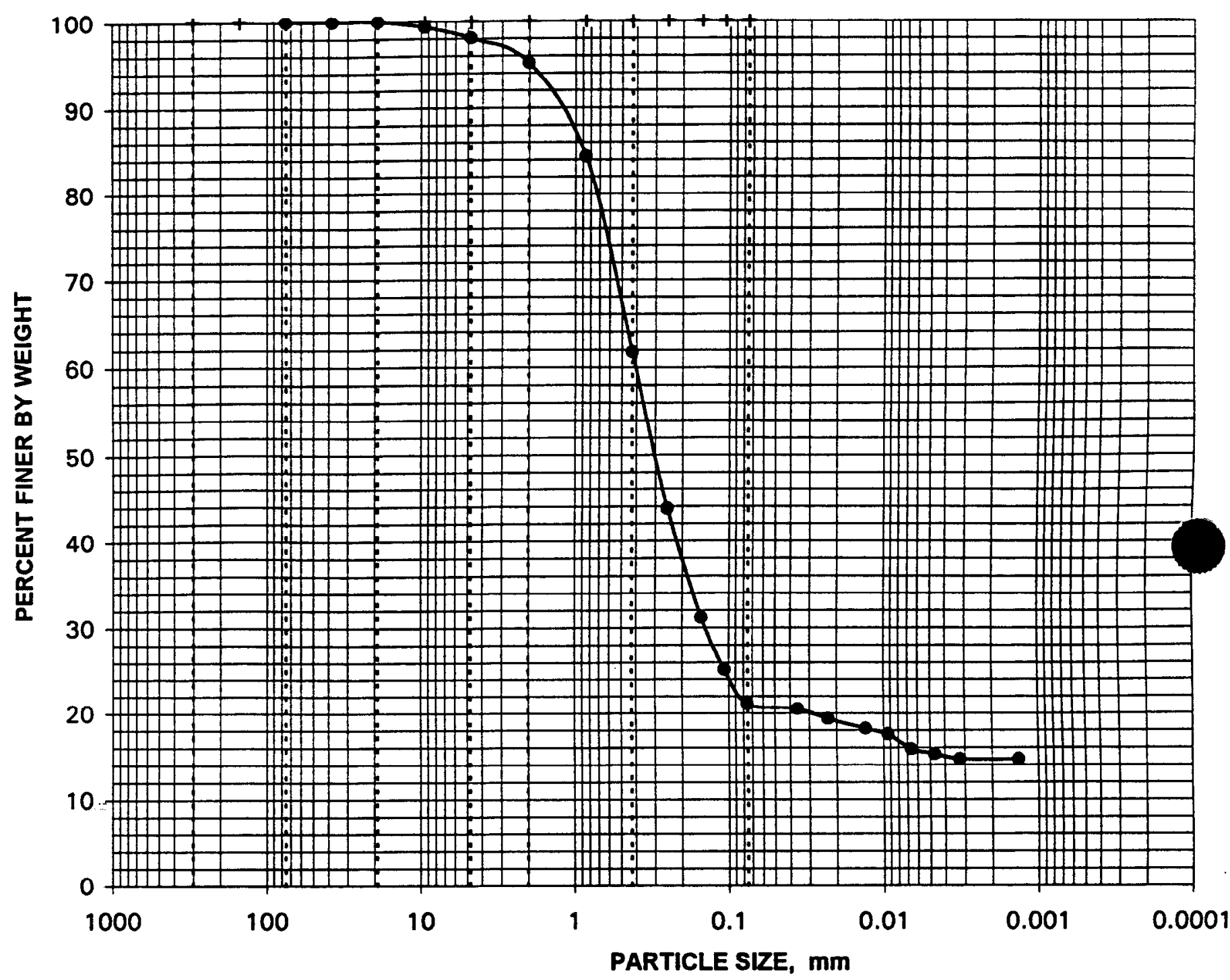

CLIENT SAMPLE NO.: $\quad$ C500Q00

IT LAB SAMPLE NO.:

ETDC-7906

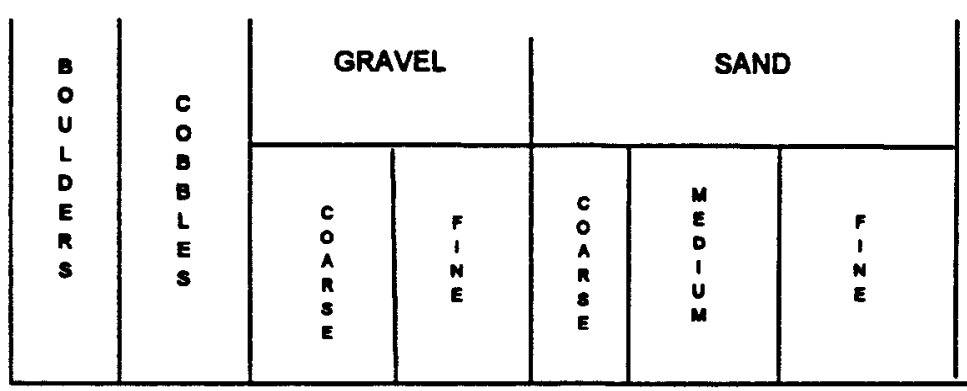

SILT 2 - 75 microns

CLAY $<2$ microns 


\section{PARTICLE-SIZE ANALYSIS}

\section{ASTM D 422}

Project Name

SAIC / Savannah River

Project No.

777304.00010500

Specific Gravity $=2.6352$
Client Sample No. $\quad \mathrm{C600000}$

IT Lab Sample No. ETDC-7907

Moisture Content $=14.5 \%$

besed on dry sample woight

SIEVE ANALYSIS

\begin{tabular}{|c|c|c|c|}
\hline & $\begin{array}{c}\text { Sieve } \\
\text { No. }\end{array}$ & $\begin{array}{c}\text { Diameter } \\
\mathrm{mm}\end{array}$ & $\begin{array}{c}\text { Percent } \\
\text { Finer }\end{array}$ \\
\hline 0 & $3^{\prime \prime}$ & 75.000 & $100.0 \%$ \\
\hline A & $1.5^{\prime \prime}$ & 37.500 & $100.0 \%$ \\
R & $0.75^{\prime \prime}$ & 19.000 & $98.2 \%$ \\
S & $0.375^{\prime \prime}$ & 9.500 & $97.2 \%$ \\
E & $\# 4$ & 4.750 & $95.5 \%$ \\
\cline { 2 - 4 } & $\# 10$ & 2.000 & $92.9 \%$ \\
\hline
\end{tabular}

\begin{tabular}{|c|c|c|c|}
\hline & $\begin{array}{c}\text { Sieve } \\
\text { No. }\end{array}$ & $\begin{array}{c}\text { Diameter } \\
\mathrm{mm}\end{array}$ & $\begin{array}{c}\text { Percent } \\
\text { Finer }\end{array}$ \\
\cline { 2 - 4 } F & $\# 20$ & 0.850 & $80.7 \%$ \\
\hline N & $\# 40$ & 0.425 & $58.7 \%$ \\
\hline E & $\# 60$ & 0.250 & $42.0 \%$ \\
\cline { 2 - 4 } & $\# 100$ & 0.149 & $30.2 \%$ \\
\hline & $\# 140$ & 0.106 & $24.5 \%$ \\
\hline & $\# 200$ & 0.075 & $20.7 \%$ \\
\hline
\end{tabular}

HYDROMETER ANALYSIS

\begin{tabular}{|c|c|c|}
\hline \multirow{3}{*}{$\begin{array}{l}H \\
Y\end{array}$} & $\begin{array}{c}\text { Diameter } \\
\mathrm{mm}\end{array}$ & $\begin{array}{c}\text { Percent } \\
\text { Finer }\end{array}$ \\
\hline & & \\
\hline & & \\
\hline $\mathbf{R}$ & 0.03567 & $20.0 \%$ \\
\hline 0 & 0.02236 & $19.4 \%$ \\
\hline M & 0.01301 & $18.2 \%$ \\
\hline E & 0.00924 & $17.0 \%$ \\
\hline E & 0.00656 & $16.4 \%$ \\
\hline$\vec{R}$ & 0.00465 & $15.8 \%$ \\
\hline & 0.00319 & $15.2 \%$ \\
\hline & 0.00133 & $14.6 \%$ \\
\hline
\end{tabular}




\section{SAIC / Savannah River}

\section{U.S. STANDARD SIEVE SIZES}

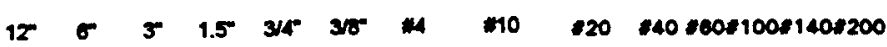

HYDROMETER

cos steve

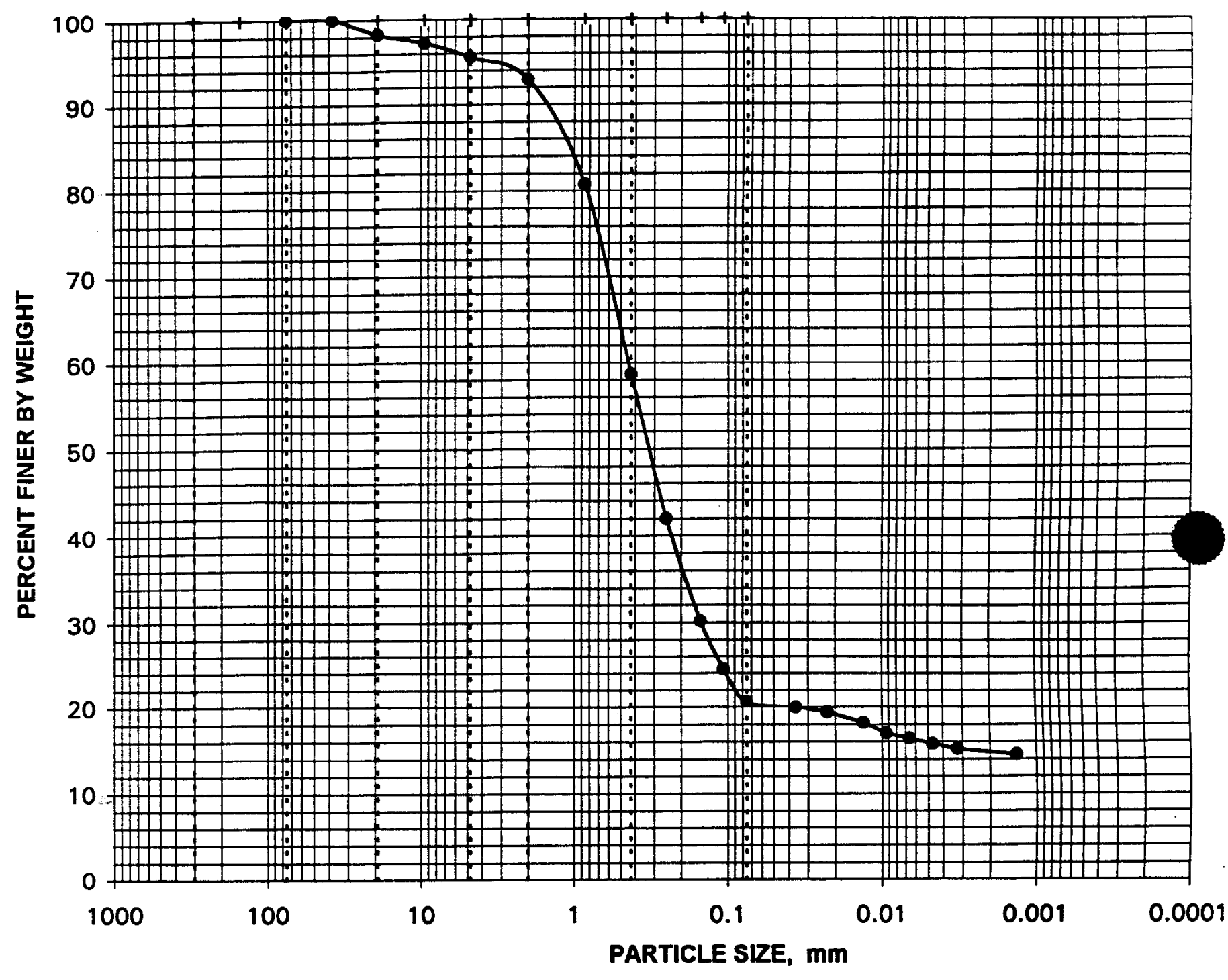

CLIENT SAMPLE NO.: $\quad \mathrm{C600Q00}$

IT LAB SAMPLE NO.:

ETDC-7907

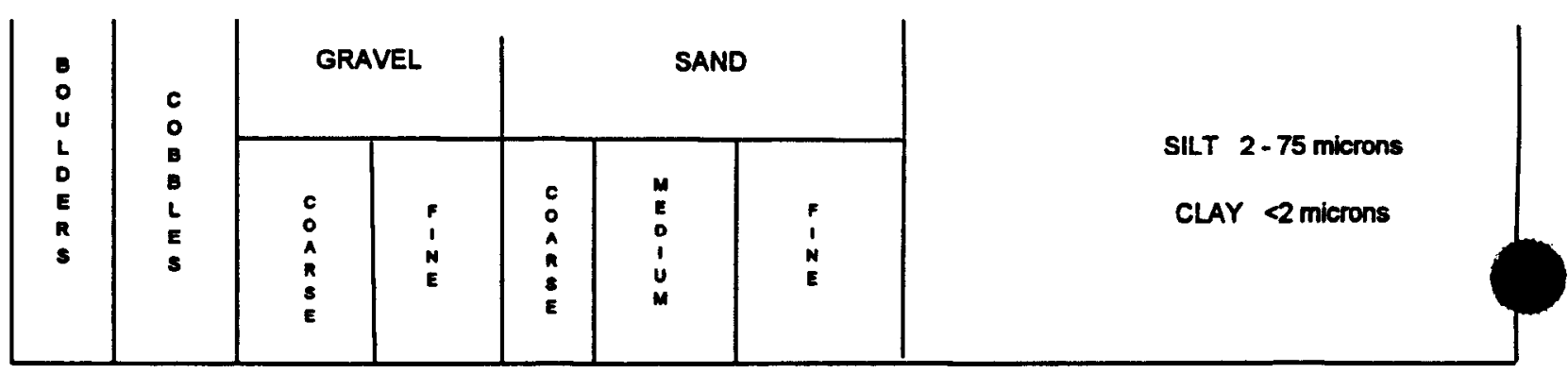




\section{PARTICLE-SIZE ANALYSIS \\ ASTM D 422}
Project Name
SAIC / SAVANNAH RIVER
Client Sample No.
C700000
Project No.
777304.00010500
IT Lab Sample No.
ETDC-7908
Specific Gravity $=2.6508$
Moisture Content $=12.6 \%$
besod on dry semplo woight

SIEVE ANALYSIS

\begin{tabular}{|c|c|c|c|}
\hline & $\begin{array}{c}\text { Sieve } \\
\text { No. }\end{array}$ & $\begin{array}{c}\text { Diameter } \\
\mathrm{mm}\end{array}$ & $\begin{array}{c}\text { Percent } \\
\text { Finer }\end{array}$ \\
\hline O & $3^{\prime \prime}$ & 75.000 & $100.0 \%$ \\
\hline A & $1.5^{\prime \prime}$ & 37.500 & $100.0 \%$ \\
\cline { 2 - 4 } S & $0.75^{\prime \prime}$ & 19.000 & $92.5 \%$ \\
\hline E & $0.375^{\prime \prime}$ & 9.500 & $90.9 \%$ \\
\hline & $\# 4$ & 4.750 & $90.2 \%$ \\
\hline
\end{tabular}

\begin{tabular}{|c|c|c|c|}
\hline & $\begin{array}{c}\text { Sieve } \\
\text { No. }\end{array}$ & $\begin{array}{c}\text { Diameter } \\
\mathrm{mm}\end{array}$ & $\begin{array}{c}\text { Percent } \\
\text { Finer }\end{array}$ \\
\cline { 2 - 4 } F & $\# 20$ & 0.850 & $77.7 \%$ \\
\hline N & $\# 40$ & 0.425 & $57.5 \%$ \\
\hline E & $\# 60$ & 0.250 & $40.0 \%$ \\
\cline { 2 - 4 } & $\# 100$ & 0.149 & $27.9 \%$ \\
\cline { 2 - 4 } & $\# 140$ & 0.106 & $22.4 \%$ \\
\hline & $\# 200$ & 0.075 & $19.0 \%$ \\
\hline
\end{tabular}

HYDROMETER ANALYSIS

\begin{tabular}{|c|c|c|}
\hline & $\begin{array}{c}\text { Diameter } \\
\mathrm{mm}\end{array}$ & $\begin{array}{c}\text { Percent } \\
\text { Finer }\end{array}$ \\
\hline$H$ & & \\
\hline & & \\
$D$ & & $18.7 \%$ \\
$R$ & 0.03483 & $17.5 \%$ \\
\hline & 0.02220 & $16.4 \%$ \\
\hline M & 0.01287 & $15.8 \%$ \\
\hline$T$ & 0.00913 & $15.2 \%$ \\
\hline E & 0.00648 & $14.6 \%$ \\
\hline$R$ & 0.00459 & $13.4 \%$ \\
\hline & 0.00316 & $12.9 \%$ \\
\hline
\end{tabular}




\section{SAIC I SAVANNAH RIVER}

U.S. STANDARD SIEVE SIZES

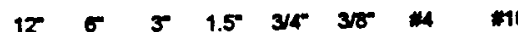

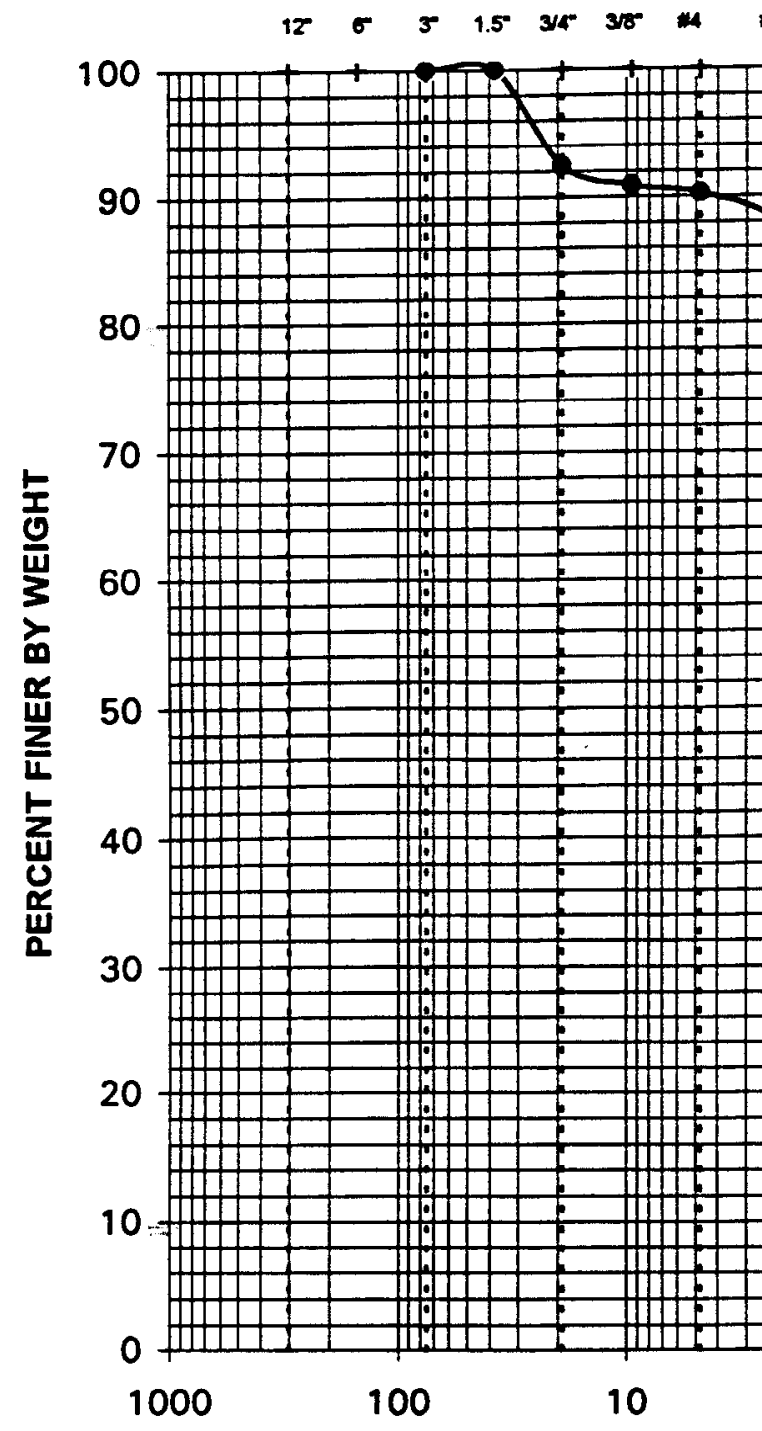

HYDROMETER

Cos SEVE

\begin{abstract}
$\$ 20 \quad 40 \$ 00 \div 100 \$ 1401200$
\end{abstract}

\section{.}

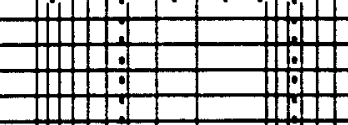

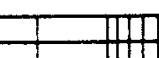
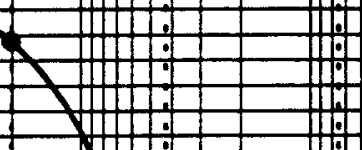

$1+$

Hig
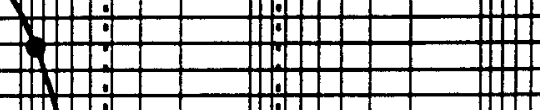

HTH

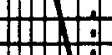

$+1 \cdot$

+

$1+1 t^{2}$
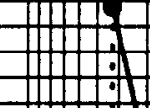

$+19$

101 i

$+1+2$

$+4$

+ +1:

+11:

$71 \%$

+

1010 '

+70 -

$+7$

$+\mathrm{H}:$

曲拉

+10:

1

0.1

10

\begin{tabular}{lllll} 
& & & & \\
\hline
\end{tabular}

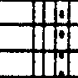

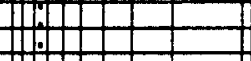

\begin{tabular}{lll} 
& & \\
\hline & &
\end{tabular}

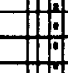

$10-12$

$+$

$+00$

$+$

\begin{tabular}{lllll} 
& & & \\
\hline & & & & \\
\hline
\end{tabular}

10

$+$

$+21$

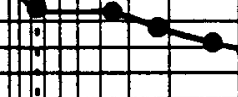

$1+9$

10 - 田

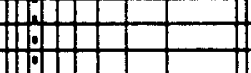

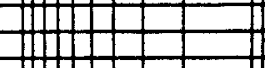

पा1+1:-

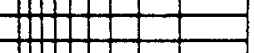

+ 車

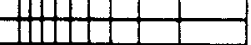

$+1012-2$

+

$+1012:-12$

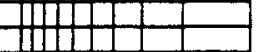

$+$

$+1$
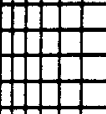

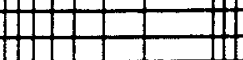

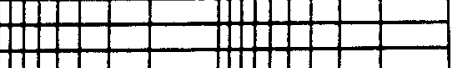

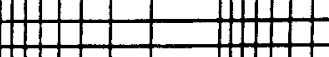

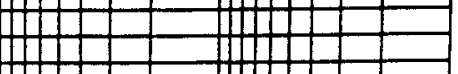

\begin{tabular}{llllll} 
& & & & \\
\hline & & & & &
\end{tabular}
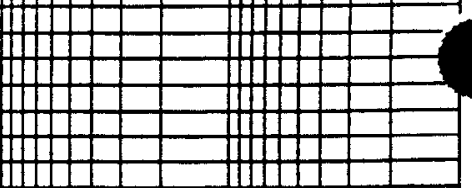

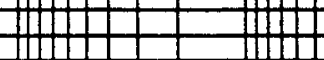

$+$

+1 + - $10+1+5$
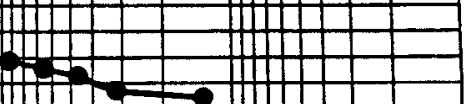

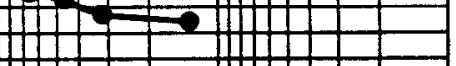

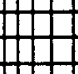

\begin{tabular}{lllll} 
& & & \\
\hline
\end{tabular}

\begin{tabular}{lllll}
\hline+4 & & & & \\
\hline
\end{tabular}

$\begin{array}{lll}0.01 & 0.001 & 0.0001\end{array}$

PARTICLE SIZE, mm

CLIENT SAMPLE NO.: $\quad \mathbf{C 7 0 0 Q 0 0}$

IT LAB SAMPLE NO.:

ETDC-7908

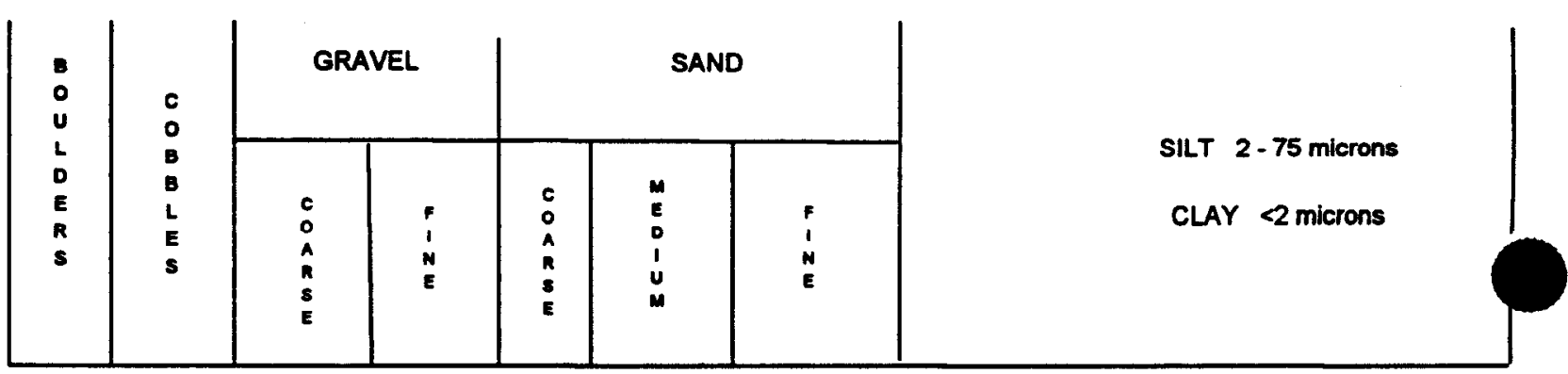




\section{PARTICLE-SIZE ANALYSIS \\ ASTM D 422}

Project Name SAIC / Savannah River

Project No. $\quad \mathbf{7 7 7 3 0 4 . 0 0 0 1 0 5 0 0}$

Specific Gravity $=2.6394$
Client Sample No. $\quad$ C800000

IT Lab Sample No. ETDC-7909

Moisture Content $=13.1 \%$

based on dry sample woight

SIEVE ANAL YSIS

\begin{tabular}{|c|c|c|c|}
\hline & $\begin{array}{c}\text { Sieve } \\
\text { No. }\end{array}$ & $\begin{array}{c}\text { Diameter } \\
\mathrm{mm}\end{array}$ & $\begin{array}{c}\text { Percent } \\
\text { Finer }\end{array}$ \\
\cline { 2 - 4 } O & $3^{\prime \prime}$ & 75.000 & $100.0 \%$ \\
\hline A & $1.5^{\prime \prime}$ & 37.500 & $100.0 \%$ \\
\cline { 2 - 4 } S & $0.75^{\prime \prime}$ & 19.000 & $95.0 \%$ \\
\cline { 2 - 4 } E & $0.375^{\prime \prime}$ & 9.500 & $90.4 \%$ \\
\cline { 2 - 4 } & $\# 4$ & 4.750 & $88.8 \%$ \\
\cline { 2 - 4 } & $\# 10$ & 2.000 & $85.7 \%$ \\
\hline
\end{tabular}

\begin{tabular}{|c|c|c|c|}
\hline & $\begin{array}{c}\text { Sieve } \\
\text { No. }\end{array}$ & $\begin{array}{c}\text { Diameter } \\
\mathrm{mm}\end{array}$ & $\begin{array}{c}\text { Percent } \\
\text { Finer }\end{array}$ \\
\cline { 2 - 4 } F & $\# 20$ & 0.850 & $76.2 \%$ \\
\hline N & $\# 40$ & 0.425 & $55.2 \%$ \\
\hline & $\# 60$ & 0.250 & $38.9 \%$ \\
\cline { 2 - 4 } & $\# 100$ & 0.149 & $27.4 \%$ \\
\hline & $\# 140$ & 0.106 & $22.0 \%$ \\
\hline 200 & 0.075 & $18.7 \%$ \\
\hline
\end{tabular}

HYDROMETER ANALYSIS

\begin{tabular}{|c|c|c|}
\hline & $\begin{array}{c}\text { Diameter } \\
\mathrm{mm}\end{array}$ & $\begin{array}{c}\text { Percent } \\
\text { Finer }\end{array}$ \\
\hline$H$ & & \\
\hline$Y$ & & \\
\hline & & $18.2 \%$ \\
\hline & 0.03581 & $17.6 \%$ \\
\hline$M$ & 0.02274 & $17.0 \%$ \\
\hline$E$ & 0.01318 & $15.3 \%$ \\
\hline & 0.00939 & $14.2 \%$ \\
\hline R & 0.00668 & $13.6 \%$ \\
\cline { 2 - 3 } & 0.00473 & $13.6 \%$ \\
\hline & 0.00328 & $11.9 \%$ \\
\hline
\end{tabular}




\section{SAIC / Savannah River}

U.S. STANDARD SIEVE SIZES

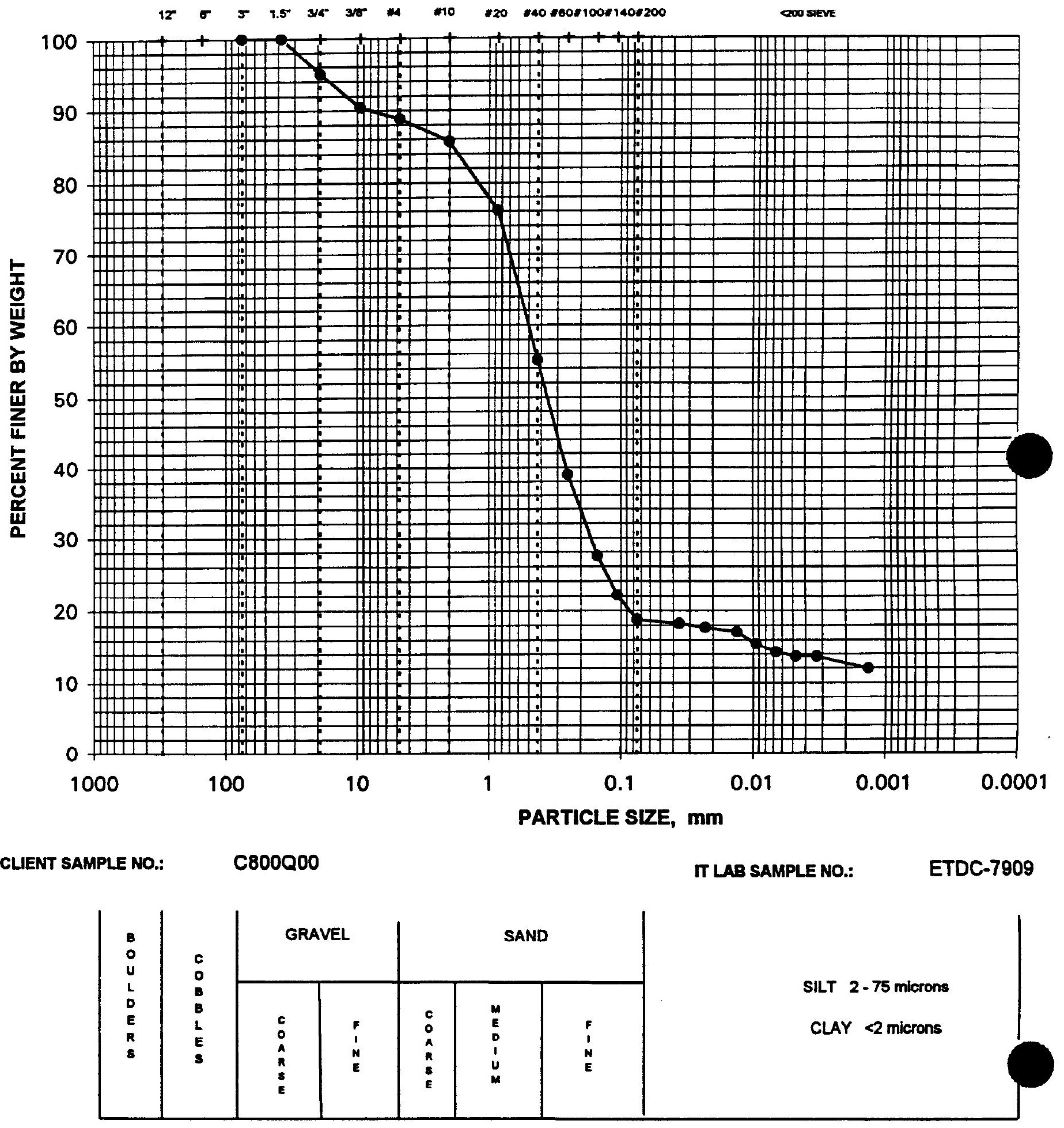

HYDROMETER

Q00 sieve 


\section{Series \\ 14 Day UCS Results}




\section{UNCONFINED COMPRESSIVE STRENGTH - ASTM D 2166 14 DAY CURE}

Project Name SAIC / SAVANNAH RIVER

Project No. $\quad 777304.00010500$

Specimen Mold Date

Specimen Test Date

STRESS AT FAILURE, psi

STRAIN AT FAILURE, \%

AXIAL DEVIATOR

$\frac{\text { STRAIN, } \%}{0.00} \frac{\text { STRESS, psi }}{0.0}$

0.10

0.20

0.30

0.40

0.50

0.76

1.01

1.26

1.51

1.76

2.01

2.27

2.52

2.77

3.02

3.27
$11 / 11 / 98$

$11 / 25 / 98$

389.5

2.8
Client Sample No.

C311F00

IT Lab Specimen No. ETDC-7805

Specimen Height, in.

3.9735

Specimen Diameter, in.

2.0045

Specimen Weight, $g$.

Moisture Content, \%

383.70

28.7

Wet Unit Weight, pcf.

116.6

Dry Unit Weight, pcf.

90.6

Rate of Strain, in./min.

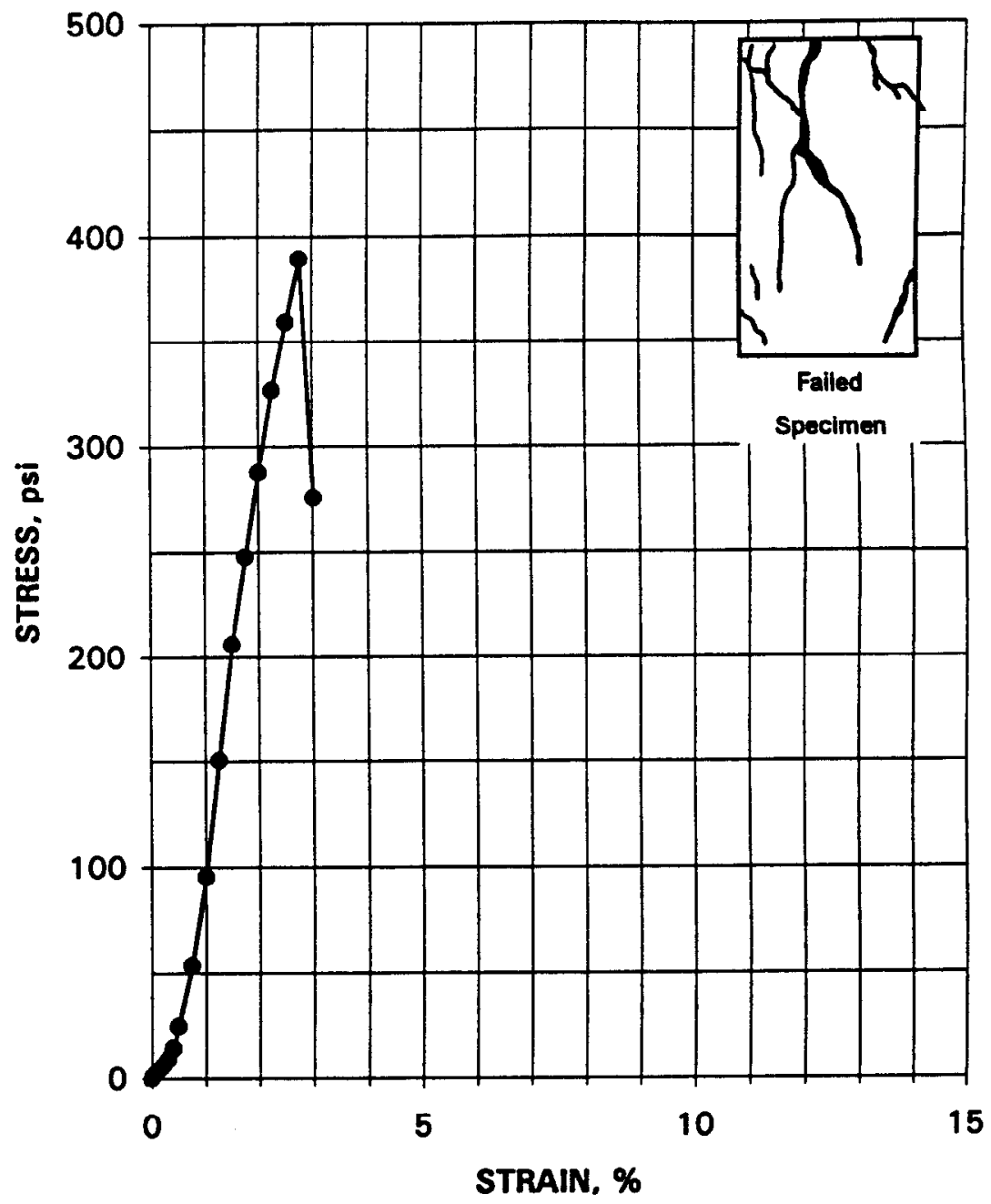




\section{UNCONFINED COMPRESSIVE STRENGTH - ASTM D 2166 14 DAY CURE}

Project Name SAIC / SAVANNAH RIVER

Project No. $\quad 777304.00010500$

Specimen Mold Date

Specimen Test Date

STRESS AT FAILURE, psi STRAIN AT FAILURE, \%

\begin{tabular}{ccc}
$\begin{array}{c}\text { AXIAL } \\
\text { STRAIN, \% }\end{array}$ & & $\begin{array}{c}\text { DEVIATOR } \\
\text { STRESS, pSi }\end{array}$ \\
\cline { 1 - 1 } 0.00 & & 0.0 \\
0.10 & & 1.6 \\
0.20 & & 2.8 \\
0.30 & & 5.7 \\
0.40 & & 9.8 \\
0.50 & & 15.1 \\
0.75 & & 53.9 \\
1.00 & & 102.6 \\
1.26 & & 145.1 \\
1.51 & & 190.5 \\
1.76 & & 224.4 \\
2.01 & 172.5 \\
2.26 & 179.4 \\
2.51 & 183.0
\end{tabular}

$11 / 11 / 98$

$11 / 25 / 98$

224.4

1.8
Client Sample No. C321F00

IT Lab Specimen No. ETDC-7806

Specimen Height, in.

3.9803

Specimen Diameter, in.

2.0073

Specimen Weight, $g$.

380.45

Moisture Content, \%

31.9

Wet Unit Weight, pcf.

115.1

Dry Unit Weight, pcf.

87.3

Rate of Strain, in./min.

0.0300

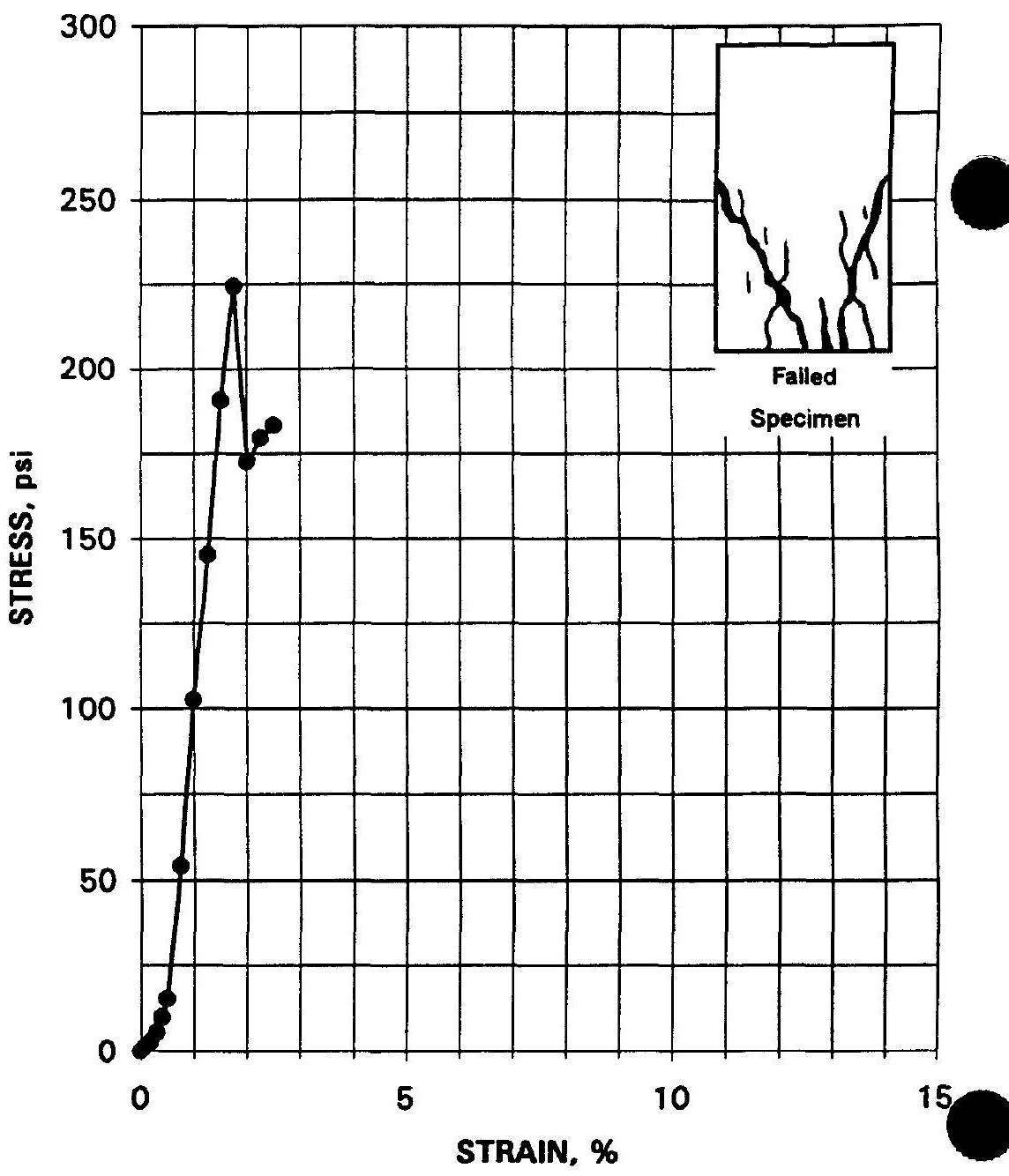




\section{UNCONFINED COMPRESSIVE STRENGTH - ASTM D 2166 14 DAY CURE}

\begin{tabular}{|c|c|}
\hline Project Name & SAIC / SAVA \\
\hline Project No. & 777304.00010 \\
\hline $\begin{array}{l}\text { Specimen Mo } \\
\text { Specimen Tes }\end{array}$ & \\
\hline STRESS AT F & URE, psi \\
\hline AXIAL & DEVIATOR \\
\hline STRAIN, \% & STRESS, psi \\
\hline 0.00 & 0.0 \\
\hline 0.10 & 1.0 \\
\hline 0.20 & 2.2 \\
\hline 0.30 & 2.8 \\
\hline 0.40 & 4.7 \\
\hline 0.50 & 8.5 \\
\hline 0.76 & 24.9 \\
\hline 1.01 & 72.3 \\
\hline 1.26 & 121.3 \\
\hline 1.51 & 169.5 \\
\hline 1.76 & 213.3 \\
\hline 2.02 & 262.8 \\
\hline 2.27 & 304.4 \\
\hline 2.52 & 338.2 \\
\hline 2.77 & 363.6 \\
\hline 2.90 & 367.4 \\
\hline 3.02 & 360.8 \\
\hline 3.28 & 347.3 \\
\hline 3.53 & 232.8 \\
\hline
\end{tabular}

$11 / 11 / 98$
$11 / 25 / 98$

367.4

2.9
Client Sample No. C331F00

IT Lab Specimen No. ETDC-7807

Specimen Height, in.

Specimen Diameter, in.

2.0027

Specimen Weight, $\mathbf{g}$.

372.74

Moisture Content, \%

32.6

Wet Unit Weight, pcf.

113.6

Dry Unit Weight, pef.

85.7

Rate of Strain, in./min.

0.0300

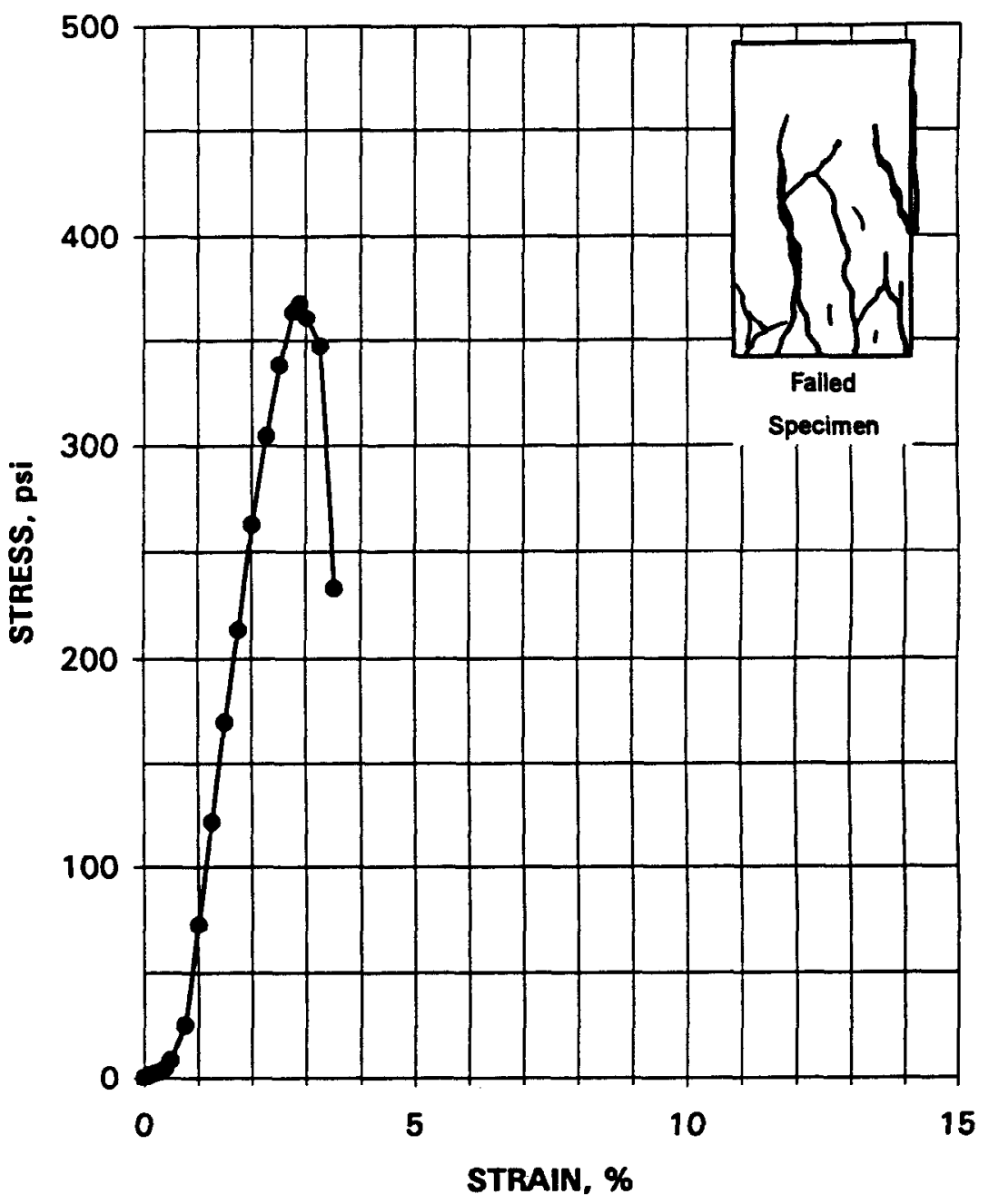




\section{UNCONFINED COMPRESSIVE STRENGTH - ASTM D 2166 14 DAY CURE}

Project Name SAIC / SAVANNAH RIVER

Project No. $\quad 777304.00010500$

Specimen Mold Date

Specimen Test Date

STRESS AT FAILURE, psi STRAIN AT FAILURE, \%

\begin{tabular}{ccc}
$\begin{array}{c}\text { AXIAL } \\
\text { STRAIN, \% }\end{array}$ & & $\begin{array}{c}\text { DEVIATOR } \\
\text { STRESS, psi }\end{array}$ \\
\cline { 1 - 1 } \cline { 3 - 3 } 0.00 & & 0.0 \\
0.10 & & 0.9 \\
0.20 & & 2.2 \\
0.30 & & 3.8 \\
0.40 & & 6.0 \\
0.50 & & 10.7 \\
0.76 & & 34.6 \\
1.01 & & 79.6 \\
1.26 & & 131.9 \\
1.51 & & 193.6 \\
1.76 & & 245.6 \\
2.01 & & 300.5 \\
2.27 & & 349.6 \\
2.52 & 400.8 \\
2.77 & 444.4 \\
2.90 & 451.8 \\
3.02 & 425.4 \\
3.27 & 324.5 \\
3.52 & 239.7
\end{tabular}

Client Sample No. C341F00

IT Lab Specimen No. ETDC-7808

Specimen Height, in.

3.9723

Specimen Diameter, in.

2.0057

Specimen Weight, $\mathrm{o}$.

379.13

Moisture Content, \%

31.2

Wet Unit Weight, pcf.

115.1

Dry Unit Weight, pcf.

87.7

Rate of Strain, in./min.

0.0300

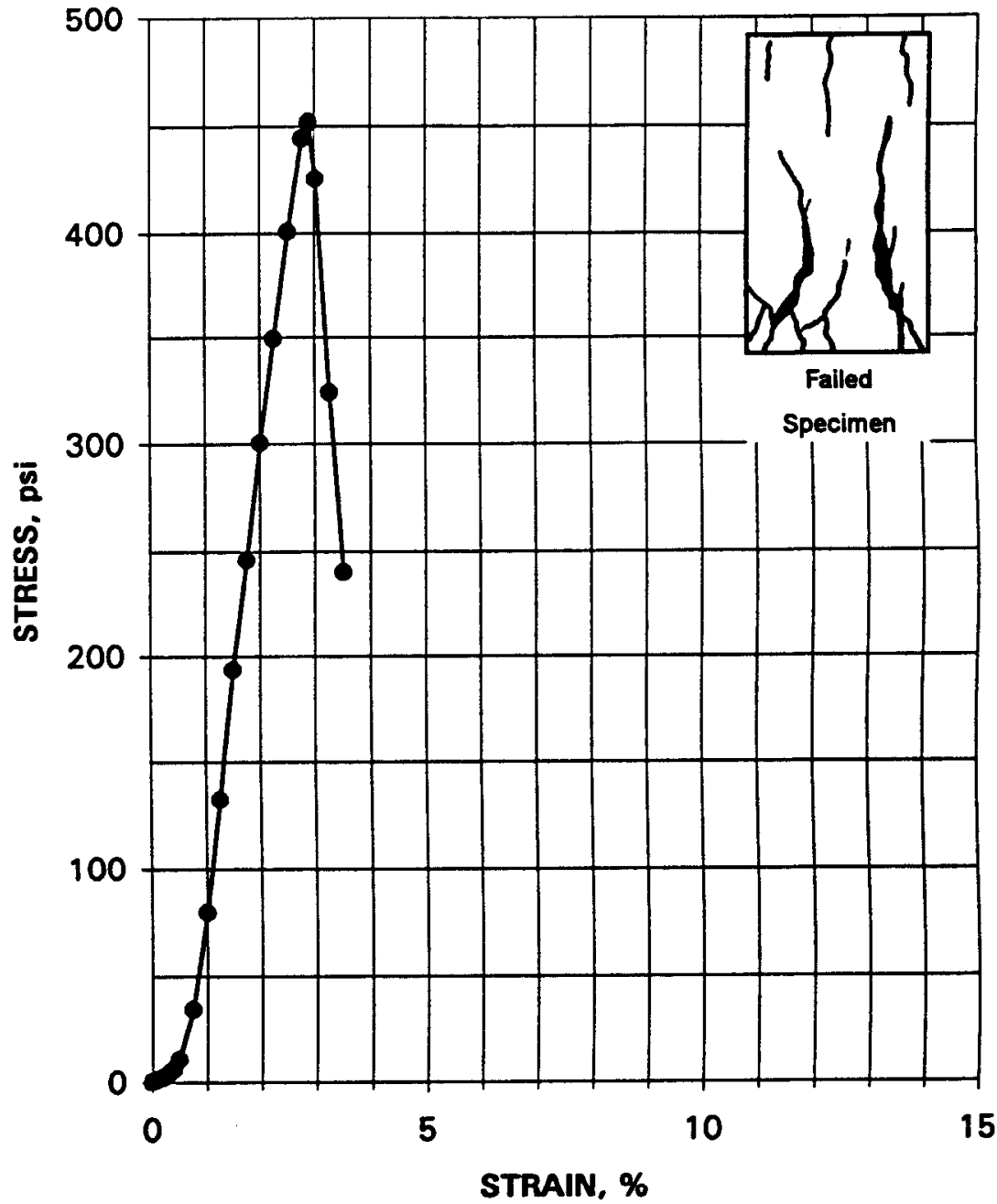




\section{UNCONFINED COMPRESSIVE STRENGTH - ASTM D 2166 14 DAY CURE}

Project Name SAIC / SAVANNAH RIVER

Project No. 777304.00010500

Specimen Mold Date
Specimen Test Date

STRESS AT FAILURE, psi

STRAIN AT FAILURE, \%

\begin{tabular}{ccc}
$\begin{array}{c}\text { AXIAL } \\
\text { STRAIN, \% }\end{array}$ & & $\begin{array}{c}\text { DEVIATOR } \\
\text { STRESS, psi }\end{array}$ \\
\cline { 1 - 1 } 0.00 & 0.0 \\
0.10 & & 1.6 \\
0.20 & & 5.1 \\
0.30 & & 11.4 \\
0.40 & & 21.5 \\
0.51 & & 35.4 \\
0.76 & & 81.0 \\
1.01 & & 121.1 \\
1.26 & & 155.9 \\
1.52 & & 189.6 \\
1.77 & & 205.3 \\
2.02 & & 232.5 \\
2.28 & 243.1 \\
2.53 & 251.4 \\
2.78 & 248.9 \\
3.03 & 65.3
\end{tabular}

Client Sample No. C411F0O

IT Lab Specimen No. ETDC-7809

Specimen Height, in.

3.9545

Specimen Diameter, in.

2.0020

Specimen Weight, $g$.

375.60

Moisture Content, \%

31.9

Wet Unit Weight, pcf.

115.0

Dry Unit Weight, pcf.

87.2

Rate of Strain, in./min.

0.0300

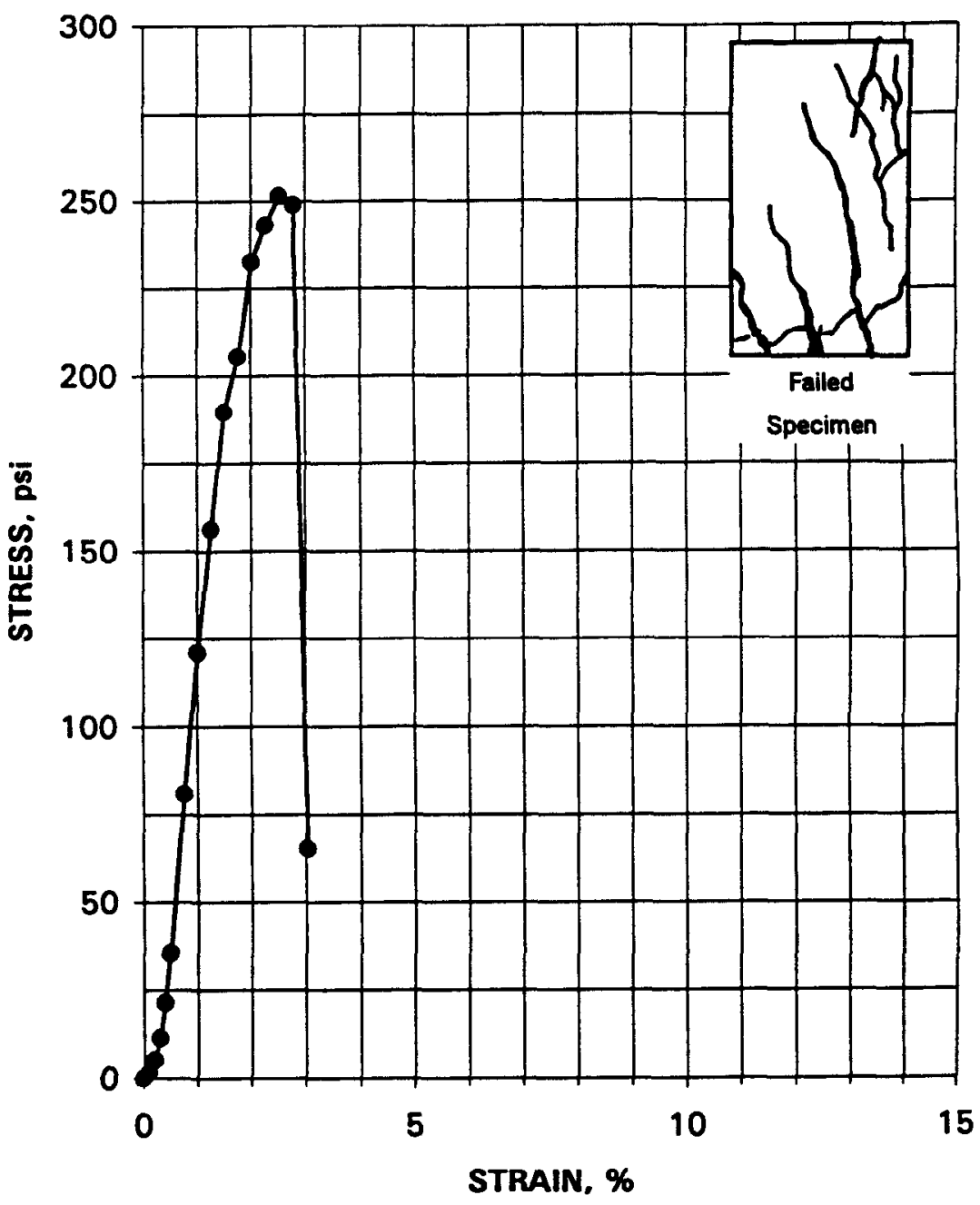




\section{UNCONFINED COMPRESSIVE STRENGTH - ASTM D 2166 14 DAY CURE}

Project Name SAIC / SAVANNAH RIVER Project No. 777304.00010500

Specimen Mold Date

Specimen Test Date

STRESS AT FAILURE, psi

STRAIN AT FAILURE, \%

AXIAL DEVIATOR

\begin{tabular}{ccc} 
STRAIN, \% & & STRESS, pSi \\
\cline { 1 - 1 } 0.00 & & 0.0 \\
0.10 & & 1.3 \\
0.20 & & 2.8 \\
0.30 & & 4.4 \\
0.40 & & 8.5 \\
0.50 & & 11.3 \\
0.75 & & 40.7 \\
1.01 & & 95.3 \\
1.26 & & 151.7 \\
1.51 & & 211.0 \\
1.76 & & 263.2 \\
2.01 & & 325.3 \\
2.26 & & 388.0 \\
2.51 & & 432.2 \\
2.77 & & 467.0 \\
3.02 & 481.7 \\
3.07 & 481.8 \\
3.27 & 375.5 \\
3.52 & 94.4
\end{tabular}

Client Sample No. C421F00

IT Lab Specimen No. ETDC-7810

Specimen Height, in.

3.9770

Specimen Diameter, in.

2.0087

Specimen Weight, $g$.

375.60

Moisture Content, \%

25.5

Wet Unit Weight, pcf.

113.6

90.5

Rate of Strain, in./min.

0.0300

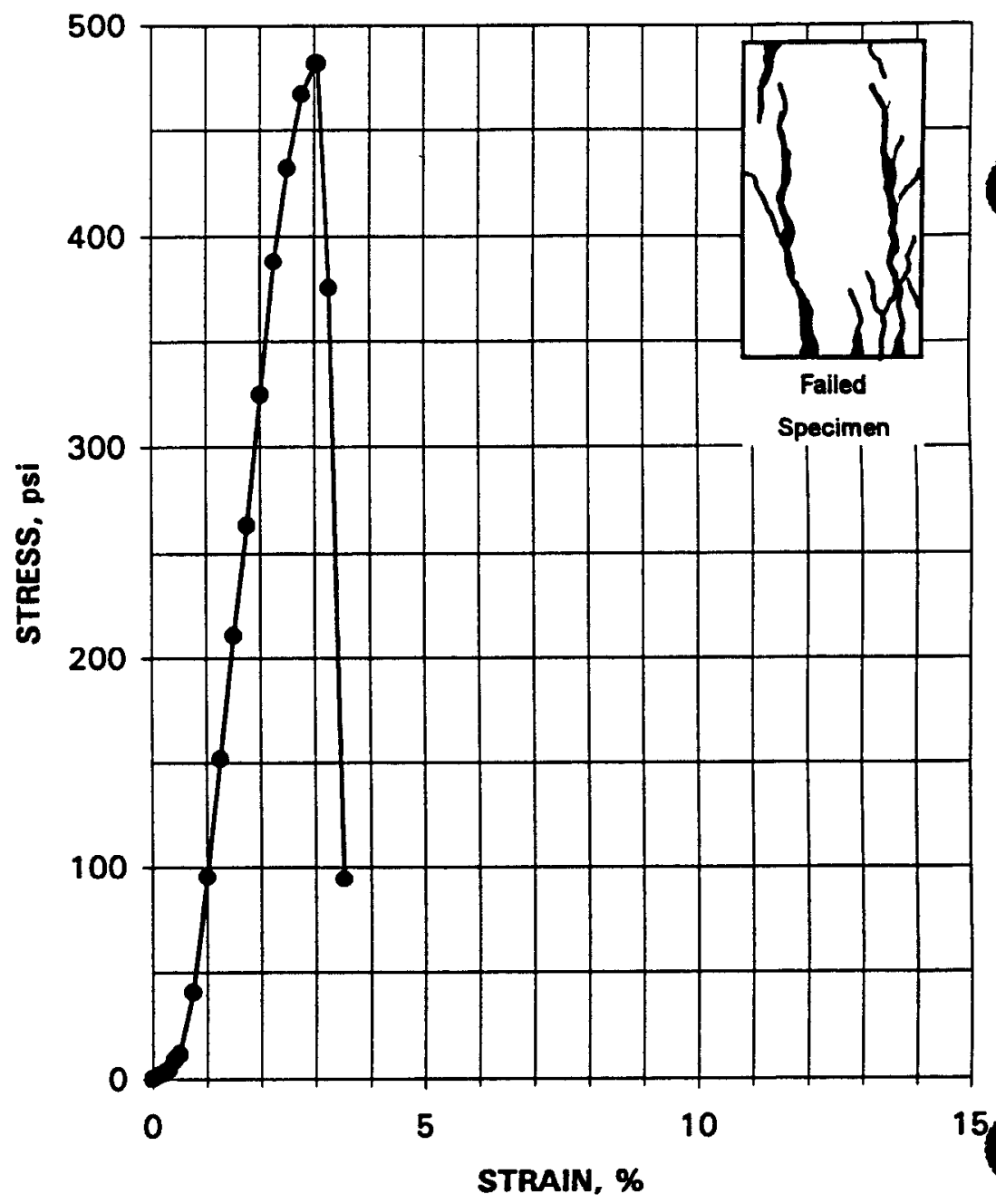




\section{UNCONFINED COMPRESSIVE STRENGTH - ASTM D 2166 14 DAY CURE}

$\begin{array}{ll}\text { Project Name } & \text { SAIC / SAVANNAH RIVER } \\ \text { Project No. } & \mathbf{7 7 7 3 0 4 . 0 0 0 1 0 5 0 0}\end{array}$

Specimen Mold Date

Specimen Test Date

STRESS AT FAILURE, psi

STRAIN AT FAILURE, \%

$\begin{array}{ccc}\begin{array}{ccc}\text { AXIAL } \\ \text { STRAIN, \% }\end{array} & & \begin{array}{c}\text { DEVIATOR } \\ \text { STRESS, pSi }\end{array} \\ 0.00 & 0.0 \\ 0.10 & & 1.3 \\ 0.20 & & 4.7 \\ 0.30 & & 11.4 \\ 0.40 & & 22.7 \\ 0.50 & & 43.2 \\ 0.75 & & 87.2 \\ 1.01 & & 123.4 \\ 1.26 & & 154.1 \\ 1.51 & & 190.9 \\ 1.76 & & 223.4 \\ 2.01 & 250.8 \\ 2.26 & 271.9 \\ 2.51 & 283.9 \\ 2.77 & 293.0 \\ 3.02 & 300.9 \\ 3.14 & 302.0 \\ 3.27 & 94.8\end{array}$

$11 / 11 / 98$

$11 / 25 / 98$

302.0

3.1
Client Sample No. C431F00

IT Lab Specimen No. ETDC-7811

Specimen Height, in.

3.9772

Specimen Diameter, in.

2.0035

Specimen Weight, $g$.

359.96

Moisture Content, \%

37.7

Wet Unit Weight, pcf.

Dry Unit Weight, pef.

109.4

79.4

Rate of Strain, in./min.

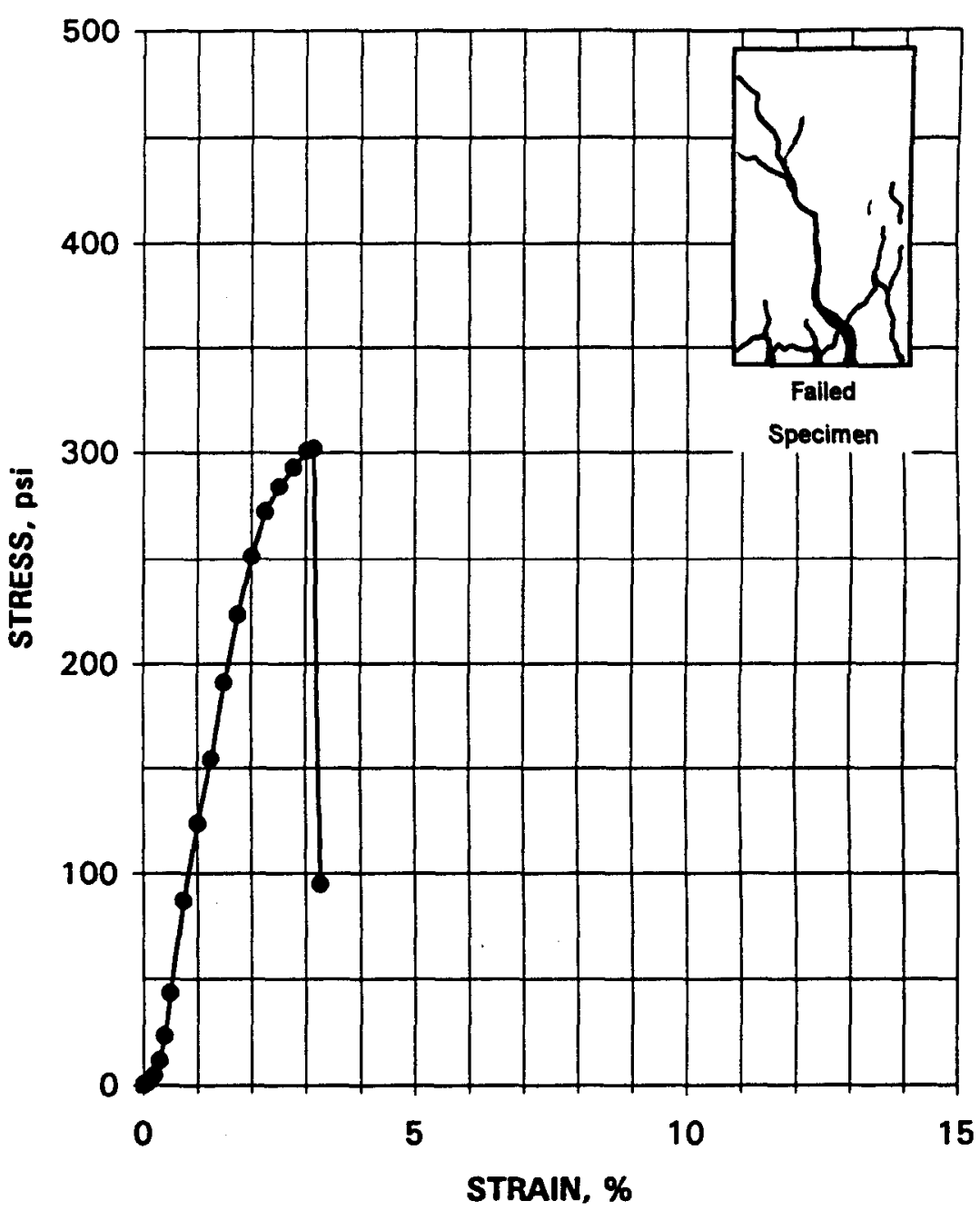




\section{UNCONFINED COMPRESSIVE STRENGTH - ASTM D 2166 14 DAY CURE}

Project Name SAIC / SAVANNAH RIVER

Project No. $\quad 777304.00010500$

Specimen Mold Date

Specimen Test Date

STRESS AT FAILURE, psi

STRAIN AT FAILURE, \%

AXIAL DEVIATOR

$\frac{\text { STRAIN, \% }}{0.00} \frac{\text { STRESS, psi }}{0.0}$

0.10

1.3

0.20

3.5

0.30

9.5

0.40

29.6

0.50

52.6

0.75

1.01

1.26

1.51

1.76

2.0 .1

2.26

2.52

2.64

2.77

3.02
$11 / 11 / 98$

$11 / 25 / 98$

289.0

2.6
Client Sample No.

C441F00

IT Lab Specimen No. ETDC-7812

Specimen Height, in.

Specimen Diameter, in.

Specimen Weight, $g$.

Moisture Content, \%

Wet Unit Weight, pef.

Dry Unit Weight, pcf.

Rate of Strain, in./min.

3.9753

2.0060

363.54

37.1

110.3

80.4

0.0300

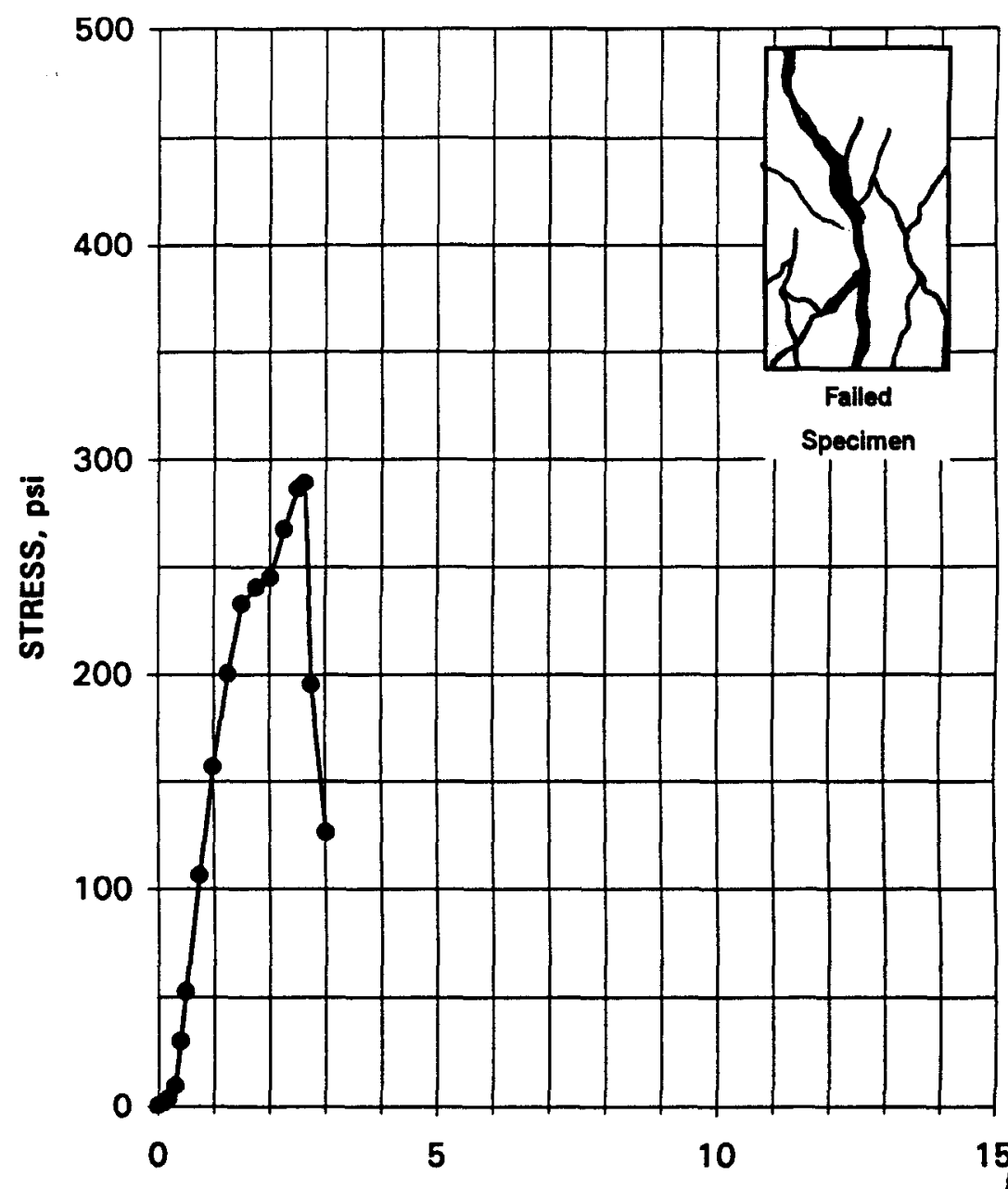

STRAIN, \% 


\section{UNCONFINED COMPRESSIVE STRENGTH - ASTM D 2166 14 DAY CURE}

Project Name SAIC / SAVANNAH RIVER

Project No. 777304.00010500

Specimen Mold Date

Specimen Test Date

STRESS AT FAILURE, psi

STRAIN AT FAILURE, $\%$

AXIAL

STRAIN, \% $\frac{\text { STRESS, psi }}{0.00}$

0.10

0.20

0.30

0.40

0.50

0.75

1.00

1.25

1.50

1.75

2.01

2.26

2.51

2.76

3.01

3.26

3.51
$11 / 11 / 98$

$11 / 25 / 98$

419.9

2.8
Client Sample No. C511F00

IT Lab Specimen No. ETDC-7813

Specimen Height, in.

3.9895

Specimen Diameter, in.

2.0010

Specimen Weight, $\mathbf{g}$.

382.28

Moisture Content, \%

29.4

Wet Unit Weight, pcf.

Dry Unit Weight, pcf.

116.1

89.7

Rate of Strain, in./min.

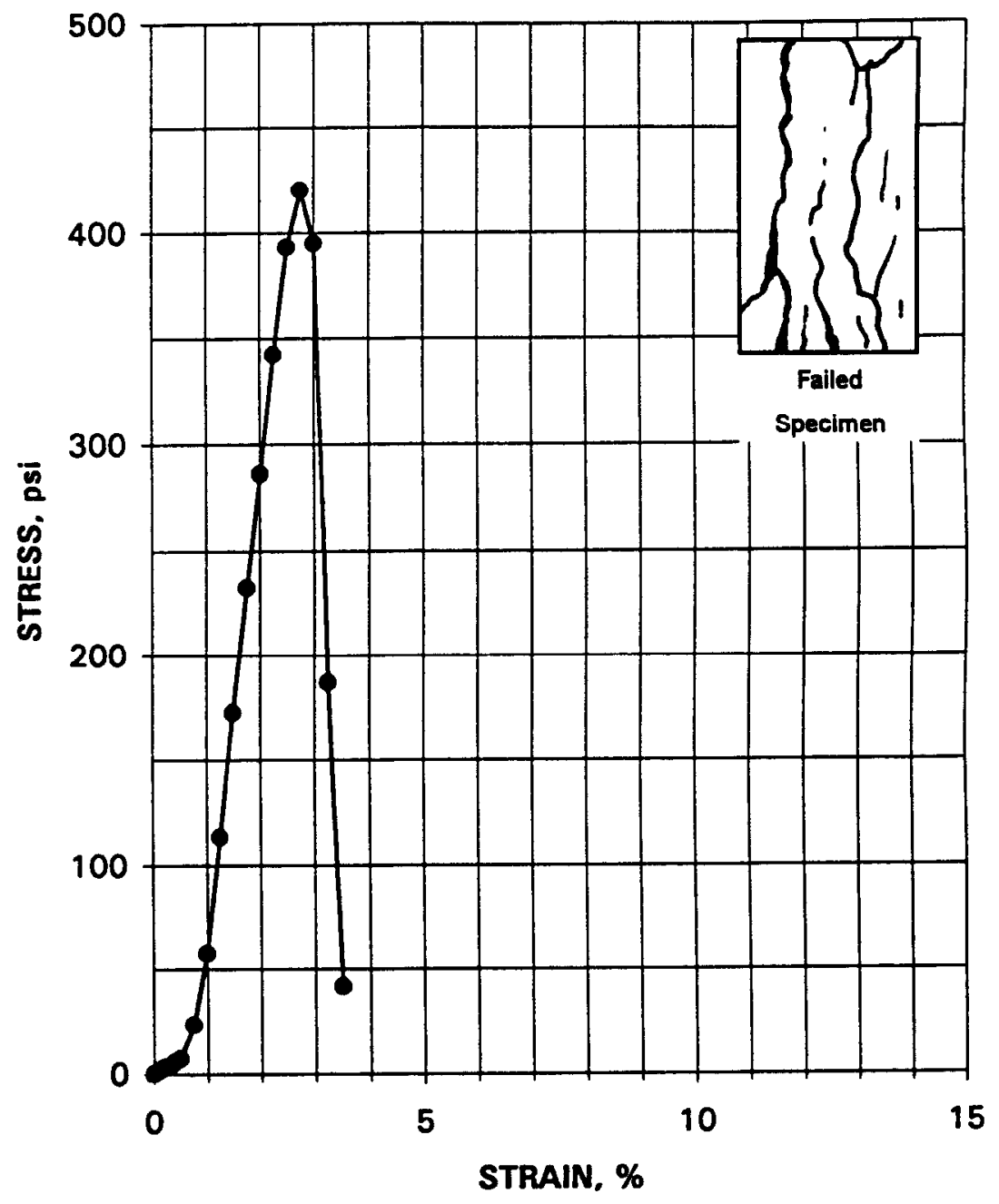




\section{UNCONFINED COMPRESSIVE STRENGTH - ASTM D 2166 14 DAY CURE}

Project Name SAIC / SAVANNAH RIVER

Project No. $\quad 777304.00010500$

Specimen Mold Date

Specimen Test Date

STRESS AT FAILURE, psi

STRAIN AT FAILURE, \%

\begin{tabular}{ccc}
$\begin{array}{ccc}\text { AXIAL } \\
\text { STRAIN, \% }\end{array}$ & & $\begin{array}{c}\text { DEVIATOR } \\
\text { STRESS, psi }\end{array}$ \\
\cline { 1 - 1 } 0.00 & & 0.0 \\
0.10 & & 1.9 \\
0.20 & & 3.8 \\
0.30 & & 4.7 \\
0.40 & & 7.3 \\
0.50 & & 11.4 \\
0.75 & & 28.0 \\
1.00 & & 61.8 \\
1.25 & & 120.2 \\
1.50 & & 179.0 \\
1.76 & & 218.4 \\
2.01 & & 271.3 \\
2.26 & 333.2 \\
2.51 & 384.3 \\
2.76 & 404.9 \\
2.83 & 405.2 \\
3.01 & 175.3
\end{tabular}

$11 / 11 / 98$

$11 / 25 / 98$

405.2

2.8
Client Sample No. C521F0O

IT Lab Specimen No. ETDC-7814

Specimen Height, in.

3.9882

Specimen Diameter, in.

Specimen Weight, $g$.

2.0038

Moisture Content, \%

Wet Unit Weight, pef.

Dry Unit Weight, pcf.

Rate of Strain, in./min.

389.72

27.1

118.1

92.9

0.0300

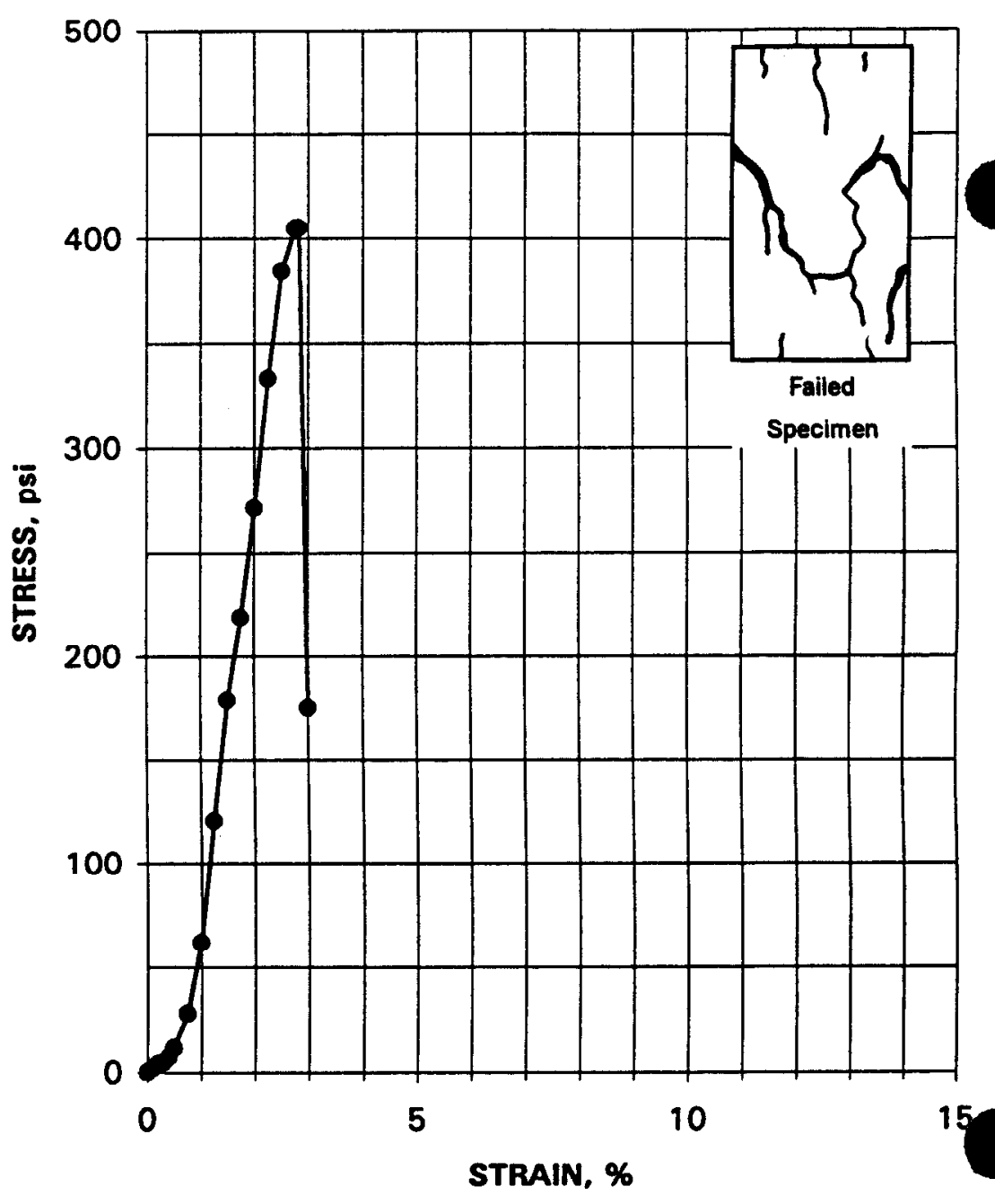




\section{UNCONFINED COMPRESSIVE STRENGTH - ASTM D 2166 14 DAY CURE}

Project Name SAIC / SAVANNAH RIVER

Project No. $\quad 777304.00010500$

Specimen Mold Date

Specimen Test Date

STRESS AT FAILURE, psi

STRAIN AT FAILURE, \%

\begin{tabular}{ccc}
$\begin{array}{c}\text { AXIAL } \\
\text { STRAIN, \% }\end{array}$ & & $\begin{array}{c}\text { DEVIATOR } \\
\text { STRESS, psi }\end{array}$ \\
\cline { 1 - 1 } 0.00 & & 0.0 \\
0.10 & & 1.3 \\
0.20 & & 1.9 \\
0.30 & & 2.9 \\
0.40 & & 8.2 \\
0.50 & & 22.1 \\
0.75 & & 62.1 \\
1.00 & & 118.6 \\
1.25 & & 170.3 \\
1.50 & & 214.7 \\
1.75 & 258.4 \\
2.00 & 302.6 \\
2.25 & 331.0 \\
2.50 & 336.4 \\
2.75 & 315.1 \\
3.00 & 107.9
\end{tabular}

$11 / 11 / 98$

$11 / 25 / 98$

336.4

2.5
Client Sample No. C531F00

IT Lab Specimen No. ETDC-7815

Specimen Height, in. 4.0020

Specimen Diameter, in. 2.0020

Specimen Weight, g. 370.48

Moisture Content, \% 34.6

Wet Unit Weight, pcf.

112.1

Dry Unit Weight, pcf.

83.2

Rate of Strain, in./min.

0.0300

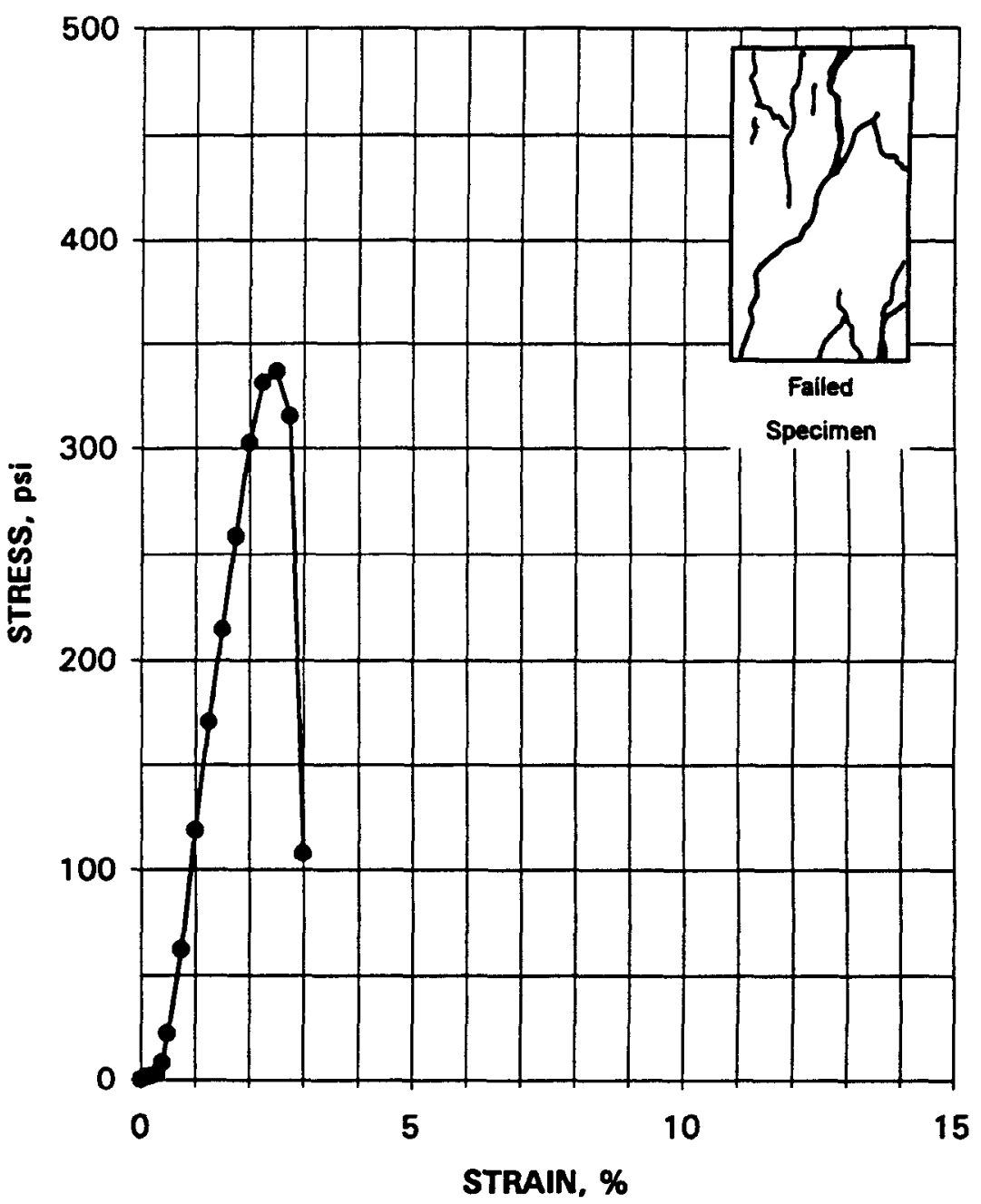




\section{UNCONFINED COMPRESSIVE STRENGTH - ASTM D 2166 14 DAY CURE}

Project Name SAIC / SAVANNAH RIVER

Project No. $\quad 777304.00010500$

Specimen Mold Date

Specimen Test Date

STRESS AT FAILURE, psi

STRAIN AT FAILURE, \%

AXIAL DEVIATOR

\begin{tabular}{ccc} 
STRAIN, $\%$ & STRESS, pSi \\
\cline { 1 - 1 } 0.00 & & 0.0 \\
0.10 & & 1.3 \\
0.20 & & 1.9 \\
0.30 & & 3.5 \\
0.40 & & 6.9 \\
0.50 & & 17.0 \\
0.75 & & 43.0 \\
1.00 & & 87.4 \\
1.25 & & 133.5 \\
1.51 & & 180.8 \\
1.76 & & 222.1 \\
2.01 & & 248.2 \\
2.26 & & 271.1 \\
2.51 & & 296.0 \\
2.76 & & 312.8 \\
3.01 & & 328.2 \\
3.26 & 340.8 \\
3.51 & 345.2 \\
3.59 & 345.8 \\
3.76 & 332.1 \\
4.01 & 275.6 \\
4.27 & 235.5 \\
4.52 & 81.0
\end{tabular}

$11 / 11 / 98$

$11 / 25 / 98$

345.8

3.6
Client Sample No. C541F00

IT Lab Specimen No. ETDC-7816

Specimen Height, in.

3.9857

Specimen Diameter, in.

2.0055

Specimen Weight, $g$.

377.04

Moisture Content, \%

32.0

Wet Unit Weight, pcf.

114.1

Dry Unit Weight, pcf.

86.4

Rate of Strain, in./min.

0.0300

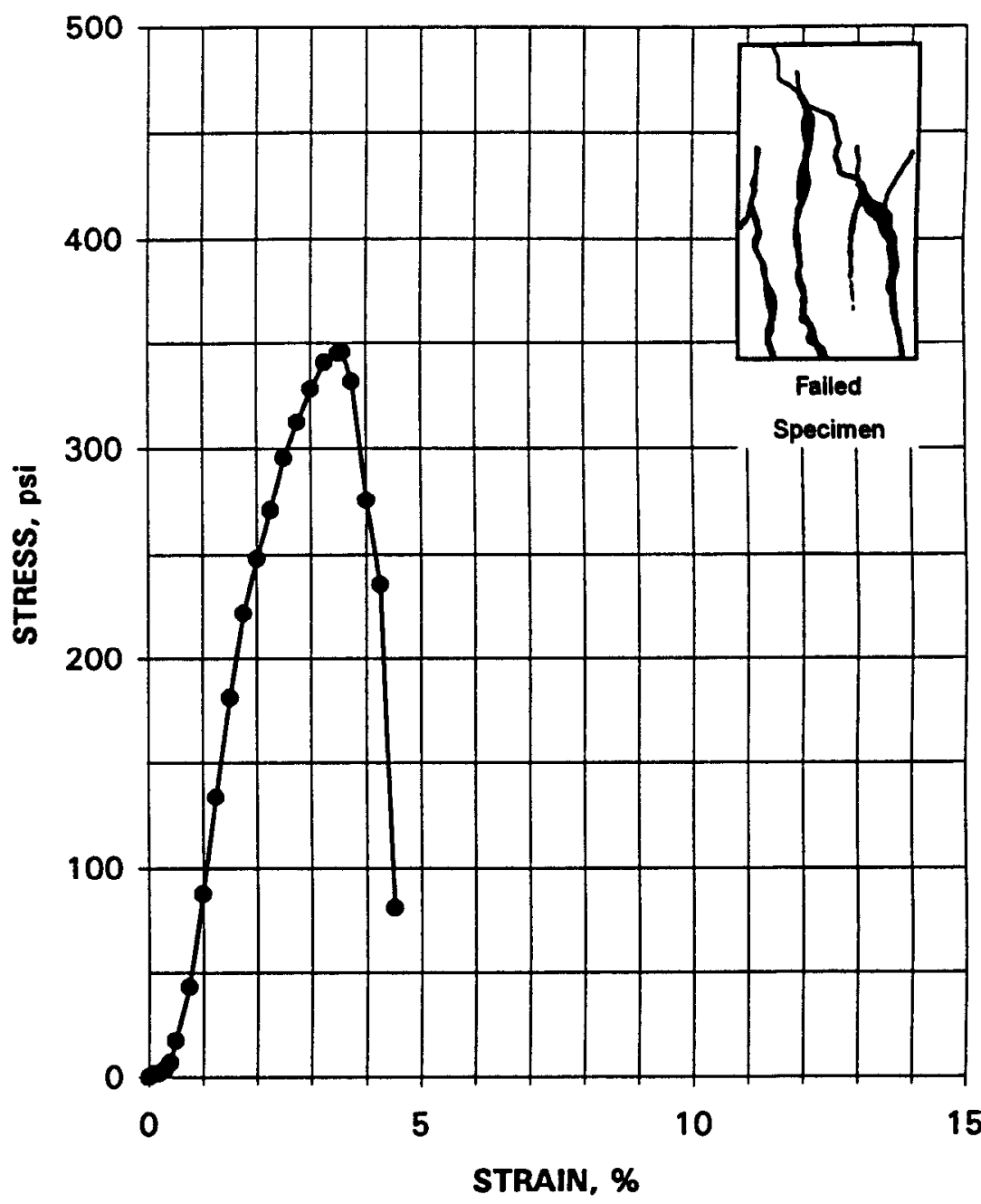




\section{UNCONFINED COMPRESSIVE STRENGTH - ASTM D 2166 14 DAY CURE}

Project Name SAIC / SAVANNAH RIVER

Project No. $\quad 777304.00010500$

Specimen Mold Date

Specimen Test Date

STRESS AT FAILURE, psi

STRAIN AT FAILURE, \%

\begin{tabular}{ccc}
$\begin{array}{c}\text { AXIAL } \\
\text { STRAIN, \% }\end{array}$ & & $\begin{array}{c}\text { DEVIATOR } \\
\text { STRESS, psi }\end{array}$ \\
\cline { 1 - 1 } 0.00 & & 0.0 \\
0.10 & & 1.0 \\
0.20 & & 1.9 \\
0.30 & & 3.2 \\
0.40 & & 6.0 \\
0.50 & & 8.2 \\
0.75 & & 27.2 \\
1.00 & & 65.9 \\
1.25 & & 115.7 \\
1.50 & & 164.3 \\
1.75 & & 208.6 \\
2.00 & & 251.2 \\
2.25 & & 301.5 \\
2.50 & 349.2 \\
2.75 & 370.3 \\
3.00 & 381.4 \\
3.10 & 382.2 \\
3.25 & 71.2
\end{tabular}

$11 / 11 / 98$

$11 / 25 / 98$

382.2

3.1
Client Sample No. C611F00

IT Lab Specimen No. ETDC-7817

Specimen Height, in.

3.9982

Specimen Diameter, in.

1.9998

Specimen Weight, 0.

384.62

Moisture Content, \%

Wet Unit Weight, pcf.

116.7

Dry Unit Weight, pcf.

Rate of Strain, in./min.

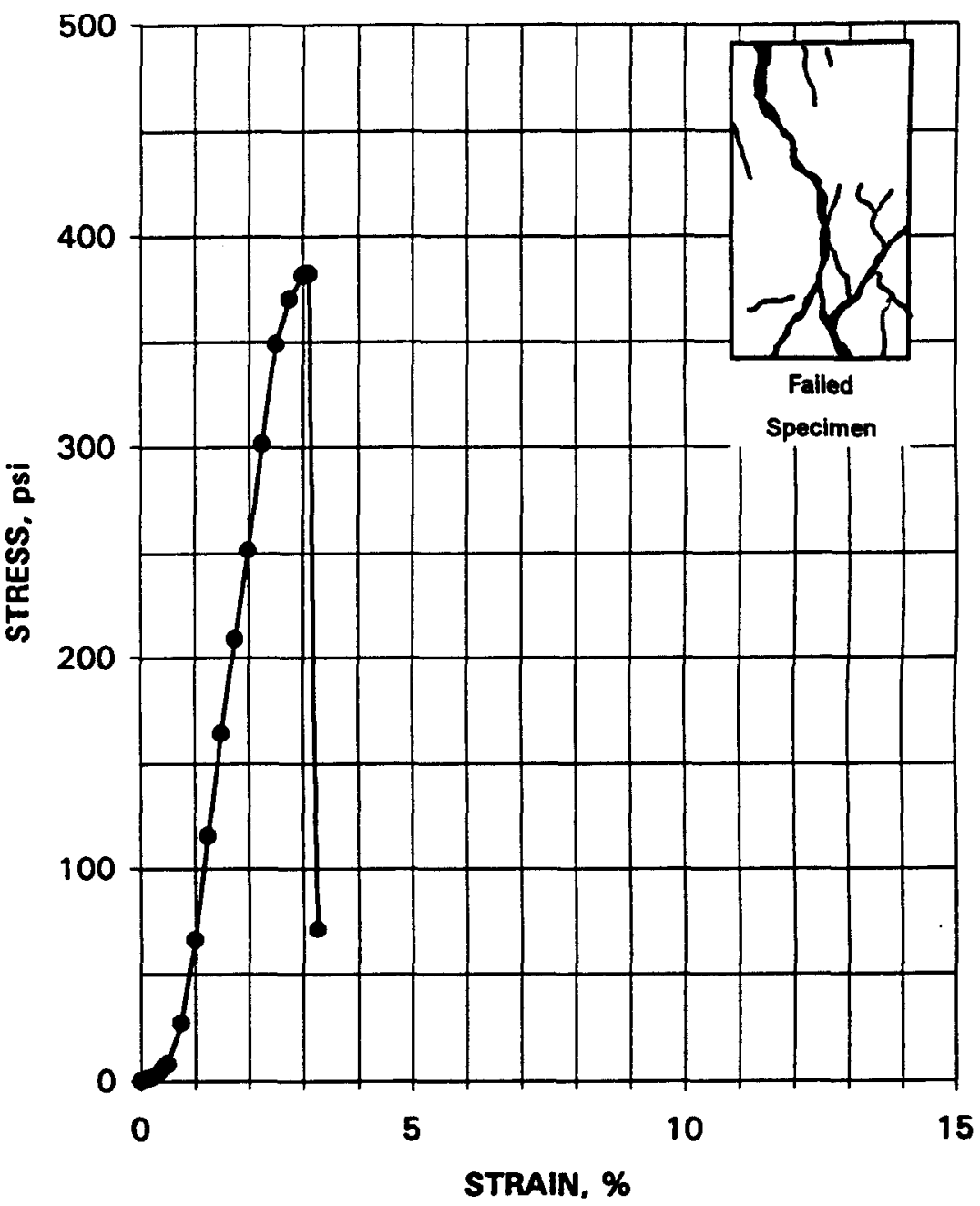




\section{UNCONFINED COMPRESSIVE STRENGTH - ASTM D 2166 14 DAY CURE}

\begin{tabular}{lc} 
Project Name & SAIC / SAVA \\
Project No. & 777304.00010 \\
Specimen Mold Date \\
Specimen Test Date \\
STRESS AT FAILURE, psi \\
STRAIN AT FAILURE, \% \\
\multicolumn{2}{|}{} \\
AXIAL & DEVIATOR \\
STRAIN, \% & STRESS, psi \\
\hline 0.00 & 0.0 \\
0.10 & 0.9 \\
0.20 & 2.8 \\
0.30 & 4.7 \\
0.40 & 10.1 \\
0.50 & 27.4 \\
0.75 & 93.5 \\
1.01 & 158.9 \\
1.26 & 222.8 \\
1.51 & 273.3 \\
1.76 & 300.8 \\
2.01 & 318.6 \\
2.26 & 332.3 \\
2.39 & 335.3 \\
2.51 & 327.1 \\
2.76 & 290.0 \\
3.02 & 149.2 \\
&
\end{tabular}

Client Sample No. C621F00

IT Lab Specimen No. ETDC-7818

$11 / 11 / 98$

$11 / 25 / 98$

335.3

2.4
Specimen Height, in.

Specimen Diameter, in.

Specimen Weight, $\mathbf{g}$.

Moisture Content, \%

Wet Unit Weight, pcf.

Dry Unit Weight, pcf.

Rate of Strain, in./min.

3.9797

2.0073

395.47

26.1

119.6

94.9

0.0300

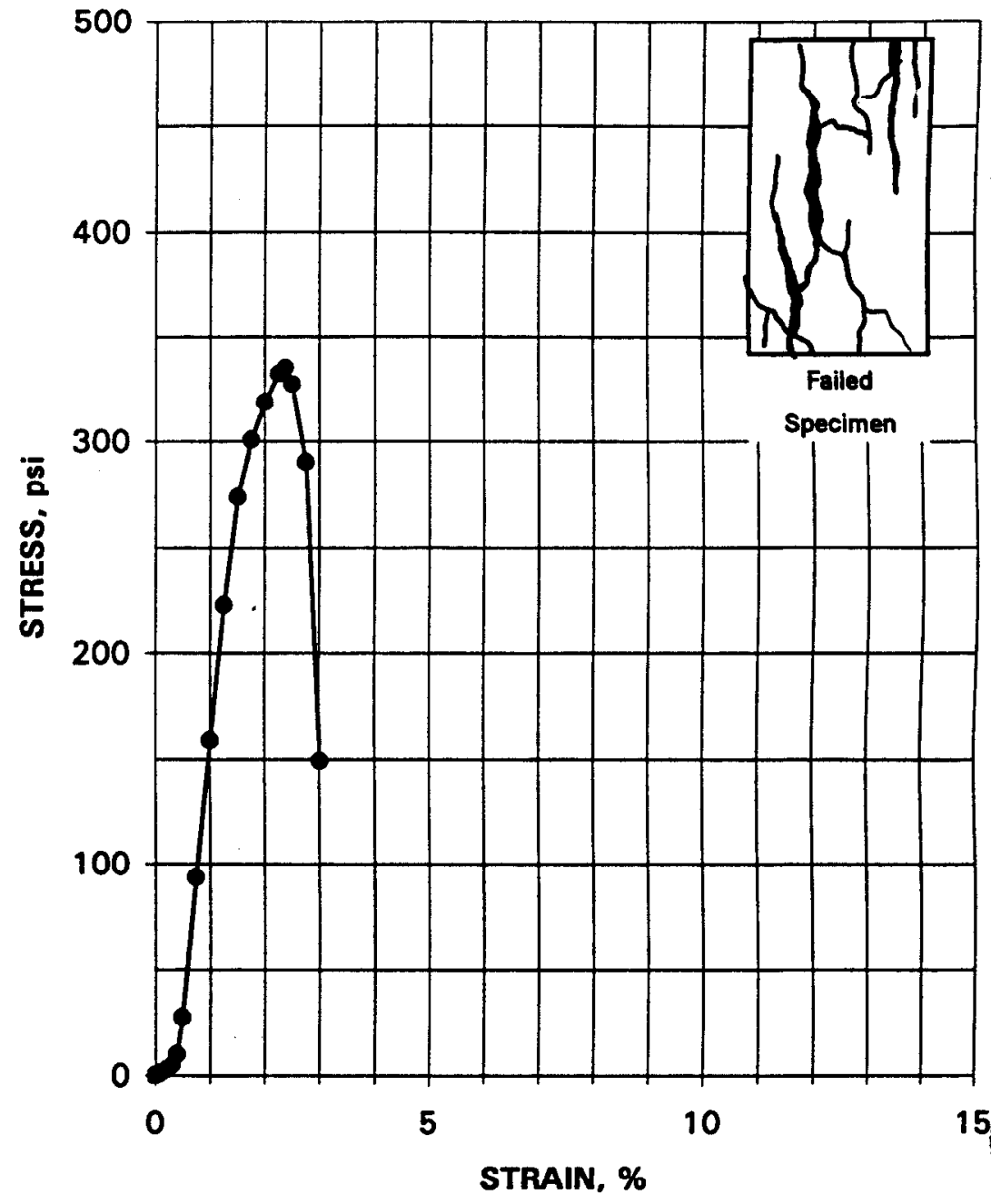




\section{UNCONFINED COMPRESSIVE STRENGTH - ASTM D 2166 14 DAY CURE}

Project Name SAIC / SAVANNAH RIVER

Project No. 777304.00010500

Specimen Mold Date

Specimen Test Date

STRESS AT FAILURE, psi

STRAIN AT FAILURE, \%

\begin{tabular}{ccc}
$\begin{array}{c}\text { AXIAL } \\
\text { STRAIN, \% }\end{array}$ & & $\begin{array}{c}c \\
\text { DEVIATOR } \\
\text { STRESS, psi }\end{array}$ \\
\cline { 1 - 1 } 0.00 & & 0.0 \\
0.10 & & 1.3 \\
0.20 & & 3.8 \\
0.30 & & 6.0 \\
0.40 & & 10.1 \\
0.49 & & 14.2 \\
0.74 & & 36.0 \\
0.99 & & 80.9 \\
1.24 & & 119.3 \\
1.48 & & 165.0 \\
1.73 & & 201.1 \\
1.98 & & 243.6 \\
2.23 & & 269.7 \\
2.47 & & 280.5 \\
2.57 & & 280.9 \\
2.72 & 273.9 \\
2.97 & 240.2 \\
3.22 & 198.1 \\
3.46 & 170.3 \\
3.71 & 154.5
\end{tabular}

$11 / 11 / 98$

$11 / 25 / 98$

280.9

2.6
Client Sample No. C631F00

IT Lab Specimen No. ETDC-7819

Specimen Height, in.

4.0413

Specimen Diameter, in.

2.0015

Specimen Weight, $\boldsymbol{o}$.

Moisture Content, \%

373.82

34.1

Wet Unit Weight, pef.

Dry Unit Weight, pcf.

112.0

83.5

Rate of Strain, in./min.

0.0300

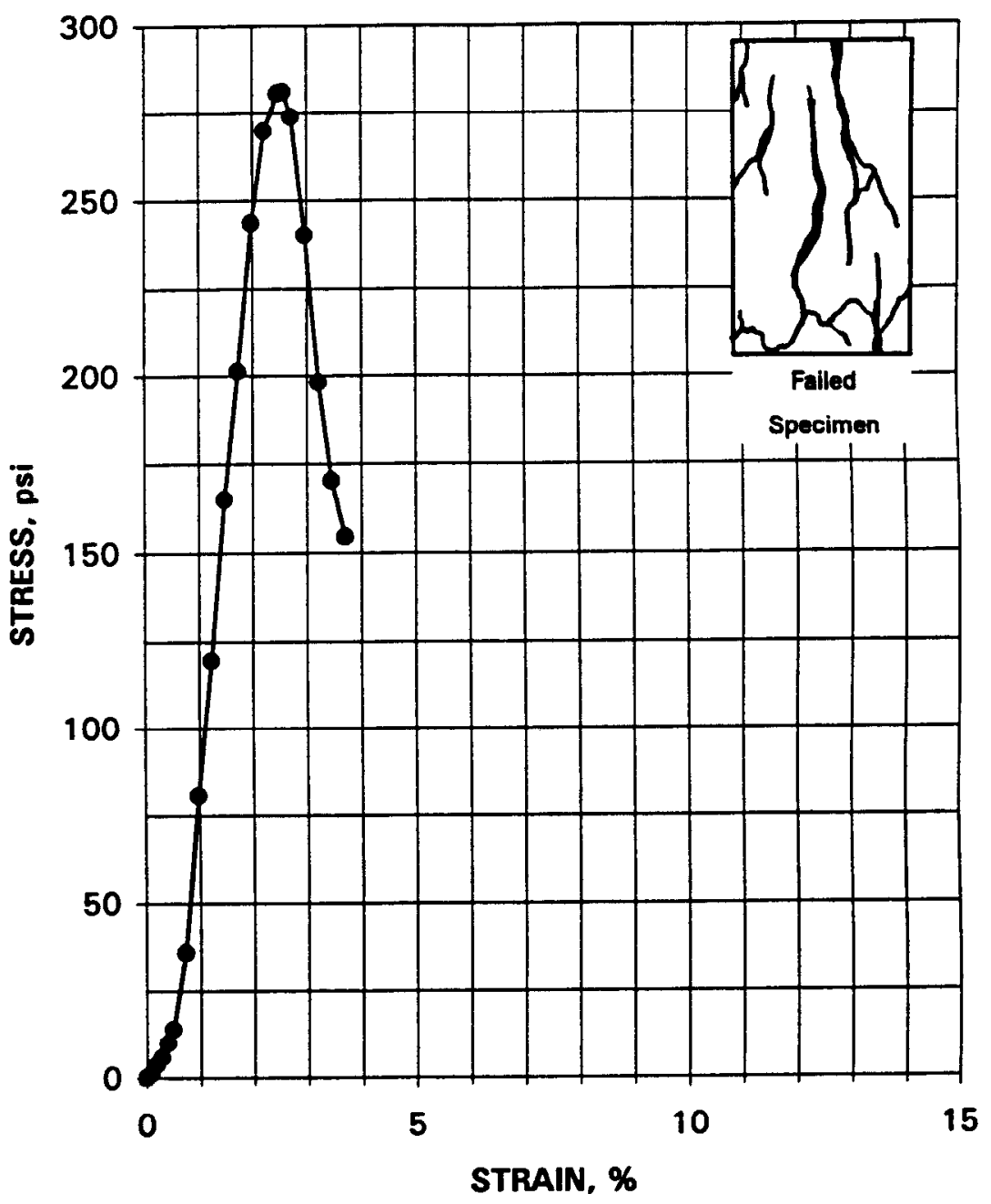




\section{UNCONFINED COMPRESSIVE STRENGTH - ASTM D 2166 14 DAY CURE}

Project Name

Project No.

SAIC / SAVANNAH RIVER

777304.00010500

Specimen Mold Date

Specimen Test Date

STRESS AT FAILURE, psi

STRAIN AT FAILURE, \%

\begin{tabular}{ccc}
$\begin{array}{c}\text { AXIAL } \\
\text { STRAIN, \% }\end{array}$ & & $\begin{array}{c}\text { DEVIATOR } \\
\text { STRESS, psi }\end{array}$ \\
\cline { 1 - 1 } 0.00 & & 0.0 \\
0.10 & & 1.9 \\
0.20 & & 3.2 \\
0.30 & & 4.7 \\
0.40 & & 7.6 \\
0.50 & & 9.4 \\
0.75 & & 21.3 \\
1.00 & & 64.5 \\
1.25 & & 121.1 \\
1.50 & & 177.8 \\
1.75 & & 236.3 \\
2.00 & & 279.7 \\
2.25 & 326.0 \\
2.38 & 378.9 \\
2.50 & 422.2 \\
2.76 & 427.9 \\
2.88 & 361.0 \\
3.26 & 216.8
\end{tabular}

$11 / 11 / 98$

$11 / 25 / 98$

427.9

2.8
Client Sample No. C641F00

IT Lab Specimen No. ETDC-7820

Specimen Height, in.

Specimen Diameter, in.

3.9922

Specimen Weight, $g$.

2.0070

Moisture Content, \%

377.09

32.1

Wet Unit Weight, pef.

Dry Unit Weight, pcf.

113.8

86.1

Rate of Strain, in./min.

0.0300

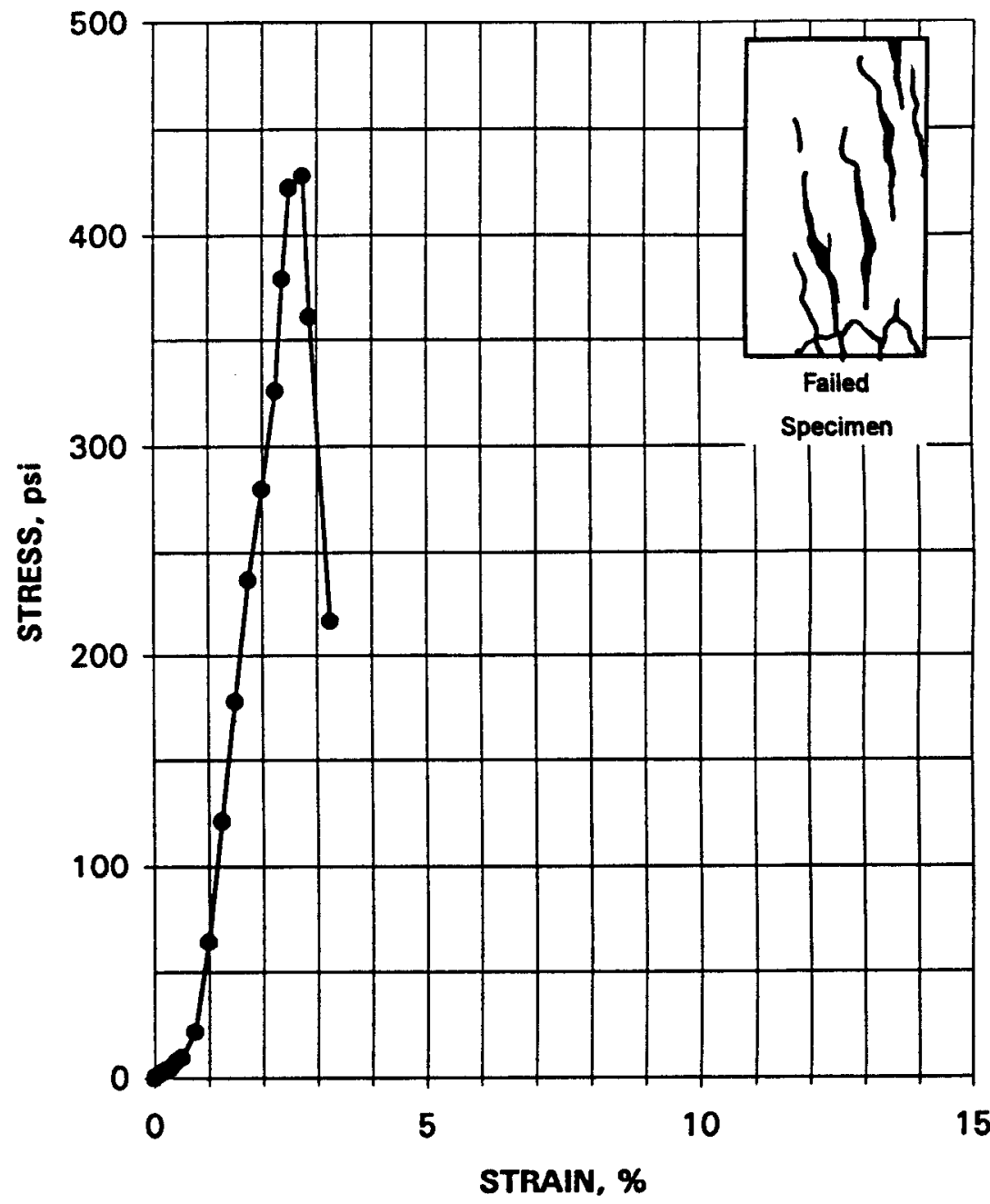




\section{Series}

\section{Day UCS Results}




\section{UNCONFINED COMPRESSIVE STRENGTH - ASTM D 2166 28-DAY CURE}

Project Name SAIC/SAVANNAH RIVER

Project No. $\quad 777304.00010500$

Specimen Mold Date

Specimen Test Date

STRESS AT FAILURE, psi

STRAIN AT FAILURE, $\%$

AXIAL DEVIATOR

\begin{tabular}{ccc} 
STRAIN, \% & STRESS, psi \\
\cline { 1 - 1 } 0.00 & 0.0 \\
0.10 & & 2.9 \\
0.20 & & 5.7 \\
0.30 & & 9.5 \\
0.40 & & 15.2 \\
0.51 & & 23.0 \\
0.76 & 52.2 \\
1.01 & 102.6 \\
1.26 & 160.9 \\
1.52 & 213.8 \\
1.77 & 243.2 \\
2.02 & 295.1 \\
2.27 & 330.8 \\
2.53 & 368.3 \\
2.78 & 399.7 \\
3.03 & 67.6
\end{tabular}

$11 / 11 / 98$

$12 / 9 / 98$

399.7

2.8
Client Sample No. C312F00

IT Lab Specimen No. ETDC-7885

Specimen Height, in.

Specimen Diameter, in.

3.9573

Specimen Weight, $\boldsymbol{g}$.

Moisture Content, \%

2.0042

383.11

28.2

Wet Unit Weight, pcf.

Dry Unit Weight, pef.

116.9

91.2

Rate of Strain, in./min.

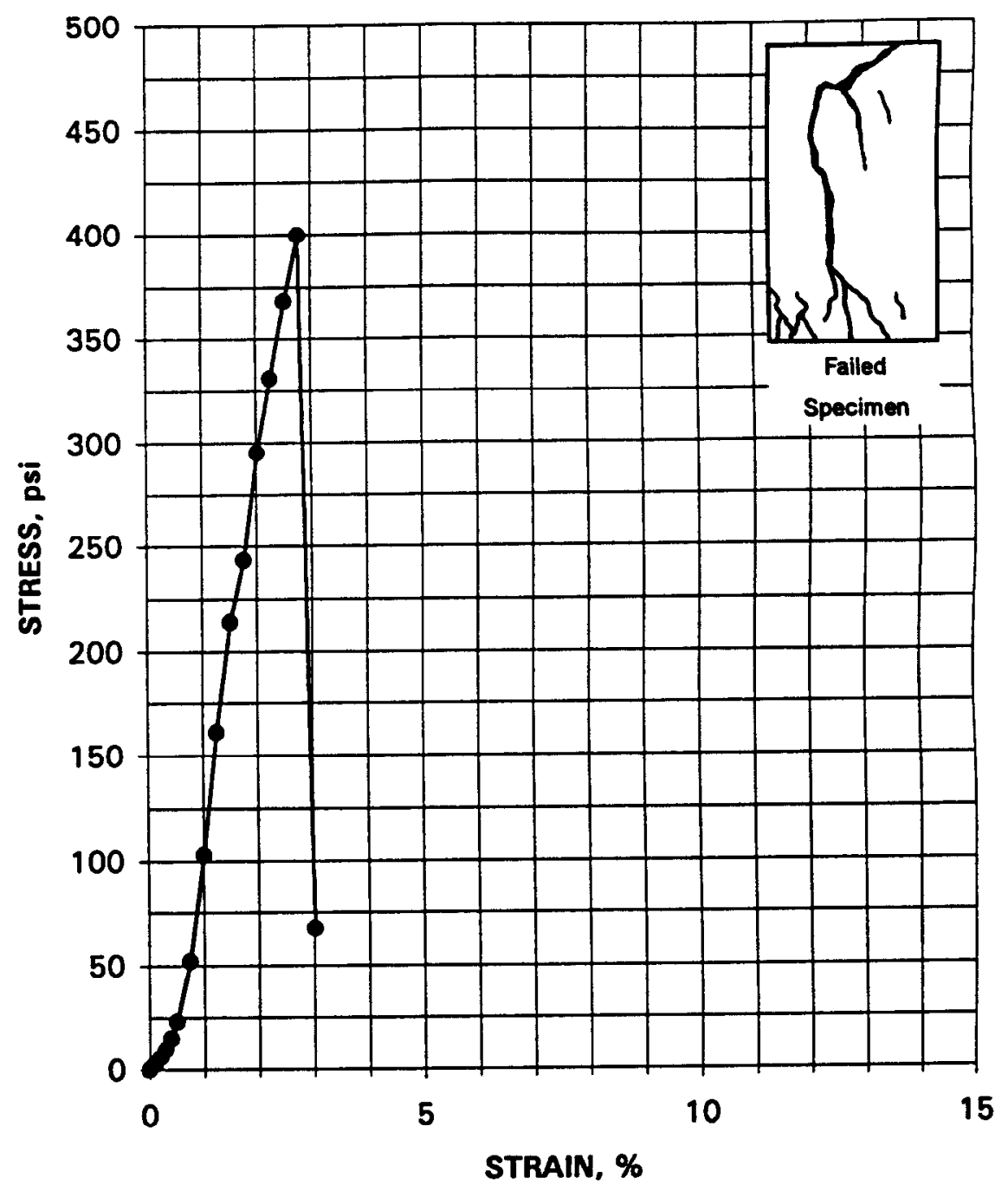




\section{UNCONFINED COMPRESSIVE STRENGTH - ASTM D 2166 28-DAY CURE}

Project Name SAIC/SAVANNAH RIVER

Project No. $\quad 777304.00010500$

Specimen Mold Date

Specimen Test Date

STRESS AT FAILURE, psi STRAIN AT FAILURE, \%

\begin{tabular}{ccc}
$\begin{array}{c}\text { AXIAL } \\
\text { STRAIN, \% }\end{array}$ & $\begin{array}{c}\text { DEVIATOR } \\
\text { STRESS, psi }\end{array}$ \\
\cline { 1 - 3 } 0.00 & & 0.0 \\
0.10 & & 1.3 \\
0.20 & & 3.2 \\
0.30 & & 5.4 \\
0.40 & & 8.5 \\
0.50 & & 11.3 \\
0.75 & & 25.8 \\
1.01 & & 46.1 \\
1.26 & & 85.0 \\
1.51 & & 125.6 \\
1.76 & & 163.8 \\
2.01 & 189.8 \\
2.26 & 202.9 \\
2.52 & 261.6 \\
2.77 & 318.8 \\
3.02 & 347.7 \\
3.27 & 300.91 \\
3.52 & 157.85
\end{tabular}

Client Sample No. C322F00

IT Lab Specimen No. ETDC-7886

Specimen Height, in.

Specimen Diameter, in.

3.9743

Specimen Weight, $g$.

2.0058

385.95

Moisture Content, \%

29.0

Wet Unit Weight, pcf.

117.1

Dry Unit Weight, pcf.

90.7

Rate of Strain, in./min.

0.0240

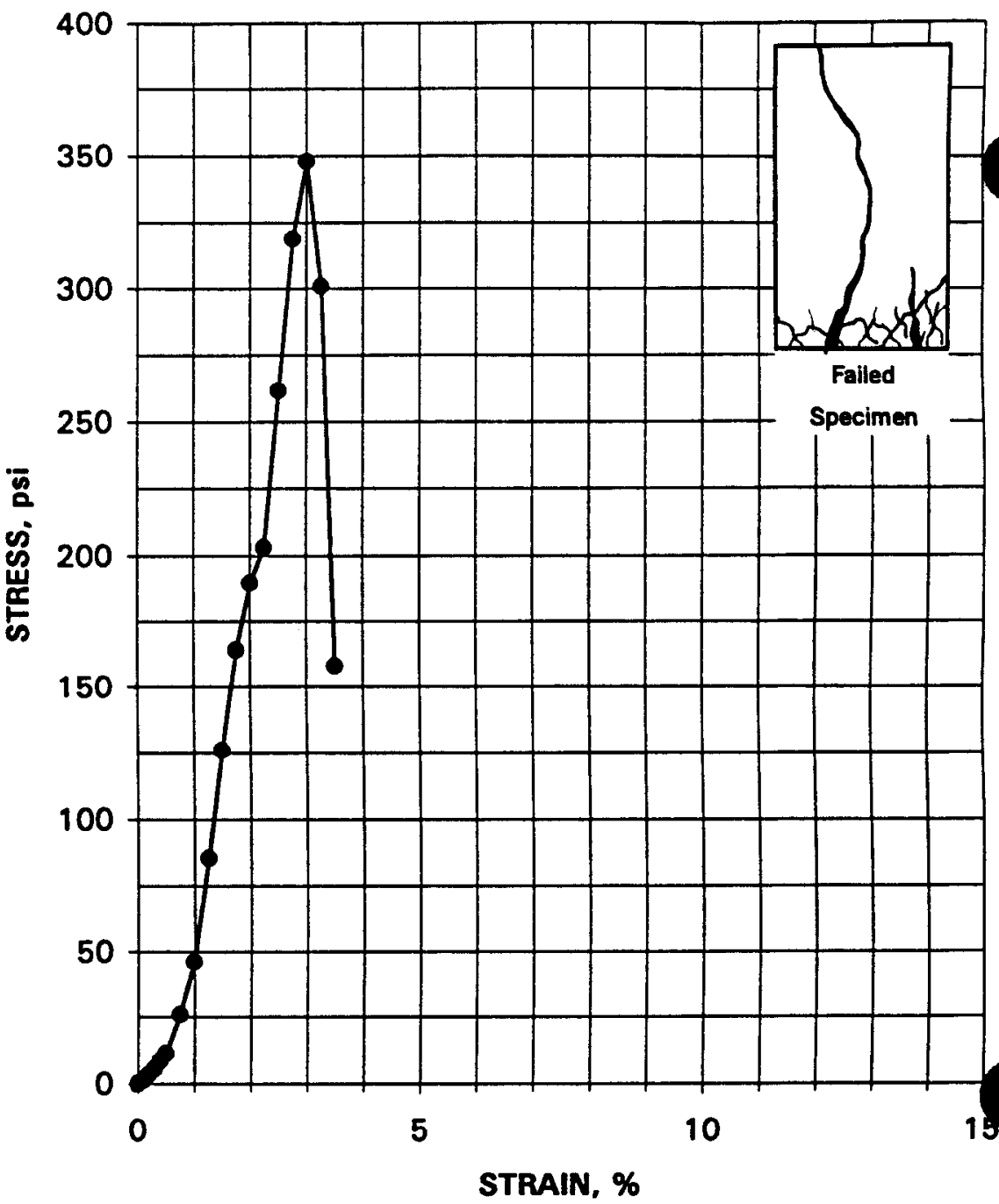




\section{UNCONFINED COMPRESSIVE STRENGTH - ASTM D 2166 28-DAY CURE}

\begin{abstract}
Project Name SAIC/SAVANNAH RIVER
Project No. $\quad 777304.00010500$
\end{abstract}

Specimen Mold Date

Specimen Test Date

STRESS AT FAILURE, psi

STRAIN AT FAILURE, \%

\begin{tabular}{cc}
$\begin{array}{c}\text { AXIAL } \\
\text { STRAIN, \% }\end{array}$ & $\begin{array}{c}\text { DEVIATOR } \\
\text { STRESS, psi }\end{array}$ \\
\cline { 2 - 3 } 0.00 & 0.0 \\
0.10 & 1.3 \\
0.20 & 2.2 \\
0.30 & 3.8 \\
0.40 & 6.7 \\
0.51 & 10.5 \\
0.76 & 25.6 \\
1.01 & 39.5 \\
1.26 & 71.8 \\
1.52 & 113.1 \\
1.77 & 148.9 \\
2.02 & 181.9 \\
2.28 & 222.0 \\
2.53 & 262.2 \\
2.78 & 295.6 \\
3.03 & 327.0 \\
3.29 & 357.63 \\
3.54 & 381.31 \\
3.79 & 388.91 \\
4.05 & 381.46 \\
4.30 & 377.71 \\
4.55 & 351.43 \\
4.80 & 215.65
\end{tabular}

Client Sample No. C332F00

IT Lab Specimen No. ETDC-7887

Specimen Height, in.

3.9550

Specimen Diameter, in.

1.9977

Specimen Weight, $g$.

372.17

Moisture Content, \%

Wet Unit Weight, pcf.

Dry Unit Weight, pcf.

114.4

86.4

Rate of Strain, in./min.

0.0240

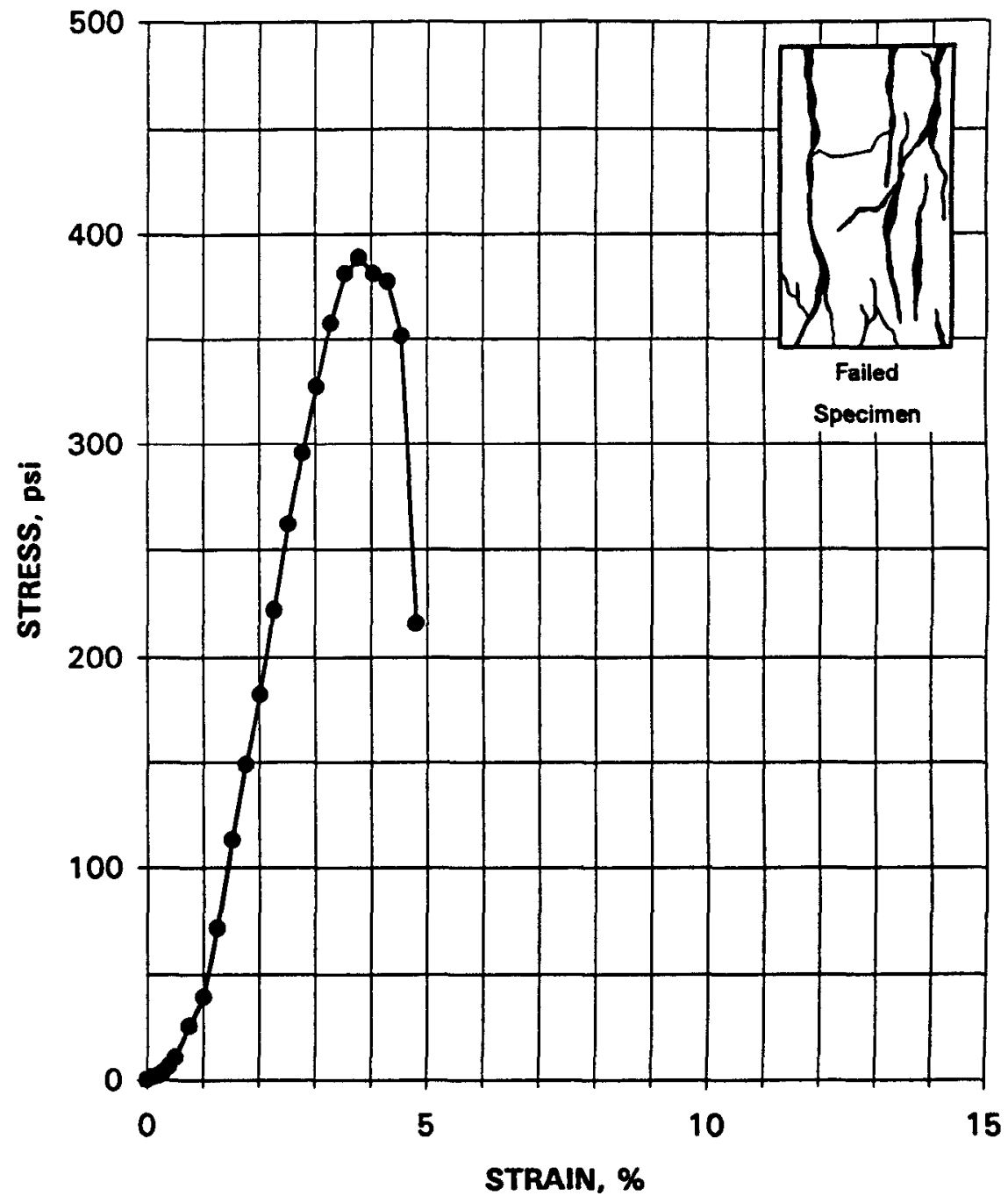




\section{UNCONFINED COMPRESSIVE STRENGTH - ASTM D 2166 28-DAY CURE}

Project Name SAIC/SAVANNAH RIVER

Project No. $\quad 777304.00010500$

\section{Specimen Mold Date}

Specimen Test Date

STRESS AT FAILURE, pSi

STRAIN AT FAILURE, $\%$

\begin{tabular}{cc}
$\begin{array}{c}\text { AXIAL } \\
\text { STRAIN, \% }\end{array}$ & $\begin{array}{c}\text { DEVIATOR } \\
\text { STRESS, psi }\end{array}$ \\
\cline { 2 - 3 } 0.00 & 0.0 \\
0.10 & 1.3 \\
0.20 & 2.2 \\
0.30 & 3.5 \\
0.40 & 4.7 \\
0.50 & 6.0 \\
0.75 & 11.3 \\
1.00 & 30.9 \\
1.25 & 69.5 \\
1.50 & 120.9 \\
1.76 & 173.2 \\
2.01 & 233.4 \\
2.26 & 276.9 \\
2.51 & 335.8 \\
2.76 & 387.7 \\
3.01 & 410.0 \\
3.26 & 224.00 \\
3.51 & 188.11
\end{tabular}

$11 / 11 / 98$

$12 / 9 / 98$

410.0

3.0
Client Sample No. C342F00

IT Lab Specimen No. ETDC-7888

Specimen Height, in.

3.9870

Specimen Diameter, in.

2.0090

Specimen Weight, $\boldsymbol{g}$.

379.62

Moisture Content, \%

Wet Unit Weight, pcf.

31.0

114.4

Dry Unit Weight, pcf.

87.3

Rate of Strain, in./min.

0.0240

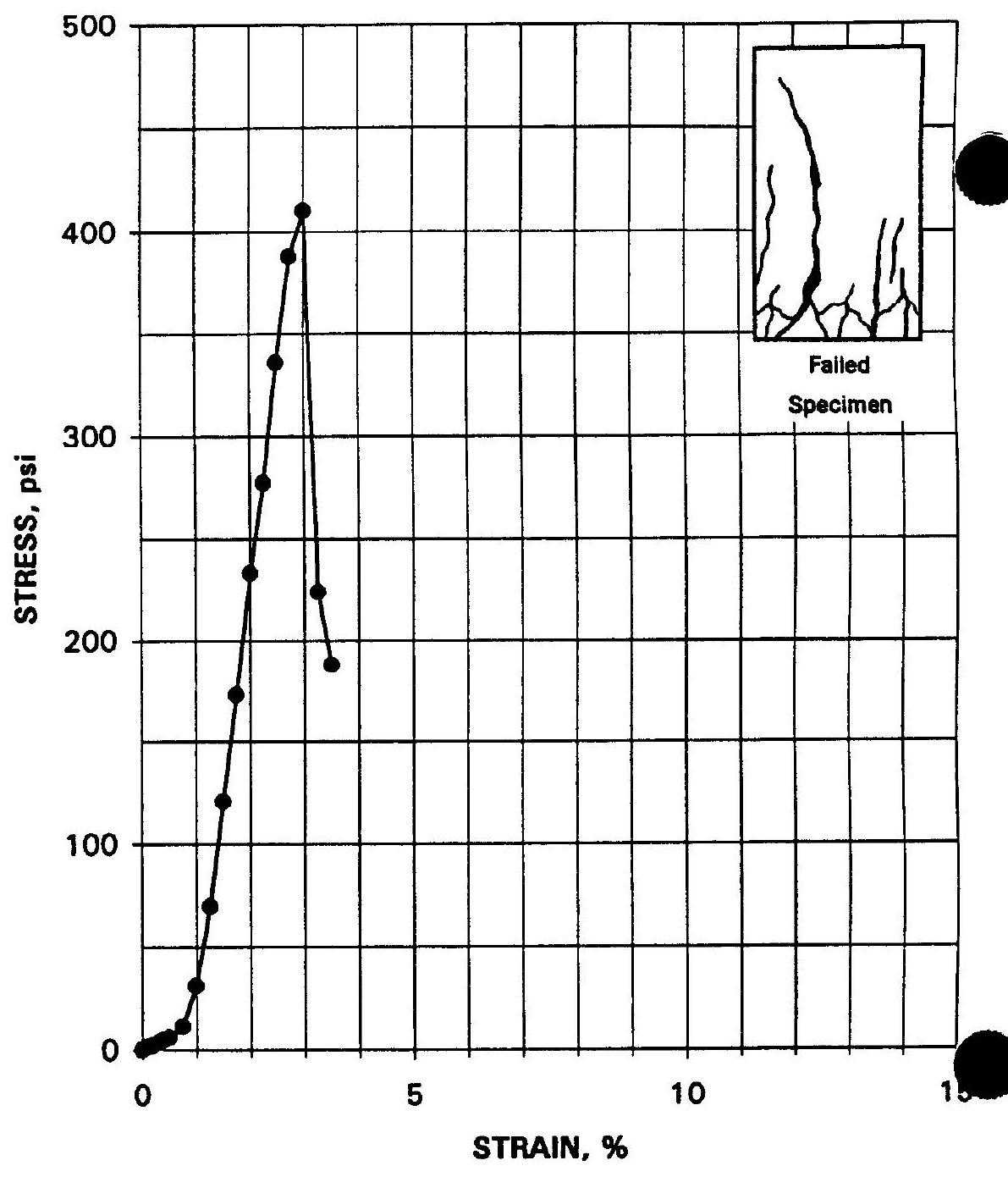




\section{UNCONFINED COMPRESSIVE STRENGTH - ASTM D 2166 28-DAY CURE}

Project Name SAIC/SAVANNAH RIVER

Project No. $\quad 777304.00010500$

Specimen Mold Date

Specimen Test Date

STRESS AT FAILURE, psi

STRAIN AT FAILURE, \%

AXIAL DEVIATOR

$\frac{\text { STRAIN, \% }}{0.00} \frac{\text { STRESS, psi }}{0.0}$

$0.10 \quad 1.6$

$0.20 \quad 2.8$

$0.30 \quad 4.4$

$0.40 \quad 6.0$

$0.50 \quad 8.8$

$0.75 \quad 19.8$

$1.00 \quad 41.7$

$1.25 \quad 72.0$

$1.51 \quad 108.6$

$1.76 \quad 139.5$

$2.01 \quad 149.7$

$2.26 \quad 165.1$

$2.51 \quad 184.7$

$2.76 \quad 204.0$

$3.01 \quad 227.4$

$3.51 \quad 236.96$

$4.01 \quad 232.08$

$3.76 \quad 233.60$

$4.52 \quad 235.10$

$5.02 \quad 235.37$

$5.52 \quad 233.53$

$6.02 \quad 230.56$

$6.52 \quad 175.17$

$7.02 \quad 51.63$
$11 / 11 / 98$

$12 / 9 / 98$

237.0

3.5
Client Sample No. C412F00

IT Lab Specimen No. ETDC-7889

Specimen Height, in.

3.9862

Specimen Diameter, in.

2.0045

Specimen Weight, $\mathbf{g}$.

375.96

Moisture Content, \%

31.7

Wet Unit Weight, pcr.

113.9

Dry Unit Weight, pcf.

86.4

Rate of Strain, in./min.

0.0240

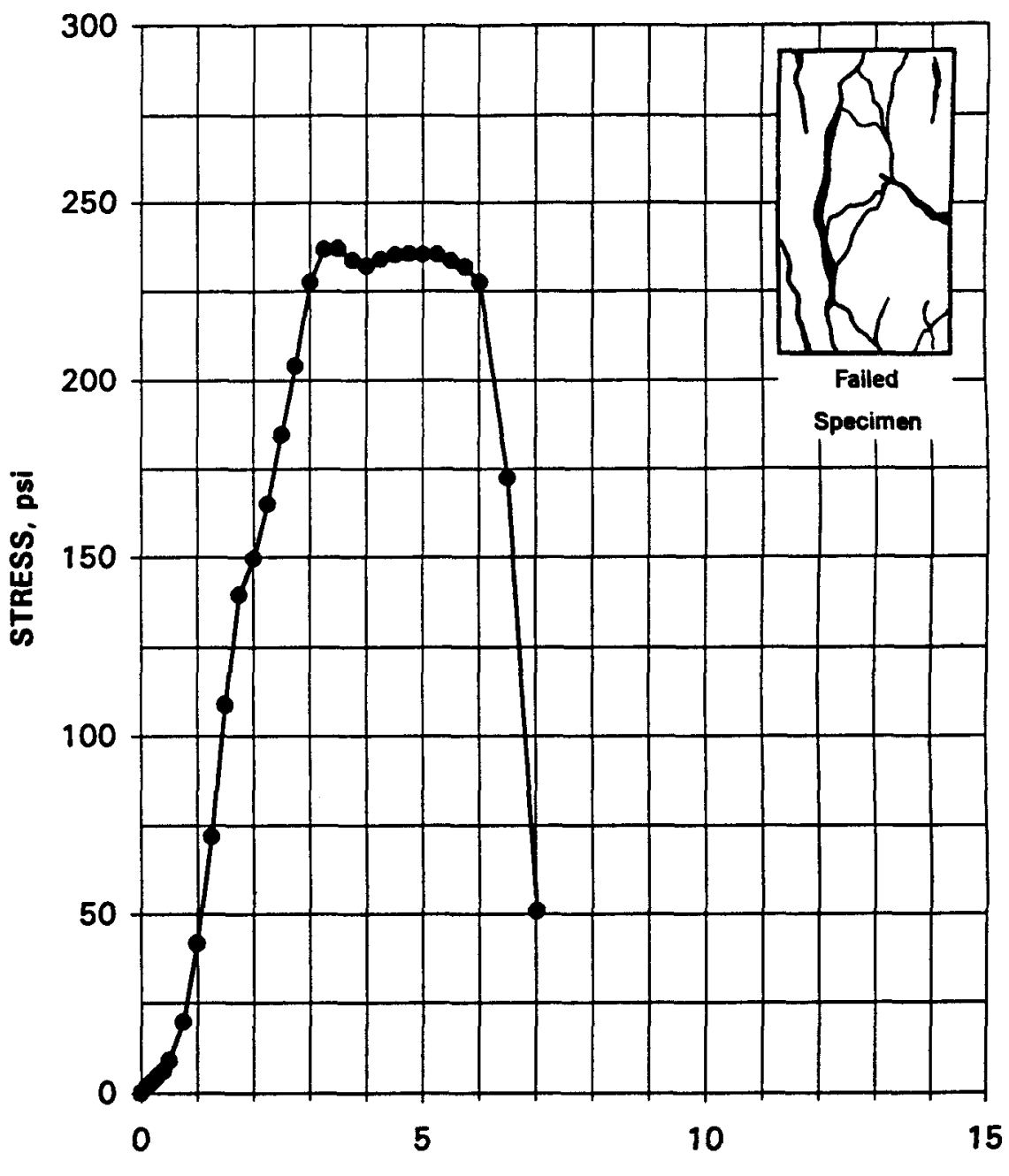

STRAIN, $\%$ 


\section{UNCONFINED COMPRESSIVE STRENGTH - ASTM D 2166 28-DAY CURE}

$\begin{array}{ll}\text { Project Name } & \text { SAIC/SAVANNAH RIVER } \\ \text { Project No. } & 777304.00010500\end{array}$

Specimen Mold Date

Specimen Test Date

STRESS AT FAILURE, psi

STRAIN AT FAILURE, \%

AXIAL

$\frac{\text { STRAIN, \% }}{0.00} \frac{\text { STRESS, psi }}{0.0}$

0.10

1.6

0.20

2.8

0.30

4.7

0.40

6.0

0.50

8.2

0.75

22.0

1.01

46.3

1.26

1.51

1.76

2.01

2.26

2.51

2.76

3.02

3.27

3.52

3.64

3.77
$11 / 11 / 98$

$12 / 9 / 98$

487.0

3.6
Client Sample No. C422F0O

IT Lab Specimen No. ETDC-7890

Specimen Height, in.

3.9788

Specimen Diameter, in.

2.0070

Specimen Weight, $\boldsymbol{g}$.

Moisture Content, \%

393.02

25.5

Wet Unit Weight, pcf.

Dry Unit Weight, pcf.

119.0

94.8

Rate of Strain, in./min.

0.0240

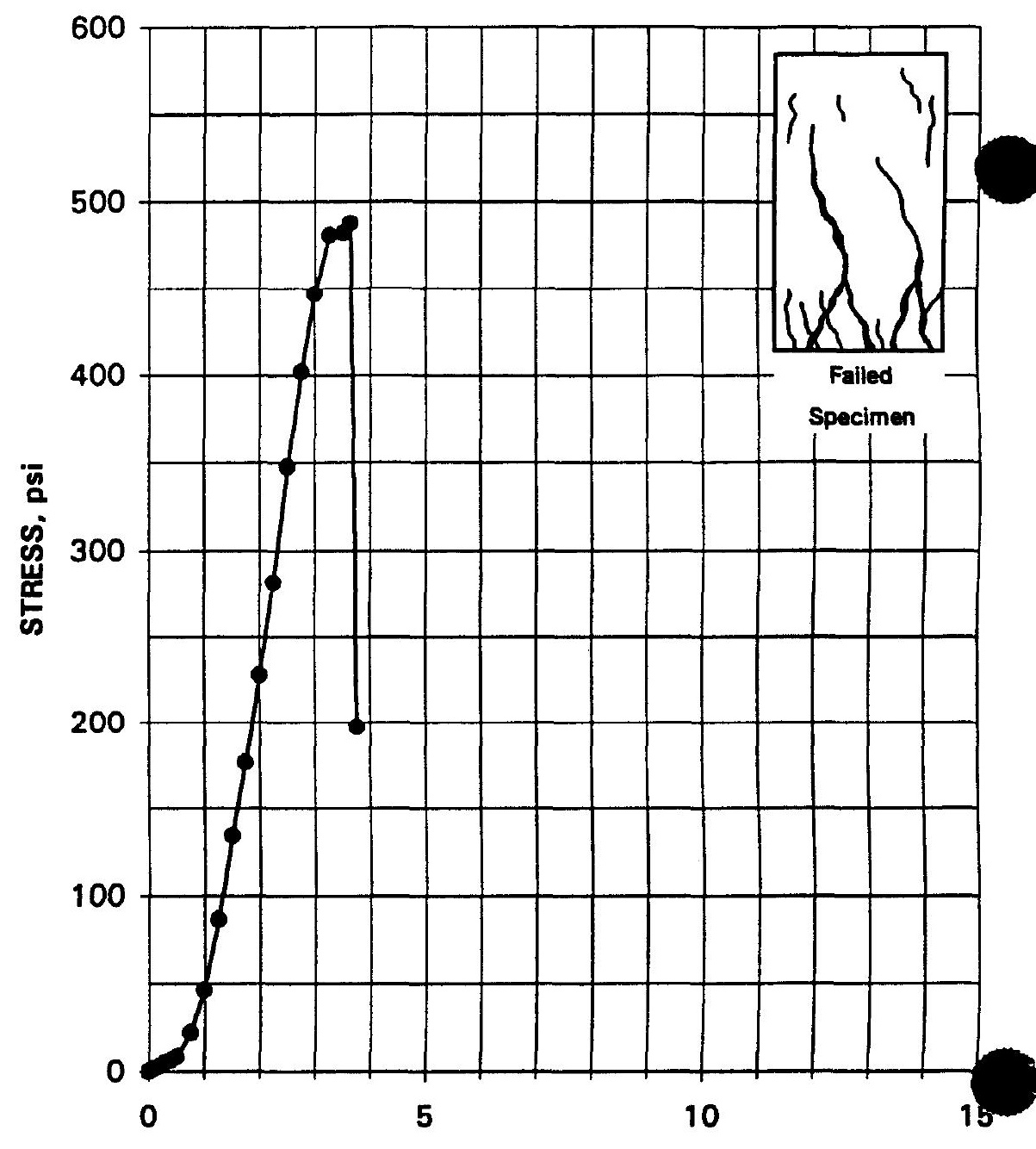

STRAIN, \% 


\section{UNCONFINED COMPRESSIVE STRENGTH - ASTM D 2166 28-DAY CURE}

Project Name SAIC/SAVANNAH RIVER

Project No. 777304.00010500

Specimen Mold Date

Specimen Test Date

STRESS AT FAILURE, psi

STRAIN AT FAILURE, \%

\begin{tabular}{ccc}
$\begin{array}{c}\text { AXIAL } \\
\text { STRAIN, } \%\end{array}$ & & $\begin{array}{c}\text { DEVIATOR } \\
\text { STRESS, psi }\end{array}$ \\
\cline { 1 - 1 } 0.00 & & 0.0 \\
0.10 & & 1.3 \\
0.20 & & 1.9 \\
0.30 & & 2.8 \\
0.40 & & 4.7 \\
0.50 & & 6.6 \\
0.76 & & 15.0 \\
1.01 & & 36.9 \\
1.26 & & 73.6 \\
1.51 & & 113.5 \\
1.76 & & 145.4 \\
2.02 & & 177.2 \\
2.27 & & 215.7 \\
2.52 & & 257.3 \\
2.77 & & 290.0 \\
3.02 & & 315.9 \\
3.28 & 336.8 \\
3.53 & 351.7 \\
3.78 & 357.8 \\
4.03 & 360.5 \\
4.28 & 360.8 \\
4.54 & 357.7 \\
4.79 & 359.8 \\
4.91 & & 360.2 \\
5.04 & & 354.0 \\
5.29 & & 131.8 \\
& &
\end{tabular}

$11 / 11 / 98$

$12 / 9 / 98$

360.8

4.3
Client Sample No. C432F0O

IT Lab Specimen No. ETDC-7891

Specimen Height, in.

3.9675

Specimen Diameter, in.

2.0083

Specimen Weight, $g$.

Moisture Content, \%

358.07

37.6

108.6

78.9

Dry Unit Weight, pcf.

0.0300

Rate of Strain, in./min.

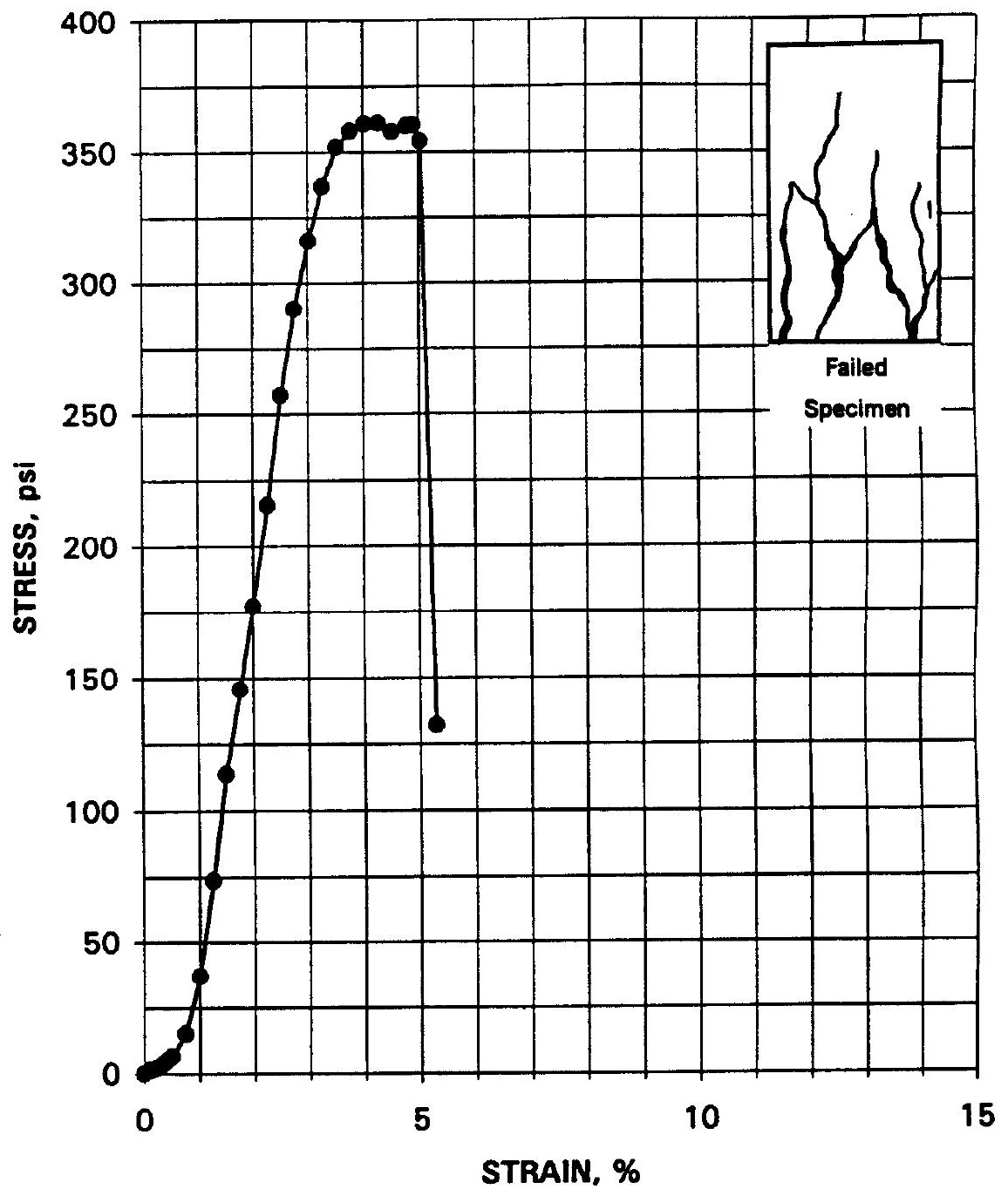




\section{UNCONFINED COMPRESSIVE STRENGTH - ASTM D 2166 28-DAY CURE}

Project Name SAIC/SAVANNAH RIVER

Project No. $\quad 777304.00010500$

Specimen Mold Date

Specimen Test Date

STRESS AT FAILURE, pSi STRAIN AT FAILURE, \%

\begin{tabular}{ccc}
$\begin{array}{c}\text { AXIAL } \\
\text { STRAIN, \% }\end{array}$ & & $\begin{array}{c}\text { DEVIATOR } \\
\text { STRESS, pSi }\end{array}$ \\
\cline { 1 - 1 } 0.00 & & 0.0 \\
0.10 & & 1.3 \\
0.20 & & 2.8 \\
0.30 & & 4.7 \\
0.40 & & 6.6 \\
0.50 & & 10.7 \\
0.75 & & 46.9 \\
1.01 & & 109.9 \\
1.26 & & 164.4 \\
1.51 & & 219.5 \\
1.76 & & 252.4 \\
2.01 & & 306.1 \\
2.26 & & 346.3 \\
2.51 & 380.2 \\
2.77 & 403.4 \\
3.02 & 407.6 \\
3.14 & 410.7 \\
3.27 & 320.5 \\
3.52 & 246.4 \\
3.77 & 209.6
\end{tabular}

Client Sample No. C442F00

IT Lab Specimen No. ETDC-7892

Specimen Height, in.

Specimen Diameter, in.

3.9762

2.0097

Specimen Weight, $g$.

364.54

Moisture Content, \%

Wet Unit Weight, pef.

36.0

110.1

Dry Unit Weight, pcf.

81.0

Rate of Strain, in./min.

0.0300

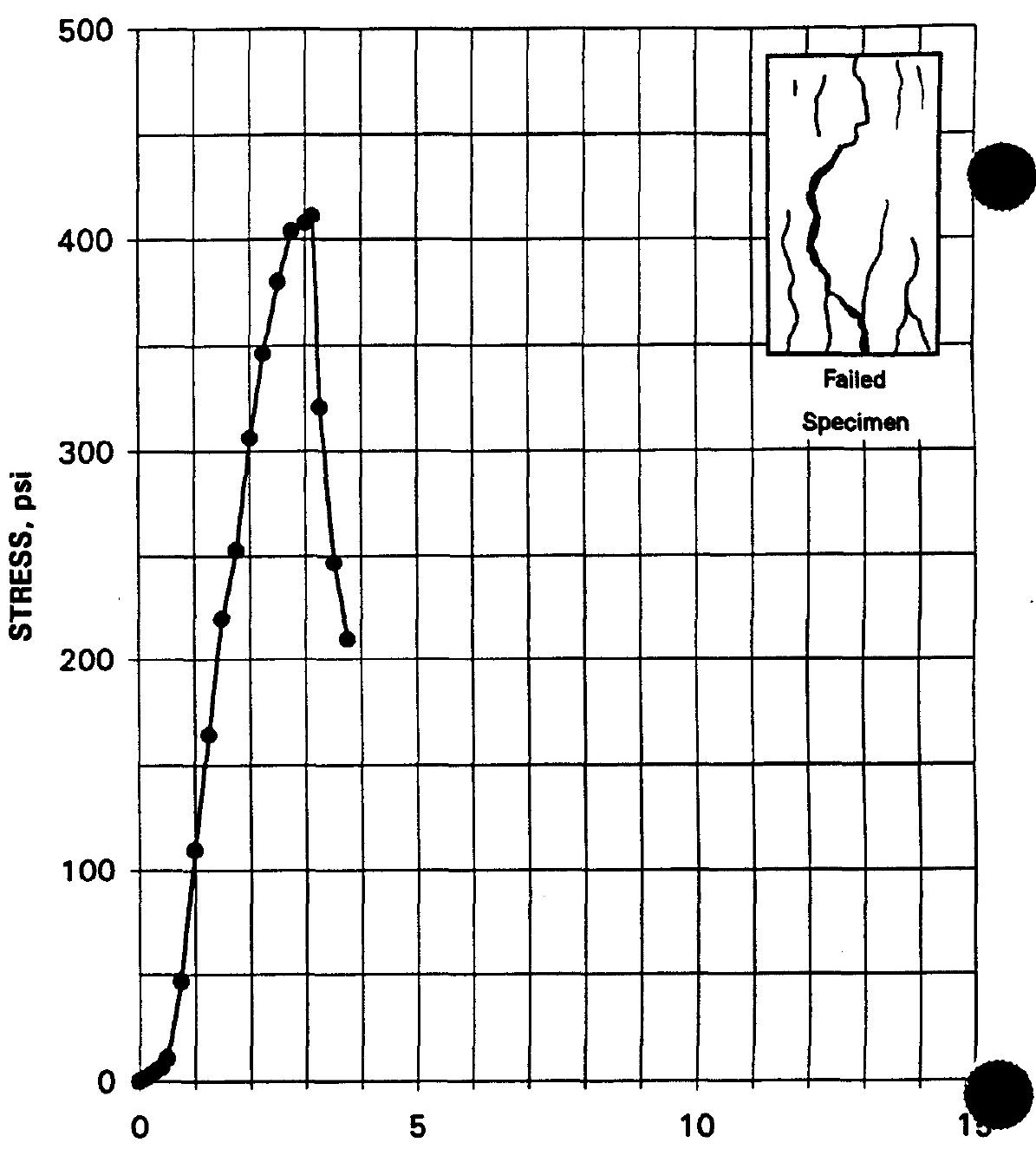

STRAIN, \% 


\section{UNCONFINED COMPRESSIVE STRENGTH - ASTM D 2166 28-DAY CURE}

Project Name SAIC/SAVANNAH RIVER

Project No. 777304.00010500

Specimen Mold Date

Specimen Test Date

STRESS AT FAILURE, psi

STRAIN AT FAILURE, \%

\begin{tabular}{ccc}
$\begin{array}{c}\text { AXIAL } \\
\text { STRAIN, } \%\end{array}$ & $\begin{array}{c}\text { DEVIATOR } \\
\text { STRESS, psi }\end{array}$ \\
\cline { 1 - 1 } 0.00 & 0.0 \\
0.10 & & 1.3 \\
0.20 & & 2.8 \\
0.30 & & 4.7 \\
0.40 & & 7.6 \\
0.50 & & 12.0 \\
0.75 & & 35.5 \\
1.00 & & 71.4 \\
1.25 & & 108.1 \\
1.51 & & 156.4 \\
1.76 & & 186.4 \\
2.01 & & 226.0 \\
2.26 & & 265.6 \\
2.51 & 297.3 \\
2.76 & 292.8 \\
3.01 & 291.7 \\
3.26 & 311.2 \\
3.51 & 327.8 \\
3.76 & 341.2 \\
4.02 & 351.0 \\
4.27 & 354.6 \\
4.52 & 353.1 \\
4.77 & 339.5 \\
4.89 & 352.9 \\
5.02 & 269.8 \\
5.27 & 30.0 \\
& &
\end{tabular}

$11 / 11 / 98$

$12 / 9 / 98$

354.6

4.3
Client Sample No. C512F00

IT Lab Specimen No. ETDC-7893

Specimen Height, in.

3.9848

Specimen Diameter, in.

2.0063

Specimen Weight, $g$.

384.57

Moisture Content, \%

27.5

Wet Unit Weight, pcf.

Dry Unit Weight, pct.

116.3

91.2

Rate of Strain, in./min.

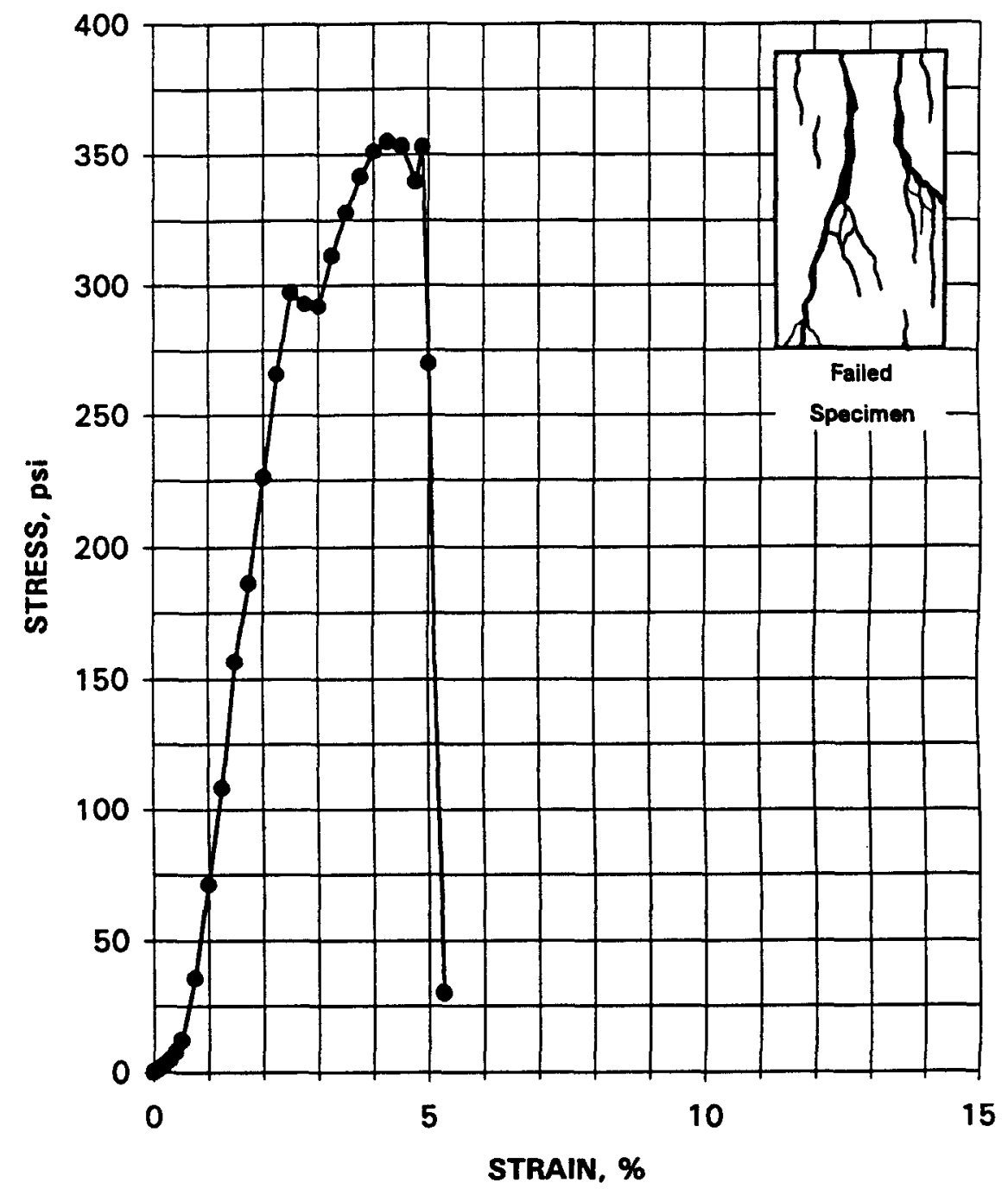




\section{UNCONFINED COMPRESSIVE STRENGTH - ASTM D 2166 28-DAY CURE}

Project Name SAIC/SAVANNAH RIVER

Project No. $\quad 777304.00010500$

Specimen Mold Date

Specimen Test Date

STRESS AT FAILURE, psi STRAIN AT FAILURE, \%

\begin{tabular}{ccc}
$\begin{array}{c}\text { AXIAL } \\
\text { STRAIN, \% }\end{array}$ & \multicolumn{1}{c}{$\begin{array}{c}\text { DEVIATOR } \\
\text { STRESS, psi }\end{array}$} \\
\cline { 1 - 1 } 0.00 & & 0.0 \\
0.10 & & 0.9 \\
0.20 & & 2.8 \\
0.30 & & 4.4 \\
0.40 & & 6.3 \\
0.51 & & 8.8 \\
0.76 & & 20.7 \\
1.01 & & 48.5 \\
1.26 & & 95.5 \\
1.52 & & 160.6 \\
1.77 & & 230.7 \\
2.02 & & 275.0 \\
2.27 & 316.0 \\
2.53 & 368.5 \\
2.78 & 408.1 \\
3.03 & 400.6 \\
3.16 & 420.3 \\
3.28 & 338.1 \\
3.54 & 272.0 \\
3.79 & 244.2
\end{tabular}

Client Sample No. C522F00

IT Lab Specimen No. ETDC-7894

Specimen Height, in.

3.9588

Specimen Diameter, in.

2.0070

Specimen Weight, $\mathrm{g}$.

389.92

Moisture Content, \%

26.2

Wet Unit Weight, pcf.

118.6

Dry Unit Weight, pct.

94.0

Rate of Strain, in./min.

0.0300

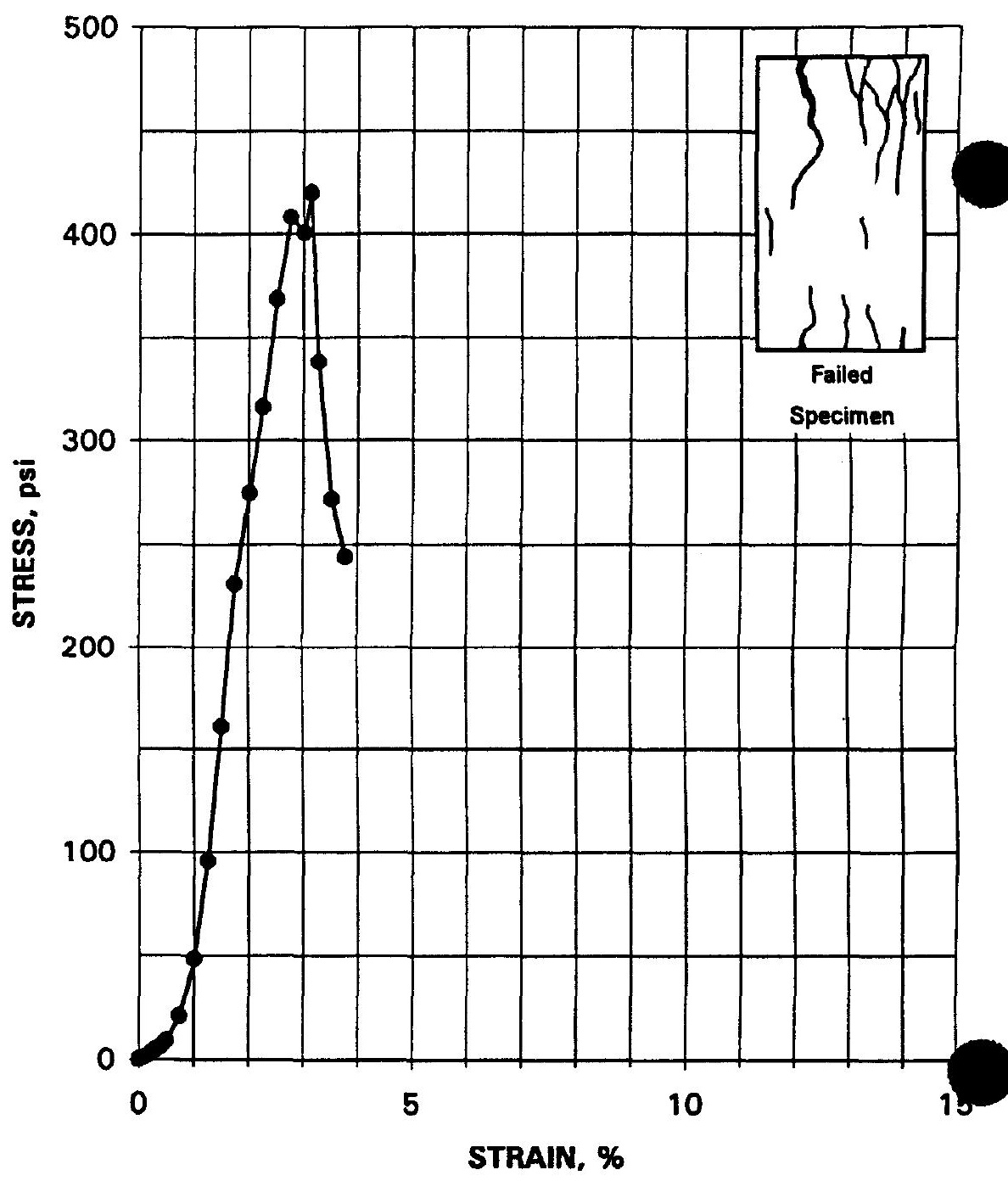




\section{UNCONFINED COMPRESSIVE STRENGTH - ASTM D 2166 28-DAY CURE}

\section{Project Name SAIC/SAVANNAH RIVER}

Project No. $\quad 777304.00010500$

Specimen Mold Date

Specimen Test Date

STRESS AT FAILURE, psi

STRAIN AT FAILURE, \%

\begin{tabular}{ccc}
$\begin{array}{c}\text { AXIAL } \\
\text { STRAIN, } \%\end{array}$ & & $\begin{array}{c}\text { DEVIATOR } \\
\text { STRESS, psi }\end{array}$ \\
\cline { 1 - 1 } 0.00 & & 0.0 \\
0.10 & & 1.3 \\
0.20 & & 2.8 \\
0.30 & & 4.7 \\
0.40 & & 6.6 \\
0.50 & & 9.4 \\
0.75 & & 27.6 \\
1.00 & & 69.6 \\
1.25 & & 112.5 \\
1.50 & & 161.5 \\
1.75 & & 198.4 \\
2.00 & & 241.0 \\
2.25 & & 287.4 \\
2.50 & 313.5 \\
2.75 & 341.7 \\
3.00 & 351.5 \\
3.25 & 281.7 \\
3.50 & 128.6
\end{tabular}

$11 / 11 / 98$

$12 / 9 / 98$

351.5

3.0
Client Sample No. C532F00

IT Lab Specimen No. ETDC-7895

Specimen Height, in.

4.0000

Specimen Diameter, in.

Specimen Weight, $\mathbf{g}$.

Moisture Content, \%

2.0057

371.22

34.1

Wet Unit Weight, pcf.

Dry Unit Weight, pcf.

111.9

83.5

Rate of Strain, in./min.

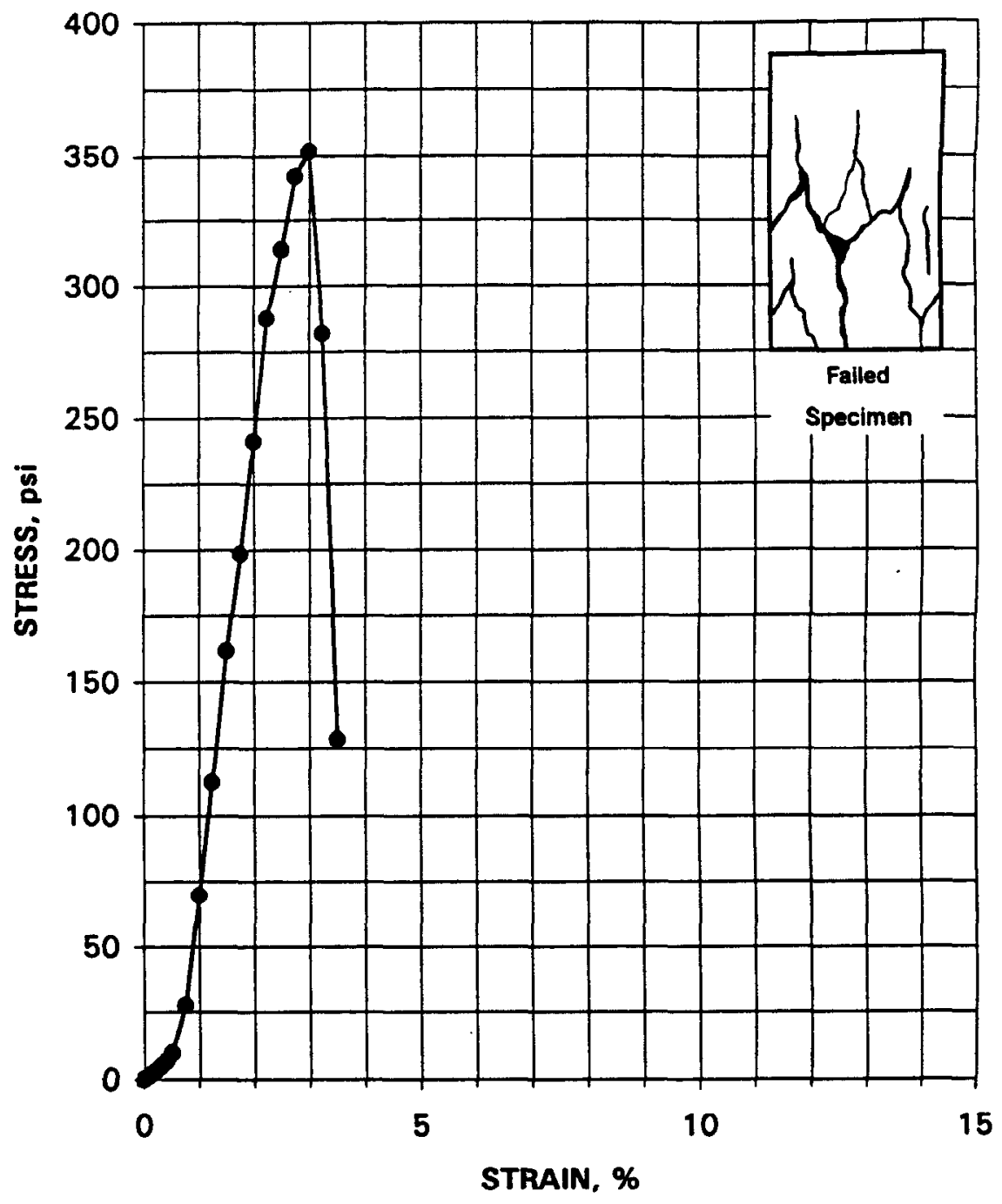




\section{UNCONFINED COMPRESSIVE STRENGTH - ASTM D 2166 28-DAY CURE}

Project Name SAIC/SAVANNAH RIVER

Project No. $\quad 777304.00010500$

Specimen Mold Date

Specimen Test Date

STRESS AT FAILURE, psi STRAIN AT FAILURE, \%

AXIAL

STRAIN, \%

0.00

0.10

0.20

0.30

0.40

0.50

0.76

1.01

1.26

1.51

1.76

2.01

2.27

2.52

2.77

3.02

3.27

3.35

3.52

3.78

4.03
DEVIATOR

STRESS, pSi

1.3

2.5

4.7

7.6

12.3

23.3

39.6

65.5

105.4

151.6

200.7

247.4

286.7

322.7

348.6

360.6

361.0

343.1

309.5

211.5
$11 / 11 / 98$

$12 / 9 / 98$

361.0

3.3
Client Sample No. C542FOO

IT Lab Specimen No. ETDC-7896

Specimen Height, in.

Specimen Diameter, in.

3.9717

Specimen Weight, g.

2.0023

Moisture Content, \%

385.01

28.0

Wet Unit Weight, pcf.

117.3

Dry Unit Weight, pcf.

91.6

Rate of Strain, in./min.

0.0300

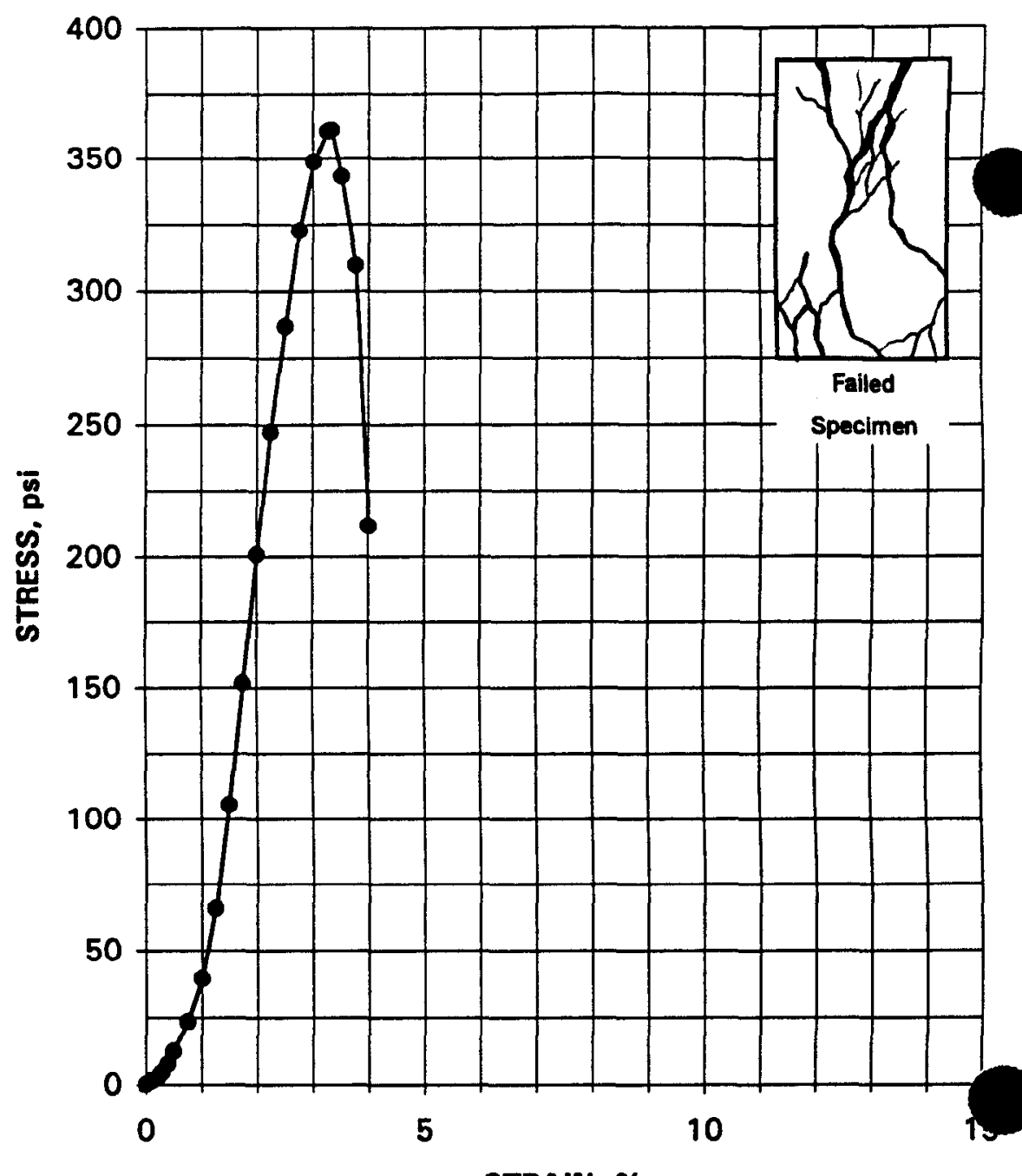

STRAIN, \% 


\section{UNCONFINED COMPRESSIVE STRENGTH - ASTM D 2166 28-DAY CURE}

Project Name SAIC/SAVANNAH RIVER

Project No. $\quad 777304.00010500$

Specimen Mold Date

Specimen Test Date

STRESS AT FAILURE, psi

STRAIN AT FAILURE, \%

\begin{tabular}{rr}
$\begin{array}{rr}\text { AXIAL } \\
\text { STRAIN, } \%\end{array}$ & $\begin{array}{r}\text { DEVIATOR } \\
\text { STRESS, psi }\end{array}$ \\
\cline { 1 - 1 } 0.00 & 0.0 \\
0.10 & 1.0 \\
0.20 & 1.9 \\
0.30 & 3.2 \\
0.40 & 6.0 \\
0.50 & 10.4 \\
0.75 & 27.8 \\
1.00 & 69.0 \\
1.25 & 123.8 \\
1.50 & 172.7 \\
1.75 & 211.3 \\
2.00 & 246.9 \\
2.26 & 269.0 \\
2.51 & 307.1 \\
2.76 & 338.5 \\
3.01 & 360.1 \\
3.26 & 370.0 \\
3.51 & 379.1 \\
3.76 & 372.9 \\
4.01 & 372.6 \\
4.26 & 376.8 \\
4.51 & 378.5 \\
4.76 & 353.3 \\
5.01 & 311.6 \\
5.26 & 229.4 \\
5.51 & 155.4
\end{tabular}

$11 / 11 / 98$

$12 / 9 / 98$

379.1

3.5
Client Sample No. C612F00

IT Lab Specimen No. ETDC-7897

Specimen Height, in.

3.9903

Specimen Diameter, in.

2.0005

Specimen Weight, $g$.

Moisture Content, \%

384.46

27.9

Wet Unit Weight, pcf.

116.8

Dry Unit Weight, pcf.

91.3

Rate of Strain, in./min.

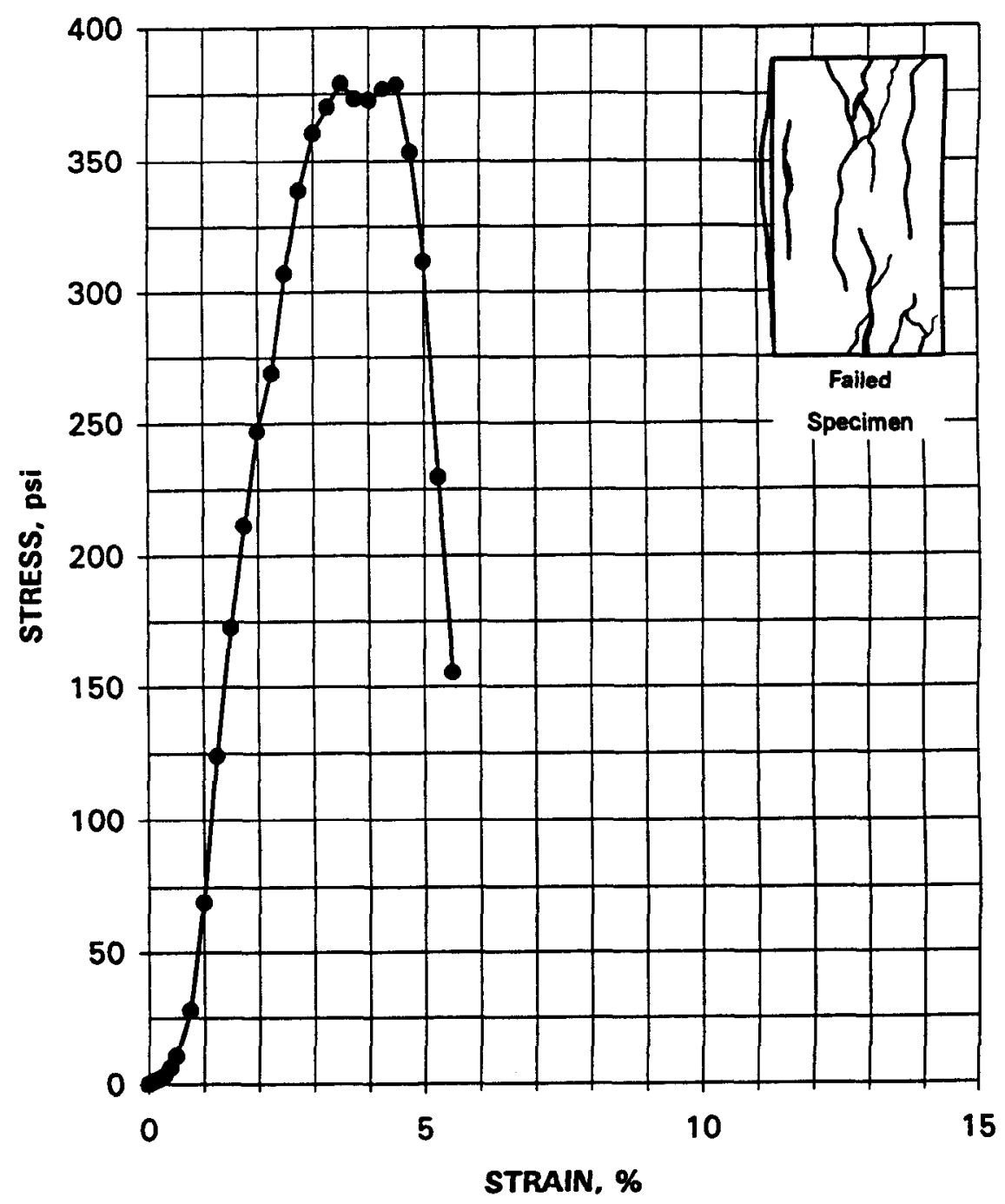




\section{UNCONFINED COMPRESSIVE STRENGTH - ASTM D 2166 28-DAY CURE}

$\begin{array}{ll}\text { Project Name } & \text { SAIC/SAVANNAH RIVER } \\ \text { Project No. } & 777304.00010500\end{array}$

Specimen Mold Date

Specimen Test Date

STRESS AT FAILURE, psi STRAIN AT FAILURE, \%

\begin{tabular}{cr}
$\begin{array}{c}\text { AXIAL } \\
\text { STRAIN, \% }\end{array}$ & $\begin{array}{r}\text { DEVIATOR } \\
\text { STRESS, psi }\end{array}$ \\
\cline { 1 - 2 } 0.00 & 0.0 \\
0.10 & 1.3 \\
0.20 & 3.8 \\
0.30 & 7.6 \\
0.40 & 11.4 \\
0.50 & 17.0 \\
0.76 & 39.9 \\
1.01 & 72.2 \\
1.26 & 114.8 \\
1.51 & 154.8 \\
1.76 & 197.1 \\
2.02 & 238.5 \\
2.27 & 266.4 \\
2.52 & 297.2 \\
2.77 & 326.6 \\
3.03 & 350.1 \\
3.28 & 381.3 \\
3.53 & 402.1 \\
3.78 & 418.1 \\
4.03 & 420.3 \\
4.16 & 424.3 \\
4.29 & 335.8 \\
4.54 & 284.4 \\
4.79 & 33.8
\end{tabular}

$11 / 11 / 98$

$12 / 9 / 98$

424.3

4.2
Client Sample No. C622F00

IT Lab Specimen No. ETDC-7898

Specimen Height, in.

Specimen Diameter, in.

3.9667

Specimen Weight, $g$.

Moisture Content, \%

Wet Unit Weight, pcf.

Dry Unit Weight, pcf.

Rate of Strain, in./min.

2.0043

389.68

26.4

118.6

93.9

0.0300

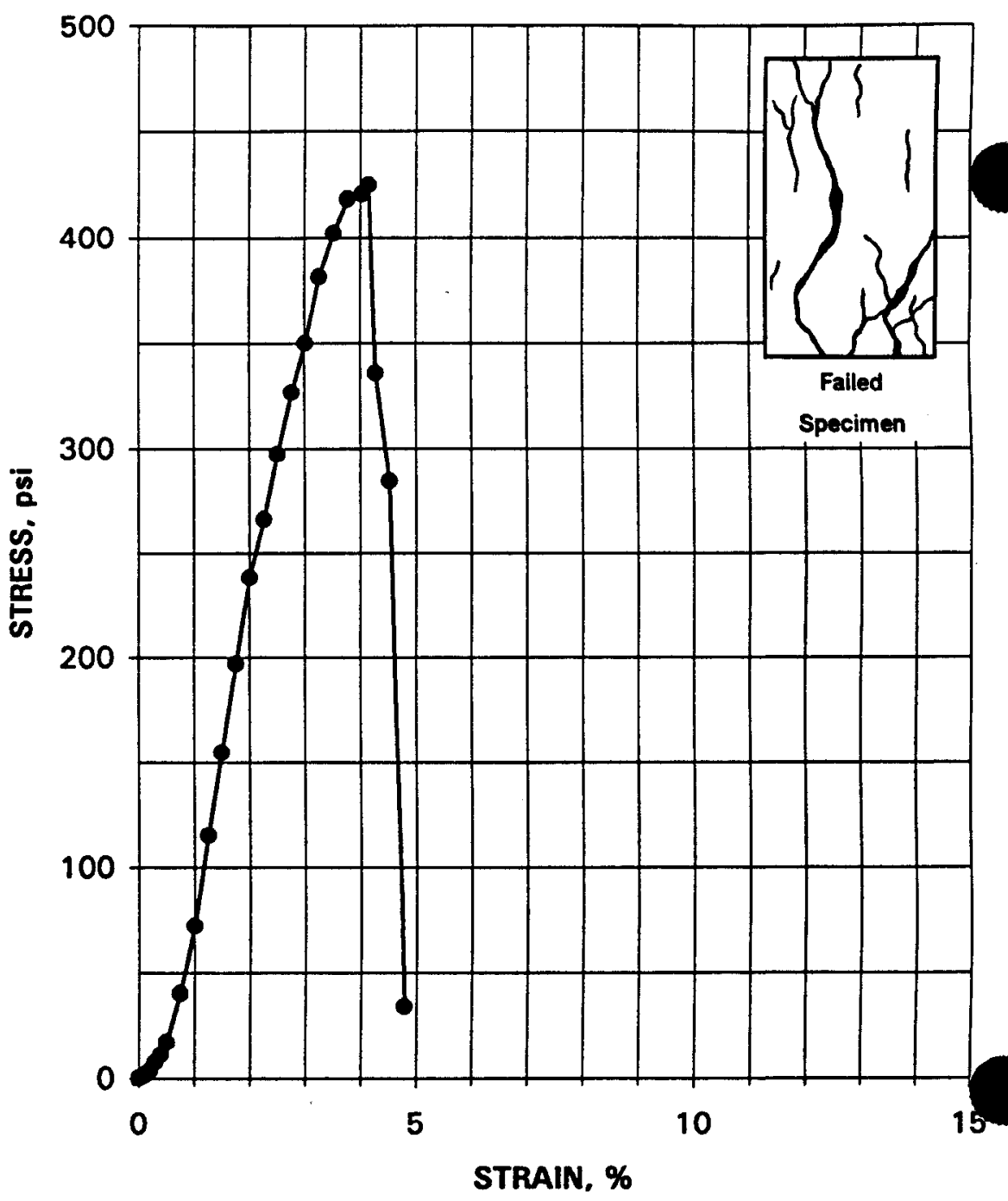




\section{UNCONFINED COMPRESSIVE STRENGTH - ASTM D 2166 28-DAY CURE}

\begin{tabular}{|c|c|c|c|c|}
\hline Project Name & \multicolumn{2}{|c|}{ SAIC/SAVANNAH RIVER } & Client Sample No. C & C632F00 \\
\hline Project No. & \multicolumn{2}{|c|}{777304.00010500} & IT Lab Specimen No. E & ETDC-7899 \\
\hline \multicolumn{2}{|c|}{ Specimen Mold Date } & $11 / 11 / 98$ & Specimen Height, in. & 3.9785 \\
\hline \multirow{2}{*}{\multicolumn{2}{|c|}{ Specimen Test Date }} & $12 / 9 / 98$ & Specimen Diameter, in. & 1.9993 \\
\hline & & & Specimen Weight, $g$. & 366.83 \\
\hline \multicolumn{2}{|c|}{ STRESS AT FAILURE, psi } & 378.6 & Moisture Content, \% & 34.1 \\
\hline \multirow{2}{*}{\multicolumn{2}{|c|}{ STRAIN AT FAILURE, $\%$}} & 2.9 & Wet Unit Weight, pcf. & 111.9 \\
\hline & & & Dry Unit Weight, pcf. & 83.4 \\
\hline AXIAL & \multicolumn{2}{|c|}{ DEVIATOR } & Rate of Strain, in./min. & 0.0300 \\
\hline
\end{tabular}

\begin{tabular}{ccc} 
STRAIN, $\%$ & STRESS, psi \\
\cline { 1 - 1 } 0.00 & & 0.0 \\
0.10 & & 1.3 \\
0.20 & & 2.9 \\
0.30 & & 4.8 \\
0.40 & & 7.3 \\
0.50 & & 10.1 \\
0.75 & & 27.8 \\
1.01 & & 76.3 \\
1.26 & & 134.9 \\
1.51 & & 201.7 \\
1.76 & & 259.7 \\
2.01 & & 291.8 \\
2.26 & & 348.4 \\
2.51 & & 368.0 \\
2.76 & 377.2 \\
2.89 & 378.6 \\
3.02 & 367.9 \\
3.27 & 96.1
\end{tabular}

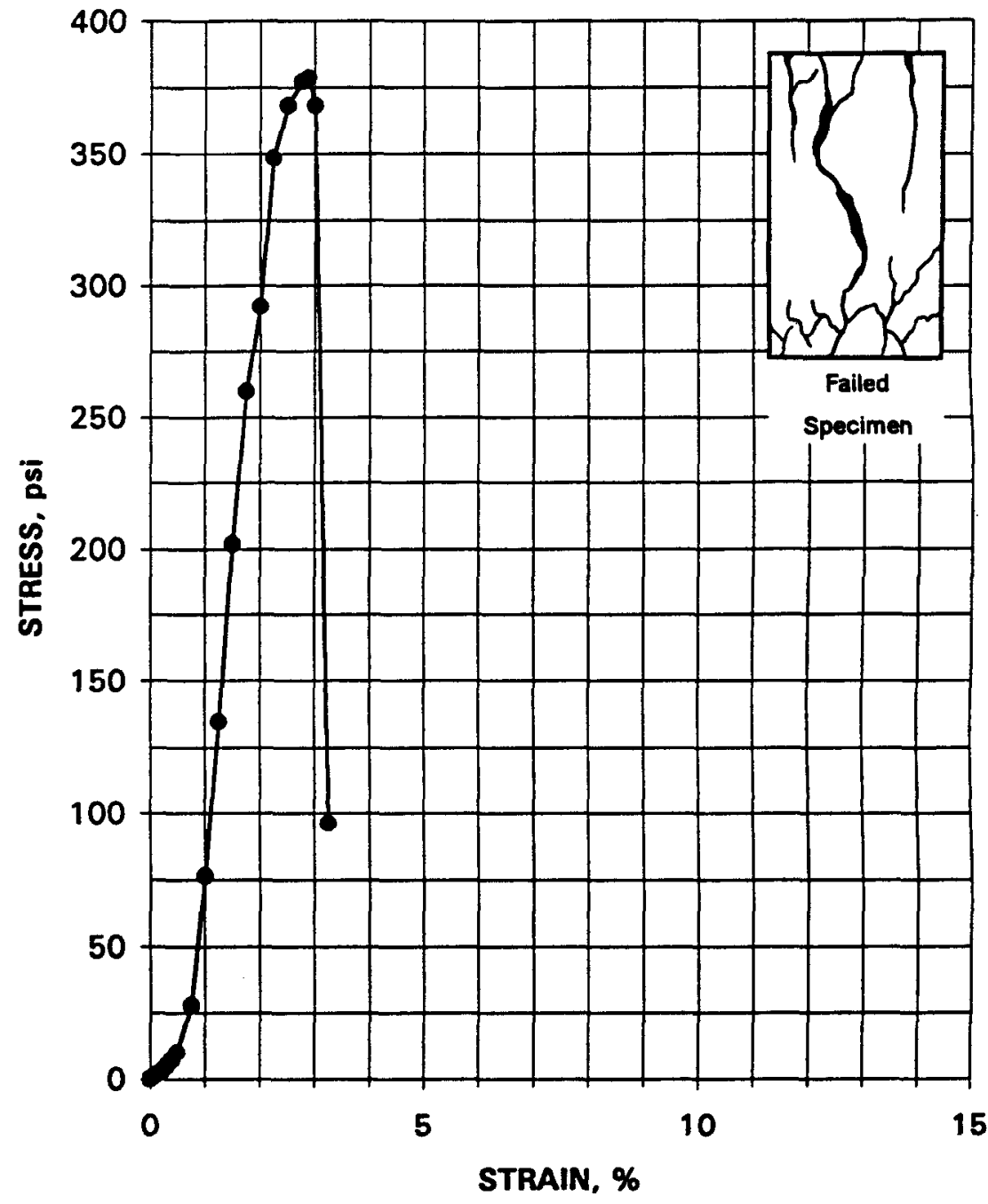




\section{UNCONFINED COMPRESSIVE STRENGTH - ASTM D 2166 28-DAY CURE}

Project Name SAIC/SAVANNAH RIVER

Project No. $\quad 777304.00010500$

Specimen Mold Date

Specimen Test Date

STRESS AT FAILURE, psi STRAIN AT FAILURE, \%

AXIAL DEVIATOR

$\frac{\text { STRAIN, \% }}{0.00} \frac{\text { STRESS, psi }}{0.0}$

0.10

0.9

0.20

0.30

0.40

0.50

0.76

1.01

1.26

1.51

1.76

2.04

2.27

2.52

2.77

3.02

3.27

3.52

3.78

4.03

4.28
1.9

3.5

6.3

8.5

23.2

59.7

114.2

185.4

265.6

327.8

392.4

456.0

486.5

493.5

504.1

311.8

269.9

256.8

227.4
$11 / 11 / 98$

$12 / 9 / 98$

504.1

3.3
Client Sample No. C642F0O

IT Lab Specimen No. ETDC-7900

Specimen Height, in.

3.9718

Specimen Diameter, in.

2.0077

Specimen Weight, $\mathbf{g}$.

Moisture Content, \%

374.14

31.2

Wet Unit Weight, pcf.

Dry Unit Weight, pef.

113.4

86.4

Rate of Strain, in./min.

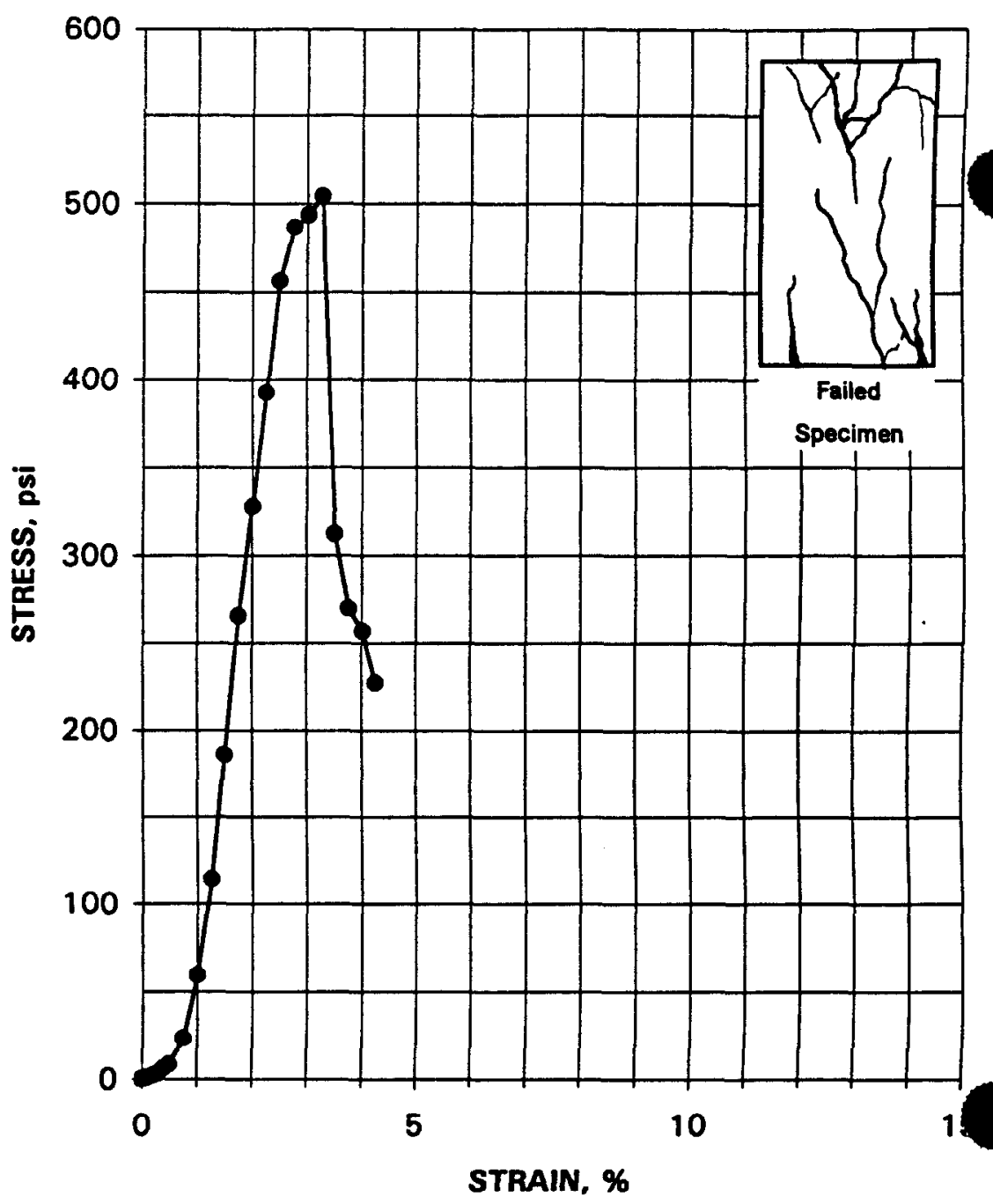




\section{UNCONFINED COMPRESSIVE STRENGTH - ASTM D 2166 28 DAY CURE, 90 DAY IMMERSION}

\begin{tabular}{lc} 
Project Name & SAIC / SAVA \\
Project No. & 777304.00010 \\
Specimen Mold Date \\
Specimen Test Date \\
STRESS AT FAILURE, psi \\
STRAIN AT FAILURE, \% \\
\multicolumn{3}{c}{ AXIAL } & DEVIATOR \\
STRAIN, $\%$ & STRESS, psi \\
\hline 0.00 & 0.0 \\
0.10 & 2.5 \\
0.20 & 4.4 \\
0.30 & 4.4 \\
0.40 & 6.3 \\
0.50 & 10.1 \\
0.75 & 50.5 \\
1.00 & 114.7 \\
1.25 & 181.0 \\
1.51 & 249.2 \\
1.76 & 320.2 \\
2.01 & 398.3 \\
2.26 & 440.9 \\
2.51 & 100.9
\end{tabular}

$11 / 11 / 98$
$3 / 9 / 99$
440.9
2.3
Client Sample No. C313F00

IT Lab Specimen No. ETDC-7837

Specimen Height, in.

3.9860

Specimen Diameter, in.

2.0000

Specimen Weight, $g$.

Moisture Content, \%

390.62

30.7

Wet Unit Weight, pcf.

118.6

Dry Unit Weight, pct.

90.7

Rate of Strain, in./min.

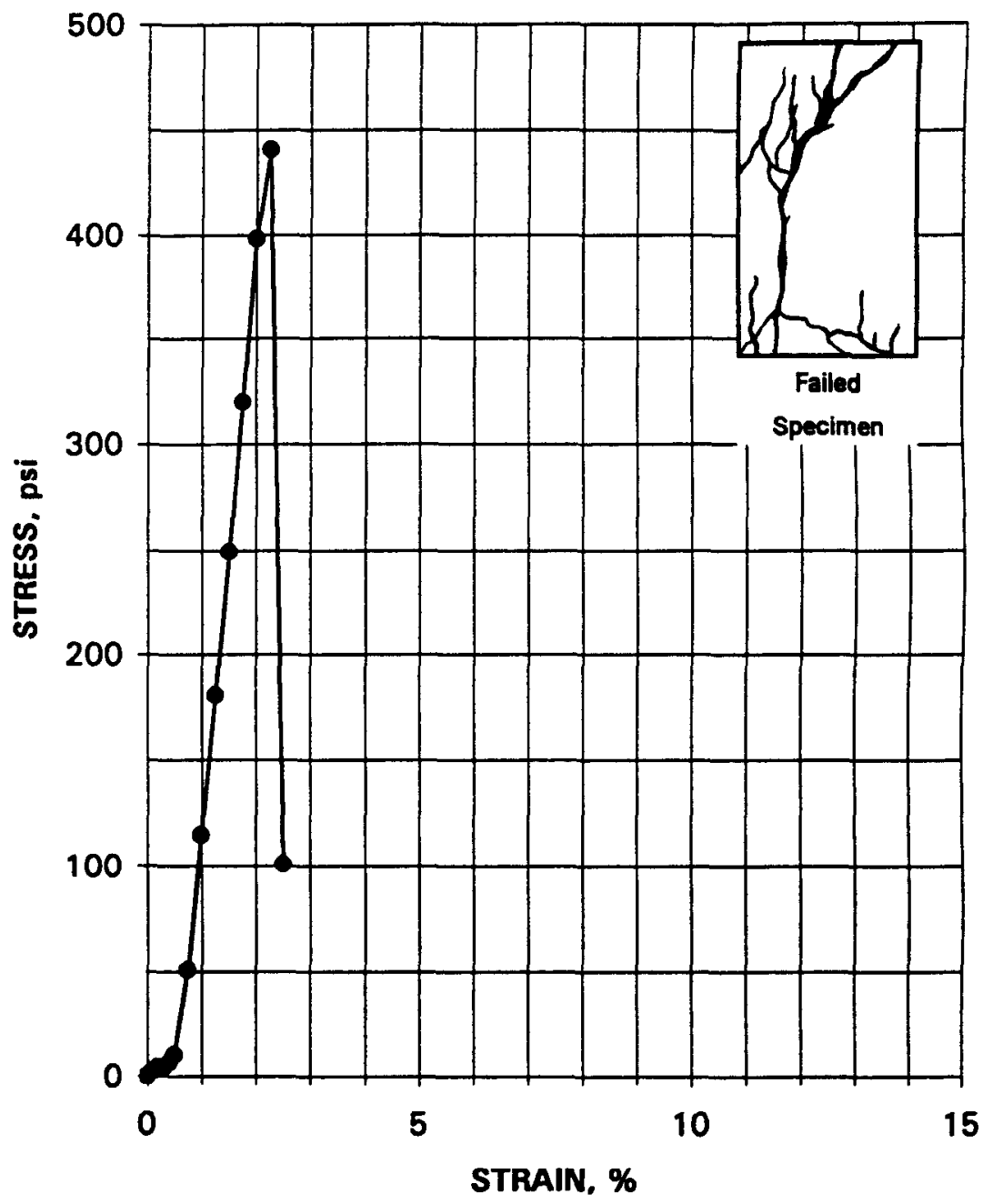




\section{Series}

\section{Day Immersion UCS Results}




\section{UNCONFINED COMPRESSIVE STRENGTH - ASTM D 2166 28 DAY CURE, 90 DAY IMMERSION}

Project Name SAIC / SAVANNAH RIVER

Project No. $\quad 777304.00010500$

Specimen Mold Date

Specimen Test Date

STRESS AT FAILURE, psi

STRAIN AT FAILURE, \%

\begin{tabular}{ccc}
$\begin{array}{ccc}\text { AXIAL } \\
\text { STRAIN, \% }\end{array}$ & & $\begin{array}{c}\text { DEVIATOR } \\
\text { STRESS, psi }\end{array}$ \\
\cline { 1 - 1 } 0.00 & & 0.0 \\
0.10 & & 3.5 \\
0.20 & & 6.0 \\
0.30 & & 5.7 \\
0.40 & & 7.0 \\
0.50 & & 15.9 \\
0.75 & & 80.7 \\
1.01 & & 141.1 \\
1.26 & & 189.5 \\
1.51 & & 241.1 \\
1.76 & & 285.6 \\
2.01 & & 326.7 \\
2.26 & & 365.2 \\
2.51 & & 399.7 \\
2.76 & & 422.8 \\
3.02 & & 439.6 \\
3.27 & 455.8 \\
3.52 & 478.9 \\
3.77 & 496.0 \\
4.02 & 514.0 \\
4.27 & 528.6 \\
4.52 & 537.5 \\
4.78 & 541.0 \\
5.03 & 551.0 \\
5.28 & 560.4 \\
5.53 & 574.3 \\
5.78 & & 580.0 \\
& &
\end{tabular}

$11 / 11 / 98$

$3 / 9 / 99$

581.9

5.9
Client Sample No. C323F00

IT Lab Specimen No. ETDC-7838

Specimen Height, in.

3.9767

Specimen Diameter, in.

1.9985

Specimen Weight, $\mathbf{g}$.

396.65

Moisture Content, \%

27.4

Wet Unit Weight, pef.

Dry Unit Weight, pef.

120.6

94.7

Rate of Strain, in./min.

0.0360

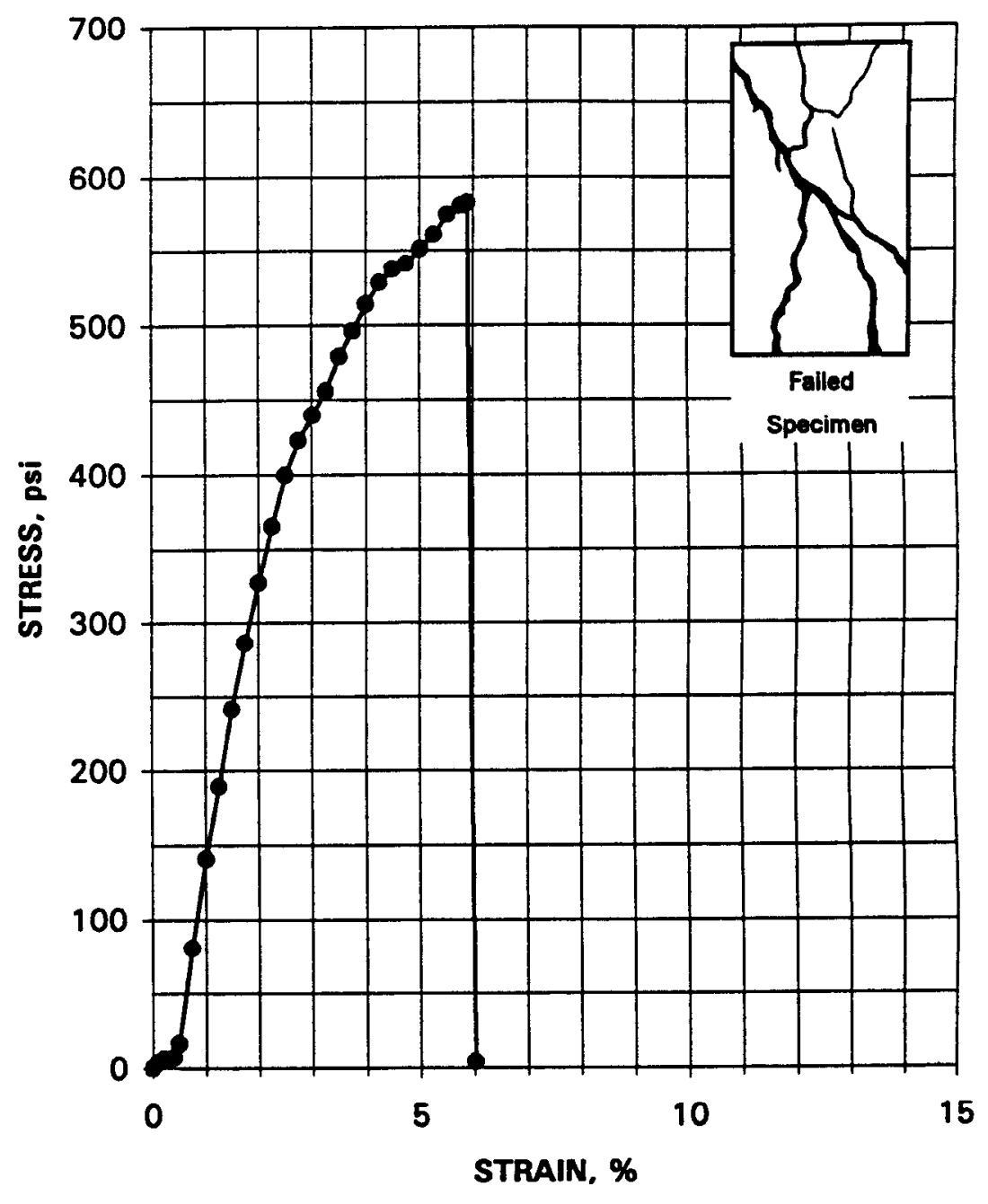




\section{UNCONFINED COMPRESSIVE STRENGTH - ASTM D 2166 28 DAY CURE, 90 DAY IMMERSION}

\begin{tabular}{lc} 
Project Name & SAIC / SAVAN \\
Project No. & 777304.00010 \\
Specimen Mold Date \\
Specimen Test Date \\
STRESS AT FAILURE, psi \\
STRAIN AT FAILURE, \% \\
\multicolumn{2}{|}{} \\
AXIAL & DEVIATOR \\
STRAIN, \% & STRESS, psi \\
\hline 0.00 & 0.0 \\
0.10 & 3.2 \\
0.20 & 10.8 \\
0.30 & 26.4 \\
0.40 & 48.0 \\
0.50 & 74.0 \\
0.75 & 140.6 \\
1.01 & 206.3 \\
1.26 & 260.0 \\
1.51 & 310.2 \\
1.76 & 354.6 \\
2.01 & 392.1 \\
2.26 & 430.1 \\
2.52 & 462.6 \\
2.77 & 444.6 \\
3.02 & 288.4 \\
3.27 & 18.2 \\
\multicolumn{2}{l}{}
\end{tabular}

Client Sample No. C333F00

IT Lab Specimen No. ETDC-7839
$11 / 11 / 98$
$3 / 9 / 99$
462.6
2.5
Specimen Height, in.
3.9792
Specimen Diameter, in.
1.9975
Specimen Weight, $\mathbf{g}$.
378.57
Moisture Content, \%
34.9
Wet Unit Weight, pct.
115.4
Dry Unit Weight, pcf.
85.6
Rate of Strain, in./min.
0.0360

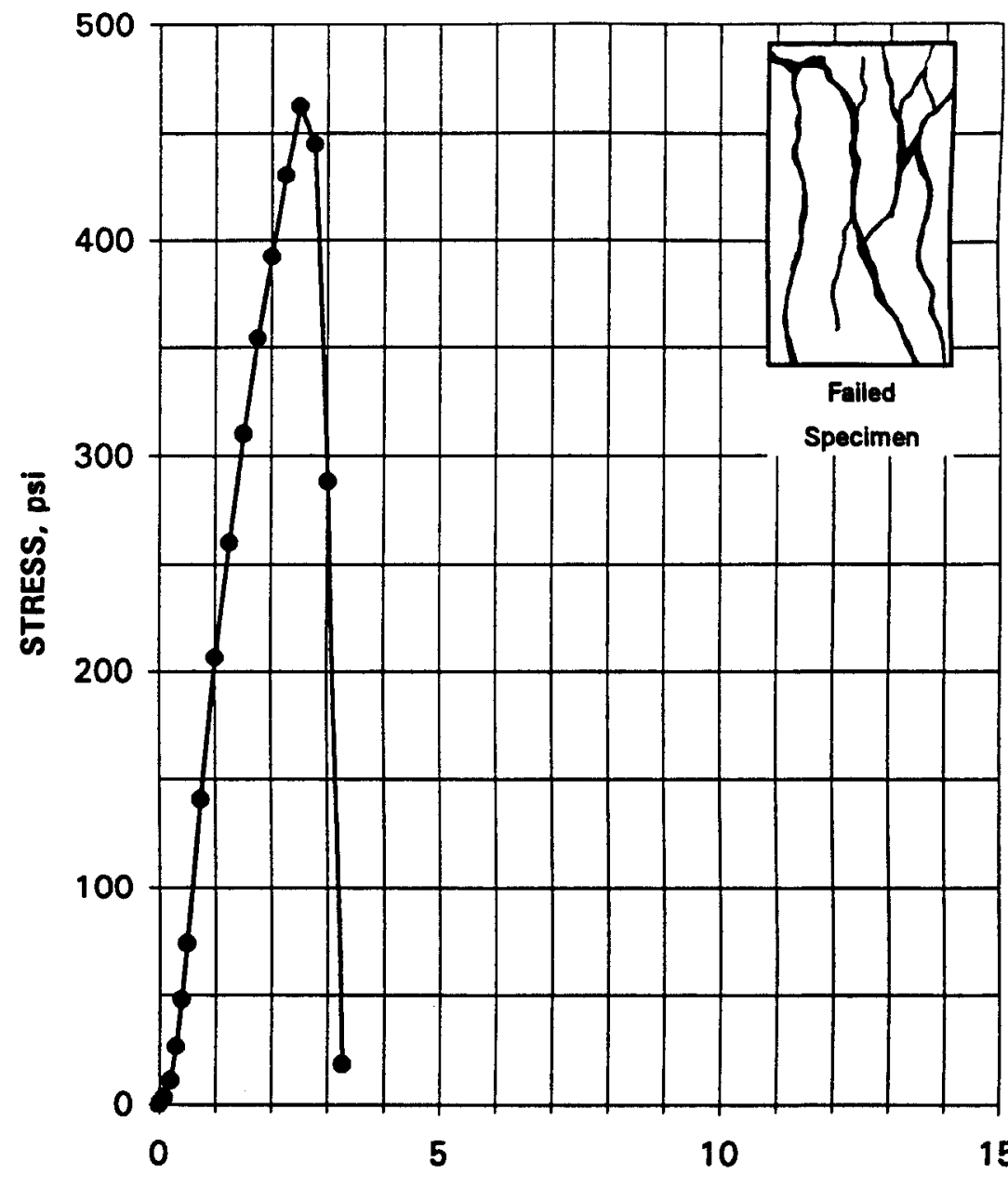

STRAIN, \% 


\section{UNCONFINED COMPRESSIVE STRENGTH - ASTM D 2166 28 DAY CURE, 90 DAY IMMERSION}

Project Name SAIC / SAVANNAH RIVER

Project No. $\quad 777304.00010500$

Specimen Mold Date

Specimen Test Date

STRESS AT FAILURE, psi

STRAIN AT FAILURE, \%

AXIAL DEVIATOR

$\begin{array}{ccc}\text { STRAIN, } \% & \text { STRESS, psi } \\ 0.00 & 0.0 \\ 0.10 & & 2.5 \\ 0.20 & & 4.4 \\ 0.30 & & 10.8 \\ 0.40 & & 17.4 \\ 0.50 & & 23.0 \\ 0.75 & & 48.8 \\ 1.00 & & 91.7 \\ 1.25 & & 148.5 \\ 1.50 & & 202.8 \\ 1.75 & & 251.0 \\ 2.00 & & 294.5 \\ 2.25 & & 343.0 \\ 2.50 & & 385.8 \\ 2.75 & & 426.1 \\ 3.00 & & 461.1 \\ 3.25 & & 448.8 \\ 3.50 & & 474.9 \\ 3.75 & 474.6 \\ 3.87 & 478.0 \\ 4.00 & 437.7 \\ 4.25 & 435.4 \\ 4.50 & 428.5 \\ 4.75 & 401.7 \\ 5.00 & & 379.8 \\ 5.25 & & 353.3\end{array}$

$11 / 11 / 98$

$3 / 9 / 99$

478.0

3.9
Client Sample No. C343F00

IT Lab Specimen No. ETDC-7840

Specimen Height, in.

3.9998

Specimen Diameter, in.

2.0032

Specimen Weight, $\boldsymbol{g}$.

384.10

Moisture Content, \%

33.1

Wet Unit Weight, pcf.

115.7

Dry Unit Weight, pcf.

86.9

Rate of Strain, in./min.

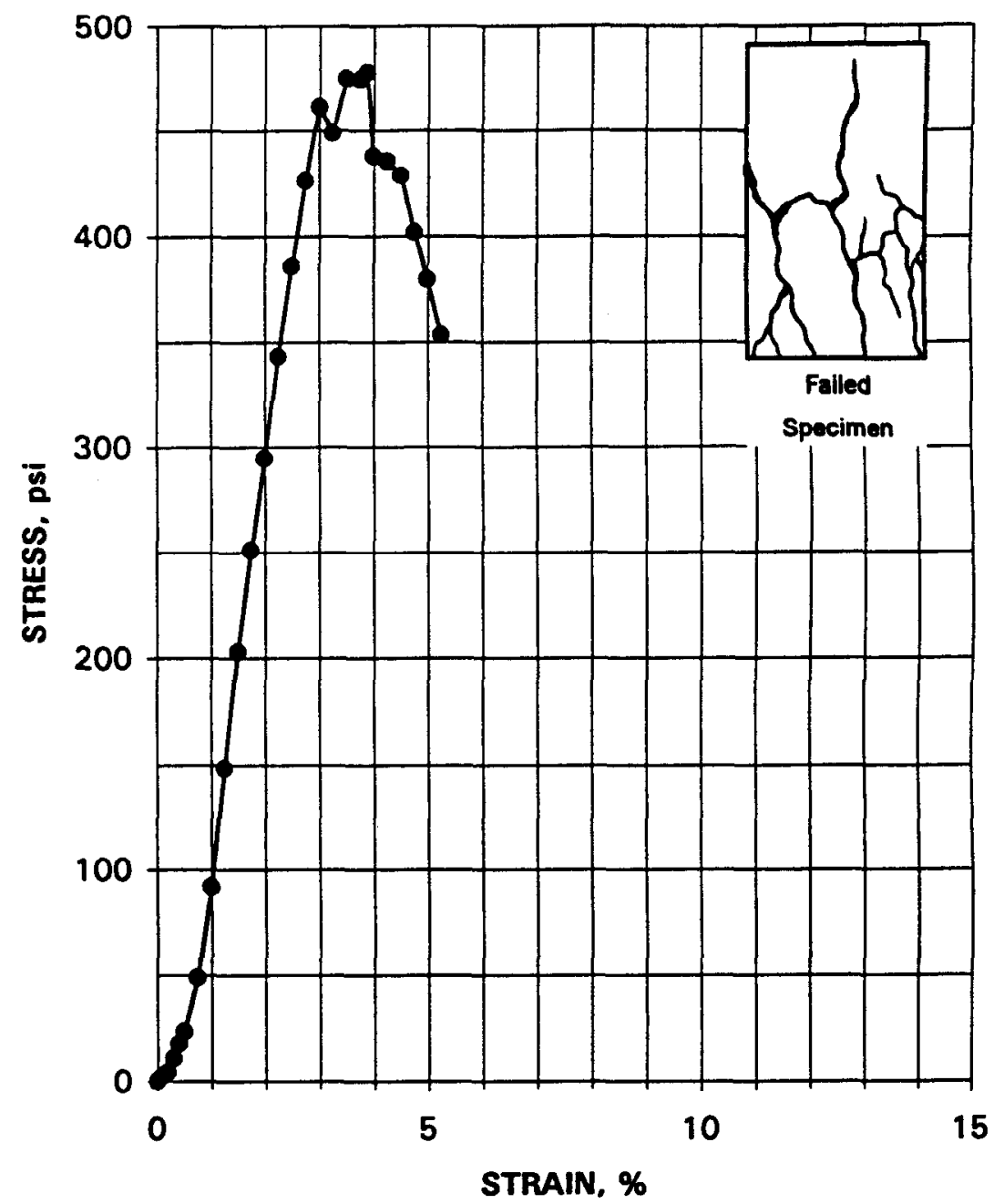




\section{UNCONFINED COMPRESSIVE STRENGTH - ASTM D 2166 28 DAY CURE, 90 DAY IMMERSION}

\begin{tabular}{lc} 
Project Name & SAIC / SAVAN \\
Project No. & 777304.00010 \\
Specimen Mold Date \\
Specimen Test Date \\
STRESS AT FAILURE, psi \\
STRAIN AT FAILURE, \% \\
\multicolumn{3}{|}{} \\
AXIAL & DEVIATOR \\
STRAIN, \% & STRESS, psi \\
\hline 0.00 & 0.0 \\
0.10 & 11.7 \\
0.20 & 26.3 \\
0.30 & 43.7 \\
0.40 & 62.7 \\
0.51 & 83.8 \\
0.76 & 141.9 \\
1.01 & 214.2 \\
1.27 & 274.2 \\
1.52 & 316.7 \\
1.77 & 348.0 \\
2.02 & 372.4 \\
2.28 & 354.3 \\
2.53 & 324.6 \\
2.78 & 31.8
\end{tabular}

Client Sample No. C413FOO

IT Lab Specimen No. ETDC-7841

Specimen Height, in.

3.9530

Specimen Diameter, in.

2.0017

Specimen Weight, $g$.

382.53

Moisture Content, \%

33.7

Wet Unit Weight, pcf.

117.0

Dry Unit Weight, pcf.

87.5

Rate of Strain, in./min.

0.0360

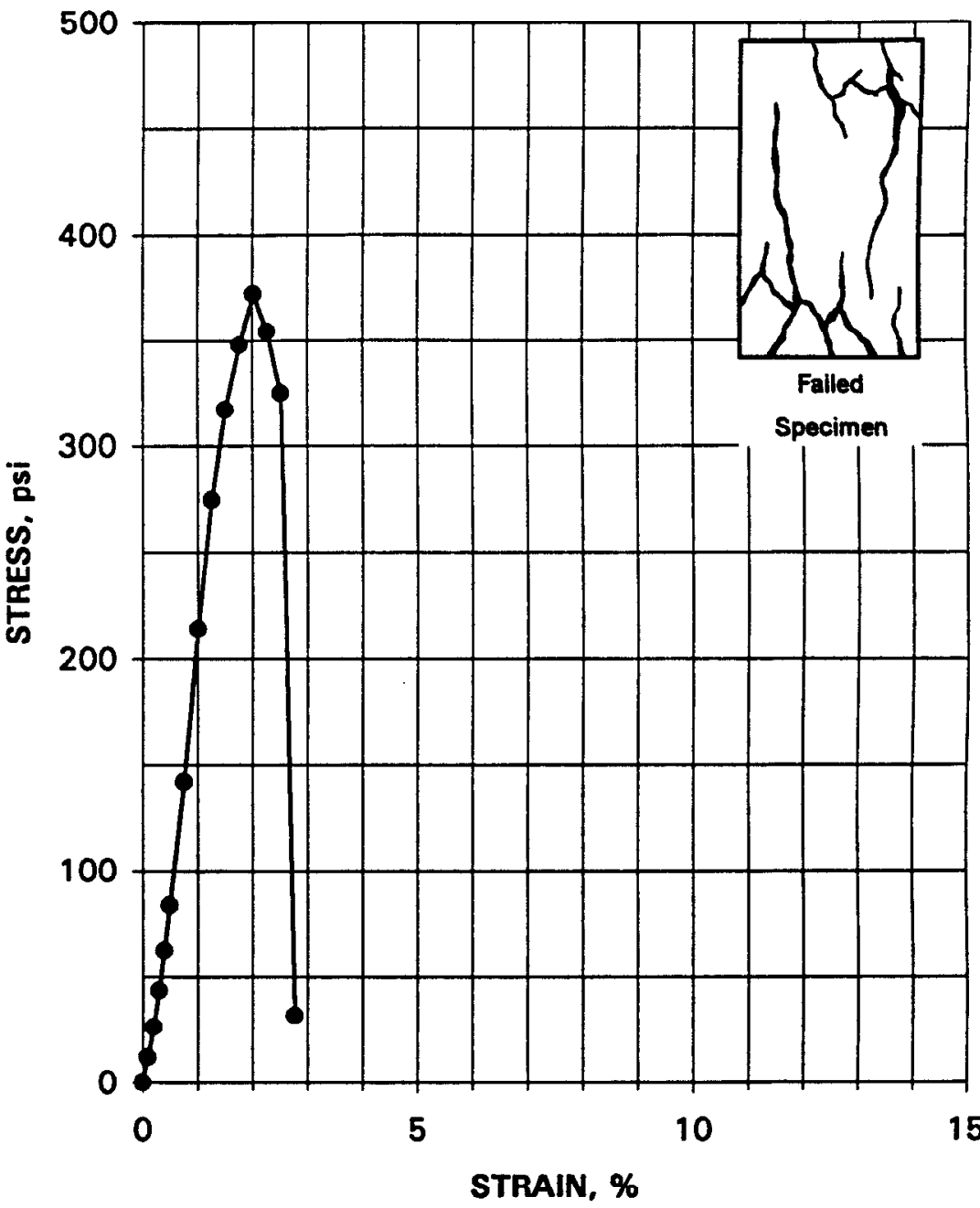




\section{UNCONFINED COMPRESSIVE STRENGTH - ASTM D 2166 28 DAY CURE, 90 DAY IMMERSION}

\begin{tabular}{lc} 
Project Name & SAIC / SAVA \\
Project No. & 777304.00010 \\
Specimen Mold Date \\
Specimen Test Date \\
STRESS AT FAILURE, psi \\
STRAIN AT FAILURE, \% \\
\multicolumn{3}{c}{} \\
AXIAL & DEVIATOR \\
STRAIN, \% & STRESS, psi \\
\cline { 2 - 2 } 0.00 & 0.0 \\
0.10 & 2.8 \\
0.20 & 13.0 \\
0.30 & 34.7 \\
0.40 & 58.0 \\
0.50 & 87.9 \\
0.75 & 151.4 \\
1.00 & 182.4 \\
1.25 & 212.6 \\
1.51 & 236.7 \\
1.76 & 205.0 \\
2.01 & 229.2 \\
2.26 & 244.4 \\
2.51 & 234.6 \\
2.76 & 71.1 \\
\end{tabular}

Client Sample No. C423F0O

IT Lab Specimen No. ETDC-7842

Specimen Height, in.

3.9880

Specimen Diameter, in.

2.0055

Specimen Weight, $g$.

383.24

Moisture Content, \%

33.5

Wet Unit Weight, pef.

Dry Unit Weight, pcf.

115.1

86.2

Rate of Strain, in./min.

0.0360






\section{UNCONFINED COMPRESSIVE STRENGTH - ASTM D 2166 28 DAY CURE, 90 DAY IMMERSION}

\begin{tabular}{|c|c|}
\hline Project Name & SAIC / SAV \\
\hline Project No. & 777304.00010 \\
\hline Specimen Mo & \\
\hline STRESS AT F & LURE, psi \\
\hline STRAIN AT F & URE, \% \\
\hline AXIAL & DEVIATOR \\
\hline STRAIN, \% & STRESS, psi \\
\hline 0.00 & 0.0 \\
\hline 0.10 & 3.8 \\
\hline 0.20 & 5.7 \\
\hline 0.30 & 15.8 \\
\hline 0.40 & 29.7 \\
\hline 0.50 & 44.3 \\
\hline 0.75 & 96.5 \\
\hline 1.01 & 156.0 \\
\hline 1.26 & 197.0 \\
\hline 1.51 & 233.7 \\
\hline 1.76 & 277.8 \\
\hline 2.01 & 306.6 \\
\hline 2.26 & 327.9 \\
\hline 2.51 & 344.4 \\
\hline 2.77 & 352.4 \\
\hline 2.89 & 355.1 \\
\hline 3.02 & 347.5 \\
\hline 3.27 & 250.8 \\
\hline 3.52 & 212.1 \\
\hline 3.77 & 54.1 \\
\hline
\end{tabular}

Client Sample No. C433F00

IT Lab Specimen No. ETDC-7843

Specimen Height, in.

Specimen Diameter, in.

3.9818

$3 / 9 / 99$

355.1

Specimen Weight, $\mathbf{o}$.

2.0020

379.62

Moisture Content, \%

41.6

Wet Unit Weight, pcf.

115.0

Dry Unit Weight, pcf.

81.2

Rate of Strain, in./min.

0.0360

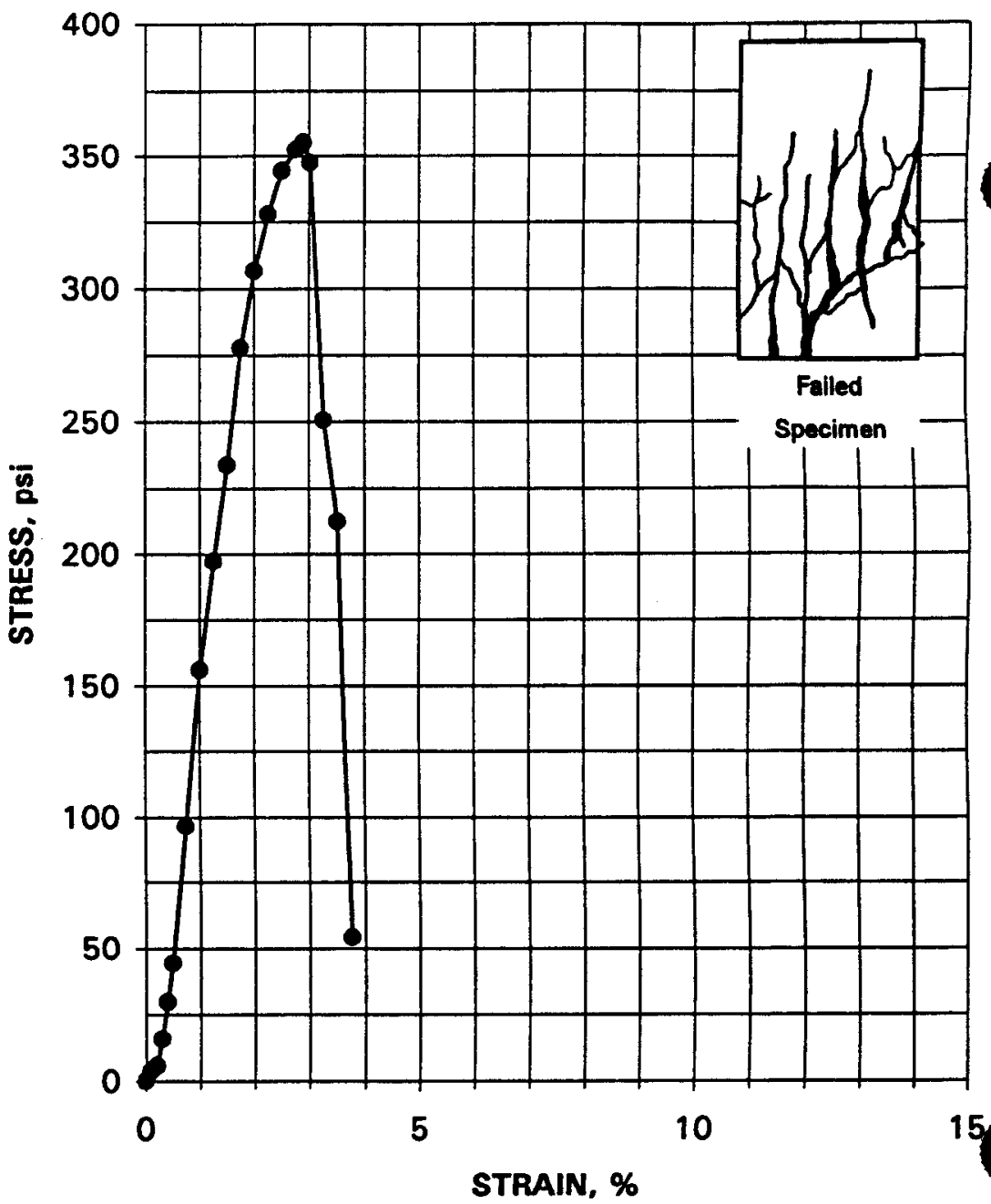




\section{UNCONFINED COMPRESSIVE STRENGTH - ASTM D 2166 28 DAY CURE, 90 DAY IMMERSION}

Project Name SAIC / SAVANNAH RIVER

Project No. $\quad 777304.00010500$

Specimen Mold Date

Specimen Test Date

STRESS AT FAILURE, psi

STRAIN AT FAILURE, \%

AXIAL

DEVIATOR

STRAIN, \%

0.00

STRESS, psi

0.10

2.5

0.20

6.3

0.30

11.3

0.40

0.50

0.76

20.0

34.4

1.01

81.5

1.26

1.51

142.6

199.3

1.76

255.8

2.02

2.27

342.0

2.52

2.77

3.02

3.27

3.53

3.78

4.03
$11 / 11 / 98$

$3 / 9 / 99$

460.8

3.0
Client Sample No. C443F00

IT Lab Specimen No. ETDC-7844

Specimen Height, in.

3.9682

Specimen Diameter, in.

2.0122

Specimen Weight, $g$.

367.07

Moisture Content, \%

39.7

Wet Unit Weight, pef.

Dry Unit Weight, pcf.

110.8

Rate of Strain, in./min.

79.3

0.0360

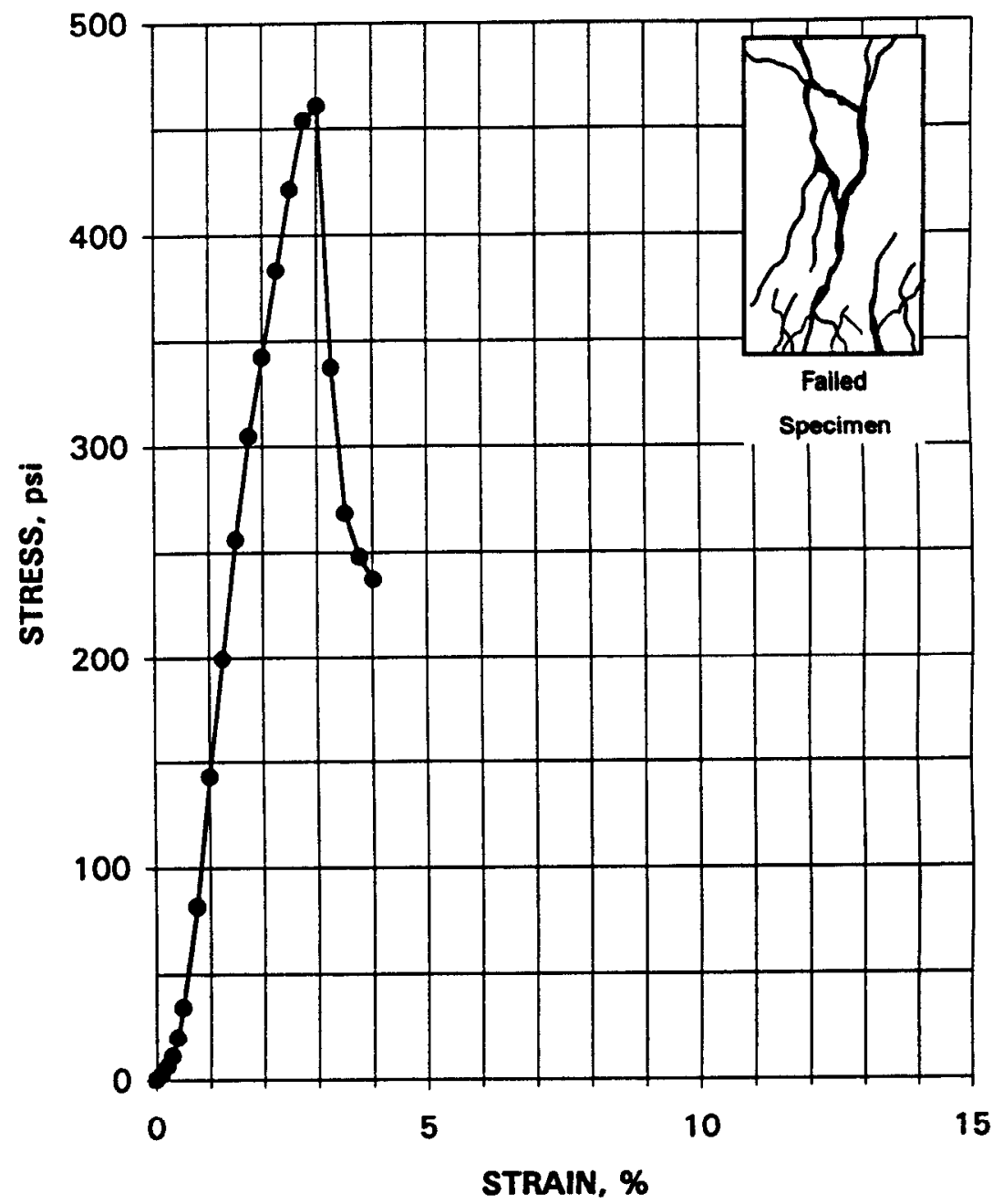




\section{UNCONFINED COMPRESSIVE STRENGTH - ASTM D 2166 28 DAY CURE, 90 DAY IMMERSION}

Project Name SAIC / SAVANNAH RIVER

Project No. $\quad 777304.00010500$

Specimen Mold Date

Specimen Test Date

STRESS AT FAILURE, psi

STRAIN AT FAILURE, \%

AXIAL DEVIATOR

$\frac{\text { STRAIN, \% }}{0.00} \frac{\text { STRESS, psi }}{0.0}$

$0.10 \quad 3.8$

$0.20 \quad 4.8$

$0.30 \quad 9.8$

$0.40 \quad 17.4$

$0.50 \quad 27.9$

$0.76 \quad 64.1$

$1.01 \quad 113.0$

$1.26 \quad 158.3$

$1.51 \quad 198.3$

$1.76 \quad 233.4$

$2.02 \quad 269.3$

$2.27 \quad 303.1$

$2.52 \quad 328.7$

$2.77 \quad 339.3$

$3.03 \quad 342.1$

$3.15 \quad 342.3$

$3.28 \quad 312.3$

$3.53 \quad 54.0$
$11 / 11 / 98$

$3 / 9 / 99$

342.3

3.2
Client Sample No. C513F00

IT Lab Specimen No. ETDC-7845

Specimen Height, in.

3.9667

Specimen Diameter, in.

2.0007

Specimen Weight, $\mathrm{g}$.

388.49

Moisture Content, \%

31.8

Wet Unit Weight, pcf.

117.9

Dry Unit Weight, pcf.

89.4

Rate of Strain, in./min.

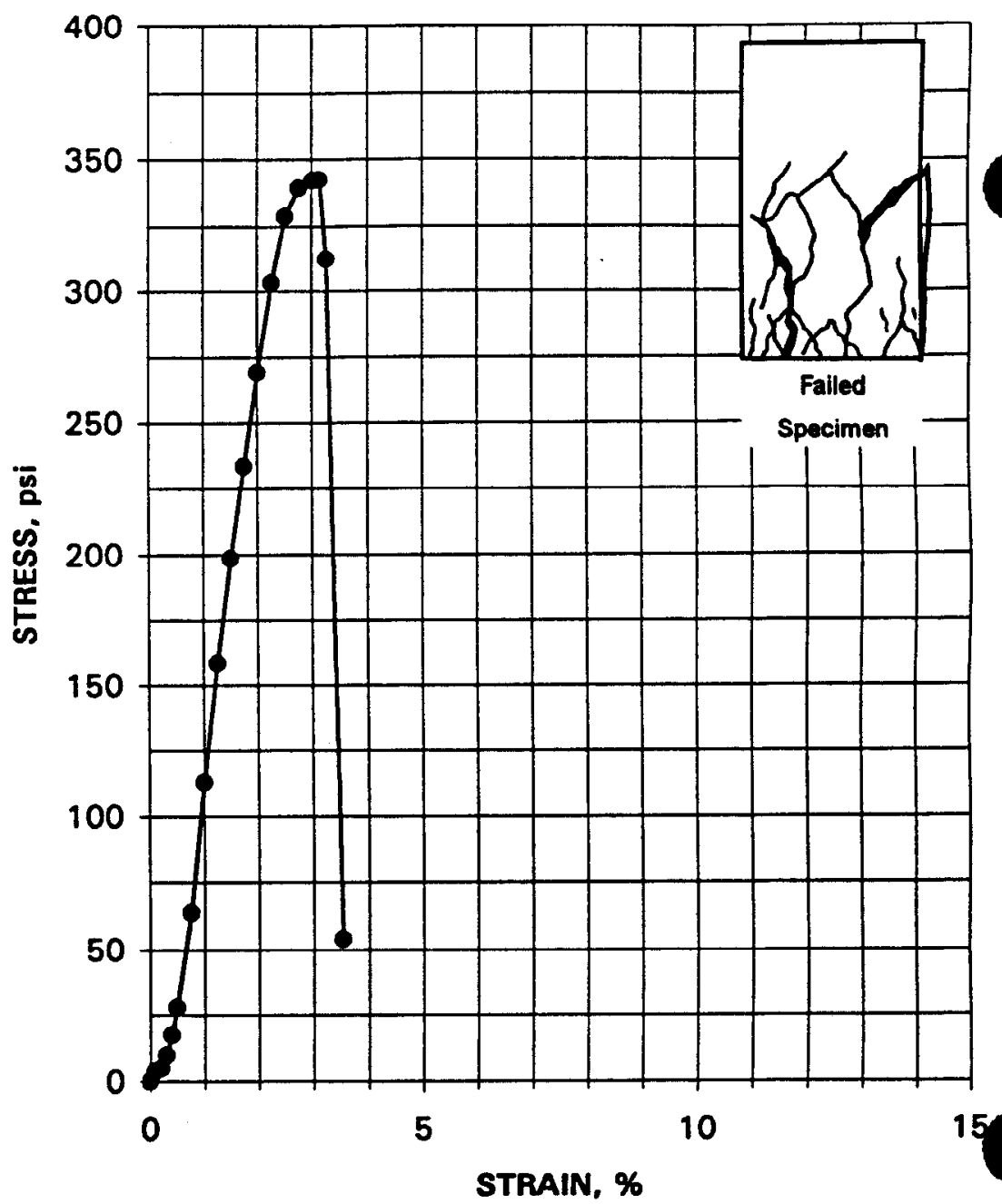




\section{UNCONFINED COMPRESSIVE STRENGTH - ASTM D 2166 28 DAY CURE, 90 DAY IMMERSION}

Project Name SAIC / SAVANNAH RIVER

Project No. $\quad 777304.00010500$

Specimen Mold Date

Specimen Test Date

STRESS AT FAILURE, psi

STRAIN AT FAILURE, \%

AXIAL DEVIATOR

$\frac{\text { STRAIN, \% }}{0.00} \frac{\text { STRESS, psi }}{0.0}$

$0.10 \quad 2.9$

$0.20 \quad 10.1$

0.30

0.40

0.50

0.76

1.01

1.26

1.51

1.76

2.01

2.27

2.52

2.77

3.02

3.27

3.52

3.65

3.78

4.03

4.28

4.53
$11 / 11 / 98$

$3 / 9 / 99$

481.8

3.6
22.5

36.7

49.6

93.9

127.3

174.0

222.3

267.9

314.5

353.7

387.1

421.0

450.1

468.9

479.9

481.8

464.0

433.9

338.8

142.8
Client Sample No. C523F00

IT Lab Specimen No. ETDC-7846

Specimen Height, in.

3.9750

Specimen Diameter, in.

2.0027

Specimen Weight, $g$.

391.78

Moisture Content, \%

28.0

Wet Unit Weight, pct.

118.4

Dry Unit Weight, pcf.

92.5

Rate of Strain, in./min.

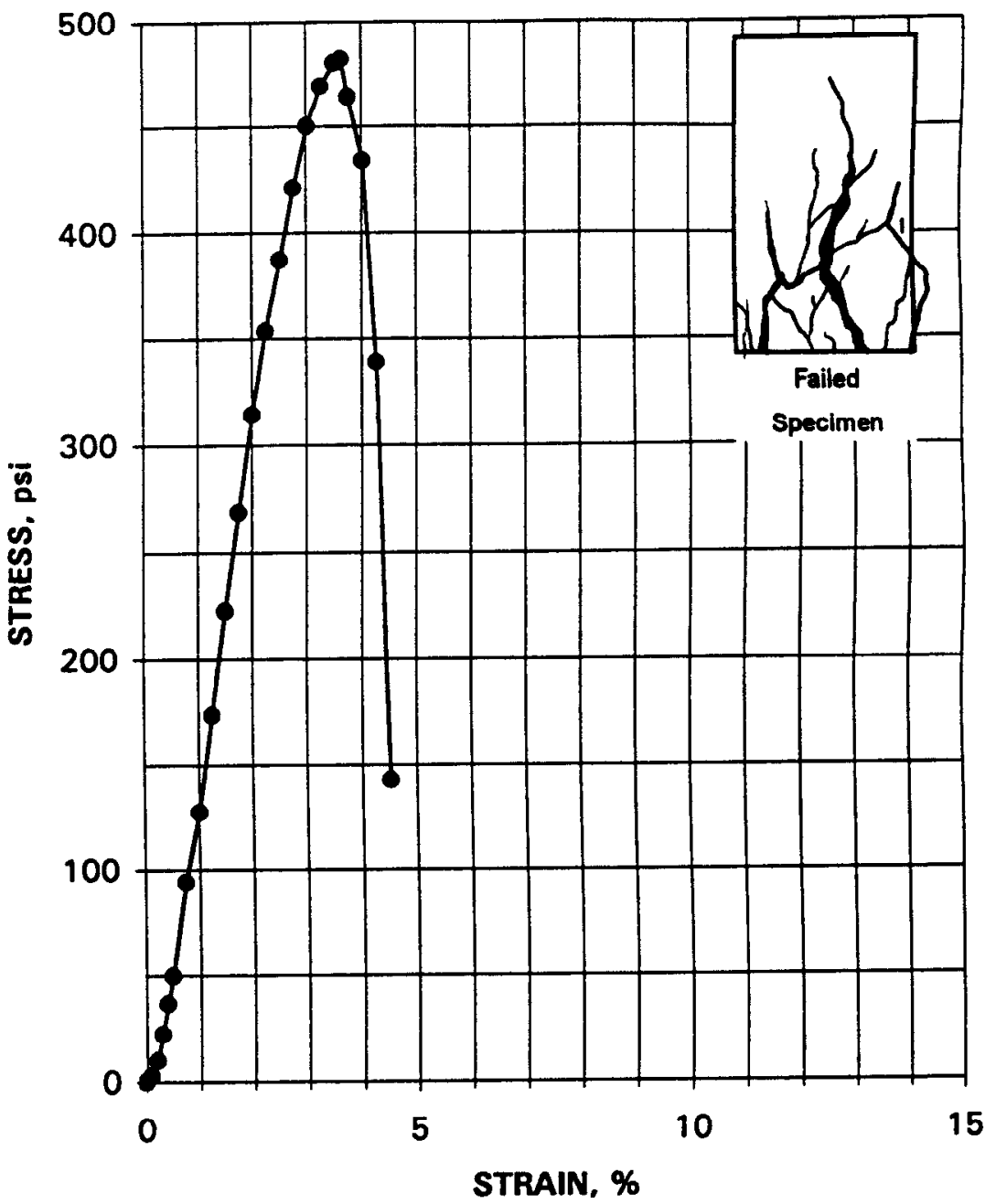




\section{UNCONFINED COMPRESSIVE STRENGTH - ASTM D 2166 28 DAY CURE, 90 DAY IMMERSION}

\begin{tabular}{lc} 
Project Name & SAIC / SAVAN \\
Project No. & 777304.00010 \\
Specimen Mold Date \\
Specimen Test Date \\
STRESS AT FAILURE, psi \\
STRAIN AT FAILURE, \% \\
\multicolumn{3}{|}{} \\
AXIAL & DEVIATOR \\
STRAIN, \% & STRESS, psi \\
\hline 0.00 & 0.0 \\
0.10 & 5.7 \\
0.20 & 17.1 \\
0.30 & 31.4 \\
0.40 & 45.3 \\
0.50 & 61.7 \\
0.75 & 114.5 \\
1.00 & 165.8 \\
1.25 & 223.2 \\
1.50 & 283.6 \\
1.75 & 339.5 \\
2.00 & 377.5 \\
2.25 & 410.1 \\
2.49 & 439.1 \\
2.74 & 464.0 \\
2.99 & 464.0 \\
3.12 & 472.4 \\
3.24 & 188.2 \\
&
\end{tabular}

$11 / 11 / 98$

$3 / 9 / 99$

472.4

3.1
Client Sample No. C533F00

IT Lab Specimen No. ETDC-7847

Specimen Height, in.

4.0135

Specimen Diameter, in.

2.0015

Specimen Weight, $g$.

375.26

Moisture Content, \%

37.3

Wet Unit Weight, pcf.

Dry Unit Weight, pef.

82.2

Rate of Strain, in./min.

0.0360

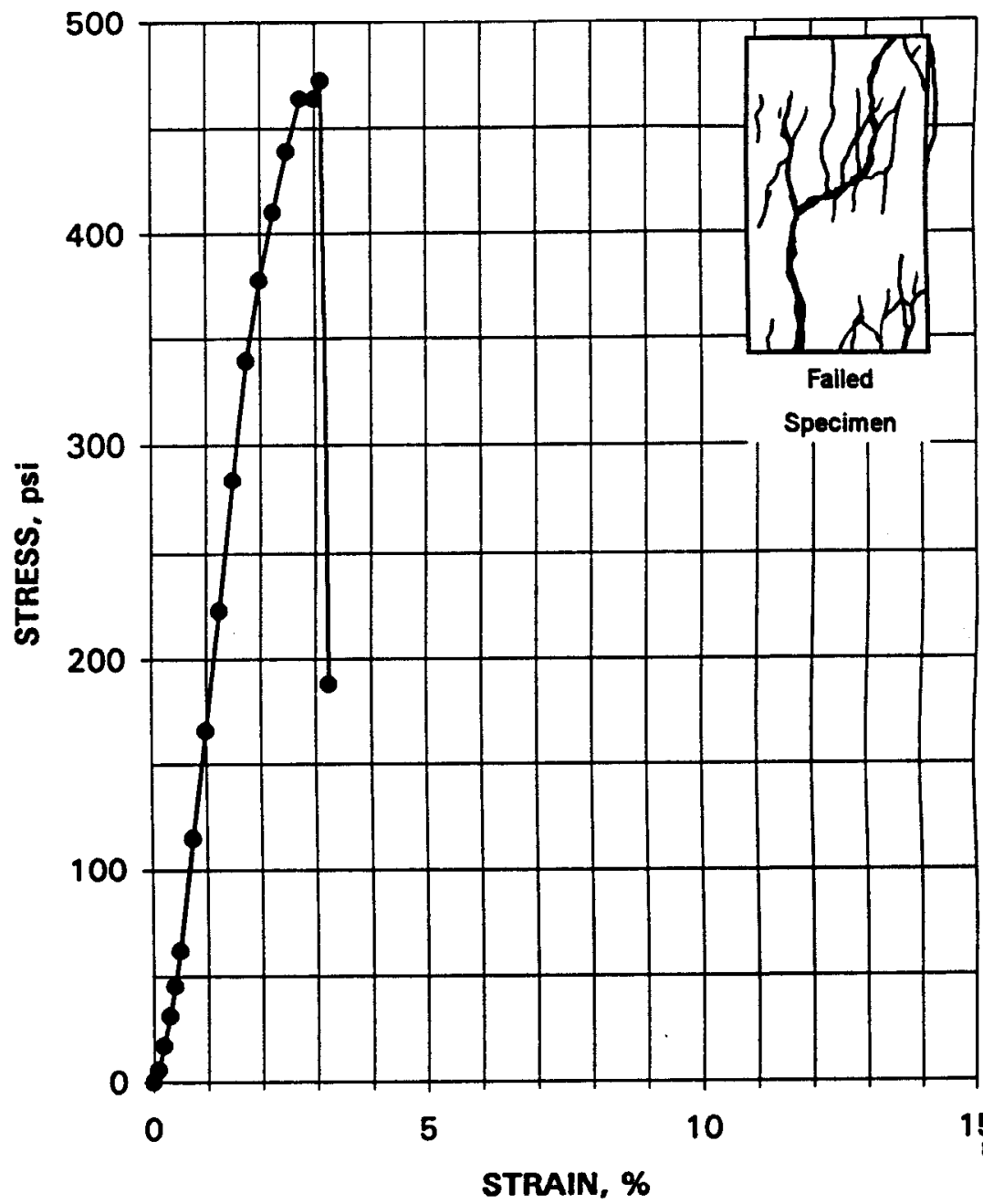




\section{UNCONFINED COMPRESSIVE STRENGTH - ASTM D 2166 28 DAY CURE, 90 DAY IMMERSION}

Project Name SAIC / SAVANNAH RIVER

Project No. 777304.00010500

\begin{tabular}{|c|c|}
\hline \multicolumn{2}{|c|}{$\begin{array}{l}\text { Specimen Mold Date } \\
\text { Specimen Test Date }\end{array}$} \\
\hline \multicolumn{2}{|c|}{ STRESS AT FAILURE, psi } \\
\hline AXIAL & DEVIATOR \\
\hline STRAIN, \% & STRESS, psi \\
\hline 0.00 & 0.0 \\
\hline 0.10 & 3.8 \\
\hline 0.20 & 18.6 \\
\hline 0.30 & 47.6 \\
\hline 0.40 & 77.8 \\
\hline 0.50 & 137.1 \\
\hline 0.75 & 193.3 \\
\hline 1.00 & 267.3 \\
\hline 1.25 & 341.2 \\
\hline 1.50 & 428.8 \\
\hline 1.75 & 506.6 \\
\hline 2.00 & 570.1 \\
\hline 2.13 & 590.1 \\
\hline 2.25 & 562.7 \\
\hline 2.50 & 115.6 \\
\hline
\end{tabular}

Client Sample No. C543F00

IT Lab Specimen No. ETDC-7848

$11 / 11 / 98$

$3 / 9 / 99$

590.1

2.1
Specimen Height, in.

Specimen Diameter, in.

Specimen Weight, $g$.

Moisture Content, \%

Wet Unit Weight, pef.

Dry Unit Weight, pcf.

Rate of Strain, in./min.
3.9968

2.0068

379.95

34.2

113.4

84.5

0.0360

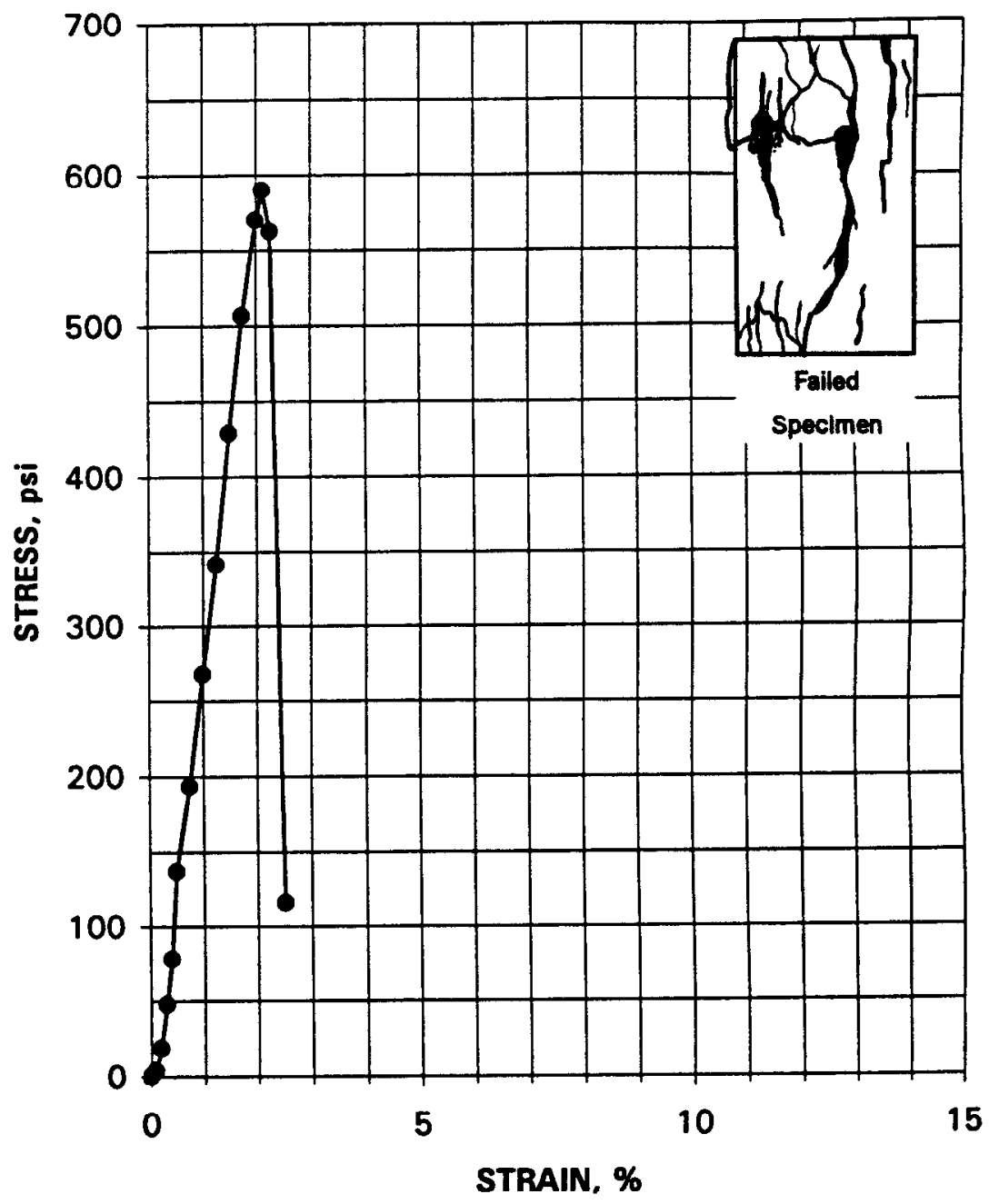




\section{UNCONFINED COMPRESSIVE STRENGTH - ASTM D 2166 28 DAY CURE, 90 DAY IMMERSION}

Project Name SAIC / SAVANNAH RIVER

Project No. $\quad 777304.00010500$

Specimen Mold Date

Specimen Test Date

STRESS AT FAILURE, psi

STRAIN AT FAILURE, \%

\begin{tabular}{ccc}
$\begin{array}{ccc}\text { AXIAL } \\
\text { STRAIN, \% }\end{array}$ & & $\begin{array}{c}\text { DEVIATOR } \\
\text { STRESS, psi }\end{array}$ \\
\cline { 1 - 1 } 0.00 & & 0.0 \\
0.10 & & 7.3 \\
0.20 & & 14.6 \\
0.30 & & 25.0 \\
0.40 & & 35.4 \\
0.50 & & 50.2 \\
0.75 & & 91.0 \\
1.00 & & 136.1 \\
1.25 & & 170.8 \\
1.50 & & 199.8 \\
1.75 & & 229.2 \\
2.00 & & 258.8 \\
2.26 & & 277.1 \\
2.51 & & 287.2 \\
2.76 & 287.1 \\
3.01 & 288.5 \\
3.13 & 289.6 \\
3.26 & 254.3 \\
3.51 & 42.0
\end{tabular}

Client Sample No. C613F00

IT Lab Specimen No. ETDC-7849

Specimen Height, in.

3.9903

Specimen Diameter, in.

2.0028

Specimen Weight, $\mathrm{g}$.

391.23

Moisture Content, \%

31.1

Wet Unit Weight, pcf.

118.4

Dry Unit Weight, pcf.

90.3

Rate of Strain, in./min.

0.0360

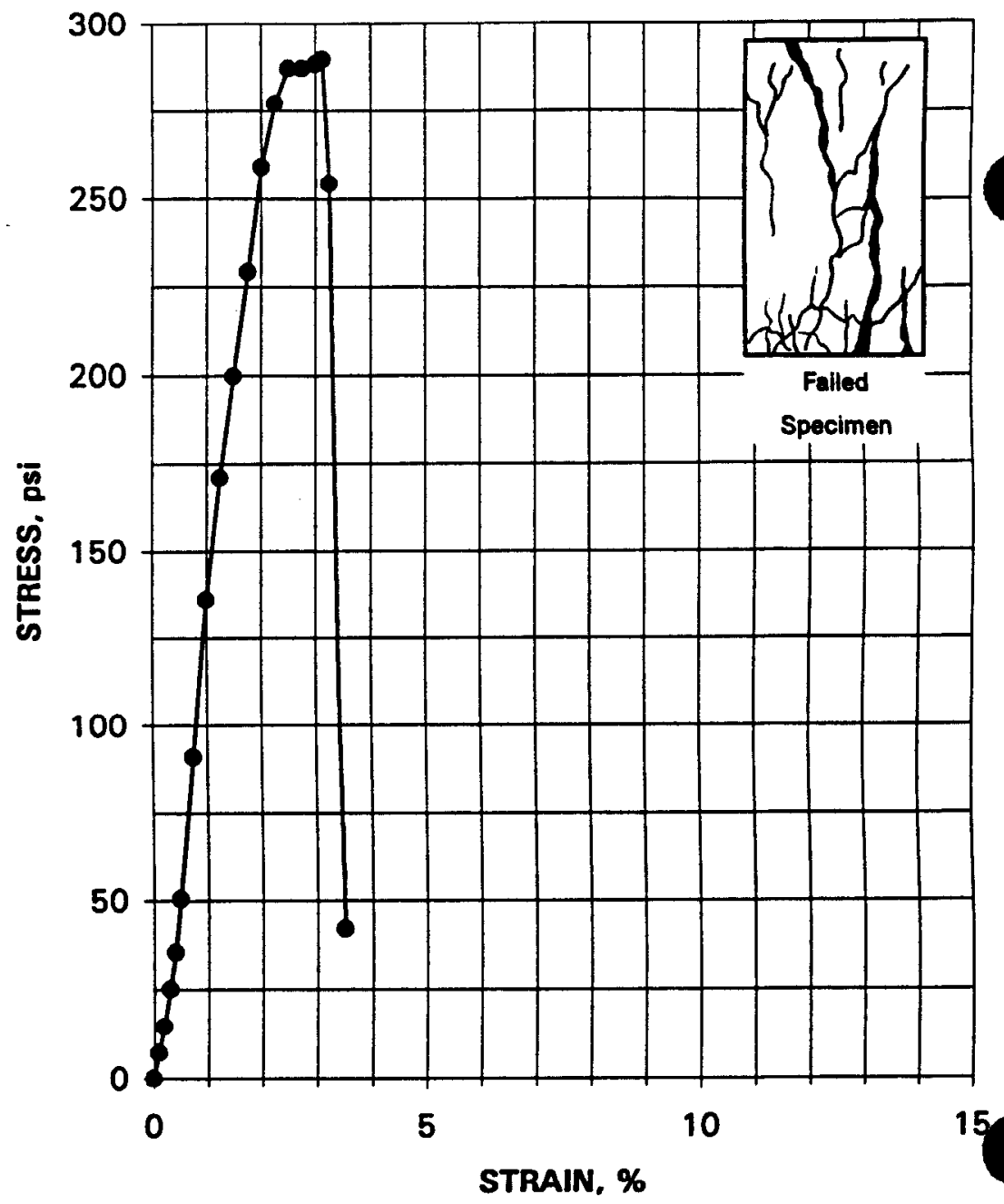




\section{UNCONFINED COMPRESSIVE STRENGTH - ASTM D 2166 28 DAY CURE, 90 DAY IMMERSION}

Project Name SAIC / SAVANNAH RIVER

Project No. $\quad 777304.00010500$

Specimen Mold Date

Specimen Test Date

STRESS AT FAILURE, psi

STRAIN AT FAILURE, $\%$

\begin{tabular}{ccc}
$\begin{array}{ccc}\text { AXIAL } \\
\text { STRAIN, \% }\end{array}$ & & $\begin{array}{c}\text { DEVIATOR } \\
\text { STRESS, psi }\end{array}$ \\
\cline { 1 - 1 } 0.00 & & 0.0 \\
0.10 & & 6.0 \\
0.20 & & 12.6 \\
0.30 & & 20.5 \\
0.40 & & 30.3 \\
0.50 & & 41.6 \\
0.75 & & 94.9 \\
1.00 & & 153.2 \\
1.25 & & 210.6 \\
1.50 & & 270.6 \\
1.75 & & 329.9 \\
2.00 & & 382.1 \\
2.25 & & 423.2 \\
2.50 & & 456.0 \\
2.75 & & 480.1 \\
3.00 & 491.4 \\
3.25 & 488.0 \\
3.51 & 496.8 \\
3.76 & 501.6 \\
4.01 & 509.4 \\
4.26 & 516.6 \\
4.51 & 526.7 \\
4.76 & 531.7 \\
5.01 & 514.1 \\
5.26 & & 1.5
\end{tabular}

$11 / 11 / 98$

$3 / 9 / 99$

531.7

4.8

Client Sample No. C623F00

IT Lab Specimen No. ETDC-7850

Specimen Height, in.

3.9942

Specimen Diameter, in.

2.0058

Specimen Weight, $g$.

394.99

Moisture Content, \%

28.8

Wet Unit Weight, pcf.

119.0

Dry Unit Weight, pcf.

92.4

Rate of Strain, in./min.

0.0360

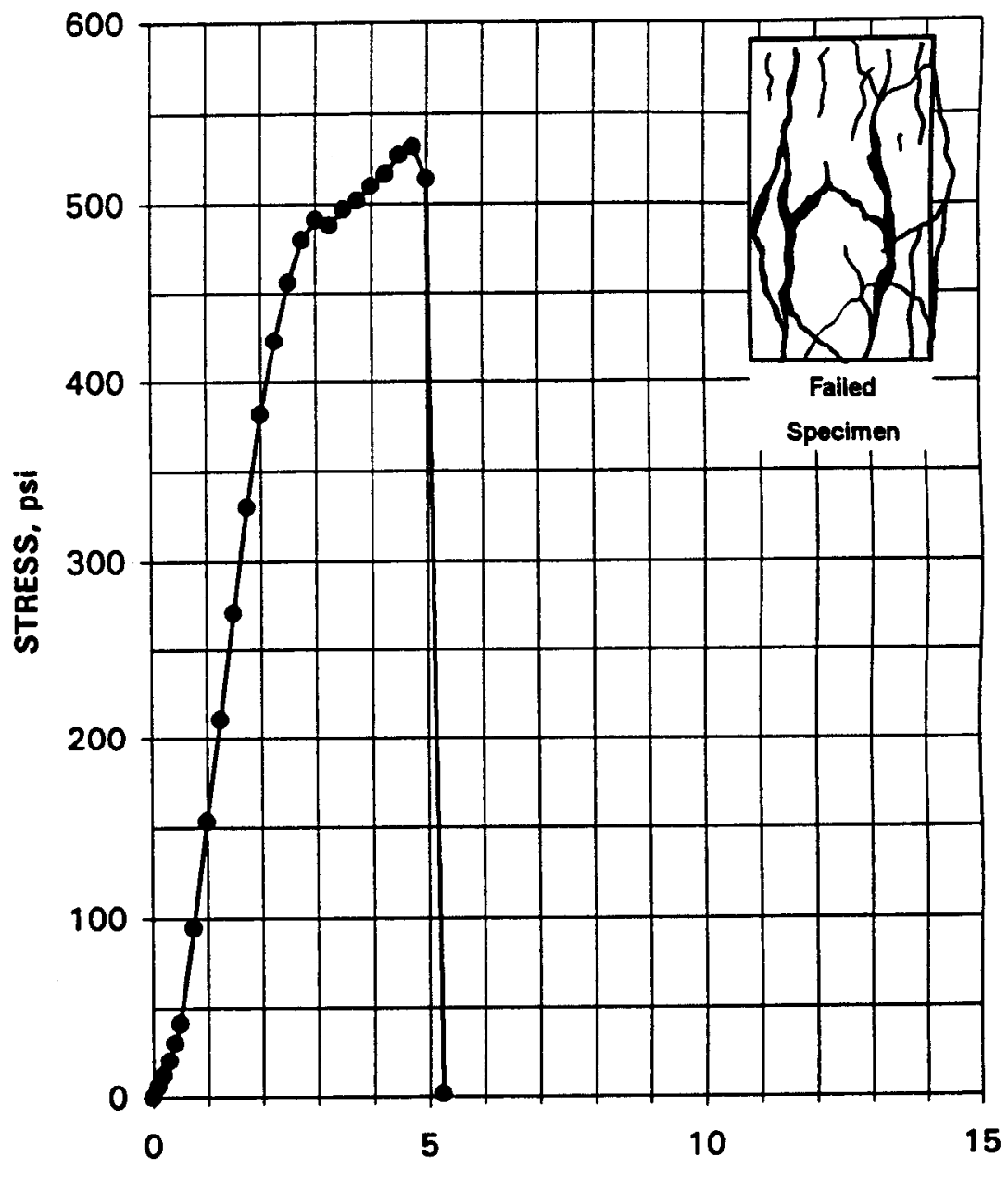

STRAIN, \% 


\section{UNCONFINED COMPRESSIVE STRENGTH - ASTM D 2166 28 DAY CURE, 90 DAY IMMERSION}

Project Name SAIC / SAVANNAH RIVER

Project No. $\quad 777304.00010500$

Specimen Mold Date

Specimen Test Date

STRESS AT FAILURE, psi

STRAIN AT FAILURE, \%

\begin{tabular}{ccc}
$\begin{array}{c}\text { AXIAL } \\
\text { STRAIN, \% }\end{array}$ & & $\begin{array}{c}\text { DEVIATOR } \\
\text { STRESS, psi }\end{array}$ \\
\cline { 1 - 1 } 0.00 & & 0.0 \\
0.10 & & 8.3 \\
0.20 & & 24.8 \\
0.30 & & 44.8 \\
0.40 & & 64.1 \\
0.50 & & 84.3 \\
0.75 & & 140.4 \\
1.00 & & 203.4 \\
1.25 & & 255.4 \\
1.50 & & 300.6 \\
1.74 & 336.8 \\
1.99 & 362.4 \\
2.24 & 364.0 \\
2.49 & 365.2 \\
2.74 & 55.8
\end{tabular}

$11 / 11 / 98$

$3 / 9 / 99$

365.2

2.5
Client Sample No. C633F00

IT Lab Specimen No. ETDC-7851

Specimen Height, in.

4.0198

Specimen Diameter, in.

1.9993

Specimen Weight, $\mathbf{g}$.

376.10

Moisture Content, \%

37.2

Wet Unit Weight, pcf.

113.0

Dry Unit Weight, pcf.

82.3

Rate of Strain, in./min.

0.0360

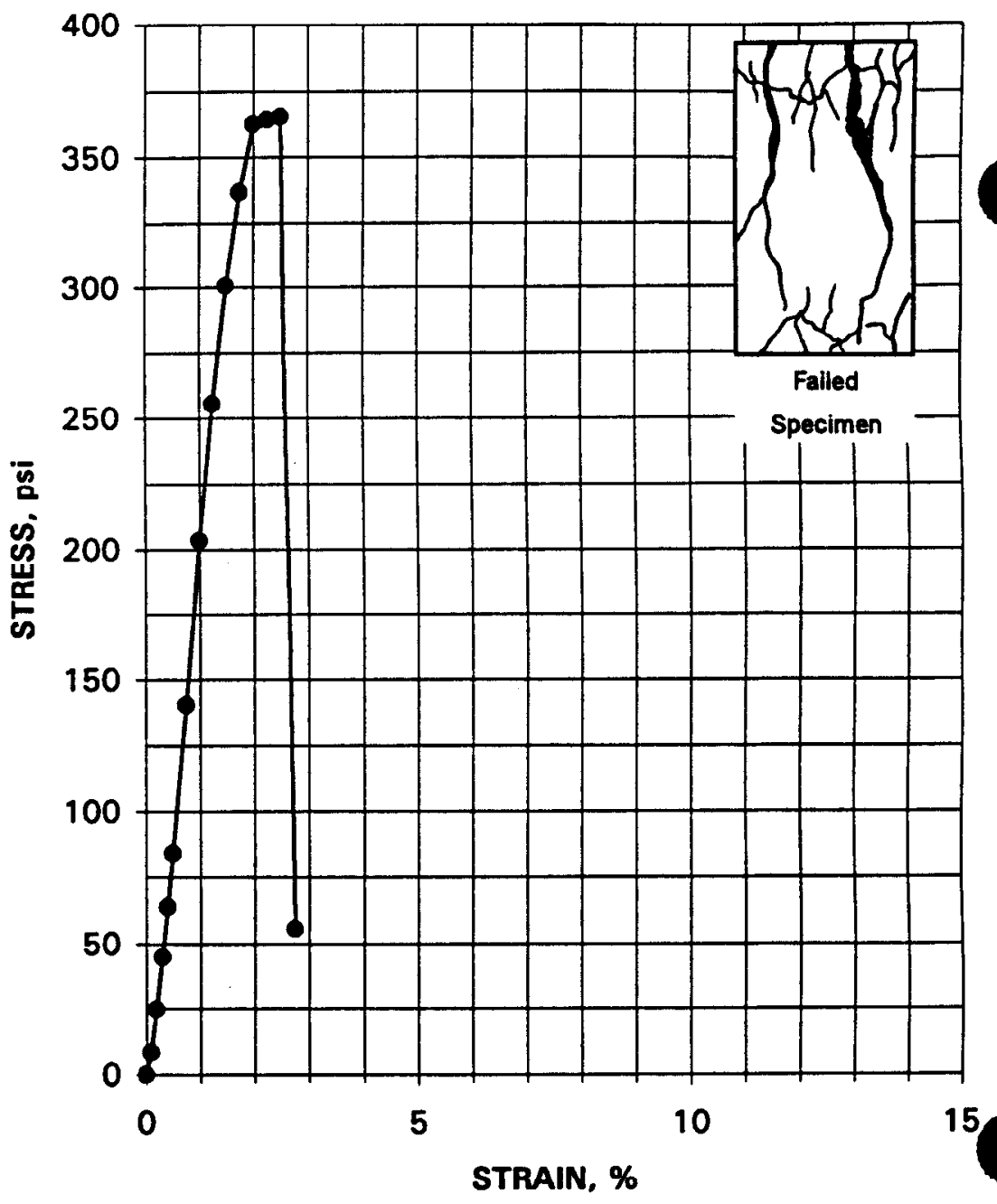




\section{UNCONFINED COMPRESSIVE STRENGTH - ASTM D 2166 28 DAY CURE, 90 DAY IMMERSION}

Project Name SAIC / SAVANNAH RIVER

Project No. $\quad 777304.00010500$

Specimen Mold Date

Specimen Test Date

STRESS AT FAILURE, psi

STRAIN AT FAILURE, \%

\begin{tabular}{ccc}
$\begin{array}{c}\text { AXIAL } \\
\text { STRAIN, } \%\end{array}$ & \multicolumn{1}{c}{$\begin{array}{c}\text { DEVIATOR } \\
\text { STRESS, psi }\end{array}$} \\
\cline { 1 - 1 } \cline { 3 - 3 } 0.00 & & 0.0 \\
0.10 & & 4.1 \\
0.20 & & 9.8 \\
0.30 & & 16.7 \\
0.40 & & 24.8 \\
0.50 & & 34.9 \\
0.75 & & 71.4 \\
1.00 & & 120.6 \\
1.25 & & 178.3 \\
1.50 & & 234.2 \\
1.75 & & 286.6 \\
2.00 & & 329.8 \\
2.26 & & 368.8 \\
2.51 & & 395.2 \\
2.76 & & 416.0 \\
3.01 & 436.4 \\
3.26 & 457.2 \\
3.51 & 465.5 \\
3.76 & 484.4 \\
4.01 & 505.5 \\
4.26 & 517.2 \\
4.31 & 517.2 \\
4.51 & 492.6 \\
4.76 & 445.3 \\
5.01 & 426.2 \\
5.26 & 417.9 \\
5.51 & & 382.1 \\
& &
\end{tabular}

$11 / 11 / 98$

$3 / 9 / 99$

517.2

4.3
Client Sample No. C634F00

IT Lab Specimen No. ETDC-7852

Specimen Height, in.

3.9902

Specimen Diameter, in.

2.0082

Specimen Weight, $\mathbf{g}$.

Moisture Content, \%

382.27

34.3

Wet Unit Weight, pcf.

116.1

Dry Unit Weight, pcf.

86.4

Rate of Strain, in./min.

0.0360

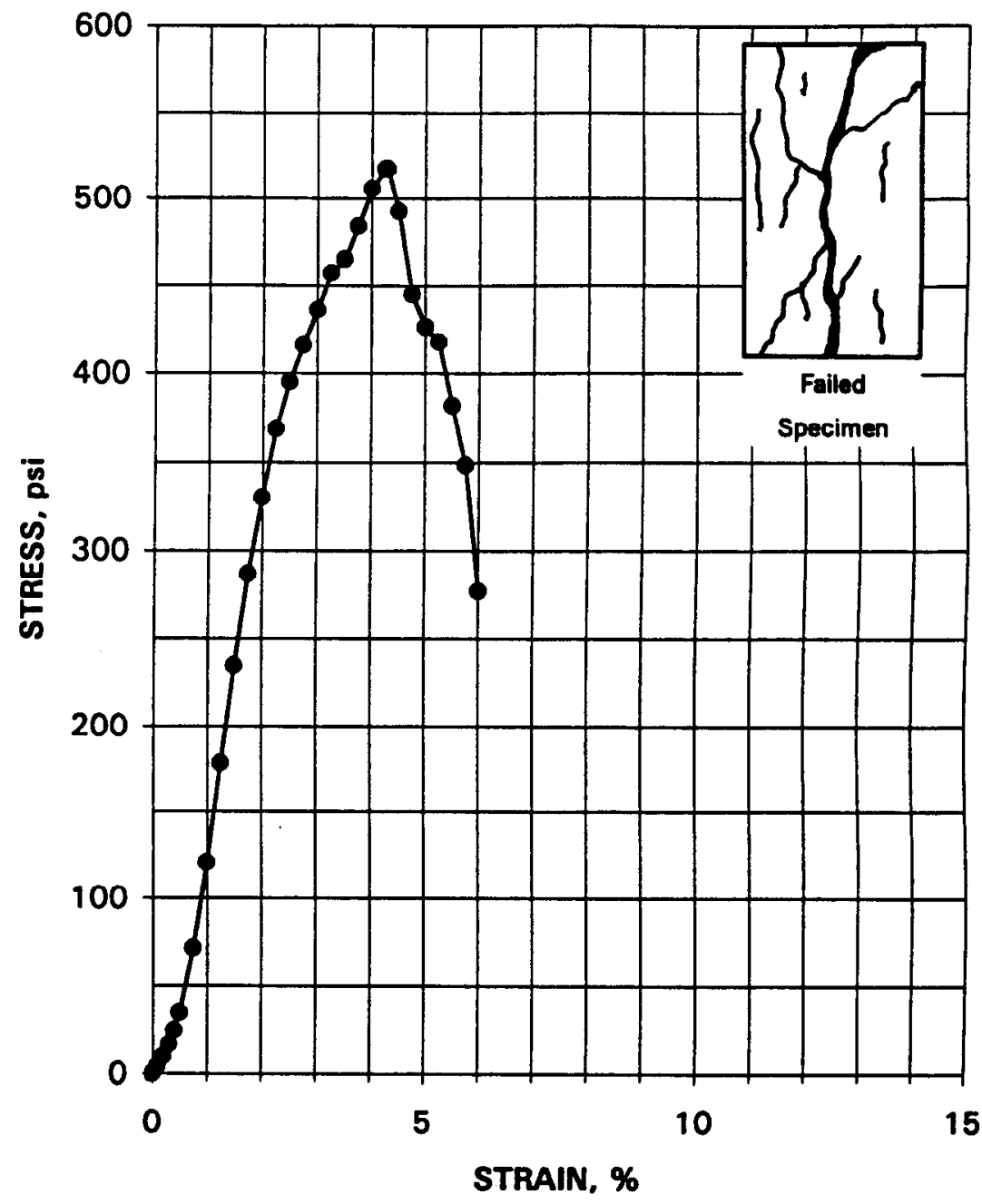




\section{Series}

28 Day Expansion/Shrinkage Results 


\section{One-Dimensional Expansion, Shrinkage, and Uplift Pressure of Soil-Lime Mixtures ASTM D 3877}

\section{8-Day Cure}

Geo Lab Knoxville Lab

\begin{tabular}{ccccc} 
Sample No. & Sample No. & Expansion, \% & Expansion Result & Shrinkage, \% \\
\hline \hline ETDC-7821 & C312K00 & $-0.049 \%$ & No expansion & -0.24 \\
ETDC-7822 & C322K00 & $-0.280 \%$ & No expansion & Insufficient sample \\
ETDC-7823 & C332K00 & $-0.007 \%$ & No expansion & -1.34 \\
ETDC-7824 & C342K00 & $-0.027 \%$ & No expansion & -1.36 \\
ETDC-7825 & C412K00 & $-0.033 \%$ & No expansion & Insufficient sample \\
ETDC-7826 & C422K00 & $-0.063 \%$ & No expansion & -0.79 \\
ETDC-7827 & C432K00 & $-0.299 \%$ & No expansion & -1.35 \\
ETDC-7828 & C442K00 & Insufficient sample & Not available & -2.29 \\
ETDC-7829 & C512K00 & $-0.168 \%$ & No expansion & -3.38 \\
ETDC-7830 & C522K00 & $-0.250 \%$ & No expansion & -0.44 \\
ETDC-7831 & C532K00 & $-0.048 \%$ & No expansion & -2.89 \\
ETDC-7832 & C542K00 & $-0.048 \%$ & No expansion & -1.09 \\
ETDC-7833 & C612K0O & $-0.035 \%$ & No expansion & -4.29 \\
ETDC-7834 & C622K00 & $-0.041 \%$ & No expansion & -0.71 \\
ETDC-7835 & C632K00 & $-0.123 \%$ & No expansion & -0.32 \\
ETDC-7836 & C642K00 & $-0.187 \%$ & No expansion & -1.01
\end{tabular}




\section{Series \\ 28 Day Permeability Results}




\section{8-DAY HYDRAULIC CONDUCTIVITY / PERMEABILITY ASTM D 5084}

PROJECT NAME: SAIC / Savannah River PROJECT NO. 777304.00010500
CLIENT SAMPLE NO.

IT LAB SAMPLE NO.
C412G00

ETDC-7869

\section{Specimen diameter, $\mathrm{cm}$}

Specimen length, $\mathrm{cm}$

Wet weight of specimen, $\mathbf{g}$.

Specimen cross-sect. area, $\mathrm{cm}^{\wedge} 2$

Water content, \%

Wet unit weight, pcf

Dry unit weight, pcf

Estimated degree of saturation, \%

Estimated spec. gravity of solids

\begin{tabular}{|l|l|}
\hline INITIAL FINAL \\
\hline 7.59
\end{tabular}

7.92

651.85

660.19

45.2648

31.1

33.9

113.5

86.6

90.6

2.65
Hydraulic gradient

17.8

Min. consolidation stress, psi

Max. consolidation stress, psi

2.0

4.0

2.0

Permeant Fluid

Sample molded

Sample set up

Four tests performed
Deaired DI Water

$11 / 11 / 98$

$12 / 7 / 98$

\section{PERMEABILITY vs TIME}

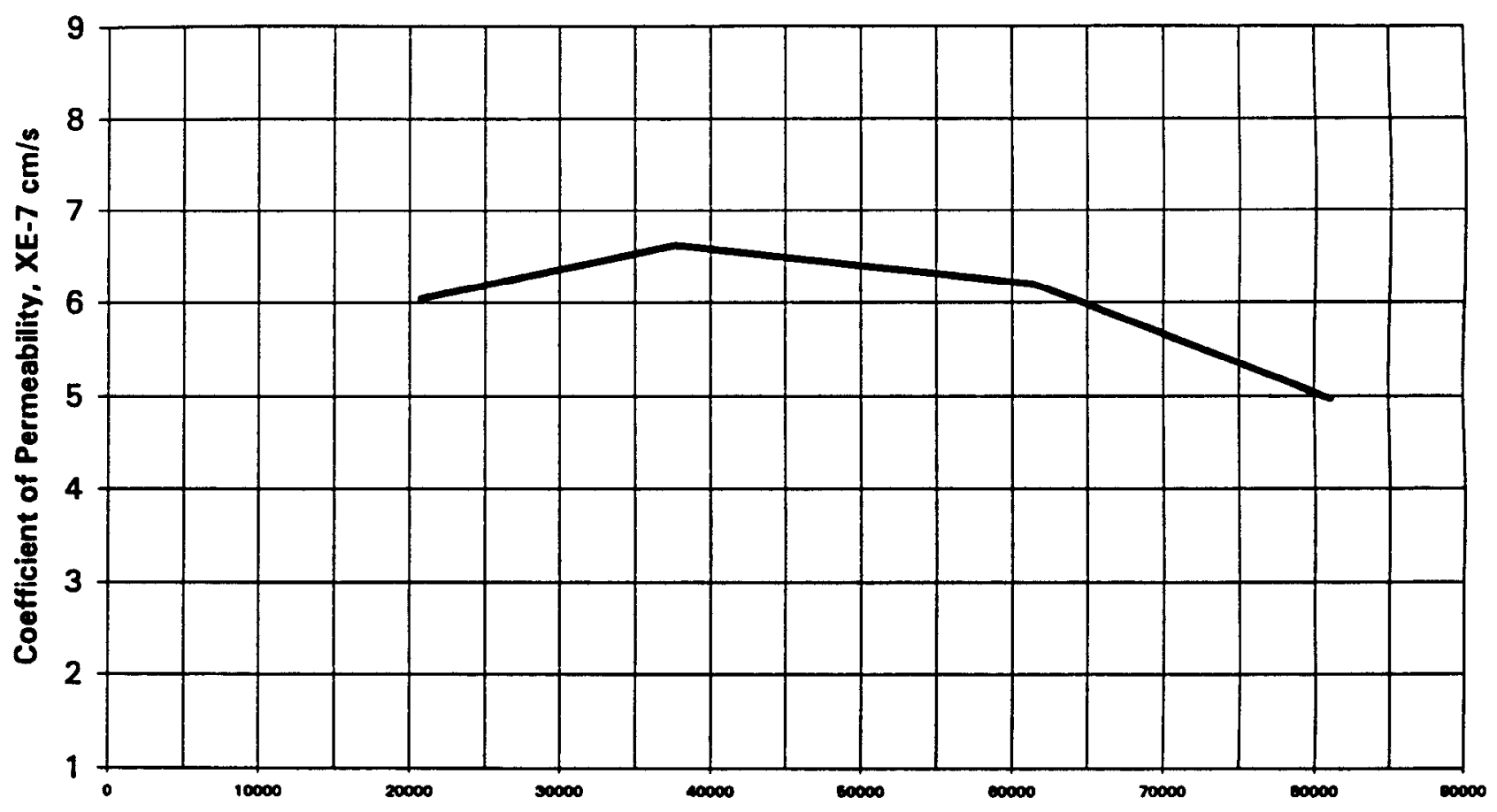

Cumulative Elapsed Time, seconds 


\section{8-DAY HYDRAULIC CONDUCTIVITY / PERMEABILITY ASTM D 5084}

PROJECT NAME: $\quad$ SAIC / Savannah River

PROJECT NO.
CLIENT SAMPLE NO. C422G00

IT LAB SAMPLE NO. ETDC-7870

\section{\begin{tabular}{|l|l|l|l|}
\hline INITIAL & FINAL \\
\hline
\end{tabular}}

Specimen diameter, $\mathrm{cm}$

Specimen length, $\mathrm{cm}$

Wet weight of specimen, $g$.

7.58

7.17

594.81

45.1034

Specimen cross-sect. area, $\mathrm{cm}^{\wedge} 2$

Water content, $\%$

30.2

114.8

88.2

Dry unit weight, pcf

91.4

2.65

\section{Estimated spec. gravity of solids}

\subsection{5}

Hydraulic gradient

19.6

598.62

27.6

Min. consolidation stress, psi

Max. consolidation stress, psi

2.0

4.0

2.0

Permeant Fluid

Deaired DI Water

Sample molded

Sample set up

$11 / 11 / 98$

$12 \Pi / 98$

\section{PERMEABILITY vS TIME}

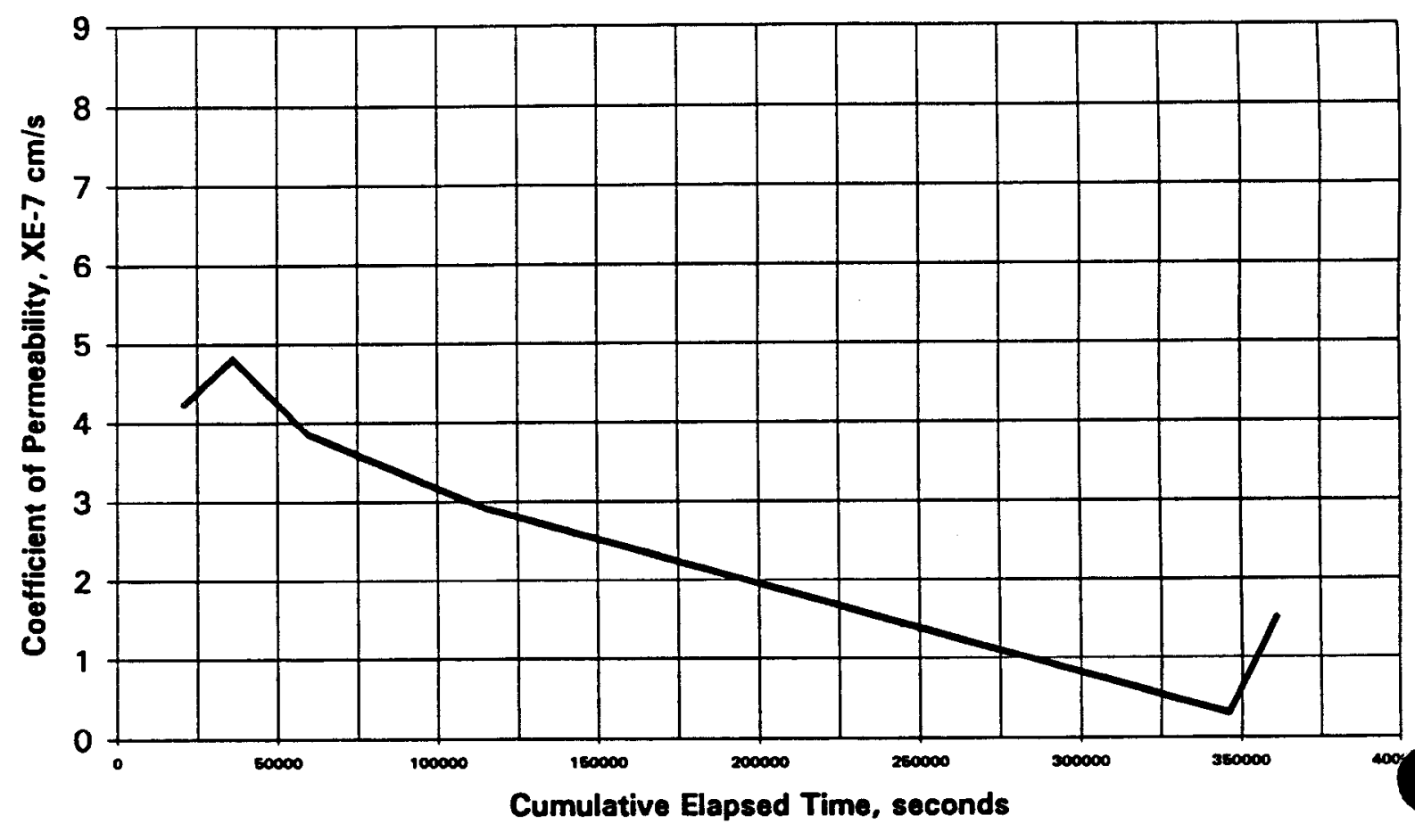




\section{8-DAY HYDRAULIC CONDUCTIVITY / PERMEABILITY ASTM D 5084}

PROJECT NAME: SAIC / Savannah River

PROJECT NO.
CLIENT SAMPLE NO. C432G00

IT LAB SAMPLE NO. ETDC-7871

INITIAL FINAL

Specimen diameter, $\mathrm{cm}$

Specimen length, $\mathrm{cm}$

Wet weight of specimen, $g$.

Specimen cross-sect. area, $\mathrm{cm}^{\wedge} 2$

Water content, \%

Wet unit weight, pcf

Dry unit weight, pcf

Estimated degree of saturation, \%

Estimated spec. gravity of solids

\subsection{9}

9.81

$776.04 \quad 785.5$

45.2800

38.2

109.1

78.9

92.4

2.65

40.7
Hydraulic gradient

Min. consolidation stress, psi

14.3

2.0

Max. consolidation stress, psi

4.0

Total backpressure, psi

Permeant Fluid

2.0

Sample molded

Sample set up

Deaired DI Water

$11 / 11 / 98$

$12 / 7 / 98$

Five tests performed

\section{PERMEABILITY vs TIME}

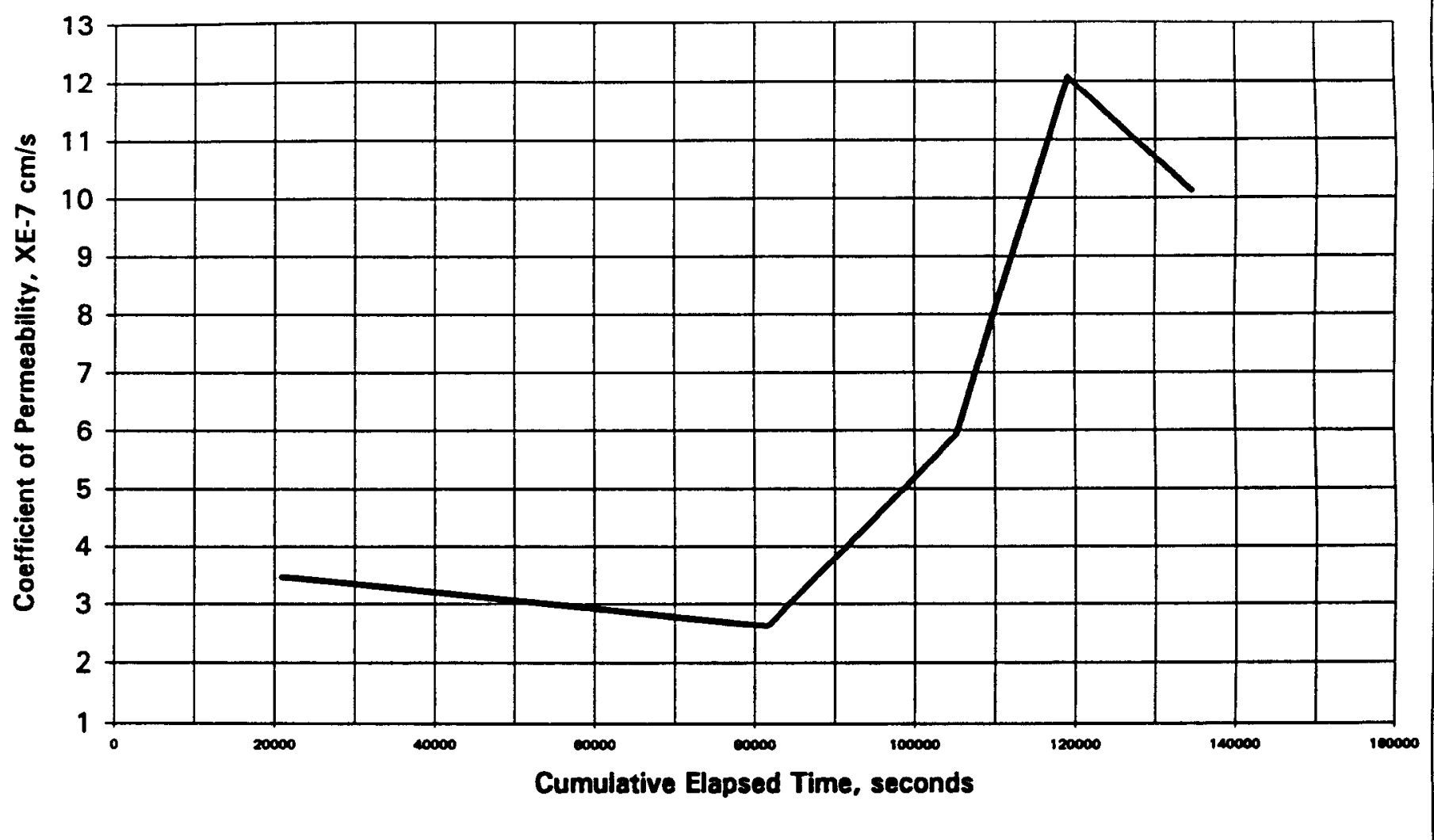




\section{8-DAY HYDRAULIC CONDUCTIVITY / PERMEABILITY ASTM D 5084}

PROJECT NAME: SAIC / Savannah River PROJECT NO. $\quad 777304.00010500$

\author{
CLIENT SAMPLE NO. C442G00 \\ IT LAB SAMPLE NO. ETDC-7872
}

\begin{tabular}{|l|l|}
\hline INITIAL & FINAL \\
\hline 7.61 &
\end{tabular}

Specimen diameter, $\mathrm{cm}$

Specimen length, $\mathrm{cm}$

Wet weight of specimen, $g$.

Specimen cross-sect. area, $\mathrm{cm}^{-2} 2$

Water content, \%

Wet unit weight, pcf

Dry unit weight, pcf

Estimated degree of saturation, \%

Estimated spec. gravity of solids
7.61

8.40

668.3

45.5226

37.7

109.0

79.2

91.8

2.65

\subsection{8}

39.2

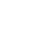

\section{Coefficient of Permeability, $\mathrm{cm} / \mathrm{s}$}

\subsection{E-08}

\section{PERMEABILITY vs TIME}

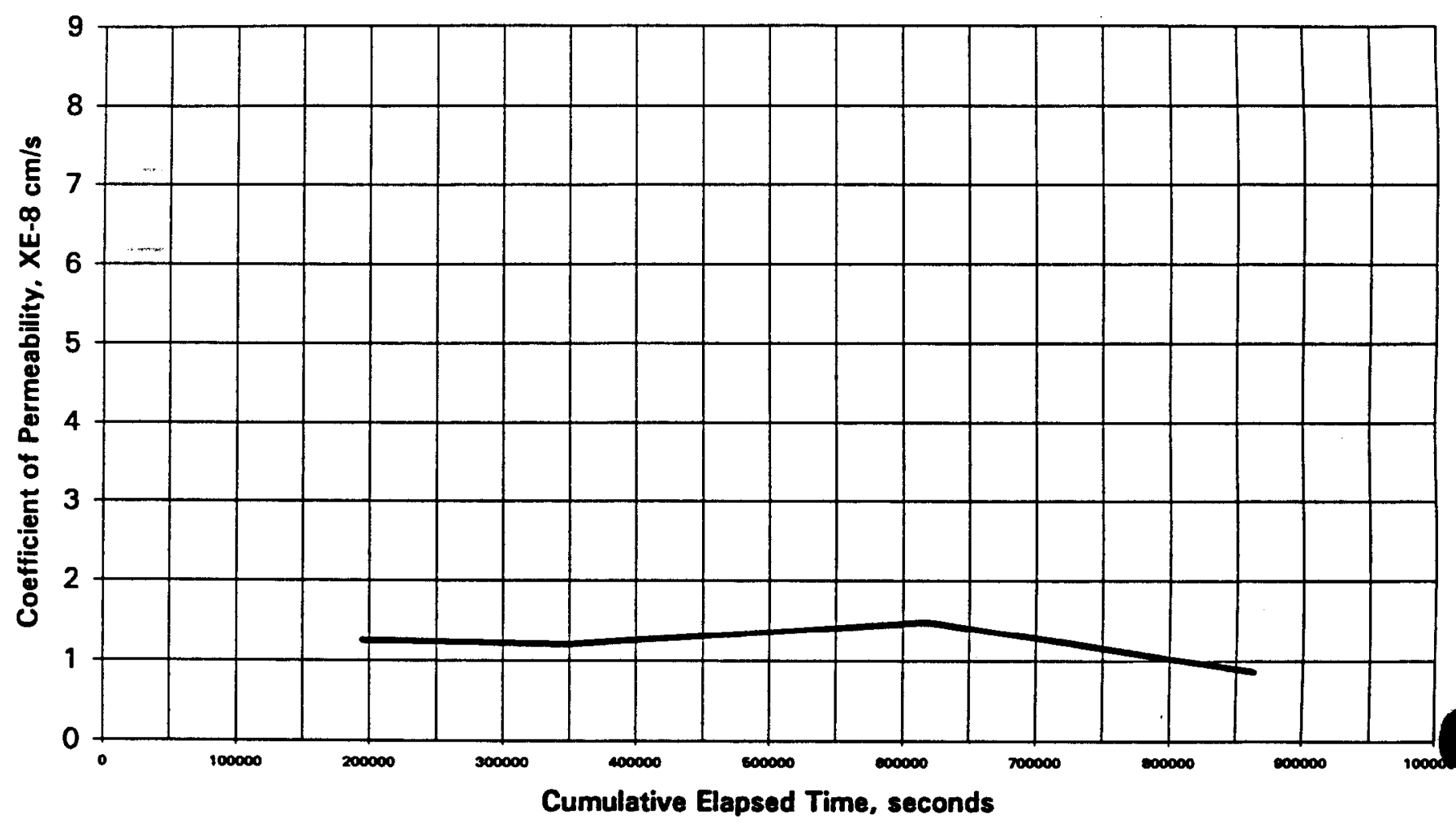




\section{8-DAY HYDRAULIC CONDUCTIVITY / PERMEABILITY ASTM D 5084}

\section{PROJECT NAME: SAIC / Savannah River \\ PROJECT NO. $\quad 777304.00010500$

\author{
CLIENT SAMPLE NO. C512G00
} \\ IT LAB SAMPLE NO. ETDC-7873}

\begin{tabular}{|c|c|c|c|c|}
\hline \multirow[b]{2}{*}{ Specimen diameter, $\mathrm{cm}$} & INITIAL & \multirow[t]{2}{*}{ FINAL } & & \\
\hline & 7.60 & & & \\
\hline Specimen length, $\mathrm{cm}$ & 6.92 & & Hydraulic gradient & 20.3 \\
\hline Wet weight of specimen, $g$. & 580.86 & 585.75 & Min. consolidation stress, psi & 2.0 \\
\hline Specimen cross-sect. area, $\mathrm{cm}^{\wedge} 2$ & 45.4012 & & Max. consolidation stress, psi & 5.0 \\
\hline Water content, $\%$ & 28.6 & 30.3 & Total backpressure, psi & 2.0 \\
\hline Wet unit weight, pcf & 115.4 & & Permeant Fluid Deaire & DI Water \\
\hline Dry unit weight, pcf & 89.8 & & Sample molded & $11 / 11 / 98$ \\
\hline Estimated degree of saturation, \% & 89.8 & & Sample set up & $12 / 7 / 98$ \\
\hline Estimated spec. gravity of solids & 2.65 & & Four tests performed & \\
\hline
\end{tabular}

\section{PERMEABILITY vS TIME}

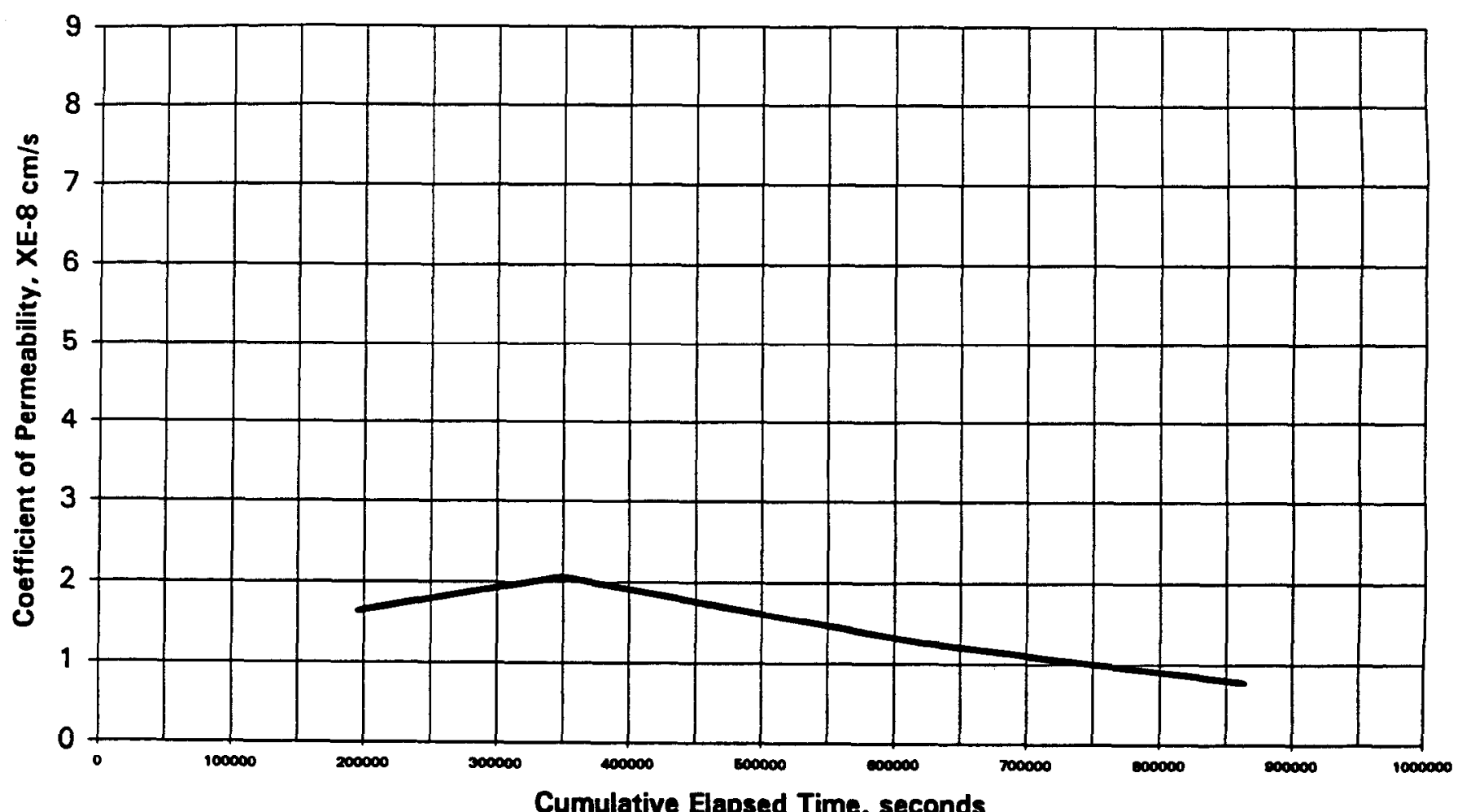

Cumulative Elapsed Time, seconds 


\section{8-DAY HYDRAULIC CONDUCTIVITY / PERMEABILITY ASTM D 5084}

$\begin{array}{llll}\text { PROJECT NAME: } & \text { SAIC / Savannah River } & \text { CLIENT SAMPLE NO. } & \text { C522G00 } \\ \text { PROJECT NO. } & 777304.00010500 & \text { IT LAB SAMPLE NO. } & \text { ETDC-7874 }\end{array}$

Specimen diameter, $\mathrm{cm}$

Specimen length, $\mathrm{cm}$

Wet weight of specimen, $g$.

Specimen cross-sect. area, $\mathrm{cm}^{-2} 2$

Water content, \%

Wet unit weight, pcf

Dry unit weight, pcf

Estimated degree of saturation, \%

Estimated spec. gravity of solids

\begin{tabular}{|l|}
\hline INITIAL \\
\hline 7.59 \\
6.96 \\
591.87 \\
45.2194 \\
24.7 \\
117.4 \\
94.1 \\
86.4 \\
2.65
\end{tabular}

\section{FINAL}

597.48
28.7

Hydraulic gradient

Min. consolidation stress, psi

20.2

Max. consolidation stress, psi

Total backpressure, psi

Permeant Fluid

Sample molded

Sample set up

Four tests performed
2.0

5.0

2.0

Deaired DI Water

$11 / 11 / 98$

$12 / 7 / 98$

\section{Coefficient of Permeability, cm/s} $1.4 \mathrm{E}-08$

\section{PERMEABILITY vs TIME}

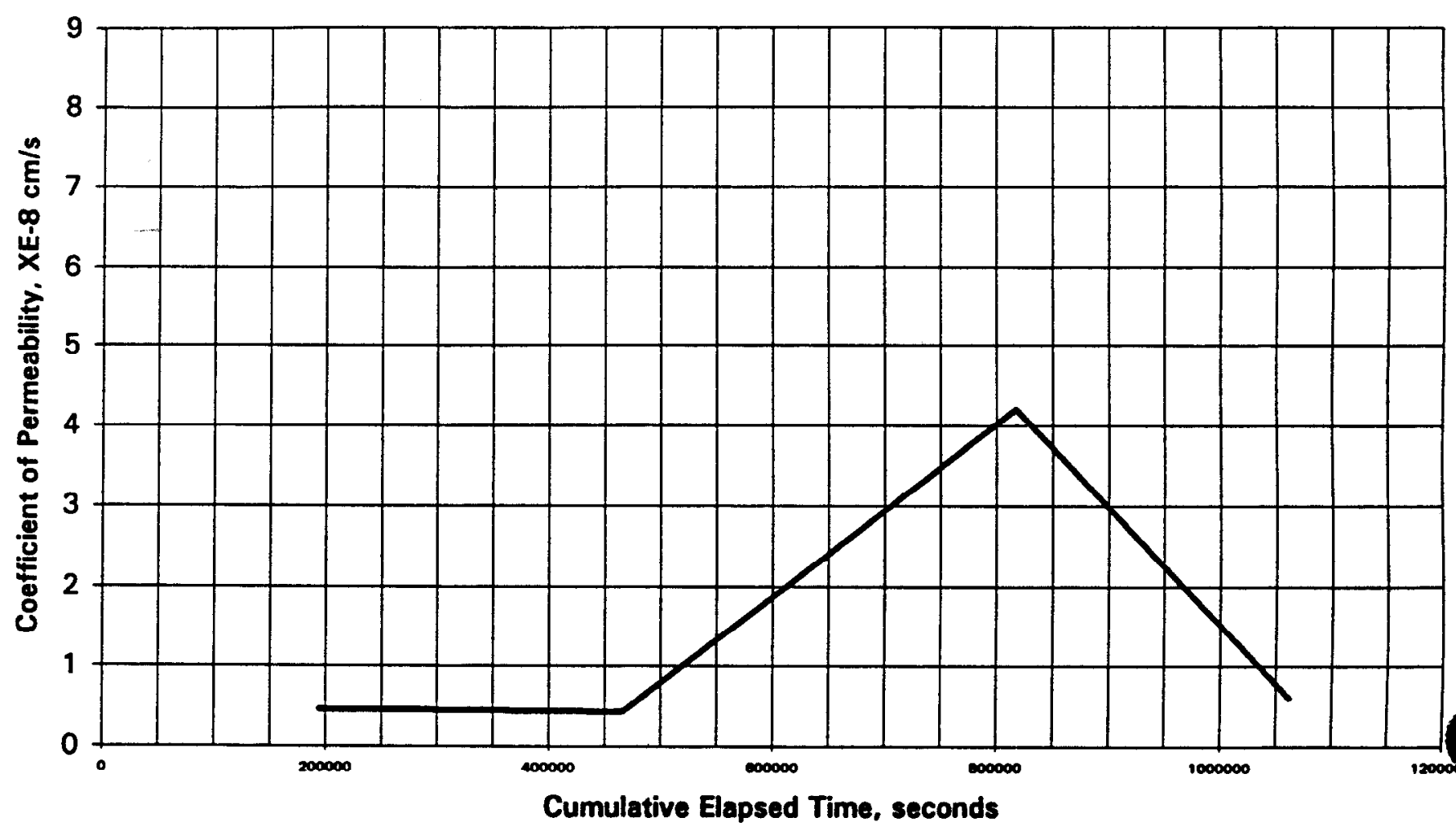




\section{8-DAY HYDRAULIC CONDUCTIVITY / PERMEABILITY ASTM D 5084}

\section{PROJECT NAME: SAIC / Savannah River PROJECT NO. 777304.00010500}

\section{CLIENT SAMPLE NO. C532G00 \\ IT LAB SAMPLE NO. ETDC-7875}

INITIAL FINAL

Specimen diameter, $\mathrm{cm}$

7.59

Specimen length, $\mathrm{cm}$

Wet weight of specimen, $g$.

9.16

Specimen cross-sect. area, $\mathrm{cm}^{-2} 2$

Water content, \%

Wet unit weight, pcf

Dry unit weight, pcf

Estimated degree of saturation, \%

Estimated spec. gravity of solids
$752.54 \quad 759.62$

45.2497

33.2

113.4

85.1

93.3

2.65

34.5
Hydraulic gradient

Min. consolidation stress, psi

Max. consolidation stress, psi

Total backpressure, psi

Permeant Fluid

Sample molded

Sample set up

Four tests performed
15.4

2.0

5.0

2.0

Deaired DI Water

$11 / 11 / 98$

$12 / 7 / 98$

\section{Coefficient of Permeability, cm/s}

\section{PERMEABILITY vs TIME}

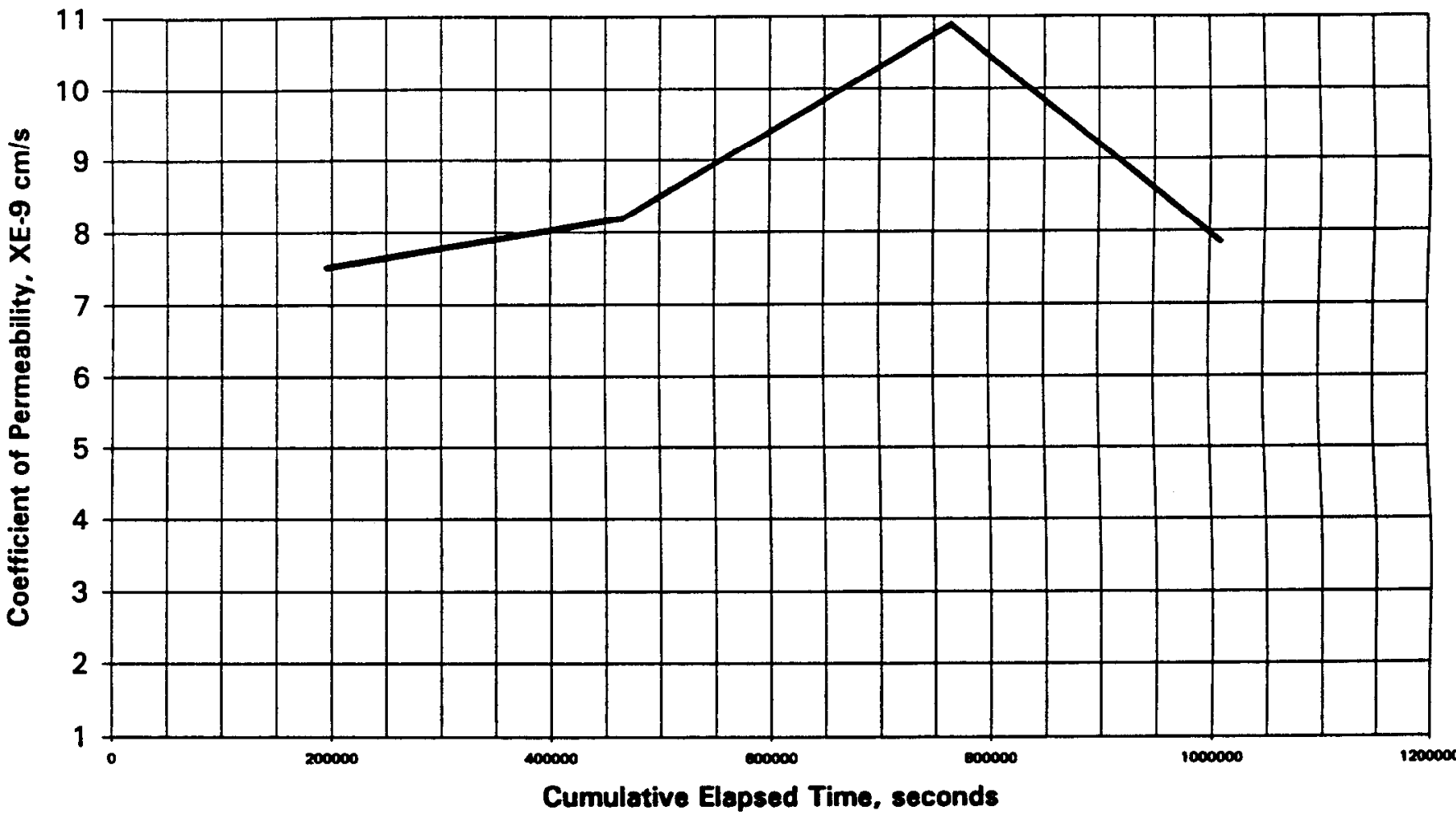




\section{8-DAY HYDRAULIC CONDUCTIVITY / PERMEABILITY ASTM D 5084}
PROJECT NAME:
SAIC / Savannah River
CLIENT SAMPLE NO. C542G00
PROJECT NO.
777304.00010500
IT LAB SAMPLE NO.
ETDC-7876

\section{Specimen diameter, $\mathrm{cm}$}

Specimen length, $\mathrm{cm}$

Wet weight of specimen, $g$.

Specimen cross-sect. area, $\mathrm{cm}^{\wedge} 2$

Water content, \%

Wet unit weight, pcf

Dry unit weight, pcf

Estimated degree of saturation, \%

Estimated spec. gravity of solids

\section{INITIAL FINAL}

7.60

8.93

737.79

745.76

45.3962

31.5

113.6

86.4

91.2

2.65

33.3

Hydraulic gradient

Min. consolidation stress, psi

Max. consolidation stress, psi

15.8

2.0

5.0

Total backpressure, psi

Permeant Fluid

Deaired DI Water

Sample molded

$11 / 11 / 98$

Sample set up

$12 / 7 / 98$

Six tests performed

\section{PERMEABILITY vs TIME}

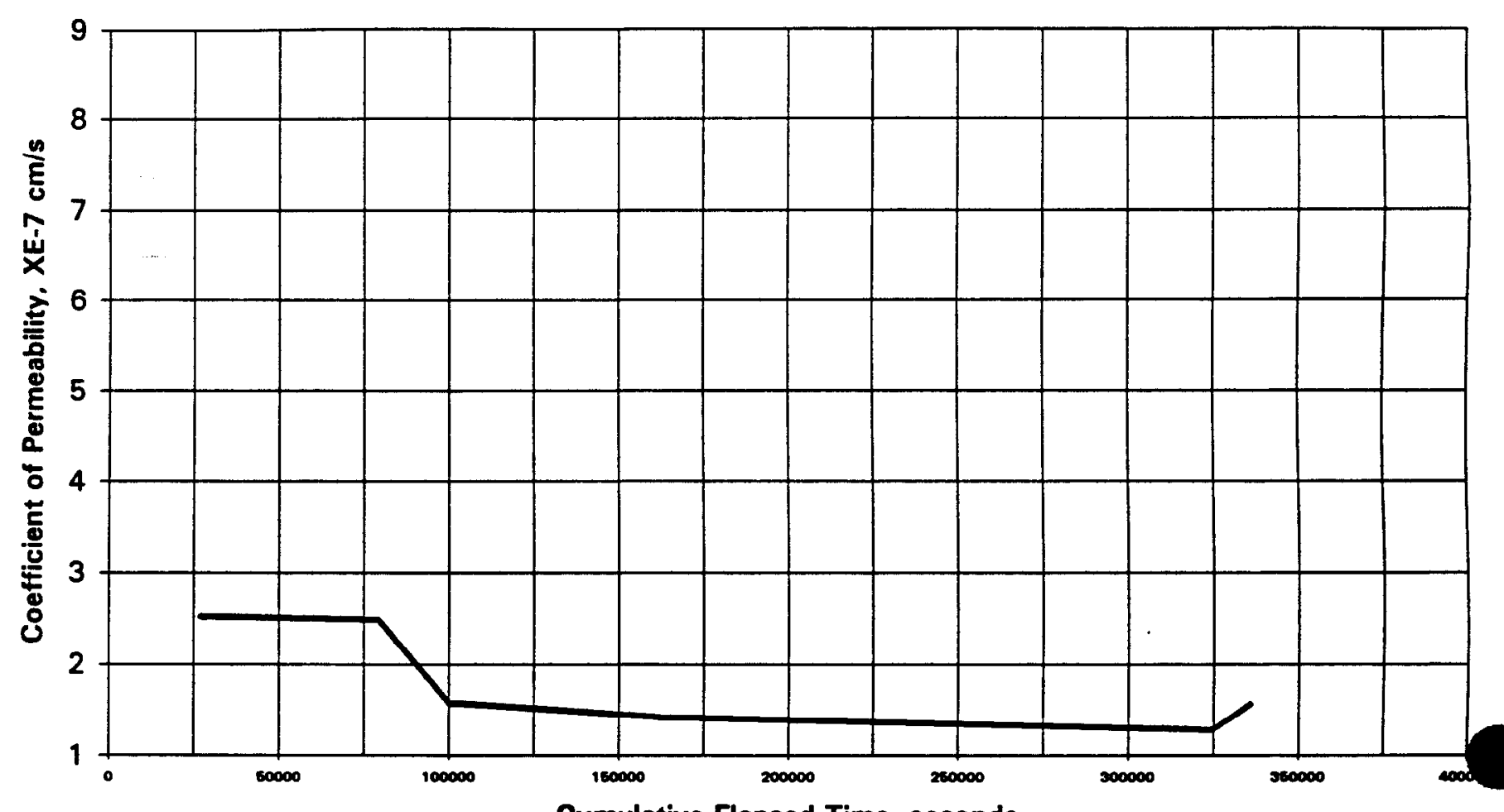

Cumulative Elapsed Time, seconds 


\section{8-DAY HYDRAULIC CONDUCTIVITY / PERMEABILITY ASTM D 5084}

$\begin{array}{ll}\text { PROJECT NAME: } & \text { SAIC / Savannah River } \\ \text { PROJECT NO. } & 777304.00010500\end{array}$
CLIENT SAMPLE NO.

IT LAB SAMPLE NO.
C612G00

ETDC-7877
Specimen diameter, $\mathrm{cm}$

\begin{tabular}{|l|l|}
\hline INITIAL FINAL \\
\hline 7.58
\end{tabular}
Specimen length, $\mathrm{cm}$
7.57
Wet weight of specimen, $g$.
634.35
643.69
Specimen cross-sect. area, $\mathrm{cm}^{`} 2$
45.0933
Water content, \%
24.5
116.0
Wet unit weight, pcf
Dry unit weight, pcf
93.2
Estimated degree of saturation, \% 83.8
Estimated spec. gravity of solids
2.65
Hydraulic gradient
18.6
Min. consolidation stress, psi $\quad 2.0$
Max. consolidation stress, psi $\quad 5.0$
31.1
Total backpressure, psi
2.0
Permeant Fluid
Sample molded
Deaired DI Water
Sample set up
$11 / 11 / 98$
Five tests performed

\section{Coefficient of Permeability, cm/s $1.5 E-07$}

\section{PERMEABILITY vs TIME}

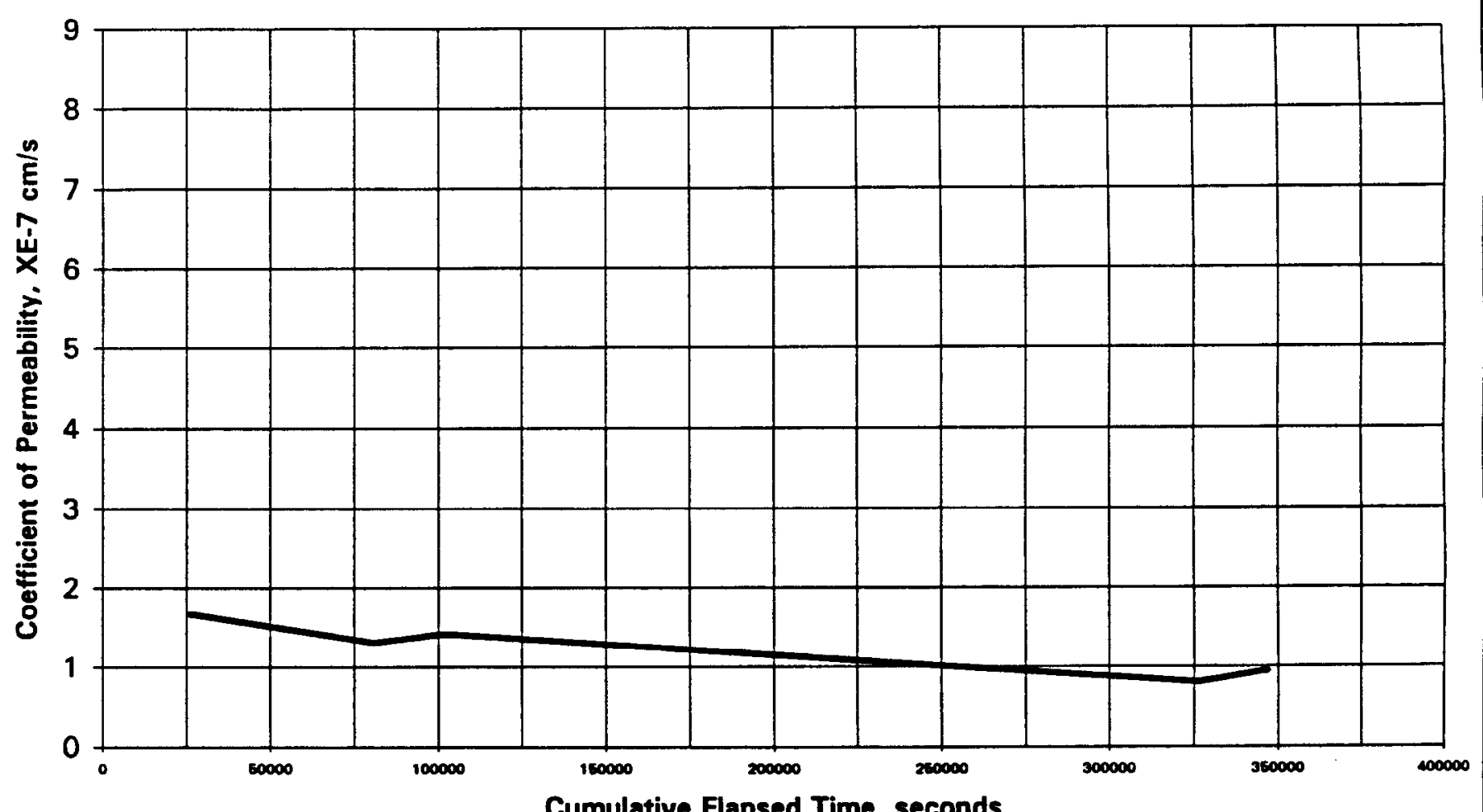

Cumulative Elapsed Time, seconds 


\section{8-DAY HYDRAULIC CONDUCTIVITY / PERMEABILITY ASTM D 5084}
PROJECT NAME:
SAIC / Savannah River
CLIENT SAMPLE NO.
C622G00
PROJECT NO.
777304.00010500
IT LAB SAMPLE NO.
ETDC-7878

\begin{tabular}{|c|c|c|c|c|}
\hline \multirow[b]{2}{*}{ Specimen diameter, $\mathrm{cm}$} & INITIAL & FINAL & & \\
\hline & \multicolumn{4}{|l|}{7.57} \\
\hline $\begin{array}{l}\text { Specimen length, cm } \\
\text { Wet weight of specimen, g. }\end{array}$ & $\begin{array}{l}7.09 \\
563.94\end{array}$ & 610.48 & $\begin{array}{l}\text { Hydraulic gradient } \\
\text { Min. consolidation stress, psi }\end{array}$ & $\begin{array}{l}19.8 \\
2.0\end{array}$ \\
\hline Specimen cross-sect. area, $\mathrm{cm}^{-} 2$ & 45.0631 & & Max. consolidation stress, psi & 4.0 \\
\hline Water content, $\%$ & 18.9 & 28.8 & Total backpressure, psi & 2.0 \\
\hline Wet unit weight, pcf & 110.1 & & Permeant Fluid Deaire & DI Water \\
\hline Dry unit weight, pcf & 92.6 & & Sample moided & $11 / 11 / 98$ \\
\hline Estimated degree of saturation, \% & 63.8 & & Sample set up & $12 / 7 / 98$ \\
\hline Estimated spec. gravity of solids & 2.65 & & Six tests performed & \\
\hline
\end{tabular}

\section{PERMEABILITY vS TIME}

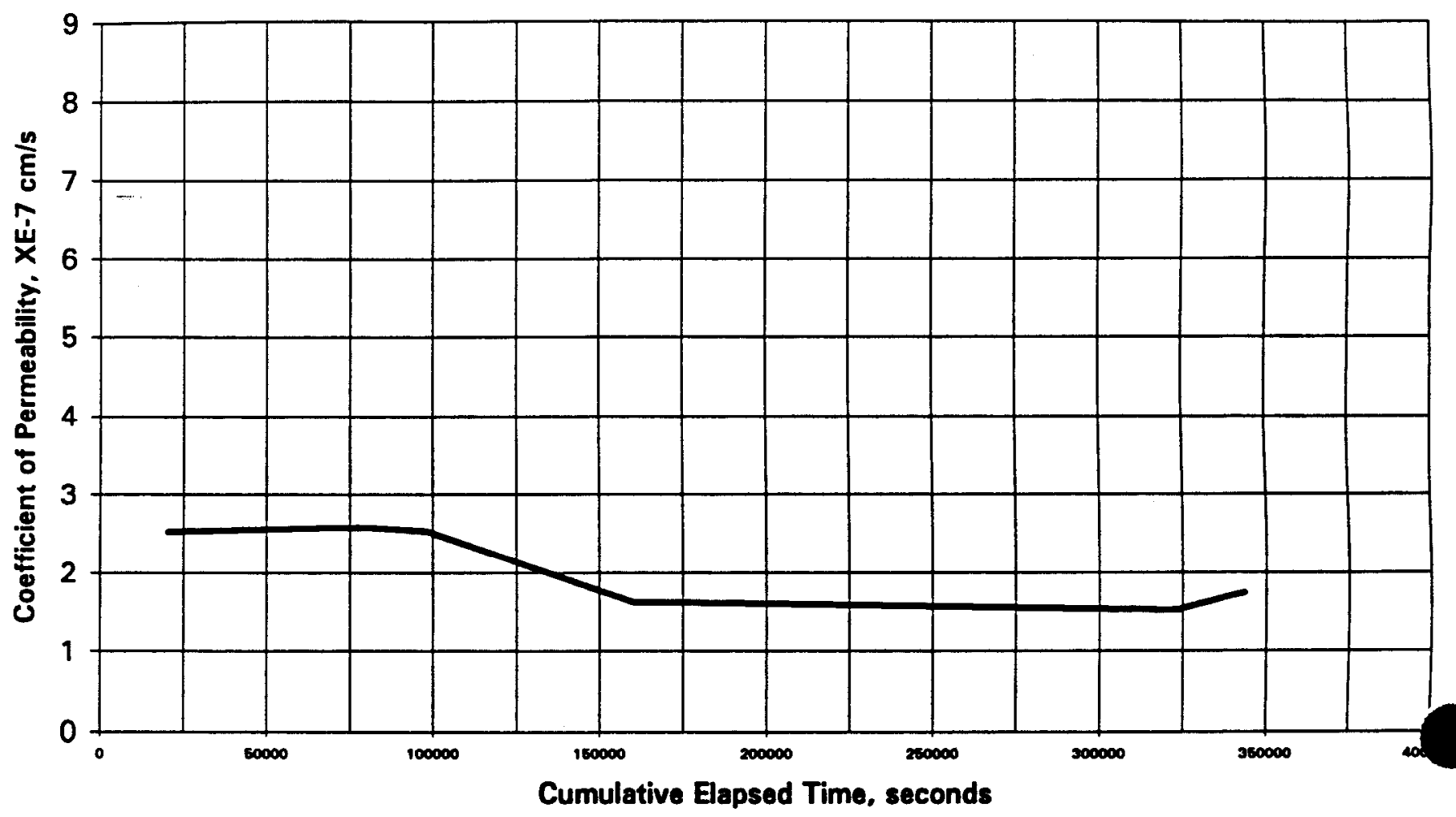




\section{8-DAY HYDRAULIC CONDUCTIVITY / PERMEABILITY ASTM D 5084}
PROJECT NAME:
SAIC / Savannah River
CLIENT SAMPLE NO.
C632G00
PROJECT NO.
777304.00010500
IT LAB SAMPLE NO. ETDC-7879

Specimen diameter, $\mathrm{cm}$

Specimen length, $\mathrm{cm}$

Wet weight of specimen, $\mathbf{g}$.

Specimen cross-sect. area, $\mathrm{cm}^{`} 2$

Water content, \%

Wet unit weight, pcf

Dry unit weight, pef

Estimated degree of saturation, \%

Estimated spec. gravity of solids

INITIAL FINAL

\subsection{9}

9.72

800.91

45.2446

32.3

113.7

85.9

92.7

2.65

812.2

34.8
Hydraulic gradient

14.5

Min. consolidation stress, psi

Max. consolidation stress, psi

2.0

4.0

Total backpressure, psi

Permeant Fluid

2.0

Sample molded

Deaired DI Water

Sample set up

$11 / 11 / 98$

$12 / 7 / 98$

Five tests performed

\section{PERMEABILITY vs TIME}

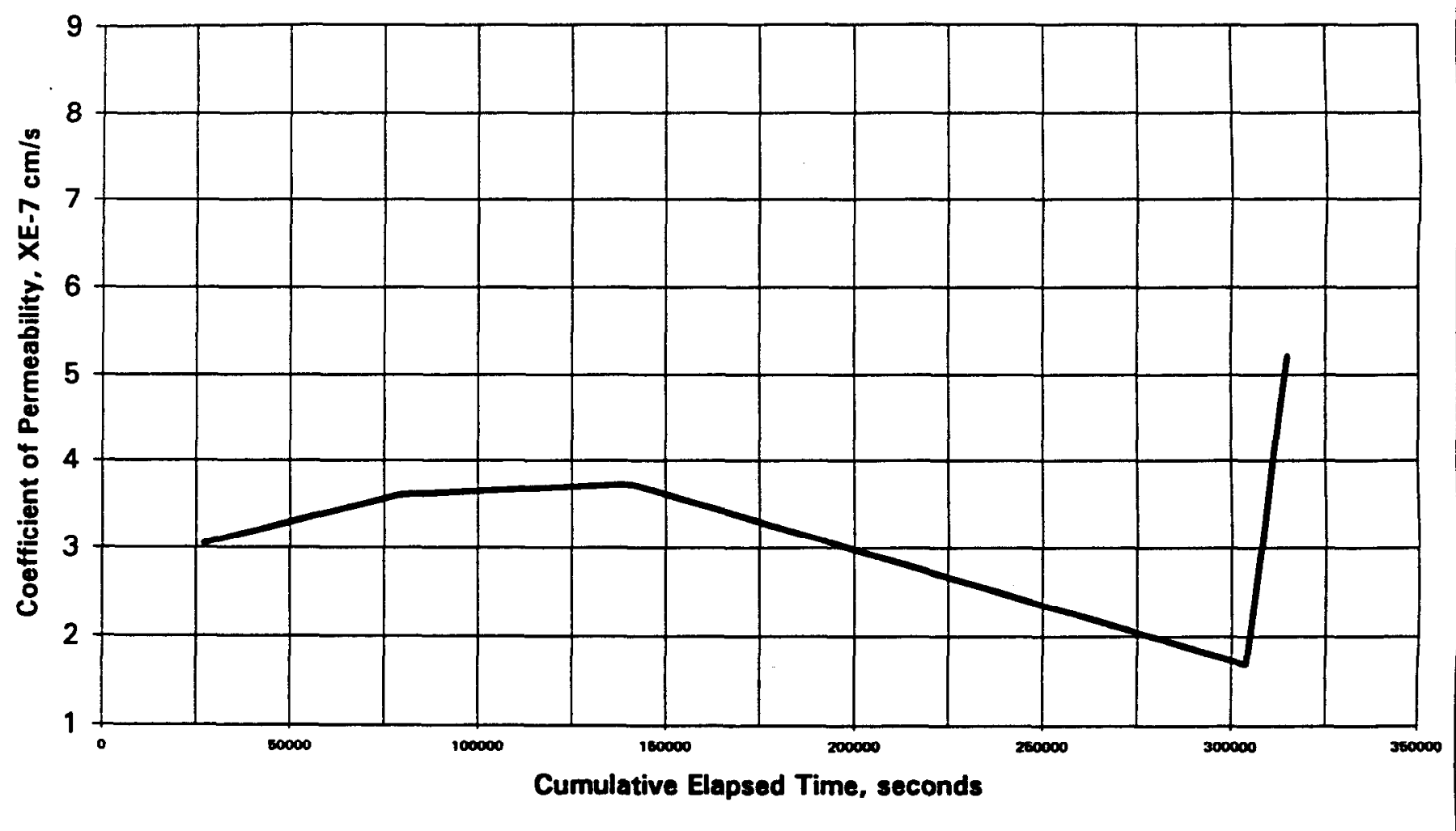




\section{8-DAY HYDRAULIC CONDUCTIVITY / PERMEABILITY ASTM D 5084}

PROJECT NAME: SAIC / Savannah River PROJECT NO.

777304.00010500
CLIENT SAMPLE NO.

IT LAB SAMPLE NO.
C642G00

ETDC-7880

\section{Specimen diameter, $\mathrm{cm}$}

Specimen length, $\mathrm{cm}$

Wet weight of specimen, $g$.

Specimen cross-sect. area, $\mathrm{cm}^{`} 2$

Water content, \%

Wet unit weight, pcf

Dry unit weight, pcf

Estimated degree of saturation, \%

Estimated spec. gravity of solids

INITIAL FINAL

\subsection{9}

9.20

759.35

768.13

45.3002

26.8

113.8

34.1

89.7

84.1

2.65
Hydraulic gradient

15.3

Min. consolidation stress, psi

2.0

5.0

Max. consolidation stress, psi

2.0

Permeant Fluid

Deaired DI Water

Sample molded

$11 / 11 / 98$

Sample set up

$12 / 7 / 98$

Five tests performed

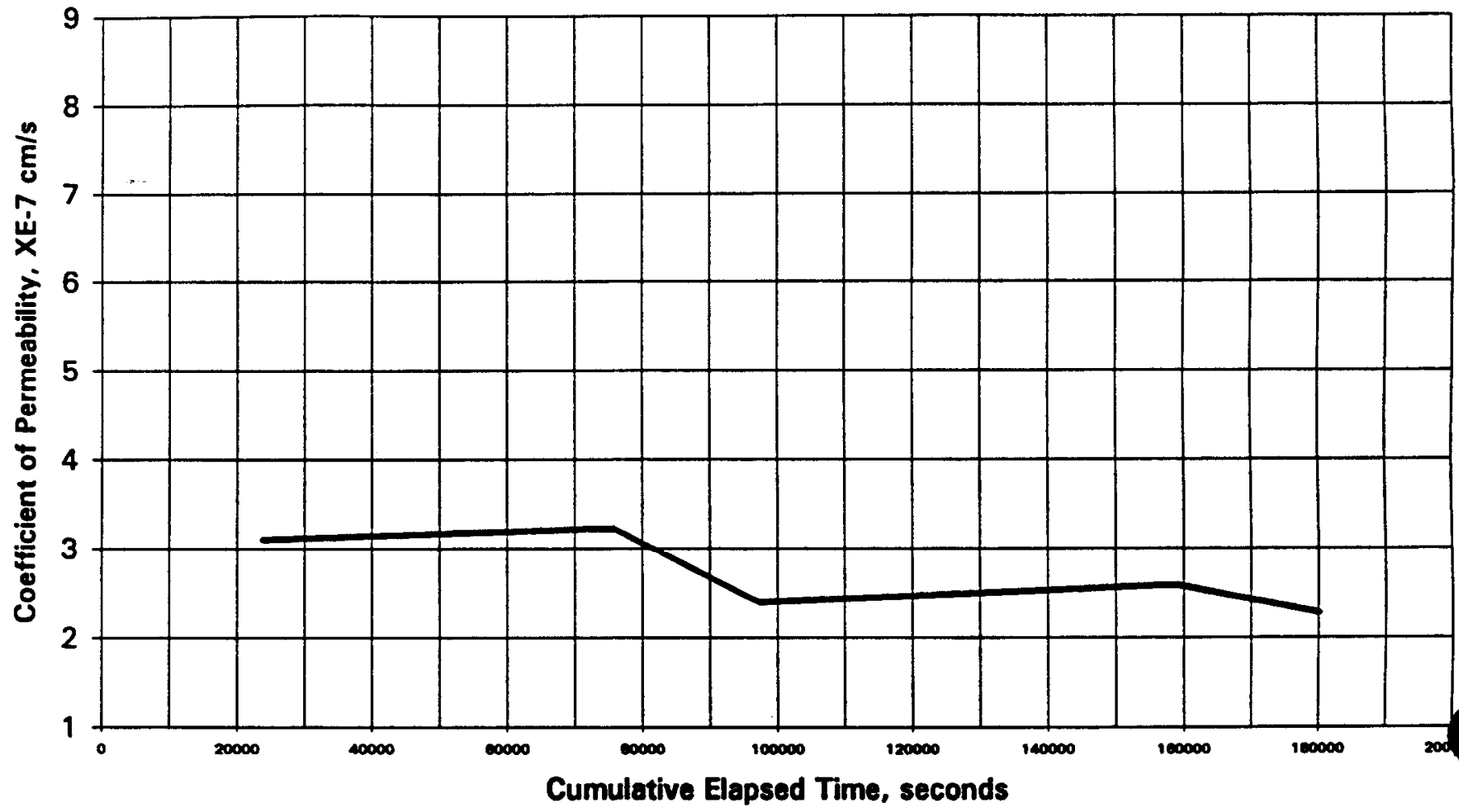




\section{8-DAY HYDRAULIC CONDUCTIVITY / PERMEABILITY ASTM D 5084}
PROJECT NAME: SAIC / Savannah River
CLIENT SAMPLE NO. C312G00
PROJECT NO.
777304.00010500
IT LAB SAMPLE NO.
ETDC-7881

\begin{tabular}{|c|c|c|c|c|}
\hline \multirow[b]{2}{*}{ Specimen diameter, cm } & INITIAL & \multirow[t]{2}{*}{ FINAL } & & \\
\hline & 7.55 & & & \\
\hline Specimen length, cm & 6.68 & & Hydraulic gradient & 21.1 \\
\hline Wet weight of specimen, $\mathrm{g}$ & 572.19 & 572.23 & Min. consolidation stress, psi & 2.0 \\
\hline Specimen cross-sect. area, $\mathrm{cm}^{\wedge} 2$ & 44.7463 & & Max. consolidation stress, psi & 4.0 \\
\hline Water content, $\%$ & 26.9 & 30.3 & Total backpressure, psi & 2.0 \\
\hline Wet unit weight, pcf & 119.4 & & Permeant Fluid Deaire & DI Water \\
\hline Dry unit weight, pcf & 94.1 & & Sample molded & $11 / 11 / 98$ \\
\hline Estimated degree of saturation, \% & 94.0 & & Sample set up & $12 / 7 / 98$ \\
\hline Estimated spec. gravity of solids & 2.65 & & Six tests performed & \\
\hline
\end{tabular}

\section{PERMEABILITY vS TIME}

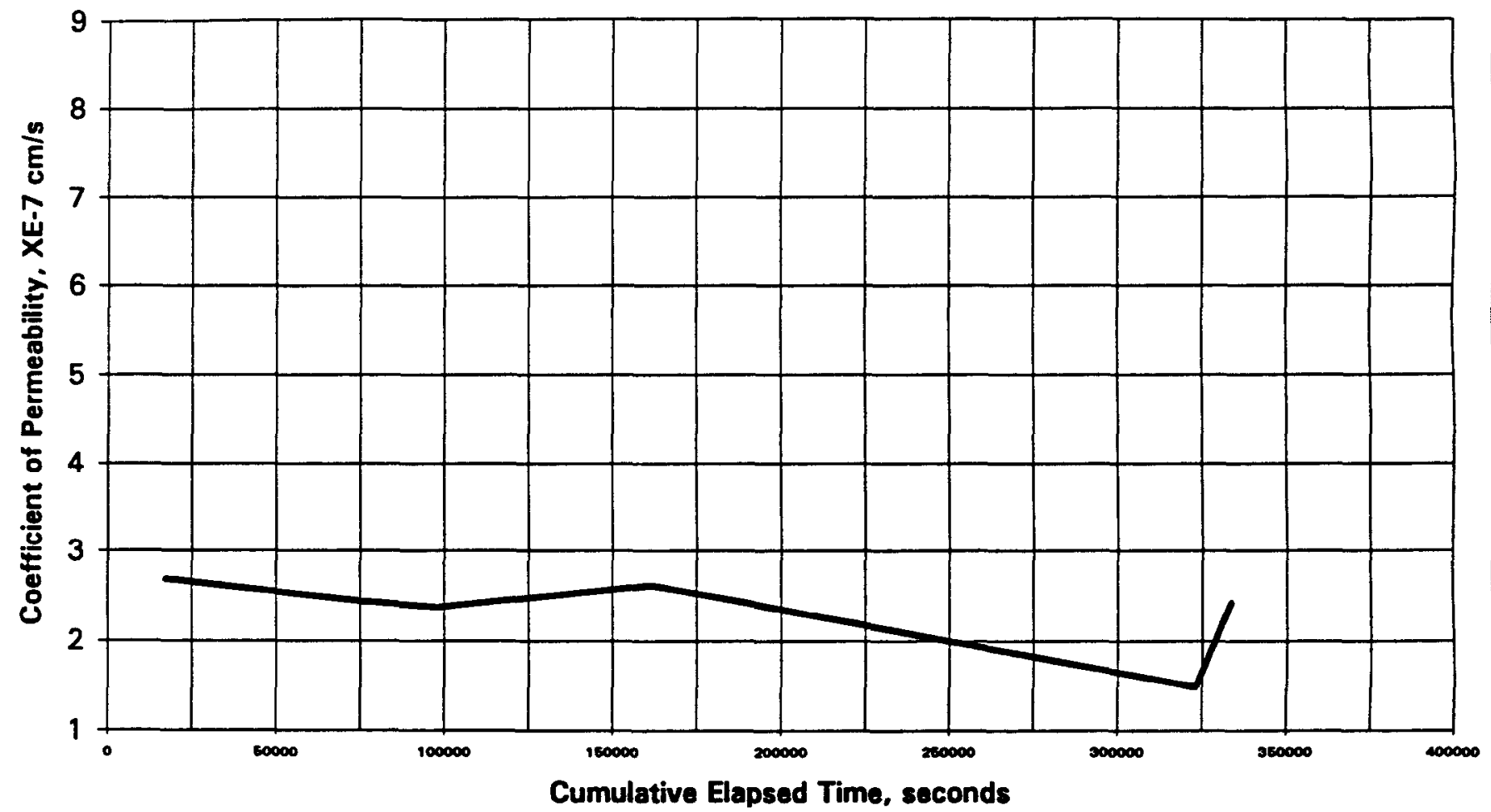




\section{8-DAY HYDRAULIC CONDUCTIVITY / PERMEABILITY ASTM D 5084}

PROJECT NAME: SAIC / Savannah River

PROJECT NO. $\quad \mathbf{7 7 7 3 0 4 . 0 0 0 1 0 5 0 0}$

\section{CLIENT SAMPLE NO. C322G00 \\ IT LAB SAMPLE NO. ETDC-7882}
Specimen diameter, $\mathrm{cm}$
INITIAL FINAL
Specimen length, $\mathrm{cm}$

\subsection{9}
Wet weight of specimen, $g$.
7.13
$611.92 \quad 617.94$
Hydraulic gradient
19.7
45.1891
Specimen cross-sect. area, $\mathrm{cm}^{-2} 2$
23.8
27.5
Min. consolidation stress, psi
2.0
Water content, \%
118.5
Wet unit weight, pcf
95.7
Dry unit weight, pcf
86.6
Max. consolidation stress, psi
4.0
Total backpressure, psi
2.0
Permeant Fluid
Deaired DI Water
Sample molded
$11 / 11 / 98$
Sample set up
$12 / 7 / 98$
$\begin{array}{ll}\text { Estimated spec. gravity of solids } & \mathbf{2 . 6 5}\end{array}$
Five tests performed

Coefficient of Permeability, cm/s 3.1E-07

\section{PERMEABILITY vs TIME}

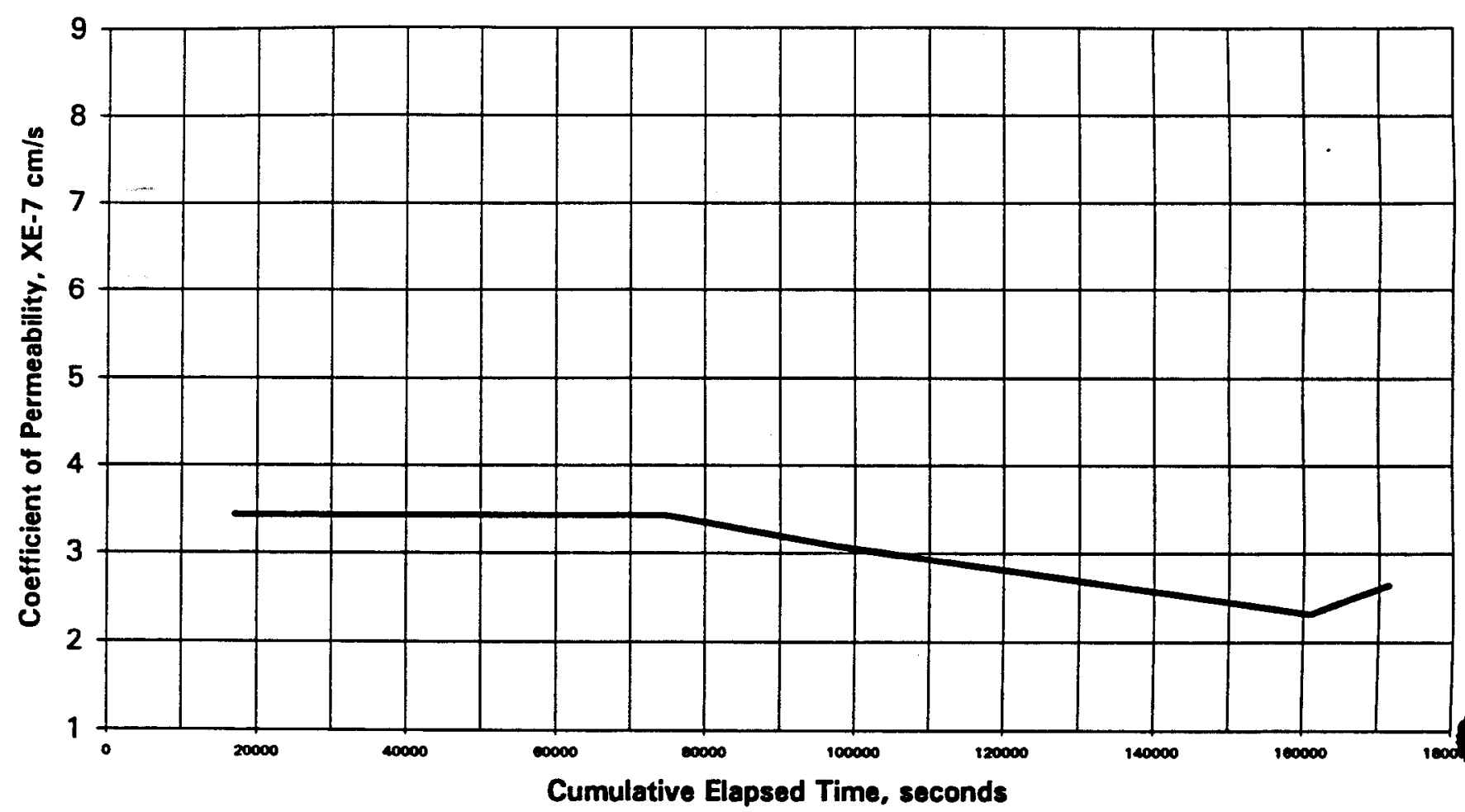




\section{8-DAY HYDRAULIC CONDUCTIVITY / PERMEABILITY ASTM D 5084}
PROJECT NAME:
SAIC / Savannah River
CLIENT SAMPLE NO.
C332G00
PROJECT NO.
777304.00010500
IT LAB SAMPLE NO.
ETDC-7883

\begin{tabular}{lll} 
& INITIAL & FINAL \\
\cline { 2 - 3 } & 7.58 & \\
Specimen diameter, cm & 8.88 & \\
Specimen length, cm & 727.73 & 736.88 \\
Wet weight of specimen, $\mathrm{g}$. & 45.1337 & \\
Specimen cross-sect. area, $\mathrm{cm}^{-2} 2$ & 43.4 & 34.9 \\
Water content, \% & 33.4 & \\
Wet unit weight, pcf & 113.3 & \\
Dry unit weight, pcf & 85.0 & \\
Estimated degree of saturation, \% & 93.4 & \\
Estimated spec. gravity of solids & 2.65
\end{tabular}

Hydraulic gradient

Min. consolidation stress, psi

15.8

Max. consolidation stress, psi

2.0

Total backpressure, psi

4.0

Permeant Fluid

Sample molded

Sample set up

Five tests performed
Deaired DI Water

11/11/98

$12 / 7 / 98$

\section{PERMEABILITY vs TIME}

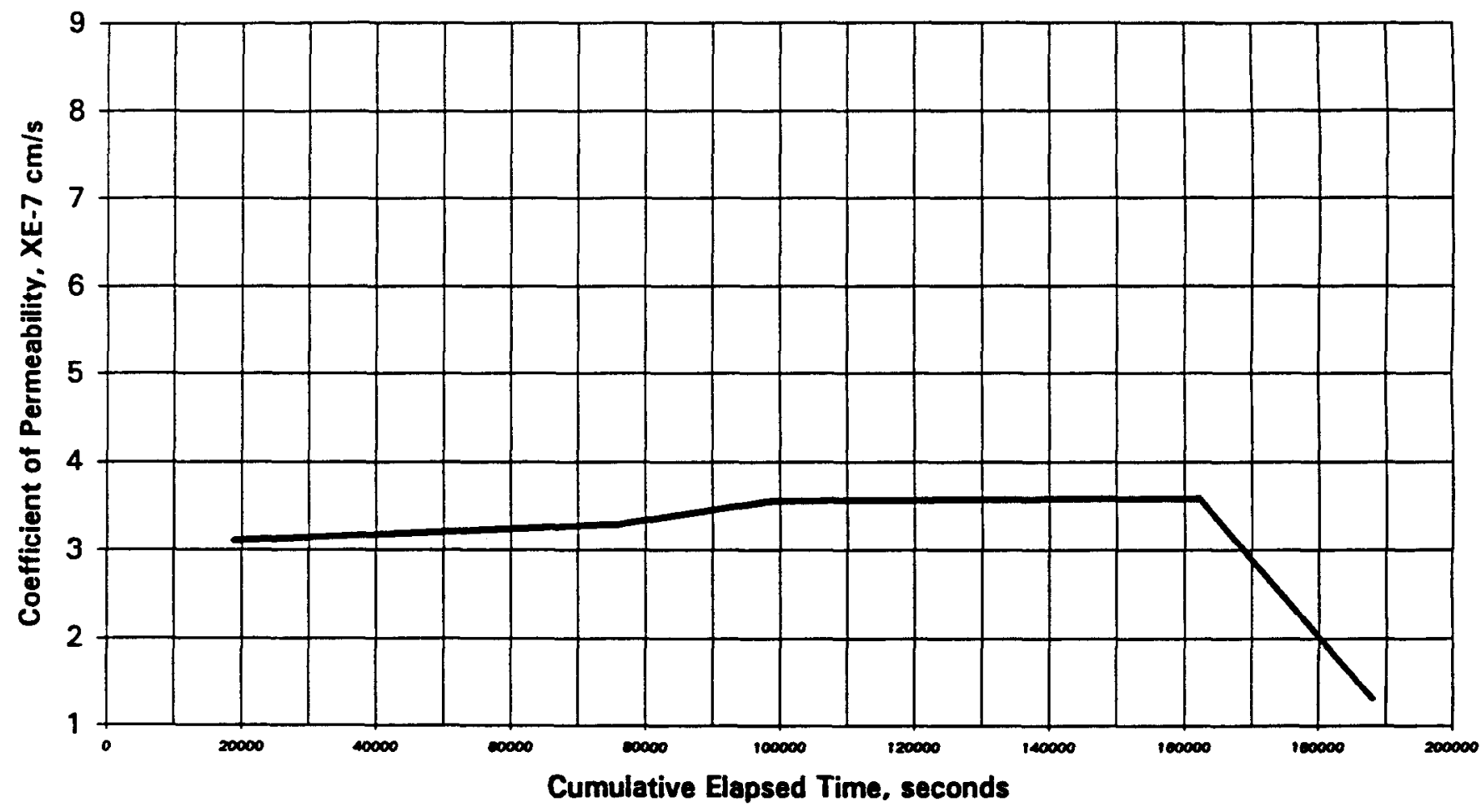




\section{8-DAY HYDRAULIC CONDUCTIVITY / PERMEABILITY ASTM D 5084}

PROJECT NAME: SAIC / Savannah River

PROJECT NO. $\quad 777304.00010500$
CLIENT SAMPLE NO. C342G00

IT LAB SAMPLE NO. ETDC-7884

\section{Specimen diameter, $\mathrm{cm}$}

Specimen length, $\mathrm{cm}$

Wet weight of specimen, $g$.

Specimen cross-sect. area, $\mathrm{cm} ` 2$

Water content, $\%$

Wet unit weight, pcf

Dry unit weight, pcf

Estimated degree of saturation, \%

Estimated spec. gravity of solids

INITIAL FINAL

7.60

8.79

$732.89 \quad 739.85$

45.3103

29.2

114.9

32.9

88.9

89.9

2.65
Hydraulic gradient

16.0

Min. consolidation stress, psi

2.0

Max. consolidation stress, psi

4.0

Total backpressure, psi

Permeant Fluid

Sample molded

Sample set up

Six tests performed
Deaired DI Water

$11 / 11 / 98$

$12 / 7 / 98$

\section{PERMEABILITY vS TIME}

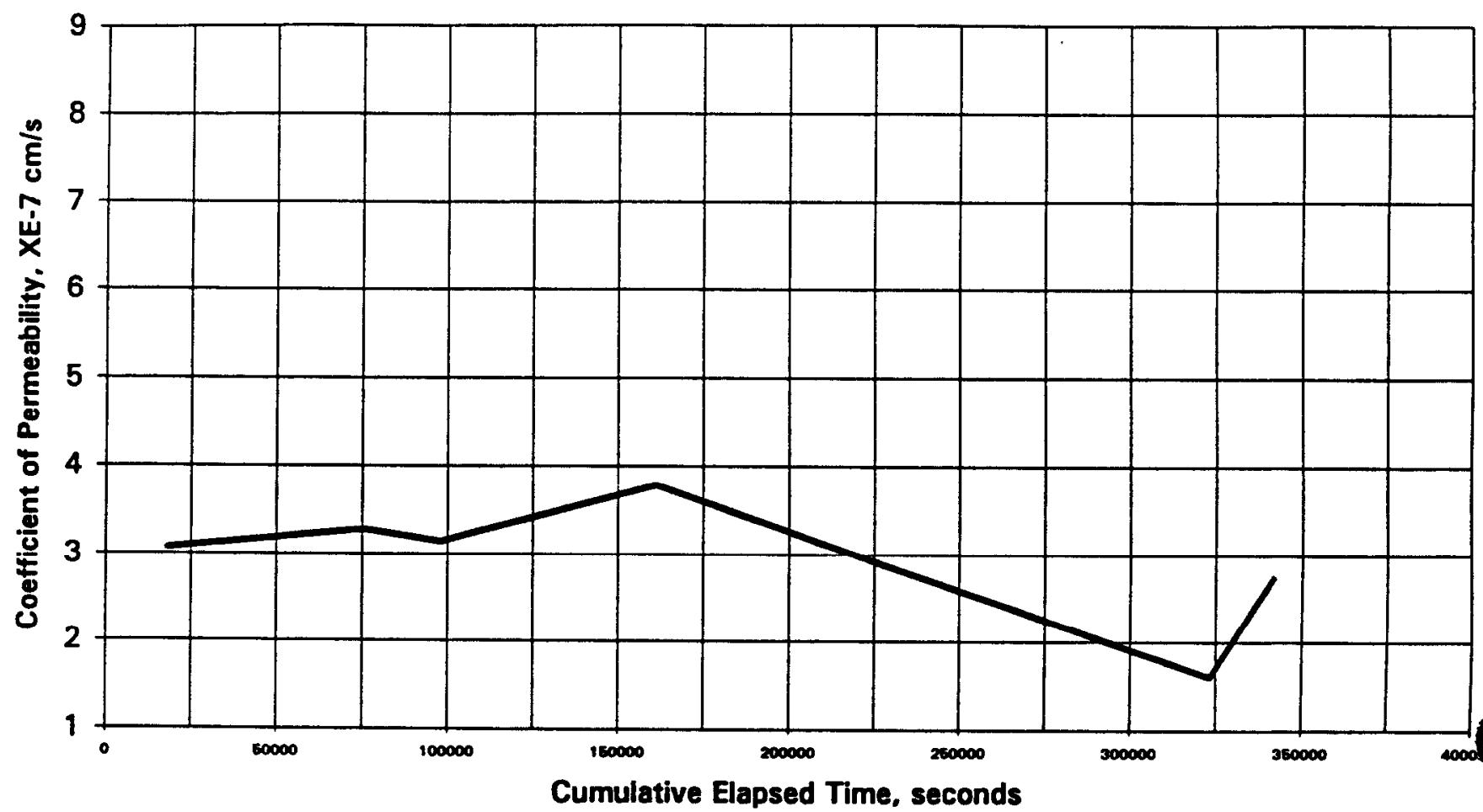




\section{Series 90 Day Immersion Permeability Results}




\section{0-DAY SATURATED HYDRAULIC CONDUCTIVITY / PERMEABILITY ASTM D 5084}

PROJECT NAME:

SAIC / Savannah River PROJECT NO.
CLIENT SAMPLE NO.

C313G00

IT LAB SAMPLE NO.

ETDC-7853

\begin{tabular}{lll} 
& \\
Specimen diameter, $\mathrm{cm}$ & INITIAL FINAL \\
\hline
\end{tabular}

Specimen length, $\mathrm{cm}$

Wet weight of specimen, $g$.

10.07

389.2

Specimen cross-sect. area, $\mathrm{cm}^{-2} 2$

Water content, \%

Wet unit weight, pcf

Dry unit weight, pcf

Estimated degree of saturation, \%

Estimated spec. gravity of solids

Initial conditions are post-soak, pre-test
20.2953

$31.1 \quad 30.8$

118.9

90.7

100.0

2.65
Hydraulic gradient

Min. consolidation stress, psi

34.9

2.0

$\begin{array}{ll}\text { Max. consolidation stress, psi } & 7.0 \\ \text { Total backpressure, psi } & 3.0\end{array}$ $\begin{array}{ll}\text { Max. Consolidation stress, psi } & 7.0 \\ \text { Total backpressure, psi } & 3.0\end{array}$

Permeant Fluid

Deaired DI Water

Sample molded

$11 / 11 / 98$

Sample set up

$3 / 9 / 99$

Four tests performed

\section{PERMEABILITY vS TIME}

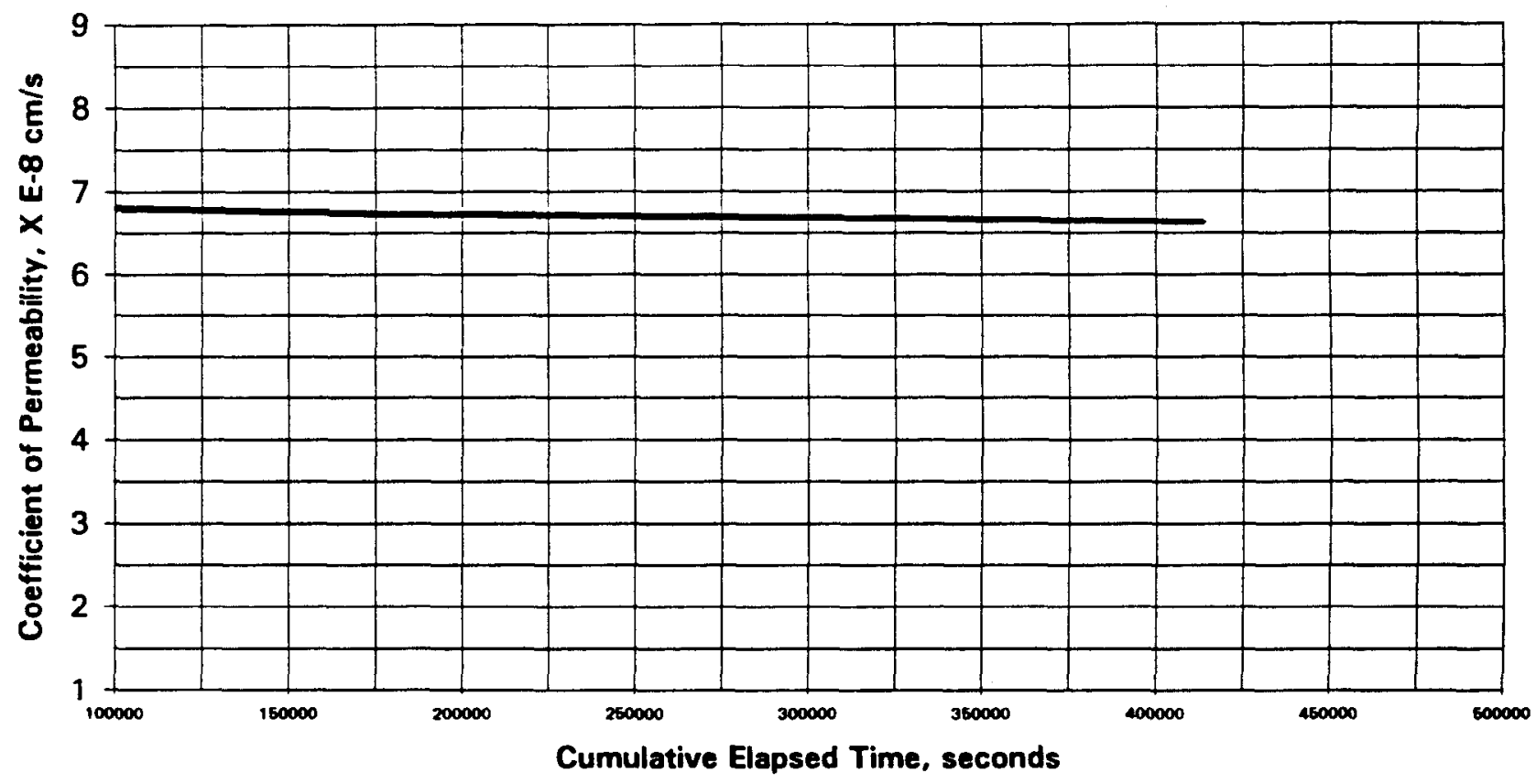




\section{0-DAY SATURATED HYDRAULIC CONDUCTIVITY / PERMEABILITY ASTM D 5084}

PROJECT NAME: PROJECT NO.
SAIC / Savannah River

777304.00010500
CLIENT SAMPLE NO.

IT LAB SAMPLE NO.
C323G00

ETDC-7854

\begin{tabular}{lll|}
\cline { 2 - 3 } & INITIAL & FINAL \\
\cline { 2 - 3 } Specimen diameter, cm & 5.10 & \\
Specimen length, cm & 10.09 & \\
Wet weight of specimen, $\mathrm{g}$. & 396.1 & 396.47 \\
Specimen cross-sect. area, cm 2 & 20.4613 & \\
Water content, \% & 28.0 & 28.1 \\
Wet unit weight, pcf & 119.7 & \\
Dry unit weight, pcf & 93.5 & \\
Estimated degree of saturation, \% & 96.6 & \\
Estimated spec. gravity of solids & 2.65 & \\
Initial conditions are post-soak, pre-test & &
\end{tabular}

Hydraulic gradient

34.9

Min. consolidation stress, psi

Max. consolidation stress, psi

2.0

7.0

3.0

Permeant Fluid

Sample molded

Sample set up

Five tests performed
Deaired DI Water

$11 / 11 / 98$

$3 / 9 / 99$

\section{\begin{tabular}{|ll|}
\hline Coefficient of Permeability, $\mathrm{cm} / \mathrm{s}$ & $6.2 \mathrm{E}-07$ \\
\hline
\end{tabular}}

\section{PERMEABILITY vS TIME}

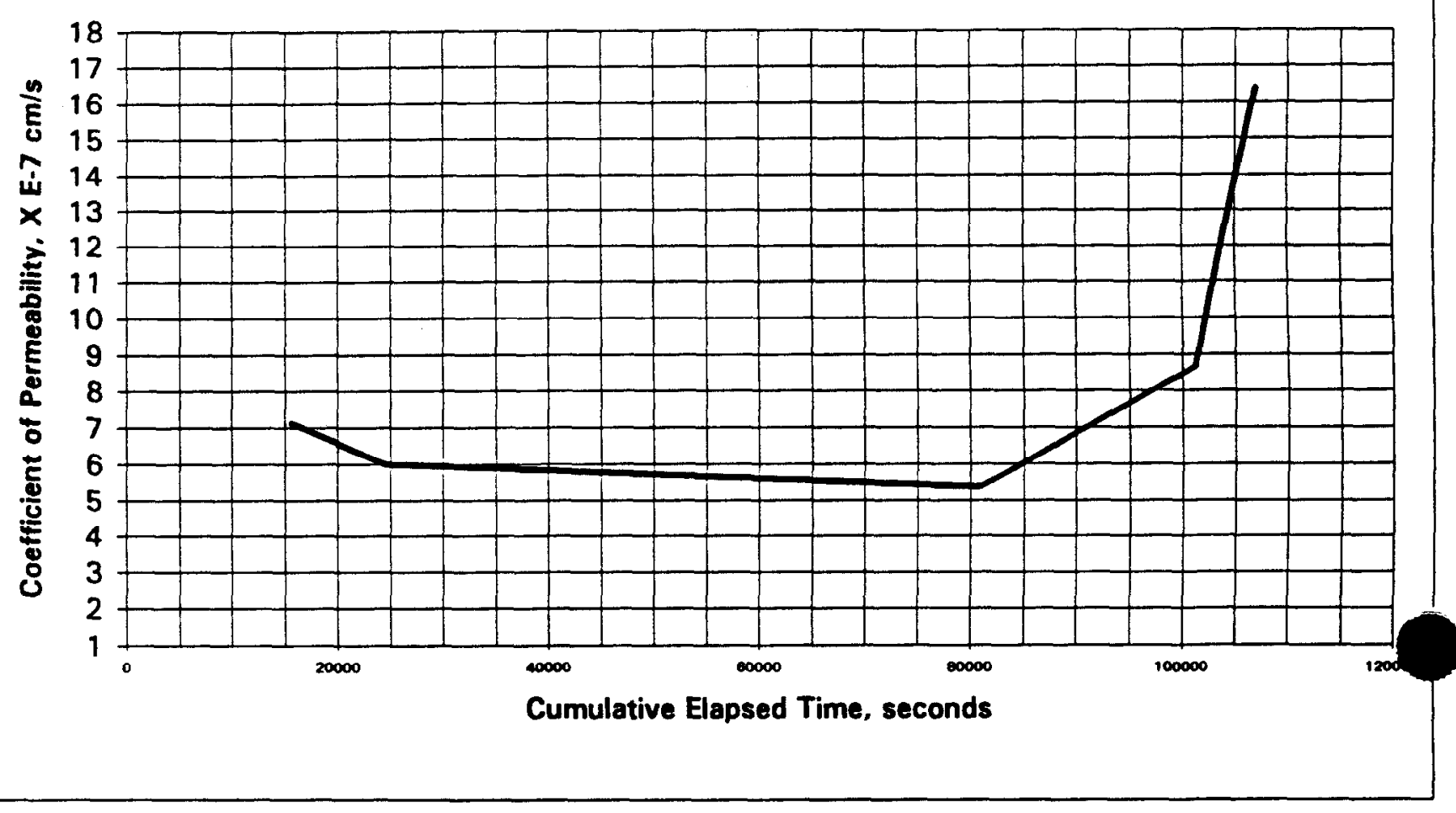




\section{0-DAY SATURATED HYDRAULIC CONDUCTIVITY / PERMEABILITY ASTM D 5084} $\begin{array}{ll}\text { PROJECT NAME: } & \text { SAIC / Savannah River } \\ \text { PROJECT NO. } & 777304.00010500\end{array}$
CLIENT SAMPLE NO.

C333G00

IT LAB SAMPLE NO.

ETDC-7855

\begin{tabular}{llllll} 
& INITIAL & FINAL & & \\
\cline { 2 - 3 } Specimen diameter, cm & 5.04 & & & \\
Specimen length, cm & 10.11 & & Hydraulic gradient & 34.8 \\
Wet weight of specimen, 9. & 376.7 & 376.3 & Min. consolidation stress, psi & 2.0 \\
Specimen cross-sect. area, cm & -2 & 19.9520 & & Max. consolidation stress, psi & 7.0 \\
Water content, \% & 35.4 & 35.2 & Total backpressure, psi & 3.0 \\
Wet unit weight, pcf & 116.6 & & Permeant Fluid & Deaired DI Water \\
Dry unit weight, pcf & 86.1 & & Sample molded & $11 / 11 / 98$ \\
Estimated degree of saturation, \% & 101.8 & & Sample set up & $3 / 8 / 99$
\end{tabular}

Initial conditions are post-soak, pre-test

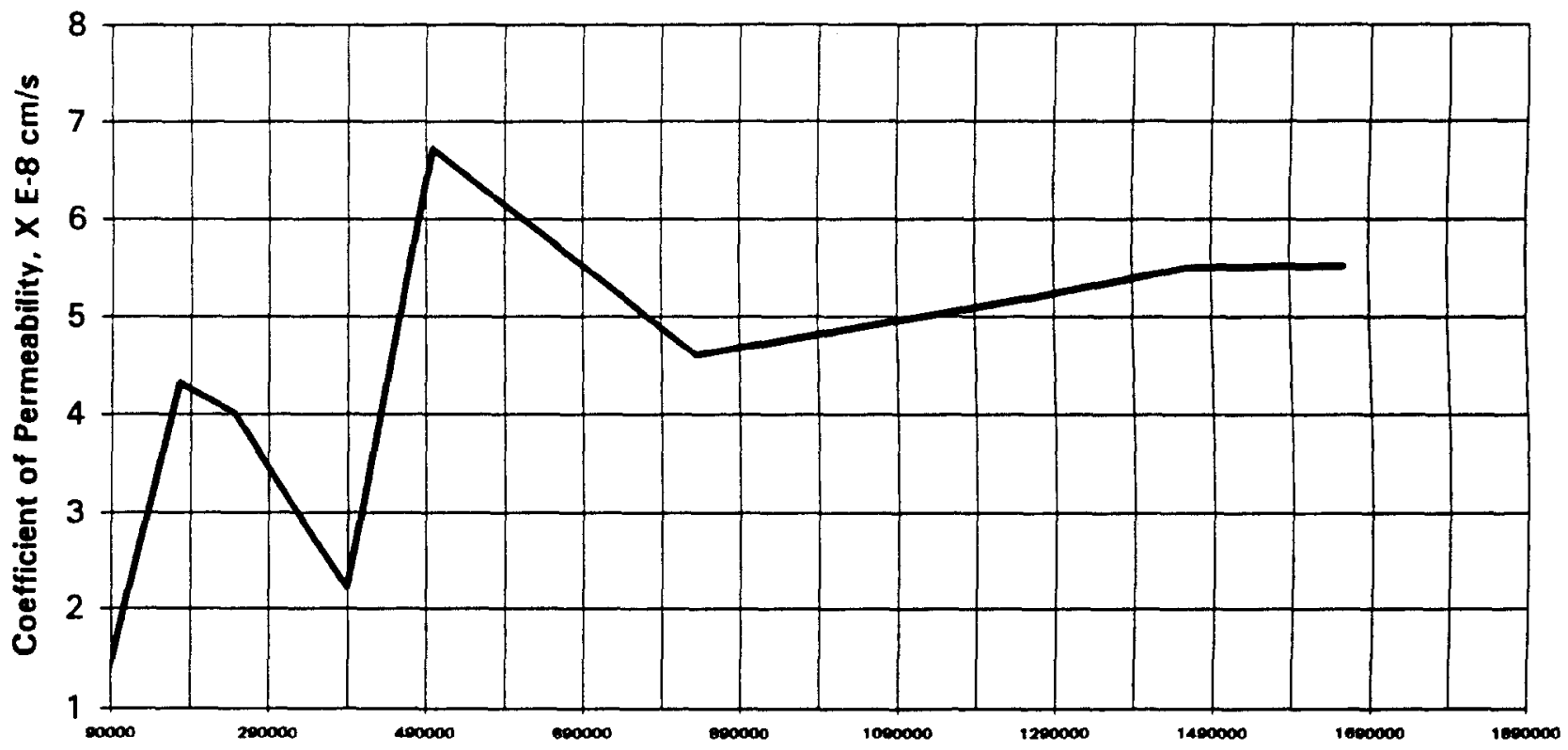

Cumulative Elapsed Time, seconds 


\section{0-DAY SATURATED HYDRAULIC CONDUCTIVITY / PERMEABILITY ASTM D 5084}

PROJECT NAME: SAIC / Savannah River PROJECT NO.
CLIENT SAMPLE NO.

C343G00

IT LAB SAMPLE NO.

ETDC-7856
INITIAL
FINAL

Specimen diameter, $\mathrm{cm}$

5.10

Specimen length, $\mathrm{cm}$

10.11

383.39

383.72

20.4376

32.7

32.8

115.9

87.3

96.8

2.65

\section{Estimated spec. gravity of solids}

Hydraulic gradient

34.8

Min. consolidation stress, psi

2.0

Max. consolidation stress, psi

5.0

5.0

Permeant Fluid

Deaired DI Water

Sample molded

$11 / 11 / 98$

Sample set up

$3 / 8 / 99$

Initial conditions are post-soak, pre-test

\section{\begin{tabular}{|ll}
\hline Coefficient of Permeability, cm/s & $3.0 \mathrm{E}-07$ \\
\hline
\end{tabular}}

\section{PERMEABILITY vs TIME}

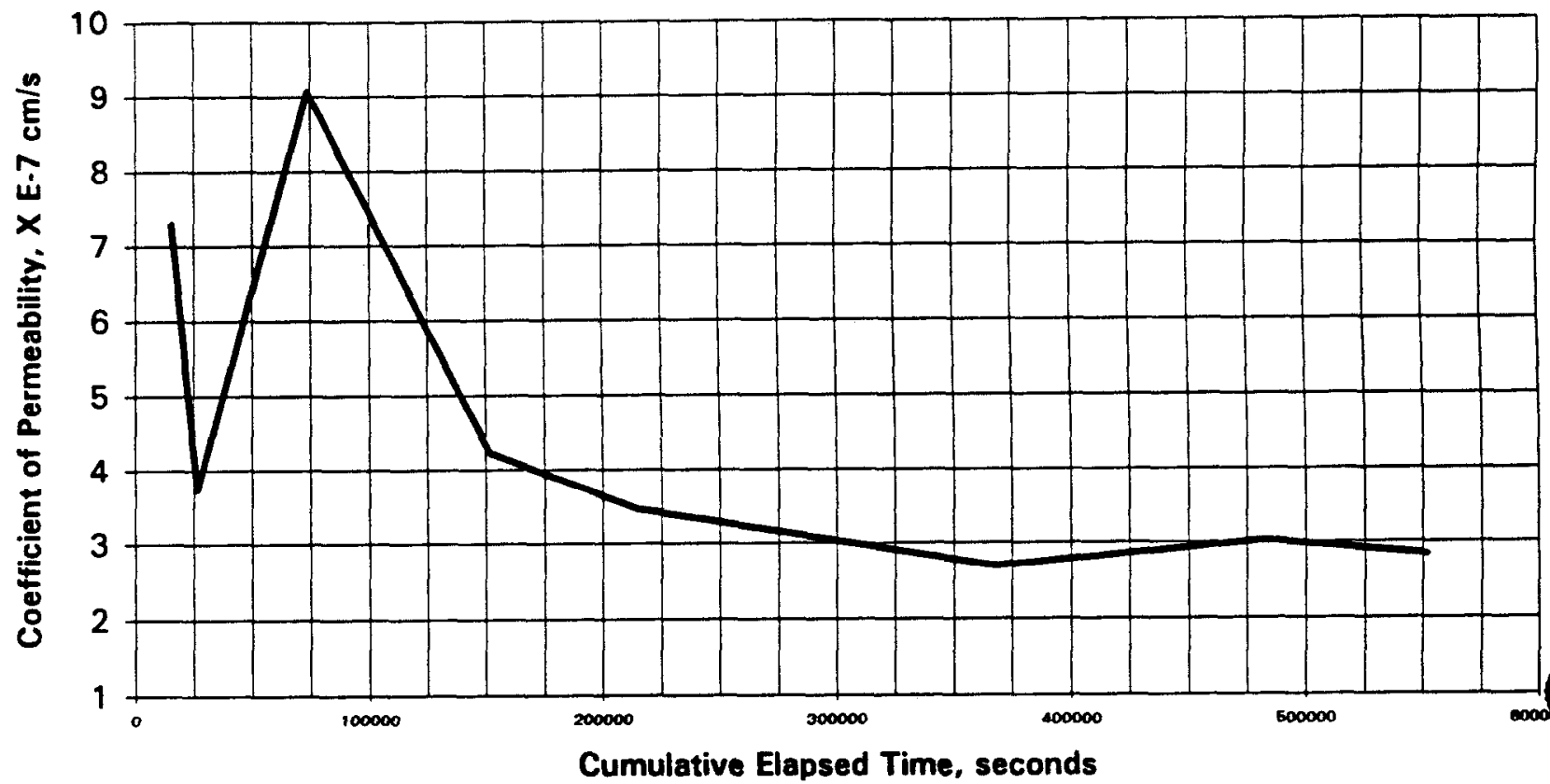




\section{0-DAY SATURATED HYDRAULIC CONDUCTIVITY / PERMEABILITY ASTM D 5084}

PROJECT NAME:

SAIC / Savannah River

PROJECT NO.
CLIENT SAMPLE NO. $\quad$ C413G00

IT LAB SAMPLE NO. ETDC-7857

\begin{tabular}{|c|c|c|c|c|}
\hline \multirow[b]{2}{*}{ Specimen diameter, cm } & INITIAL & FINAL & & \\
\hline & \multicolumn{4}{|l|}{5.09} \\
\hline Specimen length, $\mathrm{cm}$ & 10.09 & & Hydraulic gradient & 34.9 \\
\hline Wet weight of specimen, $g$. & 382.78 & 382.74 & Min. consolidation stress, psi & 2.0 \\
\hline Specimen cross-sect. area, $\mathrm{cm}^{\wedge} 2$ & 20.3224 & & Max. consolidation stress, psi & 3.0 \\
\hline Water content, $\%$ & 34.7 & 34.6 & Total backpressure, psi & 7.0 \\
\hline Wet unit weight, pcf & 116.6 & & Permeant Fluid Deaire & DI Water \\
\hline Dry unit weight, pcf & 86.6 & & Sample molded & $11 / 11 / 98$ \\
\hline Estimated degree of saturation, \% & 100.8 & & Sample set up & $3 / 8 / 99$ \\
\hline Estimated spec. gravity of solids & 2.65 & & Seven tests performed & \\
\hline
\end{tabular}

Initial conditions are post-soak, pre-test

\section{PERMEABILITY VS TIME}

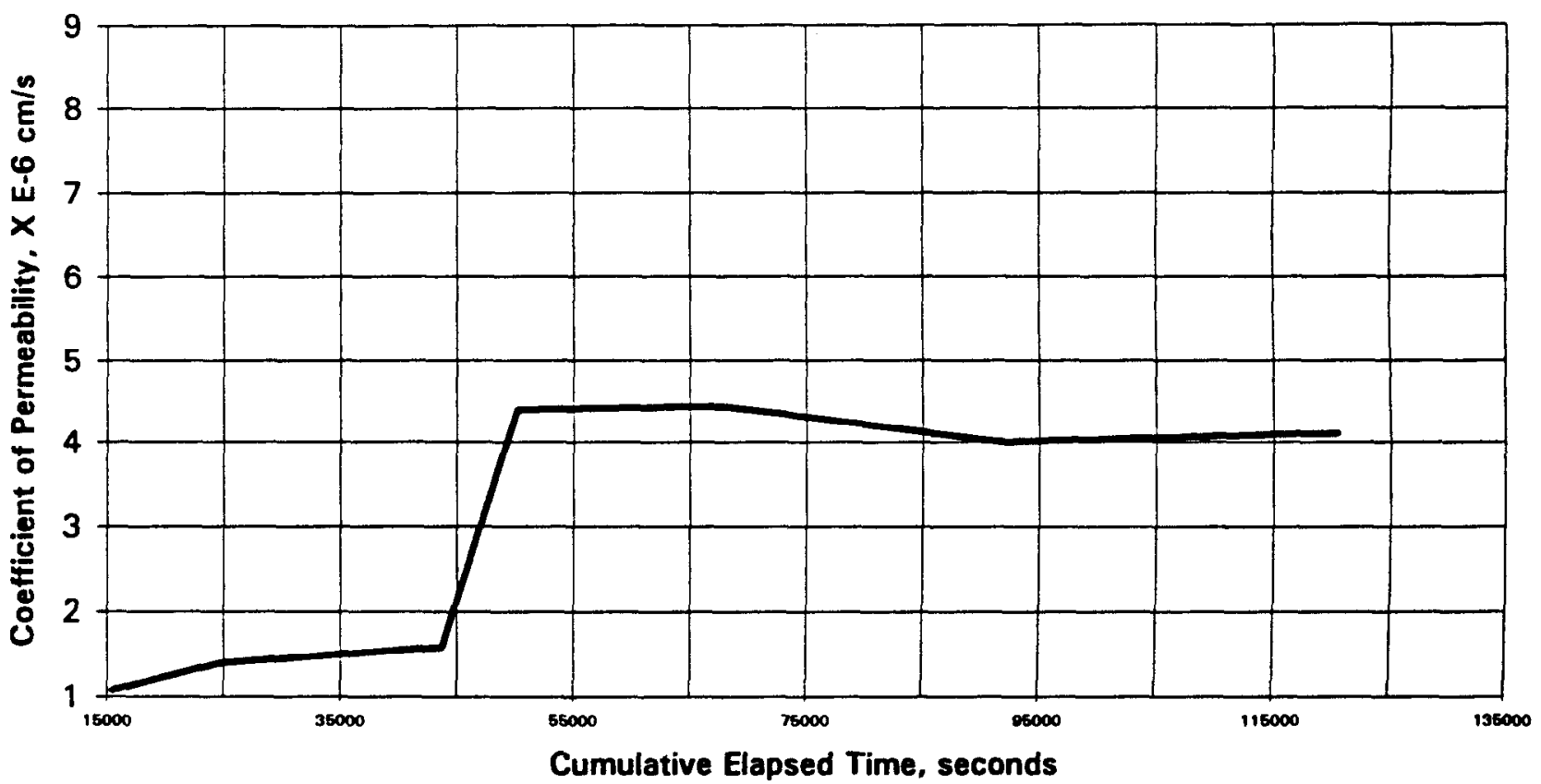




\section{0-DAY SATURATED HYDRAULIC CONDUCTIVITY / PERMEABILITY ASTM D 5084}

PROJECT NAME: SAIC / Savannah River PROJECT NO. $\quad \mathbf{7 7 7 3 0 4 . 0 0 0 1 0 5 0 0}$

\section{CLIENT SAMPLE NO. C423G00 \\ IT LAB SAMPLE NO. ETDC-7858}

Specimen diameter, $\mathrm{cm}$

Specimen length, $\mathrm{cm}$

Wet weight of specimen, $g$.

Specimen cross-sect. area, $\mathrm{cm}^{\wedge} 2$

Water content, \%

Wet unit weight, pcf

Dry unit weight, pcf

Estimated degree of saturation, \%

Estimated spec. gravity of solids

Initial conditions are post-soak, pre-test

INITIAL FINAL

\subsection{0}

10.10

381.59

384.98

20.4003

31.5

115.6

87.9

94.8

2.65
Hydraulic gradient

34.8

Min. consolidation stress, psi

Max. consolidation stress, psi

2.0

4.0

6.0

Deaired DI Water

\section{Sample molded}

$11 / 11 / 98$

Sample set up

$3 / 8 / 99$

\section{Coefficient of Permeability, cm/s} 1.2E-06

\section{PERMEABILITY vS TIME}

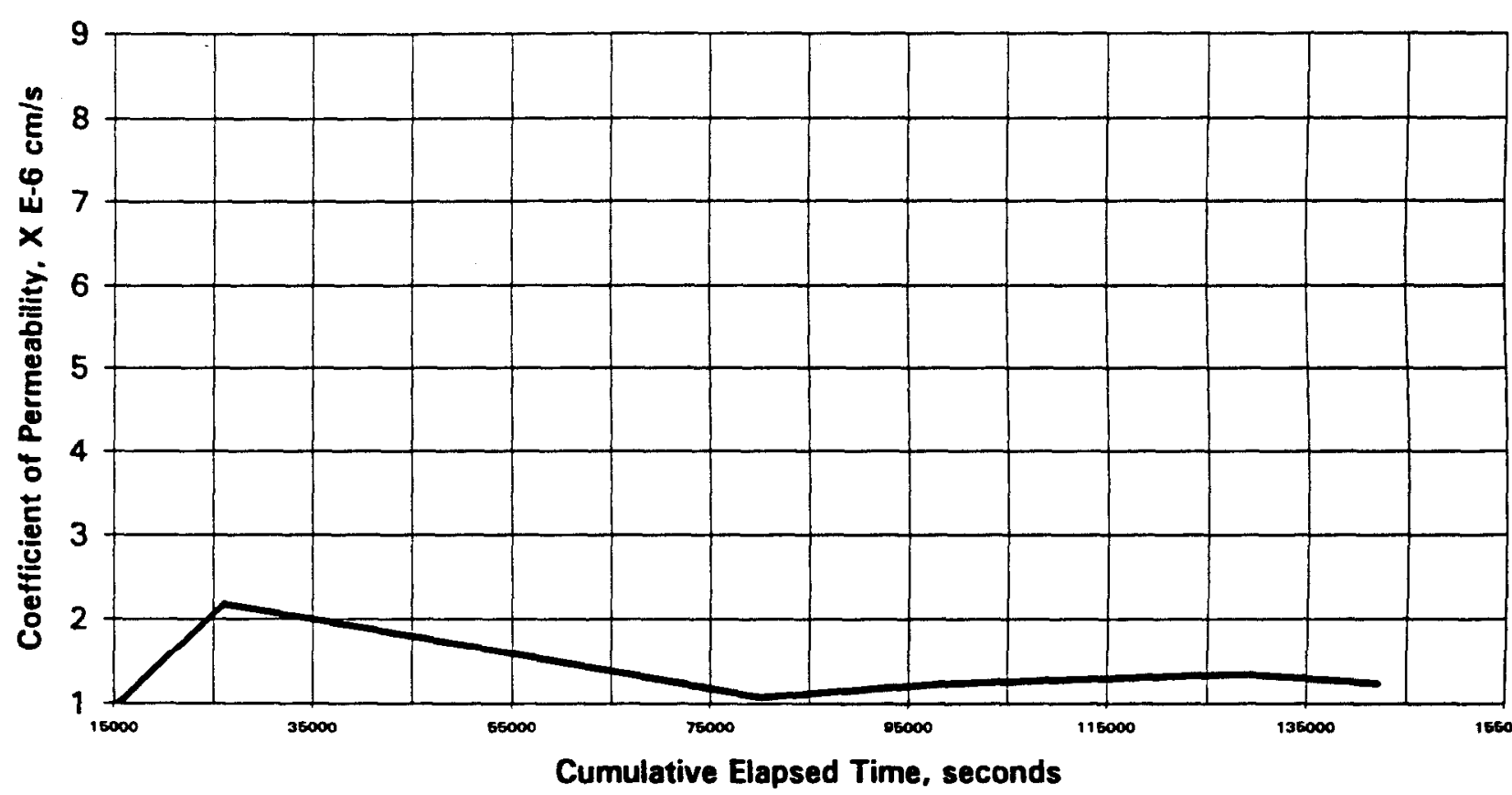




\section{0-DAY SATURATED HYDRAULIC CONDUCTIVITY / PERMEABILITY ASTM D 5084}

PROJECT NAME: SAIC / Savannah River

PROJECT NO. $\quad 777304.00010500$
CLIENT SAMPLE NO. C433G00

IT LAB SAMPLE NO. ETDC-7859
Specimen diameter, $\mathrm{cm}$

Specimen length, $\mathrm{cm}$

Wet weight of specimen, $g$.

Specimen cross-sect. area, $\mathrm{cm}^{\wedge} 2$

Water content, \%

Wet unit weight, pcf

Dry unit weight, pcf

Estimated degree of saturation, \%

Estimated spec. gravity of solids

Initial conditions are post-soak, pre-test

INITIAL FINAL

\subsection{0}

10.08

$365.06 \quad 365.46$

20.4003

40.9

110.9

78.7

98.3

2.65

41.0
Hydraulic gradient

34.9

Min. consolidation stress, psi

Max. consolidation stress, psi

2.0

4.0

Total backpressure, psi

Permeant Fluid

6.0

Sample molded

Sample set up

Thirteen tests performed
Deaired DI Water

$11 / 11 / 98$

$3 / 8 / 99$

\section{\begin{tabular}{|ll|}
\hline Coefficient of Permeability, $\mathrm{cm} / \mathrm{s}$ & $2.8 \mathrm{E}-06$ \\
\hline
\end{tabular}}

\section{PERMEABILITY vs TIME}

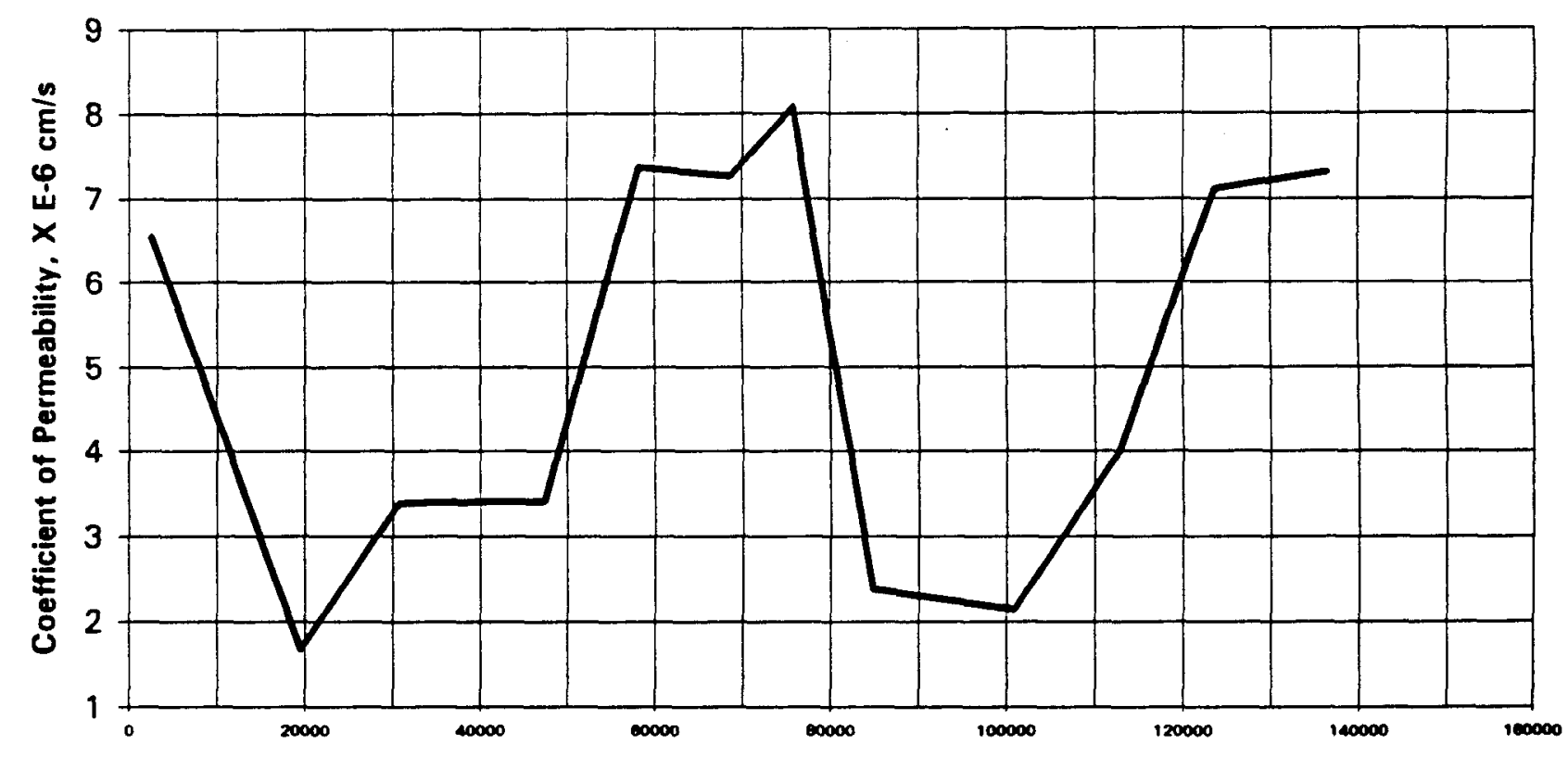

Cumulative Elapsed Time, seconds 


\section{0-DAY SATURATED HYDRAULIC CONDUCTIVITY / PERMEABILITY ASTM D 5084}

PROJECT NAME:

SAIC / Savannah River

777304.00010500

PROJECT NO.
CLIENT SAMPLE NO.

C443G00

Specimen diameter, $\mathrm{cm}$

Specimen length, $\mathrm{cm}$

Wet weight of specimen, $g$.

Specimen cross-sect. area, $\mathrm{cm}^{\wedge} 2$

Water content, \%

Wet unit weight, pcf

Dry unit weight, pcf

Estimated degree of saturation, \%

Estimated spec. gravity of solids

Initial conditions are post-soak, pre-test
IT LAB SAMPLE NO. ETDC-7860

\begin{tabular}{|ll|}
\hline Coefficient of Permeability, cm/s & $7.5 E-07$ \\
\hline
\end{tabular}

\section{PERMEABILITY vS TIME}

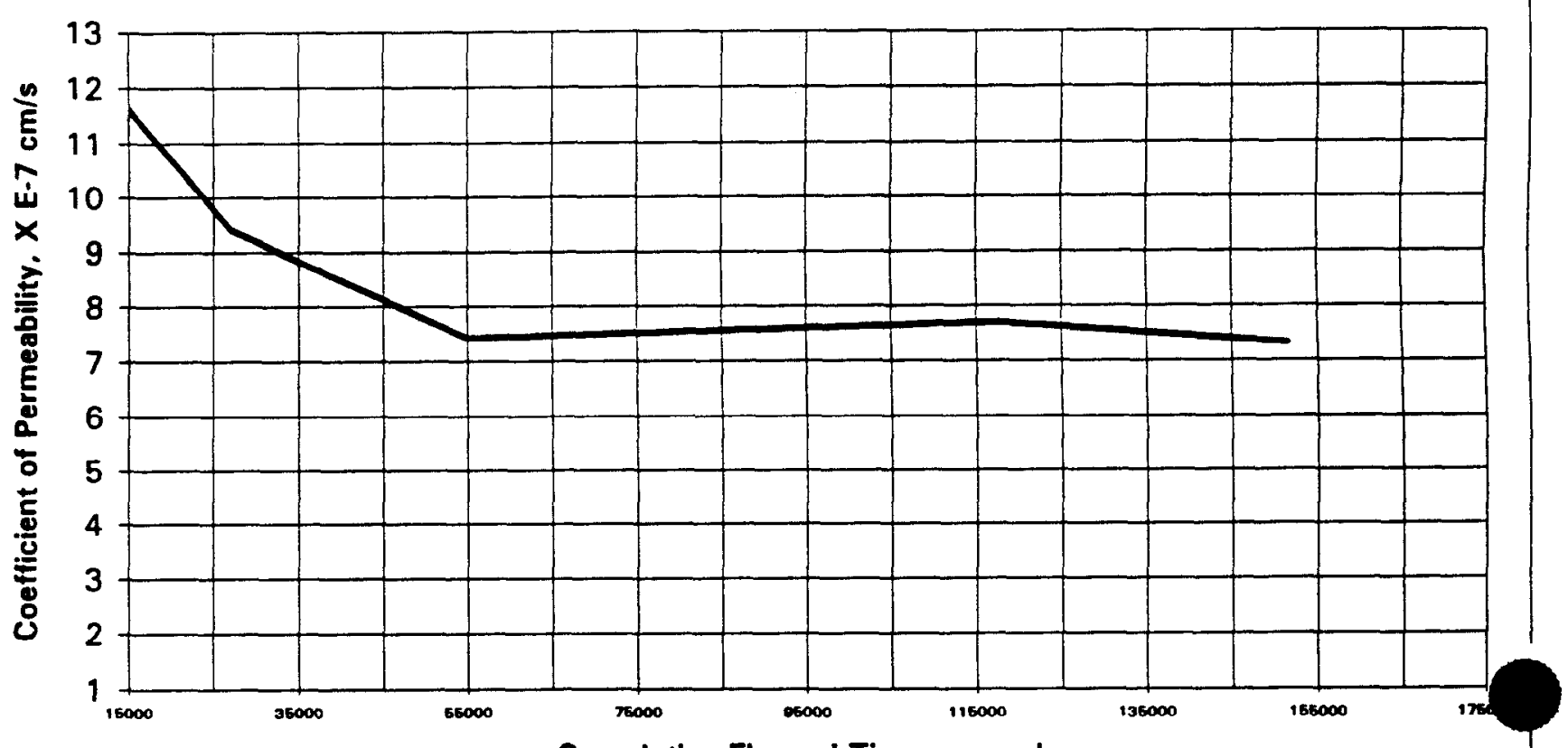

Cumulative Elapsed Time, seconds 


\section{0-DAY SATURATED HYDRAULIC CONDUCTIVITY / PERMEABILITY ASTM D 5084}

PROJECT NAME: SAIC / Savannah River PROJECT NO.
CLIENT SAMPLE NO.

IT LAB SAMPLE NO.
C513G00

ETDC-7861
Specimen diameter, $\mathrm{cm}$

Specimen length, $\mathrm{cm}$

Wet weight of specimen, $g$.

Specimen cross-sect. area, $\mathrm{cm}^{-2} 2$

Water content, \%

Wet unit weight, pcf

Dry unit weight, pcf

Estimated degree of saturation, \% 100.5

Estimated spec. gravity of solids $\quad 2.65$

\begin{tabular}{|l|l|}
\hline INITIAL & FINAL \\
\hline
\end{tabular}

5.09

10.03

$387.85 \quad 387.99$

20.3325

31.7

118.7

31.7

90.2
Hydraulic gradient

28.1

Min. consolidation stress, psi

Max. consolidation stress, psi

2.0

3.0

7.0

Permeant Fluid

Sample molded

Deaired DI Water

Sample set up

$11 / 11 / 98$

$3 / 9 / 99$

Five tests performed

Initial conditions are post-soak, pre-test

\section{PERMEABILITY vs TIME}

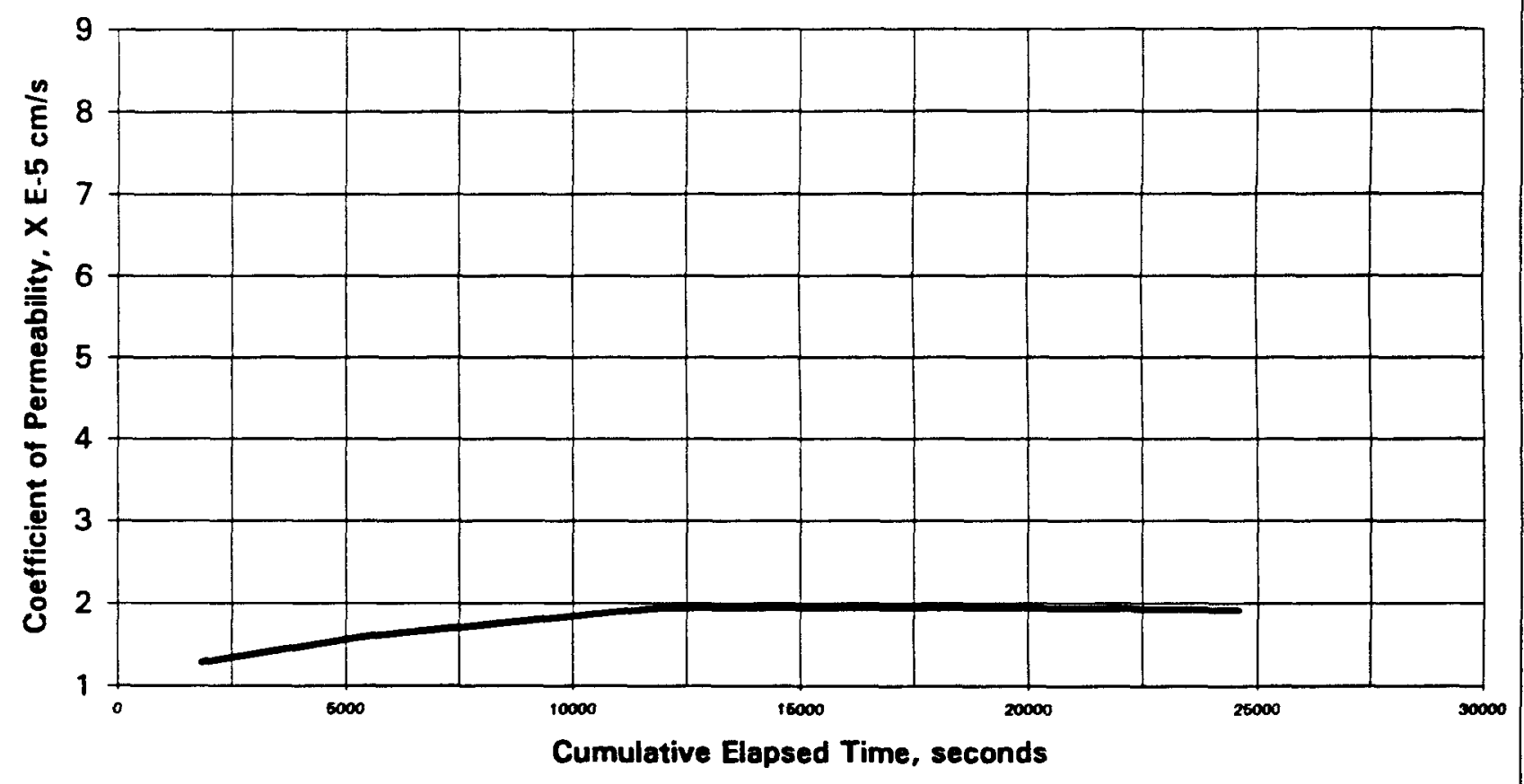




\section{0-DAY SATURATED HYDRAULIC CONDUCTIVITY / PERMEABILITY ASTM D 5084}

PROJECT NAME:

SAIC / Savannah River PROJECT NO.
CLIENT SAMPLE NO. C523G00

IT LAB SAMPLE NO. ETDC-7862
Specimen diameter, $\mathrm{cm}$

Specimen length, $\mathrm{cm}$

Wet weight of specimen, $g$.

Specimen cross-sect. area, $\mathrm{cm}^{-} 2$

Water content, \%

Wet unit weight, pcf

Dry unit weight, pcf

Estimated degree of saturation, \%

Estimated spec. gravity of solids

Initial conditions are post-soak, pre-test

\begin{tabular}{l}
\hline INITIAL FINAL \\
\hline 5.09
\end{tabular}

10.17

395.1

20.3698

28.9

119.1

92.4

96.8

2.65
Hydraulic gradient 395.73

\section{1}

Min. consolidation stress, psi

34.6 Max. consolidation stress, psi

2.0

6.0

4.0

Permeant Fluid

Deaired DI Water

Sample molded

$11 / 11 / 98$

Sample set up

$3 / 9 / 99$

Four tests performed

\section{PERMEABILITY vs TIME}

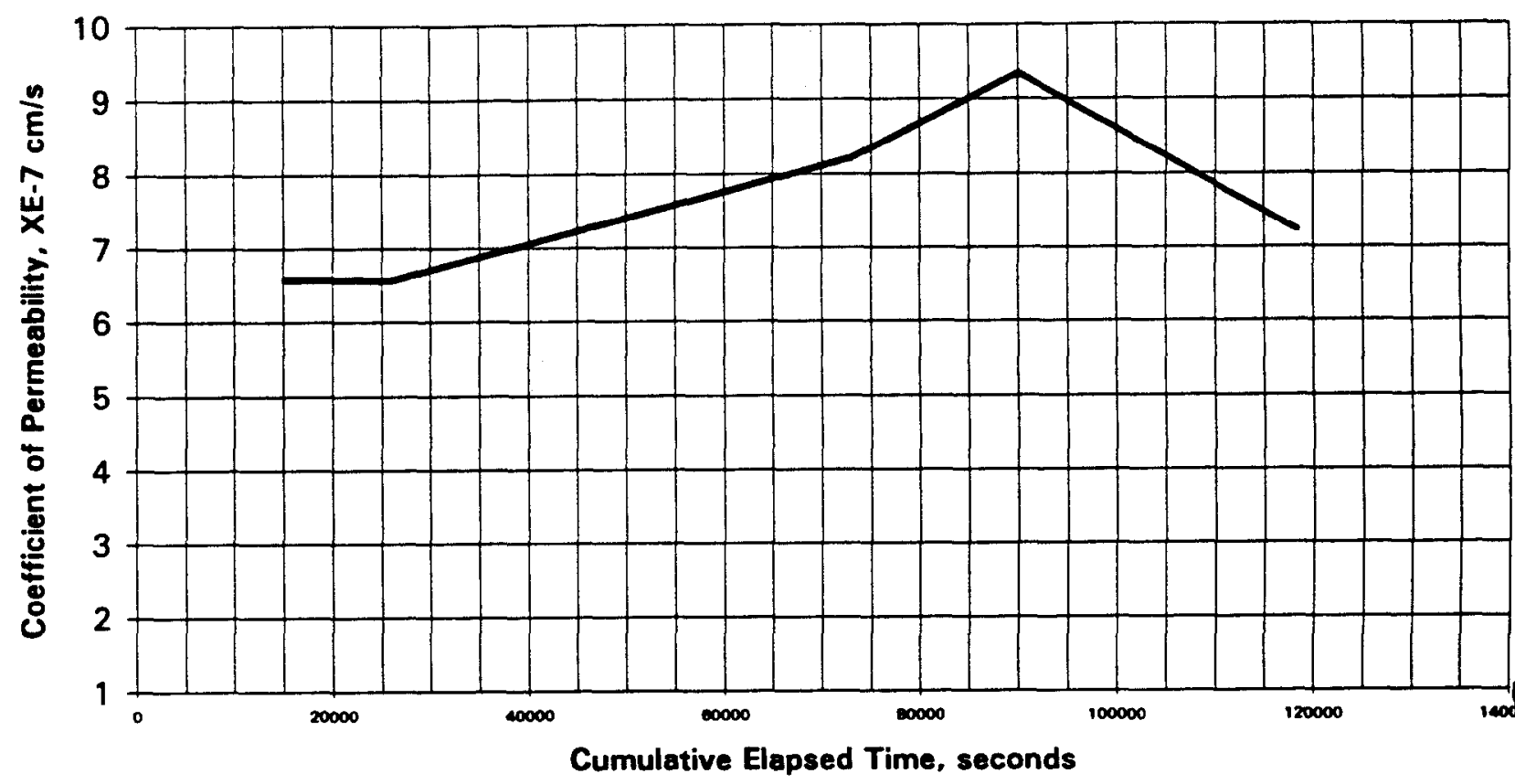




\section{0-DAY SATURATED HYDRAULIC CONDUCTIVITY / PERMEABILITY ASTM D 5084}

PROJECT NAME: PROJECT NO.

SAIC / Savannah River 777304.00010500
CLIENT SAMPLE NO.

IT LAB SAMPLE NO.
C533G00

ETDC-7863

\begin{tabular}{|c|c|c|c|c|}
\hline \multirow[b]{2}{*}{ Snecimen diameter $\mathrm{cm}$} & INITIAL & FINAL & & \\
\hline & \multicolumn{4}{|l|}{5.10} \\
\hline Specimen length, cm & \multicolumn{2}{|l|}{10.15} & \multicolumn{2}{|l|}{ Hydraulic gradient } \\
\hline Wet weight of specimen, $\mathrm{g}$ & 375.31 & 374.96 & \multicolumn{2}{|l|}{ Min. consolidation stress, psi } \\
\hline Specimen cross-sect. area, $\mathrm{cm}^{\wedge} 2$ & \multicolumn{2}{|l|}{20.4036} & \multicolumn{2}{|l|}{ Max. consolidation stress, psi } \\
\hline Water content, $\%$ & 37.2 & 37.1 & Total backpressure, psi & 3.0 \\
\hline Wet unit weight, pcf & \multicolumn{2}{|l|}{113.1} & Permeant Fluid Deaire & Deaired DI Water \\
\hline Dry unit weight, pcf & \multicolumn{2}{|l|}{82.5} & Sample molded & $11 / 11 / 98$ \\
\hline Estimated degree of saturation, \% & \multicolumn{2}{|l|}{98.0} & Sample set up & \multirow[t]{2}{*}{$3 / 9 / 99$} \\
\hline Estimated spec. gravity of solids & \multicolumn{2}{|l|}{2.65} & Seven tests performed & \\
\hline
\end{tabular}

Initial conditions are post-soak, pre-test

\section{PERMEABILITY vs TIME}

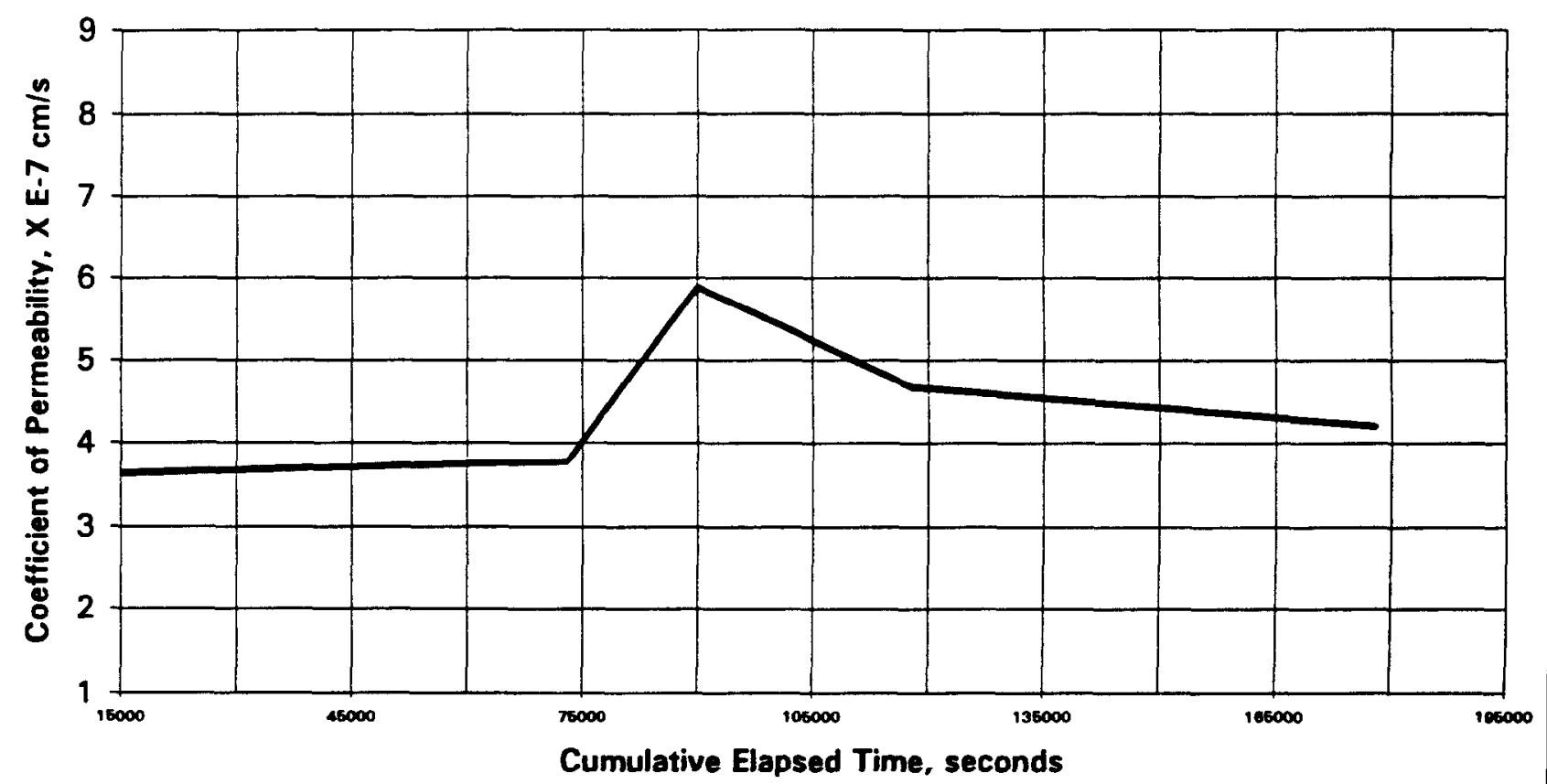




\section{0-DAY SATURATED HYDRAULIC CONDUCTIVITY / PERMEABILITY ASTM D 5084}

PROJECT NAME: SAIC / Savannah River PROJECT NO. $\quad 777304.00010500$
CLIENT SAMPLE NO. C543G00

IT LAB SAMPLE NO. ETDC-7864

\begin{tabular}{|c|c|c|c|c|}
\hline \multirow[b]{2}{*}{ Specimen diameter, $\mathrm{cm}$} & INITIAL & FINAL & & \\
\hline & \multicolumn{4}{|l|}{5.10} \\
\hline Specimen length, cm & 10.16 & & Hydraulic gradient & 34.6 \\
\hline Wet weight of specimen, $g$ & 381.78 & 382.28 & Min. consolidation stress, psi & 2.0 \\
\hline Specimen cross-sect. area, $\mathrm{cm}=2$ & 20.4511 & & Max. consolidation stress, psi & 7.0 \\
\hline Water content, $\%$ & 34.2 & 34.4 & Tota! backpressure, psi & 3.0 \\
\hline Wet unit weight, pcf & 114.7 & & Permeant Fluid Deaire & DI Water \\
\hline Dry unit weight, pcf & 85.5 & & Sample molded & $11 / 11 / 98$ \\
\hline Estimated degree of saturation, $\%$ & 96.8 & & Sample set up & $3 / 8 / 99$ \\
\hline Estimated spec. gravity of solids & 2.65 & & Five tests performed & \\
\hline
\end{tabular}

Initial conditions are post-soak, pre-test

\section{\begin{tabular}{|ll}
\hline Coefficient of Permeability, cm/s & $1.3 E-07$ \\
\hline
\end{tabular}}

\section{PERMEABILITY vS TIME}

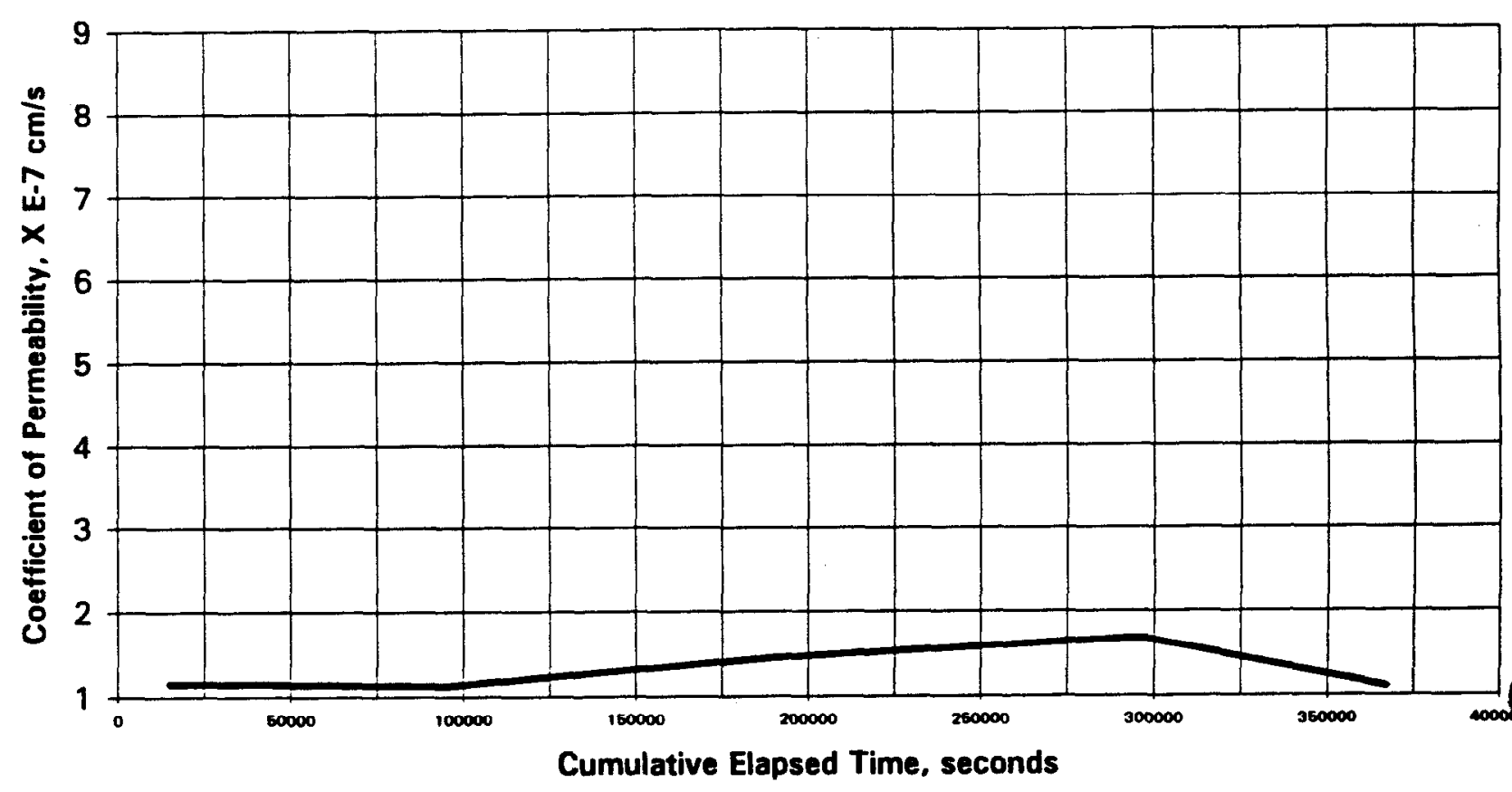




\section{0-DAY SATURATED \\ HYDRAULIC CONDUCTIVITY / PERMEABILITY ASTM D 5084}

PROJECT NAME: SAIC / Savannah River PROJECT NO. $\quad 777304.00010500$
CLIENT SAMPLE NO. C613G00

IT LAB SAMPLE NO. ETDC-7865

\begin{tabular}{|c|c|c|c|c|}
\hline \multirow[b]{2}{*}{ Specimen diameter, cm } & INITIAL & FINAL & & \\
\hline & \multicolumn{4}{|l|}{5.10} \\
\hline Specimen length, $\mathrm{cm}$ & 7.09 & & Hydraulic gradient & 49.6 \\
\hline Wet weight of specimen, $g$ & 265 & 269.7 & Min. consolidation stress, psi & 2.0 \\
\hline Specimen cross-sect. area, $\mathrm{cm}^{`} 2$ & 20.4477 & & Max. consolidation stress, psi & 4.0 \\
\hline Water content, \% & 35.8 & 32.0 & Tota! backpressure, psi & 6.0 \\
\hline Wet unit weight, pcf & 114.1 & & Permeant Fluid Deaire & DI Water \\
\hline Dry unit weight, pcf & 84.0 & & Sample molded & $11 / 11 / 98$ \\
\hline Estimated degree of saturation, $\%$ & 97.9 & & Sample set up & 3/8/99 \\
\hline Estimated spec. gravity of solids & 2.65 & & Eight tests performed & \\
\hline
\end{tabular}

Initial conditions are post-soak, pre-test

\section{PERMEABILITY vS TIME}

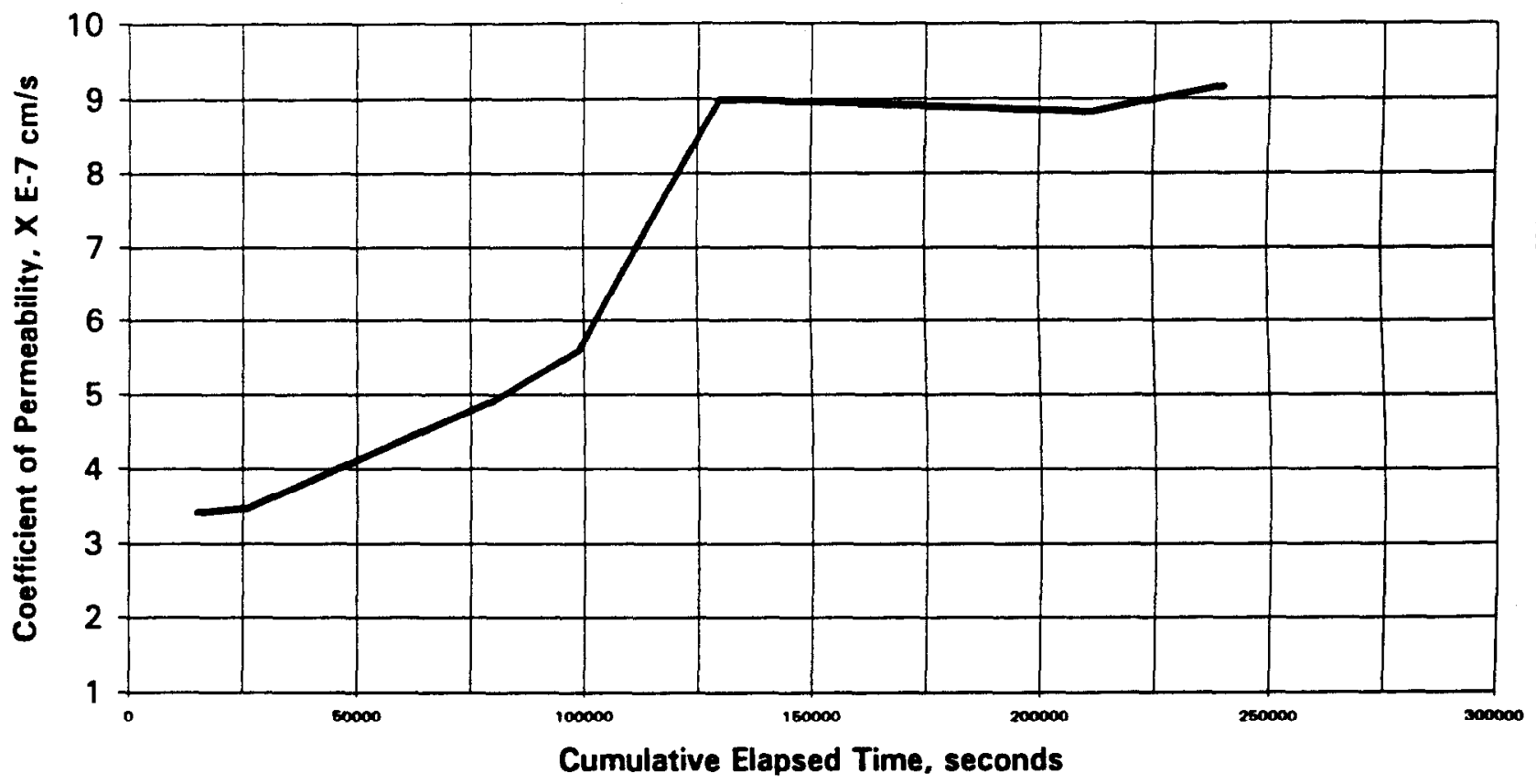




\section{0-DAY SATURATED HYDRAULIC CONDUCTIVITY / PERMEABILITY ASTM D 5084}

PROJECT NAME: SAIC / Savannah River PROJECT NO.
777304.00010500
CLIENT SAMPLE NO.

C623G00

IT LAB SAMPLE NO.

ETDC-7866
Specimen diameter, $\mathrm{cm}$

Specimen length, $\mathrm{cm}$

Wet weight of specimen, $g$.

Specimen cross-sect. area, $\mathrm{cm}^{-2}$

Water content, \%

Wet unit weight, pcf

Dry unit weight, pef

Estimated degree of saturation, \%

Estimated spec. gravity of solids

Initial conditions are post-soak, pre-test

\begin{tabular}{ll|}
\hline INITIAL & FINAL \\
\hline 5.09 & \\
10.09 & \\
392.34 & 392.71 \\
20.3799 & \\
29.3 & 29.4 \\
119.1 & \\
92.1 & \\
97.5 & \\
2.65 &
\end{tabular}

Hydraulic gradient $\quad 34.9$

Min. consolidation stress, psi 2.0

Max. consolidation stress, psi $\quad 7.0$

Total backpressure, psi $\quad 3.0$

Permeant Fluid

Deaired DI Water

Sample molded

Sample set up

$11 / 11 / 98$

$3 / 8 / 99$

\section{Coefficient of Permeability. cm/s}

\section{PERMEABILITY vS TIME}

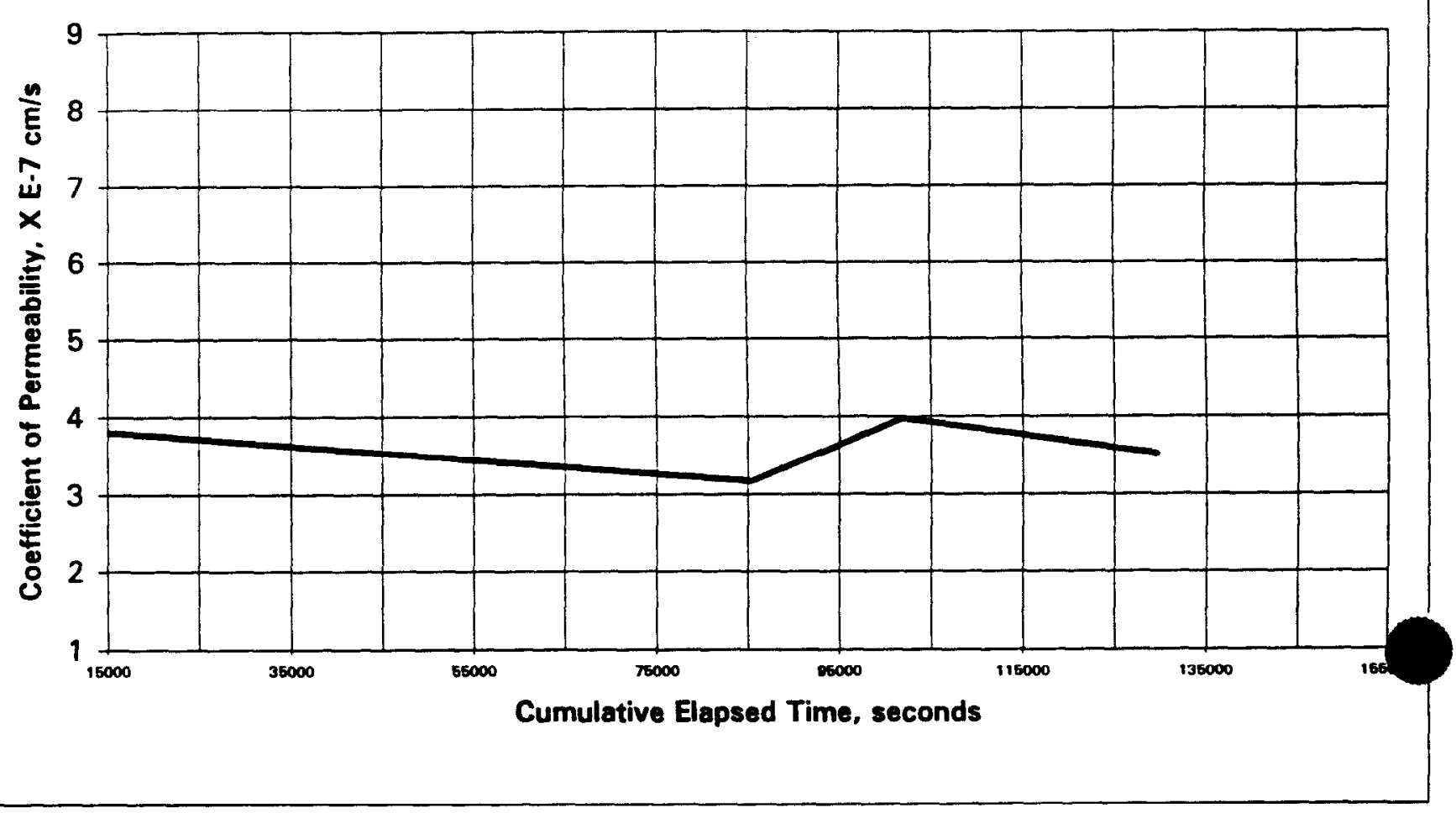




\section{0-DAY SATURATED HYDRAULIC CONDUCTIVITY / PERMEABILITY ASTM D 5084}

PROJECT NAME: SAIC / Savannah River PROJECT NO.
CLIENT SAMPLE NO. IT LAB SAMPLE NO.
C633G00

ETDC-7867

\begin{tabular}{llll}
\cline { 2 - 3 } & INITIAL & FINAL & \\
\cline { 2 - 3 } Specimen diameter, $\mathrm{cm}$ & 5.10 & & \\
Specimen length, $\mathrm{cm}$ & 10.13 & & Hydraulic gradient \\
Wet weight of specimen, g. & 374.18 & 374.79 & Min. consolidation stress, \\
Specimen cross-sect. area, $\mathrm{cm}^{-2}$ & 20.4240 & & Max. consolidation stress, \\
Water content, \% & 37.8 & 38.0 & Total backpressure, psi \\
Wet unit weight, pcf & 112.9 & & Permeant Fluid \\
Dry unit weight, pcf & 81.9 & Sample molded \\
Estimated degree of saturation, \% & 98.3 & Sample set up \\
Estimated spec. gravity of solids & 2.65 & Five tests performed \\
Initial conditions are post-soak, pre-test & & \\
\hline
\end{tabular}

\section{PERMEABILITY vS TIME}






\section{0-DAY SATURATED HYDRAULIC CONDUCTIVITY / PERMEABILITY ASTM D 5084}

PROJECT NAME:

SAIC / Savannah River

PROJECT NO.
CLIENT SAMPLE NO. C643G00

IT LAB SAMPLE NO. ETDC-7868
Specimen diameter, $\mathrm{cm}$

Specimen length, $\mathrm{cm}$

Wet weight of specimen, $g$.

Specimen cross-sect. area, $\mathrm{cm}^{-} 2$

Water content, \%

Wet unit weight, pcf

Dry unit weight, pcf

Estimated degree of saturation, \%

Estimated spec. gravity of solids

Initial conditions are post-soak, pre-test

INITIAL FINAL

\subsection{0}

10.13

381.27

381.56

20.4003

34.8

34.9

115.2

85.5

98.4

2.65

Hydraulic gradient

34.7

Min. consolidation stress, psi

2.0

Max. consolidation stress, psi

7.0

Tota! backpressure, psi

3.0

Permeant Fluid

Deaired DI Water

Sample molded

$11 / 11 / 98$

Sample set up

$3 / 8 / 99$

Ten tests performed

\section{\begin{tabular}{|ll|}
\hline Coefficient of Permeability. $\mathrm{cm} / \mathrm{s}$ & $2.4 \mathrm{E}-07$ \\
\hline
\end{tabular}}

\section{PERMEABILITY vs TIME}

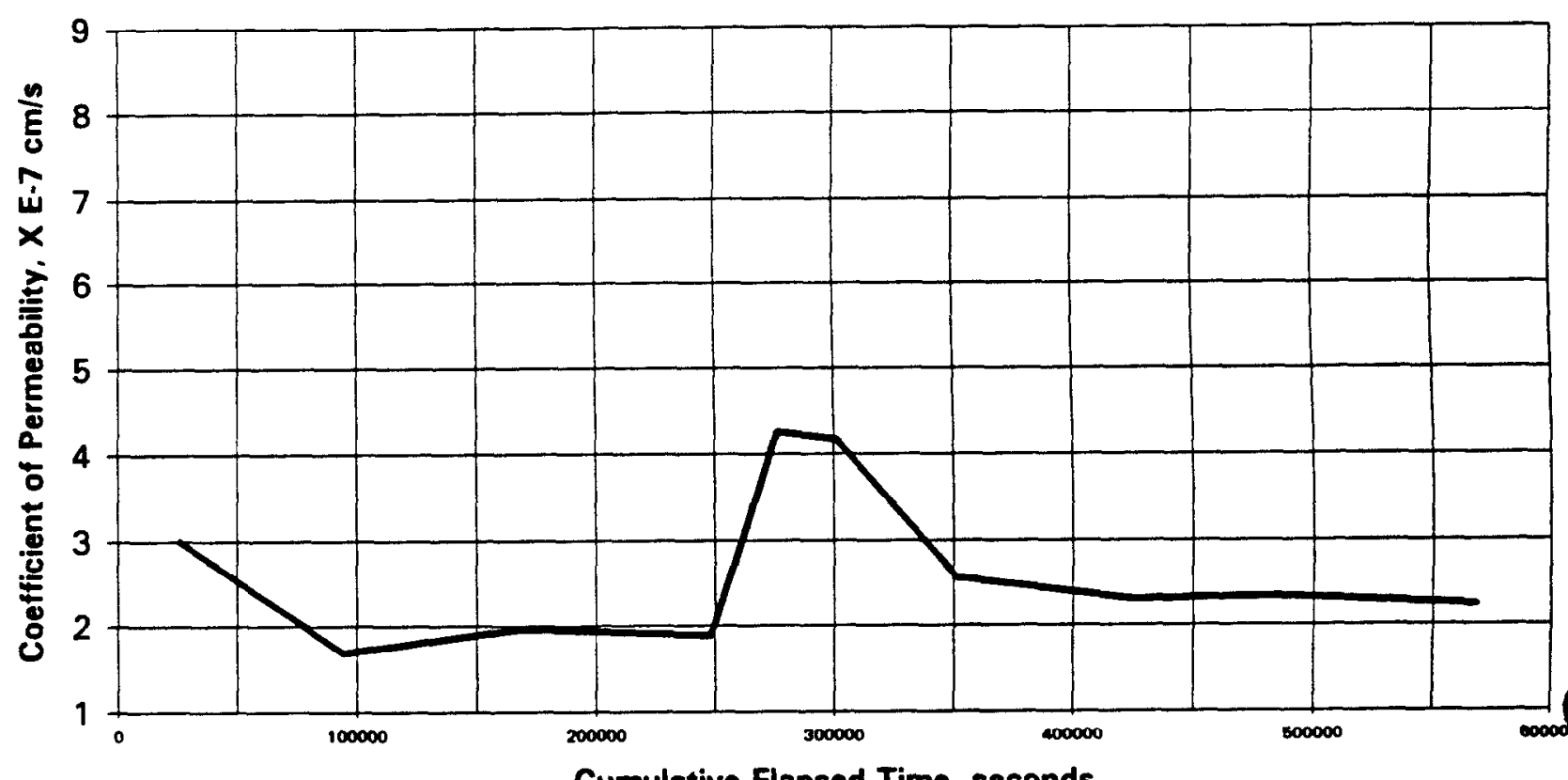

Cumulative Elapsed Time, seconds 


\section{Series \\ Leach Index}




\section{Gross Alpha Leaching for the Coarse Fraction from CRSB Soll}

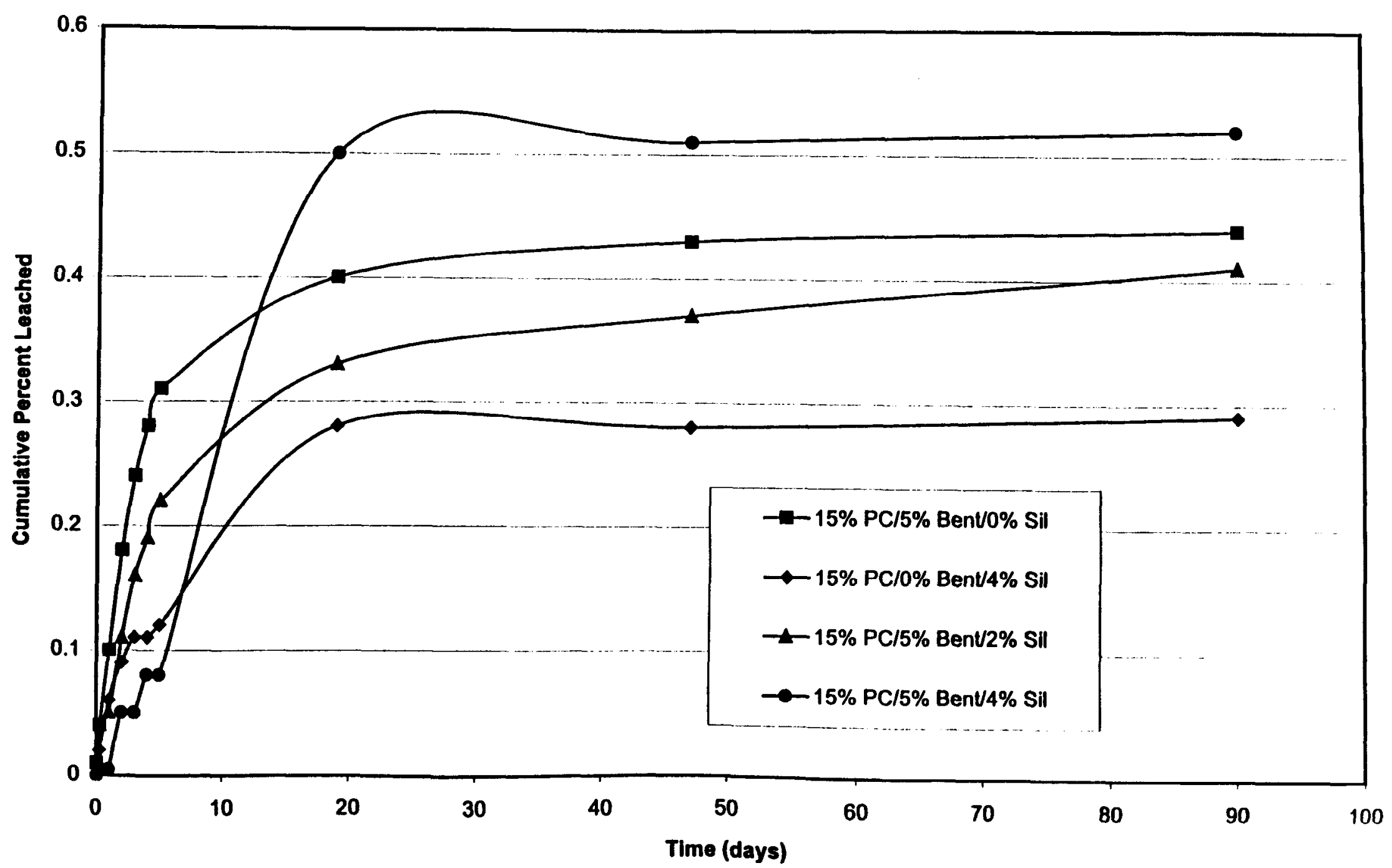


Gross Beta Leaching for the Coarse Fraction from the CSRB Soil

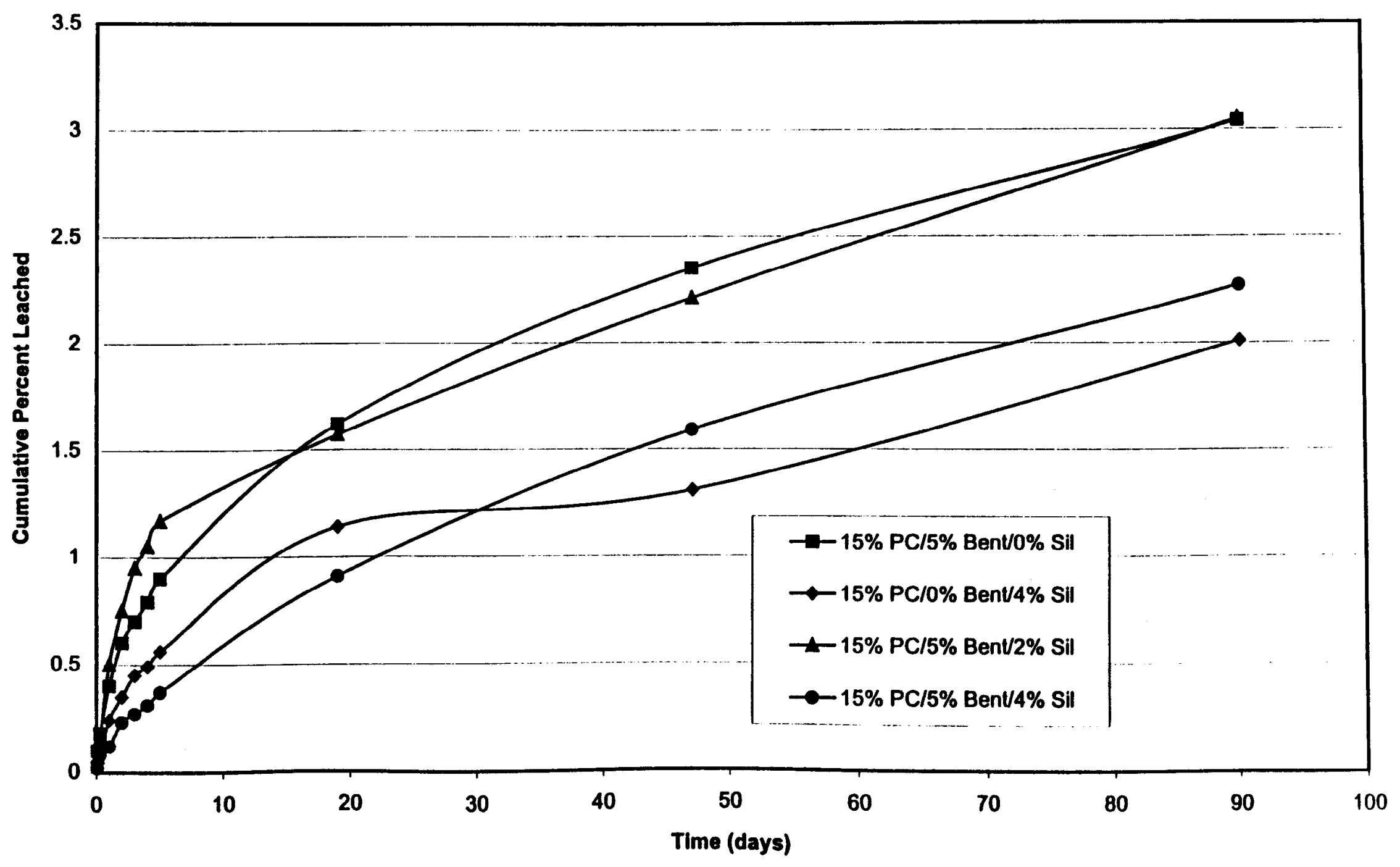




\section{American Nuclear Society - 16.1 Static Leach Test}

Project: SRS Reactor Seepage Basin Stabilization

Sample Description: $\quad$ C312

Analyst: PRL

Date: $\quad$ 15-Apr-99

Contaminant:

Gross Alpha

Initial Contaminant Conc. (pCi/g):

Initial Contaminant Level (pCi):

Monolith Height (cm):

5184

10

176.72

Monolith Surface Area $\left(\mathrm{cm}^{\wedge} 2\right)$ :

196.35

Sample Weight (g):

384

Monolith Volume $\left(\mathrm{cm}^{\wedge} 3\right)$ :

Monolith Diameter (cm):

5

Leachate Volume $(\mathrm{mL})$ :

1800

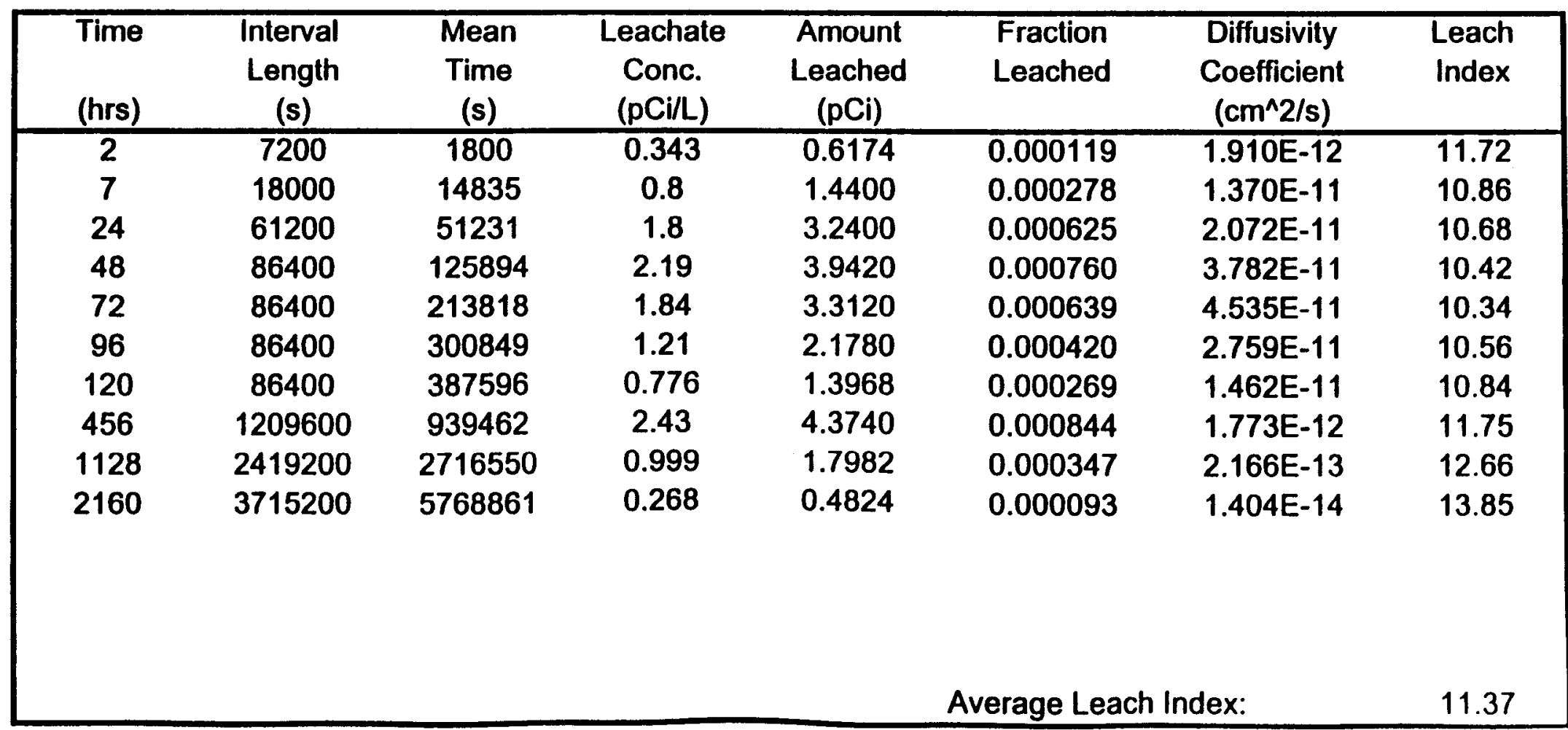




\section{American Nuclear Society - 16.1 Static Leach Test}

Project: $\quad$ SRS Reactor Seepage Basin Stabilization

Sample Description: $\quad$ C312

Analyst: PRL

Date:

15-Apr-99

Contaminant:

Gross Beta

Initial Contaminant Conc. (pCi/g):

170

65280

Monolith Height (cm):

Monolith Surface Area ( $\left.\mathrm{cm}^{\wedge} 2\right)$ :

Monolith Volume $\left(\mathrm{cm}^{\wedge} 3\right)$ :
10

176.72

196.35
Sample Weight (g):

Monolith Diameter (cm): Leachate Volume (mL):
384

5

1800

\begin{tabular}{|c|c|c|c|c|c|c|c|}
\hline $\begin{array}{l}\text { Time } \\
\text { (hrs) }\end{array}$ & $\begin{array}{l}\text { Interval } \\
\text { Length } \\
\text { (s) }\end{array}$ & $\begin{array}{c}\text { Mean } \\
\text { Time } \\
\text { (s) }\end{array}$ & $\begin{array}{l}\text { Leachate } \\
\text { Conc. } \\
\text { (pCi/L) }\end{array}$ & $\begin{array}{l}\text { Amount } \\
\text { Leached } \\
\text { (pCi) }\end{array}$ & $\begin{array}{l}\text { Fraction } \\
\text { Leached }\end{array}$ & $\begin{array}{l}\text { Diffusivity } \\
\text { Coefficient } \\
\text { (cm^2/s) }\end{array}$ & $\begin{array}{l}\text { Leach } \\
\text { Index }\end{array}$ \\
\hline 2 & 7200 & 1800 & 36.3 & 65.3400 & 0.001001 & $1.349 \mathrm{E}-10$ & 9.87 \\
\hline 7 & 18000 & 14835 & 28.1 & 50.5800 & 0.000775 & $1.066 \mathrm{E}-10$ & 9.97 \\
\hline 24 & 61200 & 51231 & 81.6 & 146.8800 & 0.002250 & $2.686 \mathrm{E}-10$ & 9.57 \\
\hline 48 & 86400 & 125894 & 72.8 & 131.0400 & 0.002007 & 2.636E-10 & 9.58 \\
\hline 72 & 86400 & 213818 & 35.5 & 63.9000 & 0.000979 & 1.064E-10 & 9.97 \\
\hline 96 & 86400 & 300849 & 31.5 & 56.7000 & 0.000869 & $1.179 \mathrm{E}-10$ & 9.93 \\
\hline 120 & 86400 & 387596 & 39.4 & 70.9200 & 0.001086 & 2.377E-10 & 9.62 \\
\hline 456 & 1209600 & 939462 & 263 & 473.4000 & 0.007252 & $1.310 \mathrm{E}-10$ & 9.88 \\
\hline 1128 & 2419200 & 2716550 & 264 & 475.2000 & 0.007279 & $9.540 E-11$ & 10.02 \\
\hline 2160 & 3715200 & 5768861 & 249 & 448.2000 & 0.006866 & $7.641 \mathrm{E}-11$ & 10.12 \\
\hline & & & & \multicolumn{3}{|c|}{ Average Leach Index: } & 9.85 \\
\hline
\end{tabular}




\section{American Nuclear Society - 16.1 Static Leach Test}

Project: $\quad$ SRS Reactor Seepage Basin Stabilization

Sample Description: $\quad$ C322

$\begin{array}{lll}\text { Analyst: } & \text { PRL } & \text { 15-Apr-99 }\end{array}$

Contaminant: Gross Alpha

Initial Contaminant Conc. (pCi/g):

13.5

Initial Contaminant Level (pCi):

Monolith Height (cm):

5279.445

Monolith Surface Area $\left(\mathrm{cm}^{\wedge} 2\right)$ :

176.72

Monolith Volume $\left(\mathrm{cm}^{\wedge} 3\right)$ :

196.35

\section{Sample Weight $(g): \quad 391.07$}

Monolith Diameter $(\mathrm{cm}):$

Leachate Volume $(\mathrm{mL}): \quad 1800$

\begin{tabular}{|c|c|c|c|c|c|c|c|}
\hline $\begin{array}{l}\text { Time } \\
\text { (hrs) }\end{array}$ & $\begin{array}{l}\text { Interval } \\
\text { Length } \\
\text { (s) }\end{array}$ & $\begin{array}{l}\text { Mean } \\
\text { Time } \\
(s)\end{array}$ & $\begin{array}{l}\text { Leachate } \\
\text { Conc. } \\
\text { (pCi/L) }\end{array}$ & $\begin{array}{l}\text { Amount } \\
\text { Leached } \\
\text { (pCi) }\end{array}$ & $\begin{array}{l}\text { Fraction } \\
\text { Leached }\end{array}$ & $\begin{array}{l}\text { Diffusivity } \\
\text { Coefficient } \\
\left(\mathrm{cm}^{\wedge} 2 / \mathrm{s}\right)\end{array}$ & $\begin{array}{l}\text { Leach } \\
\text { Index }\end{array}$ \\
\hline 2 & 7200 & 1800 & 0.254 & 0.4572 & 0.000087 & $1.010 E-12$ & 12.00 \\
\hline 7 & 18000 & 14835 & 0.432 & 0.7776 & 0.000147 & 3.853E-12 & 11.41 \\
\hline 24 & 61200 & 51231 & 0.932 & 1.6776 & 0.000318 & 5.357E-12 & 11.27 \\
\hline 48 & 86400 & 125894 & 0.879 & 1.5822 & 0.000300 & 5.875E-12 & 11.23 \\
\hline 72 & 86400 & 213818 & 0.599 & 1.0782 & 0.000204 & 4.633E-12 & 11.33 \\
\hline 96 & 86400 & 300849 & 0.125 & 0.2250 & 0.000043 & 2.839E-13 & 12.55 \\
\hline 120 & 86400 & 387596 & 0.24 & 0.4320 & 0.000082 & 1.348E-12 & 11.87 \\
\hline 456 & 1209600 & 939462 & 4.63 & 8.3340 & 0.001579 & 6.206E-12 & 11.21 \\
\hline 1128 & 2419200 & 2716550 & 0.1 & 0.1800 & 0.000034 & $2.093 E-15$ & 14.68 \\
\hline 2160 & 3715200 & 5768861 & 0.475 & 0.8550 & 0.000162 & $4.252 E-14$ & 13.37 \\
\hline & & & & & \multicolumn{2}{|c|}{ Average Leach Index: } & 12.09 \\
\hline
\end{tabular}




\section{American Nuclear Society - 16.1 Static Leach Test}

Project: $\quad$ SRS Reactor Seepage Basin Stabilization

Sample Description: $\quad$ C322

Analyst: $\quad$ PRL

Date:

15-Apr-99

Contaminant:

Gross Beta

Initial Contaminant Conc. (pCi/g):

170

Initial Contaminant Level (pCi):

Monolith Height (cm):

66481.9

Monolith Surface Area ( $\left.\mathrm{cm}^{\wedge} 2\right)$ :

Monolith Volume $\left(\mathrm{cm}^{\wedge} 3\right)$ :

10

176.72

196.35
Sample Weight (g):

Monolith Diameter (cm):

Leachate Volume $(\mathrm{mL})$ :
391.07

5

1800

\begin{tabular}{|c|c|c|c|c|c|c|c|}
\hline $\begin{array}{l}\text { Time } \\
\text { (hrs) }\end{array}$ & $\begin{array}{l}\text { Interval } \\
\text { Length } \\
\text { (s) }\end{array}$ & $\begin{array}{l}\text { Mean } \\
\text { Time } \\
\text { (s) }\end{array}$ & $\begin{array}{l}\text { Leachate } \\
\text { Conc. } \\
\text { (pCi/L) }\end{array}$ & $\begin{array}{l}\text { Amount } \\
\text { Leached } \\
\text { (pCi) }\end{array}$ & $\begin{array}{l}\text { Fraction } \\
\text { Leached }\end{array}$ & $\begin{array}{l}\text { Diffusivity } \\
\text { Coefficient } \\
\text { (cm^2/s) }\end{array}$ & $\begin{array}{l}\text { Leach } \\
\text { Index }\end{array}$ \\
\hline 2 & 7200 & 1800 & 17.8 & 32.0400 & 0.000482 & $3.128 \mathrm{E}-11$ & 10.50 \\
\hline 7 & 18000 & 14835 & 10.8 & 19.4400 & 0.000292 & $1.518 \mathrm{E}-11$ & 10.82 \\
\hline 24 & 61200 & 51231 & 61.4 & 110.5200 & 0.001662 & 1.466E-10 & 9.83 \\
\hline 48 & 86400 & 125894 & 41 & 73.8000 & 0.001110 & 8.060E-11 & 10.09 \\
\hline 72 & 86400 & 213818 & 35.7 & 64.2600 & 0.000967 & $1.038 E-10$ & 9.98 \\
\hline 96 & 86400 & 300849 & 14.2 & 25.5600 & 0.000384 & 2.310E-11 & 10.64 \\
\hline 120 & 86400 & 387596 & 26 & 46.8000 & 0.000704 & 9.979E-11 & 10.00 \\
\hline 456 & 1209600 & 939462 & 215 & 387.0000 & 0.005821 & 8.439E-11 & 10.07 \\
\hline 1128 & 2419200 & 2716550 & 61.7 & 111.0600 & 0.001671 & $5.024 E-12$ & 11.30 \\
\hline 2160 & 3715200 & 5768861 & 258 & 464.4000 & 0.006985 & $7.910 \mathrm{E}-11$ & 10.10 \\
\hline & & & & \multicolumn{3}{|c|}{ Average Leach Index: } & 10.33 \\
\hline
\end{tabular}




\section{American Nuclear Society - 16.1 Static Leach Test}

Project: $\quad$ SRS Reactor Seepage Basin Stabilization

Sample Description:

C332

Analyst: PRL

Date:

15-Apr-99

Contaminant:

Gross Alpha

Initial Contaminant Conc. (pCi/g):

13.5

Initial Contaminant Level (pCi):

Monolith Height $(\mathrm{cm})$ :

Monolith Surface Area $\left(\mathrm{cm}^{\wedge} 2\right)$ :

Monolith Volume $\left(\mathrm{cm}^{\wedge} 3\right)$ :

5054.265

10

176.72

196.35
Sample Weight (g):

Monolith Diameter (cm): Leachate Volume ( $\mathrm{mL})$ :
374.39

5

1800

\begin{tabular}{|c|c|c|c|c|c|c|c|}
\hline $\begin{array}{l}\text { Time } \\
\text { (hrs) }\end{array}$ & $\begin{array}{l}\text { Interval } \\
\text { Length } \\
\text { (s) }\end{array}$ & $\begin{array}{l}\text { Mean } \\
\text { Time } \\
\text { (s) }\end{array}$ & $\begin{array}{l}\text { Leachate } \\
\text { Conc. } \\
\text { (pCi/L) }\end{array}$ & $\begin{array}{c}\text { Amount } \\
\text { Leached } \\
(p C i)\end{array}$ & $\begin{array}{l}\text { Fraction } \\
\text { Leached }\end{array}$ & $\begin{array}{l}\text { Diffusivity } \\
\text { Coefficient } \\
\left(\mathrm{cm}^{\wedge} 2 / \mathrm{s}\right)\end{array}$ & $\begin{array}{l}\text { Leach } \\
\text { Index }\end{array}$ \\
\hline 2 & 7200 & 1800 & 0.354 & 0.6372 & 0.000126 & $2.140 \mathrm{E}-12$ & 11.67 \\
\hline 7 & 18000 & 14835 & 0.741 & 1.3338 & 0.000264 & 1.237E-11 & 10.91 \\
\hline 24 & 61200 & 51231 & 0.347 & 0.6246 & 0.000124 & 8.102E-13 & 12.09 \\
\hline 48 & 86400 & 125894 & 1.57 & 2.8260 & 0.000559 & 2.045E-11 & 10.69 \\
\hline 72 & 86400 & 213818 & 1.37 & 2.4660 & 0.000488 & 2.645E-11 & 10.58 \\
\hline 96 & 86400 & 300849 & 1.09 & 1.9620 & 0.000388 & 2.355E-11 & 10.63 \\
\hline 120 & 86400 & 387596 & 0.679 & 1.2222 & 0.000242 & 1.178E-11 & 10.93 \\
\hline 456 & 1209600 & 939462 & 2.99 & 5.3820 & 0.001065 & 2.824E-12 & 11.55 \\
\hline 1128 & 2419200 & 2716550 & 1.22 & 2.1960 & 0.000434 & 3.399E-13 & 12.47 \\
\hline 2160 & 3715200 & 5768861 & 1.19 & 2.1420 & 0.000424 & 2.911E-13 & 12.54 \\
\hline & & & & & \multicolumn{2}{|c|}{ Average Leach Index: } & 11.40 \\
\hline
\end{tabular}




\section{American Nuclear Society - 16.1 Static Leach Test}

Project: SRS Reactor Seepage Basin Stabilization

Sample Description:

C332

Analyst: $\quad$ PRL

Date:

15-Apr-99

Contaminant:

Gross Beta

Initial Contaminant Conc. (pCi/g):

170

Initial Contaminant Level ( $\mathrm{pCi})$ :

63646.3

Monolith Height $(\mathrm{cm})$ :

Monolith Surface Area (cm^2):

Monolith Volume $\left(\mathrm{cm}^{\wedge} 3\right)$ :

10

176.72

196.35
Sample Weight (g):

Monolith Diameter $(\mathrm{cm})$ :

Leachate Volume $(\mathrm{mL})$ :
374.39

5

1800

\begin{tabular}{|c|c|c|c|c|c|c|c|}
\hline $\begin{array}{l}\text { Time } \\
\text { (hrs) }\end{array}$ & $\begin{array}{l}\text { Interval } \\
\text { Length } \\
\text { (s) }\end{array}$ & $\begin{array}{c}\text { Mean } \\
\text { Time } \\
\text { (s) }\end{array}$ & $\begin{array}{l}\text { Leachate } \\
\text { Conc. } \\
\text { (pCi/L) }\end{array}$ & $\begin{array}{l}\text { Amount } \\
\text { Leached } \\
\text { (pCi) }\end{array}$ & $\begin{array}{l}\text { Fraction } \\
\text { Leached }\end{array}$ & $\begin{array}{l}\text { Diffusivity } \\
\text { Coefficient } \\
\text { (cm^2/s) }\end{array}$ & $\begin{array}{l}\text { Leach } \\
\text { Index }\end{array}$ \\
\hline 2 & 7200 & 1800 & 25.8 & 46.4400 & 0.000730 & $7.170 \mathrm{E}-11$ & 10.14 \\
\hline 7 & 18000 & 14835 & 30.5 & 54.9000 & 0.000863 & $1.321 \mathrm{E}-10$ & 9.88 \\
\hline 24 & 61200 & 51231 & 119 & 214.2000 & 0.003365 & $6.009 \mathrm{E}-10$ & 9.22 \\
\hline 48 & 86400 & 125894 & 89.9 & 161.8200 & 0.002542 & $4.228 \mathrm{E}-10$ & 9.37 \\
\hline 72 & 86400 & 213818 & 72.4 & 130.3200 & 0.002048 & 4.658E-10 & 9.33 \\
\hline 96 & 86400 & 300849 & 33 & 59.4000 & 0.000933 & $1.361 E-10$ & 9.87 \\
\hline 120 & 86400 & 387596 & 43.3 & 77.9400 & 0.001225 & 3.020E-10 & 9.52 \\
\hline 456 & 1209600 & 939462 & 143 & 257.4000 & 0.004044 & 4.073E-11 & 10.39 \\
\hline 1128 & 2419200 & 2716550 & 224 & 403.2000 & 0.006335 & $7.225 E-11$ & 10.14 \\
\hline 2160 & 3715200 & 5768861 & 296 & 532.8000 & 0.008371 & $1.136 \mathrm{E}-10$ & 9.94 \\
\hline & & & & \multicolumn{3}{|c|}{ Average Leach Index: } & 9.78 \\
\hline
\end{tabular}




\section{American Nuclear Society - 16.1 Static Leach Test}

Project: SRS Reactor Seepage Basin Stabilization

Sample Description: $\quad$ C342

Analyst: PRL

Date:

15-Apr-99

Contaminant:

Gross Alpha

Initial Contaminant Conc. (pCi/g):

Initial Contaminant Level (pCi):

Monolith Height $(\mathrm{cm})$ :

Monolith Surface Area $\left(\mathrm{cm}^{\wedge} 2\right)$ :

Monolith Volume $\left(\mathrm{cm}^{\wedge} 3\right)$ :

13.5

5036.85

10

176.72

196.35

\begin{tabular}{|c|c|c|c|c|c|c|c|}
\hline $\begin{array}{l}\text { Time } \\
\text { (hrs) }\end{array}$ & $\begin{array}{l}\text { Interval } \\
\text { Length } \\
\text { (s) }\end{array}$ & $\begin{array}{c}\text { Mean } \\
\text { Time } \\
\text { (s) }\end{array}$ & $\begin{array}{l}\text { Leachate } \\
\text { Conc. } \\
\text { (pCi/L) }\end{array}$ & $\begin{array}{l}\text { Amount } \\
\text { Leached } \\
(\mathrm{pCi})\end{array}$ & $\begin{array}{l}\text { Fraction } \\
\text { Leached }\end{array}$ & $\begin{array}{l}\text { Diffusivity } \\
\text { Coefficient } \\
\text { (cm^2/s) }\end{array}$ & $\begin{array}{l}\text { Leach } \\
\text { Index }\end{array}$ \\
\hline 2 & 7200 & 1800 & 0.1 & 0.1800 & 0.000036 & $1.720 \mathrm{E}-13$ & 12.76 \\
\hline 7 & 18000 & 14835 & 0.133 & 0.2394 & 0.000048 & $4.012 E-13$ & 12.40 \\
\hline 24 & 61200 & 51231 & 0.1 & 0.1800 & 0.000036 & 6.775E-14 & 13.17 \\
\hline 48 & 86400 & 125894 & 0.94 & 1.6920 & 0.000336 & 7.381E-12 & 11.13 \\
\hline 72 & 86400 & 213818 & 0.231 & 0.4158 & 0.000083 & 7.571E-13 & 12.12 \\
\hline 96 & 86400 & 300849 & 0.61 & 1.0980 & 0.000218 & $7.428 \mathrm{E}-12$ & 11.13 \\
\hline 120 & 86400 & 387596 & 0.116 & 0.2088 & 0.000041 & $3.461 \mathrm{E}-13$ & 12.46 \\
\hline 456 & 1209600 & 939462 & 11.9 & 21.4200 & 0.004253 & $4.504 \mathrm{E}-11$ & 10.35 \\
\hline 1128 & 2419200 & 2716550 & 0.1 & 0.1800 & 0.000036 & $2.299 \mathrm{E}-15$ & 14.64 \\
\hline 2160 & 3715200 & 5768861 & 0.708 & 1.2744 & 0.000253 & $1.038 \mathrm{E}-13$ & 12.98 \\
\hline & & & & & \multicolumn{2}{|c|}{ Average Leach Index: } & 12.31 \\
\hline
\end{tabular}




\section{American Nuclear Society - 16.1 Static Leach Test}

Project: $\quad$ SRS Reactor Seepage Basin Stabilization

Sample Description: $\quad$ C342

$\begin{array}{llll}\text { Analyst: PRL } & \text { Date: } & \text { 15-Apr-99 }\end{array}$

Contaminant: Gross Beta

Initial Contaminant Conc. ( $\mathrm{pCi} / \mathrm{g})$ :

170

Initial Contaminant Level (pCi):

64625.5

Monolith Height (cm):

10

Monolith Surface Area $\left(\mathrm{cm}^{\wedge} 2\right)$ :

Monolith Volume $\left(\mathrm{cm}^{\wedge} 3\right)$ :

176.72

196.35

Sample Weight (g):

380.15

Monolith Diameter (cm):

Leachate Volume $(\mathrm{mL})$ :

\begin{tabular}{|c|c|c|c|c|c|c|c|}
\hline $\begin{array}{l}\text { Time } \\
\text { (hrs) }\end{array}$ & $\begin{array}{l}\text { Interval } \\
\text { Length } \\
\text { (s) }\end{array}$ & $\begin{array}{l}\text { Mean } \\
\text { Time } \\
(\mathbf{s})\end{array}$ & $\begin{array}{l}\text { Leachate } \\
\text { Conc. } \\
\text { (pCi/L) }\end{array}$ & $\begin{array}{l}\text { Amount } \\
\text { Leached } \\
\text { (pCi) }\end{array}$ & $\begin{array}{l}\text { Fraction } \\
\text { Leached }\end{array}$ & $\begin{array}{l}\text { Diffusivity } \\
\text { Coefficient } \\
\text { (cm^2/s) }\end{array}$ & $\begin{array}{l}\text { Leach } \\
\text { Index }\end{array}$ \\
\hline 2 & 7200 & 1800 & 7.55 & 13.5900 & 0.000210 & $5.955 E-12$ & 11.23 \\
\hline 7 & 18000 & 14835 & 19.9 & 35.8200 & 0.000554 & 5.456E-11 & 10.26 \\
\hline 24 & 61200 & 51231 & 17.2 & 30.9600 & 0.000479 & $1.218 \mathrm{E}-11$ & 10.91 \\
\hline 48 & 86400 & 125894 & 37.4 & 67.3200 & 0.001042 & $7.098 E-11$ & 10.15 \\
\hline 72 & 86400 & 213818 & 14.6 & 26.2800 & 0.000407 & 1.837E-11 & 10.74 \\
\hline 96 & 86400 & 300849 & 14.7 & 26.4600 & 0.000409 & $2.620 E-11$ & 10.58 \\
\hline 120 & 86400 & 387596 & 22.2 & 39.9600 & 0.000618 & $7.699 \mathrm{E}-11$ & 10.11 \\
\hline 456 & 1209600 & 939462 & 192 & 345.6000 & 0.005348 & $7.122 E-11$ & 10.15 \\
\hline 1128 & 2419200 & 2716550 & 246 & 442.8000 & 0.006852 & $8.452 E-11$ & 10.07 \\
\hline 2160 & 3715200 & 5768861 & 243 & 437.4000 & 0.006768 & $7.426 \mathrm{E}-11$ & 10.13 \\
\hline & & & & \multicolumn{3}{|c|}{ Average Leach Index: } & 10.43 \\
\hline
\end{tabular}


Gross Alpha Leaching for the Fine Fraction from CRSB Soil

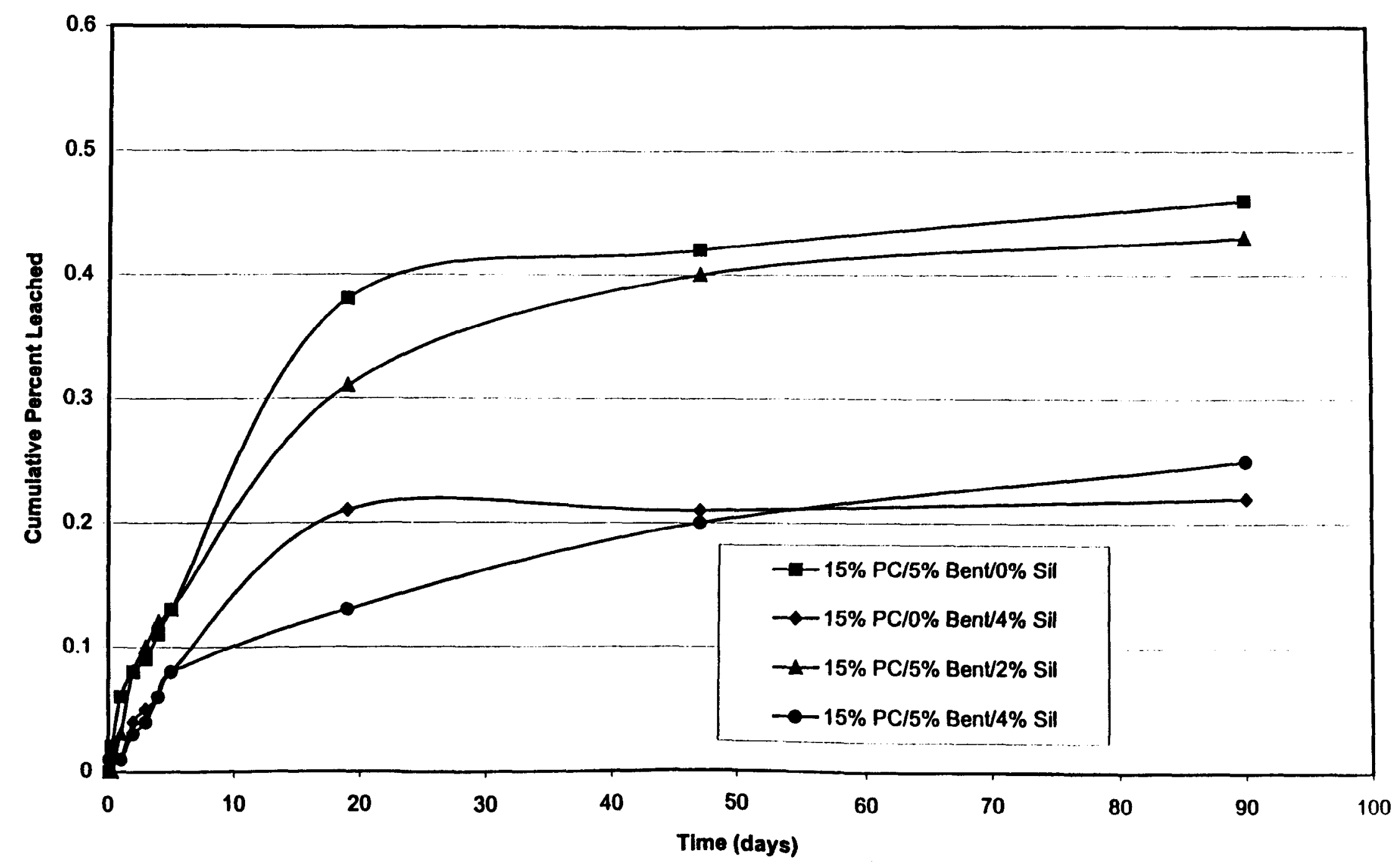




\section{Gross Beta Leaching for the Fine Fraction from the CSRB Soil}

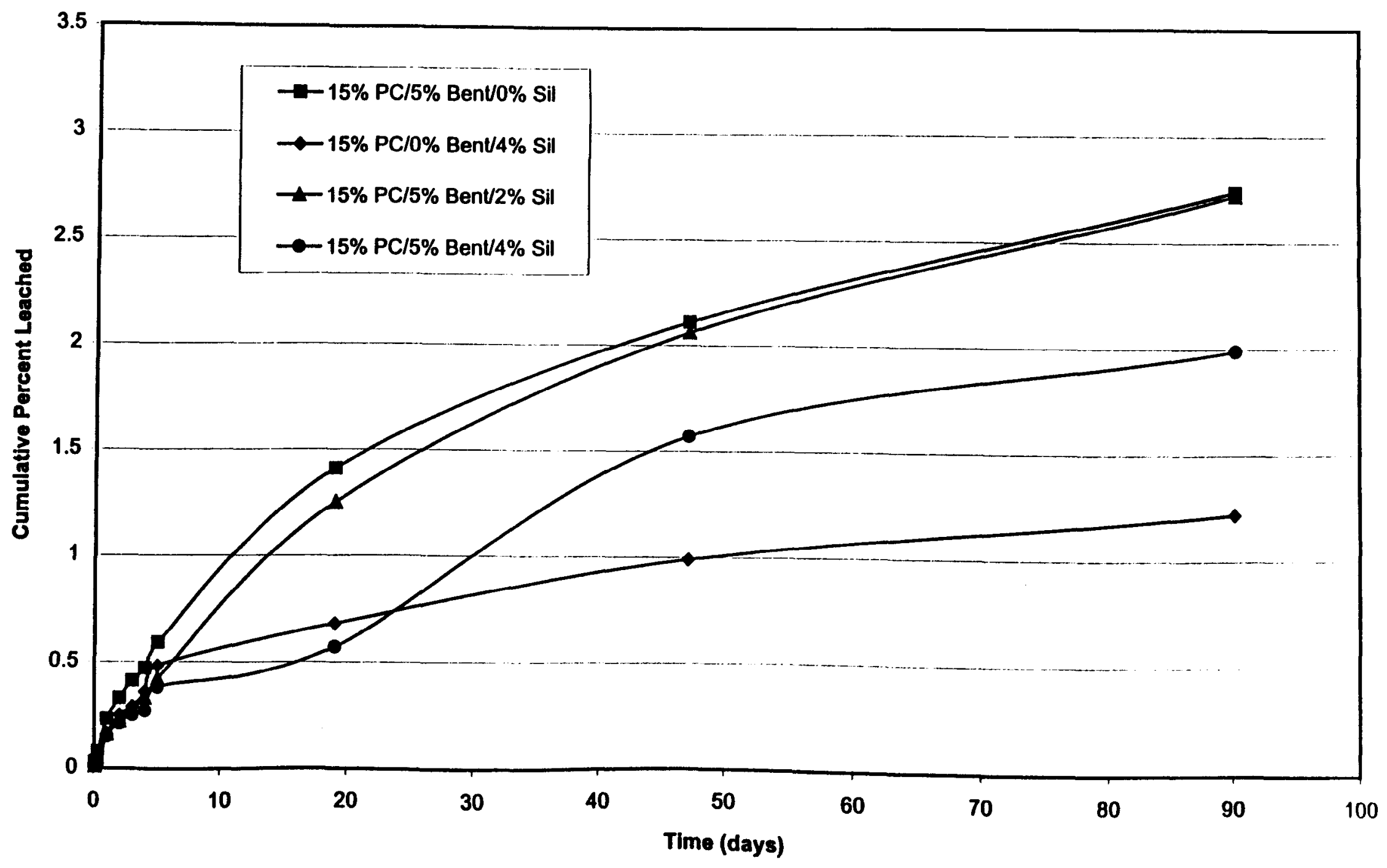




\section{American Nuclear Society - 16.1 Static Leach Test}

Project: $\quad$ SRS Reactor Seepage Basin Stabilization

Sample Description:

C412

Analyst: PRL

Date:

15-Apr-99

Contaminant:

Gross Alpha

Initial Contaminant Conc. (pCi/g):

16.1

6006.91

Initial Contaminant Level (pCi):

10

Monolith Height (cm):

176.72

196.35

Sample Weight (g):

373.1

Monolith Diameter $(\mathrm{cm})$ :

5

Monolith Surface Area $\left(\mathrm{cm}^{\wedge} 2\right)$ :

Monolith Volume $\left(\mathrm{cm}^{\wedge} 3\right)$ :

Leachate Volume $(\mathrm{mL})$ :

1800

\begin{tabular}{|c|c|c|c|c|c|c|c|}
\hline $\begin{array}{l}\text { Time } \\
\text { (hrs) }\end{array}$ & $\begin{array}{l}\text { Interval } \\
\text { Length } \\
\text { (s) }\end{array}$ & $\begin{array}{l}\text { Mean } \\
\text { Time } \\
\text { (s) }\end{array}$ & $\begin{array}{l}\text { Leachate } \\
\text { Conc. } \\
\text { (pCi/L) }\end{array}$ & $\begin{array}{l}\text { Amount } \\
\text { Leached } \\
\text { (pCi) }\end{array}$ & $\begin{array}{l}\text { Fraction } \\
\text { Leached }\end{array}$ & $\begin{array}{l}\text { Diffusivity } \\
\text { Coefficient } \\
\left(\mathrm{cm}^{\wedge} 2 / \mathrm{s}\right)\end{array}$ & $\begin{array}{l}\text { Leach } \\
\text { Index }\end{array}$ \\
\hline 2 & 7200 & 1800 & 1.28 & 2.3040 & 0.000384 & $1.981 \mathrm{E}-11$ & 10.70 \\
\hline 7 & 18000 & 14835 & 0.609 & 1.0962 & 0.000182 & $5.914 \mathrm{E}-12$ & 11.23 \\
\hline 24 & 61200 & 51231 & 0.548 & 0.9864 & 0.000164 & $1.431 E-12$ & 11.84 \\
\hline 48 & 86400 & 125894 & 0.261 & 0.4698 & 0.000078 & $4.001 E-13$ & 12.40 \\
\hline 72 & 86400 & 213818 & 2.14 & 3.8520 & 0.000641 & $4.568 E-11$ & 10.34 \\
\hline 96 & 86400 & 300849 & 1.09 & 1.9620 & 0.000327 & $1.668 \mathrm{E}-11$ & 10.78 \\
\hline 120 & 86400 & 387596 & 0.64 & 1.1520 & 0.000192 & $7.407 \mathrm{E}-12$ & 11.13 \\
\hline 456 & 1209600 & 939462 & 6.13 & 11.0340 & 0.001837 & $8.403 E-12$ & 11.08 \\
\hline 1128 & 2419200 & 2716550 & 1.28 & 2.3040 & 0.000383558 & $2.64852 E-13$ & 12.58 \\
\hline 2160 & 3715200 & 5768861 & 1.5 & 2.7000 & 0.000449482 & $3.27504 \mathrm{E}-13$ & 12.48 \\
\hline & & & & & \multicolumn{2}{|c|}{ Average Leach Index: } & 11.46 \\
\hline
\end{tabular}




\section{American Nuclear Society - 16.1 Static Leach Test}

Project: $\quad$ SRS Reactor Seepage Basin Stabilization

Sample Description: $\quad$ C412

Analyst: PRL

Date:

15-Apr-99

Contaminant:

Gross Beta

Initial Contaminant Conc. (pCi/g):

224

83574.4

Monolith Height (cm):

Monolith Surface Area ( $\left.\mathrm{cm}^{\wedge} 2\right)$ :

Monolith Volume $\left(\mathrm{cm}^{\wedge} 3\right)$ :
10

176.72

196.35
Sample Weight (g):

Monolith Diameter $(\mathrm{cm})$ : Leachate Volume $(\mathrm{mL})$ :
373.1

5

1800

\begin{tabular}{|c|c|c|c|c|c|c|c|}
\hline $\begin{array}{l}\text { Time } \\
\text { (hrs) }\end{array}$ & $\begin{array}{l}\text { Interval } \\
\text { Length } \\
\text { (s) }\end{array}$ & $\begin{array}{c}\text { Mean } \\
\text { Time } \\
(\mathbf{s})\end{array}$ & $\begin{array}{l}\text { Leachate } \\
\text { Conc. } \\
\text { (pCi/L) }\end{array}$ & $\begin{array}{l}\text { Amount } \\
\text { Leached } \\
\text { (pCi) }\end{array}$ & $\begin{array}{l}\text { Fraction } \\
\text { Leached }\end{array}$ & $\begin{array}{l}\text { Diffusivity } \\
\text { Coefficient } \\
\left(\mathrm{cm}^{\wedge} 2 / \mathrm{s}\right)\end{array}$ & $\begin{array}{l}\text { Leach } \\
\text { Index }\end{array}$ \\
\hline 2 & 7200 & 1800 & 39.1 & 70.3800 & 0.000842 & $9.550 \mathrm{E}-11$ & 10.02 \\
\hline 7 & 18000 & 14835 & 26.1 & 46.9800 & 0.000562 & 5.612E-11 & 10.25 \\
\hline 24 & 61200 & 51231 & 82.3 & 148.1400 & 0.001773 & 1.667E-10 & 9.78 \\
\hline 48 & 86400 & 125894 & 57.9 & 104.2200 & 0.001247 & 1.017E-10 & 9.99 \\
\hline 72 & 86400 & 213818 & 56.4 & 101.5200 & 0.001215 & 1.639E-10 & 9.79 \\
\hline 96 & 86400 & 300849 & 58.6 & 105.4800 & 0.001262 & $2.490 E-10$ & 9.60 \\
\hline 120 & 86400 & 387596 & 28.3 & 50.9400 & 0.000610 & 7.482E-11 & 10.13 \\
\hline 456 & 1209600 & 939462 & 307 & 552.6000 & 0.006612 & $1.089 E-10$ & 9.96 \\
\hline 1128 & 2419200 & 2716550 & 325 & 585.0000 & 0.007000 & 8.821E-11 & 10.05 \\
\hline 2160 & 3715200 & 5768861 & 290 & 522.0000 & 0.006246 & $6.324 E-11$ & 10.20 \\
\hline & & & & \multicolumn{3}{|c|}{ Average Leach Index: } & 9.98 \\
\hline
\end{tabular}




\section{American Nuclear Society - 16.1 Static Leach Test}

Project: $\quad$ SRS Reactor Seepage Basin Stabilization

Sample Description: $\quad$ C422

Analyst: $\quad$ PRL

Date: $\quad$ 15-Apr-99

Contaminant:

Gross Alpha

Initial Contaminant Conc. (pCi/g):

Initial Contaminant Level (pCi):

Monolith Height (cm):

Monolith Surface Area ( $\left.\mathrm{cm}^{\wedge} 2\right)$ :

Monolith Volume $\left(\mathrm{cm}^{\wedge} 3\right)$ :

16.1

6148.751

10

176.72

196.35

\begin{tabular}{|c|c|c|c|c|c|c|c|}
\hline $\begin{array}{l}\text { Time } \\
\text { (hrs) }\end{array}$ & $\begin{array}{l}\text { Interval } \\
\text { Length } \\
\text { (s) }\end{array}$ & $\begin{array}{l}\text { Mean } \\
\text { Time } \\
\text { (s) }\end{array}$ & $\begin{array}{l}\text { Leachate } \\
\text { Conc. } \\
\text { (pCi/L) }\end{array}$ & $\begin{array}{c}\text { Amount } \\
\text { Leached } \\
(p C i)\end{array}$ & $\begin{array}{l}\text { Fraction } \\
\text { Leached }\end{array}$ & $\begin{array}{l}\text { Diffusivity } \\
\text { Coefficient } \\
\left(\mathrm{cm}^{\wedge} 2 / \mathrm{s}\right)\end{array}$ & $\begin{array}{l}\text { Leach } \\
\text { Index }\end{array}$ \\
\hline 2 & 7200 & 1800 & 0.4 & 0.7200 & 0.000117 & $1.847 \mathrm{E}-12$ & 11.73 \\
\hline 7 & 18000 & 14835 & 0.562 & 1.0116 & 0.000165 & 4.807E-12 & 11.32 \\
\hline 24 & 61200 & 51231 & 0.739 & 1.3302 & 0.000216 & 2.483E-12 & 11.61 \\
\hline 48 & 86400 & 125894 & 0.736 & 1.3248 & 0.000215 & 3.036E-12 & 11.52 \\
\hline 72 & 86400 & 213818 & 0.678 & 1.2204 & 0.000198 & 4.376E-12 & 11.36 \\
\hline 96 & 86400 & 300849 & 0.528 & 0.9504 & 0.000155 & $3.734 E-12$ & 11.43 \\
\hline 120 & 86400 & 387596 & 0.261 & 0.4698 & 0.000076 & 1.176E-12 & 11.93 \\
\hline 456 & 1209600 & 939462 & 3.24 & 5.8320 & 0.000948 & 2.240E-12 & 11.65 \\
\hline 1128 & 2419200 & 2716550 & 0.1 & 0.1800 & 0.000029 & $1.543 E-15$ & 14.81 \\
\hline 2160 & 3715200 & 5768861 & 0.188 & 0.3384 & 0.000055 & 4.910E-15 & 14.31 \\
\hline & & & & & \multicolumn{2}{|c|}{ Average Leach Index: } & 12.17 \\
\hline
\end{tabular}




\section{American Nuclear Society - 16.1 Static Leach Test}

Project: $\quad$ SRS Reactor Seepage Basin Stabilization

Sample Description: $\quad$ C422

Analyst: $\quad$ PRL

Date: $\quad$ 15-Apr-99

Contaminant:

Gross Beta

Initial Contaminant Conc. (pCi/g):

224

85547.84

10

Monolith Height (cm):

Monolith Surface Area $\left(\mathrm{cm}^{\wedge} 2\right)$ :

Monolith Volume $\left(\mathrm{cm}^{\wedge} 3\right)$ :
176.72 196.35
Sample Weight (g):

Monolith Diameter (cm): Leachate Volume $(\mathrm{mL})$ :
381.91

\section{5}

1800

\begin{tabular}{|c|c|c|c|c|c|c|c|}
\hline $\begin{array}{l}\text { Time } \\
\text { (hrs) }\end{array}$ & $\begin{array}{l}\text { Interval } \\
\text { Length } \\
\text { (s) }\end{array}$ & $\begin{array}{c}\text { Mean } \\
\text { Time } \\
\text { (s) }\end{array}$ & $\begin{array}{c}\text { Leachate } \\
\text { Conc. } \\
\text { (pCi/L) }\end{array}$ & $\begin{array}{l}\text { Amount } \\
\text { Leached } \\
\text { (pCi) }\end{array}$ & $\begin{array}{l}\text { Fraction } \\
\text { Leached }\end{array}$ & $\begin{array}{l}\text { Diffusivity } \\
\text { Coefficient } \\
\text { (cm^^/s) }\end{array}$ & $\begin{array}{l}\text { Leach } \\
\text { Index }\end{array}$ \\
\hline 2 & 7200 & 1800 & 32.1 & 57.7800 & 0.000675 & $6.143 \mathrm{E}-11$ & 10.21 \\
\hline 7 & 18000 & 14835 & 21.4 & 38.5200 & 0.000450 & 3.600E-11 & 10.44 \\
\hline 24 & 61200 & 51231 & 65.9 & 118.6200 & 0.001387 & $1.020 \mathrm{E}-10$ & 9.99 \\
\hline 48 & 86400 & 125894 & 53.9 & 97.0200 & 0.001134 & 8.413E-11 & 10.08 \\
\hline 72 & 86400 & 213818 & 19.9 & 35.8200 & 0.000419 & $1.948 \mathrm{E}-11$ & 10.71 \\
\hline 96 & 86400 & 300849 & 29.5 & 53.1000 & 0.000621 & $6.022 E-11$ & 10.22 \\
\hline 120 & 86400 & 387596 & 13.5 & 24.3000 & 0.000284 & 1.625E-11 & 10.79 \\
\hline 456 & 1209600 & 939462 & 84.9 & 152.8200 & 0.001786 & 7.947E-12 & 11.10 \\
\hline 1128 & 2419200 & 2716550 & 149 & 268.2000 & 0.003135 & $1.769 \mathrm{E}-11$ & 10.75 \\
\hline 2160 & 3715200 & 5768861 & 108 & 194.4000 & 0.002272 & 8.371E-12 & 11.08 \\
\hline & & & & \multicolumn{3}{|c|}{ Average Leach Index: } & 10.54 \\
\hline
\end{tabular}




\section{American Nuclear Society - 16.1 Static Leach Test}

Project: $\quad$ SRS Reactor Seepage Basin Stabilization

Sample Description: $\quad$ C432

$\begin{array}{llll}\text { Analyst: } & \text { PRL } & \text { Date: } & \text { 15-Apr-99 }\end{array}$

Contaminant: Gross Alpha

Initial Contaminant Conc. (pCi/g):

Initial Contaminant Level (pCi):

16.1

5802.44

Monolith Height (cm):

10

Monolith Surface Area (cm^2):

176.72

Monolith Volume $\left(\mathrm{cm}^{\wedge} 3\right)$ :

196.35

Sample Weight (g):

360.4

Monolith Diameter $(\mathrm{cm})$ :

Leachate Volume $(\mathrm{mL})$ :

5

1800

\begin{tabular}{|c|c|c|c|c|c|c|c|}
\hline $\begin{array}{l}\text { Time } \\
\text { (hrs) }\end{array}$ & $\begin{array}{l}\text { Interval } \\
\text { Length } \\
\text { (s) }\end{array}$ & $\begin{array}{c}\text { Mean } \\
\text { Time } \\
\text { (s) }\end{array}$ & $\begin{array}{c}\text { Leachate } \\
\text { Conc. } \\
\text { (pCi/L) } \\
\end{array}$ & $\begin{array}{l}\text { Amount } \\
\text { Leached } \\
\text { (pCi) }\end{array}$ & $\begin{array}{l}\text { Fraction } \\
\text { Leached }\end{array}$ & $\begin{array}{l}\text { Diffusivity } \\
\text { Coefficient } \\
\text { (cm^2/s) }\end{array}$ & $\begin{array}{l}\text { Leach } \\
\text { Index }\end{array}$ \\
\hline 2 & 7200 & 1800 & 0.509 & 0.9162 & 0.000158 & $3.358 \mathrm{E}-12$ & 11.47 \\
\hline 7 & 18000 & 14835 & 0.514 & 0.9252 & 0.000159 & $4.515 \mathrm{E}-12$ & 11.35 \\
\hline 24 & 61200 & 51231 & 0.534 & 0.9612 & 0.000166 & $1.456 \mathrm{E}-12$ & 11.84 \\
\hline 48 & 86400 & 125894 & 0.582 & 1.0476 & 0.000181 & $2.132 E-12$ & 11.67 \\
\hline 72 & 86400 & 213818 & 1.24 & 2.2320 & 0.000385 & $1.644 \mathrm{E}-11$ & 10.78 \\
\hline 96 & 86400 & 300849 & 0.597 & 1.0746 & 0.000185 & 5.361E-12 & 11.27 \\
\hline 120 & 86400 & 387596 & 0.1 & 0.1800 & 0.000031 & $1.938 \mathrm{E}-13$ & 12.71 \\
\hline 456 & 1209600 & 939462 & 6.05 & 10.8900 & 0.001877 & 8.772E-12 & 11.06 \\
\hline 1128 & 2419200 & 2716550 & 2.93 & 5.2740 & 0.000909 & $1.487 \mathrm{E}-12$ & 11.83 \\
\hline 2160 & 3715200 & 5768861 & 0.954 & 1.7172 & 0.000296 & $1.420 \mathrm{E}-13$ & 12.85 \\
\hline & & & & & \multicolumn{2}{|c|}{ Average Leach Index: } & 11.68 \\
\hline
\end{tabular}




\section{American Nuclear Society - 16.1 Static Leach Test}

Project: $\quad$ SRS Reactor Seepage Basin Stabilization

Sample Description: $\quad$ C432

$\begin{array}{llll}\text { Analyst: PRL } & \text { Date: } & \text { 15-Apr-99 }\end{array}$

Contaminant: Gross Beta

Initial Contaminant Conc. (pCi/g): 224

Initial Contaminant Level (pCi): $\quad \mathbf{8 0 7 2 9 . 6}$

Monolith Height (cm):

10

Monolith Surface Area (cm^2):

Monolith Volume $\left(\mathrm{cm}^{\wedge} 3\right)$ :

Sample Weight (g):

Monolith Diameter (cm):

Leachate Volume $(\mathrm{mL})$ :

5
1800

1800

\begin{tabular}{|c|c|c|c|c|c|c|c|}
\hline $\begin{array}{l}\text { Time } \\
\text { (hrs) }\end{array}$ & $\begin{array}{l}\text { Interval } \\
\text { Length } \\
\text { (s) }\end{array}$ & $\begin{array}{l}\text { Mean } \\
\text { Time } \\
\text { (s) }\end{array}$ & $\begin{array}{c}\text { Leachate } \\
\text { Conc. } \\
\text { (pCi/L) }\end{array}$ & $\begin{array}{l}\text { Amount } \\
\text { Leached } \\
\text { (pCi) }\end{array}$ & $\begin{array}{l}\text { Fraction } \\
\text { Leached }\end{array}$ & $\begin{array}{l}\text { Diffusivity } \\
\text { Coefficient } \\
\text { (cm^2/s) }\end{array}$ & $\begin{array}{l}\text { Leach } \\
\text { Index }\end{array}$ \\
\hline 2 & 7200 & 1800 & 57.6 & 103.6800 & 0.001284 & $2.221 \mathrm{E}-10$ & 9.65 \\
\hline 7 & 18000 & 14835 & 29.4 & 52.9200 & 0.000656 & 7.631E-11 & 10.12 \\
\hline 24 & 61200 & 51231 & 77.9 & 140.2200 & 0.001737 & $1.600 \mathrm{E}-10$ & 9.80 \\
\hline 48 & 86400 & 125894 & 68.8 & 123.8400 & 0.001534 & $1.539 \mathrm{E}-10$ & 9.81 \\
\hline 72 & 86400 & 213818 & 53 & 95.4000 & 0.001182 & $1.551 E-10$ & 9.81 \\
\hline 96 & 86400 & 300849 & 39 & 70.2000 & 0.000870 & $1.182 \mathrm{E}-10$ & 9.93 \\
\hline 120 & 86400 & 387596 & 33.3 & 59.9400 & 0.000742 & $1.110 \mathrm{E}-10$ & 9.95 \\
\hline 456 & 1209600 & 939462 & 200 & 360.0000 & 0.004459 & 4.952E-11 & 10.31 \\
\hline 1128 & 2419200 & 2716550 & 367 & 660.6000 & 0.008183 & $1.205 E-10$ & 9.92 \\
\hline 2160 & 3715200 & 5768861 & 293 & 527.4000 & 0.006533 & $6.918 E-11$ & 10.16 \\
\hline & & & & \multicolumn{3}{|c|}{ Average Leach Index: } & 9.95 \\
\hline
\end{tabular}




\section{American Nuclear Society - 16.1 Static Leach Test}

Project: $\quad$ SRS Reactor Seepage Basin Stabilization

Sample Description: $\quad$ C442

Analyst: $\quad$ PRL

Date:

15-Apr-99

Contaminant: Gross Alpha

Initial Contaminant Conc. (pCi/g):

16.1

Initial Contaminant Level (pCi):

5885.516

Monolith Height (cm):

10

Monolith Surface Area $\left(\mathrm{cm}^{\wedge} 2\right)$ :

Monolith Volume $\left(\mathrm{cm}^{\wedge} 3\right)$ :

196.35

\begin{tabular}{|c|c|c|c|c|c|c|c|}
\hline $\begin{array}{l}\text { Time } \\
\text { (hrs) }\end{array}$ & $\begin{array}{l}\text { Interval } \\
\text { Length } \\
\text { (s) }\end{array}$ & $\begin{array}{c}\text { Mean } \\
\text { Time } \\
(\mathrm{s}) \\
\end{array}$ & $\begin{array}{l}\text { Leachate } \\
\text { Conc. } \\
\text { (pCi/L) }\end{array}$ & $\begin{array}{l}\text { Amount } \\
\text { Leached } \\
(\mathrm{pCi})\end{array}$ & $\begin{array}{l}\text { Fraction } \\
\text { Leached }\end{array}$ & $\begin{array}{l}\text { Diffusivity } \\
\text { Coefficient } \\
\left(\mathrm{cm}^{\wedge} 2 / \mathrm{s}\right)\end{array}$ & $\begin{array}{l}\text { Leach } \\
\text { Index }\end{array}$ \\
\hline 2 & 7200 & 1800 & 0.162 & 0.2916 & 0.000050 & $3.306 \mathrm{E}-13$ & 12.48 \\
\hline 7 & 18000 & 14835 & 0.565 & 1.0170 & 0.000173 & 5.303E-12 & 11.28 \\
\hline 24 & 61200 & 51231 & 0.572 & 1.0296 & 0.000175 & 1.624E-12 & 11.79 \\
\hline 48 & 86400 & 125894 & 0.201 & 0.3618 & 0.000061 & $2.472 E-13$ & 12.61 \\
\hline 72 & 86400 & 213818 & 1.13 & 2.0340 & 0.000346 & 1.327E-11 & 10.88 \\
\hline 96 & 86400 & 300849 & 0.547 & 0.9846 & 0.000167 & 4.375E-12 & 11.36 \\
\hline 120 & 86400 & 387596 & 0.221 & 0.3978 & 0.000068 & $9.200 E-13$ & 12.04 \\
\hline 456 & 1209600 & 939462 & 0.813 & 1.4634 & 0.000249 & $1.540 E-13$ & 12.81 \\
\hline 1128 & 2419200 & 2716550 & 2.43 & 4.3740 & 0.000743 & 9.943E-13 & 12.00 \\
\hline 2160 & 3715200 & 5768861 & 1.62 & 2.9160 & 0.000495 & $3.979 E-13$ & 12.40 \\
\hline & & & & & \multicolumn{2}{|c|}{ Average Leach Index: } & 11.96 \\
\hline
\end{tabular}




\section{American Nuclear Society - 16.1 Static Leach Test}

Project: $\quad$ SRS Reactor Seepage Basin Stabilization

Sample Description: $\quad$ C442

$\begin{array}{llll}\text { Analyst: PRL } & \text { Date: Apr-99 }\end{array}$

Contaminant: Gross Beta

Initial Contaminant Conc. (pCi/g):

224

81885.44

Monolith Height (cm):

Monolith Surface Area $\left(\mathrm{cm}^{\wedge} 2\right)$ :

Monolith Volume (cm^3):
10

176.72

196.35
Sample Weight (g):

Monolith Diameter (cm):

Leachate Volume $(\mathrm{mL})$ :
365.56

5

1800

\begin{tabular}{|c|c|c|c|c|c|c|c|}
\hline $\begin{array}{l}\text { Time } \\
\text { (hrs) }\end{array}$ & $\begin{array}{l}\text { Interval } \\
\text { Length } \\
\text { (s) }\end{array}$ & $\begin{array}{c}\text { Mean } \\
\text { Time } \\
\text { (s) }\end{array}$ & $\begin{array}{l}\text { Leachate } \\
\text { Conc. } \\
\text { (pCi/L) }\end{array}$ & $\begin{array}{l}\text { Amount } \\
\text { Leached } \\
(p C i)\end{array}$ & $\begin{array}{l}\text { Fraction } \\
\text { Leached }\end{array}$ & $\begin{array}{l}\text { Diffusivity } \\
\text { Coefficient } \\
\text { (cm^^/s) }\end{array}$ & $\begin{array}{l}\text { Leach } \\
\text { Index }\end{array}$ \\
\hline 2 & 7200 & 1800 & 24 & 43.2000 & 0.000528 & $3.748 \mathrm{E}-11$ & 10.43 \\
\hline 7 & 18000 & 14835 & 20.5 & 36.9000 & 0.000451 & $3.606 \mathrm{E}-11$ & 10.44 \\
\hline 24 & 61200 & 51231 & 39.3 & 70.7400 & 0.000864 & $3.959 E-11$ & 10.40 \\
\hline 48 & 86400 & 125894 & 20.6 & 37.0800 & 0.000453 & 1.341E-11 & 10.87 \\
\hline 72 & 86400 & 213818 & 34.4 & 61.9200 & 0.000756 & $6.352 E-11$ & 10.20 \\
\hline 96 & 86400 & 300849 & 29 & 52.2000 & 0.000637 & $6.352 E-11$ & 10.20 \\
\hline 120 & 86400 & 387596 & 21.1 & 37.9800 & 0.000464 & 4.332E-11 & 10.36 \\
\hline 456 & 1209600 & 939462 & 69.1 & 124.3800 & 0.001519 & $5.746 \mathrm{E}-12$ & 11.24 \\
\hline 1128 & 2419200 & 2716550 & 456 & 820.8000 & 0.010024 & $1.809 E-10$ & 9.74 \\
\hline 2160 & 3715200 & 5768861 & 190 & 342.0000 & 0.004177 & $2.828 \mathrm{E}-11$ & 10.55 \\
\hline & & & & \multicolumn{3}{|c|}{ Average Leach Index: } & 10.44 \\
\hline
\end{tabular}


Gross Beta Leaching for the Bulk Fraction \#1 from the CSRB Soil

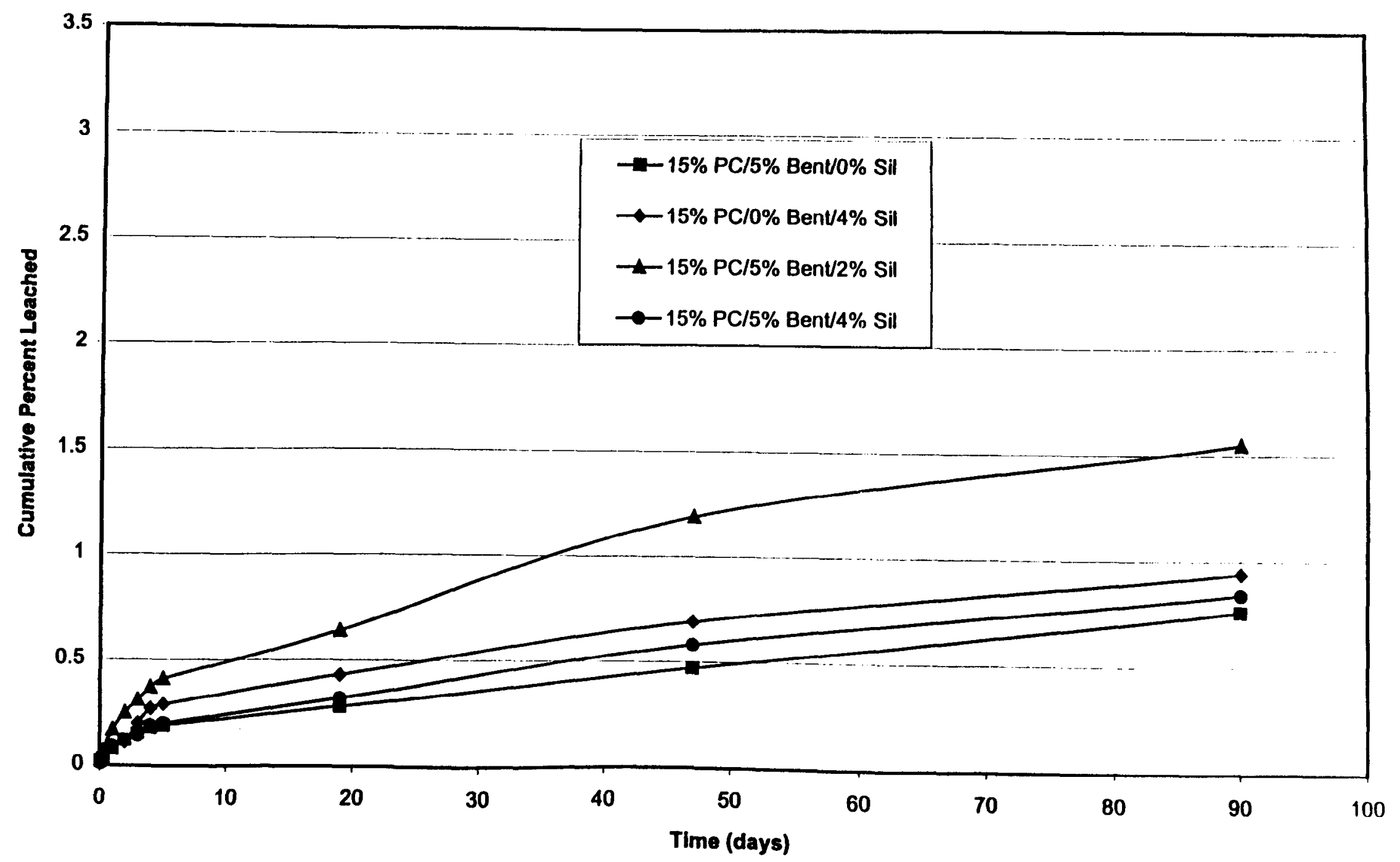


Gross Alpha Leaching for the Bulk Fraction \#1 from the CRSB Soil

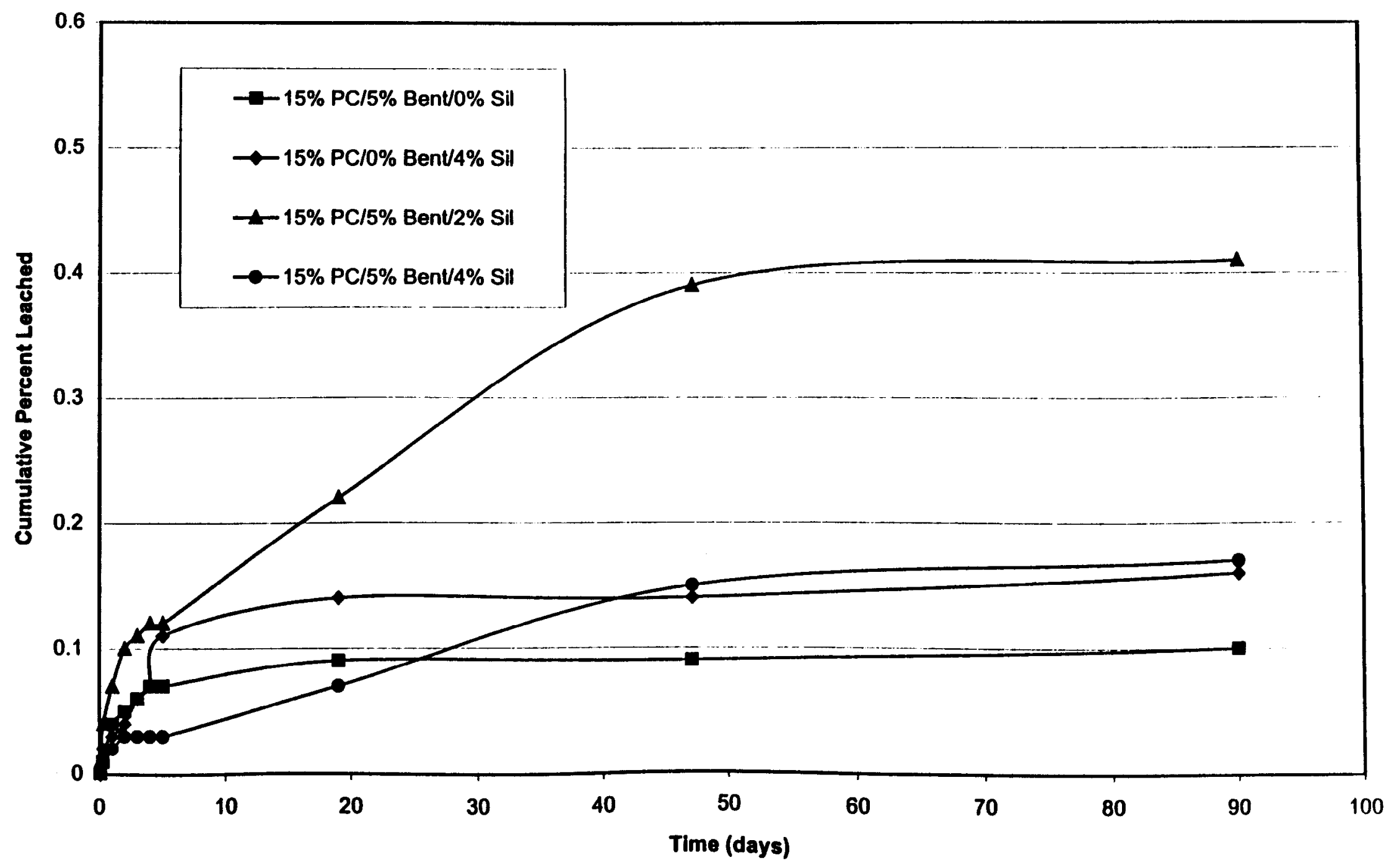




\section{American Nuclear Society - 16.1 Static Leach Test}

Project: $\quad$ SRS Reactor Seepage Basin Stabilization

Sample Description: $\quad$ C512

$\begin{array}{llll}\text { Analyst: PRL } & \text { Date: Apr-99 }\end{array}$

Contaminant: Gross Alpha

Initial Contaminant Conc. (pCi/g): $\quad 24.3$

Initial Contaminant Level (pCi): $\quad 9170.091$

Monolith Height (cm): $\quad 10$

Monolith Surface Area $\left(\mathrm{cm}^{\wedge} 2\right): \quad 176.72$

Monolith Volume $\left(\mathrm{cm}^{\wedge} 3\right)$ : $\quad 196.35$

Sample Weight $(g)$ :

377.37

Monolith Diameter $(\mathrm{cm}):$

Leachate Volume $(\mathrm{mL}): \quad 1800$

\begin{tabular}{|c|c|c|c|c|c|c|c|}
\hline $\begin{array}{l}\text { Time } \\
\text { (hrs) }\end{array}$ & $\begin{array}{l}\text { Interval } \\
\text { Length } \\
\text { (s) }\end{array}$ & $\begin{array}{c}\text { Mean } \\
\text { Time } \\
\text { (s) }\end{array}$ & $\begin{array}{c}\text { Leachate } \\
\text { Conc. } \\
\text { (pCi/L) }\end{array}$ & $\begin{array}{l}\text { Amount } \\
\text { Leached } \\
\text { (pCi) }\end{array}$ & $\begin{array}{l}\text { Fraction } \\
\text { Leached }\end{array}$ & $\begin{array}{l}\text { Diffusivity } \\
\text { Coefficient } \\
\text { (cm^2/s) }\end{array}$ & $\begin{array}{l}\text { Leach } \\
\text { Index }\end{array}$ \\
\hline 2 & 7200 & 1800 & 0.055 & 0.0990 & 0.000011 & $1.570 \mathrm{E}-14$ & $\overline{13.80}$ \\
\hline 7 & 18000 & 14835 & 0.372 & 0.6696 & 0.000073 & $9.469 \mathrm{E}-13$ & 12.02 \\
\hline 24 & 61200 & 51231 & 1.49 & 2.6820 & 0.000292 & $4.538 E-12$ & 11.34 \\
\hline 48 & 86400 & 125894 & 0.74 & 1.3320 & 0.000145 & $1.380 \mathrm{E}-12$ & 11.86 \\
\hline 72 & 86400 & 213818 & 0.613 & 1.1034 & 0.000120 & $1.608 \mathrm{E}-12$ & 11.79 \\
\hline 96 & 86400 & 300849 & 0.153 & 0.2754 & 0.000030 & $1.410 E-13$ & 12.85 \\
\hline 120 & 86400 & 387596 & 0.1 & 0.1800 & 0.000020 & 7.759E-14 & 13.11 \\
\hline 456 & 1209600 & 939462 & 0.819 & 1.4742 & 0.000161 & 6.436E-14 & 13.19 \\
\hline 1128 & 2419200 & 2716550 & 0.1 & 0.1800 & 0.000020 & $6.936 \mathrm{E}-16$ & 15.16 \\
\hline 2160 & 3715200 & 5768861 & 0.9 & 1.6200 & 0.000177 & $5.059 \mathrm{E}-14$ & 13.30 \\
\hline & & & & \multicolumn{3}{|c|}{ Average Leach Index: } & 12.84 \\
\hline
\end{tabular}




\section{American Nuclear Society - 16.1 Static Leach Test}

Project: $\quad$ SRS Reactor Seepage Basin Stabilization

Sample Description: $\quad$ C512

Analyst: PRL

Date:

15-Apr-99

Contaminant:

Gross Beta

Initial Contaminant Conc. (pCi/g):

284

107173.08

Monolith Height (cm):

Monolith Surface Area $\left(\mathrm{cm}^{\wedge} 2\right)$ :

Monolith Volume $\left(\mathrm{cm}^{\wedge} 3\right)$ :
10

176.72

196.35
Sample Weight (g):

Monolith Diameter (cm):

Leachate Volume $(\mathrm{mL})$ :
377.37

5

1800

\begin{tabular}{|c|c|c|c|c|c|c|c|}
\hline $\begin{array}{l}\text { Time } \\
\text { (hrs) }\end{array}$ & $\begin{array}{l}\text { Interval } \\
\text { Length } \\
\text { (s) }\end{array}$ & $\begin{array}{c}\text { Mean } \\
\text { Time } \\
\text { (s) }\end{array}$ & $\begin{array}{l}\text { Leachate } \\
\text { Conc. } \\
\text { (pCi/L) }\end{array}$ & $\begin{array}{l}\text { Amount } \\
\text { Leached } \\
\text { (pCi) }\end{array}$ & $\begin{array}{l}\text { Fraction } \\
\text { Leached }\end{array}$ & $\begin{array}{l}\text { Diffusivity } \\
\text { Coefficient } \\
\left(\mathrm{cm}^{\wedge} 2 / \mathrm{s}\right)\end{array}$ & $\begin{array}{l}\text { Leach } \\
\text { Index }\end{array}$ \\
\hline 2 & 7200 & 1800 & 9.58 & 17.2440 & 0.000161 & $3.486 \mathrm{E}-12$ & 11.46 \\
\hline 7 & 18000 & 14835 & 8.03 & 14.4540 & 0.000135 & 3.230E-12 & 11.49 \\
\hline 24 & 61200 & 51231 & 30.5 & 54.9000 & 0.000512 & $1.392 E-11$ & 10.86 \\
\hline 48 & 86400 & 125894 & 25 & 45.0000 & 0.000420 & 1.153E-11 & 10.94 \\
\hline 72 & 86400 & 213818 & 14.8 & 26.6400 & 0.000249 & 6.864E-12 & 11.16 \\
\hline 96 & 86400 & 300849 & 18 & 32.4000 & 0.000302 & 1.429E-11 & 10.85 \\
\hline 120 & 86400 & 387596 & 9.28 & 16.7040 & 0.000156 & $4.892 E-12$ & 11.31 \\
\hline 456 & 1209600 & 939462 & 54.2 & 97.5600 & 0.000910 & 2.064E-12 & 11.69 \\
\hline 1128 & 2419200 & 2716550 & 111 & 199.8000 & 0.001864 & $6.257 E-12$ & 11.20 \\
\hline 2160 & 3715200 & 5768861 & 174 & 313.2000 & 0.002922 & $1.384 E-11$ & 10.86 \\
\hline & & & & \multicolumn{3}{|c|}{ Average Leach Index: } & 11.18 \\
\hline
\end{tabular}




\section{American Nuclear Society - 16.1 Static Leach Test}

Project: $\quad$ SRS Reactor Seepage Basin Stabilization

Sample Description: $\quad$ C522

$\begin{array}{llll}\text { Analyst: PRL } & \text { Date: }\end{array}$

Contaminant: Gross Alpha

Initial Contaminant Conc. (pCi/g): $\quad 24.3$

Initial Contaminant Level (pCi): $\quad 9541.395$

Monolith Height (cm): 10

Monolith Surface Area $\left(\mathrm{cm}^{\wedge} 2\right)$ : $\quad 176.72$

Monolith Volume $\left(\mathrm{cm}^{\wedge} 3\right)$ : $\quad 196.35$

Leachate Volume $(\mathrm{mL})$ : $\quad 1800$

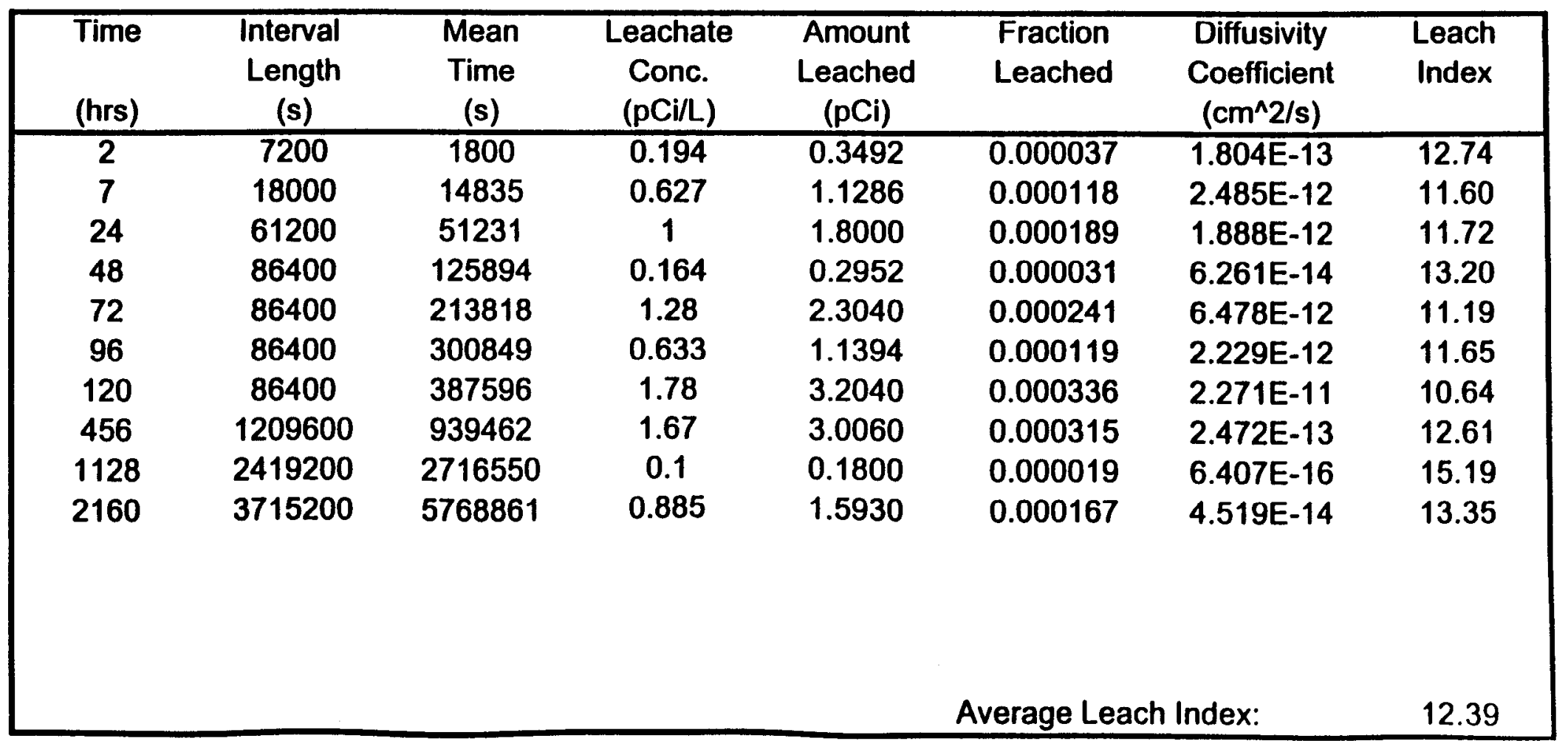




\section{American Nuclear Society - 16.1 Static Leach Test}

Project: SRS Reactor Seepage Basin Stabilization

Sample Description: $\quad$ C522

$\begin{array}{llll}\text { Analyst: PRL } & \text { Date: 15-Apr-99 }\end{array}$

Contaminant: Gross Beta

Initial Contaminant Conc. ( $\mathrm{pCi} / \mathrm{g})$ :

284

Initial Contaminant Level (pCi):

111512.6

Monolith Height (cm):

Monolith Surface Area ( $\left.\mathrm{cm}^{\wedge} 2\right)$ :

10

176.72

Monolith Volume $\left(\mathrm{cm}^{\wedge} 3\right)$ :

196.35

392.65

Monolith Diameter (cm):

5 Leachate Volume $(\mathrm{mL})$ :

1800

\begin{tabular}{|c|c|c|c|c|c|c|c|}
\hline $\begin{array}{l}\text { Time } \\
\text { (hrs) }\end{array}$ & $\begin{array}{l}\text { Interval } \\
\text { Length } \\
\text { (s) }\end{array}$ & $\begin{array}{l}\text { Mean } \\
\text { Time } \\
\text { (s) }\end{array}$ & $\begin{array}{l}\text { Leachate } \\
\text { Conc. } \\
\text { (pCi/L) }\end{array}$ & $\begin{array}{l}\text { Amount } \\
\text { Leached } \\
\text { (pCi) }\end{array}$ & $\begin{array}{l}\text { Fraction } \\
\text { Leached }\end{array}$ & $\begin{array}{c}\text { Diffusivity } \\
\text { Coefficient } \\
\left(\mathrm{cm}^{\wedge} 2 / \mathrm{s}\right)\end{array}$ & $\begin{array}{l}\text { Leach } \\
\text { Index }\end{array}$ \\
\hline 2 & 7200 & 1800 & 10.7 & 19.2600 & 0.000173 & $4.017 \mathrm{E}-12$ & $\overline{11.40}$ \\
\hline 7 & 18000 & 14835 & 6.77 & 12.1860 & 0.000109 & $2.121 \mathrm{E}-12$ & 11.67 \\
\hline 24 & 61200 & 51231 & 35.7 & 64.2600 & 0.000576 & $1.762 \mathrm{E}-11$ & 10.75 \\
\hline 48 & 86400 & 125894 & 16.4 & 29.5200 & 0.000265 & 4.584E-12 & 11.34 \\
\hline 72 & 86400 & 213818 & 57.3 & 103.1400 & 0.000925 & $9.504 E-11$ & 10.02 \\
\hline 96 & 86400 & 300849 & 37.6 & 67.6800 & 0.000607 & $5.758 E-11$ & 10.24 \\
\hline 120 & 86400 & 387596 & 13.1 & 23.5800 & 0.000211 & $9.004 \mathrm{E}-12$ & 11.05 \\
\hline 456 & 1209600 & 939462 & 88.5 & 159.3000 & 0.001429 & $5.082 \mathrm{E}-12$ & 11.29 \\
\hline 1128 & 2419200 & 2716550 & 163 & 293.4000 & 0.002631 & $1.246 \mathrm{E}-11$ & 10.90 \\
\hline 2160 & 3715200 & 5768861 & 155 & 279.0000 & 0.002502 & $1.015 \mathrm{E}-11$ & 10.99 \\
\hline & & & & \multicolumn{3}{|c|}{ Average Leach Index: } & 10.97 \\
\hline
\end{tabular}




\section{American Nuclear Society - 16.1 Static Leach Test}

Project: $\quad$ SRS Reactor Seepage Basin Stabilization

Sample Description: $\quad$ C532

$\begin{array}{llll}\text { Analyst: PRL } & \text { Date: } & \text { 15-Apr-99 }\end{array}$

Contaminant: Gross Alpha

Initial Contaminant Conc. (pCi/g):

Initial Contaminant Level ( $\mathrm{pCi})$ :

24.3

9010.683

Monolith Height (cm):

10

Monolith Surface Area ( $\left.\mathrm{cm}^{\wedge} 2\right)$ :

Monolith Volume $\left(\mathrm{cm}^{\wedge} 3\right)$ :

176.72

196.35

Sample Weight (g):

Monolith Diameter (cm):

Leachate Volume $(\mathrm{mL})$ :
370.81

5

1800

\begin{tabular}{|c|c|c|c|c|c|c|c|}
\hline $\begin{array}{l}\text { Time } \\
\text { (hrs) }\end{array}$ & $\begin{array}{l}\text { Interval } \\
\text { Length } \\
\text { (s) }\end{array}$ & $\begin{array}{l}\text { Mean } \\
\text { Time } \\
\text { (s) }\end{array}$ & $\begin{array}{l}\text { Leachate } \\
\text { Conc. } \\
\text { (pCi/L) }\end{array}$ & $\begin{array}{l}\text { Amount } \\
\text { Leached } \\
\text { (pCi) }\end{array}$ & $\begin{array}{l}\text { Fraction } \\
\text { Leached }\end{array}$ & $\begin{array}{l}\text { Diffusivity } \\
\text { Coefficient } \\
\text { (cm^2/s) }\end{array}$ & $\begin{array}{l}\text { Leach } \\
\text { Index }\end{array}$ \\
\hline 2 & 7200 & 1800 & 0.422 & 0.7596 & 0.000084 & $9.570 \mathrm{E}-13$ & 12.02 \\
\hline 7 & 18000 & 14835 & 1.47 & 2.6460 & 0.000294 & $1.531 \mathrm{E}-11$ & 10.81 \\
\hline 24 & 61200 & 51231 & 1.6 & 2.8800 & 0.000320 & $5.420 E-12$ & 11.27 \\
\hline 48 & 86400 & 125894 & 1.54 & 2.7720 & 0.000308 & $6.190 \mathrm{E}-12$ & 11.21 \\
\hline 72 & 86400 & 213818 & 0.453 & 0.8154 & 0.000090 & $9.097 E-13$ & 12.04 \\
\hline 96 & 86400 & 300849 & 0.302 & 0.5436 & 0.000060 & $5.689 \mathrm{E}-13$ & 12.24 \\
\hline 120 & 86400 & 387596 & 0.236 & 0.4248 & 0.000047 & 4.476E-13 & 12.35 \\
\hline 456 & 1209600 & 939462 & 5 & 9.0000 & 0.000999 & $2.484 E-12$ & 11.60 \\
\hline 1128 & 2419200 & 2716550 & 8.59 & 15.4620 & 0.001715963 & 5.30098E-12 & 11.28 \\
\hline 2160 & 3715200 & 5768861 & 0.865 & 1.5570 & 0.000172795 & 4.84009E-14 & 13.32 \\
\hline & & & & & \multicolumn{2}{|c|}{ Average Leach Index: } & 11.81 \\
\hline
\end{tabular}




\section{American Nuclear Society - 16.1 Static Leach Test}

Project: $\quad$ SRS Reactor Seepage Basin Stabilization

Sample Description: $\quad$ C532

$\begin{array}{llll}\text { Analyst: PRL } & \text { Date: }\end{array}$

Contaminant: Gross Beta

Initial Contaminant Conc. (pCi/g): $\quad 284$

Initial Contaminant Level (pCi): $\quad 105310.04$

Monolith Height (cm): 10

Monolith Surface Area (cm^2): $\quad 176.72$

Monolith Volume $\left(\mathrm{cm}^{\wedge} 3\right)$ : $\quad 196.35$

Sample Weight $(\mathrm{g}): \quad 370.81$

Monolith Diameter (cm): $\quad 5$ Leachate Volume $(\mathrm{mL})$ : $\quad 1800$

\begin{tabular}{|c|c|c|c|c|c|c|c|}
\hline $\begin{array}{l}\text { Time } \\
\text { (hrs) }\end{array}$ & $\begin{array}{l}\text { Interval } \\
\text { Length } \\
\text { (s) }\end{array}$ & $\begin{array}{c}\text { Mean } \\
\text { Time } \\
(s) \\
\end{array}$ & $\begin{array}{l}\text { Leachate } \\
\text { Conc. } \\
\text { (pCi/L) }\end{array}$ & $\begin{array}{l}\text { Amount } \\
\text { Leached } \\
\text { (pCi) }\end{array}$ & $\begin{array}{l}\text { Fraction } \\
\text { Leached }\end{array}$ & $\begin{array}{l}\text { Diffusivity } \\
\text { Coefficient } \\
\text { (cm^^/s) }\end{array}$ & $\begin{array}{l}\text { Leach } \\
\text { Index }\end{array}$ \\
\hline 2 & 7200 & 1800 & 16.5 & 29.7000 & 0.000282 & $1.071 \mathrm{E}-11$ & 10.97 \\
\hline 7 & 18000 & 14835 & 25.1 & 45.1800 & 0.000429 & 3.269E-11 & 10.49 \\
\hline 24 & 61200 & 51231 & 56.5 & 101.7000 & 0.000966 & 4.948E-11 & 10.31 \\
\hline 48 & 86400 & 125894 & 50.8 & 91.4400 & 0.000868 & 4.931E-11 & 10.31 \\
\hline 72 & 86400 & 213818 & 32 & 57.6000 & 0.000547 & 3.323E-11 & 10.48 \\
\hline 96 & 86400 & 300849 & 34.9 & 62.8200 & 0.000597 & 5.562E-11 & 10.25 \\
\hline 120 & 86400 & 387596 & 23.3 & 41.9400 & 0.000398 & $3.194 \mathrm{E}-11$ & 10.50 \\
\hline 456 & 1209600 & 939462 & 133 & 239.4000 & 0.002273 & 1.287E-11 & 10.89 \\
\hline 1128 & 2419200 & 2716550 & 326 & 586.8000 & 0.005572 & $5.590 \mathrm{E}-11$ & 10.25 \\
\hline 2160 & 3715200 & 5768861 & 206 & 370.8000 & 0.003521 & $2.010 E-11$ & 10.70 \\
\hline & & & & \multicolumn{3}{|c|}{ Average Leach Index: } & 10.52 \\
\hline
\end{tabular}




\section{American Nuclear Society - 16.1 Static Leach Test}

Project: $\quad$ SRS Reactor Seepage Basin Stabilization

Sample Description: $\quad$ C542

$\begin{array}{llll}\text { Analyst: } & \text { PRL } & \text { 15-Apr-99 }\end{array}$

Contaminant: Gross Alpha

Initial Contaminant Conc. (pCi/g): $\quad 24.3$

Initial Contaminant Level (pCi): $\quad 9145.062$

Monolith Height (cm):

10

Monolith Surface Area $\left(\mathrm{cm}^{\wedge} 2\right)$ : $\quad 176.72$

Monolith Volume $\left(\mathrm{cm}^{\wedge} 3\right)$ : $\quad 196.35$

\begin{tabular}{|c|c|c|c|c|c|c|c|}
\hline $\begin{array}{l}\text { Time } \\
\text { (hrs) }\end{array}$ & $\begin{array}{l}\text { Interval } \\
\text { Length } \\
\text { (s) }\end{array}$ & $\begin{array}{l}\text { Mean } \\
\text { Time } \\
\text { (s) }\end{array}$ & $\begin{array}{l}\text { Leachate } \\
\text { Conc. } \\
\text { (pCi/L) }\end{array}$ & $\begin{array}{c}\text { Amount } \\
\text { Leached } \\
\text { (pCi) }\end{array}$ & $\begin{array}{l}\text { Fraction } \\
\text { Leached }\end{array}$ & $\begin{array}{l}\text { Diffusivity } \\
\text { Coefficient } \\
\left(\mathrm{cm}^{\wedge} 2 / \mathrm{s}\right)\end{array}$ & $\begin{array}{l}\text { Leach } \\
\text { Index }\end{array}$ \\
\hline 2 & 7200 & 1800 & 0.1 & 0.1800 & 0.000020 & 5.217E-14 & 13.28 \\
\hline 7 & 18000 & 14835 & 0.468 & 0.8424 & 0.000092 & 1.507E-12 & 11.82 \\
\hline 24 & 61200 & 51231 & 0.533 & 0.9594 & 0.000105 & 5.839E-13 & 12.23 \\
\hline 48 & 86400 & 125894 & 0.172 & 0.3096 & 0.000034 & 7.497E-14 & 13.13 \\
\hline 72 & 86400 & 213818 & 0.106 & 0.1908 & 0.000021 & 4.836E-14 & 13.32 \\
\hline 96 & 86400 & 300849 & 0.165 & 0.2970 & 0.000032 & $1.649 E-13$ & 12.78 \\
\hline 120 & 86400 & 387596 & 0.1 & 0.1800 & 0.000020 & 7.802E-14 & 13.11 \\
\hline 456 & 1209600 & 939462 & 1.71 & 3.0780 & 0.000337 & $2.821 E-13$ & 12.55 \\
\hline 1128 & 2419200 & 2716550 & 4.26 & 7.6680 & 0.000838 & $1.266 \mathrm{E}-12$ & 11.90 \\
\hline 2160 & 3715200 & 5768861 & 0.801 & 1.4418 & 0.000158 & $4.029 E-14$ & 13.39 \\
\hline & & & & & \multicolumn{2}{|c|}{ Average Leach Index: } & 12.75 \\
\hline
\end{tabular}


Project: $\quad$ SRS Reactor Seepage Basin Stabilization

Sample Description: $\quad$ C542

Analyst: $\quad$ PRL

Date: $\quad$ 15-Apr-99

Contaminant:

Gross Beta

Initial Contaminant Conc. (pCi/g):

Initial Contaminant Level (pCi):

Monolith Height (cm):

Monolith Surface Area $\left(\mathrm{cm}^{\wedge} 2\right)$ :

Monolith Volume $\left(\mathrm{cm}^{\wedge} 3\right)$ :

284
106880.56
10
176.72
196.35

Sample Weight (g):

376.34

Monolith Diameter $(\mathrm{cm}):$ Leachate Volume (mL):

1800

\begin{tabular}{|c|c|c|c|c|c|c|c|}
\hline $\begin{array}{l}\text { Time } \\
\text { (hrs) }\end{array}$ & $\begin{array}{l}\text { Interval } \\
\text { Length } \\
\text { (s) }\end{array}$ & $\begin{array}{l}\text { Mean } \\
\text { Time } \\
\text { (s) }\end{array}$ & $\begin{array}{l}\text { Leachate } \\
\text { Conc. } \\
\text { (pCi/L) }\end{array}$ & $\begin{array}{l}\text { Amount } \\
\text { Leached } \\
\text { (pCi) }\end{array}$ & $\begin{array}{l}\text { Fraction } \\
\text { Leached }\end{array}$ & $\begin{array}{l}\text { Diffusivity } \\
\text { Coefficient } \\
\left(\mathrm{cm}^{\wedge} 2 / \mathrm{s}\right)\end{array}$ & $\begin{array}{l}\text { Leach } \\
\text { Index }\end{array}$ \\
\hline 2 & 7200 & 1800 & 7.88 & 14.1840 & 0.000133 & $2.372 \mathrm{E}-12$ & 11.62 \\
\hline 7 & 18000 & 14835 & 12.7 & 22.8600 & 0.000214 & 8.124E-12 & 11.09 \\
\hline 24 & 61200 & 51231 & 32.6 & 58.6800 & 0.000549 & $1.599 E-11$ & 10.80 \\
\hline 48 & 86400 & 125894 & 18.3 & 32.9400 & 0.000308 & $6.213 E-12$ & 11.21 \\
\hline 72 & 86400 & 213818 & 13.5 & 24.3000 & 0.000227 & 5.742E-12 & 11.24 \\
\hline 96 & 86400 & 300849 & 26.6 & 47.8800 & 0.000448 & 3.137E-11 & 10.50 \\
\hline 120 & 86400 & 387596 & 10.1 & 18.1800 & 0.000170 & 5.827E-12 & 11.23 \\
\hline 456 & 1209600 & 939462 & 71.1 & 127.9800 & 0.001197 & $3.571 E-12$ & 11.45 \\
\hline 1128 & 2419200 & 2716550 & 154 & 277.2000 & 0.002594 & 1.211E-11 & 10.92 \\
\hline 2160 & 3715200 & 5768861 & 154 & 277.2000 & 0.002594 & 1.090E-11 & 10.96 \\
\hline & & & & \multicolumn{3}{|c|}{ Average Leach Index: } & 11.14 \\
\hline
\end{tabular}


Gross Alpha Leaching for the Bulk Fraction \#2 from the CRSB Soil

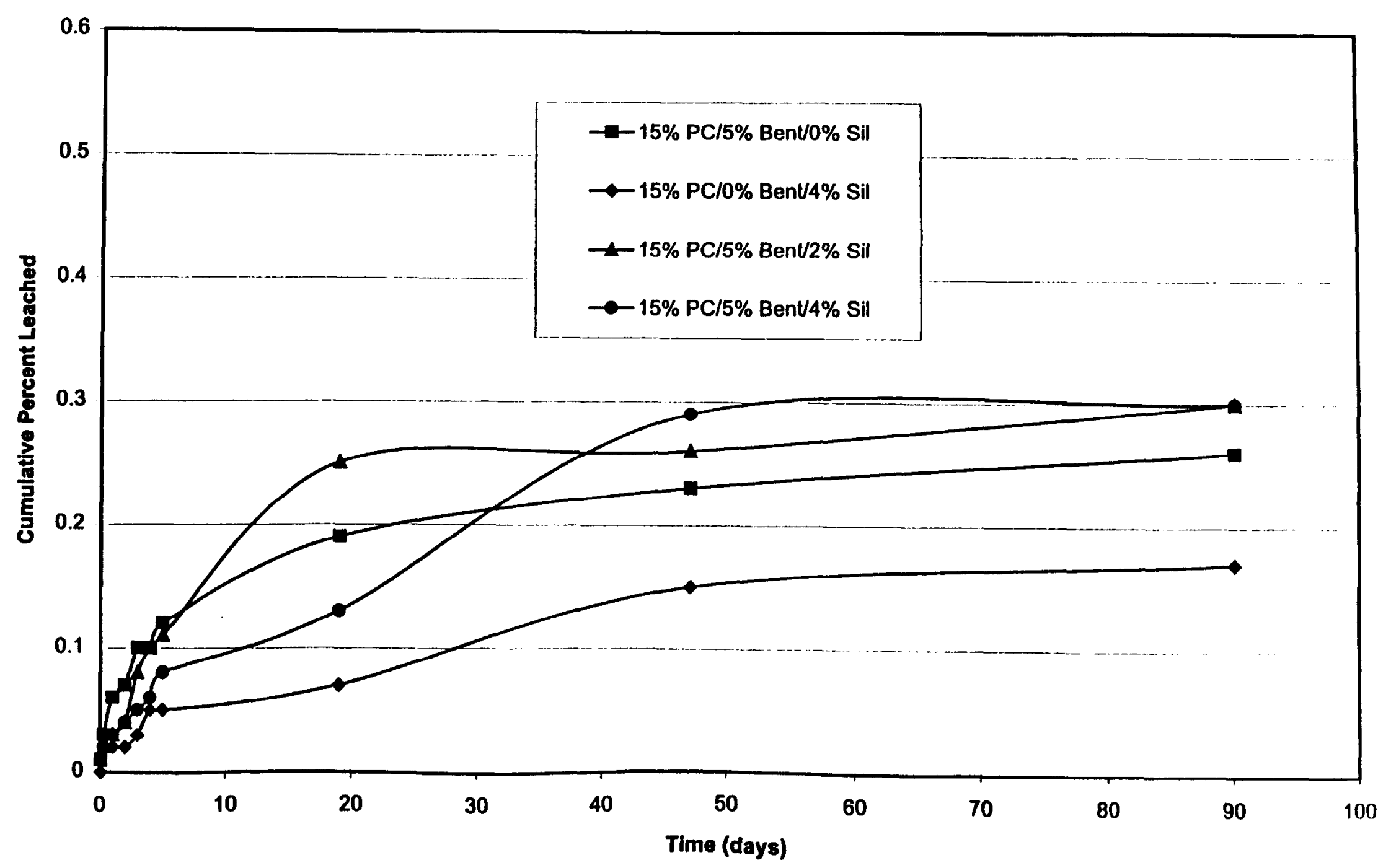




\section{Gross Beta Leaching for the Bulk Fraction \#2 from the CSRB Soll}

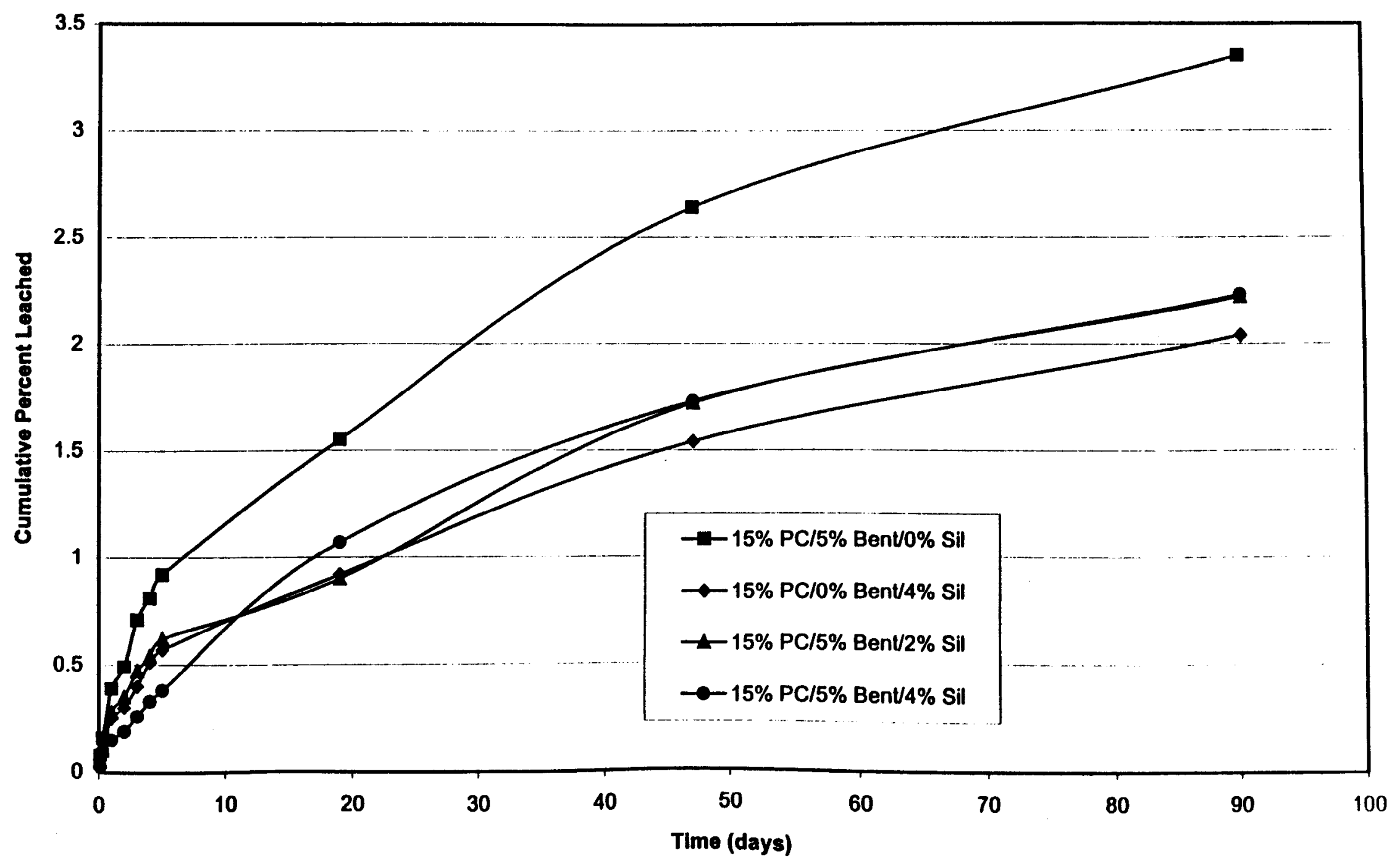




\section{American Nuclear Society - 16.1 Static Leach Test}

Project: SRS Reactor Seepage Basin Stabilization

Sample Description: $\quad \mathrm{C612}$

$\begin{array}{llll}\text { Analyst: PRL } & \text { Date: } & \text { 15-Apr-99 }\end{array}$

Contaminant: Gross Alpha

Initial Contaminant Conc. (pCi/g): $\quad 23.2$

Initial Contaminant Level (pCi): $\quad 8990$

Monolith Height (cm): $\quad 10$

Monolith Surface Area (cm^2): $\quad 176.72$

Monolith Volume $\left(\mathrm{cm}^{\wedge} 3\right)$ :

196.35

Sample Weight (g): $\quad 387.5$

Monolith Diameter $(\mathrm{cm}):$

Leachate Volume $(\mathrm{mL})$ : $\quad 1800$

\begin{tabular}{|c|c|c|c|c|c|c|c|}
\hline $\begin{array}{l}\text { Time } \\
\text { (hrs) }\end{array}$ & $\begin{array}{l}\text { Interval } \\
\text { Length } \\
\text { (s) }\end{array}$ & $\begin{array}{l}\text { Mean } \\
\text { Time } \\
\text { (s) }\end{array}$ & $\begin{array}{l}\text { Leachate } \\
\text { Conc. } \\
\text { (pCi/L) }\end{array}$ & $\begin{array}{l}\text { Amount } \\
\text { Leached } \\
\text { (pCi) }\end{array}$ & $\begin{array}{l}\text { Fraction } \\
\text { Leached }\end{array}$ & $\begin{array}{l}\text { Diffusivity } \\
\text { Coefficient } \\
\left(\mathrm{cm}^{\wedge} 2 / \mathrm{s}\right)\end{array}$ & $\begin{array}{l}\text { Leach } \\
\text { Index }\end{array}$ \\
\hline 2 & 7200 & 1800 & 0.575 & 1.0350 & 0.000115 & $1.785 \mathrm{E}-12$ & 11.75 \\
\hline 7 & 18000 & 14835 & 0.866 & 1.5588 & 0.000173 & 5.339E-12 & 11.27 \\
\hline 24 & 61200 & 51231 & 1.47 & 2.6460 & 0.000294 & 4.596E-12 & 11.34 \\
\hline 48 & 86400 & 125894 & 0.76 & 1.3680 & 0.000152 & $1.515 \mathrm{E}-12$ & 11.82 \\
\hline 72 & 86400 & 213818 & 1.47 & 2.6460 & 0.000294 & $9.624 \mathrm{E}-12$ & 11.02 \\
\hline 96 & 86400 & 300849 & 0.1 & 0.1800 & 0.000020 & $6.266 \mathrm{E}-14$ & 13.20 \\
\hline 120 & 86400 & 387596 & 0.91 & 1.6380 & 0.000182 & $6.685 \mathrm{E}-12$ & 11.17 \\
\hline 456 & 1209600 & 939462 & 3.53 & 6.3540 & 0.000707 & $1.244 \mathrm{E}-12$ & 11.91 \\
\hline 1128 & 2419200 & 2716550 & 1.73 & 3.1140 & 0.000346 & $2.160 \mathrm{E}-13$ & 12.67 \\
\hline 2160 & 3715200 & 5768861 & 1.44 & 2.5920 & 0.000288 & $1.348 \mathrm{E}-13$ & 12.87 \\
\hline & & & & \multicolumn{3}{|c|}{ Average Leach Index: } & 11.90 \\
\hline
\end{tabular}




\section{American Nuclear Society - 16.1 Static Leach Test}

Project: SRS Reactor Seepage Basin Stabilization

Sample Description: $\quad$ C612

Analyst: $\quad$ PRL

Date: $\quad$ 15-Apr-99

Contaminant:

Gross Beta

Initial Contaminant Conc. (pCi/g):

182

Initial Contaminant Level (pCi):

Monolith Height (cm):

70525

Monolith Surface Area $\left(\mathrm{cm}^{\wedge} 2\right)$ :

10

Monolith Volume $\left(\mathrm{cm}^{\wedge} 3\right)$ :

176.72

196.35

Sample Weight (g):

387.5

Monolith Diameter (cm):

5

Leachate Volume $(\mathrm{mL})$ :

1800

\begin{tabular}{|c|c|c|c|c|c|c|c|}
\hline $\begin{array}{l}\text { Time } \\
\text { (hrs) }\end{array}$ & $\begin{array}{l}\text { Interval } \\
\text { Length } \\
\text { (s) }\end{array}$ & $\begin{array}{c}\text { Mean } \\
\text { Time } \\
(s)\end{array}$ & $\begin{array}{l}\text { Leachate } \\
\text { Conc. } \\
\text { (pCi/L) }\end{array}$ & $\begin{array}{l}\text { Amount } \\
\text { Leached } \\
\text { (pCi) }\end{array}$ & $\begin{array}{l}\text { Fraction } \\
\text { Leached }\end{array}$ & $\begin{array}{l}\text { Diffusivity } \\
\text { Coefficient } \\
\text { (cm²/s) }\end{array}$ & $\begin{array}{l}\text { Leach } \\
\text { Index }\end{array}$ \\
\hline 2 & 7200 & 1800 & 31.2 & 56.1600 & 0.000796 & $8.540 \mathrm{E}-11$ & 10.07 \\
\hline 7 & 18000 & 14835 & 31.3 & 56.3400 & 0.000799 & $1.133 E-10$ & 9.95 \\
\hline 24 & 61200 & 51231 & 89.5 & 161.1000 & 0.002284 & $2.768 \mathrm{E}-10$ & 9.56 \\
\hline 48 & 86400 & 125894 & 41.6 & 74.8800 & 0.001062 & $7.374 E-11$ & 10.13 \\
\hline 72 & 86400 & 213818 & 84.8 & 152.6400 & 0.002164 & $5.204 \mathrm{E}-10$ & 9.28 \\
\hline 96 & 86400 & 300849 & 38.9 & 70.0200 & 0.000993 & $1.541 \mathrm{E}-10$ & 9.81 \\
\hline 120 & 86400 & 387596 & 44.8 & 80.6400 & 0.001143 & 2.633E-10 & 9.58 \\
\hline 456 & 1209600 & 939462 & 245 & 441.0000 & 0.006253 & $9.738 E-11$ & 10.01 \\
\hline 1128 & 2419200 & 2716550 & 427 & 768.6000 & 0.010898 & $2.138 E-10$ & 9.67 \\
\hline 2160 & 3715200 & 5768861 & 278 & 500.4000 & 0.007095 & $8.161 E-11$ & 10.09 \\
\hline & & & & \multicolumn{3}{|c|}{ Average Leach Index: } & 9.81 \\
\hline
\end{tabular}




\section{American Nuclear Society - 16.1 Static Leach Test}

Project: $\quad$ SRS Reactor Seepage Basin Stabilization

Sample Description: $\quad \mathrm{C622}$

$\begin{array}{llll}\text { Analyst: PRL } & \text { 15-Apr-99 }\end{array}$

Contaminant: Gross Alpha

Initial Contaminant Conc. (pCi/g): $\quad 23.2$

Initial Contaminant Level (pCi): $\quad 9033.384$

Monolith Height $(\mathrm{cm})$ :

Monolith Surface Area $\left(\mathrm{cm}^{\wedge} 2\right)$ :

Monolith Volume $\left(\mathrm{cm}^{\wedge} 3\right)$ :

10

176.72

196.35
Sample Weight (g):

Monolith Diameter (cm):

Leachate Volume $(\mathrm{mL})$ :
389.37

5

1800

\begin{tabular}{|c|c|c|c|c|c|c|c|}
\hline $\begin{array}{l}\text { Time } \\
\text { (hrs) }\end{array}$ & $\begin{array}{l}\text { Interval } \\
\text { Length } \\
\text { (s) }\end{array}$ & $\begin{array}{c}\text { Mean } \\
\text { Time } \\
\text { (s) }\end{array}$ & $\begin{array}{l}\text { Leachate } \\
\text { Conc. } \\
\text { (pCi/L) }\end{array}$ & $\begin{array}{c}\text { Amount } \\
\text { Leached } \\
\text { (pCi) }\end{array}$ & $\begin{array}{l}\text { Fraction } \\
\text { Leached }\end{array}$ & $\begin{array}{l}\text { Diffusivity } \\
\text { Coefficient } \\
\text { (cm^2/s) }\end{array}$ & $\begin{array}{l}\text { Leach } \\
\text { Index }\end{array}$ \\
\hline 2 & 7200 & 1800 & 0.25 & 0.4500 & 0.000050 & $3.342 \mathrm{E}-13$ & 12.48 \\
\hline 7 & 18000 & 14835 & 0.544 & 0.9792 & 0.000108 & 2.087E-12 & 11.68 \\
\hline 24 & 61200 & 51231 & 0.107 & 0.1926 & 0.000021 & $2.412 E-14$ & 13.62 \\
\hline 48 & 86400 & 125894 & 0.0216 & 0.0389 & 0.000004 & $1.212 E-15$ & 14.92 \\
\hline 72 & 86400 & 213818 & 0.811 & 1.4598 & 0.000162 & $2.901 \mathrm{E}-12$ & 11.54 \\
\hline 96 & 86400 & 300849 & 0.568 & 1.0224 & 0.000113 & $2.002 E-12$ & 11.70 \\
\hline 120 & 86400 & 387596 & 0.217 & 0.3906 & 0.000043 & 3.765E-13 & 12.42 \\
\hline 456 & 1209600 & 939462 & 1.14 & 2.0520 & 0.000227 & $1.285 E-13$ & 12.89 \\
\hline 1128 & 2419200 & 2716550 & 4.05 & 7.2900 & 0.000807007 & 1.17245E-12 & 11.93 \\
\hline 2160 & 3715200 & 5768861 & 0.63 & 1.1340 & 0.000125534 & $2.55456 E-14$ & 13.59 \\
\hline & & & & & \multicolumn{2}{|c|}{ Average Leach Index: } & 12.68 \\
\hline
\end{tabular}




\section{American Nuclear Society - 16.1 Static Leach Test}

Project: $\quad$ SRS Reactor Seepage Basin Stabilization

Sample Description:

$\mathrm{C} 622$

Analyst: $\quad$ PRL

Date:

15-Apr-99

Contaminant:

Gross Beta

Initial Contaminant Conc. (pCi/g):

182

Initial Contaminant Level ( $\mathrm{pCi})$ :

70865.34

Monolith Height (cm):

10

Monolith Surface Area $\left(\mathrm{cm}^{\wedge} 2\right)$ :

Monolith Volume $\left(\mathrm{cm}^{\wedge} 3\right)$ :

176.72

196.35

Sample Weight (g):

Monolith Diameter $(\mathrm{cm})$ :

Leachate Volume $(\mathrm{mL})$ :
389.37

5

1800

\begin{tabular}{|c|c|c|c|c|c|c|c|}
\hline $\begin{array}{l}\text { Time } \\
\text { (hrs) }\end{array}$ & $\begin{array}{l}\text { Interval } \\
\text { Length } \\
\text { (s) }\end{array}$ & $\begin{array}{l}\text { Mean } \\
\text { Time } \\
\text { (s) }\end{array}$ & $\begin{array}{l}\text { Leachate } \\
\text { Conc. } \\
\text { (pCill) }\end{array}$ & $\begin{array}{l}\text { Amount } \\
\text { Leached } \\
(p C i)\end{array}$ & $\begin{array}{l}\text { Fraction } \\
\text { Leached }\end{array}$ & $\begin{array}{l}\text { Diffusivity } \\
\text { Coefficient } \\
\left(\mathrm{cm}^{\wedge} 2 / \mathrm{s}\right)\end{array}$ & $\begin{array}{l}\text { Leach } \\
\text { Index }\end{array}$ \\
\hline 2 & 7200 & 1800 & 24.7 & 44.4600 & 0.000627 & $5.301 \mathrm{E}-11$ & 10.28 \\
\hline 7 & 18000 & 14835 & 19 & 34.2000 & 0.000483 & 4.136E-11 & 10.38 \\
\hline 24 & 61200 & 51231 & 54.9 & 98.8200 & 0.001394 & $1.032 E-10$ & 9.99 \\
\hline 48 & 86400 & 125894 & 19.3 & 34.7400 & 0.000490 & 1.572E-11 & 10.80 \\
\hline 72 & 86400 & 213818 & 39.1 & 70.3800 & 0.000993 & 1.096E-10 & 9.96 \\
\hline 96 & 86400 & 300849 & 44.5 & 80.1000 & 0.001130 & 1.997E-10 & 9.70 \\
\hline 120 & 86400 & 387596 & 22.7 & 40.8600 & 0.000577 & 6.695E-11 & 10.17 \\
\hline 456 & 1209600 & 939462 & 139 & 250.2000 & 0.003531 & 3.104E-11 & 10.51 \\
\hline 1128 & 2419200 & 2716550 & 245 & 441.0000 & 0.006223 & $6.972 E-11$ & 10.16 \\
\hline 2160 & 3715200 & 5768861 & 194 & 349.2000 & 0.004928 & $3.936 E-11$ & 10.40 \\
\hline & & & & \multicolumn{3}{|c|}{ Average Leach Index: } & 10.24 \\
\hline
\end{tabular}




\section{American Nuclear Society - 16.1 Static Leach Test}

Project: $\quad$ SRS Reactor Seepage Basin Stabilization

Sample Description: $\quad$ C632

$\begin{array}{llll}\text { Analyst: PRL } & \text { Date: } & \text { 15-Apr-99 }\end{array}$

Contaminant: Gross Alpha

Initial Contaminant Conc. (pCi/g):

$23.2 \quad$ Sample Weight (g):

367.95

Initial Contaminant Level (pCi):

Monolith Height (cm):

8536.44

Monolith Surface Area $\left(\mathrm{cm}^{\wedge} 2\right)$ :

10

176.72

Monolith Diameter $(\mathrm{cm})$ :

5

Monolith Volume $\left(\mathrm{cm}^{\wedge} 3\right)$ :

196.35

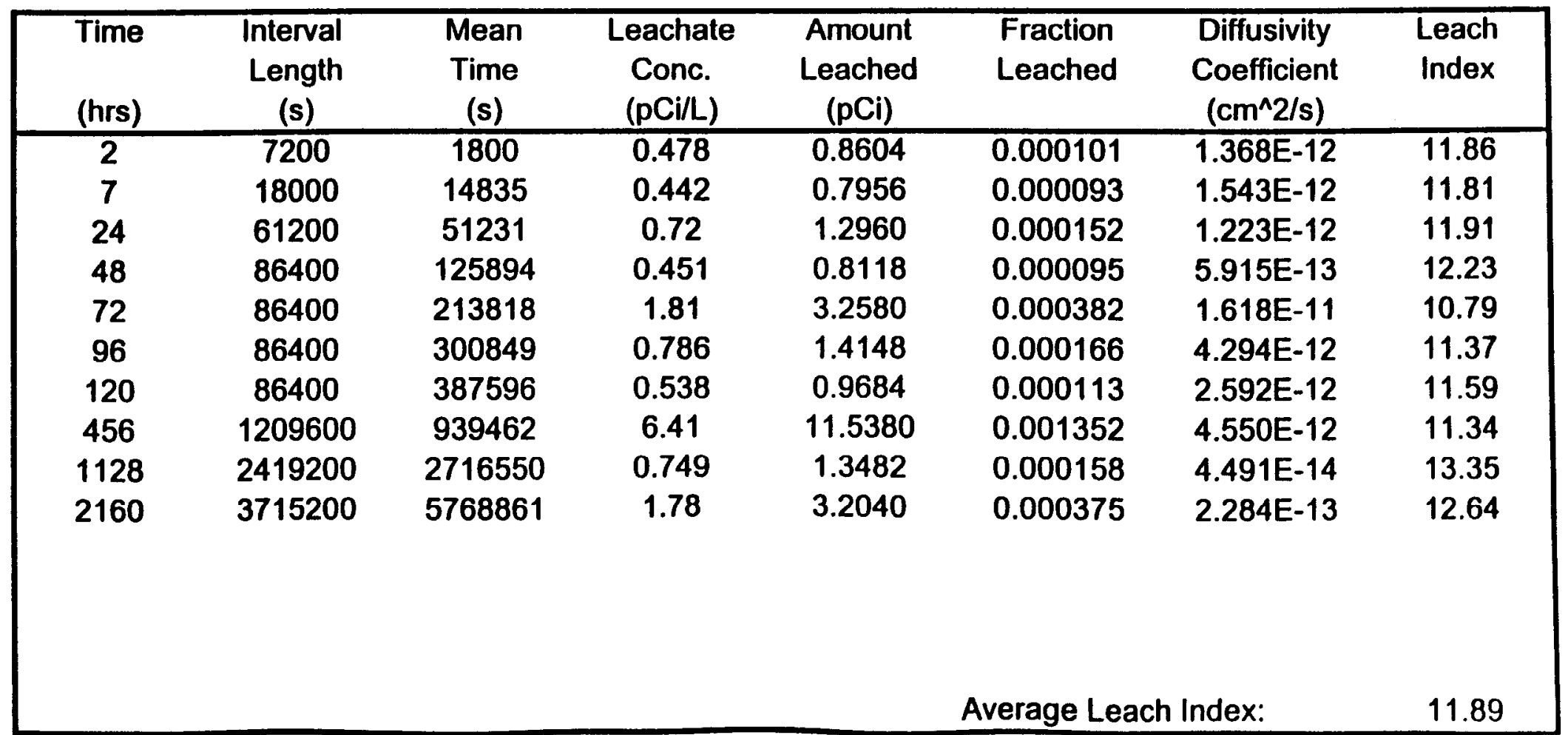




\section{American Nuclear Society - 16.1 Static Leach Test}

Project: $\quad$ SRS Reactor Seepage Basin Stabilization

Sample Description: $\quad \mathrm{C632}$

$\begin{array}{llll}\text { Analyst: PRL } & \text { Date: Apr-99 }\end{array}$

Contaminant: Gross Beta

Initial Contaminant Conc. (pCi/g): $\quad 182$

Initial Contaminant Level (pCi):

Monolith Height (cm):

66966.9

Monolith Surface Area $\left(\mathrm{cm}^{\wedge} 2\right)$ :

Monolith Volume $\left(\mathrm{cm}^{\wedge} 3\right)$ :

10

176.72

196.35

\begin{tabular}{|c|c|c|c|c|c|c|c|}
\hline $\begin{array}{l}\text { Time } \\
\text { (hrs) }\end{array}$ & $\begin{array}{l}\text { Interval } \\
\text { Length } \\
\text { (s) }\end{array}$ & $\begin{array}{c}\text { Mean } \\
\text { Time } \\
\text { (s) }\end{array}$ & $\begin{array}{l}\text { Leachate } \\
\text { Conc. } \\
\text { (pCi/L) }\end{array}$ & $\begin{array}{l}\text { Amount } \\
\text { Leached } \\
\text { (pCi) }\end{array}$ & $\begin{array}{l}\text { Fraction } \\
\text { Leached }\end{array}$ & $\begin{array}{l}\text { Diffusivity } \\
\text { Coefficient } \\
\left(\mathrm{cm}^{\wedge} 2 / \mathrm{s}\right)\end{array}$ & $\begin{array}{l}\text { Leach } \\
\text { Index }\end{array}$ \\
\hline 2 & 7200 & 1800 & 17 & 30.6000 & 0.000457 & $2.812 \mathrm{E}-11$ & 10.55 \\
\hline 7 & 18000 & 14835 & 18.6 & 33.4800 & 0.000500 & 4.439E-11 & 10.35 \\
\hline 24 & 61200 & 51231 & 67.4 & 121.3200 & 0.001812 & $1.741 \mathrm{E}-10$ & 9.76 \\
\hline 48 & 86400 & 125894 & 27.7 & 49.8600 & 0.000745 & $3.626 \mathrm{E}-11$ & 10.44 \\
\hline 72 & 86400 & 213818 & 44.3 & 79.7400 & 0.001191 & $1.575 \mathrm{E}-10$ & 9.80 \\
\hline 96 & 86400 & 300849 & 24.8 & 44.6400 & 0.000667 & $6.946 \mathrm{E}-11$ & 10.16 \\
\hline 120 & 86400 & 387596 & 29.1 & 52.3800 & 0.000782 & $1.232 E-10$ & 9.91 \\
\hline 456 & 1209600 & 939462 & 105 & 189.0000 & 0.002822 & $1.984 \mathrm{E}-11$ & 10.70 \\
\hline 1128 & 2419200 & 2716550 & 305 & 549.0000 & 0.008198 & $1.210 \mathrm{E}-10$ & 9.92 \\
\hline 2160 & 3715200 & 5768861 & 187 & 336.6000 & 0.005026 & 4.095E-11 & 10.39 \\
\hline & & & & \multicolumn{3}{|c|}{ Average Leach Index: } & 10.20 \\
\hline
\end{tabular}




\section{American Nuclear Society - 16.1 Static Leach Test}

Project: $\quad$ SRS Reactor Seepage Basin Stabilization

Sample Description: $\quad$ C642

$\begin{array}{llll}\text { Analyst: PRL } & \text { Date: }\end{array}$

Contaminant: Gross Alpha

Initial Contaminant Conc. (pCi/g): $\quad 23.2$

Initial Contaminant Level (pCi): $\quad 8772.384$

Monolith Height $(\mathrm{cm})$ :

Monolith Surface Area (cm^2): $\quad 176.72$

Monolith Volume (cm^3): $\quad 196.35$

Sample Weight (g):

Monolith Diameter $(\mathrm{cm}): \quad 5$

Leachate Volume $(\mathrm{mL}): \quad 1800$

\begin{tabular}{|c|c|c|c|c|c|c|c|}
\hline $\begin{array}{l}\text { Time } \\
\text { (hrs) }\end{array}$ & $\begin{array}{l}\text { Interval } \\
\text { Length } \\
\text { (s) }\end{array}$ & $\begin{array}{c}\text { Mean } \\
\text { Time } \\
\text { (s) }\end{array}$ & $\begin{array}{c}\text { Leachate } \\
\text { Conc. } \\
\text { (pCi/L) }\end{array}$ & $\begin{array}{c}\text { Amount } \\
\text { Leached } \\
(\mathrm{pCi})\end{array}$ & $\begin{array}{l}\text { Fraction } \\
\text { Leached }\end{array}$ & $\begin{array}{l}\text { Diffusivity } \\
\text { Coefficient } \\
\text { (cm^2/s) }\end{array}$ & $\begin{array}{l}\text { Leach } \\
\text { Index }\end{array}$ \\
\hline 2 & 7200 & 1800 & 0.367 & 0.6606 & 0.000075 & $7.637 \mathrm{E}-13$ & 12.12 \\
\hline 7 & 18000 & 14835 & 0.365 & 0.6570 & 0.000075 & $9.961 \mathrm{E}-13$ & 12.00 \\
\hline 24 & 61200 & 51231 & 0.781 & 1.4058 & 0.000160 & $1.362 E-12$ & 11.87 \\
\hline 48 & 86400 & 125894 & 0.305 & 0.5490 & 0.000063 & $2.562 E-13$ & 12.59 \\
\hline 72 & 86400 & 213818 & 0.783 & 1.4094 & 0.000161 & $2.868 \mathrm{E}-12$ & 11.54 \\
\hline 96 & 86400 & 300849 & 0.561 & 1.0098 & 0.000115 & $2.071 \mathrm{E}-12$ & 11.68 \\
\hline 120 & 86400 & 387596 & 0.886 & 1.5948 & 0.000182 & $6.656 \mathrm{E}-12$ & 11.18 \\
\hline 456 & 1209600 & 939462 & 2.51 & 4.5180 & 0.000515 & $6.606 \mathrm{E}-13$ & 12.18 \\
\hline 1128 & 2419200 & 2716550 & 7.41 & 13.3380 & 0.001520 & $4.162 \mathrm{E}-12$ & 11.38 \\
\hline 2160 & 3715200 & 5768861 & 0.456 & 0.8208 & 0.000094 & $1.419 \mathrm{E}-14$ & 13.85 \\
\hline & & & & & \multicolumn{2}{|c|}{ Average Leach Index: } & 12.04 \\
\hline
\end{tabular}




\section{American Nuclear Society - 16.1 Static Leach Test}

Project: $\quad$ SRS Reactor Seepage Basin Stabilization

Sample Description: $\quad C 642$

Analyst: $\quad$ PRL

Date:

15-Apr-99

Contaminant:

Gross Beta

Initial Contaminant Conc. (pCi/g):

182

Initial Contaminant Level (pCi):

68817.84

Monolith Height (cm):

10

Monolith Surface Area $\left(\mathrm{cm}^{\wedge} 2\right)$ :

176.72

Monolith Volume $\left(\mathrm{cm}^{\wedge} 3\right)$ :

196.35

Sample Weight (g):

Monolith Diameter $(\mathrm{cm})$ :

Leachate Volume $(\mathrm{mL})$ :
378.12

5

1800

\begin{tabular}{|c|c|c|c|c|c|c|c|}
\hline $\begin{array}{l}\text { Time } \\
\text { (hrs) }\end{array}$ & $\begin{array}{l}\text { Interval } \\
\text { Length } \\
\text { (s) }\end{array}$ & $\begin{array}{l}\text { Mean } \\
\text { Time } \\
\text { (s) }\end{array}$ & $\begin{array}{l}\text { Leachate } \\
\text { Conc. } \\
\text { (pCi/L) }\end{array}$ & $\begin{array}{l}\text { Amount } \\
\text { Leached } \\
\text { (pCi) }\end{array}$ & $\begin{array}{l}\text { Fraction } \\
\text { Leached }\end{array}$ & $\begin{array}{l}\text { Diffusivity } \\
\text { Coefficient } \\
\text { (cm^2/s) }\end{array}$ & $\begin{array}{l}\text { Leach } \\
\text { Index }\end{array}$ \\
\hline 2 & 7200 & 1800 & 11.2 & 20.1600 & 0.000293 & $1.156 \mathrm{E}-11$ & 10.94 \\
\hline 7 & 18000 & 14835 & 21.4 & 38.5200 & 0.000560 & $5.564 \mathrm{E}-11$ & 10.25 \\
\hline 24 & 61200 & 51231 & 24.3 & 43.7400 & 0.000636 & 2.143E-11 & 10.67 \\
\hline 48 & 86400 & 125894 & 15.6 & 28.0800 & 0.000408 & $1.089 E-11$ & 10.96 \\
\hline 72 & 86400 & 213818 & 25.8 & 46.4400 & 0.000675 & $5.059 \mathrm{E}-11$ & 10.30 \\
\hline 96 & 86400 & 300849 & 27.5 & 49.5000 & 0.000719 & 8.087E-11 & 10.09 \\
\hline 120 & 86400 & 387596 & 20.2 & 36.3600 & 0.000528 & $5.622 E-11$ & 10.25 \\
\hline 456 & 1209600 & 939462 & 263 & 473.4000 & 0.006879 & $1.178 \mathrm{E}-10$ & 9.93 \\
\hline 1128 & 2419200 & 2716550 & 251 & 451.8000 & 0.006565 & $7.759 E-11$ & 10.11 \\
\hline 2160 & 3715200 & 5768861 & 194 & 349.2000 & 0.005074 & $4.174 E-11$ & 10.38 \\
\hline & & & & \multicolumn{3}{|c|}{ Average Leach Index: } & 10.39 \\
\hline
\end{tabular}




\section{ATTACHMENT \\ Appendix C}

\section{Chain-of-Custody \& Request-for-Analysis}


INTERNATIONAL

TECHNOLOGY

CORPORATION

Project Name/No. 1 SALC 77730400018500 Shipment Date $711 / 23 / 98$
ANALYSIS REQUEST AND CHAIN OF CUSTODY RECORD * Sample Team Members 2 Profit Center No. 3

Lab Destination 8 ETOC

Project Manager 4 PnuL Loth

Purchase Order No. 6

Required Report Date 11 12/4/98

\begin{tabular}{|c|c|c|c|c|c|c|}
\hline $\begin{array}{l}\text { Samplo } 14 \\
\text { Numbor }\end{array}$ & $\begin{array}{c}\text { 8ample } 15 \\
\text { Description/Type }\end{array}$ & $\begin{array}{l}\text { Date/Time } \\
\text { Collected }\end{array}$ & $\begin{array}{c}\text { Container } \\
\text { Typa }\end{array}$ & \begin{tabular}{|l} 
Bample 18 \\
Volume
\end{tabular} & $\begin{array}{r}\text { Pre- } 19 \\
\text { servative } \\
\end{array}$ & $\begin{array}{c}\text { Requested Testing } 20 \\
\text { Program }\end{array}$ \\
\hline $\mathrm{C}_{3} \| \mathrm{FOO}$ & STAS. SBiL & $11 / .1 / 98$ & $2^{\prime \prime} \times 4^{\prime \prime}$ mord & $200 M c$ & NONE & ucs (14 oay) \\
\hline 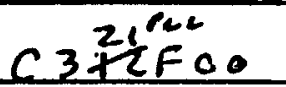 & & & & & & \\
\hline C.331 Foo & & & & & & \\
\hline C 341 Foo & & & & & & \\
\hline C411Foo & & & & & & \\
\hline C421 Foo & & & & & & \\
\hline$C 431$ Foo & & & & & & \\
\hline$C 441$ Foo & $\downarrow$ & $\downarrow$ & $\downarrow$ & $V$ & $\downarrow$ & $\downarrow$ \\
\hline
\end{tabular}

Project Contact/Phone 12

Carrier/Waybill No. ${ }^{13}$
Reference Document No. 426618 Page 1 of 2

Bill to: 5

Special Instructions: 23

Possible Hazard Identification: 24

Nonhazard [l Flammable

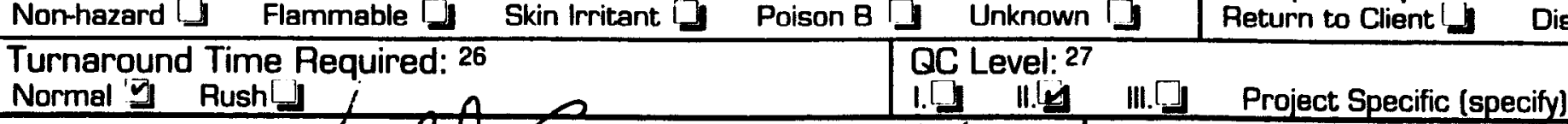

\begin{tabular}{|c|c|c|c|}
\hline $\begin{array}{l}\text { 1. Relinquished by } \\
\text { (Signature/Affiliation) }\end{array}$ & $\begin{array}{l}\text { Date: } 19301 / 23 / 98 \\
\text { Time: } 1030\end{array}$ & 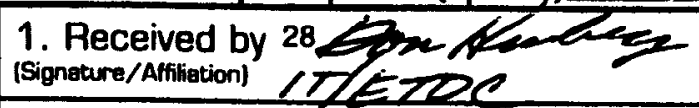 & $\begin{array}{l}\text { Date: } / /-23-98 \\
\text { Time: } / 100\end{array}$ \\
\hline $\begin{array}{l}\text { 2. Relinquished by } \\
\text { [Signature/Affitiation] }\end{array}$ & $\begin{array}{l}\text { Date: } \\
\text { Time: }\end{array}$ & $\begin{array}{l}\text { 2. Received by } \\
\text { (Signature/Affliation) }\end{array}$ & $\begin{array}{l}\text { Date: } \\
\text { Time: } \\
\end{array}$ \\
\hline $\begin{array}{l}\text { 3. Relinquished by } \\
\text { (Signeare/Affliation) }\end{array}$ & $\begin{array}{l}\text { Date: } \\
\text { Time: }\end{array}$ & $\begin{array}{l}\text { 3. Received by } \\
\text { [Signature/Afribition) }\end{array}$ & $\begin{array}{l}\text { Date: } \\
\text { Time: }\end{array}$ \\
\hline
\end{tabular}

Comments: ${ }^{20}(2)$ samples marked C421 F00 
TI INTERNATIONAL

OLOGY

CORPORATION

Project Name SAle
ANALYSIS RERUEST AND CHAIN OF CUSTODY RECORD (cont.) *

Project No. 777304, 00010500
Reference Document No. ${ }^{30} 4 \angle 6618$ Page 2 of 2

Samples Shipment Date $'$ '../23/28

\begin{tabular}{|c|c|c|c|c|c|c|c|c|}
\hline $\begin{array}{l}\text { Sample } 14 \\
\text { Number }\end{array}$ & $\begin{array}{c}\text { Sample } 15 \\
\text { Description/Type }\end{array}$ & $\begin{array}{l}\text { Date/Time } 16 \\
\text { Colloctod } \\
\end{array}$ & $\begin{array}{c}\text { Container't } \\
\text { Type }\end{array}$ & \begin{tabular}{|l|}
$\begin{array}{l}\text { Sample 18 } \\
\text { Volume }\end{array}$ \\
\end{tabular} & $\begin{array}{r}\text { Pro-19 } \\
\text { semvative } \\
\end{array}$ & $\begin{array}{c}\text { Requested Tosting } 20 \\
\text { Program }\end{array}$ & $\begin{array}{l}\text { Condition on } 21 \\
\text { Receint }\end{array}$ & $\begin{array}{l}\text { Disposal } 22 \\
\text { Record No. }\end{array}$ \\
\hline C511Foo & STAB. SOLL. & 1111198 & $244^{\prime \prime} \operatorname{recos}$ & $200 \mathrm{mc}$ & كميرم & ves (14 eav) & ETDC 7813 & \\
\hline$C 521$ Foo & & 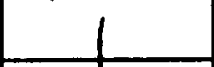 & 1 & & & 1 & ETDC 7814 & 3 \\
\hline es31foo & & & & & & & ETDC $\quad 7815$ & \\
\hline es4, Foo & & & & & & & ETDC 7816 & \\
\hline e $6 / 1$ Foo & & & & & & & FTnR: $\quad 7817$ & 11 \\
\hline $\mathrm{C}_{621} \mathrm{FoO}$ & & & & & & & ETDC 7818 & \\
\hline C631F00 & & & & & & & 7819 & \\
\hline C64lFoo & $\downarrow$ & $\downarrow$ & $\downarrow$ & $t$ & $\downarrow$ & $\downarrow$ & ETDC 7820 & \\
\hline$=$ & & & & & & & 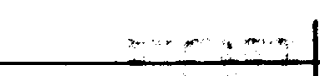 & \\
\hline & - & & & & & & $=$ & \\
\hline & & & & & & & & \\
\hline & & & & & & & $\varepsilon=1$ & 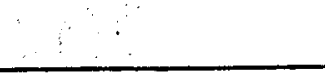 \\
\hline & & & & & & & $3: 3$ & $\because$ \\
\hline & & & & & & & & \\
\hline & & & & & & & 3 & \\
\hline & & & & & & & & \\
\hline & & & & & & & & \\
\hline & & & & & & & 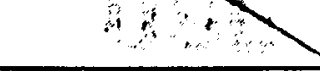 & \\
\hline & & & & & & & & \\
\hline & & & & & & & & \\
\hline
\end{tabular}


INTERNATIONAI

TECHNOLOGY

CORPORATION

Project Name/No. 3 SAIC 777304 Sample Team Members 2

Profit Center No. 3

Project Manager ${ }^{4} P$, LoA/2

Purchase Order No. 6

Required Report Date 11
ANALYSIS REQUEST AND CHAIN OF CUSTODY RECORD*

Samples Shipment Date ${ }^{7} u / 23 / 98$

Lab Destination 8 . ETOC

Lab Contact ${ }^{9}$ R. LoLE

Project Contact/Phone 12

Carrier/Waybill No. ${ }^{13}$
Reference Document No. 426623 Page 1 of 2

Bill to: ${ }^{5}$

Report to: 10

ONE CONTAINER PER LINE

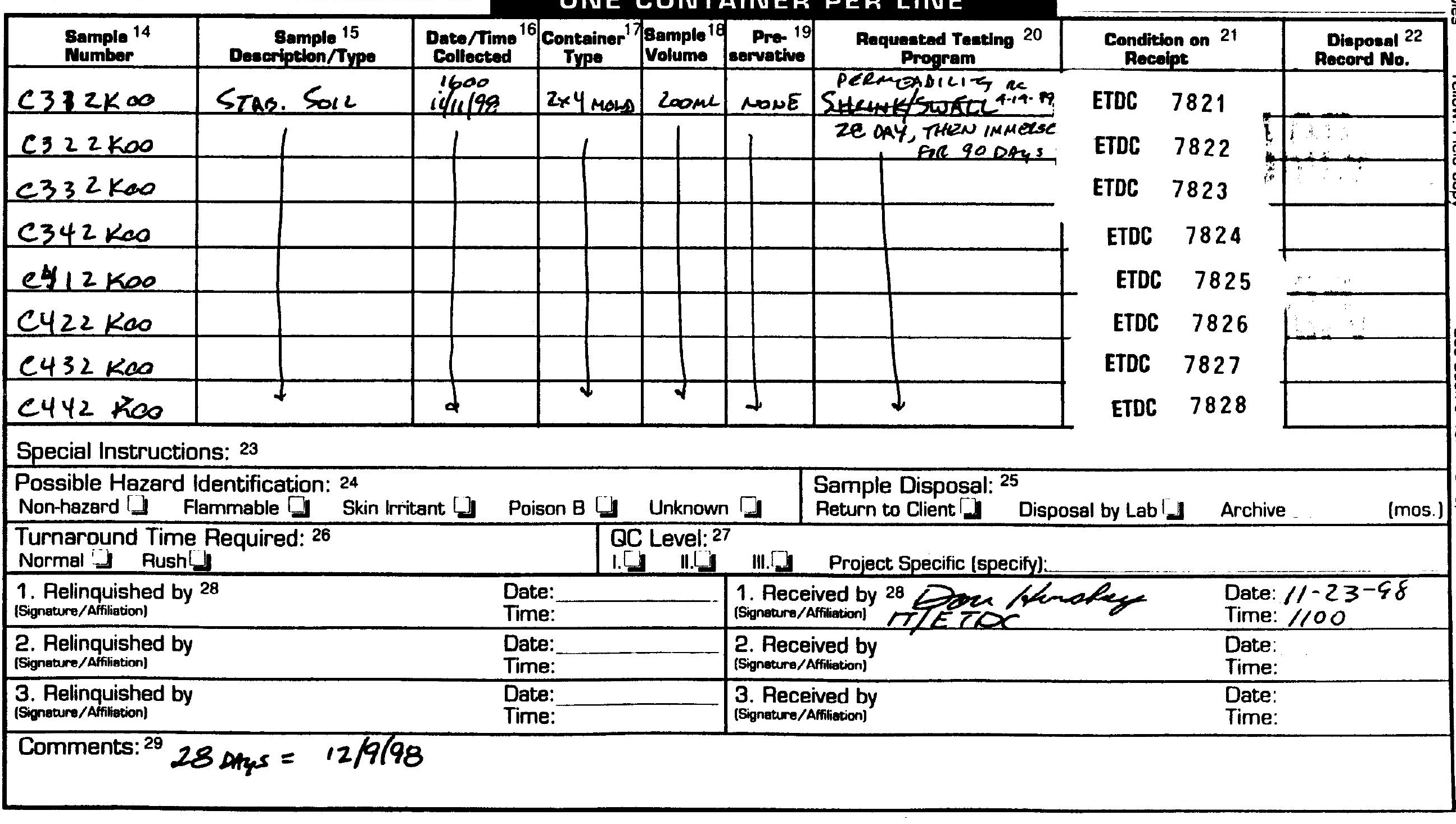


[1. INTERNATIONAL

TECHNOLOGY

CORPORATION

Project Name SAle
ANALYSIS REQUEST AND CHAIN OF CUSTODY RECORD (cont.) *

Project No. ZZ7304
Reference Document No. ${ }^{30} 426623$

Page 2 of 2

Samples Shipment Date il/23/28

ONE CONTAINER PER LINE

\begin{tabular}{|c|c|c|c|c|c|c|c|c|}
\hline $\begin{array}{l}\text { Sample } 14 \\
\text { Number }\end{array}$ & $\begin{array}{c}\text { Sample } 15 \\
\text { Deeceription/Type } \\
\end{array}$ & \begin{tabular}{|l} 
Date/Time \\
Collocted
\end{tabular} & $\begin{array}{c}\text { Container } \\
\text { Type }\end{array}$ & $\begin{array}{l}\text { Sample } 18 \\
\text { Volume }\end{array}$ & $\begin{array}{r}\text { Pro-19 } \\
\text { semvative } \\
\end{array}$ & $\begin{array}{l}\text { Requested Testing } 20 \\
\text { Program Mftes- }\end{array}$ & $\begin{array}{c}\text { Condition on } 21 \\
\text { Receipt }\end{array}$ & $\begin{array}{l}\text { Disposal } 22 \\
\text { Rocord No. } \\
\end{array}$ \\
\hline C512koo & STAS, SOlL & i2000 & $2 \times 4$ mans & $200 \mathrm{ml}$ & Raned & SERMencistat & ETDC $\quad 7829$ & \\
\hline$C S^{22}=1000$ & & 27 & & 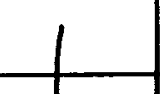 & & $\begin{array}{l}28006, \text { TrE } \\
9000 \% \text { cmeaxse }\end{array}$ & ETDC 7830 & 5 \\
\hline$c 5321000$ & & & & & & & ETDC 7831 & 264 \\
\hline$C 542600$ & & & & & & & ETDC 7832 & La \\
\hline $\mathrm{CG} 12 \mathrm{KOO}$ & & & & & & & ETDC 7833 & 3,129 \\
\hline e6221<00 & & & & & & & ETDC $\quad 7834$ & \\
\hline $6632 k 00$ & & & & & & & ETDC $\quad 7835$ & $3:$ \\
\hline e642K00 & $\downarrow$ & 7 & $\downarrow$ & $\downarrow$ & t & $\downarrow$ & ETDC $\quad 7836$ & $\therefore \quad 4$ \\
\hline & & & & & & & Nong & 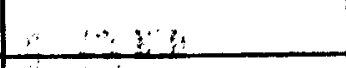 \\
\hline & & & & & & & f & 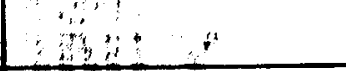 \\
\hline & & & & & & & 396406 & \\
\hline & & & & & & & Hon & 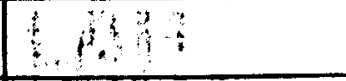 \\
\hline & & & & & & & isto & 40 \\
\hline & & & & & & & $4=0$ & 4123 \\
\hline & & & & & & & $\$ 1+1$ & 30 \\
\hline & & & & & & & & \\
\hline & & & & & & & In! & , \\
\hline & & & & & & & Neton & $\because:$ \\
\hline & & & & & & & & \\
\hline & & & & & & & & \\
\hline
\end{tabular}


INTERNATIONAI

TECHNOLOGY

CORPORATION

Project Name/No. 1 ZZZ304/SAlC Sample Team Members 2

Profit Center No. 3

Project Manager ${ }^{4}$ P. Coxa

Purchase Order No. 6

Required Report Date 11
ANALYSIS REQUEST AND CHAIN OF CUSTODY RECORD *

Samples Shipment Date $7 \mathrm{M} / 23 / 83$

Lab Destination 8 ETDC

Lab Contact ${ }^{9} R$. Covs

Project Contact/Phone 12

Carrier/Waybill No. ${ }^{13}$
Reference Document No. 426620 Page 1 of 2

Bill to: 5

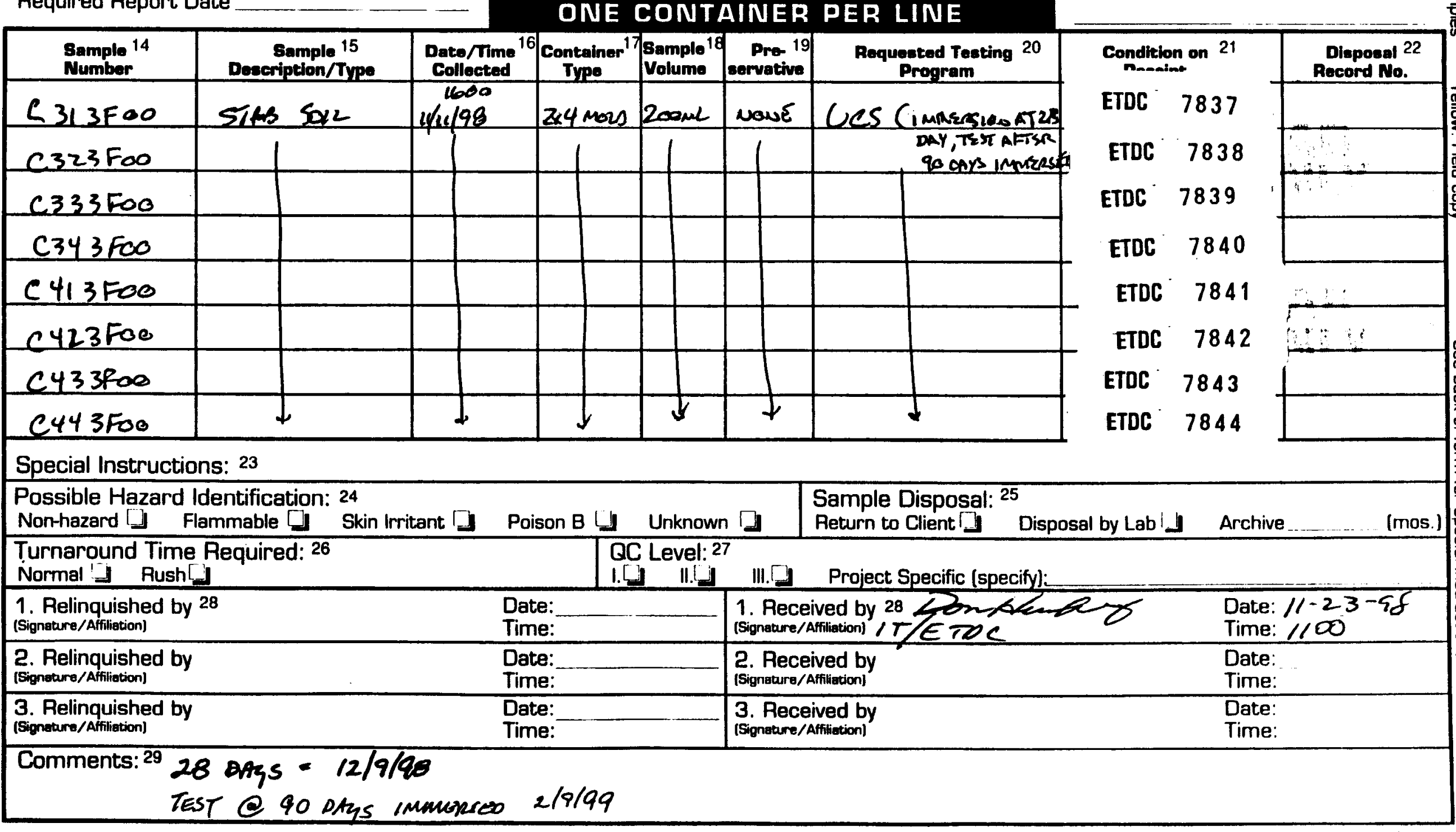


INTERNATIONAL

TECLNOLOGY

CORPORATION

Project Name 777304
ANALYSIS REQUEST AND CHAIN OF CUSTODY RECORD (cont.)*

Project No.SAle
Reference Document No. ${ }^{30} 4266^{20}$ Page 2 of 2

Samples Shipment Date $i c / 29 / 2 \theta$

\section{ONE CONTAINER PER LINE}

\begin{tabular}{|c|c|c|c|c|c|c|c|c|}
\hline $\begin{array}{l}\text { Sample } 14 \\
\text { Number }\end{array}$ & $\begin{array}{c}\text { Sample } 15 \\
\text { Dascription/Type }\end{array}$ & $\begin{array}{l}\text { Dato/Time } 16 \\
\text { Collocted }\end{array}$ & $\begin{array}{c}\text { Container } 17 \\
\text { Type }\end{array}$ & $\begin{array}{l}\text { Sample } 18 \\
\text { Volume } \\
\end{array}$ & $\begin{array}{r}\text { Pro-19 } \\
\text { sorvativo } \\
\end{array}$ & $\begin{array}{l}\text { Requestod Testing } 20 \\
\text { Program }\end{array}$ & $\begin{array}{c}\text { Condition on } 21 \\
\text { Recoipt }\end{array}$ & $\begin{array}{l}\text { Disposal } 22 \\
\text { Record No. } \\
\end{array}$ \\
\hline C513Foo & STAS SOLL & $\begin{array}{r}1600 \\
11 / 4 / 98 \\
\end{array}$ & $2 \times 4$ rens & $200 \mathrm{me}$ & Fon & UeS Cimmsesian & ETDC 7845 & \\
\hline C523F0O & 1 & 8. & & & & 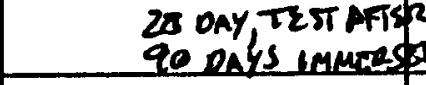 & ETDC 7846 & \\
\hline C533Foo & & & & & & 1 & ETDC 7847 & 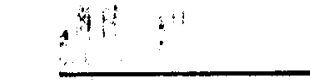 \\
\hline est3fos & & & & & & & ETDC 7848 & \\
\hline C613Foe & & & & & & & ETDC 7849 & 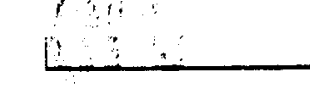 \\
\hline C623F00 & & & & & & & ETDC 7850 & \\
\hline C633 Fos & & & & & & & ETDC 7851 & \\
\hline C643Foo & $\downarrow$ & $\downarrow$ & $\downarrow$ & $\downarrow$ & $\downarrow$ & $\downarrow$ & ETOC 7852 & \\
\hline & & & & & & & 18 & \\
\hline & & & & & & & & \\
\hline & & & & & & & 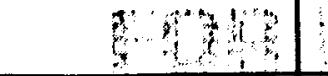 & 3 \\
\hline & & & & & & & 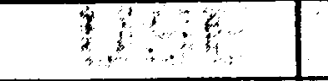 & a? \\
\hline & & & & & & & 80002 & \\
\hline & & & & & & & 850 & \\
\hline & & & & & & & & \\
\hline & & & & & & & $3 \times x$ & \\
\hline & & & & & & & 9. & \\
\hline & & & & & & & & \\
\hline & & & & & & & & \\
\hline
\end{tabular}


INTERNATIONAL

TECHNOLOGY

CORPORATION

Project Name/No. 1 SAlC 777304 Sample Team Members 2

Profit Center No. 3

Project Manager 4 P. LEne

Purchase Order No. 6

Required Report Date ${ }^{11}$
2

\section{ANALYSIS REQUEST AND} CHAIN OF CUSTODY RECORD *

Samples Shipment Date $711 / 23 / 28$

Lab Destination EFOC

Lab Contact 9 R. Corio

Project Contact/Phone 12

Carrier/Waybill No. ${ }^{13}$
Reference Document No. 426622 Page 1 of 2

Bill to: ${ }^{5}$

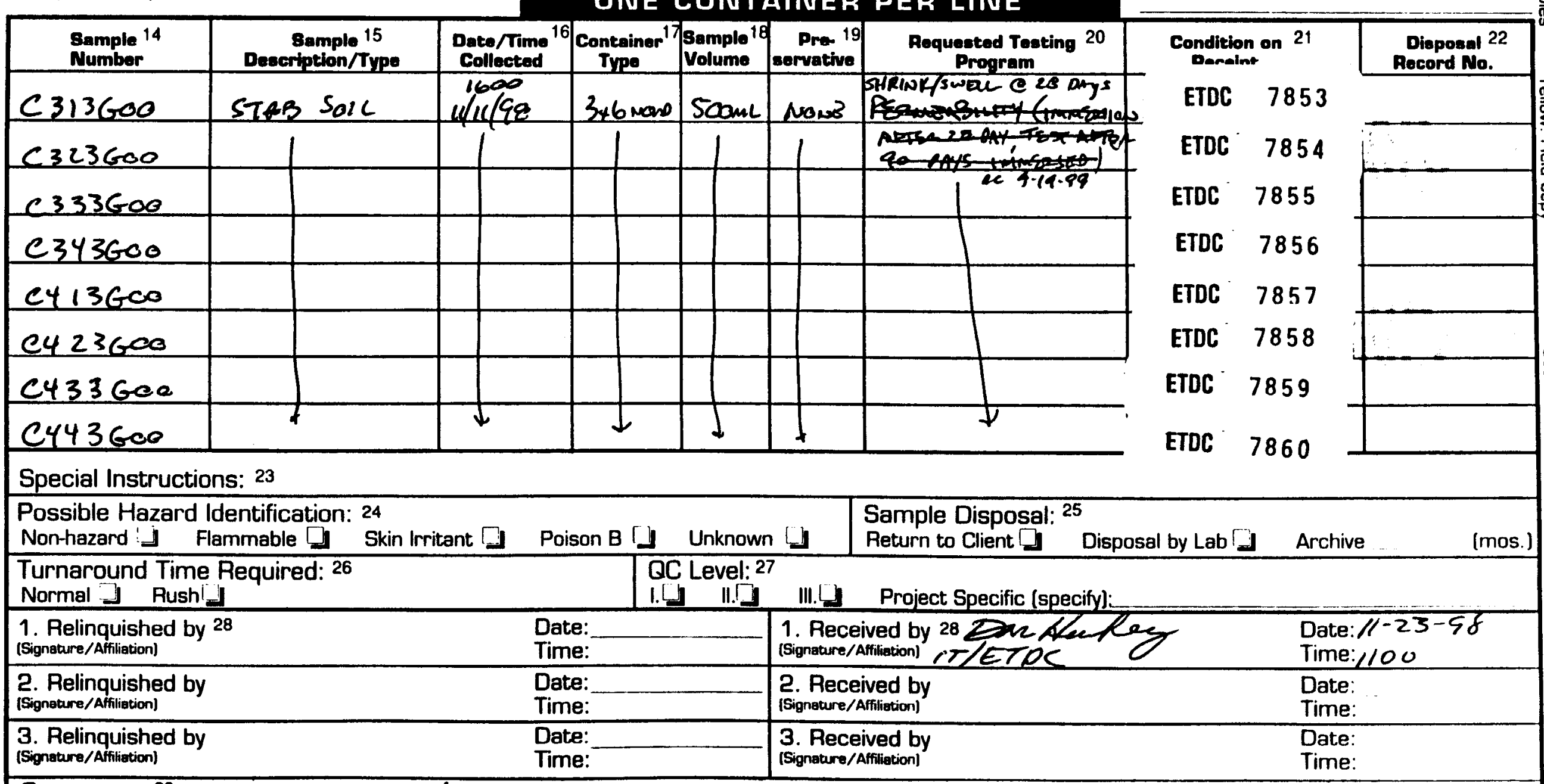

\section{ONE CONTAINER PER LINE}

सRINK/swal e 28 ang $^{5}$

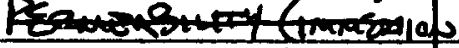

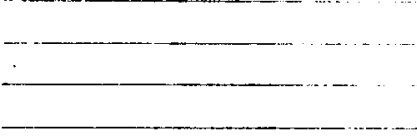

Report to: 10

1

Comments: 29 Deys $=12 / 9 / 98$ 
INTERNATIONAL

TECHNOLOGY

CORPORATION

Project Name SALC
ANALYSIS REQUEST AND CHAIN OF CUSTODY RECORD (cont.) *

Project No. ZZZ7304.
Reference Document No. ${ }^{30} 426622$ Page 2 of 2

Samples Shipment Date il/2s/28.

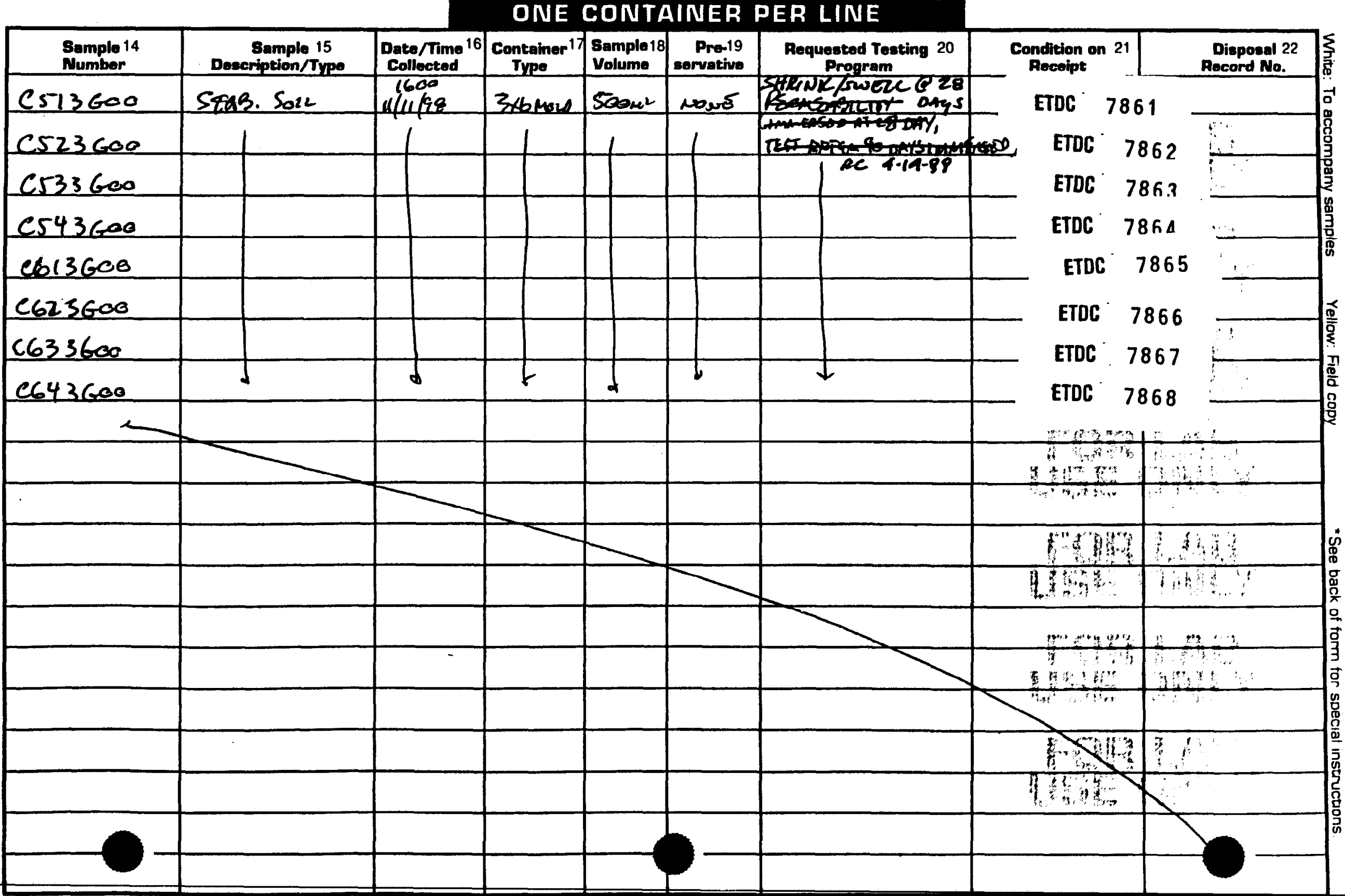


Project Name/No. 1 Sample Team Members 2 Profit Center No. 3 Project Manager 4 Purchase Order No. 6 Required Report Date 11

\section{ANALYSIS REQUEST AND} CHAIN OF CUSTODY RECORD *

Samples Shipment Date 7

Lab Destination 8

Lab Contact 9

Project Contact/Phone 12

Carrier/Waybill No. 13
Reference Document No. 42662 Page 1 of 2

Bill to: 5

Report to: 10
ONE CONTAINER PER LINE

\begin{tabular}{|c|c|c|c|c|c|c|}
\hline $\begin{array}{l}\text { Samplo } 14 \\
\text { Numbor }\end{array}$ & $\begin{array}{c}\text { Sample } 15 \\
\text { Description/Type }\end{array}$ & $\begin{array}{l}\text { Date/Time } \\
\text { Collected }\end{array}$ & $\begin{array}{c}\text { Containar } \\
\text { Type }\end{array}$ & $\begin{array}{l}\text { Sample } \\
\text { Volume }\end{array}$ & $\begin{array}{c}\text { Pro } 19 \\
\text { sorvative }\end{array}$ & $\begin{array}{l}\text { Pequested Toating } 20 \\
\text { Program }\end{array}$ \\
\hline C4 12G0O & S7AS Soll & $\begin{array}{l}1600 \\
1411 / 98\end{array}$ & 36 mas & Somal & mans & 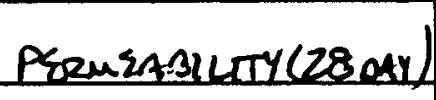 \\
\hline C4 2260O & & & & & I & \\
\hline 2432600 & & & & & & \\
\hline $\mathrm{C}_{4} \mathrm{~S}_{3 \mathrm{GO}}$ & & & & & & \\
\hline CSIRGOO & & & & & & \\
\hline CSZ2600 & & & & & & \\
\hline $\mathrm{CS} 32 \mathrm{GaO}$ & & & & & & \\
\hline $\operatorname{es~} 42600$ & $\checkmark$ & 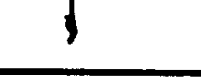 & & $\downarrow$ & $\downarrow$ & $\downarrow$ \\
\hline
\end{tabular}

Condition on 21

Receipt

ETDC 7869

ETDC 7870

ETDC 7871

ETDC 7872

ETDC 7873

ETDC 7874

ETDC 7875

ETDC 7876

Special Instructions: 23

Possible Hazard Identification: 24

Non-hazard $\square$ Flammable Iy Skin Irritant [J Poison B D Unknown

Turnaround Time Required: 26

Normal ${ }^{-2}$ Rush $\left.{ }^{-}\right]$

1. Relinquished by 28 (Signatura/Affiliztion) 2. Relinquished by
(Signeture/Affiliation)

3. Relinquished by

[Signeture/Afflistion]

Sample Disposal: 25

Return to Client $\beth$ Disposal by Lab I Archive

Dieposal 22 Record No.

Comments: 29

28 DMs - $12 / 9 / 98$

Project Specific (specify

Date:
Time:
Date:
Time:
Date:
Time:

1. Received by 28 (Signature/Affilietion)

2. Received by (Signeture/Affiliotion)

3. Received by (Signeture/Affitation)
Date: $/ 1-23.98$

Time: $/ 100$

Date:

Time:

Date

Time: 
INTERNATIONAL

TECTNOLOGY

CORPORATION

Project Name SAlC
ANALYSIS REQUEST AND CHAIN OF CUSTODY RECORD (cont.)*

Project No. 777304
Reference Document No. ${ }^{30} 426621$ Page 2 of 2

Samples Shipment Date ... $1 / 23 / 18$
ONE CONTAINER PER LINE

\begin{tabular}{|c|c|c|c|c|c|c|c|c|}
\hline $\begin{array}{l}\text { Sample } 14 \\
\text { Number }\end{array}$ & $\begin{array}{c}\text { Sample } 15 \\
\text { Description/Type }\end{array}$ & $\begin{array}{l}\text { Date/Time } \\
\text { Collected }\end{array}$ & $\begin{array}{c}\text { Container } \\
\text { Type }\end{array}$ & $\begin{array}{l}\text { Sample } 18 \\
\text { Volume }\end{array}$ & $\begin{array}{r}\text { Pro-19 } \\
\text { servative } \\
\end{array}$ & $\begin{array}{c}\text { Pequostod Tosting } 20 \\
\text { Program }\end{array}$ & $\begin{array}{c}\text { Condition on } 21 \\
\text { Darsalot }\end{array}$ & $\begin{array}{c}\text { Dispowal } 22 \\
\text { Rocord No. }\end{array}$ \\
\hline$C_{612600}$ & Stos solc & $\begin{array}{l}1600 \\
141460\end{array}$ & $3 \times 6$ How & $500 \mathrm{me}$ & Mans & Pormonsilety (2anx) & ETDC 7877 & \\
\hline$C 6 z^{2}-600$ & & 1 & 1 & 7 & & & ETDC 7878 & Di \\
\hline $\mathrm{C} 632 \mathrm{GOO}$ & & & & & & & ETDC 7879 & 23 \\
\hline$C_{6} 42600$ & & & & & & & ETDC $\quad 7880$ & \\
\hline$c 312600$ & & & & & & & ETDC 7881 & $5: 0^{\circ}$ \\
\hline$c 322600$ & & & & & & & ETDC & \\
\hline$C 332600$ & & & & & & & 7000 & 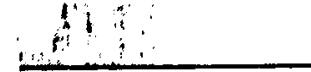 \\
\hline C.342600 & J & $\downarrow$ & $\downarrow$ & $\downarrow$ & $\downarrow$ & $\downarrow$ & 8883 & \\
\hline & & & & & & & 1884 & \\
\hline & & & & & & & 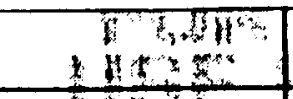 & 3 \\
\hline & & & & & & & 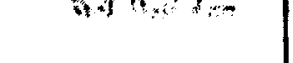 & \\
\hline & & & & & & & 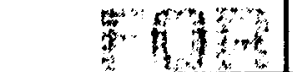 & $4 \%$ \\
\hline & & & & & & & 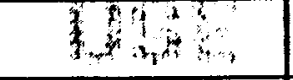 & $\because \ldots$ \\
\hline & & & & & & & & \\
\hline & & & & & & & L & $41:$ \\
\hline & & & & & & & & \\
\hline & & & & & & & 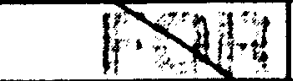 & 14 \\
\hline & & & & & & & $4,11 \%$ & \\
\hline & & & & & & & & \\
\hline & & & & & & & & \\
\hline
\end{tabular}


INTERNATIONAL

CORPORATION

Project Name/No. 1 SALC 777304 Sample Team Members 2

Profit Center No. 3

Project Manager ${ }^{4} P$. colpe

Purchase Order No. 6

Required Report Date 11
TECHNOLOGY

ANALYSIS REQUEST AND CHAIN OF CUSTODY RECORD*

Samples Shipment Date 7 12/23/98

Lab Destination 8 ETDC

Lab Contact ${ }^{9}$ RaLPW CoL $\bar{\theta}$

Project Contact/Phone 12

Carrier/Waybill No. ${ }^{13}$

ONE CONTAINER PER LINE

\begin{tabular}{|c|c|c|c|c|c|c|}
\hline $\begin{array}{l}\text { Sample } 14 \\
\text { Number }\end{array}$ & $\begin{array}{c}\text { 8ample } 15 \\
\text { Description/Type }\end{array}$ & $\begin{array}{l}\text { Date/Time } \\
\text { Collected }\end{array}$ & $\begin{array}{c}\text { Container } \\
\text { Type }^{17} \\
\end{array}$ & \begin{tabular}{|l}
$\begin{array}{l}\text { Sample } \\
\text { Volume }\end{array}$ \\
\end{tabular} & \begin{tabular}{|c|} 
Pro- 19 \\
servative
\end{tabular} & $\begin{array}{c}\text { Requestad Teating } 20 \\
\text { Program }\end{array}$ \\
\hline C3Z广F⿻o & Stacs Soll & 11/468 & $2^{x} \times 4^{2}$ mors & $20 \mathrm{oul}$ & NoNB & $\operatorname{ces}(28$ DAY) \\
\hline C322faa & 1 & 1 & 1 & & 1 & 1 \\
\hline C332Foo & & & & & & \\
\hline e $342 F 00$ & & & & & & \\
\hline$C 412 f 00$ & & & & & & \\
\hline C4L2 FoO & & & & & & \\
\hline$C_{43} 2$ Foo & & & & & & \\
\hline $\mathrm{CH}_{42 \mathrm{~F}} \mathrm{OO}$ & d & $\sqrt{ }$ & $\downarrow$ & $\downarrow$ & $\downarrow$ & $\downarrow$ \\
\hline
\end{tabular}
(Signeture/Affliotion)
Special Instructions: ${ }^{23}$

Possible Hazard Identification: 24

Non-hazard [] Flammable [D Skin Irritant [J Poison B [] Unknown []

Turnaround Time Required: 26

Normal y Rushi]

1. Relinquished by 28 (Signature/Affiliation)

2. Relinquistíed by

3. Relinquished by

(Signeture/Afiliation)

Sample Disposal: 25

Return to Client $\mid$ Disposal by Lab $\$ Archive

Condition on 21

Recoiot

ETDC 7885

ETDC 7886

ETDC 7887

ETDC 7888

ETDC 7889

FTDC 7890

ETDC 7891

ETDC 7892

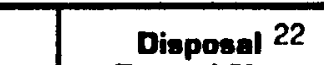
Record No.

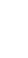
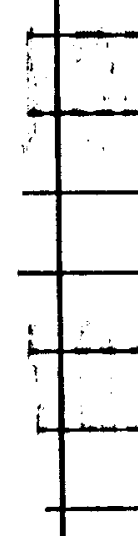

Comments: 29

28 oms $=12 / 9 / 98$ 


\section{四四 \\ TECENOLOGY \\ CORPORATION}

Project Name SAIC
ANALYSIS REQUEST AND CHAIN OF CUSTODY RECORD (cont.)*

Project No. 777304
Reference Document No. ${ }^{30} 426619$ Page 2 of 2

Samples Shipment Date $1 / 23 / 28$

ONE CONTAINER PER LINE

\begin{tabular}{|c|c|c|c|c|c|c|c|c|}
\hline $\begin{array}{l}\text { Sample } 14 \\
\text { Number } \\
\end{array}$ & $\begin{array}{c}\text { Sample } 15 \\
\text { Description/Type }\end{array}$ & \begin{tabular}{|l}
$\begin{array}{l}\text { Date/Time } \\
\text { Colloctad }\end{array}$ \\
\end{tabular} & $\begin{array}{c}\text { Container } 17 \\
\text { Type }\end{array}$ & $\begin{array}{l}\text { Sample } 18 \\
\text { Volume }\end{array}$ & $\begin{array}{r}\text { Pro-19 } \\
\text { servative } \\
\end{array}$ & $\begin{array}{c}\text { Requested Testing } 20 \\
\text { Program }\end{array}$ & $\begin{array}{c}\text { Condition on } 21 \\
\text { Receipt }\end{array}$ & $\begin{array}{c}\text { Disposal } 22 \\
\text { Rocord No. }\end{array}$ \\
\hline $\mathrm{CS} 12 \mathrm{FoO}$ & STAS. Soke & $11 / 11 / 98$ & 2"x4" $\mathrm{nas}$ & Legure & NoNE & $\operatorname{ces}(28$ esy) & ETDC $\quad 7893$ & \\
\hline $\mathrm{C} 522 \mathrm{FoO}$ & 1 & 1 & 1 & 1 & 1 & & ETDC 7894 & $\vdots$ \\
\hline C532 Foo & & & & & & & ETDC $\quad 7895$ & 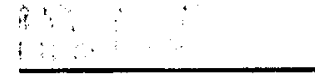 \\
\hline C542 Foo & & & & & & & ETOC 7896 & \\
\hline C612 Foo & & & & & & & ETDC 7897 & \\
\hline C622 Foo & & & & & & & ETDC 7898 & \\
\hline C632 Foo & & & & & & & ETDC 7899 & \\
\hline $0642 F 00$ & $\downarrow$ & $\downarrow$ & $\downarrow$ & $\downarrow$ & $\downarrow$ & $\downarrow$ & cton & \\
\hline & & & & & & & 7900 & \\
\hline & & & & & & & 43 & \\
\hline & & & & & & & & \\
\hline & & & & & & & 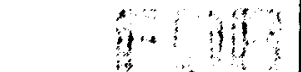 & $\because$ \\
\hline & & & & & & & 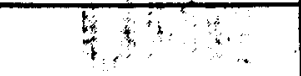 & $\cdots$ \\
\hline & & & & & & & $3, \cdots, \ldots$ & $x$ \\
\hline & & & & & & & 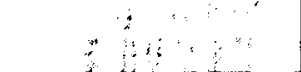 & \\
\hline & & & & & & & & \\
\hline & & & & & & & 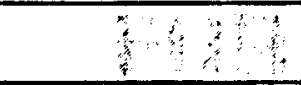 & \\
\hline & & & & & & & 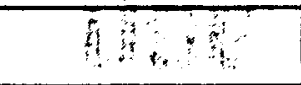 & \\
\hline & & & & & & & & \\
\hline & & & & & & & & \\
\hline
\end{tabular}



IU INTERNATIONAL

Project Name/No. 1 SAIC 77730400010500 Samples Shipment Date ${ }^{7}$ _L/ $24 / 48$
ANALYSIS REQUEST AND CHAIN OF CUSTODY RECORD *

0500

Lab Destination 8

Lab Contact 9

Project Contact/Phone 12

Carrier/Waybill No. ${ }^{13}$
Reference Document No. 426624 Page 1 of 2

Bill to: 5
Project Manager 4 P. C CAA

Purchase Order No. 6
Profit Center No. 3

Required Report Date 11

$$
\text { R. Cale }
$$

\begin{tabular}{|c|c|c|c|c|c|c|}
\hline $\begin{array}{l}\text { Sampla } 14 \\
\text { Numbar } \\
\end{array}$ & $\begin{array}{c}\text { Bampla } 15 \\
\text { Description/Type }\end{array}$ & $\begin{array}{l}\text { Dato/Time } \\
\text { Collocted } \\
\end{array}$ & \begin{tabular}{|c|} 
Container \\
Type
\end{tabular} & $\begin{array}{l}\text { Sample } \\
\text { Volume } \\
\end{array}$ & $\begin{array}{r}\text { Pro- } 19 \\
\text { servative } \\
\end{array}$ & $\begin{array}{c}\text { Pequestod Tosting } 20 \\
\text { Program }\end{array}$ \\
\hline C100Q00 & Soll & $\begin{array}{l}0800 \\
11 / 24198\end{array}$ & $\begin{array}{l}\text { Bas } \\
1-\text { Gxians }\end{array}$ & $1-L$ & 10, & 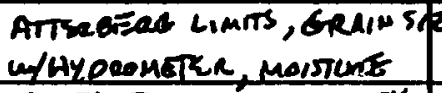 \\
\hline$C 200900$ & & & & & & 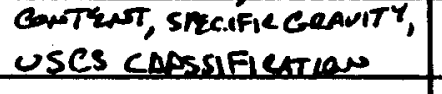 \\
\hline$C_{300 Q 00}$ & & & & & & \\
\hline C400Q00 & & & & & & \\
\hline C 500 Q00 & & & & & & \\
\hline CGoo Goo & & & & & & \\
\hline C700900 & & & & & & \\
\hline$C 800 Q 00$ & $\checkmark$ & $\downarrow$ & $\downarrow$ & $\downarrow$ & $\sqrt{ }$ & $\downarrow$ \\
\hline
\end{tabular}

Report to: 10

Special Instructions: 23

Possible Hazard Identification: 24

Non-hazard [] Flammable [] Skin Irritant J Poison B U Unknown L

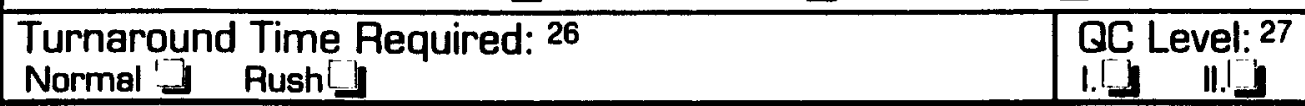

Normal $y$ Rushld

1. Relinquished by 28 (Signature/Affiliation)

\begin{tabular}{l} 
Date: \\
Time: \\
\hline Date: \\
Time: \\
\hline Date: \\
Time:
\end{tabular}

Sample Disposal: ${ }^{25}$

Return to Client [J] Disposal by Lab 1 Archive \begin{tabular}{c|c}
$\begin{array}{c}\text { Condition on } 21 \\
\text { Recelpt }\end{array}$ & $\begin{array}{c}\text { Diaposal } 22 \\
\text { Record No. }\end{array}$ \\
\hline
\end{tabular} ETDC 7902

ETDC 7903

ETDC 7904

ETDC 7905

ETDC 7906

ETDC 7907

ETDC 7908

ETDC 7909 2. Relinquished by

3. Relinquished by (Signature/Affiliation)

III.TI Project Specific (specify):

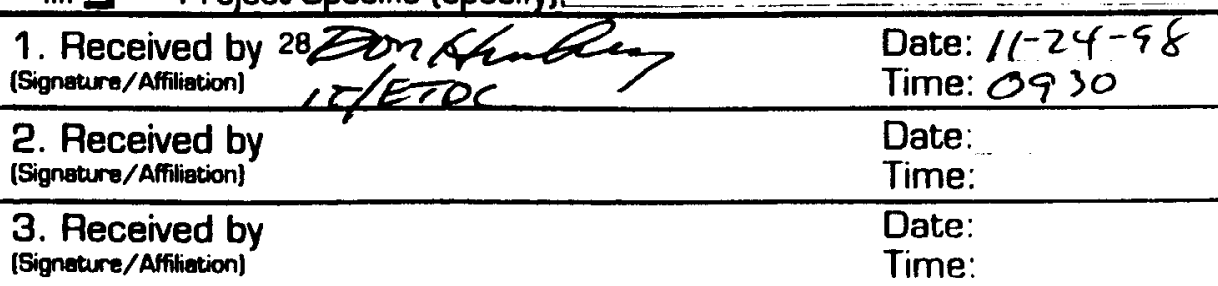

Comments: 29 
Project Name SALC
ANALYSIS REQUEST AND CHAIN OF CUSTODY RECORD (cont.)*

Project No. Z77304.00010500
Reference Document No. ${ }^{30} 426624$

Page 2 of 2

Samples Shipment Date iv/24/98

\section{ONE CONTAINER PER LINE}

\begin{tabular}{|c|c|c|c|c|c|c|c|c|}
\hline $\begin{array}{l}\text { Sample } 14 \\
\text { Number }\end{array}$ & $\begin{array}{c}\text { Sample } 15 \\
\text { Description/Type }\end{array}$ & $\begin{array}{l}\text { Date/Time } 16 \\
\text { Collected }\end{array}$ & $\begin{array}{c}\text { Container } 17 \\
\text { Type }\end{array}$ & $\begin{array}{l}\text { Sample } 18 \\
\text { Volume }\end{array}$ & $\begin{array}{r}\text { Pro-19 } \\
\text { sorvative }\end{array}$ & $\begin{array}{l}\text { Requeated Testing } 20 \\
\text { Program }\end{array}$ & $\begin{array}{c}\text { Condition on } 21 \\
\text { Remalist }\end{array}$ & $\begin{array}{l}\text { Disposal } 22 \\
\text { Record No. }\end{array}$ \\
\hline KlooG00 & Sole & $\begin{array}{c}0800 \\
1 / 24 / 29 \\
\end{array}$ & $\begin{array}{c}1 \text { - Gxecen } \\
\text { sis }\end{array}$ & $l-L$ & pares & 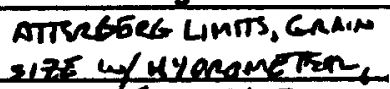 & ETDC 7910 & \\
\hline K200G00 & 1 & 1 & 1 & 1 & 1 & $\begin{array}{l}\text { Moisunef content, } \\
\text { Spegific GRAVITY. }\end{array}$ & ETDC & \\
\hline$K 300900$ & & & & & & USCS CUCSIFICATION & 7912 & \\
\hline KYe0G00 & & & & & & & ETDC 7913 & \\
\hline$K 700900$ & & & & & & & ETDC 7914 & \\
\hline K800900. & $\downarrow$ & $\downarrow$ & $\downarrow$ & $\downarrow$ & $d$ & $\downarrow$ & ETDC & \\
\hline- & & & & & & & & 1 \\
\hline & & & & & & & exs & $111 \%$ \\
\hline & & & & & & & Len & \\
\hline & & & & & & & $4+4 x^{3}$ & \\
\hline & & & & & & & & \\
\hline & & & & & & & 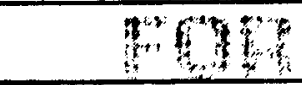 & 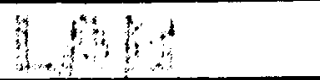 \\
\hline & & & & & & & 整, & $4 b^{3} b^{4}$ \\
\hline & & & & & & & & $3 \mathrm{n} 2$ \\
\hline & & & & & & & & \\
\hline & & & & & & & & \\
\hline & & & & & & & 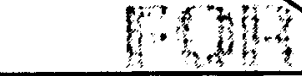 & \\
\hline & & & & & & & 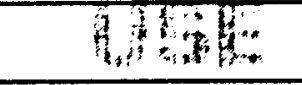 & \\
\hline & & & & & & & & \\
\hline & & & & & & & & \\
\hline
\end{tabular}


INIERNATIONAL

TECHNOLOGY

CORPORATION
ANALYSIS REQUEST AND CHAIN OF CUSTODY RECORD *
Reference Document No. 426868 Page 1 of

Bill to: 5

Project Name/No. 1 SALC/SeS 777304 Samples Shipment Date 7 Sample Team Members 2

Lab Destination 8 ETDC

Profit Center No. 3

Project Manager 4 F. Low? Lab Contact 9 R. Con

Purchase Order No. 6

Project Contact/Phone 12

Carrier/Waybill No. ${ }^{13}$

Report to: 10

Required Report Date 11

ONE CONTAINER PER LINE

\begin{tabular}{|c|c|c|c|c|c|c|}
\hline $\begin{array}{l}\text { Sampla } 14 \\
\text { Number }\end{array}$ & $\begin{array}{c}\text { Sample } 15 \\
\text { Description/Type }\end{array}$ & $\begin{array}{l}\text { Date//Tima } \\
\text { Collected } \\
\end{array}$ & $\begin{array}{c}\text { Container } \\
\text { Type }\end{array}$ & $\begin{array}{l}\text { Sample }{ }^{18} \\
\text { Volume }\end{array}$ & $\begin{array}{r}\text { Pre } 19 \\
\text { servative } \\
\end{array}$ & $\begin{array}{c}\text { Pequested Testing } 20 \\
\text { Program }\end{array}$ \\
\hline K311Foo & STAS. Soil & $11 / 25 / 98$ & $2 \times 4$ mons & $200 \mathrm{~mL}$ & Non & ues \\
\hline$k 321 F 00$ & 1 & & & & 1 & \\
\hline K331 F00 & & & & & & \\
\hline$k 341$ Foo & & & & & & \\
\hline$K y_{1}, F_{00}$ & & & & & & \\
\hline $1<421 F 00$ & & & & & & \\
\hline K431 FCo & & & & & & \\
\hline$|<4 y| F_{0}$ & $\downarrow$ & t & $\downarrow$ & 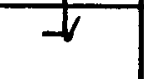 & 3 & $\downarrow$ \\
\hline
\end{tabular}

Condition on 21 Receiot

ETOC 7948

ETDC 7949

ETDC 7950

ETDC 7951

ETDC 7952

ETDC 7953

ETDC 7954

ETDC 7955

Special Instructions: 23

Possible Hazard Identification: 24

Nonhazard [」] Flammable [马 Skin Irritant $] \quad$ Poison B [] Unknown $\square$

\begin{tabular}{l|l}
\hline Turnaround Time Required: 26 & QC Level: 27
\end{tabular}

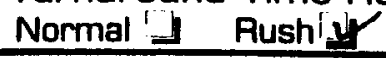

1. Relinquished bignare/Affiation)
(Signaturat

III.[.] Project Specific (specify):

Sample Disposal: 25

Return to Client!d Disposal by Lab I Archive

Dioposal 22

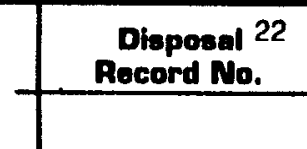
(Signature/Affiliation)

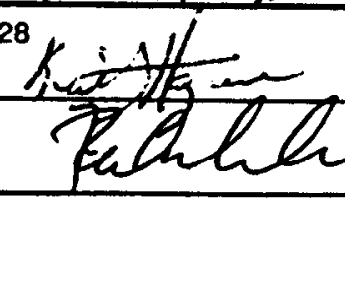

Date: $2-11-98$

2. Received by (Signature/Affilution)

Time: $0 \% 0$,

\section{Received by}

Date: $12-11.98$

ITETOC Date:

0955

Comments: ${ }^{29}$

16 DAY AgE ON DeC. 11, 1988 
INTERNATIONAL

CORPORATION 777304.000 IOSOHAIN OF CUSTODY RECORD *

Project Name/No. ${ }_{1} \mathrm{Sa}, \mathrm{C}$ WS RC Samples Shipment Date 7

Sample Team Members 2 Bennett

Profit Center No. ${ }^{3} 6585001$

Project Manager Pau X LeaK

Purchase Order No. 6

Required Report Date 11

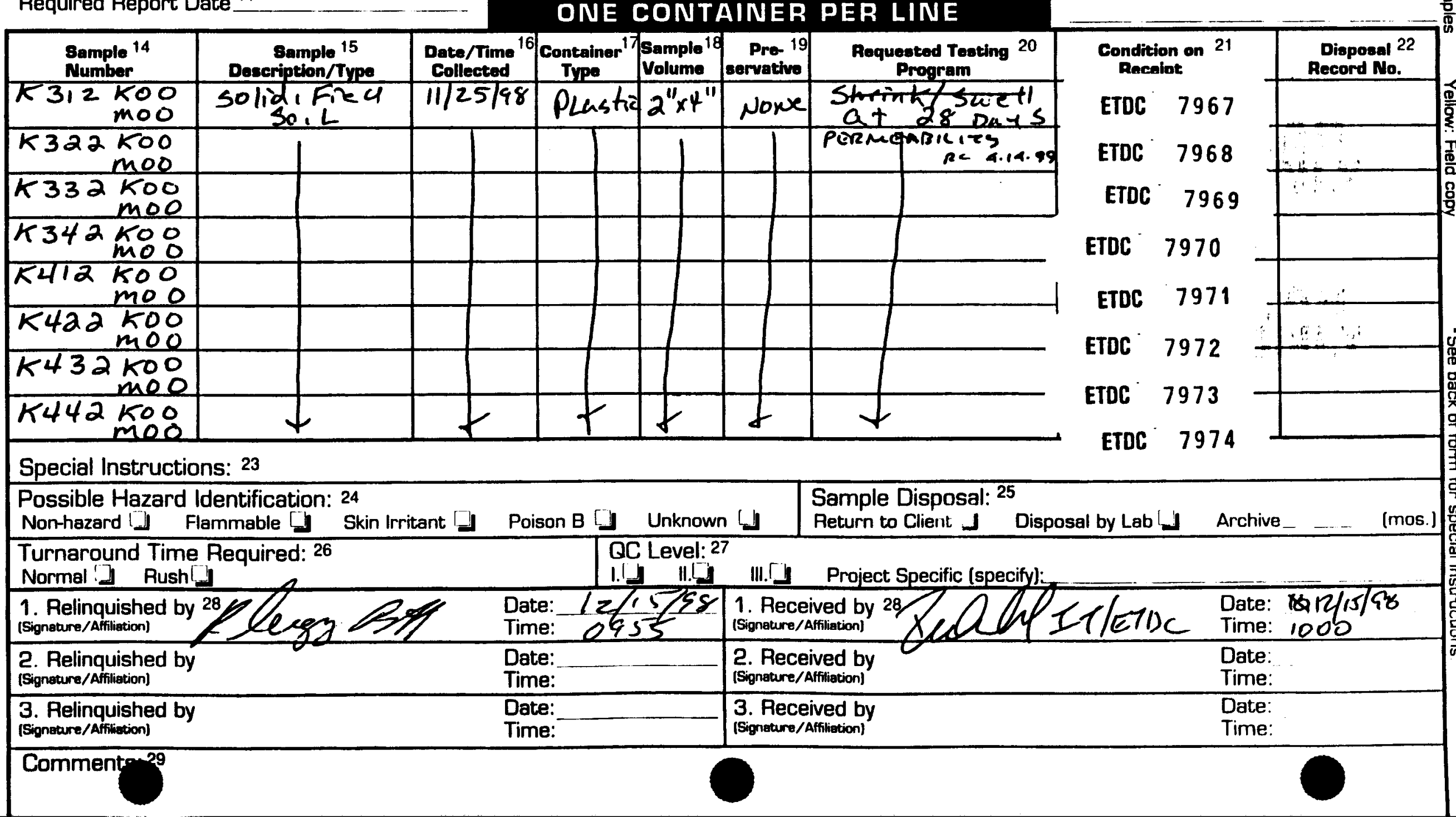

Reference Document No. 426869 Page 1 of 3

Bill to: $5777304: 000 / 0500$ 麇
Lab Destination 8 ET DC

Lab Contact 9 RALPA Cole

Project Contact/Phone ${ }^{2}$ Paul Le uK - Report to: ${ }^{10}$ Pa KL LeaK Carrier/Waybill No. ${ }_{-}^{13}$ HAND

ONE CONTAINER PER LINE 
NTERNATIONAI

TECHNOLOGY

CORPORATION

Project Name SaIC WSRC
ANALYSIS REQUEST AND CHAIN OF CUSTODY RECORD (cont.) *

Project No. 777304
Reference Document No. ${ }^{30} 426869$ Page 2 of 3

Samples Shipment Date

ONE CONTAINER PER LINE

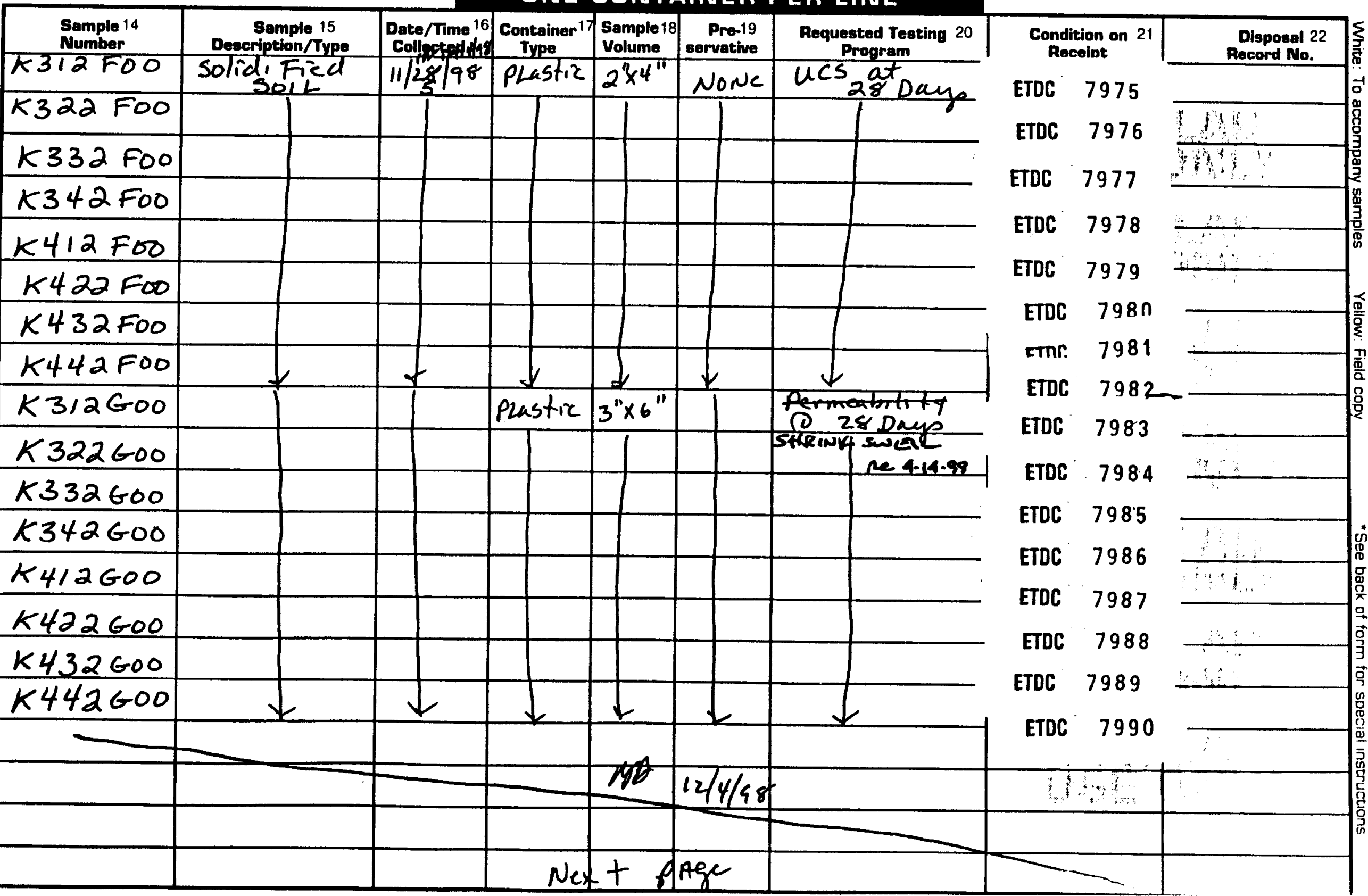




\section{th TECHNOLOGY} CORPORATION
ANALYSIS REQUEST AND CHAIN OF CUSTODY RECORD *

Project Name/No. $177730 \%$.000i0500 Samples Shipment Date 7

Sample Team Members 2

Profit Center No. 3

Project Manager ${ }^{4}$ P. CoAR

Purchase Order No. 6

Required Report Date 11

Lab Destination 8

Lab Contact 9

Project Contact/Phone 12

R. Cole

Carrier/Waybill No. ${ }^{13}$
Reference Document No. 426631 Page 1 of

Bill to: 5

\section{Report to:}



Comments: ${ }^{29}$ 
DNTERNATIONAI

TECHNOLOGY

CORPORATION

Project Name SAIC/SRS
ANALYSIS REQUEST AND CHAIN OF CUSTODY RECORD (cont.)*

Project No. 777304.00010500
Reference Document No. ${ }^{30} 426631$ Page 2 of 2

Samples Shipment Date $12 / 23 / 98$

\begin{tabular}{|c|c|c|c|c|c|c|c|c|}
\hline $\begin{array}{l}\text { Sample } 14 \\
\text { Number } \\
\end{array}$ & $\begin{array}{c}\text { Sample } 15 \\
\text { Deacription/Type }\end{array}$ & $\begin{array}{l}\text { Dato/Time }{ }^{16} \\
\text { Collected }\end{array}$ & $\begin{array}{c}\text { Container }{ }^{17} \\
\text { Type } \\
\end{array}$ & \begin{tabular}{|l|}
$\begin{array}{l}\text { Sample 18 } \\
\text { Volume }\end{array}$ \\
\end{tabular} & $\begin{array}{r}\begin{array}{r}\text { Pro.19 } \\
\text { servative }\end{array} \\
\end{array}$ & $\begin{array}{c}\text { Pequestad Testing } 20 \\
\text { Program }\end{array}$ & $\begin{array}{c}\text { Condition on } 21 \\
\text { Receipt }\end{array}$ & $\begin{array}{l}\text { Disposal } 22 \\
\text { Record No. } \\
\end{array}$ \\
\hline$P 100900$ & soic & $12 / 21 / 900020$ & \begin{tabular}{|c|} 
Gose \\
sing
\end{tabular} & $1-2$ & Nond & 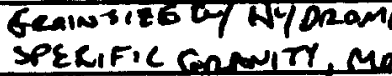 & TISN ETDC 80 & 015 \\
\hline P200900 & 1 & 10825 & 1 & 1 & & Atruburac umits & ETDC 801 & 13 \\
\hline$P 300 Q 00$ & & era & & & & & ETDC 8017 & $7 \quad a^{5}$ \\
\hline P400Q00 & & Dios & & & & & ETDC: $8 \cap 18$ & 3 \\
\hline P500Q00 & & 090 & & & & & ETDC 8019 & $4 \quad \mathrm{c}$ \\
\hline P600Q0e & & Coss & & & & & ETDC 8020 & \\
\hline Pfocqeo & & 0920 & & & & & ETDC 8021 & $\therefore 3$ \\
\hline P8OOQPe & $\downarrow$ & $\$ \quad 0.25$ & $\downarrow$ & $\downarrow$ & $\downarrow$ & & ETDC 8022 & $4: 5$ \\
\hline & & & & & & & $x+3 y$ & $3 \quad 43$. \\
\hline & & & & & & & 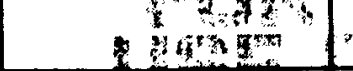 & 30 \\
\hline & & & & & & & 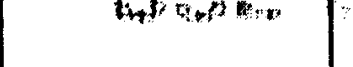 & 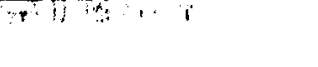 \\
\hline & & & & & & & 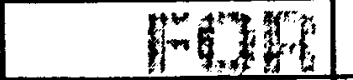 & 13 \\
\hline & & & & & & & 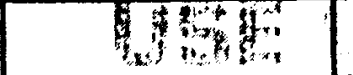 & 319,8 \\
\hline & & & & & & & tin 190 & $\approx 5$ \\
\hline & & & & & & & 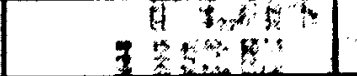 & $3 \times$ \\
\hline & & & & & & & $\tan x \sin 20$ & \\
\hline & & & & & & & quth & 1 \\
\hline & & & & & & & 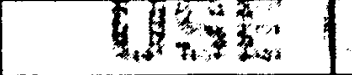 & 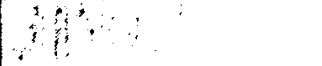 \\
\hline & & & & & & & & \\
\hline & & & & & & & & \\
\hline
\end{tabular}



(1) CORPORATION
ANALYSIS REQUEST AND CHAIN OF CUSTODY RECORD *

Project Name/No. 1 SALC 777304.0010100 Samples Shipment Date 7 1/19/99 Sample Tearn Members 2

Profit Center No. 3

Project Manager ${ }^{4}$ P. Lone

Purchase Order No. 6

Required Report Date 11

Lab Destination 8 GEo

Lab Contact ${ }^{9}$ R. Cas

Project Contact/Phone 12

Carrier/Waybill No. 13 Report to: 10

ONE CONTAINER PER LINE

\begin{tabular}{|c|c|c|c|c|c|c|}
\hline $\begin{array}{l}\text { Bemple } 14 \\
\text { Number }\end{array}$ & $\begin{array}{c}\text { 8ample } 15 \\
\text { Description/Type }\end{array}$ & $\begin{array}{l}\text { Dato/Timo } \\
\text { Collected } \\
\end{array}$ & $\begin{array}{c}\text { Container } \\
\text { Type }\end{array}$ & $\begin{array}{l}\text { Sampla } 18 \\
\text { Volume } \\
\end{array}$ & $\begin{array}{r}\text { Pre- } 19 \\
\text { servathe } \\
\end{array}$ & $\begin{array}{c}\text { Requestod Tosting } 20 \\
\text { Program }\end{array}$ \\
\hline P313F00 & STAS SOLL & $1 / 191990810$ & $2 \times 4$ man & $200 \mathrm{Ml}$ & NOMÉ & 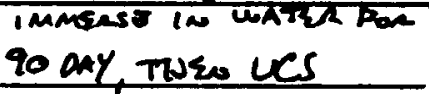 \\
\hline P323 FCO & & & 1 & 1 & & 1 \\
\hline P333Foo & & & & & & \\
\hline P343Foo & & & & & & \\
\hline$P 413$ Foo & & & & & & \\
\hline P423Foo & & & & & & \\
\hline P433Foo & & & & & & \\
\hline P443F00 & $\downarrow$ & $d$ & $V$ & V & $\sqrt{ }$ & $\downarrow$ \\
\hline
\end{tabular}

Special Instructions: 23

Possible Hazard Identification: 24

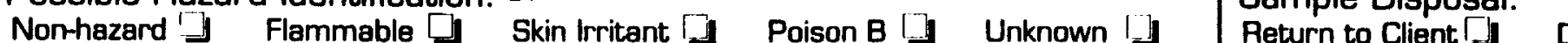

Turnaround Time Required: 26

Normal '] Rush! I]

1. Relinquished by 28

[Signature/Affiliation]

2. Relinquished by

(Signecure/Affilietion)

3. Relinquished by

(Signeare/Afflietion)

Comments: 29

Reference Document No. 426647

Page 1 of 2

Bill to: 5$$
-
$$

-

\begin{tabular}{|c|c|}
\hline \multicolumn{2}{|c|}{$\begin{array}{c}\text { Condition on } \\
\text { Recaint }\end{array}$} \\
\hline ETDC & 8023 \\
\hline ETDC & 8024 \\
\hline ETDC & 8025 \\
\hline ETDC & 8026 \\
\hline ETDC & 8027 \\
\hline ETDC & 8028 \\
\hline ETDC & 8029 \\
\hline ETDC & 8030 \\
\hline
\end{tabular}

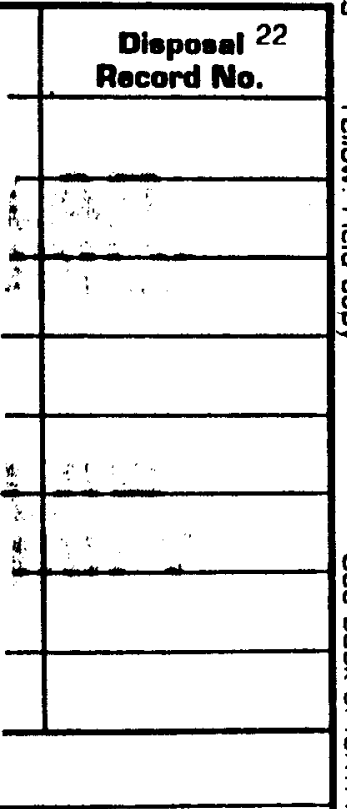

Sample Disposal: 25

Return to Client $[d$ Disposal by Lab $\mid$ Archive (mos.) QC Level: 27

Date:

Time:

Date:

Time:

Date:

Time:
1. Received by 28 (Signature/Affiliation)

\section{Received by} (Signeaure/Affiliection)

3. Received by

[Signoture/Afflistion)
Date: / $19-99$

Time: 0900

Date:

Time:

Date:

Time: 

INTERNATIONAL
TECHNOLOGY
CORPORATION

Project Name SALC
ANALYSIS REQUEST AND CHAIN OF CUSTODY RECORD (cont.) *

Project No. Z7Z30\%, oolaroso
Reference Document No. ${ }^{30} 426647$ Page 2 of 2

Samples Shipment Date ' l/19/99 ONE CONTAINER PER LINE

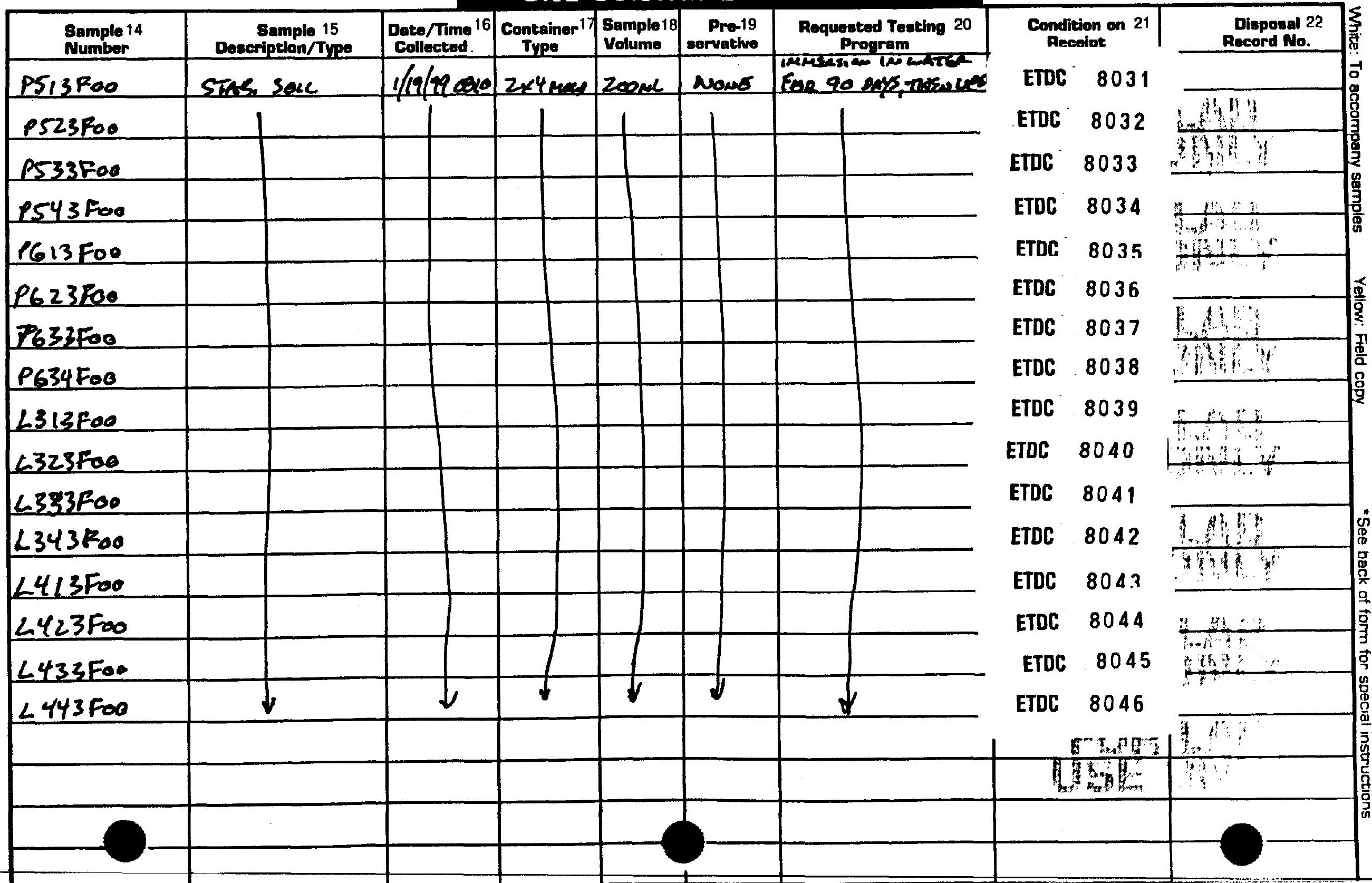


J. INIERNATIONAL TECHINOLOGY

CORPORATION

Project Name/No. 1 SAKC 777304 Sample Team Members 2

Profit Center No. 3

Project Manager ${ }^{4} P$. CoTAR

Purchase Order No. 6

Required Report Date 11
ANALYSIS REQUEST AND CHAIN OF CUSTODY RECORD *
Reference Document No. 426648 Page 1 of 2

Bill to: 5

oolosooa Samples Shipment Date $7 \quad 1 / 19 / 99$

\author{
Lab Destination 8 Geo \\ Lab Contact 9 L. Ca $\angle 5$
}

Project Contact/Phone 12

Carrier/Waybill No. 13

\section{ONE CONTAINER PER LINE}

\begin{tabular}{|c|c|c|c|c|c|c|}
\hline $\begin{array}{l}\text { Sample } 14 \\
\text { Number } \\
\end{array}$ & $\begin{array}{c}\text { 8ample } 15 \\
\text { Description/Type }\end{array}$ & $\begin{array}{l}\text { Date/Time } \\
\text { Collected }\end{array}$ & $\begin{array}{c}\text { Container } \\
\text { Type }\end{array}$ & $\begin{array}{l}\text { Sample } \\
\text { Volume }\end{array}$ & $\begin{array}{c}\text { Pro } 19 \\
\text { servative } \\
\end{array}$ & $\begin{array}{c}\text { Pequested Toating } 20 \\
\text { Propram }\end{array}$ \\
\hline$P 312 F \infty$ & sTne. Soll & Heqa9o85 & $2 \times 4$ man & $200 \mathrm{ml}$ & noes & ucs $Q 28 \mathrm{DAy}$ \\
\hline$P 322 F 00$ & & & & & & \\
\hline P332P00 & & & & & & \\
\hline 12342500 & & & & & & \\
\hline$P_{4} / 2 F_{00}$ & & & & & & \\
\hline 1422500 & & & & & & \\
\hline P432For & & & & & & \\
\hline PYYLFaO & $\Phi$ & a) & & $y$ & $\psi$ & $\downarrow$ \\
\hline
\end{tabular}

Special Instructions: $\mathbf{2 3}$

Possible Hazard Identification: 24

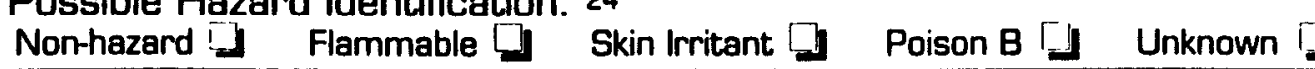
\begin{tabular}{|l|l}
\hline Turnaround Time Required: 26 & QC Level: 27
\end{tabular}

Normal !] Rush[y

1. Relinquished by 28 [Signature/Affiliation] 1. 11.5

\section{Relinquished by} (Signature/Affiliation)

3. Relinquished by (Signeture/Affilistion)

Comments: 29
Qarmint

ETDC 8047

ETDC 8048

ETDC 8049

ETDC 8050

ETDC 8051

ETDC 8052

ETDC 8053

ETDC 8054
Condition on 21

Sample Disposal: ${ }^{25}$

Return to Client L Disposal by Lab!J Archive

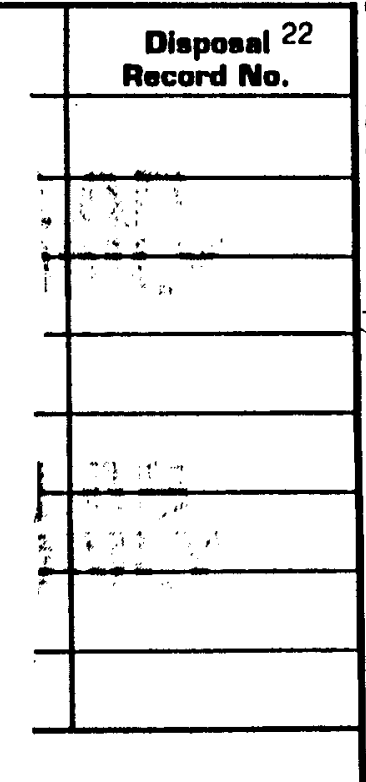

(mos.)

Date:
Time:
Date:
Time:
Date:
Time:

III. Project Specific (specify):

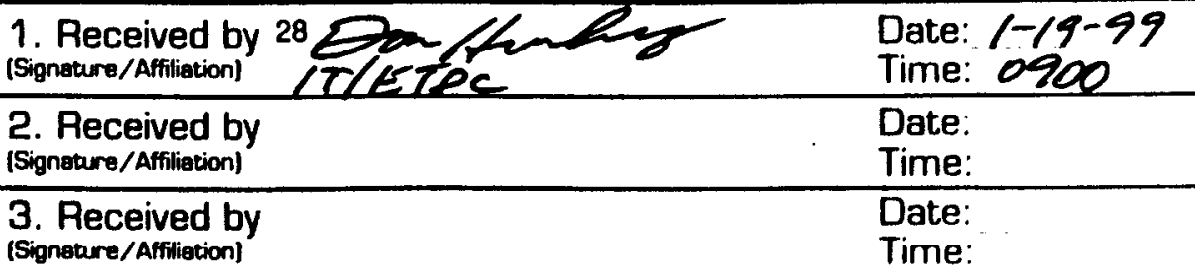


I NTERNATIONAL

TECHNOLOGY

CORPORATION

Project Name SAuC 777304.00105000
ANALYSIS REQUEST AND CHAIN OF CUSTODY RECORD (cont.)*
Reference Document No. ${ }^{30} 4$ z66 68

Page 2 of 2

Samples Shipment Date _ _/1/9/99

Project No.

ONE CONTAINER PER LINE

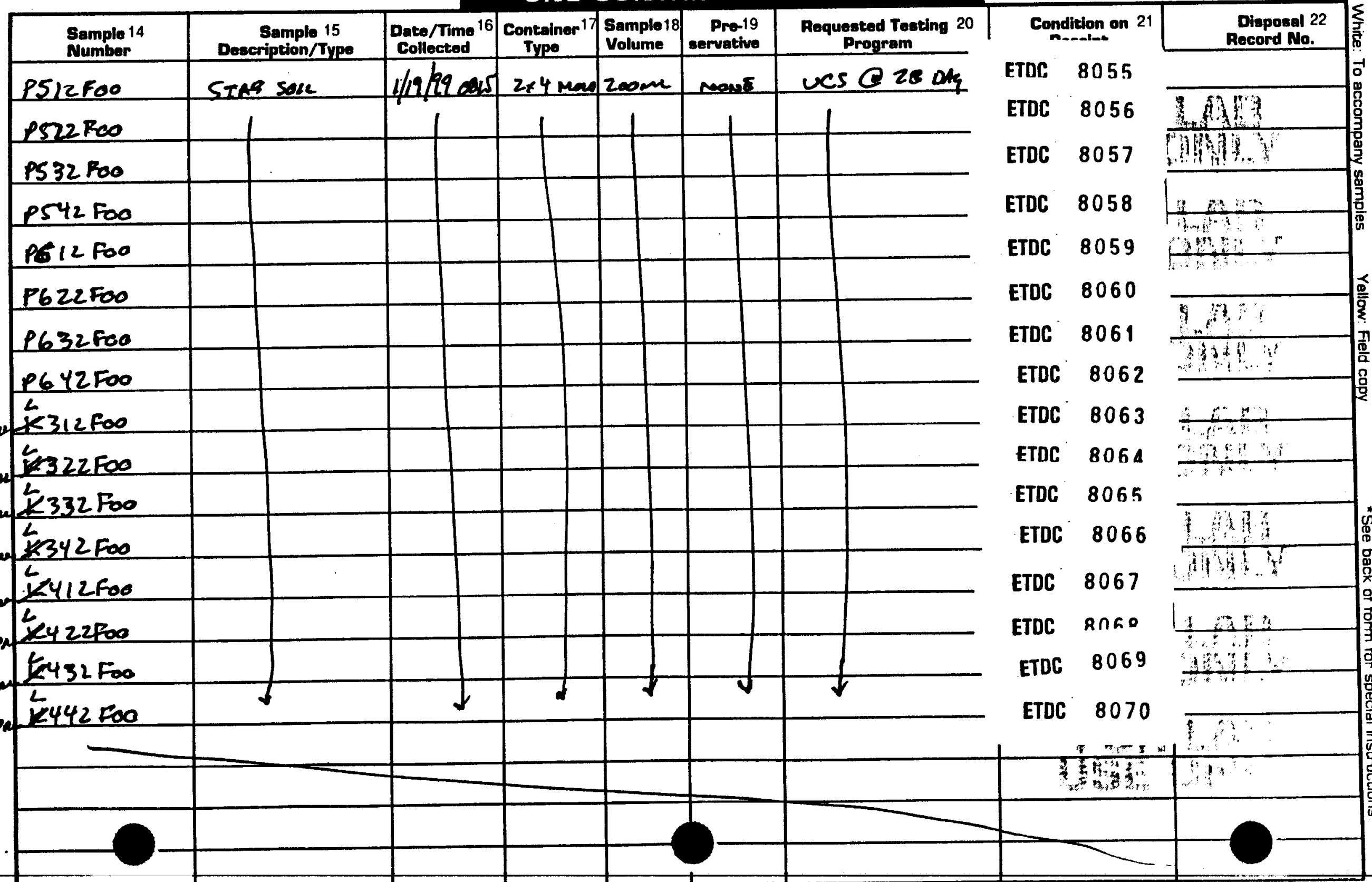


ANALYSIS REQUEST AND CHAIN OF CUSTODY RECORD*

Project Name/No. I SALC $77330 \%$. 00105000 Samples Shipment Date? 1/19/99

Lab Destination 8 GEO

Lab Contact 9 K. Cas

Profit Center No. ${ }^{3}$

Project Manager ${ }^{4}$ P. LSBR

Purchase Order No. 6

Required Report Date 11
Project Contact/Phone 12
Carrier/Waybill No. ${ }^{13}$
Reference Document No. 426649 Page 1 of $z$

Bill to: 5

\begin{tabular}{|c|c|c|c|c|c|c|}
\hline $\begin{array}{l}\text { Bample } 14 \\
\text { Number }\end{array}$ & $\begin{array}{c}\text { Bample } 15 \\
\text { Deseription/Type }\end{array}$ & $\begin{array}{l}\text { Date/Time } \\
\text { Collocted } \\
\end{array}$ & $\begin{array}{c}\text { Containor } \\
\text { Type }\end{array}$ & $\begin{array}{l}\text { Sample } 18 \\
\text { Volume } \\
\end{array}$ & $\begin{array}{l}\text { Pre. } 19 \\
\text { eervative } \\
\end{array}$ & $\begin{array}{c}\text { Pequestod Tosting } \\
\text { Program } 20\end{array}$ \\
\hline$P 312 K 00$ & sfres. Sole & $1 / 2 y / 99$ os30 & $2 x^{2} /$ maes & Loome & nons & Hyopgive conductury \\
\hline$P 322 R \circ O$ & & & & & & \\
\hline P332KaO & & & & & & \\
\hline $8342 \mathrm{KoO}$ & & & & & & \\
\hline$p \$ 12 K 00$ & & & & & & \\
\hline$P 422 K 00$ & & & & & & \\
\hline $1432 \mathrm{kaO}$ & & & & & & \\
\hline PपY KaO & $\downarrow$ & $\downarrow$ & $\downarrow$ & $\boldsymbol{\Psi}$ & $\downarrow$ & $\psi$ \\
\hline
\end{tabular}

\section{ONE CONTAINER PER LINE}

Special Instructions: 23

Possible Hazard Identification: 24

Nonhazard $\square \quad$ Flammable $\square$ Skin Irritant $\square \quad$ Poison B $\square \quad$ Unknown $\square$

\begin{tabular}{|l|l}
\hline Turnaround Time Required: 26 & QC Level: 27
\end{tabular}

Normal ] Rush[] 1.] …]

1. Relinquished by 28 (Signature/Afriliation)

Date:

Time:

2. Relinquished by (Signeture/Affiliation)

Date:

Time:

3. Relinquished by

(Signeture/Afflietion)

Date:

Time:
Sample Disposal: 25

Return to Client Disposal by Lab ل Archive Condition on $21 \quad$ Dleposal 22 Deriaint: Aecord No.

ETDC 8071

ETDC 8072

ETDC 8073

ETDC. 8074

ETDC 8075

ETDC 8076

ETDC $\quad 8077$

ETDC 8078

III. Project Specific (specify)

Comments: 29

\begin{tabular}{|c|c|}
\hline $\begin{array}{l}\text { 1. Received by } 28207 \\
\text { (Signature/Affilistion) }\end{array}$ & $\begin{array}{l}\text { Date: } / 79-59 \\
\text { Time: } 0900\end{array}$ \\
\hline $\begin{array}{l}\text { 2. Received by } \\
\text { [Signature/Affliation) }\end{array}$ & $\begin{array}{l}\text { Date: } \\
\text { Time: }\end{array}$ \\
\hline $\begin{array}{l}\text { 3. Received by } \\
\text { (Signeture/Affliotion) }\end{array}$ & $\begin{array}{l}\text { Date: } \\
\text { Time: }\end{array}$ \\
\hline
\end{tabular}


Project Name SALC
ANALYSIS REQUEST AND CHAIN OF CUSTODY RECORD (cont.) *

Project No. 777304.00105000
Reference Document No.30 426649 Page $z$ of 2

Samples Shipment Date $1 / 19 / 29$ ONE CONTAINER PER LINE

\begin{tabular}{|c|c|c|c|c|c|c|c|c|}
\hline $\begin{array}{l}\text { 8ample } 14 \\
\text { Mumber } \\
\end{array}$ & $\begin{array}{c}\text { Sample } 15 \\
\text { Description/Type } \\
\end{array}$ & \begin{tabular}{|l|}
$\begin{array}{l}\text { Dato/Time } \\
\text { Colloctad }\end{array}$ \\
\end{tabular} & $\begin{array}{c}\text { Container } 17 \\
\text { Type }\end{array}$ & \begin{tabular}{|l|}
$\begin{array}{l}\text { Sample 18 } \\
\text { Volume }\end{array}$ \\
\end{tabular} & $\begin{array}{r}\text { Pro-19 } \\
\text { Sorvathve }\end{array}$ & $\begin{array}{c}\text { Requested Testing } 20 \\
\text { Program } \\
\end{array}$ & Condition on 21 & $\begin{array}{l}\text { Disposal } 22 \\
\text { Record No. } \\
\end{array}$ \\
\hline PS12 Koo & STAB SOLL & $1 / 19 / 99009$ & $2 \times 4$ mase & $200 \mathrm{me}$ & Napes & 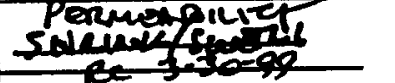 & ETDC $\quad 8079$ & \\
\hline $1522 \mathrm{kOO}$ & & & & & & & ETDC 8080 & (4) 19 \\
\hline P532K00 & & & & & & & ETDC 8081 & $978 \ldots$ \\
\hline$P S 4=K a O$ & & & & & & & ETDC 8082 & \\
\hline Ph/2keo & & & & & & & ETDC $\quad 8083$ & \\
\hline P62ZK00 & & & & & & & 8084 & \\
\hline P632K00 & & & & & & & 8085 & \\
\hline PG4 KKOO & & & & & & & ETDC 8086 & \\
\hline 2312600 & & & & & & & ETDC $8 \cap 87$ & \\
\hline$\angle 322 \mathrm{KoO}$ & & & & & , & & ETDC 8088 & \\
\hline $6332 \mathrm{kOO}$ & & & & & & & 8089 & \\
\hline$\angle 342600$ & & & & & & & 8090 & \\
\hline$\angle 412800$ & & & & & & & ETDC 8091 & \\
\hline$\angle 422 K 00$ & & & & & & & ETDC $\quad 8092$ & \\
\hline $2432 k 00$ & & & & & & & ETDC 8093 & \\
\hline$\angle 442 k 00$ & $\gamma$ & 7 & $\downarrow$ & $\downarrow$ & $\downarrow$ & & 8094 & \\
\hline & & & & & & & 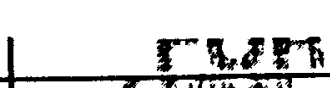 & 1.113 \\
\hline & & & & & & & 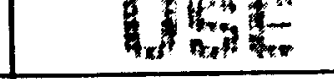 & 139 \\
\hline & & & & & & & & \\
\hline & & & & & & & & \\
\hline
\end{tabular}




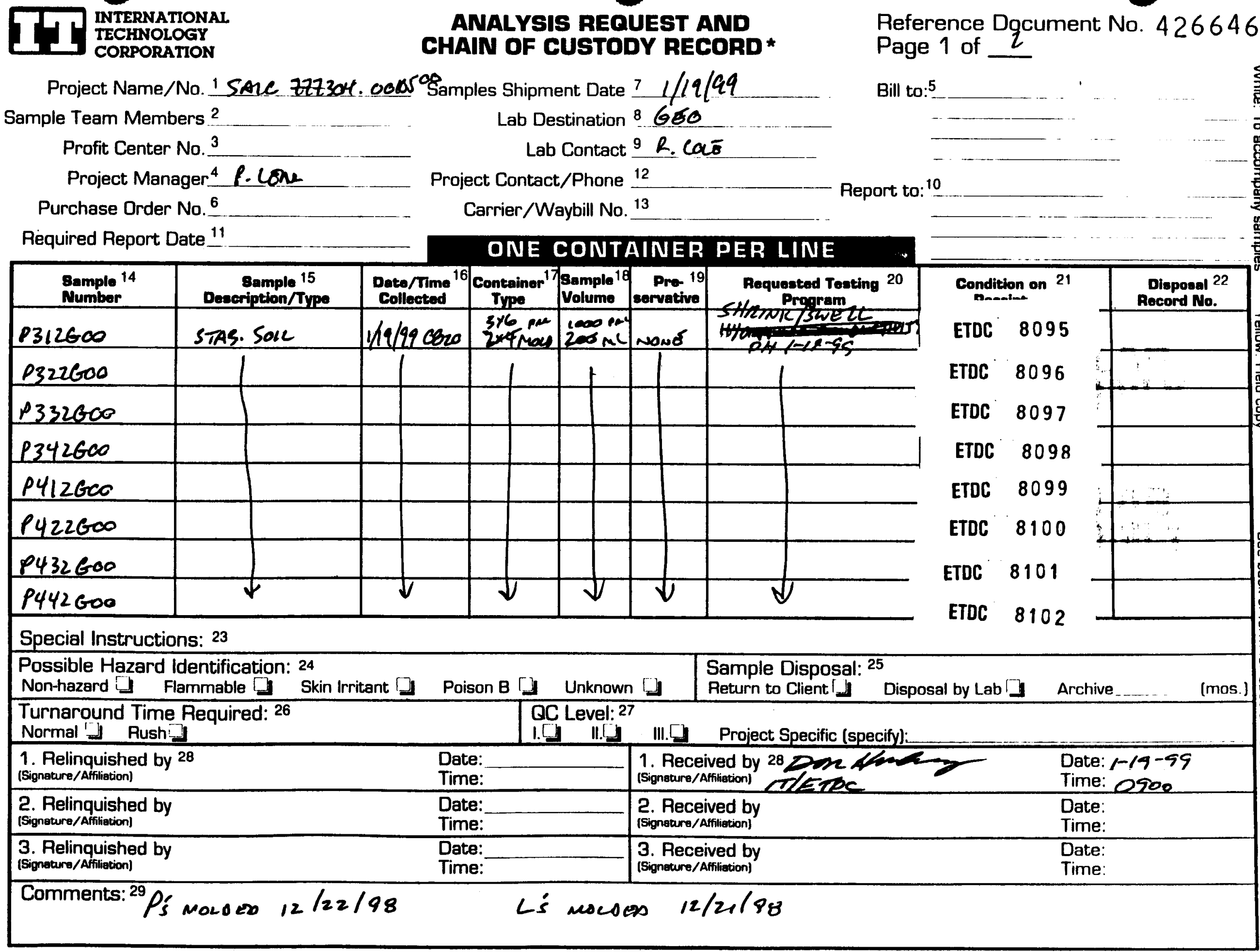


INTERNATIONA

TECINNOLOGY

CORPORATION

Project Name $7 Z Z 3$ o4
ANALYSIS RERUEST AND CHAIN OF CUSTODY RECORD (cont.)*

Project No. $00 / 05000$

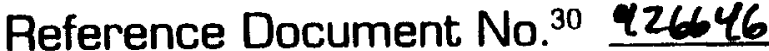
Page 2 of $\underline{2}$

Samples Shipment Date $1 / 19 / 99$
ONE CONTAINER PER LINE

\begin{tabular}{|c|c|c|c|c|c|c|}
\hline $\begin{array}{l}\text { Sample } 14 \\
\text { Number } \\
\end{array}$ & $\begin{array}{c}\text { Sample } 15 \\
\text { Doecription/Type }\end{array}$ & $\begin{array}{l}\text { Date/Time } \\
\text { Colloctod }\end{array}$ & $\begin{array}{c}\text { Conteiner }{ }^{17} \\
\text { Type }\end{array}$ & $\begin{array}{l}\text { Sample } 18 \\
\text { Volume }\end{array}$ & $\begin{array}{r}\text { Pre-19 } \\
\text { sorvative } \\
\end{array}$ & $\begin{array}{c}\text { Requested Tosting } 20 \\
\text { Propram }\end{array}$ \\
\hline 1512600 & Stag. Sall & 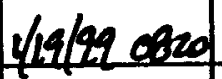 & $360 \mathrm{~cm}$ & 200 one & $\operatorname{son} \sigma$ & Hyocunue Conperet \\
\hline P522600 & 1 & 1 & 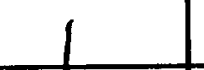 & & & \\
\hline P532600 & & & & & & \\
\hline 1542600 & & & & & & \\
\hline$p 612600$ & & & & & & \\
\hline P622600 & & & & & & \\
\hline 7672600 & & & & & & \\
\hline 1642600 & & & & & & \\
\hline $13126=0$ & & & & & & \\
\hline 2322600 & & & & & & \\
\hline$\angle 332600$ & & & & & & \\
\hline$\angle 342600$ & & & & & & \\
\hline$\angle 412000$ & & & & & & \\
\hline$\angle 422600$ & & & & & & \\
\hline 6432600 & & & & & & \\
\hline 24426.00 & $\downarrow$ & $\boldsymbol{\psi}$ & $\downarrow$ & $\downarrow$ & $\downarrow$ & $b$ \\
\hline se & & & & & & \\
\hline & & & & & & \\
\hline & & & & & & \\
\hline
\end{tabular}

\begin{tabular}{l}
$\begin{array}{l}\text { Condition on } 21 \\
\text { ETDC } 8103\end{array}$ \\
ETDC 8104 \\
ETDC 8105 \\
ETDC 8106 \\
ETDC 8107 \\
ETDC 8108 \\
ETDC 8109 \\
ETDC 8110 \\
ETDC 8111 \\
ETDC 8112 \\
ETDC 8113 \\
ETDC 8114 \\
ETDC 8115 \\
ETDC 8116 \\
\hline ETDC 8117 \\
ETDC 8118 \\
\hline 8
\end{tabular}


INTERNATIONAL

TECKNOLOGY

CORPORATION

Project Name/No. 1 ZzZ304 . 00/05000 Sample Team Members ${ }^{2}$

Profit Center No. 3

Project Manager 4 P. Loxa.

Purchase Order No. 6

Required Report Date 11

\section{ANALYSIS REQUEST AND} CHAIN OF CUSTODY RECORD*

Samples Shipment Date $? / / 9 / 99$

Lab Destination 8 GEo.

Lab Contact 9 R. CorE

Project Contact/Phone 12

Carrier/Waybill No. 13
Reference Document No. 426645 Page 1 of 2

Bill to: 5

\section{ONE CONTAINER PER LINE}

\begin{tabular}{|c|c|c|c|c|c|c|c|c|c|}
\hline $\begin{array}{l}\text { Bample } 14 \\
\text { Number }\end{array}$ & $\begin{array}{c}\text { Sample } 15 \\
\text { Description/Type }\end{array}$ & $\begin{array}{l}\text { Dato/Timo } \\
\text { Collectod }\end{array}$ & $\begin{array}{c}\text { Container } \\
\text { Type }\end{array}$ & $\begin{array}{l}\text { Sample } 19 \\
\text { Volume }\end{array}$ & $\begin{array}{l}\text { Proo } 19 \\
\text { servative }\end{array}$ & $\begin{array}{l}\text { Requestod Tosting } 20 \\
\text { Program }\end{array}$ & $\begin{array}{l}\text { Condition on } \\
\text { Domatort }\end{array}$ & & $\begin{array}{l}\text { Disposall } 22 \\
\text { Pecord No. }\end{array}$ \\
\hline$P 313600$ & SFAR, SOLC & $1 / 19 / 920025$ & 3ybras & $1000 \mu$ & nane & 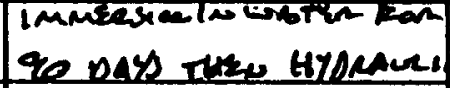 & ETDC 81 & 19 & \\
\hline 1323600 & 1 & 1 & 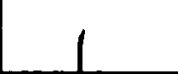 & 1 & & Conovertut74 & ETDC 81 & 120 & 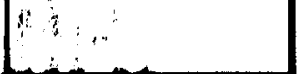 \\
\hline P333600 & & & & & & & ETDC 81 & 21 & $\because$ \\
\hline 8343600 & & & & & & & ETDC & & \\
\hline$p 413600$ & & & & & & & ETDC & 3 & 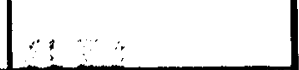 \\
\hline p423G00 & & & & & & & ETDC & 4 & $\therefore:$ \\
\hline 2433600 & & & & & & & 8125 & & \\
\hline 1443600 & t & $\downarrow$ & $\downarrow$ & $\downarrow$ & $\boldsymbol{\nabla}$ & $\downarrow$ & 812 & & \\
\hline Special Instruc & s: 23 & & & & & & & & \\
\hline $\begin{array}{l}\text { Possible Hazar } \\
\text { Non-hazard } \rrbracket\end{array}$ & $\begin{array}{l}\text { entification: } \\
\text { mmable } \square\end{array}$ & nt & $\operatorname{son} B \square$ & Unknown & ב & $\begin{array}{l}\text { Sample Disposal: }{ }^{25} \\
\text { Return to Client } \square \text { Dispc }\end{array}$ & sal by Lab J] & Archive & (mos.) \\
\hline $\begin{array}{l}\text { Turnaround Tir } \\
\text { Normal }] \text { Rus }\end{array}$ & Required: 26 & & \begin{tabular}{l|l} 
& $Q C$ \\
& $1 . Q$ \\
\end{tabular} & \begin{tabular}{|l} 
Level: 27 \\
11.4 \\
\end{tabular} & il. & Project Specific (specify): & & & \\
\hline $\begin{array}{l}\text { 1. Relinquished } \\
\text { [Signatura/Afribation] }\end{array}$ & & $\begin{array}{l}\text { Dat: } \\
\text { Tim }\end{array}$ & & & $\begin{array}{l}\text { 1. Recei } \\
\text { (signasure/A) }\end{array}$ & 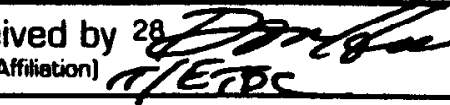 & $\sum$ & $\begin{array}{l}\text { Date: } \\
\text { Time: } \\
\end{array}$ & $\begin{array}{l}1-19-99 \\
0900\end{array}$ \\
\hline 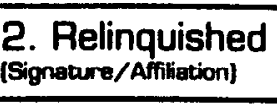 & & $\begin{array}{l}\text { Date } \\
\text { Timm }\end{array}$ & & & $\begin{array}{l}\text { 2. Recei } \\
\text { isignaurre/AI }\end{array}$ & $\begin{array}{l}\text { lived by } \\
\text { affinitions }\end{array}$ & & $\begin{array}{l}\text { Date: } \\
\text { Time: }\end{array}$ & \\
\hline $\begin{array}{l}\text { 3. Relinquished } \\
\text { (Signeare/Affistion) }\end{array}$ & & $\begin{array}{l}\text { Dat } \\
\text { Tim }\end{array}$ & & & $\begin{array}{l}\text { 3. Recei } \\
\text { (Signeture/A }\end{array}$ & $\begin{array}{l}\text { ived by } \\
\text { affihetionl }\end{array}$ & & $\begin{array}{l}\text { Date: } \\
\text { Time: }\end{array}$ & \\
\hline
\end{tabular}

Comments: 29 
DNTERNATIONA

TECHNOLOGY

CORPORATION

Project Name 777304
ANALYSIS REQUEST AND CHAIN OF CUSTODY RECORD (cont.)*

Project No. O0/0rooo
Reference Document No. ${ }^{30} 426645$ Page $z$ of $z$

Samples Shipment Date i/19/99 ONE CONTAINER PER LINE

\begin{tabular}{|c|c|c|c|c|c|c|c|c|}
\hline $\begin{array}{l}\text { Sample } 14 \\
\text { Number }\end{array}$ & $\begin{array}{c}\text { Sample } 15 \\
\text { Description/Type } \\
\end{array}$ & $\begin{array}{l}\text { Date/Time } \\
\text { Collocted }\end{array}$ & $\begin{array}{c}\text { Container } \\
\text { Type }\end{array}$ & \begin{tabular}{|l|}
$\begin{array}{l}\text { Sample 18 } \\
\text { Volume }\end{array}$ \\
\end{tabular} & $\begin{array}{r}\text { Pro-19 } \\
\text { sorvativo } \\
\end{array}$ & $\begin{array}{c}\text { Requestod Testing } 20 \\
\text { Program } \\
\end{array}$ & Condition on 21 & $\begin{array}{l}\text { Disposel } 22 \\
\text { Rocord No. } \\
\end{array}$ \\
\hline$P 573600$ & Shenc. Sake & $164 / 29$ ober & $386 \mathrm{man}$ & Looper & neest & Mencesen anys the & ETDC 8127 & \\
\hline e523600 & 1 & & & & & Hyourcele cavacar & ETOC 8128 & 4 \\
\hline 1533600 & & & & & & & ETDC $\quad 8129$ & औn? \\
\hline 1543600 & & & & & & & ETDC 8130 & \\
\hline 1613600 & & & & & & & 8131 & \\
\hline 1623600 & & & & & & & ETDC 8132 & \\
\hline 1633600 & & & & & & & 8133 & : \\
\hline$p 643600$ & & & & & & & 8134 & 4 \\
\hline 2313600 & & & & & & & ETDC $\quad 8135$ & \\
\hline$\$ 323600$ & & & & & & & 8136 & \\
\hline 2333600 & & & & & & & ETDC 8137 & \\
\hline 4343600 & & & & & & & 8138 & \\
\hline 2413600 & & & & & & & 8139 & \\
\hline 2423600 & & & & & & & 8140 & \\
\hline$\angle 433600$ & & & & & & & 8141 & 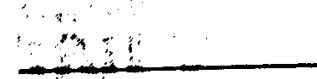 \\
\hline $24436-0$ & $b$ & $\downarrow$ & $\downarrow$ & & $\downarrow$ & $\sqrt{ }$ & 8142 & \\
\hline & & & & & & & I. & 8 \\
\hline & & & & & & & (2) & 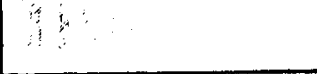 \\
\hline & & & & & & & & \\
\hline & & & & & & & & \\
\hline
\end{tabular}


ATTACHMtNT

Appendix D 
NONCONFORMANCE/VARIANCE REPORT

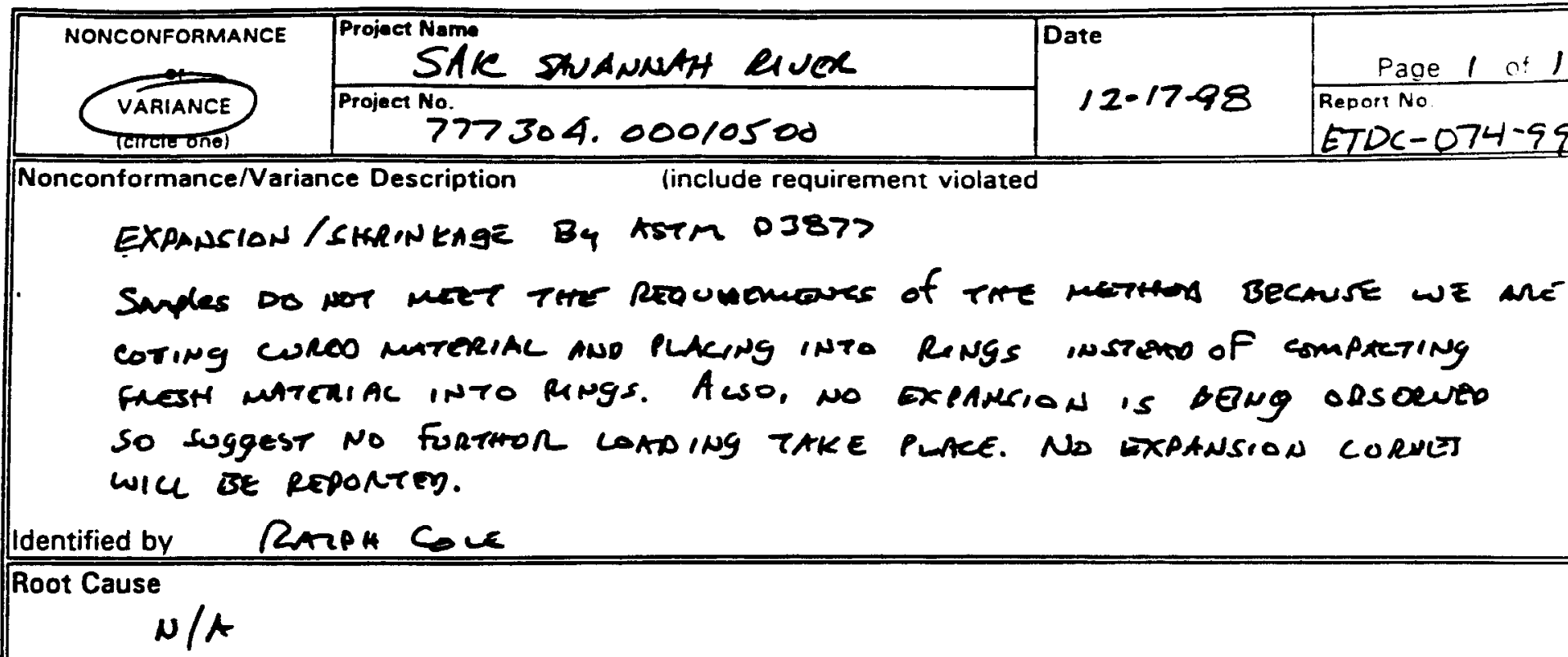

Corrective Action
N/A Delude expected completion date)
Circumstance in report.

To be completed by

Expected Completion Date

Action taken to preclude recurrence

$$
N / A
$$

To be performed by

Expected Completion Date

Client notified

(include client name, how notified, and response)

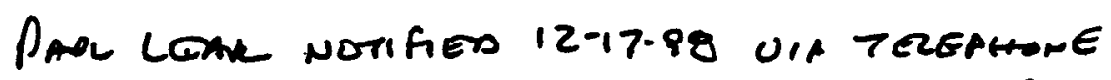

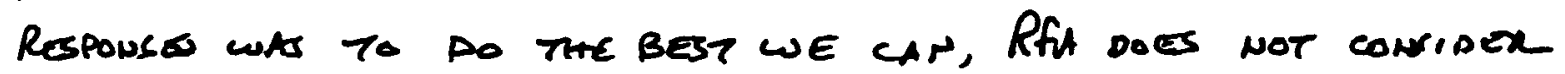
matrons Being toted

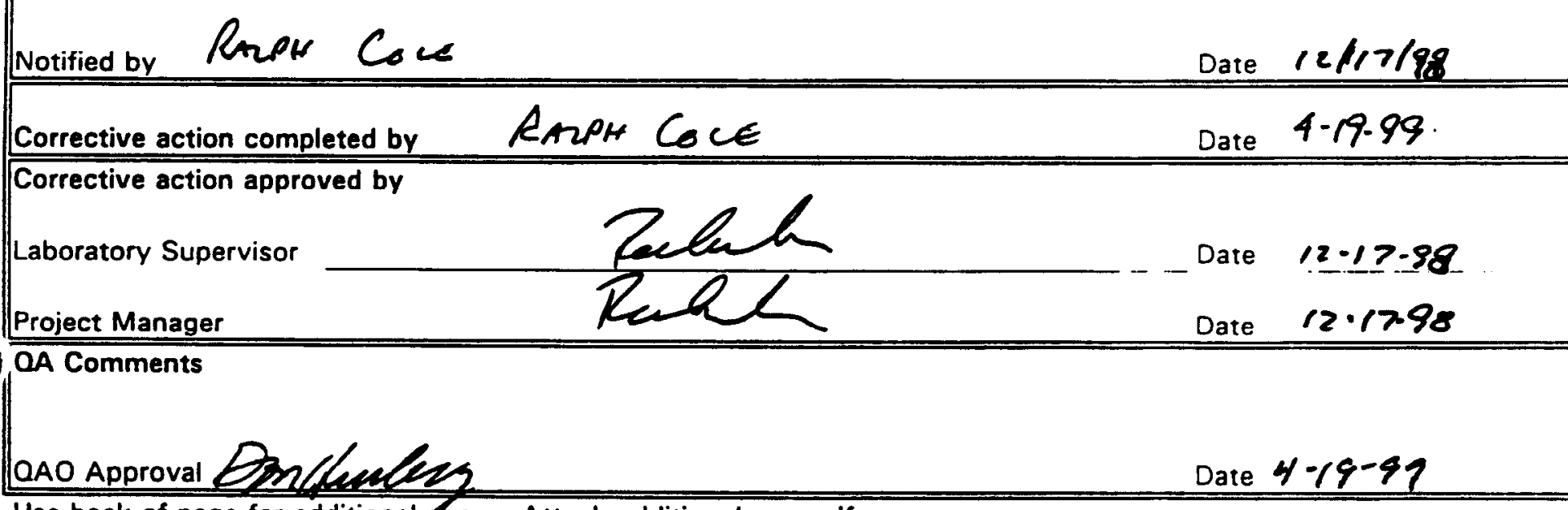

Use back of page for additional pace. Attach additional pages if necessary. 
NONCONFORMANCE/VARIANCE REPORT

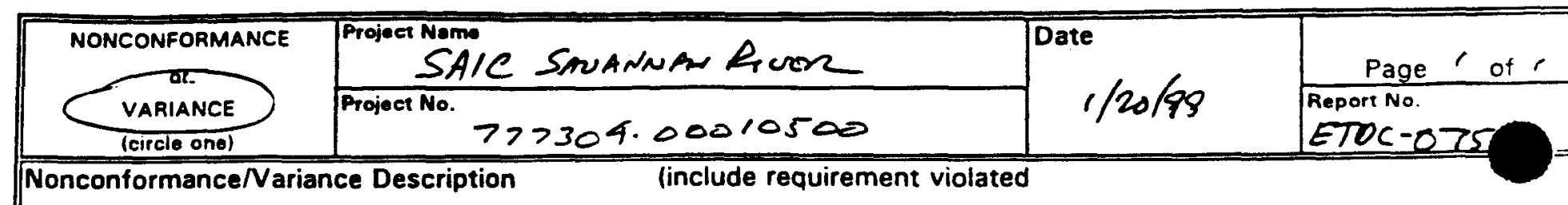

Poeminaicizy Tests By Astr D 5084

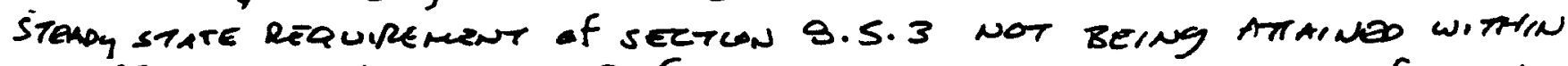

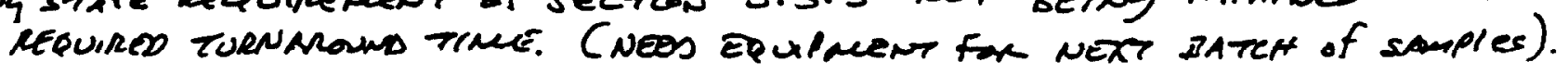

Identified by Don Husker Root Cause

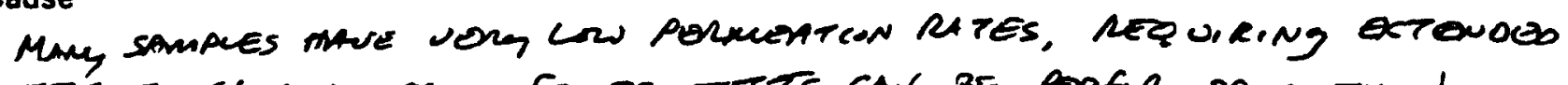

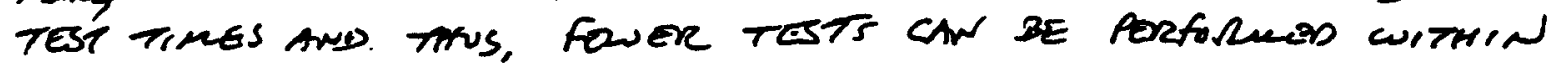
TORUARSOND TIRE.

Corrective Action (include expected completion date)

Note CONDITLON in ROPONT

To be completed by

Action taken to preclude recurrence

$N / A$
Expected Completion Date

Expected Completion Date

To be performed by

(include client name, how notified, and response)

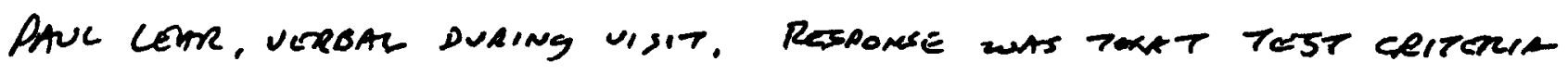

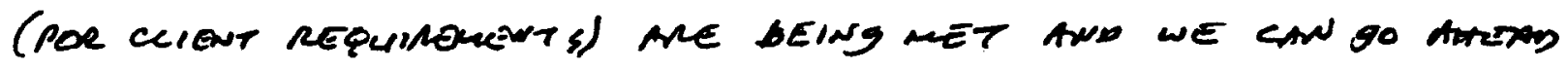
AND SZAS TESTS WrEN NECESSARY.

Notified by Rash Cree

Date $1 / 20 / 95$

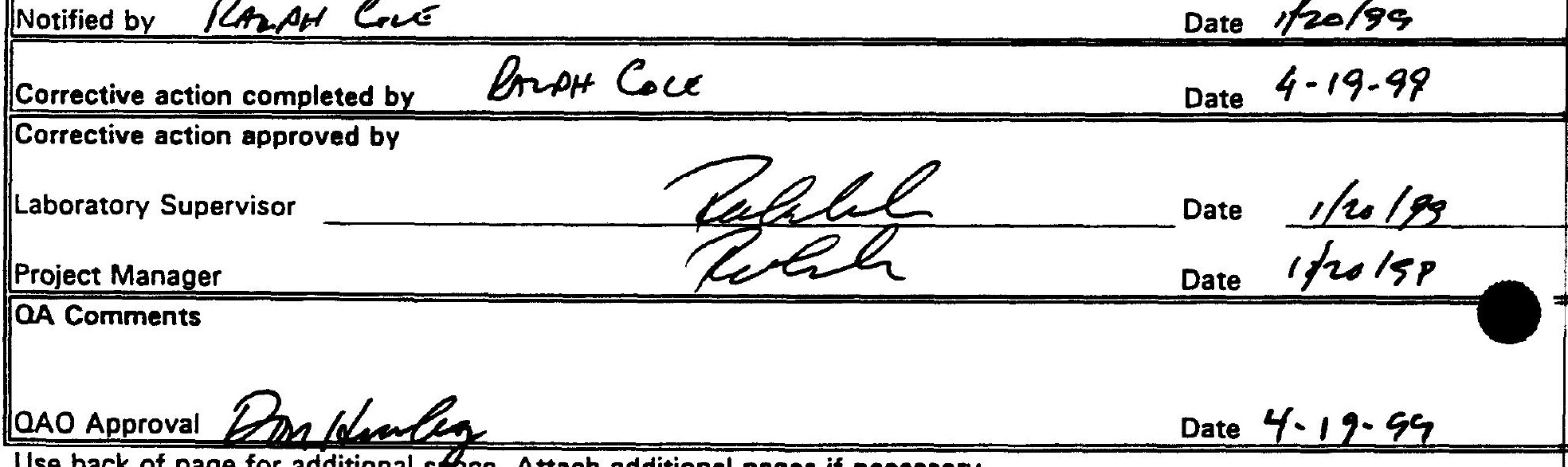

Use back of page for additional space. Attach additional pages if necessary. 
Soil Stabilization/Solidification Grout Mix Design Study WSRC-RP-99-4075. Revision 1

C-, K-, L-, and P-Reactor Seepage Basins Closure (U) August 1999

Appendix B

Sampling Notes 
This page intentionally left blank. 


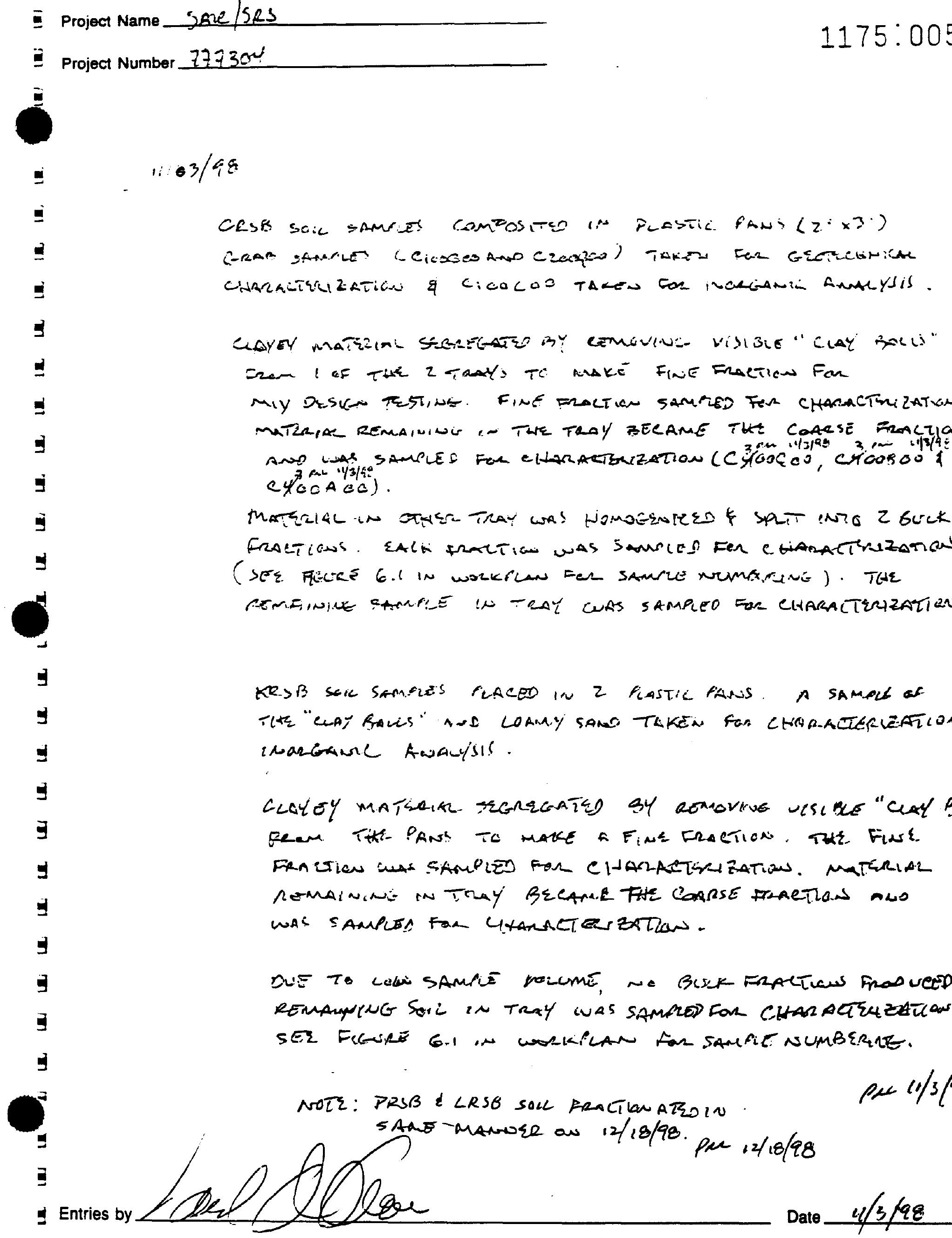


oject Name Squires

$1175: 006$

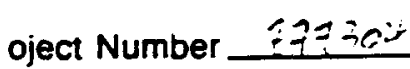

$11 / 110199$

$196(11198$

Gout mixes Far "C" Reactor bee page hasid were prepared using the Formulation design from the wist plan. Wsec-eP98-4184 Rev. O Oct 1998 page 7

Die to the amount of sample the wort plan Formulation was modified For using $2500 \mathrm{~g}$ of soil.

Water was ADDed equal to the weight of the Dir Reagents (PC: Bentonite)

ADjusted weights are as Follows

\begin{tabular}{|c|c|c|c|c|c|}
\hline \multicolumn{5}{|c|}{ SAIC WSRC Seepage Basin C Formulations } \\
\hline \multirow{2}{*}{ Formulation } & Soil & $\begin{array}{c}\text { Portland } \\
\text { Cement }\end{array}$ & $\begin{array}{c}\text { Sodium } \\
\text { Bentonite }\end{array}$ & $\begin{array}{c}\text { Sodium } \\
\text { Silicate }\end{array}$ & Water \\
\hline 1 & 2500 & 468.75 & 156.25 & 0 & 625 \\
\hline 2 & 2500 & 463.13 & 0 & 123.75 & 463.125 \\
\hline 3 & 2500 & 481.25 & 160 & 64.375 & 641.25 \\
\hline 4 & 2500 & 493.75 & 164.375 & 131.875 & 658.125 \\
\hline
\end{tabular}

ing

$\frac{9413198}{1100011 / 13 / 58}$

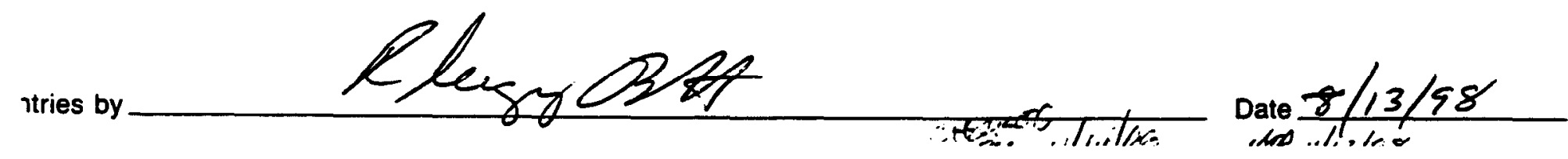


3 Project Name SAHC/SAS

$1175: 007$

Project Number T2236

Mixes were prepend in a Kitchen A lo mixer using a spade Blade. molds wen Filled in Thirds Sample Numbers Followed the plan in the work Plan.

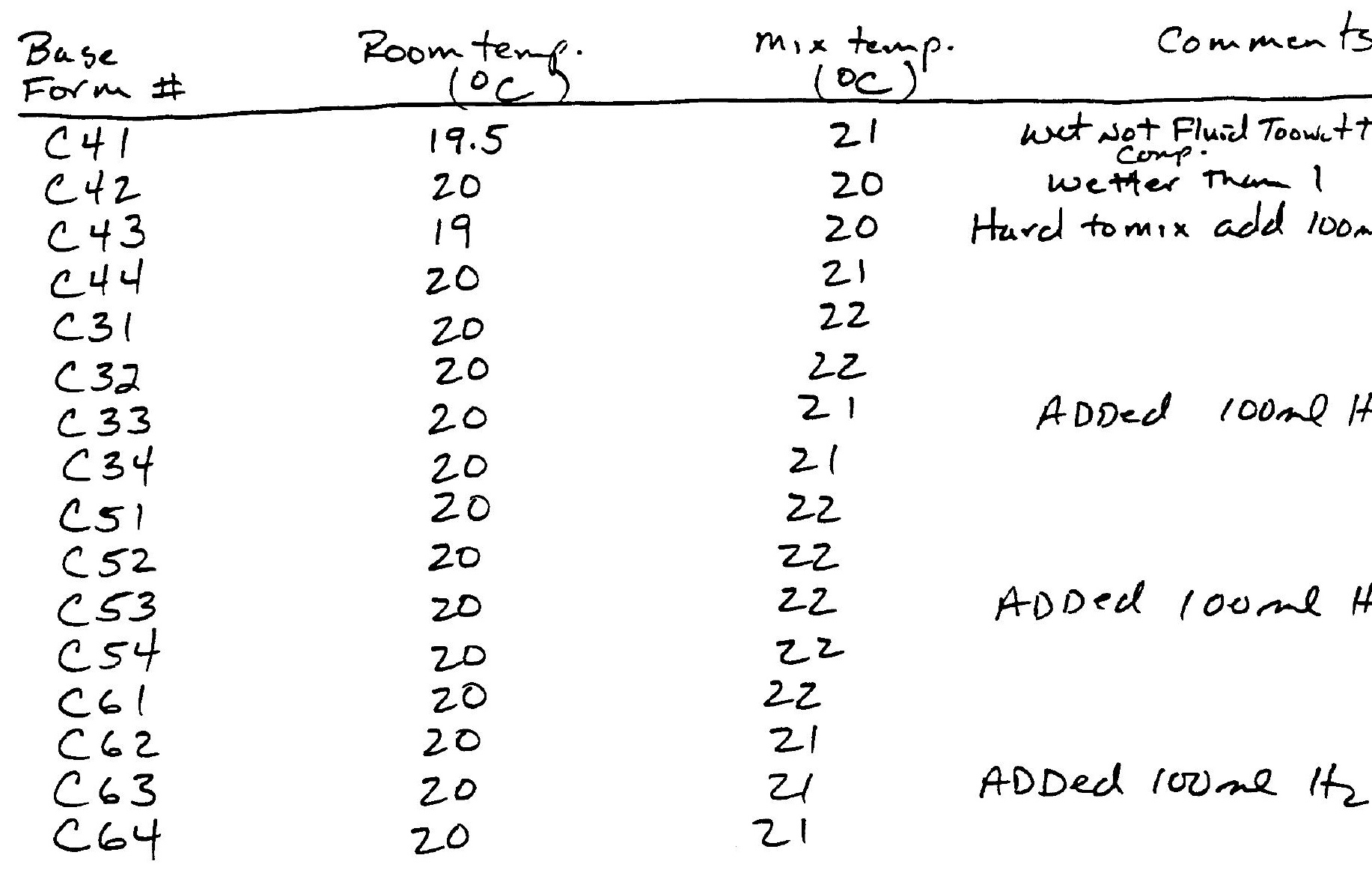

Time of set

all mixes 44.5 TSP 6 hrs

at $16 \mathrm{his}>4.5 \mathrm{TSF}$

no liquid bleed at 16 ms.

Entries by

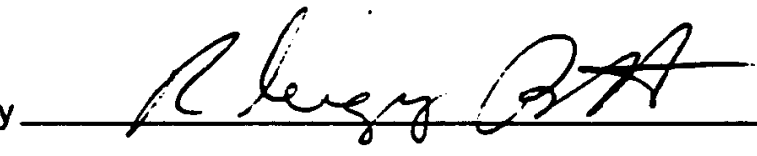

Date $11 / 13 / 98$ 
- jest Name SAre/Sas

$1175: 008$

oject Number $72730 \mathrm{H}$

RAw Density

$$
\begin{aligned}
& C 3 \text { - Vol. inch Doa } \times(4-27 / 16) \text { height } \\
& \text { Density - } 2.17 \mathrm{gl} \text { me }
\end{aligned}
$$

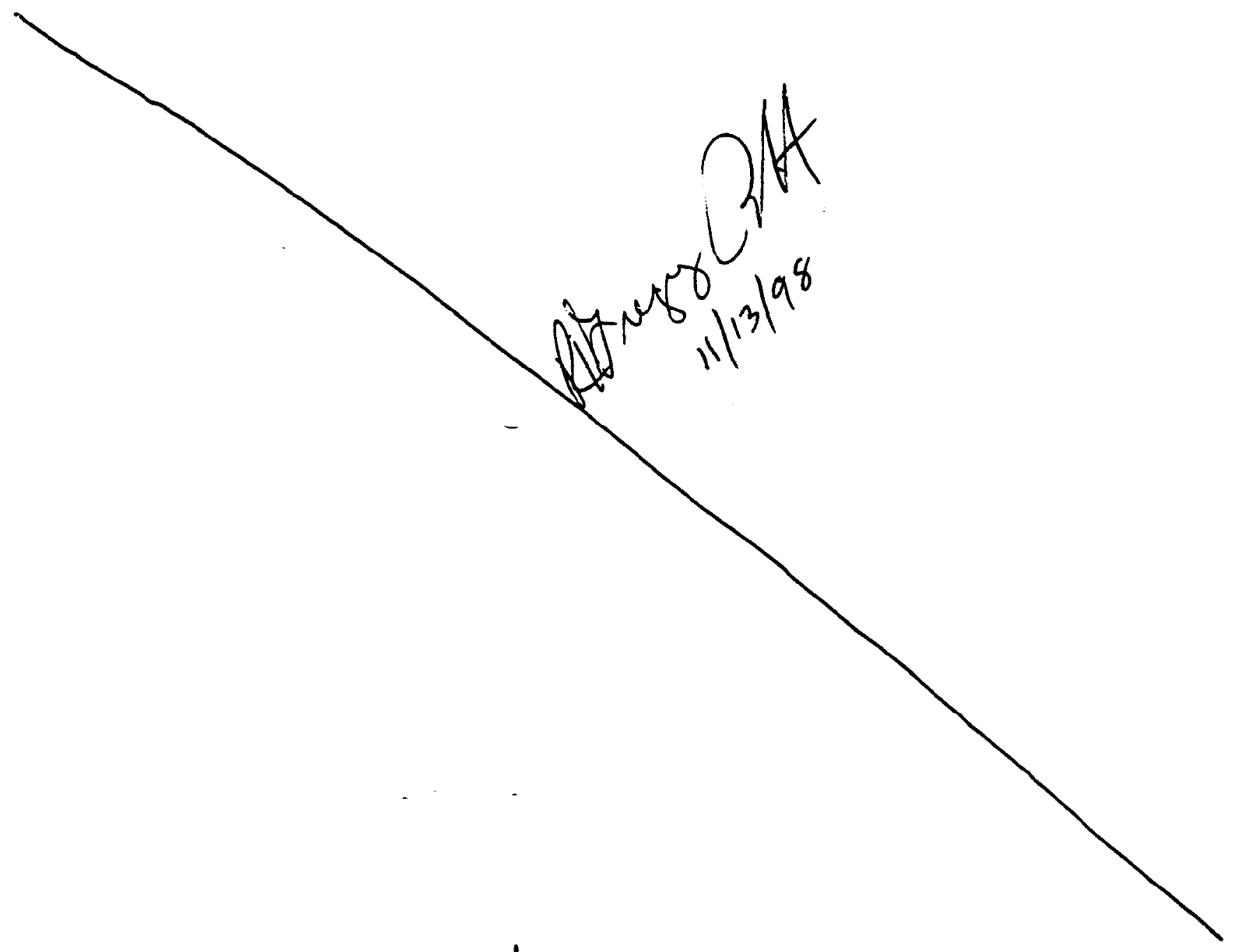

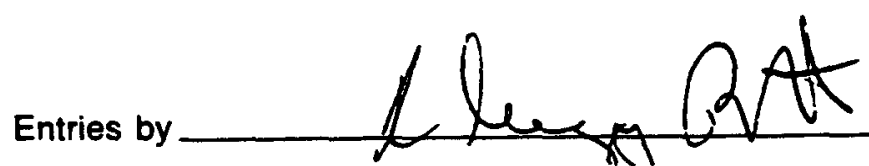

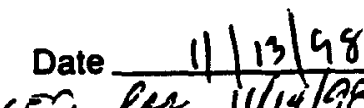


3 Project Name Sare/Ses

Project Number $=773$ or

$1175: 009$

$11 / 17 / 98$

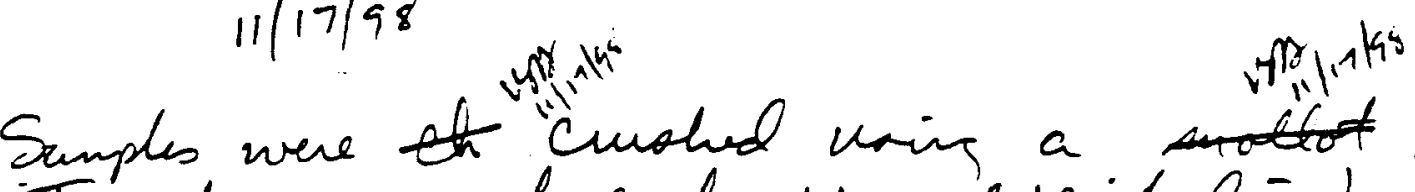

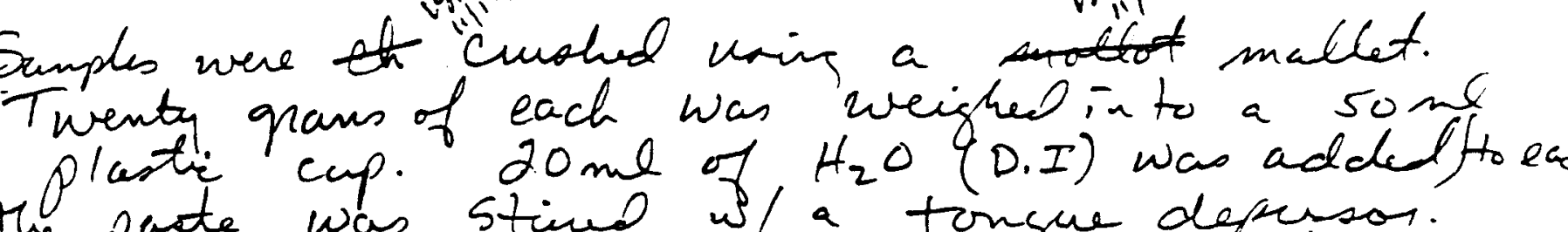
The paste was stine $\mathrm{w} / \mathrm{a}$ tongue defuses. The $\rho^{H}$ metes was calibrated wy 7810 buffers. The slope $=98 \%$ buffer che. $7 \rightarrow 7.02 \quad 10 \rightarrow 10.0$

$\begin{array}{ll}\text { sample } & P H \\ C 520 J 00 & 12.80 \\ C 340 J 00 & 12.78 \\ C 420500 & 12.81 \\ C 40 J 00 & 12.40 \\ C 530 J 00 & 12.59 \\ C 620 J 00 & 12.72 \\ C 440 J 00 & 12.72 \\ C 630 J 00 & 12.62 \\ C 320 J 00 & 12.68 \\ C 610500 & 12.32 \\ C 430500 & 12.56 \\ C 310500 & 12.30 \\ C 640 J 00 & 12.68 \\ C 510500 & 12.28 \\ C 330500 & 12.52 \\ C 540 J 00 & 12.63\end{array}$

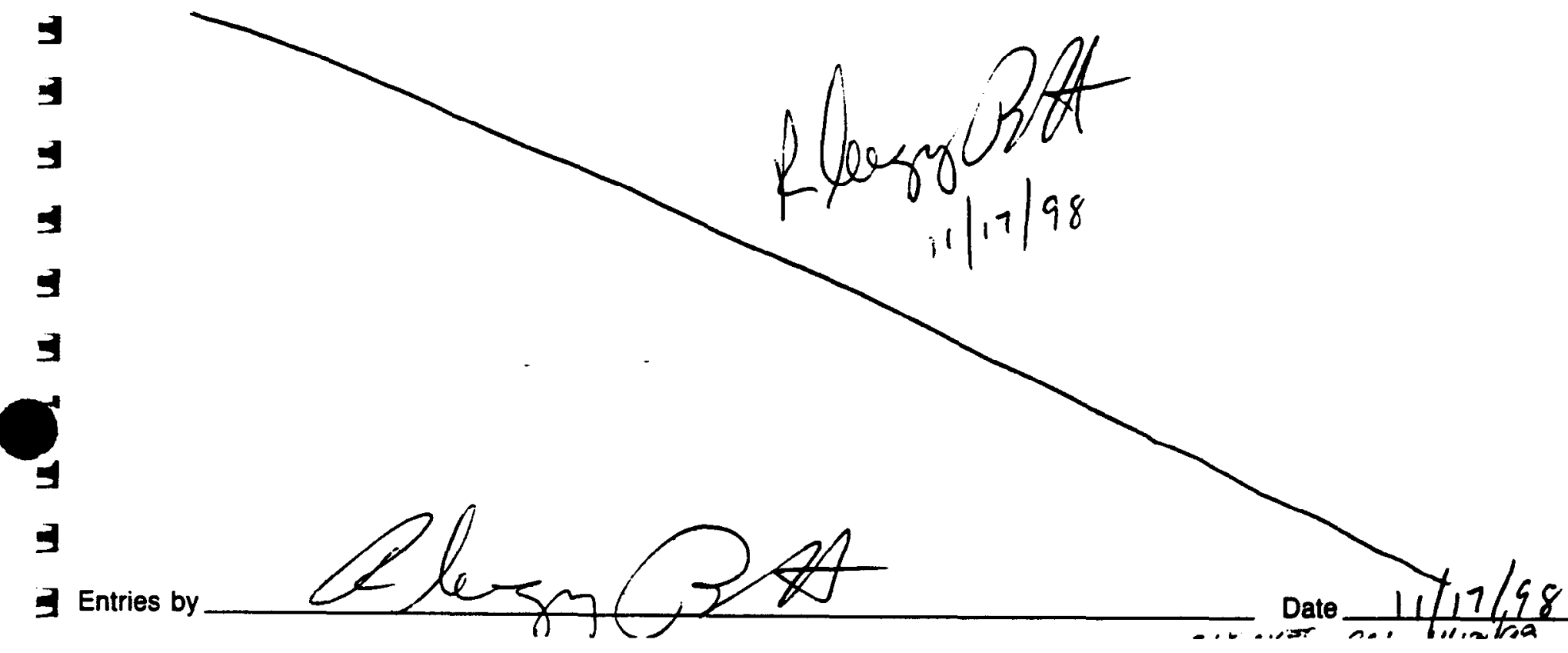




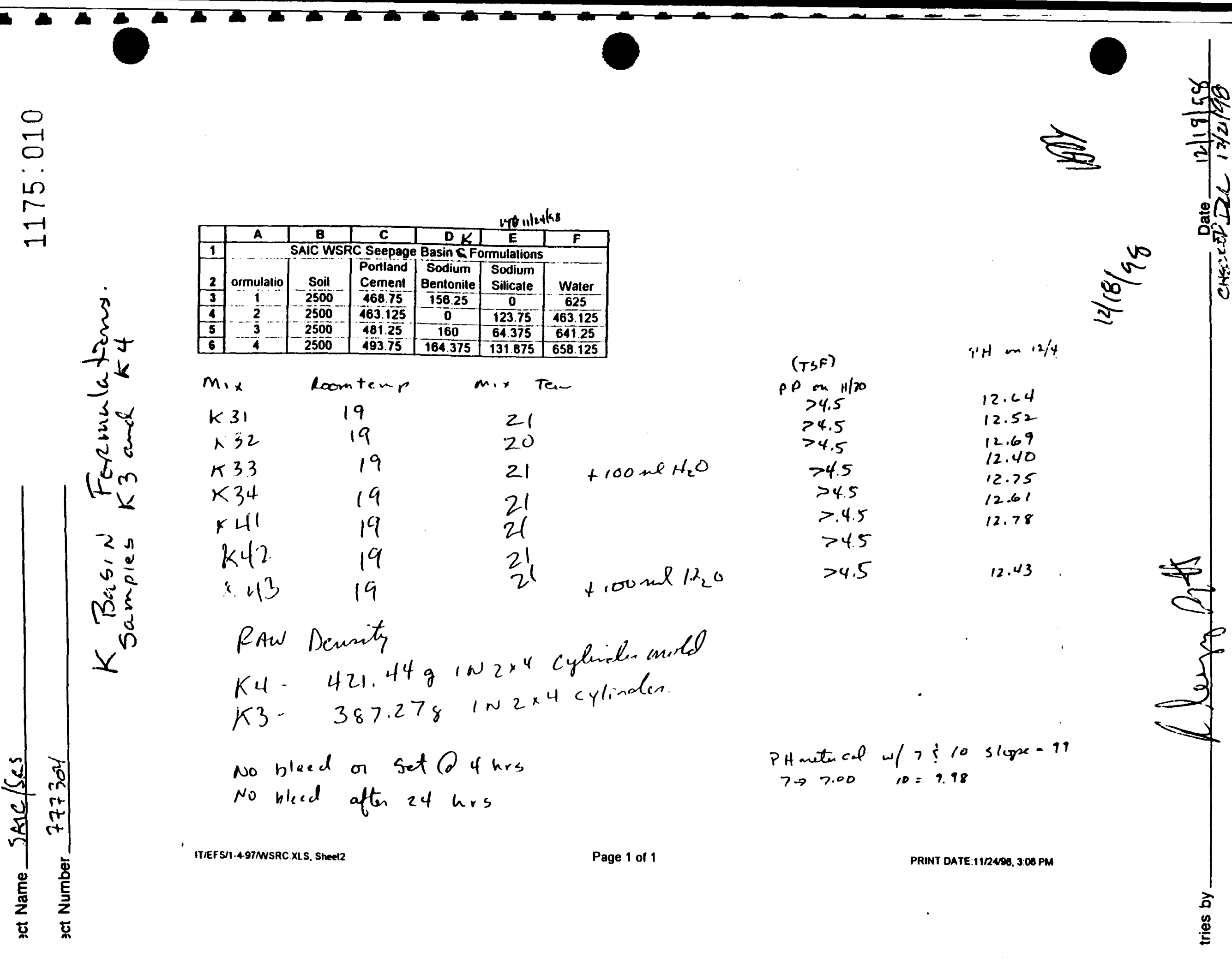


Project Name Sa lc WS RC

$1175: 01$

Project Number $27720^{2}$

ANS i6.1 Mice wits (2 8Day Ans).

$12 / 15 / 48$

Sample

$K 4421+00$

$\kappa 3121700$

253321400

mass

$K 412$ HOO

$K 4321100$

$K 322 H 00$

$K 342100$

$K 4221+00$

$$
\begin{aligned}
& 371.24 \\
& 395.89 \\
& 382.58 \\
& 376.63 \\
& 366.14 \\
& 392.15 \\
& 383.07 \\
& 386.11
\end{aligned}
$$

molds placed in 2.p lock bags unset start of Ex traction.

$12 / 17 / 98$.

Redfox fort Potential

$25 \mathrm{~g}$ of sample $m_{1} \times \mathrm{d} d \mathrm{w} 25 \mathrm{~g}$ of water Red ox measured immediately.

STd. (200 I 10) read $205 \mathrm{~ms}$ Sample K340E00 read $-10.0 \mathrm{mv}$ K410 E00 read $-7.0 \mathrm{mv}$ samples shaken on plat Foin shaker (low)

stopped (c) $091512 / 18 / 98$.

$200 \mathrm{std} \rightarrow 190 \mathrm{mv}$

K340E00 lead 0.00

$K 410$ E00 read 0.00

both Samples Emulsed so water was added. readings Did not Change.

Entries by

Date $\frac{12 / 17 / 9}{\text { pas } 1,1,100}$ 
jet Name SAiC/CRS

$1175: 012$

rect Number $27 ? 330 \mathrm{p}$

$$
\begin{aligned}
& 10 / 21 / 48
\end{aligned}
$$

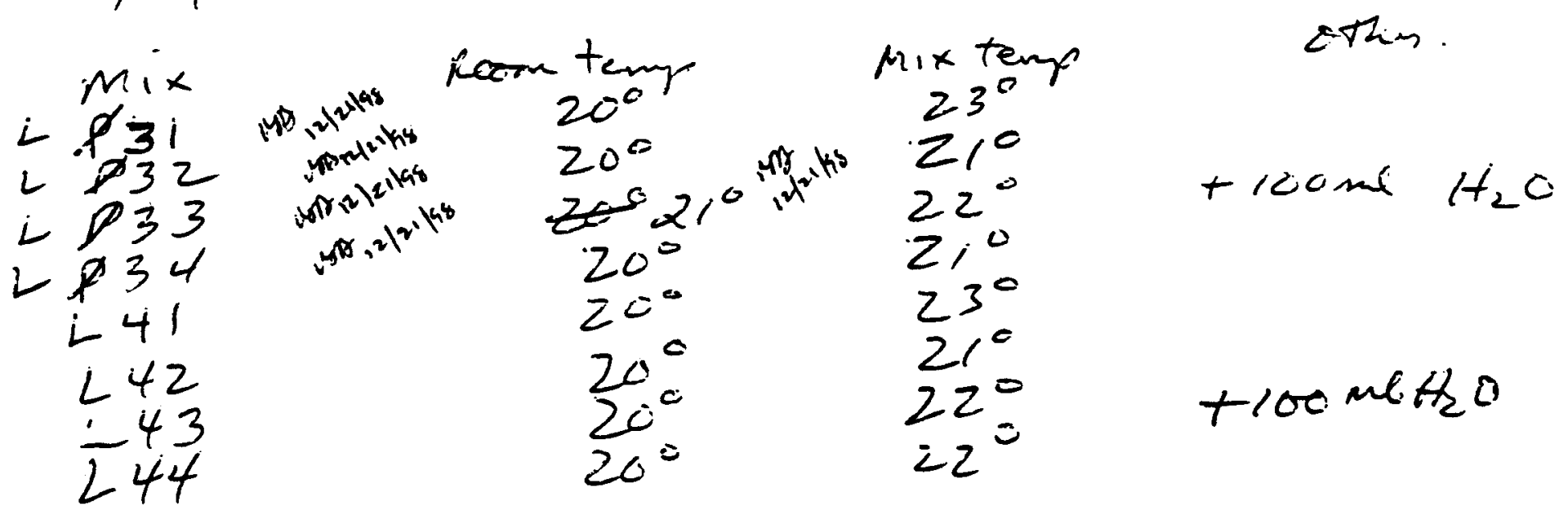

Ail nixes set less Than 4 his

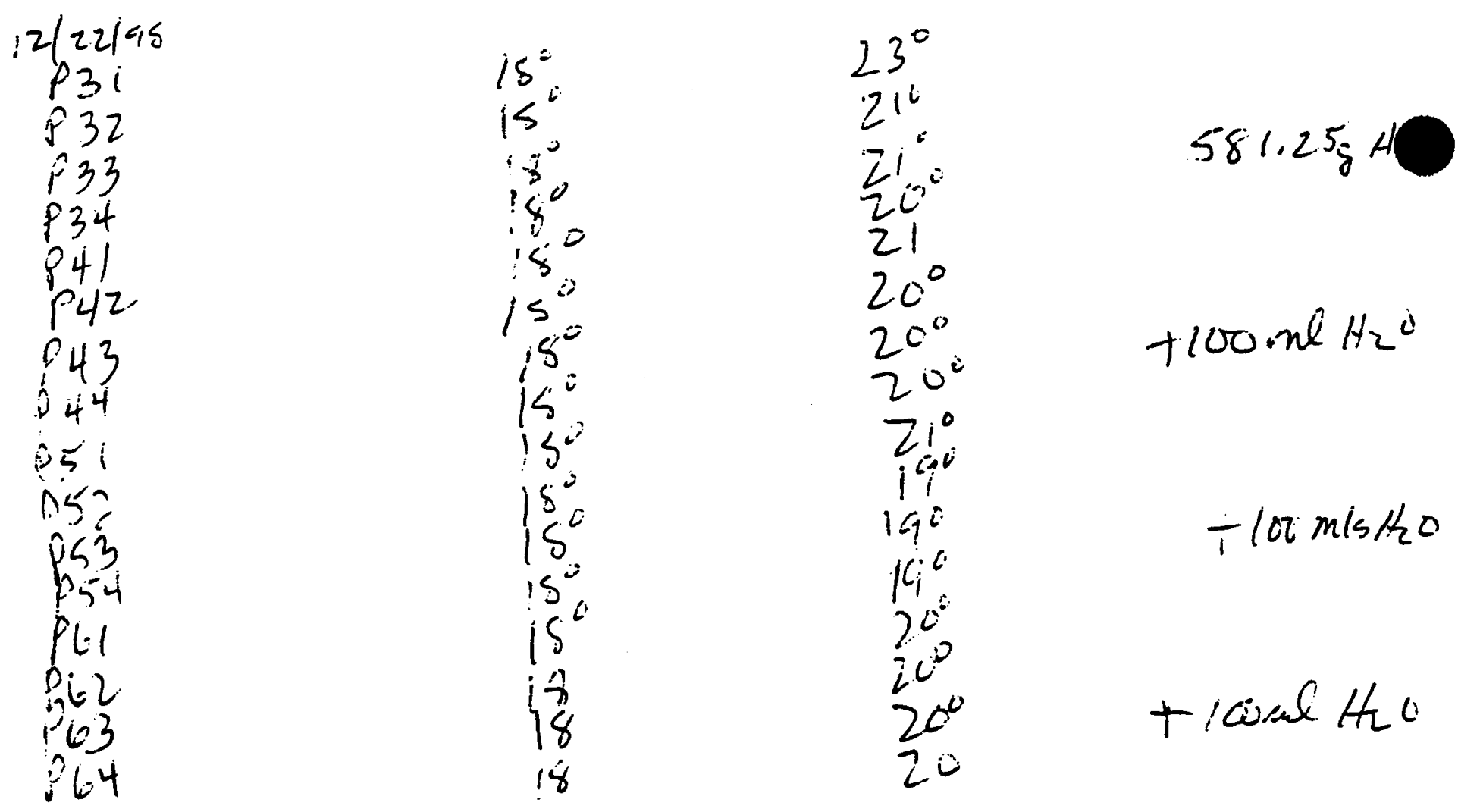

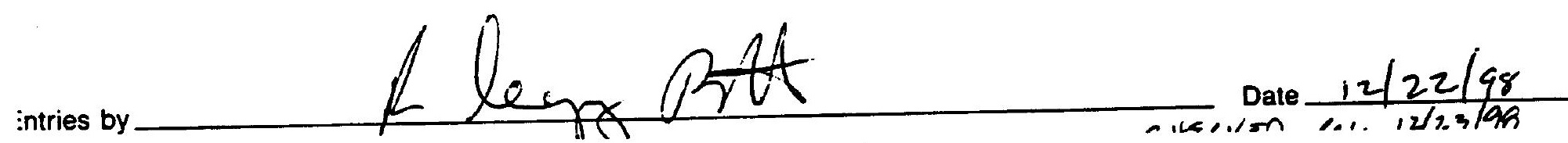


ject Number $19 ? 3: 2$

$-110 \cdot U_{-4}$

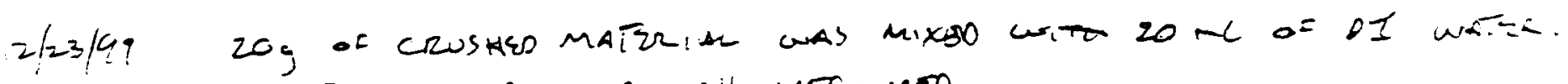
PASTE STIRZEO KANO DIV MERSUROD.

SArPLE

- P310500

P320500

P330500

P340J00

$P+10500$

$P+20500$

$P \$ 30500$

$P 140500$

P510J00

P520500

P530J00

P 540500

P6 10500

P6 2050 s

$P 630500$

PN

12.45

12.61

12.77

12.68

12.50

12.68

12.83

12.87

i2. 39

12.62

12.73

12.86

12.41

12.40

12.73

1640500

12.84

L310508

12.35

$\angle 320100$

12.73

$\angle 330500$

12.68

1340100

12.82

$\begin{array}{ll}\angle 410 J 00 & P N \\ \angle 420 J 00 & 12.52 \\ \angle 430 J 00 & 12.57 \\ \angle 440 J 00 & 12.02 \\ & 12.85\end{array}$

PN METES WAS CAMIBLATSD WITH 7 \& 10 BUFFERS CHECL BUFER REAOINES WERE 6.99 ANO 10.01 .

Pac $12 / 23 / 98$

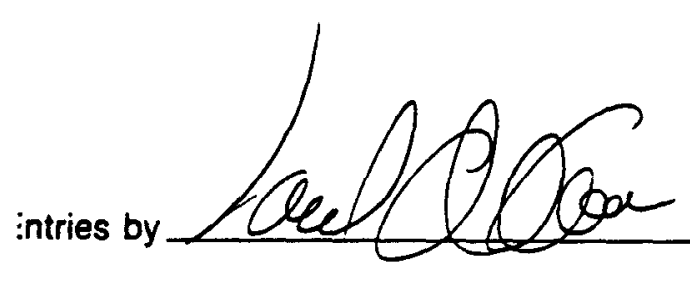

Date $12 / 23 / 98$ 
3 Project Name Stal 7 ses

3 Project Number 77 ? $30^{\circ}$

1

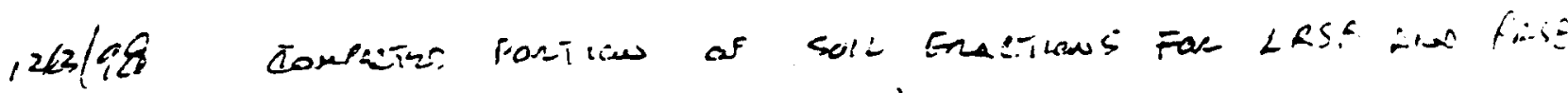

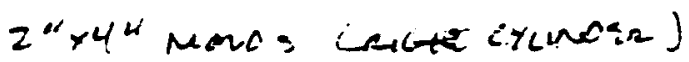

3

3
43
416.759
44
420.439

5

$y$

$\exists$

5

5

is

3

5

5

3

is

5

4

5

3

3

5

5

5

5

5

3

3

- Entries by

$$
\begin{array}{ll}
\text { P3 } & 409.629 \\
\text { P4 } & 413.799 \\
\text { P5 } & 401.569 \\
\text { PE } & 412.319
\end{array}
$$
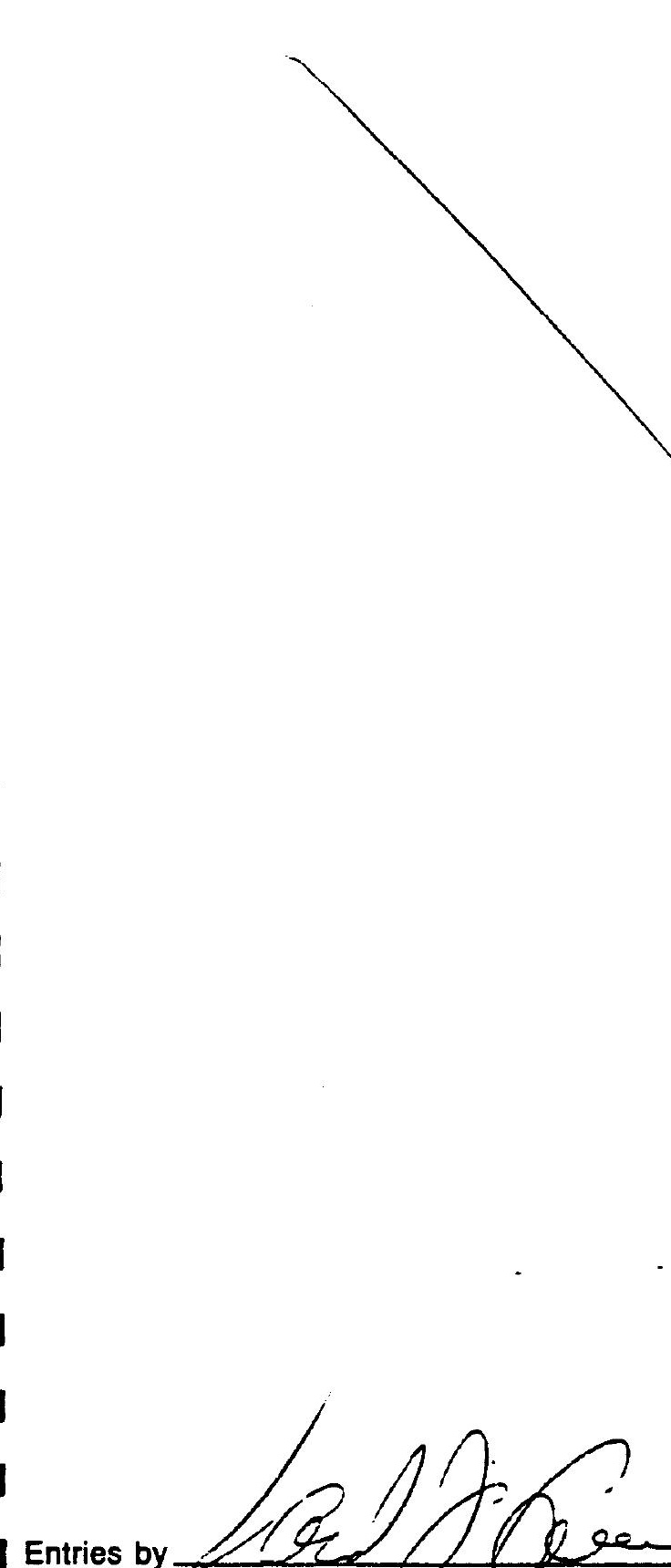

Date $12 / 23 / 98$ 


\section{Appendix C}

CSRB Sampling Map 
This page intentionally left blank. 


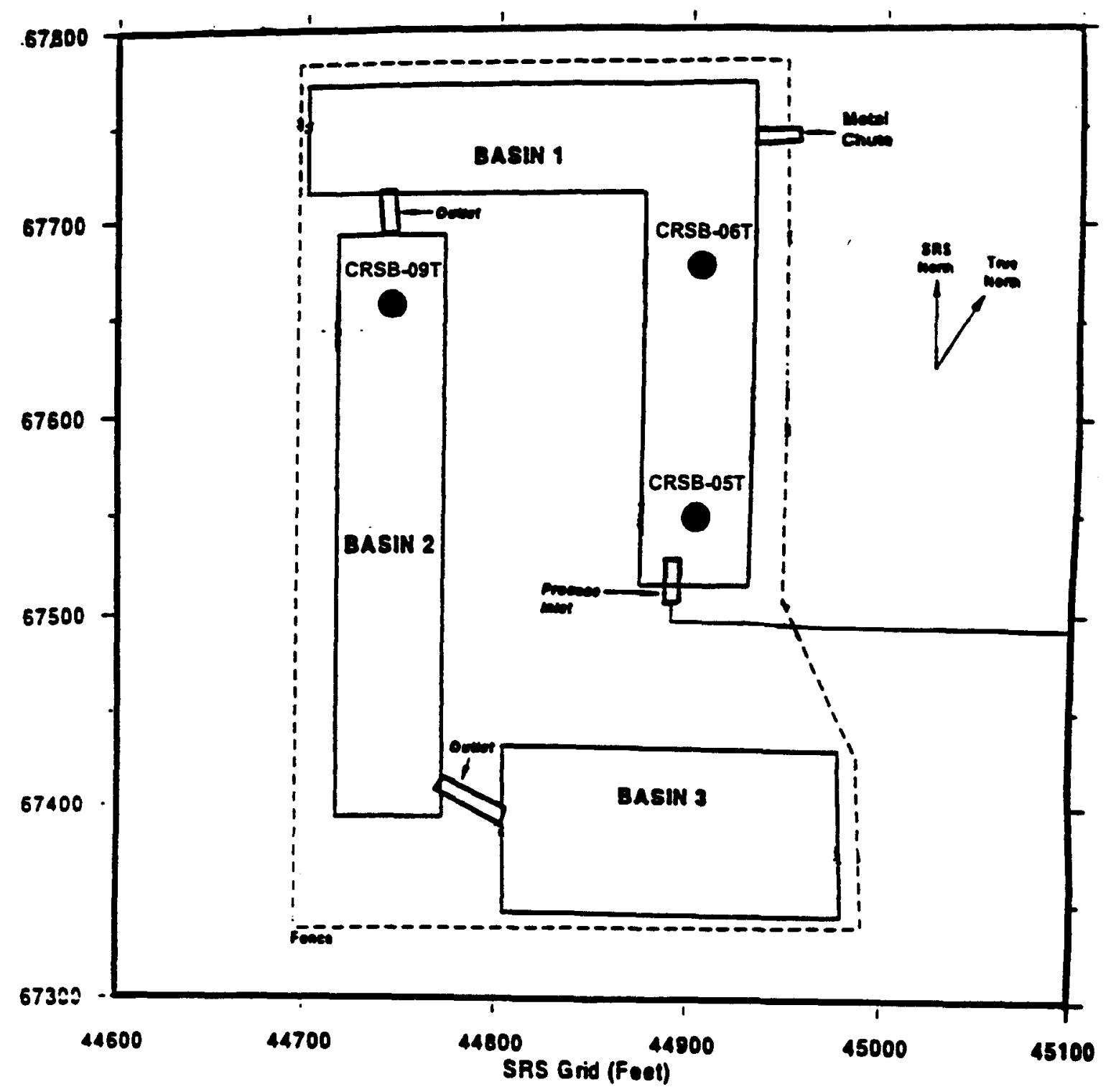

- Soil Sampling Locations

C-Reactor Seepage Basins Proposed Sampling Locations 
Soil Stabilization/Solidification Grout Mix Design Study WSRC-RP-99-4075, Revision 1

C-, K-, L-, and P-Reactor Seepage Basins Closure (U) August 1999

Appendix D

Test Procedures 
This page intentionally left blank. 
United States Department of Energy

Savannah River Site

\section{SOIL STABILIZATION/SOLIDIFICATION GROUT MIX DESIGN STUDY FOR THE \\ C-, K-, L-, AND P-REACTOR \\ SEEPAGE BASINS CLOSURE \\ PROJECT WORK PLAN (U)}

WSRC-RP-98-4184

Revision 0

October 1998

Westinghouse Savannah River Company Savannah River Site Aiken, S.C. 29808

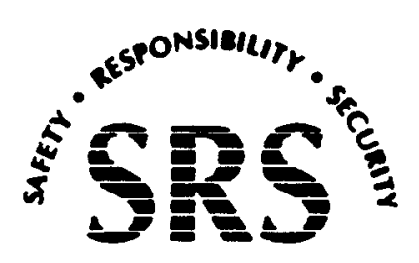




\section{TABLE OF CONTENTS}

LIST OF TABLES iii

LIST OF FIGURES

LIST OF ACRONYMS iv

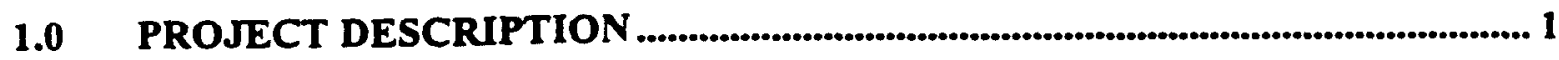

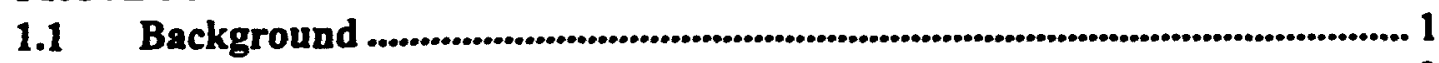

1.2 C-Reactor Seepage Basins .................................................................................... 2

1.3 K-Reactor Seepage Basin ..._._.

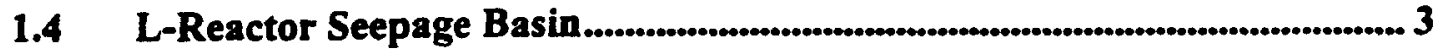

1.5 P-Reactor Seepage Basins ................................................................................. 3

1.6 Related Immobilization Investigation at LAOCB ...................................... 4

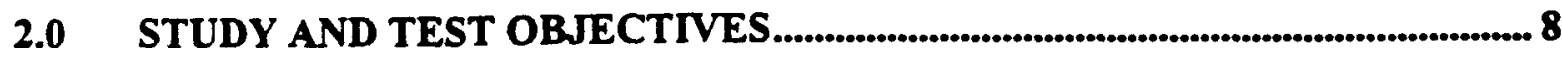

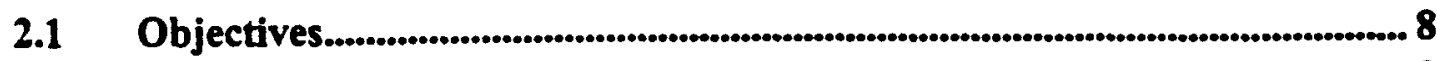

2.2 Study Performance Standards ................................................................ 8

3.0 PREPLANNING ACTIVITIES/TECHNICAL APPROACH......................... 11

4.0 EQUIPMENT, MATERIALS, AND FACILITIES ..................................... 13

4.1 Equipment .......................................................................................................... 13

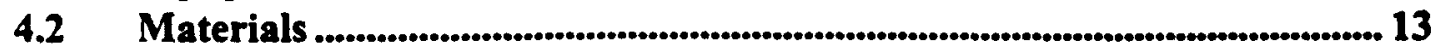

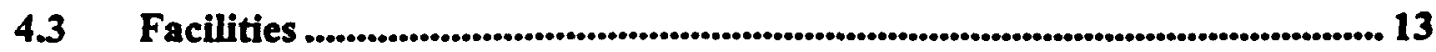

5.0 SAMPLE TRANSPORTATION AND TRACKING ................................... 15

5.1 Sample Transportation............................................................................. 15

5.2 Sample Chain-of-Custody .................................................................... 16

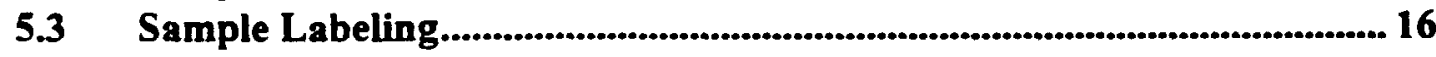

6.0 BENCH-SCALE S/S TESTING ............................................................................ 19

6.1 Phase I Testing ................................................................................................... 19

6.1.1 Treatability Testing on CRSB Soils .................................................. 19

6.1.2 Treatability Testing on KRSB Soils ................................................ 20

6.2 Phase II Testing...................................................................................................... 20

6.2.1 Treatability Testing on LRSB Soils ........................................................... 20

6.2.2 Treatability Testing on PRSB Soils.............................................................. 21

6.3 Grout Mix Design .................................................................................................... 22

6.4 Stabilization Formulation Development ..................................................... 22

6.5 Performance Testing......................................................................................... 22 
7.0 DATA MANAGEMENT AND QUALITY ASSURANCE

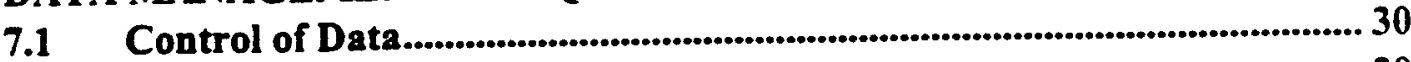

7.2 Data Review and Verification .................................................................... 30

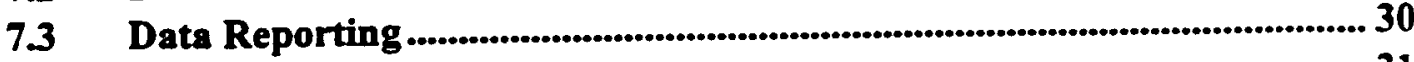

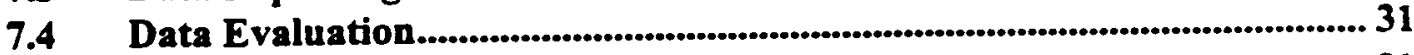

7.5 Data Interpretation ........................................................................................ 31

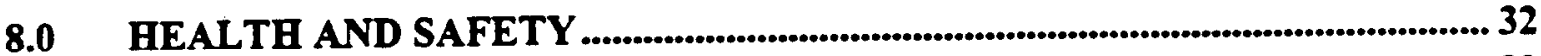

8.1 Project Description and Planning.................................................................. 32

8.2 Chemical Hygiene Plan............................................................................... 32

8.3 Key Point Cards .................................................................................................... 32

8.4 Bench-Scale Experimental Review .......................................................................33

8.5 Radiation Protection Plan ............................................................................... 33

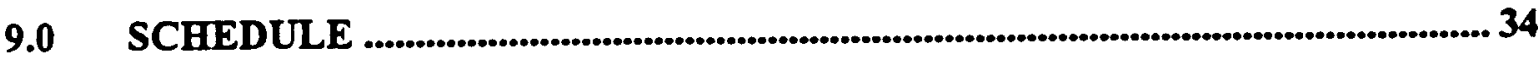

10.0 RESIDUAL WASTE MANAGEMENT ................................................................... 37

10.1 Responsibility and Training.......................................................................... 37

10.2 Sample Management And Storage ......................................................................... 38

10.3 Waste Minimization .................................................................................................... 38

10.4 Estimated Residual Quantities ............................................................... 39

10.5 Residual Characterization and Classification ............................................... 39

10.6 Residuals Storage and Disposal ............................................................................... 39

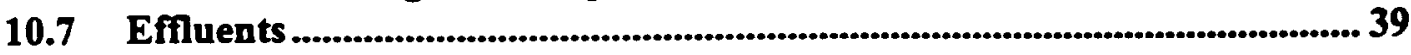

10.8 Emergency Response and Spill Management.................................................... 39

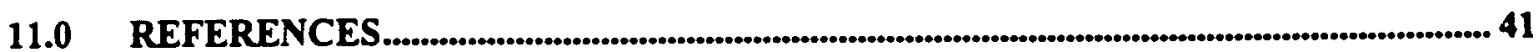

APPENDLX A Technology Development Laboratory's Chemical Hygiene Plan

APPENDIX B Technology Development Laboratory's QA Manual

APPENDIX C Specific Technology Development Laboratory Standard Operating Procedures 


\section{LIST OF TABLES}

Table 1-1 Grout Design Mixes for the Reactor Seepage Basin Soils

Table 2-1 Performance Criteria for the S/S Treated Material

Table 5-1 Treatability Testing Sample Numbering Scheme.

Table 6-1 Samples Generated During Treatability Testing of CRSB Soil Samples. 26

Table 6-2 Samples Generated During Treatability Testing of KRSB Soil Samples.

Table 6-3 Samples Generated During Treatability Testing of LRSB Soil Samples

Table 6-4 Samples Generated During Treatability Testing of KRSB Soil Samples

Table 9-1 Schedule for S/S Treatability Testing on the Reactor Seepage Basin Soils

\section{LIST OF FIGURES}

Figure 5-1 Copy of Analysis Request and Chain of Custody Record

Figure 6-1 Flowchart for the Segregation of the Reactor Seepage Basin Soils .24

Figure 6-2 Flowchart for Formulation Testing on Reactor Seepage Basin Soils 25 


\section{LIST OF ACRONYMS}

\begin{tabular}{ll} 
ACI & American Concrete Institute \\
ANS & American Nuclear Society \\
ASTM & American Society for Testing and Materials \\
CHP & Chemical Hygiene Plan \\
Co & Cobalt \\
COC & Constituents of Concern \\
CRSB & C-Area Reactor Seepage Basin \\
Cs & Cesium \\
DOE & Department of Energy \\
ETDC & Environmental Technology Development Center \\
oF & Degrees Fahrenheit \\
g & Gram \\
HASP & Health and Safety Plan \\
HEPA & High efficiency particulate air \\
HMTR & Hazardous Materials Transportation Regulations \\
IT & International Technology Corporation \\
KRSB & K-Area Reactor Seepage Basin \\
LAOCB & L-Area Oil and Chemical Basin \\
lbs/hr & Pounds per hour \\
LRSB & L-Area Reactor Seepage Basin \\
mg/kg & milligrams per kilograms \\
mL & milliliter \\
NIST & National Institute for Standards and Technology \\
NRC & Nuclear Regulatory Commission \\
OSHA & Occupational Safety and Health Administration \\
pCi/g & picoCuries per gram \\
PHSS & Project Health and Safety Summary \\
POR & Pilot operations room \\
PPE & Personal Protection Equipment \\
ppm & Parts per million \\
PRSB & P-Area Reactor Seepage Basin \\
Psia & pounds per square inch ambient \\
PTSM & Principal Threat Source Materials \\
Pu & Plutonium \\
PVC & Polyvinyl Chloride \\
QA & Quality Assurance \\
QAPP & Quality Assurance Project Plan \\
QC & Quality Control \\
RCRA & Resource Conservation and Recovery Act \\
RCS & Regulatory Compliance Specialist \\
RMA & Radiological Material Area \\
ROD & Record of Decision \\
\hline
\end{tabular}


LIST OF ACRONYMS (Continued)

rpm Revolutions per minute

RPP Radiation Protection Program

RSC Radiation Safety Committee

RSO Radiological Safery Officer

RWP Radiation Work Permit

SAIC Science Applications International Company

SCDHEC South Carolina Department of Health and Environmental Control

SOP Standard Operating Procedure

Sr Strontium

SRS Savannah River Site

S/S Stabilization/Solidification

TAL Technology Applications Laboratories

TDL Technology Development Laboratory

US DOT United States Department of Transportation

US EPA United States Environmental Protection Agency

WSRC Westinghouse Savannah River Company 


\subsection{PROJECT DESCRIPTION}

\subsection{Background}

Between 1957 and 1991, reactor seepage basins received low-level radioactive purge water from the disassembly basins present at each of the Savannah River Site (SRS) production reactors. This water purge was necessary to keep the tritium concentration in the disassembly basin water within safe levels for operating personnel. Before the use of mixed-bed deionizers and sand filters began in the 1960 s, purge water was pumped directly from the disassembly basins to the seepage basins. From 1970 to 1978 , the seepage basins for active reactors were bypassed, and the filtered, deionized purge water was discharged directly into nearby streams. In 1978, the C-Reactor Seepage Basin (CRSB), L-Reactor Seepage Basin (LRSB), and P-Reactor Seepage Basins (PRSBs) were reactivated. The K-Reactor Seepage Basin (KRSB) was used from 1957 to 1960 only. After that time, water was purged from the K-Area Disassembly Basin to the K-Area Retention Basin. The R-Area seepage basins have been filled, covered with asphalt, and are not considered in this sudy. Although still open, none of the other four basins have been active since 1991.

Although many radionuclides were discharged to the basins, almost all of the radioactivity was due to tritium. Tritium is highly mobile, and it migrated relatively quickly from the basins via leaching. Most of the remaining radioactivity is the result of small amounts of cobalt (Co)-60, strontium ( $\mathrm{Sr}$ )-90, cesium (Cs)-137, and alpha emitters such as plutonium (Pu)-239/240. Given the relatively short half-life of $\mathrm{Co}-60$, the other three isotopes are of more concern with respect to future leaching to groundwater. Under the "Plug-In Record of Decision (ROD)" approach, the selected remediation technology for CRSB, KRSB, LRSB and PRSB is that the soils beneath the basins be remediated by in-situ stabilization/solidification $(\mathrm{S} / \mathrm{S})$ treatment, backfilled with clean soil, and covered with a low-permeability soil cover system. A S/S grout mix utilizing various reagents and additives/admixtures is required for the definitive remediation design. The $S / S$ grout mix designs developed and recommended during this task will be used for in situ $S / S$ of the various constituents of concern (COCs) in the subject basins.

The CRSB, KRSB, LRSB and PRSBs are scheduled for closure during the next several years. The Plug-In Proposed Plan WSRC-RP-98-4098, Revision 0, dated June 1998 and the Plug-In Proposed Plan WSRC-RP-98-4099, Revision 0, dated June 1998, identify the selected remediation technology for closure. At present, these two documents have not been approved by the United States Environmental Protection Agency (US EPA) and the South Carolina Department of Health and Environmental Control (SCDHEC) and are still in the review cycle. 
However, Westinghouse Savannah River Company (WSRC) believes that the remediation technology recommended in these referenced documents is appropriate for the given sites. The selected remediation technology recommends that the soils beneath the basins be remediated with in-sin S/S treatment, the basins backfilled with clean soil, and covered with a lowpermeability soil cover system. The results of a previous study (Section 1.6) identified preliminary S/S grout mixes utilizing various reagents (e.g., Portland cement, bentonite, and soluble silicates) for the in-situ $\mathrm{S} / \mathrm{S}$ treatment of the basin soils. A final, recommended $\mathrm{S} / \mathrm{S}$ grout mix will be developed for each of the four reactor seepage basins using the results of this task.

To develop the final, recommended S/S grout mix, a phased approach will be taken. Initial treatability testing on the CSRB and KRSB soils will involve four grout formulations of Portland cement, bentonite, and soluble silicates in various combinations. Each formulation will be tested on soil material to encompass the expected variation in lithology and geotechnical properties within the reactor seepage basins. In Phase II, four formulations will be tested on the LRSB and PRSB soils, again using soil material which encompasses the expected variation in lithology and geotechnical properties for each area.

\section{$1.2 \quad$ C-Reactor Seepage Basins}

The CRSBs are three unlined basins which were constructed in the late 1950s and operated from 1959 to 1970. Wastewater from the reactor disassembly basin was discharged into the southeastern end of L-shaped Basin No. 1. This L-shaped basin slopes to the north and west where a cascade overflow pipe located in the top of the basin wall connects it to Basin No. 2. Basin No. 2 also has a similar cascade overflow into Basin No. 3 located in its southeastern comer. Basin No. 3 would have received wastewater if Basins No. 1 and 2 were nearly full.

Radiation surveys and shallow soil sampling indicate that Basin No. 3 was used sparingly. Grouting is not included in the remedial action plan for the soil Basin No. 3. Therefore, Basin No. 3 will not be discussed further in this document.

From 1970 to 1978, disassembly basin wastewater was mixed with large volumes of heat exchanger cooling water and discharged to streams. After improvements in the treatment of disassembly basin wastewater by the addition of deionization and filtering treatment processes, wastewater discharge to the seepage basins resumed in 1987. The basins have not received wastewater since 1987. 
The Principal Threat Source Materials (PTSM) are Cs- 137 and Co-60. Basin No. 1 contains the highest concentrations of these radioactive sources, with the highest detected concentrations found in the 4 to $6 \mathrm{ft}$ depth range below the existing basin bottom. Basin No. 2 contains the radioactive sources, but at lesser concentrations. The highest detected concentrations were found in the 1 to $4 \mathrm{ft}$ depth range below the basin's existing bottom. Carbon-14, though not a PTSM, was detected in all three basins. Co-60 was detected in Basin No. 1 in the 0 to $1 \mathrm{ft}$ depth range. Cobalt-60 has a short half-life ( 5 1// yrs). Because it decays in a relatively short time compared to the other PTSMs, it will not be considered in this study.

\section{$1.3 \quad$ K-Reactor Seepage Basin}

The KRSB was constructed in 1957 as an unlined basin to receive low-level radioactive wastewater from disassembly basin purges at the K-Reactor. The K-Reactor disassembly basin discharged by gravity to the east side of the KRSB.

The PTSM are Cs-137 and Sr-90. Approximately 83 percent of the non-volatile beta activity is in the top $1 \mathrm{ft}$ of soil, 90 percent in the top $3 \mathrm{ft}$ of soil, and at least 95 percent of the activity is above a depth of 7 feet.

\subsection{L-Reactor Seepage Basin}

The LRSB is an unlined, L-shaped earthen basin used for the disposal of low-level radioactive purge water from the L-Reactor disassembly basin. The LRSB received purge water from 1958 until 1968, when L-Reactor was placed on warm standby. Between 1985 and 1988, mixed bed deionizers and sand filters intercepted the purge water prior to discharge to the LRSB. In 1991, L-Reactor was placed in shutdown status.

In 1978, four soil samples were obtained from inside the LRSB. These samples were found to contain Co-60 (1,052.2 picoCuries per gram [pCi/g]), Cs-137 (258 pCi/g) and Sr-90 (70.6 pCi/g).

\subsection{P-Reactor Seepage Basins}

The PRSB consists of three unlined basins located to the west of the P-Reactor perimeter fence. Two 400 foot underground process sewer lines extended from the P-Reactor disassembly basin to Basin 1. When Basin 1 filled to capacity, it overflowed through a 12-inch metal sewer line into Basin 2. Likewise, Basin 2 overflowed into Basin 3. A "hot chute", a 20 to 25 foot long 
polyvinyl chloride (PVC) pipe, is located in the north wall of Basin 2 and extends the ouiside of the basin perimeter fence into the basin.

The basins were used from 1957 to 1970 to dispose of low-level radioactive process purge water from the reactor disassembly basins. From 1970 to 1978, the seepage basins were bypassed. and filtered deionized purge water was discharged directly into Steel Creek. In 1978, discharge of process purge water to the basins was resumed. The basins have not received wastewater since the P-Reactor was shut down for repairs in 1991.

In 1972, radionuclides were analyzed in a limited number of soil cores taken to a depth of 1.5 feet. Maximum Cs-137 activities of 7,100 pCi/g were noted. In 1976, five surface soil samples (4-inch deep) were taken from each seepage basin. The maximum Cs-137 activity was observed to be $4,400 \mathrm{pCi} / \mathrm{g}$ and the maximum Sr-89/90 concentration was $600 \mathrm{pCi} / \mathrm{g}$. In 1978, sixteen borings were advanced to a maximum depth of 20 feet. These samples were analyzed for Co-60, Cs-137, and Sr-90. The highest activities were measured in Basins 1 and 2, with elevated activity leveis in the top 5 feet.

\subsection{Related Immobilization Investigation At LAOCB}

A soil stabilization Final Corrective Measure/Remedial Action has been proposed to stabilize waste materials within the L-Area Oil and Chemical Basin (LAOCB). A laboratory-scale immobilization study was conducted to evaluate the effectiveness of $S / S$ reagents in immobilizing radionuclides present in the LAOCB. The similarity of the soils and COCs present at the LAOCB and reactor seepage basins suggest that immobilization studies for the LAOCB are very relevant to addressing the soil stabilization to the C-, K-, L- and P-Area reactor seepage basins.

The results of the LAOCB study provide a firm foundation for the immobilization studies proposed at CRSB, KRSB, LRSB, and PRSB. The radionuclides of concern in the LAOCB included all the PTSMs identified in the C-, K-, L- and P-Area reactor seepage basins: Cs-137, Sr-90, and Pu-239/240. The LAOCB also contained additional radionuclides not identified as PTSMs in the reactor seepage basins: (americium-241; Co-60; and uranium-234, -235, and -238) (WSRC, 1996).

The LAOCB soil characteristics were defined using samples obtained using a remote vibracore sampler. An organic-rich sludge/sediment layer is present at the surface to a depth of $0.5 \mathrm{ft}$. Beneath this layer is a very fine to medium grained sandy siltsilty sand layer, which includes 
various percentages of clay (WSRC, 1996). The soils in the four reactor seepage basins are similar to the LAOCB soils.

The LRSB is proximate to the LAOCB and has the same types of soils. The soils in the vicinity of the CRSB, KRSB and PRSB are classified as belonging to the Udorthent and Blanton soil series (WSRC, 1992, 1997, 1998). Udorthents are disturbed soils, derived as spoil or refuse from excavations and major construction activities. Udorthents generally include gravel, sand, and clay. The Blanton soil series is the predominant type at SRS, covering 21 percent of the SRS area. (WSRC, 1992) Blantons are moderately permeable soils found in sandy and loamy sediments of the Coastal Plains (WSRC, 1998). The walls C-, K-, L- and P-Area reactor seepage basins were visually inspected, and the lithologies were found to be similar, further supporting the LAOCB study applicability.

The LAOCB immobilization study was conducted in phases, including reagent screening, reagent optimization, and extended performance testing. The purpose of the screening phase of the study was to evaluate mixing characteristics and performance of six possible mixtures. The mixtures evaluated included mixes of Portland Cement, Portland Cement/Flyash, Portland Cement/Bentonite, Portland Cement/Silicate, Portland Cement/Hematite and Portland Cement/Apatite. Based on the results of reagent screening, three reagent combinations were selected for further study: Portland Cement, Portland Cement/Bentonite, and Portland Cement/Silicate (WSRC, 1996).

The objective of the second phase was to determine the optimum dosage ratios of the three selected reagents. High mixes appeared to reduce the leachability of COCs more appreciably than low mixes. Consequently, high mixes were retained for further evaluation in extended performance testing.

The objective of the extended performance testing was to determine long-term performance of soils treated with the high mixes of the three selected reagent combinations. Significant results of this phase of testing are as follows:

- The unconfined compressive strengths of all three mixes exceeded the Nuclear Regulatory Commission (NRC) guidelines (compressive strength of at least 500 psia).

- The compressive strength of all three mixtures were significantly reduced by the 90-day water immersion. The greatest reduction in average compressive strength was seen in the Portland Cement sample which was reduced by 89 percent to 450 psia. 
- The three mixtures all effectively reduced the leachability of gross alphabeta (reduced the leachability to below 20 percent).

The study results suggest that both bentonite and silicate additives significantly increase the effectiveness of the Portland Cement at reducing the leachability of radionuclides of concern. Based on these findings, this work plan defines the detailed confimatory tests to determine which mixes would meet the performance requirements.

The LAOCB study recommended mixes using a combination of Portland cement, bentonite, and sodium silicate be tested to determine the mixture which will be most effective for the least cost. Dosage ratios of 0.15 Portland cement to 0.05 bentonite to 0.04 silicate (fraction of total weight), respectively, were recommended for further evaluation in the pilot study. Because of the relatively high cost of sodium silicate, it was also recommended that a lower sodium silicate dosage ratio of 0.02 be evaluated to determine how much benefit is achieved by adding more sodium silicate.

Based on the results of the LAOCB study, four grout mixes have been identified for evaluation in this study: Portland cement and bentonite; Portland cement and sodium silicate; Portland cement, bentonite and sodium silicate (low); and Portland cement, bentonite, and sodium silicate (high). The specific formulations for each mix are shown in Table 1-1. 
Soil Stabilization/Solidification Grout Mix Design Study for the

WSRC-RP-98 $\leadsto 184$. Rev. 0

C-, K-, L, and P-Reactor Seepage Basins Closure (U)

October 1998

Table 1-1 Grout Design Mixes for the Reactor Seepage Basin Soils

\begin{tabular}{|c|c|c|c|c|c|c|c|}
\hline \multirow{2}{*}{ Formulation } & Soil & \multicolumn{2}{|c|}{ Portland Cement } & \multicolumn{2}{|c|}{ Sodium Bentonite } & \multicolumn{2}{|c|}{ Sodium Silicate } \\
\cline { 2 - 8 } & $\mathbf{( g )}$ & $\mathbf{( g )}$ & $\begin{array}{c}\text { (\% by } \\
\text { weight) }\end{array}$ & $\begin{array}{c}\text { (g) } \\
\text { (g) }\end{array}$ & $\begin{array}{c}\text { (\%) by } \\
\text { weight) }\end{array}$ & (g) \\
\hline 1 & 4,000 & 750 & 15 & 250 & 5 & 0 & 0 \\
\hline 2 & 4,000 & 741 & 15 & 0 & 0 & 198 & 4 \\
\hline 3 & 4,000 & 770 & 15 & 256 & 5 & 103 & 2 \\
\hline 4 & 4,000 & 790 & 15 & 263 & 5 & 211 & 4 \\
\hline
\end{tabular}

7 


\subsection{STUDY AND TEST OBJECTINES}

\section{$2.1 \quad$ Objectives}

The purpose of this study is to perform bench-scale (laboratory-scale) studies to develop grout mix designs which can stabilize and solidify the radiological contaminants in the CRSB, KRSB. LRSB, and PRSB soils. One or more S/S grout mix designs may be required, as appropriate, for each seepage basin site. The grout mixes developed and recommended during this task will be used for in-situ S/S. It is expected that the depth of S/S will not exceed $20 \mathrm{ft}$ below the existing basin bottoms and in most cases will probably be less than $10 \mathrm{ft}$. The study will be implemented in two phases. The first phase of work, designated Phase I, will be implemented between November 1998 and March 1999 using the CRSB and KRSB soils. With Phase I substantially complete, Phase II will be implemented between February 1999 and June 1999 using the LRSB and PRSB soils.

Initial treatability testing (Phase I) on the CRSB and KRSB soils will involve four formulations involving Portland cement, bentonite, and soluble silicates in various combinations. Each formulation will be replicated on soil materials which reflect the expected variability in lithology and geotechnical properties within a given reactor seepage basin. In Phase II, the same four formulations will be tested on the LRSB and PRSB soils, again on soils which represent the expected variability in lithology and geotechnical properties within a given reactor seepage basin.

The grout mix recommended for each reactor seepage basin will be the one that will produce a stabilized waste monolith (grouted waste) which meets performance requirements/acceptance criteria (Section 2.2). Acceptable construction tolerances and mixing process constraints (if any) will be defined. Field optimization tests are not expected to be necessary as long as the construction tolerances, PTSMs, and mixing process constraints identified as a result of this study are not exceeded.

\subsection{Study Performance Standards}

The primary goal of the S/S treatment is to minimize the mobility of the PTSMs. S/S is a performance-based remedial approach since it does not reduce the total mass of the PTSMs and cannot in itself achieve concentration-based remedial objectives. The performance standards for the S/S treatment of the soils underneath the CRSB, KRSB, LRSB, and PRSB are given in Table 2-1. 
Additional performance parameters such as total carbonation activity, sulfates, sulfides. sulfite. chloride, nitrate, nitrite, nitric compounds, boric compounds, and oxidation reduction potential had been considered for the $S / S$ treated material. Total carbonation activity would be required in demonstrate that the stabilized material contains sufficient carbonation to maintain an alkaline $\mathrm{pH}$. The Portland cement (15 percent of the total treated weight) added as part of the S/S grout mix will produce a sufficient total carbonation activity level to satisfy this criteria. Therefore, the determination of total carbonation activity on the $\mathrm{S} / \mathrm{S}$ treated material will not supply any useful information.

When high salt content wastes are stabilized, the sulfate, sulfide, sulfite, sulfide, nitrate, nitrite. and nitric and boric compound levels are of concern, as the presence of these chemical species in high (>50,000 milligram/kilogram [mg/kg]) levels can affect the physical properties of the final $\mathrm{S} / \mathrm{S}$ treated material. However, there is no indication that the soils underneath the CRSB and KRSB contain high levels of the chemical species. Furthermore, previous $S / S$ treatability work on the LAOCB soils indicated minimal anion leaching from similar $\mathrm{S} / \mathrm{S}$ treated soils using similar reagents. Therefore, performance criteria for these chemical species would not influence the physical stability of the S/S treated material. Soils will be analyzed to verify that none of these compounds are present at levels which would affect the $\mathrm{S} / \mathrm{S}$ treated material.

Certain contaminants, such as arsenic, chromium, and selenium, can easily exist in a number of valance states. The immobilization of these contaminants can be affected by the oxidation/ reduction potential of the S/S treated material. The immobilization of the PTSMs are not affected by their valance state, therefore the oxidation/reduction potential of the $\mathrm{S} / \mathrm{S}$ treated material will not affect its performance. Therefore, performance criteria for these parameters will not be determined in this study. 
Table 2-1 Performance Criteria for the S/S Treated Material

\begin{tabular}{|c|c|c|}
\hline Parameter & Criteria & Standard Test Method \\
\hline Batching Weight Precision & $\leq 5 \%$ & NIST traceable \\
\hline Batching Mix Ratio Variation & $\leq 0.5 \mathrm{gal} / \mathrm{b}$ & NIST traceable \\
\hline $\begin{array}{l}\text { Unconfined Compressive } \\
\text { Strength at } 14 \text { days Cure }\end{array}$ & $>100 \mathrm{psi}$ & ASTM D 2166 \\
\hline $\begin{array}{l}\text { Unconfined Compressive } \\
\text { Strength at } 28 \text { days Cure }\end{array}$ & $>100$ psi & ASTM D 2166 \\
\hline $\begin{array}{l}\text { Unconfined Compressive } \\
\text { Strength after Water } \\
\text { Immersion for } 90 \text { days }\end{array}$ & $>100 \mathrm{psi}$ & $\begin{array}{l}\text { ASTM D 2166, immersion per } \\
\text { NRC guidelines }\end{array}$ \\
\hline $\begin{array}{l}\text { Hydraulic Conductivity at } 28 \\
\text { days Cure }\end{array}$ & $<1 \times 10 \mathrm{E}-08 \mathrm{~cm} / \mathrm{sec}$ & ASTM D 5084 \\
\hline $\begin{array}{l}\text { Hydraulic Conductivity after } \\
\text { Water Immersion for } 90 \text { days }\end{array}$ & $<1 \times 10 \mathrm{E}-08 \mathrm{~cm} / \mathrm{sec}$ & $\begin{array}{l}\text { ASTM D 5084, immersion per } \\
\text { NRC guidelines }\end{array}$ \\
\hline $\begin{array}{l}\text { Leachability Indices for Gross } \\
\text { Alpha, Nonvolatile Beta }\end{array}$ & $>8.0$ & ANS 16.1 \\
\hline $\begin{array}{l}\text { Detection Limit for Gross } \\
\text { Alpha }\end{array}$ & $6 \mathrm{pCi} / \mathrm{ml}$ & Gas Flow \\
\hline $\begin{array}{l}\text { Detection Limits for } \\
\text { Nonvolatile Beta }\end{array}$ & $15 \mathrm{pCi} / \mathrm{ml}$ & Gas Flow \\
\hline $\begin{array}{l}\text { Temperature at Core and } \\
\text { Surface }\end{array}$ & $<100^{\circ} \mathrm{F}$ & ASTM C 1064 \\
\hline $\mathrm{pH}$ & $>10.0$ & ASTM D 4972 \\
\hline Shrink/Swell & $\begin{array}{c}<25 \% \text { of } 28 \text {-day UCS failure } \\
\text { strain }\end{array}$ & ASTM D 3877 \\
\hline Bleed Fluid & $<0.5 \%$ & ANS 55.1 or EPA 9095 \\
\hline Volume Increase & $<50 \%$ & None \\
\hline Hazardous dust/vapor release & -- & Per OSHA Standards \\
\hline $\begin{array}{l}\text { Gas generation due to } \\
\text { chemical, biological, organic, } \\
\text { thermal, and radiation sources }\end{array}$ & $\begin{array}{c}\text { Lesser of: } 0.5 \mathrm{moles} / \mathrm{cf} / \mathrm{yr} \text { or } \\
800 \mathrm{moles} / \mathrm{cf} \text { total }\end{array}$ & $\begin{array}{l}\text { Per } 10 \text { CFR } 60 \text { or Per } \\
\text { NUREG-CR-2333 }\end{array}$ \\
\hline Initial Set Time & $>6$ hours & ASTM C 19I \\
\hline Final Set Time & $<180$ days & ASTM C 191 \\
\hline False Set & Prohibited & ASTM C 191 \\
\hline Flash Set & Prohibited & ASTM C 191 \\
\hline
\end{tabular}




\subsection{PREPLANNING ACTIVITIESTECHNICAL APPROACH}

The technical approach to the S/S treatability testing on the soil from the four reactor seepage basins is based on the similarity of the chemical nature of the contaminants of concem and the method of contamination for each reactor seepage basin. Therefore, the S/S grout mix design for the reactor seepage basins will not have to compensate for variability in radionuclides, heavy metals, organic, or inorganic constituents. The major variability which the S/S mix design must compensate for is the variability in lithology which may be present within the reactor seepage basins in each of the four areas.

The samples used for the LAOCB treatability testing consisted of reddish and tan sandy soil, reddish and tan sandy soil $(80 \%)$ mixed with small amounts of olive green clay $(20 \%)$, and olive green clay $(80 \%)$ mixed with reddish sandy soil $(20 \%)$. Since the reactor seepage basins are located in areas of similar lithology to the LAOCB, it is likely that a similar range in soil material will be encountered for the CRSB, KRSB, LRSB, and PRSB soils. The S/S grout mix design must be demonstrated to handle this range in lithological properties.

The technical approach to the treatability testing will be to divide or segregate the soils from each reactor seepage basin into fractions, which represent the range of lithology represented by the sample material. This will be accomplished by physically segregating the soil sample material from each reactor seepage basin to fractions which are primarily coarse (sandy), fractions which are primarily fine (clayey), and two fractions which represent the overall bulk combination of coarse and fine soil material (bulk fractions). The bulk fraction samples will represent the typical or expected lithological conditions, while the fine and coarse fraction samples will represent the worst case extremes.

For the CRSB and PRSB soils, sufficient material has been collected to allow the physical segregation discussed above. For the KRSB and LRSB soils, the quantity of soil available for performing the tests is limited. Therefore, the KRSB and LRSB soils will be segregated into only coarse and fine fraction samples, representing the extremes in lithology for the these reactor seepage basins.

The fractions resulting from the physical segregation of the soil material from each reactor seepage basin will be used in the S/S grout mix design testing. During this testing, four grout mixes (Portland cement/Bentonite, Portland cement/high Silicate, Portland cement/Bentonite/ high Silicate, and Portland cement/low Silicate) will be used on each fraction of each reactor 
seepage basin. The S/S treated material from each soil/grnut mixing will be subjected to physic:i! and chemical testing to determine if it meets the performance criteria (Table 2.1).

- Section 6.0 discusses how this technical approach will be applied to each of the reactor seepage basin soils. 


\subsection{EQUIPMENT, MATERIALS, AND FACILITIES}

\subsection{Equipment}

The equipment to be used in the laboratory-scale S/S treatability testing includes:

- 3 foot by 6 foot metal tray,

- Stainless steel mixing bowls,

- 5 quart planetary mixer,

- 3 inch by 6 inch right cylinder plastic molds,

- 2 inch by 4 inch right cylinder plastic molds,

- Stainless steel spatulas,

- Laboratory balances, and

- Graduated cylinders.

\subsection{Materials}

The reagents to be used during the laboratory-scale S/S treatability testing includes:

- Type I Portland cement,

- Sodium bentonite, and

- Type $\mathrm{N}$ soluble silicates.

The personal protective equipment to be used during the treatability study includes:

- Nitrile gloves,

- Tyvek coveralls, and

- Tyvek boot covers.

\subsection{Facilities}

The laboratory-scale S/S treatability testing will be conducted at the International Technology, Corp. (IT) Technology Development Laboratory (TDL) in Knoxville, TN. Geotechnical testing on the CRSB and KRSB soils and the S/S treated materials will be conducted at IT's Environmental Technology Development Center (ETDC) in Oak Ridge, TN. 
The TDL and ETDC each maintain a Radioactive Materials License from the State of Tennessee and operate under the treatability exemption in 40 CFR 261.4. Both facilities have a L'.S. Dept of Agriculture Animal and Plant Health Inspection Services agreement for the importation of foreign and domestic soils.

Treatability studies and other activities are performed under the relevant permits, licenses, or exemptions. The Technology Applications Laboratories (TAL) Director is responsible for verifying that TAL facilities maintain the required permits and licenses to meet specific requirements. Access to each facility is strictly controlled.

The TDL was designed to allow bench-scale testing of physical and chemical treatment processes on radioactive, hazardous and mixed waste samples. This laboratory has four isolated negativepressure cubicles that contain fume hoods and laboratory benches constructed of materials selected for easy decontamination. Each cubicle's ventilation system is equipped with both high efficiency particulate air (HEPA) and activated carbon bed housings to allow proper selection of effluent controls. The drains from the sinks in this laboratory are connected to a collection tank.

The ETDC has features that ensure the handling of mixed and hazardous waste materials will not have an adverse impact on personnel safety or the environment. The mixed waste treatability testing laboratories have been designed and constructed to facilitate cleanup and decontamination activities. Local ventilation through HEPA filters is supported by area ventilation systems that utilize a double HEPA filtration system prior to discharge. Liquid discharges from treatment processes are collected into holding tanks for analysis prior to discharge. Personnel contamination monitoring stations are in place to prevent the spread of radioactive contamination to uncontrolled areas.

Instrumentation and geotechnical laboratories used for the analyses of radioactive, hazardous, and mixed waste samples are maintained in a controlled area.

An instrument calibration laboratory is used for calibration of portable radiation protection instrumentation. This laboratory is equipped with a variety of National Institute for Standards and Technology (NIST) traceable sealed and plated sources and test equipment. Access and radiation control features include warning lights, radiological postings, key restrictions and shielding. 


\subsection{SAMPLE TRANSPORTATION AND TRACKING}

\subsection{Sample Transportation}

Soil samples from CRSB, KRSB, LRSB, and PRSB will be transported by licensed shipper from the Radiological Material Area (RMA) at Building 772-7B, SRS, to the ETDC. Chains of custody will be used to ensure that samples are properly identified, including boring number. depth below ground surface sample was taken, and the date. A Shipping Order, per United States Department of Transportation (US DOT) requirements, is required for off-site shipment and will be provided by the SRS Hazardous Materials Transportation Regulator (HMTR).

Chain of custody forms will accompany the sample shipment. Upon receipt at the ETDC, the soil samples will each be given a unique sample number and logged into the ETDC sample inventory system. The unique sample number will be used to track materials such as samples, test-residues, test products and reagents throughout the project.

Upon receipt, the outside of each container will be smeared to measure extemal contamination, and the dose rate at the surface of the containers will be measured. Until the contaminated soil is characterized, the gross alpha and non-volatile beta radiation data received from WSRC will be used for assigning radiological categories to the drum and any sub-samples taken from the containers. The containers will be then moved to the pilot operations room (POR) at the ETDC. This room is designed for pilot-scale testing and has high air turnover. The air exhausted from the POR is treated by HEPA filters and vapor phase carbon before discharge. In the POR, the containers will be opened to visibly assess the condition of the contents. A grab sample of soil will be collected and analyzed for gross alpha/beta activity as a comparison to data supplied by WSRC.

After the container is opened, the contaminated soil will be dumped into a three foot wide by six foot long metal tray with other soil material from the same seepage basin. The soil material will be separated in fractions representing the variability of lithology and geotechnical properties present in the soil from each reactor seepage basin (See Sections 3.0, 6.1.1 and 6.2.1 for further details). The remaining contaminated soil will be mixed by hand using a garden hoe until it is visually uniform. Any debris or oversize material (greater than 6 inches) will be removed from the soil and stored separately. The homogenized soil fractions will be put in five-gallon buckets and will be transferred to the TDL for bench-scale studies. 
Procedures for laboratory sample receipt. custody, coding, tracking, hanuiling. and storage are in: Standard Operating Procedure (SOP) PDGADM0005, Appendix C.

\subsection{Sample Chain-Of-Custody}

A strict chain-of-custody record shall be maintained for all samples received at the TDL or the ETDC. The treatability facilities are controlled-access facilities, with multiple secured sample storage areas.

Sample transfers external to the treatability facility will be accompanied by a combined Analysis Request and Chain-of-Custody Record (Figure 5-1). Samples transferred to the treatability analytical department or Geotechnical Laboratory will also be accompanied by an Analysis Request and Chain-of-Custody Record. Instructions for completing the form are on the back of the form.

Copies of all Analysis Request and Chain-of-Custody Records will be maintained in the project file.

\subsection{Sample Labeling}

Samples collected will have a unique sample identification which describes the reactor seepage basin, the soil fraction or subsample, the formulation, applicable cure period, testing to be conducted on the sample, and any subsample required for the applicable testing. Table 5-1 details the sample numbering categories and their applicable codes.

For example, Sample Number C422F00 would be the unconfined compressive strength sample, cured 28 days, from the cement + bentonite + low silicate formulation from the fine fraction of the CRSB soil material. Likewise, Sample L612H02 would be the second leachate subsample from the ANS 16.1 leachability test on the treated material, cured 28 days, from the cement + bentonite formulation on the bulk fraction of the LRSB soil material. 


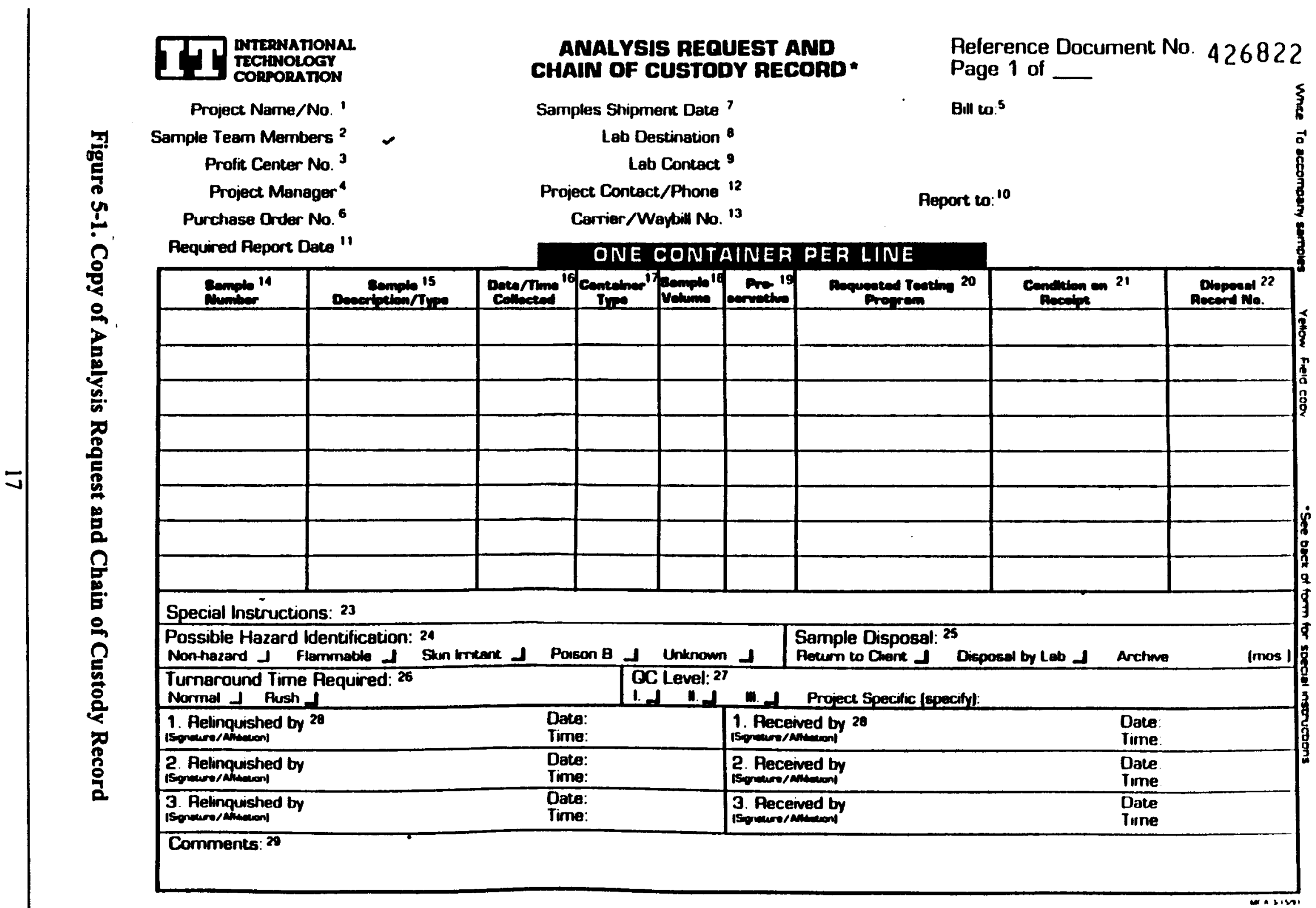


Table 5-1 Treatability Testing Sample Numbering Scheme

\begin{tabular}{|c|c|c|c|}
\hline Category & Code & Category & Code \\
\hline \multirow[t]{4}{*}{ Reactor } & $C=C R S B$ & \multirow[t]{17}{*}{ Test } & $A=C s-137 . S r-90$. Pu-239.240 \\
\hline & $K=K R S B$ & & B = gross alpha nonvolatile bera \\
\hline & $L=$ LRSB & & $C=$ Inorganics \\
\hline & $P=P R S B$ & & $D=$ Total cabonation activity \\
\hline \multirow[t]{8}{*}{ Subsample } & $1=G_{a b} \mid$ & & E = Oxidationreduction polential \\
\hline & $2=G r a b 2$ & & $F=$ Unconfined Compressive Strength \\
\hline & $3=$ Coarse & & $G=$ Hydraulic Conductivity \\
\hline & $4=$ Fine & & $H=$ ANS 16.1 Leachability \\
\hline & $5=$ Typical 1 & & $1=$ Temperature \\
\hline & $6=$ Typical 2 & & $\mathrm{~J}=\mathrm{pH}$ \\
\hline & $7=$ Typical 3 & & $K=$ Shrink/Swell \\
\hline & $8=$ Typical 4 & & $L=$ Bleed Volume \\
\hline \multirow[t]{5}{*}{ Formulation } & $0=$ Not Applicable & & $M=$ Volume increase \\
\hline & $1=P C+$ bentonite & & $N=$ Senting \\
\hline & $2=$ Cement + bentonite + low silicate & & $Q=$ Geotechnical \\
\hline & $3=C$ ement + bentonite + high silicate & & \\
\hline & $4=$ Cement + high silicate & & \\
\hline \multirow[t]{11}{*}{ Cure Period } & $0=$ Not Applicable & \multirow{2}{*}{$\begin{array}{c}\text { Test } \\
\text { Subsample }\end{array}$} & 00 - when test not " $\mathrm{H}$ " \\
\hline & $1=14$ days & & $01=2$ hours \\
\hline & $2=28$ days & & $02=7$ hours \\
\hline & $3=90$ days immersed & & $03=1$ day \\
\hline & & & $04=2$ days \\
\hline & & & $05=3$ days \\
\hline & & & $06=4$ days \\
\hline & & & $07=5$ days \\
\hline & & & $08=19$ days \\
\hline & & & $09=47$ days \\
\hline & . & & $10=90$ days \\
\hline
\end{tabular}




\subsection{BENCH-SCALE S/S TESTING}

The bench-scale treatability testing will demonstrate how the treated material from the selected $\mathrm{S} / \mathrm{S}$ grout mix designs performs with respect to the performance criteria listed in Table 2.1. The technical approach used for selecting the soil fractions for $\mathrm{S} / \mathrm{S}$ testing is described in Section 3.0.

\subsection{Phase I Testing}

The first phase of the S/S treatability study on the reactor seepage basin soils will involve the soils from the CRSBs and KRSB (due to the early scheduled closure for these basins). The flowcharts in Figures 6.1 and 6.2 demonstrate the progression of the treatability testing.

\subsubsection{Treatability Testing on CRSB Soils}

Step 1. The soil material from the CRSB will be dumped into a three foot wide by six foot long metal tray. Two 1-quart samples which appear representative of the soil material in the tray will be collected for geotechnical and characterization analysis (Table 6-1).

Step 2. The soil material will be segregated by lithological properties into samples for formulation mix design testing. The objective of this segregation is to produce samples for mix design testing which bracket the anticipated range in lithologic properties. This will ensure that the performance of the mix designs is evaluated against the potential variability in lithology within the CRSB.

Clayey material will be segregated and combined to make a fine fraction sample for mix design testing. Coarse material will be segregated and combined to make a coarse fraction sample for mix design testing. The remaining material will be homogenized and two bulk fraction samples for mix design testing will be prepared. Two one-quart samples will be collected for geotechnical analysis, if there is a sufficient quantity of soil remaining.

Step 3. The four fractions (coarse; fine, and 2 bulk) will be used for formulation mix design testing. Four formulations will be made on each fraction (Table 1-1). 


\subsubsection{Treatability Testing on KRSB Soils}

Step 1. The soil material from the KRSB will be dumped into a three foot wide by six foot long metal tray. Two 1-quart samples which appear representative of the soil material in the tray will be collected for geotechnical and characterization analysis (Table 6-2).

Step 2. The soil material will be segregated by lithological properties into samples for formulation mix design testing. The objective of this segregation is to produce samples for mix design testing which bracket the anticipated range in lithologic properties. This will ensure that the performance of the mix designs is evaluated against the potential variability in lithology within the KRSB.

Clayey material will be segregated and combined to make a fine fraction sample for mix design testing. Coarse material will be segregated and combined to make a coarse fraction sample for mix design testing. The remaining material will be homogenized and two I-quart samples will be collected for geotechnical analysis.

Step 3. The two fractions (coarse and fine) segregated above will be used for formulation mix design testing. Four formulations will be made on each fraction (Table 1-1).

\subsection{Phase II Testing}

The second phase of the S/S treatability study on the reactor seepage basin soils will involve the soils from the LRSB and PRSB. The second phase will begin during the latter part of the Phase I testing (see Section 9 for the schedule). The flowcharts in Figures 6.1 and 6.2 demonstrate the progression of the treatability testing.

\subsubsection{Treatability Testing on LRSB Soils}

Step 1. The soil material from the LRSB will be dumped into a three foot wide by six foot long metal tray. Two 1-quart samples which appear representative of the soil material in the tray will be collected for geotechnical and characterization analysis (Table 6-3).

Step 2. The soil material will be segregated by lithological properties into samples for formulation mix design testing. The objective of this segregation is to produce samples for mix design testing which bracket the anticipated range in lithologic properties. This will ensure that 
the performance of the mix designs is evaluated against the potential variability in lithology within the LRSB.

Clayey material will be segregated and combined to make a fine fraction sample for mix design testing. Coarse material will be segregated and combined to make a coarse fraction sample for mix design testing. The remaining material will be homogenized and two 1 -quart samples will be collected for geotechnical analysis.

Step 3. The fractions (coarse and fine) segregated above will be used for formulation mix design testing. Four formulations will be made on each fraction (Table 1-1).

\subsubsection{Treatability Testing on PRSB Soils}

Step 1. The soil material from the PRSB will be dumped into a three foot wide by six foot long metal tray. Two 1-quart samples which appear representative of the soil material in the tray will be collected for geotechnical and characterization analysis (Table 6-4).

Step 2. The soil material will be segregated by lithological properties into samples for formulation mix design testing. The objective of this segregation is to produce samples for mix design testing which bracket the anticipated range in lithologic properties. This will ensure that the performance of the mix designs is evaluated against the potential variability in lithology within the PRSB.

Clayey material will be segregated and combined to make a fine fraction sample for mix design testing. Coarse material will be segregated and combined to make a coarse fraction sample for mix design testing. The remaining material will be homogenized and two bulk fraction samples for mix design testing. If sufficient material remains, two l-quart samples collected for geotechnical analysis.

Step 3. The four fractions (coarse, fine, and 2 bulk) will be used for formulation mix design testing. Four formulations will be made on each fraction (Table 1-1). 


\subsection{Grout Mix Design}

The S/S grout mixes for each basin using the CRSB, KRSB, LRSB. and PRSB soils. selected S S additives (admixture), and other $\mathrm{S} / \mathrm{S}$ grout mix components will be developed as presented below. During Phase I, four mixes will be considered for CRSB and KRSB. They are:

- Portland Cement/Bentonite

- Portland Cement/ Silicate

- Portland Cement/Bentonite/high Silicate

- Portland Cement/ Bentonite/low Silicate

In developing the mixes, the components (including cementitious and pozzolanic components and their chemical constituents) will be of high quality and of known composition and commercially available. The manufacturer's technical product information and certification of compliance including conformance determined per American Society of Testing and Materials (ASTM) and American Concrete Institute ( $\mathrm{ACl}$ ) test standards will be included as an appendix to the final report. All cementitious and pozzolanic components shall be chemically stable and shall be environmentally nonhazardous before and after their $\mathrm{S} / \mathrm{S}$ applications.

\subsection{Stabilization Formulation Development}

Each formulation will be mixed in a planetary mixer. A 4,000 $\mathrm{g}$ aliquot of seepage basin soil will be added to the mixing bowl. The dry reagents will be weighed out, blended, and mixed into 1,000 milliliters (ml) of water. Soluble silicate or an additional $200 \mathrm{ml}$ of water will be added to the reagent slurry. The reagent slurry will then be added to the soil in the mixing bowl. The soil and reagent slurry will be mixed at 30-40 revolutions per minute (rpm) for 1 to 2 minutes to produce a homogeneous treated material.

The treated material will be poured into appropriate molds for performance testing, vibrated to remove air pockets, and capped to minimize moisture loss. The molded material will be allowed to cure at ambient laboratory temperature (approximately $72^{\circ} \mathrm{F}$ ) in sealed coolers.

\subsection{Performance Testing}

The cured treated material will be subjected to the performance criteria testing listed in Tables 6.1, 6.3, 6.4 and 6.5 for Step 3. The testing listed is the same as performance criteria (Table 2.1) 
for the final stabilized waste material. with the exception of the criteria for the batching. hazardous dust/vapor release and the gas generation. The results from the performance testing will provide information on the leachability, durability, hydraulic conductivity, strength. and long-term durability of the S/S treated soil material. The methods for performanice testing are listed in the tables. 

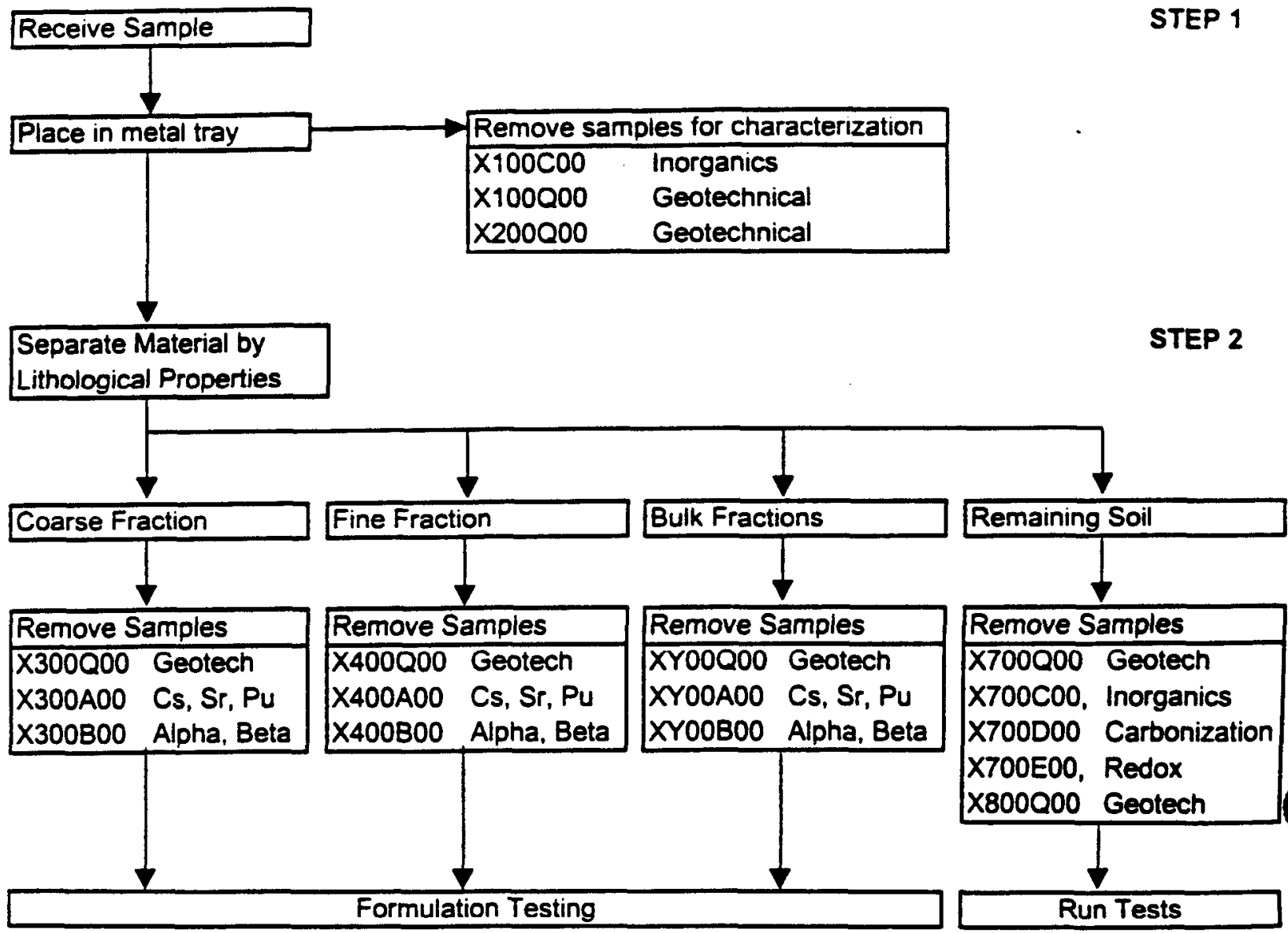

$X=C, K, L$, or $P$ (Reactor)

$Y=5$ or 6 (Subsample)

Figure 6-1. Flowchart for the Segregation of the Reactor Seepage Basin Soils 


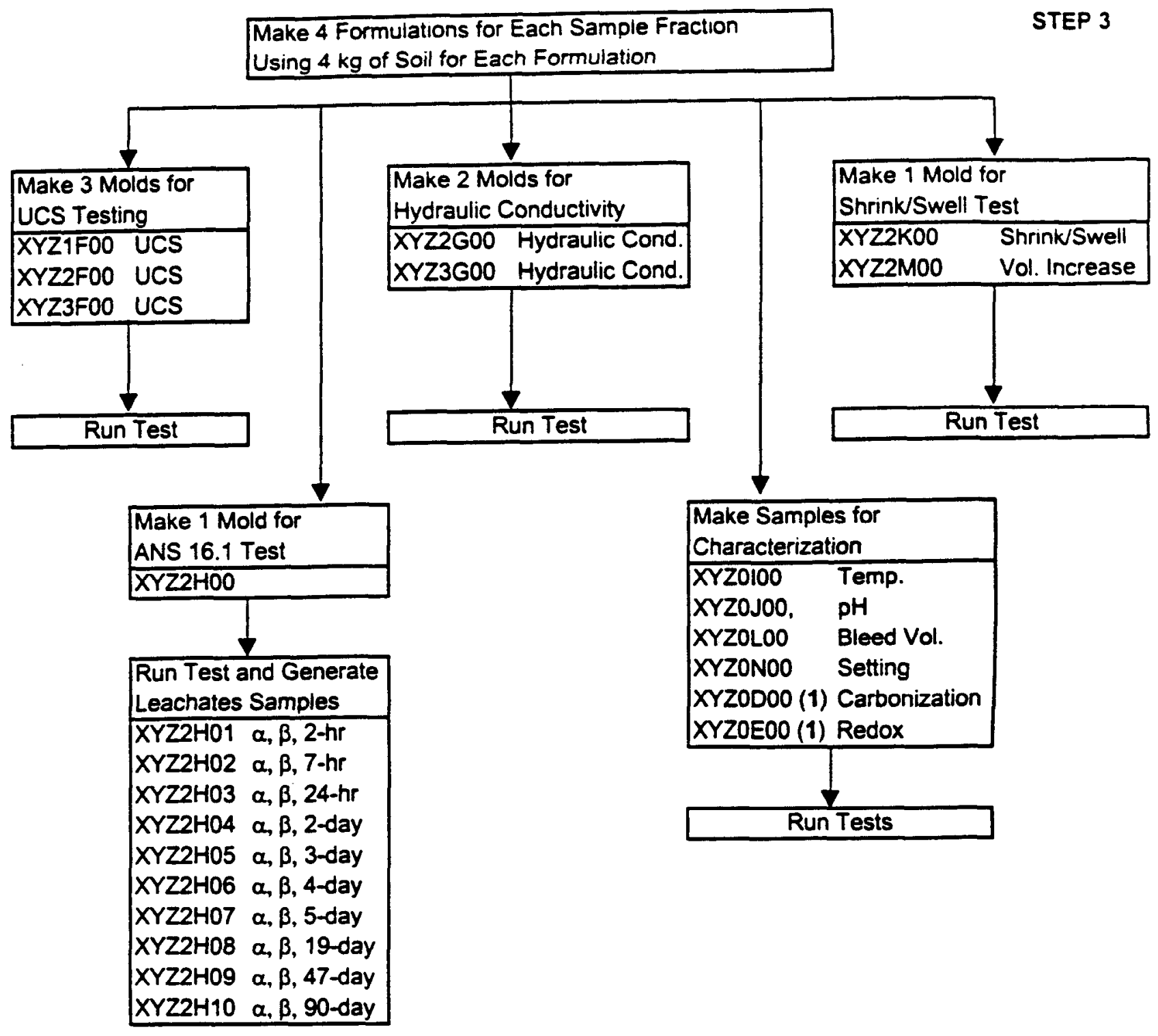

(1) Only on two randomly selected formulations

$X=C, K, L$, or $P$ (Reactor)

$Y=3,4,5$, or 6 (Subsample)

$Z=1,2,3$, or 4 (Formulation)

UCS = Unconfined Compressive Strength

Figure 6-2. Flowchart for Formulation Testing on Reactor Seepage Basin Soils 
Table 6-1 Samples Generated During Treatability Testing of CRSB Soil Samples

\begin{tabular}{|c|c|c|c|c|c|}
\hline \multirow[t]{2}{*}{ Testing Parameters } & \multirow[t]{2}{*}{ Method } & \multicolumn{4}{|c|}{ Number of Tests } \\
\hline & & Total & Step 1 & Step 2 & Step 3 \\
\hline Aterberg Limits & ASTM D 4318 & 8 & 2 & 6 & \\
\hline Grain Size Distribution & ASTM D422 & 8 & 2 & 6 & \\
\hline Moisture Content & ASTM D2216 & 8 & 2 & 6 & \\
\hline Specific Gravity & ASTM D854 & 8 & 2 & 6 & \\
\hline USCS Soil Classification & ASTM D2487 & 8 & 2 & 6 & \\
\hline Cesium-137 & Gamma Spectroscopy & 4 & & 4 & \\
\hline Plutonium 239,240 & Alpha Spectroscopy & 4 & & 4 & \\
\hline Strontium 90 & Gas Flow & 4 & & 4 & \\
\hline Gross Alpha (For ANS 16.1) & Gas Flow & 164 & & 4 & 160 \\
\hline Nonvolarile Beta (For ANS 16.1) & Gas Flow & 164 & & 4 & 160 \\
\hline Leachability Index & ANS 16.1 & 16 & & & 16 \\
\hline Total Boric Compounds & TBD & 1 & 1 & & \\
\hline Total Carbonation Activity & TBD & 2 & 1 & & 1 \\
\hline Chloride Compounds & SW-846 Method 9056 & 1 & 1 & & \\
\hline Nitrate & SW-846 Method 9056 & 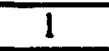 & 1 & & \\
\hline Nitric Compounds & TBD & 1 & 1 & & \\
\hline Nitrite & ASTM D9056 & 1 & 1 & & \\
\hline Oxidation/Reduction State & ASTM D1498 & 2 & $T$ & & 1 \\
\hline Sulfate & SW-846 Method 9056 & 1 & $I$ & & \\
\hline Sulfide & SW-846 Method 9034 & 1 & $I$ & & \\
\hline UCS@14 days cure & ASTM D2166 & 16 & & & 16 \\
\hline UCS @ 28 days cure & ASTM D2166 & 16 & & & 16 \\
\hline UCS @ 90 days immersion & ASTM D2166 & 16 & & & 16 \\
\hline Hydraulic Conductivity@28 days & ASTM D5084 & 16 & & & 16 \\
\hline $\begin{array}{l}\text { Hydraulic Conductivity @ } 90 \text { days } \\
\text { immersion }\end{array}$ & ASTM D5084 & 16 & & & 16 \\
\hline Temperanure & ASTM C1064 & 16 & & & 16 \\
\hline $\mathrm{pH}$ & SW-846 Method 9045C & 16 & & & 16 \\
\hline Shrinkage/Swell & ASTM D3877 & 16 & & & 16 \\
\hline Bleed Fluid & SW-846 Method 9095 & 16 & & & 16 \\
\hline Set Time & ASTM C191 & 16 & & & 16 \\
\hline
\end{tabular}




\section{Table 6-2 Samples Generated During Treatability Testing of KRSB Soil Samples}

\begin{tabular}{|c|c|c|c|c|c|}
\hline \multirow{2}{*}{ Testing Parameters } & \multirow[t]{2}{*}{ Method } & \multicolumn{4}{|c|}{ Number of Tests } \\
\hline & & Total & Step 1 & Step 2 & Step 3 \\
\hline Atterberg Limits & ASTM D 4318 & 6 & 2 & 4 & \\
\hline Grain Size Distribution & ASTM D422 & 6 & 2 & 4 & \\
\hline Moisture Content & ASTM D2216 & 6 & 2 & 4 & \\
\hline Specific Gravity & ASTM D854 & 6 & 2 & 4 & \\
\hline USCS Soil Classification & ASTM D2487 & 6 & 2 & 4 & \\
\hline Cesium-137 & Gamma Spectroscopy & 2 & & 2 & \\
\hline Plutonium 239,240 & Alpha Spectroscopy & 2 & & 2 & \\
\hline Strontium 90 & Gas Flow & 2 & & 2 & \\
\hline Gross Alpha (For ANS 16.1) & Gas Flow & 82 & & 2 & 80 \\
\hline Nonvolatile Beta (For ANS 16.1) & Gas Flow & 82 & & 2 & 80 \\
\hline Leachability Index & ANS 16.1 & 8 & & & 8 \\
\hline Total Boric Compounds & IBD & 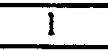 & 1 & & \\
\hline Total Carbonation Activity & TBD & 2 & $\mathrm{~T}$ & & 1 \\
\hline Chloride Compounds & SW-846 Method 9056 & I & 1 & & \\
\hline Nitrate & SW-846 Method 9056 & $T$ & 1 & & \\
\hline Nitric Compounds & TBD & $T$ & 1 & & \\
\hline Nitrite & ASTM D9056 & $T$ & $T$ & & \\
\hline Oxidation/Reduction State & ASTM D1498 & 2 & 1 & & 1 \\
\hline Sulfate & SW-846 Method 9056 & $T$ & 1 & & \\
\hline Sulfide & SW-846 Method 9034 & 1 & 1 & & \\
\hline UCS @ 14 days cure & ASTM D2166 & 8 & & & 8 \\
\hline UCS @ 28 days cure & ASTM D2166 & 8 & & & 8 \\
\hline UCS @ 90 days immersion & ASTM D2166 & 8 & & & 8 \\
\hline Hydraulic Conductivity @ 28 days & ASTM DS084 & 8 & & & 8 \\
\hline $\begin{array}{l}\text { Hydraulic Conductivity @ } 90 \text { days } \\
\text { immersion }\end{array}$ & ASTM D5084 & 8 & & & 8 \\
\hline Temperature & ASTM C1064 & 8 & & & 8 \\
\hline $\mathrm{pH}$ & SW-846 Methad 9045C & 8 & & & 8 \\
\hline Shrinkage/Swell & ASTM D3877 & 8 & & & 8 \\
\hline Bleed Fluid & SW-846 Method 9095 & 8 & & & 8 \\
\hline Set Time & ASTM C191 & 8 & & & 8 \\
\hline
\end{tabular}


Table 6-3 Samples Generated During Treatability Testing of LRSB Soil Samples

\begin{tabular}{|c|c|c|c|c|c|}
\hline \multirow[t]{2}{*}{ Testing Parameters } & \multirow[t]{2}{*}{ Method } & \multicolumn{4}{|c|}{ Number of Tests } \\
\hline & & Total & Step 1 & Step 2 & Step 3 \\
\hline Atterberg Limits & ASTM D 4318 & 8 & 2 & 6 & \\
\hline Grain Size Distribution & ASTM D422 & 8 & 2 & 6 & \\
\hline Moisture Content & ASTM D2216 & 8 & 2 & 6 & \\
\hline Specific Gravity & ASTM D854 & 8 & 2 & 6 & \\
\hline USCS Soil Classification & ASTM D2487 & 8 & 2 & 6 & \\
\hline Cesium-137 & Gamma Spectroscopy & 4 & & 4 & \\
\hline Plutonium 239,240 & Alpha Spectroscopy & 4 & & 4 & \\
\hline Strontium 90 & Gas Flow & 4 & & 4 & \\
\hline Gross Alpha (For ANS 16.1) & Gas Flow & 164 & & 4 & 160 \\
\hline Nonvolatile Beta (For ANS 16.1) & Gas Flow & 164 & & 4 & 160 \\
\hline Leachability Index & ANS 16.1 & 16 & & & 16 \\
\hline Total Boric Compounds & TBD & 1 & 1 & & \\
\hline Total Carbonation Activity & TBD & 2 & 1 & & 1 \\
\hline Chloride Compounds & SW-846 Method 9056 & 1 & 1 & & \\
\hline Nitrate & SW-846 Method 9056 & 1 & $\mathrm{~T}$ & & \\
\hline Nitric Compounds & TBD & 1 & 1 & & \\
\hline Nitrite & ASTM D9056 & 1 & 1 & & \\
\hline Oxidation/Reduction State & ASTM DI498 & 2 & 1 & & 1 \\
\hline Sulfate & SW-846 Method 9056 & 1 & 1 & & \\
\hline Sulfide & SW-846 Method 9034 & 1 & 1 & & \\
\hline UCS @ 14 days cure & ASTM D2166 & 16 & & & 16 \\
\hline UCS@ 28 days cure & ASTM D2166 & 16 & & & 16 \\
\hline UCS@90 days immersion & ASTM D2166 & 16 & & & 16 \\
\hline Hydraulic Conductivity@28 days & ASTM DS084 & 16 & & & 16 \\
\hline $\begin{array}{l}\text { Hydraulic Conductivity @ } 90 \text { days } \\
\text { immersion }\end{array}$ & ASTM D5084 & 16 & & & 16 \\
\hline Temperature & ASTM Cl064 & 16 & & & 16 \\
\hline $\mathrm{pH}$ & SW-846 Method $9045 \mathrm{C}$ & 16 & & & 16 \\
\hline Shrinkage/Swell & ASTM D3877 & 16 & & & 16 \\
\hline Bleed Fluid & SW-846 Method 9095 & 16 & & & 16 \\
\hline Set Time & ASTM CI91 & 16 & & & 16 \\
\hline
\end{tabular}


Soil Stabilization/Solidification Grout Mix Design Study for the

\section{Table 6-4 Samples Generated During Treatability Testing of PRSB Soil Samples}

\begin{tabular}{|l|l|c|c|c|c|}
\hline \multirow{2}{*}{ Testing Parameters } & \multirow{2}{*}{ Method } & \multicolumn{3}{|c|}{ Number of Tests } \\
\cline { 2 - 6 } & & & & & \\
& & Total & Step 1 & Step 2 & Step 3 \\
\hline Atterberg Limits & ASTM D 4318 & 6 & 2 & 4 & \\
\hline Grain Size Distribution & ASTM D422 & 6 & 2 & 4 & \\
\hline Moisture Content & ASTM D2216 & 6 & 2 & 4 & \\
\hline Specific Gravity & ASTM D854 & 6 & 2 & 4 & \\
\hline USCS Soil Classification & ASTM D2487 & 6 & 2 & 4 & \\
\hline Cesium-137 & Gamma Spectroscopy & 2 & & 2 & \\
\hline Plutonium 239,240 & Alpha Spectroscopy & 2 & & 2 & \\
\hline Strontium 90 & Gas Flow & 2 & & 2 & \\
\hline Gross Alpha (For ANS 16.1) & Gas Flow & 82 & & 2 & 80 \\
\hline Nonvolatile Beta (For ANS 16.1) & Gas Flow & 82 & & 2 & 80 \\
\hline Leachability Index & ANS 16.1 & 8 & & & 8 \\
\hline Total Boric Compounds & TBD & 1 & 1 & & \\
\hline Total Carbonation Activity & IBD & 2 & 1 & & 1 \\
\hline Chloride Compounds & SW-846 Method 9056 & 1 & 1 & & \\
\hline Nitrate & SW-846 Method 9056 & 1 & 1 & & \\
\hline Nitric Compounds & TBD & 1 & 1 & & \\
\hline Nitrite & ASTM D9056 & 1 & 1 & & \\
\hline Oxidation/Reduction State & ASTM D1498 & 2 & 1 & & 1 \\
\hline Sulfate & SW-846 Method 9056 & 1 & 1 & & \\
\hline Sulfide & SW-846 Method 9034 & 1 & 1 & & \\
\hline UCS @ 14 days cure & ASTM D2166 & 8 & & & 8 \\
\hline UCS @ 28 days cure & ASTM D2166 & 8 & & & 8 \\
\hline USC @ 90 days immersion & ASTM D2166 & 8 & & & 8 \\
\hline Hydraulic Conductivity @ 28 days & ASTM D5084 & 8 & & & 8 \\
\hline Hydraulic Conductivity @ 90 days & ASTM D5084 & 8 & & & 8 \\
\hline immersion & & & & & \\
\hline Temperanure & ASTM C1064 & 8 & & & 8 \\
\hline pH & SW-846 Method 9045C & 8 & & & 8 \\
\hline Shrinkage/Swell & ASTM D38;7 & 8 & & & 8 \\
\hline Bleed Fluid & SW-846 Method 9095 & 8 & & & 8 \\
\hline Set Time & ASTM C191 & 8 & & & 8 \\
\hline & & & & \\
\hline
\end{tabular}




\subsection{DATA MANAGEMENT AND QUALITY ASSURANCE}

The treatability testing for the CRSB, KRSB, LRSB, and PRSB soils will be conducted under IT's Technical Application Laboratories Quality Assurance (QA) program, as outlined in it's Quality Assurance Manual (Appendix B). Data management for the treatability testing will include control of data, review of analytical and testing results, and manual compilation. organization and entry of data into Excel spreadsheets for presentation and analysis.

\subsection{Control Of Data}

Sampling logs and laboratory notebooks will be controlled as per the laboratory SOP's TDL1503, "Analytical Logbook Recording Procedures," and TDLI504, "Laboratory Notebook Recording Procedures."

All data collected, including suppliet/manufacturet information, data collection forms/sheets and laboratory analytical reports, will be sent to the project file. The project manager will have control of the project file.

\subsection{Data Review And Verification}

Data obtained from sampling logs, laboratory notebooks, data collection forms/sheets and testing laboratories will be reviewed for correctness and reasonableness prior to use. Data from commercial laboratories will also be reviewed with respect to laboratory internal Quality Control (QC) results to assess quality of data to assure data quality objectives are met and determine any data qualification needs.

One hundred percent of data transcriptions will be checked.

All calculations performed by software will be verified independently, either by hand or by entering data of known result into the software.

\subsection{Data Reporting}

Data collected for each formulation will be grouped and tabulated for summary presentation and examination.

Key process data from the formulations will also be tabulated for summary presentation, examination and overall process variable/performance determination. The final stabilized waste 
characteristics for the formulations will be tabulated for summary presentation. examination and overall process waste characteristics determination.

\subsection{Data Evaluation}

Data obtained from sampling logs, laboratory notebooks, data collection formsisheets and testing laboratories will be reviewed for correctness and reasonableness prior to use. Results from commercial laboratories will also be reviewed with respect to laboratory internal QC results to assess quality of data to assure data quality objectives are met and determine any data qualification needs.

Formulation development results will be evaluated for consistency with expected trends with key variables.

Data from performance testing will be evaluated by comparing results of replicate formulations of the mixes listed in Table 1-1. Data is expected to be predictable, based on formulation development results, reproducible, and provide consistent results within a reasonable variation. Where possible, results from replicate formulations will be evaluated statistically to determine mean values and standard deviations.

\subsection{Data Interpretation}

Data interpretation will be performed by senior technical personnel on the project. Interpretation will be aided by the generation of performance indicators and variable relationships so that trends may be determined. Data trends are used to predict effects on performance due to changes in key variables or operating conditions. Treatment criteria for evaluation of formulations are listed in Table 2.1. Results are expected to be reproducible and provide consistent results within a reasonable variation. 


\subsection{HEALTH AND SAFETY}

The health and safety programs in place at the IT/TAL comply with the Occupational Safety and Health Administration (OSHA) standard for laboratories, 29 CFR 1910.1450, Occupational Exposures to Hazardous Chemicals in Laboratories and the Tennessee State Regulations for Protection Against Radiation. The following procedures and programs have been implemented in order to assure compliance with these regulatory requirements.

\subsection{Project Description And Planning}

The Project Description and Planning Summary Forms are used to document project-specific requirements, including health and safety requirements, and communicate those requirements to project staff. One of the forms, the Project Health and Safety Summary (PHSS), describes sample and equipment hazards, procedural requirements, and personal protective equipment (PPE) requirements. The PHSS will specify the need for keypoint cards, system reviews, work permits, and/or license amendments. The PHSS is approved by the Health and Safety Professional.

\subsection{Chemical Hygiene Plan}

Laboratory operations at the IT/TAL laboratories are conducted under the requirements of the facility Chemical Hygiene Plan (CHP). The CHP includes the necessary work practices, procedures and policies to ensure that employees are protected from all potentially hazardous chemicals in use in their work area. The CHP, supplied in Appendix A, describes basic safe work practices, training and hazard communication requirements, procedures for work with regulated chemicals, the use of engineering controls and personal protective equipment, emergency procedures, medical surveillance, inspections and record keeping requirements. The CHP serves as the umbrella under which routine laboratory operations are conducted. Health and safety aspects of projects involving unique or higher hazard situations may be addressed in projectspecific health and safety plans (HASP).

\subsection{Key Point Cards}

Key Point Cards are used to document equipment hazards, emergency shut down procedures and procedure or project specific hazards. The Key Point Card is the primary safety checklist for all TDL laboratory activities. It must be completed prior to performing any treatability testing or 
analytical procedure. The complete Key Point Card is posted in the work area where the actuvity. will occur.

\subsection{Bench-Scale Experimental Review}

A safety review is required before initiating work with new and/or unfamiliar chemicals and reaction setups. This review is performed with the supervisor and other key personnel who are familiar with the type of experiment. A Bench-Scale Experimental Review Checklist form is be reviewed with the appropriate supervisor.

\subsection{Radiation Protection Plan}

Both the ETDC and the TDL are licensed by the Tennessee Division of Radiological Health (TDRH) to perform analytical procedures and treatability testing on radioactively contaminated materials. These activities are performed in accordance with the state approved Radiation Protection Program (RPP). The elements of the RPP include training, exposure limits, monitoring and survey requirements, exposure control and radioactive material control.

As described above, the PHSS documents if the project involves radioactive materials and the associated requirements for receiving and working with the materials. The facility Radiation Safety Officer (RSO) will determine if a license amendment is required and will issue an Authorization Number for receipt of the materials once it is verified that the samples meet license limits. Samples are screened and classified upon receipt to assess radiological hazards and to verify sample activity. The RSO specifies work requirements including the need for a Radiation Work Permit (RWP), special monitoring, project-specific training, and PPE. Pilot scale operations involving radioactive materials are reviewed and approved by the Radiation Safety Committee (RSC).

In addition to project specific requirements, routine radiological surveys and personnel monitoring are performed to verify the effectiveness of radiation hazard control measures. 


\subsection{SCHEDULE}

The project has several milestones associated with completion of tasks. The project tasks with estimated times for completion are listed in the Table 9-1.

When the initial Phase I performance testing results are available for the S/S treated CRSB and KRSB soils, a briefing package will be produced, summarizing the data and its significance toward the leachability, durability, hydraulic conductivity, strength, and long-term durability of the S/S treated material. A workshop covering this briefing package will be scheduled at the SRS.

At the completion of Phase I of the S/S treatability testing, a report will be prepared detailing observations and procedures from the treatability study, and the results of the performance tests. This report will include specific recommendations concerning full-scale treatment of the soils. The report will include, but is not limited to the following sections:

\section{Project Description}

- Introduction

- Waste units description

- Soil characteristics

\section{Study Objectives}

- Objectives

- Performance criteria

\section{Study Approach}

- Soil characterization

- Grout mix design

- Formulation development

- Performance testing

- Deviations from the work plan

Data Analysis and Interpretation

- Soil characterization 
- Grout mix design/formulation development

- Performance testing

\section{- Full-Scale Delivery Systems}

- Reagents

- Delivery systems

Conclusions and Recommendations

- Summary of findings

- Recommendations

At the completion of Phase II of the S/S treatability testing, the final report will be revised and reissued to include the Phase II results, conclusions, and recommendations. 
Table 9-1 Schedule for S/S Treatability Testing on the Reactor Seepage Basin Soils

\begin{tabular}{|l|c|}
\hline \multicolumn{1}{|c|}{ Schedule Milestone } & Date \\
\hline $\begin{array}{l}\text { Receipt of CRSB, KRSB, LRSB, and PRSB Samples } \\
\text { From SRS }\end{array}$ & November 4, 1998 \\
\hline Initiation of Phase I Treatability Testing & November 8, 1998 \\
\hline Submittal of Draft Phase I Briefing Package to WSRC & December 21, 1998 \\
\hline Submittal of Final Phase I Briefing Package to WSRC & January 11,1999 \\
\hline Submittal of Draft Phase I Report To WSRC & February 2, 1999 \\
\hline Initiation of Phase II Treatability Testing & February 8, 1999 \\
\hline Submittal of Final Phase I Report to WSRC & March 21, 1999 \\
\hline Submittal of Draft Phase II Report to WSRC & May 10, 1999 \\
\hline Submittal of Final Phase II Report to WSRC & July 5, 1999 \\
\hline
\end{tabular}




\subsection{RESIDUAL WASTE MANAGEMENT}

This project involves treatability testing of soil from two Reactor Seepage Basins. provided to IT. When testing is completed, all residuals will be containerized and transponed back to the SRS.

IT's Waste Management program was developed to ensure that throughout the stabilization demonstration, waste minimization technologies are utilized to the fullest and that residuals generated at the end of the demonstration are properly managed. In addition, the program ensures that the treatability testing is conducted in full compliance with the regulations of the Tennessee Division of Solid Waste Management and the TDRH.

The elements of this plan include:

- Responsibilities and training

- Sample management and storage

Waste Minimization

- Estimated residual quantities

- Residuals characterization and classification

- Residual storage and disposal

- Effluents

- Emergency response and spill management.

\subsection{RESPONSIBILITY AND TRAINING}

IT's Regulatory Compliance Specialist (RCS) will oversee the receipt, on-site management and return of the soil samples and residuals for both the TDL and ETDC, thus ensuring full accountability for the sample between the bench-scale and pilot-scale efforts. Employees of the TDL and the ETDC have received facility-specific training in Resource Conservation and Recovery Act (RCRA) hazardous waste compliance, radioactive materials handling, and U.S. DOT hazardous materials general awareness. 


\subsection{SAMPLE MANAGEMENT AND STORAGE}

The soil samples will be received at the ETDC in Oak Ridge directly from SRS. The containers containing the sample material will be stored in "Isolator" drums as a means of secondary containment and will be kept in Room 1. Shortly after receipt, the samples will be sampled for geotechnical testing and the remaining soil homogenized per the Work Plan. Portions of the untreated soil material will be transported by IT vehicle with chain-of-custody documentation and appropriate US DOT labeling and packaging to the TDL in Knoxville for bench-scale testing. All samples will be returned from the TDL to the ETDC in a similar manner after this phase of the study is complete. No samples will be retained at the TDL. Transfers will be coordinated, prepared, recorded, and supervised by the RCS. Liquids will be held in secondary containment capable of holding 100 percent of their volume to prohibit the spread of contamination in the event of a spill. See Section 10.8, Emergency Response and Spill Management, for more details.

\subsection{WASTE MINIMIZATION}

The ETDC Waste Minimization Policy is " . . to reduce all radioactive, hazardous, and nonhazardous wastes to the minimum levels technically and economically practical while maintaining full compliance with all federal and state waste regulations." ETDC's Waste Minimization Policy is driven by management's awareness of IT's environmental responsibility, public image, long-term liability, and waste management costs. The policy is implemented by the concerted efforts of the IT technical staff. IT has demonstrated that performing initial benchscale treatability tests with reduced sample quantities, utilizing effective nonhazardous chemical substitutes whenever possible, and considering beneficial reuse or reclamation options are all effective means to minimize the quantity of hazardous and radioactive wastes generated. These and other waste minimization techniques have been incorporated into the Work Plan to eliminate hazardous wastes and reduce radioactive waste volume.

Because much of waste minimization is simply increased efficiency, several IT preliminary procedures ensure that the pilot-scale treatment testing is performed as efficiently as possible. A fraction of the sample will first be treated in a bench-scale system similar to the pilot-scale system. These bench scale results will be used to refine the operating parameters of the IT pilot system. Before sample is introduced into the pilot system, wipe samples are taken and inspection of the equipment is performed to certify that the system is free of prior contaminants. 


\subsection{ESTIMATED RESIDUAL QUANTITIES}

Previous project experience enables IT to estimate the total volume and types of residuals expected from testing these samples. Based on information received from SAIC and WSRC and the IT Work Plan, the volume of unused soil, treating residuals, and laboratory generated waste will be approximately 40 gallons per reactor basin.

\subsection{RESIDUAL CHARACTERIZATION AND CLASSIFICATION}

Since all of the unused samples and residuals will be returned to the SRS, there will be no need to characterize and classify the residuals, other than for correct and proper shipping. The RCS will classify the residuals based on data provided by WSRC, process knowledge, and posttreatment analyses.

\subsection{RESIDUALS STORAGE AND DISPOSAL}

All residuals will be properly containerized in appropriate shipping containers, segregated, and accumulated in the ETDC. The residuals will be shipped back to SRS via a licensed shipper. Eventual disposition will be the responsibility of WSRC.

\subsection{EFFLUENTS}

Emissions of radioactive materials in effluents from the ETDC are regulated by the TDRH and the Oak Ridge Public Works. No radioactive liquids are discharged from the facility to the ground or sewer. Gaseous emissions are discharged following treatment through vents that are continuously sampled for radioactive materials to ensure compliance with the Tennessee SRPAR.

Based on Tennessee Air Pollution Control Regulations and on ETDC's off-gas treatment system, no air discharge permit or associated monitoring is required.

\subsection{EMERGENCY RESPONSE AND SPILL MANAGEMENT}

The TDL and the ETDC have a current Emergency Action and Fire Prevention Plan that is designed to adequately cover projects such as this demonstration. This plan is to be implemented in the case of any emergency or spill at the TDL or the ETDC that may adversely affect human health or the environment. Preparedness or prevention as well as emergency response provisions are addressed in these emergency plans in the event of a fire/ explosion, spill/release, or natural 
disaster. State and local agencies requested to provide emergency assistance are listed with their phone numbers in the Plan, and these names and numbers are posted adjacent to facilit: telephones. Both TDL and ETDC personnel are currently trained and familiar with the procedures of the Plan. IT's project-specific HSP identifies potential hazards associated with the project, thereby increasing the worker's preparedness and prevention awareness. Trained Primary and Alternate Emergency Coordinators have been assigned for 24-hour response, and emergency response equipment is readily accessible in all work areas. 


\subsection{REFERENCES}

WSRC. 1992. Phase II RCRA Facilin' Investigation'Remedial Investigation Plan for the P-Alica - Burning/Rubble Pit (13I-P) U). WSRC-RP-91-1123, Rev. 1, Westinghouse Savannah River Company, Savannah River Site, Aiken, SC 29808.

WSRC. 1996. Laboratory-Scale Immobilization Study Report for the L-Area Oil and Chemical Basin 904-83G (U). WSRC-RP-95-15, Rev. 0, Westinghouse Savannah River Company, Savannah River Site, Aiken, SC 29808.

WSRC. 1997. Remedial Investigation Report and Baseline Risk Assessment for the K-Reactor Seepage Basins (U). WSRC-RP-96-871, Rev. 1, Westinghouse Savannah River Company, Savannah River Site, Aiken, SC 29808.

WSRC. 1998. ASCADTM RI Work Plan for the C-Reactor Seepage Basins (904-66G, 904-67G, and 904-68G) (U). WSRC-RP-97-431, Rev. 1.1, Westinghouse Savannah River Company, Savannah River Site, Aiken, SC 29808. 
Soil Stabilization/Solidification Grout Mix Design Study WSRC-RP-99-4075, Revision 1

C-, K-, L-, and P-Reactor Seepage Basins Closure (U) August 1999

\section{Appendix E}

Analytical Methods 
This page intentionally left blank. 


\section{PROCESS DEVELOPMENT GROUP}

\section{Standard Operating Procedure}

Title:

Prepared by:

Reviewed by:

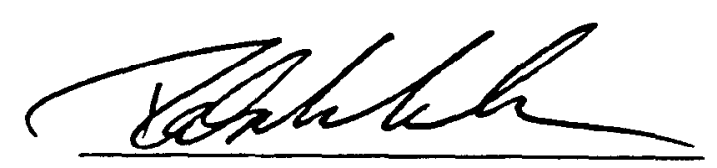

Operations approval:

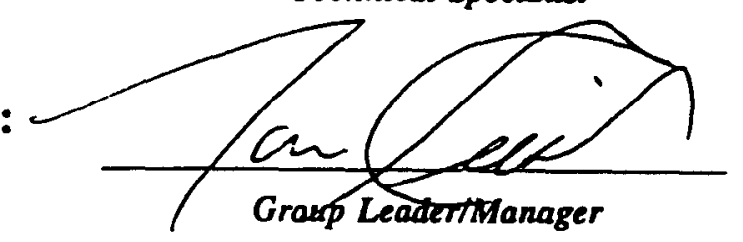

Interim approval:

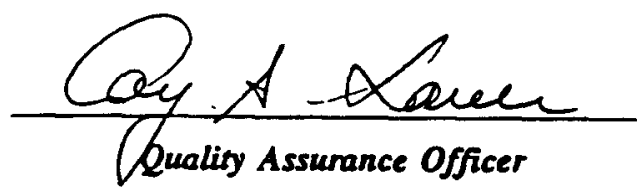

Technical Specialist

\section{Particle-Size Distribution of Soils}

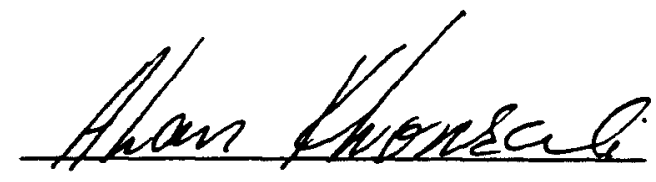

Date:

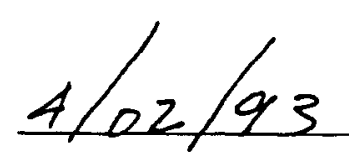

Date: $\quad 1-05-83$

Date:

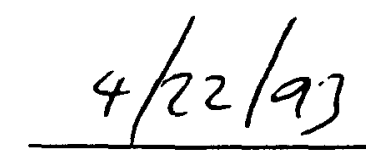

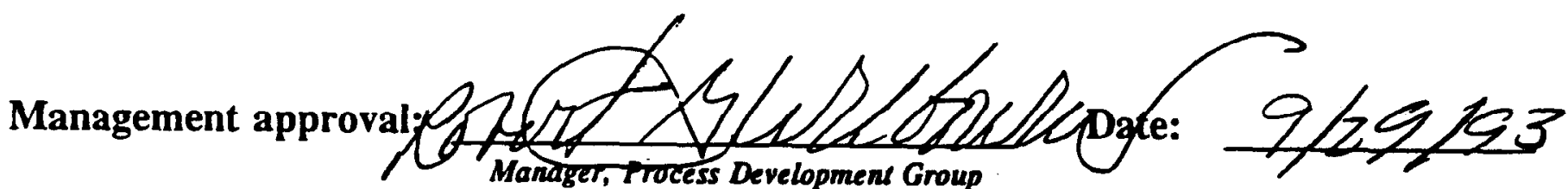

\section{APPROVED FOR INTERIM USE}

\section{CONTROLLED COPY NO.}


SOP NO. PDEGEOOO2O

REVISION NO. 00

DATE: SeP -. 1993

PAGE 2 OF 19

Particle-Size Distribution of Soils

1.0 Scope and objectives

1.1 This procedure covers the determination of the particle size distribution of soils and is in general accordance with ASTM D 422 .

1.2 This procedure covers two methods for quantifying particle sizes. For particles coarser than the No.200 $(75 \mathrm{\mu m})$ sieve, the particle size is determined by sieving; for particles finer than the No.200 sieve, the particle size distribution is calculated from hydrometer data.

1.3 This method is a necessary index test for soils, for it presents the relative proportions of different sizes of particles. From this test it is possible to determine the size ranges likely to control the engineering properties of the soil.

1.4 Values stated in SI units shall be regarded as standard except as noted.

2.0 Definitions

2.1 ASTM - American Society of Testing and Materials

2.2 Coarse Fraction - The portion of the soil specimen retained on the No.10 sieve.

2.3 Composite Analysis - A particle size test combining the sieve and hydrometer (sedimentation) analyses.

2.4 Fine Fraction - The portion of the soil specimen finer than the No.10 sieve.

2.5 Hydrometer Analysis - Same as sedimentation analysis. The portion of the test procedure covering the silt and clay sample fraction.

2.6 Hygroscopic Moisture - The amount of water, expressed as a percentage, not removed from a soil specimen by air drying, or by oven drying at a temperature less than 110 $\pm 5^{\circ} \mathrm{C}$. 
SOP NO. PDGGEOO 220

REVISION NO. 00

DATE: Sept. 1393

PAGE 3 OF 19

3.0 Methodology

3.1 Summary - The sieve and hydrometer portions of this method combine to cover a range of particle sizes from 3.5 inch diameter cobbles to clays in the 1 micron range. Sieving is performed on the portion of the test specimen coarser than the No. 200 sieve. Particles are separated into different size ranges using a series of sieves with standard openings. A sedimentation procedure is used for the silt and clay portion of the specimen. The density of the suspension is determined using a soil hydrometer and the readings are converted to particle size. Final results are customarily presented graphically as cumulative percent finer than diameters.

3.2 Interferences or Potential Problem Areas

3.2.1 Sample portions prepared for sedimentation analysis normally require chemical dispersion prior to mechanical dispersion. Sodium hexametaphosphate solution is the most common dispersion agent. Solutions of this salt, if acidic, will gradually revert or hydrolyze to the orthophosphate form resulting in decreased dispersive action. Fresh dispersant solution shall be prepared, or the solution $\mathrm{pH}$ adjusted to 8 or 9 using sodium carbonate, at least every 30 days.

3.2.2 The hydrometer readings recorded in the sedimentation analysis are an indirect measurement of the quantity of particles in suspension and are subject to errors due to turbidity, density currents, and soluble minerals contained in the soil sample. Results determined after the 5 minute reading are normally reliable. However, other factors influence the settling velocities of particles finer than 1 micron, nullifying several of the assumptions made by stoke's Law. Results below the 1 micron size interval may not be valid.

3.2.3 Hydrometer specimens containing organic debris frequently foam excessively during mechanical agitation. Some foams may have the consistency of shaving cream and be long lasting, hiding the portion of the hydrometer stem at which readings are taken. An Ivory soap sliver dipped quickly into the foam, or a few drops of isopropyl alcohol will usually hasten the dissipation of the foam. If these measures do not dissipate the 
SOP NO. PDGG:00020

foam quickly, it will be necessary to allow the foam to dissipate on its own, and hydrometer readings started when feasible. Write "foam" in any data entry blocks where readings were not obtained due to excessive foam.

3.3 Sample Requirements, Handling, and Preservation

All samples submitted for analysis must be of sufficient volume as to allow ample material for index tests and multiple specimen preparation.

3.3.2 Samples submitted for analysis should be sufficiently large as to yield quantities of material retained on the No.10 sieve according to the following table.

\begin{tabular}{||cc|c|}
\hline \begin{tabular}{|c|} 
Nominal Dia. of Largest \\
Particles, in. (mm)
\end{tabular} & $\begin{array}{l}\text { Approximate } \\
\text { Minimum Mass of } \\
\text { Portion. o. }\end{array}$ \\
\hline $3 / 8$ & $(9.5)$ & 500 \\
\hline $3 / 4$ & $(19.0)$ & 1000 \\
\hline 1 & $(25.4)$ & 2000 \\
\hline $11 / 2$ & $(38.1)$ & 3000 \\
\hline 2 & $(50.8)$ & 4000 \\
\hline 3 & $(76.2)$ & 5000 \\
\hline
\end{tabular}

3.3.3 The portion passing the No.10 sieve shall weigh at least 120 grams for sandy soils and at least 60 grams for silty and clayey soils.

3.3.4 Separation of the soil particles may be made using the No.4, the No.40, or the No. 200 sieve instead of the No.10 sieve as required by material or client specifications.

3.3.5 Specimens shall be prepared from representative samples as soon as practicable after sample receipt.

3.3.6 Samples may be sealed against moisture loss and stored for up to six months in an area not subject to temperature extremes (lower than $1^{\circ} \mathrm{C}$ or higher than $35^{\circ} \mathrm{C}$ ). 
SOP NO. PDGGEOOO2O

REVI IION NO.

DATE.

Sept. 1993

PAGE

5 OF 19

\subsection{Required Equipment and Reagents}

3.4.1 Balance - A mechanical or electronic balance accurate to 0.02 grams or $0.1 \%$, whichever is greater. Readability shall be to 0.01 grams for weights below $1 \mathrm{~kg}$, to 0.1 gram for weights between $1 \mathrm{~kg}$ and $4 \mathrm{~kg}$, and to 1 gram for weights above $4 \mathrm{~kg}$.

3.4.2 Stirring Apparatus - A mechanically operated stirring device in which an electric motor turns a vertical shaft at a speed of not less than 10,000 rpm without load. The shaft shall be equipped with a replaceable stirring paddle made of metal, plastic, or hard rubber. The shaft shall be of such length that the stirring paddle will operate not less than $\frac{1}{4}$ inch nor more than $1 \frac{1}{2}$ inches above the bottom of the dispersion cup. A special dispersion cup equipped with four opposing sets of wire baffles shall be provided to hold the sample being dispersed.

3.4.3 Drying oven - An oven, either gravity or forced convection, capable of maintaining an internal temperature of $110 \pm 5 \circ \mathrm{C}$.

3.4.4 Thermometer - A thermometer capable of measuring temperatures over the range prevailing during the hydrometer test shall be used. Thermometers shall be graduated in $0.5^{\circ} \mathrm{C}$ divisions and be accurate to $\pm 1^{\circ} \mathrm{C}$.

3.4.5 Hydrometer - An ASTM soil hydrometer, graduated to read in either specific gravity or grams per liter of suspension. The hydrometer shall conform to the requirements of ASTM E 100 for type $151 \mathrm{H}$ or $152 \mathrm{H}$. Dimensions of both types are the same; the scales, however, differ.

3.4.6 Sedimentation Cylinder - A volumetric glass cylinder approximately 18 inches in height and $2 \frac{1}{2}$ inches in diameter. The cylinder shall have a volume of $1000 \mathrm{ml}$ with an inside diameter such that the $1000 \mathrm{ml}$ mark is $36 \pm 2 \mathrm{~cm}$ from the inside bottom.

3.4.7 Sieves - A series of square-mesh woven-wire cloth sieves conforming to the requirements of ASTM E 11. 
3.4.8 Water Bath or constant Temperature Room - A water bath or constant temperature room for maintaining the soil suspension at a constant temperature during the hydrometer analysis. An insulated tank capable of maintaining the temperature of the suspension at a constant temperature at or near $20^{\circ} \mathrm{C}$ is satisfactory. The hydrometer analysis may be performed in a room of controlled temperature.

3.4.9 Mortar and Rubber-Covered Pestle - A large ceramic mortar and rubber-covered pestle or soil mill with rubber-covered bars (Hogentogler model s9131 or equivalent).

3.4.10 Sieve Shaker - A mechanical device designed to hold a stack of sieves and shake the stack in an orbital motion.

3.4.11 Dispersing Agent - A solution of sodium hexametaphosphate prepared by thoroughly mixing 40 grams of commercial grade sodium hexametaphosphate into sufficient deionized water to form 1 liter of solution.

3.4.12 Miscellaneous equipment - Miscellaneous equipment, including but not limited to, spatulas, aluminum pans, wash bottles, size 12 rubber stopper, etc.

3.5 Calibration Requirements

3.5.1 Balance calibrations shall be verified quarterly in accordance with SOP PDGADMO002.

3.5.2 Thermometer calibrations shall be verified annually in accordance with SOP PDGADMO008.

3.5.3 Sieves shall be maintained in accordance with SOP PDGGEO0023.

3.5.4 Sieve shakers shall be calibrated in accordance with SOP PDGGEO0032.

3.5.5 Hydrometer calibrations shall be verified in accordance with SOP PDGGEO0033.

3.6 Quality Control Requirements

3.6.1 Balances - Brass weights shall be used to verify the accuracy of each balance before use or 
daily, whichever is less, and the result recorded in the balance log book. Brass weights shall meet oIML Class $\boldsymbol{M}_{2}$ specifications or better and shall be checked quarterly in accordance with SOP PDGADMO003.

3.6.2 Ovens - Temperatures of ovens in use shall be recorded daily in accordance with SOP PDGADMO006.

3.6.3 Visually inspect the integrity of sieves before use. Ensure loose particles are dislodged from the wire mesh and that the number of blocked openings is not greater than $5 \%$ of the mesh area. No. 200 and finer sieves shall be replaced annually. Other sieves shall be replaced as required.

3.6.4 Nonconformance/variance reports - In accordance with SOP PDGADMO009, nonconformance/variance reports shall be used to document any discrepancies in the performance or specifications of this procedure.

3.7 Health and Safety - Sample material containing hazardous, radiological, or mixed contaminants shall be handled in accordance with current facility Health and Safety directives. Engineering controls shall be used whenever possible to reduce personnel exposure to sample hazards (i.e., sample prepared inside vented hood space, sample extruder enclosure adequately ventilated, etc.). Personal protective equipment shall be used as necessary to prevent personnel exposure to hazards. As a minimum, appropriate protective gloves shall be worn by personnel handiing contaminated sample material, in addition to safety glasses required of all personnel in the laboratory at all times. When in doubt as to the hazards involved in any operation, consult with the Geotechnical Laboratory Manager or Health and Safety officer before proceeding.

\subsection{Sample Preparation}

3.8.1 Air-dry the soil sample to be tested until friable. Alternatively, the sample may be oven dried at a temperature not exceeding $60^{\circ} \mathrm{C}$. Record the weight of the dry soil as "dry weight of soil". uncorrected for hygroscopic moisture.

3.8.2 Thoroughly disaggregate the dry soil lumps by grinding in a mortar with rubber-covered pestle 
DATE : Sept. 1993

PAGE

or soil mill with rubber-covered bars. Sieve the ground soil over a No.10 sieve. Regrind the portion retained on the sieve and pass over the sieve again. Take care not to lose any soil particles while handling.

3.8.3 Place the portion of the sample retained on the No. 10 sieve in a covered container and add sufficient water to cover the specimen.

3.8.4 Remove a portion of the material passing the No. 10 sieve weighing approximately 60 grams for clayey soils, to 120 grams for sandy soils. Record the weight of the soil removed as "weight of hydrometer specimen" uncorrected for hygroscopic moisture. Place the soil in a beaker of at least $250 \mathrm{ml}$ capacity and cover with approximately $120 \mathrm{ml}$ of the sodium hexametaphosphate solution. All hydrometer specimens to be run at the same time should contain the same amount of sodium hexametaphosphate solution. Allow to soak for at least 16 hours.

3.8.5 Determine the hygroscopic moisture content of the air-dried soil passing the No.10 sieve. Weigh a portion of the remaining soil of at least 15 grams, once the hydrometer portion has been weighed. This sample shall be dried in an oven at $110 \pm 5^{\circ} \mathrm{C}$ and the water content determined in accordance with SOP PDGGEO0019.

\subsection{Coarse Sieve Analysis}

3.9.1 After the material retained on the No.10 sieve has soaked overnight, wash the entire portion on a No. 10 sieve with a water spray to remove small particles adhering the coarse fraction. Retain and label the wash water for disposal.

3.9 .2 Oven dry the washed specimen in an oven at 110 $\pm 5^{\circ} \mathrm{C}$ to a constant weight.

3.9.3 Arrange a nest of sieves covering the sizes of the particles to be tested. Place the coarsest sieve on top and other sieves in decreasing order. Place a sieve pan on the bottom of the sieve stack. Pour the dry coarse fraction over a nest of sieves to separate the specimen into a series of fractions. Place a sieve cover on top of the top sieve. Separation of particles may be 
achieved using the 3 inch, 2 inch, 13inch, 1 inch, $\frac{3}{4}$ inch, * inch, No.4, and No.10 sieves. This arrangement may be modified as necessary to meet project specifications or the properties of the test sample.

3.9.4 Sieving of the sample shall be performed by means of a lateral and vertical motion of the sieve, followed by a jarring action. This will ensure continuous sample movement over the entire surface of the sieve. At no time shall the sample fragments be forced through the sieve, or manipulated in any way by hand. The sieving operation shall continue until no more than 1 mass $\&$ of the material retained on a sieve passes that sieve during 1 minute of sieving. When mechanical sieving is employed, a check on the accuracy of the sieving operation may be secured by following the practices covered in the hand method of sieving.

3.9.5 At the end of the sieving operation, successively remove and invert each sieve on a flat surface and brush the retained particles out of the sieve mesh. Weigh and record the weight of the particles retained on each sieve.

3.10.1 Hydrometer readings are based on distilled or demineralized water. The sodium hexametaphosphate dispersing solution added to the fine fraction results in a liquid having a specific gravity appreciably greater than that of distilled or demineralized water. The hydrometer correction measures the difference between the hydrometer reading in the dispersant and the reading in water for subtraction from data readings.

3.10 .2 Prepare a $1000 \mathrm{ml}$ solution of sodium hexametaphosphate by pouring $120 \mathrm{ml}$ of the 40 g/liter solution in a glass sedimentation cylinder. Add demineralized water until the contents reaches the $1000 \mathrm{ml}$ mark. Place a No.12 or No.13 rubber stopper over the open end of the cylinder and agitate by hand, turning the cylinder upside down and back several times. Place the cylinder in the constant-temperature water bath or in the area where the sedimentation tests will be performed. Once the 
temperature of the solution becomes constant, insert the hydrometer. Allow the temperature of the hydrometer to equilibrate and record the hydrometer reading as observed at the top of the meniscus formed on the stem. Record this reading as the hydrometer correction factor. For hydrometer type $151 \mathrm{H}$ the correction factor is the difference between this reading and one; for hydrometer type $152 \mathrm{H}$ the correction factor is the difference between the reading and zero.

\subsection{Sedimentation Analysis}

3.11 .1 Upon completion of the soaking period, thoroughly disperse the sample using the stirring apparatus. Transfer the beaker contents into the dispersion cup. Ensure that no residue remains in the beaker by flushing the contents using distilled or demineralized water. If necessary, add distilled or demineralized water to the dispersion cup such that it is approximately half full. Stir the soil-water slurry for a period of 1 minute.

3.11.2 Immediately transfer the soil/water mixture into a glass sedimentation cylinder. Add distilled or demineralized water to the $1000 \mathrm{ml}$ mark.

3.11.3 Block the open end of the glass cylinder using a rubber stopper. Agitate the mixture by turning the cylinder upside down and back for a period of 1 minute counting for approximately 60 turns. Each upside down-and-back motion counts as two turns.

3.11.4 After the cylinder containing the soil/water slurry has been shaken for 60 seconds, place the glass cylinder in a convenient location (in the water bath or on a lab bench not subject to vibration). Draw the bottom of the rubber stopper across the rim of the cylinder to scrape off particles adhering to the stopper. start the elapsed time at the end of the hand agitation period and record hydrometer readings at elapsed times of $0.5,1,2,5,15,30,60,250$, and 1440 minutes. Time intervals may be varied as needed, or as required to produce results corresponding to specific particle diameters.

3.11.5 Take hydrometer readings by inserting the hydrometer into the soil/water mixture 
SOP NO. PDGCEO0020

REVISION NO.

DATE: Sept. 1993

PAGE 11 OF 19

approximately 25 to 30 seconds prior to the reading time. Lower the hydrometer slowly into the suspension to avoid disturbing settling particles in the solution. Lower the hydrometer to the approximate depth expected for the reading. Wait until the hydrometer is stable and not touching the side of the cylinder. Read the hydrometer at the top of the meniscus formed on the hydrometer stem. Record the reading. slowly remove the hydrometer from the soil/water suspension and place it with a spinning motion in a graduate of clean distilled or demineralized water. Avoid leaving the hydrometer in a specimen cylinder for more than a few minutes to prevent particles from accumulating on the hydrometer bulb. If large floating particles adhere to the hydrometer after it is withdrawn from the specimen, wipe the hydrometer with a paper towel before placing it in the cylinder of water.

3.11.6 At every reading interval after the 30 second reading, record the temperature of the suspension by inserting a thermometer into the suspension. Take care not to agitate the suspension beyond that necessary to obtain the temperature. Temperatures after the 1 hour reading may be recorded from a thermometer immersed in a sedimentation cylinder containing dilute dispersant.

3.11.7 Upon completion of all the required hydrometer readings, transfer the contents of the sedimentation cylinder into a No.200 sieve and wash with tap water until the wash water is clear. Place the residue in a drying pan and dry overnight in an oven at a temperature of $110 \pm 5^{\circ} \mathrm{C}$.

3.11.8 Perform a sieve analysis of the portion retained on the No. 200 sieve in accordance with section 3.9 using No. 20 , No.40, No.60, No.100, No.140, and No. 200 sieves. The number and size of sieves used may be adjusted to suit client or material requirements.

\subsection{Calculations}

3.12.1 Correct the weight of material used for the hydrometer analysis and the total specimen weight for hygroscopic moisture by dividing 
these weights by 1 plus the hygroscopic moisture content expressed as a fraction.

\subsection{2 .2 Coarse Fraction Sieve Analysis}

3.12.2.1 Calculate the percentage of the specimen passing the No.10 sieve by dividing the weight of material passing the No.10 sieve by the total weight of material originally separated on the No. 10 sieve. Multiply the result by 100 . To determine the weight passing the No.10 sieve, subtract the quantity retained on the No.10 sieve from the original weight.

3.12.2.2 To determine the total weight of material passing the No.4 sieve, add to the weight of the soil passing the No.10 sieve the quantity of the fraction passing the No.4 sieve and retained on the No.10 sieve. For the remaining sieves, determine the percentages passing in a similar manner.

3.12.2.3 To calculate the total percentage passing for each sieve, divide the total quantity passing, as described above, by the total weight of the sample. Multiply the result by 100 .

\subsubsection{Percentages of soil in Suspension}

3.12.3.1 Determine the weight of a total sample represented by the weight of soil used in the hydrometer test, by dividing the oven-dry weight used by the percentage passing the No.10 sieve, and multiplying the result by 100 . This represents the weight $W$ in the equation for percentage remaining in suspension.

3.12.3.2 The percentage of soil remaining in suspension at the level where the hydrometer is measuring the density of the suspension may be determined as follows:

3.12.2.7.1 For hydrometer type 151H:

$$
P=\left[\frac{100,000}{W} * \frac{G}{\left(G-G_{1}\right)}\right]\left(R-G_{1}\right)
$$


3.12.2.7.2 For hydrometer type 152H:

$$
P=\frac{R a}{W} * 100
$$

where:

$a=$ correction factor to be applied to the reading of hydrometer $152 \mathrm{H}$. Factor computed based on a specific gravity of 2.65. (Correction factors are provided in Table 1),

$P=$ percentage of soil remaining in suspension at the level at which the hydrometer measures the density of the suspension,

$R$ = hydrometer reading with correction applied (see section 3.8.5),

$W=$ oven-dry weight of soil in a total test sample represented by quantity of soil dispersed (see section 3.9.6), g,

$G$ = specific gravity of the soil particles as determined by the method appropriate to the sample being tested,

$G_{1}=$ specific gravity of the liquid in which soil particles are suspended. Use numerical value of one in both instances in the equation. In the first instance any possible variation produces no significant effect, and in the second instance, the correction factor $R$ is based on a value of one for $G_{1}$.

3.12.4 Particle Diameters - The diameter of a particle corresponding to the percentage indicated by a given hydrometer reading is calculated according to stokes' law. The result of this determination is based on the premise that a particle of this diameter was at the surface of the suspension at the beginning of sedimentation and had settled to the level at which the hydrometer is measuring the density of the suspension. The particle size calculated represents the diameter of a sphere that would fall at the same rate as the soil particles. This assumption is validated by stokes' law in that it considers the terminal velocity of a single sphere falling in an infinity of liquid. Calculate the diameter of soil particles as follows: 
SOP NO.

REVISION

DATE :

PAGE
PDGG $=00020$

NO.

Sept. 1993 14 OF 19

$$
D=K \sqrt{\frac{L}{T}}
$$

where:

$D=$ diameter of particle, $\mathrm{mm}$

$K=$ constant depending on the temperature of the suspension and the specific gravity of the soil particles. Values of $K$ for a range of temperatures and specific gravities are given in Table 2 . The value of $K$ does not change for a series of readings constituting a test, while values of $L$ and $T$ do vary.

$L$ = Effective depth. The distance from the surface of the suspension to the level at which the density of the suspension is being measured, cm. (For a given hydrometer and sedimentation cylinder, values vary according to the hydrometer readings. Values of effective depth are given in Table 3 .

$T$ = interval of time from beginning of sedimentation to the taking of the reading, min.

\subsubsection{Fine Sieve Analysis}

3.12.5.1 For sieve analysis values of portion finer than the No.10 sieve (the hydrometer portion), first determine the weight of the portion that would have been retained on the No.10 sieve had it not been removed. This weight equals the total percentage retained on the No. 10 sieve (100 minus total percentage passing) times the weight of the total sample represented by the weight of soil used and the result divided by 100 .

3.12.5.2 Calculate the total weight of the specimen passing the No. 200 sieve. Add weights retained on all the sieves (including the No.10) and subtract the result from the weight of the total dry specimen.

3.12.5.3 Calculate the total weights passing each of the other sieves as described in section 3.12 .2 . 
3.12.5.4 Calculate the total percentages passing by dividing the total quantity passing $(3.12 .5 .3)$ by the total dry weight of the specimen, and multiply the result by 100 .

3.12 .6 Once the hydrometer analysis is complete, a graph of the test results shall be prepared by plotting the diameters of the particles on a logarithmic scale as the abscissa and the percentages finer than the corresponding diameters to an arithmetic scale as the ordinate. The preparation of the graph may be omitted if the hydrometer test is not performed. In this case, any values may be obtained directly from the tabulated data.

3.12.7 A validated computer spreadsheet is currently in use for calculating and graphing all required data.

4.0 Forms - A copy of the data recording form used in conjunction with this procedure is included in Appendix $A$.

5.0 References

\subsection{ASTM Procedures}

5.1.1 D 422 - Method for Particle-Size Analysis of Soils

5.1.2 E 11 - Specifications for Wire-Cloth Sieves for Testing purposes

5.1.3 E 100 - Specification for ASTM Hydrometers

5.2 IT Standard Operating Procedures

5.2.1 PDGADMO002 - Balance Calibration and Maintenance

5.2.2 PDGADMO003 - Use and Maintenance of Class $S$ and Brass Weights

5.2.3 PDGADMO006 - Verification of Oven and Refrigerator Temperatures

5.2.4 PDGADMOOOB - Verification of Thermometer Calibration

5.2.5 PDGADMOO09 - Nonconformance/Variance Reports

5.2.6 PDGGEO0019 - MOisture Content of Soil and 
Geotechnical Purposes

5.2.7 PDGGE00023 - Sieve Specification, Calibration, and Inspection

5.2.8 PDGGE00032 - Calibration of Sieve Shakers

5.2.9 PDGGEO0033 - Calibration of Soil Hydrometers 
TABLE 1

Values of Correction Factor, a, for Different Specific Gravities of Soil Particles

\begin{tabular}{|c|c|}
\hline Specific Gravity & Correction Factor \\
\hline 2.95 & 0.94 \\
\hline 2.90 & 0.95 \\
\hline 2.85 & 0.96 \\
\hline 2.80 & 0.97 \\
\hline 2.75 & 0.98 \\
\hline 2.70 & 0.99 \\
\hline 2.65 & 1.00 \\
\hline 2.60 & 1.01 \\
\hline 2.55 & 1.02 \\
\hline 2.50 & 1.03 \\
\hline 2.45 & 1.05 \\
\hline
\end{tabular}




\section{Table 2}

Values of $K$ Used in Equation for Computing Particle Diameters

\begin{tabular}{|cccccc|c|c|c|c|c|}
\hline \hline $\begin{array}{c}\text { Temp. } \\
{ }^{\circ} \mathrm{C}\end{array}$ & 2.45 & 2.50 & 2.55 & 2.60 & 2.65 & 2.70 & 2.75 & 2.80 & 2.85 \\
\hline 16 & 0.01530 & 0.01505 & 0.01481 & 0.01457 & 0.01435 & 0.01414 & 0.01394 & 0.01374 & 0.01356 \\
\hline 17 & 0.01511 & 0.01486 & 0.01462 & 0.01439 & 0.01417 & 0.01396 & 0.01376 & 0.01356 & 0.01338 \\
\hline 18 & 0.01492 & 0.01467 & 0.01443 & 0.01421 & 0.01399 & 0.01378 & 0.01359 & 0.01339 & 0.01321 \\
\hline 19 & 0.01474 & 0.01449 & 0.01425 & 0.01403 & 0.01382 & 0.01361 & 0.01342 & 0.01323 & 0.01305 \\
\hline 20 & 0.01456 & 0.01431 & 0.01408 & 0.01386 & 0.01365 & 0.01344 & 0.01325 & 0.01307 & 0.01289 \\
\hline 21 & 0.01438 & 0.01414 & 0.01391 & 0.01369 & 0.01348 & 0.01328 & 0.01309 & 0.01291 & 0.01273 \\
\hline 22 & 0.01421 & 0.01397 & 0.01374 & 0.01353 & 0.01332 & 0.01312 & 0.01294 & 0.01276 & 0.01258 \\
\hline 23 & 0.01404 & 0.01381 & 0.01358 & 0.01337 & 0.01317 & 0.01297 & 0.01279 & 0.01261 & 0.01243 \\
\hline 24 & 0.01388 & 0.01365 & 0.01342 & 0.01321 & 0.01301 & 0.01282 & 0.01264 & 0.01246 & 0.01229 \\
\hline 25 & 0.01372 & 0.01349 & 0.01327 & 0.01306 & 0.01286 & 0.01267 & 0.01249 & 0.01232 & 0.01215 \\
\hline 26 & 0.01357 & 0.01334 & 0.01312 & 0.01291 & 0.01272 & 0.01253 & 0.01235 & 0.01218 & 0.01201 \\
\hline 27 & 0.01342 & 0.01319 & 0.01297 & 0.01277 & 0.01258 & 0.01239 & 0.01221 & 0.01204 & 0.01188 \\
\hline 28 & 0.01327 & 0.01304 & 0.01283 & 0.01264 & 0.01244 & 0.01255 & 0.01208 & 0.01191 & 0.01175 \\
\hline 29 & 0.01312 & 0.01290 & 0.01269 & 0.01249 & 0.01230 & 0.01212 & 0.01195 & 0.01178 & 0.01162 \\
\hline 30 & 0.01298 & 0.01276 & 0.01256 & 0.01236 & 0.01217 & 0.01199 & 0.01182 & 0.01165 & 0.01149 \\
\hline
\end{tabular}


SOP NO. PIIGGEOOO20

REVISION

N. 00

DATE: SEpt. 1993

PAGE

19 OF 19

\section{Table 3}

Values of $L$, Effective Depth

\begin{tabular}{|c|c|c|c|c|c|}
\hline \multicolumn{2}{|c|}{ Hydrometer $151 \mathrm{H}$} & \multicolumn{4}{|c|}{ Hydrometer $152 \mathrm{H}$} \\
\hline $\begin{array}{c}\text { Actual } \\
\text { Hydrometer } \\
\text { Reading }\end{array}$ & $\begin{array}{c}\text { Efective } \\
\text { Depth, } L \text {, } \\
\mathrm{cm}\end{array}$ & $\begin{array}{l}\text { Actual } \\
\text { Hydrometer } \\
\text { Reading }\end{array}$ & $\begin{array}{c}\text { Efective } \\
\text { Depth, } L \text {, } \\
\text { cm }\end{array}$ & $\begin{array}{l}\text { Actual } \\
\text { Hydrometer } \\
\text { Reading }\end{array}$ & $\begin{array}{c}\text { Efective } \\
\text { Depth, } L \text {, } \\
\text { cm }\end{array}$ \\
\hline 1.000 & 16.3 & $\overline{0}$ & 16.3 & 31 & 11.2 \\
\hline 1.001 & 16.0 & 1 & 16.1 & 32 & 11.1 \\
\hline 1.002 & 15.8 & 2 & 16.0 & 33 & 10.9 \\
\hline 1.003 & 15.5 & 3 & 15.8 & 34 & 10.7 \\
\hline 1.004 & 15.2 & 4 & 15.6 & 35 & 10.6 \\
\hline 1.005 & 15.0 & 5 & 15.5 & 36 & 10.4 \\
\hline 1.006 & 14.7 & 6 & 15.3 & 37 & 10.2 \\
\hline 1.007 & 14.4 & 7 & 15.2 & 38 & 10.1 \\
\hline 1.008 & 14.2 & 8 & 15.0 & 39 & 9.9 \\
\hline 1.009 & 13.9 & 9 & 14.8 & 40 & 9.7 \\
\hline 1.010 & 13.7 & 10 & 14.7 & 41 & 9.6 \\
\hline 1.011 & 13.4 & 11 & 14.5 & 42 & 9.4 \\
\hline 1.012 & 13.1 & 12 & 14.3 & 43 & 9.2 \\
\hline 1.013 & 12.9 & 13 & 14.2 & 44 & 9.1 \\
\hline 1.014 & 12.6 & 14 & 14.0 & 45 & 8.9 \\
\hline 1.015 & 12.3 & 15 & 13.8 & 46 & 8.8 \\
\hline 1.016 & 12.1 & 16 & 13.7 & 47 & 8.6 \\
\hline 1.017 & 11.8 & 17 & 13.5 & 48 & 8.4 \\
\hline 1.018 & 11.5 & 18 & 13.3 & 49 & 8.3 \\
\hline 1.019 & 11.3 & 19 & 13.2 & 50 & 8.1 \\
\hline 1.020 & 11.0 & 20 & 13.0 & 51 & 7.9 \\
\hline 9.021 & 10.7 & 21 & 12.9 & 52 & 7.8 \\
\hline 1.022 & 10.5 & 22 & 12.7 & 53 & 7.6 \\
\hline 1.023 & 10.2 & 23 & 12.5 & 54 & 7.4 \\
\hline 1.024 & 10.0 & 24 & 12.4 & 55 & 7.3 \\
\hline 1.025 & 9.7 & 25 & 12.2 & 56 & 7.1 \\
\hline 1.026 & 9.4 & 26 & 12.0 & 57 & 7.0 \\
\hline 1.027 & 8.2 & 27 & 11.9 & 58 & 6.8 \\
\hline 1.028 & 8.9 & 28 & 11.7 & 59 & 6.6 \\
\hline 1.029 & 8.6 & 29 & 11.5 & 60 & 6.5 \\
\hline 1.030 & 8.4 & 30 & 11.4 & & \\
\hline 1.031 & 8.1 & & & & \\
\hline 1.032 & 7.8 & & & & \\
\hline 1.033 & 7.6 & & & & \\
\hline 1.034 & 7.3 & & & & \\
\hline 1.035 & 7.0 & - & & & \\
\hline 1.036 & 6.8 & & & & \\
\hline 1.037 & 6.5 & & & & \\
\hline 1.038 & 6.2 & & & & \\
\hline
\end{tabular}


APPENDIX A

\section{DATA COLLECTION FORM}


SOP NO. PDGGEO0020

REVISIO: NO. 00

DATE: Sept. 1993

PAGE 19 OF 19

Computet Entry By Dote

Entry/tronsezipt in Cnock مu: :

Projeet Name

Project Number

IT/ETDC Sampie No

Coarse sieves by:

Date :

PARTICLE SIZE

ANALYSIS ASTM D422 SOP PDCGEO0020
Fine sieves by

Date

\begin{tabular}{|l|l|l|l|}
\hline \multicolumn{1}{l|}{} & \multicolumn{1}{c|}{$\begin{array}{c}\text { Sieve } \\
\text { Opening } \\
\text { mm }\end{array}$} & $\begin{array}{c}\text { Sieve } \\
\text { No. }\end{array}$ & $\begin{array}{l}\text { Cumulative Wt of } \\
\text { Mat'1 Retained, 8. }\end{array}$ \\
\hline C & 75 & $3^{*}$ & \\
\cline { 2 - 5 } O & 37.5 & $1.5^{\circ}$ & \\
\cline { 2 - 5 } A & 19 & $3 / 4^{*}$ & \\
\cline { 2 - 5 } R & 9.5 & $3 / 8$ & \\
\cline { 2 - 5 } & 4.75 & 14 & \\
\hline E & 2 & 110 & \\
\hline
\end{tabular}

Hydrometer Analysis Performed By

\begin{tabular}{|c|c|c|c|c|c|}
\hline & $\begin{array}{l}\text { Date } \\
199\end{array}$ & $\begin{array}{l}\text { Clock } \\
\text { Time }\end{array}$ & $\begin{array}{l}\text { Elapsed } \\
\text { Time }\end{array}$ & $\begin{array}{c}\text { Temp } \\
\text { C }\end{array}$ & $\begin{array}{c}\text { Hydrometer } \\
\text { Reading }\end{array}$ \\
\hline \multirow{3}{*}{ H } & & & $0 \mathrm{~min}$. & & \\
\hline & & . & 0.5 & & \\
\hline & & & 1 & & \\
\hline D & & & 2 & & \\
\hline $\mathbf{R}$ & & & 5 & & \\
\hline 0 & & & is & & \\
\hline$M$ & & & 30 & & \\
\hline $\mathbf{E}$ & & & 60 & & \\
\hline$T$ & & & 120 & & \\
\hline$E$ & & & 250 & & \\
\hline \multirow[t]{2}{*}{$\mathbf{R}$} & & & 1440 & & \\
\hline & & & & & \\
\hline
\end{tabular}

\begin{tabular}{|c|c|c|c|}
\hline & $\begin{array}{c}\text { Sieve } \\
\text { Opening } \\
\text { mm }\end{array}$ & $\begin{array}{l}\text { Sieve } \\
\text { No. }\end{array}$ & $\begin{array}{l}\text { Cumulative Wt of } \\
\text { Mat'l Retained, } 8 \text {. }\end{array}$ \\
\hline \multirow[b]{2}{*}{$F$} & 0.85 & 120 & \\
\hline & 0.425 & $\# 40$ & \\
\hline \multirow{4}{*}{$\begin{array}{l}\mathbf{N} \\
\mathbf{E}\end{array}$} & 0.25 & 160 & \\
\hline & 0.150 & $\# 100$ & \\
\hline & 0.106 & $\$ 140$ & \\
\hline & 0.075 & $\$ 200$ & \\
\hline
\end{tabular}

Sample Prepped By

Date

\begin{tabular}{|l|l|}
\hline $\begin{array}{l}\text { A Calculated Dry Wt of Hydrometer } \\
\text { Sample, g (B/P) }\end{array}$ & \\
\hline B Wt of Air-Dried Sample, Hydrometer & \\
\hline C Calc Total Dry Sample Wt, \& (D/I+P) & \\
\hline D Total Wt of Air-Dry Soil, \& & \\
\hline E ASTM Hydrometer Type & ISIH \\
\hline F Hydrometer Serial Number & \\
\hline G Thermometer Serial Number & \\
\hline H Hydrometer Correction Factor & \\
\hline K HYGROSCOPIC MOISTURE & CONTENT \\
\hline L Tare Number & \\
\hline M Tore Wieght, grams & \\
\hline N Wi Air-Dry Sample + Tare, \&. & \\
\hline O W/ Oven-Dried Sample + Tare, B. & \\
\hline P Hygroseopic Moisture Content, \% & \\
\hline
\end{tabular}

Hydrometer Readings Recorded as follows: ASTM 151H - (Scale reading-11) 100 


\title{
Standard Test Method for Particle-Size Analysis of Soils ${ }^{1}$
}

\begin{abstract}
This madard is issued uoder the fired designation D 422; the aumber immediately following the desipantion indicales the year of originel edoption or, in the case of revision, the year of ins revisios. A aumber in perentheses indicates the year of lest reapproval. A

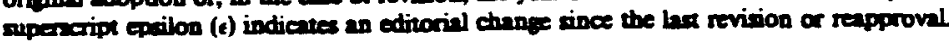

" Nork-Section 19 was added editorially in Seprember 1990.

\section{Scope}

1.1 This test method covers the quantitative determination of the distribution of particle sizes in soils. The distribution of particle sizes larger than $75 \mu \mathrm{m}$ (retained on the No. 200 sieve) is determined by sieving, while the distribution of particle sizes smaller than $75 \mu \mathrm{m}$ is determined by a sedimentation process, using a hydrometer to secure the necessary data (Notes 1 and 2).

Nore 1 -Separation may be made on the No. $4(4.75-\mathrm{mm})$, No. 40 $(425 \mu \mathrm{m})$, or No. $200(75-\mu \mathrm{m})$ sieve instead of the No. 10. For whatever sieve used, the size shall be indicated in the report.

NOTE 2-Two types of dispersion devices are provided: (1) a high-speed mechanical stirrer, and (2) air dispersion. Extensive investigations indicate that air-dispersion devices produce a more positive dispersion of plastic soils below the 20-4m size and appreciably less degradation on all sizes when used with sandy soils. Because of the definite advantages favoring air dispersion, its use is recommended. The results from the two types of devices differ in magnitude, depending upon soil type, leading to marked differences in particle size distribution, especially for sizes finer than $20 \mu \mathrm{m}$.

\section{Referenced Documents}

\subsection{ASTM Standards:}

D421 Practice for Dry Preparation of Soil Samples for Particle-Size Analysis and Determination of Soil Constants ${ }^{2}$

E 11 Specification for Wire-Cloth Sieves for Testing Purposes ${ }^{3}$

E 100 Specification for ASTM Hydrometers ${ }^{4}$

\section{Apparatus}

3.1 Balances-A balance sensitive to $0.01 \mathrm{~g}$ for weighing the material passing a No. $10(2.00-\mathrm{mm})$ sieve, and a balance sensitive to $0.1 \%$ of the mass of the sample to be weighed for weighing the material retained on a No. 10 sieve.

3.2 Stirring Apparatus-Either apparatus A or B may be used.

3.2.1 Apparatus A shall consist of a mechanically oper-

\footnotetext{
I This test method is under the jurisdiction of ASTM Committee D-18 on Soil and Rock and is the direct responsibility of Subcommittee D18.03 on Texture, Plasticity, and Density Characteristics of Soils.

Current edition approved Nov. 21, 1963. Originally published 1935. Replaces D $422-62$.

2 Annual Book of ASTM Standards, Vol 04.08

3 Anmual Book of ASTM Standards, Vol 14.02.

- Annual Book of ASTM Slandards, Vol 14.03.
}

ated stirring device in which a suitably mounted electric motor turns a vertical shaft at a speed of not less than 10000 Ipm without load. The shaft shall be equipped with a replaceable stirring paddle made of metal, plastic, or hard rubber, as shown in Fig. 1. The shaft shall be of such length that the stirring paddle will operate not less than $3 / 4$ in. (19.0 $\mathrm{mm}$ ) nor more than $1 / 2$ in. (38.1 mm) above the bottom of the dispersion cup. A special dispersion cup conforming to either of the designs shown in Fis. 2 shall be provided to hold the sample while it is being dispersed.

3.2.2 Apparatus B shall consist of an air-jet dispersion cup (Note 3) conforming to the general details shown in Fig. 3 (Notes 4 and 5).

NOTE 3-The amount of air required by an air-jet dispersion cup is of the order of $2 \mathrm{t}^{3} / \mathrm{min}$; some small air compressors are not capable of supplying sufficient air to operate a cup.

NoTe 4-Another air-type dispersion device, known as a dispersion tube, developed by Chu and Davidson at lowa State Colleze, has been shown to give results equivalent to those secured by the air-jet dispersion cups. When it is used, soaking of the sample can be done in the sedimentation cylinder, thus eliminating the need for transferring the slurry. When the air-dispersion rube is used, it shall be $s 0$ indicated in the report.

NotE 5-Water may condense in air lines when not in use. This water must be removed, either by using a water trap on the air line, or by blowing the water out of the line before using any of the air for dispersion purposes.

3.3 Hydrometer-An ASTM hydrometer, graduated to read in either specific gravity of the suspension or grams per litre of suspension, and conforming to the requirements for hydrometers $151 \mathrm{H}$ or $152 \mathrm{H}$ in Specifications E 100. Dimensions of both hydrometers are the same, the scale being the only item of difference.

3.4 Sedimentation Cylinder-A glass cylinder essentially $18 \mathrm{in} .(457 \mathrm{~mm})$ in height and $21 / 2$ in. $(63.5 \mathrm{~mm})$ in diameter, and marked for a volume of $1000 \mathrm{~mL}$. The inside diameter shall be such that the $1000-\mathrm{mL}$ mark is $36 \pm 2 \mathrm{~cm}$ from the bottom on the inside.

3.5 Thermometer-A thermometer accurate to $1^{\circ} \mathrm{F}$ $\left(0.5^{\circ} \mathrm{C}\right)$.

3.6 Sieves-A series of sieves, of square-mesh woven-wire cloth, conforming to the requirements of Specification E 11. A full set of sieves includes the following (Note 6):

\footnotetext{
S Detailed working drawings for this cup are available at a nominal cos from the American Society for Testing and Materials, 1916 Race St., Philndelphin, PA 19103. Order Adjunct No. 12-404220-00.
} 

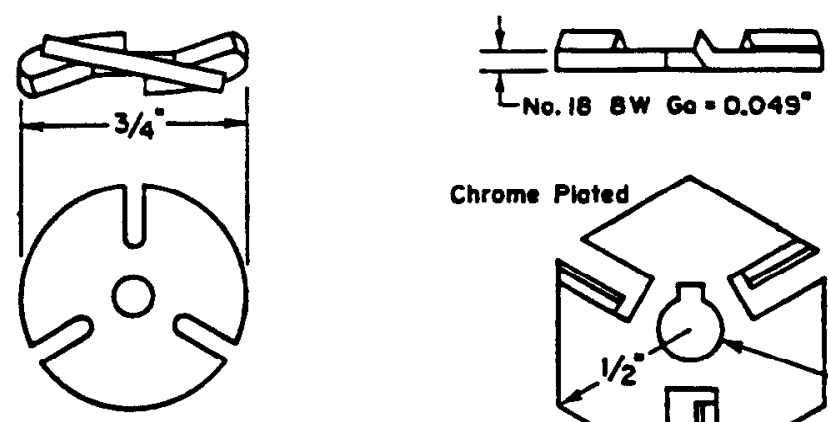

tric

000

b a

tard

igth

19.0

a of

$\mathrm{g}$ to

iold

sion

Fig.

sp is

le of

sion

been

rinn

$x$ in

This

or by

- for

1 to

per

i for

ren-

the

ially

ster,

eter

the

wire

: 11 .

from

a. PA

(a)

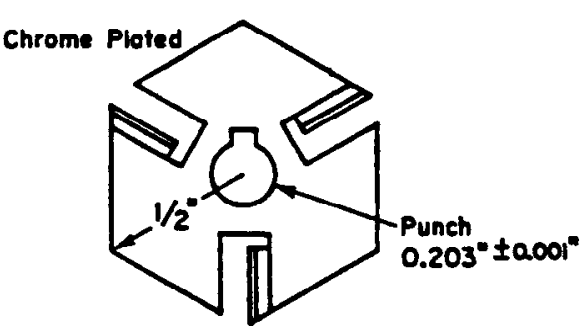

(b)

\begin{tabular}{|c|c|c|c|c|c|}
\hline in. & $\begin{array}{l}0.001 \\
0.03\end{array}$ & $\begin{array}{l}0.049 \\
1.24\end{array}$ & $\begin{array}{l}0.203 \\
5.16\end{array}$ & $\begin{array}{l}* \\
12.7\end{array}$ & $\begin{array}{l}7 / 4 \\
19.0\end{array}$ \\
\hline
\end{tabular}

Fle 1 Dotil of Stining Paddles

$$
\begin{aligned}
& \text { 3-in. }(75-\mathrm{mm}) \\
& \text { 2-in. }(50-\mathrm{mm}) \\
& 1 \text {-in. }(37.5-\mathrm{mm}) \\
& 1 \text {-in. }(25.0-\mathrm{mm}) \\
& \text { *-in. }(19.0-\mathrm{mm}) \\
& \text { K-in. (9.5-mm) } \\
& \text { No. } 4 \text { (4.75-mm) }
\end{aligned}
$$

Note 6-A set of sieves giving uniform spacing of points for the graph, as required in Section 17, may be used if desired. This set consists of the following sieves:

$$
\begin{aligned}
& \text { 3-in. (75-mm) } \\
& \text { 14h-in. (37.5-mm) } \\
& \text { W-in. (19.0-mm) } \\
& \text { W-in. }(9.5-\mathrm{mm}) \\
& \text { No. } 4(4.75-\mathrm{mm}) \\
& \text { No. } 8 \text { (2.36-mm) }
\end{aligned}
$$

No. $16(1.18-\mathrm{mm})$

No. $30(600-\mu m)$

No. $50(300-\mu m)$

No. $100(150-\mu \mathrm{m})$

No. $200(75-\mu m)$

3.7 Water Bath or Constant-Temperature Room-A water bath or constant-temperarure room for maintaining the soil suspension at a constant temperature during the hydrometer analysis. A satisfactory water tank is an insulated tank that maintains the temperature of the suspension at a convenient constant temperature at or near $68^{\circ} \mathrm{F}\left(20^{\circ} \mathrm{C}\right)$. Such a device is illustrated in Fig. 4. In cases where the work is performed in a room at an automatically controlled constant temperature, the water bath is not necessary.

3.8 Beaker-A beaker of $250-\mathrm{mL}$ capacity.

3.9 Timing Device-A watch or clock with a second hand.

\section{Dispersing Agent}

4.1 A solution of sodium hexametaphosphate (sometimes called sodium metaphosphate) shall be used in distilled or demineralized water, at the rate of $40 \mathrm{~g}$ of sodium hexametaphosphate/litre of solution (Note 7).

NoTE 7-Solutions of this salt, if acidic, slowly revert or hydrolyze back to the orthophosphate form with a resultant decrease in dispersive action. Solutions should be prepared frequently (at least once a month) or adjusted to pH of 8 or 9 by means of sodium carbonate. Bottles containing solutions should have the date of preparation marked on them.

4.2 All water used shall be either distilled or demineralized water. The water for a hydrometer test shall

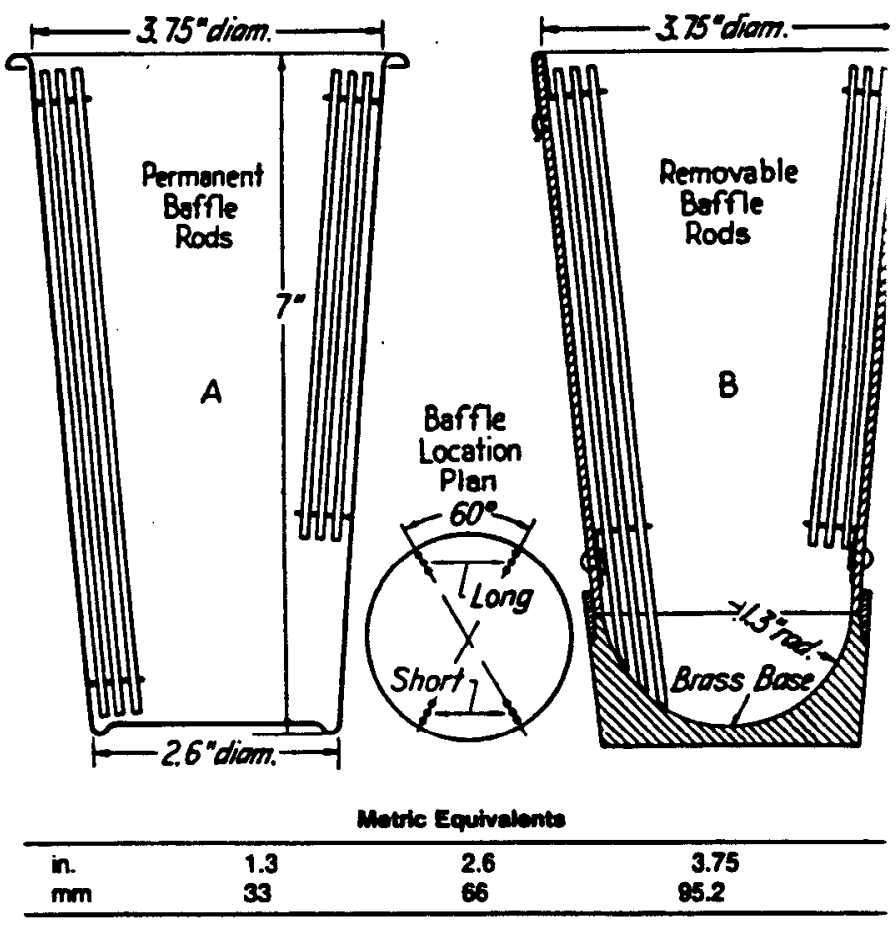

FIG. 2 Diapersion Cupe of Apparatus

be brought to the temperature that is expected to prevai during the hydrometer test. For example, if the sedimenta tion cylinder is to be placed in the water bath, the distilled 0 : demineralized water to be used shall be brought to the temperature of the controlled water bath; or, if the sedimen. tation cylinder is used in a room with controlled tempera. ture, the water for the test shall be at the temperature of the room. The basic temperature for the hydrometer test is $68^{\circ} \mathrm{F}$ $\left(20^{\circ} \mathrm{C}\right)$. Small variations of temperature do not introduce differences that are of practical significance and do no prevent the use of corrections derived as prescribed. 

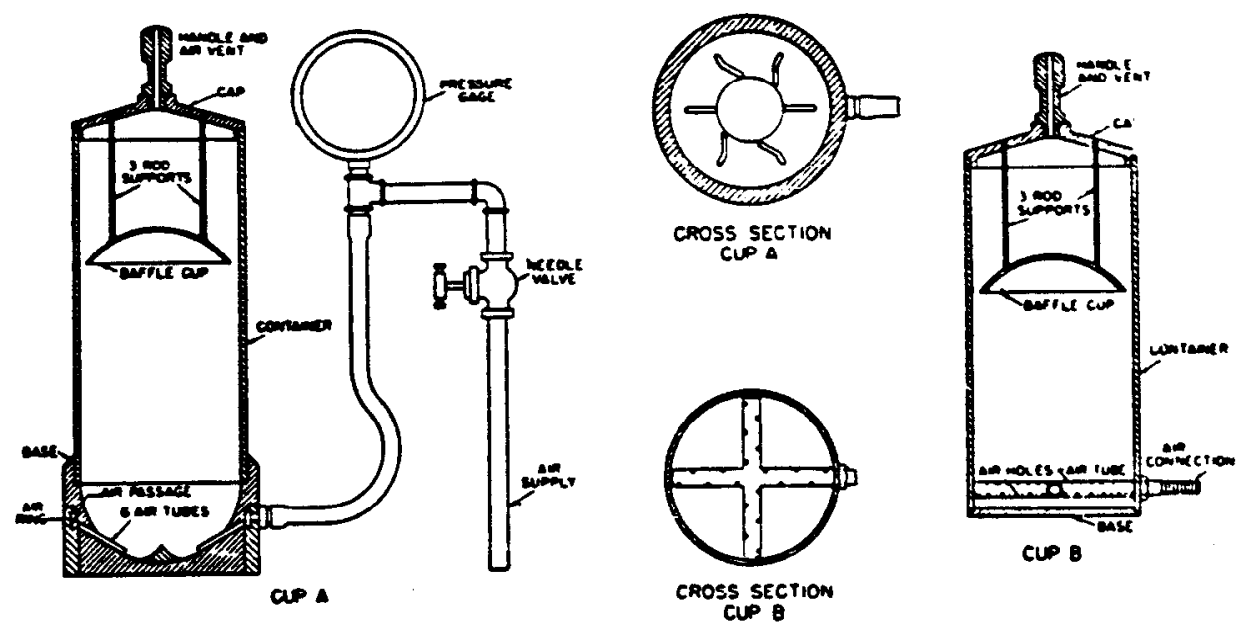

FIC. 3 Airatet Dispersion Cups of Apparatus B

\section{Test Sample}

5.1 Prepare the test sample for mechanical analysis as outlined in Practice D 421. During the preparation procedure the sample is divided into two portions. One portion contains only particles retained on the No. $10(2.00-\mathrm{mm})$ sieve while the other portion contains only particles passing the No. 10 sieve. The mass of air-dried soil selected for purpose of tests, as prescribed in Practice D 421, shall be sufficient to yield quantities for mechanical analysis as follows:

5.1.1 The size of the portion retained on the No. 10 sieve shall depend on the maximum size of particle, according to the following schedule:

Nominal Diameter of
Largest Particles,
in. (mm)
th $(9.5)$
$1 / 4(19.0)$
1 (25.4)
$11 / 2(38.1)$
$2 \quad(50.8)$
$3 \quad(76.2)$

Approximate Minimum
Mass of Portion, 8
500
1000
2000
3000
4000
5000

5.1.2 The size of the portion passing the No. 10 sieve shall be approximately $115 \mathrm{~g}$ for sandy soils and approximately 65 $\mathrm{g}$ for silt and clay soils.

5.2 Provision is made in Section 5 of Practice D 421 for weighing of the air-dry soil selected for purpose of tests, the separation of the soil on the No. 10 sieve by dry-sieving and washing, and the weighing of the washed and dried fraction retained on the No. 10 sieve. From these two masses the percentages retained and passing the No. 10 sieve can be calculated in accordance with 12.1.

Note 8-A check on the mass values and the thoroughness of pulverization of the clods may be secured by weighing the portion passing the No. 10 sieve and adding this velue to the mass of the washed and oven-dried portion retained on the No. 10 sieve.

\section{SIEVE ANALYSIS OF PORTION RETAINED ON NO. 10 $(2.00-\mathrm{mm})$ SIEVI}

\section{Procedure}

6.1 Separate the portion retained on the No. $10(2.00-$ $\mathrm{mm}$ ) sieve into a series of fractions using the 3-in. (75-mm),

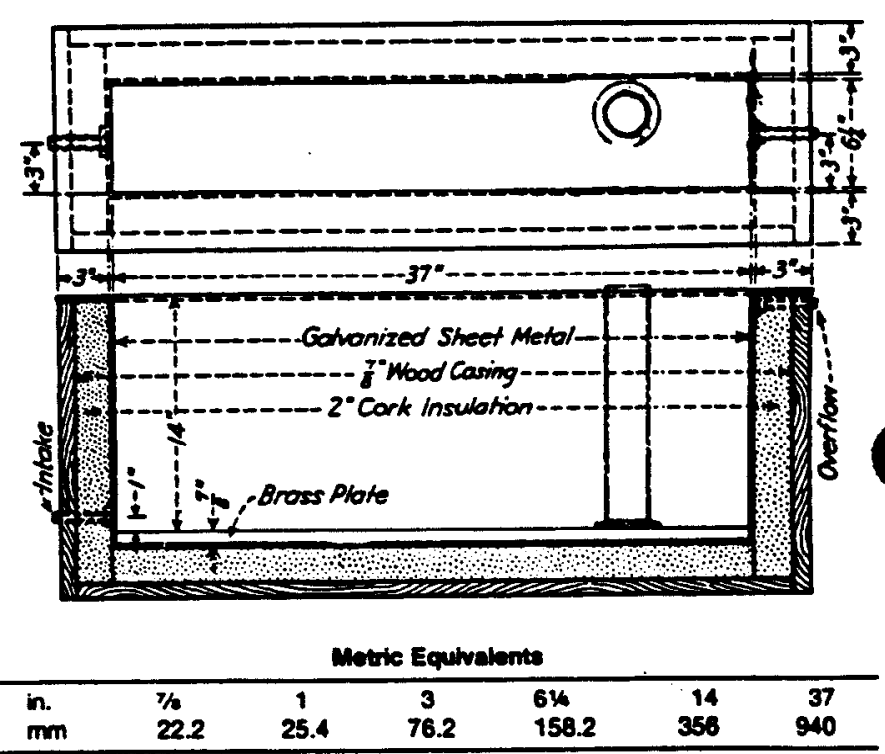

FG. 4 Insulatod Water Bath

2-in. (50-mm), 11/2-in. (37.5-mm), 1-in. (25.0-mm), $3 / 6$-in. (19.0-mm), 3/6-in. (9.5-mm), No. 4 (4.75-mm), and No. 10 sieves, or as many as may be needed depending on the sample, or upon the specifications for the material under test.

6.2 Conduct the sieving operation by means of a lateral and vertical motion of the sieve, accompanied by a jarring action in order to keep the sample moving continuously over the surface of the sieve. In no case turn or manipulate fragments in the sample through the sieve by hand. Continue sieving until not more than 1 mass \% of the residue on a sieve passes that sieve during $1 \mathrm{~min}$ of sieving. When mechanical sieving is used, test the thoroughness of sieving by using the hand method of sieving as described above.

6.3 Determine the mass of each fraction on a balance conforming to the requirements of 3.1. At the end of weighing, the sum of the masses retained on all the siever used should equal closely the original mass of the quantity sieved. 
HYDROMETER AND SIEVE ANALYSIS OF PORTION PASSING THE NO. $10(2.00-\mathrm{mm})$ SIEVE

\section{Determination of Composite Correction for Hydrometer Reading}

7.1 Equations for percentages of soil remaining in suspension, as given in 14.3, are based on the use of distilled or demineralized water. A dispersing agent is used in the water, however, and the specific gravity of the resulting liquid is appreciably greater than that of distilled or demineralized water.

7.1.1 Both soil hydrometers are calibrated at $68^{\circ} \mathrm{F}\left(20^{\circ} \mathrm{C}\right)$, and variations in temperature from this standard temperature produce inaccuracies in the actual bydrometer readings. The amount of the inaccuracy increases as the variation from the standard temperature increases.

7.1.2 Hydrometers are graduated by the manufacturer to be read at the bottom of the meniscus formed by the liquid on the stem. Since it is not possible to secure readings of soil suspensions at the bottom of the meniscus, readings must be taken at the top and a correction applied.

7.1.3 The net amount of the corrections for the three items enumerated is designated as the composite correction, and may be determined experimentally.

7.2 For convenience, a graph or table of composite corrections for a series of $1^{\circ}$ temperature differences for the range of expected test temperatures may be prepared and used as needed. Measurement of the composite corrections may be made at two temperatures spanning the range of expected test temperatures, and corrections for the intermediate temperatures calculated assuming a straight-line relationship between the two observed values.

7.3 Prepare $1000 \mathrm{~mL}$ of liquid composed of distilled or demineralized water and dispersing agent in the same proportion as will prevail in the sedimentation (bydrometer) test. Place the liquid in a sedimentation cyclinder and the cylinder in the constant-temperature water bath, set for one of the two temperatures to be used. When the temperature of the liquid becomes constant, insert the hydrometer, and, after a short interval to permit the hydrometer to come to the temperature of the liquid, read the hydrometer at the top of the meniscus formed on the stem. For hydrometer $151 \mathrm{H}$ the composite correction is the difference between this reading and one; for hydrometer $152 \mathrm{H}$ it is the difference between the reading and zero. Bring the liquid and the hydrometer to the other temperature to be used, and secure the composite correction as before.

\section{Hygroscopic Moisture}

8.1 When the sample is weighed for the bydrometer test, weigh out an auxiliary portion of from 10 to $15 \mathrm{~g}$ in a small metal or glass container, dry the sample to a constant mass in an oven at $230 \pm 9^{\circ} \mathrm{F}\left(110 \pm 5^{\circ} \mathrm{C}\right)$, and weigh again. Record the masses.

\section{Dispersion of Soil Sample}

9.1 When the soil is mostly of the clay and silt sizes, weigh out a sample of air-dry soil of approximately $50 \mathrm{~g}$. When the soil is mostly sand the sample should be approximately 100 g.
9.2 Place the sample in the $250-\mathrm{mL}$ beaker and cover r $125 \mathrm{~mL}$ of sodium hexametaphosphate solution $(40 \mathrm{~g}$ Stir until the soil is thoroughly wetred. Allow to soak for least $16 \mathrm{~b}$.

9.3 At the end of the soaking period. disperse the sam further, using either stirring apparatus A or B. If stir apparatus $A$ is used, transfer the soil - water siurry from beaker into the special dispersion cup shown in Fig. washing any residue from the beaker into the cup a distilled or demineralized water (Note 9). Add distilled demineralized water, if necessary, so that the cup is $m$ than balf full. Stir for a period of $1 \mathrm{~min}$.

Nore 9-A large size syringe is a convenient device for bandling water in the washing operation. Other devices include the wash-w bottie and a hose with nozle connected to a pressurized distilled $w$ tank.

9.4 If stirring apparatus B (Fig 3) is used, remove cover cap and connect the cup to a compressed air supply means of a rubber hose. A air gage must be on the 1 between the cup and the control valve. Open the cont valve so that the gage indicates 1 psi $(7 \mathrm{kPa})$ pressure (N 10). Transfer the soil - water slurry from the beaker to air-jet dispersion cup by washing with distilled demineralized water. Add distilled or demineralized water necessary, so that the total volume in the cup is $250 \mathrm{~mL}$, no more.

Nore 10-The initial air pressure of I poi is required to prevent soil - water mixture from entering the air-jet chamber when the mixt is transferred to the dispersion cup.

9.5 Place the cover cap on the cup and open the control valve until the gage pressure is $20 \mathrm{psi}$ (140 kP Disperse the soil according to the following schedule:

$\begin{array}{cc}\text { Plasticity Index } & \text { Dieperion Period, } \\ \text { Under } 5 & 5 \\ 6 \text { to } 20 & 10 \\ \text { Over } 20 & 15\end{array}$

Soils containing large percentages of mica need be dispers for only $1 \mathrm{~min}$. After the dispersion period, reduce the $\mathrm{g}$ pressure to 1 psi preparatory to transfer of soil - water slu to the sedimentation cylinder.

\section{Hydrometer Test}

10.1 Immediately after dispersion, transfer the soil - wa slurry to the glass sedimentation cylinder, and add distil or demineralized water until the total volume is $1000 \mathrm{~mL}$

10.2 Using the palm of the hand over the open end of cylinder (or a rubber stopper in the open end), turn cylinder upside down and back for a period of $1 \mathrm{~min}$ complete the agitation of the slurry (Note 11). At the end 1 min set the cylinder in a convenient location and ta hydrometer readings at the following intervals of ti (measured from the beginning of sedimentation), or as ma as may be needed, depending on the sample or the specifi tion for the material under test: $2,5,15,30,60,250$, a $1440 \mathrm{~min}$. If the controlled water bath is used, the sedime tation cylinder should be placed in the bath between the and 5-min readings.

Nore 11-The number of turns during this minute should approximately 60 , counting the turn upside down and back as two tur 
Any soil remaining in the bottom of the cylinder during the firs few turns should be loosened by vigorous shaking of the cylinder while it is in the inverted position.

10.3 When it is desired to take a hydrometer reading, carefully insert the hydrometer about 20 to $25 \mathrm{~s}$ before the reading is due to approximately the depth it will have when the reading is taken. As soon as the reading is taken, carefully remove the hydrometer and place it with a spinning motion in a graduate of clean distilled or demineralized water.

Nore 12-It is important to remove the hydrometer immediately after each reading Readings shall be taken at the top of the meniscus formed by the suspension around the stem, since it is not possible to secure readings at the bottom of the meniscus.

10.4 After each reading, take the temperature of the suspension by inserting the thermometer into the suspension.

\section{Sieve Andysis}

11.1 After taking the final hydrometer reading, transfer the suspension to a No. $200(75-\mu \mathrm{m})$ sieve and wash with tap water until the wash water is clear. Transfer the material on the No. 200 sieve to a suitable container, dry in an oven at $230 \pm 9^{\circ} \mathrm{F}\left(110 \pm 5^{\circ} \mathrm{C}\right)$ and make a sieve analysis of the portion retained, using as many sieves as desired, or required for the material, or upon the specification of the material under test.

\section{CALCULATIONS AND REPORT}

12. Sieve Analysis Values for the Portion Coarser than the No. $10(2.00-\mathrm{mm})$ Sieve

12.1 Calculate the percentage passing the No. 10 sieve by dividing the mass passing the No. 10 sieve by the mass of soil originally split on the No. 10 sieve, and multiplying the result by 100 . To obtain the mass passing the No. 10 sieve, subtract the mass retained on the No. 10 sieve from the original mass.

12.2 To secure the total mass of soil passing the No. 4 $(4.75-\mathrm{mm})$ sieve, add to the mass of the material passing the No. 10 sieve the mass of the fraction passing the No. 4 sieve and retained on the No. 10 sieve. To secure the total mass of soil passing the $3 /$-in. (9.5-mm) sieve, add to the total mass of soil passing the No. 4 sieve, the mass of the fraction passing the $3 / 2$-in. sieve and retained on the No. 4 sieve. For the remaining sieves, continue the calculations in the same manner.

12.3 To determine the total percentage passing for each sieve, divide the total mass passing (see 12.2) by the total mass of sample and multiply the result by 100 .

\section{Hygroscopic Moisture Correction Factor}

13.1 The hydroscopic moisture correction factor is the ratio between the mass of the oven-dried sample and the air-dry mass before drying. It is a number less than one, except when there is no hygroscopic moisture.

\section{Percentages of Soil in Suspension}

14.1 Calculate the oven-dry mass of soil used in the hydrometer analysis by multiplying the air-dry mass by the hygroscopic moisture correction factor.

14.2 Calculate the mass of a total sample represented by the mass of soil used in the hydrometer test, by dividing the oven-dry mass used by the percentage passing the No. 10
TABLE 1 Vahos of Comection Fector, a, for Diflorom Specific Cravilies of Soll Partictes"

\begin{tabular}{cc}
\hline Specinc Grovity & Correctoon Factor \\
\hline 2.95 & 0.94 \\
2.90 & 0.95 \\
2.85 & 0.96 \\
2.80 & 0.97 \\
2.75 & 0.90 \\
2.70 & 0.99 \\
2.65 & 1.00 \\
2.60 & 1.01 \\
2.55 & 1.02 \\
2.50 & 1.03 \\
2.45 & 1.05 \\
\hline
\end{tabular}

$A$ For we in equetion for percentage of scil remeining in suspenseon whon usng Hycrometer 152H.

$(2.00-\mathrm{mm})$ sieve, and multiplying the result by 100 . This value is the weight $W$ in the equation for percentage remaining in suspension.

14.3 The percentage of soil remaining in suspension at the level at which the hydrometer is measuring the density of the suspension may be calculated as follows (Note 13): For hydrometer $151 \mathrm{H}$ :

$$
P=\left[(100000 / W) \times G /\left(G-G_{1}\right)\right]\left(R-G_{1}\right)
$$

Note 13-The bracketed portion of the equation for hydrometer $151 \mathrm{H}$ is consiant for a series of readings and may be calculated first and then multiplied by the portion in the parentheses.

For hydrometer $152 \mathrm{H}$ :

$$
P=(R a / W) \times 100
$$

where:

$a=$ correction faction to be applied to the reading of hydrometer 152H. (Values shown on the scale ar computed using a specific gravity of 2.65. Correction factors are given in Table 1),

$P=$ percentage of soil remaining in suspension at the level at which the hydrometer measures the density of the suspension,

$R=$ hydrometer reading with composite correction applied (Section 7),

$W=$ oven-dry mass of soil in a total test sample represented by mass of soil dispersed (see 14.2), $B$,

$G=$ specific gravity of the soil particles, and

$G_{l}=$ specific gravity of the liquid in which soil particles are suspended. Use numerical value of one in both instances in the equation. In the first instance any possible variation produces no significant effect, and in the second instance, the composite correction for $R$ is based on a value of one for $G_{1}$.

\section{Diameter of Soil Particles}

15.1 The diameter of a particle corresponding to the percentage indicated by a given hydrometer reading shall be calculated according to Stokes' law (Note 14), on the basis that a particle of this diameter was at the surface of the suspension at the beginning of sedimentation and had settled to the level at which the hydrometer is measuring the density of the suspension. According to Stokes' law:

$$
D=\sqrt{\left[30 n / 980\left(G-G_{1}\right)\right] \times L / T}
$$

where:

$D$ = diameter of particle, $\mathbf{m m}$, 


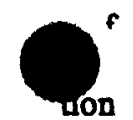

evel

the

ap-

pre-

; are

roth

any

and

Jr $R$

the

u be

zasis

the

tled

usity $n=$ coefficient of viscosity of the suspending medium (in this case water) in poises (varies with changes in temperature of the suspending medium),

$L=$ distance from the surface of the suspension to the level at which the density of the suspension is being measured, $\mathrm{cm}$. (For a given hydrometer and sedimentation cylinder, values vary according to the hydrometer readings. This distance is known as effective depth (Table 2)),

$T=$ interval of time from beginning of sedimentation to the taking of the reading, min,

$G=$ specific gravity of soil particles, and

$G_{1}=$ specific gravity (relative density) of suspending medium (value may be used as 1.000 for all practical purposes).

NoTE 14-Since Stokes' law considers the terminal velocity of a single sphere falling in an infinity of liquid, the sizes calculated represent the diameter of spheres that would fall at the same rate as the soil particles.

15.2 For convenience in calculations the above equation may be written as follows:

$$
D=K \sqrt{L / T}
$$

where:

$K=$ constant depending on the temperature of the suspension and the specific gravity of the soil particles. Values of $K$ for a range of temperatures and specific gravities are given in Table 3. The value of $K$ does not change for a series of readings constituting a test, while values of $L$ and $T$ do vary.

15.3 Values of $D$ may be computed with sufficient accuracy, using an ordinary 10 -in. slide rule.

Note 15 -The value of $L$ is divided by $T$ using the $A$ - and $B$-scales, the square root being indicated on the $D$-scale. Without ascertaining the value of the square root it may be multiplied by $K$, using either the $C$ - or Cl-scale.

\section{Sieve Analysis Values for Portion Finer than No. 10} (2.00-mm) Sieve

16.1 Calculation of percentages passing the various sieves used in sieving the portion of the sample from the hydrometer test involves several steps. The first step is to calculate the mass of the fraction that would have been retained on the No. 10 sieve had it not been removed. This mass is equal to the total percentage retained on the No. 10 sieve ( 100 minus total percentage passing) times the mass of the total sample represented by the mass of soil used (as calculated in 14.2), and the result divided by 100 .

16.2 Calculate next the total mass passing the No. 200 sieve. Add together the fractional masses retained on all the sieves, including the No. 10 sieve, and subtract this sum from the mass of the total sample (as calculated in 14.2).

16.3 Calculate next the total masses passing each of the other sieves, in a manner similar to that given in 12.2 .

16.4 Calculate last the total percentages passing by dividing the total mass passing (as calculated in 16.3) by the total mass of sample (as calculated in 14.2), and multiply the result by 100 .

\section{Graph}

17.1 When the hydrometer analysis is performed, a graph
TABLE 2 Vawes of Effective Depth Based on Hydrometer and Sedimentution Cylinder of Specified SizesA

\begin{tabular}{|c|c|c|c|c|c|}
\hline \multicolumn{2}{|c|}{ Hyorometer $151 \mathrm{H}$} & \multicolumn{4}{|c|}{ Hyorometer $152 \mathrm{H}$} \\
\hline $\begin{array}{l}\text { Acoued } \\
\text { Hycrometer } \\
\text { Rasading }\end{array}$ & $\begin{array}{l}\text { Efroctive } \\
\text { Depth. } \\
\text { L, cm }\end{array}$ & $\begin{array}{l}\text { Actud } \\
\text { Hycrometer } \\
\text { Reanding }\end{array}$ & $\begin{array}{l}\text { Eflectove } \\
\text { Depor. } \\
\text { L. con }\end{array}$ & $\begin{array}{l}\text { Actual } \\
\text { Hycrometer } \\
\text { Reading }\end{array}$ & $\begin{array}{l}\text { Eftective } \\
\text { Depts. } \\
\text { L. con }\end{array}$ \\
\hline $\begin{array}{l}1.000 \\
1.001 \\
1.002 \\
1.003 \\
1.004 \\
1.005\end{array}$ & $\begin{array}{l}16.3 \\
16.0 \\
15.8 \\
15.5 \\
15.2 \\
15.0\end{array}$ & $\begin{array}{l}0 \\
1 \\
2 \\
3 \\
4 \\
5\end{array}$ & $\begin{array}{l}16.3 \\
16.1 \\
16.0 \\
15.8 \\
15.6 \\
15.5\end{array}$ & $\begin{array}{l}31 \\
32 \\
33 \\
34 \\
35\end{array}$ & $\begin{array}{l}11.2 \\
11.1 \\
10.9 \\
10.7 \\
10.6\end{array}$ \\
\hline $\begin{array}{l}1.006 \\
1.007 \\
1.008 \\
1.009 \\
1.010\end{array}$ & $\begin{array}{l}14.7 \\
14.4 \\
14.2 \\
13.9 \\
13.7\end{array}$ & $\begin{array}{c}6 \\
7 \\
8 \\
9 \\
10\end{array}$ & $\begin{array}{l}15.3 \\
15.2 \\
15.0 \\
14.8 \\
14.7\end{array}$ & $\begin{array}{l}36 \\
37 \\
38 \\
39 \\
40\end{array}$ & $\begin{array}{c}10.4 \\
10.2 \\
10.1 \\
9.9 \\
9.7\end{array}$ \\
\hline $\begin{array}{l}1.011 \\
1.012 \\
1.013 \\
1.014 \\
1.015\end{array}$ & $\begin{array}{l}13.4 \\
13.1 \\
12.9 \\
12.6 \\
12.3\end{array}$ & $\begin{array}{l}11 \\
12 \\
13 \\
14 \\
15\end{array}$ & $\begin{array}{l}14.5 \\
14.3 \\
14.2 \\
14.0 \\
13.8\end{array}$ & $\begin{array}{l}41 \\
42 \\
43 \\
44 \\
45\end{array}$ & $\begin{array}{l}9.6 \\
9.4 \\
9.2 \\
9.1 \\
8.9\end{array}$ \\
\hline $\begin{array}{l}1.016 \\
1.017 \\
1.018 \\
1.019 \\
1.020\end{array}$ & $\begin{array}{l}12.1 \\
11.8 \\
11.5 \\
11.3 \\
11.0\end{array}$ & $\begin{array}{l}16 \\
17 \\
18 \\
19 \\
20\end{array}$ & $\begin{array}{l}13.7 \\
13.5 \\
13.3 \\
13.2 \\
13.0\end{array}$ & $\begin{array}{l}46 \\
47 \\
48 \\
49 \\
50\end{array}$ & $\begin{array}{l}8.8 \\
8.6 \\
8.4 \\
8.3 \\
8.1\end{array}$ \\
\hline $\begin{array}{l}1.021 \\
1.022 \\
1.023 \\
1.024 \\
1.025\end{array}$ & $\begin{array}{c}10.7 \\
10.5 \\
10.2 \\
10.0 \\
9.7\end{array}$ & $\begin{array}{l}21 \\
22 \\
23 \\
24 \\
25\end{array}$ & $\begin{array}{l}12.9 \\
12.7 \\
12.5 \\
12.4 \\
12.2\end{array}$ & $\begin{array}{l}51 \\
52 \\
53 \\
54 \\
55\end{array}$ & $\begin{array}{l}7.9 \\
7.8 \\
7.6 \\
7.4 \\
7.3\end{array}$ \\
\hline $\begin{array}{l}1.026 \\
1.027 \\
1.028 \\
1.029 \\
1.030\end{array}$ & $\begin{array}{l}9.4 \\
9.2 \\
8.9 \\
8.6 \\
8.4\end{array}$ & $\begin{array}{l}26 \\
27 \\
28 \\
29 \\
30\end{array}$ & $\begin{array}{l}12.0 \\
11.9 \\
11.7 \\
11.5 \\
11.4\end{array}$ & $\begin{array}{l}58 \\
57 \\
58 \\
59 \\
60\end{array}$ & $\begin{array}{l}7.1 \\
7.0 \\
6.8 \\
6.6 \\
6.5\end{array}$ \\
\hline $\begin{array}{l}1.031 \\
1.032 \\
1.033 \\
1.034 \\
1.035 \\
1.036 \\
1.037 \\
1.038\end{array}$ & $\begin{array}{l}8.1 \\
7.8 \\
7.6 \\
7.3 \\
7.0 \\
6.8 \\
6.5 \\
6.2\end{array}$ & & & & \\
\hline
\end{tabular}

4 Values of effective depth are calculated from the equation:

$$
L=L_{1}+V_{2}\left[L_{2}-\left(V_{1} / A\right)\right]
$$

where:

$L=$ effective depth, $\mathrm{cm}$,

$L_{1}$ - distance slong the reem of the bydrometer from the top of the bulb to th mark for a bydrometer reading $\mathrm{cm}$.

$L_{2}=$ overall length of the hydrometer bulb, $\mathrm{cm}$.

$V_{\mathrm{B}}=$ volume of hydrometer bulb, $\mathrm{cm}^{3}$, and

$A$ - crow-sectional erea of sedimentation cylinder, $\mathrm{cm}^{2}$

Values used in calculating the velues in Tabie 2 ere as follown:

For both mydromoters, $151 \mathrm{H}$ and $152 \mathrm{H}$ :

$L_{2}=14.0 \mathrm{~cm}$

$V_{1}=67.0 \mathrm{~cm}^{3}$

$A=27.8 \mathrm{~cm}^{2}$

For tyerometer $151 \mathrm{H}$ :

$L_{1}=10.5 \mathrm{~cm}$ for a rading of 1.000

$=2.3 \mathrm{~cm}$ for a readins of 1.031

For motromoter 152H:

$L_{1}=10.5 \mathrm{~cm}$ for a reading of $0 \mathrm{e}$ hitre

- $2.3 \mathrm{~cm}$ for a readins of 50 elitre

of the test results shall be made, plotting the diameters of th particles on a logarithmic scale as the abscissa and th percentages smaller than the corresponding diameters to ar 
TABLE 3 Vehes of $K$ for Une in Equation for Computing Diamoue of Partich in Hydrometer Analyais

\begin{tabular}{|c|c|c|c|c|c|c|c|c|c|}
\hline \multirow{2}{*}{$\begin{array}{c}\text { Temperatere. } \\
{ }^{\circ} \mathrm{C}\end{array}$} & \multicolumn{9}{|c|}{ Soecitic Grovity of Sal Perticios } \\
\hline & 2.45 & 2.50 & 2.55 & 2.60 & 2.65 & 2.70 & 2.75 & 2.80 & 2.85 \\
\hline $\begin{array}{l}16 \\
17 \\
18 \\
19 \\
20\end{array}$ & $\begin{array}{l}0.01510 \\
0.01511 \\
0.01492 \\
0.01474 \\
0.01456\end{array}$ & $\begin{array}{l}0.01505 \\
0.01486 \\
0.01467 \\
0.01449 \\
0.01431\end{array}$ & $\begin{array}{l}0.01481 \\
0.01462 \\
0.01445 \\
0.01425 \\
0.01408\end{array}$ & $\begin{array}{l}0.01457 \\
0.01439 \\
0.01421 \\
0.01408 \\
0.01386\end{array}$ & $\begin{array}{l}0.01435 \\
0.01417 \\
0.01399 \\
0.01382 \\
0.01355\end{array}$ & $\begin{array}{l}0.01414 \\
0.01396 \\
0.01378 \\
0.01361 \\
0.01344\end{array}$ & $\begin{array}{l}0.01394 \\
0.01376 \\
0.01359 \\
0.01342 \\
0.01325\end{array}$ & $\begin{array}{c}0.01374 \\
0.01356 \\
0.01339 \\
0.1323 \\
0.01307\end{array}$ & $\begin{array}{l}0.01356 \\
0.01338 \\
0.01321 \\
0.01305 \\
0.01289\end{array}$ \\
\hline $\begin{array}{l}21 \\
22 \\
23 \\
24 \\
25\end{array}$ & $\begin{array}{l}0.01438 \\
0.01421 \\
0.01404 \\
0.01388 \\
0.01372\end{array}$ & $\begin{array}{l}0.01414 \\
0.01397 \\
0.01381 \\
0.01385 \\
0.01349\end{array}$ & $\begin{array}{l}0.01391 \\
0.01374 \\
0.01358 \\
0.01342 \\
0.01327\end{array}$ & $\begin{array}{l}0.01369 \\
0.01353 \\
0.01337 \\
0.01321 \\
0.01305\end{array}$ & $\begin{array}{l}0.01348 \\
0.01332 \\
0.01317 \\
0.01301 \\
0.01286\end{array}$ & $\begin{array}{l}0.01328 \\
0.01312 \\
0.01297 \\
0.01282 \\
0.01287\end{array}$ & $\begin{array}{l}0.01309 \\
0.01294 \\
0.01279 \\
0.01264 \\
0.01249\end{array}$ & $\begin{array}{l}0.01291 \\
0.01276 \\
0.01261 \\
0.01246 \\
0.01232\end{array}$ & $\begin{array}{l}0.01273 \\
0.01258 \\
0.01243 \\
0.01229 \\
0.01215\end{array}$ \\
\hline $\begin{array}{l}26 \\
27 \\
28 \\
29 \\
30\end{array}$ & $\begin{array}{l}0.01357 \\
0.01342 \\
0.01327 \\
0.01312 \\
0.01298\end{array}$ & $\begin{array}{l}0.01334 \\
0.01319 \\
0.01304 \\
0.01290 \\
0.01276\end{array}$ & $\begin{array}{l}0.01312 \\
0.01297 \\
0.01283 \\
0.01289 \\
0.01256\end{array}$ & $\begin{array}{l}0.01291 \\
0.01277 \\
0.01264 \\
0.01249 \\
0.01236\end{array}$ & $\begin{array}{l}0.01272 \\
0.01258 \\
0.01244 \\
0.01250 \\
0.01217\end{array}$ & $\begin{array}{l}0.01253 \\
0.01239 \\
0.01255 \\
0.01212 \\
0.01199\end{array}$ & $\begin{array}{l}0.01235 \\
0.01221 \\
0.01206 \\
0.01195 \\
0.01182\end{array}$ & $\begin{array}{l}0.01218 \\
0.01204 \\
0.01191 \\
0.01178 \\
0.01165\end{array}$ & $\begin{array}{l}0.01201 \\
0.01188 \\
0.01175 \\
0.01162 \\
0.01149\end{array}$ \\
\hline
\end{tabular}

arithmetic scale as the ordinate. When the hydrometer analysis is not made on a portion of the soil, the preparation of the graph is optional, since values may be secured directly from tabulated data.

\section{Report}

18.1 The report shall include the following:

18.1.1 Maximum size of particles,

18.1.2 Percentage passing (or retained on) each sieve, which may be tabulated or presented by plotting on a graph (Note 16),

18.1.3 Description of sand and gravel particles:

18.1.3.1 Shape-rounded or angular,

18.1.3.2 Hardness-hard and durable, sof, or weathered and friable,

18.1.4 Specific gravity, if unusually high or low,

18.1.5 Any difficulty in dispersing the fraction passing the No. $10(2.00-\mathrm{mm})$ sieve, indicating any change in type and amount of dispersing agent, and

18.1.6 The dispersion device used and the length of the dispersion period.

NoTe 16-This tabulation of graph represents the gradation of the sample tested. If particles larger than those contained in the sample were removed before testing, the report shall so state giving the amount and maximum size.

18.2 For materials tested for compliance with definite specifications, the fractions called for in such specifications shall be reported. The fractions smaller than the No. 10 sieve shall be read from the graph.

18.3 For materials for which compliance with definite specifications is not indicated and when the soil is composed almost entirely of particles passing the No. $4(4.75-\mathrm{mm})$ sieve, the results read from the graph may be reported as follows:
(I) Gravel, pasing 3-in. and retained on No. 4 neve

(2) Sand, paring No. 4 sieve and retained on No. 200 sieve

(a) Coarse and pasting No. 4 sieve and retrined on No. 10 sieve

(b) Medium and, parsing No. 10 sieve and retained on No. 40 sieve

(c) Fine and paraing No. 40 sieve and retrined on No. $\ldots \ldots \ldots \ldots$ 200 sieve

(3) Silt size, 0.074 to $0.005 \mathrm{~mm}$

(d) Clay size, sinollex than $0.005 \mathrm{~mm}$

Colloids, smaller than $0.001 \mathrm{~mm}$

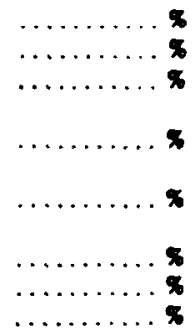

18.4 For materials for which compliance with definite specifications is not indicated and when the soil contains material retained on the No. 4 sieve sufficient to require a sieve analysis on that portion, the results may be reported as follows (Note 17):

\section{SIEVE ANALYSIS}

\section{Sieve Sire}

3-in.

2-in.

$1 y_{2}$-in.

1 -in.

*ain.

*-in.

No. $4(4.75-\mathrm{mm})$

No. $10(2.00-\mathrm{mm})$

No. $40(425-\mu m)$

No. $200(75-\mu \mathrm{m})$

\section{HYDROMETER ANALYSIS}

$0.074 \mathrm{~mm}$

$0.005 \mathrm{~mm}$

$0.001 \mathrm{~mm}$

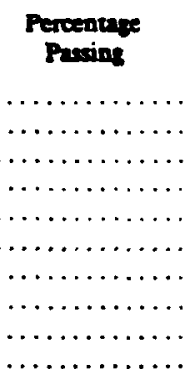

$\ldots \ldots \ldots \ldots$

NoTE 17-No. $8(2.36-\mathrm{mm})$ and No. $50(300-\mu \mathrm{m})$ sieves may be substituted for No. 10 and No. 40 sieves.

\section{Keywords}

19.1 grain-size; hydrometer analysis; hygroscopic moisture; particle-size; sieve analysis

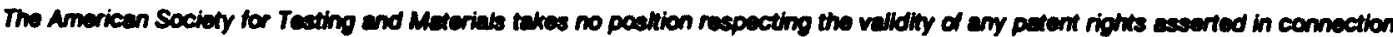

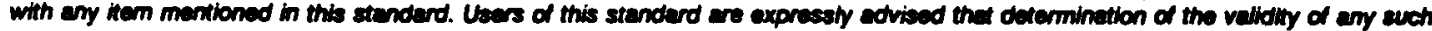
petert nights, and the sist of intringament of such rights, ere entiraly thair own responalbithy.
}

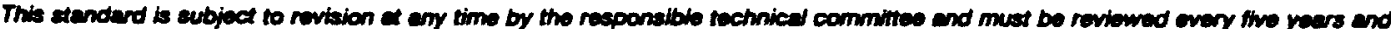

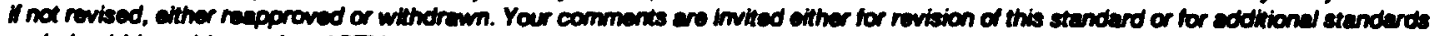

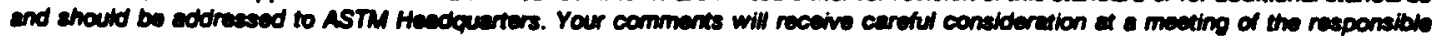

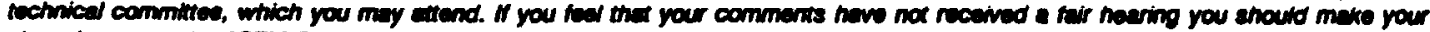
views known to the ASTM Comnitree on Stenderds, 1916 Rece ST., Philadelphile, PA 19103. 


\section{PROCESS DEVELOPMENT GROUP}

\section{Standard Operating Procedure}

Title:

Atterberg Limits: Liquid Limit and Plastic Limit

Prepared by:

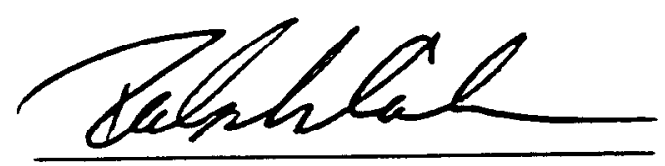

Date: $\quad 4-02-93$

Reviewed by:

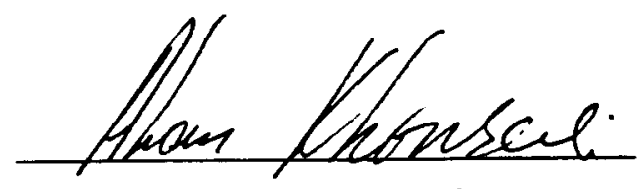

Date:

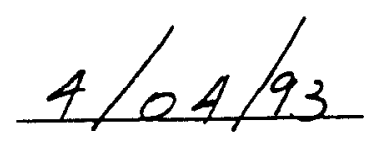

Operations approval:
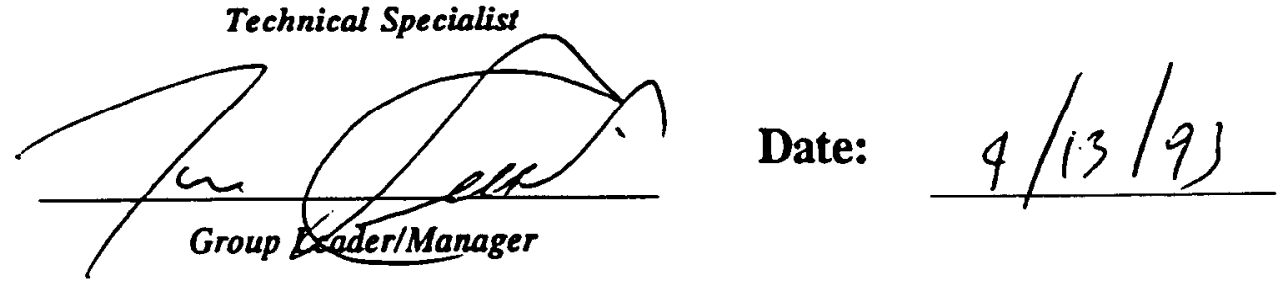

Interim approval:

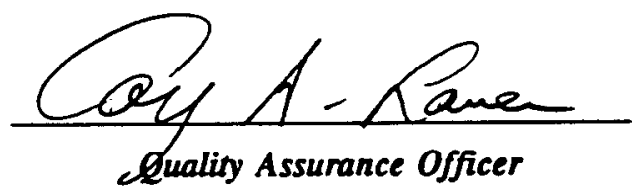

Date: $\quad 9 / 28 / 93$

Management approval:

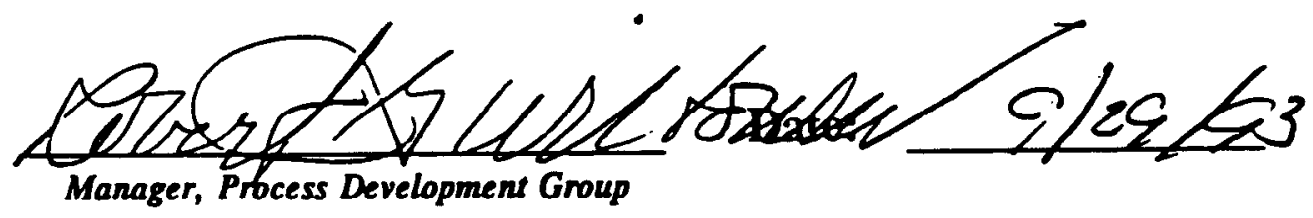

\section{APPROVED FOR INTERIMUSE}

\section{CONTROLLED COPY NO___}

Environmental Technology Development Center

P.O. Box 4339 - 1570 Bear Creek Road • Oak Ridge. Tennessee 37830 • 615-482-6497 • FAX: 615-482-1890 
SC? NO. PDGGE00021

REJISION NO.

DAIE: Sept. 1993

PAGE 2 OF 13

\section{Atterberg Limits: Liquid Limit and Plastic Limit}

\subsection{Scope and objectives}

1.1 This test method covers the determination of the Iiquid limit, plastic limit, and plasticity index of cohesive soils and is in general accordance with ASTM D 4318 .

1.2 The liquid limit, plastic limit, and plasticity index define the boundaries of consistency for plastic soils and are primary tools for soil classification.

1.3 This test method requires judgements be made by the person performing the tests based on previous experience with similar soils. This procedure assumes that the person performing the test has attained a degree of competence beyond the entry level in geotechnical testing procedures. Persons who are not familiar with the equipment or general procedures contained herein shall not perform this procedure without direct supervision of a qualified associate.

1.4 Values stated in SI units shall be regarded as standard except as noted.

\subsection{Definitions}

2.1 ASTM - American Society of Testing and Materials

2.2 Atterberg Limits - originally, seven "limits of consistency" of fine-grained soils were defined by Albert Atterberg. Current usage of the term refers only to liquid limit, plastic limit, and occasionally, shrinkage limit.

2.3 Consistency - The relative ease with which a soil can be deformed.

2.4 Liquid Limit (LL) - The liquid limit of a soil is defined as the moisture content defining the boundary between the liquid and plastic states of the soil.

2.5 Plastic Limit (PL) - The plastic limit is defined as the moisture content defining the boundary between the plastic and brittle solid states of the soil.

2.6 Plastic soil - ${ }^{-}$A soil which exhibits plasticity over a wide range of moisture contents and will retain its 
2.7 Plasticity Index (PI) - The plasticity index is defined as the range of water contents over which the soil exhibits plasticity.

3.0 Methodology

\subsection{Summary}

3.1.1 The soil sample is processed to remove all particles coarser than the No.40 (425 micron) sieve. The liquid limit is determined by spreading the moist sample in a standard brass cup, dividing the soil pat using a grooving tool, and dropping the brass cup repeatedly on a standard base material from a standard height. The number of drops necessary to close the groove in the soil pat is recorded and the moisture content of the soil pat determined. Trials are performed until the groove closes over a $\frac{1}{2}$ inch width at 20-30 drops of the brass cup and a correction factor applied to the result to determine the moisture content corresponding to a blow count of 25. An alternative method determines the moisture content corresponding to the 25-blow intercept of a bestfit line drawn through the results of three trials at differing blow counts. The moisture content corresponding to groove closure at 25 blows is taken as the liquid limit.

3.1.2 The plastic limit is determined by rolling an ellipsoidal mass of soil into $/ / 6$-inch diameter threads on a glass plate. The soil is pressed back together and re-rolled until the soil crumbles before reaching a diameter of $y$-inch. The plastic limit is calculated as the moisture content at which the soil thread crumbles.

3.1.3 The plasticity index is numerically equal to the difference between the liquid limit and the plastic limit.

3.2 Interferences or Potential Problem Areas - Soils that are prepared by initial oven-drying of the material may alter the Atterberg results of naturally occurring soils.

\subsection{Sample Requirements and Handling}

3.3.1 All samples submitted for analysis must yield approximately 200-300 grams of material finer than the No.40 (425 micron) sieve. Because only the soil 
SOP NC. PDGGEOOO21

REVIS:ON NO. OO

DATE: Sept. 1993

PAGE 4 OF 13

fraction finer than the No.40 sieve is used to determine the Atterberg limits, coarse-grained soils require substantially greater initial sample sizes than do predominately clay samples.

3.3.2 Samples may be sealed against moisture loss and stored for up to six months in an area not subject to temperature extremes (lower than $1^{\circ} \mathrm{C}$ or higher than $35^{\circ} \mathrm{C}$ ).

\subsection{Required Equipment and Reagents}

3.4.1 Balance - An electronic or mechanical balance accurate to 0.02 grams or $\pm 0.1 \%$, whichever is greater.

3.4.2 Drying oven - An oven, either gravity or forcedconvection, capable of maintaining an internal temperature of $110 \pm 5{ }^{\circ} \mathrm{C}$.

3.4.3 Liquid Limit Device - Liquid limit trials are performed using a mechanical device consisting of a brass cup suspended from a carriage designed to control the drop of the cup onto a hard synthetic base. The device may be manually operated or powered by an electric motor. ELE/Soiltest models CL-204, CL-205, or equivalent are suitable.

3.4.4 Grooving Tool - A grooving tool made of noncorrosive metal is used to place a groove in the soil pat. The tool may be either the Cassagrande flat-type or the wedge-type as specified by USACE EM-1110-2-1906 Appendix III (respectively, ELE/Soiltest model $C L-220$ and model CL-218A, or equivalent). The consistency of the soil will determine the choice of tool for a particular soil.

3.4.5 Gauge Block - A metal $10 \pm 0.05 \mathrm{~mm}$ thick is used to determine the height of drop of the brass cup. The gauge block may be incorporated into the grooving tool,

3.4.6 Porcelain Dish - A porcelain dish approximately 5-6 inches in diameter is used to $\mathrm{mix}$ and store the soil while curing.

3.4.7 Spatula - A spatula with a metal blade approximately $\frac{3}{4}$ inches wide and 4-5 inches long is used to mix and spread the soil in the brass cup. 
3.4.8 Ground Glass Plate - A glass plate approximately 12 inches square and $\frac{1}{4}$ inch thick with a sandblasted or ground upper surface is used for rolling plastic limit material.

3.4.9 Sieve - An 8 inch diameter No.40 (425 micron) stainless steel mesh sieve is used to separate coarse particles from the test soil.

3.4.10 water - All water shall be ASTM TYpe II or better.

3.4.11 Miscellaneous equipment - Miscellaneous equipment, including but not limited to, moisture tins, wash bottle, rubber spatula, etc., as required.

3.5 Calibration Requirements

3.5.1 Balance calibrations shall be verified annually in accordance with SOP PDGADMO002.

3.5.2 Liquid limit devices shall be calibrated annually in accordance with SOP PDGGEO0022, and daily or before use, whichever is greater, as follows: Lift the cup by turning the hand crank until the cup follower is at the top of the cam. Insert a $10 \mathrm{~mm}$ gauge block between the cup and base. Turn the hand crank several times. If the cup does not rise the drop height is too low. If the cup rises and drops, producing a loud ringing sound, the height of the drop is too high. Adjust the cup drop height to 10 $\pm 0.2 \mathrm{~mm}$, if needed, by loosening the adjustment screw and moving the carriage in or out as necessary to produce the desired drop height. Tighten the adjustment screw and recheck the drop height. The drop height is right if a soft click is heard while turning the hand crank with the gauge block in position. The drop height may also be verified by raising the cup, inserting the gauge block, and visually, or with feeler gauges, determining the height of the cup above the gauge block.

\subsection{Quality Control Requirements}

3.6.1 Balances - Brass weights shall be used to verify the accuracy of each balance before use or daily, whichever is less, and the result recorded in the balance log book. Brass weights shall meet OIML Class $M_{2}$ specifications or better and shall be checked monthly in accordance with SOP PDGADMO003. 
SOP NO. PDGGEOOO2I

REVISIC N NO. 00

DATE: Sept. 1993

PAGE 6 OF 13

3.6.2 Ovens - Temperatures of ovens in use shall be recorded daily in accordance with SOP PDGADMO006.

3.6.3 Grooving tools shall be checked annually for proper dimensions and excessive tip wear.

3.6.4 Sieves shall be maintained in accordance with sop PDGGEO0023.

3.6.5 Nonconformance/variance reports - In accordance with SOP PDGADM0009, nonconformance/variance reports shall be used to document any discrepancies in the performance or specifications of this procedure.

3.7 Health and Safety - Sample material containing hazardous, radiological, or mixed contaminants shall be handled in accordance with current facility Health and safety directives. Engineering controls shall be used whenever possible to reduce personnel exposure to sample hazards ( $i . e .$, sample prepared inside vented hood space, sample extruder enclosure adequately ventilated, etc.). Personal protective equipment shall be used as necessary to prevent personnel exposure to hazards. As a minimum, appropriate protective gloves shall be worn by personnel handling contaminated sample material, in addition to safety glasses required of all personnel in the laboratory at all times. When in doubt as to the hazards involved in any operation, consult with the Geotechnical Laboratory Manager or Health and Safety officer before proceeding.

\subsection{Specimen Preparation}

3.8.1 The laboratory manager or client shall specify the specimen preparation method to be used from one of the following methods:

3.8.1.1 Remove a portion of the soil to be tested and push through the No.40 sieve. Discard coarse particles retained on the sieve.

3.8.1.2 Air-dry the soil to be tested until friable. Grind in a mortar using a rubber-covered pestle and pass the material over the No.40 sieve. Regrind material retained on the sieve until no appreciable soil passes the sieve.

3.8.1.3 When initial sample size is small and additional analyses are requested (moisture content, particle-size, specific gravity, 
etc.), oven-dry the entire sample and remove material finer than the No.40 sieve from the soil not used in the other analyses. Ovendrying may alter the test results for certain soils. This procedure shall be used with caution.

3.8.2 Thoroughly mix the soil passing the No.40 sieve in the porcelain dish and wet or dry the soil to a moisture content that will allow groove closure at 25-30 drops of the liquid limit cup. Store the specimen in a covered container for at least 16 hours or overnight to allow all soil particles to achieve moisture equilibrium.

\subsection{One-point Liquid Limit}

3.9.1 After the soil has seasoned for at least 16 hours, thoroughly remix the soil to be tested. Using the metal spatula, press the soil into the brass cup taking care to avoid entrapping air. Spread the soil to a thickness of approximately $10 \mathrm{~mm}$ using as few strokes of the spatula as possible. The upper surface of the soil pat in the cup shall be essentially flat and horizontal.

3.9.2 Bisect the soil pat from the highest point to the lowest point of the cup using the grooving tool. Draw the grooving tool beveled edge first in an arc, maintaining the tool handle perpendicular to the cup. If the soil cannot be cut in one stroke of the grooving tool without tearing, several strokes may be used in alternating directions and at increasing cutting depths. Take care to prevent sliding of the soil pat against the bottom of the cup.

3.9.3 Ensure that the bottom of the cup and surface of the base are free of debris. Raise and drop the cup by turning the handle at a rate of approximately 2 drops per second until the two halves of the soil pat come into contact along a distance of $\frac{1}{2}$ inch.

3.9.4 Verify the validity of the trial by observing that both halves of the soil pat have flowed approximately equal amounts. A bulge in one half of the soil pat probably indicates the presence of entrapped air and is justification for a retrial. Likewise, ensure that the soil has flowed and not slipped along the soil/metal boundary of the cup. If a retrial is necessary, remove the soil from the 
SOP NO. F JGGEO0021

REVISION NO. 00

DATE: $\quad \leqslant$ ept. 1993

PAGE 8 OF 13

3.9.5 If the number of blows necessary to close the groove along a distance of $\frac{1}{2}$ inch is more than 30 , add a small amount of water to the soil and $\mathrm{mix}$ thoroughly. Repeat trials at successively higher moisture contents until the number of blows required to close the groove along a distance of $\frac{1}{2}$ inch is between 20 and 30 drops.

3.9.6 If the number of blows necessary to close the groove along a distance of $\frac{1}{2}$ inch is less than 20 , spread the soil in a thin layer on the ground glass plate and allow to dry. Frequent stirring is required to ensure uniform drying and at no time shall the soil be allowed to crust. Repeat trials at successively lower moisture contents until the number of blows required to close the groove along a distance of $\frac{3}{2}$ inch is between 20 and 30 drops.

3.9.7 Once groove closure has been established at a blow count of 20-30 blows, record the number of blows. Remove a section of soil from the cup approximately $\frac{1}{2}$ inch wide and running the breadth of the cup. Place this section in a moisture tin and perform a moisture content analysis in accordance with sop PDGGEO0019.

3.9.8 Perform another trial at the same moisture content to verify the result. If the second attempt yields a drop count differing by more than 2 blows, remix the entire soil portion and perform additional trials until two successive trials yield blow counts not differing by more than two blows. Some soils require lengthy mixing to achieve duplicable results.

\subsection{Multi-Point Liquid Limit}

3.10.1 Considerable judgement may be necessary when adjusting the moisture content of the soil to the desired consistency and in recognizing potential problems as they occur. Inexperienced operators will usually benefit from performing the multipoint test.

3.10.2 Proceed as in 3.9 except adjust the initial moisture content of the specimen to 25-35 blows during initial mixing. A minimum of three trials 
covering a blow count range of 15 to 35 are required. One trial shall be performed resulting in a blow count of 25-35, another trial resulting in a blow count of 20-30, and another trial resulting in a blow count of 15-25. Begin trials with the blow count in the range of 25-35. Add small amounts of water to the soil and mix thoroughly between trials.

\subsection{Plastic Iimit}

3.11.1 Remove a portion of soil used in the liquid limit test weighing approximately 30 grams. This material may be obtained after the initial mixing or after completion of the liquid limit test. For expediency, the soil should not be so moist as to be sticky but should deform easily under light finger pressure without cracking. Divide this portion into two approximately equal portions and put one half into an air-tight container or cover with a moist towel.

3.11.2 Form the soil into an ellipsoidal mass and roll on the ground glass plate into a $1 / 6$ inch diameter thread. Regroup all the thread sections and press together into an ellipsoidal mass. Continue rolling and regrouping the threads until the soil begins to fracture and crumble before it reaches a diameter of $\%$ inch. When the amount of fractured soil exceeds one-half of the total amount being tested, place all thread sections and crumbs in a moisture tin and perform a moisture content determination in accordance with SOP PDGGEO0019.

3.11.3 Perform the same procedure of rolling and rerolling for the duplicate portion set aside above. Perform plastic limit tests until two results differ by no more than $1.5 \%$ moisture content.

\subsection{Calculations}

3.12.1 Calculate the one-point liquid limit, $L L$, as follows:

$$
L L=K\left(W_{N}\right)
$$

where:

$$
\begin{aligned}
& K . \quad=\text { a factor given in Table } 1 \\
& W_{N}=\text { water content corresponding to } N \\
& N=\text { number of blows }
\end{aligned}
$$


SOP NO. PDGGEOOO21

REVISION NO. 00

DATE: Sept. 1993

PAGE 10 OF 13

The liquid limit is the average of the two trial liquid limit values. Report results to the nearest whole number.

3.12.2 Multi-point liquid limit - Plot the relationship between the moisture content of the liquid limit specimens to the number of blows on a semilogarithmic graph with moisture content in percent on the abscissa and the number of blows on the logarithmic ordinate. Draw a best fit straight line through the points. Take the moisture content corresponding to the 25 blow intercept as the liquid limit.

3.12.3 The plastic limit, $P L$, is equal to the average moisture content of the plastic limit trials.

3.12.4 Calculate the plasticity index, PI, as follows: $P I=L L-P L$

where:

$$
\begin{aligned}
& I L=\text { liquid limit } \\
& P L=\text { plastic limit }
\end{aligned}
$$

3.12.5 Report liquid limit and plasticity index results rounded to whole numbers unless otherwise requested by the client. Plastic limit is not normally reported.

4.0 Forms - A copy of the data form used in conjunction with this procedure is included in Appendix $A$.

5.0 References

5.1 ASTM Procedure: D 4318 - Liquid Limit, Plastic Limit, and Plasticity Index of Soils

5.2 United States Army Corps of Engineers Procedure: EM-1110-2-1906, Appendix III, Liquid and Plastic Limits

5.3 IT standard Operating Procedures

5.3.1 PDGADM0002 - Balance Calibration and Maintenance

5.3.2 PDGADMO003 - Use and Maintenance of Class $S$ and Brass Weights

5.3.3 PDGADMOOO6 - Verification of Oven and Refrigerator Temperatures 
SOP NO. PDGGEOOO21 REVISIOI. NO.

00

DATE:

sept. 1993

PAGE

13 OF 13 
APPENDIX A

DATA COLLECTION FORM 
SOP NO. PDGGEO0021

REVI: ION NO.

DATE:

Sept. 1993

PAGE

11 OF 13

5.3.4 PDGADMO009 - Nonconformance/Variance Reports

5.3.5 PDGGEO0019 - Moisture Content of Soil and Rock

5.3.6 PDGGE00022 - Calibration and Inspection of Liquid Limit Device

5.3.7 PDGGEO0023 - Sieve Specification, Calibration, and Inspection 
SOP NO. PDGGEOOO21

REV: SION NO. 00

DATE : Sept. 1993

PAGE 12 OF 13

\section{TABLE I}

Factors for Obtaining Liquid Limit from Water Content and Number of Drops at Groove Closure

\begin{tabular}{|c|c|}
\hline $\begin{array}{c}\text { Number of Drops } \\
\text { (Factor for Liquid Limit) }\end{array}$ & R \\
\hline 20 & 0.974 \\
\hline 21 & 0.979 \\
\hline 22 & 0.985 \\
\hline 23 & 0.990 \\
\hline 24 & 0.995 \\
\hline 25 & 1.000 \\
\hline 26 & 1.005 \\
\hline 27 & 1.009 \\
\hline 28 & 1.014 \\
\hline 29 & 1.018 \\
\hline 30 & 1.022 \\
\hline
\end{tabular}


SOP NO. PDGGEO0021

REVISIC S NO. 00

DATE: Sept. 1993

PAGE 13 OF 13

ITT NTERNATONAL TECHNOLOGY CORPORATION
Specimon Prep by:

Terted by

Celc. Entry by:

Checked by:
Dete
Dere
Dete
Dete

\section{ATTERBERG LIMITS}

ASTM D 4318 SOP PDGGEOOOZ1

Project

Project No.

Sample No.

\begin{tabular}{|l|c|c|c|c|c|}
\hline Test No. & 1 & 2 & 3 & 4 & 5 \\
\hline Blow Range & $25-35$ & $20-30$ & $15-25$ & & \\
\hline Tare No. & & & & & \\
\hline Tare Wt. g & & & & & \\
\hline Wt Tare + Wet Soil,g & & & & & \\
\hline Wt Tare + Dry Soil, g & & & & & \\
\hline Wt of Water, g & & & & & \\
\hline Wt of Dry Soil, g & & & & & \\
\hline Water Content, \% & & & & & \\
\hline No. Blows & & & & & \\
\hline N Factor (if used) & & & & & \\
\hline Liquid Limit & & & & & \\
\hline
\end{tabular}

\begin{tabular}{|l|c|c|c|}
\hline \multicolumn{3}{|c|}{ PLASTIC LIMIT } & 1 \\
\hline Test No. & 1 & 2 & 3 \\
\hline Tare Name & & & \\
\hline Tare Wt. $g$ & & & \\
\hline Wt Tare + Wet Soil, g & & & \\
\hline Wt Tare + Dry Soil, $g$ & & & \\
\hline Wt of Water, 9 & & & \\
\hline Wt of Dry Soil, $g$ & & & \\
\hline Water Content, \% & & & \\
\hline
\end{tabular}

RESULTS SUMMARY

\section{LIQUID LIMIT}

PLASTIC LIMIT

PLASTICITY INDEX

USCS SYMBOL
FLOW CHART

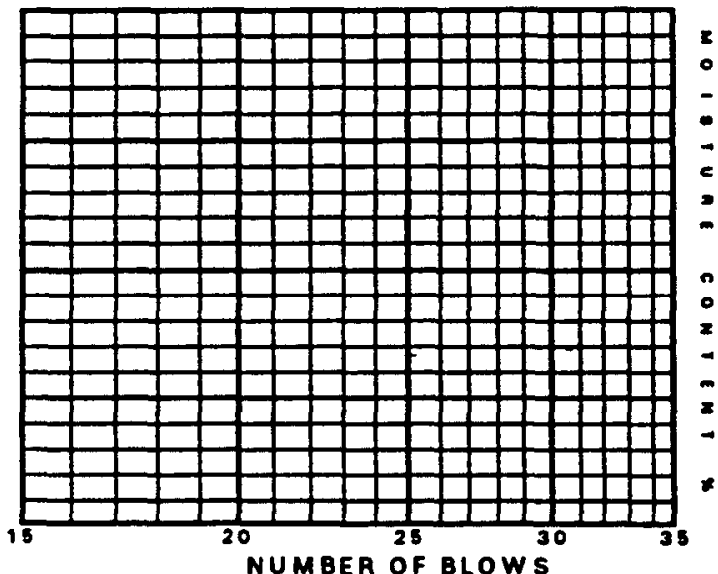

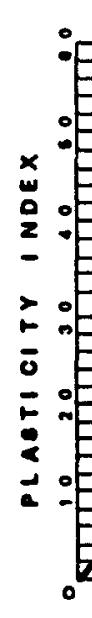

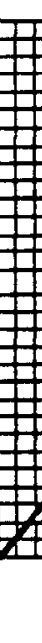

PLASTICITY CHART

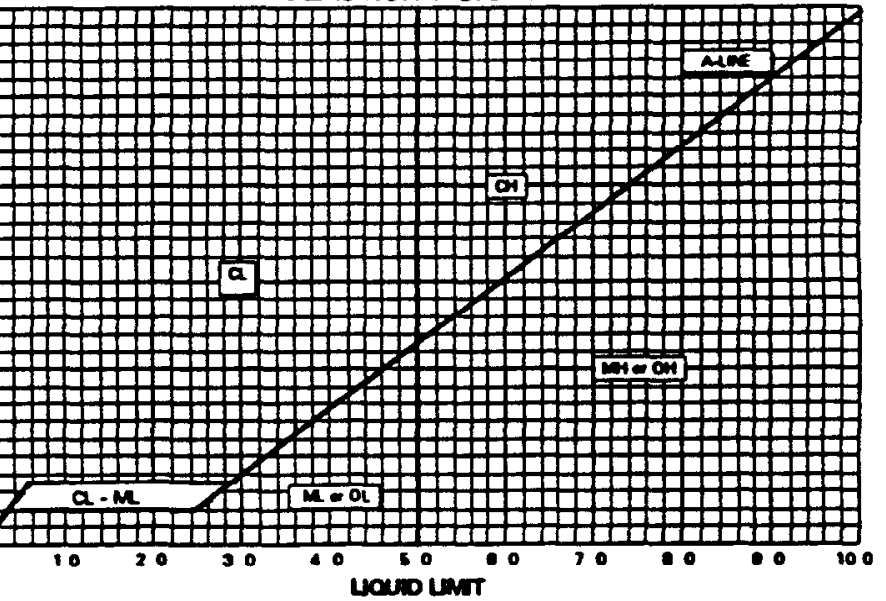




\title{
Standard Test Method for

\begin{abstract}
This andind is imed under the fxed designetion D 4318; the number immedintely following the desionstion indicates the year of

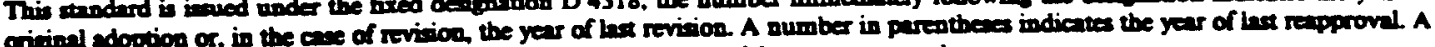

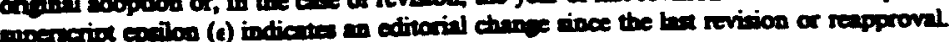

\section{Scope}

F.1.1 This test method covers the determination of the tuid limit, plastic limit, and the plasticity index of soils as 3. fined in Section 3.

1.1.1 Two procedures for preparing test specimens are tivided as follows: Wet preparation procedure, as described 3i10.1. Dry preparation procedure, as described in 10.2. The tibcedure to be used shall be specified by the requesting fithority. If no procedure is specified, use the wet preparaiton procedure.

1.1.2 Two methods for determining the liquid limit are Tovided as follows: Method A, Multipoint test as described

7 Sections 11 and 12. Method B, One-point test as described in Sections 14 and 15 . The method to be used shall be tooified by the requesting authority. If no method is fivecified, use Method A.

FiY.1.3 The plastic limit test procedure is described in Uctions 16, 17, and 18. The plastic limit test is performed on ifterial prepared for the liquid limit test.

M.1.4 The procedure for calculating the plasticity index is 1 tren in Section 19.

1.2 The liquid limit and plastic limit of soils (along with inte shrinkage limit) are often collectively referred to as the 7 titerberg limits. These limits distinguished the boundaries of the several consistency states of plastic soils.

o1:3 The multipoint hquid limit method is generally more Thecise than the one-point method. It is recommended that Whe multipoint method be used in cases where results may be fibject to dispute, or where greater precision is required.

f 511.4 Because the one-point method requires the operator to 3 fidge when the test specimen is approximately at its liquid timit, it is particularly not recommended for use by inexpeFitenced operators.

fil:5 The correlations on which the calculations of the Whie-point method are based may not be valid for certain 3 foils, such as organic soils or soils from a marine environFif It is strongly recommended that the liquid limit of Mhese soils be determined by the multipoint method.

4. 1.6 The liquid and plastic limits of many soils that have Feen allowed to dry before testing may be considerably 3iffiferent from values obtained on undried samples. If the ff. fquid and plastic limits of soils are used to correlate or the engineering behavior of soils in their natural at?

307 This test method is under the juriadiction of ASTM Commitice D-18 on Soil 2. Rock and is the direce responsibility of Subcommittec D18.03 on Texture, Whicity and Density Chancteristice of Soits

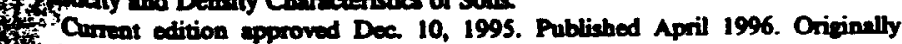
Fintiatud as D 4318 - 83. Lat previous edtition D 4318 - 95 .

- A Summary of Changes section appears at the ead of standard. moist state, samples should not be permitted to dry before testing unless dath on dried samples are specifically desired.

1.7 The composition and concentration of soluble salts in a soil affect the values of the liquid and plastic limits as well as the water content values of soils (see Method D 2216). Special consideration should therefore be given to soils from a marine environment or other sources where high soluble salt concentrations may be present. The degree to which the salts present in these soils are diluted or concentrated must be given careful consideration.

1.8 Since the tests described herein are performed only on that portion of a soil which passes the $425-\mu \mathrm{m}$ (No. 40) sieve, the relative contribution of this portion of the soil to the properties of the sample as a whole must be considered when using these tests to evaluate properties of a soil.

1.9 The values statod in acceptable metric units are to be regarded as the standard. The values given in parentheses are for information only.

1.10 This standard does not purpon 10 address all of the safety concerns, if any, associated with its use. It is the responsibility of the user of this standard to establish appropriate safety and health practices and determine the a bility of regulatory limitations prior to use.

\section{Referenced Documents}

2.1 ASTM Standards:

C 670 Practice for Preparing Precision and Bias Statements for Test Methods for Construction Materials ${ }^{2}$

C 702 Methods for Reducing Field Samples of Aggregate to Testing Size ${ }^{3}$

D75 Practice for Sampling Aggregates ${ }^{2}$

D420 Practice for Investigating and Sampling Soil and Rock for Engineering Purposes 2

D653 Terminology Relating to Soil, Rock, and Contained Fluids ${ }^{2}$

D 1241 Specification for Materials for Soil-Aggregate Subbase, Base, and Surface Courses ${ }^{2}$

D2216 Test Method for Laboratory Determination Water (Moisture) Content of Soil and Rock ${ }^{2}$

D2487 Classification of Soils for Engineering Purposen (Unified Soil Classification System) ${ }^{2}$

D2488 Practice for Description and Identification of Soils (Visual-Manual Procedure)

D 3282 Practice for Classification of Soils and Soil-Ag. gregate Mixtures for Highway Construction Purposes ${ }^{3}$

2 Anumal Book of ASTM Standards, Vol 04.02

innual Book of ASTM Standards, Vol 04.08. 
D4753 Specification for Evaluating. Selecting. and Specifving Balances and Scales for Use in Soil and Rock Testing ${ }^{3}$

E 11 Specification for Wire-Cloth Sieves for Testing Purposes ${ }^{2}$

\section{Terminology}

\subsection{Definitions:}

3.1.1 The definitions of terms in this test method are in accordance with Terminology D 653 .

\subsection{Description of Terms Specific to This Standard:}

3.2.1 Atterberg limits-Originally, six "limits of consistency" of fine-grained soils were defined by Albern Atterberg: the upper limit of viscous flow, the liquid limit. the sticky limit, the cohesion limit. the plastic limit, and the shrinkage limit. In current engineering usage, the term usually refers only to the liquid limit, plastic limit, and in some references, the shrinkage limit.

3.2.2 consistency - the relative ease with which a soil can be deformed.

3.2.3 liquid limit $\left(L L, w_{V}\right)$-the water content, in percent, of a soil at the arbitrarily defined boundary between the semi-liquid and plastic states.

3.2.3.1 Discussion-The undrained shear strength of soil at the liquid limit is considered to be approximately $2 \mathrm{kPa}$ (0.28 psi).

3.2.4 plastic limit ( $\left.P L, w_{p}\right)$-the water content, in percent, of a soil at the boundary between the plastic and semi-solid states.

3.2.5 plastic soil-a soil which has a range of water content over which it exhibits plasticity and which will retain its shape on drying.

3.2.6 plasticity index ( $P I)$ - the range of water content over which a soil behaves plastically. Numerically, it is the difference between the liquid limit and the plastic limit.

3.2.7 liquidity index - the ratio, expressed as a percentage of (1) the natural water content of a soil minus its plastic limit, to (2) its plasticity index.

3.2.8 activity number $(A)$-the ratio of (1) the plasticity index of a soil to (2) the percent by weight of particles having an equivalent diameter smaller than $0.002 \mathrm{~mm}$.

\section{Summary of Test Method}

4.1 The sample is processed to remove any material retained on a $425-\mu \mathrm{m}$ (No. 40 ) sieve. The liquid limit is determined by performing trials in which a portion of the sample is spread in a brass cup, divided in two by a grooving tool, and then allowed to flow together from the shocks caused by repeatedly dropping the cup in a standard mechanical device. The multipoint liquid limit, Method A. requires three or more trials over a range of water contents to be performed and the data from the trials plotted or calculated to make a relationship from which the liquid limit is determined. The one-point liquid limit, Method B, uses the data from two trials at one water content multiplied by a correction factor to determine the liquid limit.

4.2 The plastic limit is determined by alternately pressing together and rolling into a $3.2-\mathrm{mm}$ ( $1 / 8$-in.) diameter thread a small portion of plastic soil until its water content is reduced to a point at which the thread crumbles and can no longer be pressed together and rerolled. The water content of the soil at this point is reponed as the plastic limit.

4.3 The plasticity index is calculated as the differen is between the liquid limit and the plastic limit.

\section{Significance and Use}

5.1 This test method is used as an integral pan of severa engineering classification systems to characterize the fine. grained fractions of soils (see Test Method D 2487 and Practice D 3282) and to specify the fine-grained fraction of construetion materials (see Specification D 1241). The liquid limit. plastic limit, and plasticity index of soils are also used extensively, either individually or together. with other soil properties to correlate with engineering behavior such as compressibility, permeability. compactibility. shrink-swell and shear strength.

5.2 The liquid and plastic limits of a soil can be used with the natural water content of the soil to express its relative consistency or liquidity index and can be used with the percentage finer than $2-\mu \mathrm{m}$ size to determine its activily number.

5.3 These methods are sometimes used to evaluate the weathering characteristics of clay-shale materials. Wheo subjected to repeated wetting and drying cycles, the liquid limits of these materials tend to increase. The amount of increase is considered to be a measure of a shale's susceptibility to weathering.

5.4 The liquid limit of a soil containing substantial amounts of organic matter decreases dramatically when the soil is oven-dried before testing. Comparison of the liquid limit of a sample before and after oven-drying can therefore be used as a qualitative measure of organic matter content of a soil.

\section{Apparatus}

6.1 Liquid Limit Device-A mechanical device consisting of a brass cup suspended from a carriage designed to control its drop onto a hard nubber base. Figure 1 shows the essential features and critical dimensions of the device. The device may be operated by either a hand crank or electric motor.

6.1.1 Base-A hard rubber base having a D Durometer hardness of 80 to 90 , and a resilience such that an $8-\mathrm{mm}$ (5/16-in.) diameter polished steel ball, when dropped from a height of $25 \mathrm{~cm}$ (9.84 in.) will have an average rebound of at least $77 \%$ but no more than $90 \%$. Conduct resilience tests on the finished base with the feet attached. Details for measuring the resilience of the base are given in Appendix A.

6.1.2 Rubber Feet, supporting the base, designed to provide isolation of the base from the work surface, and having an $A$ Durometer hardness no greater than 60 as measured on the finished feet attached to the base.

6.1.3 Cup, brass, with a weight, including cup hanger, of 185 to $215 \mathrm{~g}$.

6.1.4 Cam-designed to raise the cup smoothly and continuously to its maximum height, over a distance of at least $180^{\circ}$ of cam rotation, without developing an upward or downward velocity of the cup when the cam follower leavesthe cam. (The preferred cam motion is a uniformly accelerated lift curve.)

Note 1 -The cam and follower design in Fig. 1 is for uniformly accelerated (parabolic) motion after contact and assures that the cup hos no velocity at drop off. Other cam designs also provide this feature and 


\begin{tabular}{|c|c|c|c|c|c|c|c|c|c|c|c|}
\hline ETER & $A^{8}$ & $B^{2}$ & $c^{2}$ & $E$ & $F$ & G & $H$ & $J^{2}$ & $k^{2}$ & $L^{2}$ & $M^{2}$ \\
\hline th & $\begin{array}{l}54 \\
\pm 0.5\end{array}$ & $\begin{array}{l}2 \\
\pm 0.1\end{array}$ & $\begin{array}{l}27 \\
\pm 0.5\end{array}$ & $\begin{array}{l}56 \\
\pm 2.0\end{array}$ & 32 & 10 & 16 & $\begin{array}{l}60 \\
+1.0\end{array}$ & $\begin{array}{l}50 \\
\pm 2.0\end{array}$ & $\begin{array}{l}150 \\
\pm 2.0\end{array}$ & $\begin{array}{l}125 \\
\pm \quad 2.0\end{array}$ \\
\hline IETTER & $N$ & $\boldsymbol{P}$ & $\boldsymbol{R}$ & $T$ & $u^{8}$ & $V$ & $w$ & 2 & & & \\
\hline 19 & 24 & 28 & 24 & 45 & $\begin{array}{l}47 \\
\pm 1.0\end{array}$ & 3.8 & 13 & 6.5 & & & \\
\hline
\end{tabular}

ESSENTIAL DIMENSIONS
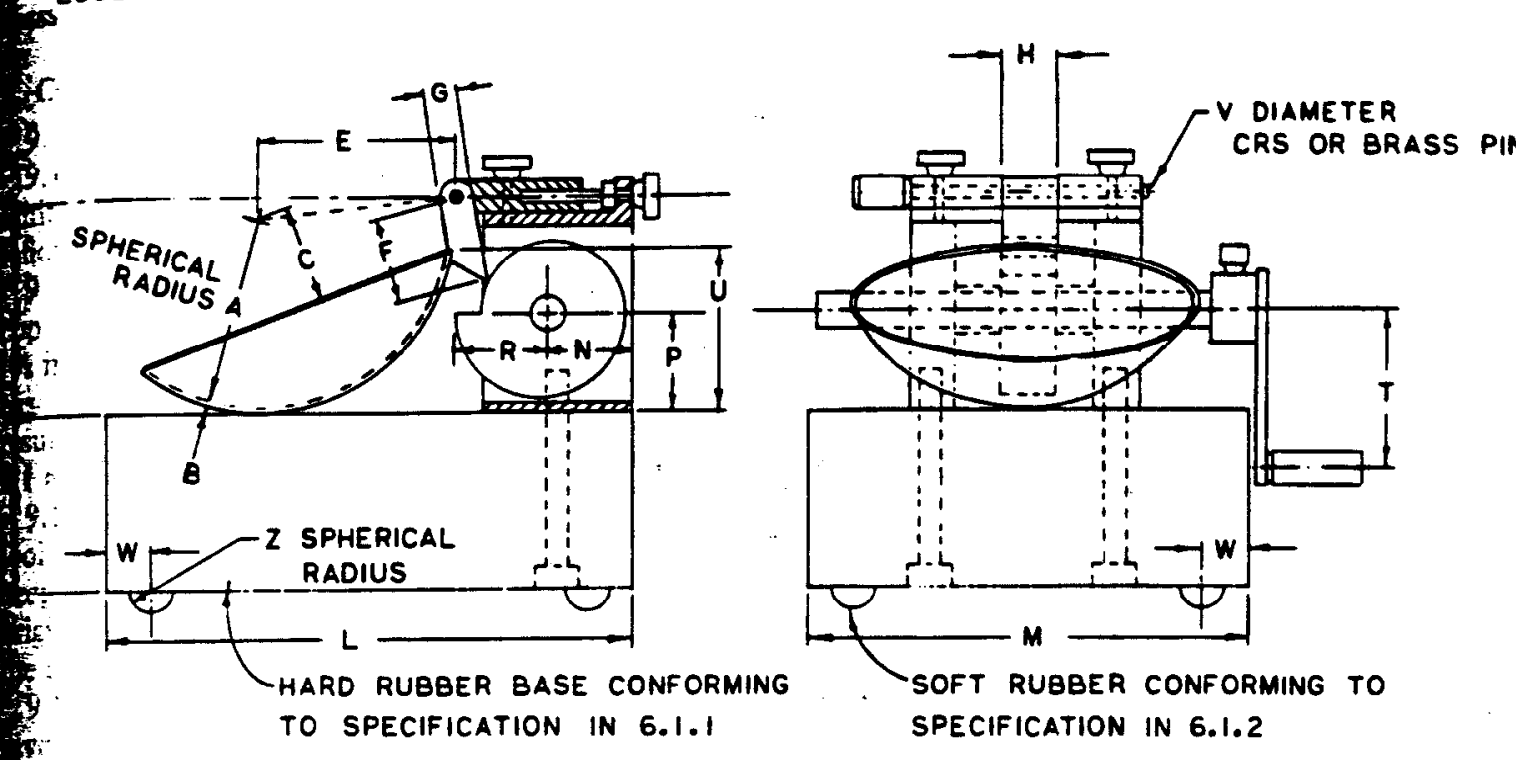

\begin{tabular}{|c|c|}
\hline CAM & CAM \\
ANGLE & RADIUS \\
DEGREES & \\
\hline
\end{tabular}

\begin{tabular}{l|l|}
\hline 0 & $0.742 R$ \\
\hline
\end{tabular}

(5

Fe. 1 Hend-Operated Lquid Linit Device

If be used. However, if the cam-follower lift pattern is not known, if velocity at drop off can be assured by carefully filing or machining ream and follower so that the cup beight remains constant over the 20 to $45^{\circ}$ of cam rotation.

6.1.5 Carriage, constructed in a way that allows conveitint but secure adjustment of the height of drop of the cup $610 \mathrm{~mm}$ (0.394 in.), and designed such that the cup and hanger assembly is only attached to the carriage by fans of a removable pin.

6.1.6 Motor Drive (Optional)-As an alternative to the and crank shown in Fig. 1, the device may be equipped with motor to turn the cam. Such a motor must turn the cam at 0.1 revolutions per second and must be isolated from the If of the device by rubber mounts or in some other way if prevents vibration from the motor being transmitted to 1. N-OFF switch and a means of conveniently positioning the in for height of drop adjustments. The results obtained ing a motor-driven device must not differ from those fotained using a manually operated device.

6.2 Flat Grooving Tool-A tool made of plastic or oncorroding-metal having the dimensions shown in Fig. 2. the design of the tool may vary as long as the essential yensions are maintained. The tool may, but need not, iforporate the gage for adjusting the height of drop of the ifuid limit device.

Nore 2-Prior to the adoption of this test method, a curved grooving was specified as part of the apparatus for performing the liquid limit The curved tool is not considered to be as accurate as the flat tool described in 6.2 since it does not control the depth of the so liquid limit cup. However, there sre some datn which indicate th typically the liquid limit is slightly increased when the int tool is used instead of the curved tool.

6.3 Gage-A metal gage block for adjusting the height of drop of the cup, having the dimensions shown in Fig. 3. The design of the tool may vary provided the gage will rest securely on the base without being susceptible to rocking and the edge which contacts the cup during adjustment i straight, at least $10 \mathrm{~mm}(3 / \mathrm{s}$ in.) wide, and without bevel of radius.

6.4 Containers-Small corrosion-resistant containers with snug-fitting lids for water content specimens. Aluminum of stainless steel cans $2.5 \mathrm{~cm}$ (1 in.) high by $5 \mathrm{~cm}(2$ in.) in diameter are appropriate.

6.5 Balance, conforming to Specification D 4753, Clas GP1.

6.6 Storage Container-A container in which to store the prepared soil specimen that will not contaminate the speof imen in any way, and will prevent moisture loss. A porcelain glass, or plastic dish about $11.4 \mathrm{~cm}(41 / 2$ in.) in diameter and a plastic bag large enough to enclose the dish and be folded over is adequate.

6.7 Ground Glass Plate-A ground glass plate at least 30 $\mathrm{cm}$ (12 in.) square by $1 \mathrm{~cm}(3 / \mathrm{h} \mathrm{in.)} \mathrm{thick} \mathrm{for} \mathrm{rolling} \mathrm{plasti}$ limit threads.

6.8 Spatula-A spatula or pill knife having a blad it $2 \mathrm{~cm}(3 / 4 \mathrm{in}$.) wide, and about 10 to $13 \mathrm{~cm}(3$ to $4 \mathrm{in}$. 6.9 Sieve-A 20.3-cm (8-in.) diameter, 425- $\mu \mathrm{m}$ (No. 40) 


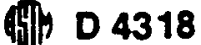

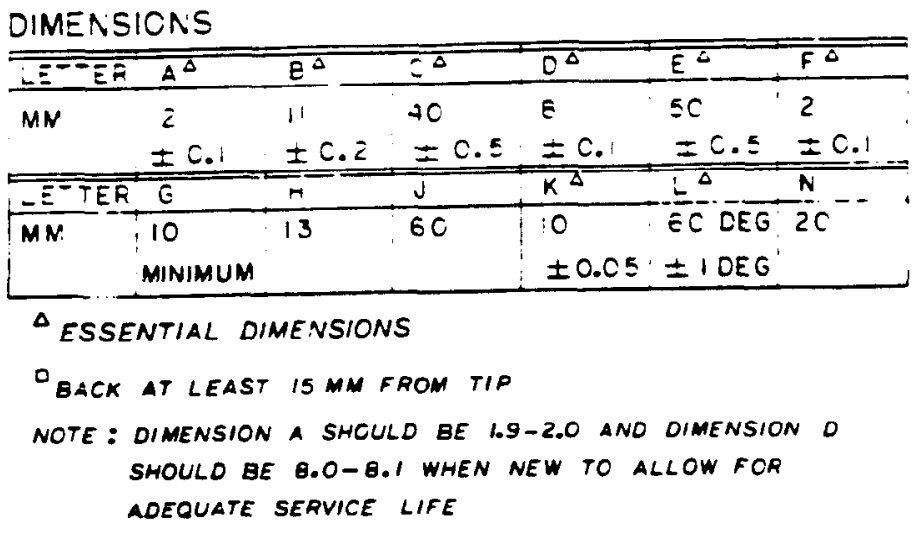
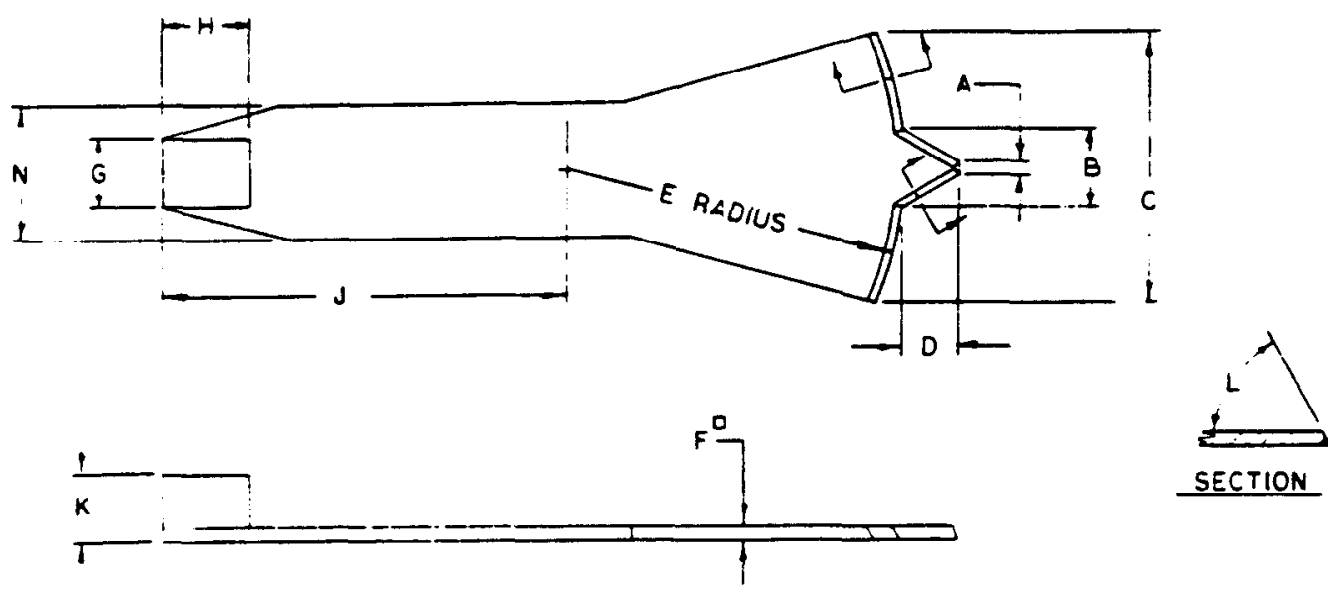

FIG. 2 Grooving Tool (Optional Height-of-Drop Gage Artached)
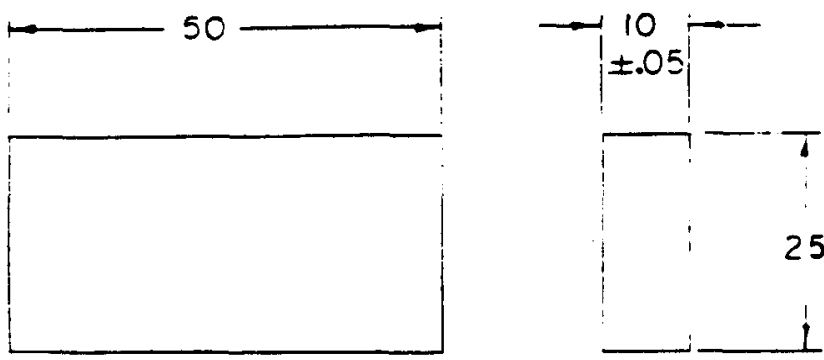

DIMENSIONS IN MILLIMETRES

FIG. 3 Height of Drop Gage

sieve conforming to the requirements of Specification $E$ II and having a $\mathrm{rm}$ at least $5 \mathrm{~cm}(2 \mathrm{in}$.) above the mesh. A 2$\mathrm{mm}$ (No. 10 ) sieve meeting the same requirements may also be needed.

6.10 Wash Bottle, or similar container for adding controlled amounts of water to soil and washing fines from coarse particles.

6.11 Drying Oven, thermostatically controlled, preferably of the forced-draft type, capable of continuously maintaining a temperature of $110 \pm 5^{\circ} \mathrm{C}\left(230 \pm 9^{\circ} \mathrm{F}\right)$ throughout the drying chamber.

6.12 Washing Pan, round. flat-bottomed, at least $7.6 \mathrm{~cm}$
( 3 in.) deep, and slightly larger at the bottom than a $20.3-\mathrm{cm}$ (8-in.) diameter sieve.

\section{Reagents and Materials}

7.1 Purity of Water-Where distilled water is referred to in this test method, either distilled or demineralized water may be used.

\section{Sampling}

8.1 Samples may be taken from any location that satisfies testing needs. However, Methods C 702, Practice D 75, and Practice D 420 should be used as guides for selecting and preserving samples from various types of sampling opertions. Samples which will be prepared using the wet prepartion procedure (10.1) must be kept at their natural wates content prior to preparation.

8.2 Where sampling operations have preserved the natural stratification of a sample, the various strata must be kept. separated and tests performed on the particular stratum of interest with as little contamination as possible from other strata. Where a mixture of materials will be used in construction, combine the various components in such proportions that the resultant sample represents the actual construction case.

8.3 Where data from this test method are to be used for correlation with other laboratory or field test data, use the 
Imaterial as used for these tests where possible.

5. L Obtain a representative portion from the total sample -ient to provide 150 to $200 \mathrm{~g}$ of material passing the 425 . (No. 40) sieve. Free flowing samples may be roducod by inethods of quartering or splitting. Cohesive samples - Ti be mixed thoroughly in a pan with a spatula, or scoop If representative portion scooped from the total mass by fling one or more sweeps with a scoop through the mixed 2

$x^{\infty}$

Talibration of Apparatus

H Inspection of Wear:

* I. I Liquid Limit Device-Determine that the liquid iil device is clean and in good working order. Check the injwing specific points.

7.1.1 Wear of Base-The spot on the base where the ftikakes contact should be wom no greater than $10 \mathrm{~mm}$ ifi.) in diameter. If the wear spot is greater than this, the ttan be machined to remove the worn spot provided the fifficing does not make the base thinner than specified in and the other dimensional relationships are maintained. 31.1.2 Wear of Cup-Replace the cup when the grooving I has wom a depression in the cup $0.1 \mathrm{~mm}(0.004 \mathrm{in}$.) 17p or when the rim of the cup has been reduced to half its Hethal thickness. Verify that the cup is firmly attached to 1. cirp hanger.

9.1.1.3 Wear of Cup Hanger-Verify that the cup hanger Hot does not bind and is not worn to an extent that allows whe than $3 \mathrm{~mm}(1 / \mathrm{s}$ in.) side-to-side movement of the lowest Jignt on the rim.

9.1.1.4 Wear of Cam-The cam shall not be worn to an itith that the cup drops before the cup hanger (cam iflower) loses contact with the cam.

Tit 1.2 Grooving Tools-Inspect grooving tools for wear on ituquent and regular basis. The rapidity of wear depends on T material from which the tool is made and the types of oils being tested. Soils containing a large proportion of sand fiticles may cause rapid wear of grooving tools; therefore, jein testing these materials, tools should be inspected more ifquently than for other soils.

Nore 3-The width of the tip of grooving tools is conveniently pect using a pocket-sized measuring magnifier equipped with a Whimetre scale. Magnifiers of this type are available from most Dintory supply companies. The depth of the tip of grooving tools can checked using the depth measuring feature of vernier calipers.
9.2 Adjustment of Height of Drop-Adjust the beight of drop of the cup so that the point on the cup that comes in contact with the base rises to a height of $10 \pm 0.2 \mathrm{~mm}$. See Fis 4 for proper location of the gage relative to th during adjustment.

Nore 4-A convenient procedure for adjusting the height of drop is as follows phace a piece of masioing tape across the outside bottom of th cup parlled with the axis of the cup hanger pivor. The edge of the tap awny from the cup haneer should bisect the spot on the cup that contact the base. For new cups, placing a picce of carbon paper on the base and allowing the cup to drop several times will mert the contact spor. Arack the cup to the device and turn the crank until the cup is raised to it maximum beight. Stide the beight gage under the cup from the front and obverve whether the gage contacts the cup or the tape. (See Fig 4.) If the tupe and cup are both contacted, the beint of drop is approxi mately correct. If not, adjust the cup until simulnneous contact is made Check adjustment by turnine the crnak at 2 revolutions per second whil bolding the gage in position aginst the tape and cup. If a frint ringing of cticking sound is heard without the cup rising from the gage, the adjustment is correct. If no ringins is beard or if the cup rises from the gage, readjust the beight of drop. If the cup rocks on the gnoe during thi checkins operation, the com follower pivot is excowively worn and th. worn parts should be replaced. Always remove tape after completion of adjustment operntion.

\section{Preparation of Test Specimens}

10.1 Wet Preparation-Except where the dry method of specimen preparation is specified (10.2), prepare specimens for test as described in the following sections.

10.1.1 Samples Passing the 425- $1 \mathrm{~m}$ (No. 40) Sieve:

10.1.1.1 When by visual and manual procedures it is determined that the sample has little or no material retained on a $425-\mu m$ (No. 40) sieve, prepare a specimen of 150 to $200 \mathrm{~g}$ by mixing thoroughly with distilled or demine water on the glass plate using the spatula. If desired, sc soil in a storage dish with a small amount of water to soften the soil before the start of mixing. Adjust the water content of the soil to bring it to a consistency that would require 25 to 35 blows of the liquid limit device to close the groove (Note 5).

10.1.1.2 If, during mixing, a small percentage of material is encountered that would be retained on a $425-\mu \mathrm{m}$ (No. 40) sieve, remove these particles by hand (if possible). If it is impractical to remove the coarser material by hand, remove small percentages (less than about $15 \%$ ) of coarser material by working the specimen through a $425-\mu m$ sieve using a piece of rubber sheeting, rubber stopper, or other convenien

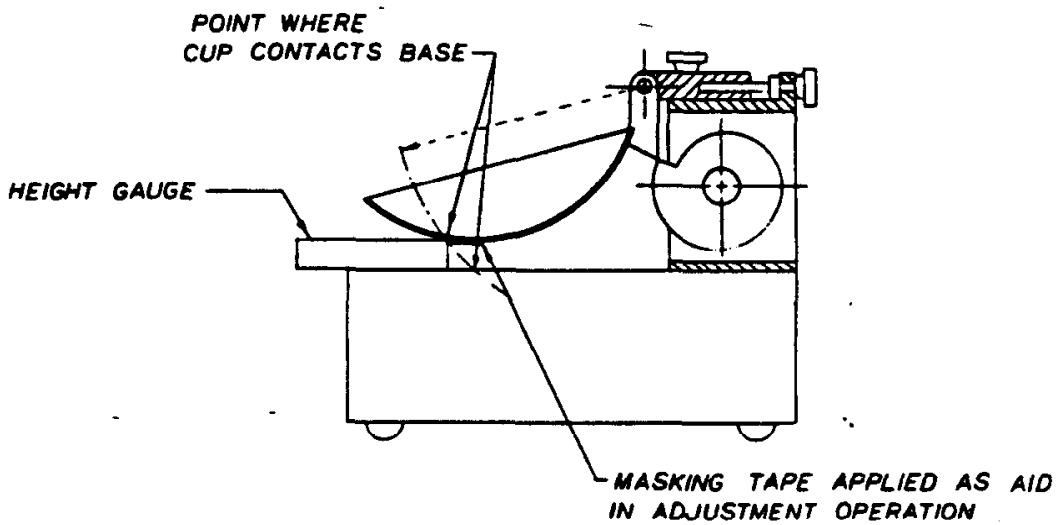

Fic. 4 Calibration for Helght of Drop 


\section{K Series 28 Day UCS Results}




\section{UNCONFINED COMPRESSIVE STRENGTH - ASTM D 2166 28-DAY CURE}

$\begin{array}{lr}\begin{array}{l}\text { Project Name } \\ \text { Project No. }\end{array} & 777304.00010500 \\ & \\ \text { Specimen Mold Date } & 11 / 25 / 98 \\ \text { Specimen Test Date } & 12 / 23 / 98 \\ & 500.7 \\ \text { STRESS AT FAILURE, psi } & 3.4\end{array}$

\begin{tabular}{ccc}
$\begin{array}{c}\text { AXIAL } \\
\text { STRAIN, \% }\end{array}$ & & $\begin{array}{c}\text { DEVIATOR } \\
\text { STRESS, psi }\end{array}$ \\
\cline { 1 - 1 } 0.00 & & 0.0 \\
0.10 & & 1.3 \\
0.20 & & 4.1 \\
0.30 & & 8.2 \\
0.40 & & 12.7 \\
0.50 & & 26.2 \\
0.75 & & 69.7 \\
1.00 & & 119.5 \\
1.25 & & 174.1 \\
1.50 & & 235.9 \\
1.74 & & 299.6 \\
1.99 & & 368.6 \\
2.24 & & 431.4 \\
2.49 & & 465.6 \\
2.74 & & 476.4 \\
2.99 & & 485.1 \\
3.24 & 499.8 \\
3.36 & 500.7 \\
3.49 & 489.0 \\
3.74 & 370.9 \\
3.99 & 53.4
\end{tabular}

Client Sample No. K312F0O

IT Lab Specimen No. ETDC-7975

Specimen Height, in.

4.0130

Specimen Diameter, in.

2.0020

Specimen Weight, $g$.

394.06

Moisture Content, \%

24.4

Wet Unit Weight, pcf.

118.9

Dry Unit Weight, pcf.

95.5

Rate of Strain, in./min.

0.0240

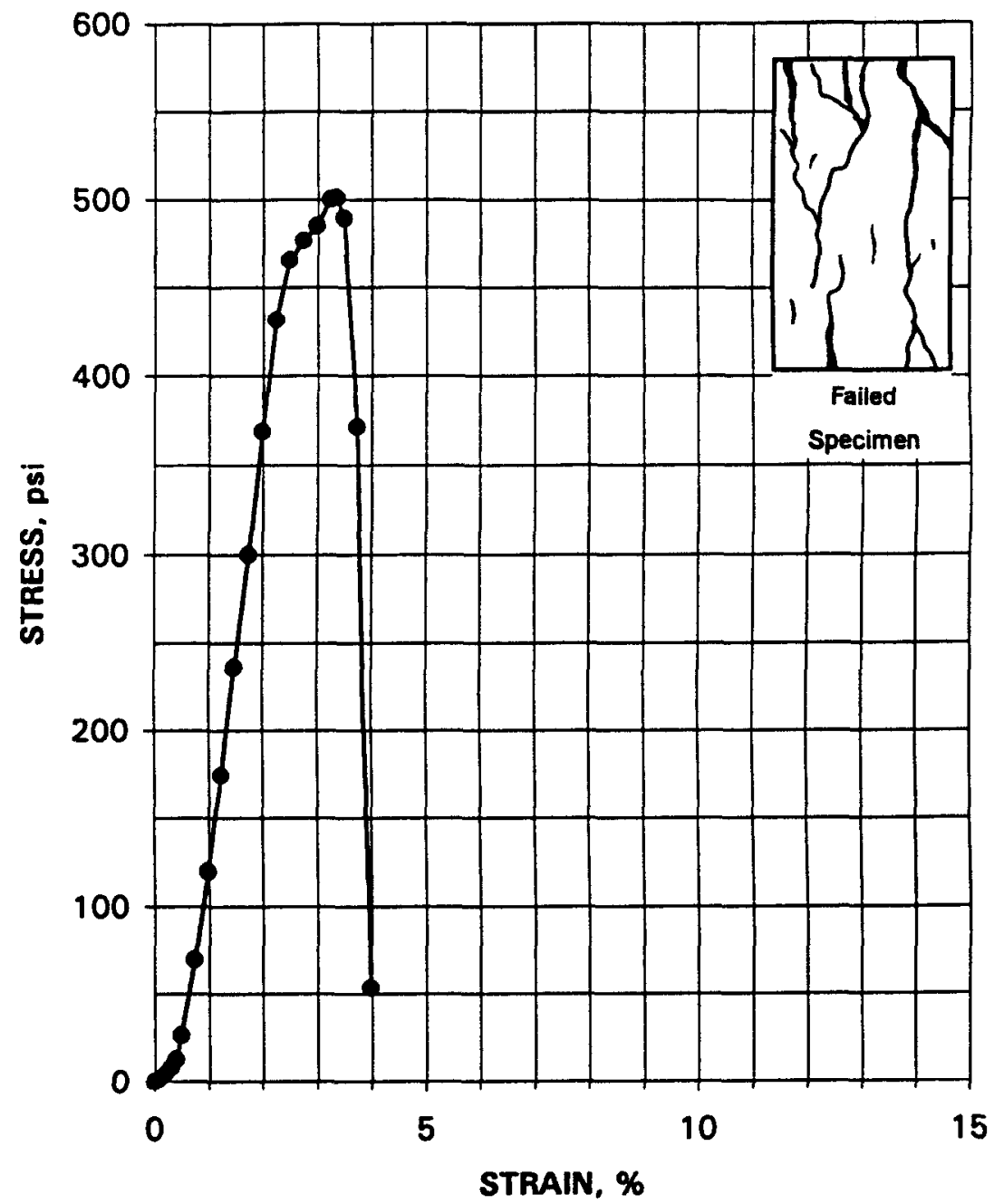




\section{UNCONFINED COMPRESSIVE STRENGTH - ASTM D 2166 28-DAY CURE}

\begin{tabular}{lc} 
Project Name & SAIC/SAVAN \\
Project No. & 777304.00010 \\
\multicolumn{3}{l}{ Specimen Mold Date } \\
Specimen Test Date \\
STRESS AT FAILURE, psi \\
STRAIN AT FAILURE, \% \\
\multicolumn{3}{|}{} \\
AXIAL & DEVIATOR \\
STRAIN, \% & STRESS, psi \\
\hline 0.00 & 0.0 \\
0.10 & 1.6 \\
0.20 & 4.4 \\
0.30 & 8.9 \\
0.40 & 11.1 \\
0.50 & 19.3 \\
0.75 & 56.1 \\
1.00 & 137.7 \\
1.25 & 213.8 \\
1.51 & 273.6 \\
1.76 & 336.0 \\
2.01 & 414.8 \\
2.26 & 501.2 \\
2.51 & 558.1 \\
2.76 & 609.8 \\
3.01 & 613.1 \\
3.14 & 619.1 \\
3.26 & 70.6 \\
\multicolumn{2}{l}{} \\
ST &
\end{tabular}

$11 / 25 / 98$

$12 / 23 / 98$

619.1

3.1
Client Sample No. K322F00

IT Lab Specimen No. ETDC-7976

Specimen Height, in.

Specimen Diameter, in.

2.0025

Specimen Weight, $g$.

392.08

Moisture Content, \%

Wet Unit Weight, pcf.

119.0

Dry Unit Weight, pcf.

Rate of Strain, in./min.

0.0240

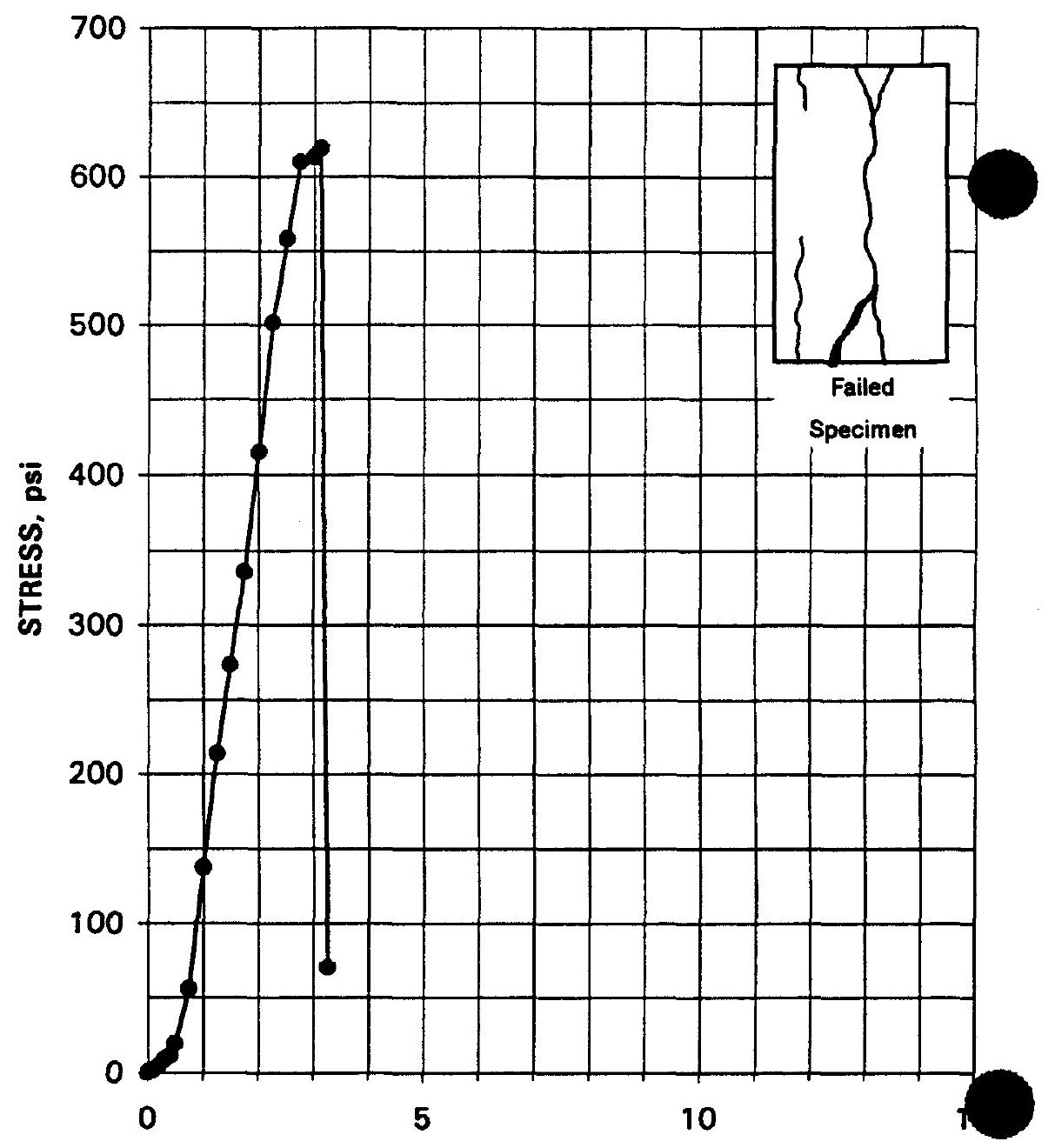

STRAIN, \% 


\section{UNCONFINED COMPRESSIVE STRENGTH - ASTM D 2166 28-DAY CURE}

$\begin{array}{ll}\text { Project Name } & \text { SAIC/SAVANNAH RIVER } \\ \text { Project No. } & 777304.00010500\end{array}$

Specimen Mold Date

Specimen Test Date

STRESS AT FAILURE, psi

STRAIN AT FAILURE, \%

AXIAL DEVIATOR

$\frac{\text { STRAIN, \% }}{0.00} \frac{\text { STRESS, psi }}{0.0}$

$0.10 \quad 1.3$

$0.20 \quad 3.5$

0.30

6.0

0.40

8.5

0.50

12.9

0.75

33.0

1.00

1.25

1.50

1.75

2.00

2.25

2.50

2.75

3.00

3.25

3.50

3.53

3.75
$11 / 25 / 98$

$12 / 23 / 98$

532.7

3.5
Client Sample No. K332F00

IT Lab Specimen No. ETDC-7977

Specimen Height, in.

3.9955

Specimen Diameter, in.

2.0048

Specimen Weight, $\mathbf{g}$.

380.58

Moisture Content, \%

28.8

Wet Unit Weight, pcf.

115.0

Dry Unit Weight, pct.

89.2

Rate of Strain, in./min.

0.0240

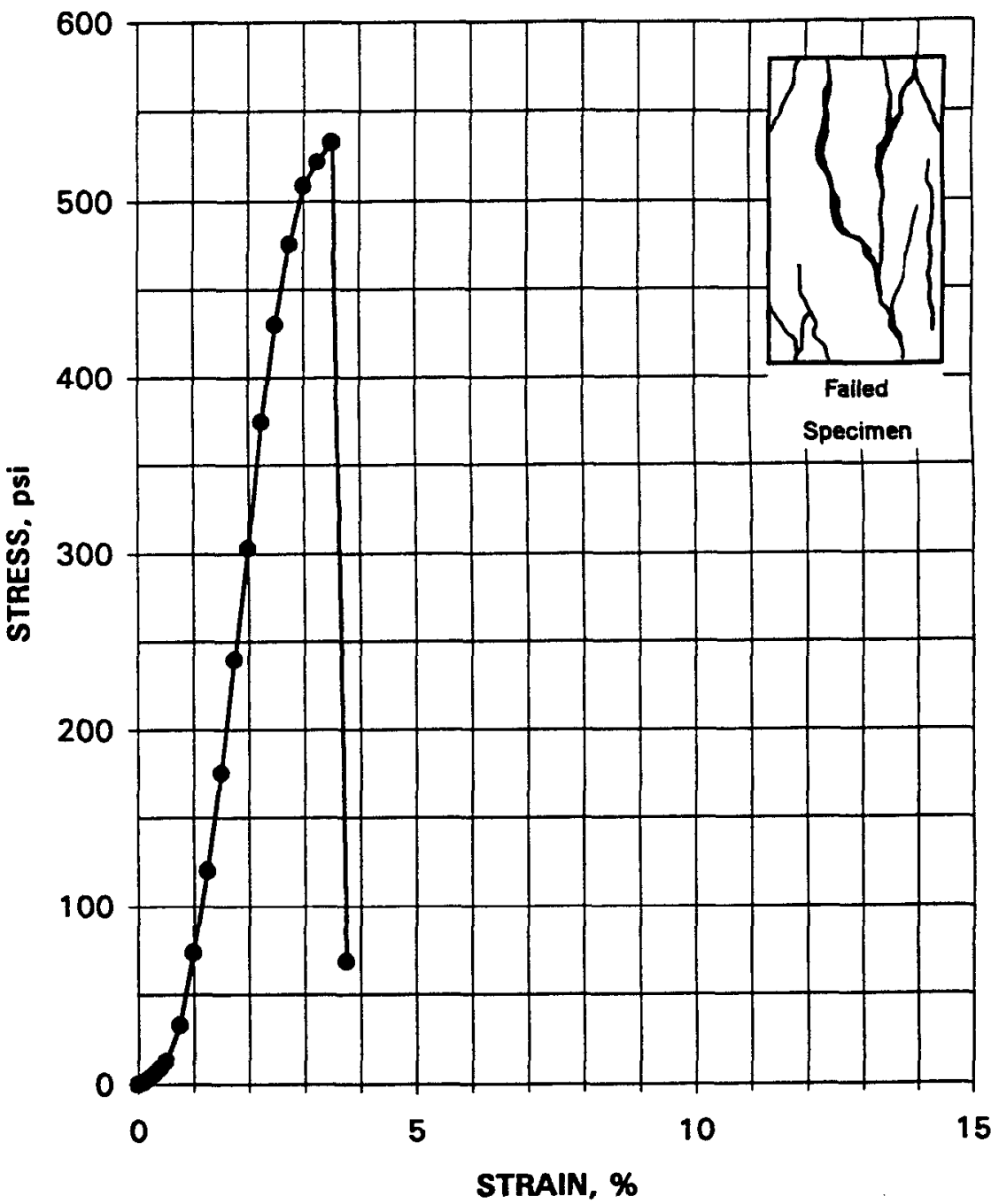




\section{UNCONFINED COMPRESSIVE STRENGTH - ASTM D 2166 28-DAY CURE}

Project Name SAIC/SAVANNAH RIVER

Project No. $\quad 777304.000105$

Specimen Mold Date

Specimen Test Date

STRESS AT FAILURE, psi

STRAIN AT FAILURE, \%

\begin{tabular}{ccc}
$\begin{array}{ccc}\text { AXIAL } \\
\text { STRAIN, \% }\end{array}$ & & $\begin{array}{c}\text { DEVIATOR } \\
\text { STRESS, psi }\end{array}$ \\
\cline { 1 - 1 } 0.00 & & 0.0 \\
0.10 & & 1.6 \\
0.20 & & 2.8 \\
0.29 & & 4.7 \\
0.39 & & 7.2 \\
0.49 & & 11.9 \\
0.74 & & 35.0 \\
0.98 & & 88.3 \\
1.23 & & 169.3 \\
1.47 & & 250.3 \\
1.72 & & 338.9 \\
1.96 & & 429.2 \\
2.21 & & 499.3 \\
2.45 & & 540.1 \\
2.57 & 541.0 \\
2.70 & 497.7 \\
2.94 & 436.8 \\
3.19 & 416.5 \\
3.43 & 396.5 \\
3.68 & 343.6 \\
3.92 & 62.4
\end{tabular}

Client Sample No. K342F00

IT Lab Specimen No. ETDC-7978

Specimen Height, in.

4.0780

Specimen Diameter, in.

2.0100

Specimen Weight, $\boldsymbol{g}$.

386.61

Moisture Content, \%

28.1

Wet Unit Weight, pcf.

113.8

Dry Unit Weight, pcf.

88.9

Rate of Strain, in./min.

0.0240

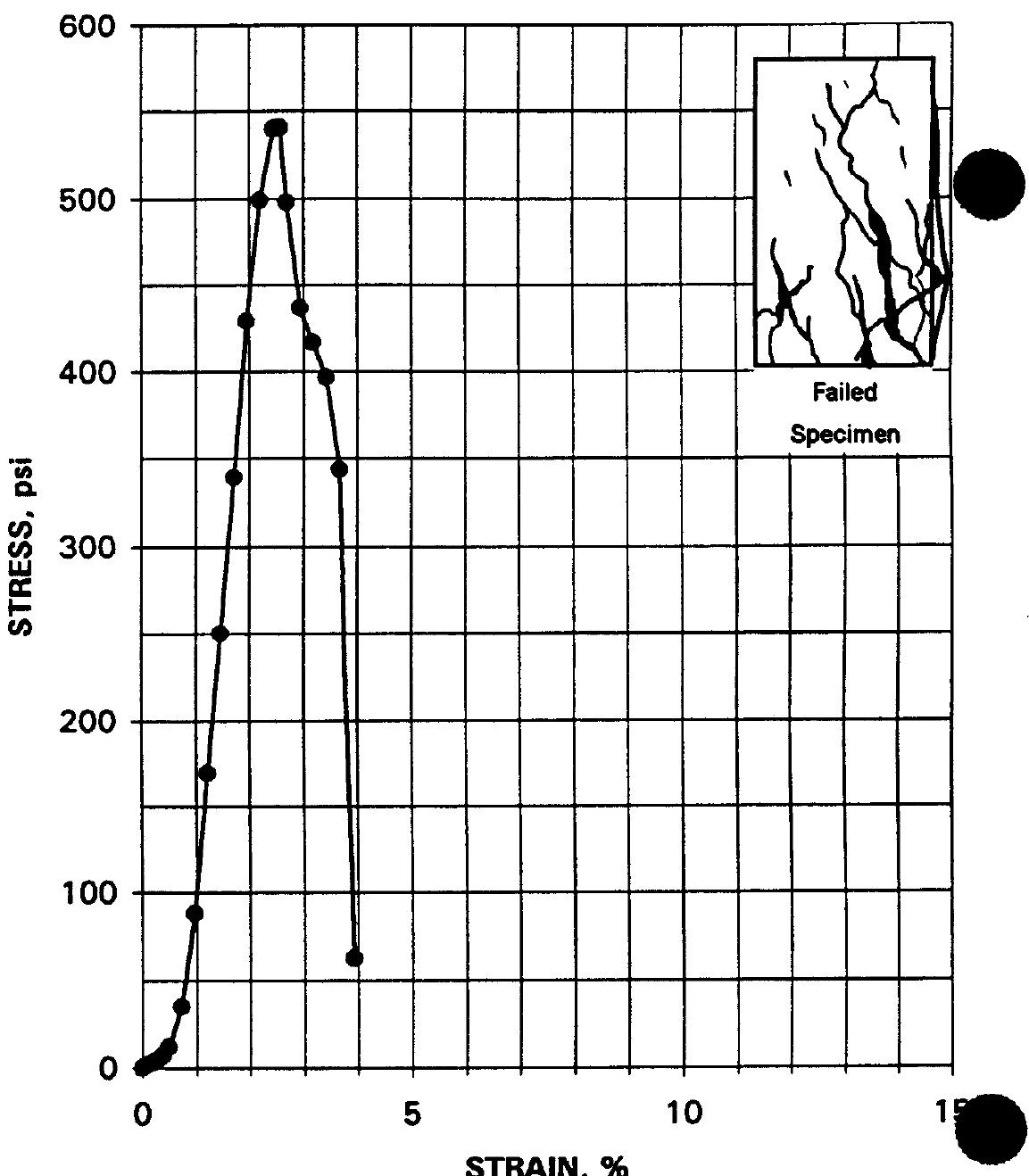




\section{UNCONFINED COMPRESSIVE STRENGTH - ASTM D 2166 28-DAY CURE}

Project Name SAIC/SAVANNAH RIVER

Project No. $\quad 777304.00010500$

\begin{abstract}
Specimen Mold Date
\end{abstract}
Specimen Test Date

STRESS AT FAILURE, psi STRAIN AT FAILURE, \%

\begin{tabular}{|c|c|}
\hline $\begin{array}{c}\text { AXIAL } \\
\text { STRAIN, \% }\end{array}$ & $\begin{array}{l}\text { DEVIATOR } \\
\text { STRESS, psi }\end{array}$ \\
\hline 0.00 & 0.0 \\
\hline 0.10 & 1.3 \\
\hline 0.20 & 3.5 \\
\hline 0.30 & 7.6 \\
\hline 0.40 & 13.0 \\
\hline 0.50 & 19.9 \\
\hline 0.75 & 50.1 \\
\hline 1.00 & 100.0 \\
\hline 1.25 & 150.9 \\
\hline 1.50 & 201.8 \\
\hline 1.76 & 256.5 \\
\hline 2.01 & 299.5 \\
\hline 2.26 & 334.4 \\
\hline 2.51 & 370.4 \\
\hline 2.76 & 380.0 \\
\hline 3.01 & 397.5 \\
\hline 3.26 & 412.1 \\
\hline 3.31 & 412.2 \\
\hline 3.51 & 391.4 \\
\hline 3.76 & 300.5 \\
\hline 4.01 & 54.9 \\
\hline
\end{tabular}

Client Sample No.

K412F0O

IT Lab Specimen No.

ETDC-7979

Specimen Height, in.

Specimen Diameter, in.

2.0020

Specimen Weight, $g$.

374.50

Moisture Content, \%

31.3

Wet Unit Weight, pcf.

113.7

Dry Unit Weight, pcf.

86.6

Rate of Strain, in./min.

0.0240

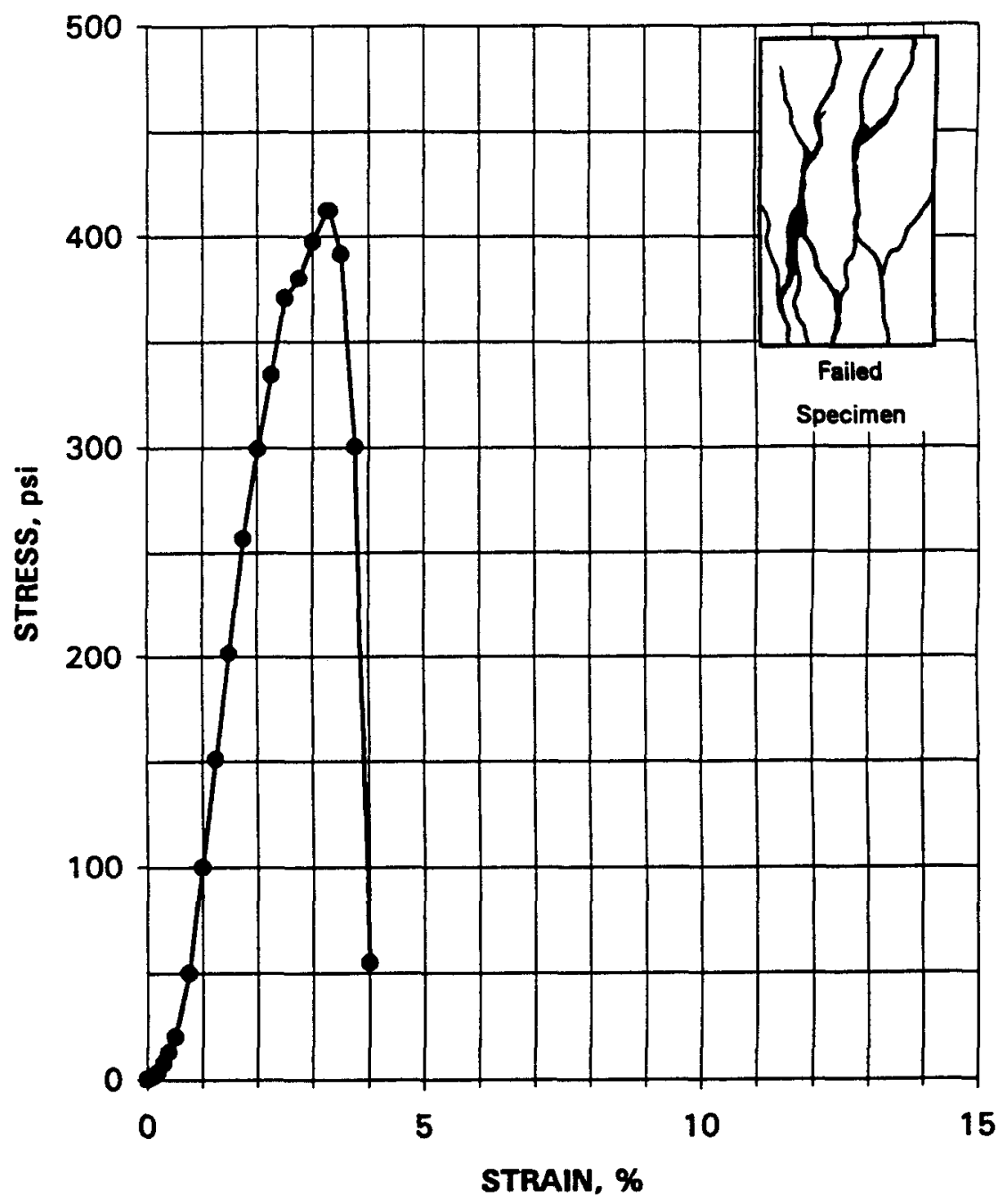




\section{UNCONFINED COMPRESSIVE STRENGTH - ASTM D 2166 28-DAY CURE}

\begin{tabular}{lc} 
Project Name & SAIC/SAVAN \\
Project No. & 777304.00010 \\
Specimen Mold Date \\
Specimen Test Date \\
STRESS AT FAILURE, psi \\
STRAIN AT FAILURE, \% \\
\multicolumn{3}{c}{ AXIAL } & DEVIATOR \\
STRAIN, \% & STRESS, psi \\
\hline 0.00 & 0.0 \\
0.10 & 1.6 \\
0.20 & 3.1 \\
0.30 & 5.3 \\
0.40 & 8.2 \\
0.50 & 11.3 \\
0.75 & 34.4 \\
1.00 & 72.7 \\
1.25 & 128.3 \\
1.49 & 180.8 \\
1.74 & 229.0 \\
1.99 & 289.0 \\
2.24 & 360.7 \\
2.49 & 372.4 \\
2.74 & 333.4 \\
2.99 & 248.4 \\
3.24 & 182.2
\end{tabular}

Client Sample No. K422F00

IT Lab Specimen No. ETDC-7980

Specimen Height, in.

4.0145

Specimen Diameter, in.

2.0093

Specimen Weight, g.

388.24

Moisture Content, \%

30.6

Wet Unit Weight, pcf.

116.2

Dry Unit Weight, pef.

89.0

Rate of Strain, in./min.

0.0240

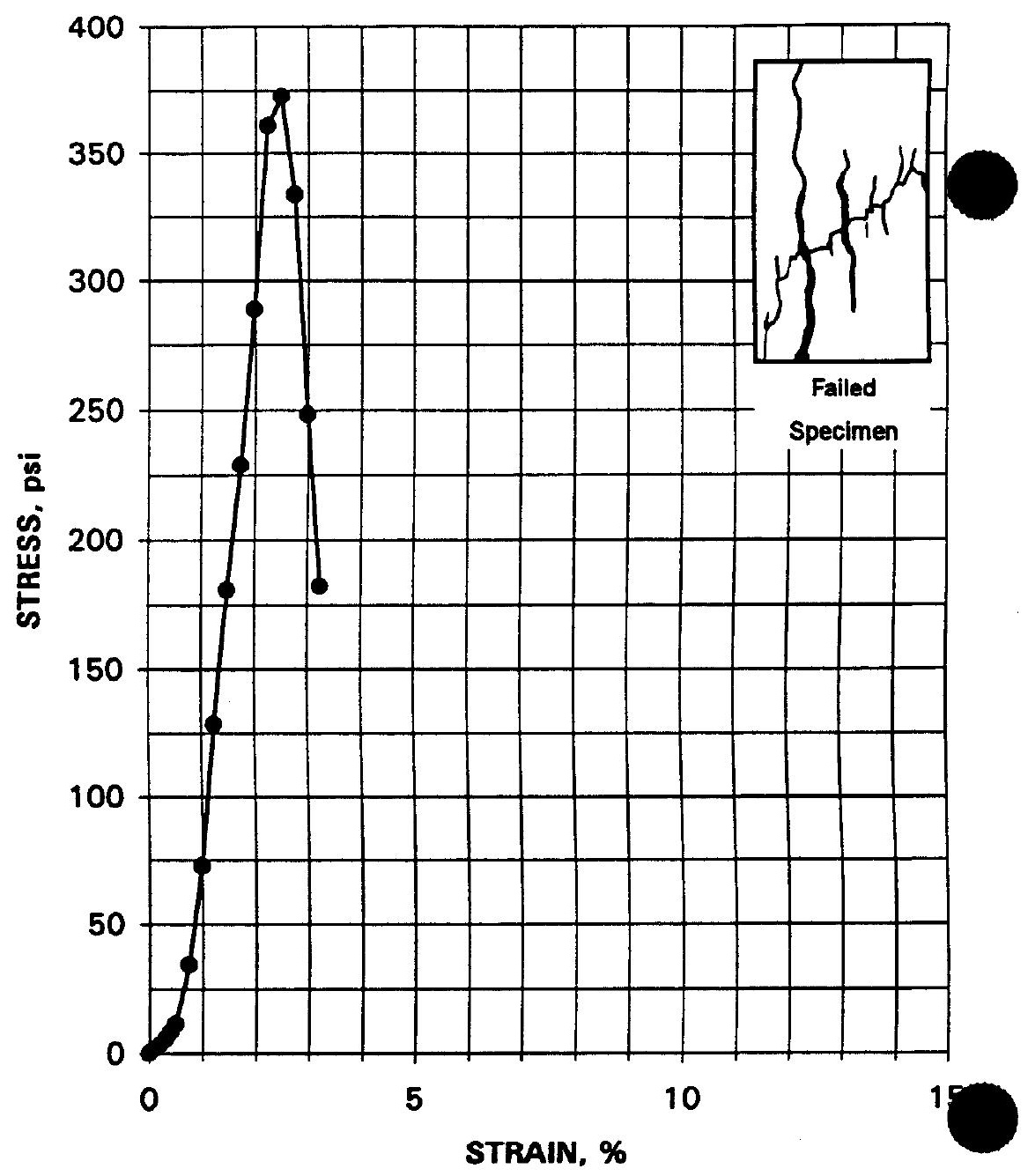




\section{UNCONFINED COMPRESSIVE STRENGTH - ASTM D 2166 28-DAY CURE}

$\begin{array}{ll}\text { Project Name } & \text { SAIC/SAVANNAH RIVER } \\ \text { Project No. } & 777304.00010500\end{array}$

\begin{tabular}{|c|c|}
\hline \multicolumn{2}{|c|}{ Specimen Mold Date } \\
\hline \multicolumn{2}{|c|}{ STRESS AT FAILURE, psi } \\
\hline AXIAL & DEVIATOR \\
\hline STRAIN, \% & STRESS, psi \\
\hline 0.00 & 0.0 \\
\hline 0.10 & 1.6 \\
\hline 0.20 & 3.5 \\
\hline 0.30 & 6.6 \\
\hline 0.40 & 10.3 \\
\hline 0.50 & 15.0 \\
\hline 0.75 & 30.9 \\
\hline 1.00 & 66.7 \\
\hline 1.25 & 114.4 \\
\hline 1.50 & 162.2 \\
\hline 1.75 & 211.0 \\
\hline 2.00 & 255.2 \\
\hline 2.25 & 281.9 \\
\hline 2.50 & 300.5 \\
\hline $\begin{array}{l}2.75 \\
2.88\end{array}$ & $\begin{array}{l}316.3 \\
318.3\end{array}$ \\
\hline 3.00 & 264.2 \\
\hline 3.25 & 118.8 \\
\hline
\end{tabular}

Client Sample No. K432FOO

IT Lab Specimen No. ETDC-7981

Specimen Height, in.

3.9977

Specimen Diameter, in.

2.0110

Specimen Weight, $g$.

365.78

Moisture Content, \%

36.5

Wet Unit Weight, pcf.

109.8

Dry Unit Weight, pcf.

80.4

Rate of Strain, in./min.

0.0240

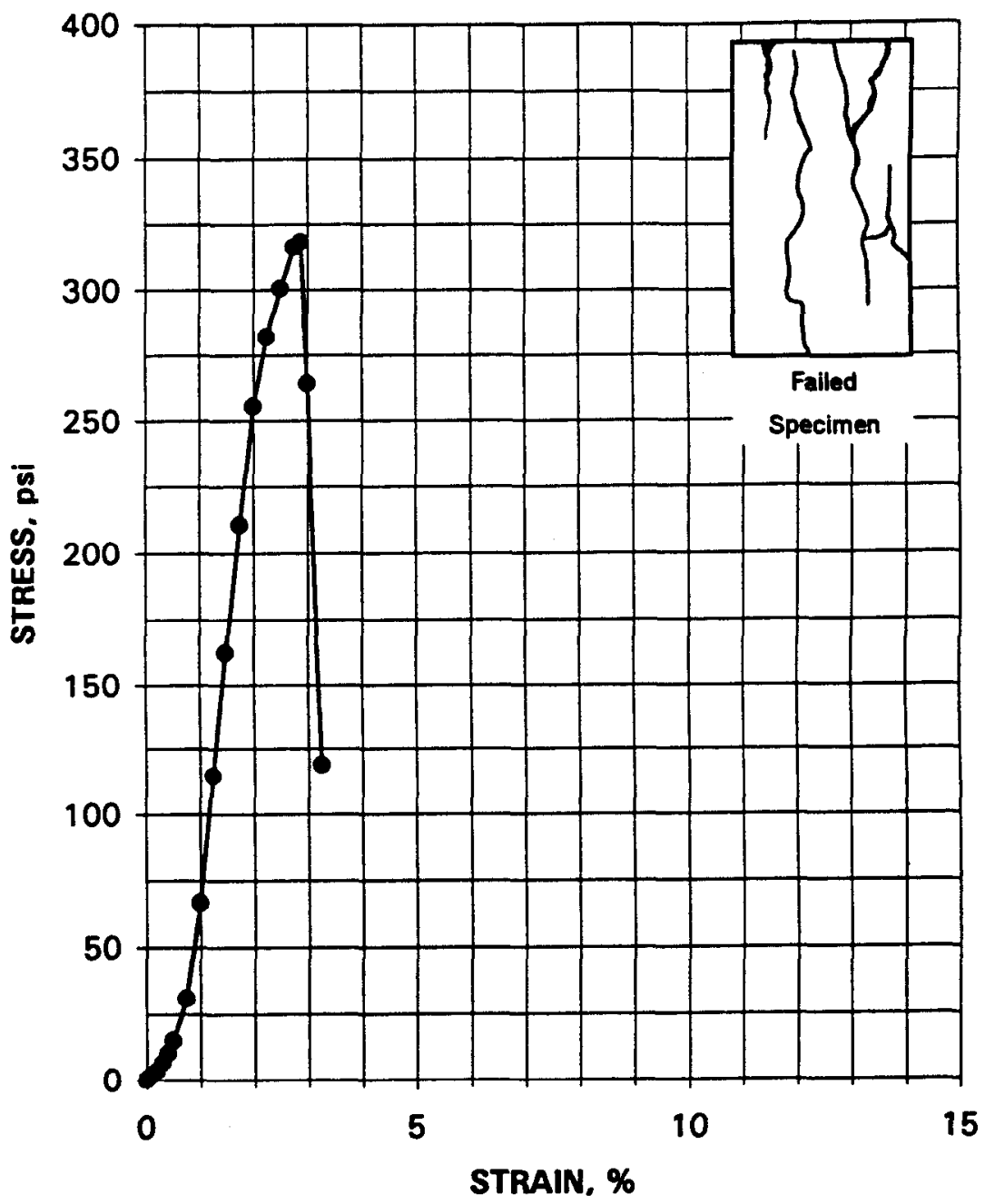




\section{UNCONFINED COMPRESSIVE STRENGTH - ASTM D 2166 28-DAY CURE}

Project Name SAIC/SAVANNAH RIVER

Project No. $\quad 777304.00010500$

Specimen Mold Date

Specimen Test Date

STRESS AT FAILURE, psi

STRAIN AT FAILURE, \%

AXIAL

\begin{tabular}{|c|c|}
\hline STRAIN, \% & STRESS, psi \\
\hline 0.00 & 0.0 \\
\hline 0.10 & 1.3 \\
\hline 0.20 & 2.8 \\
\hline 0.30 & 5.4 \\
\hline 0.40 & 9.8 \\
\hline 0.50 & 14.5 \\
\hline 0.74 & 40.8 \\
\hline 0.99 & 102.9 \\
\hline 1.24 & 187.9 \\
\hline 1.49 & 267.1 \\
\hline 1.74 & 347.8 \\
\hline 1.99 & 424.0 \\
\hline 2.23 & 446.7 \\
\hline 2.36 & 462.2 \\
\hline 2.48 & 311.2 \\
\hline 2.73 & 173.7 \\
\hline
\end{tabular}

$11 / 25 / 98$

$12 / 23 / 98$

462.2

2.4
Client Sample No. K442F00

IT Lab Specimen No. ETDC-7982

Specimen Height, in.

4.0300

Specimen Diameter, in.

2.0073

Specimen Weight, $g$.

Moisture Content, $\%$

373.62

35.1

Wet Unit Weight, pcf.

Dry Unit Weight, pcf.

111.6

Rate of Strain, in./min.

82.6

0.0240

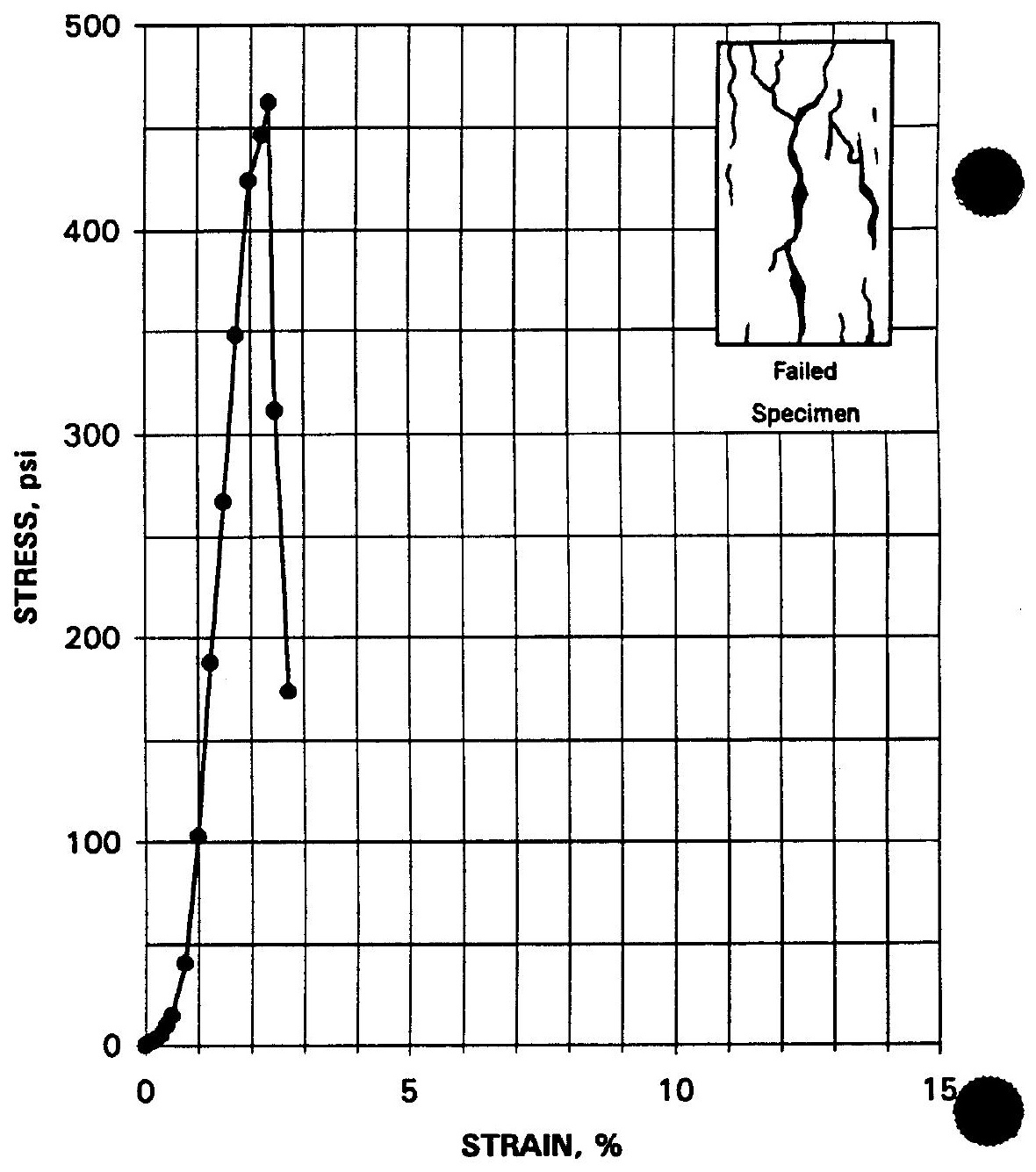




\section{K Series 90 Day Immersion UCS Results}




\section{UNCONFINED COMPRESSIVE STRENGTH - ASTM D 2166 28 DAY CURE, 90 DAY SATURATION}

\begin{tabular}{lc} 
Project Name & SAIC / SAVA \\
Project No. & 777304.00010 \\
\multicolumn{3}{l}{} \\
Specimen Mold Date \\
Specimen Test Date \\
STRESS AT FAILURE, psi \\
STRAIN AT FAILURE, \% \\
\multicolumn{3}{|}{} \\
AXIAL & DEVIATOR \\
STRAIN, \% & STRESS, psi \\
\hline 0.00 & 0.0 \\
0.10 & 5.1 \\
0.20 & 12.7 \\
0.30 & 26.4 \\
0.40 & 46.0 \\
0.50 & 66.6 \\
0.75 & 134.1 \\
1.00 & 195.2 \\
1.25 & 260.5 \\
1.50 & 324.2 \\
1.75 & 393.5 \\
2.00 & 468.0 \\
2.24 & 521.6 \\
2.49 & 534.6 \\
2.62 & 539.5 \\
2.74 & 533.2 \\
2.99 & 20.1 \\
\multicolumn{2}{c}{}
\end{tabular}

Client Sample No. K313F00

IT Lab Specimen No. ETDC-7991

Specimen Height, in.

4.0095

Specimen Diameter, in.

1.9992

Specimen Weight, $g$.

402.53

Moisture Content, \%

26.6

Wet Unit Weight, pcf.

121.9

Dry Unit Weight, pcf.

96.2

Rate of Strain, in./min.

0.0400

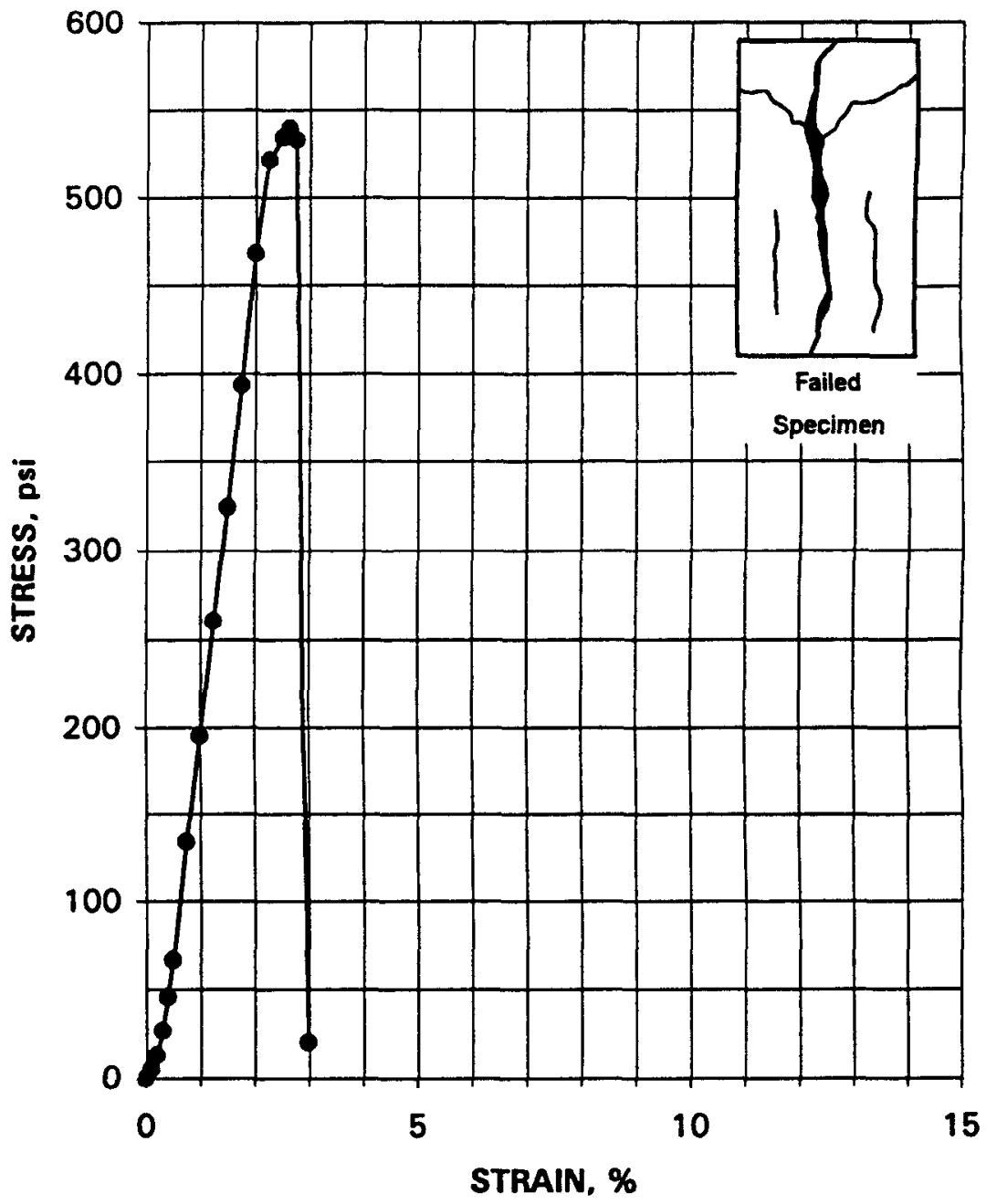




\section{UNCONFINED COMPRESSIVE STRENGTH - ASTM D 2166 28 DAY CURE, 90 DAY SATURATION}

Project Name SAIC / SAVANNAH RIVER

Project No. 777304.00010500

Specimen Mold Date

Specimen Test Date

STRESS AT FAILURE, psi

STRAIN AT FAILURE, \%

\begin{tabular}{ccc}
$\begin{array}{c}\text { AXIAL } \\
\text { STRAIN, \% }\end{array}$ & & $\begin{array}{c}\text { DEVIATOR } \\
\text { STRESS, psi }\end{array}$ \\
\cline { 1 - 1 } 0.00 & & 0.0 \\
0.10 & & 7.2 \\
0.20 & & 13.5 \\
0.30 & & 21.7 \\
0.40 & & 28.3 \\
0.50 & & 36.7 \\
0.75 & & 72.7 \\
1.00 & & 120.6 \\
1.25 & & 192.5 \\
1.50 & & 259.5 \\
1.75 & & 323.3 \\
2.00 & & 390.8 \\
2.25 & 470.9 \\
2.50 & 546.3 \\
2.75 & 585.1 \\
2.88 & 593.3 \\
3.00 & 340.3 \\
3.25 & 169.7
\end{tabular}

Client Sample No. K323F00

IT Lab Specimen No. ETDC-7992

Specimen Height, in.

3.9962

Specimen Diameter, in.

2.0088

Specimen Weight, $g$.

397.93

Moisture Content, \%

24.8

Wet Unit Weight, pcf.

119.7

Dry Unit Weight, pcf.

95.9

Rate of Strain, in./min.

0.0440

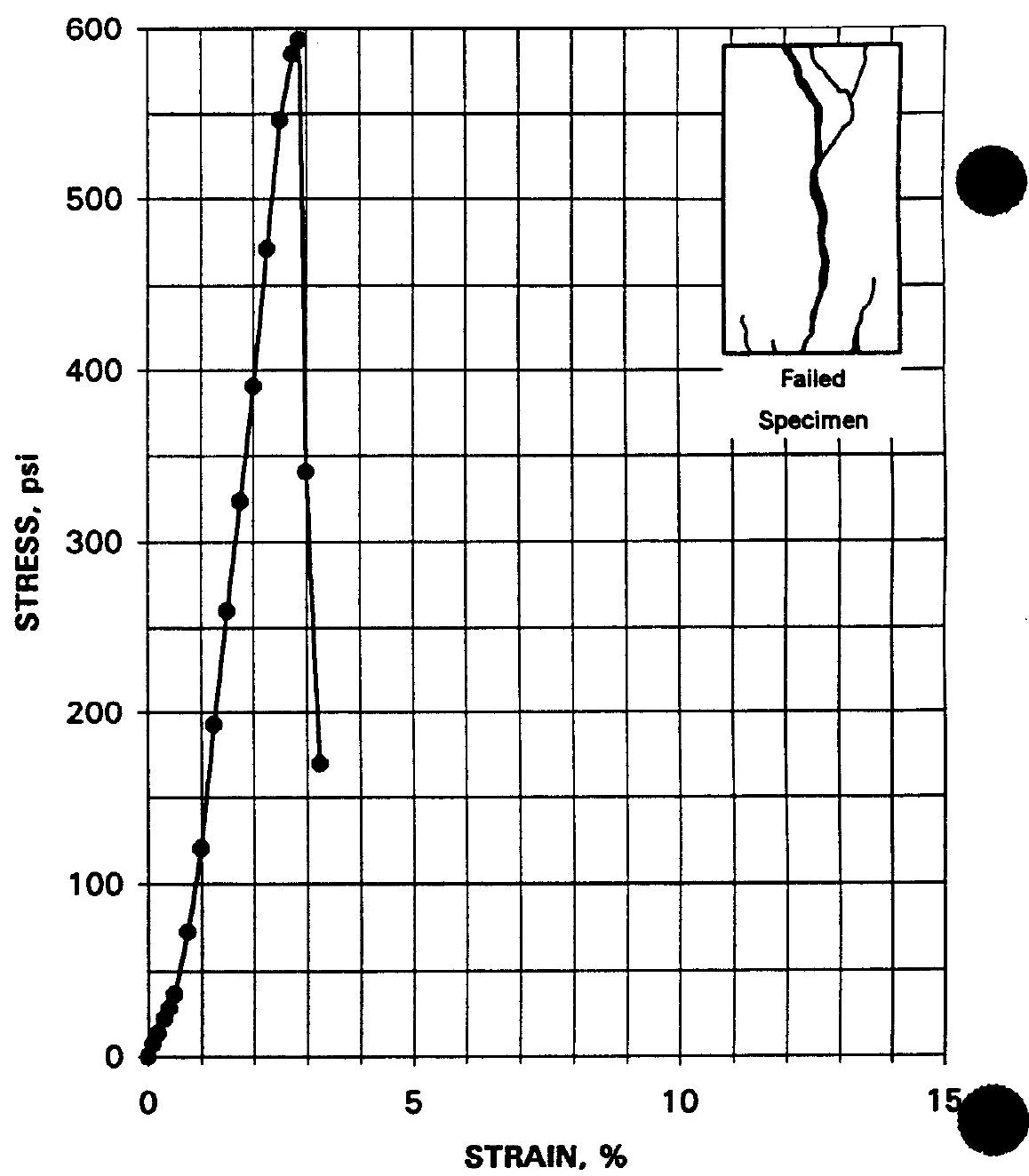




\section{UNCONFINED COMPRESSIVE STRENGTH - ASTM D 2166 28 DAY CURE, 90 DAY SATURATION}

Project Name SAIC / SAVANNAH RIVER

Project No. 777304.00010500

Specimen Mold Date

Specimen Test Date

STRESS AT FAILURE, psi

STRAIN AT FAILURE, \%

AXIAL DEVIATOR

$\frac{\text { STRAIN, \% }}{0.00} \frac{\text { STRESS, pSi }}{0.0}$

0.10

5.0

0.20

9.8

0.30

21.7

0.40

36.8

0.50

57.5

0.75

121.0

1.00

1.25

1.50

1.75

1.87

2.00

2.25
$11 / 25 / 98$

$3 / 23 / 99$

448.4

1.9
Client Sample No. K333F00

IT Lab Specimen No. ETDC-7993

Specimen Height, in.

Specimen Diameter, in.

4.0040

Specimen Weight, $g$.

2.0078

Moisture Content, \%

385.96

31.2

Wet Unit Weight, pcf.

116.0

Dry Unit Weight, pcf.

88.4

Rate of Strain, in./min.

0.0440

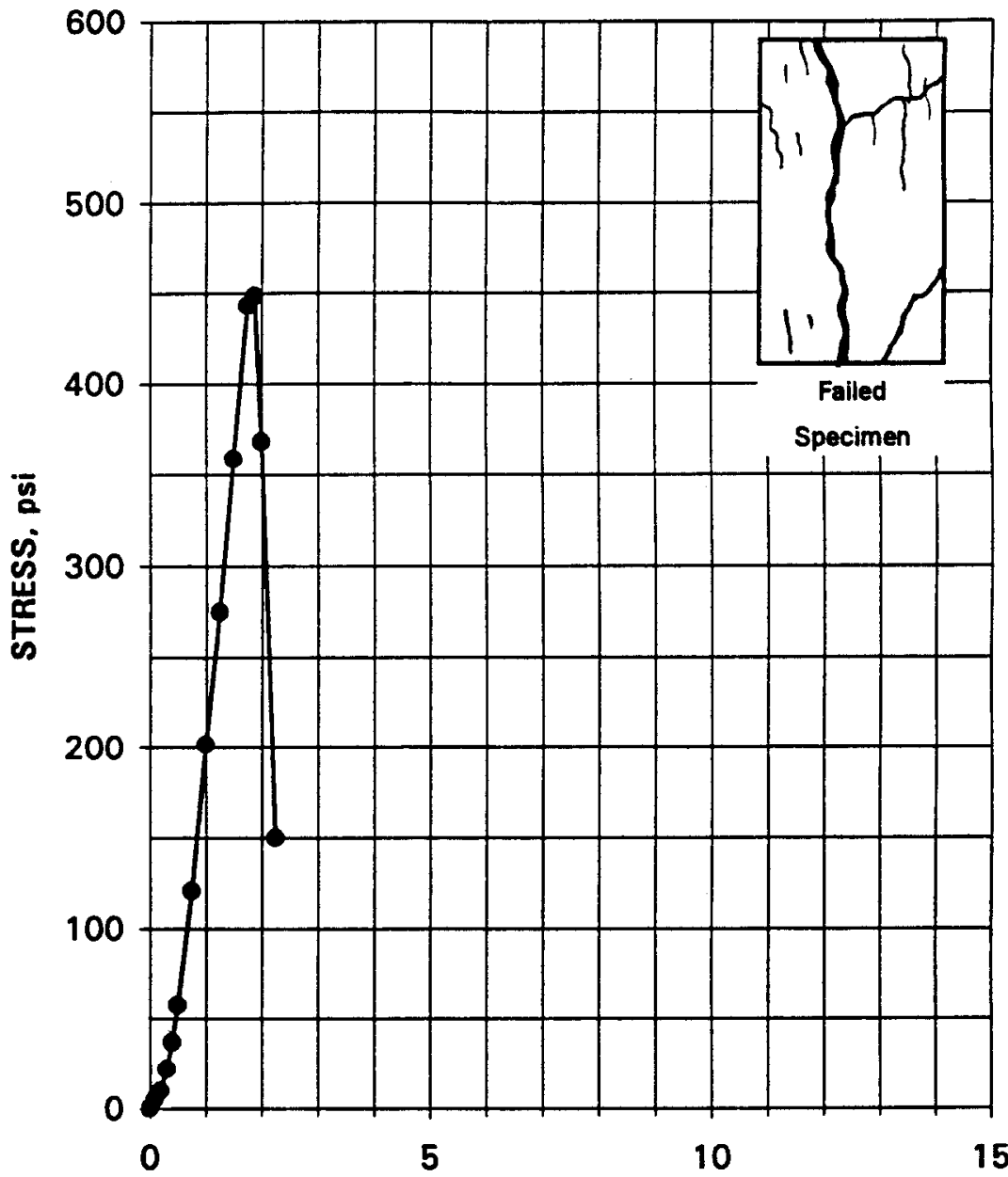

STRAIN, \% 


\section{UNCONFINED COMPRESSIVE STRENGTH - ASTM D 2166 28 DAY CURE, 90 DAY SATURATION}

Project Name SAIC / SAVANNAH RIVER

Project No. $\quad 777304.00010500$

Specimen Mold Date

Specimen Test Date

STRESS AT FAILURE, psi

STRAIN AT FAILURE, \%

AXIAL DEVIATOR

\begin{tabular}{|c|c|}
\hline STRAIN，\% & STRESS, psi \\
\hline 0.00 & 0.0 \\
\hline 0.10 & 15.2 \\
\hline 0.20 & 36.7 \\
\hline 0.30 & 57.8 \\
\hline 0.40 & 77.9 \\
\hline 0.50 & 102.1 \\
\hline 0.75 & 167.2 \\
\hline 0.99 & 236.4 \\
\hline 1.24 & 301.8 \\
\hline 1.49 & 358.5 \\
\hline 1.74 & 417.0 \\
\hline 1.99 & 463.2 \\
\hline 2.24 & 469.1 \\
\hline 2.36 & 477.8 \\
\hline 2.49 & 421.6 \\
\hline 2.74 & 416.5 \\
\hline 2.98 & 407.8 \\
\hline 3.23 & 352.8 \\
\hline 3.48 & 327.4 \\
\hline 3.73 & 317.7 \\
\hline
\end{tabular}

$11 / 25 / 98$

$3 / 23 / 99$

477.8

2.4
Client Sample No. K343F00

IT Lab Specimen No. ETDC-7994

Specimen Height, in.

4.0218

Specimen Diameter, in.

2.0050

Specimen Weight, $g$.

387.28

Moisture Content, \%

29.9

Wet Unit Weight, pcf.

Dry Unit Weight, pcf.

116.2

89.4

Rate of Strain, in./min.

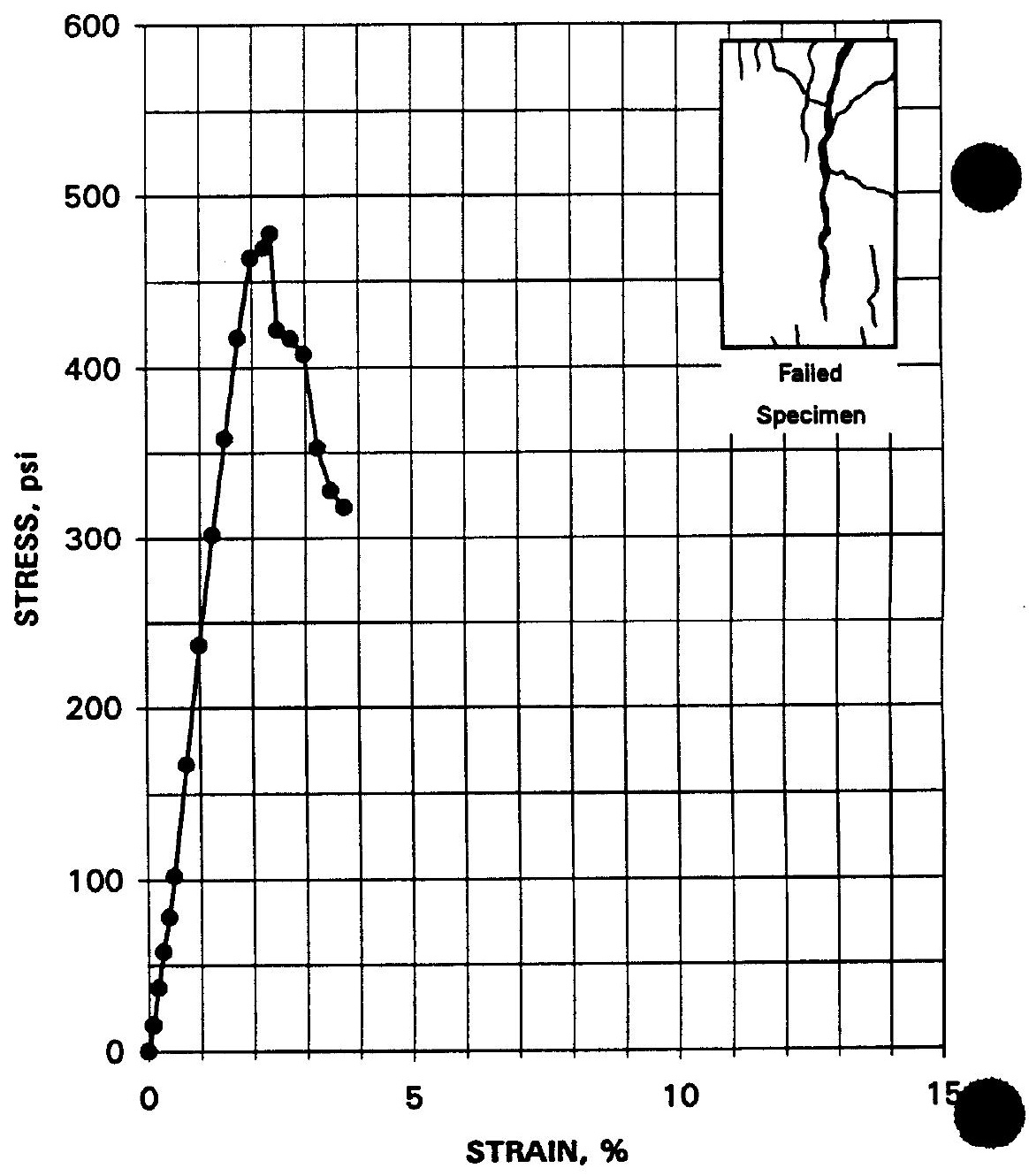




\section{UNCONFINED COMPRESSIVE STRENGTH - ASTM D 2166 28 DAY CURE, 90 DAY SATURATION}

Project Name SAIC / SAVANNAH RIVER

Project No. $\quad 777304.00010500$

Specimen Mold Date

Specimen Test Date

STRESS AT FAILURE, psi STRAIN AT FAILURE, $\%$

\begin{tabular}{ccc}
$\begin{array}{cc}\text { AXIAL } \\
\text { STRAIN, \% }\end{array}$ & \multicolumn{1}{c}{$\begin{array}{c}\text { DEVIATOR } \\
\text { STRESS, psi }\end{array}$} \\
\cline { 1 - 1 } 0.00 & & 0.0 \\
0.10 & & 5.1 \\
0.20 & & 8.8 \\
0.30 & & 13.9 \\
0.40 & & 19.9 \\
0.50 & & 24.6 \\
0.75 & & 46.2 \\
1.00 & & 76.2 \\
1.25 & & 108.5 \\
1.50 & & 149.4 \\
1.75 & & 201.6 \\
2.00 & & 242.0 \\
2.24 & 258.1 \\
2.49 & 216.7 \\
2.74 & 215.2 \\
2.99 & 211.6 \\
3.24 & 208.9 \\
3.49 & 207.4 \\
3.74 & 186.5 \\
3.99 & 64.1
\end{tabular}

Client Sample No. K413F00

IT Lab Specimen No. ETDC-7995

Specimen Height, in.

4.0098

Specimen Diameter, in.

2.0055

Specimen Weight, $g$.

384.10

Moisture Content, \%

33.9

Wet Unit Weight, pef.

115.5

Dry Unit Weight, pef.

86.3

Rate of Strain, in./min.

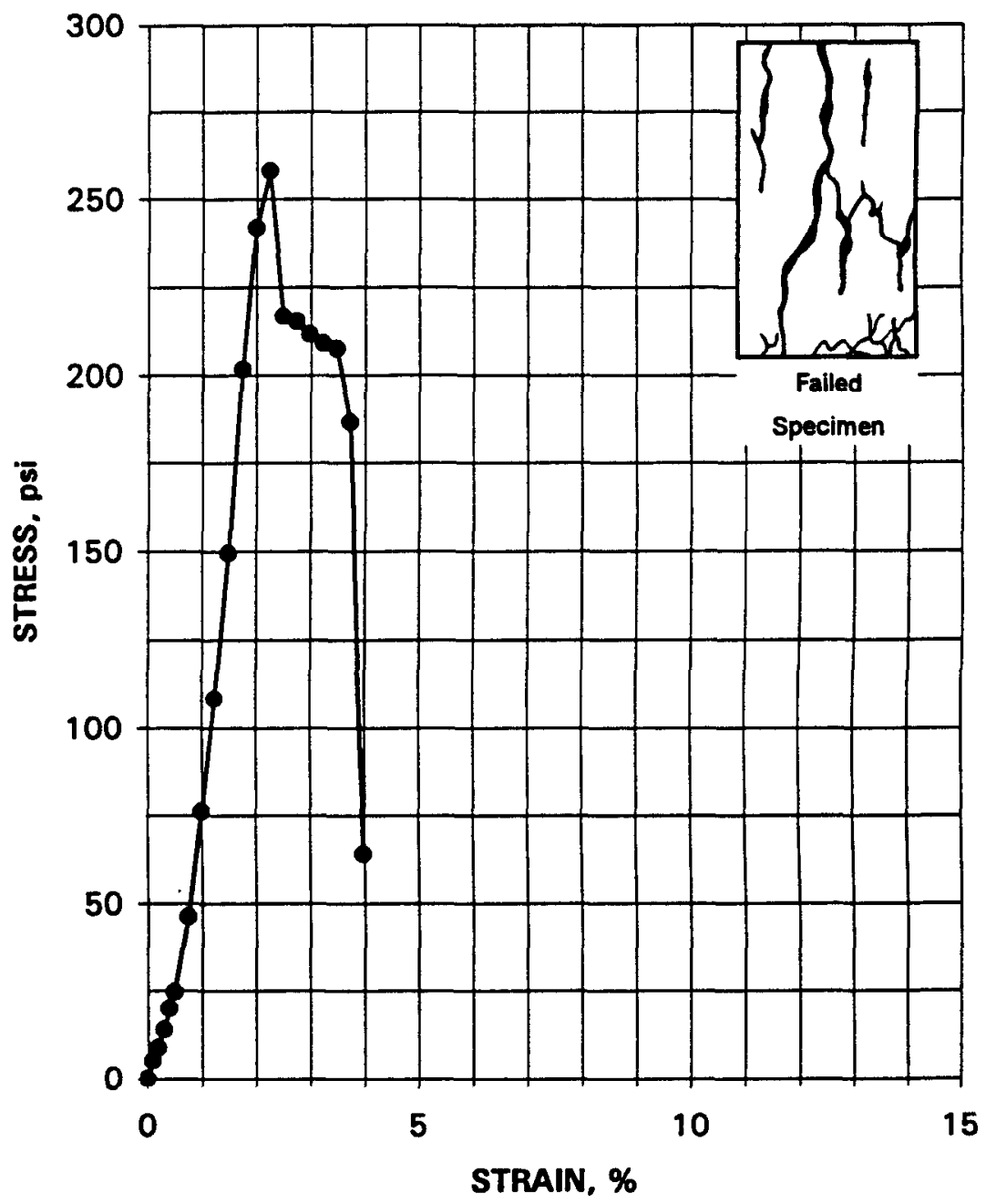




\section{UNCONFINED COMPRESSIVE STRENGTH - ASTM D 2166 28 DAY CURE, 90 DAY SATURATION}

Project Name SAIC / SAVANNAH RIVER

Project No. 777304.00010500

Specimen Mold Date

Specimen Test Date

STRESS AT FAILURE, psi

STRAIN AT FAILURE, \%

\begin{tabular}{ccc}
$\begin{array}{c}\text { AXIAL } \\
\text { STRAIN, \% }\end{array}$ & & $\begin{array}{c}\text { DEVIATOR } \\
\text { STRESS, psi }\end{array}$ \\
\cline { 1 - 1 } 0.00 & & 0.0 \\
0.10 & & 5.4 \\
0.20 & & 16.4 \\
0.30 & & 27.0 \\
0.40 & & 42.1 \\
0.50 & & 58.7 \\
0.74 & & 110.1 \\
0.99 & & 183.2 \\
1.24 & & 255.6 \\
1.49 & & 326.0 \\
1.74 & & 397.4 \\
1.86 & 410.2 \\
1.98 & 229.2 \\
2.23 & 198.2
\end{tabular}

$11 / 25 / 98$

$3 / 23 / 99$

410.2

1.9
Client Sample No. K423F00

IT Lab Specimen No. ETDC-7996

Specimen Height, in.

4.0323

Specimen Diameter, in.

2.0098

Specimen Weight, $g$.

393.49

Moisture Content, \%

31.5

117.2

89.1

Dry Unit Weight, pcf.

0.0400

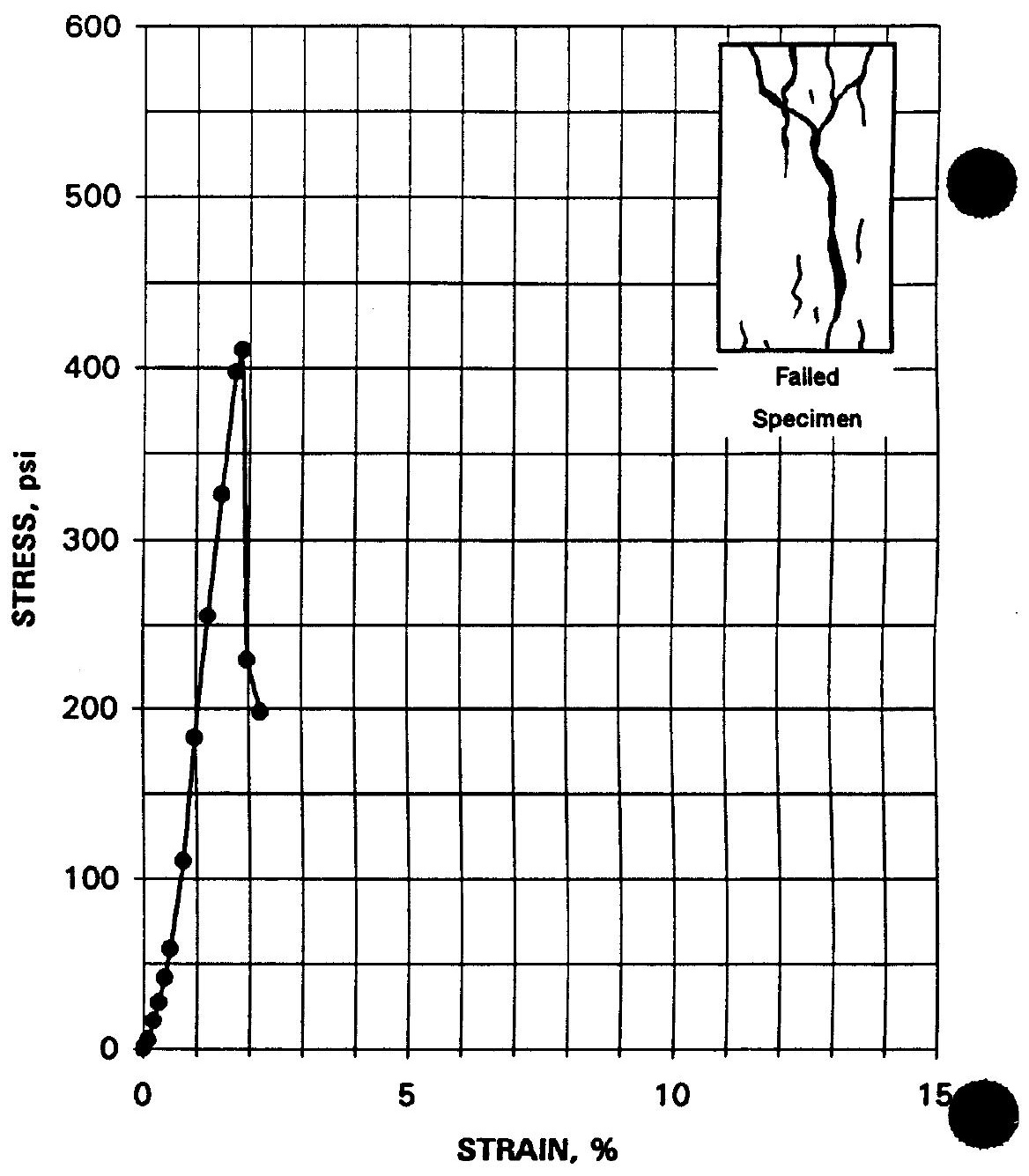




\section{UNCONFINED COMPRESSIVE STRENGTH - ASTM D 2166 28 DAY CURE, 90 DAY SATURATION}

Project Name SAIC / SAVANNAH RIVER

Project No. $\quad 777304.00010500$

Specimen Mold Date

Specimen Test Date

STRESS AT FAILURE, psi

STRAIN AT FAILURE, \%

\begin{tabular}{ccc}
$\begin{array}{c}\text { AXIAL } \\
\text { STRAIN, \% }\end{array}$ & $\begin{array}{c}\text { DEVIATOR } \\
\text { STRESS, psi }\end{array}$ \\
\cline { 1 - 1 } 0.00 & & 0.0 \\
0.10 & & 6.3 \\
0.20 & & 15.4 \\
0.30 & & 22.9 \\
0.40 & & 32.6 \\
0.50 & & 45.4 \\
0.75 & & 79.1 \\
1.00 & & 107.5 \\
1.25 & & 159.2 \\
1.50 & & 223.9 \\
1.75 & & 279.9 \\
2.00 & 318.7 \\
2.25 & 330.2 \\
2.37 & 333.5 \\
2.50 & 50.3
\end{tabular}

Client Sample No. K433F00

IT Lab Specimen No. ETDC-7997

Specimen Height, in.

4.0040

Specimen Diameter, in.

2.0110

Specimen Weight, $g$.

369.98

Moisture Content, \%

40.6

Wet Unit Weight, pcf.

110.8

Dry Unit Weight, pcf.

78.8

Rate of Strain, in./min.

0.0400

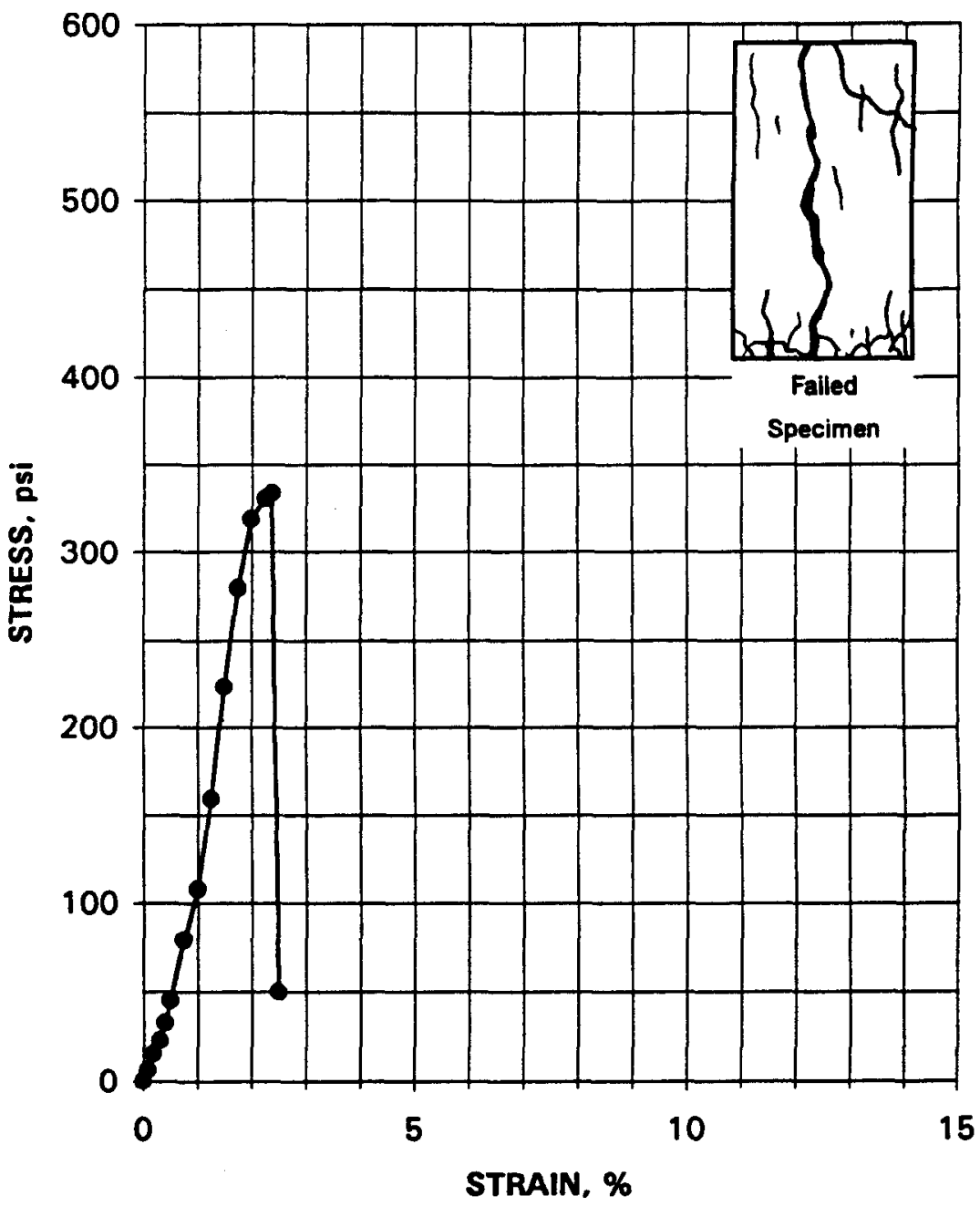




\section{UNCONFINED COMPRESSIVE STRENGTH - ASTM D 2166 28 DAY CURE, 90 DAY SATURATION}

Project Name SAIC / SAVANNAH RIVER

Project No. $\quad 777304.00010500$

Specimen Mold Date

Specimen Test Date

STRESS AT FAILURE, psi

STRAIN AT FAILURE, \%

\begin{tabular}{ccc}
$\begin{array}{c}\text { AXIAL } \\
\text { STRAIN, \% }\end{array}$ & & $\begin{array}{c}\text { DEVIATOR } \\
\text { STRESS, psi }\end{array}$ \\
\cline { 1 - 1 } 0.00 & & 0.0 \\
0.10 & & 5.7 \\
0.20 & & 19.5 \\
0.30 & & 36.8 \\
0.39 & & 53.7 \\
0.49 & & 79.1 \\
0.74 & & 163.5 \\
0.99 & & 264.6 \\
1.23 & & 366.8 \\
1.48 & 452.7 \\
1.73 & 525.3 \\
1.97 & 158.4
\end{tabular}

Client Sample No. K443F00

IT Lab Specimen No. ETDC-7998

Specimen Height, in.

Specimen Diameter, in.

4.0532

Specimen Weight, $g$.

2.0087

376.10

Moisture Content, \%

37.0

Wet Unit Weight, pef.

Dry Unit Weight, pcf.

111.6

Rate of Strain, in./min.

81.4

0.0400

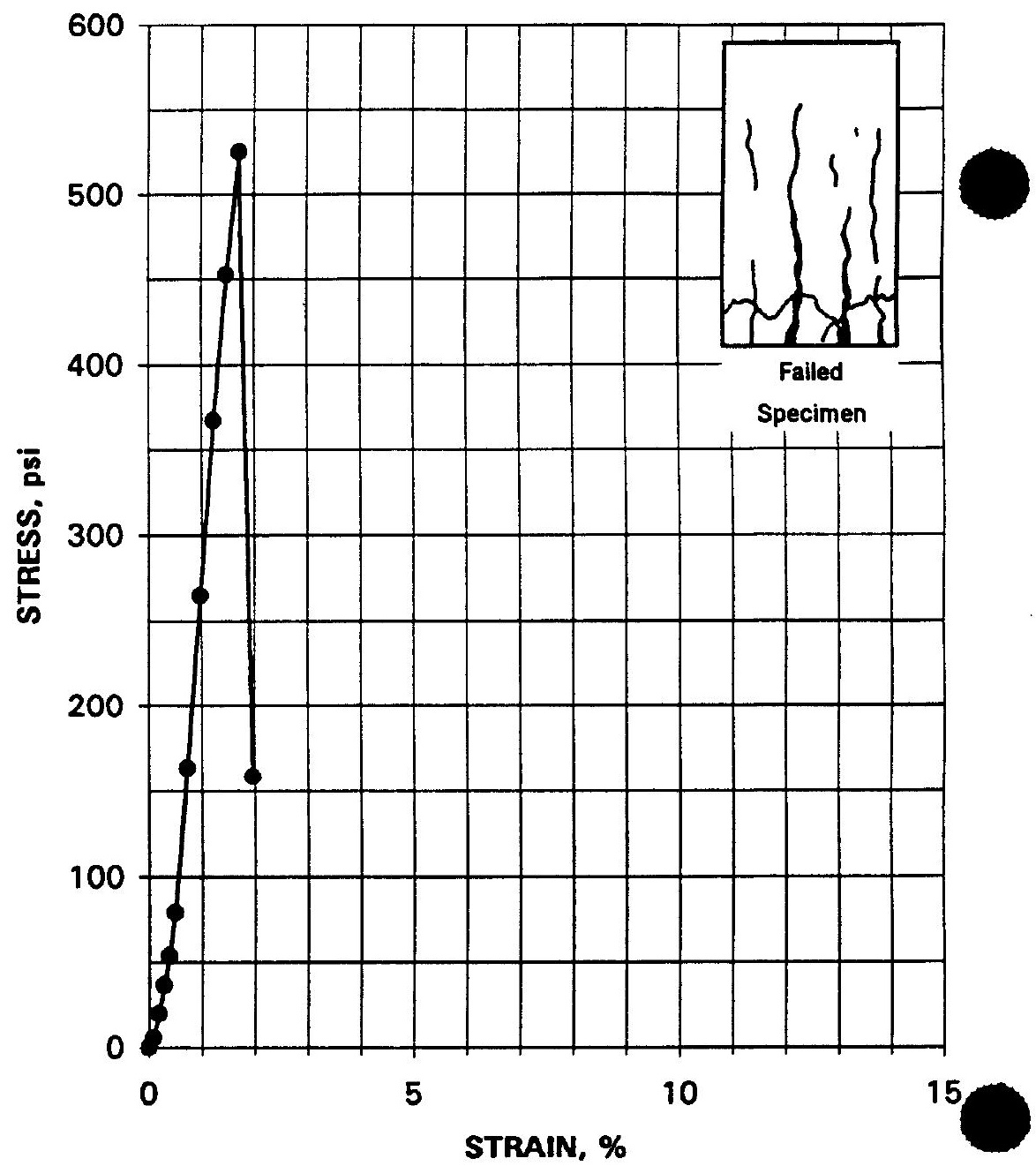




\section{K Series}

28 Day Expansion/Shrinkage Results 


\section{One-Dimensional Expansion, Shrinkage, and Uplift Pressure of Soil-Lime Mixtures ASTM D 3877}

\section{8-Day Cure}

\begin{tabular}{ccccc}
$\begin{array}{c}\text { Geo Lab } \\
\text { Sample No. }\end{array}$ & $\begin{array}{c}\text { Knoxville Lab } \\
\text { Sample No. }\end{array}$ & Expansion, \% & Expansion Result & Shrinkage, \% \\
\hline \hline ETDC-7967 & K312K00 & -0.001 & No expansion & -0.64 \\
ETDC-7968 & K322K00 & 0.000 & No expansion & Insufficient sample \\
ETDC-7969 & K332K00 & -0.003 & No expansion & -4.11 \\
ETDC-7970 & K342K00 & -0.001 & No expansion & -1.06 \\
ETDC-7971 & K412K00 & -0.001 & No expansion & -0.39 \\
ETDC-7972 & K422K00 & 0.000 & No expansion & -0.34 \\
ETDC-7973 & K432K00 & -0.001 & No expansion & Insufficient sample \\
ETDC-7974 & K442K00 & -0.004 & No expansion & -0.2
\end{tabular}




\section{K Series \\ 28 Day Permeability Results}




\section{8-DAY HYDRAULIC CONDUCTIVITY / PERMEABILITY ASTM D 5084}

PROJECT NAME: SAIC / Savannah River

PROJECT NO. $\quad 777304.00010500$

\section{CLIENT SAMPLE NO. K312G00 \\ IT LAB SAMPLE NO. ETDC-7983}

\begin{tabular}{|c|c|c|c|c|}
\hline \multirow[b]{2}{*}{ Specimen diameter, $\mathrm{cm}$} & INITIAL & FINAL & & \\
\hline & \multicolumn{4}{|l|}{5.08} \\
\hline Specimen length, cm & 10.13 & & Hydraulic gradient & 20.8 \\
\hline Wet weight of specimen, $g$ & 393.12 & 400.46 & Min. consolidation stress, psi & 2.0 \\
\hline Specimen cross-sect. area, $\mathrm{cm}^{-} 2$ & 20.2683 & & Max. consolidation stress, psi & 6.0 \\
\hline Water content, \% & 25.0 & 27.3 & Tota! backpressure, psi & 3.0 \\
\hline Wet unit weight, pcf & 119.5 & & Permeant Fluid Deair & DI Water \\
\hline Dry unit weight, pcf & 95.6 & & Sample molded & $11 / 25 / 98$ \\
\hline Estimated degree of saturation, \% & 90.6 & & Sample set up & $12 / 23 / 98$ \\
\hline Estimated spec. gravity of solids & 2.65 & & Six tests performed & \\
\hline
\end{tabular}

\section{PERMEABILITY vS TIME}

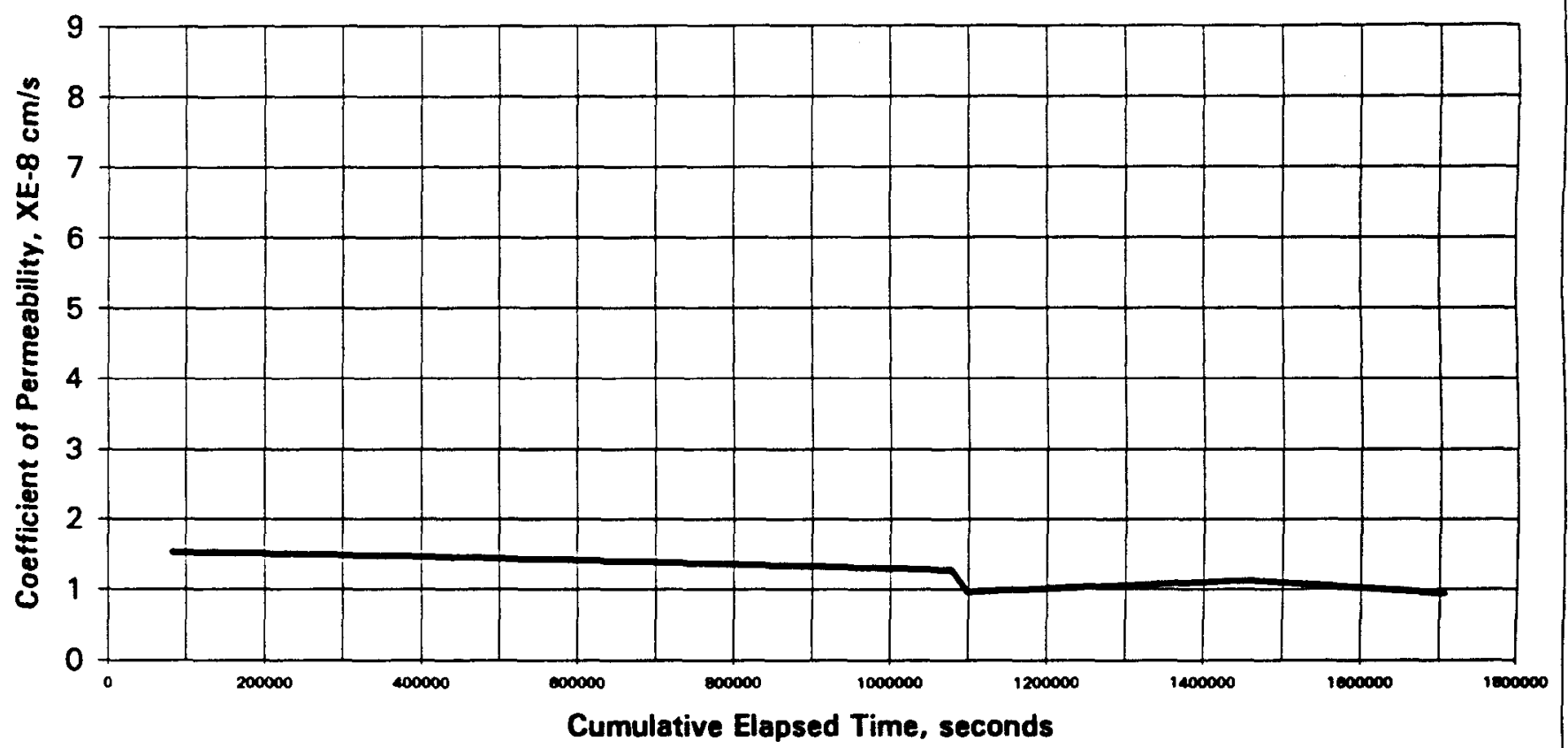




\section{8-DAY HYDRAULIC CONDUCTIVITY / PERMEABILITY ASTM D 5084}

PROJECT NAME: SAIC / Savannah River

PROJECT NO. $\quad 777304.00010500$
CLIENT SAMPLE NO.
K322G00
IT LAB SAMPLE NO.
ETDC-7984

\begin{tabular}{|c|c|c|c|c|}
\hline \multirow[b]{2}{*}{ Specimen diameter, $\mathrm{cm}$} & INITIAL & FINAL & & \multirow[b]{3}{*}{20.7} \\
\hline & \multicolumn{2}{|l|}{5.08} & & \\
\hline Specimen length, $\mathrm{cm}$ & \multicolumn{2}{|l|}{10.19} & Hydraulic gradient & \\
\hline Wet weight of specimen, $\mathrm{g}$ & 392.63 & 400.08 & Min. consolidation stress, psi & 2.0 \\
\hline Specimen cross-sect. area, $\mathrm{cm}^{-2} 2$ & \multicolumn{2}{|l|}{20.3055} & Max. consolidation stress, psi & 8.0 \\
\hline Water content, \% & 23.1 & 25.5 & Tota! backpressure, psi & 2.0 \\
\hline Wet unit weight, pcf & \multicolumn{2}{|l|}{118.5} & Permeant Fluid & Deaired DI Water \\
\hline Dry unit weight, pcf & \multicolumn{2}{|l|}{96.2} & Sample molded & $11 / 25 / 98$ \\
\hline Estimated degree of saturation, $\%$ & \multicolumn{2}{|l|}{85.2} & Sample set up & \multirow[t]{2}{*}{$12 / 23 / 98$} \\
\hline Estimated spec. gravity of solids & \multicolumn{2}{|l|}{2.65} & Five tests performed & \\
\hline
\end{tabular}

\begin{tabular}{|ll|}
\hline Coefficient of Permeability, cm/s & $3.0 E-09$ \\
\hline
\end{tabular}

\section{PERMEABILITY VS TIME}

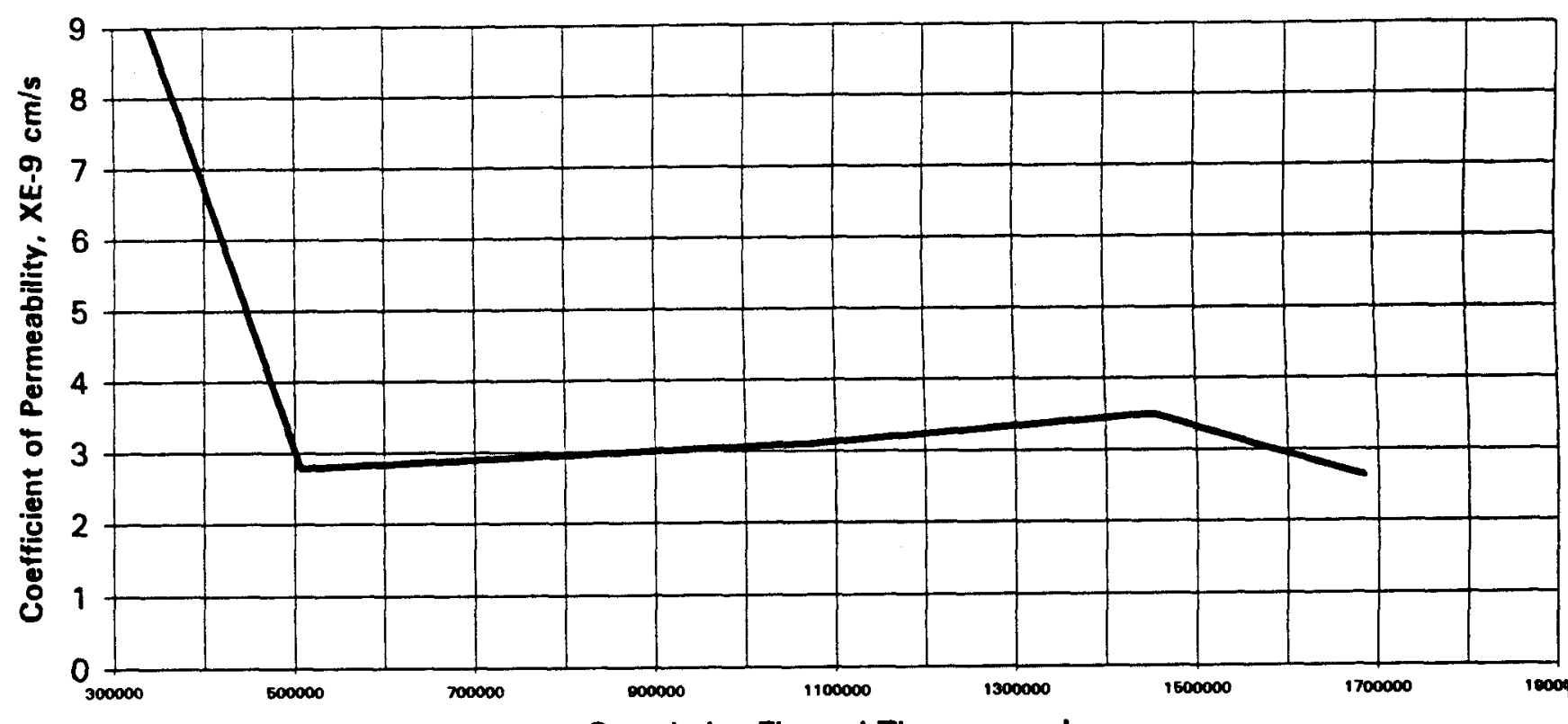

Cumulative Elapsed Time, seconds 


\section{8-DAY HYDRAULIC CONDUCTIVITY / PERMEABILITY ASTM D 5084}

PROJECT NAME: SAIC / Savannah River

PROJECT NO. $\quad 777304.00010500$
CLIENT SAMPLE NO. K332G00

IT LAB SAMPLE NO. ETDC-7985
Specimen diameter, $\mathrm{cm}$

Specimen length, $\mathrm{cm}$

Wet weight of specimen, $g$.

Specimen cross-sect. area, $\mathrm{cm}^{\wedge} 2$

Water content, \%

Wet unit weight, pcf

Dry unit weight, pcf

Estimated degree of saturation, \%

Estimated spec. gravity of solids

\begin{tabular}{l}
\hline INITIAL FINAL \\
\hline 5.08
\end{tabular}

10.19

382.69

387.1

20.2818

29.7

115.6

89.1

92.0

2.65
Hydraulic gradient

13.8

Min. consolidation stress, psi

2.0

4.0

2.0

Max. consolidation stress, psi

Total backpressure, psi

Permeant Fluid

Deaired DI Water

Sample molded

$11 / 25 / 98$

Sample set up

$12 / 23 / 98$

\section{PERMEABILITY vS TIME}

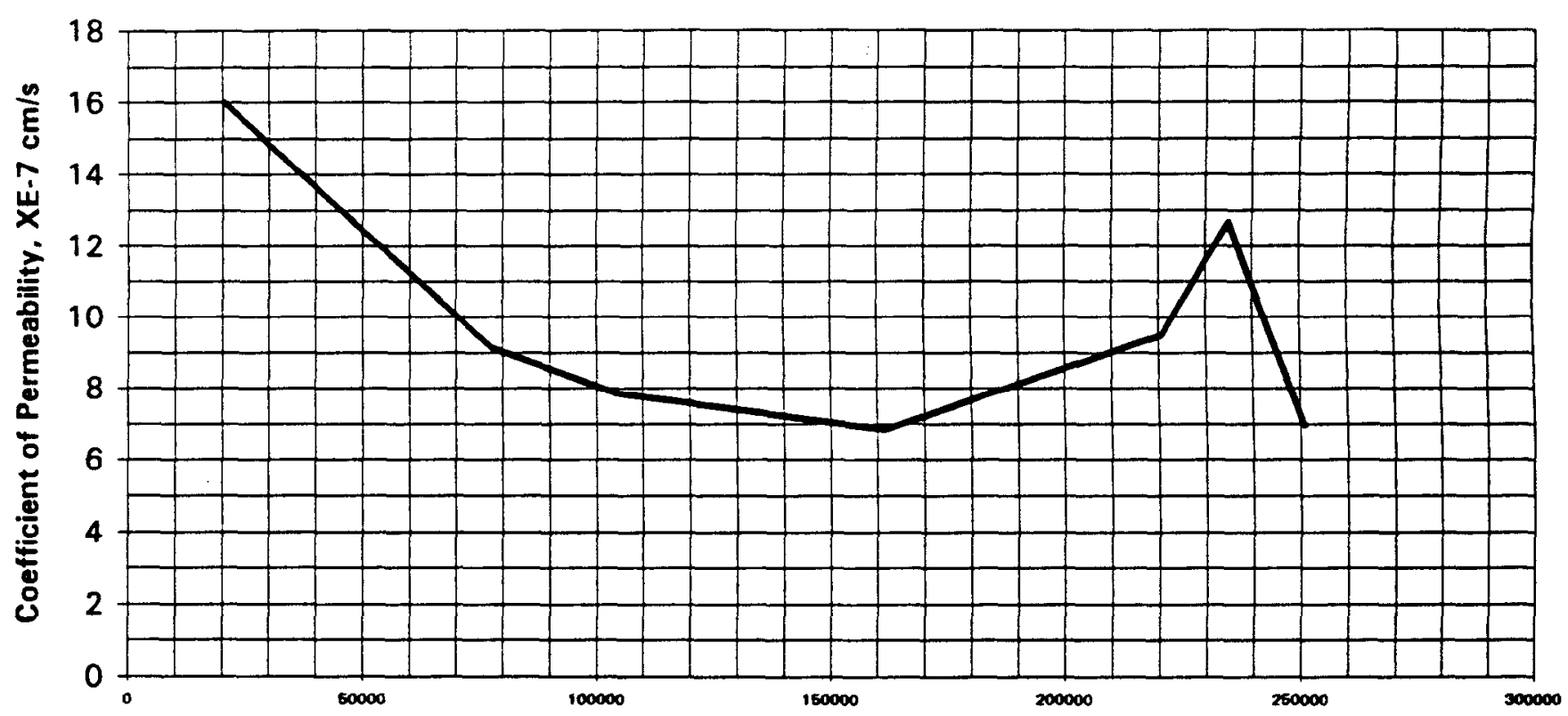

Cumulative Elapsed Time, seconds 


\section{8-DAY HYDRAULIC CONDUCTIVITY / PERMEABILITY ASTM D 5084}

PROJECT NAME:

PROJECT NO.

SAIC / Savannah River

777304.00010500

\section{CLIENT SAMPLE NO. K342G00 \\ IT LAB SAMPLE NO. ETDC-7986}

\begin{tabular}{lllll} 
& INITIAL & FINAL & & \\
\cline { 2 - 3 } Specimen diameter, cm & 5.12 & & & \\
Specimen length, cm & 10.22 & & Hydraulic gradient & 13.8 \\
Wet weight of specimen, g. & 383.2 & 388.96 & Min. consolidation stress, psi & 2.0 \\
Specimen cross-sect. area, cm ^2 & 20.5530 & & Max. consolidation stress, psi & 4.0 \\
Water content, \% & 28.1 & 30.0 & Tota! backpressure, psi & 2.0 \\
Wet unit weight, pcf & 113.9 & & Permeant Fluid & Deaired DI Water \\
Dry unit weight, pcf & 88.9 & & Sample molded & $11 / 25 / 98$ \\
Estimated degree of saturation, \% & 86.5 & & Sample set up & $12 / 23 / 98$ \\
Estimated spec. gravity of solids & 2.65 & & Five tests performed &
\end{tabular}

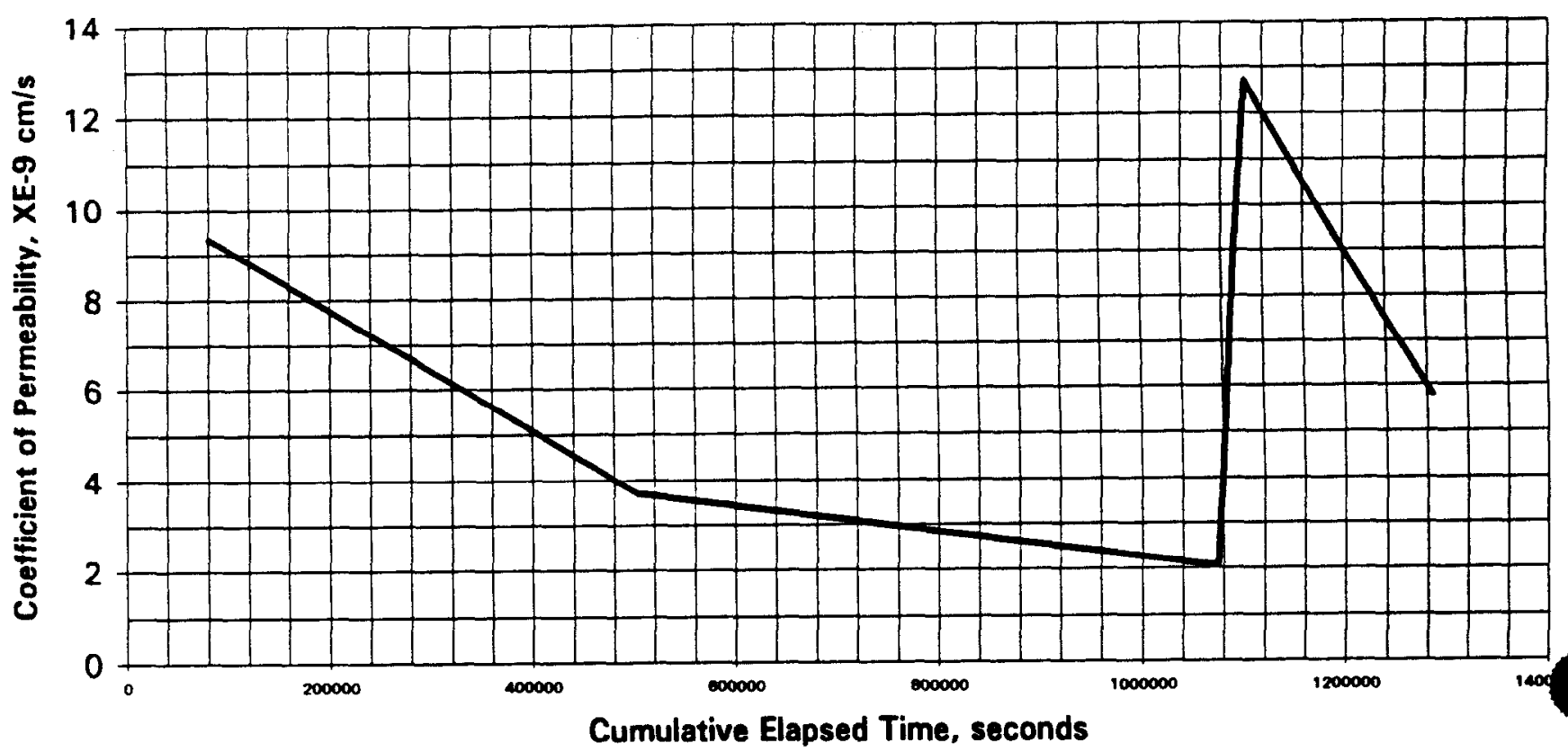




\section{8-DAY HYDRAULIC CONDUCTIVITY / PERMEABILITY ASTM D 5084}

PROJECT NAME: SAIC / Savannah River

PROJECT NO.
777304.00010500
CLIENT SAMPLE NO. K412G00

IT LAB SAMPLE NO. ETDC-7987

\section{Specimen diameter, $\mathrm{cm}$}

Specimen length, $\mathrm{cm}$

Wet weight of specimen, $g$.

Specimen cross-sect. area, $\mathrm{cm}^{\wedge} 2$

Water content, \%

Wet unit weight, pcf

Dry unit weight, pcf

Estimated degree of saturation, \%

Estimated spec. gravity of solids

\begin{tabular}{ll}
\hline INITIAL \\
\hline 5.08 & FINAL \\
\hline
\end{tabular}

10.15

$376.86 \quad 383.39$

20.2751

30.7

114.4

87.5

91.4

2.65

\section{0}

Hydraulic gradient

20.8

Min. consolidation stress, psi

2.0

Max. consolidation stress, psi

7.0

3.0

Total backpressure, psi

Permeant Fluid

Deaired DI Water

Sample molded

$11 / 25 / 98$

Sample set up

$12 / 23 / 98$
Five tests performed

\section{PERMEABILITY vS TIME}

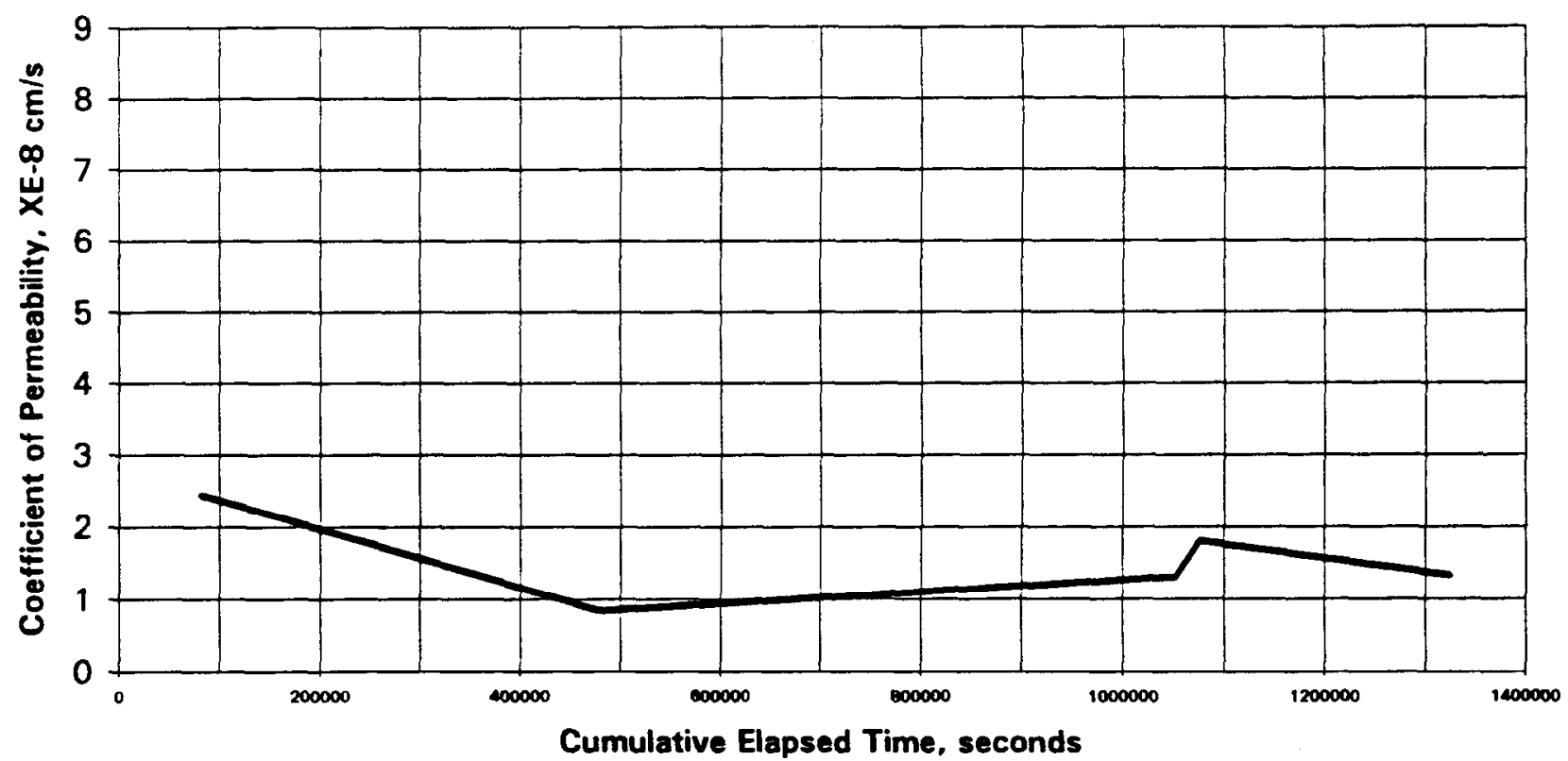

Cumulative Elapsed Time, seconds 


\section{8-DAY HYDRAULIC CONDUCTIVITY / PERMEABILITY ASTM D 5084}

PROJECT NAME: SAIC / Savannah River PROJECT NO. $\quad \mathbf{7 7 7 3 0 4 . 0 0 0 1 0 5 0 0}$
CLIENT SAMPLE NO. K422G00

IT LAB SAMPLE NO. ETDC-7988
Specimen diameter, $\mathrm{cm}$

Specimen length, $\mathrm{cm}$

Wet weight of specimen, $g$.

Specimen cross-sect. area, $\mathrm{cm}^{-2} 2$

Water content, $\%$

Wet unit weight, pcf

Dry unit weight, pcf

Estimated degree of saturation, \%

Estimated spec. gravity of solids

\begin{tabular}{l}
\hline INITIAL FINAL \\
\hline 5.09
\end{tabular}

10.17

386.26

389.97

20.3630

30.5

31.7

116.5

89.3

94.7

2.65
Hydraulic gradient 13.8

Min. consolidation stress, psi

2.0

Max. consolidation stress, psi 4.0

Total backpressure, psi

Permeant Fluid

2.0

Sample molded

Deaired DI Water

Sample set up

$11 / 25 / 98$

$12 / 23 / 98$

Five tests performed

\section{PERMEABILITY vs TIME}

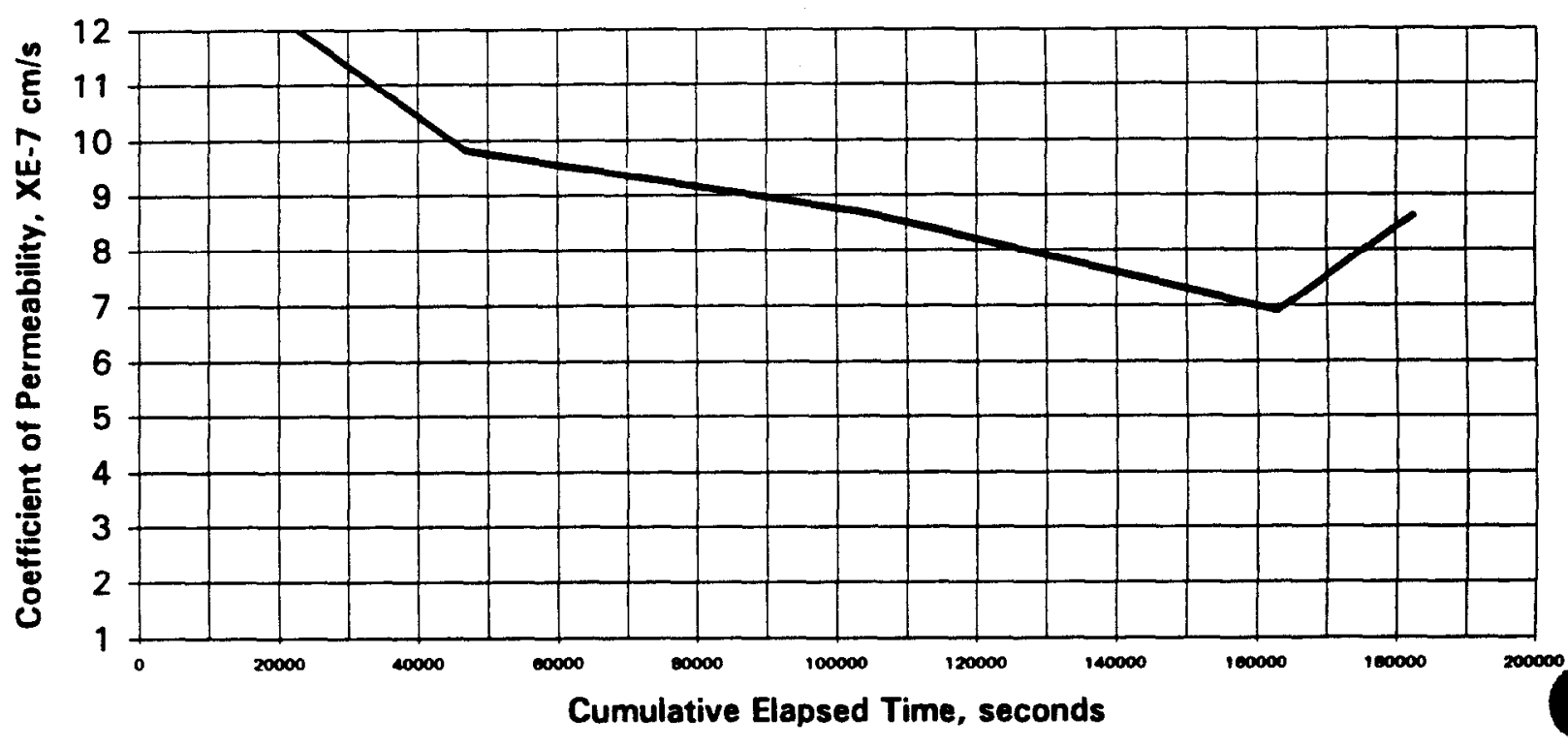




\section{8-DAY HYDRAULIC CONDUCTIVITY / PERMEABILITY ASTM D 5084}

PROJECT NAME: SAIC / Savannah River PROJECT NO. $\quad 777304.00010500$ $\begin{array}{ll}\text { CLIENT SAMPLE NO. } & \mathrm{K} 432 \mathrm{GO0} \\ \text { IT LAB SAMPLE NO. } & \text { ETDC-7989 }\end{array}$

\begin{tabular}{|c|c|c|c|c|}
\hline & INITIAL & FINAL & & \\
\hline Specimen diameter, $\mathrm{cm}$ & 5.09 & & & \\
\hline Specimen length, cm & 10.18 & & Hydraulic gradient & 27.6 \\
\hline Wet weight of specimen, $g$ & 368.73 & 374.34 & Min. consolidation stress, psi & 2.0 \\
\hline Specimen cross-sect. area, $\mathrm{cm}^{\wedge} 2$ & 20.3528 & & Max. consolidation stress, psi & 7.0 \\
\hline Water content, $\%$ & 36.8 & 38.9 & Total backpressure, psi & 3.0 \\
\hline Wet unit weight, pcf & 111.1 & & Permeant Fluid Deaire & DI Water \\
\hline Dry unit weight, pcf & 81.2 & & Sample molded & $11 / 25 / 98$ \\
\hline Estimated degree of saturation, $\%$ & 94.0 & & Sample set up & $12 / 23 / 98$ \\
\hline Estimated spec. gravity of solids & 2.65 & & Six tests performed & \\
\hline
\end{tabular}

\begin{tabular}{|ll|}
\hline Coefficient of Permeability, cm/s & $1.9 \mathrm{E}-09$ \\
\hline
\end{tabular}

Result is average of last two tests.

\section{PERMEABILITY vS TIME}

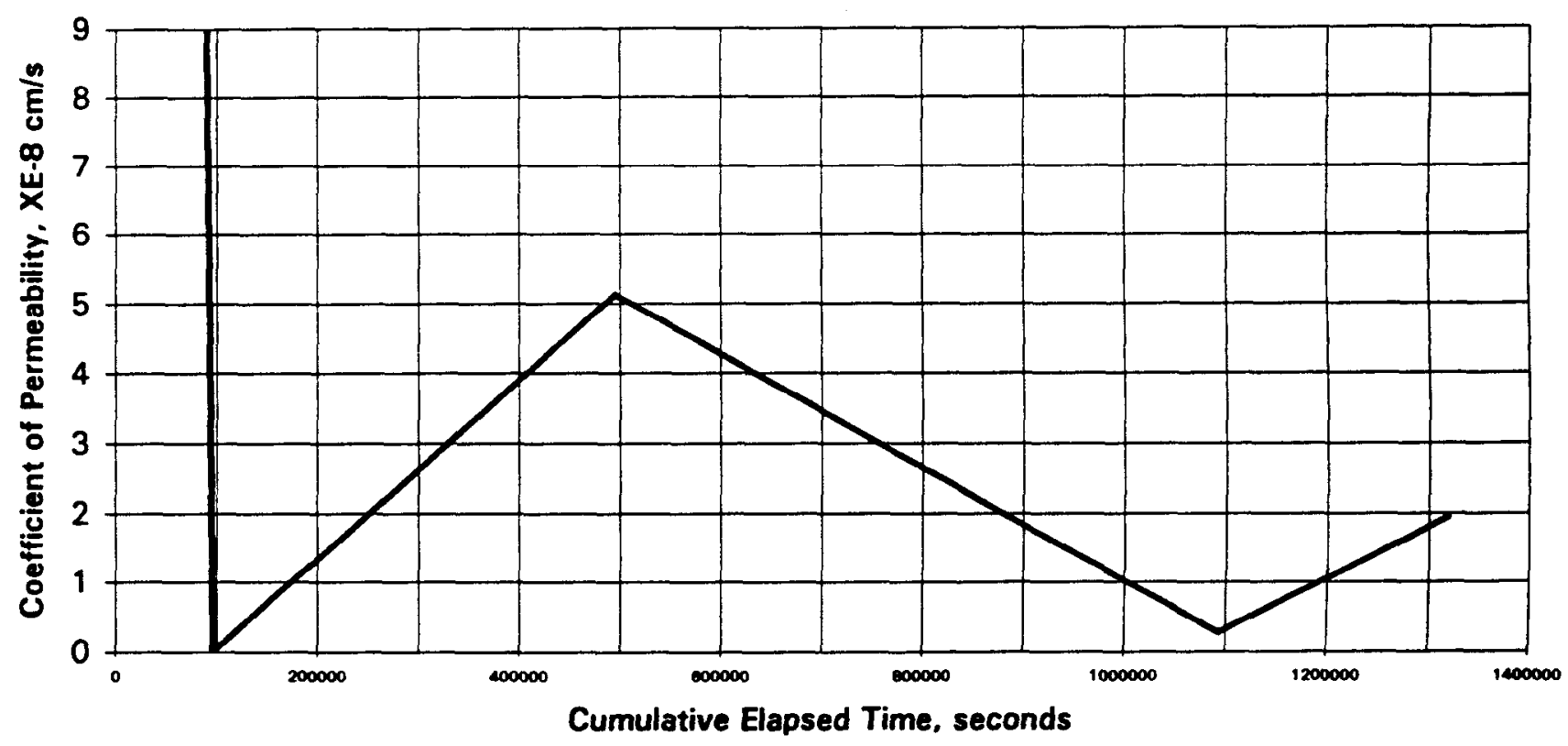




\section{8-DAY HYDRAULIC CONDUCTIVITY / PERMEABILITY ASTM D 5084}

PROJECT NAME: SAIC / Savannah River

PROJECT NO. $\quad 777304.00010500$
CLIENT SAMPLE NO. K442G00

IT LAB SAMPLE NO. ETDC-7990

\begin{tabular}{|c|c|c|c|c|}
\hline \multirow[b]{2}{*}{ Specimen diameter, $\mathrm{cm}$} & INITIAL & FINAL & \multirow[b]{3}{*}{ Hvdraulic aradient } & \\
\hline & 5.10 & & & \\
\hline Specimen length, $\mathrm{cm}$ & 10.25 & & & 13.7 \\
\hline Wet weight of specimen, $g$ & 371.68 & 376.28 & Min. consolidation stress, psi & 2.0 \\
\hline Specimen cross-sect. area, $\mathrm{cm}^{\wedge} 2$ & 20.4511 & & Max. Consolidation stress, psi & 6.0 \\
\hline Water content, $\%$ & 35.4 & 37.1 & Total backpressure, psi & 3.0 \\
\hline Wet unit weight, pcf & 110.7 & & Permeant Fluid Deaire & DI Water \\
\hline Dry unit weight, pcf & 81.7 & & Sample molded & $11 / 25 / 98$ \\
\hline Estimated degree of saturation, $\%$ & 91.6 & & Sample set up & $12 / 23 / 98$ \\
\hline Estimated spec. gravity of solids & 2.65 & & Six tests performed & \\
\hline
\end{tabular}

\section{PERMEABILITY vs TIME}

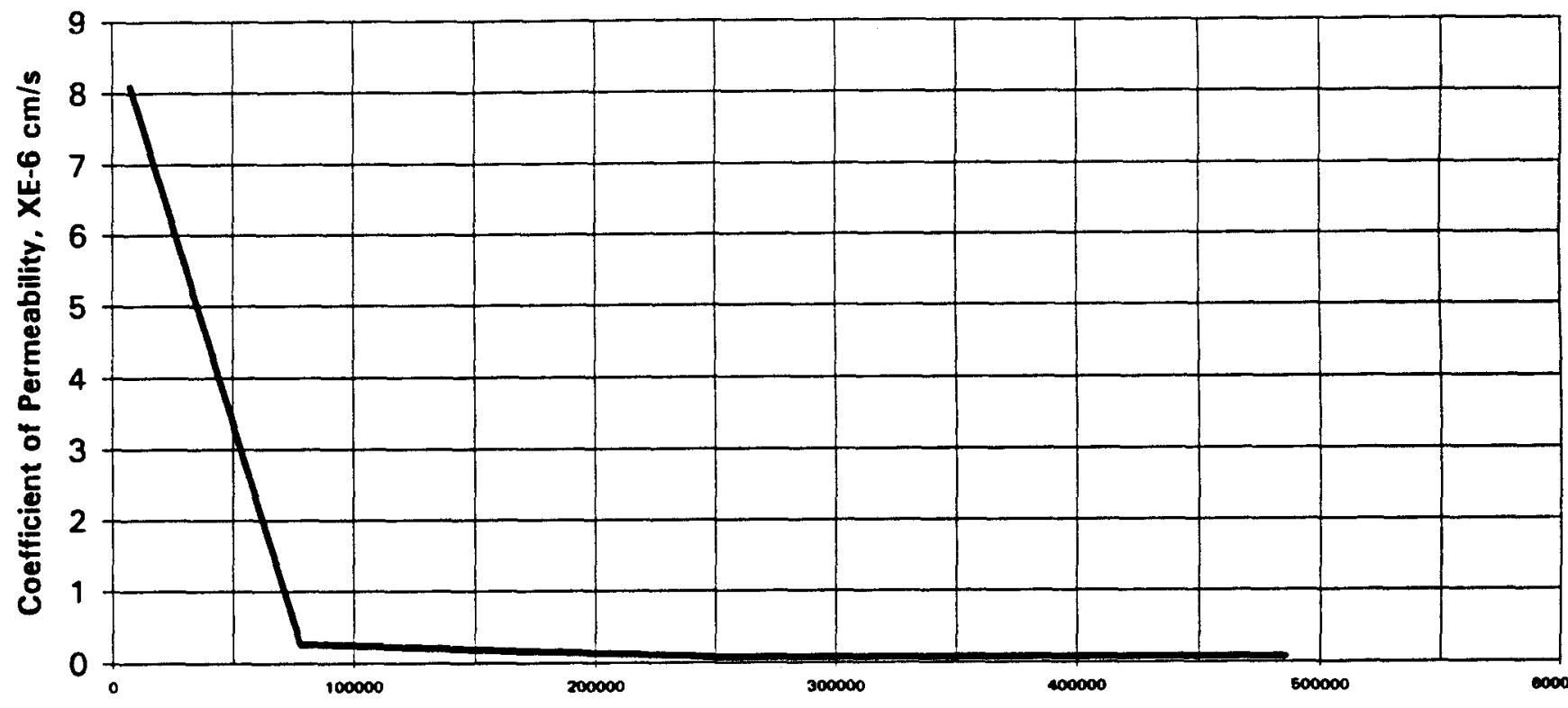

Cumulative Elapsed Time, seconds 


\section{$K$ Series \\ 90 Day Immersion Permeability Results}




\section{0-DAY SATURATED HYDRAULIC CONDUCTIVITY / PERMEABILITY ASTM D 5084}

PROJECT NAME:

PROJECT NO.

777304.00010500
CLIENT SAMPLE NO. K313G00

IT LAB SAMPLE NO. ETDC-7999

\section{INITIAL FINAL}

Specimen diameter, $\mathrm{cm}$

7.60

Specimen length, $\mathrm{cm}$

7.62

Wet weight of specimen, $g$.

$666.83 \quad 666.83$

45.3204

Specimen cross-sect. area, $\mathrm{cm}^{-2} 2$

Water content, \%

Wet unit weight, pcf

Dry unit weight, pcf

Estimated degree of saturation, \%

Estimated spec. gravity of solids

2.6500
Hydraulic gradient

18.5

Min. consolidation stress, psi

2.0

Max. consolidation stress, psi

4.0

\section{0}

Total backpressure, psi

Permeant Fluid

Sample molded

Deaired DI Water

Sample set up

$11 / 25 / 99$

Six tests performed

\subsection{E-06}

\section{PERMEABILITY vs TIME}

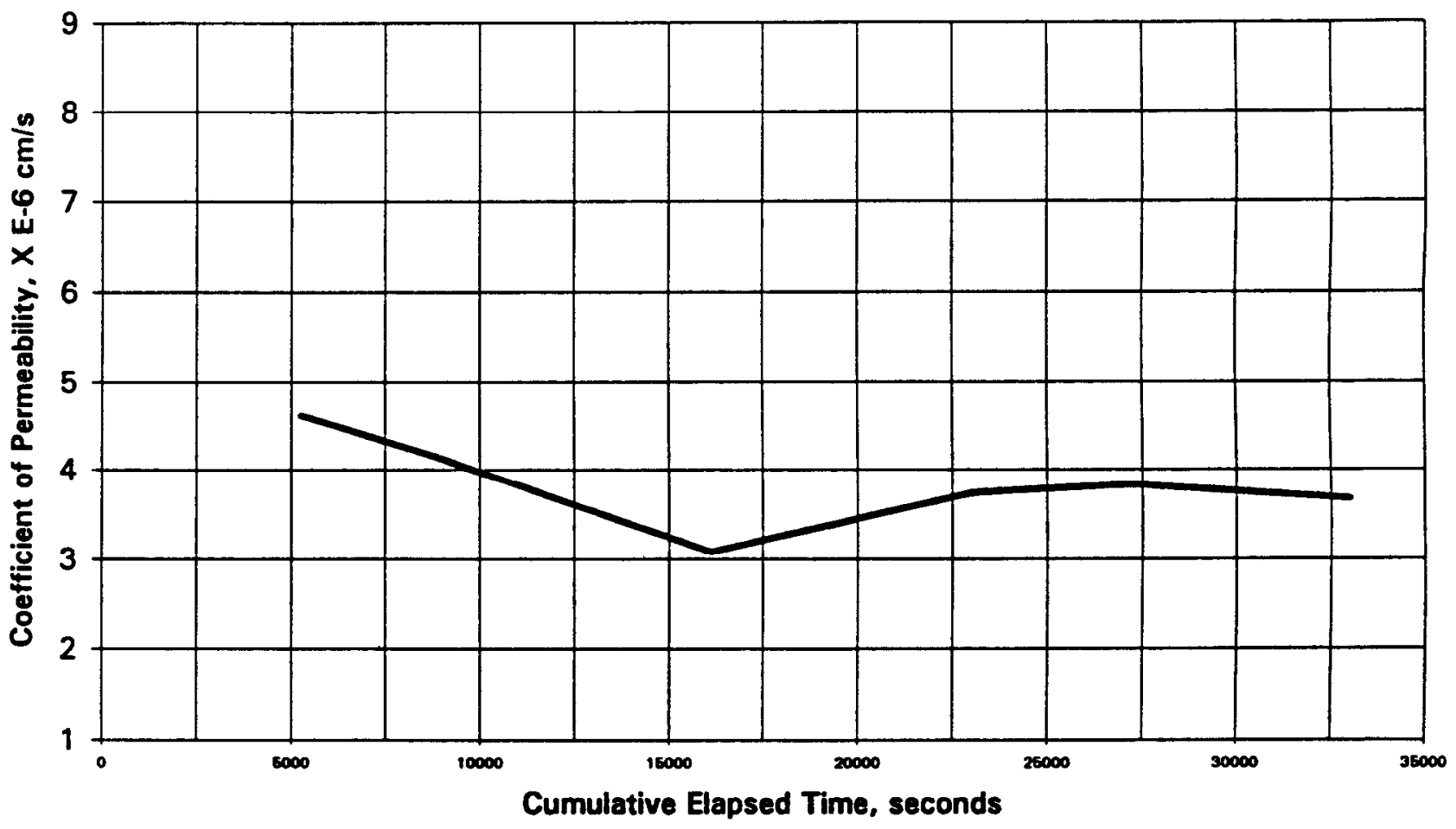




\section{0-DAY SATURATED HYDRAULIC CONDUCTIVITY / PERMEABILITY ASTM D 5084}

PROJECT NAME: SAIC / Savannah River PROJECT NO.
777304.00010500
CLIENT SAMPLE NO.

IT LAB SAMPLE NO.
K323G00

ETDC-8000

\section{Specimen diameter, $\mathrm{cm}$}

Specimen length, $\mathrm{cm}$

Wet weight of specimen, $g$.

Specimen cross-sect. area, $\mathrm{cm}^{-2} 2$

Water content, \%

Wet unit weight, pcf

Dry unit weight, pcf

Estimated degree of saturation, \%

Estimated spec. gravity of solids

Initial conditions are post-soak, pre-test

\begin{tabular}{ll}
\hline INITIAL & FINAL \\
\hline 7.59 &
\end{tabular}

6.79

587.98

45.2295

23.9

119.5

96.4

88.6

2.65

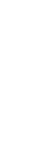

Hydraulic gradient

590.36

24.4

24.4

Permeant Fluid

Sample molded

Sample set up

\section{Coefficient of Permeability, cm/s}

Min. consolidation stress, psi

Max. consolidation stress, psi

31.1

2.0

5.0

3.0

Deaired DI Water

$11 / 25 / 99$

$3 / 23 / 99$

\section{Eight tests performed}

\section{PERMEABILITY vS TIME}

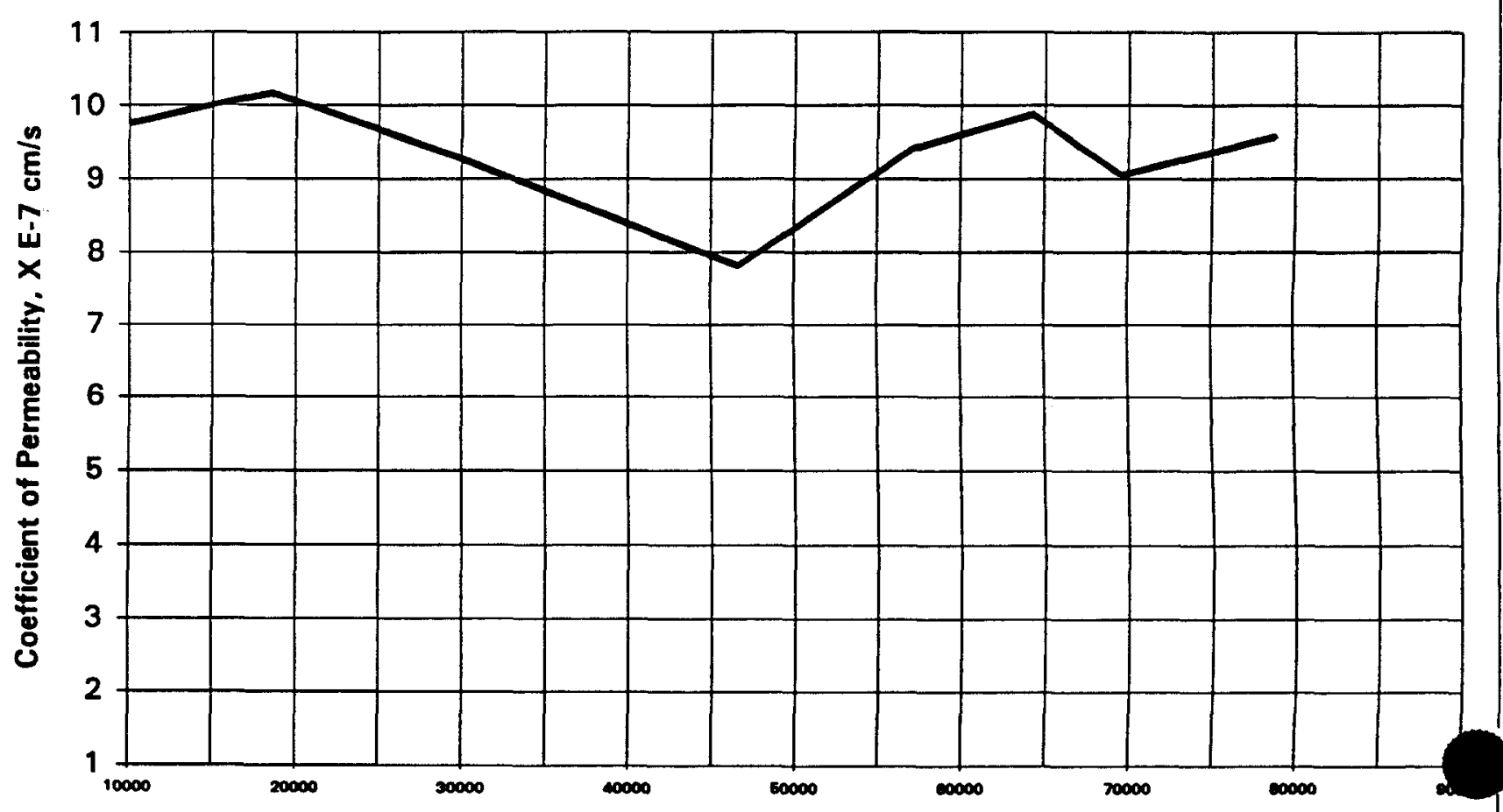

Cumulative Elapsed Time, seconds 


\section{0-DAY SATURATED \\ HYDRAULIC CONDUCTIVITY / PERMEABILITY}

\section{ASTM D 5084}

PROJECT NAME: SAIC / Savannah River

PROJECT NO.
777304.00010500
CLIENT SAMPLE NO. K333G00

IT LAB SAMPLE NO. ETDC-8001
Specimen diameter, $\mathrm{cm}$

Specimen length, $\mathrm{cm}$

Wet weight of specimen, $g$.

Specimen cross-sect. area, $\mathrm{cm}^{`} 2$

Water content, \%

Wet unit weight, pcf

Dry unit weight, pcf

Estimated degree of saturation, \%

Estimated spec. gravity of solids

Initial conditions are post-soak, pre-test

INITIAL FINAL

\subsection{0}

9.06

$763.78 \quad 765.03$

45.3153

31.5

\section{7}

116.1

88.3

95.5

2.65

Hydraulic gradient

15.5

Min. consolidation stress, psi

2.0

Max. consolidation stress, psi 4.0

Total backpressure, psi

4.0

Permeant Fluid

Deaired DI Water

Sample molded

$11 / 25 / 99$

Sample set up

$3 / 23 / 99$

Five tests performed

\begin{tabular}{|ll}
\hline Coefficient of Permeability, $\mathrm{cm} / \mathrm{s}$ & $1.0 \mathrm{E}-06$
\end{tabular}

\section{PERMEABILITY VS TIME}

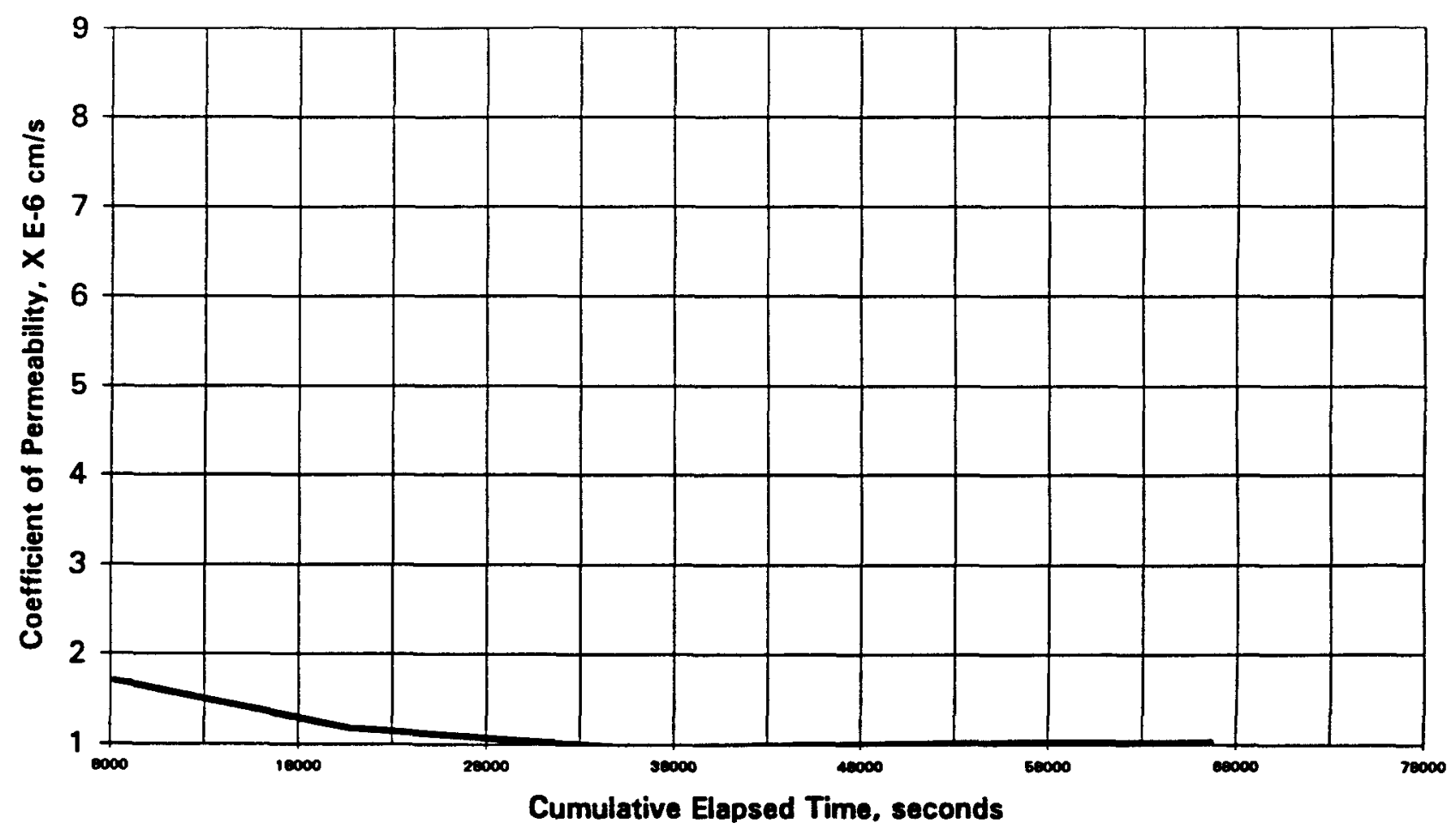




\section{0-DAY SATURATED HYDRAULIC CONDUCTIVITY / PERMEABILITY ASTM D 5084}

PROJECT NAME: SAIC / Savannah River PROJECT NO.
777304.00010500

\section{CLIENT SAMPLE NO. K343G00 \\ IT LAB SAMPLE NO. ETDC-8002}

\section{Specimen diameter, $\mathrm{cm}$}

Specimen length, $\mathrm{cm}$

Wet weight of specimen, $g$.

Specimen cross-sect. area, $\mathrm{cm}^{`} 2$ Water content, \%

Wet unit weight, pcf

Dry unit weight, pef

Estimated degree of saturation, $\%$

Estimated spec. gravity of solids

Initial conditions are post-soak, pre-test

INITIAL FINAL

\subsection{8}

9.28

781

Hydraulic gradient

7.6

45.1841

27.0

116.2

91.5

88.6

2.65
784.46

Min. consolidation stress, psi

2.0

Max. consolidation stress, psi $\quad 3.0$

\section{6}

Total backpressure, psi

Permeant Fluid

5.0

Sample molded

Deaired DI Water

Sample set up

$11 / 25 / 99$

Six tests performed

\section{Coefficient of Permeability, cm/s 1.2E-05}

\section{PERMEABILITY vs TIME}

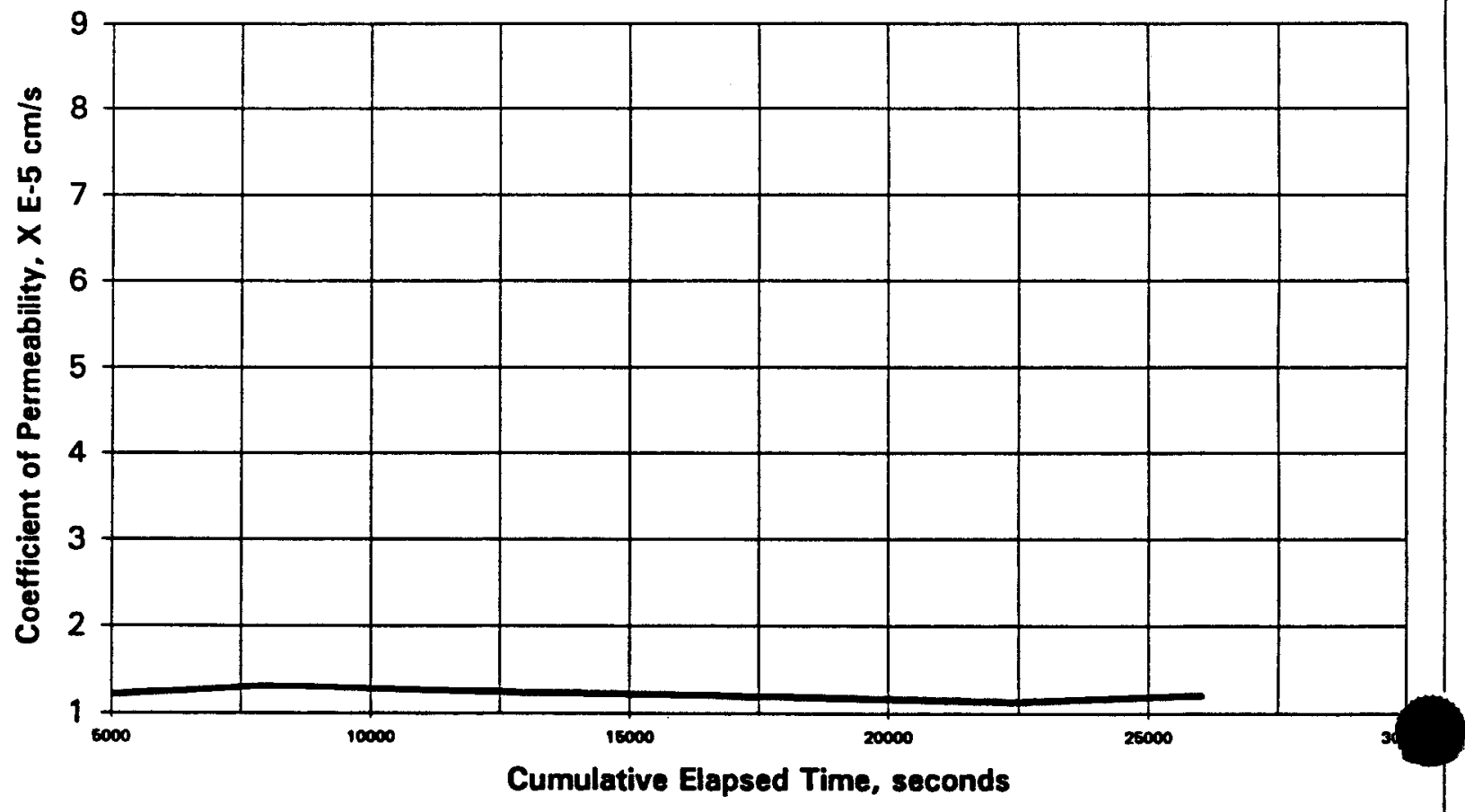




\section{0-DAY SATURATED HYDRAULIC CONDUCTIVITY / PERMEABILITY} ASTM D 5084

PROJECT NAME: SAIC / Savannah River PROJECT NO. $\begin{array}{ll}\text { CLIENT SAMPLE NO. } & \text { K413G00 } \\ \text { IT LAB SAMPLE NO. } & \text { ETDC- } 8003\end{array}$
Specimen diameter, $\mathrm{cm}$

Specimen length, $\mathrm{cm}$

Wet weight of specimen, $g$.

Specimen cross-sect. area, $\mathrm{cm}^{\star} 2$

Water content, \%

Wet unit weight, pef

Dry unit weight, pcf

Estimated degree of saturation, \%

Estimated spec. gravity of solids

Initial conditions are post-sosk, pre-test

\begin{tabular}{|l|l|}
\hline INITIAL \\
\hline 7.59
\end{tabular}

8.40

$700.9 \quad 702.59$

Hydraulic gradient

16.8

45.2749

33.4

33.7

Min. consolidation stress, psi

2.0

Max. consolidation stress, psi

4.0

115.1

86.3

Total backpressure, psi

4.0

Permeant Fluid

Deaired DI Water

96.5

2.65

Sample molded

$11 / 25 / 99$

Sample set up

$3 / 23 / 99$

\section{Coefficient of Permeability, cm/s $3.1 E-06$}

\section{PERMEABILITY vs TIME}

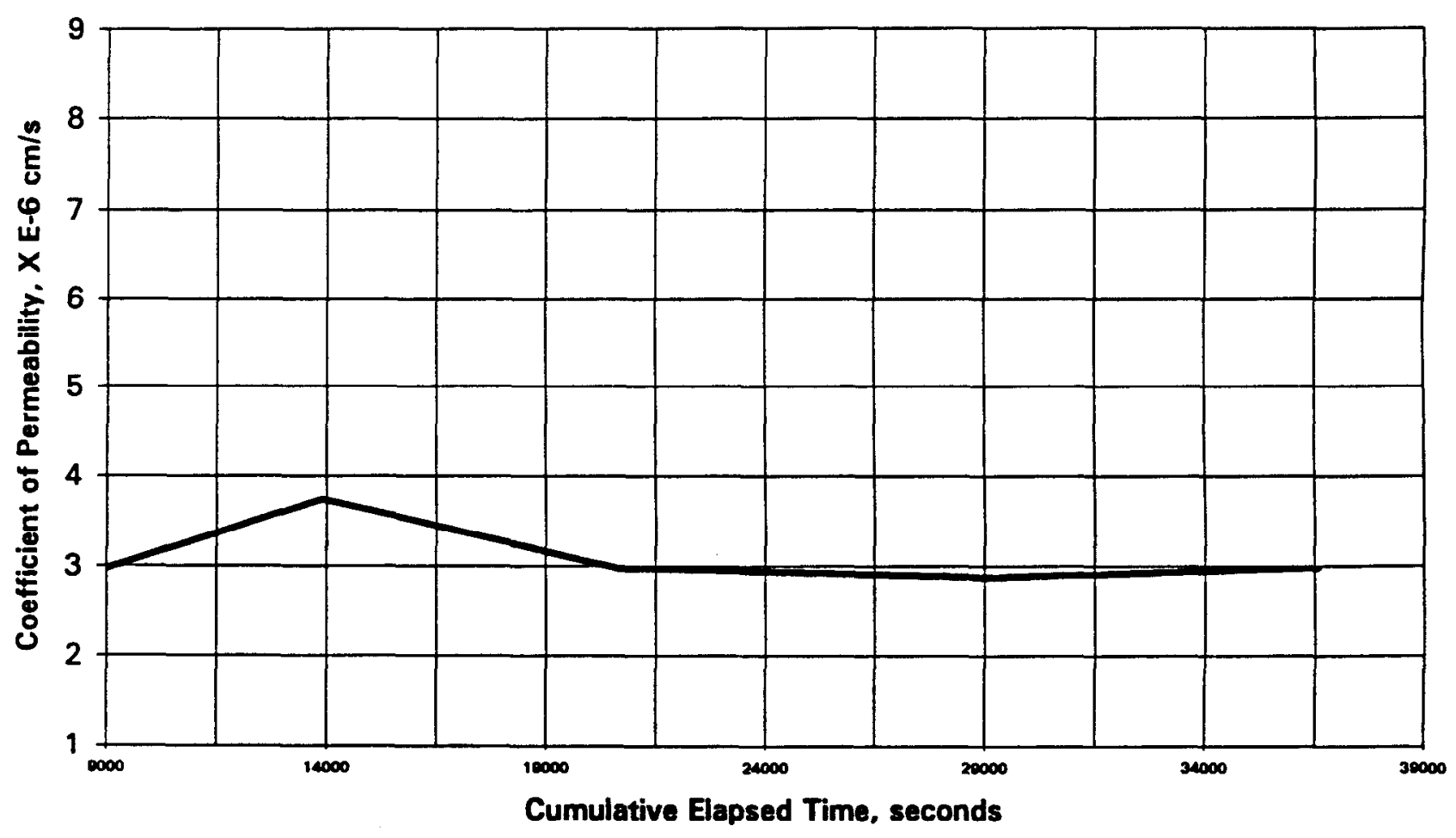




\section{0-DAY SATURATED
HYDRAULIC CONDUCTIVITY / PERMEABILITY
ASTM D 5084}

PROJECT NAME: SAIC / Savannah River

PROJECT NO. 777304.00010500
CLIENT SAMPLE NO. K423G00

IT LAB SAMPLE NO. ETDC-8004

\section{Specimen diameter, $\mathrm{cm}$}

Specimen length, $\mathrm{cm}$

Wet weight of specimen, $\boldsymbol{g}$.

Specimen cross-sect. area, $\mathrm{cm}^{-2}$

Water content, \%

Wet unit weight, pcf

Dry unit weight, pcf

Estimated degree of saturation, \%

Estimated spec. gravity of solids

Initial conditions are post-soak, pre-test

\begin{tabular}{ll|}
\hline INITIAL & FINAL \\
\hline 7.60 & \\
7.22 & \\
603.42 & 604.37 \\
45.3759 & \\
29.8 & 30.0 \\
115.0 & \\
88.6 & \\
91.1 & \\
2.65 &
\end{tabular}

Hydraulic gradient

29.3

Min. consolidation stress, psi

2.0

Max. consolidation stress, psi

5.0

Total backpressure, psi

Permeant Fluid

Sample molded

Deaired DI Water

Sample set up

$11 / 25 / 99$

Six tests performed

\section{Coefficient of Permeability, cm/s $1.3 E-06$}

\section{PERMEABILITY vS TIME}

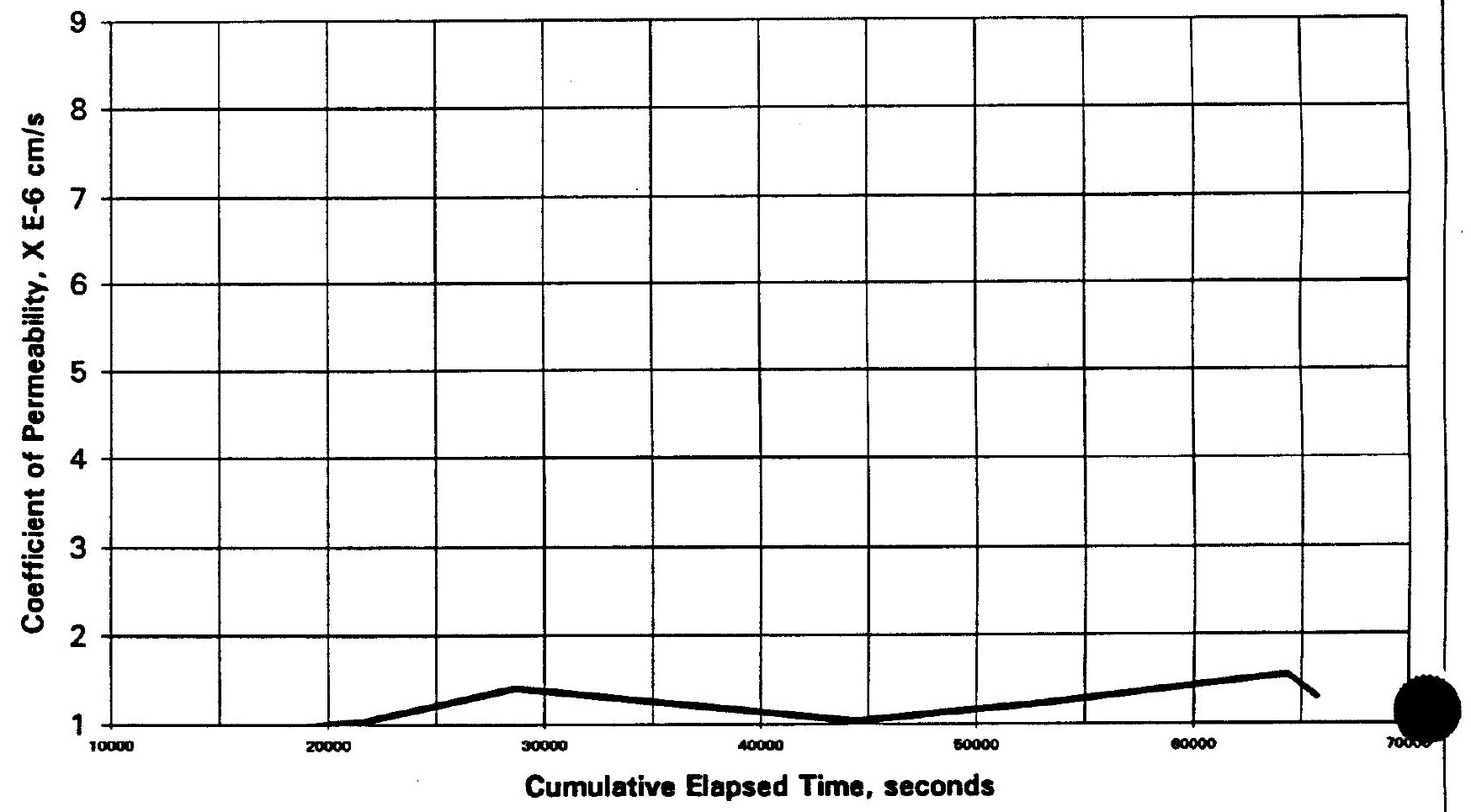




\section{0-DAY SATURATED HYDRAULIC CONDUCTIVITY / PERMEABILITY ASTM D 5084}

PROJECT NAME: SAIC / Savannah River PROJECT NO.
777304.00010500
CLIENT SAMPLE NO. K433G00

IT LAB SAMPLE NO. ETDC-8005

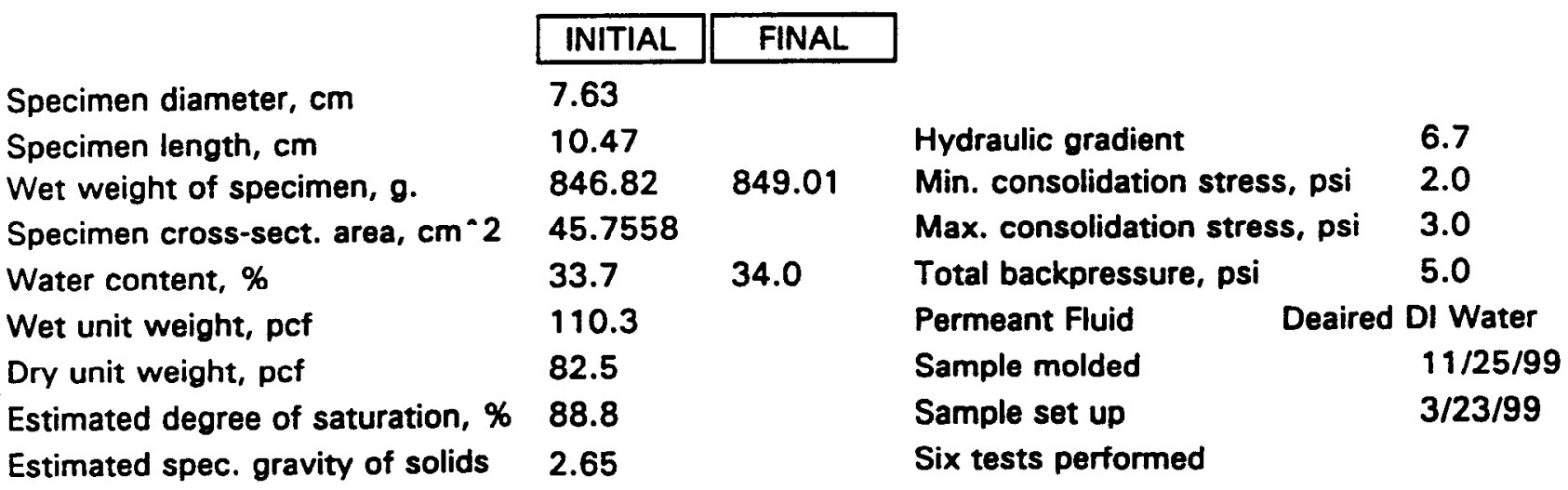

Initial conditions are post-soak, pre-test

\section{PERMEABILITY vs TIME}

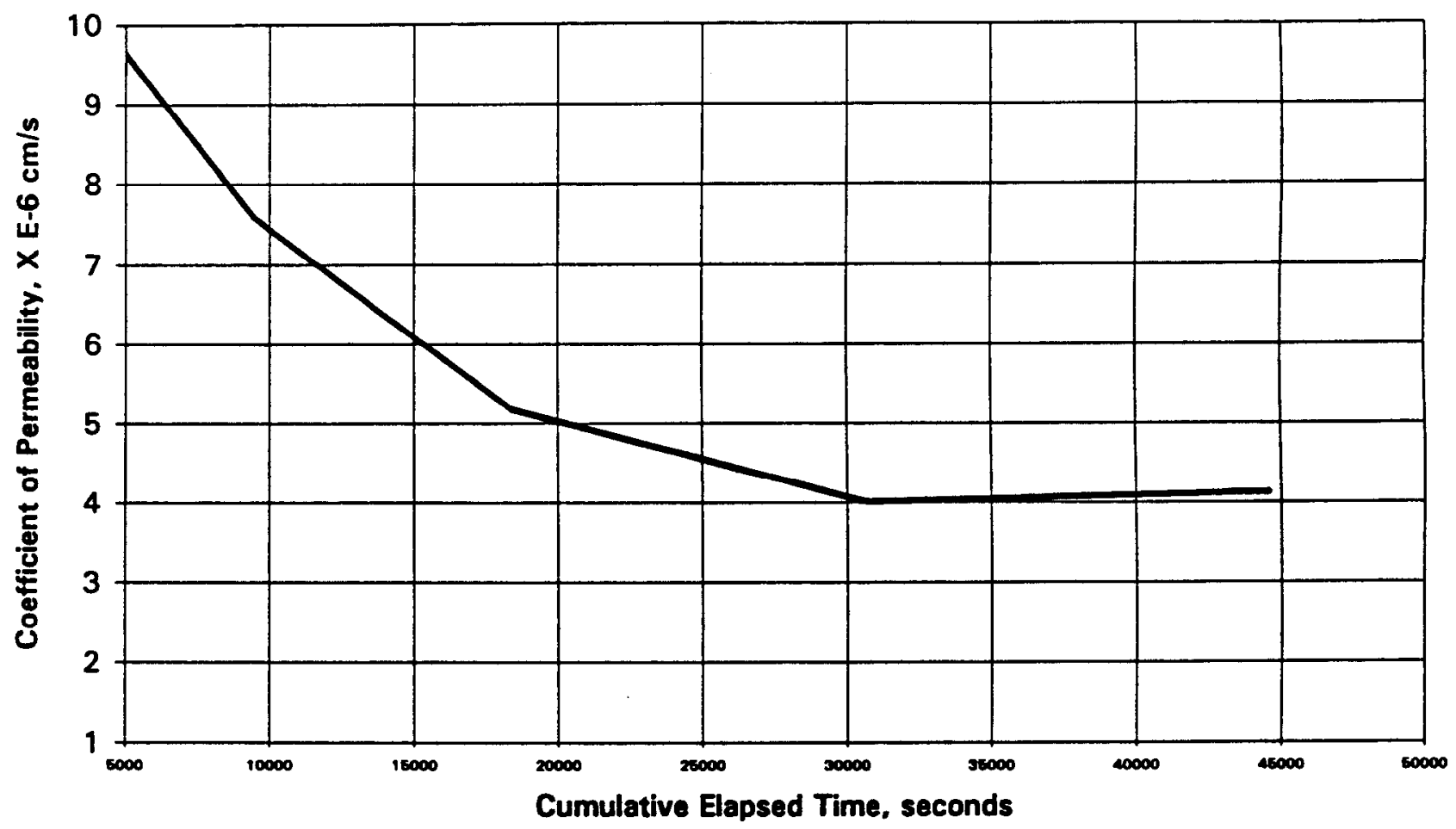




\section{0-DAY SATURATED HYDRAULIC CONDUCTIVITY / PERMEABILITY ASTM D 5084} $\begin{array}{ll}\text { PROJECT NAME: } & \text { SAIC / Savannah River } \\ \text { PROJECT NO. } & 777304.00010500\end{array}$
CLIENT SAMPLE NO. K443G00

IT LAB SAMPLE NO. ETDC-8006

INITIAL FINAL

Specimen diameter, $\mathrm{cm}$

Specimen length, $\mathrm{cm}$

Wet weight of specimen, $g$.

Specimen cross-sect. area, $\mathrm{cm}^{\wedge} 2$

Water content, \%

Wet unit weight, pcf

Dry unit weight, pcf

Estimated degree of saturation, \%

Estimated spec. gravity of solids

Initial conditions are post-soak, pre-test

\subsection{0}

10.54

853.04

45.3507

35.6

111.4

82.1

93.1

2.6500
Hydraulic gradient

855.42

Min. consolidation stress, psi

Max. consolidation stress, psi

36.0

Total backpressure, psi

Permeant Fluid

Sample molded

Sample set up

Five tests performed
20.0

2.0

5.0

3.0

Deaired DI Water

$11 / 25 / 99$

$3 / 23 / 99$

\section{Coefficient of Permeability, cm/s 2.5E-06}

\section{PERMEABILITY vS TIME}

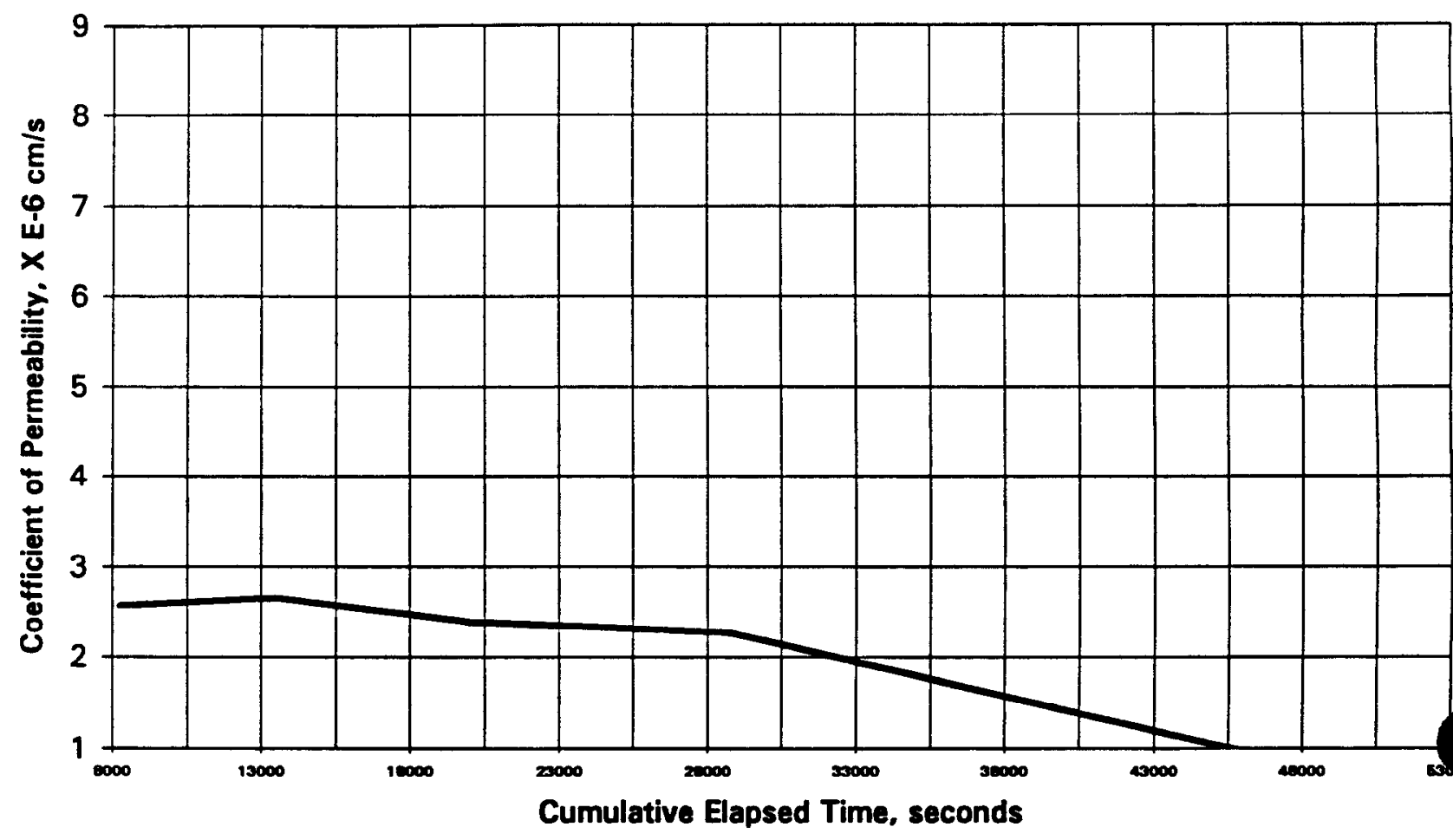


$K$ Series

Leach Index 


\section{Gross Alpha Leaching for the Coarse Fraction from KRSB Soil}

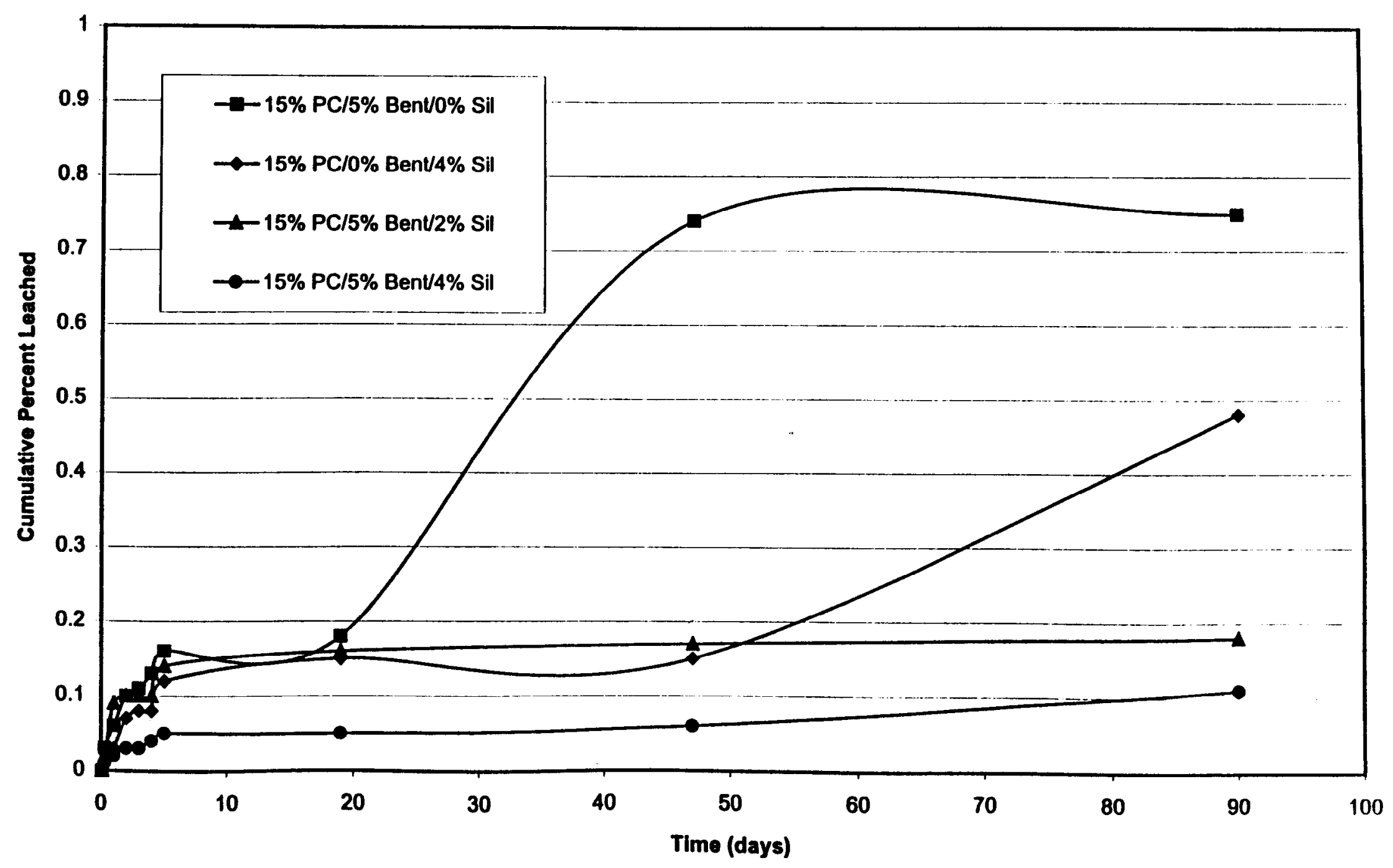


Gross Beta Leaching for the Coarse Fraction from the KSRB Soll

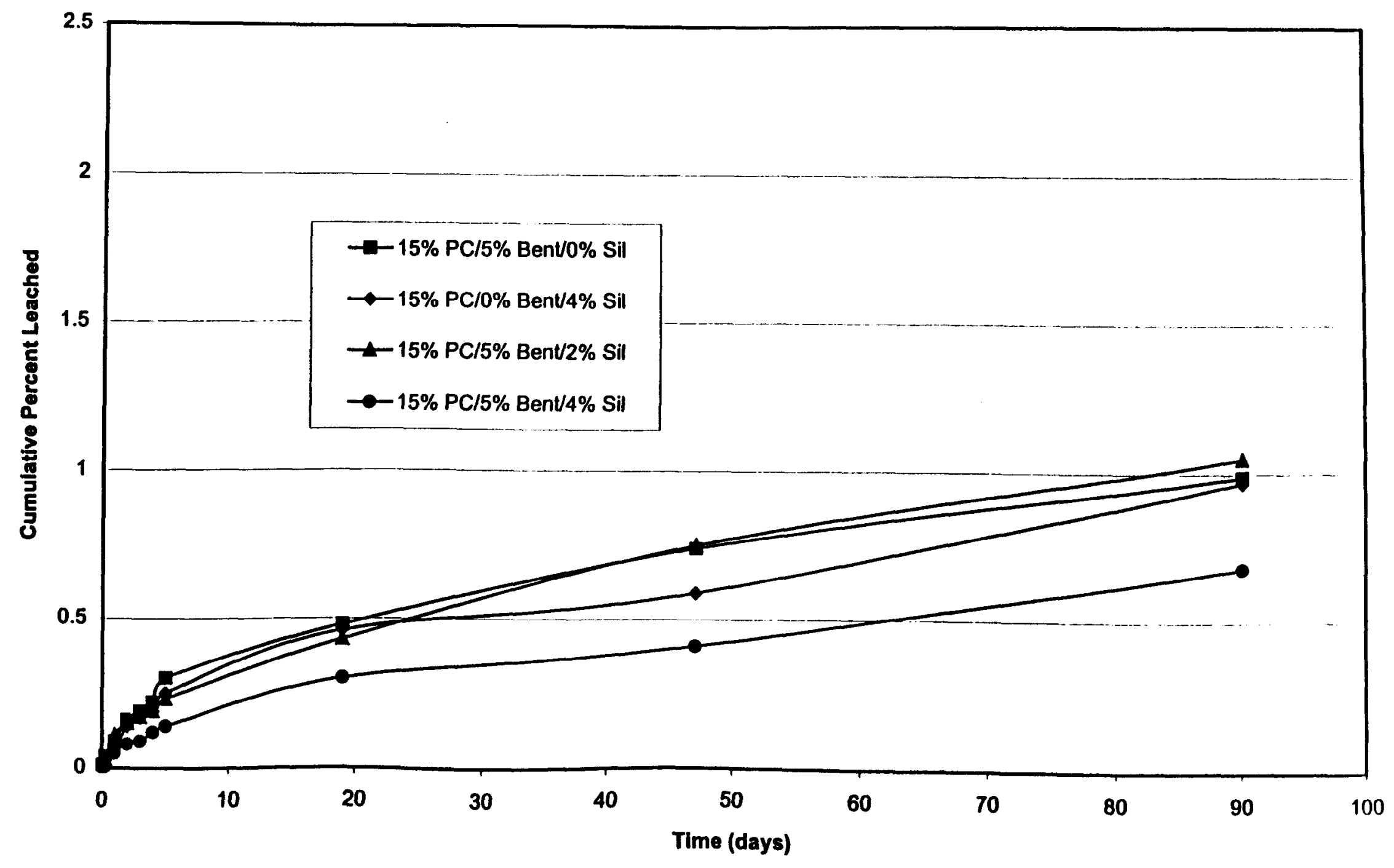




\section{American Nuclear Society - 16.1 Static Leach Test}

Project: $\quad$ SRS Reactor Seepage Basin Stabilization

Sample Description: $\quad \mathrm{K} 312$

Analyst: PRL

Date:

15-Apr-99

Contaminant:

Gross Alpha

Initial Contaminant Conc. (pCi/g):

Initial Contaminant Level (pCi):

Monolith Height (cm):

21.1

8353.279

10

176.72

196.35

Monolith Volume $\left(\mathrm{cm}^{\wedge} 3\right)$ :

Monolith Diameter $(\mathrm{cm})$ :

Leachate Volume $(\mathrm{mL})$ :
395.89

5

1800

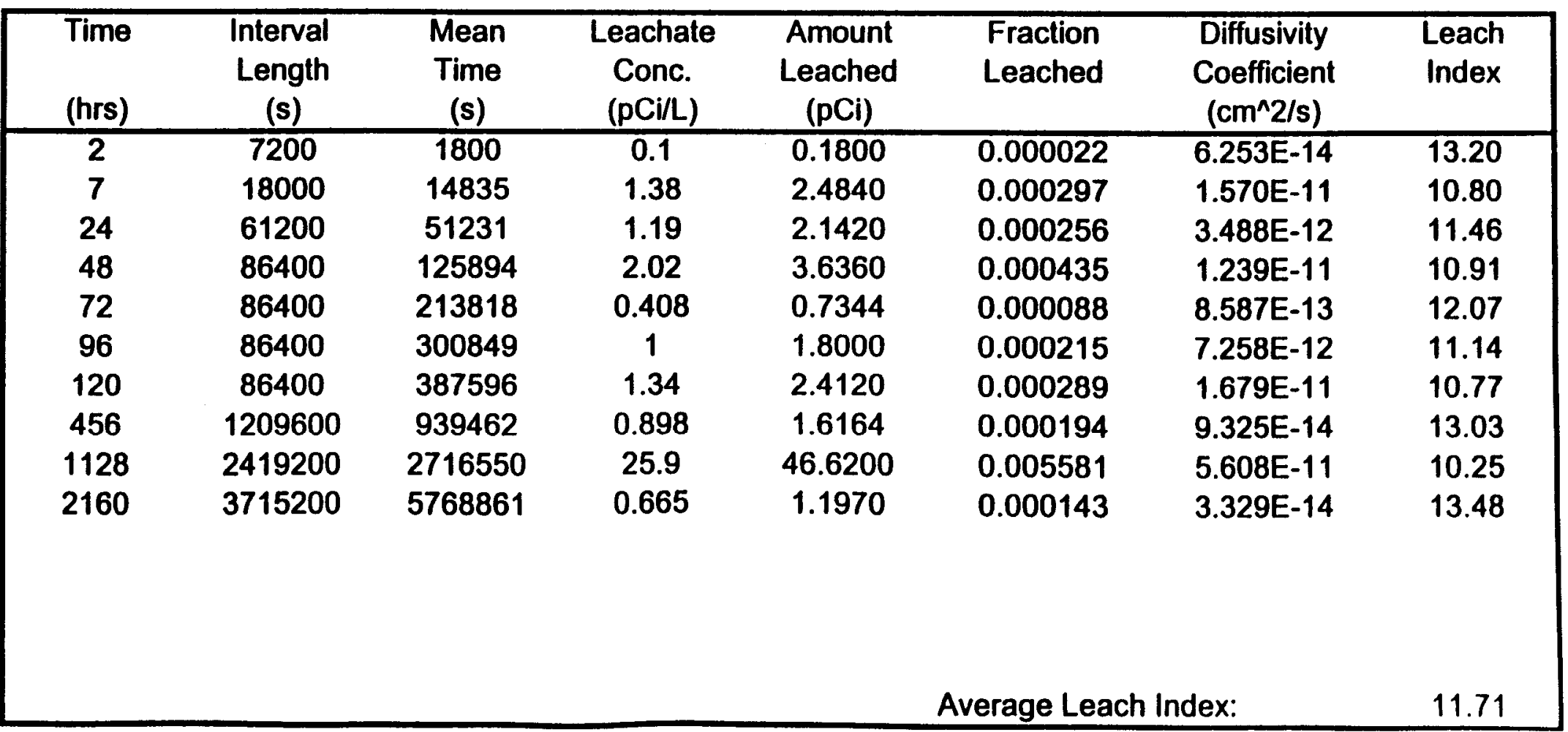




\section{American Nuclear Society - 16.1 Static Leach Test}

Project: $\quad$ SRS Reactor Seepage Basin Stabilization

Sample Description: $\quad \mathrm{K} 312$

Analyst: PRL

Date:

15-Apr-99

Contaminant:

Gross Beta

Initial Contaminant Conc. (pCi/g):

704

278706.56

Initial Contaminant Level ( $\mathrm{pCi}$ :

Monolith Height (cm):

Monolith Surface Area $\left(\mathrm{cm}^{\wedge} 2\right)$ :

Monolith Volume $\left(\mathrm{cm}^{\wedge} 3\right)$ :
10

176.72

196.35

\begin{tabular}{|c|c|c|c|c|c|c|c|}
\hline $\begin{array}{l}\text { Time } \\
\text { (hrs) }\end{array}$ & $\begin{array}{l}\text { Interval } \\
\text { Length } \\
\text { (s) }\end{array}$ & $\begin{array}{l}\text { Mean } \\
\text { Time } \\
(s)\end{array}$ & $\begin{array}{l}\text { Leachate } \\
\text { Conc. } \\
\text { (pCi/L) }\end{array}$ & $\begin{array}{l}\text { Amount } \\
\text { Leached } \\
\text { (pCi) }\end{array}$ & $\begin{array}{l}\text { Fraction } \\
\text { Leached }\end{array}$ & $\begin{array}{l}\text { Diffusivity } \\
\text { Coefficient } \\
\left(\mathrm{cm}^{\wedge} 2 / \mathrm{s}\right)\end{array}$ & $\begin{array}{l}\text { Leach } \\
\text { Index }\end{array}$ \\
\hline 2 & 7200 & 1800 & 18.8 & 33.8400 & 0.000121 & $1.985 E-12$ & 11.70 \\
\hline 7 & 18000 & 14835 & 38.7 & 69.6600 & 0.000250 & 1.109E-11 & 10.95 \\
\hline 24 & 61200 & 51231 & 83.1 & 149.5800 & 0.000537 & $1.528 \mathrm{E}-11$ & 10.82 \\
\hline 48 & 86400 & 125894 & 112 & 201.6000 & 0.000723 & 3.422E-11 & 10.47 \\
\hline 72 & 86400 & 213818 & 38.4 & 69.1200 & 0.000248 & $6.833 E-12$ & 11.17 \\
\hline 96 & 86400 & 300849 & 45.4 & 81.7200 & 0.000293 & $1.344 \mathrm{E}-11$ & 10.87 \\
\hline 120 & 86400 & 387596 & 130 & 234.0000 & 0.000840 & $1.420 E-10$ & 9.85 \\
\hline 456 & 1209600 & 939462 & 274 & 493.2000 & 0.001770 & $7.799 \mathrm{E}-12$ & 11.11 \\
\hline 1128 & 2419200 & 2716550 & 405 & 729.0000 & 0.002616 & $1.232 \mathrm{E}-11$ & 10.91 \\
\hline 2160 & 3715200 & 5768861 & 392 & 705.6000 & 0.002532 & $1.039 \mathrm{E}-11$ & 10.98 \\
\hline & & & & \multicolumn{3}{|c|}{ Average Leach Index: } & 10.88 \\
\hline
\end{tabular}

Monolith Diameter $(\mathrm{cm}):$

Leachate Volume (mL): $\quad 1800$ 


\section{American Nuclear Society - 16.1 Static Leach Test}

Project: SRS Reactor Seepage Basin Stabilization

Sample Description: $\quad$ K322

Analyst: PRL

Date: 15-Apr-99

Contaminant:

Gross Alpha

Initial Contaminant Conc. (pCi/g):

21.1

Initial Contaminant Level (pCi):

Monolith Height $(\mathrm{cm})$ :

8274.365

10

Monolith Surface Area $\left(\mathrm{cm}^{\wedge} 2\right)$ :

Monolith Volume (cm^3):

176.72

196.35

Sample Weight (g):

Monolith Diameter $(\mathrm{cm})$ :

Leachate Volume $(\mathrm{mL})$ :
392.15

5

1800

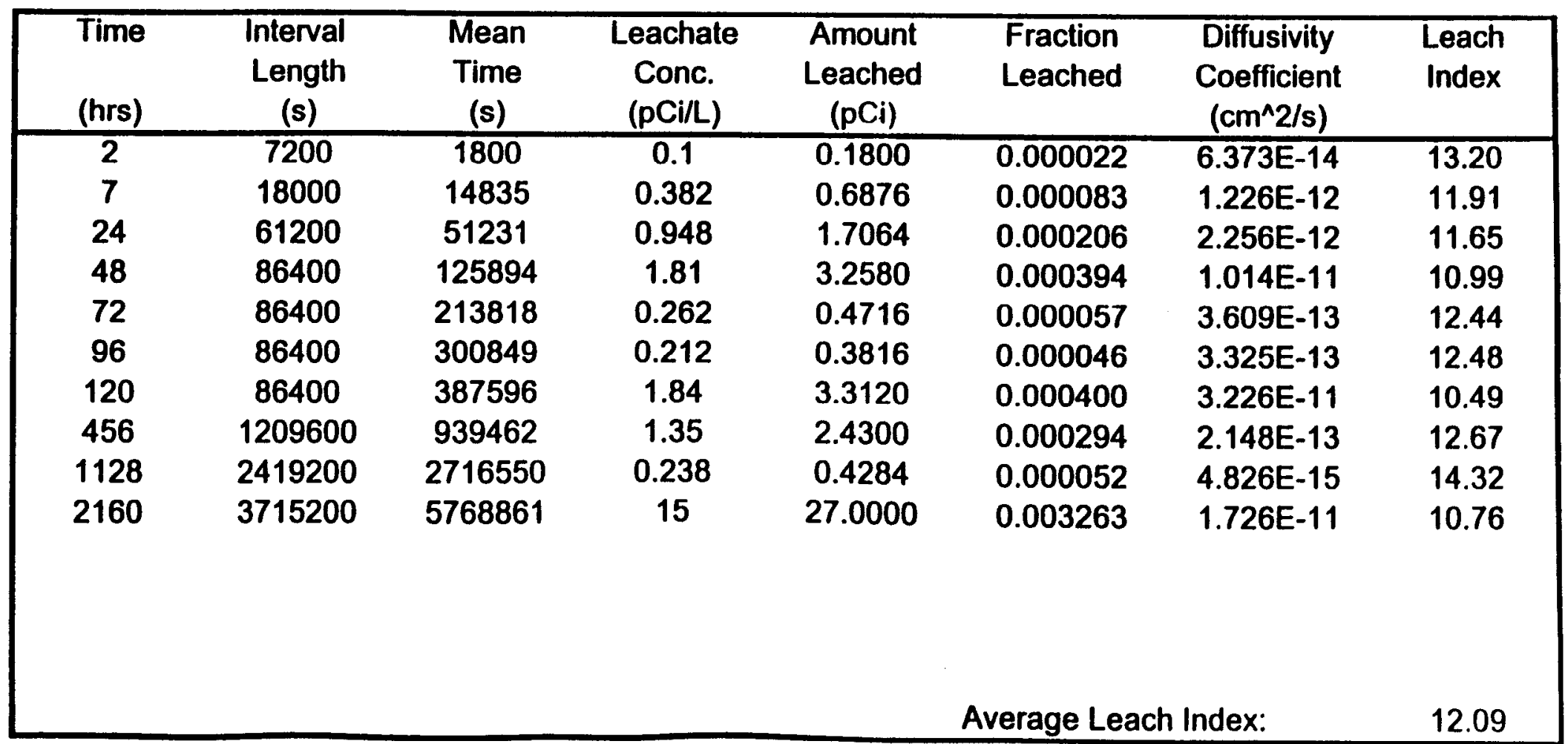




\section{American Nuclear Society - 16.1 Static Leach Test}

Project: $\quad$ SRS Reactor Seepage Basin Stabilization

Sample Description: $\quad$ K322

$\begin{array}{llll}\text { Analyst: PRL } & \text { Date: } & \text { 15-Apr-99 }\end{array}$

Contaminant: Gross Beta

Initial Contaminant Conc. (pCi/g): $\quad 704$

Initial Contaminant Level (pCi): $\quad 276073.6$

Monolith Height (cm):

Monolith Surface Area ( $\left.\mathrm{cm}^{\wedge} 2\right)$ :

Monolith Volume $\left(\mathrm{cm}^{\wedge} 3\right)$ :

Sample Weight (g):

Monolith Diameter (cm): Leachate Volume $(\mathrm{mL})$ :
392.15

5

1800

\begin{tabular}{|c|c|c|c|c|c|c|c|}
\hline $\begin{array}{l}\text { Time } \\
\text { (hrs) }\end{array}$ & $\begin{array}{l}\text { Interval } \\
\text { Length } \\
\text { (s) }\end{array}$ & $\begin{array}{l}\text { Mean } \\
\text { Time } \\
\text { (s) }\end{array}$ & $\begin{array}{l}\text { Leachate } \\
\text { Conc. } \\
\text { (pCi/L) }\end{array}$ & $\begin{array}{l}\text { Amount } \\
\text { Leached } \\
\text { (pCi) }\end{array}$ & $\begin{array}{l}\text { Fraction } \\
\text { Leached }\end{array}$ & $\begin{array}{l}\text { Diffusivity } \\
\text { Coefficient } \\
\text { (cm^2/s) }\end{array}$ & $\begin{array}{l}\text { Leach } \\
\text { Index }\end{array}$ \\
\hline 2 & 7200 & 1800 & 16.9 & 30.4200 & 0.000110 & $1.635 \mathrm{E}-12$ & 11.79 \\
\hline 7 & 18000 & 14835 & 23.1 & 41.5800 & 0.000151 & 4.028E-12 & 11.39 \\
\hline 24 & 61200 & 51231 & 55 & 99.0000 & 0.000359 & 6.822E-12 & 11.17 \\
\hline 48 & 86400 & 125894 & 124 & 223.2000 & 0.000808 & 4.275E-11 & 10.37 \\
\hline 72 & 86400 & 213818 & 37.6 & 67.6800 & 0.000245 & 6.677E-12 & 11.18 \\
\hline 96 & 86400 & 300849 & 33.5 & 60.3000 & 0.000218 & 7.457E-12 & 11.13 \\
\hline 120 & 86400 & 387596 & 86.2 & 155.1600 & 0.000562 & 6.361E-11 & 10.20 \\
\hline 456 & 1209600 & 939462 & 332 & 597.6000 & 0.002165 & 1.167E-11 & 10.93 \\
\hline 1128 & 2419200 & 2716550 & 199 & 358.2000 & 0.001297 & 3.031E-12 & 11.52 \\
\hline 2160 & 3715200 & 5768861 & 575 & 1035.0000 & 0.003749 & $2.278 \mathrm{E}-11$ & 10.64 \\
\hline & & & & & \multicolumn{2}{|c|}{ Average Leach Index: } & 11.03 \\
\hline
\end{tabular}




\section{American Nuclear Society - 16.1 Static Leach Test}

Project: $\quad$ SRS Reactor Seepage Basin Stabilization

Sample Description: $\quad \mathrm{K} 332$

Analyst: PRL 15-Apr-99

Contaminant: Gross Alpha

Initial Contaminant Conc. (pCi/g):

21.1

8072.438

10

Monolith Height (cm):

Monolith Surface Area $\left(\mathrm{cm}^{\wedge} 2\right)$ :

Monolith Volume $\left(\mathrm{cm}^{\wedge} 3\right)$ :
176.72

196.35

\begin{tabular}{|c|c|c|c|c|c|c|c|}
\hline $\begin{array}{l}\text { Time } \\
\text { (hrs) }\end{array}$ & $\begin{array}{l}\text { Interval } \\
\text { Length } \\
\text { (s) }\end{array}$ & $\begin{array}{l}\text { Mean } \\
\text { Time } \\
\text { (s) }\end{array}$ & $\begin{array}{l}\text { Leachate } \\
\text { Conc. } \\
\text { (pCi/L) }\end{array}$ & $\begin{array}{l}\text { Amount } \\
\text { Leached } \\
\text { (pCi) }\end{array}$ & $\begin{array}{l}\text { Fraction } \\
\text { Leached }\end{array}$ & $\begin{array}{l}\text { Diffusivity } \\
\text { Coefficient } \\
\left(\mathrm{cm}^{\wedge} 2 / \mathrm{s}\right)\end{array}$ & $\begin{array}{l}\text { Leach } \\
\text { Index }\end{array}$ \\
\hline 2 & 7200 & 1800 & 0.1 & 0.1800 & 0.000022 & $6.696 \mathrm{E}-14$ & 13.17 \\
\hline 7 & 18000 & 14835 & 0.962 & 1.7316 & 0.000215 & 8.171E-12 & 11.09 \\
\hline 24 & 61200 & 51231 & 3.05 & 5.4900 & 0.000680 & $2.454 \mathrm{E}-11$ & 10.61 \\
\hline 48 & 86400 & 125894 & 0.175 & 0.3150 & 0.000039 & 9.960E-14 & 13.00 \\
\hline 72 & 86400 & 213818 & 0.1 & 0.1800 & 0.000022 & $5.524 \mathrm{E}-14$ & 13.26 \\
\hline 96 & 86400 & 300849 & 0.1 & 0.1800 & 0.000022 & $7.772 E-14$ & 13.11 \\
\hline 120 & 86400 & 387596 & 1.69 & 3.0420 & 0.000377 & $2.860 E-11$ & 10.54 \\
\hline 456 & 1209600 & 939462 & 0.832 & 1.4976 & 0.000186 & $8.571 E-14$ & 13.07 \\
\hline 1128 & 2419200 & 2716550 & 0.52 & 0.9360 & 0.000116 & $2.420 E-14$ & 13.62 \\
\hline 2160 & 3715200 & 5768861 & 0.341 & 0.6138 & 0.000076 & $9.372 E-15$ & 14.03 \\
\hline & & & & & \multicolumn{2}{|c|}{ Average Leach Index: } & 12.55 \\
\hline
\end{tabular}




\section{American Nuclear Society - 16.1 Static Leach Test}

Project: SRS Reactor Seepage Basin Stabilization

Sample Description: $\quad \mathrm{K} 332$

$\begin{array}{llll}\text { Analyst: PRL } & \text { Date: } & \text { 15-Apr-99 }\end{array}$

Contaminant: Gross Beta

Initial Contaminant Conc. (pCi/g):

704

Initial Contaminant Level (pCi):

269336.32

Monolith Height (cm):

Monolith Surface Area ( $\left.\mathrm{cm}^{\wedge} 2\right)$ :

Monolith Volume $\left(\mathrm{cm}^{\wedge} 3\right)$ :

10

176.72

196.35
Sample Weight (g):

Monolith Diameter $(\mathbf{c m})$ : Leachate Volume $(\mathrm{mL})$ :
382.58

5

1800

\begin{tabular}{|c|c|c|c|c|c|c|c|}
\hline $\begin{array}{l}\text { Time } \\
\text { (hrs) }\end{array}$ & $\begin{array}{l}\text { Interval } \\
\text { Length } \\
\text { (s) }\end{array}$ & $\begin{array}{c}\text { Mean } \\
\text { Time } \\
\text { (s) }\end{array}$ & $\begin{array}{l}\text { Leachate } \\
\text { Conc. } \\
\text { (pCi/L) }\end{array}$ & $\begin{array}{l}\text { Amount } \\
\text { Leached } \\
\text { (pCi) }\end{array}$ & $\begin{array}{l}\text { Fraction } \\
\text { Leached }\end{array}$ & $\begin{array}{l}\text { Diffusivity } \\
\text { Coefficient } \\
\left(\mathrm{cm}^{\wedge} 2 / \mathrm{s}\right)\end{array}$ & $\begin{array}{l}\text { Leach } \\
\text { Index }\end{array}$ \\
\hline 2 & 7200 & 1800 & 10.1 & 18.1800 & 0.000067 & $6.136 \mathrm{E}-13$ & 12.21 \\
\hline 7 & 18000 & 14835 & 40.9 & 73.6200 & 0.000273 & 1.327E-11 & 10.88 \\
\hline 24 & 61200 & 51231 & 116 & 208.8000 & 0.000775 & $3.188 E-11$ & 10.50 \\
\hline 48 & 86400 & 125894 & 61.1 & 109.9800 & 0.000408 & $1.091 E-11$ & 10.96 \\
\hline 72 & 86400 & 213818 & 30.5 & 54.9000 & 0.000204 & 4.616E-12 & 11.34 \\
\hline 96 & 86400 & 300849 & 25.2 & 45.3600 & 0.000168 & 4.433E-12 & 11.35 \\
\hline 120 & 86400 & 387596 & 61 & 109.8000 & 0.000408 & 3.347E-11 & 10.48 \\
\hline 456 & 1209600 & 939462 & 292 & 525.6000 & 0.001951 & $9.484 E-12$ & 11.02 \\
\hline 1128 & 2419200 & 2716550 & 483 & 869.4000 & 0.003228 & $1.876 \mathrm{E}-11$ & 10.73 \\
\hline 2160 & 3715200 & 5768861 & 446 & 802.8000 & 0.002981 & $1.440 E-11$ & 10.84 \\
\hline & & & & \multicolumn{3}{|c|}{ Average Leach Index: } & 11.03 \\
\hline
\end{tabular}




\section{American Nuclear Society - 16.1 Static Leach Test}

Project: $\quad$ SRS Reactor Seepage Basin Stabilization

Sample Description: $\quad \mathrm{K} 342$

$\begin{array}{llll}\text { Analyst: PRL } & \text { Date: Apr-99 }\end{array}$

Contaminant: Gross Alpha

Initial Contaminant Conc. (pCi/g):

Initial Contaminant Level (pCi):

Monolith Height (cm):

21.1

8082.777

10

Monolith Surface Area $\left(\mathrm{cm}^{\wedge} 2\right)$ :

Monolith Volume $\left(\mathrm{cm}^{\wedge} 3\right)$ :
176.72

196.35

\begin{tabular}{|c|c|c|c|c|c|c|c|}
\hline $\begin{array}{l}\text { Time } \\
\text { (hrs) }\end{array}$ & $\begin{array}{l}\text { Interval } \\
\text { Length } \\
\text { (s) }\end{array}$ & $\begin{array}{l}\text { Mean } \\
\text { Time } \\
\text { (s) }\end{array}$ & $\begin{array}{l}\text { Leachate } \\
\text { Conc. } \\
\text { (pCi/L) }\end{array}$ & $\begin{array}{c}\text { Amount } \\
\text { Leached } \\
(\mathrm{pCi})\end{array}$ & $\begin{array}{l}\text { Fraction } \\
\text { Leached }\end{array}$ & $\begin{array}{l}\text { Diffusivity } \\
\text { Coefficient } \\
\left(\mathrm{cm}^{\wedge} 2 / \mathrm{s}\right)\end{array}$ & $\begin{array}{l}\text { Leach } \\
\text { Index }\end{array}$ \\
\hline 2 & 7200 & 1800 & 0.1 & 0.1800 & 0.000022 & $6.679 \mathrm{E}-14$ & 13.18 \\
\hline 7 & 18000 & 14835 & 0.254 & 0.4572 & 0.000057 & $5.682 E-13$ & 12.25 \\
\hline 24 & 61200 & 51231 & 0.572 & 1.0296 & 0.000127 & $8.608 E-13$ & 12.07 \\
\hline 48 & 86400 & 125894 & 0.393 & 0.7074 & 0.000088 & $5.010 \mathrm{E}-13$ & 12.30 \\
\hline 72 & 86400 & 213818 & 0.161 & 0.2898 & 0.000036 & $1.428 \mathrm{E}-13$ & 12.85 \\
\hline 96 & 86400 & 300849 & 0.1 & 0.1800 & 0.000022 & $7.752 E-14$ & 13.11 \\
\hline 120 & 86400 & 387596 & 0.546 & 0.9828 & 0.000122 & 2.977E-12 & 11.53 \\
\hline 456 & 1209600 & 939462 & 0.1 & 0.1800 & 0.000022 & $1.235 E-15$ & 14.91 \\
\hline 1128 & 2419200 & 2716550 & 0.372 & 0.6696 & 0.000083 & $1.236 \mathrm{E}-14$ & 13.91 \\
\hline 2160 & 3715200 & 5768861 & 2.12 & 3.8160 & 0.000472 & 3.613E-13 & 12.44 \\
\hline & & & & & \multicolumn{2}{|c|}{ Average Leach Index: } & 12.85 \\
\hline
\end{tabular}




\section{American Nuclear Society - 16.1 Static Leach Test}

Project: $\quad$ SRS Reactor Seepage Basin Stabilization

Sample Description: $\quad \mathrm{K} 342$

Analyst: $\quad$ PRL

Date:

15-Apr-99

Contaminant:

Gross Beta

Initial Contaminant Conc. (pCi/g):

704

Initial Contaminant Level (pCi):

269681.28

Monolith Height (cm):

10

Monolith Surface Area ( $\left.\mathrm{cm}^{\wedge} 2\right)$ :

176.72

Monolith Volume $\left(\mathrm{cm}^{\wedge} 3\right)$ :

196.35

Sample Weight (g):

383.07

Monolith Diameter (cm):

5

Leachate Volume $(\mathrm{mL})$ :

1800

\begin{tabular}{|c|c|c|c|c|c|c|c|}
\hline $\begin{array}{l}\text { Time } \\
\text { (hrs) }\end{array}$ & $\begin{array}{l}\text { Interval } \\
\text { Length } \\
\text { (s) }\end{array}$ & $\begin{array}{l}\text { Mean } \\
\text { Time } \\
(s)\end{array}$ & $\begin{array}{l}\text { Leachate } \\
\text { Conc. } \\
\text { (pCi/L) }\end{array}$ & $\begin{array}{l}\text { Amount } \\
\text { Leached } \\
\text { (pCi) }\end{array}$ & $\begin{array}{l}\text { Fraction } \\
\text { Leached }\end{array}$ & $\begin{array}{l}\text { Diffusivity } \\
\text { Coefficient } \\
\text { (cm^2/s) }\end{array}$ & $\begin{array}{l}\text { Leach } \\
\text { Index }\end{array}$ \\
\hline 2 & 7200 & 1800 & 6.17 & 11.1060 & 0.000041 & $2.284 E-13$ & 12.64 \\
\hline 7 & 18000 & 14835 & 8.8 & 15.8400 & 0.000059 & 6.127E-13 & 12.21 \\
\hline 24 & 61200 & 51231 & 59.1 & 106.3800 & 0.000394 & 8.255E-12 & 11.08 \\
\hline 48 & 86400 & 125894 & 45.8 & 82.4400 & 0.000306 & $6.112 \mathrm{E}-12$ & 11.21 \\
\hline 72 & 86400 & 213818 & 21.2 & 38.1600 & 0.000142 & 2.224E-12 & 11.65 \\
\hline 96 & 86400 & 300849 & 38.8 & 69.8400 & 0.000259 & $1.048 E-11$ & 10.98 \\
\hline 120 & 86400 & 387596 & 23.4 & 42.1200 & 0.000156 & $4.912 E-12$ & 11.31 \\
\hline 456 & 1209600 & 939462 & 245 & 441.0000 & 0.001635 & $6.659 E-12$ & 11.18 \\
\hline 1128 & 2419200 & 2716550 & 165 & 297.0000 & 0.001101 & $2.183 E-12$ & 11.66 \\
\hline 2160 & 3715200 & 5768861 & 412 & 741.6000 & 0.002750 & 1.226E-11 & 10.91 \\
\hline & & & & \multicolumn{3}{|c|}{ Average Leach Index: } & 11.48 \\
\hline
\end{tabular}




\section{Gross Alpha Leaching for the Fine Fraction from KRSB Soil}

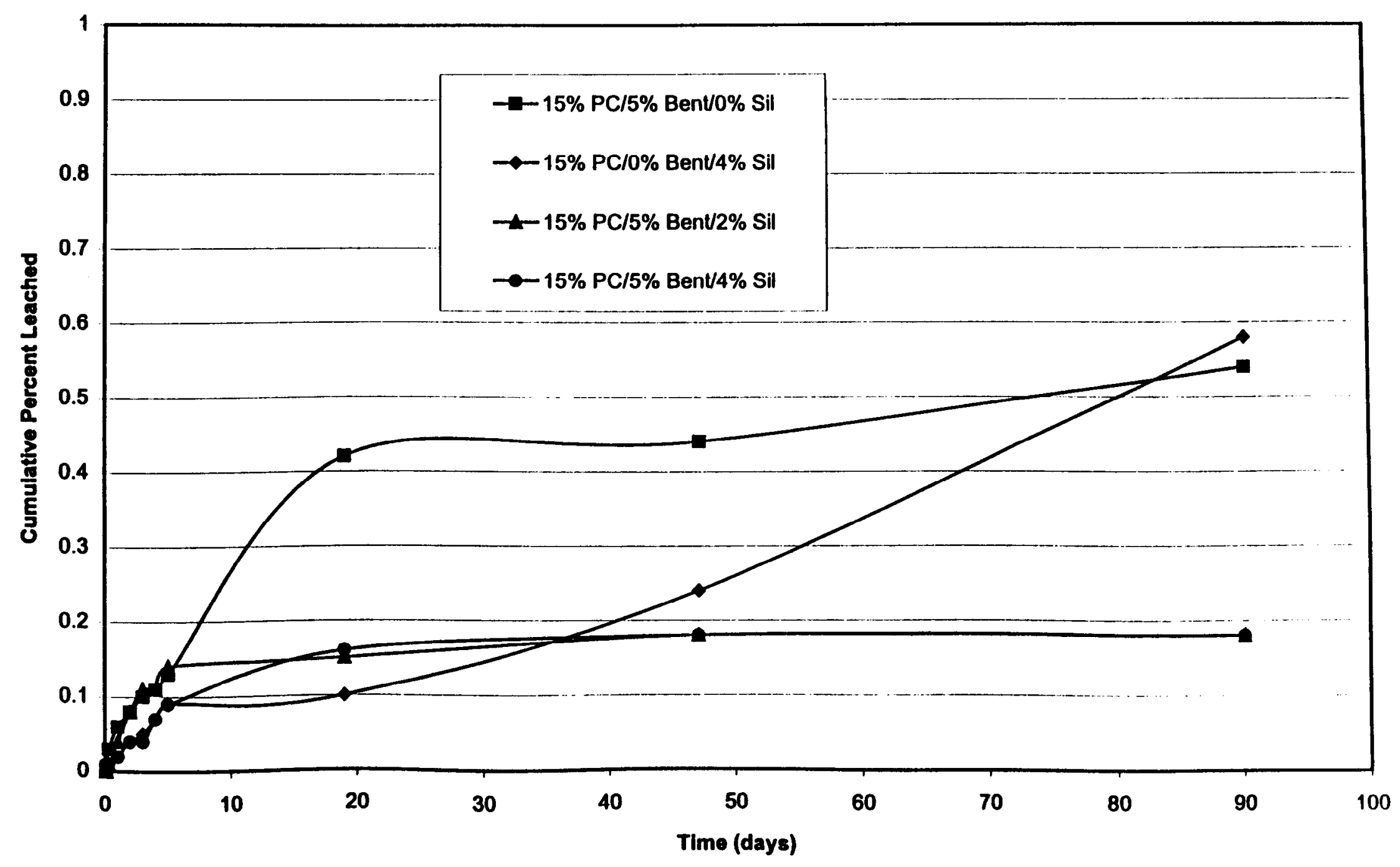


Gross Beta Leaching for the Fine Fraction from the KSRB Soil

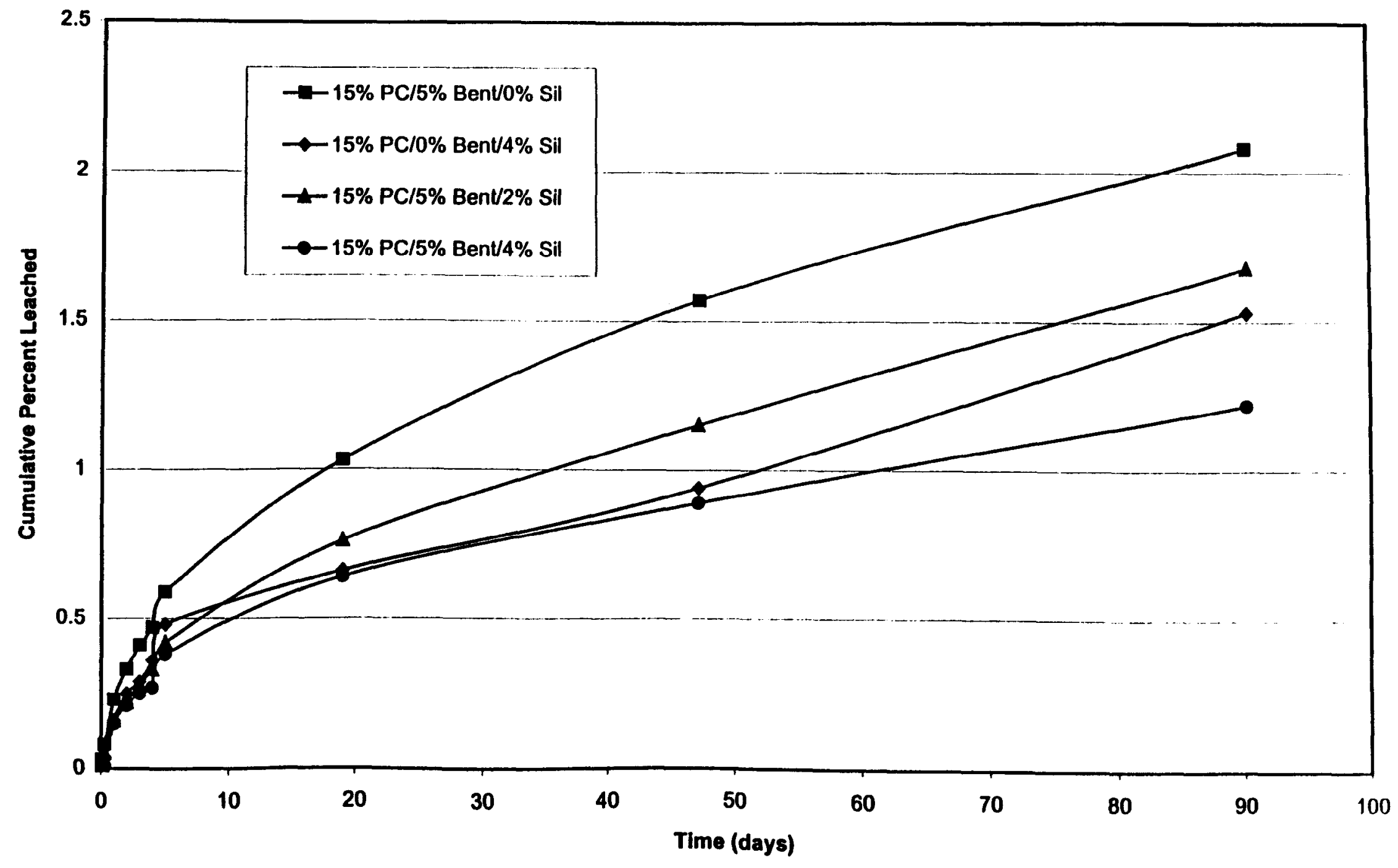




\section{American Nuclear Society - 16.1 Static Leach Test}

Project: $\quad$ SRS Reactor Seepage Basin Stabilization

Sample Description: $\quad \mathrm{K} 412$

$\begin{array}{llll}\text { Analyst: } & \text { PRL } & \text { Date: Apr-99 }\end{array}$

Contaminant: Gross Alpha

Initial Contaminant Conc. (pCi/g):

Initial Contaminant Level (pCi):

Monolith Height (cm):

12.9

4852.077

10

Monolith Surface Area (cm²):

Monolith Volume $\left(\mathrm{cm}^{\wedge} 3\right)$ :
176.72

196.35
Sample Weight (g):

Monolith Diameter $(\mathrm{cm})$ :

Leachate Volume $(\mathrm{mL})$ :
376.13

5

1800

\begin{tabular}{|c|c|c|c|c|c|c|c|}
\hline $\begin{array}{l}\text { Time } \\
\text { (hrs) }\end{array}$ & $\begin{array}{l}\text { Interval } \\
\text { Length } \\
\text { (s) }\end{array}$ & $\begin{array}{l}\text { Mean } \\
\text { Time } \\
\text { (s) }\end{array}$ & $\begin{array}{c}\text { Leachate } \\
\text { Conc. } \\
\text { (pCi/L) }\end{array}$ & $\begin{array}{c}\text { Amount } \\
\text { Leached } \\
\text { (pCi) }\end{array}$ & $\begin{array}{l}\text { Fraction } \\
\text { Leached }\end{array}$ & $\begin{array}{l}\text { Diffusivity } \\
\text { Coefficient } \\
\left(\mathrm{cm}^{\wedge} 2 / \mathrm{s}\right)\end{array}$ & $\begin{array}{l}\text { Leach } \\
\text { Index }\end{array}$ \\
\hline 2 & 7200 & 1800 & 0.1 & 0.1800 & 0.000037 & $1.853 \mathrm{E}-13$ & 12.73 \\
\hline 7 & 18000 & 14835 & 0.596 & 1.0728 & 0.000221 & 8.681E-12 & 11.06 \\
\hline 24 & 61200 & 51231 & 1.02 & 1.8360 & 0.000378 & 7.596E-12 & 11.12 \\
\hline 48 & 86400 & 125894 & 0.341 & 0.6138 & 0.000127 & $1.047 E-12$ & 11.98 \\
\hline 72 & 86400 & 213818 & 0.532 & 0.9576 & 0.000197 & 4.327E-12 & 11.36 \\
\hline 96 & 86400 & 300849 & 0.476 & 0.8568 & 0.000177 & $4.874 E-12$ & 11.31 \\
\hline 120 & 86400 & 387596 & 0.501 & 0.9018 & 0.000186 & $6.956 \mathrm{E}-12$ & 11.16 \\
\hline 456 & 1209600 & 939462 & 7.74 & 13.9320 & 0.002871 & 2.053E-11 & 10.69 \\
\hline 1128 & 2419200 & 2716550 & 0.576 & 1.0368 & 0.000214 & 8.220E-14 & 13.09 \\
\hline 2160 & 3715200 & 5768861 & 2.59 & 4.6620 & 0.000961 & 1.497E-12 & 11.82 \\
\hline & & & & \multicolumn{3}{|c|}{ Average Leach Index: } & 11.63 \\
\hline
\end{tabular}




\section{American Nuclear Society - 16.1 Static Leach Test}

Project: $\quad$ SRS Reactor Seepage Basin Stabilization

Sample Description: $\quad \mathrm{K} 412$

Analyst: $\quad$ PRL

Date:

15-Apr-99

Contaminant:

Gross Beta

Initial Contaminant Conc. (pCi/g):

293

Initial Contaminant Level (pCi):

110206.09

Monolith Height (cm):

10

Monolith Surface Area $\left(\mathrm{cm}^{\wedge} 2\right)$ :

176.72

Monolith Volume $\left(\mathrm{cm}^{\wedge} 3\right)$ :

196.35

Sample Weight (g):

Monolith Diameter $(\mathrm{cm})$ :

Leachate Volume (mL):
376.13

5

1800

\begin{tabular}{|c|c|c|c|c|c|c|c|}
\hline $\begin{array}{l}\text { Time } \\
\text { (hrs) }\end{array}$ & $\begin{array}{l}\text { Interval } \\
\text { Length } \\
\text { (s) }\end{array}$ & $\begin{array}{c}\text { Mean } \\
\text { Time } \\
\text { (s) }\end{array}$ & $\begin{array}{l}\text { Leachate } \\
\text { Conc. } \\
\text { (pCi/L) }\end{array}$ & $\begin{array}{l}\text { Amount } \\
\text { Leached } \\
\text { (pCi) }\end{array}$ & $\begin{array}{l}\text { Fraction } \\
\text { Leached }\end{array}$ & $\begin{array}{l}\text { Diffusivity } \\
\text { Coefficient } \\
\left(\mathrm{cm}^{\wedge} 2 / \mathrm{s}\right)\end{array}$ & $\begin{array}{l}\text { Leach } \\
\text { Index }\end{array}$ \\
\hline 2 & 7200 & 1800 & 16.2 & 29.1600 & 0.000265 & $9.428 \mathrm{E}-12$ & 11.03 \\
\hline 7 & 18000 & 14835 & 30 & 54.0000 & 0.000490 & $4.264 E-11$ & 10.37 \\
\hline 24 & 61200 & 51231 & 92.2 & 165.9600 & 0.001506 & $1.203 \mathrm{E}-10$ & 9.92 \\
\hline 48 & 86400 & 125894 & 66.7 & 120.0600 & 0.001089 & 7.763E-11 & 10.11 \\
\hline 72 & 86400 & 213818 & 43.2 & 77.7600 & 0.000706 & 5.531E-11 & 10.26 \\
\hline 96 & 86400 & 300849 & 42.3 & 76.1400 & 0.000691 & $7.461 E-11$ & 10.13 \\
\hline 120 & 86400 & 387596 & 67.7 & 121.8600 & 0.001106 & $2.462 E-10$ & 9.61 \\
\hline 456 & 1209600 & 939462 & 272 & 489.6000 & 0.004443 & $4.915 \mathrm{E}-11$ & 10.31 \\
\hline 1128 & 2419200 & 2716550 & 330 & 594.0000 & 0.005390 & $5.230 E-11$ & 10.28 \\
\hline 2160 & 3715200 & 5768861 & 308 & 554.4000 & 0.005031 & 4.102E-11 & 10.39 \\
\hline & & & & \multicolumn{3}{|c|}{ Average Leach Index: } & 10.24 \\
\hline
\end{tabular}




\section{American Nuclear Society - 16.1 Static Leach Test}

Project: $\quad$ SRS Reactor Seepage Basin Stabilization

Sample Description: $\quad \mathrm{K} 422$

Analyst: PRL

Date:

15-Apr-99

Contaminant:

Gross Alpha

Initial Contaminant Conc. (pCi/g):

Initial Contaminant Level (pCi):

Monolith Height (cm):

12.9

4980.819

Monolith Surface Area $\left(\mathrm{cm}^{\wedge} 2\right)$ :

Monolith Volume $\left(\mathrm{cm}^{\wedge} 3\right)$ :
10

176.72

196.35
Sample Weight (g):

Monolith Diameter (cm):

Leachate Volume $(\mathrm{mL})$ :
386.11

5

1800

\begin{tabular}{|c|c|c|c|c|c|c|c|}
\hline $\begin{array}{l}\text { Time } \\
\text { (hrs) }\end{array}$ & $\begin{array}{l}\text { Interval } \\
\text { Length } \\
\text { (s) }\end{array}$ & $\begin{array}{c}\text { Mean } \\
\text { Time } \\
\text { (s) }\end{array}$ & $\begin{array}{c}\text { Leachate } \\
\text { Conc. } \\
\text { (pCi/L) }\end{array}$ & $\begin{array}{l}\text { Amount } \\
\text { Leached } \\
\text { (pCi) }\end{array}$ & $\begin{array}{l}\text { Fraction } \\
\text { Leached }\end{array}$ & $\begin{array}{l}\text { Diffusivity } \\
\text { Coefficient } \\
\text { (cm^2/s) }\end{array}$ & $\begin{array}{l}\text { Leach } \\
\text { Index }\end{array}$ \\
\hline 2 & 7200 & 1800 & 0.26 & 0.4680 & 0.000094 & $1.189 \mathrm{E}-12$ & 11.92 \\
\hline 7 & 18000 & 14835 & 0.1 & 0.1800 & 0.000036 & 2.319E-13 & 12.63 \\
\hline 24 & 61200 & 51231 & 0.1 & 0.1800 & 0.000036 & $6.928 \mathrm{E}-14$ & 13.16 \\
\hline 48 & 86400 & 125894 & 0.739 & 1.3302 & 0.000267 & 4.665E-12 & 11.33 \\
\hline 72 & 86400 & 213818 & 0.2 & 0.3600 & 0.000072 & $5.803 E-13$ & 12.24 \\
\hline 96 & 86400 & 300849 & 0.46 & 0.8280 & 0.000166 & 4.320E-12 & 11.36 \\
\hline 120 & 86400 & 387596 & 0.501 & 0.9018 & 0.000181 & $6.601 E-12$ & 11.18 \\
\hline 456 & 1209600 & 939462 & 0.277 & 0.4986 & 0.000100 & 2.496E-14 & 13.60 \\
\hline 1128 & 2419200 & 2716550 & 4.05 & 7.2900 & 0.001464 & 3.857E-12 & 11.41 \\
\hline 2160 & 3715200 & 5768861 & 9.31 & 16.7580 & 0.003365 & $1.835 \mathrm{E}-11$ & 10.74 \\
\hline & & & & & \multicolumn{2}{|c|}{ Average Leach Index: } & 11.96 \\
\hline
\end{tabular}




\section{American Nuclear Society - 16.1 Static Leach Test}

Project: SRS Reactor Seepage Basin Stabilization

Sample Description: K422

Analyst: $\quad$ PRL

Date: 15-Apr-99

Contaminant:

Gross Beta

Initial Contaminant Conc. (pCi/g):

293

Initial Contaminant Level (pCi):

113130.23

Monolith Height (cm):

10

Monolith Surface Area ( $\left.\mathrm{cm}^{\wedge} 2\right)$ :

176.72

Monolith Volume $\left(\mathrm{cm}^{\wedge} 3\right)$ :

196.35

Sample Weight (g):

Monolith Diameter (cm):

Leachate Volume $(\mathrm{mL})$ :
386.11

5

1800

\begin{tabular}{|c|c|c|c|c|c|c|c|}
\hline $\begin{array}{l}\text { Time } \\
\text { (hrs) } \\
\end{array}$ & $\begin{array}{l}\text { Interval } \\
\text { Length } \\
\text { (s) } \\
\end{array}$ & $\begin{array}{l}\text { Mean } \\
\text { Time } \\
(\mathbf{s}) \\
\end{array}$ & $\begin{array}{c}\text { Leachate } \\
\text { Conc. } \\
\text { (pCi/L) } \\
\end{array}$ & $\begin{array}{c}\text { Amount } \\
\text { Leached } \\
(p C i) \\
\end{array}$ & $\begin{array}{l}\text { Fraction } \\
\text { Leached }\end{array}$ & $\begin{array}{c}\text { Diffusivity } \\
\text { Coefficient } \\
\left(\mathrm{cm}^{\wedge} 2 / \mathrm{s}\right) \\
\end{array}$ & $\begin{array}{l}\text { Leach } \\
\text { Index }\end{array}$ \\
\hline 2 & 7200 & 1800 & 10.1 & 18.1800 & 0.000161 & $3.478 \mathrm{E}-12$ & 11.46 \\
\hline 7 & 18000 & 14835 & 14.1 & 25.3800 & 0.000224 & 8.938E-12 & 11.05 \\
\hline 24 & 61200 & 51231 & 79.1 & 142.3800 & 0.001259 & 8.403E-11 & 10.08 \\
\hline 48 & 86400 & 125894 & 54.6 & 98.2800 & 0.000869 & $4.936 \mathrm{E}-11$ & 10.31 \\
\hline 72 & 86400 & 213818 & 27.5 & 49.5000 & 0.000438 & 2.127E-11 & 10.67 \\
\hline 96 & 86400 & 300849 & 38.2 & 68.7600 & 0.000608 & 5.774E-11 & 10.24 \\
\hline 120 & 86400 & 387596 & 78.6 & 141.4800 & 0.001251 & $3.150 \mathrm{E}-10$ & 9.50 \\
\hline 456 & 1209600 & 939462 & 112 & 201.6000 & 0.001782 & 7.908E-12 & 11.10 \\
\hline 1128 & 2419200 & 2716550 & 179 & 322.2000 & 0.002848 & $1.460 \mathrm{E}-11$ & 10.84 \\
\hline 2160 & 3715200 & 5768861 & 368 & 662.4000 & 0.005855 & 5.557E-11 & 10.26 \\
\hline & & & & \multicolumn{3}{|c|}{ Average Leach Index: } & 10.55 \\
\hline
\end{tabular}




\section{American Nuclear Society - 16.1 Static Leach Test}

Project: $\quad$ SRS Reactor Seepage Basin Stabilization

Sample Description: K432

Analyst: PRL

Date:

15-Apr-99

Contaminant:

Gross Alpha

Initial Contaminant Conc. (pCi/g):

12.9

Initial Contaminant Level (pCi):

4723.206

Monolith Height $(\mathrm{cm})$ :

10

Monolith Surface Area ( $\left.\mathrm{cm}^{\wedge} 2\right)$ :

176.72

Monolith Volume $\left(\mathrm{cm}^{\wedge} 3\right)$ :

196.35

Sample Weight (g):

Monolith Diameter (cm):

Leachate Volume (mL):
366.14

5

1800

\begin{tabular}{|c|c|c|c|c|c|c|c|}
\hline $\begin{array}{l}\text { Time } \\
\text { (hrs) }\end{array}$ & $\begin{array}{l}\text { Interval } \\
\text { Length } \\
\text { (s) }\end{array}$ & $\begin{array}{c}\text { Mean } \\
\text { Time } \\
\text { (s) }\end{array}$ & $\begin{array}{l}\text { Leachate } \\
\text { Conc. } \\
\text { (pCi/L) }\end{array}$ & $\begin{array}{c}\text { Amount } \\
\text { Leached } \\
(\mathrm{pCi})\end{array}$ & $\begin{array}{l}\text { Fraction } \\
\text { Leached }\end{array}$ & $\begin{array}{c}\text { Diffusivity } \\
\text { Coefficient } \\
\left(\mathrm{cm}^{\wedge} 2 / \mathrm{s}\right)\end{array}$ & $\begin{array}{l}\text { Leach } \\
\text { Index }\end{array}$ \\
\hline 2 & 7200 & 1800 & 0.1 & 0.1800 & 0.000038 & $1.956 \mathrm{E}-13$ & 12.71 \\
\hline 7 & 18000 & 14835 & 0.1 & 0.1800 & 0.000038 & $2.579 E-13$ & 12.59 \\
\hline 24 & 61200 & 51231 & 0.783 & 1.4094 & 0.000298 & 4.724E-12 & 11.33 \\
\hline 48 & 86400 & 125894 & 1.18 & 2.1240 & 0.000450 & 1.323E-11 & 10.88 \\
\hline 72 & 86400 & 213818 & 0.624 & 1.1232 & 0.000238 & $6.282 E-12$ & 11.20 \\
\hline 96 & 86400 & 300849 & 0.1 & 0.1800 & 0.000038 & $2.270 \mathrm{E}-13$ & 12.64 \\
\hline 120 & 86400 & 387596 & 0.712 & 1.2816 & 0.000271 & 1.483E-11 & 10.83 \\
\hline 456 & 1209600 & 939462 & 0.386 & 0.6948 & 0.000147 & $5.389 E-14$ & 13.27 \\
\hline 1128 & 2419200 & 2716550 & 0.701 & 1.2618 & 0.000267 & $1.285 E-13$ & 12.89 \\
\hline 2160 & 3715200 & 5768861 & 0.1 & 0.1800 & 0.000038 & $2.354 E-15$ & 14.63 \\
\hline
\end{tabular}




\section{American Nuclear Society - 16.1 Static Leach Test}

Project: $\quad$ SRS Reactor Seepage Basin Stabilization

Sample Description: K432

$\begin{array}{llll}\text { Analyst: PRL } & \text { Date: }\end{array}$

Contaminant: Gross Beta

Initial Contaminant Conc. (pCi/g):

293

Initial Contaminant Level (pCi):

107279.02

Monolith Height (cm):

Monolith Surface Area ( $\left.\mathrm{cm}^{\wedge} 2\right)$ :

176.72

Monolith Volume $\left(\mathrm{cm}^{\wedge} 3\right)$ :

196.35

Sample Weight (g):

Monolith Diameter $(\mathrm{cm})$ :

Leachate Volume $(\mathrm{mL})$ :
366.14

5

1800

\begin{tabular}{|c|c|c|c|c|c|c|c|}
\hline $\begin{array}{l}\text { Time } \\
\text { (hrs) }\end{array}$ & $\begin{array}{l}\text { Interval } \\
\text { Length } \\
\text { (s) }\end{array}$ & $\begin{array}{l}\text { Mean } \\
\text { Time } \\
(s)\end{array}$ & $\begin{array}{l}\text { Leachate } \\
\text { Conc. } \\
\text { (pCi/L) }\end{array}$ & $\begin{array}{l}\text { Amount } \\
\text { Leached } \\
\text { (pCi) }\end{array}$ & $\begin{array}{l}\text { Fraction } \\
\text { Leached }\end{array}$ & $\begin{array}{l}\text { Diffusivity } \\
\text { Coefficient } \\
\left(\mathrm{cm}^{\wedge} 2 / \mathrm{s}\right)\end{array}$ & $\begin{array}{l}\text { Leach } \\
\text { Index }\end{array}$ \\
\hline 2 & 7200 & 1800 & 6.85 & 12.3300 & 0.000115 & $1.779 \mathrm{E}-12$ & 11.75 \\
\hline 7 & 18000 & 14835 & 24.1 & 43.3800 & 0.000404 & $2.904 E-11$ & 10.54 \\
\hline 24 & 61200 & 51231 & 66.6 & 119.8800 & 0.001117 & $6.625 E-11$ & 10.18 \\
\hline 48 & 86400 & 125894 & 31.4 & 56.5200 & 0.000527 & $1.816 E-11$ & 10.74 \\
\hline 72 & 86400 & 213818 & 38.2 & 68.7600 & 0.000641 & $4.564 \mathrm{E}-11$ & 10.34 \\
\hline 96 & 86400 & 300849 & 27.4 & 49.3200 & 0.000460 & $3.304 E-11$ & 10.48 \\
\hline 120 & 86400 & 387596 & 56 & 100.8000 & 0.000940 & $1.778 \mathrm{E}-10$ & 9.75 \\
\hline 456 & 1209600 & 939462 & 201 & 361.8000 & 0.003373 & $2.832 E-11$ & 10.55 \\
\hline 1128 & 2419200 & 2716550 & 234 & 421.2000 & 0.003926 & $2.775 E-11$ & 10.56 \\
\hline 2160 & 3715200 & 5768861 & 317 & 570.6000 & 0.005319 & 4.586E-11 & 10.34 \\
\hline & & & & \multicolumn{3}{|c|}{ Average Leach Index: } & 10.52 \\
\hline
\end{tabular}




\section{American Nuclear Society - 16.1 Static Leach Test}

Project: $\quad$ SRS Reactor Seepage Basin Stabilization

Sample Description: K442

$\begin{array}{llll}\text { Analyst: } & \text { PRL } & \text { Date: 15-Apr-99 }\end{array}$

Contaminant: Gross Alpha

Initial Contaminant Conc. (pCi/g): $\quad 12.9$

Initial Contaminant Level (pCi): $\quad 4788.996$

Monolith Height (cm):

Monolith Surface Area ( $\left.\mathrm{cm}^{\wedge} 2\right)$ :

Monolith Volume $\left(\mathrm{cm}^{\wedge} 3\right)$ :

10

176.72

196.35

\begin{tabular}{|c|c|c|c|c|c|c|c|}
\hline $\begin{array}{l}\text { Time } \\
\text { (hrs) }\end{array}$ & $\begin{array}{l}\text { Interval } \\
\text { Length } \\
\text { (s) }\end{array}$ & $\begin{array}{c}\text { Mean } \\
\text { Time } \\
\text { (s) }\end{array}$ & $\begin{array}{c}\text { Leachate } \\
\text { Conc. } \\
\text { (pCi/L) } \\
\end{array}$ & $\begin{array}{c}\text { Amount } \\
\text { Leached } \\
\text { (pCi) }\end{array}$ & $\begin{array}{l}\text { Fraction } \\
\text { Leached }\end{array}$ & $\begin{array}{l}\text { Diffusivity } \\
\text { Coefficient } \\
\text { (cm^2/s) }\end{array}$ & $\begin{array}{l}\text { Leach } \\
\text { Index }\end{array}$ \\
\hline 2 & 7200 & 1800 & 0.205 & 0.3690 & 0.000077 & $7.995 \mathrm{E}-13$ & 12.10 \\
\hline 7 & 18000 & 14835 & 0.1 & 0.1800 & 0.000038 & $2.509 E-13$ & 12.60 \\
\hline 24 & 61200 & 51231 & 0.1 & 0.1800 & 0.000038 & $7.495 E-14$ & 13.13 \\
\hline 48 & 86400 & 125894 & 0.601 & 1.0818 & 0.000226 & $3.338 \mathrm{E}-12$ & 11.48 \\
\hline 72 & 86400 & 213818 & 0.145 & 0.2610 & 0.000054 & 3.300E-13 & 12.48 \\
\hline 96 & 86400 & 300849 & 0.595 & 1.0710 & 0.000224 & $7.818 E-12$ & 11.11 \\
\hline 120 & 86400 & 387596 & 0.637 & 1.1466 & 0.000239 & 1.154E-11 & 10.94 \\
\hline 456 & 1209600 & 939462 & 1.86 & 3.3480 & 0.000699 & 1.217E-12 & 11.91 \\
\hline 1128 & 2419200 & 2716550 & 0.468 & 0.8424 & 0.000176 & 5.570E-14 & 13.25 \\
\hline 2160 & 3715200 & 5768861 & 0.1 & 0.1800 & 0.000038 & $2.290 E-15$ & 14.64 \\
\hline & & & & & \multicolumn{2}{|c|}{ Average Leach Index: } & 12.36 \\
\hline
\end{tabular}




\section{American Nuclear Society - 16.1 Static Leach Test}

Project: $\quad$ SRS Reactor Seepage Basin Stabilization

Sample Description: $\quad$ K442

$\begin{array}{llll}\text { Analyst: } & \text { PRL } & \text { Date: } & \text { 15-Apr-99 }\end{array}$

Contaminant: Gross Beta

Initial Contaminant Conc. (pCi/g):

Initial Contaminant Level (pCi):

293

108773.32

Monolith Height (cm):

Monolith Surface Area $\left(\mathrm{cm}^{\wedge} 2\right)$ :

Monolith Volume $\left(\mathrm{cm}^{\wedge} 3\right)$ :

10

176.72

196.35

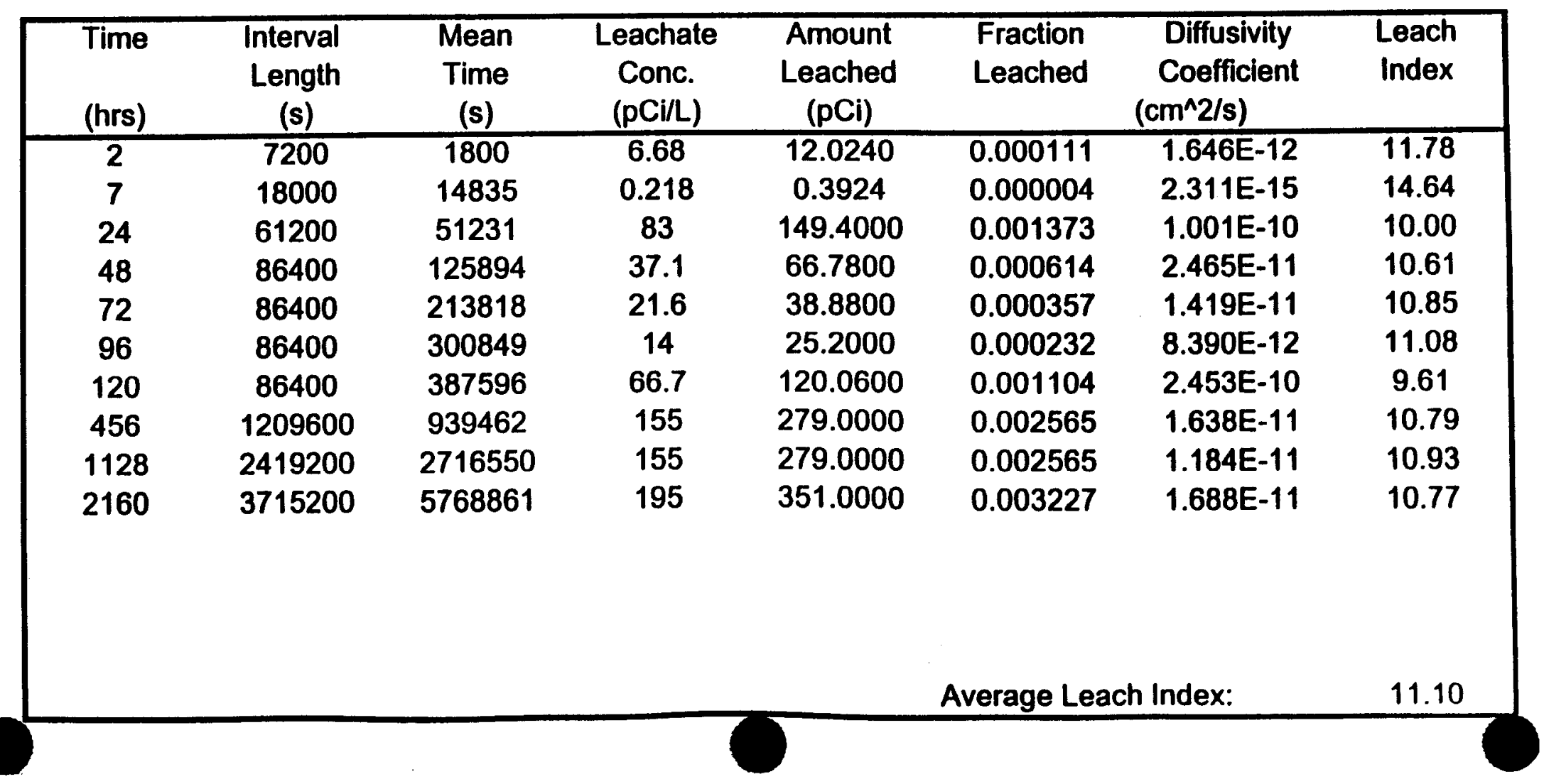


L Series

Leach Index 
Gross Alpha Leaching for the Coarse Fraction from LRSB Soil

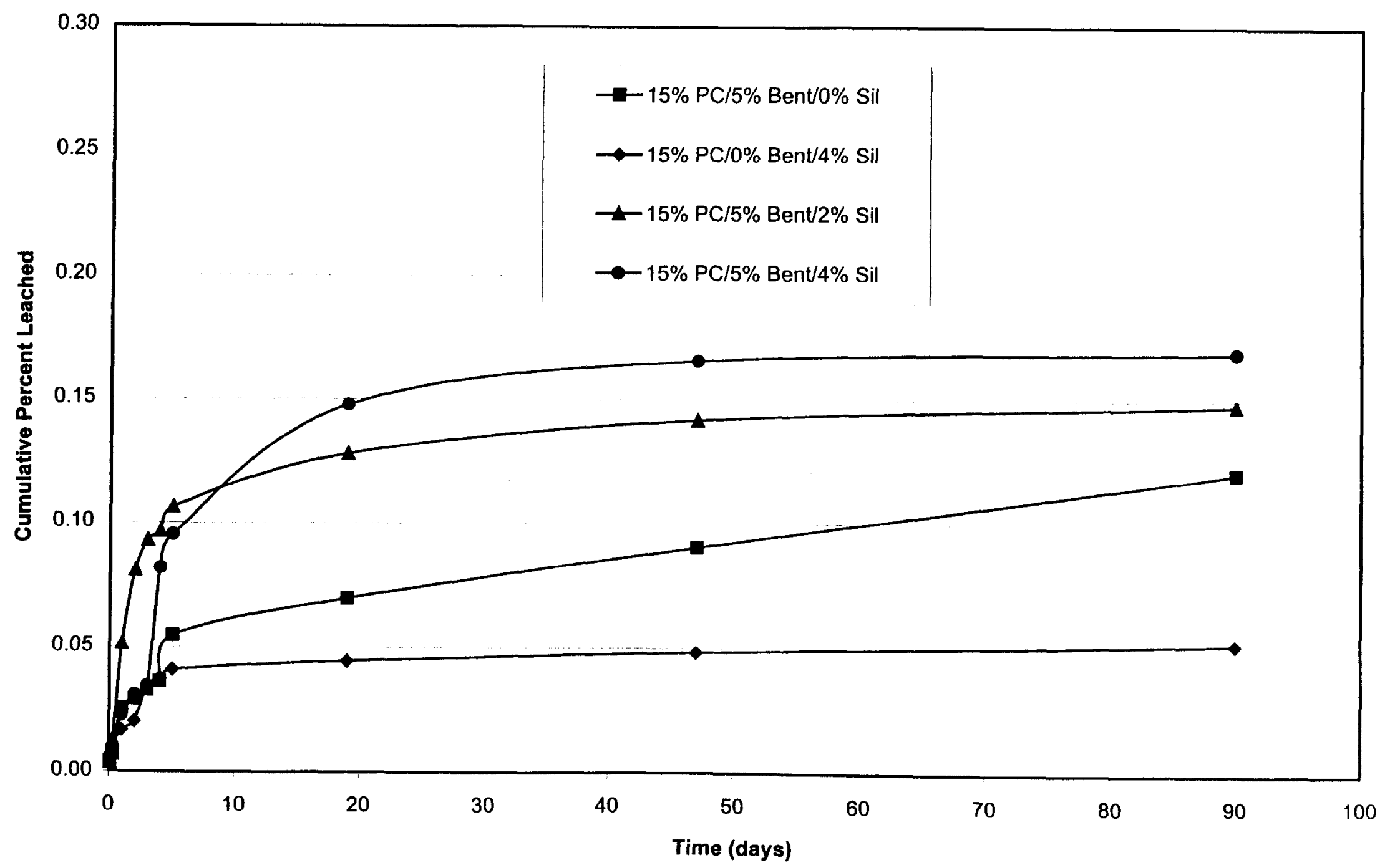




\section{American Nuclear Society - 16.1 Static Leach Test}

Project: $\quad$ SRS Reactor Seepage Basin Stabilization

Sample Description: $\quad$ L312

$\begin{array}{lll}\text { Analyst: PRL Date: 25-May-99 } & \text { Da }\end{array}$

Contaminant: Gross Alpha

Initial Contaminant Conc. (pCi/g): $\quad 13.4$

Initial Contaminant Level (pCi): $\quad 5057.562$

Monolith Height (cm):

10

Monolith Surface Area (cm²): $\quad 176.72$

196.35

Monolith Volume $\left(\mathrm{cm}^{\wedge} 3\right)$ :

\begin{tabular}{|cccccccc|}
\hline Time & $\begin{array}{c}\text { Interval } \\
\text { Length } \\
(\mathrm{hrs})\end{array}$ & $\begin{array}{c}\text { Mean } \\
\text { Time } \\
(\mathrm{s})\end{array}$ & $\begin{array}{c}\text { Leachate } \\
\text { Conc. } \\
(\mathrm{pCi} / \mathrm{L})\end{array}$ & $\begin{array}{c}\text { Amount } \\
\text { Leached } \\
(\mathrm{pCi})\end{array}$ & $\begin{array}{c}\text { Fraction } \\
\text { Leached }\end{array}$ & $\begin{array}{c}\text { Diffusivity } \\
\text { Coefficient } \\
\left(\mathrm{cm}^{\wedge} 2 / \mathrm{s}\right)\end{array}$ & $\begin{array}{c}\text { Leach } \\
\text { Index }\end{array}$ \\
\hline 2 & 7200 & 1800 & 0.1 & 0.1800 & 0.000036 & $1.706 \mathrm{E}-13$ & 12.77 \\
7 & 18000 & 14835 & 0.1 & 0.1800 & 0.000036 & $2.249 \mathrm{E}-13$ & 12.65 \\
24 & 61200 & 51231 & 0.516 & 0.9288 & 0.000184 & $1.789 \mathrm{E}-12$ & 11.75 \\
48 & 86400 & 125894 & 0.1 & 0.1800 & 0.000036 & $8.285 \mathrm{E}-14$ & 13.08 \\
72 & 86400 & 213818 & 0.1 & 0.1800 & 0.000036 & $1.407 \mathrm{E}-13$ & 12.85 \\
96 & 86400 & 300849 & 0.1 & 0.1800 & 0.000036 & $1.980 \mathrm{E}-13$ & 12.70 \\
120 & 86400 & 387596 & 0.519 & 0.9342 & 0.000185 & $6.871 \mathrm{E}-12$ & 11.16 \\
456 & 1209600 & 939462 & 0.425 & 0.7650 & 0.000151 & $5.698 \mathrm{E}-14$ & 13.24 \\
1128 & 2419200 & 2716550 & 0.582 & 1.0476 & 0.000207 & $7.724 \mathrm{E}-14$ & 13.11 \\
2160 & 3715200 & 5768861 & 0.844 & 1.5192 & 0.000300 & $1.463 \mathrm{E}-13$ & 12.83 \\
& & & & & & & \\
\end{tabular}




\section{American Nuclear Society - 16.1 Static Leach Test}

Project: $\quad$ SRS Reactor Seepage Basin Stabilization

Sample Description: $\quad$ L322

Analyst: $\quad$ PRL

Date: $\quad$ 15-Apr-99

Contaminant:

Gross Alpha

Initial Contaminant Conc. (pCi/g):

13.4

Initial Contaminant Level (pCi):

Monolith Height (cm):

5183.12

10

Monolith Surface Area $\left(\mathrm{cm}^{\wedge} 2\right)$ :

176.72

Monolith Volume $\left(\mathrm{cm}^{\wedge} 3\right)$ :

196.35

Sample Weight (g):

Monolith Diameter $(\mathrm{cm})$ :

Leachate Volume $(\mathrm{mL})$ :
386.8

5

1800






\section{American Nuclear Society - 16.1 Static Leach Test}

Project: $\quad$ SRS Reactor Seepage Basin Stabilization

Sample Description:

L332

Analyst: $\quad \mathrm{PRL}$

Date:

25-May-99

Contaminant:

Gross Alpha

Initial Contaminant Conc. (pCi/g):

Initial Contaminant Level (pCi):

Monolith Height $(\mathrm{cm})$ :

13.4

4958

Monolith Surface Area $\left(\mathrm{cm}^{\wedge} 2\right)$ :

10

176.72

Monolith Volume $\left(\mathrm{cm}^{\wedge} 3\right)$ :

196.35

Sample Weight (g):

Monolith Diameter $(\mathrm{cm})$ :

Leachate Volume $(\mathrm{mL})$ :
370

5

1800

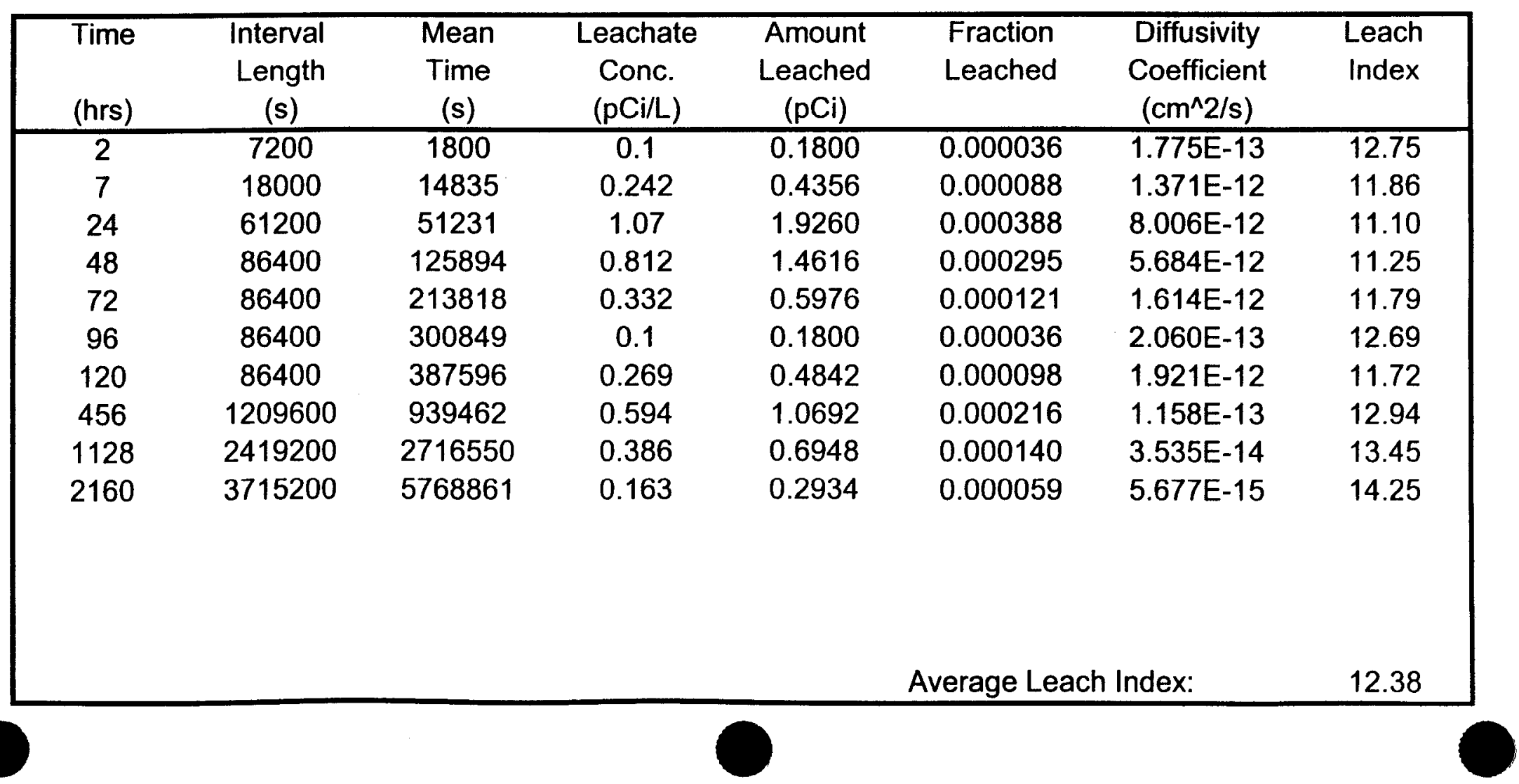




\section{American Nuclear Society - 16.1 Static Leach Test}

Project: SRS Reactor Seepage Basin Stabilization

Sample Description:

L342

Analyst: PRL

Date:

25-May-99

Contaminant:

Gross Alpha

Initial Contaminant Conc. (pCi/g):

Initial Contaminant Level $(\mathrm{pCi})$ :

Monolith Height $(\mathrm{cm})$ :

Monolith Surface Area $\left(\mathrm{cm}^{\wedge} 2\right)$ :

Monolith Volume $\left(\mathrm{cm}^{\wedge} 3\right)$ :

13.4

5011.332

10

176.72

196.35
Sample Weight (g):

Monolith Diameter $(\mathrm{cm})$ :

Leachate Volume $(\mathrm{mL})$ :
373.98

5

1800

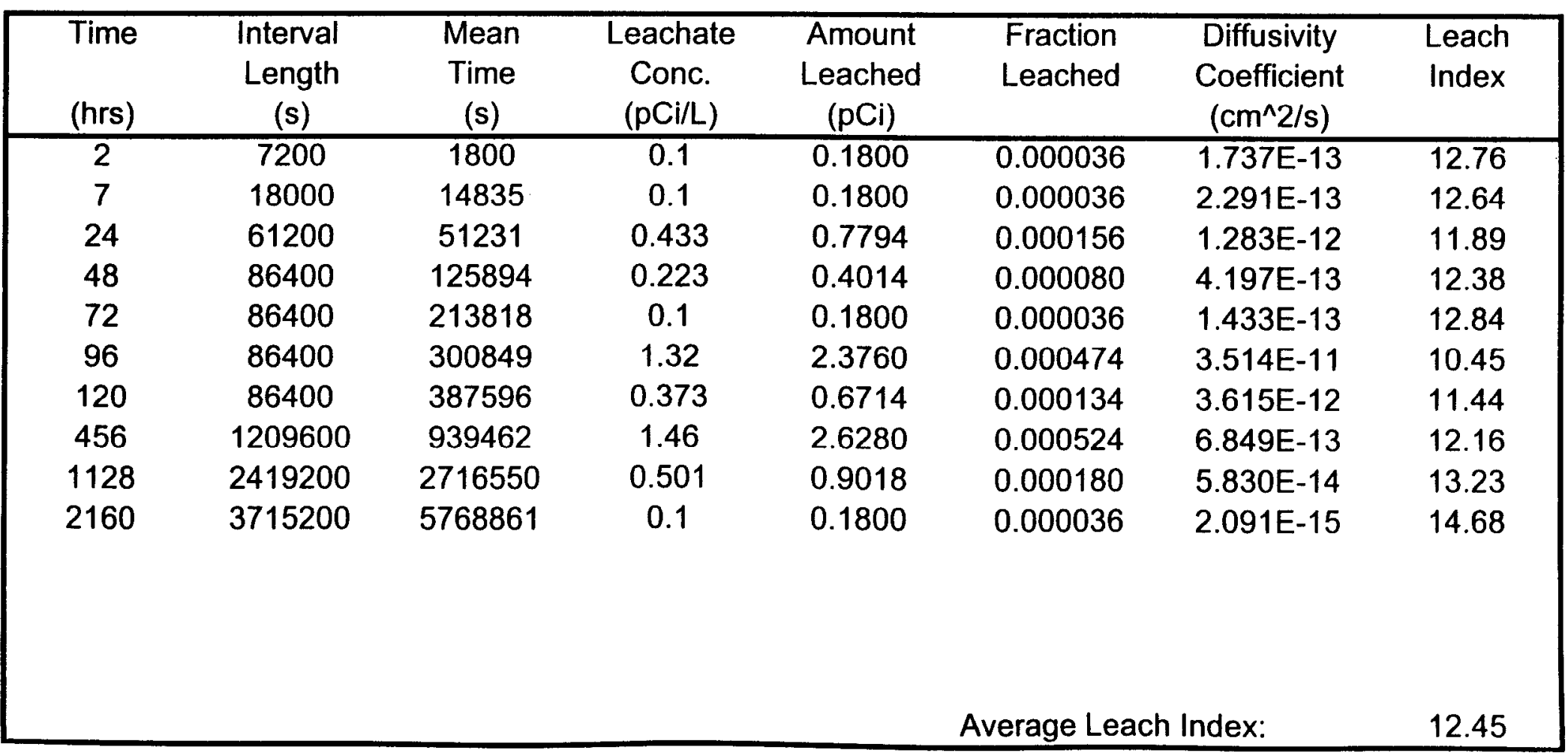


Gross Alpha Leaching for the Fine Fraction from LRSB Soil

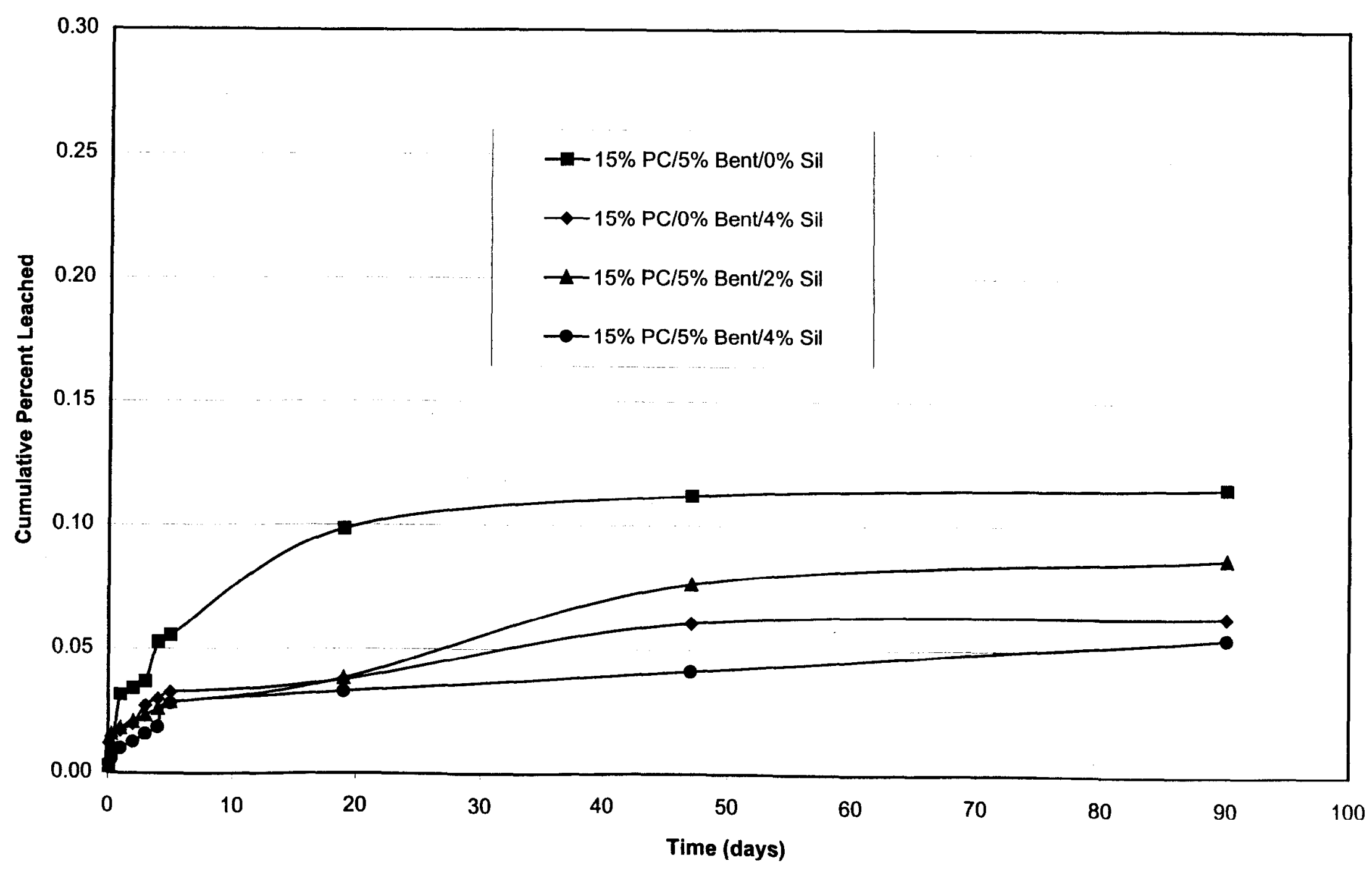

0

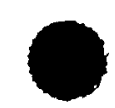




\section{American Nuclear Society - 16.1 Static Leach Test}

Project: $\quad$ SRS Reactor Seepage Basin Stabilization

Sample Description:

L412

Analyst: $\quad$ PRL

Date:

25-May-99

Contaminant:

Gross Alpha

Initial Contaminant Conc. (pCi/g):

17.9

6734.159

Initial Contaminant Level (pCi):

Monolith Height (cm):

10

Monolith Surface Area $\left(\mathrm{cm}^{\wedge} 2\right)$ :

176.72

Monolith Volume $\left(\mathrm{cm}^{\wedge} 3\right)$ :

196.35

Sample Weight (g):

Monolith Diameter $(\mathrm{cm})$ :

Leachate Volume $(\mathrm{mL})$ :
376.21

5

1800

\begin{tabular}{|cccccccc|}
\hline Time & $\begin{array}{c}\text { Interval } \\
\text { Length } \\
(\mathrm{s})\end{array}$ & $\begin{array}{c}\text { Mean } \\
\text { Time } \\
(\mathrm{s})\end{array}$ & $\begin{array}{c}\text { Leachate } \\
\text { Conc. } \\
(\mathrm{pCi} / \mathrm{L})\end{array}$ & $\begin{array}{c}\text { Amount } \\
\text { Leached } \\
(\mathrm{pCi})\end{array}$ & $\begin{array}{c}\text { Fraction } \\
\text { Leached }\end{array}$ & $\begin{array}{c}\text { Diffusivity } \\
\text { Coefficient } \\
\left(\mathrm{cm}^{\wedge} 2 / \mathrm{s}\right)\end{array}$ & $\begin{array}{c}\text { Leach } \\
\text { Index }\end{array}$ \\
\hline 2 & 7200 & 1800 & 0.1 & 0.1800 & 0.000027 & $9.622 \mathrm{E}-14$ & 13.02 \\
7 & 18000 & 14835 & 0.178 & 0.3204 & 0.000048 & $4.020 \mathrm{E}-13$ & 12.40 \\
24 & 61200 & 51231 & 0.9 & 1.6200 & 0.000241 & $3.070 \mathrm{E}-12$ & 11.51 \\
48 & 86400 & 125894 & 0.1 & 0.1800 & 0.000027 & $4.673 \mathrm{E}-14$ & 13.33 \\
72 & 86400 & 213818 & 0.1 & 0.1800 & 0.000027 & $7.937 \mathrm{E}-14$ & 13.10 \\
96 & 86400 & 300849 & 0.593 & 1.0674 & 0.000159 & $3.927 \mathrm{E}-12$ & 11.41 \\
120 & 86400 & 387596 & 0.1 & 0.1800 & 0.000027 & $1.439 \mathrm{E}-13$ & 12.84 \\
456 & 1209600 & 939462 & 1.61 & 2.8980 & 0.000430 & $4.612 \mathrm{E}-13$ & 12.34 \\
1128 & 2419200 & 2716550 & 0.506 & 0.9108 & 0.000135 & $3.293 \mathrm{E}-14$ & 13.48 \\
2160 & 3715200 & 5768861 & 0.123 & 0.2214 & 0.000033 & $1.752 \mathrm{E}-15$ & 14.76 \\
& & & & & & & \\
& & & & & & & \\
\end{tabular}




\section{American Nuclear Society - 16.1 Static Leach Test}

Project: $\quad$ SRS Reactor Seepage Basin Stabilization

Sample Description: $\quad$ L422

Analyst: $\quad \mathrm{PRL}$

Date:

25-May-99

Contaminant:

Gross Alpha

Initial Contaminant Conc. (pCi/g):

17.9

Initial Contaminant Level ( $\mathrm{pCi}$ :

Monolith Height (cm):

6643.585

Monolith Surface Area $\left(\mathrm{cm}^{\wedge} 2\right)$ :

10

Monolith Volume $\left(\mathrm{cm}^{\wedge} 3\right)$ :

176.72

196.35

\begin{tabular}{|cccccccc|}
\hline Time & $\begin{array}{c}\text { Interval } \\
\text { Length } \\
(\mathrm{hrs})\end{array}$ & $\begin{array}{c}\text { Mean } \\
\text { Time } \\
(\mathrm{s})\end{array}$ & $\begin{array}{c}\text { Leachate } \\
\text { Conc. } \\
(\mathrm{pCi} / \mathrm{L})\end{array}$ & $\begin{array}{c}\text { Amount } \\
\text { Leached } \\
(\mathrm{pCi})\end{array}$ & $\begin{array}{c}\text { Fraction } \\
\text { Leached }\end{array}$ & $\begin{array}{c}\text { Diffusivity } \\
\text { Coefficient } \\
\left(\mathrm{cm}^{\wedge} / \mathrm{s}\right)\end{array}$ & $\begin{array}{c}\text { Leach } \\
\text { Index }\end{array}$ \\
\hline 2 & 7200 & 1800 & 0.433 & 0.7794 & 0.000117 & $1.853 \mathrm{E}-12$ & 11.73 \\
7 & 18000 & 14835 & 0.1 & 0.1800 & 0.000027 & $1.304 \mathrm{E}-13$ & 12.88 \\
24 & 61200 & 51231 & 0.1 & 0.1800 & 0.000027 & $3.894 \mathrm{E}-14$ & 13.41 \\
48 & 86400 & 125894 & 0.1 & 0.1800 & 0.000027 & $4.802 \mathrm{E}-14$ & 13.32 \\
72 & 86400 & 213818 & 0.268 & 0.4824 & 0.000073 & $5.857 \mathrm{E}-13$ & 12.23 \\
96 & 86400 & 300849 & 0.1 & 0.1800 & 0.000027 & $1.147 \mathrm{E}-13$ & 12.94 \\
120 & 86400 & 387596 & 0.1 & 0.1800 & 0.000027 & $1.478 \mathrm{E}-13$ & 12.83 \\
456 & 1209600 & 939462 & 0.185 & 0.3330 & 0.000050 & $6.257 \mathrm{E}-15$ & 14.20 \\
1128 & 2419200 & 2716550 & 0.852 & 1.5336 & 0.000231 & $9.593 \mathrm{E}-14$ & 13.02 \\
2160 & 3715200 & 5768861 & 0.1 & 0.1800 & 0.000027 & $1.190 \mathrm{E}-15$ & 14.92 \\
& & & & & & & \\
& & & & & & & \\
& & & & & & & \\
\end{tabular}




\section{American Nuclear Society - 16.1 Static Leach Test}

Project: $\quad$ SRS Reactor Seepage Basin Stabilization

Sample Description: $\quad$ L432

$\begin{array}{llll}\text { Analyst: PRL } & \text { Date: } & & \end{array}$

Contaminant:

Gross Alpha

Initial Contaminant Conc. (pCi/g):

17.9

Initial Contaminant Level (pCi):

6925.331

Monolith Height $(\mathrm{cm})$ :

10

Monolith Surface Area $\left(\mathrm{cm}^{\wedge} 2\right)$ :

176.72

196.35

Sample Weight (g):

Monolith Diameter $(\mathrm{cm})$ :

Leachate Volume $(\mathrm{mL})$ :

1800

Monolith Volume $\left(\mathrm{cm}^{\wedge} 3\right)$ :

\begin{tabular}{|c|c|c|c|c|c|c|c|}
\hline $\begin{array}{l}\text { Time } \\
\text { (hrs) }\end{array}$ & $\begin{array}{l}\text { Interval } \\
\text { Length } \\
\text { (s) }\end{array}$ & $\begin{array}{c}\text { Mean } \\
\text { Time } \\
\text { (s) }\end{array}$ & $\begin{array}{l}\text { Leachate } \\
\text { Conc. } \\
\text { (pCi/L) }\end{array}$ & $\begin{array}{l}\text { Amount } \\
\text { Leached } \\
(\mathrm{pCi})\end{array}$ & $\begin{array}{c}\text { Fraction } \\
\text { Leached }\end{array}$ & $\begin{array}{c}\text { Diffusivity } \\
\text { Coefficient } \\
\text { (cm^2/s) }\end{array}$ & $\begin{array}{l}\text { Leach } \\
\text { Index }\end{array}$ \\
\hline 2 & 7200 & 1800 & 0.1 & 0.1800 & 0.000026 & $9.098 \mathrm{E}-14$ & 13.04 \\
\hline 7 & 18000 & 14835 & 0.493 & 0.8874 & 0.000128 & $2.916 \mathrm{E}-12$ & 11.54 \\
\hline 24 & 61200 & 51231 & 0.1 & 0.1800 & 0.000026 & $3.584 \mathrm{E}-14$ & 13.45 \\
\hline 48 & 86400 & 125894 & 0.1 & 0.1800 & 0.000026 & 4.419E-14 & 13.35 \\
\hline 72 & 86400 & 213818 & 0.1 & 0.1800 & 0.000026 & 7.505E-14 & 13.12 \\
\hline 96 & 86400 & 300849 & 0.1 & 0.1800 & 0.000026 & 1.056E-13 & 12.98 \\
\hline 120 & 86400 & 387596 & 0.1 & 0.1800 & 0.000026 & $1.360 \mathrm{E}-13$ & 12.87 \\
\hline 456 & 1209600 & 939462 & 0.371 & 0.6678 & 0.000096 & $2.316 \mathrm{E}-14$ & 13.64 \\
\hline 1128 & 2419200 & 2716550 & 1.47 & 2.6460 & 0.000382 & $2.628 \mathrm{E}-13$ & 12.58 \\
\hline 2160 & 3715200 & 5768861 & 0.407 & 0.7326 & 0.000106 & $1.814 \mathrm{E}-14$ & 13.74 \\
\hline & & & & & \multicolumn{2}{|c|}{ Average Leach Index: } & 13.03 \\
\hline
\end{tabular}




\section{American Nuclear Society - 16.1 Static Leach Test}

Project: $\quad$ SRS Reactor Seepage Basin Stabilization

Sample Description: L442

Analyst: $\quad$ PRL

Date:

25-May-99

Contaminant:

Gross Alpha

Initial Contaminant Conc. (pCi/g):

17.9

Initial Contaminant Level (pCi):

6545.851

Monolith Height (cm):

10

Monolith Surface Area $\left(\mathrm{cm}^{\wedge} 2\right)$ :

176.72

Monolith Volume $\left(\mathrm{cm}^{\wedge} 3\right)$ :

196.35

\begin{tabular}{|c|c|c|c|c|c|c|c|}
\hline $\begin{array}{l}\text { Time } \\
\text { (hrs) }\end{array}$ & $\begin{array}{l}\text { Interval } \\
\text { Length } \\
\text { (s) }\end{array}$ & $\begin{array}{l}\text { Mean } \\
\text { Time } \\
(\mathrm{s}) \\
\end{array}$ & $\begin{array}{c}\text { Leachate } \\
\text { Conc. } \\
\text { (pCi/L) }\end{array}$ & $\begin{array}{c}\text { Amount } \\
\text { Leached } \\
(\mathrm{pCi})\end{array}$ & $\begin{array}{l}\text { Fraction } \\
\text { Leached }\end{array}$ & $\begin{array}{l}\text { Diffusivity } \\
\text { Coefficient } \\
\left(\mathrm{cm}^{\wedge} 2 / \mathrm{s}\right)\end{array}$ & $\begin{array}{l}\text { Leach } \\
\text { Index }\end{array}$ \\
\hline 2 & 7200 & 1800 & 0.1 & 0.1800 & 0.000027 & $1.018 \mathrm{E}-13$ & 12.99 \\
\hline 7 & 18000 & 14835 & 0.1 & 0.1800 & 0.000027 & 1.343E-13 & 12.87 \\
\hline 24 & 61200 & 51231 & 0.156 & 0.2808 & 0.000043 & $9.762 E-14$ & 13.01 \\
\hline 48 & 86400 & 125894 & 0.1 & 0.1800 & 0.000027 & $4.946 \mathrm{E}-14$ & 13.31 \\
\hline 72 & 86400 & 213818 & 0.116 & 0.2088 & 0.000032 & $1.130 \mathrm{E}-13$ & 12.95 \\
\hline 96 & 86400 & 300849 & 0.1 & 0.1800 & 0.000027 & 1.182E-13 & 12.93 \\
\hline 120 & 86400 & 387596 & 0.348 & 0.6264 & 0.000096 & $1.844 E-12$ & 11.73 \\
\hline 456 & 1209600 & 939462 & 0.176 & 0.3168 & 0.000048 & $5.833 E-15$ & 14.23 \\
\hline 1128 & 2419200 & 2716550 & 0.302 & 0.5436 & 0.000083 & $1.242 E-14$ & 13.91 \\
\hline 2160 & 3715200 & 5768861 & 0.495 & 0.8910 & 0.000136 & $3.003 E-14$ & 13.52 \\
\hline & & & & & \multicolumn{2}{|c|}{ Average Leach Index: } & 13.15 \\
\hline
\end{tabular}




\section{Series \\ Index Test Results}




\section{MOISTURE CONTENT}

PROJECT NAME

SAIC - Savannah River
PROJECT NUMBER

777304.00010500

\begin{tabular}{|c|c|c|c|c|}
\hline $\begin{array}{c}\text { IT LAB } \\
\text { SAMPLE NO. }\end{array}$ & $\begin{array}{c}\text { CLIENT } \\
\text { SAMPLE NO. }\end{array}$ & $\begin{array}{l}\text { MOISTURE, \% } \\
\text { ASTM D } 2216\end{array}$ & $\begin{array}{c}\text { MOISTURE , \% } \\
\text { SW846 }\end{array}$ & $\begin{array}{l}\text { SOLIDS, \% } \\
\text { SW846 }\end{array}$ \\
\hline ETDC-8009 & L100000 & 14.7 & 12.8 & 87.2 \\
\hline ETDC-8010 & L200000 & 15.1 & 13.1 & 86.9 \\
\hline ETDC-8011 & L300000 & 15.1 & 13.1 & 86.9 \\
\hline ETDC-8012 & L400000 & 15.9 & 13.7 & 86.3 \\
\hline ETDC-8013 & $\mathrm{L} 700000$ & 14.6 & 12.8 & 87.2 \\
\hline ETDC-8014 & L800000 & 14.9 & 12.9 & 87.1 \\
\hline & & & & \\
\hline & & & & \\
\hline & & & & \\
\hline & & & & \\
\hline & & & & \\
\hline & & & & \\
\hline & & & & \\
\hline & & & & \\
\hline & & & & \\
\hline & & & & \\
\hline & & & & \\
\hline & & & & \\
\hline & & & & \\
\hline & & & & \\
\hline
\end{tabular}

ASTM D 2216 results are based on dry sample weight. SW846 results are based on wet sample weight.

Solids content is determined by subtracting the SW846 moisture $(\%)$ from 100 . 


\section{SPECIFIC GRAVITY ASTM D 854}

PROJECT NAME:

SAIC - Savannah River
PROJECT NUMBER:

777304.00010500

IT LAB SAMPLE NO.

CLIENT SAMPLE NO.

SPECIFIC GRAVITY

\begin{tabular}{|c|c|c|}
\hline ETDC-8009 & L100000 & 2.6370 \\
\hline ETDC-8010 & L200000 & 2.6255 \\
\hline ETDC-8011 & L300000 & 2.6279 \\
\hline ETDC-8012 & L400000 & 2.6335 \\
\hline ETDC-8013 & L700000 & 2.6289 \\
\hline ETDC-8014 & L800000 & 2.6336 \\
\hline & & \\
\hline & & \\
\hline & & \\
\hline & & \\
\hline & & \\
\hline & & \\
\hline & & \\
\hline & & \\
\hline & & \\
\hline
\end{tabular}




\section{ATTERBERG LIMITS ASTM D 4318}

PROJECT NAME:

SAIC - Savannah River
PROJECT NO.

777304.00010500

\section{ATTERBERG LIMITS RESULTS}

\begin{tabular}{|c|c|c|c|c|c|c|}
\hline \multicolumn{2}{|c|}{ LAB SAMPLE NO. } & FIELD SAMPLE NO. & LIQUID LIMIT & PLASTIC LIMIT & PLASTICITY INDEX & USCS SYMBOL \\
\hline ETDC-8009 & $\bullet$ & L100000 & 34 & 17 & 17 & $\mathrm{CL}$ \\
\hline ETDC-8010 & $\cdot$ & L200000 & 32 & 17 & 15 & $\overline{\mathrm{CL}}$ \\
\hline ETDC-8011 & $\square$ & L300Q00 & 36 & 16 & 20 & $\mathrm{CL}$ \\
\hline ETDC-8012 & 0 & L400000 & 36 & 17 & 19 & $\overline{C L}$ \\
\hline ETDC-8013 & + & $\mathrm{L} 700000$ & 34 & 17 & 17 & $\mathrm{CL}$ \\
\hline ETDC-8014 & 0 & L800000 & 33 & 17 & 16 & $\overline{C L}$ \\
\hline & - & & & & & \\
\hline & $x$ & & & & & \\
\hline & $\Delta$ & & & & & \\
\hline & * & & & & & \\
\hline
\end{tabular}

- NP = Nonplastic

\section{PLASTICITY CHART}

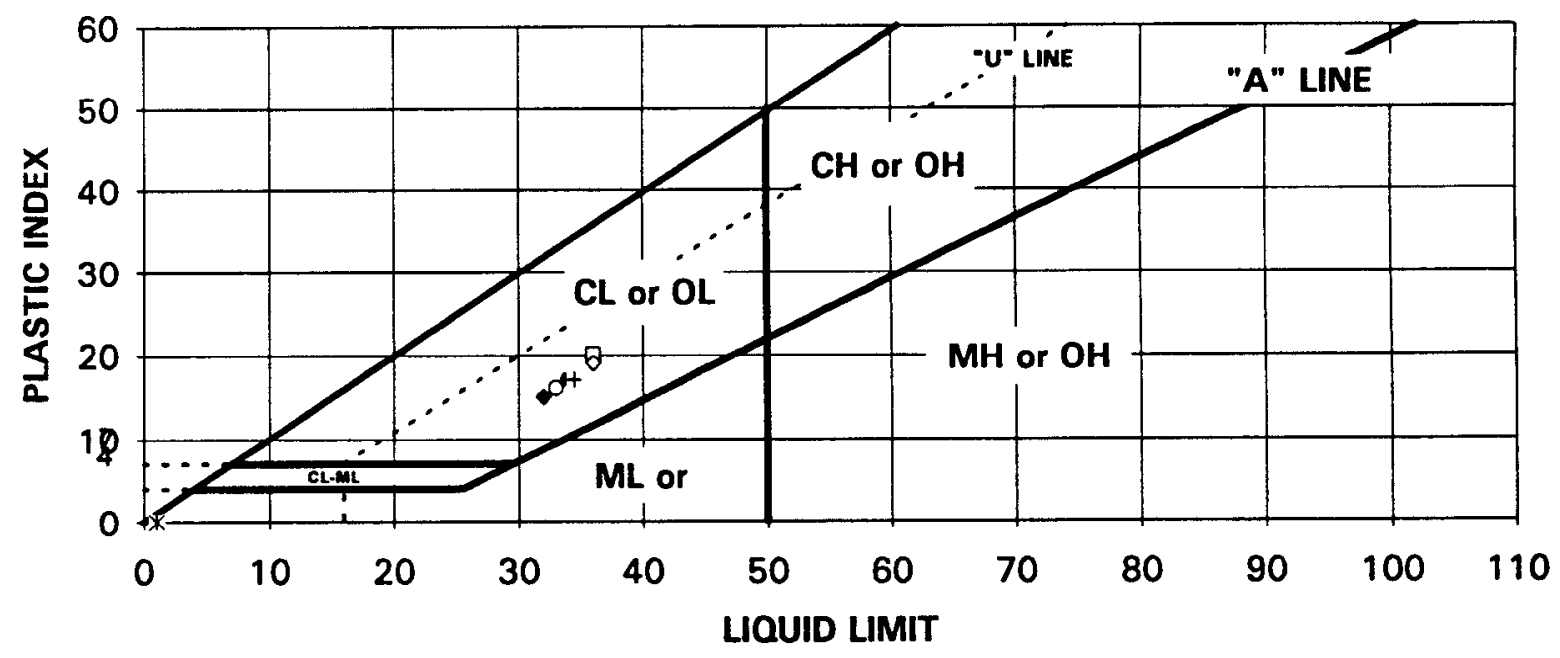




\section{SOIL CLASSIFICATION \\ ASTM D 2487}

\begin{tabular}{|c|c|c|c|c|}
\hline & $\begin{array}{c}\text { ETDC } \\
\text { NUMBER: }\end{array}$ & $\begin{array}{l}\text { CLIENT } \\
\text { NUMBER: }\end{array}$ & $\begin{array}{l}\text { GROUP } \\
\text { SYMBOL: }\end{array}$ & $\begin{array}{l}\text { CLASSIFICATION- } \\
\text { GROUP NAME: }\end{array}$ \\
\hline SAMPLE 1: & ETDC-8009 & L100000 & SC & CLAYEY SAND \\
\hline SAMPLE 2: & ETDC-8010 & L200000 & sc & CLAYEY SAND \\
\hline SAMPLE 3: & ETDC-8011 & L300000 & SC & CLAYEY SAND \\
\hline SAMPLE 4: & ETDC-8012 & $L 400000$ & sc & CLAYEY SAND \\
\hline SAMPLE 5: & ETDC-8013 & $L 700000$ & sc & CLAYEY SAND \\
\hline SAMPLE 6: & ETDC-8014 & L800000 & SC & CLAYEY SAND \\
\hline SAMPLE 7: & & & & \\
\hline SAMPLE 8: & & & & \\
\hline SAMPLE 9: & & & & \\
\hline SAMPLE 10: & & & & \\
\hline
\end{tabular}




\section{SOIL CLASSIFICATION ASTM D 2487}

PROJECT NAME: $\quad$ SAIC - Savannah River

PROJECT NO:

777304.00010500

\begin{tabular}{|c|c|c|c|c|c|}
\hline & SAMPLE 1 & SAMPLE 2 & SAMPLE 3 & SAMPLE 4 & SAMPLE 5 \\
\hline ETDC NUMBER: & ETDC-8009 & ETDC-8010 & ETDC-8011 & ETDC-8012 & ETDC-8013 \\
\hline CLIENT NUMBER: & L100000 & $\mathbf{L 2 0 0 0 0 0}$ & $\mathrm{L} 300000$ & $L 400000$ & $\mathrm{~L} 700000$ \\
\hline $\begin{array}{l}\text { (COARSE-GRAVELS, SANDS) } \\
\% \text { RETAINED ON \# } 200 \text { SIEVE: }\end{array}$ & 65.8 & 68.0 & 72.6 & 66.2 & 66.0 \\
\hline $\begin{array}{l}\text { (FINE-SILTS, CLAYS) } \\
\% \text { PASSING \# } 200 \text { SIEVE: }\end{array}$ & 34.2 & 32.0 & 27.4 & 33.8 & 34.0 \\
\hline$\%$ GRAVEL: & 0.8 & 0.2 & 0.4 & 0.4 & 0.5 \\
\hline$\%$ SAND: & 64.9 & 67.7 & 72.2 & 65.8 & 65.5 \\
\hline '60 IN MM (DIAMETER AT 60\% PASSING): & 0.24774 & 0.34188 & 0.50697 & 0.27507 & 0.26483 \\
\hline J30 IN MM (DIAMETER AT 30\% PASSING): & 0.01251 & 0.05091 & 0.11525 & 0.02056 & 0.02527 \\
\hline D10 IN MM (DIAMETER AT 10\% PASSING): & 0.00056 & 0.00061 & 0.00067 & 0.00055 & 0.00061 \\
\hline Cc: & 442.49 & 557.38 & 761.37 & 500.55 & 436.39 \\
\hline Cu: & 1.13 & 12.36 & 39.35 & 2.80 & 3.97 \\
\hline LIQUID LIMIT: & 34 & 32 & 36 & 36 & 34 \\
\hline PLASTIC INDEX: & 17 & 15 & 20 & 19 & 17 \\
\hline ATTERBERG CLASSIFICATION*: & $\mathrm{CL}$ & $\mathbf{C L}$ & $C L$ & $\mathrm{CL}$ & $\mathrm{CL}$ \\
\hline & SAMPLE 6 & SAMPLE 7 & SAMPLE 8 & SAMPLE 9 & SAMPLE 10 \\
\hline ETDC NUMBER: & ETDC-8014 & & & & \\
\hline CLIENT NUMBER: & L800000 & & & & \\
\hline $\begin{array}{l}\text { (COARSE-GRAVELS, SANDS) } \\
\% \text { RETAINED ON \# } 200 \text { SIEVE: }\end{array}$ & 65.4 & & & & \\
\hline $\begin{array}{l}\text { (FINE-SILTS, CLAYS) } \\
\% \text { PASSING \# } 200 \text { SIEVE: }\end{array}$ & 34.6 & & & & \\
\hline \% GRAVEL: & 0.7 & & & & \\
\hline \% SAND: & 64.8 & & & & \\
\hline D60 IN MM (DIAMETER AT 60\% PASSING): & 0.26137 & & & & \\
\hline D3O IN MM (DIAMETER AT 30\% PASSING): & 0.02933 & & & & \\
\hline D10 IN MM (DIAMETER AT 10\% PASSING): & 0.00058 & & & & \\
\hline Cc: & 448.40 & & & & \\
\hline Cu: & 5.65 & & & & \\
\hline IQUID LIMIT: & 33 & & & & \\
\hline LASTIC INDEX: & 16 & & & & \\
\hline ATTERBERG CLASSIFICATION*: & $C L$ & & & & \\
\hline
\end{tabular}




\section{PARTICLE-SIZE ANALYSIS ASTM D 422}

$\begin{array}{llll}\text { Project Name } & \text { SAIC - Savannah River } & \text { Client Sample No. } & \text { L100000 } \\ \text { Project No. } & 777304.00010500 & \text { IT Lab Sample No. } & \text { ETDC-8009 } \\ \text { Specific Gravity }=2.6370 & \begin{array}{l}\text { Moisture Content }=\quad 14.7 \% \\ \text { based on dry sample woight }\end{array}\end{array}$

SIEVE ANALYSIS

\begin{tabular}{|c|c|c|c|}
\hline & $\begin{array}{c}\text { Sieve } \\
\text { No. }\end{array}$ & $\begin{array}{c}\text { Diameter } \\
\mathrm{mm}\end{array}$ & $\begin{array}{c}\text { Percent } \\
\text { Finer }\end{array}$ \\
\cline { 2 - 4 } $\mathrm{O}$ & $3^{n}$ & 75.000 & $100.0 \%$ \\
$\mathrm{~A}$ & $1.5^{\prime \prime}$ & 37.500 & $100.0 \%$ \\
$\mathrm{R}$ & $0.75^{\prime \prime}$ & 19.000 & $100.0 \%$ \\
$\mathrm{~S}$ & $0.375^{\prime \prime}$ & 9.500 & $99.2 \%$ \\
$\mathrm{E}$ & $\# 4$ & 4.750 & $99.2 \%$ \\
\cline { 2 - 4 } & $\# 4$ & 2.000 & $98.3 \%$ \\
\hline
\end{tabular}

\begin{tabular}{|c|c|c|c|}
\hline & $\begin{array}{c}\text { Sieve } \\
\text { No. }\end{array}$ & $\begin{array}{c}\text { Diameter } \\
\mathrm{mm}\end{array}$ & $\begin{array}{c}\text { Percent } \\
\text { Finer }\end{array}$ \\
\cline { 2 - 4 } F & $\# 20$ & 0.850 & $93.5 \%$ \\
\cline { 2 - 4 } N & $\# 40$ & 0.425 & $78.3 \%$ \\
\cline { 2 - 4 } E & $\# 60$ & 0.250 & $60.4 \%$ \\
\cline { 2 - 4 } & $\# 100$ & 0.149 & $43.5 \%$ \\
\cline { 2 - 4 } & $\# 140$ & 0.106 & $37.4 \%$ \\
\hline & $\# 200$ & 0.075 & $34.2 \%$ \\
\hline
\end{tabular}

\section{HYDROMETER ANALYSIS}

\begin{tabular}{|c|c|c|}
\hline & $\begin{array}{c}\text { Diameter } \\
\mathrm{mm}\end{array}$ & $\begin{array}{c}\text { Percent } \\
\text { Finer }\end{array}$ \\
\hline$H$ & 0.06785 & $33.6 \%$ \\
\hline$Y$ & 0.04819 & $32.2 \%$ \\
\hline & 0.03422 & $31.5 \%$ \\
\hline$R$ & 0.02174 & $30.8 \%$ \\
\hline & 0.01260 & $30.1 \%$ \\
\hline & 0.00899 & $27.9 \%$ \\
\hline$T$ & 0.00641 & $26.5 \%$ \\
\hline & 0.00455 & $25.8 \%$ \\
$R$ & 0.00313 & $24.3 \%$ \\
\cline { 2 - 3 } & 0.00132 & $23.6 \%$ \\
\hline & & \\
\cline { 2 - 3 } & &
\end{tabular}




\section{SAIC - Savannah River}

U.S. STANDARD SIEVE SIZES

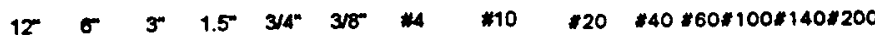

HYDROMETER

RoO SIEVE



CliENT SAMPLE NO: $\quad$ L100Q00
IT LAB SAMPLE NO.:

ETDC-8009

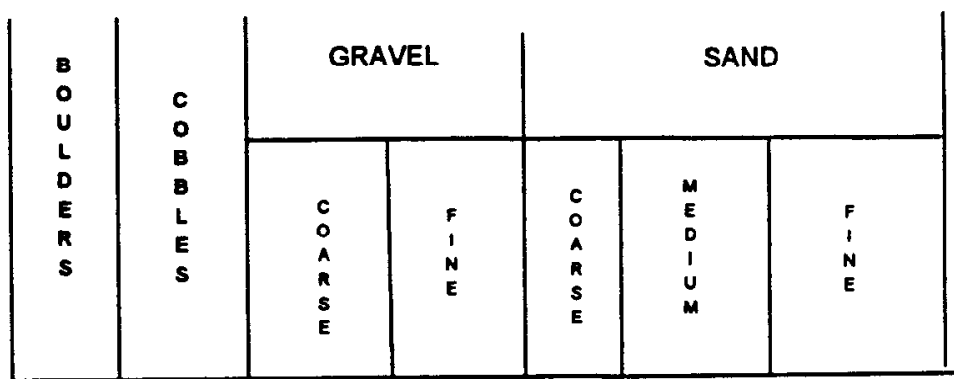

SILT 2 - 75 microns

CLAY $<2$ microns 


\section{PARTICLE-SIZE ANALYSIS ASTM D 422}

$\begin{array}{llll}\text { Project Name } & \text { SAIC - Savannah River } & \text { Client Sample No. } & \text { L200000 } \\ \text { Project No. } & 777304.00010500 & \text { IT Lab Sample No. } & \text { ETDC-8010 } \\ \text { Specific Gravity }= & 2.6255 & \begin{array}{l}\text { Moisture Content }= \\ \text { based on dry sample weight }\end{array}\end{array}$

SIEVE ANALYSIS

\begin{tabular}{|c|c|c|c||}
\hline & $\begin{array}{c}\text { Sieve } \\
\text { Co. }\end{array}$ & $\begin{array}{c}\text { Diameter } \\
\mathrm{mm}\end{array}$ & $\begin{array}{c}\text { Percent } \\
\text { Finer }\end{array}$ \\
\cline { 2 - 4 } O & $3^{\prime \prime}$ & 75.000 & $100.0 \%$ \\
\hline R & $1.5^{n}$ & 37.500 & $100.0 \%$ \\
\cline { 2 - 4 } S & $0.75^{n}$ & 19.000 & $100.0 \%$ \\
\cline { 2 - 4 } E & $0.375^{n}$ & 9.500 & $100.0 \%$ \\
\cline { 2 - 4 } & $\# 4$ & 4.750 & $99.8 \%$ \\
\hline & $\# 10$ & 2.000 & $98.8 \%$ \\
\hline
\end{tabular}

\begin{tabular}{|c|c|c|c|}
\hline & $\begin{array}{c}\text { Sieve } \\
\text { No. }\end{array}$ & $\begin{array}{c}\text { Diameter } \\
\mathrm{mm}\end{array}$ & $\begin{array}{c}\text { Percent } \\
\text { Finer }\end{array}$ \\
\cline { 2 - 4 } $\mathrm{F}$ & $\# 20$ & 0.850 & $91.5 \%$ \\
\hline $\mathrm{N}$ & $\# 40$ & 0.425 & $69.0 \%$ \\
\hline \multirow{4}{*}{$\mathrm{E}$} & $\# 60$ & 0.250 & $50.1 \%$ \\
\cline { 2 - 4 } & $\# 100$ & 0.149 & $38.8 \%$ \\
\hline & $\# 140$ & 0.106 & $34.5 \%$ \\
\hline & $\# 200$ & 0.075 & $32.0 \%$ \\
\hline
\end{tabular}

HYDROMETER ANALYSIS

\begin{tabular}{|c|c|c|}
\hline \multirow{11}{*}{$\begin{array}{l}H \\
Y \\
\text { D } \\
R \\
O \\
M \\
E \\
T \\
E \\
R\end{array}$} & $\begin{array}{c}\text { Diameter } \\
\mathrm{mm}\end{array}$ & $\begin{array}{c}\text { Percent } \\
\text { Finer } \\
\end{array}$ \\
\hline & 0.06815 & $30.5 \%$ \\
\hline & 0.04840 & $29.8 \%$ \\
\hline & 0.03437 & $29.1 \%$ \\
\hline & 0.02183 & $28.4 \%$ \\
\hline & 0.01268 & $27.1 \%$ \\
\hline & 0.00899 & $26.4 \%$ \\
\hline & 0.00643 & $24.4 \%$ \\
\hline & 0.00456 & $23.0 \%$ \\
\hline & 0.00310 & $22.3 \%$ \\
\hline & 0.00133 & $21.7 \%$ \\
\hline
\end{tabular}




\section{SAIC - Savannah River}

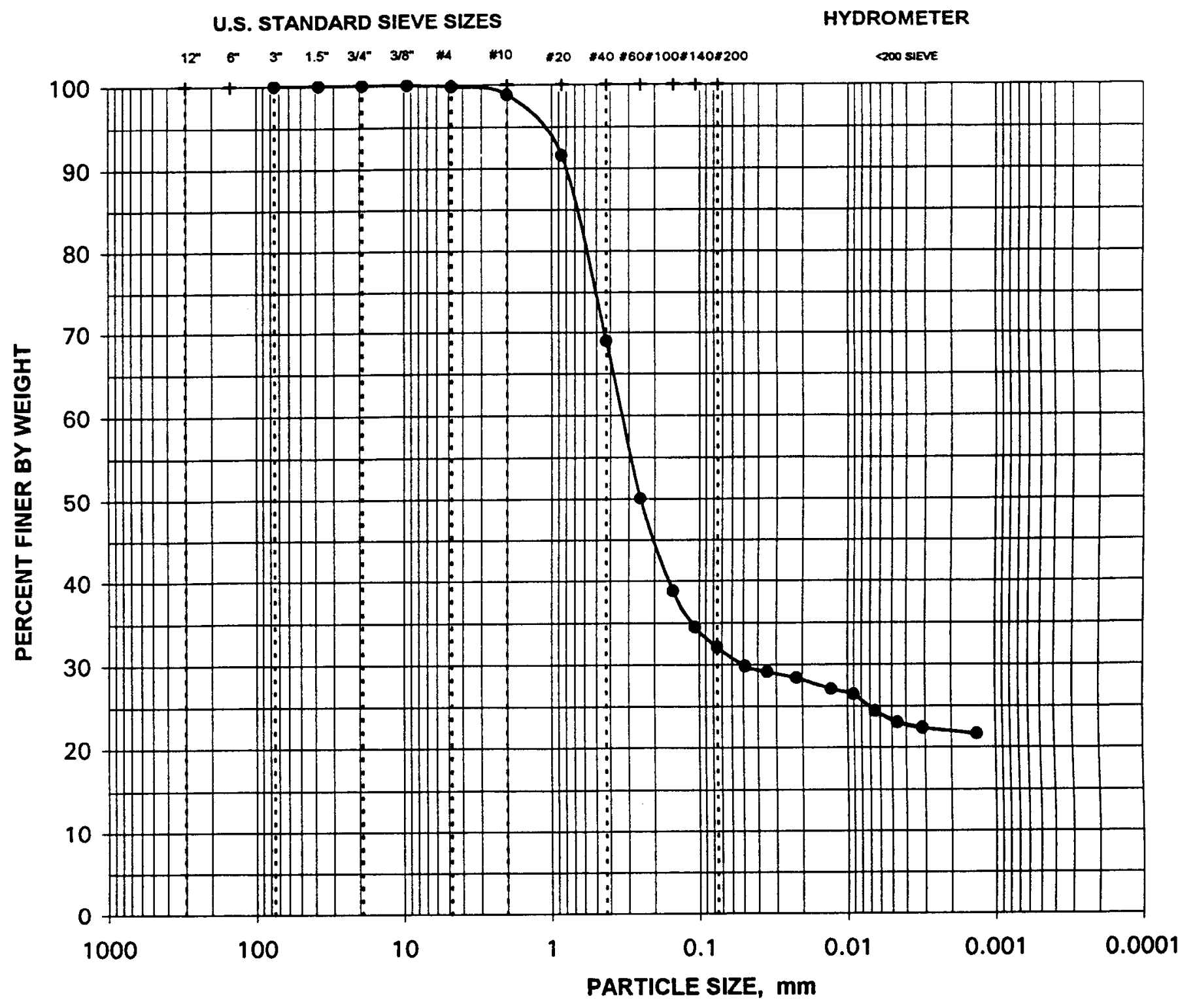

CLIENT SAMPLE NO:: L200Q00

IT LAB SAMPLE NO.:

ETDC-8010

\begin{tabular}{|c|c|c|c|c|c|c|c|}
\hline \multirow{2}{*}{$\begin{array}{l}B \\
O \\
U \\
L \\
D \\
E \\
R \\
S\end{array}$} & \multirow{2}{*}{$\begin{array}{l}C \\
0 \\
B \\
B \\
L \\
E \\
S\end{array}$} & \multicolumn{2}{|c|}{ GRAVEL } & \multicolumn{3}{|c|}{ SAND } & SILT 2 - 75 microns \\
\hline & & $\begin{array}{l}\mathrm{C} \\
0 \\
\hat{R} \\
\mathbf{S} \\
\mathrm{E}\end{array}$ & $\begin{array}{l}F \\
1 \\
\text { N }\end{array}$ & $\begin{array}{l}\mathbf{C} \\
\hat{O} \\
\hat{R} \\
\mathbf{S} \\
\mathbf{E}\end{array}$ & $\begin{array}{l}\mathbf{M} \\
\mathbf{E} \\
\mathbf{D} \\
\mathbf{U} \\
\mathbf{M}\end{array}$ & $\begin{array}{l}\mathbf{F} \\
\vdots \\
\mathbf{N} \\
\mathbf{E}\end{array}$ & CLAY <2 microns \\
\hline
\end{tabular}




\begin{tabular}{|c|c|c|c|}
\hline Project Name & SAIC - Savannah River & Client Sample No. & L300Q00 \\
\hline Project No. & 777304.00010500 & IT Lab Sample No. & ETDC-8011 \\
\hline Specific Gravity & 2.6279 & $\begin{array}{l}\text { Moisture Content }= \\
\text { based on dry sample w }\end{array}$ & $15.1 \%$ \\
\hline
\end{tabular}

SIEVE ANALYSIS

\begin{tabular}{|c|c|c|c|}
\hline & $\begin{array}{c}\text { Sieve } \\
\text { No. }\end{array}$ & $\begin{array}{c}\text { Diameter } \\
\mathrm{mm}\end{array}$ & $\begin{array}{c}\text { Percent } \\
\text { Finer }\end{array}$ \\
$\mathrm{C}$ & $3^{n}$ & 75.000 & $100.0 \%$ \\
$\mathrm{~A}$ & $1.5^{n}$ & 37.500 & $100.0 \%$ \\
$\mathrm{R}$ & $0.75^{n}$ & 19.000 & $100.0 \%$ \\
$\mathrm{~S}$ & $0.375^{n}$ & 9.500 & $100.0 \%$ \\
$\mathrm{E}$ & $\# 4$ & 4.750 & $99.6 \%$ \\
\cline { 2 - 4 } & $\# 4$ & 2.000 & $98.5 \%$ \\
\hline
\end{tabular}

\begin{tabular}{|c|c|c|c|}
\hline & $\begin{array}{c}\text { Sieve } \\
\text { No. }\end{array}$ & $\begin{array}{c}\text { Diameter } \\
\mathrm{mm}\end{array}$ & $\begin{array}{c}\text { Percent } \\
\text { Finer }\end{array}$ \\
\cline { 2 - 4 } F & $\# 20$ & 0.850 & $83.4 \%$ \\
\hline N & $\# 40$ & 0.425 & $54.4 \%$ \\
\cline { 2 - 4 } E & $\# 60$ & 0.250 & $40.7 \%$ \\
\cline { 2 - 4 } & $\# 100$ & 0.149 & $32.6 \%$ \\
\cline { 2 - 4 } & $\# 140$ & 0.106 & $29.3 \%$ \\
\hline & $\# 200$ & 0.075 & $27.4 \%$ \\
\hline
\end{tabular}

HYDROMETER ANALYSIS

\begin{tabular}{|c|c|c|}
\hline & $\begin{array}{c}\text { Diameter } \\
\mathrm{mm}\end{array}$ & $\begin{array}{c}\text { Percent } \\
\text { Finer }\end{array}$ \\
\hline $\mathrm{H}$ & & \\
\cline { 2 - 3 } $\mathrm{D}$ & 0.04922 & $26.2 \%$ \\
$\mathrm{R}$ & 0.03495 & $25.5 \%$ \\
\hline O & 0.02220 & $24.8 \%$ \\
\hline M & 0.01287 & $24.1 \%$ \\
\hline T & 0.00917 & $22.1 \%$ \\
\hline E & 0.00651 & $21.5 \%$ \\
\hline$R$ & 0.00462 & $20.8 \%$ \\
\hline & 0.00314 & $20.1 \%$ \\
\hline & 0.00134 & $20.1 \%$ \\
\hline
\end{tabular}


HYDROMETER

Q00 SIEVE

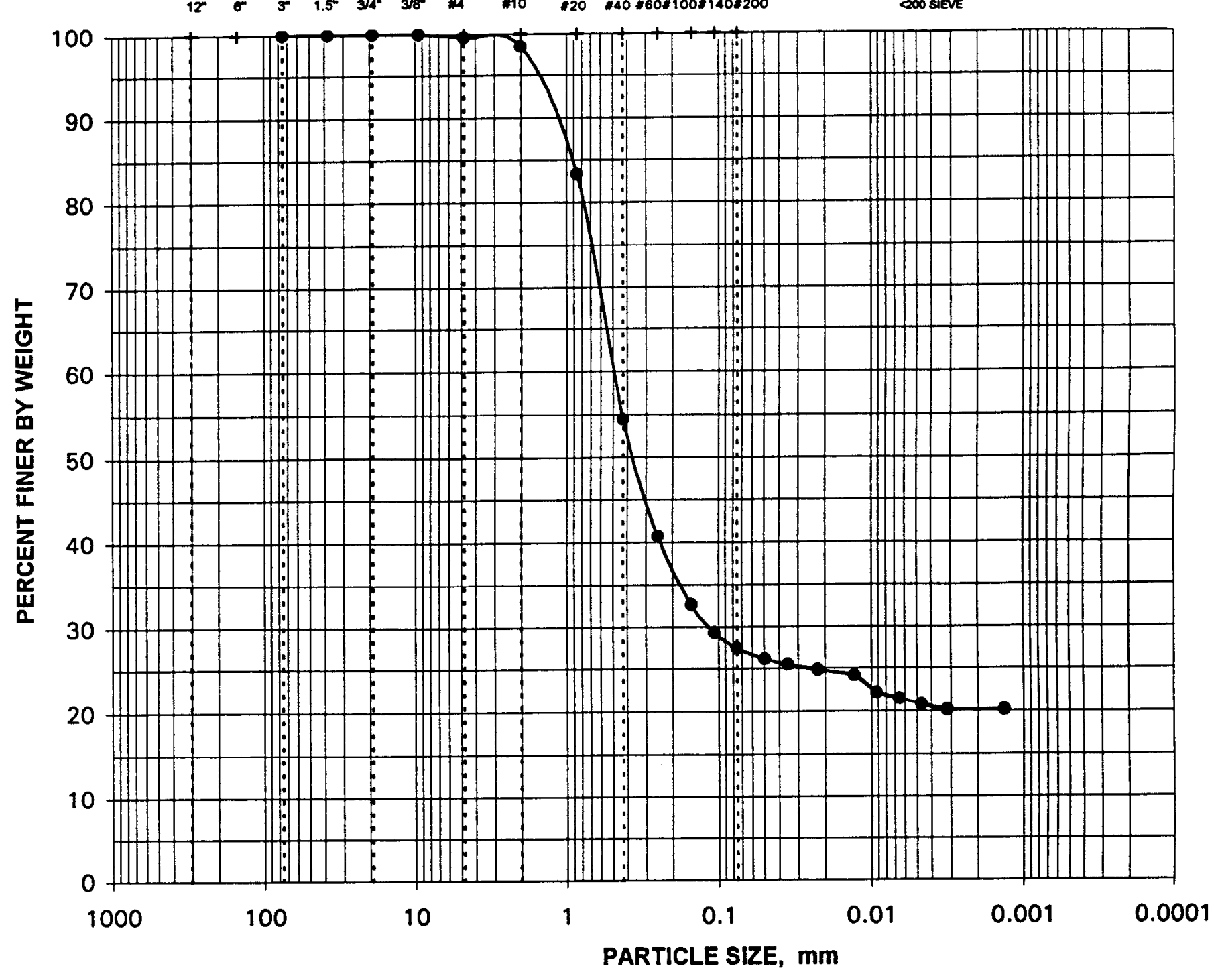

CLIENT SAMPLE NO.: $\quad$ L300Q00

IT LAB SAMPLE NO.:

ETDC-8011

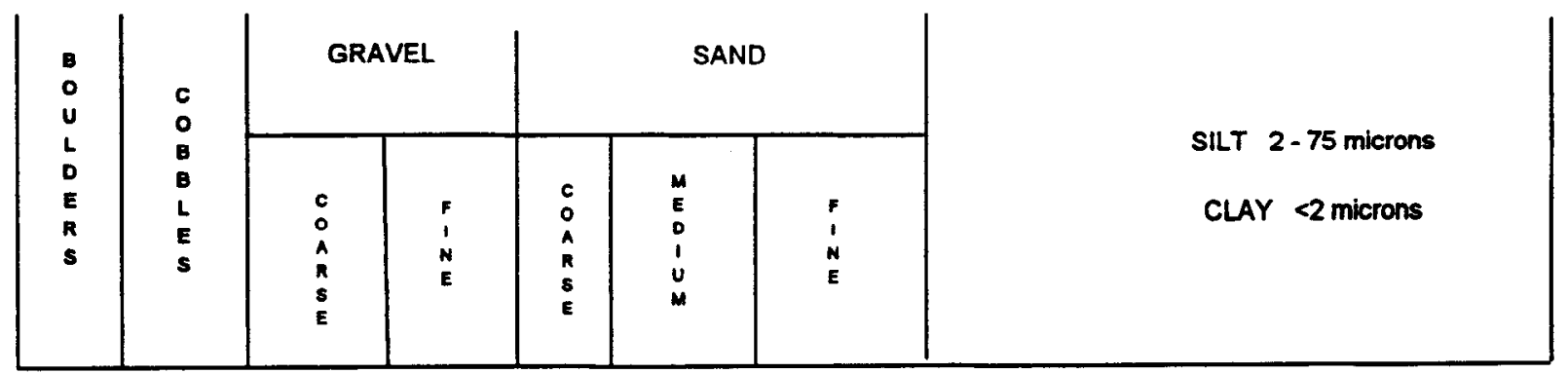




\section{PARTICLE-SIZE ANALYSIS \\ ASTM D 422}

\begin{tabular}{|c|c|c|c|}
\hline Project Name & SAIC - Savannah River & Client Sample No. & L400000 \\
\hline Project No. & 777304.00010500 & IT Lab Sample No. & ETDC-8012 \\
\hline Specific Gravity $=$ & 2.6335 & $\begin{array}{l}\text { Moisture Content = } \\
\text { besod on dry sample weight }\end{array}$ & $15.9 \%$ \\
\hline
\end{tabular}

SIEVE ANALYSIS

\begin{tabular}{|c|c|c|c|}
\hline & $\begin{array}{c}\text { Sieve } \\
\text { No. }\end{array}$ & $\begin{array}{c}\text { Diameter } \\
\mathrm{mm}\end{array}$ & $\begin{array}{c}\text { Percent } \\
\text { Finer }\end{array}$ \\
\cline { 2 - 4 } $\mathrm{O}$ & $3^{n}$ & 75.000 & $100.0 \%$ \\
$\mathrm{~A}$ & $1.5^{\prime \prime}$ & 37.500 & $100.0 \%$ \\
$\mathrm{R}$ & $0.75^{\prime \prime}$ & 19.000 & $100.0 \%$ \\
$\mathrm{~S}$ & $0.375^{\prime \prime}$ & 9.500 & $100.0 \%$ \\
$\mathrm{E}$ & $\# 4$ & 4.750 & $99.6 \%$ \\
\cline { 2 - 4 } & $\# 4$ & 2.000 & $98.3 \%$ \\
\hline
\end{tabular}

\begin{tabular}{|c|c|c|c|}
\hline & $\begin{array}{c}\text { Sieve } \\
\text { No. }\end{array}$ & $\begin{array}{c}\text { Diameter } \\
\mathrm{mm}\end{array}$ & $\begin{array}{c}\text { Percent } \\
\text { Finer }\end{array}$ \\
\cline { 2 - 4 } $\mathrm{F}$ & $\# 20$ & 0.850 & $91.3 \%$ \\
\hline $\mathrm{N}$ & $\# 40$ & 0.425 & $75.1 \%$ \\
\cline { 2 - 4 } $\mathrm{E}$ & $\# 60$ & 0.250 & $57.5 \%$ \\
\cline { 2 - 4 } & $\# 100$ & 0.149 & $41.9 \%$ \\
\cline { 2 - 4 } & $\# 140$ & 0.106 & $36.7 \%$ \\
\hline & $\# 200$ & 0.075 & $33.8 \%$ \\
\hline
\end{tabular}

HYDROMETER ANALYSIS

\begin{tabular}{|c|c|c|}
\hline & $\begin{array}{c}\text { Diameter } \\
\mathrm{mm}\end{array}$ & $\begin{array}{c}\text { Percent } \\
\text { Finer }\end{array}$ \\
\hline$H$ & & \\
\cline { 2 - 3 } & & $32.2 \%$ \\
\hline$R$ & 0.04798 & $30.9 \%$ \\
\hline O & 0.03407 & $30.2 \%$ \\
\hline M & 0.02164 & $28.8 \%$ \\
\hline T & 0.01260 & $28.1 \%$ \\
\hline E & 0.00895 & $27.4 \%$ \\
\hline$R$ & 0.00634 & $25.4 \%$ \\
\hline & 0.00453 & $24.0 \%$ \\
\hline & 0.00307 & $24.0 \%$ \\
\hline
\end{tabular}




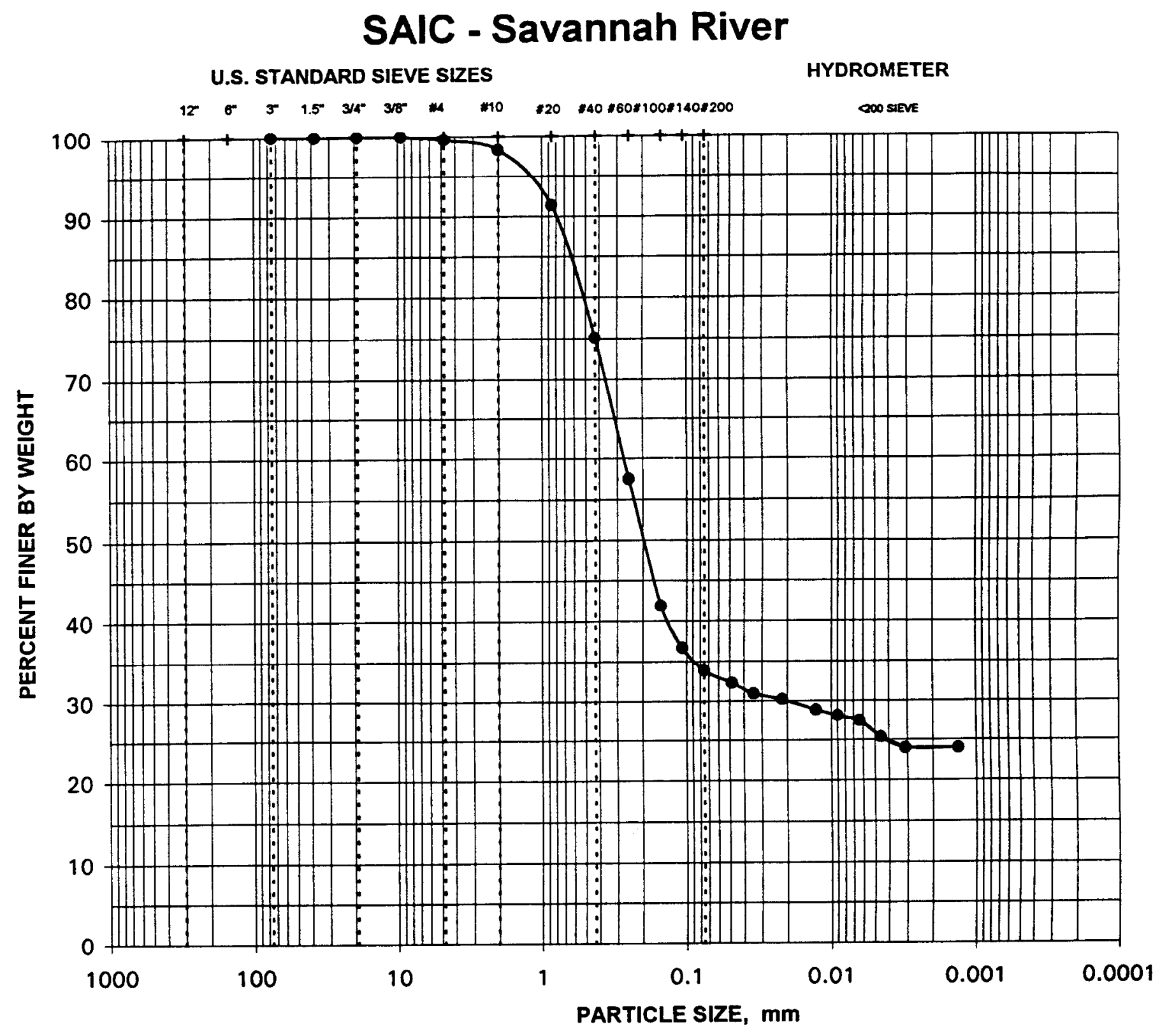

CLIENT SAMPLE NO:: $\quad$ L400Q00

IT LAB SAMPLE NO.:

ETDC-8012

\begin{tabular}{|c|c|c|c|c|c|c|c|}
\hline \multirow{2}{*}{$\begin{array}{l}B \\
0 \\
U \\
L \\
D \\
E \\
R \\
S\end{array}$} & \multirow{2}{*}{$\begin{array}{l}\text { C } \\
\text { D } \\
B \\
B \\
L \\
\text { E } \\
\text { S }\end{array}$} & \multicolumn{2}{|c|}{ GRAVEL } & \multicolumn{3}{|c|}{ SAND } & \multirow{2}{*}{$\begin{array}{l}\text { SILT } 2 \text { - } 75 \text { microns } \\
\text { CLAY }<2 \text { microns }\end{array}$} \\
\hline & & $\begin{array}{l}c \\
0 \\
\hat{A} \\
\mathbf{S} \\
\mathbf{E}\end{array}$ & $\begin{array}{l}\text { F } \\
\text { N } \\
\text { E }\end{array}$ & $\begin{array}{l}\mathrm{C} \\
0 \\
\hat{R} \\
\mathbf{R} \\
\mathrm{E}\end{array}$ & $\begin{array}{l}M \\
E \\
D \\
I \\
U \\
M\end{array}$ & $\begin{array}{l}F \\
i \\
N \\
E\end{array}$ & \\
\hline
\end{tabular}




\section{PARTICLE-SIZE ANALYSIS \\ ASTM D 422}

$\begin{array}{llll}\text { Project Name } & \text { SAIC }- \text { Savannah River } & \text { Client Sample No. } & \text { L700000 } \\ \text { Project No. } & 777304.00010500 & \text { IT Lab Sample No. } & \text { ETDC-8013 } \\ \text { Specific Gravity }=2.6289 & \begin{array}{l}\text { Moisture Content }= \\ \text { based on dry sample woight }\end{array}\end{array}$

SIEVE ANALYSIS

\begin{tabular}{|c|c|c|c|}
\hline & $\begin{array}{c}\text { Sieve } \\
\text { No. }\end{array}$ & $\begin{array}{c}\text { Diameter } \\
\mathrm{mm}\end{array}$ & $\begin{array}{c}\text { Percent } \\
\text { Finer }\end{array}$ \\
\cline { 2 - 4 } $\mathrm{O}$ & $3^{\prime \prime}$ & 75.000 & $100.0 \%$ \\
\cline { 2 - 4 } $\mathrm{A}$ & $1.5^{\prime \prime}$ & 37.500 & $100.0 \%$ \\
\cline { 2 - 4 } S & $0.75^{n}$ & 19.000 & $100.0 \%$ \\
\cline { 2 - 4 } E & $0.375^{\prime \prime}$ & 9.500 & $100.0 \%$ \\
\cline { 2 - 4 } & $\# 4$ & 4.750 & $99.5 \%$ \\
\hline & $\# 10$ & 2.000 & $98.6 \%$ \\
\hline
\end{tabular}

\begin{tabular}{|c|c|c|c|}
\hline \multirow{4}{*}{ F } & $\begin{array}{c}\text { Sieve } \\
\text { No. }\end{array}$ & $\begin{array}{c}\text { Diameter } \\
\mathrm{mm}\end{array}$ & $\begin{array}{c}\text { Percent } \\
\text { Finer }\end{array}$ \\
\cline { 2 - 4 } I & $\# 20$ & 0.850 & $92.2 \%$ \\
\hline N & $\# 40$ & 0.425 & $76.2 \%$ \\
\hline E & $\# 60$ & 0.250 & $58.5 \%$ \\
\cline { 2 - 4 } & $\# 100$ & 0.149 & $42.4 \%$ \\
\hline & $\# 140$ & 0.106 & $36.9 \%$ \\
\hline 200 & 0.075 & $34.0 \%$ \\
\hline
\end{tabular}

HYDROMETER ANALYSIS

\begin{tabular}{|c|c|c|}
\hline \multirow[b]{2}{*}{$\mathrm{H}$} & $\begin{array}{c}\text { Diameter } \\
\mathrm{mm}\end{array}$ & $\begin{array}{l}\text { Percent } \\
\text { Finer }\end{array}$ \\
\hline & & \\
\hline \multirow{2}{*}{$\begin{array}{l}Y \\
D \\
R\end{array}$} & 0.04799 & $31.2 \%$ \\
\hline & 0.03408 & $30.5 \%$ \\
\hline 0 & 0.02164 & $29.8 \%$ \\
\hline \multirow{6}{*}{$\begin{array}{c}M \\
\text { E } \\
T \\
E \\
R\end{array}$} & 0.01240 & $28.3 \%$ \\
\hline & 0.00887 & $26.2 \%$ \\
\hline & 0.00630 & $25.4 \%$ \\
\hline & 0.00442 & $24.0 \%$ \\
\hline & 0.00311 & $23.2 \%$ \\
\hline & 0.00132 & $21.8 \%$ \\
\hline
\end{tabular}


U.S. STANDARD SIEVE SIZES

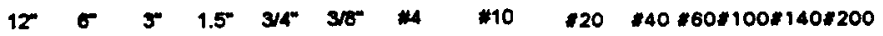

HYDROMETER

Q00 SIEve



CLIENT SAMPLE NO.: $\quad$ L700Q00

IT LAB SAMPLE NO.:

ETDC-8013

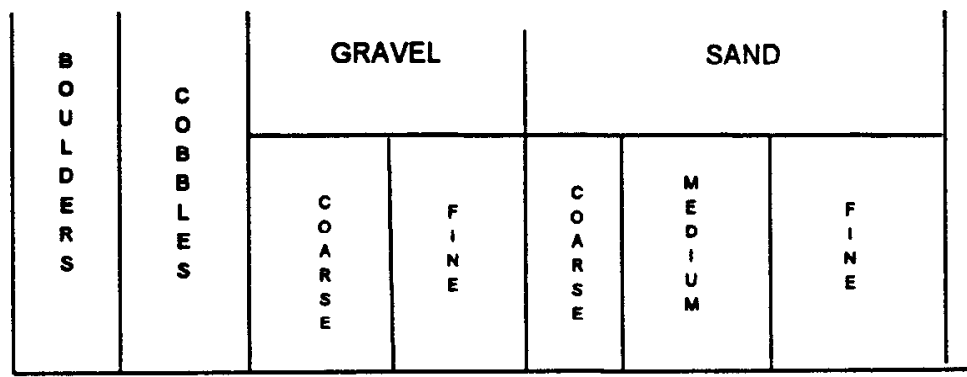

SILT 2 - 75 microns

CLAY $<2$ microns 


\section{PARTICLE-SIZE ANALYSIS \\ ASTM D 422}

$\begin{array}{llll}\text { Project Name } & \text { SAIC - Savannah River } & \text { Client Sample No. } & \text { L800000 } \\ \text { Project No. } & 777304.00010500 & \text { IT Lab Sample No. } & \text { ETDC-8014 } \\ \text { Specific Gravity }=2.6336 & \begin{array}{l}\text { Moisture Content }=\quad 14.9 \% \\ \text { based on dry sample woight }\end{array}\end{array}$

SIEVE ANALYSIS

\begin{tabular}{|c|c|c|c|}
\hline & $\begin{array}{c}\text { Sieve } \\
\text { No. }\end{array}$ & $\begin{array}{c}\text { Diameter } \\
\mathrm{mm}\end{array}$ & $\begin{array}{c}\text { Percent } \\
\text { Finer }\end{array}$ \\
\cline { 2 - 4 } $\mathrm{O}$ & $3^{n}$ & 75.000 & $100.0 \%$ \\
$\mathrm{~A}$ & $1.5^{\prime \prime}$ & 37.500 & $100.0 \%$ \\
$\mathrm{R}$ & $0.75^{n}$ & 19.000 & $100.0 \%$ \\
$\mathrm{~S}$ & $0.375^{\prime \prime}$ & 9.500 & $99.5 \%$ \\
$\mathrm{E}$ & $\# 4$ & 4.750 & $99.3 \%$ \\
\hline & $\# 10$ & 2.000 & $98.4 \%$ \\
\hline
\end{tabular}

\begin{tabular}{|c|c|c|c|}
\hline & $\begin{array}{c}\text { Sieve } \\
\text { No. }\end{array}$ & $\begin{array}{c}\text { Diameter } \\
\mathrm{mm}\end{array}$ & $\begin{array}{c}\text { Percent } \\
\text { Finer }\end{array}$ \\
\cline { 2 - 4 } F & $\# 20$ & 0.850 & $93.0 \%$ \\
\hline N & $\# 40$ & 0.425 & $76.9 \%$ \\
\hline \multirow{4}{*}{ E } & $\# 60$ & 0.250 & $58.8 \%$ \\
\cline { 2 - 4 } & $\# 100$ & 0.149 & $43.0 \%$ \\
\cline { 2 - 4 } & $\# 140$ & 0.106 & $37.5 \%$ \\
\hline & $\# 200$ & 0.075 & $34.6 \%$ \\
\hline
\end{tabular}

HYDROMETER ANALYSIS

\begin{tabular}{|c|c|c|}
\hline & $\begin{array}{c}\text { Diameter } \\
\mathrm{mm}\end{array}$ & $\begin{array}{c}\text { Percent } \\
\text { Finer }\end{array}$ \\
\cline { 2 - 3 } $\mathrm{H}$ & & \\
\hline $\mathrm{Y}$ & & $31.0 \%$ \\
\hline$R$ & 0.04799 & $30.3 \%$ \\
\hline & 0.03367 & $29.5 \%$ \\
\hline M & 0.02138 & $28.8 \%$ \\
\hline E & 0.01237 & $27.4 \%$ \\
\hline T & 0.00880 & $25.9 \%$ \\
\hline R & 0.00628 & $24.5 \%$ \\
\cline { 2 - 3 } & 0.00445 & $23.8 \%$ \\
\cline { 2 - 3 } & 0.00310 & $22.3 \%$ \\
\hline
\end{tabular}




\section{SAIC - Savannah River}

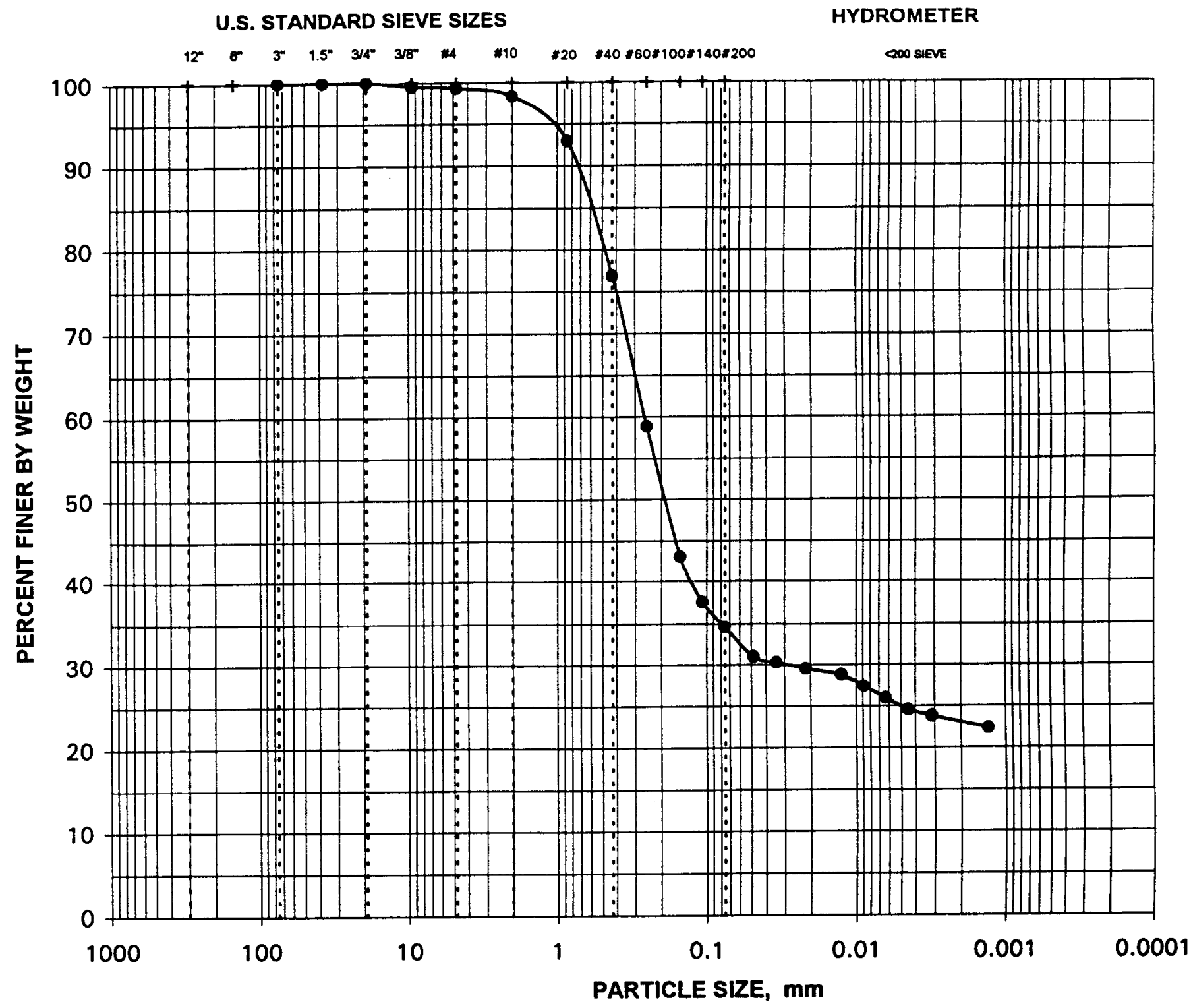

CLIENT SAMPLE NO:: L800Q00

IT LAB SAMPLE NO.:

ETDC-8014

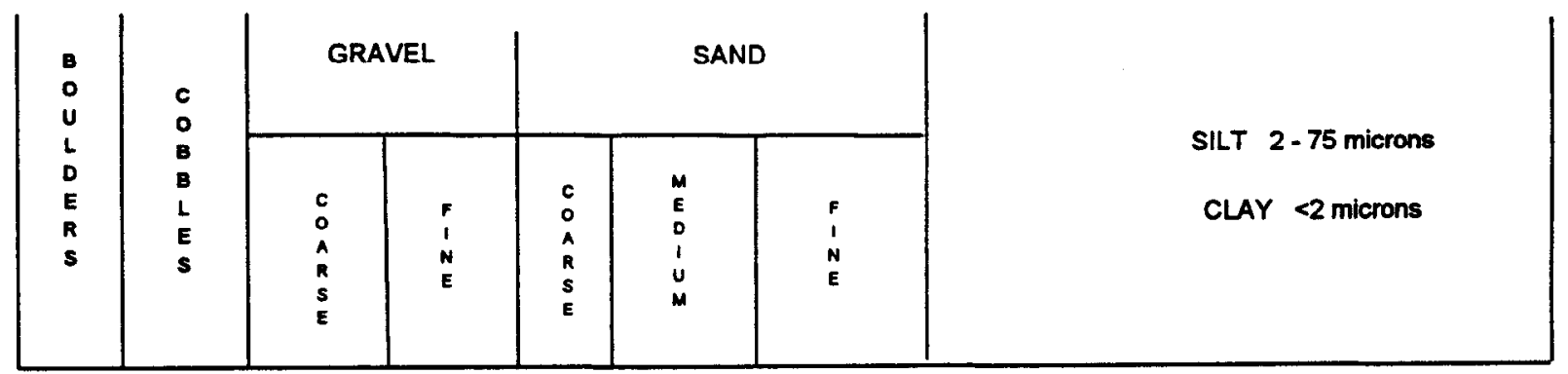




\section{Series \\ 28 Day UCS Results}




\section{UNCONFINED COMPRESSIVE STRENGTH - ASTM D 2166 28-DAY CURE}

Project Name SAIC - Savannah River

Project No. $\quad 777304.00010500$

Specimen Mold Date

Specimen Test Date

STRESS AT FAILURE, psi

STRAIN AT FAILURE, \%

\begin{tabular}{ccc}
$\begin{array}{c}\text { AXIAL } \\
\text { STRAIN, \% }\end{array}$ & & $\begin{array}{c}\text { DEVIATOR } \\
\text { STRESS, psi }\end{array}$ \\
\cline { 1 - 1 } 0.00 & & 0.0 \\
0.10 & & 2.5 \\
0.20 & & 6.3 \\
0.30 & & 13.8 \\
0.40 & & 23.7 \\
0.50 & & 37.1 \\
0.75 & & 80.0 \\
1.01 & & 141.5 \\
1.26 & & 205.9 \\
1.51 & & 265.9 \\
1.76 & & 329.9 \\
2.01 & & 379.7 \\
2.26 & & 413.7 \\
2.51 & 433.1 \\
2.76 & 441.4 \\
3.02 & 453.4 \\
3.27 & 466.8 \\
3.52 & 471.3 \\
3.77 & 457.4 \\
4.02 & 316.0 \\
4.27 & 75.3
\end{tabular}

Client Sample No. L312F0O

IT Lab Specimen No. ETDC-8063

Specimen Height, in.

3.9783

Specimen Diameter, in.

2.0152

Specimen Weight, $g$.

376.72

Moisture Content, \%

29.3

Wet Unit Weight, pcf.

113.1

Dry Unit Weight, pcf.

87.5

Rate of Strain, in./min.

0.0400

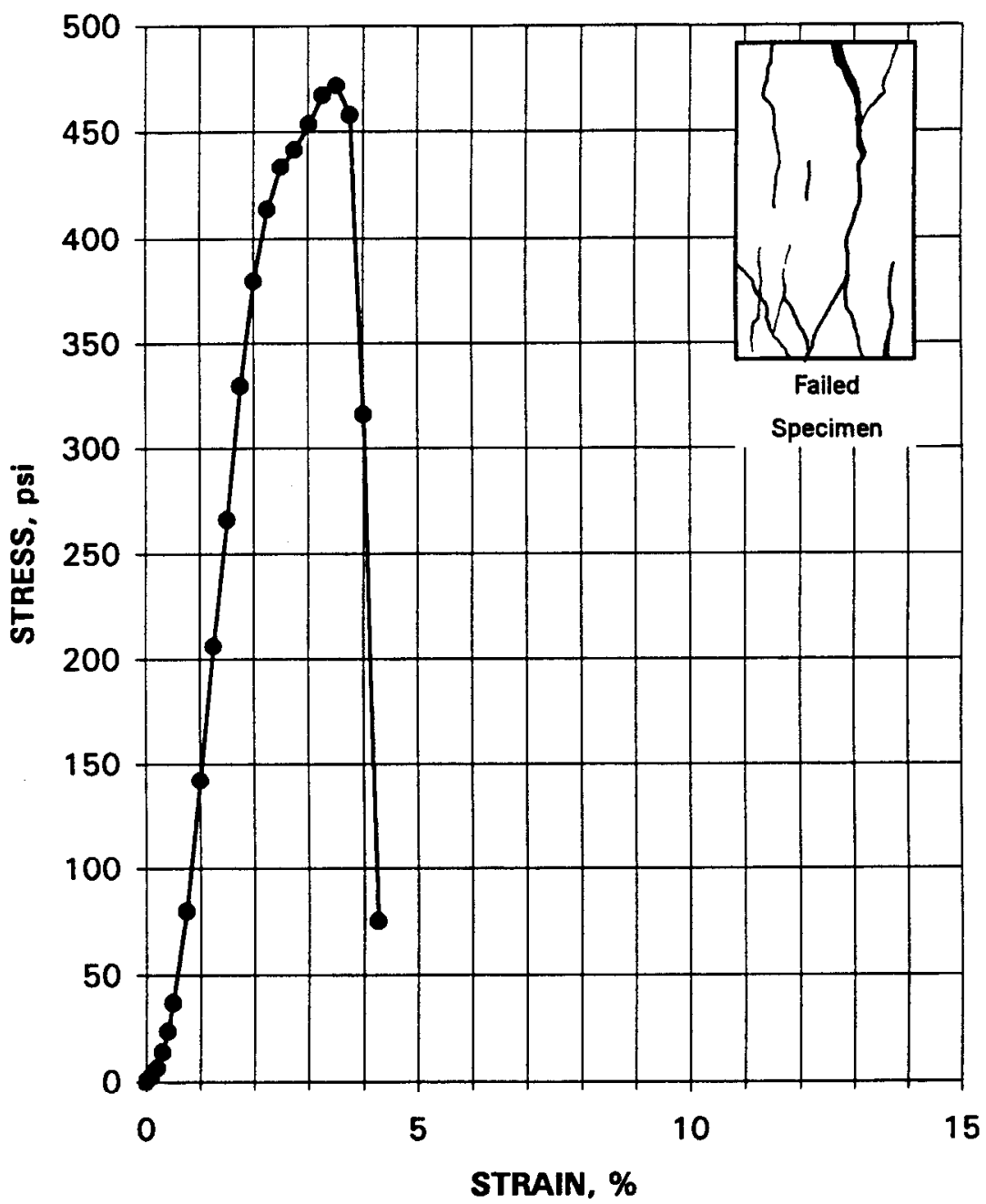




\section{UNCONFINED COMPRESSIVE STRENGTH - ASTM D 2166 28-DAY CURE}

$\begin{array}{ll}\text { Project Name } & \text { SAIC - Savannah River } \\ \text { Project No. } & 777304.00010500\end{array}$

Specimen Mold Date

Specimen Test Date

STRESS AT FAILURE, psi

STRAIN AT FAILURE, \%

AXIAL DEVIATOR

$\frac{\text { STRAIN, \% }}{0.00} \frac{\text { STRESS, psi }}{0.0}$

$0.10 \quad 2.8$

$0.20 \quad 8.2$

$0.30 \quad 17.6$

$0.40 \quad 33.0$

$0.50 \quad 55.6$

$0.75 \quad 147.6$

$1.00 \quad 228.2$

$1.25 \quad 307.4$

$1.50 \quad 406.5$

$1.75 \quad 474.9$

$2.00 \quad 545.8$

$2.25 \quad 615.1$

$2.51 \quad 639.6$

$2.76 \quad 600.2$

$3.01 \quad 578.2$

$3.26 \quad 569.3$

$3.51 \quad 550.8$

$3.76 \quad 499.2$

$4.01 \quad 436.7$

$4.26 \quad 392.4$
$1 / 20 / 99$

639.6

2.5
Client Sample No. L322F00

IT Lab Specimen No. ETDC-8064

Specimen Height, in.

3.9915

Specimen Diameter, in.

2.0082

Specimen Weight, $g$.

390.43

Moisture Content, \%

27.1

Wet Unit Weight, pcf.

117.7

Dry Unit Weight, pcf.

92.6

Rate of Strain, in./min.

0.0400

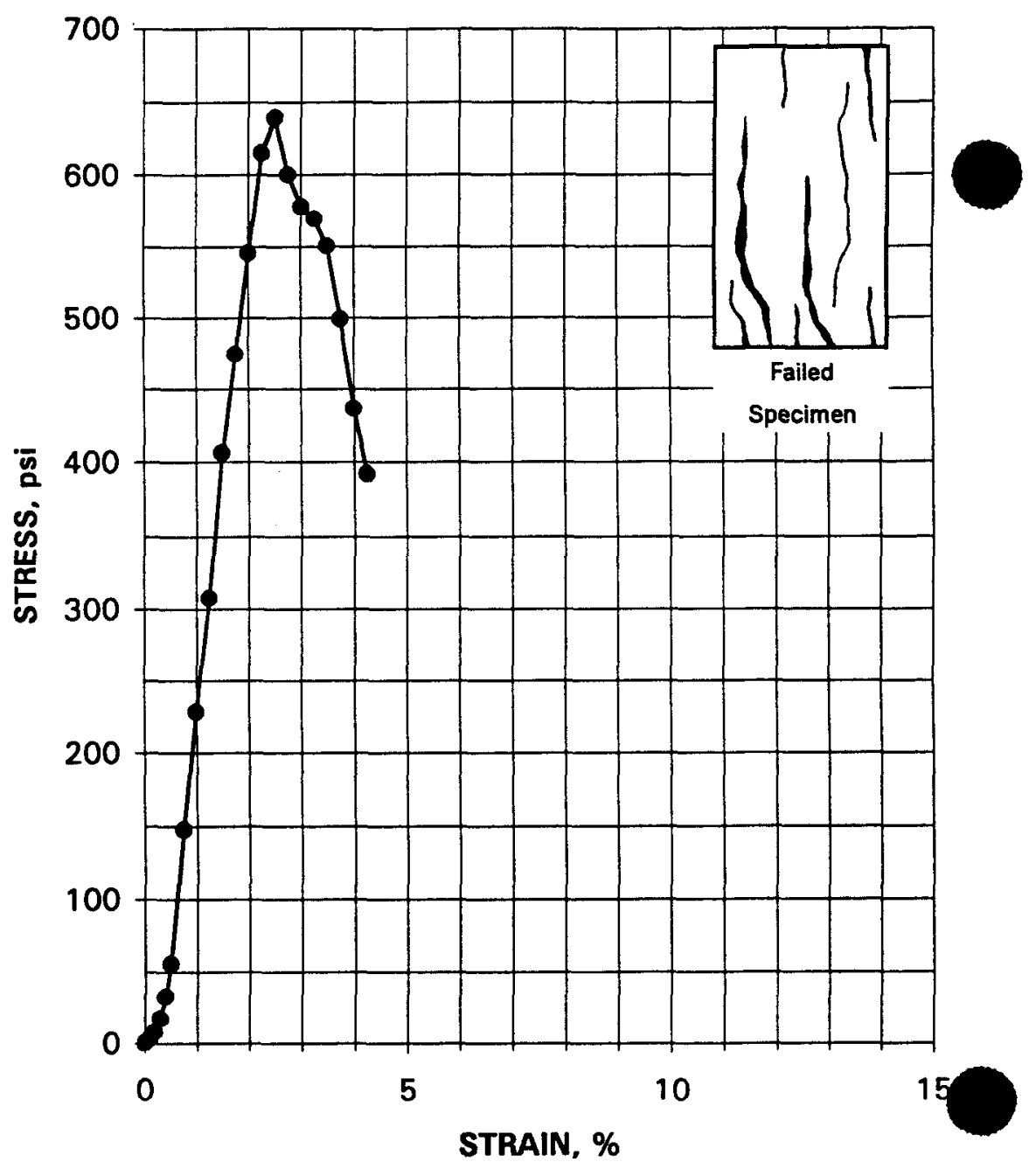




\section{UNCONFINED COMPRESSIVE STRENGTH - ASTM D 2166 28-DAY CURE}

Project Name SAIC - Savannah River

Project No. 777304.00010500

Specimen Mold Date

Specimen Test Date

STRESS AT FAILURE, psi

STRAIN AT FAILURE, \%

AXIAL DEVIATOR

\begin{tabular}{ccc} 
STRAIN, \% & STRESS, psi \\
\cline { 1 - 1 } 0.00 & & 0.0 \\
0.10 & & 3.5 \\
0.20 & & 14.0 \\
0.30 & & 33.6 \\
0.40 & & 62.0 \\
0.50 & & 96.4 \\
0.75 & & 205.0 \\
1.00 & & 307.4 \\
1.25 & & 407.0 \\
1.50 & & 521.5 \\
1.75 & & 607.6 \\
2.00 & & 154.5 \\
2.25 & & 99.1
\end{tabular}

Client Sample No. L332F00

IT Lab Specimen No. ETDC-8065

Specimen Height, in.

4.0012

Specimen Diameter, in.

2.0017

Specimen Weight, $g$.

369.41

Moisture Content, \%

Wet Unit Weight, pef.

111.8

Dry Unit Weight, pcf.

83.9

Rate of Strain, in./min.

0.0400

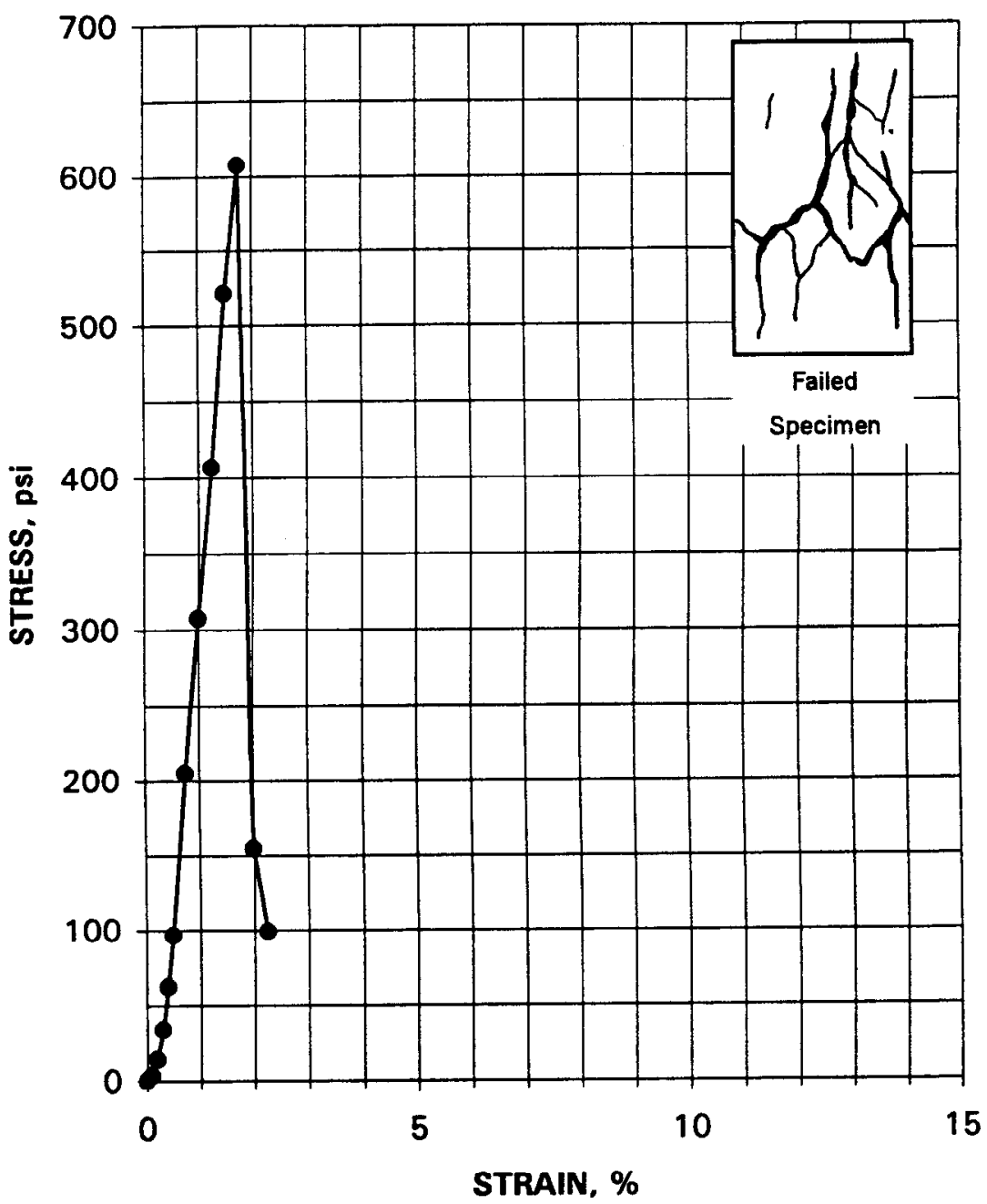




\section{UNCONFINED COMPRESSIVE STRENGTH - ASTM D 2166 28-DAY CURE}

Project Name SAIC - Savannah River

Project No. $\quad 777304.00010500$

Specimen Mold Date

Specimen Test Date

STRESS AT FAILURE, psi

STRAIN AT FAILURE, \%

AXIAL DEVIATOR

$\frac{\text { STRAIN, \% }}{0.00} \frac{\text { STRESS, psi }}{0.0}$

$0.10 \quad 4.1$

$0.20 \quad 10.4$

$0.30 \quad 20.5$

$0.40 \quad 44.5$

$0.50 \quad 73.1$

$0.75 \quad 157.2$

$1.00 \quad 233.9$

$1.24 \quad 310.5$

$1.49 \quad 394.9$

$1.74 \quad 455.2$

$1.99 \quad 496.0$

$2.24 \quad 509.6$

$2.49 \quad 330.1$

$2.74 \quad 314.5$

$2.99 \quad 188.9$
$1 / 20 / 99$

509.6

2.2
Client Sample No. L342FOO

IT Lab Specimen No. ETDC-8066

Specimen Height, in.

4.0193

Specimen Diameter, in.

2.0052

Specimen Weight, $g$.

373.56

Moisture Content, \%

32.0

Wet Unit Weight, pef.

112.1

Dry Unit Weight, pcf.

84.9

Rate of Strain, in./min.

0.0400

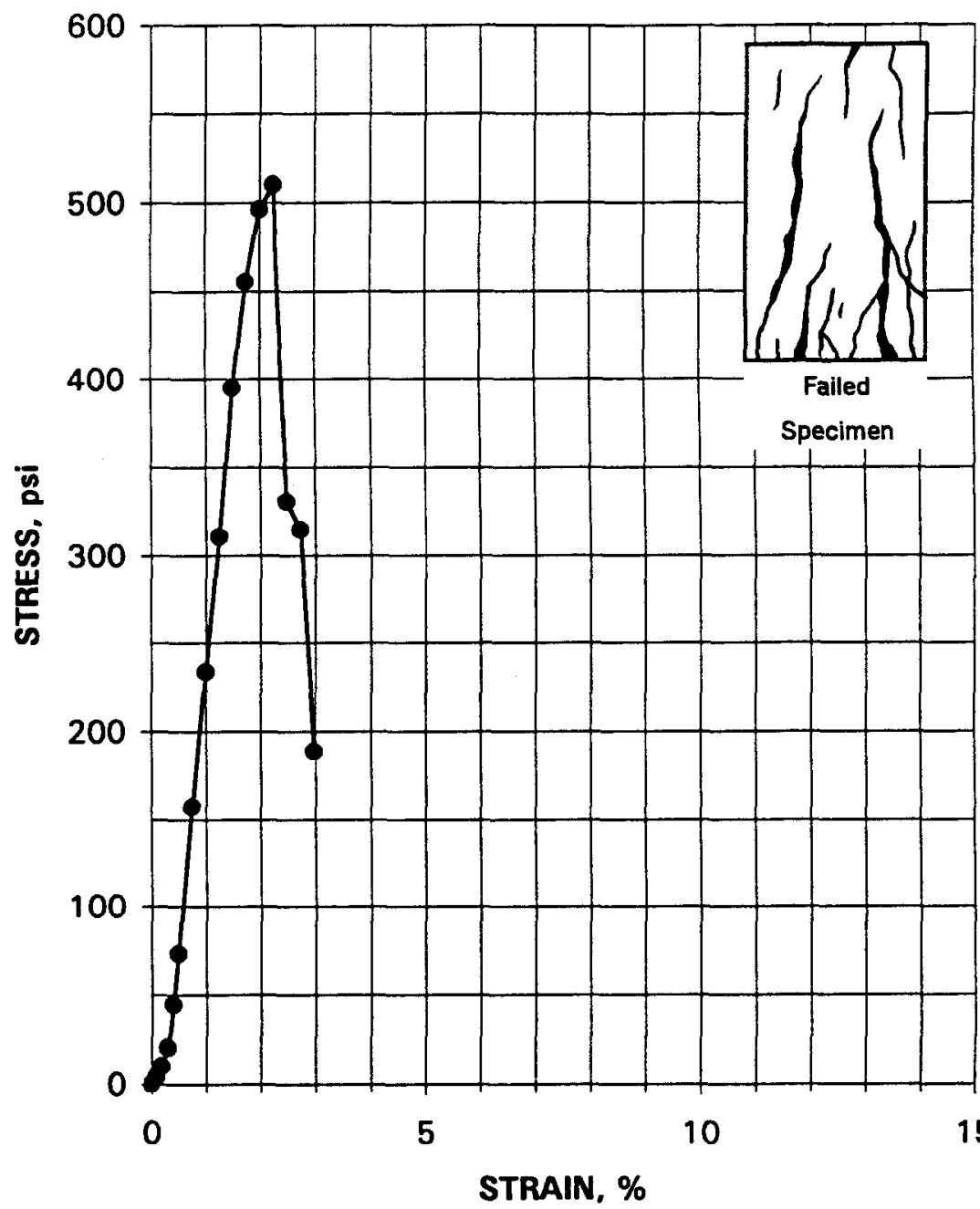




\section{UNCONFINED COMPRESSIVE STRENGTH - ASTM D 2166 28-DAY CURE}

$\begin{array}{ll}\text { Project Name } & \text { SAIC - Savannah River } \\ \text { Project No. } & 777304.00010500\end{array}$

Specimen Mold Date

Specimen Test Date

STRESS AT FAILURE, psi STRAIN AT FAILURE, \%

\begin{tabular}{ccc}
$\begin{array}{c}\text { AXIAL } \\
\text { STRAIN, \% }\end{array}$ & & $\begin{array}{c}\text { DEVIATOR } \\
\text { STRESS, psi }\end{array}$ \\
\cline { 1 - 1 } 0.00 & & 0.0 \\
0.10 & & 3.2 \\
0.20 & & 9.9 \\
0.30 & & 24.2 \\
0.40 & & 43.2 \\
0.50 & & 67.9 \\
0.75 & & 143.1 \\
1.01 & & 223.7 \\
1.26 & & 297.1 \\
1.51 & & 356.4 \\
1.76 & & 407.2 \\
2.01 & & 433.1 \\
2.26 & & 463.8 \\
2.51 & 490.6 \\
2.77 & 96.2
\end{tabular}

Client Sample No. L412FOO

IT Lab Specimen No. ETDC-8067

Specimen Height, in.

Specimen Diameter, in.

Specimen Weight, $g$.

371.62

Moisture Content, \%

Wet Unit Weight, pcf.

113.6

Dry Unit Weight, pcf.

Rate of Strain, in./min.

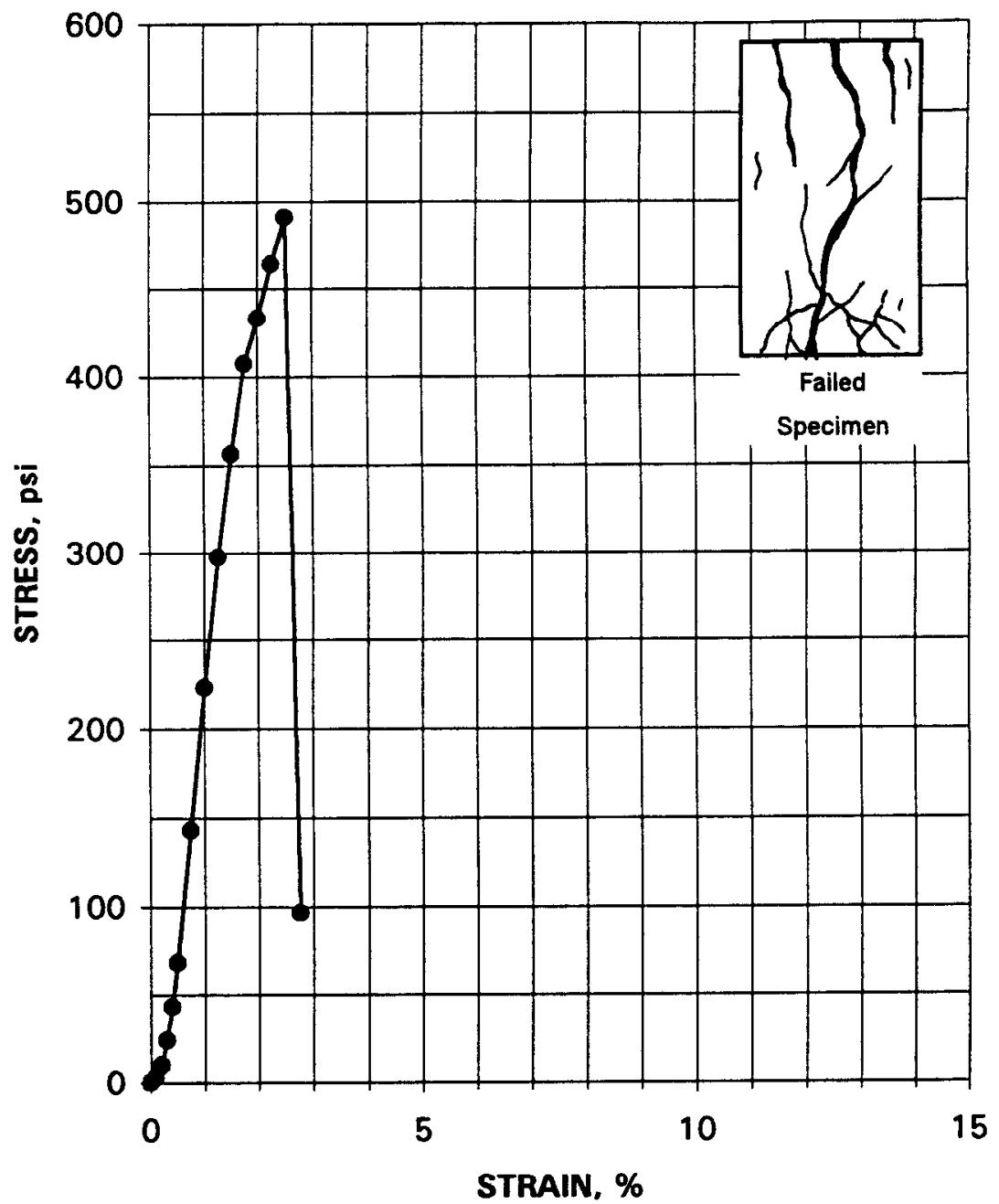




\section{UNCONFINED COMPRESSIVE STRENGTH - ASTM D 2166 28-DAY CURE}

$\begin{array}{ll}\text { Project Name } & \text { SAIC - Savannah River } \\ \text { Project No. } & 777304.00010500\end{array}$

Specimen Mold Date

Specimen Test Date

STRESS AT FAILURE, psi

STRAIN AT FAILURE, \%

AXIAL

STRAIN, \%

$\begin{array}{lcc}0.00 & 0.0 \\ 0.10 & & 4.5 \\ 0.20 & & 14.9 \\ 0.30 & & 38.4 \\ 0.40 & & 66.9 \\ 0.50 & & 101.7 \\ 0.75 & & 167.1 \\ 0.99 & & 222.5 \\ 1.24 & & 275.7 \\ 1.49 & & 343.7 \\ 1.74 & & 376.6 \\ 1.99 & & 238.0 \\ 2.24 & & 217.2\end{array}$

Client Sample No. L422FOO

IT Lab Specimen No. ETDC-8068

Specimen Height, in.

4.0208

Specimen Diameter, in.

2.0000

Specimen Weight, $g$.

390.02

Moisture Content, \%

27.6

Wet Unit Weight, pcf.

117.6

Dry Unit Weight, pcf.

92.2

Rate of Strain, in./min.

0.0400

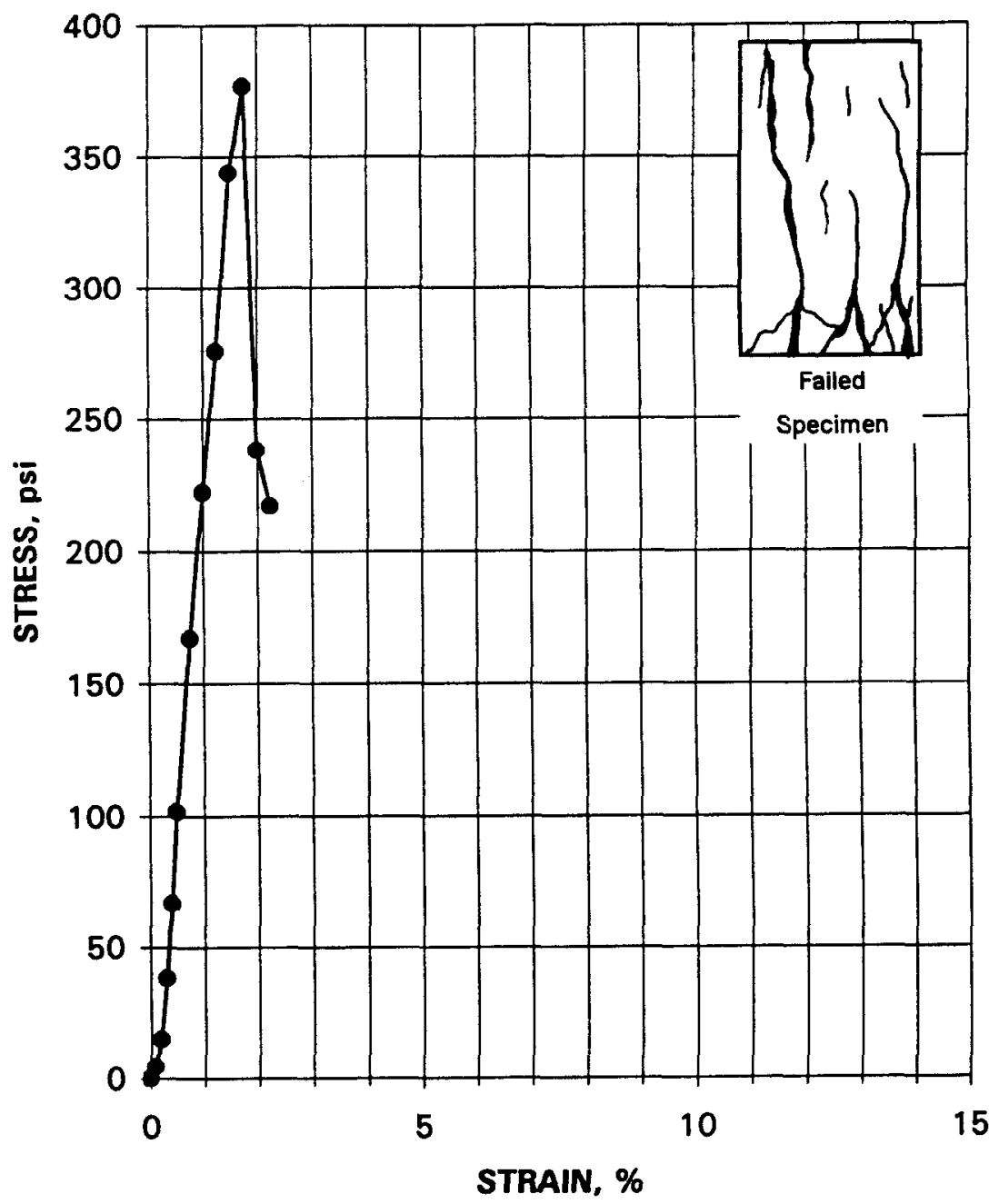




\section{UNCONFINED COMPRESSIVE STRENGTH - ASTM D 2166 28-DAY CURE}

\begin{tabular}{lc}
$\begin{array}{l}\text { Project Name } \\
\text { Project No. }\end{array}$ & SAIC - Savan \\
\multicolumn{3}{l}{ 777304.00010 } \\
Specimen Mold Date \\
Specimen Test Date \\
STRESS AT FAILURE, psi \\
STRAIN AT FAILURE, \% \\
\multicolumn{3}{|}{} \\
AXIAL & DEVIATOR \\
STRAIN, \% & STRESS, psi \\
\cline { 2 - 2 } 0.00 & 0.0 \\
0.10 & 3.5 \\
0.20 & 13.0 \\
0.30 & 23.5 \\
0.40 & 39.6 \\
0.50 & 96.5 \\
0.75 & 167.6 \\
1.00 & 243.1 \\
1.25 & 320.7 \\
1.50 & 406.3 \\
1.75 & 417.5 \\
1.99 & 282.4 \\
2.24 & 166.3
\end{tabular}

Client Sample No. L432FOO

IT Lab Specimen No. ETDC-8069

Specimen Height, in.

4.0105

Specimen Diameter, in.

2.0008

Specimen Weight, $g$.

366.60

Moisture Content, \%

Wet Unit Weight, pcf.

Dry Unit Weight, pcf.

Rate of Strain, in./min.

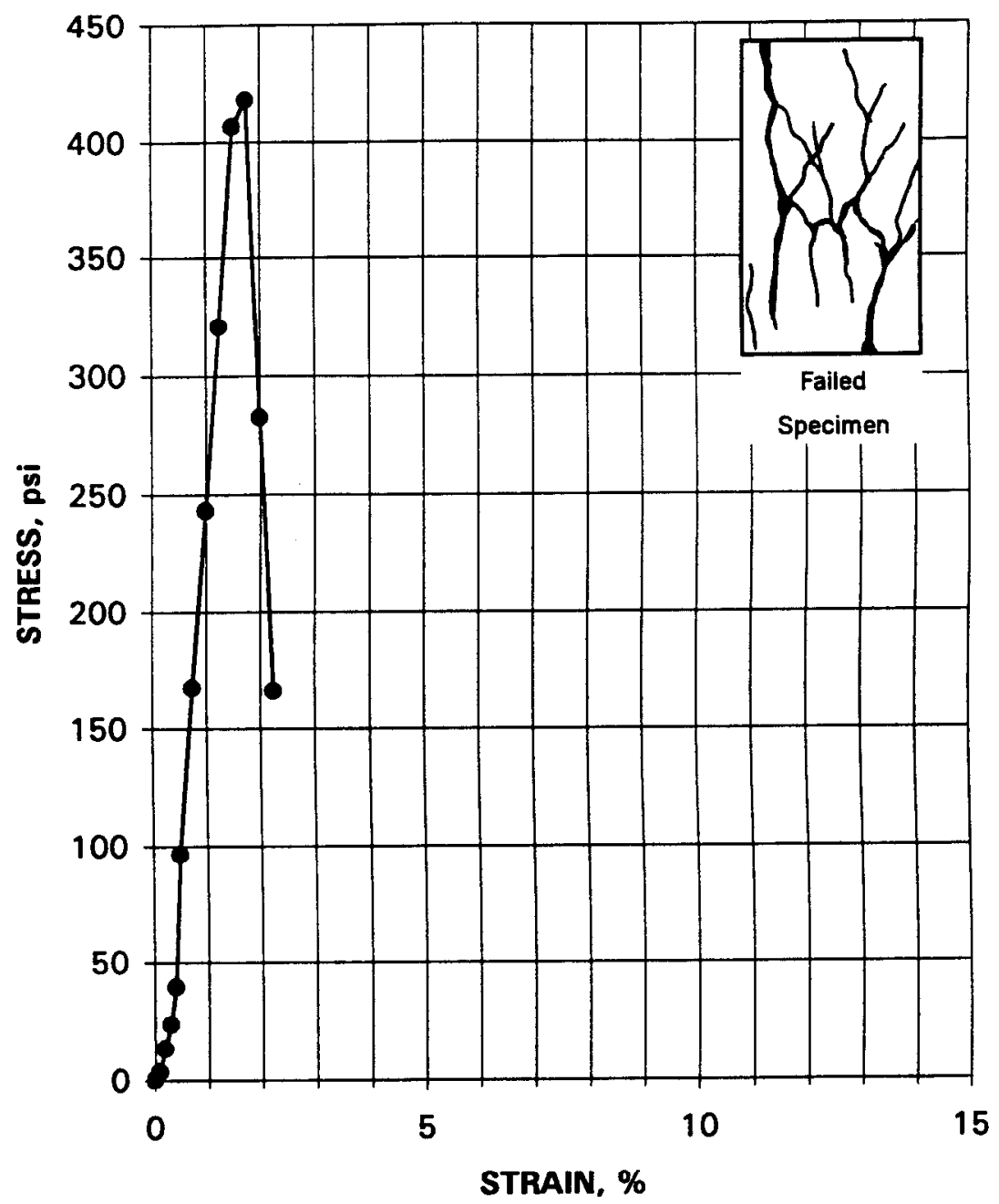




\section{UNCONFINED COMPRESSIVE STRENGTH - ASTM D 2166 28-DAY CURE}

$\begin{array}{ll}\text { Project Name } & \text { SAIC - Savannah River } \\ \text { Project No. } & 777304.00010500\end{array}$

Specimen Mold Date

Specimen Test Date

STRESS AT FAILURE, psi

STRAIN AT FAILURE, \%

\begin{tabular}{ccc}
$\begin{array}{c}\text { AXIAL } \\
\text { STRAIN, \% }\end{array}$ & & $\begin{array}{c}\text { DEVIATOR } \\
\text { STRESS, psi }\end{array}$ \\
\cline { 1 - 1 } 0.00 & & 0.0 \\
0.10 & & 3.2 \\
0.20 & & 8.9 \\
0.30 & & 19.6 \\
0.40 & & 38.8 \\
0.50 & & 62.8 \\
0.75 & & 132.8 \\
1.00 & & 226.3 \\
1.25 & & 297.8 \\
1.50 & & 351.1 \\
1.75 & & 418.4 \\
2.00 & & 478.3 \\
2.25 & & 527.6 \\
2.50 & & 546.3 \\
2.55 & & 546.6 \\
2.75 & & 443.5 \\
3.00 & 411.6 \\
3.25 & 343.9 \\
3.50 & & 73.1
\end{tabular}

Client Sample No. L442FOO

IT Lab Specimen No. ETDC-8070

Specimen Height, in.

4.0038

Specimen Diameter, in.

2.0038

Specimen Weight, $g$.

371.01

Moisture Content, \%

32.7

Wet Unit Weight, pcf.

112.0

Dry Unit Weight, pcf.

84.3

Rate of Strain, in./min.

0.0400

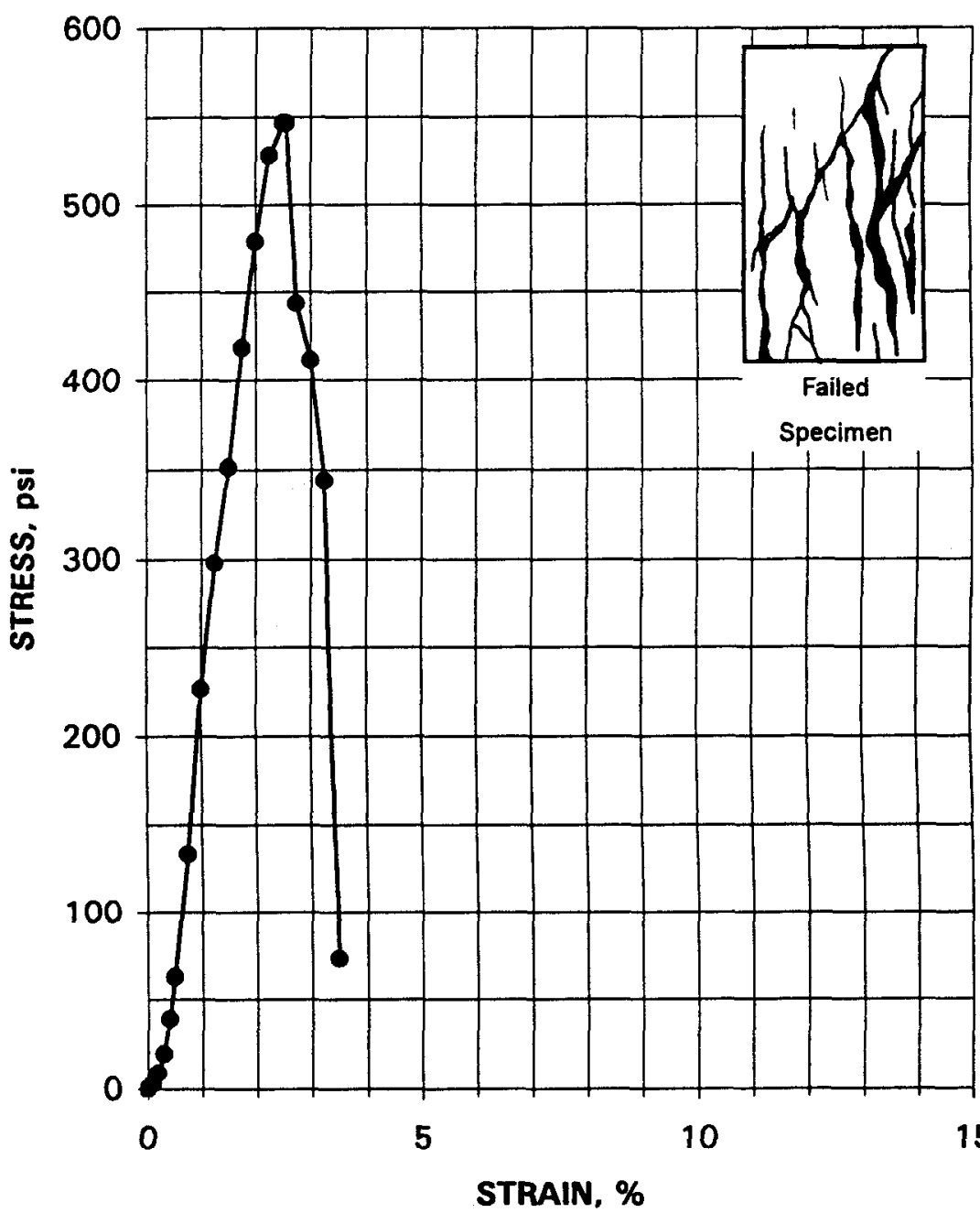




\section{Series \\ 90 Day Immersion UCS Results}




\section{UNCONFINED COMPRESSIVE STRENGTH - ASTM D 2166 28-DAY CURE, 90-DAY SATURATION}

$\begin{array}{ll}\text { Project Name } & \text { SAIC/SAVANNAH RIVER } \\ \text { Project No. } & 777304.00010500\end{array}$

Specimen Mold Date

Specimen Test Date

STRESS AT FAILURE, psi

STRAIN AT FAILURE, \%

AXIAL DEVIATOR

STRAIN, \%

$\begin{array}{lcc}0.00 & 0.0 \\ 0.10 & & 11.7 \\ 0.20 & & 22.7 \\ 0.30 & & 41.9 \\ 0.40 & & 68.0 \\ 0.50 & & 98.4 \\ 0.75 & & 180.6 \\ 1.01 & & 261.1 \\ 1.26 & & 351.5 \\ 1.51 & & 433.4 \\ 1.76 & & 513.9 \\ 2.01 & & 587.5 \\ 2.14 & 613.0 \\ 2.26 & 57.7\end{array}$

$12 / 21 / 98$

$4 / 20 / 99$

613.0

2.1
Client Sample No. L313F00

IT Lab Specimen No. ETDC-8039

Specimen Height, in.

3.9778

Specimen Diameter, in.

2.0077

Specimen Weight, $g$.

380.46

Moisture Content, \%

31.6

Wet Unit Weight, pcf.

115.1

Dry Unit Weight, pef.

87.5

Rate of Strain, in./min.

0.0400

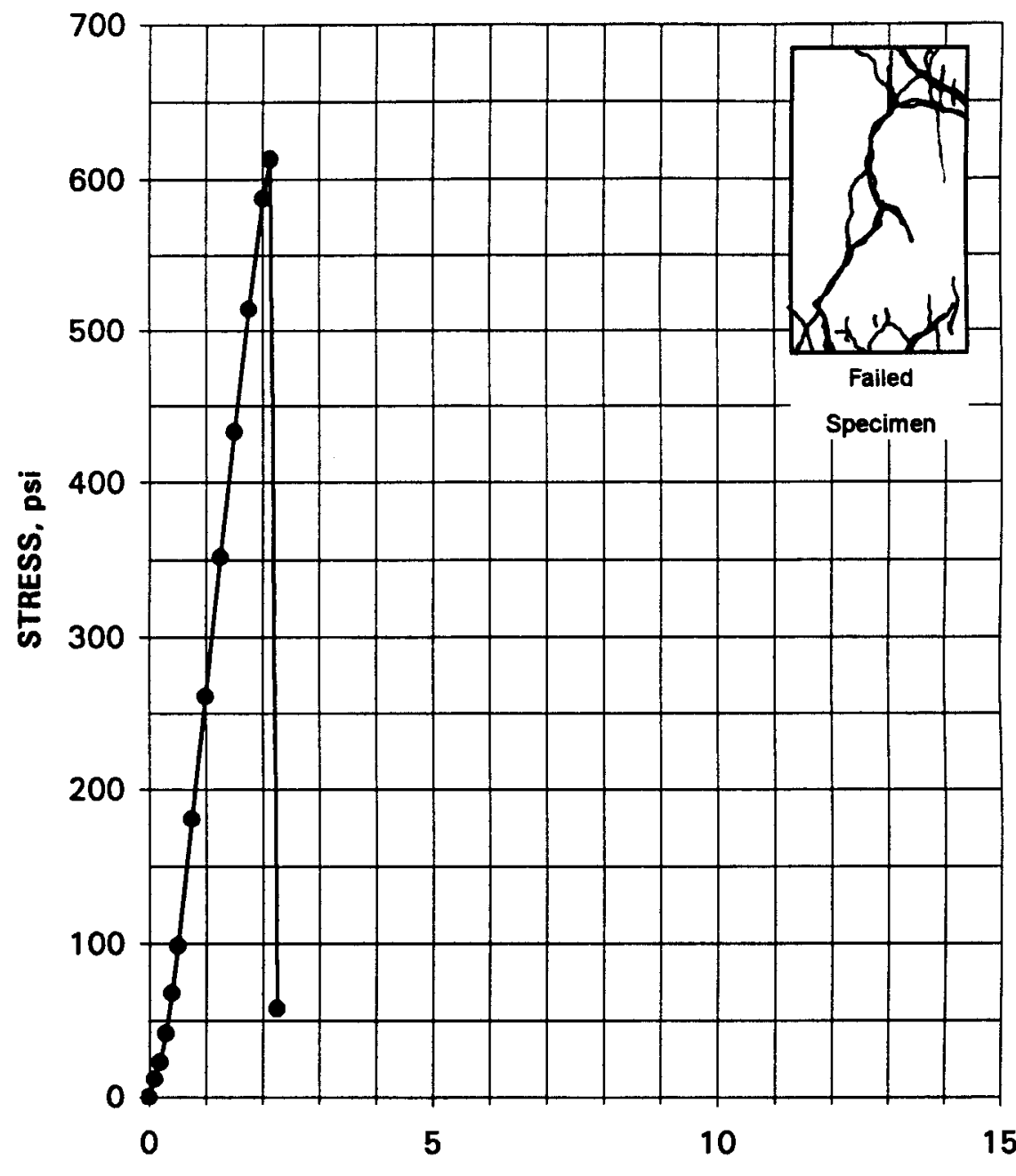

STRAIN, \% 


\section{UNCONFINED COMPRESSIVE STRENGTH - ASTM D 2166 28-DAY CURE, 90-DAY SATURATION}

\begin{tabular}{lc} 
Project Name & SAIC/SAVAN \\
Project No. & 777304.00010 \\
Specimen Mold Date \\
Specimen Test Date \\
STRESS AT FAILURE, psi \\
STRAIN AT FAILURE, \% \\
\multicolumn{3}{|}{} \\
AXIAL & DEVIATOR \\
STRAIN, \% & STRESS, psi \\
\hline 0.00 & 0.0 \\
0.10 & 8.8 \\
0.20 & 24.3 \\
0.30 & 54.1 \\
0.40 & 101.5 \\
0.50 & 157.3 \\
0.75 & 282.8 \\
1.00 & 409.6 \\
1.25 & 511.1 \\
1.49 & 645.3 \\
1.74 & 784.5 \\
1.99 & 894.7 \\
2.24 & 974.2 \\
2.37 & 982.5 \\
2.49 & 86.8 \\
\end{tabular}

Client Sample No. L323F00

IT Lab Specimen No. ETDC-8040

Specimen Height, in.

Specimen Diameter, in.

2.0087

Specimen Weight, $\mathfrak{g}$.

393.80

Moisture Content, \%

28.4

Wet Unit Weight, pcf.

117.9

Dry Unit Weight, pcf.

Rate of Strain, in./min.

0.0400

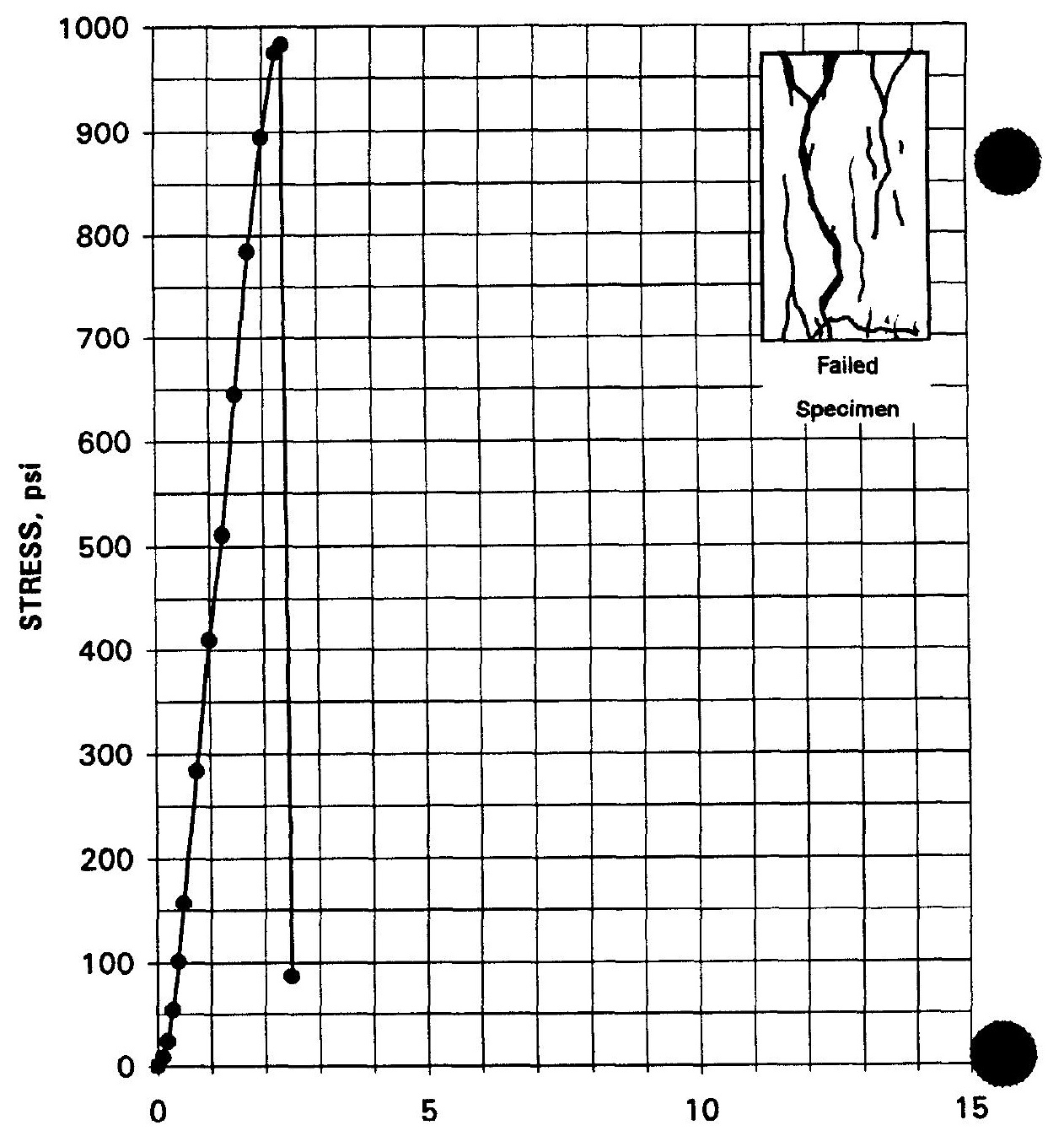

STRAIN, \% 


\section{UNCONFINED COMPRESSIVE STRENGTH - ASTM D 2166 28-DAY CURE, 90-DAY SATURATION}

Project Name SAIC/SAVANNAH RIVER

Project No. 777304.00010500

Specimen Mold Date

Specimen Test Date

STRESS AT FAILURE, psi

STRAIN AT FAILURE, \%

\begin{tabular}{ccc}
$\begin{array}{c}\text { AXIAL } \\
\text { STRAIN, \% }\end{array}$ & $\begin{array}{c}\text { DEVIATOR } \\
\text { STRESS, pSi }\end{array}$ \\
\cline { 1 - 2 } 0.00 & 0.0 \\
0.10 & 3.5 \\
0.20 & 4.7 \\
0.30 & 4.7 \\
0.40 & 7.5 \\
0.50 & 20.7 \\
0.75 & 72.0 \\
1.00 & 132.4 \\
1.25 & 187.4 \\
1.50 & 232.0 \\
1.75 & 278.8 \\
2.00 & 326.0 \\
2.24 & 360.9 \\
2.49 & 399.7 \\
2.74 & 426.9 \\
2.99 & 386.0 \\
3.24 & 352.4 \\
3.49 & 352.4 \\
3.74 & 357.5 \\
3.99 & 359.0 \\
4.24 & 301.9
\end{tabular}

Client Sample No. L333F00

IT Lab Specimen No. ETDC-8041

Specimen Height, in. $\quad 4.0092$

Specimen Diameter, in. $\quad 2.0095$

Specimen Weight, $g$.

372.80

Moisture Content, \%

37.0

Wet Unit Weight, pcf.

111.7

Dry Unit Weight, pcf.

81.6

Rate of Strain, in./min.

0.0400

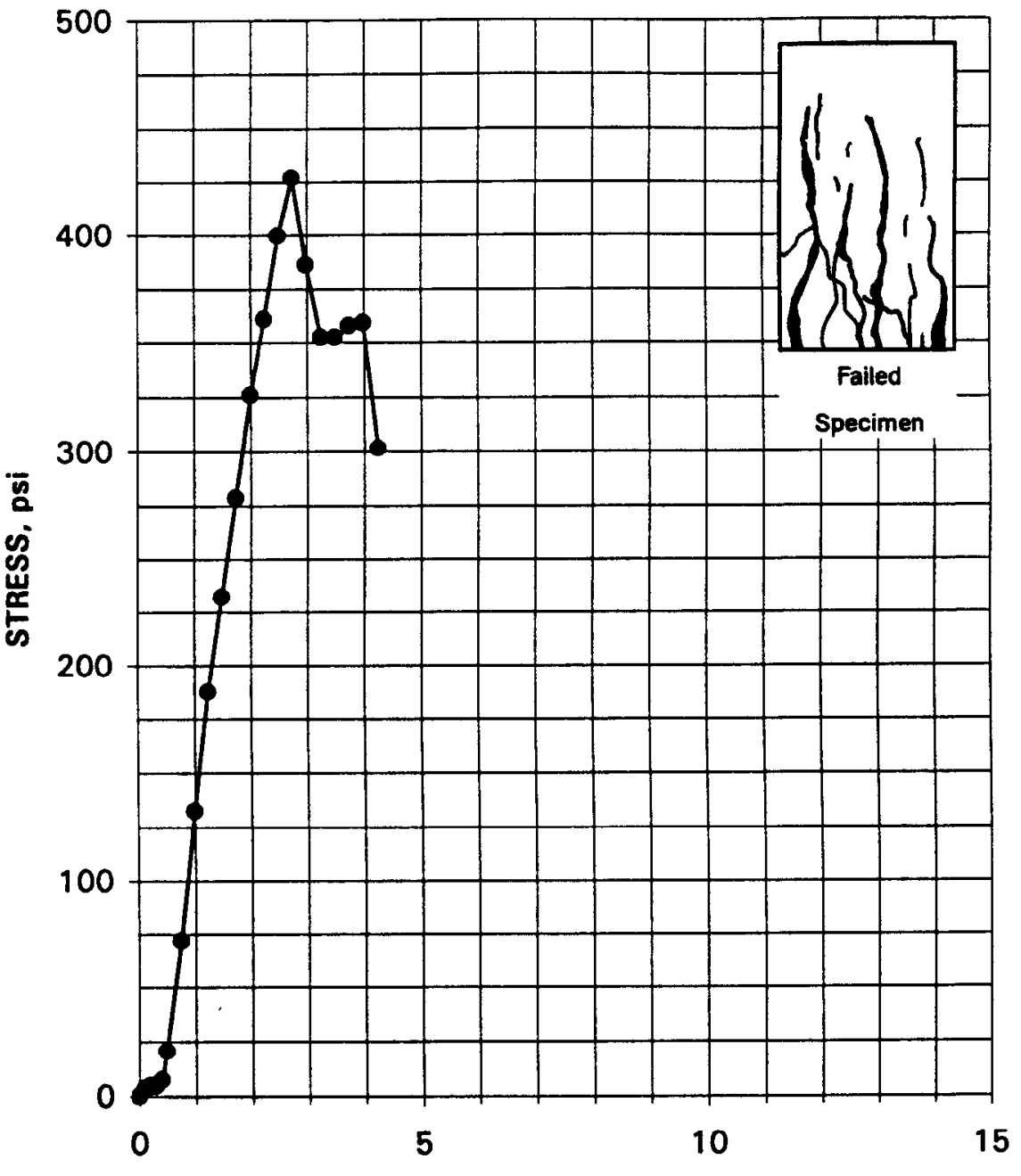

STRAIN, \% 


\section{UNCONFINED COMPRESSIVE STRENGTH - ASTM D 2166 28-DAY CURE, 90-DAY SATURATION}

\begin{tabular}{|c|c|}
\hline Project Name & SAIC/SAVAI \\
\hline Project No. & 777304.00010 \\
\hline $\begin{array}{l}\text { Specimen Mol } \\
\text { Specimen Test }\end{array}$ & $\begin{array}{l}\text { Date } \\
\text { Date }\end{array}$ \\
\hline $\begin{array}{l}\text { STRESS AT F } \\
\text { STRAIN AT FA }\end{array}$ & $\begin{array}{l}\text { LURE, psi } \\
\text { URE, \% }\end{array}$ \\
\hline AXIAL & DEVIATOR \\
\hline STRAIN, \% & STRESS, psi \\
\hline 0.00 & 0.0 \\
\hline 0.10 & 2.2 \\
\hline 0.20 & 4.4 \\
\hline 0.30 & 6.3 \\
\hline 0.40 & 9.7 \\
\hline 0.50 & 22.5 \\
\hline 0.74 & 66.5 \\
\hline 0.99 & 136.2 \\
\hline 1.24 & 225.6 \\
\hline 1.49 & 316.2 \\
\hline 1.74 & 408.2 \\
\hline 1.98 & 470.7 \\
\hline 2.23 & 555.3 \\
\hline 2.48 & 634.9 \\
\hline 2.73 & 692.4 \\
\hline 2.85 & 697.0 \\
\hline 2.98 & 400.6 \\
\hline 3.23 & 36.5 \\
\hline
\end{tabular}

Client Sample No. L343F00

IT Lab Specimen No. ETDC-8042

$12 / 21 / 98$

$4 / 20 / 99$

697.0

2.9

Specimen Height, in.

4.0307

Specimen Diameter, in.

2.0115

Specimen Weight, $\mathrm{g}$.

379.61

Moisture Content, \%

33.7

Wet Unit Weight, pcf.

112.9

Dry Unit Weight, pcf.

84.4

Rate of Strain, in./min.

0.0400

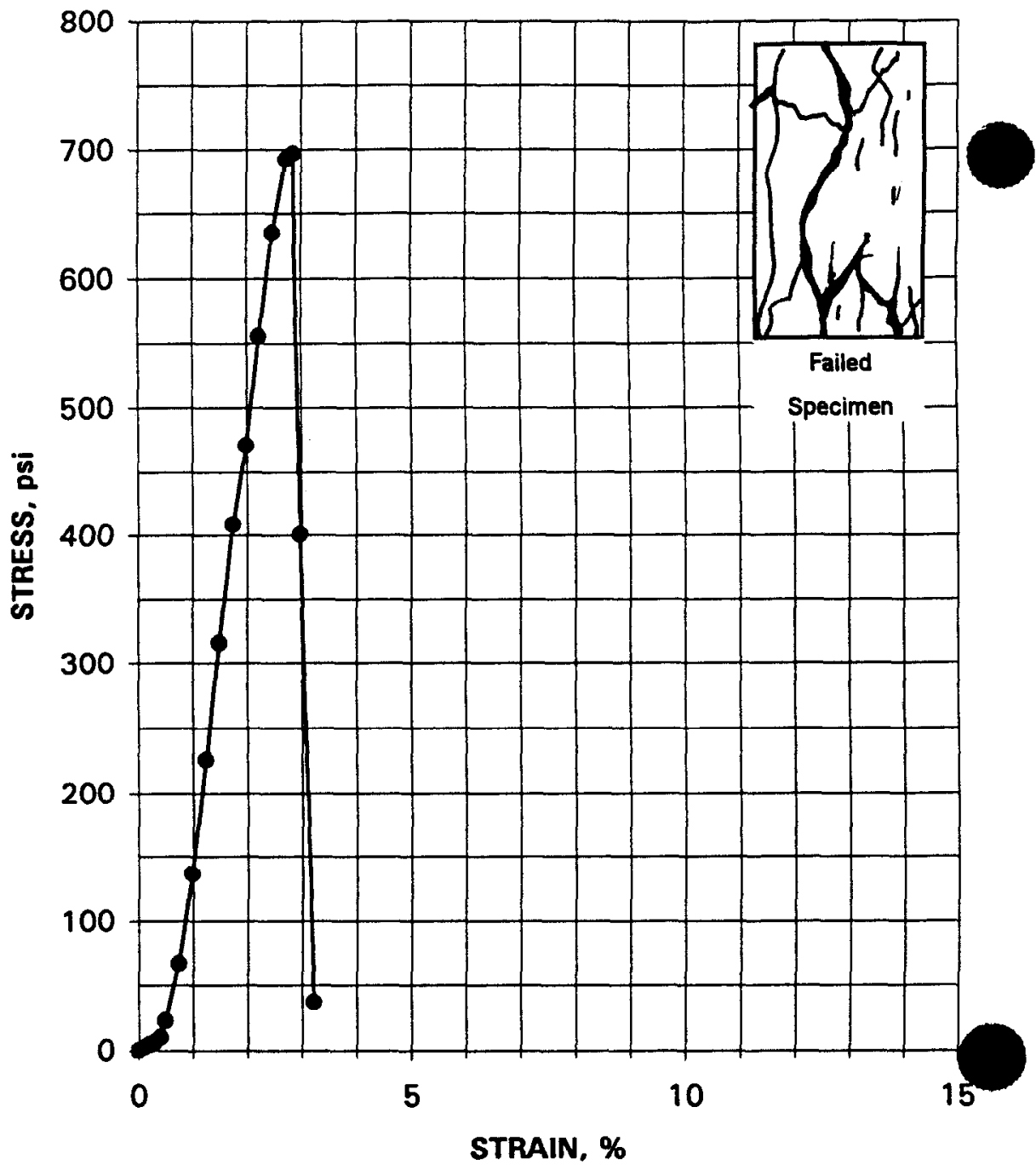




\section{UNCONFINED COMPRESSIVE STRENGTH - ASTM D 2166 28-DAY CURE, 90-DAY SATURATION}

\begin{tabular}{|c|c|}
\hline Project Name & SAIC/SAVA \\
\hline Project No. & 777304.0001 \\
\hline $\begin{array}{l}\text { Specimen Mo } \\
\text { Specimen Tes }\end{array}$ & \\
\hline $\begin{array}{l}\text { STRESS AT F } \\
\text { STRAIN AT F }\end{array}$ & $\begin{array}{l}\text { LURE, psi } \\
\text { _URE, \% }\end{array}$ \\
\hline AXIAL & DEVIATOR \\
\hline STRAIN, \% & STRESS, psi \\
\hline 0.00 & 0.0 \\
\hline 0.10 & 4.4 \\
\hline 0.30 & 5.0 \\
\hline 0.40 & 12.6 \\
\hline 0.50 & 31.8 \\
\hline 0.75 & 113.4 \\
\hline 1.00 & 192.1 \\
\hline 1.25 & 252.3 \\
\hline 1.50 & 303.4 \\
\hline 1.75 & 335.9 \\
\hline 2.00 & 357.4 \\
\hline 2.25 & 396.7 \\
\hline 2.50 & 435.8 \\
\hline 2.75 & 449.5 \\
\hline 3.00 & 457.5 \\
\hline 3.25 & 468.3 \\
\hline 3.50 & 473.5 \\
\hline 3.75 & 479.9 \\
\hline 4.00 & 336.4 \\
\hline 4.25 & 241.0 \\
\hline 4.50 & 211.9 \\
\hline 4.75 & 162.2 \\
\hline
\end{tabular}

Client Sample No. L413F0O

IT Lab Specimen No. ETDC-8043

Specimen Height, in.

3.9975

Specimen Diameter, in.

2.0055

Specimen Weight, $g$.

385.16

Moisture Content, \%

32.4

Wet Unit Weight, pcf.

116.2

Dry Unit Weight, pcf.

87.7

Rate of Strain, in./min.

0.0400

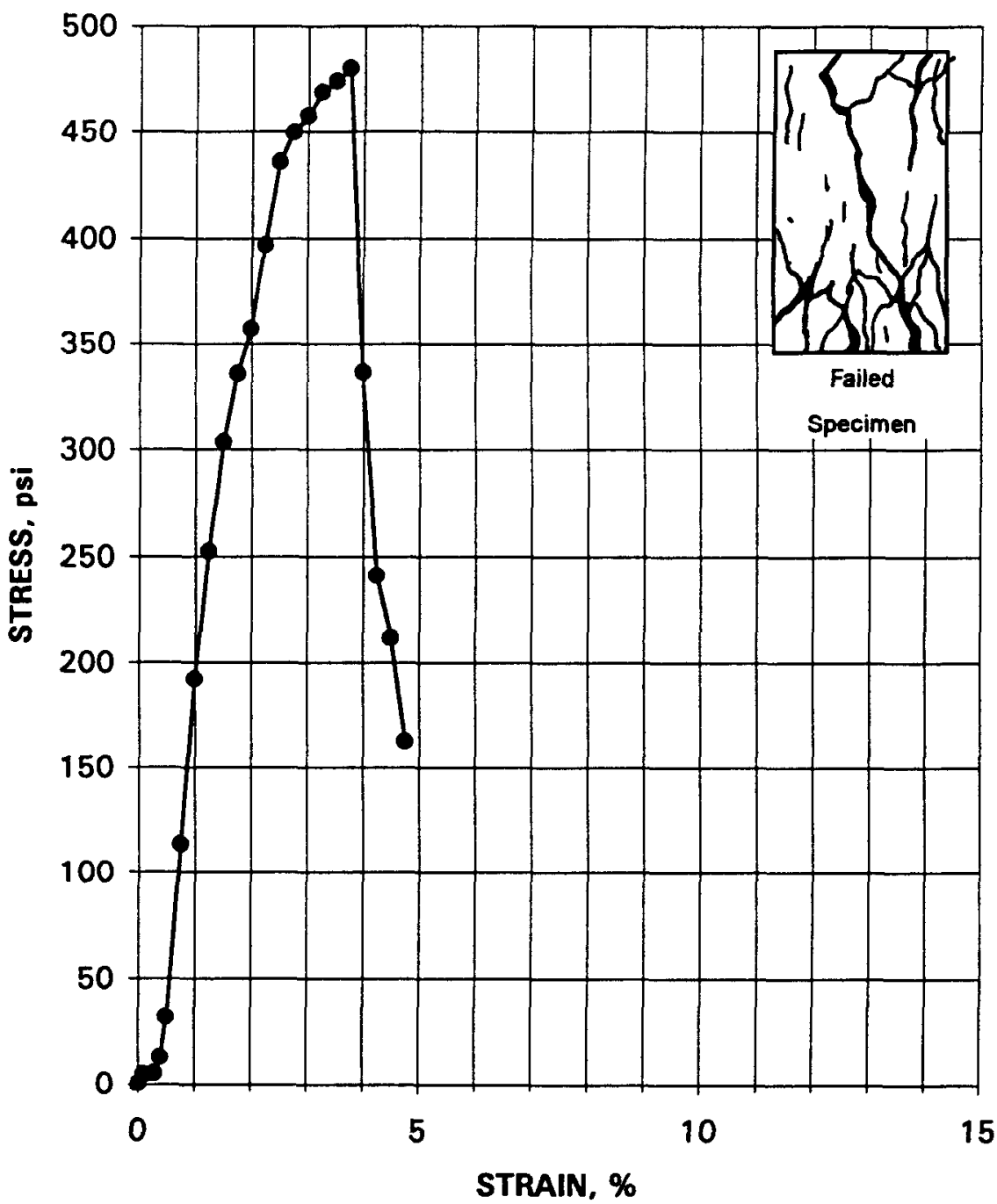




\section{UNCONFINED COMPRESSIVE STRENGTH - ASTM D 2166 28-DAY CURE, 90-DAY SATURATION}

\begin{abstract}
Project Name SAIC/SAVANNAH RIVER
Project No. $\quad 777304.00010500$
\end{abstract}

Specimen Mold Date

Specimen Test Date

STRESS AT FAILURE, psi

STRAIN AT FAILURE, \%

\begin{tabular}{cc}
$\begin{array}{c}\text { AXIAL } \\
\text { STRAIN, \% }\end{array}$ & $\begin{array}{c}\text { DEVIATOR } \\
\text { STRESS, psi }\end{array}$ \\
\cline { 1 - 1 } 0.00 & 0.0 \\
0.10 & 3.5 \\
0.20 & 4.1 \\
0.30 & 4.4 \\
0.40 & 7.9 \\
0.50 & 40.9 \\
0.75 & 144.6 \\
1.00 & 210.6 \\
1.25 & 274.7 \\
1.50 & 328.8 \\
1.75 & 376.4 \\
2.00 & 421.9 \\
2.25 & 455.7 \\
2.38 & 461.6 \\
2.50 & 294.3 \\
2.75 & 276.7 \\
3.00 & 216.5
\end{tabular}

Client Sample No. L423FOO

IT Lab Specimen No. ETDC-8044

Specimen Height, in.

3.9962

Specimen Diameter, in.

2.0070

Specimen Weight, $g$.

388.11

Moisture Content, \%

29.0

Wet Unit Weight, pcf.

117.0

Dry Unit Weight, pcf.

90.7

Rate of Strain, in./min.

0.0400

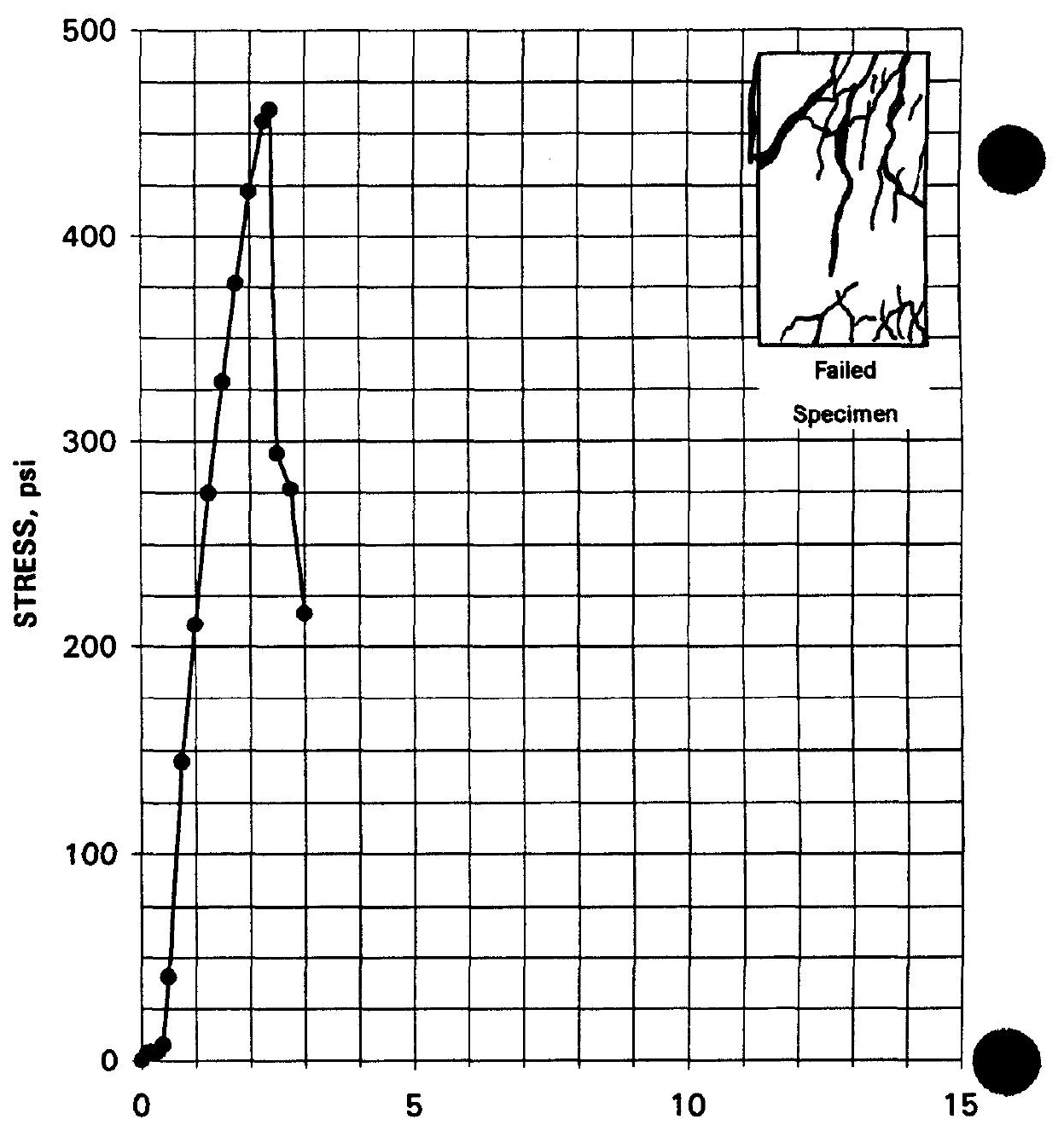

STRAIN, \% 


\section{UNCONFINED COMPRESSIVE STRENGTH - ASTM D 2166 28-DAY CURE, 90-DAY SATURATION}

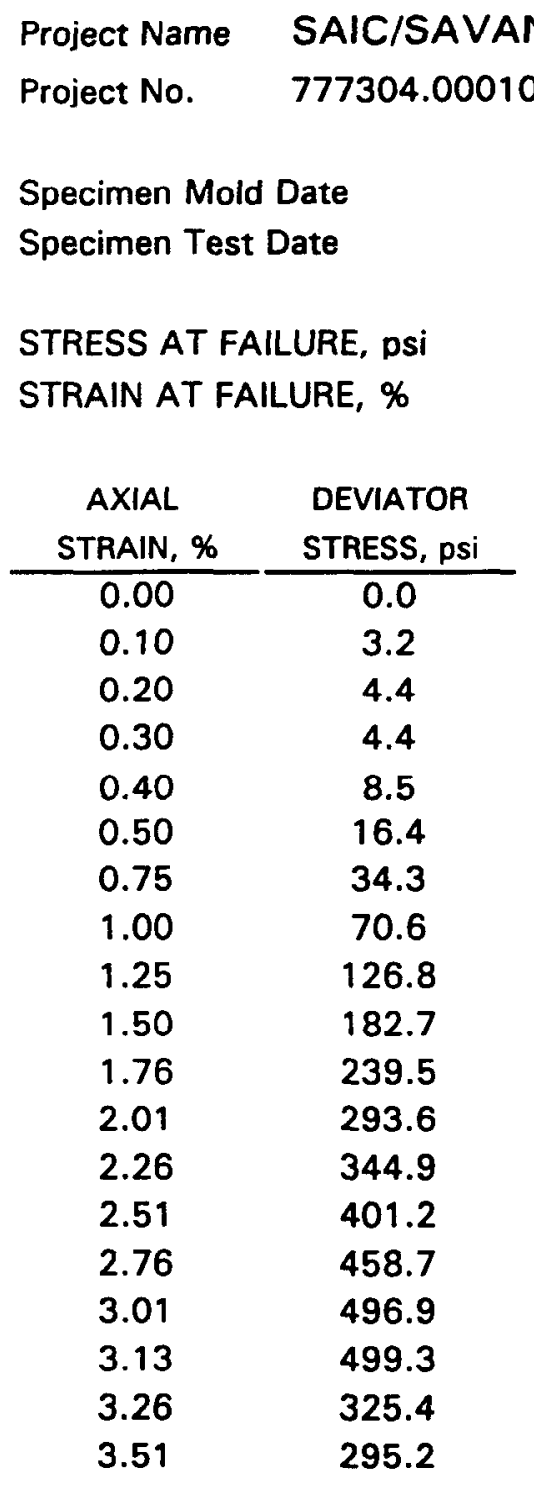

Client Sample No. L433FOO

IT Lab Specimen No. ETDC-8045
$12 / 21 / 98$
$4 / 20 / 99$

499.3

3.1
Specimen Height, in.

Specimen Diameter, in.

2.0040

Specimen Weight, g.

Moisture Content, \%

Wet Unit Weight, pcf.

Dry Unit Weight, pcf.

Rate of Strain, in./min.

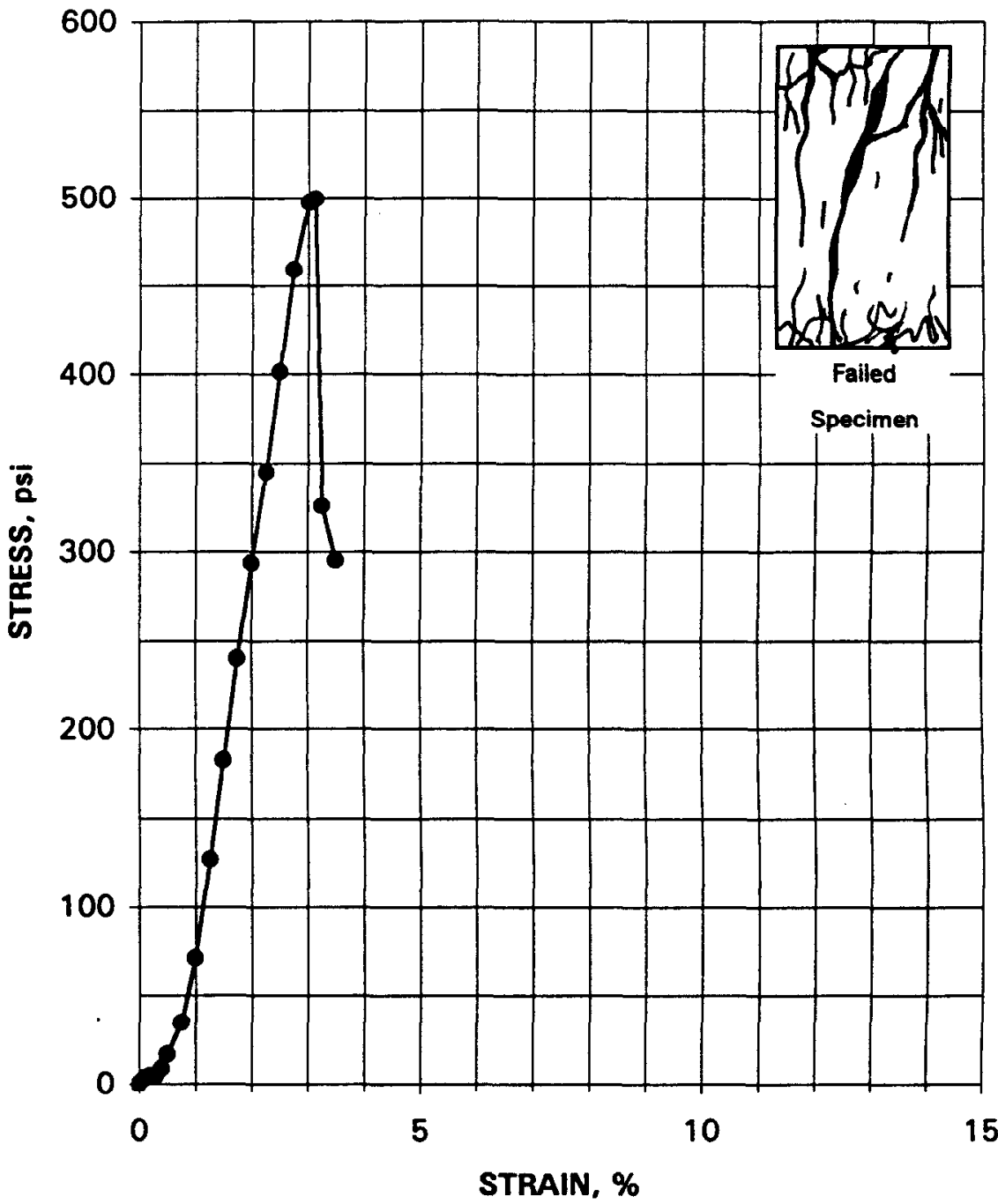




\section{UNCONFINED COMPRESSIVE STRENGTH - ASTM D 2166 28-DAY CURE, 90-DAY SATURATION}

Project Name SAIC/SAVANNAH RIVER

Project No. $\quad 777304.00010500$

Specimen Mold Date

Specimen Test Date

STRESS AT FAILURE, psi

STRAIN AT FAILURE, \%

\begin{tabular}{ccc}
$\begin{array}{c}\text { AXIAL } \\
\text { STRAIN, \% }\end{array}$ & $\begin{array}{c}\text { DEVIATOR } \\
\text { STRESS, psi }\end{array}$ \\
\cline { 1 - 1 } 0.00 & 0.0 \\
0.10 & 3.8 \\
0.20 & 4.7 \\
0.30 & 5.0 \\
0.40 & & 6.0 \\
0.50 & 6.9 \\
0.75 & & 23.8 \\
0.99 & 50.7 \\
1.24 & 84.6 \\
1.49 & 147.5 \\
1.74 & 236.0 \\
1.99 & 313.4 \\
2.24 & 393.9 \\
2.49 & 457.0 \\
2.73 & 459.8 \\
2.98 & 287.9 \\
3.23 & 264.2 \\
3.48 & 75.3
\end{tabular}

Client Sample No. L443FOO

IT Lab Specimen No. ETDC-8046

Specimen Height, in.

4.0240

Specimen Diameter, in.

2.0073

Specimen Weight, $g$.

375.02

Maisture Content, \%

34.6

Wet Unit Weight, pcf.

112.2

Dry Unit Weight, pcf.

83.4

Rate of Strain, in./min.

0.0400

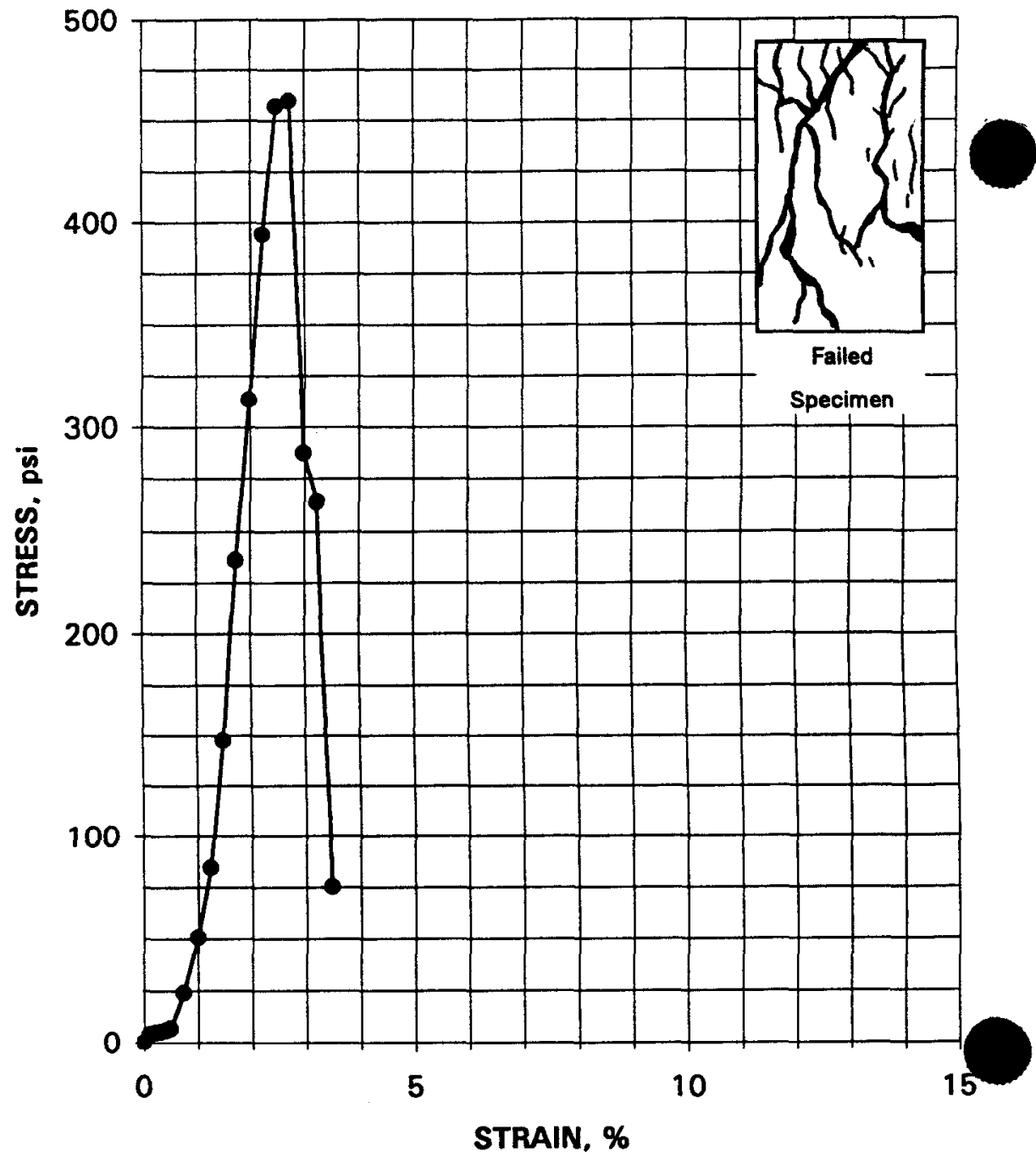




\section{Series 28 Day Expansion / Shrinkage Results}




\section{One-Dimensional Expansion, Shrinkage, and} Uplift Pressure of Soil-Lime Mixtures ASTM D 3877

\section{8-Day Cure}

Geo Lab Knoxville Lab

\begin{tabular}{ccccc} 
Sample No. & Sample No. & Expansion, \% & Expansion Result & Shrinkage, \% \\
\hline \hline ETDC-8087 & L312KOO & $-0.059 \%$ & No expansion & $0.22 \%$ \\
ETDC-8088 & L322K00 & $-0.120 \%$ & No expansion & $0.75 \%$ \\
ETDC-8089 & L332K00 & $-0.110 \%$ & No expansion & $4.13 \%$ \\
ETDC-8090 & L342K00 & $-0.070 \%$ & No expansion & $1.12 \%$ \\
ETDC-8091 & L412K00 & $-0.050 \%$ & No expansion & $2.32 \%$ \\
ETDC-8092 & L422K00 & $-0.100 \%$ & No expansion & Insuff. Sample \\
ETDC-8093 & L432KDO & $-0.440 \%$ & No expansion & $1.98 \%$ \\
ETDC-8094 & L442KDO & $-0.040 \%$ & No expansion & $1.07 \%$
\end{tabular}




\section{Series \\ 28 Day Permeability Results}




\section{8-DAY HYDRAULIC CONDUCTIVITY / PERMEABILITY ASTM D 5084}

PROJECT NAME: SAIC / Savannah River PROJECT NO. $\quad 777304.00010500$
CLIENT SAMPLE NO. L312G00

IT LAB SAMPLE NO. ETDC-8111

\section{Specimen diameter, $\mathrm{cm}$}

Specimen length, $\mathrm{cm}$

Wet weight of specimen, $g$.

Specimen cross-sect. area, $\mathrm{cm}^{-2} 2$

Water content, \%

Wet unit weight, pcf

Dry unit weight, pcf

Estimated degree of saturation, \%

Estimated spec. gravity of solids

\begin{tabular}{|l|}
\hline INITIAL FINAL \\
\hline 5.06
\end{tabular}

10.10

374.4

20.1267

29.7

115.0

88.6

90.8

2.65
Hydraulic gradient

27.9

381.38

32.1

Min. consolidation stress, psi

2.0

Max. consolidation stress, psi

8.0

3.0

Total backpressure, psi

Permeant Fluid

Deaired DI Water

Sample molded

$12 / 21 / 98$

Sample set up

$1 / 21 / 99$
Five tests performed

\section{\begin{tabular}{|ll|}
\hline Coefficient of Permeability, $\mathrm{cm} / \mathrm{s}$ & 8.7E-09 \\
\hline
\end{tabular}}

\section{PERMEABILITY vS TIME}

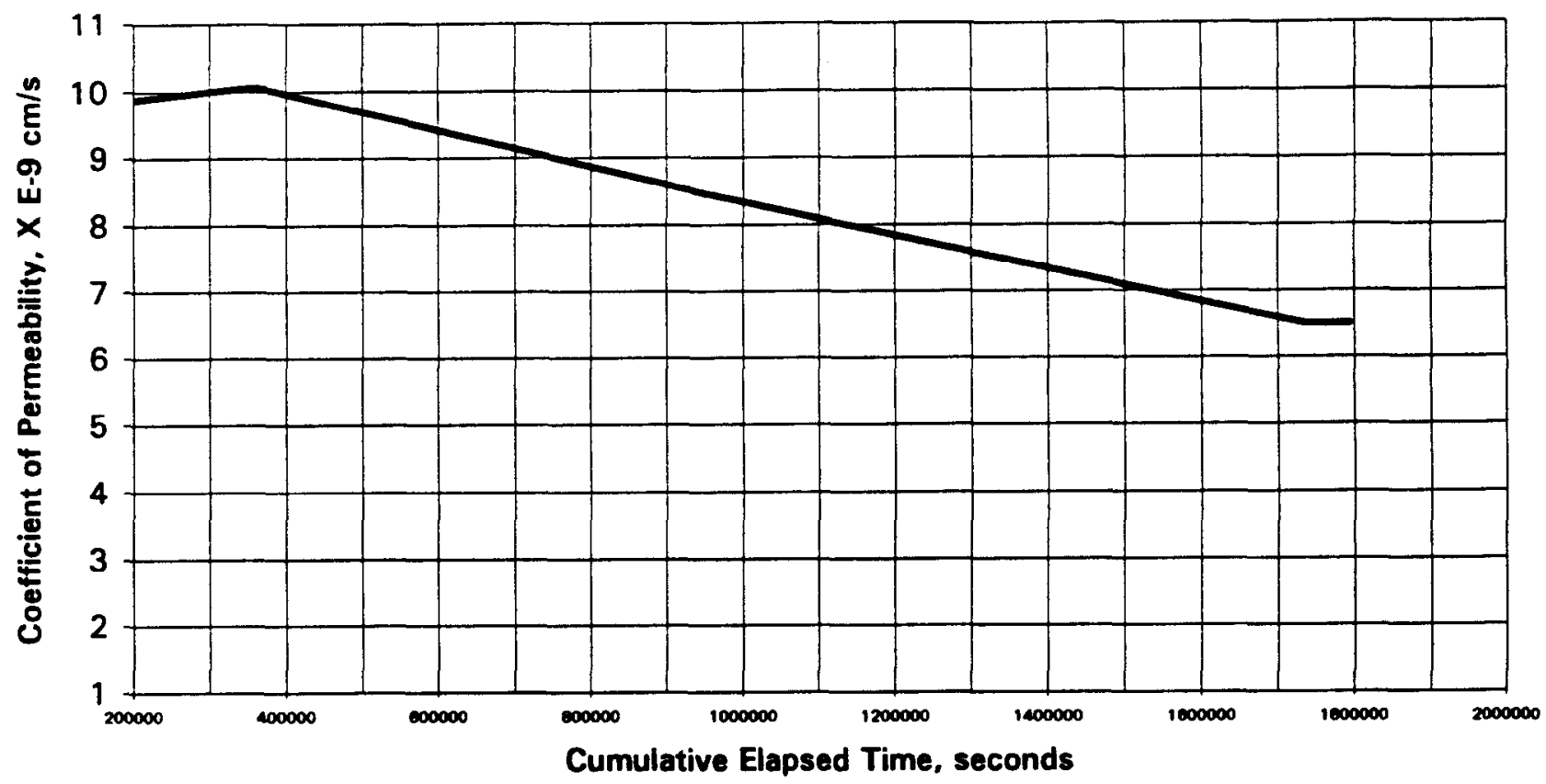




\section{8-DAY HYDRAULIC CONDUCTIVITY / PERMEABILITY ASTM D 5084}

PROJECT NAME: SAIC / Savannah River PROJECT NO. $\quad \mathbf{7 7 7 3 0 4 . 0 0 0 1 0 5 0 0}$
CLIENT SAMPLE NO. L322G00

IT LAB SAMPLE NO. ETDC-8112
Specimen diameter, $\mathrm{cm}$

\begin{tabular}{|l|l|}
\hline INITIAL FINAL \\
\hline
\end{tabular}
Specimen length, $\mathrm{cm}$

\subsection{1}
Wet weight of specimen, $g$.
10.17
$388.66 \quad 393.06$
Specimen cross-sect. area, $\mathrm{cm}^{\wedge} 2$
20.5122
Water content, \%
27.5
116.3
Wet unit weight, pcf
Dry unit weight, pcf
91.2
Estimated degree of saturation, \%
89.5
Estimated spec. gravity of solids
2.65
Hydraulic gradient
27.7
Min. consolidation stress, psi
2.0
Max. consolidation stress, psi
8.0
4.0
Total backpressure, psi
Permeant Fluid
Deaired DI Water
Sample molded
$12 / 21 / 98$
Sample set up
$1 / 21 / 99$

\section{\begin{tabular}{|ll}
\hline Coefficient of Permeability, $\mathrm{cm} / \mathrm{s}$ & $3.3 \mathrm{E}-09$ \\
\hline
\end{tabular}}

\section{PERMEABILITY vS TIME}

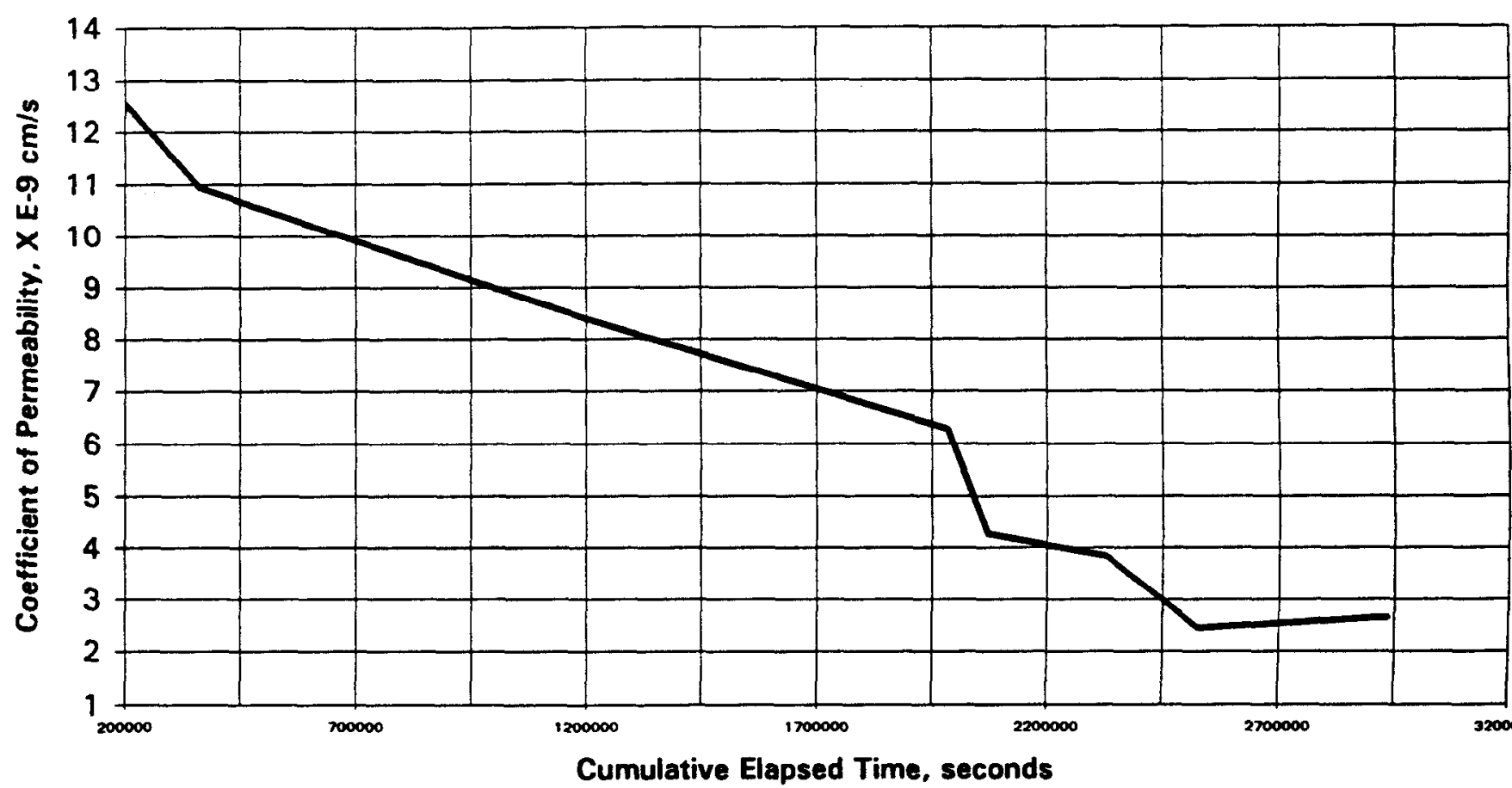




\section{8-DAY HYDRAULIC CONDUCTIVITY / PERMEABILITY ASTM D 5084}

PROJECT NAME: SAIC / Savannah River

PROJECT NO. $\quad 777304.00010500$
CLIENT SAMPLE NO. L332G00

IT LAB SAMPLE NO. ETDC-8113

\section{Specimen diameter, $\mathrm{cm}$}

Specimen length, $\mathrm{cm}$

Wet weight of specimen, $g$.

Specimen cross-sect. area, $\mathrm{cm}^{`} 2$

Water content, \%

Wet unit weight, pcf

Dry unit weight, pcf

Estimated degree of saturation, \%

Estimated spec. gravity of solids

INITIAL FINAL

\subsection{0}

10.16

$365.24 \quad 371.5$

20.4274

35.5

109.9

37.8

81.1

90.5

2.65
Hydraulic gradient

34.6

Min. consolidation stress, psi

Max. consolidation stress, psi

2.0

10.0

3.0

Total backpressure, psi

Permeant Fluid

Sample molded

Deaired DI Water

Sample set up

$12 / 21 / 98$

$1 / 21 / 99$

Seven tests performed

\section{PERMEABILITY vS TIME}

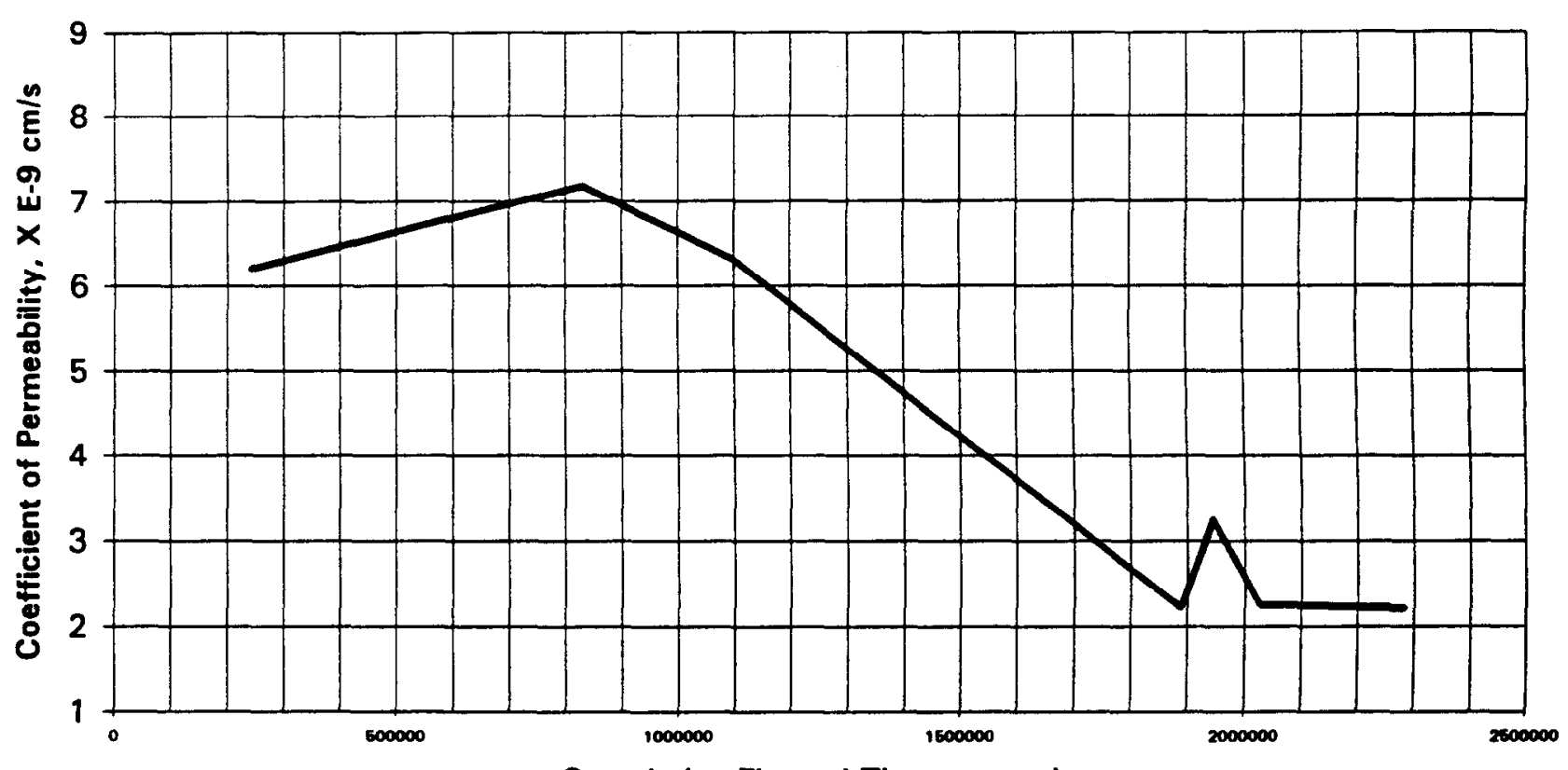

Cumulative Elapsed Time, seconds 


\section{8-DAY HYDRAULIC CONDUCTIVITY / PERMEABILITY ASTM D 5084}

PROJECT NAME: SAIC / Savannah River

PROJECT NO. $\quad \mathbf{7 7 7 3 0 4 . 0 0 0 1 0 5 0 0}$

\section{CLIENT SAMPLE NO. L342G00 \\ IT LAB SAMPLE NO. ETDC-8114}

Specimen diameter, $\mathrm{cm}$

Specimen length, $\mathrm{cm}$

Wet weight of specimen, $g$.

Specimen cross-sect. area, $\mathrm{cm}^{\star} 2$

Water content, \%

Wet unit weight, pcf

Dry unit weight, pcf

Estimated degree of saturation, \%

Estimated spec. gravity of solids

\begin{tabular}{l}
\hline INITIAL FINAL \\
\hline 5.08
\end{tabular}

10.17

$374.72 \quad 377.56$

20.2615

33.6

34.6

113.5

85.0

94.1

2.65
Hydraulic gradient

34.6

Min. consolidation stress, psi

Max. consolidation stress, psi

2.0

9.0

Total backpressure, psi

Permeant Fluid

3.0

Sample molded

Deaired DI Water

Sample set up

$12 / 21 / 98$

$1 / 21 / 99$

Eight tests performed

\section{Coefficient of Permeability, cm/s $2.5 E-08$}

\section{PERMEABILITY VS TIME}

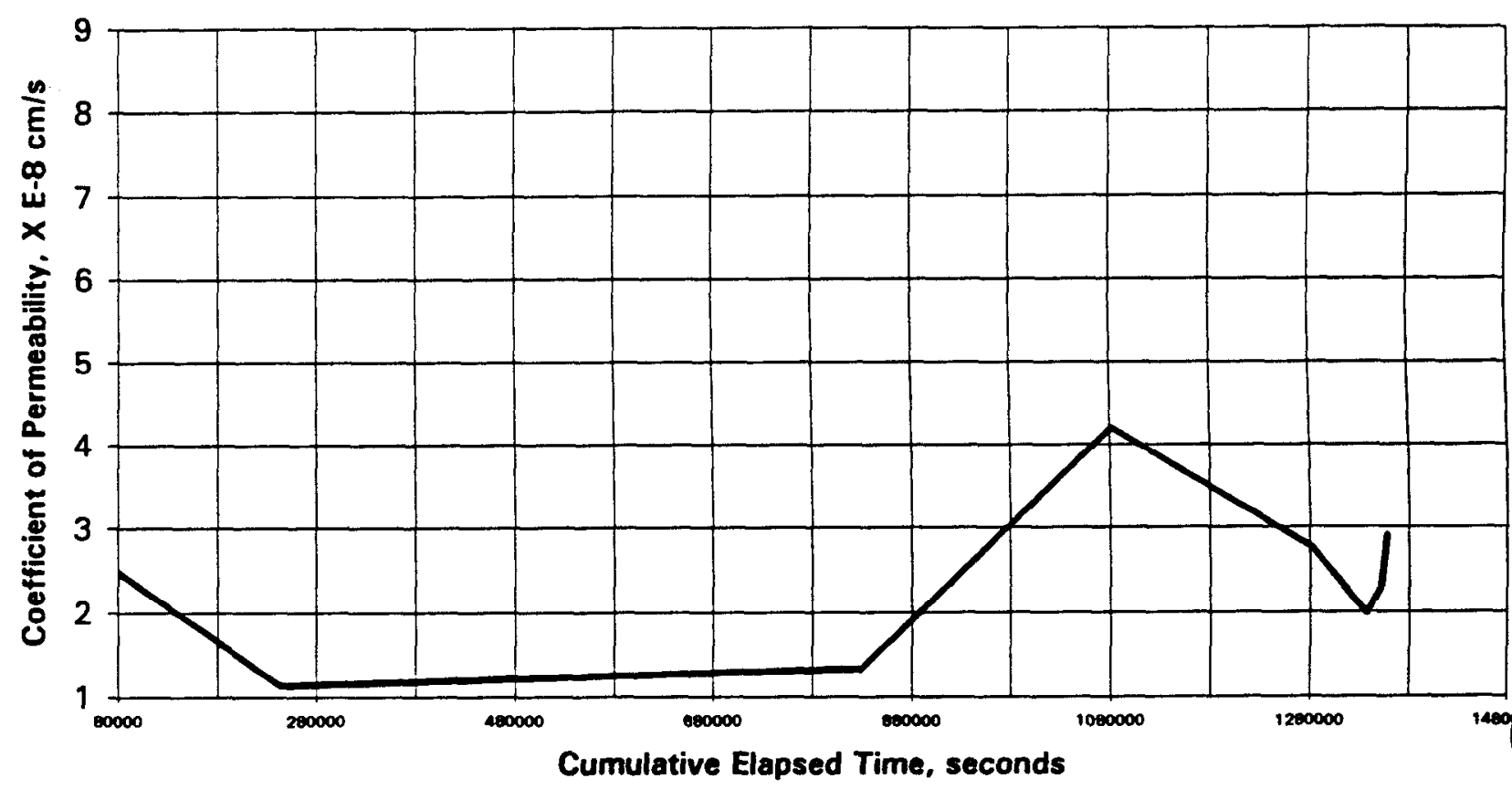




\section{8-DAY HYDRAULIC CONDUCTIVITY / PERMEABILITY ASTM D 5084}

PROJECT NAME: SAIC / Savannah River

PROJECT NO.

777304.00010500
CLIENT SAMPLE NO. L412G00

IT LAB SAMPLE NO. ETDC-8115

\begin{tabular}{llll} 
& INITIAL & FINAL \\
\cline { 2 - 2 } & 5.08 & \\
Specimen diameter, cm & 10.11 & \\
Specimen length, cm & 375.03 & 380.79 \\
Wet weight of specimen, g. & 20.2751 & \\
Specimen cross-sect. area, cm 2 & 2 & 32.3 \\
Water content, \% & 30.3 & \\
Wet unit weight, pcf & 114.3 & \\
Dry unit weight, pcf & 87.7 & \\
Estimated degree of saturation, \% & 90.6 & \\
Estimated spec. gravity of solids & 2.65 &
\end{tabular}

Hydraulic gradient

34.8

Min. consolidation stress, psi

2.0

Max. consolidation stress, psi

9.0

Total backpressure, psi

Permeant Fluid

Deaired DI Water

Sample molded

$12 / 21 / 98$

Sample set up

$1 / 21 / 99$

Six tests performed

\section{PERMEABILITY vs TIME}

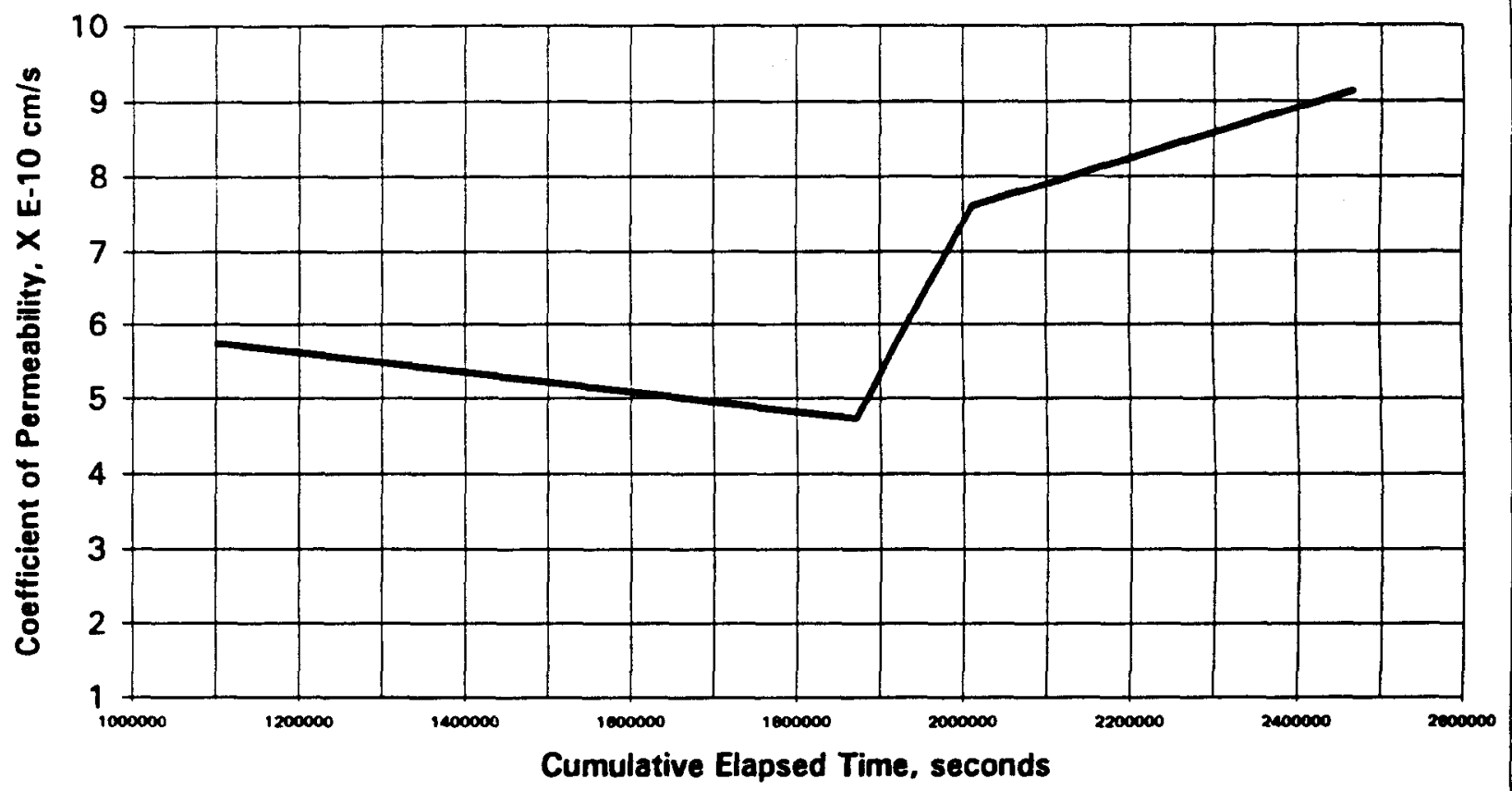




\section{8-DAY HYDRAULIC CONDUCTIVITY / PERMEABILITY ASTM D 5084}

PROJECT NAME: SAIC / Savannah River

PROJECT NO. $\quad 777304.00010500$

\section{CLIENT SAMPLE NO. L422G00 \\ IT LAB SAMPLE NO. ETDC-8116}

Specimen diameter, $\mathrm{cm}$

Specimen length, $\mathrm{cm}$

Wet weight of specimen, $g$.

Specimen cross-sect. area, $\mathrm{cm}^{-} 2$

Water content, \%

Wet unit weight, pcf

Dry unit weight, pcf

Estimated degree of saturation, \%

Estimated spec. gravity of solids

\begin{tabular}{|l|l|}
\hline INITIAL FINAL \\
\hline 5.08
\end{tabular}

10.16

$390.91 \quad 393.61$

20.2953

27.3

28.2

118.3

93.0

92.7

2.65
Hydraulic gradient

34.6

Min. consolidation stress, psi

Max. consolidation stress, psi $\quad \mathbf{9 . 0}$

Total backpressure, psi

Permeant Fluid

Sample molded

Sample set up

Five tests performed
2.0

3.0

Deaired DI Water

$12 / 21 / 98$

$1 / 21 / 99$

\section{\begin{tabular}{|ll}
\hline Coefficient of Permeability, cm/s & 7.3E-09 \\
\hline
\end{tabular}}

\section{PERMEABILITY vS TIME}

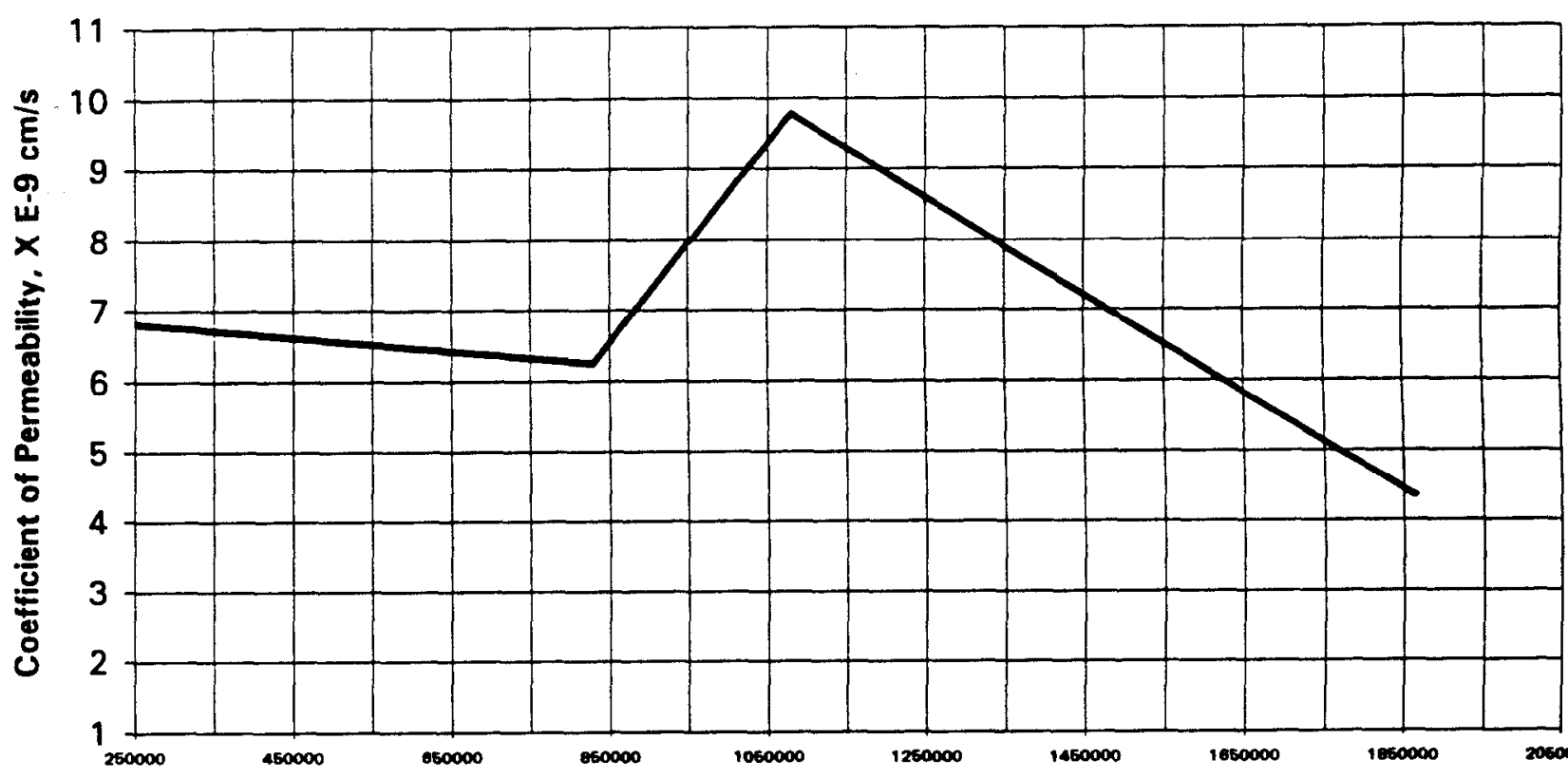

Cumulative Elapsed Time, seconds 


\section{8-DAY HYDRAULIC CONDUCTIVITY / PERMEABILITY ASTM D 5084}

PROJECT NAME:

SAIC / Savannah River

PROJECT NO.

777304.00010500
CLIENT SAMPLE NO. L432G00

IT LAB SAMPLE NO. ETDC-8117

\begin{tabular}{lll} 
& \\
Specimen diameter, $\mathrm{cm}$ & INITIAL FINAL \\
\hline
\end{tabular}

Specimen length, $\mathrm{cm}$

Wet weight of specimen, $g$.

10.16

363.49

Specimen cross-sect. area, $\mathrm{cm}^{-2} \quad 20.2548$

Water content, \%

Wet unit weight, pcf

Dry unit weight, pcf

35.4

110.3

81.5

Estimated degree of saturation, \% 90.9

Estimated spec. gravity of solids

2.65
368.8

\section{3}

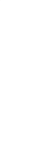

\section{Hydraulic gradient}

41.6

Min. consolidation stress, psi

Max. consolidation stress, psi

2.0

9.0

Total backpressure, psi

Permeant Fluid

3.0

Sample molded

Deaired DI Water

Sample set up

$12 / 21 / 98$

Eight tests performed

\section{PERMEABILITY vs TIME}

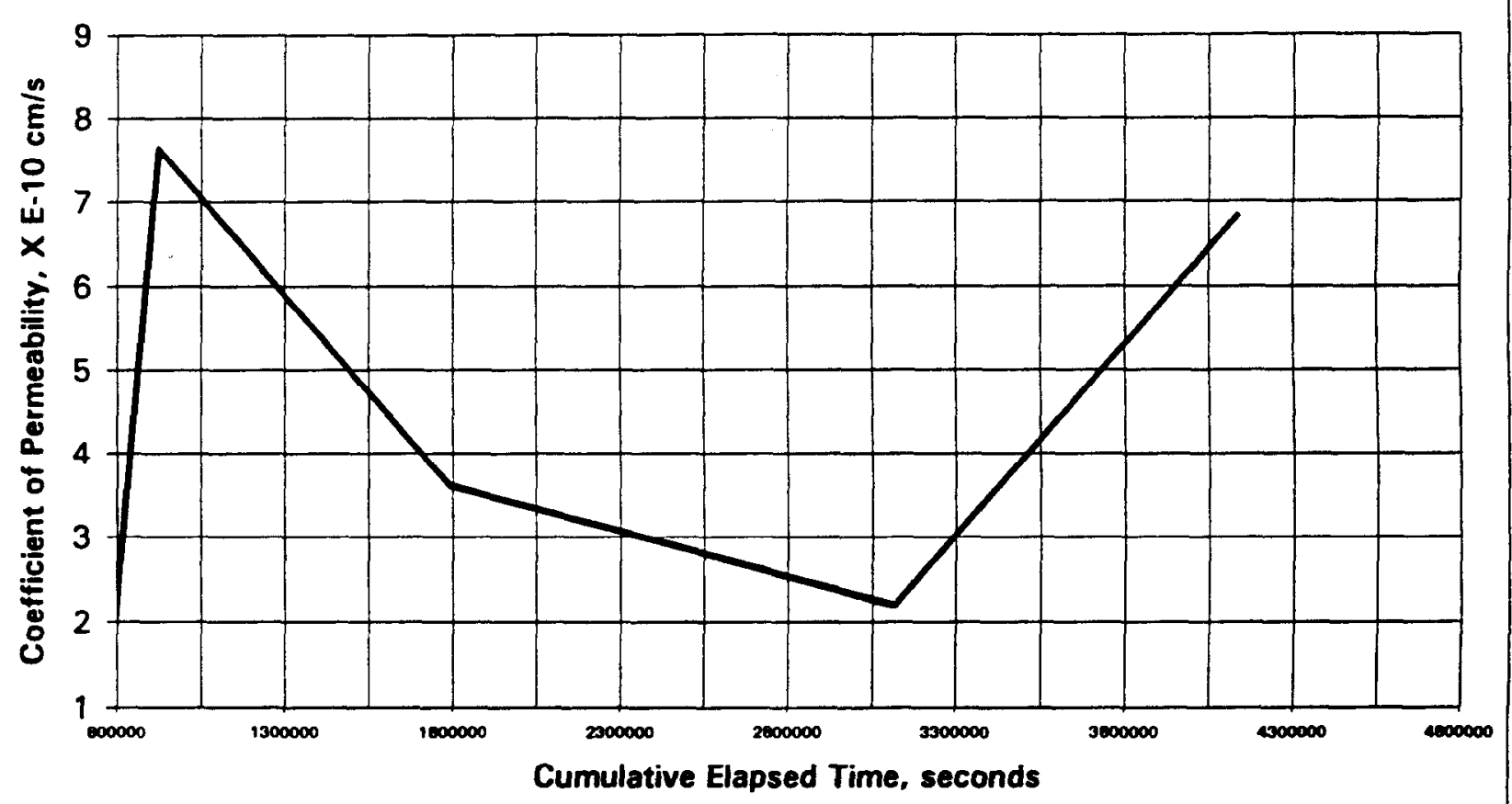




\section{8-DAY HYDRAULIC CONDUCTIVITY / PERMEABILITY ASTM D 5084}

PROJECT NAME: SAIC / Savannah River PROJECT NO. 777304.00010500
CLIENT SAMPLE NO. L442G00

IT LAB SAMPLE NO. ETDC-8118
Specimen diameter, $\mathrm{cm}$

Specimen length, $\mathrm{cm}$

Wet weight of specimen, $g$.

Specimen cross-sect. area, $\mathrm{cm}^{`} 2$

Water content, \%

Wet unit weight, pcf

Dry unit weight, pcf

Estimated degree of saturation, \%

Estimated spec. gravity of solids

\begin{tabular}{ll}
\hline INITIAL & FINAL \\
\hline 5.09 &
\end{tabular}

10.15

$378.38 \quad 381.73$

20.3224

31.9

114.6

86.8

93.5

2.65

33.1
Hydraulic gradient 27.7

Min. consolidation stress, psi

Max. consolidation stress, psi

2.0

9.0

Total backpressure, psi

Permeant Fluid

Deaired DI Water

Sample molded

$12 / 21 / 98$

Sample set up

$1 / 21 / 99$

Six tests performed

\section{\begin{tabular}{|ll}
\hline Coefficient of Permeability, cm/s & $1.9 \mathrm{E}-09$ \\
\hline
\end{tabular}}

\section{PERMEABILITY vs TIME}

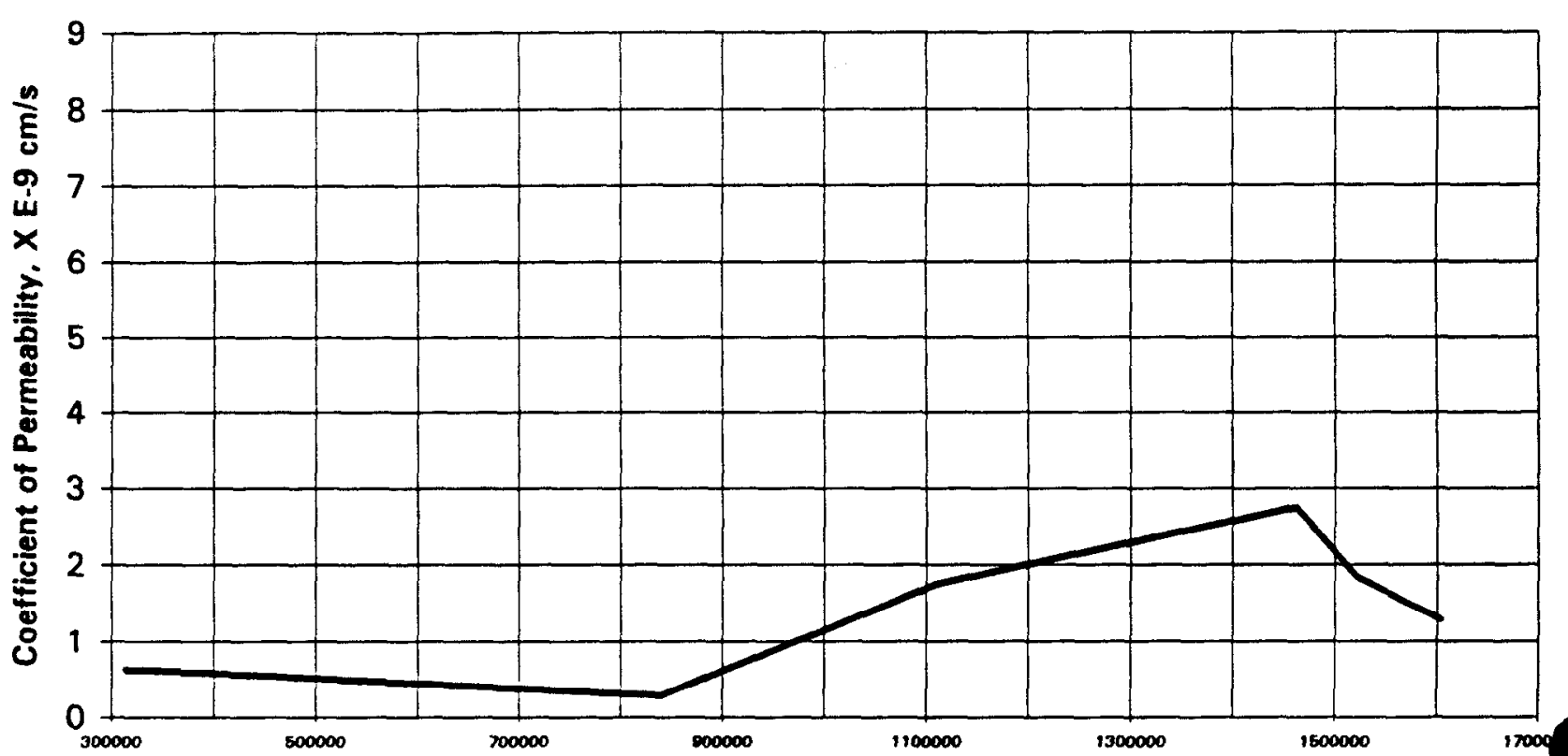

Cumulative Elapsed Time, seconds 


\section{Series \\ 90 Immersion Permeability Results}




\section{0-DAY SATURATED HYDRAULIC CONDUCTIVITY / PERMEABILITY ASTM D 5084}

PROJECT NAME:

PROJECT NO.
CLIENT SAMPLE NO. L313G00

IT LAB SAMPLE NO. ETDC-8135
SAIC / Savannah River

777304.00010500
FINAL

\subsection{8}

Specimen diameter, $\mathrm{cm}$

Specimen length, $\mathrm{cm}$

Wet weight of specimen, $g$.

7.65

641.44

45.1437

Specimen cross-sect. area, $\mathrm{cm}^{-2} 2$

Water content, \%

Wet unit weight, pcf

Dry unit weight, pcf

Estimated degree of saturation, \%

Estimated spec. gravity of solids

Initial conditions are post-soak, pre-test
116.0

91.0

88.9

2.65

\section{5 \\ 28.4}

Hydraulic gradient

646.09

(n)

Total backpressure, psi

Permeant Fluid

Min. consolidation stress, psi

18.4

Max. consolidation stress, psi

2.0

3.0

Deaired DI Water

Sample molded

$12 / 22 / 98$

Sample set up

$4 / 19 / 99$

\section{Twelve tests performed}

\section{Coefficient of Permeability, cm/s 1.4E-06}

\section{PERMEABILITY vS TIME}

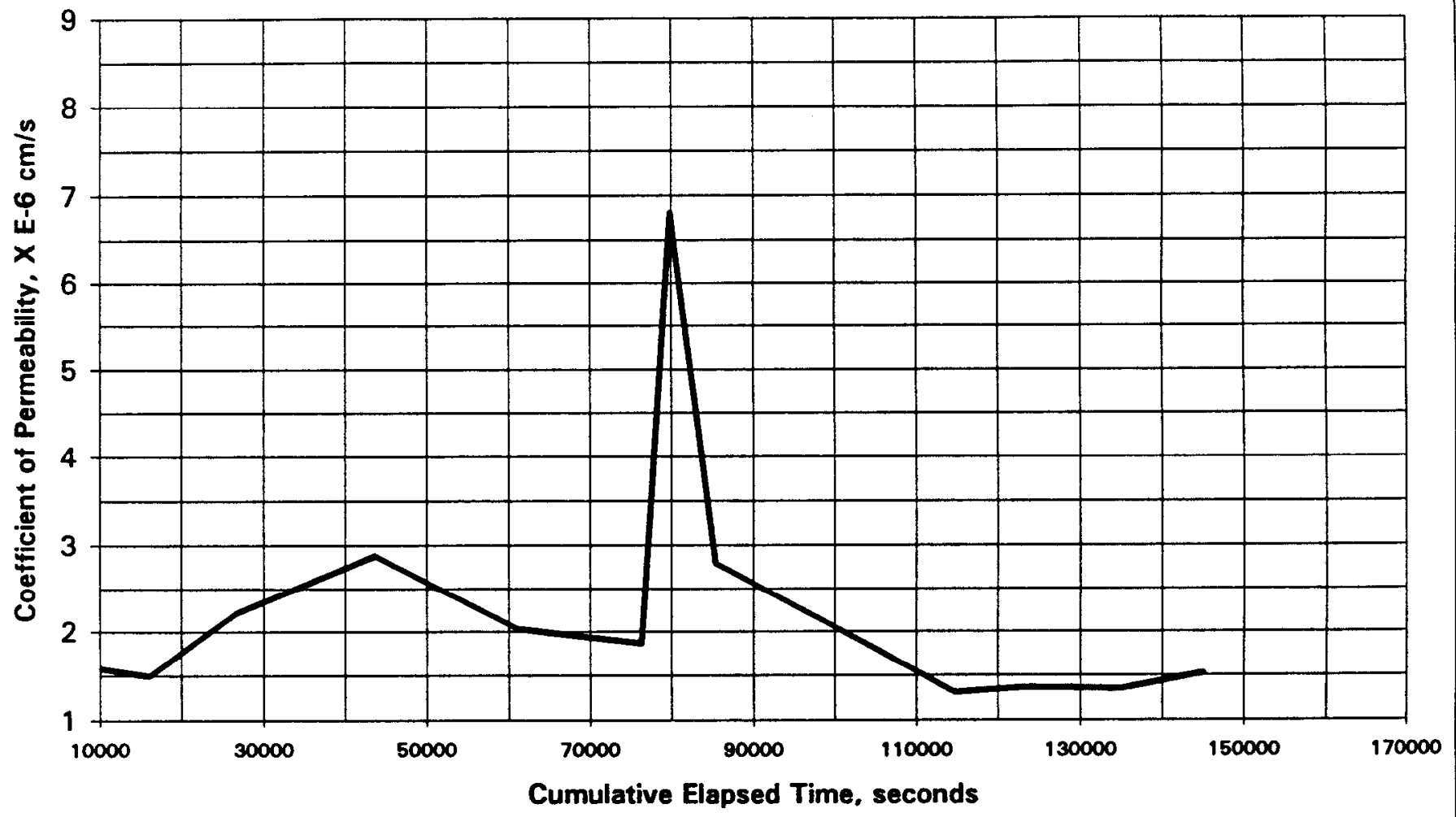




\section{0-DAY SATURATED HYDRAULIC CONDUCTIVITY / PERMEABILITY ASTM D 5084}

PROJECT NAME: SAIC / Savannah River PROJECT NO.
777304.00010500
CLIENT SAMPLE NO.

IT LAB SAMPLE NO.
L323G00

ETDC-8136

INITIAL FINAL

Specimen diameter, $\mathrm{cm}$

Specimen length, $\mathrm{cm}$

Wet weight of specimen, $g$.

Specimen cross-sect. area, $\mathrm{cm}^{\wedge} 2$

Water content, \%

Wet unit weight, pcf

Dry unit weight, pcf

Estimated degree of saturation, \%

Estimated spec. gravity of solids

Initial conditions are post-soak, pre-test

\subsection{0}

6.59

$562.39 \quad 563.61$

45.3052

28.5

117.6

91.5

93.6

2.65

28.8
Hydraulic gradient

21.4

Min. consolidation stress, psi

2.0

Max. consolidation stress, psi

3.0

7.0

Total backpressure, psi

Permeant Fluid

Deaired DI Water

Sample molded

$12 / 22 / 98$

Sample set up

$4 / 19 / 99$

Six tests performed

\section{Coefficient of Permeability, cm/s 2.6E-06}

\section{PERMEABILITY vs TIME}

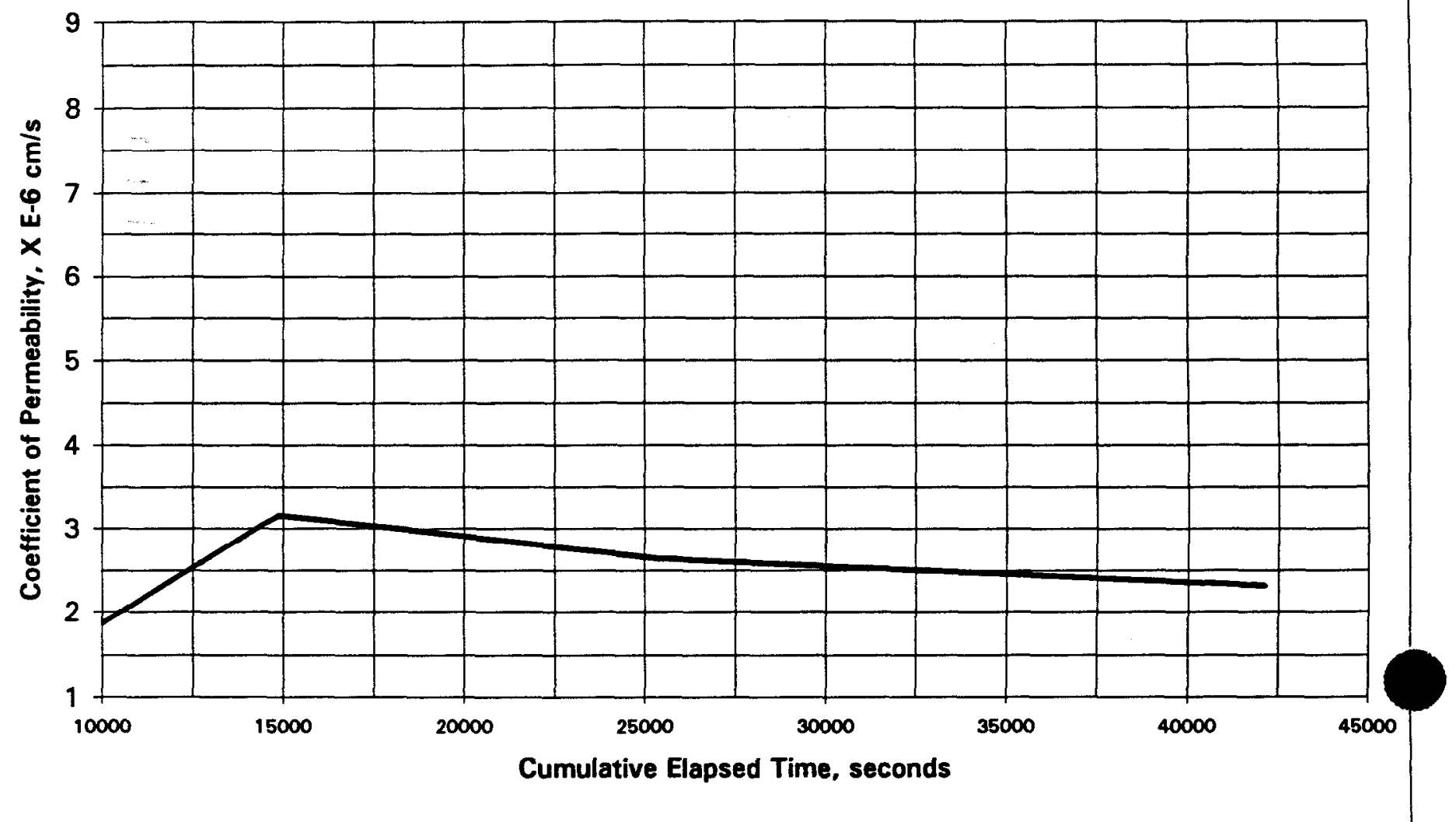




\section{0-DAY SATURATED HYDRAULIC CONDUCTIVITY / PERMEABILITY ASTM D 5084}

PROJECT NAME: SAIC / Savannah River

PROJECT NO.
CLIENT SAMPLE NO. L333G00

IT LAB SAMPLE NO. ETDC-8137
Specimen diameter, cm

Specimen length, $\mathrm{cm}$

Wet weight of specimen, $g$.

Specimen cross-sect. area, $\mathrm{cm}^{`} 2$

Water content, \%

Wet unit weight, pcf

Dry unit weight, pcf

Estimated degree of saturation, \%

Estimated spec. gravity of solids

Initial conditions are post-soak, pre-test

INITIAL FINAL

\subsection{9}

9.43

$774.62 \quad 778.4$

45.2093

31.5

32.1

113.5

86.3

91.0

2.65
Hydraulic gradient 14.9

Min. consolidation stress, psi

2.0

Max. consolidation stress, psi

3.0

7.0

Permeant Fluid

Deaired DI Water

Sample molded

$12 / 22 / 98$

Sample set up

$4 / 19 / 99$

Ten tests performed

\section{Coefficient of Permeability, $\mathrm{cm} / \mathrm{s} \quad 1.2 \mathrm{E}-06$}

\section{PERMEABILITY vs TIME}

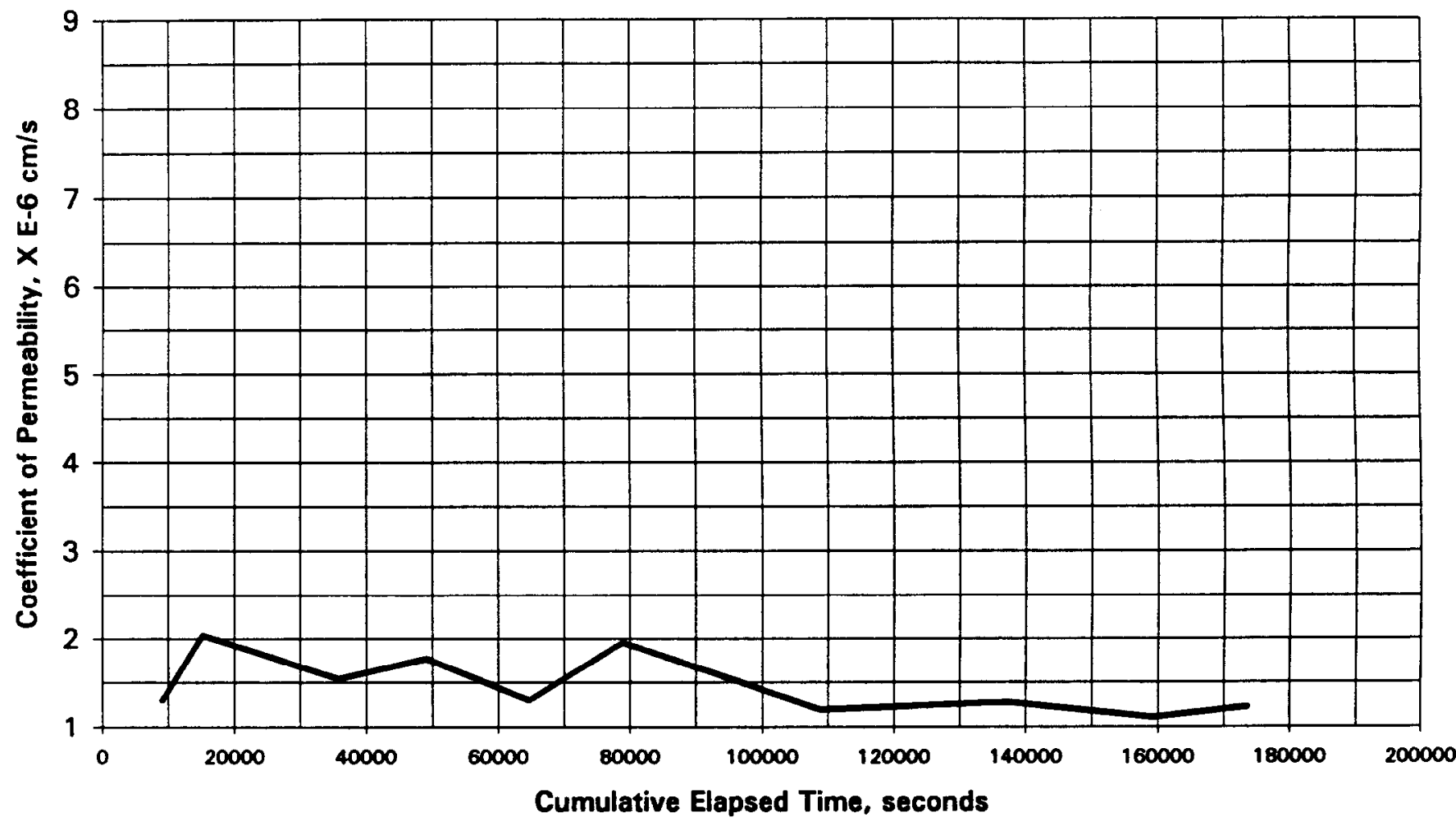




\section{0-DAY SATURATED HYDRAULIC CONDUCTIVITY / PERMEABILITY ASTM D 5084}

PROJECT NAME: $\quad$ SAIC / Savannah River PROJECT NO.
777304.00010500
CLIENT SAMPLE NO. L343G00

IT LAB SAMPLE NO. ETDC-8138

INITIAL FINAL

Specimen diameter, $\mathrm{cm}$

Specimen length, $\mathrm{cm}$

Wet weight of specimen, $g$.

Specimen cross-sect. area, $\mathrm{cm}^{-2} 2$

Water content, \%

Wet unit weight, pcf

Dry unit weight, pcf

Estimated degree of saturation, $\%$

Estimated spec. gravity of solids

Initial conditions are post-soak, pre-test

\subsection{0}

9.41

$776.08 \quad 778.25$

45.3456

32.4

113.5

32.8

85.7

92.3

2.65
Hydraulic gradient

Min. consolidation stress, psi

Max. consolidation stress, psi

Total backpressure, psi

Permeant Fluid

Sample molded

Sample set up

Seven tests performed
29.9

2.0

4.0

6.0

Deaired DI Water

12/22/98

$4 / 19 / 99$

\section{\begin{tabular}{|ll}
\hline Coefficient of Permeability, $\mathrm{cm} / \mathrm{s}$ & $1.6 \mathrm{E}-06$
\end{tabular}}

\section{PERMEABILITY vS TIME}

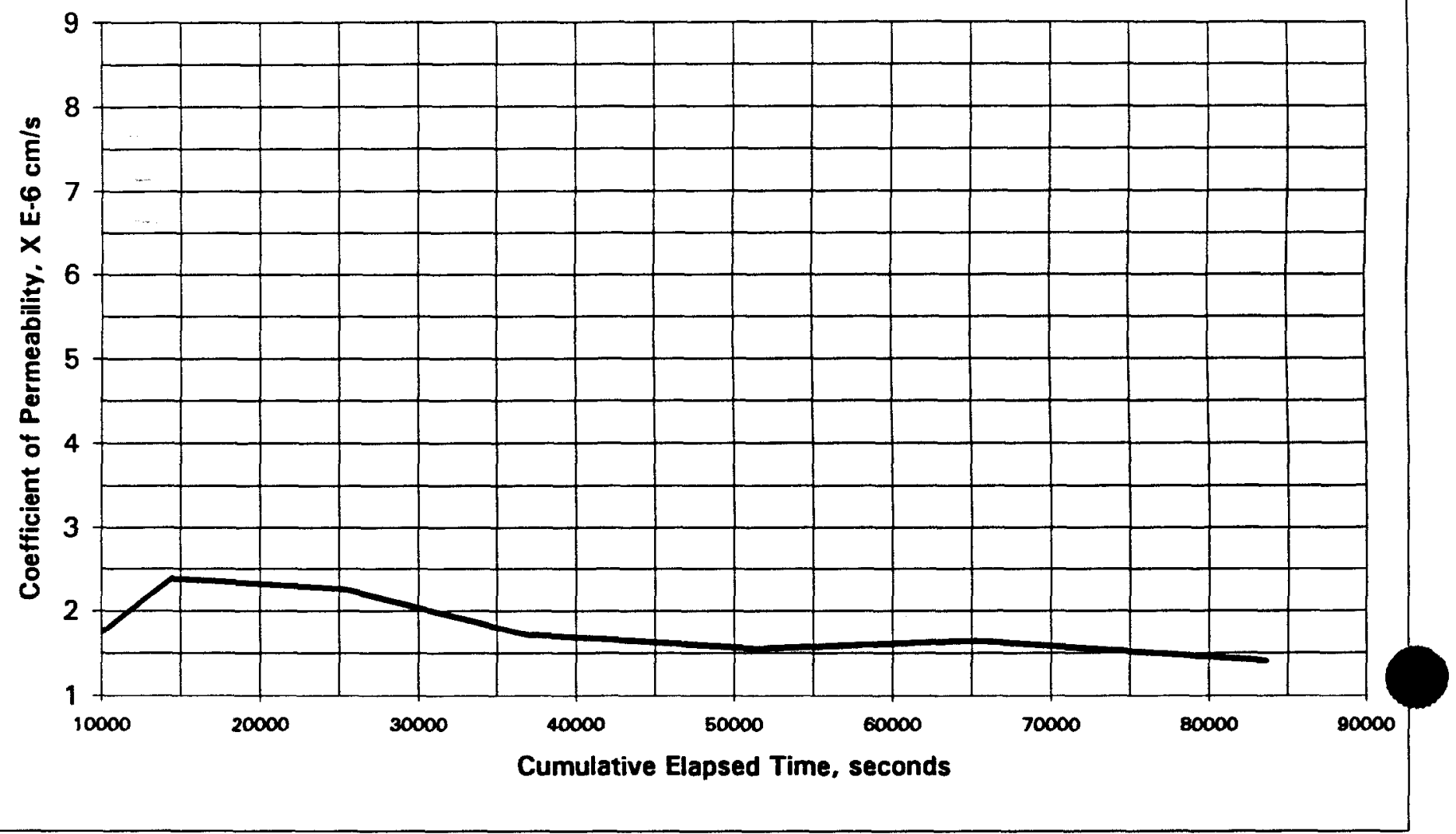




\section{0-DAY SATURATED HYDRAULIC CONDUCTIVITY / PERMEABILITY ASTM D 5084}

PROJECT NAME:

SAIC / Savannah River

PROJECT NO.
CLIENT SAMPLE NO. L413G00

IT LAB SAMPLE NO. ETDC-8139

\begin{tabular}{|l|l|}
\hline INITIAL & FINAL \\
\hline
\end{tabular}

Specimen diameter, $\mathrm{cm}$

Specimen length, $\mathrm{cm}$

7.58

Wet weight of specimen, $g$.

7.46

625.4

Specimen cross-sect. area, $\mathrm{cm}^{-2}$

Water content, \%

Wet unit weight, pcf

Dry unit weight, pcf

45.1286

28.6

115.9

90.1

90.7

2.65

Estimated spec. gravity of solids

Initial conditions are post-soak, pre-test

628.71

Hydraulic gradient

18.9

Min. consolidation stress, psi

2.0

Max. consolidation stress, psi $\quad 4.0$

Total backpressure, psi

Permeant Fluid

6.0

Sample molded

Deaired DI Water

Sample set up

$12 / 22 / 98$

Seven tests performed

\section{Coefficient of Permeability, cm/s $1.1 \mathrm{E}-06$}

\section{PERMEABILITY vS TIME}

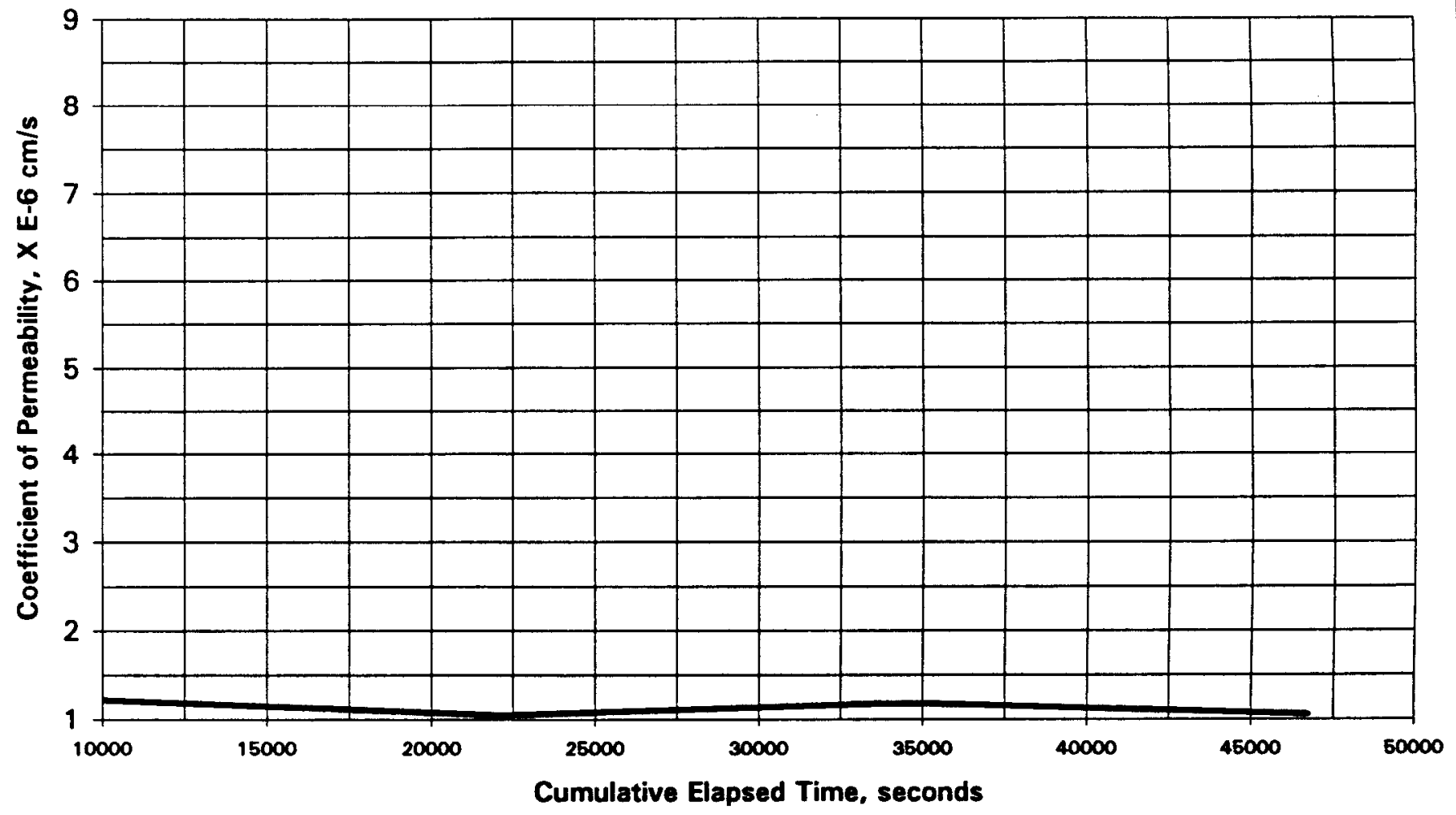




\section{0-DAY SATURATED HYDRAULIC CONDUCTIVITY / PERMEABILITY ASTM D 5084}

$\begin{array}{ll}\text { PROJECT NAME: } & \text { SAIC / Savannah River } \\ \text { PROJECT NO. } & 777304.00010500\end{array}$
CLIENT SAMPLE NO. L423G00

IT LAB SAMPLE NO. ETDC-8140

INITIAL FINAL

Specimen diameter, $\mathrm{cm}$

Specimen length, $\mathrm{cm}$

Wet weight of specimen, $g$.

Specimen cross-sect. area, $\mathrm{cm}^{-2} 2$

Water content, \%

Wet unit weight, pcf

Dry unit weight, pcf

Estimated degree of saturation, \%

Estimated spec. gravity of solids

Initial conditions are post-sosk, pre-test

\subsection{0}

5.83

$492.83 \quad 495.23$

45.3507

25.5

26.1

116.3

92.7

86.1

2.65
Hydraulic gradient

24.1

Min. consolidation stress, psi

2.0

Max. consolidation stress, psi

4.0

Total backpressure, psi

Permeant Fluid

Deaired DI Water

Sample molded

$12 / 22 / 98$

Sample set up

$4 / 19 / 99$

Seven tests performed

\section{PERMEABILITY vS TIME}






\section{0-DAY SATURATED HYDRAULIC CONDUCTIVITY / PERMEABILITY ASTM D 5084}

PROJECT NAME: SAIC / Savannah River

PROJECT NO. $\quad 777304.00010500$

\section{CLIENT SAMPLE NO. L433G00 \\ IT LAB SAMPLE NO. ETDC-8141}

\begin{tabular}{|l|l|}
\hline INITIAL FINAL \\
\hline 7.60
\end{tabular}

Specimen diameter, $\mathrm{cm}$

7.60

Specimen length, $\mathrm{cm}$

8.90

Wet weight of specimen, $\mathbf{g}$.

719.74

723.16

Specimen cross-sect. area, $\mathrm{cm}^{-2} 2$

Water content, \%

45.4164

33.7

34.3

Wet unit weight, pcf

Dry unit weight, pcf

111.1

83.1

Estimated degree of saturation, \% 90.1

Estimated spec. gravity of solids

2.65
Hydraulic gradient

15.8

Min. consolidation stress, psi

2.0

Max. consolidation stress, psi

6.0

4.0

Total backpressure, psi

Permeant Fluid

Deaired DI Water

Sample molded

$12 / 22 / 98$

Sample set up

$4 / 19 / 99$

Initial conditions are post-soak, pre-test

\section{PERMEABILITY vs TIME}

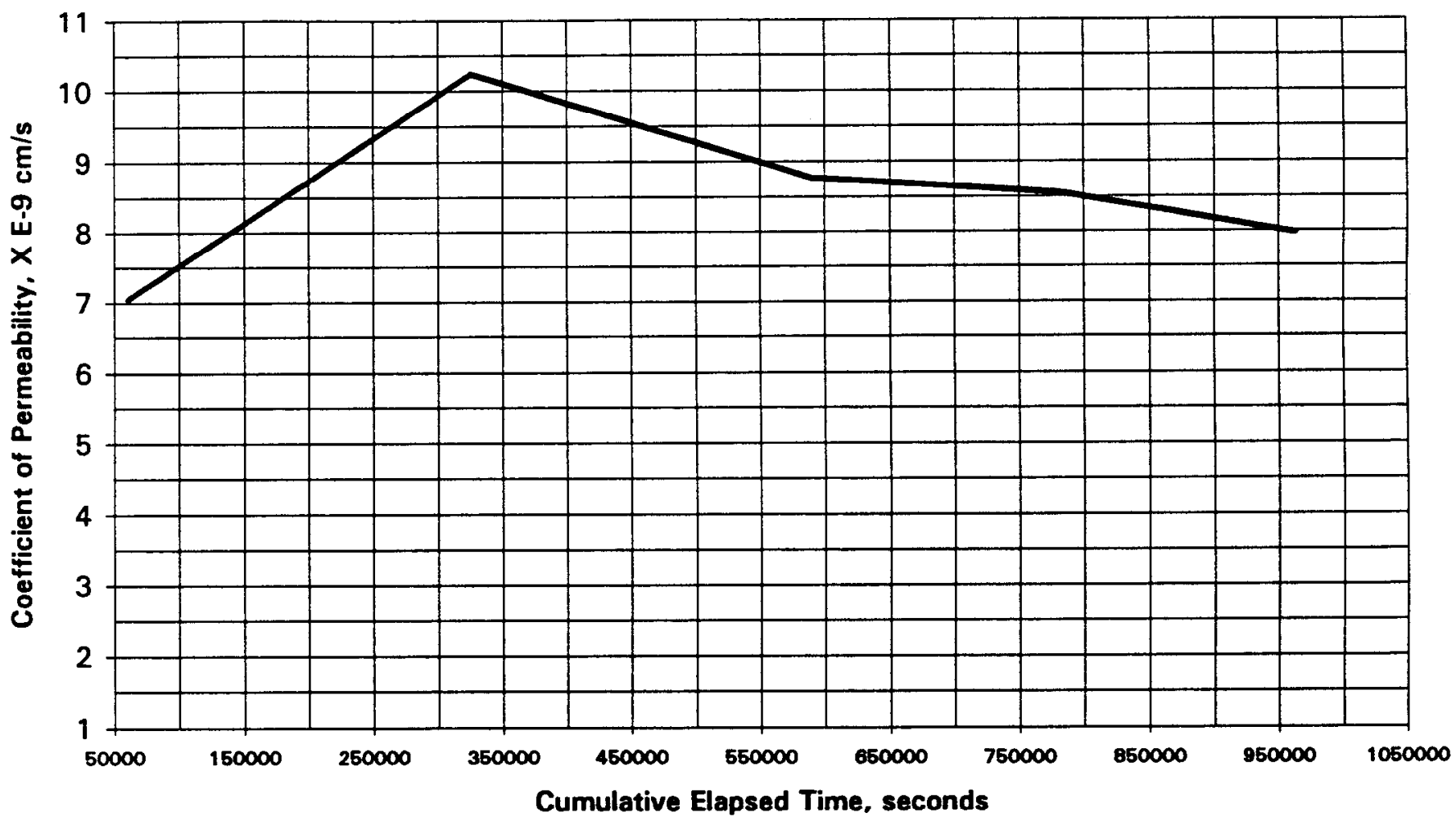




\section{0-DAY SATURATED HYDRAULIC CONDUCTIVITY / PERMEABILITY ASTM D 5084}

PROJECT NAME:

PROJECT NO.
SAIC / Savannah River

777304.00010500
CLIENT SAMPLE NO. L443G00

IT LAB SAMPLE NO. ETDC-8142

\begin{tabular}{lll|}
\cline { 2 - 2 } & INITIAL & FINAL \\
\cline { 2 - 2 } Specimen diameter, cm & 7.62 & \\
Specimen length, cm & 9.70 & \\
Wet weight of specimen, g. & 795.92 & 798.4 \\
Specimen cross-sect. area, cm -2 & 45.5885 & \\
Water content, \% & 30.1 & 30.5 \\
Wet unit weight, pcf & 112.4 & \\
Dry unit weight, pcf & 86.3 & \\
Estimated degree of saturation, \% & 87.2 & \\
Estimated spec. gravity of solids & 2.65 & \\
Initial conditions are post-soak, pre-test & &
\end{tabular}

Hydraulic gradient

14.5

Min. consolidation stress, psi

2.0

Max. consolidation stress, psi

6.0

Total backpressure, psi

6.0

Permeant Fluid

Deaired DI Water

Sample molded

$12 / 22 / 98$

Sample set up

$4 / 19 / 99$

Five tests performed

Four consecutive tests $+1-25 \%$ not achieved.

\section{PERMEABILITY vS TIME}

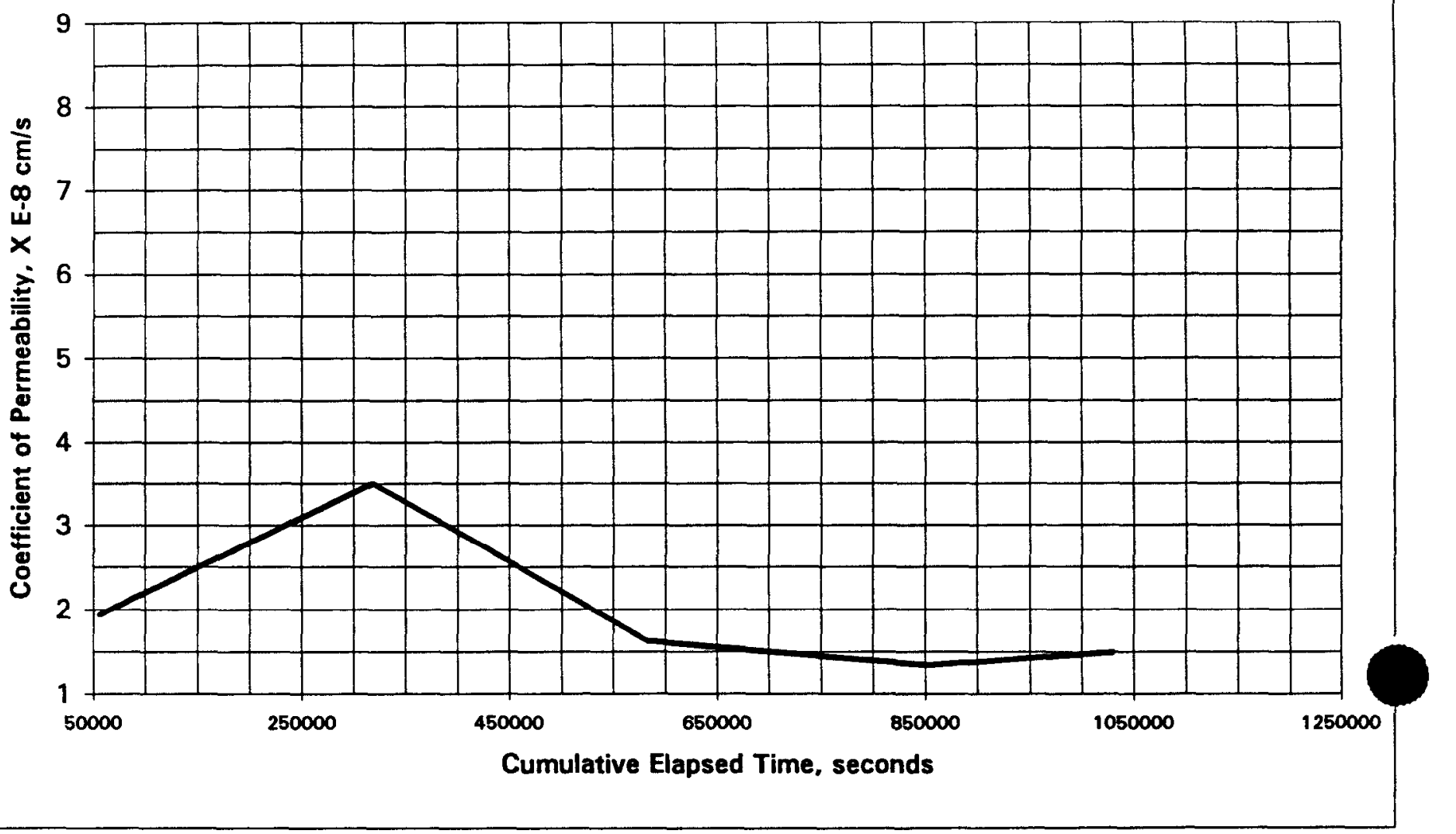


P Series

Leach Index 


\section{American Nuclear Society - 16.1 Static Leach Test}

Project: $\quad$ SRS Reactor Seepage Basin Stabilization

Sample Description: $\quad$ P412

Analyst: $\quad \mathrm{PRL}$

Date:

25-May-99

Contaminant:

Gross Beta

Initial Contaminant Conc. (pCi/g):

1340

Initial Contaminant Level (pCi):

499337.6

Monolith Height $(\mathrm{cm})$

10

Monolith Surface Area $\left(\mathrm{cm}^{\wedge} 2\right)$ :

176.72

Monolith Volume $\left(\mathrm{cm}^{\wedge} 3\right)$ :

196.35

Sample Weight (g):

Monolith Diameter (cm):

Leachate Volume $(\mathrm{mL})$ :
372.64

5

1800

\begin{tabular}{|c|c|c|c|c|c|c|c|}
\hline $\begin{array}{l}\text { Time } \\
\text { (hrs) }\end{array}$ & $\begin{array}{l}\text { Interval } \\
\text { Length } \\
\text { (s) }\end{array}$ & $\begin{array}{c}\text { Mean } \\
\text { Time } \\
\text { (s) }\end{array}$ & $\begin{array}{c}\text { Leachate } \\
\text { Conc. } \\
\text { (pCi/L) }\end{array}$ & $\begin{array}{c}\text { Amount } \\
\text { Leached } \\
(\mathrm{pCi}) \\
\end{array}$ & $\begin{array}{l}\text { Fraction } \\
\text { Leached }\end{array}$ & $\begin{array}{l}\text { Diffusivity } \\
\text { Coefficient } \\
\left(\mathrm{cm}^{\wedge} 2 / \mathrm{s}\right) \\
\end{array}$ & $\begin{array}{l}\text { Leach } \\
\text { Index }\end{array}$ \\
\hline 2 & 7200 & 1800 & 100 & 180.0000 & 0.000360 & $1.750 \mathrm{E}-11$ & 10.76 \\
\hline 7 & 18000 & 14835 & 179 & 322.2000 & 0.000645 & 7.394E-11 & 10.13 \\
\hline 24 & 61200 & 51231 & 150 & 270.0000 & 0.000541 & $1.551 \mathrm{E}-11$ & 10.81 \\
\hline 48 & 86400 & 125894 & 166 & 298.8000 & 0.000598 & $2.342 E-11$ & 10.63 \\
\hline 72 & 86400 & 213818 & 156 & 280.8000 & 0.000562 & 3.513E-11 & 10.45 \\
\hline 96 & 86400 & 300849 & 161 & 289.8000 & 0.000580 & $5.265 \mathrm{E}-11$ & 10.28 \\
\hline 120 & 86400 & 387596 & 75.1 & 135.1800 & 0.000271 & 1.476E-11 & 10.83 \\
\hline 456 & 1209600 & 939462 & 795 & 1431.0000 & 0.002866 & $2.045 \mathrm{E}-11$ & 10.69 \\
\hline 1128 & 2419200 & 2716550 & 1170 & 2106.0000 & 0.004218 & $3.202 E-11$ & 10.49 \\
\hline 2160 & 3715200 & 5768861 & 731 & 1315.8000 & 0.002635 & $1.126 \mathrm{E}-11$ & 10.95 \\
\hline & & & & & \multicolumn{2}{|c|}{ Average Leach Index: } & 10.60 \\
\hline
\end{tabular}




\section{American Nuclear Society - 16.1 Static Leach Test}

Project: $\quad$ SRS Reactor Seepage Basin Stabilization

Sample Description:

P422

Analyst: $\quad$ PRL

Date:

25-May-99

Contaminant:

Gross Beta

Initial Contaminant Conc. (pCi/g):

1340

Initial Contaminant Level (pCi):

504175

Monolith Height (cm):

10

Monolith Surface Area $\left(\mathrm{cm}^{\wedge} 2\right)$ :

176.72

Monolith Volume $\left(\mathrm{cm}^{\wedge} 3\right)$ :

196.35

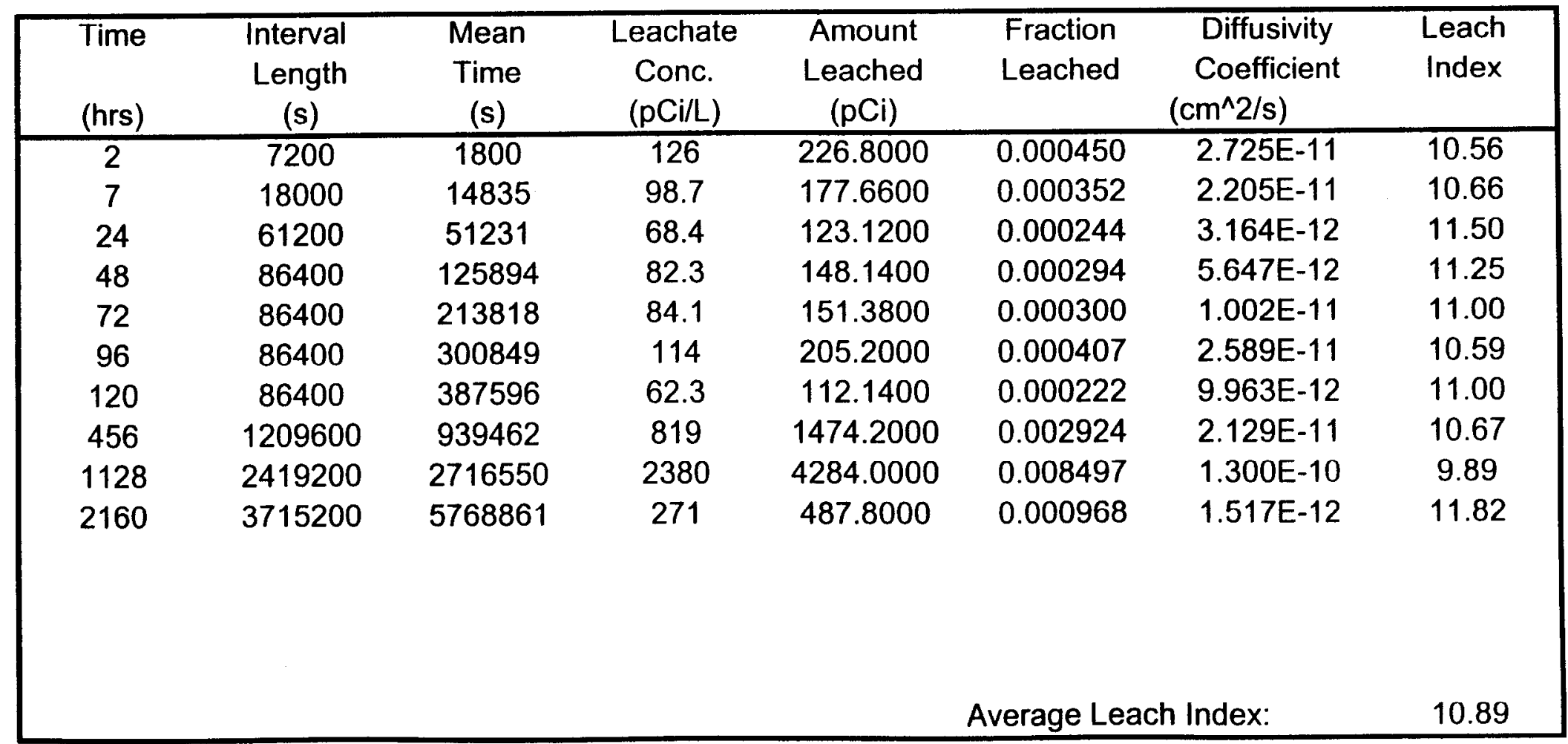




\section{American Nuclear Society - 16.1 Static Leach Test}

Project: $\quad$ SRS Reactor Seepage Basin Stabilization

Sample Description: $\quad$ P432

$\begin{array}{lll}\text { Analyst: PRL Date: 25-May-99 } & \text { Da }\end{array}$

Contaminant: Gross Beta

Initial Contaminant Conc. (pCi/g): $\quad 1340$

Initial Contaminant Level (pCi): $\quad 478353.2$

Monolith Height (cm):

10

Monolith Surface Area $\left(\mathrm{cm}^{\wedge} 2\right)$ : $\quad 176.72$

196.35

Sample Weight $(\mathrm{g})$ :

Monolith Diameter $(\mathrm{cm})$ :

Leachate Volume $(\mathrm{mL})$ :
356.98

5

1800

Monolith Volume $\left(\mathrm{cm}^{\wedge} 3\right)$ :

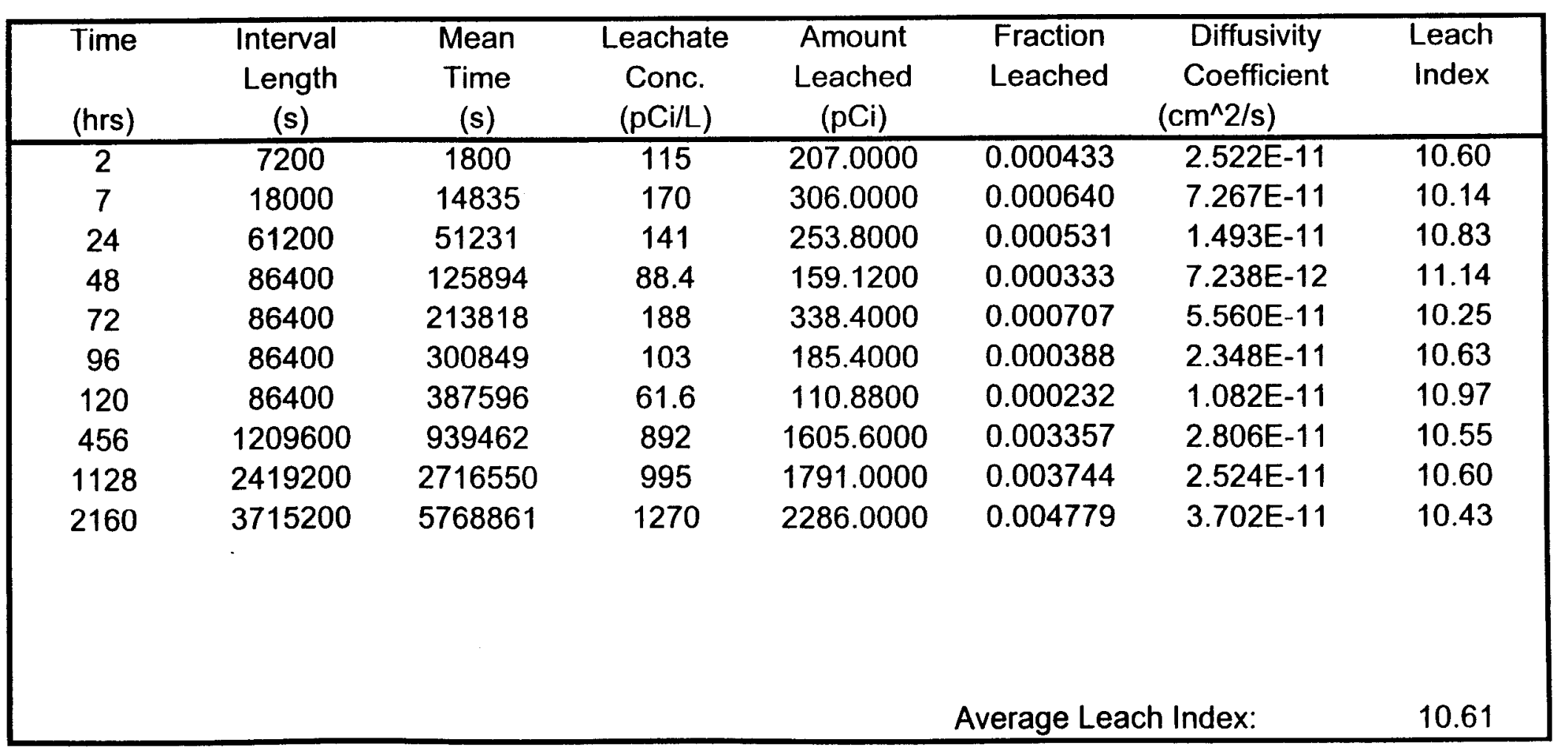




\section{American Nuclear Society - 16.1 Static Leach Test}

Project: $\quad$ SRS Reactor Seepage Basin Stabilization

Sample Description: $\quad \mathrm{P} 442$

$\begin{array}{lll}\text { Analyst: PRL 25-May-99 } & \text { Date: }\end{array}$

Contaminant: Gross Beta

Initial Contaminant Conc. (pCi/g): $\quad 1340$

Initial Contaminant Level (pCi): $\quad 488376.4$

Monolith Height $(\mathrm{cm})$ :

10

Monolith Surface Area $\left(\mathrm{cm}^{\wedge} 2\right)$ :

Monolith Volume $\left(\mathrm{cm}^{\wedge} 3\right)$ :

176.72

196.35

Sample Weight $(g)$ :

364.46

Monolith Diameter $(\mathrm{cm})$ :

5

Leachate Volume $(\mathrm{mL})$ :

1800

\begin{tabular}{|c|c|c|c|c|c|c|c|}
\hline $\begin{array}{l}\text { Time } \\
\text { (hrs) }\end{array}$ & $\begin{array}{l}\text { Interval } \\
\text { Length } \\
\text { (s) }\end{array}$ & $\begin{array}{l}\text { Mean } \\
\text { Time } \\
\text { (s) }\end{array}$ & $\begin{array}{l}\text { Leachate } \\
\text { Conc. } \\
\text { (pCi/L) }\end{array}$ & $\begin{array}{l}\text { Amount } \\
\text { Leached } \\
(\mathrm{pCi})\end{array}$ & $\begin{array}{l}\text { Fraction } \\
\text { Leached }\end{array}$ & $\begin{array}{l}\text { Diffusivity } \\
\text { Coefficient } \\
\left(\mathrm{cm}^{\wedge} 2 / \mathrm{s}\right)\end{array}$ & $\begin{array}{l}\text { Leach } \\
\text { Index }\end{array}$ \\
\hline 2 & 7200 & 1800 & 75.9 & 136.6200 & 0.000280 & $1.054 \mathrm{E}-11$ & 10.98 \\
\hline 7 & 18000 & 14835 & 23.1 & 41.5800 & 0.000085 & 1.287E-12 & 11.89 \\
\hline 24 & 61200 & 51231 & 123 & 221.4000 & 0.000453 & $1.090 \mathrm{E}-11$ & 10.96 \\
\hline 48 & 86400 & 125894 & 79.9 & 143.8200 & 0.000294 & $5.672 \mathrm{E}-12$ & 11.25 \\
\hline 72 & 86400 & 213818 & 171 & 307.8000 & 0.000630 & $4.413 E-11$ & 10.36 \\
\hline 96 & 86400 & 300849 & 31.2 & 56.1600 & 0.000115 & 2.067E-12 & 11.68 \\
\hline 120 & 86400 & 387596 & 52 & 93.6000 & 0.000192 & 7.397E-12 & 11.13 \\
\hline 456 & 1209600 & 939462 & 799 & 1438.2000 & 0.002945 & $2.160 \mathrm{E}-11$ & 10.67 \\
\hline 1128 & 2419200 & 2716550 & 890 & 1602.0000 & 0.003280 & $1.937 \mathrm{E}-11$ & 10.71 \\
\hline 2160 & 3715200 & 5768861 & 1310 & 2358.0000 & 0.004828 & $3.779 \mathrm{E}-11$ & 10.42 \\
\hline & & & & & \multicolumn{2}{|c|}{ Average Leach Index: } & 11.00 \\
\hline
\end{tabular}


Gross Alpha Leaching for the Bulk Fraction \#1 from PRSB Soil

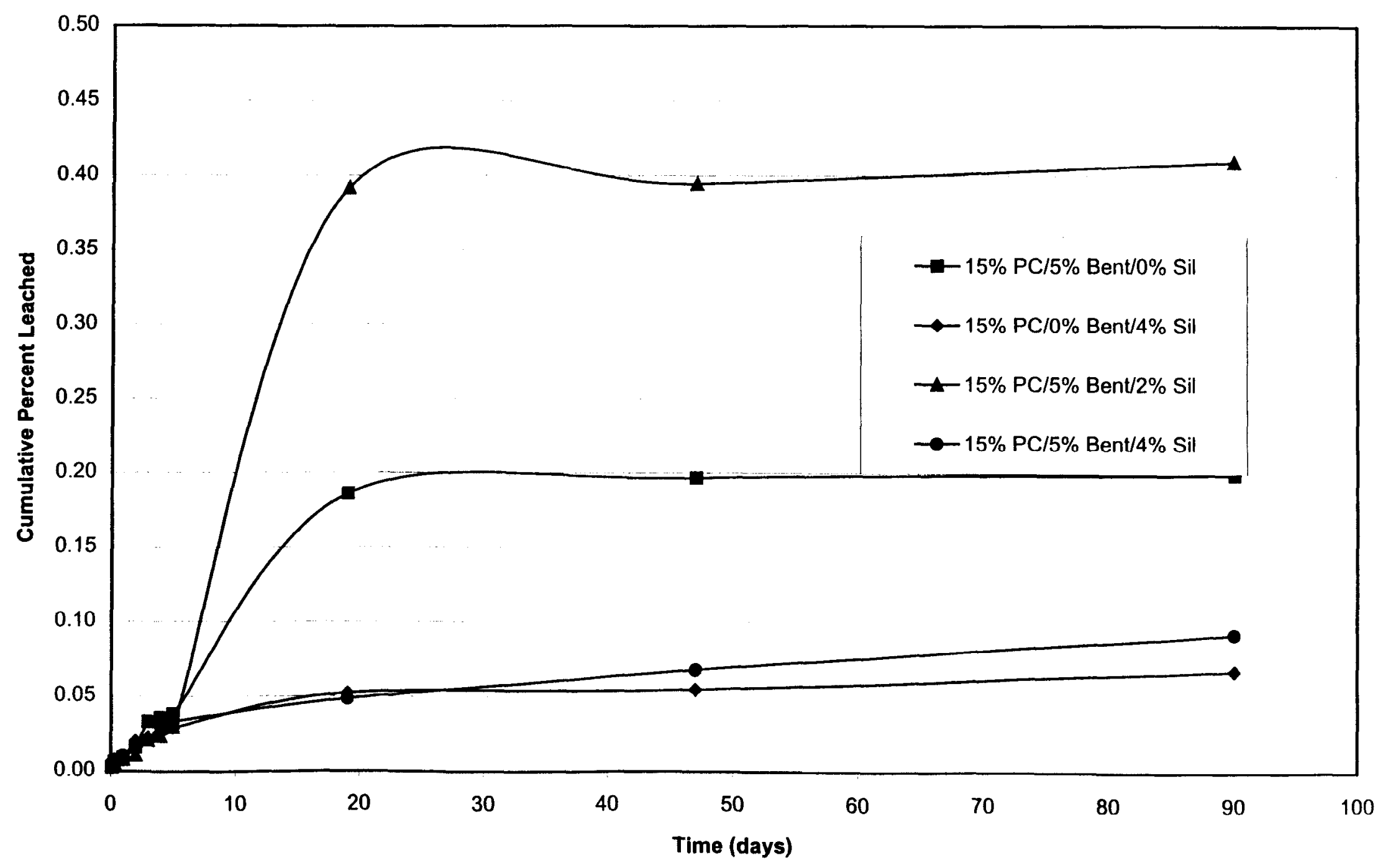




\section{American Nuclear Society - 16.1 Static Leach Test}

Project: $\quad$ SRS Reactor Seepage Basin Stabilization

Sample Description: $\quad$ P512

Analyst: $\quad \mathrm{PRL}$

Date:

25-May-99

Contaminant:

Gross Alpha

Initial Contaminant Conc. ( $\mathrm{pCi} / \mathrm{g}$ ):

17.9

Initial Contaminant Level (pCi):

Monolith Height $(\mathrm{cm})$ :

6827.955

10

Monolith Surface Area $\left(\mathrm{cm}^{\wedge} 2\right)$ :

176.72

196.35

Sample Weight (g):

381.45

Monolith Volume $\left(\mathrm{cm}^{\wedge} 3\right)$ :

Monolith Diameter $(\mathrm{cm})$ :

5

Leachate Volume $(\mathrm{mL})$ :

1800

\begin{tabular}{|c|c|c|c|c|c|c|c|}
\hline $\begin{array}{l}\text { Time } \\
\text { (hrs) }\end{array}$ & $\begin{array}{l}\text { Interval } \\
\text { Length } \\
\text { (s) }\end{array}$ & $\begin{array}{c}\text { Mean } \\
\text { Time } \\
\text { (s) }\end{array}$ & $\begin{array}{l}\text { Leachate } \\
\text { Conc. } \\
\text { (pCi/L) }\end{array}$ & $\begin{array}{c}\text { Amount } \\
\text { Leached } \\
\text { (pCi) }\end{array}$ & $\begin{array}{l}\text { Fraction } \\
\text { Leached }\end{array}$ & $\begin{array}{l}\text { Diffusivity } \\
\text { Coefficient } \\
\left(\mathrm{cm}^{\wedge} 2 / \mathrm{s}\right)\end{array}$ & $\begin{array}{l}\text { Leach } \\
\text { Index }\end{array}$ \\
\hline 2 & 7200 & 1800 & 0.1 & 0.1800 & 0.000026 & $9.359 \mathrm{E}-14$ & 13.03 \\
\hline 7 & 18000 & 14835 & 0.145 & 0.2610 & 0.000038 & $2.595 \mathrm{E}-13$ & 12.59 \\
\hline 24 & 61200 & 51231 & 0.1 & 0.1800 & 0.000026 & $3.687 \mathrm{E}-14$ & 13.43 \\
\hline 48 & 86400 & 125894 & 0.265 & 0.4770 & 0.000070 & 3.192E-13 & 12.50 \\
\hline 72 & 86400 & 213818 & 0.645 & 1.1610 & 0.000170 & $3.212 E-12$ & 11.49 \\
\hline 96 & 86400 & 300849 & 0.1 & 0.1800 & 0.000026 & $1.086 \mathrm{E}-13$ & 12.96 \\
\hline 120 & 86400 & 387596 & 0.1 & 0.1800 & 0.000026 & $1.400 \mathrm{E}-13$ & 12.85 \\
\hline 456 & 1209600 & 939462 & 5.59 & 10.0620 & 0.001474 & $5.408 \mathrm{E}-12$ & 11.27 \\
\hline 1128 & 2419200 & 2716550 & 0.408 & 0.7344 & 0.000108 & $2.083 E-14$ & 13.68 \\
\hline 2160 & 3715200 & 5768861 & 0.1 & 0.1800 & 0.000026 & 1.127E-15 & 14.95 \\
\hline
\end{tabular}




\section{American Nuclear Society - 16.1 Static Leach Test}

Project: SRS Reactor Seepage Basin Stabilization

Sample Description: P522

$\begin{array}{lll}\text { Analyst: PRL } & \text { Date: May-99 }\end{array}$

Contaminant: Gross Alpha

Initial Contaminant Conc. (pCi/g): $\quad 17.9$

Initial Contaminant Level (pCi): $\quad 6962.742$

Monolith Height $(\mathrm{cm})$ :

10

Monolith Surface Area $\left(\mathrm{cm}^{\wedge} 2\right)$ : $\quad 176.72$

Monolith Volume $\left(\mathrm{cm}^{\wedge} 3\right)$ : $\quad 196.35$

\begin{tabular}{|cccccccc|}
\hline Time & $\begin{array}{c}\text { Interval } \\
\text { Length } \\
(\mathrm{s})\end{array}$ & $\begin{array}{c}\text { Mean } \\
\text { Time } \\
(\mathrm{s})\end{array}$ & $\begin{array}{c}\text { Leachate } \\
\text { Conc. } \\
(\mathrm{pCi} / \mathrm{L})\end{array}$ & $\begin{array}{c}\text { Amount } \\
\text { Leached } \\
(\mathrm{pCi})\end{array}$ & $\begin{array}{c}\text { Fraction } \\
\text { Leached }\end{array}$ & $\begin{array}{c}\text { Diffusivity } \\
\text { Coefficient } \\
(\mathrm{cm} \text { ) } / \mathrm{s})\end{array}$ & $\begin{array}{c}\text { Leach } \\
\text { Index }\end{array}$ \\
\hline 2 & 7200 & 1800 & 0.1 & 0.1800 & 0.000026 & $9.000 \mathrm{E}-14$ & 13.05 \\
7 & 18000 & 14835 & 0.1 & 0.1800 & 0.000026 & $1.187 \mathrm{E}-13$ & 12.93 \\
24 & 61200 & 51231 & 0.1 & 0.1800 & 0.000026 & $3.545 \mathrm{E}-14$ & 13.45 \\
48 & 86400 & 125894 & 0.471 & 0.8478 & 0.000122 & $9.698 \mathrm{E}-13$ & 12.01 \\
72 & 86400 & 213818 & 0.1 & 0.1800 & 0.000026 & $7.424 \mathrm{E}-14$ & 13.13 \\
96 & 86400 & 300849 & 0.1 & 0.1800 & 0.000026 & $1.045 \mathrm{E}-13$ & 12.98 \\
120 & 86400 & 387596 & 0.156 & 0.2808 & 0.000040 & $3.275 \mathrm{E}-13$ & 12.48 \\
456 & 1209600 & 939462 & 0.892 & 1.6056 & 0.000231 & $1.324 \mathrm{E}-13$ & 12.88 \\
1128 & 2419200 & 2716550 & 0.1 & 0.1800 & 0.000026 & $1.203 \mathrm{E}-15$ & 14.92 \\
2160 & 3715200 & 5768861 & 0.507 & 0.9126 & 0.000131 & $2.785 \mathrm{E}-14$ & 13.56 \\
& & & & & & & \\
& & & & & & & \\
\end{tabular}




\section{American Nuclear Society - 16.1 Static Leach Test}

Project: $\quad$ SRS Reactor Seepage Basin Stabilization

Sample Description: P532

$\begin{array}{llll}\text { Analyst: PRL } & \text { Date: } & & \end{array}$

Contaminant: Gross Alpha Initial Contaminant Conc. (pCi/g): Initial Contaminant Level $(\mathrm{pCi})$ :

Monolith Height $(\mathrm{cm})$ :

Monolith Surface Area $\left(\mathrm{cm}^{\wedge} 2\right)$ :

Monolith Volume $\left(\mathrm{cm}^{\wedge} 3\right)$ :
17.9

17.9
6614.229
10
176.72
196.35

Sample Weight $(g)$ :

Monolith Diameter $(\mathrm{cm})$ :

Leachate Volume $(\mathrm{mL})$ :
369.51

5

1800

\begin{tabular}{|c|c|c|c|c|c|c|c|}
\hline $\begin{array}{l}\text { Time } \\
\text { (hrs) }\end{array}$ & $\begin{array}{l}\text { Interval } \\
\text { Length } \\
\text { (s) }\end{array}$ & $\begin{array}{c}\text { Mean } \\
\text { Time } \\
\text { (s) }\end{array}$ & $\begin{array}{l}\text { Leachate } \\
\text { Conc. } \\
\text { (pCi/L) }\end{array}$ & $\begin{array}{l}\text { Amount } \\
\text { Leached } \\
\text { (pCi) }\end{array}$ & $\begin{array}{l}\text { Fraction } \\
\text { Leached }\end{array}$ & $\begin{array}{c}\text { Diffusivity } \\
\text { Coefficient } \\
\left(\mathrm{cm}^{\wedge} 2 / \mathrm{s}\right)\end{array}$ & $\begin{array}{l}\text { Leach } \\
\text { Index }\end{array}$ \\
\hline 2 & 7200 & 1800 & 0.1 & 0.1800 & 0.000027 & $9.974 \mathrm{E}-14$ & 13.00 \\
\hline 7 & 18000 & 14835 & 0.1 & 0.1800 & 0.000027 & $1.315 \mathrm{E}-13$ & 12.88 \\
\hline 24 & 61200 & 51231 & 0.1 & 0.1800 & 0.000027 & $3.929 E-14$ & 13.41 \\
\hline 48 & 86400 & 125894 & 0.1 & 0.1800 & 0.000027 & $4.844 \mathrm{E}-14$ & 13.31 \\
\hline 72 & 86400 & 213818 & 0.364 & 0.6552 & 0.000099 & $1.090 \mathrm{E}-12$ & 11.96 \\
\hline 96 & 86400 & 300849 & 0.1 & 0.1800 & 0.000027 & $1.158 \mathrm{E}-13$ & 12.94 \\
\hline 120 & 86400 & 387596 & 0.227 & 0.4086 & 0.000062 & $7.685 \mathrm{E}-13$ & 12.11 \\
\hline 456 & 1209600 & 939462 & 13.3 & 23.9400 & 0.003619 & $3.263 \mathrm{E}-11$ & 10.49 \\
\hline 1128 & 2419200 & 2716550 & 0.1 & 0.1800 & 0.000027 & $1.333 \mathrm{E}-15$ & 14.88 \\
\hline 2160 & 3715200 & 5768861 & 0.552 & 0.9936 & 0.000150 & $3.658 \mathrm{E}-14$ & 13.44 \\
\hline & & & & & \multicolumn{2}{|c|}{ Average Leach Index: } & 12.84 \\
\hline
\end{tabular}




\section{American Nuclear Society - 16.1 Static Leach Test}

Project: $\quad$ SRS Reactor Seepage Basin Stabilization

Sample Description: P542

$\begin{array}{lll}\text { Analyst: PRL } & \text { Date: May-99 }\end{array}$

Contaminant: Gross Alpha Initial Contaminant Conc. (pCi/g):

Initial Contaminant Level ( $\mathrm{pCi})$ :

Monolith Height (cm):

Monolith Surface Area $\left(\mathrm{cm}^{\wedge} 2\right)$ :

Monolith Volume $\left(\mathrm{cm}^{\wedge} 3\right)$ :

17.9

6698.359

10

176.72

196.35

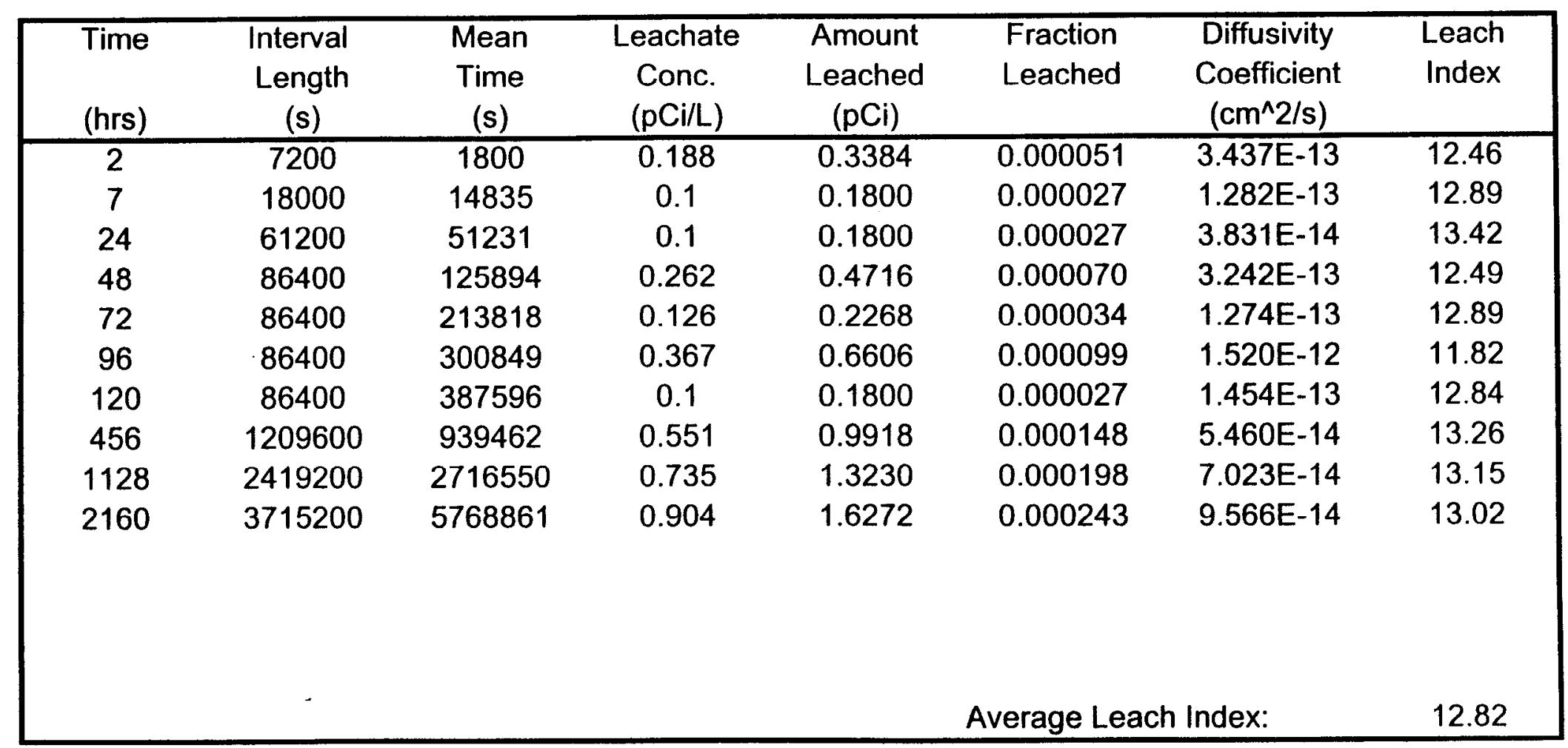




\section{Gross Beta Leaching for the Bulk Fraction \#1 from the PSRB Soil}

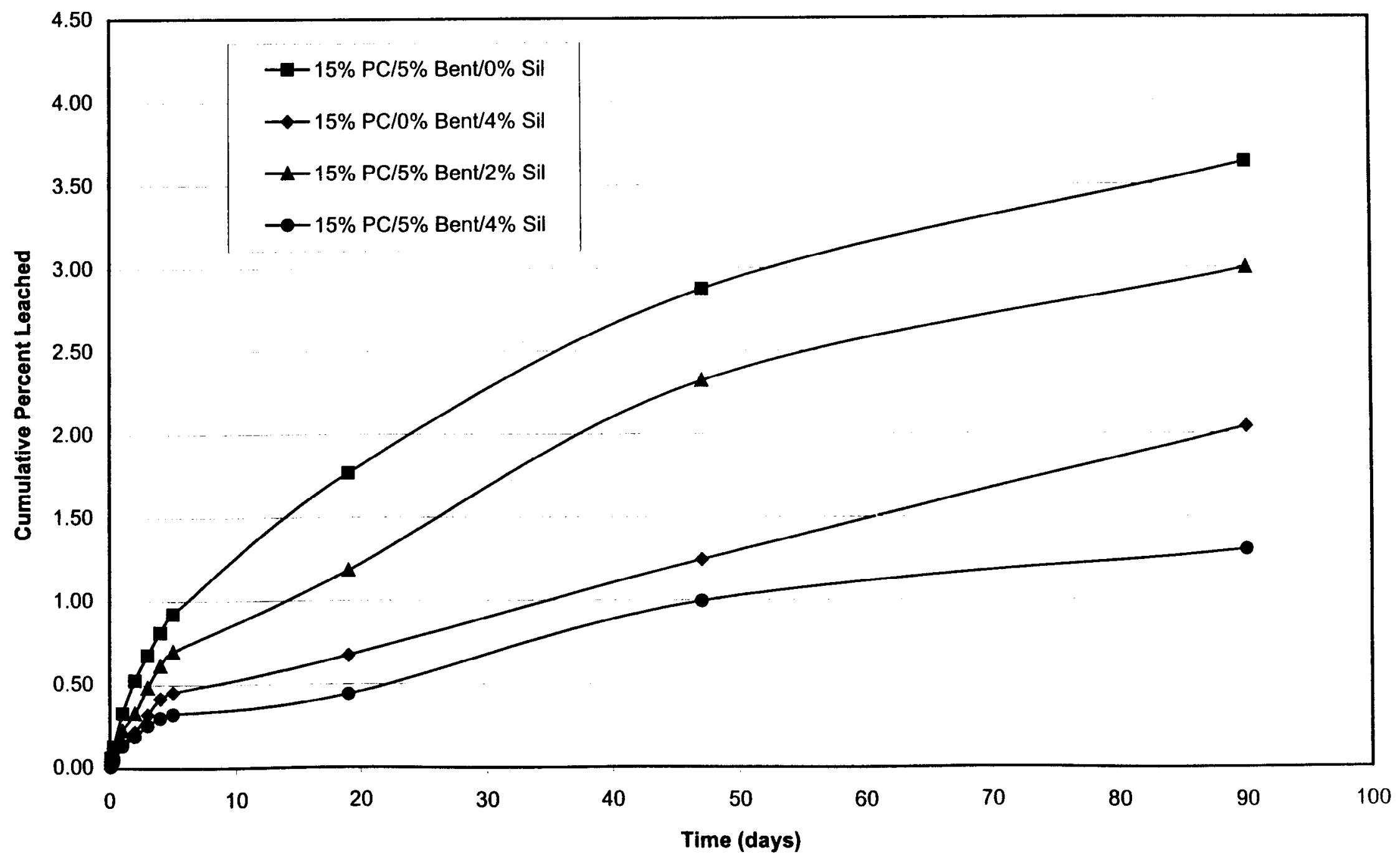




\section{American Nuclear Society - 16.1 Static Leach Test}

Project: $\quad$ SRS Reactor Seepage Basin Stabilization

Sample Description: P512

$\begin{array}{llll}\text { Analyst: PRL } & \text { Date: May-99 }\end{array}$

Contaminant: Gross Beta

Initial Contaminant Conc. (pCi/g): 338

Initial Contaminant Level (pCi): $\quad 128930.1$

Monolith Height $(\mathrm{cm})$ :

10

Monolith Surface Area $\left(\mathrm{cm}^{\wedge} 2\right)$ :

Monolith Volume $\left(\mathrm{cm}^{\wedge} 3\right)$ :

176.72

196.35

\begin{tabular}{|c|c|c|c|c|c|c|c|}
\hline $\begin{array}{l}\text { Time } \\
\text { (hrs) }\end{array}$ & $\begin{array}{l}\text { Interval } \\
\text { Length } \\
\text { (s) }\end{array}$ & $\begin{array}{c}\text { Mean } \\
\text { Time } \\
(\mathrm{s})\end{array}$ & $\begin{array}{l}\text { Leachate } \\
\text { Conc. } \\
\text { (pCi/L) }\end{array}$ & $\begin{array}{l}\text { Amount } \\
\text { Leached } \\
\text { (pCi) }\end{array}$ & $\begin{array}{l}\text { Fraction } \\
\text { Leached }\end{array}$ & $\begin{array}{l}\text { Diffusivity } \\
\text { Coefficient } \\
\left(\mathrm{cm}^{\wedge} 2 / \mathrm{s}\right)\end{array}$ & $\begin{array}{l}\text { Leach } \\
\text { Index }\end{array}$ \\
\hline 2 & 7200 & 1800 & 43.8 & 78.8400 & 0.000611 & $5.036 \mathrm{E}-11$ & 10.30 \\
\hline 7 & 18000 & 14835 & 49.5 & 89.1000 & 0.000691 & $8.481 \mathrm{E}-11$ & 10.07 \\
\hline 24 & 61200 & 51231 & 141 & 253.8000 & 0.001969 & $2.056 \mathrm{E}-10$ & 9.69 \\
\hline 48 & 86400 & 125894 & 143 & 257.4000 & 0.001996 & 2.607E-10 & 9.58 \\
\hline 72 & 86400 & 213818 & 107 & 192.6000 & 0.001494 & $2.479 E-10$ & 9.61 \\
\hline 96 & 86400 & 300849 & 96.6 & 173.8800 & 0.001349 & $2.843 E-10$ & 9.55 \\
\hline 120 & 86400 & 387596 & 79 & 142.2000 & 0.001103 & $2.450 \mathrm{E}-10$ & 9.61 \\
\hline 456 & 1209600 & 939462 & 605 & 1089.0000 & 0.008446 & $1.777 \mathrm{E}-10$ & 9.75 \\
\hline 1128 & 2419200 & 2716550 & 793 & 1427.4000 & 0.011071 & $2.207 E-10$ & 9.66 \\
\hline 2160 & 3715200 & 5768861 & 545 & 981.0000 & 0.007609 & $9.385 E-11$ & 10.03 \\
\hline & & & & & \multicolumn{2}{|c|}{ Average Leach Index: } & 9.78 \\
\hline
\end{tabular}




\section{American Nuclear Society - 16.1 Static Leach Test}

Project: SRS Reactor Seepage Basin Stabilization

Sample Description:

P522

Analyst: $\quad \mathrm{PRL}$

Gross Beta

Contaminant:

Initial Contaminant Conc. (pCi/g):
Initial Contaminant Level $(\mathrm{pCi})$ :

338

Monolith Height $(\mathrm{cm})$ :

131475.24

10

Monolith Surface Area $\left(\mathrm{cm}^{\wedge} 2\right)$ :

176.72

Monolith Volume $\left(\mathrm{cm}^{\wedge} 3\right)$ :

Date:

25-May-99

\begin{tabular}{|c|c|c|c|c|c|c|c|}
\hline $\begin{array}{l}\text { Time } \\
\text { (hrs) }\end{array}$ & $\begin{array}{l}\text { Interval } \\
\text { Length } \\
\text { (s) }\end{array}$ & $\begin{array}{l}\text { Mean } \\
\text { Time } \\
\text { (s) }\end{array}$ & $\begin{array}{l}\text { Leachate } \\
\text { Conc. } \\
\text { (pCi/L) }\end{array}$ & $\begin{array}{l}\text { Amount } \\
\text { Leached } \\
\text { (pCi) }\end{array}$ & $\begin{array}{l}\text { Fraction } \\
\text { Leached }\end{array}$ & $\begin{array}{l}\text { Diffusivity } \\
\text { Coefficient } \\
\left(\mathrm{cm}^{\wedge} 2 / \mathrm{s}\right)\end{array}$ & $\begin{array}{l}\text { Leach } \\
\text { Index }\end{array}$ \\
\hline 2 & 7200 & 1800 & 14.9 & 26.8200 & 0.000204 & $5.604 \mathrm{E}-12$ & 11.25 \\
\hline 7 & 18000 & 14835 & 36.4 & 65.5200 & 0.000498 & $4.410 \mathrm{E}-11$ & 10.36 \\
\hline 24 & 61200 & 51231 & 56.3 & 101.3400 & 0.000771 & $3.152 \mathrm{E}-11$ & 10.50 \\
\hline 48 & 86400 & 125894 & 50.8 & 91.4400 & 0.000695 & $3.164 \mathrm{E}-11$ & 10.50 \\
\hline 72 & 86400 & 213818 & 75.4 & 135.7200 & 0.001032 & $1.184 \mathrm{E}-10$ & 9.93 \\
\hline 96 & 86400 & 300849 & 71.1 & 127.9800 & 0.000973 & $1.481 \mathrm{E}-10$ & 9.83 \\
\hline 120 & 86400 & 387596 & 26 & 46.8000 & 0.000356 & $2.552 \mathrm{E}-11$ & 10.59 \\
\hline 456 & 1209600 & 939462 & 160 & 288.0000 & 0.002191 & 1.195E-11 & 10.92 \\
\hline 1128 & 2419200 & 2716550 & 417 & 750.6000 & 0.005709 & $5.868 \mathrm{E}-11$ & 10.23 \\
\hline 2160 & 3715200 & 5768861 & 581 & 1045.8000 & 0.007954 & $1.026 \mathrm{E}-10$ & 9.99 \\
\hline & & & & & \multicolumn{2}{|c|}{ Average Leach Index: } & 10.41 \\
\hline
\end{tabular}




\section{American Nuclear Society - 16.1 Static Leach Test}

Project: $\quad$ SRS Reactor Seepage Basin Stabilization

Sample Description: $\quad$ P532

$\begin{array}{lll}\text { Analyst: PRL 25-May-99 } & \text { Date: }\end{array}$

Contaminant: Gross Beta

Initial Contaminant Conc. (pCi/g): $\quad 338$

Initial Contaminant Level (pCi): $\quad 124894.38$

Monolith Height $(\mathrm{cm})$ :

10

Monolith Surface Area (cm²): $\quad 176.72$

$\begin{array}{ll}\text { Monolith Surface Area }\left(\mathrm{cm}^{\wedge} 2\right): & 176.72 \\ \text { Monolith Volume }\left(\mathrm{cm}^{\wedge} 3\right): & 196.35\end{array}$

Sample Weight (g):

Monolith Diameter $(\mathrm{cm})$ :

Leachate Volume $(\mathrm{mL})$ :

1800

\begin{tabular}{|c|c|c|c|c|c|c|c|}
\hline Time & $\begin{array}{l}\text { Interval } \\
\text { Length } \\
\text { (s) }\end{array}$ & $\begin{array}{c}\text { Mean } \\
\text { Time } \\
\text { (s) }\end{array}$ & $\begin{array}{l}\text { Leachate } \\
\text { Conc. } \\
\text { (pCi/L) }\end{array}$ & $\begin{array}{l}\text { Amount } \\
\text { Leached } \\
\text { (pCi) }\end{array}$ & $\begin{array}{l}\text { Fraction } \\
\text { Leached }\end{array}$ & $\begin{array}{c}\text { Diffusivity } \\
\text { Coefficient } \\
\left(\mathrm{cm}^{\wedge} 2 / \mathrm{s}\right)\end{array}$ & $\begin{array}{l}\text { Leach } \\
\text { Index }\end{array}$ \\
\hline 2 & 7200 & 1800 & 24.3 & 43.7400 & 0.000350 & $1.652 \mathrm{E}-11$ & 10.78 \\
\hline 7 & 18000 & 14835 & 37.1 & 66.7800 & 0.000535 & $5.077 \mathrm{E}-11$ & 10.29 \\
\hline 24 & 61200 & 51231 & 95.1 & 171.1800 & 0.001371 & $9.966 \mathrm{E}-11$ & 10.00 \\
\hline 48 & 86400 & 125894 & 70.6 & 127.0800 & 0.001017 & $6.772 \mathrm{E}-11$ & 10.17 \\
\hline 72 & 86400 & 213818 & 108 & 194.4000 & 0.001557 & $2.691 \mathrm{E}-10$ & 9.57 \\
\hline 96 & 86400 & 300849 & 90.5 & 162.9000 & 0.001304 & $2.659 \mathrm{E}-10$ & 9.58 \\
\hline 120 & 86400 & 387596 & 58.2 & 104.7600 & 0.000839 & $1.417 \mathrm{E}-10$ & 9.85 \\
\hline 456 & 1209600 & 939462 & 336 & 604.8000 & 0.004842 & $5.840 \mathrm{E}-11$ & 10.23 \\
\hline 1128 & 2419200 & 2716550 & 790 & 1422.0000 & 0.011386 & $2.334 \mathrm{E}-10$ & 9.63 \\
\hline 2160 & 3715200 & 5768861 & 468 & 842.4000 & 0.006745 & 7.375E-11 & 10.13 \\
\hline & & & & & \multicolumn{2}{|c|}{ Average Leach Index: } & 10.02 \\
\hline
\end{tabular}




\section{American Nuclear Society - 16.1 Static Leach Test}

Project: $\quad$ SRS Reactor Seepage Basin Stabilization

Sample Description:

P542

Analyst: $\quad \mathrm{PRL}$

Date:

25-May-99

Contaminant:

Gross Beta

Initial Contaminant Conc. (pCi/g):

338

Initial Contaminant Level (pCi):

126482.98

Monolith Height $(\mathrm{cm})$ :

10

Monolith Surface Area $\left(\mathrm{cm}^{\wedge} 2\right)$ :

176.72

Monolith Volume $\left(\mathrm{cm}^{\wedge} 3\right)$ :

196.35

\begin{tabular}{|cccccccc|}
\hline Time & $\begin{array}{c}\text { Interval } \\
\text { Length } \\
(\mathrm{s})\end{array}$ & $\begin{array}{c}\text { Mean } \\
\text { Time } \\
(\mathrm{s})\end{array}$ & $\begin{array}{c}\text { Leachate } \\
\text { Conc. } \\
(\mathrm{pCi} / \mathrm{L})\end{array}$ & $\begin{array}{c}\text { Amount } \\
\text { Leached } \\
(\mathrm{pCi})\end{array}$ & $\begin{array}{c}\text { Fraction } \\
\text { Leached }\end{array}$ & $\begin{array}{c}\text { Diffusivity } \\
\text { Coefficient } \\
\left(\mathrm{cm}^{\wedge} 2 / \mathrm{s}\right)\end{array}$ & $\begin{array}{c}\text { Leach } \\
\text { Index }\end{array}$ \\
\hline 2 & 7200 & 1800 & 7.04 & 12.6720 & 0.000100 & $1.352 \mathrm{E}-12$ & 11.87 \\
7 & 18000 & 14835 & 20.7 & 37.2600 & 0.000295 & $1.541 \mathrm{E}-11$ & 10.81 \\
24 & 61200 & 51231 & 63.6 & 114.4800 & 0.000905 & $4.346 \mathrm{E}-11$ & 10.36 \\
48 & 86400 & 125894 & 41.8 & 75.2400 & 0.000595 & $2.315 \mathrm{E}-11$ & 10.64 \\
72 & 86400 & 213818 & 46.9 & 84.4200 & 0.000667 & $4.949 \mathrm{E}-11$ & 10.31 \\
96 & 86400 & 300849 & 30 & 54.0000 & 0.000427 & $2.849 \mathrm{E}-11$ & 10.55 \\
120 & 86400 & 387596 & 15.9 & 28.6200 & 0.000226 & $1.031 \mathrm{E}-11$ & 10.99 \\
456 & 1209600 & 939462 & 82.4 & 148.3200 & 0.001173 & $3.424 \mathrm{E}-12$ & 11.47 \\
1128 & 2419200 & 2716550 & 389 & 700.2000 & 0.005536 & $5.517 \mathrm{E}-11$ & 10.26 \\
2160 & 3715200 & 5768861 & 216 & 388.8000 & 0.003074 & $1.532 \mathrm{E}-11$ & 10.81 \\
& & & & & & & \\
& & & & & & & \\
& & & & & & & \\
\end{tabular}


Gross Alpha Leaching for the Bulk Fraction \#2 from PRSB Soil

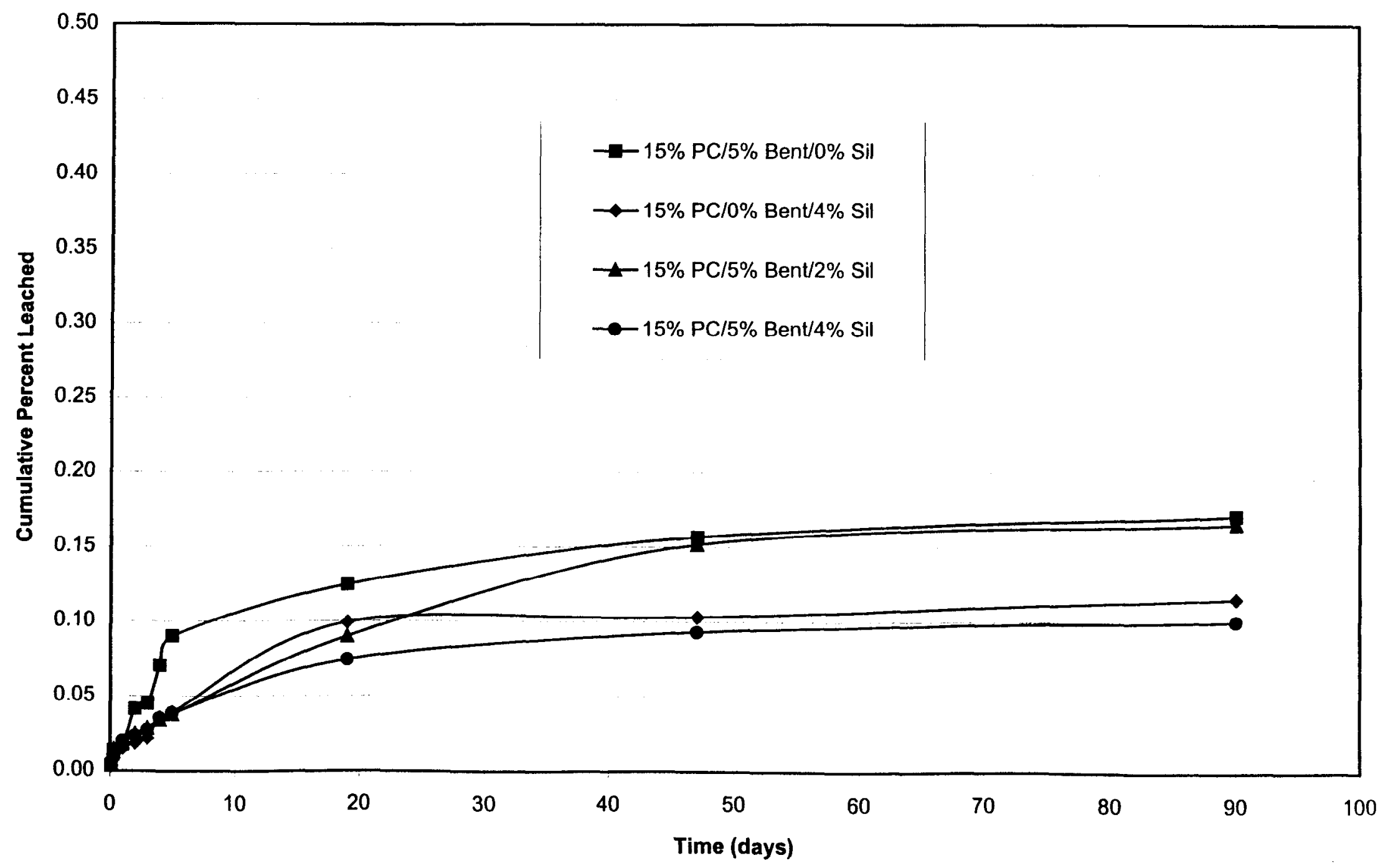




\section{American Nuclear Society - 16.1 Static Leach Test}

Project: $\quad$ SRS Reactor Seepage Basin Stabilization

Sample Description:

P612

Analyst: $\quad \mathrm{PRL}$

Date:

25-May-99

Contaminant:

Gross Alpha

Initial Contaminant Conc. (pCi/g):

13.5

Initial Contaminant Level $(\mathrm{pCi})$ :

Monolith Height (cm):

5158.35

Monolith Surface Area (cm^2):

10

Monolith Volume $\left(\mathrm{cm}^{\wedge} 3\right)$ :

196.35

Sample Weight (g):

Monolith Diameter $(\mathrm{cm})$ :

Leachate Volume $(\mathrm{mL})$ :
382.1

5

1800

\begin{tabular}{|c|c|c|c|c|c|c|c|}
\hline $\begin{array}{l}\text { Time } \\
\text { (hrs) }\end{array}$ & $\begin{array}{l}\text { Interval } \\
\text { Length } \\
\text { (s) }\end{array}$ & $\begin{array}{c}\text { Mean } \\
\text { Time } \\
\text { (s) }\end{array}$ & $\begin{array}{l}\text { Leachate } \\
\text { Conc. } \\
\text { (pCi/L) }\end{array}$ & $\begin{array}{c}\text { Amount } \\
\text { Leached } \\
(\mathrm{pCi})\end{array}$ & $\begin{array}{l}\text { Fraction } \\
\text { Leached }\end{array}$ & $\begin{array}{c}\text { Diffusivity } \\
\text { Coefficient } \\
\left(\mathrm{cm}^{\wedge} 2 / \mathrm{s}\right)\end{array}$ & $\begin{array}{l}\text { Leach } \\
\text { Index }\end{array}$ \\
\hline 2 & 7200 & 1800 & 0.1 & 0.1800 & 0.000035 & $1.640 \mathrm{E}-13$ & 12.79 \\
\hline 7 & 18000 & 14835 & 0.292 & 0.5256 & 0.000102 & 1.844E-12 & 11.73 \\
\hline 24 & 61200 & 51231 & 0.1 & 0.1800 & 0.000035 & $6.460 \mathrm{E}-14$ & 13.19 \\
\hline 48 & 86400 & 125894 & 0.695 & 1.2510 & 0.000243 & 3.847E-12 & 11.41 \\
\hline 72 & 86400 & 213818 & 0.1 & 0.1800 & 0.000035 & $1.353 E-13$ & 12.87 \\
\hline 96 & 86400 & 300849 & 0.73 & 1.3140 & 0.000255 & $1.014 \mathrm{E}-11$ & 10.99 \\
\hline 120 & 86400 & 387596 & 0.561 & 1.0098 & 0.000196 & 7.717E-12 & 11.11 \\
\hline 456 & 1209600 & 939462 & 0.983 & 1.7694 & 0.000343 & $2.930 \mathrm{E}-13$ & 12.53 \\
\hline 1128 & 2419200 & 2716550 & 0.93 & 1.6740 & 0.000325 & $1.896 \mathrm{E}-13$ & 12.72 \\
\hline 2160 & 3715200 & 5768861 & 0.416 & 0.7488 & 0.000145 & $3.416 \mathrm{E}-14$ & 13.47 \\
\hline & & & & \multicolumn{3}{|c|}{ Average Leach Index: } & 12.28 \\
\hline
\end{tabular}




\section{American Nuclear Society - 16.1 Static Leach Test}

Project: $\quad$ SRS Reactor Seepage Basin Stabilization

Sample Description: $\quad$ P622

Analyst: $\quad \mathrm{PRL}$

Date: 25-May-99

Contaminant:

Gross Alpha

Initial Contaminant Conc. (pCi/g):

13.5

Initial Contaminant Level (pCi):

Monolith Height $(\mathrm{cm})$ :

5299.29

10

Monolith Surface Area $\left(\mathrm{cm}^{\wedge} 2\right)$ :

Monolith Volume $\left(\mathrm{cm}^{\wedge} 3\right)$ :

196.35

Sample Weight (g):

Monolith Diameter (cm):

Leachate Volume $(\mathrm{mL})$ :

1800

\begin{tabular}{|c|c|c|c|c|c|c|c|}
\hline $\begin{array}{l}\text { Time } \\
\text { (hrs) }\end{array}$ & $\begin{array}{l}\text { Interval } \\
\text { Length } \\
\text { (s) }\end{array}$ & $\begin{array}{l}\text { Mean } \\
\text { Time } \\
\text { (s) }\end{array}$ & $\begin{array}{l}\text { Leachate } \\
\text { Conc. } \\
\text { (pCi/L) }\end{array}$ & $\begin{array}{l}\text { Amount } \\
\text { Leached } \\
\text { (pCi) }\end{array}$ & $\begin{array}{l}\text { Fraction } \\
\text { Leached }\end{array}$ & $\begin{array}{l}\text { Diffusivity } \\
\text { Coefficient } \\
\left(\mathrm{cm}^{\wedge} 2 / \mathrm{s}\right)\end{array}$ & $\begin{array}{l}\text { Leach } \\
\text { Index }\end{array}$ \\
\hline 2 & 7200 & 1800 & 0.1 & 0.1800 & 0.000034 & $1.554 \mathrm{E}-13$ & 12.81 \\
\hline 7 & 18000 & 14835 & 0.147 & 0.2646 & 0.000050 & 4.427E-13 & 12.35 \\
\hline 24 & 61200 & 51231 & 0.197 & 0.3546 & 0.000067 & $2.375 E-13$ & 12.62 \\
\hline 48 & 86400 & 125894 & 0.1 & 0.1800 & 0.000034 & 7.547E-14 & 13.12 \\
\hline 72 & 86400 & 213818 & 0.1 & 0.1800 & 0.000034 & $1.282 \mathrm{E}-13$ & 12.89 \\
\hline 96 & 86400 & 300849 & 0.414 & 0.7452 & 0.000141 & $3.091 \mathrm{E}-12$ & 11.51 \\
\hline 120 & 86400 & 387596 & 0.1 & 0.1800 & 0.000034 & $2.323 E-13$ & 12.63 \\
\hline 456 & 1209600 & 939462 & 1.76 & 3.1680 & 0.000598 & $8.900 E-13$ & 12.05 \\
\hline 1128 & 2419200 & 2716550 & 0.116 & 0.2088 & 0.000039 & $2.795 E-15$ & 14.55 \\
\hline 2160 & 3715200 & 5768861 & 0.371 & 0.6678 & 0.000126 & $2.574 \mathrm{E}-14$ & 13.59 \\
\hline & & & & & \multicolumn{2}{|c|}{ Average Leach Index: } & 12.81 \\
\hline
\end{tabular}




\section{American Nuclear Society - 16.1 Static Leach Test}

Project: $\quad$ SRS Reactor Seepage Basin Stabilization

Sample Description: $\quad$ P632

Analyst: $\quad \mathrm{PRL}$

Date:

25-May-99

Contaminant:

Gross Alpha

Initial Contaminant Conc. (pCi/g):

13.5

Initial Contaminant Level (pCi):

Monolith Height (cm):

4962.465

Monolith Surface Area $\left(\mathrm{cm}^{\wedge} 2\right)$ :

176.72

196.35

Sample Weight (g):

367.59

Monolith Volume $\left(\mathrm{cm}^{\wedge} 3\right)$ :

Monolith Diameter $(\mathrm{cm})$ :

5

Leachate Volume $(\mathrm{mL})$ :

1800

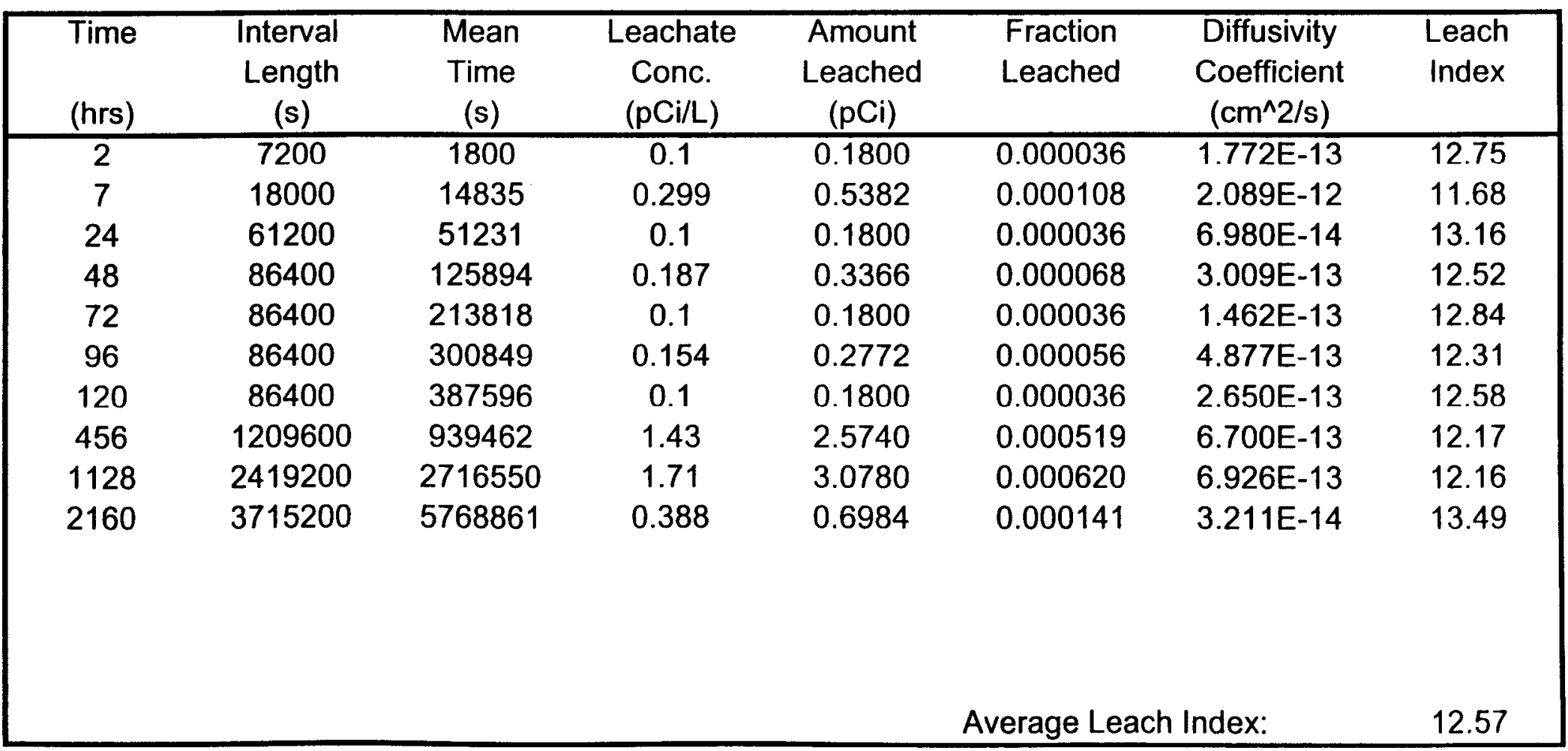




\section{American Nuclear Society - 16.1 Static Leach Test}

Project: $\quad$ SRS Reactor Seepage Basin Stabilization

Sample Description: $\quad \mathrm{P} 642$

Analyst: $\quad \mathrm{PRL}$

Date: 25-May-99

Contaminant:

Gross Alpha

Initial Contaminant Conc. (pCi/g):

13.5

Initial Contaminant Level $(\mathrm{pCi})$ :

Monolith Height $(\mathrm{cm})$ :

5055.75

10

Monolith Surface Area $\left(\mathrm{cm}^{\wedge} 2\right)$ :

Monolith Volume $\left(\mathrm{cm}^{\wedge} 3\right)$ :

176.72

196.35

Sample Weight (g):

374.5

Monolith Diameter $(\mathrm{cm})$ :

5

Leachate Volume $(\mathrm{mL})$ :

1800

\begin{tabular}{|c|c|c|c|c|c|c|c|}
\hline $\begin{array}{l}\text { Time } \\
\text { (hrs) }\end{array}$ & $\begin{array}{l}\text { Interval } \\
\text { Length } \\
\text { (s) }\end{array}$ & $\begin{array}{l}\text { Mean } \\
\text { Time } \\
\text { (s) }\end{array}$ & $\begin{array}{l}\text { Leachate } \\
\text { Conc. } \\
\text { (pCi/L) }\end{array}$ & $\begin{array}{l}\text { Amount } \\
\text { Leached } \\
(p C i)\end{array}$ & $\begin{array}{l}\text { Fraction } \\
\text { Leached }\end{array}$ & $\begin{array}{l}\text { Diffusivity } \\
\text { Coefficient } \\
\text { (cm^2/s) }\end{array}$ & $\begin{array}{l}\text { Leach } \\
\text { Index }\end{array}$ \\
\hline 2 & 7200 & 1800 & 0.158 & 0.2844 & 0.000056 & $4.261 \mathrm{E}-13$ & 12.37 \\
\hline 7 & 18000 & 14835 & 0.1 & 0.1800 & 0.000036 & $2.251 \mathrm{E}-13$ & 12.65 \\
\hline 24 & 61200 & 51231 & 0.311 & 0.5598 & 0.000111 & $6.504 \mathrm{E}-13$ & 12.19 \\
\hline 48 & 86400 & 125894 & 0.1 & 0.1800 & 0.000036 & $8.291 E-14$ & 13.08 \\
\hline 72 & 86400 & 213818 & 0.1 & 0.1800 & 0.000036 & $1.408 \mathrm{E}-13$ & 12.85 \\
\hline 96 & 86400 & 300849 & 0.223 & 0.4014 & 0.000079 & $9.853 E-13$ & 12.01 \\
\hline 120 & 86400 & 387596 & 0.1 & 0.1800 & 0.000036 & $2.553 E-13$ & 12.59 \\
\hline 456 & 1209600 & 939462 & 0.989 & 1.7802 & 0.000352 & $3.088 \mathrm{E}-13$ & 12.51 \\
\hline 1128 & 2419200 & 2716550 & 0.533 & 0.9594 & 0.000190 & $6.483 E-14$ & 13.19 \\
\hline 2160 & 3715200 & 5768861 & 0.208 & 0.3744 & 0.000074 & 8.890E-15 & 14.05 \\
\hline & & & & & \multicolumn{2}{|c|}{ Average Leach Index: } & 12.75 \\
\hline
\end{tabular}


Gross Beta Leaching for the Bulk Fraction \#2 from the PSRB Soil

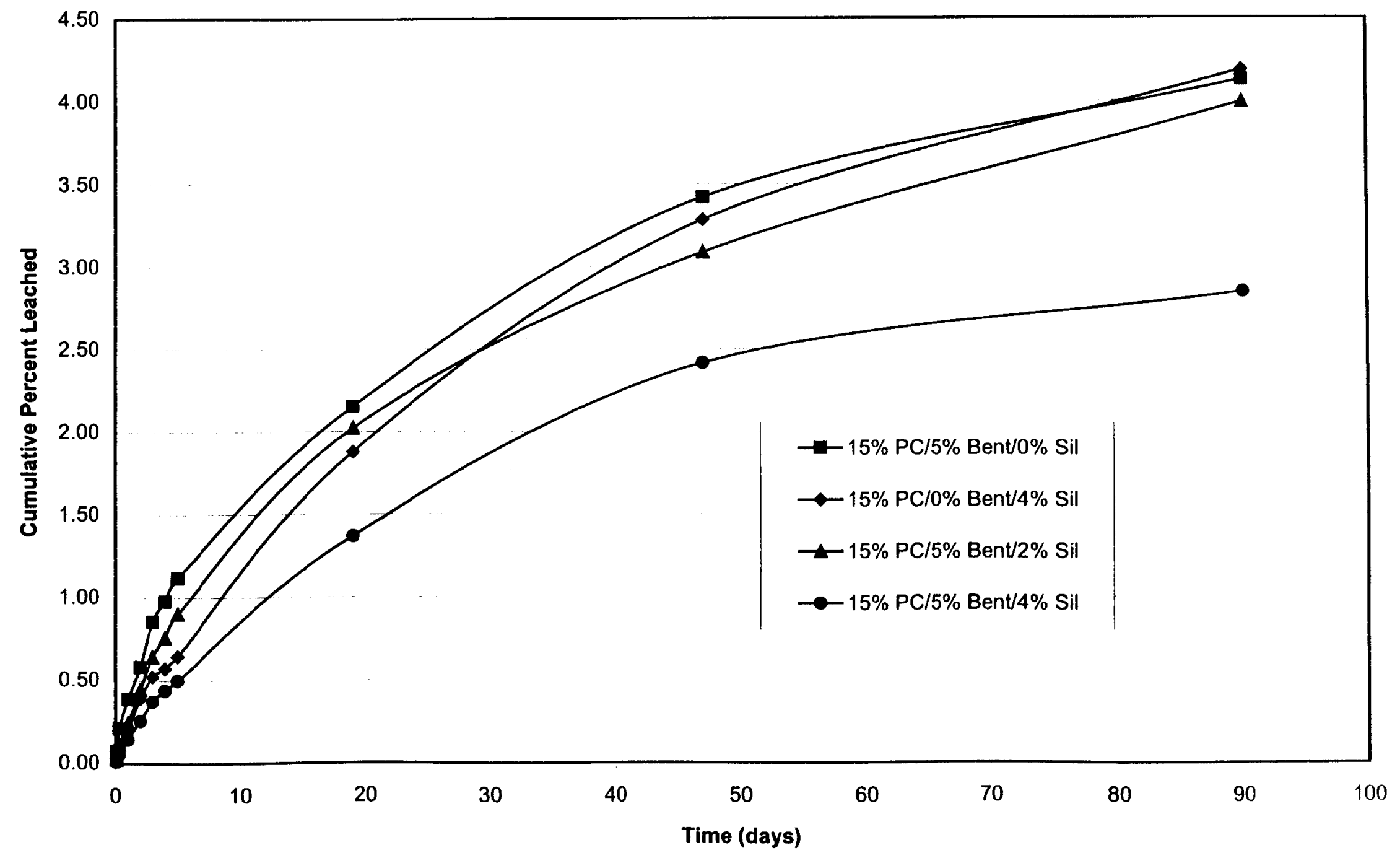




\section{American Nuclear Society - 16.1 Static Leach Test}

Project: SRS Reactor Seepage Basin Stabilization

Sample Description:

P612

Analyst: $\quad$ PRL

Date:

25-May-99

Contaminant:

Gross Beta

Initial Contaminant Conc. (pCi/g):

183

Initial Contaminant Level ( $p C i)$ :

69924.3

Monolith Height $(\mathrm{cm})$ :

10

Monolith Surface Area $\left(\mathrm{cm}^{\wedge} 2\right)$ :

176.72

Monolith Volume $\left(\mathrm{cm}^{\wedge} 3\right)$ :

196.35

Sample Weight (g):

Monolith Diameter $(\mathrm{cm})$ : Leachate Volume $(\mathrm{mL})$ :
382.1

5

1800

\begin{tabular}{|c|c|c|c|c|c|c|c|}
\hline $\begin{array}{l}\text { Time } \\
\text { (hrs) }\end{array}$ & $\begin{array}{l}\text { Interval } \\
\text { Length } \\
\text { (s) }\end{array}$ & $\begin{array}{l}\text { Mean } \\
\text { Time } \\
\text { (s) }\end{array}$ & $\begin{array}{l}\text { Leachate } \\
\text { Conc. } \\
\text { (pCi/L) }\end{array}$ & $\begin{array}{l}\text { Amount } \\
\text { Leached } \\
\text { (pCi) }\end{array}$ & $\begin{array}{l}\text { Fraction } \\
\text { Leached }\end{array}$ & $\begin{array}{c}\text { Diffusivity } \\
\text { Coefficient } \\
\left(\mathrm{cm}^{\wedge} 2 / \mathrm{s}\right) \\
\end{array}$ & $\begin{array}{l}\text { Leach } \\
\text { Index }\end{array}$ \\
\hline 2 & 7200 & 1800 & 28.7 & 51.6600 & 0.000739 & $7.351 \mathrm{E}-11$ & 10.13 \\
\hline 7 & 18000 & 14835 & 53.1 & 95.5800 & 0.001367 & $3.318 \mathrm{E}-10$ & 9.48 \\
\hline 24 & 61200 & 51231 & 69 & 124.2000 & 0.001776 & $1.674 \mathrm{E}-10$ & 9.78 \\
\hline 48 & 86400 & 125894 & 75 & 135.0000 & 0.001931 & $2.438 \mathrm{E}-10$ & 9.61 \\
\hline 72 & 86400 & 213818 & 106 & 190.8000 & 0.002729 & $8.271 \mathrm{E}-10$ & 9.08 \\
\hline 96 & 86400 & 300849 & 47.8 & 86.0400 & 0.001230 & $2.367 \mathrm{E}-10$ & 9.63 \\
\hline 120 & 86400 & 387596 & 54.7 & 98.4600 & 0.001408 & $3.993 \mathrm{E}-10$ & 9.40 \\
\hline 456 & 1209600 & 939462 & 401 & 721.8000 & 0.010323 & $2.654 \mathrm{E}-10$ & 9.58 \\
\hline 1128 & 2419200 & 2716550 & 493 & 887.4000 & 0.012691 & $2.899 \mathrm{E}-10$ & 9.54 \\
\hline 2160 & 3715200 & 5768861 & 275 & 495.0000 & 0.007079 & 8.124E-11 & 10.09 \\
\hline & & & & & \multicolumn{2}{|c|}{ Average Leach Index: } & 9.63 \\
\hline
\end{tabular}




\section{American Nuclear Society - 16.1 Static Leach Test}

Project: $\quad$ SRS Reactor Seepage Basin Stabilization

Sample Description: $\quad$ P622

$\begin{array}{llll}\text { Analyst: PRL 25-May-99 } & \text { Date: }\end{array}$

Contaminant: Gross Beta

Initial Contaminant Conc. (pCi/g):

Initial Contaminant Level (pCi):

Monolith Height $(\mathrm{cm})$ :

Monolith Surface Area $\left(\mathrm{cm}^{\wedge} 2\right)$ :

Monolith Volume $\left(\mathrm{cm}^{\wedge} 3\right)$ :
183

183
71834.82
10
176.72
196.35

Sample Weight (g):

Monolith Diameter $(\mathrm{cm})$ :

Leachate Volume $(\mathrm{mL})$ :
392.54

5

1800

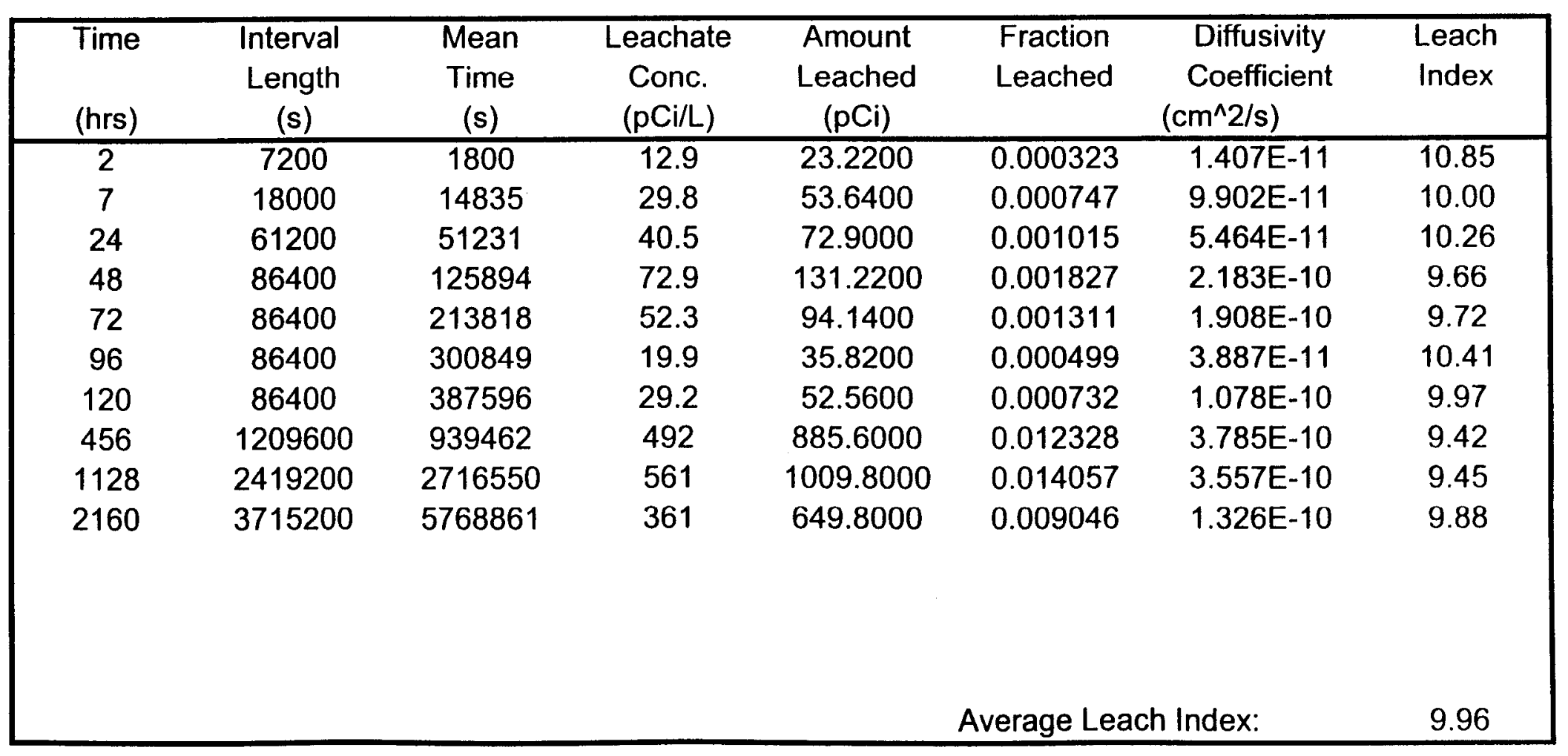




\section{American Nuclear Society - 16.1 Static Leach Test}

Project: $\quad$ SRS Reactor Seepage Basin Stabilization

Sample Description: $\quad$ P632

$\begin{array}{lll}\text { Analyst: PRL Date: } & \text { Day-99 }\end{array}$

Contaminant: Gross Beta

Initial Contaminant Conc. (pCi/g):

183

Initial Contaminant Level (pCi):

Monolith Height $(\mathrm{cm})$ :

Monolith Surface Area $\left(\mathrm{cm}^{\wedge} 2\right)$ :

Monolith Volume $\left(\mathrm{cm}^{\wedge} 3\right)$ :
67268.97

10

176.72

196.35
Sample Weight (g):

Monolith Diameter $(\mathrm{cm})$ :

Leachate Volume $(\mathrm{mL})$ :
367.59

5

1800

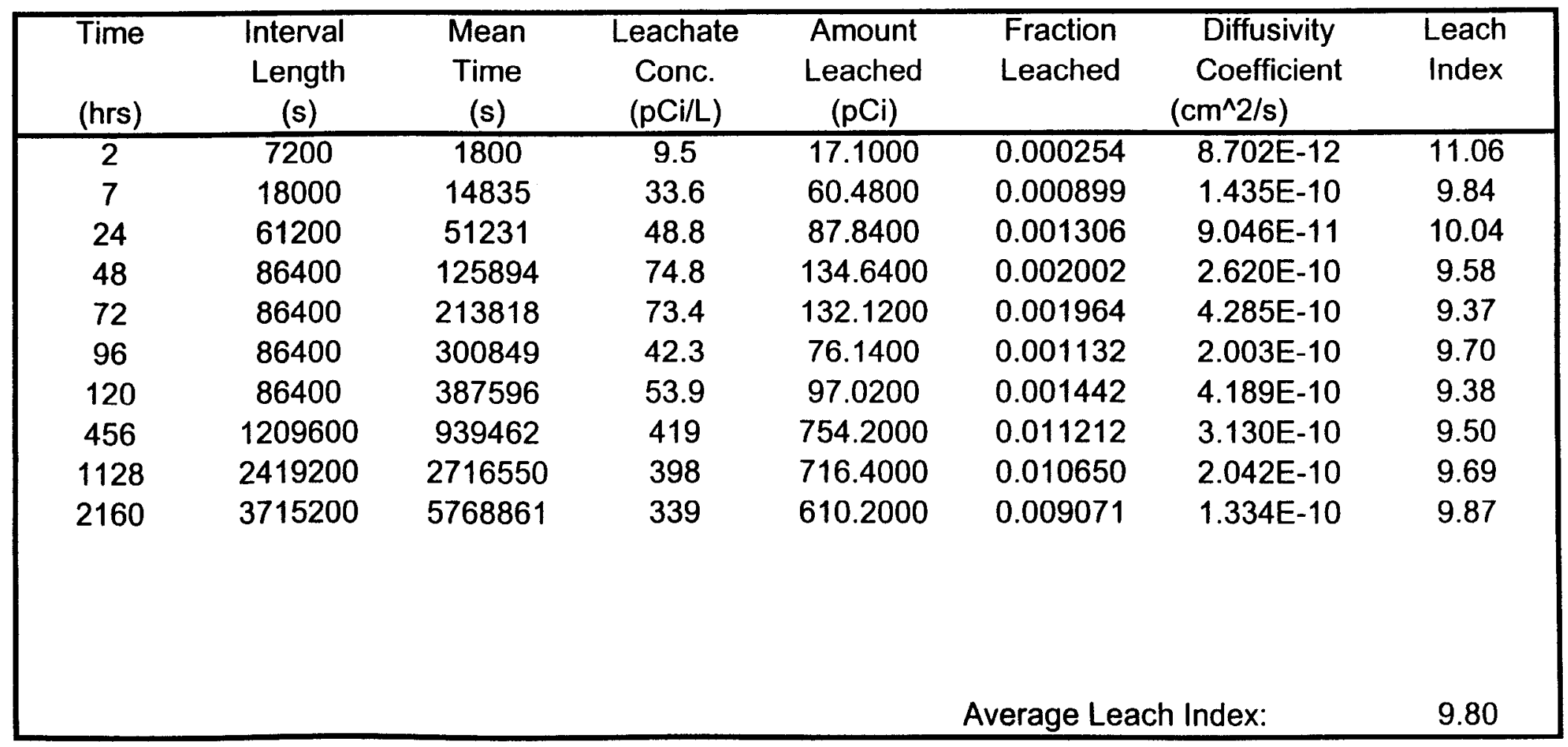




\section{American Nuclear Society - 16.1 Static Leach Test}

Project: $\quad$ SRS Reactor Seepage Basin Stabilization

Sample Description: $\quad \mathrm{P} 642$

$\begin{array}{lll}\text { Analyst: PRL 25-May-99 } & \text { Date: }\end{array}$

Contaminant: Gross Beta

Initial Contaminant Conc. (pCi/g): $\quad 183$

Initial Contaminant Level (pCi): $\quad 68533.5$

Monolith Height (cm):

$10 \quad$ Monolith Diameter $(\mathrm{cm}): \quad 5$

$\begin{array}{llll}\text { Monolith Surface Area }\left(\mathrm{cm}^{\wedge} 2\right): & 176.72 & \text { Leachate Volume }(\mathrm{mL}): & 1800\end{array}$

Monolith Volume $\left(\mathrm{cm}^{\wedge} 3\right)$ :

196.35

\begin{tabular}{|c|c|c|c|c|c|c|c|}
\hline $\begin{array}{l}\text { Time } \\
\text { (hrs) }\end{array}$ & $\begin{array}{l}\text { Interval } \\
\text { Length } \\
\text { (s) }\end{array}$ & $\begin{array}{c}\text { Mean } \\
\text { Time } \\
\text { (s) }\end{array}$ & $\begin{array}{c}\text { Leachate } \\
\text { Conc. } \\
\text { (pCi/L) }\end{array}$ & $\begin{array}{c}\text { Amount } \\
\text { Leached } \\
(\mathrm{pCi})\end{array}$ & $\begin{array}{l}\text { Fraction } \\
\text { Leached }\end{array}$ & $\begin{array}{c}\text { Diffusivity } \\
\text { Coefficient } \\
\left(\mathrm{cm}^{\wedge} 2 / \mathrm{s}\right) \\
\end{array}$ & $\begin{array}{l}\text { Leach } \\
\text { Index }\end{array}$ \\
\hline 2 & 7200 & 1800 & 4.52 & 8.1360 & 0.000119 & $1.898 \mathrm{E}-12$ & 11.72 \\
\hline 7 & 18000 & 14835 & 15.5 & 27.9000 & 0.000407 & $2.943 \mathrm{E}-11$ & 10.53 \\
\hline 24 & 61200 & 51231 & 35 & 63.0000 & 0.000919 & 4.483E-11 & 10.35 \\
\hline 48 & 86400 & 125894 & 43.3 & 77.9400 & 0.001137 & $8.460 \mathrm{E}-11$ & 10.07 \\
\hline 72 & 86400 & 213818 & 43.2 & 77.7600 & 0.001135 & $1.430 \mathrm{E}-10$ & 9.84 \\
\hline 96 & 86400 & 300849 & 25.6 & 46.0800 & 0.000672 & 7.067E-11 & 10.15 \\
\hline 120 & 86400 & 387596 & 22.9 & 41.2200 & 0.000601 & $7.285 \mathrm{E}-11$ & 10.14 \\
\hline 456 & 1209600 & 939462 & 331 & 595.8000 & 0.008694 & $1.882 \mathrm{E}-10$ & 9.73 \\
\hline 1128 & 2419200 & 2716550 & 398 & 716.4000 & 0.010453 & 1.967E-10 & 9.71 \\
\hline 2160 & 3715200 & 5768861 & 164 & 295.2000 & 0.004307 & $3.008 \mathrm{E}-11$ & 10.52 \\
\hline & & & & & \multicolumn{2}{|c|}{ Average Leach Index: } & 10.28 \\
\hline
\end{tabular}


Gross Beta Leaching for the Fine Fraction from the PSRB Soil

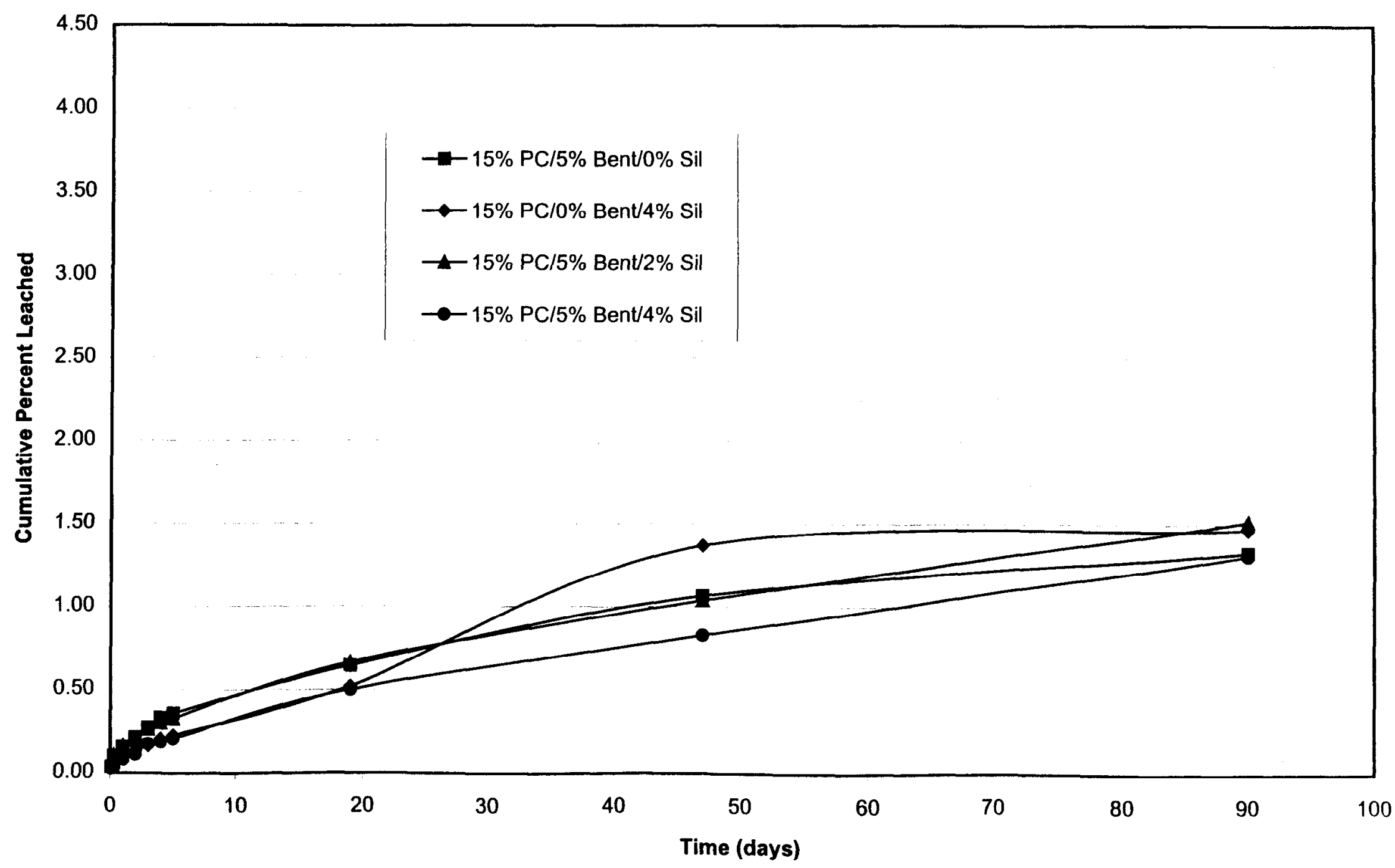




\section{P Series}

Index Test Results 


\section{American Nuclear Society - 16.1 Static Leach Test}

Project: $\quad$ SRS Reactor Seepage Basin Stabilization

Sample Description: $\quad$ P432

Analyst: $\quad \mathrm{PRL}$

Date:

25-May-99

Contaminant:

Gross Alpha

Initial Contaminant Conc. (pCi/g):

32.3

Initial Contaminant Level (pCi):

Monolith Height $(\mathrm{cm})$ :

11530.454

10

Monolith Surface Area $\left(\mathrm{cm}^{\wedge} 2\right)$ :

176.72

Monolith Volume $\left(\mathrm{cm}^{\wedge} 3\right)$ :

196.35

Sample Weight (g):

Monolith Diameter $(\mathrm{cm})$ :

Leachate Volume $(\mathrm{mL})$ :
356.98

5

1800

\begin{tabular}{|c|c|c|c|c|c|c|c|}
\hline $\begin{array}{l}\text { Time } \\
\text { (hrs) }\end{array}$ & $\begin{array}{l}\text { Interval } \\
\text { Length } \\
\text { (s) }\end{array}$ & $\begin{array}{c}\text { Mean } \\
\text { Time } \\
\text { (s) }\end{array}$ & $\begin{array}{c}\text { Leachate } \\
\text { Conc. } \\
\text { (pCi/L) }\end{array}$ & $\begin{array}{l}\text { Amount } \\
\text { Leached } \\
\text { (pCi) }\end{array}$ & $\begin{array}{l}\text { Fraction } \\
\text { Leached }\end{array}$ & $\begin{array}{l}\text { Diffusivity } \\
\text { Coefficient } \\
(\mathrm{cm} \wedge / \mathrm{s})\end{array}$ & $\begin{array}{l}\text { Leach } \\
\text { Index }\end{array}$ \\
\hline 2 & 7200 & 1800 & 0.1 & 0.1800 & 0.000016 & $3.282 \mathrm{E}-14$ & 13.48 \\
\hline 7 & 18000 & 14835 & 1.44 & 2.5920 & 0.000225 & 8.974E-12 & 11.05 \\
\hline 24 & 61200 & 51231 & 1.3 & 2.3400 & 0.000203 & 2.185E-12 & 11.66 \\
\hline 48 & 86400 & 125894 & 0.1 & 0.1800 & 0.000016 & $1.594 \mathrm{E}-14$ & 13.80 \\
\hline 72 & 86400 & 213818 & 0.595 & 1.0710 & 0.000093 & $9.584 \mathrm{E}-13$ & 12.02 \\
\hline 96 & 86400 & 300849 & 0.1 & 0.1800 & 0.000016 & $3.809 \mathrm{E}-14$ & 13.42 \\
\hline 120 & 86400 & 387596 & 0.1 & 0.1800 & 0.000016 & $4.908 \mathrm{E}-14$ & 13.31 \\
\hline 456 & 1209600 & 939462 & 7.86 & 14.1480 & 0.001227 & $3.749 \mathrm{E}-12$ & 11.43 \\
\hline 1128 & 2419200 & 2716550 & 0.1 & 0.1800 & 0.000016 & 4.387E-16 & 15.36 \\
\hline 2160 & 3715200 & 5768861 & 0.503 & 0.9054 & 0.000079 & $9.995 \mathrm{E}-15$ & 14.00 \\
\hline & & & & & \multicolumn{2}{|c|}{ Average Leach Index: } & 12.95 \\
\hline
\end{tabular}




\section{American Nuclear Society - 16.1 Static Leach Test}

Project: $\quad$ SRS Reactor Seepage Basin Stabilization

Sample Description: P442

Analyst: $\quad$ PRL

Date: 25-May-99

Contaminant:

Gross Alpha

Initial Contaminant Conc. ( $\mathrm{pCi} / \mathrm{g})$ :

Initial Contaminant Level (pCi):

Monolith Height $(\mathrm{cm})$ :

Monolith Surface Area $\left(\mathrm{cm}^{\wedge} 2\right)$ :

Monolith Volume $\left(\mathrm{cm}^{\wedge} 3\right)$ :

23.2
8455.472
10
176.72
196.35

Sample Weight (g):

Monolith Diameter $(\mathrm{cm})$ :

Leachate Volume $(\mathrm{mL})$ :
364.46

5

1800

\begin{tabular}{|c|c|c|c|c|c|c|c|}
\hline $\begin{array}{l}\text { Time } \\
\text { (hrs) }\end{array}$ & $\begin{array}{l}\text { Interval } \\
\text { Length } \\
\text { (s) }\end{array}$ & $\begin{array}{l}\text { Mean } \\
\text { Time } \\
(\mathrm{s})\end{array}$ & $\begin{array}{c}\text { Leachate } \\
\text { Conc. } \\
\text { (pCi/L) }\end{array}$ & $\begin{array}{l}\text { Amount } \\
\text { Leached } \\
(\mathrm{pCi})\end{array}$ & $\begin{array}{l}\text { Fraction } \\
\text { Leached }\end{array}$ & $\begin{array}{l}\text { Diffusivity } \\
\text { Coefficient } \\
\left(\mathrm{cm}^{\wedge} 2 / \mathrm{s}\right)\end{array}$ & $\begin{array}{l}\text { Leach } \\
\text { Index }\end{array}$ \\
\hline 2 & 7200 & 1800 & 0.1 & 0.1800 & 0.000021 & $6.103 \mathrm{E}-14$ & 13.21 \\
\hline 7 & 18000 & 14835 & 0.181 & 0.3258 & 0.000039 & 2.637E-13 & 12.58 \\
\hline 24 & 61200 & 51231 & 0.1 & 0.1800 & 0.000021 & $2.404 \mathrm{E}-14$ & 13.62 \\
\hline 48 & 86400 & 125894 & 0.227 & 0.4086 & 0.000048 & $1.527 E-13$ & 12.82 \\
\hline 72 & 86400 & 213818 & 5.45 & 9.8100 & 0.001160 & 1.495E-10 & 9.83 \\
\hline 96 & 86400 & 300849 & 0.204 & 0.3672 & 0.000043 & $2.948 E-13$ & 12.53 \\
\hline 120 & 86400 & 387596 & 0.1 & 0.1800 & 0.000021 & $9.126 \mathrm{E}-14$ & 13.04 \\
\hline 456 & 1209600 & 939462 & 8.84 & 15.9120 & 0.001882 & 8.819E-12 & 11.05 \\
\hline 1128 & 2419200 & 2716550 & 0.169 & 0.3042 & 0.000036 & $2.330 \mathrm{E}-15$ & 14.63 \\
\hline 2160 & 3715200 & 5768861 & 0.1 & 0.1800 & 0.000021 & 7.346E-16 & 15.13 \\
\hline & & & & & \multicolumn{2}{|c|}{ Average Leach Index: } & 12.84 \\
\hline
\end{tabular}




\section{American Nuclear Society - 16.1 Static Leach Test}

Project: $\quad$ SRS Reactor Seepage Basin Stabilization

Sample Description: $\quad$ P422

$\begin{array}{lll}\text { Analyst: PRL Date: 25-May-99 } & \end{array}$

Contaminant: Gross Alpha

Initial Contaminant Conc. (pCi/g): $\quad 32.3$

Initial Contaminant Level (pCi): $\quad 12152.875$

Monolith Height $(\mathrm{cm})$ :

10

Monolith Surface Area $\left(\mathrm{cm}^{\wedge} 2\right)$ :

176.72

196.35

Sample Weight (g):

376.25

Monolith Diameter $(\mathrm{cm})$ :

Leachate Volume $(\mathrm{mL})$ :

5

1800

Monolith Volume $\left(\mathrm{cm}^{\wedge} 3\right)$ :

\begin{tabular}{|c|c|c|c|c|c|c|c|}
\hline $\begin{array}{l}\text { Time } \\
\text { (hrs) }\end{array}$ & $\begin{array}{l}\text { Interval } \\
\text { Length } \\
\text { (s) }\end{array}$ & $\begin{array}{c}\text { Mean } \\
\text { Time } \\
\text { (s) }\end{array}$ & $\begin{array}{c}\text { Leachate } \\
\text { Conc. } \\
\text { (pCi/L) }\end{array}$ & $\begin{array}{l}\text { Amount } \\
\text { Leached } \\
\text { (pCi) }\end{array}$ & $\begin{array}{l}\text { Fraction } \\
\text { Leached }\end{array}$ & $\begin{array}{c}\text { Diffusivity } \\
\text { Coefficient } \\
\text { (cm^2/s) }\end{array}$ & $\begin{array}{l}\text { Leach } \\
\text { Index }\end{array}$ \\
\hline 2 & 7200 & 1800 & 0.1 & 0.1800 & 0.000015 & $2.954 \mathrm{E}-14$ & 13.53 \\
\hline 7 & 18000 & 14835 & 1.2 & 2.1600 & 0.000178 & 5.610E-12 & 11.25 \\
\hline 24 & 61200 & 51231 & 0.1 & 0.1800 & 0.000015 & 1.164E-14 & 13.93 \\
\hline 48 & 86400 & 125894 & 2.02 & 3.6360 & 0.000299 & $5.855 E-12$ & 11.23 \\
\hline 72 & 86400 & 213818 & 0.34 & 0.6120 & 0.000050 & $2.817 \mathrm{E}-13$ & 12.55 \\
\hline 96 & 86400 & 300849 & 0.1 & 0.1800 & 0.000015 & $3.429 \mathrm{E}-14$ & 13.46 \\
\hline 120 & 86400 & 387596 & 0.1 & 0.1800 & 0.000015 & $4.418 \mathrm{E}-14$ & 13.35 \\
\hline 456 & 1209600 & 939462 & 8.37 & 15.0660 & 0.001240 & $3.827 E-12$ & 11.42 \\
\hline 1128 & 2419200 & 2716550 & 0.436 & 0.7848 & 0.000065 & $7.508 \mathrm{E}-15$ & 14.12 \\
\hline 2160 & 3715200 & 5768861 & 0.1 & 0.1800 & 0.000015 & $3.556 \mathrm{E}-16$ & 15.45 \\
\hline & & & & & \multicolumn{2}{|c|}{ Average Leach Index: } & 13.03 \\
\hline
\end{tabular}




\section{American Nuclear Society - 16.1 Static Leach Test}

Project: $\quad$ SRS Reactor Seepage Basin Stabilization

Sample Description:

P412

Analyst: $\quad \mathrm{PRL}$

Date:

25-May-99

Contaminant:

Gross Alpha

Initial Contaminant Conc. (pCi/g):

32.3

Initial Contaminant Level (pCi):

Monolith Height $(\mathrm{cm})$ :

12036.272

10

Monolith Surface Area $\left(\mathrm{cm}^{\wedge} 2\right)$ :

176.72

Monolith Diameter $(\mathrm{cm})$ :

Sample Weight (g):

372.64

Monolith Volume $\left(\mathrm{cm}^{\wedge} 3\right)$ :

196.35

Leachate Volume $(\mathrm{mL})$ :

1800

\begin{tabular}{|cccccccc|}
\hline Time & $\begin{array}{c}\text { Interval } \\
\text { Length } \\
(\mathrm{s})\end{array}$ & $\begin{array}{c}\text { Mean } \\
\text { Time } \\
(\mathrm{s})\end{array}$ & $\begin{array}{c}\text { Leachate } \\
\text { Conc. } \\
(\mathrm{pCi} / \mathrm{L})\end{array}$ & $\begin{array}{c}\text { Amount } \\
\text { Leached } \\
(\mathrm{pCi})\end{array}$ & $\begin{array}{c}\text { Fraction } \\
\text { Leached }\end{array}$ & $\begin{array}{c}\text { Diffusivity } \\
\text { Coefficient } \\
(\mathrm{cm} \text { ) } / \mathrm{s})\end{array}$ & $\begin{array}{c}\text { Leach } \\
\text { Index }\end{array}$ \\
\hline 2 & 7200 & 1800 & 0.1 & 0.1800 & 0.000015 & $3.012 \mathrm{E}-14$ & 13.52 \\
7 & 18000 & 14835 & 0.1 & 0.1800 & 0.000015 & $3.972 \mathrm{E}-14$ & 13.40 \\
24 & 61200 & 51231 & 0.184 & 0.3312 & 0.000028 & $4.017 \mathrm{E}-14$ & 13.40 \\
48 & 86400 & 125894 & 2.28 & 4.1040 & 0.000341 & $7.605 \mathrm{E}-12$ & 11.12 \\
72 & 86400 & 213818 & 0.1 & 0.1800 & 0.000015 & $2.485 \mathrm{E}-14$ & 13.60 \\
96 & 86400 & 300849 & 0.1 & 0.1800 & 0.000015 & $3.496 \mathrm{E}-14$ & 13.46 \\
120 & 86400 & 387596 & 0.1 & 0.1800 & 0.000015 & $4.504 \mathrm{E}-14$ & 13.35 \\
456 & 1209600 & 939462 & 3.08 & 5.5440 & 0.000461 & $5.284 \mathrm{E}-13$ & 12.28 \\
1128 & 2419200 & 2716550 & 0.1 & 0.1800 & 0.000015 & $4.026 \mathrm{E}-16$ & 15.40 \\
2160 & 3715200 & 5768861 & 0.514 & 0.9252 & 0.000077 & $9.578 \mathrm{E}-15$ & 14.02 \\
& & & & & & & \\
\end{tabular}


Gross Alpha Leaching for the Fine Fraction from PRSB Soil

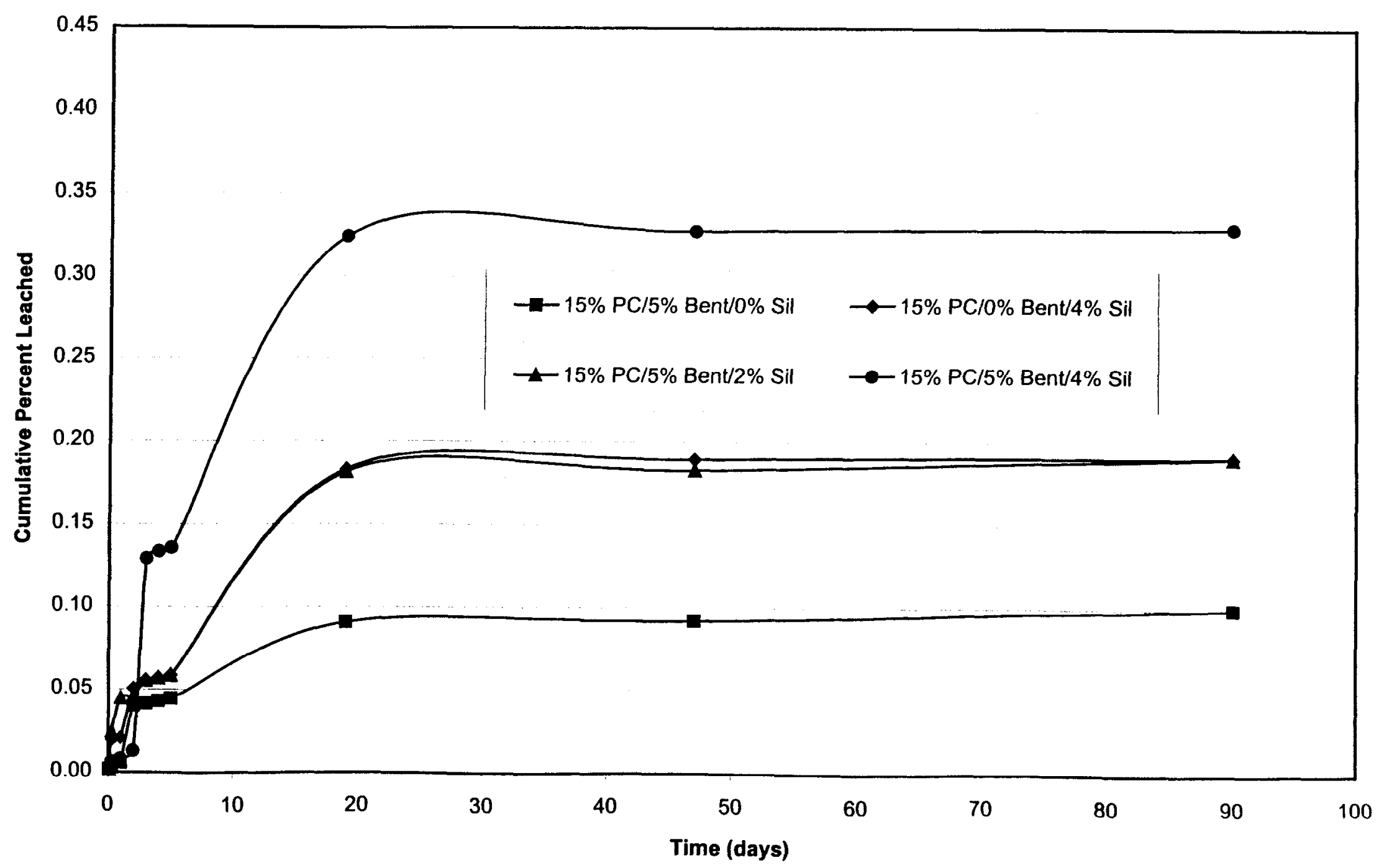




\section{American Nuclear Society - 16.1 Static Leach Test}

Project: $\quad$ SRS Reactor Seepage Basin Stabilization

Sample Description: $\quad$ P342

$\begin{array}{lll}\text { Analyst: PRL Date: 25-May-99 } & \end{array}$

Contaminant: Gross Beta

Initial Contaminant Conc. (pCi/g): 239

Initial Contaminant Level (pCi): $\quad 90506.91$

Monolith Height $(\mathrm{cm})$ :

10

Monolith Surface Area $\left(\mathrm{cm}^{\wedge} 2\right)$ :

Monolith Volume $\left(\mathrm{cm}^{\wedge} 3\right)$ :

176.72

196.35

\begin{tabular}{|c|c|c|c|c|c|c|c|}
\hline $\begin{array}{l}\text { Time } \\
\text { (hrs) }\end{array}$ & $\begin{array}{l}\text { Interval } \\
\text { Length } \\
\text { (s) }\end{array}$ & $\begin{array}{l}\text { Mean } \\
\text { Time } \\
(s)\end{array}$ & $\begin{array}{l}\text { Leachate } \\
\text { Conc. } \\
\text { (pCi/L) }\end{array}$ & $\begin{array}{l}\text { Amount } \\
\text { Leached } \\
\text { (pCi) }\end{array}$ & $\begin{array}{l}\text { Fraction } \\
\text { Leached }\end{array}$ & $\begin{array}{c}\text { Diffusivity } \\
\text { Coefficient } \\
\left(\mathrm{cm}^{\wedge} 2 / \mathrm{s}\right)\end{array}$ & $\begin{array}{l}\text { Leach } \\
\text { Index }\end{array}$ \\
\hline 2 & 7200 & 1800 & 5.67 & 10.2060 & 0.000113 & $1.712 \mathrm{E}-12$ & 11.77 \\
\hline 7 & 18000 & 14835 & 10.2 & 18.3600 & 0.000203 & $7.308 \mathrm{E}-12$ & 11.14 \\
\hline 24 & 61200 & 51231 & 19.5 & 35.1000 & 0.000388 & 7.979E-12 & 11.10 \\
\hline 48 & 86400 & 125894 & 20.1 & 36.1800 & 0.000400 & $1.045 \mathrm{E}-11$ & 10.98 \\
\hline 72 & 86400 & 213818 & 19.2 & 34.5600 & 0.000382 & 1.620E-11 & 10.79 \\
\hline 96 & 86400 & 300849 & 15.9 & 28.6200 & 0.000316 & $1.563 \mathrm{E}-11$ & 10.81 \\
\hline 120 & 86400 & 387596 & 15.5 & 27.9000 & 0.000308 & $1.914 \mathrm{E}-11$ & 10.72 \\
\hline 456 & 1209600 & 939462 & 73 & 131.4000 & 0.001452 & $5.249 \mathrm{E}-12$ & 11.28 \\
\hline 1128 & 2419200 & 2716550 & 94.3 & 169.7400 & 0.001875 & $6.332 E-12$ & 11.20 \\
\hline 2160 & 3715200 & 5768861 & 184 & 331.2000 & 0.003659 & $2.171 \mathrm{E}-11$ & 10.66 \\
\hline & & & & & \multicolumn{2}{|c|}{ Average Leach Index: } & 11.04 \\
\hline
\end{tabular}


Project: $\quad$ SRS Reactor Seepage Basin Stabilization

Sample Description: $\quad$ P322

$\begin{array}{lll}\text { Analyst: PRL Date: 25-May-99 } & \text { Da }\end{array}$

Contaminant: Gross Beta

Initial Contaminant Conc. (pCi/g): 239

Initial Contaminant Level (pCi): $\quad 94381.1$

Monolith Height $(\mathrm{cm})$ :

10

Monolith Surface Area $\left(\mathrm{cm}^{\wedge} 2\right)$ : $\quad 176.72$

Monolith Volume $\left(\mathrm{cm}^{\wedge} 3\right)$ : $\quad 196.35$

\begin{tabular}{|cccccccc|}
\hline Time & $\begin{array}{c}\text { Interval } \\
\text { Length } \\
(\mathrm{hrs})\end{array}$ & $\begin{array}{c}\text { Mean } \\
\text { Time } \\
(\mathrm{s})\end{array}$ & $\begin{array}{c}\text { Leachate } \\
\text { Conc. } \\
(\mathrm{pCi} / \mathrm{L})\end{array}$ & $\begin{array}{c}\text { Amount } \\
\text { Leached } \\
(\mathrm{pCi})\end{array}$ & $\begin{array}{c}\text { Fraction } \\
\text { Leached }\end{array}$ & $\begin{array}{c}\text { Diffusivity } \\
\text { Coefficient } \\
\left(\mathrm{cm}^{\wedge} 2 / \mathrm{s}\right)\end{array}$ & $\begin{array}{c}\text { Leach } \\
\text { Index }\end{array}$ \\
\hline 2 & 7200 & 1800 & 6.66 & 11.9880 & 0.000127 & $2.173 \mathrm{E}-12$ & 11.66 \\
7 & 18000 & 14835 & 14.2 & 25.5600 & 0.000271 & $1.302 \mathrm{E}-11$ & 10.89 \\
24 & 61200 & 51231 & 20.4 & 36.7200 & 0.000389 & $8.030 \mathrm{E}-12$ & 11.10 \\
48 & 86400 & 125894 & 20.4 & 36.7200 & 0.000389 & $9.901 \mathrm{E}-12$ & 11.00 \\
72 & 86400 & 213818 & 34.9 & 62.8200 & 0.000666 & $4.922 \mathrm{E}-11$ & 10.31 \\
96 & 86400 & 300849 & 11.6 & 20.8800 & 0.000221 & $7.650 \mathrm{E}-12$ & 11.12 \\
120 & 86400 & 387596 & 14.9 & 26.8200 & 0.000284 & $1.626 \mathrm{E}-11$ & 10.79 \\
456 & 1209600 & 939462 & 183 & 329.4000 & 0.003490 & $3.033 \mathrm{E}-11$ & 10.52 \\
1128 & 2419200 & 2716550 & 150 & 270.0000 & 0.002861 & $1.473 \mathrm{E}-11$ & 10.83 \\
2160 & 3715200 & 5768861 & 284 & 511.2000 & 0.005416 & $4.756 \mathrm{E}-11$ & 10.32 \\
& & & & & & & \\
\end{tabular}


Gross Beta Leaching for the Coarse Fraction from the PSRB Soil

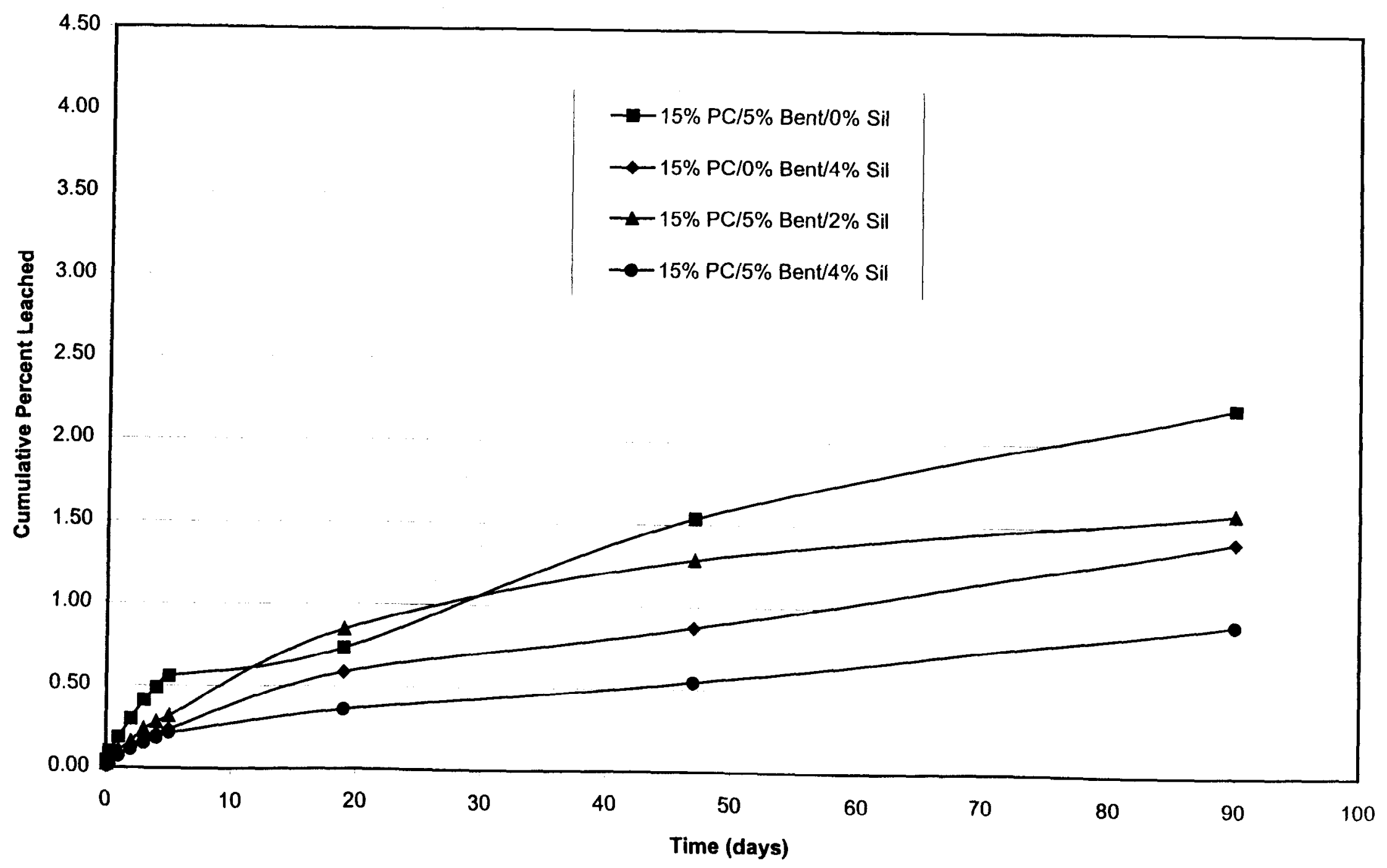




\section{American Nuclear Society - 16.1 Static Leach Test}

Project: $\quad$ SRS Reactor Seepage Basin Stabilization

Sample Description: $\quad$ P332

$\begin{array}{lll}\text { Analyst: PRL } & \text { Date: } & \end{array}$

Contaminant: Gross Beta

Initial Contaminant Conc. (pCi/g): 239

Initial Contaminant Level (pCi): $\quad 90714.84$

Monolith Height (cm): $\quad 10$

Monolith Surface Area $\left(\mathrm{cm}^{\wedge} 2\right)$ : $\quad 176.72$

Monolith Volume $\left(\mathrm{cm}^{\wedge} 3\right)$ : $\quad 196.35$

379.56

Monolith Diameter $(\mathrm{cm}): \quad 5$

Leachate Volume $(\mathrm{mL}): \quad 1800$

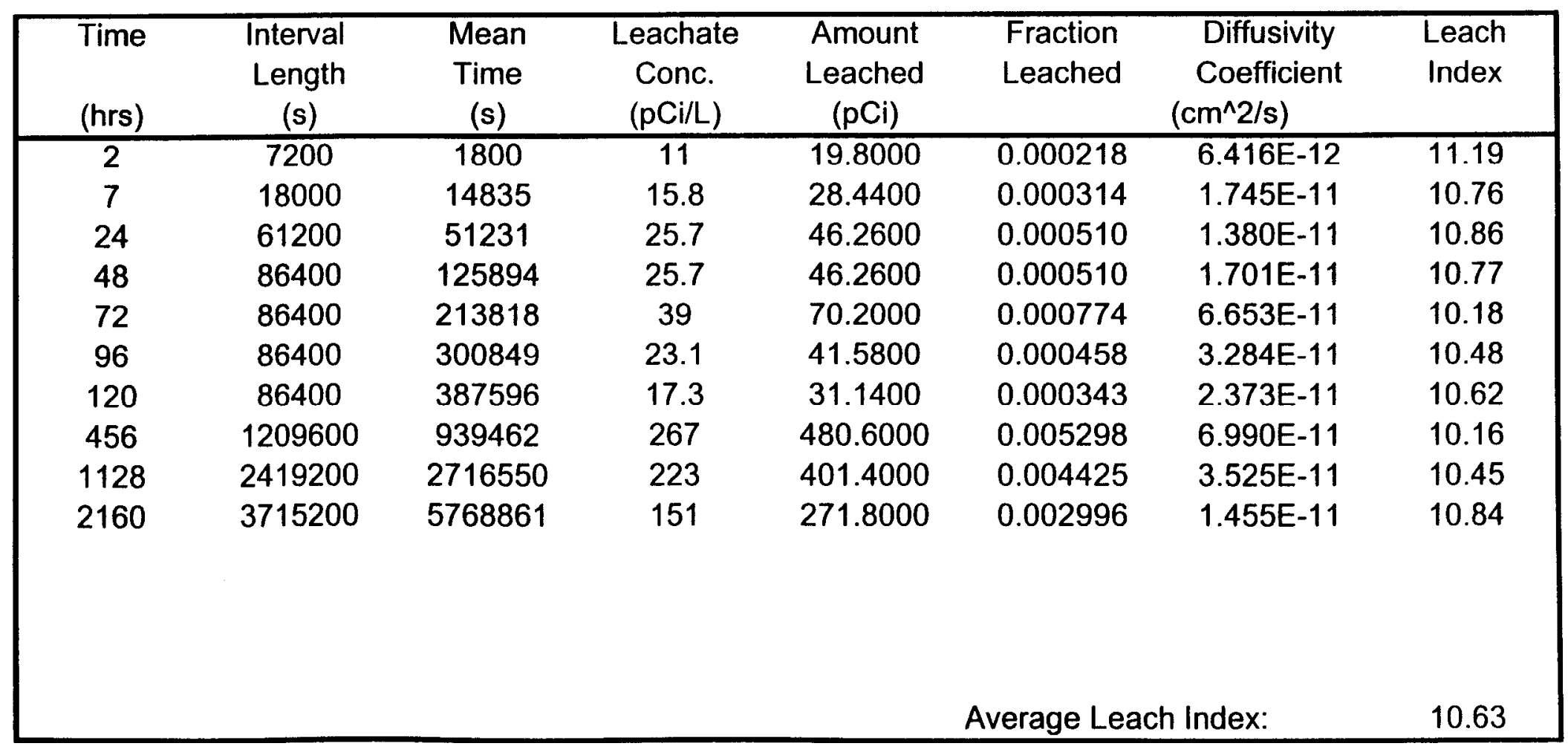




\section{American Nuclear Society - 16.1 Static Leach Test}

Project: $\quad$ SRS Reactor Seepage Basin Stabilization

Sample Description: $\quad$ P312

$\begin{array}{llll}\text { Analyst: PRL } & \text { Date: } & \text { 25-May-99 }\end{array}$

Contaminant: Gross Beta

Initial Contaminant Conc. (pCi/g):

Initial Contaminant Level (pCi):

Monolith Height $(\mathrm{cm})$ :

Monolith Surface Area $\left(\mathrm{cm}^{\wedge} 2\right)$ :

Monolith Volume $\left(\mathrm{cm}^{\wedge} 3\right)$ :
239

239
90882.14
10
176.72
196.35

380.26

Monolith Diameter $(\mathrm{cm})$ :

5

Leachate Volume $(\mathrm{mL})$ :

\begin{tabular}{|cccccccc|}
\hline Time & $\begin{array}{c}\text { Interval } \\
\text { Length } \\
(\mathrm{s})\end{array}$ & $\begin{array}{c}\text { Mean } \\
\text { Time } \\
(\mathrm{s})\end{array}$ & $\begin{array}{c}\text { Leachate } \\
\text { Conc. } \\
(\mathrm{pCi} / \mathrm{L})\end{array}$ & $\begin{array}{c}\text { Amount } \\
\text { Leached } \\
(\mathrm{pCi})\end{array}$ & $\begin{array}{c}\text { Fraction } \\
\text { Leached }\end{array}$ & $\begin{array}{c}\text { Diffusivity } \\
\text { Coefficient } \\
\left(\mathrm{cm}^{\wedge} \text { 2/s }\right)\end{array}$ & $\begin{array}{c}\text { Leach } \\
\text { Index }\end{array}$ \\
\hline 2 & 7200 & 1800 & 19.2 & 34.5600 & 0.000380 & $1.947 \mathrm{E}-11$ & 10.71 \\
7 & 18000 & 14835 & 28.2 & 50.7600 & 0.000559 & $5.540 \mathrm{E}-11$ & 10.26 \\
24 & 61200 & 51231 & 43.9 & 79.0200 & 0.000869 & $4.011 \mathrm{E}-11$ & 10.40 \\
48 & 86400 & 125894 & 56.5 & 101.7000 & 0.001119 & $8.191 \mathrm{E}-11$ & 10.09 \\
72 & 86400 & 213818 & 58 & 104.4000 & 0.001149 & $1.466 \mathrm{E}-10$ & 9.83 \\
96 & 86400 & 300849 & 39.1 & 70.3800 & 0.000774 & $9.374 \mathrm{E}-11$ & 10.03 \\
120 & 86400 & 387596 & 35.4 & 63.7200 & 0.000701 & $9.899 \mathrm{E}-11$ & 10.00 \\
456 & 1209600 & 939462 & 88 & 158.4000 & 0.001743 & $7.565 \mathrm{E}-12$ & 11.12 \\
1128 & 2419200 & 2716550 & 407 & 732.6000 & 0.008061 & $1.170 \mathrm{E}-10$ & 9.93 \\
2160 & 3715200 & 5768861 & 346 & 622.8000 & 0.006853 & $7.613 \mathrm{E}-11$ & 10.12 \\
& & & & & & & \\
& & & & & & & \\
& & & & & & & \\
\end{tabular}




\section{American Nuclear Society - 16.1 Static Leach Test}

Project: $\quad$ SRS Reactor Seepage Basin Stabilization

Sample Description: $\quad$ P342

Analyst: $\quad$ PRL

Date: $\quad$ 25-May-99

Contaminant:

Gross Alpha

Initial Contaminant Conc. (pCi/g):

19

7195.11

Initial Contaminant Level ( $p C i)$ :

Monolith Height $(\mathrm{cm})$ :

Monolith Surface Area $\left(\mathrm{cm}^{\wedge} 2\right)$ :

Monolith Volume $\left(\mathrm{cm}^{\wedge} 3\right)$ :
10

176.72

196.35
Sample Weight (g):

378.69

Monolith Diameter $(\mathrm{cm})$ :

5

Leachate Volume $(\mathrm{mL})$ :

1800

\begin{tabular}{|c|c|c|c|c|c|c|c|}
\hline $\begin{array}{l}\text { Time } \\
\text { (hrs) }\end{array}$ & $\begin{array}{l}\text { Interval } \\
\text { Length } \\
\text { (s) }\end{array}$ & $\begin{array}{l}\text { Mean } \\
\text { Time } \\
(\mathrm{s})\end{array}$ & $\begin{array}{l}\text { Leachate } \\
\text { Conc. } \\
\text { (pCi/L) }\end{array}$ & $\begin{array}{l}\text { Amount } \\
\text { Leached } \\
(\mathrm{pCi})\end{array}$ & $\begin{array}{l}\text { Fraction } \\
\text { Leached }\end{array}$ & $\begin{array}{l}\text { Diffusivity } \\
\text { Coefficient } \\
\left(\mathrm{cm}^{\wedge} 2 / \mathrm{s}\right)\end{array}$ & $\begin{array}{l}\text { Leach } \\
\text { Index }\end{array}$ \\
\hline 2 & 7200 & 1800 & 0.1 & 0.1800 & 0.000025 & $8.428 E-14$ & 13.07 \\
\hline 7 & 18000 & 14835 & 0.418 & 0.7524 & 0.000105 & $1.942 \mathrm{E}-12$ & 11.71 \\
\hline 24 & 61200 & 51231 & 0.1 & 0.1800 & 0.000025 & $3.320 \mathrm{E}-14$ & 13.48 \\
\hline 48 & 86400 & 125894 & 1.75 & 3.1500 & 0.000438 & 1.254E-11 & 10.90 \\
\hline 72 & 86400 & 213818 & 0.1 & 0.1800 & 0.000025 & 6.953E-14 & 13.16 \\
\hline 96 & 86400 & 300849 & 0.1 & 0.1800 & 0.000025 & $9.783 E-14$ & 13.01 \\
\hline 120 & 86400 & 387596 & 1.23 & 2.2140 & 0.000308 & 1.907E-11 & 10.72 \\
\hline 456 & 1209600 & 939462 & 0.794 & 1.4292 & 0.000199 & $9.826 E-14$ & 13.01 \\
\hline 1128 & 2419200 & 2716550 & 0.68 & 1.2240 & 0.000170 & $5.210 E-14$ & 13.28 \\
\hline 2160 & 3715200 & 5768861 & 0.1 & 0.1800 & 0.000025 & $1.015 E-15$ & 14.99 \\
\hline & & & & & \multicolumn{2}{|c|}{ Average Leach Index: } & 12.73 \\
\hline
\end{tabular}




\section{American Nuclear Society - 16.1 Static Leach Test}

Project: $\quad$ SRS Reactor Seepage Basin Stabilization

Sample Description: $\quad$ P332

Analyst: $\quad \mathrm{PRL}$

Date:

25-May-99

Contaminant:

Gross Alpha

Initial Contaminant Conc. (pCi/g):

19

Initial Contaminant Level ( $\mathrm{pCi})$ :

Monolith Height $(\mathrm{cm})$ :

7211.64

10

Monolith Surface Area $\left(\mathrm{cm}^{\wedge} 2\right)$ :

176.72

Monolith Volume $\left(\mathrm{cm}^{\wedge} 3\right)$ :

196.35

\begin{tabular}{|cccccccc|}
\hline Time & $\begin{array}{c}\text { Interval } \\
\text { Length } \\
(\mathrm{s})\end{array}$ & $\begin{array}{c}\text { Mean } \\
\text { Time } \\
(\mathrm{s})\end{array}$ & $\begin{array}{c}\text { Leachate } \\
\text { Conc. } \\
(\mathrm{pCi} / \mathrm{L})\end{array}$ & $\begin{array}{c}\text { Amount } \\
\text { Leached } \\
(\mathrm{pCi})\end{array}$ & $\begin{array}{c}\text { Fraction } \\
\text { Leached }\end{array}$ & $\begin{array}{c}\text { Diffusivity } \\
\text { Coefficient } \\
\left(\mathrm{cm}^{\wedge} 2 / \mathrm{s}\right)\end{array}$ & $\begin{array}{c}\text { Leach } \\
\text { Index }\end{array}$ \\
\hline 2 & 7200 & 1800 & 0.1 & 0.1800 & 0.000025 & $8.390 \mathrm{E}-14$ & 13.08 \\
7 & 18000 & 14835 & 0.475 & 0.8550 & 0.000119 & $2.496 \mathrm{E}-12$ & 11.60 \\
24 & 61200 & 51231 & 0.1 & 0.1800 & 0.000025 & $3.305 \mathrm{E}-14$ & 13.48 \\
48 & 86400 & 125894 & 0.1 & 0.1800 & 0.000025 & $4.075 \mathrm{E}-14$ & 13.39 \\
72 & 86400 & 213818 & 0.1 & 0.1800 & 0.000025 & $6.921 \mathrm{E}-14$ & 13.16 \\
96 & 86400 & 300849 & 0.1 & 0.1800 & 0.000025 & $9.738 \mathrm{E}-14$ & 13.01 \\
120 & 86400 & 387596 & 0.1 & 0.1800 & 0.000025 & $1.255 \mathrm{E}-13$ & 12.90 \\
456 & 1209600 & 939462 & 0.141 & 0.2538 & 0.000035 & $3.084 \mathrm{E}-15$ & 14.51 \\
1128 & 2419200 & 2716550 & 0.1 & 0.1800 & 0.000025 & $1.122 \mathrm{E}-15$ & 14.95 \\
2160 & 3715200 & 5768861 & 0.1 & 0.1800 & 0.000025 & $1.010 \mathrm{E}-15$ & 15.00 \\
& & & & & & & \\
& & & & & & & \\
& & & & & & & \\
\end{tabular}




\section{American Nuclear Society - 16.1 Static Leach Test}

Project: $\quad$ SRS Reactor Seepage Basin Stabilization

Sample Description: $\quad$ P322

$\begin{array}{llll}\text { Analyst: PRL Date: 25-May-99 } & \text { Dat }\end{array}$

Contaminant: Gross Alpha

Initial Contaminant Conc. ( $\mathrm{pCi} / \mathrm{g})$ :

Initial Contaminant Level ( $\mathrm{pCi})$ :

19

7503.1

Monolith Height (cm):

10

Monolith Surface Area $\left(\mathrm{cm}^{\wedge} 2\right)$ :

Monolith Volume $\left(\mathrm{cm}^{\wedge} 3\right)$ :

176.72

196.35

Sample Weight (g):

394.9

Monolith Diameter $(\mathrm{cm})$ :

5

Leachate Volume $(\mathrm{mL})$ :

1800

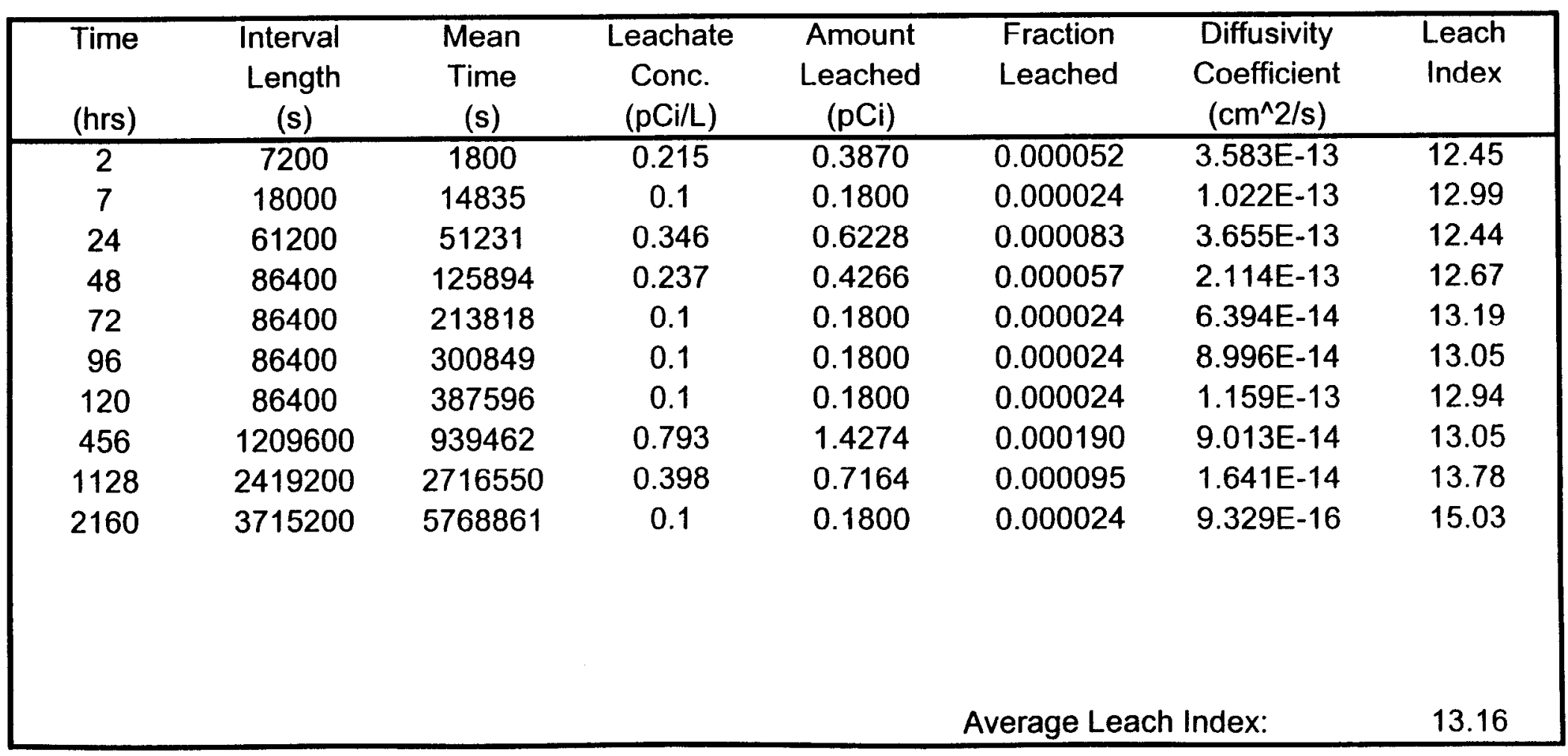




\section{American Nuclear Society - 16.1 Static Leach Test}

Project: $\quad$ SRS Reactor Seepage Basin Stabilization

Sample Description: $\quad$ P312

$\begin{array}{llll}\text { Analyst: PRL 25-May-99 } & \text { Date: }\end{array}$

Contaminant: Gross Alpha

Initial Contaminant Conc. (pCi/g):

Initial Contaminant Level (pCi):

Monolith Height $(\mathrm{cm})$ :

Monolith Surface Area $\left(\mathrm{cm}^{\wedge} 2\right)$ :

Monolith Volume $\left(\mathrm{cm}^{\wedge} 3\right)$ :

$$
\begin{gathered}
19 \\
7224.94 \\
10 \\
176.72 \\
196.35
\end{gathered}
$$

380.26

\begin{tabular}{|c|c|c|c|c|c|c|c|}
\hline $\begin{array}{l}\text { Time } \\
\text { (hrs) }\end{array}$ & $\begin{array}{l}\text { Interval } \\
\text { Length } \\
\text { (s) }\end{array}$ & $\begin{array}{l}\text { Mean } \\
\text { Time } \\
\text { (s) }\end{array}$ & $\begin{array}{c}\text { Leachate } \\
\text { Conc. } \\
\text { (pCi/L) }\end{array}$ & $\begin{array}{l}\text { Amount } \\
\text { Leached } \\
\text { (pCi) }\end{array}$ & $\begin{array}{l}\text { Fraction } \\
\text { Leached }\end{array}$ & $\begin{array}{l}\text { Diffusivity } \\
\text { Coefficient } \\
\left(\mathrm{cm}^{\wedge} 2 / \mathrm{s}\right)\end{array}$ & $\begin{array}{l}\text { Leach } \\
\text { Index }\end{array}$ \\
\hline 2 & 7200 & 1800 & 0.1 & 0.1800 & 0.000025 & $8.359 \mathrm{E}-14$ & 13.08 \\
\hline 7 & 18000 & 14835 & 0.147 & 0.2646 & 0.000037 & $2.382 E-13$ & 12.62 \\
\hline 24 & 61200 & 51231 & 0.1 & 0.1800 & 0.000025 & 3.293E-14 & 13.48 \\
\hline 48 & 86400 & 125894 & 0.2 & 0.3600 & 0.000050 & $1.624 \mathrm{E}-13$ & 12.79 \\
\hline 72 & 86400 & 213818 & 0.1 & 0.1800 & 0.000025 & $6.895 E-14$ & 13.16 \\
\hline 96 & 86400 & 300849 & 0.354 & 0.6372 & 0.000088 & $1.216 \mathrm{E}-12$ & 11.92 \\
\hline 120 & 86400 & 387596 & 0.1 & 0.1800 & 0.000025 & $1.250 \mathrm{E}-13$ & 12.90 \\
\hline 456 & 1209600 & 939462 & 0.359 & 0.6462 & 0.000089 & $1.992 E-14$ & 13.70 \\
\hline 1128 & 2419200 & 2716550 & 0.159 & 0.2862 & 0.000040 & $2.825 E-15$ & 14.55 \\
\hline 2160 & 3715200 & 5768861 & 0.309 & 0.5562 & 0.000077 & $9.607 E-15$ & 14.02 \\
\hline & & & & \multicolumn{3}{|c|}{ Average Leach Index: } & 13.22 \\
\hline
\end{tabular}

Monolith Diameter (cm):

Leachate Volume $(\mathrm{mL})$ :

5

1800 
Gross Alpha Leaching for the Coarse Fraction from PRSB Soil

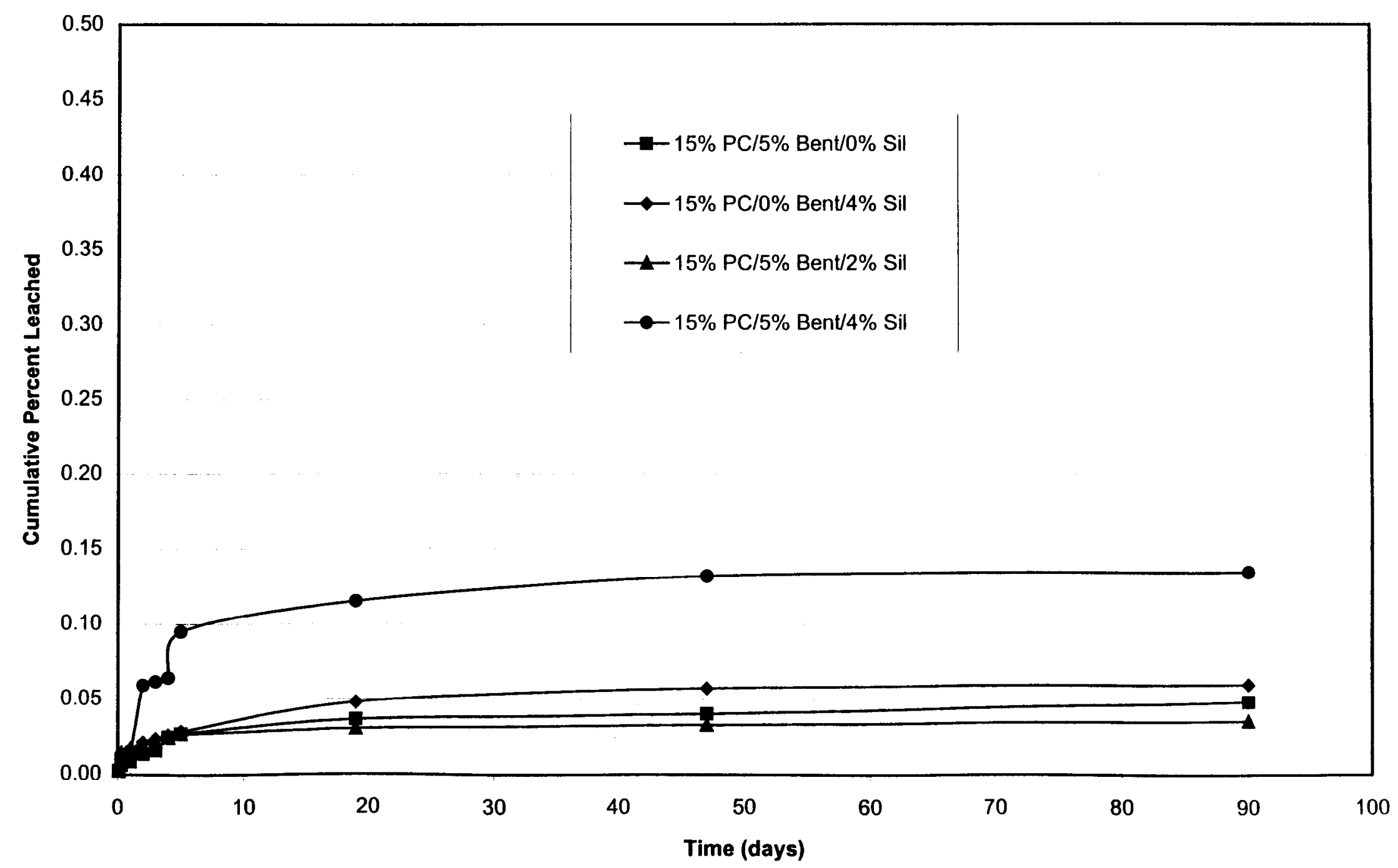




\section{MOISTURE CONTENT}

PROJECT NAME

SAIC - Savannah River
PROJECT NUMBER

777304.00010500

\begin{tabular}{|c|c|c|c|c|}
$\begin{array}{c}\text { IT LAB } \\
\text { SAMPLE NO. }\end{array}$ & $\begin{array}{c}\text { CLIENT } \\
\text { SAMPLE NO. }\end{array}$ & $\begin{array}{c}\text { MOISTURE, \% } \\
\text { ASTM D 2216 }\end{array}$ & $\begin{array}{c}\text { MOISTURE , \% } \\
\text { SW846 }\end{array}$ & $\begin{array}{c}\text { SOLIDS, \% } \\
\text { SW846 }\end{array}$ \\
\hline ETDC-8015 & P100000 & 13.1 & 11.6 & 88.4 \\
\hline ETDC-8016 & P200000 & 14.9 & 13.0 & 87.0 \\
\hline ETDC-8017 & P300000 & 14.9 & 13.0 & 87.0 \\
\hline ETDC-8018 & P400000 & 19.6 & 16.4 & 83.6 \\
\hline ETDC-8019 & P500000 & 14.9 & 13.0 & 87.0 \\
\hline ETDC-8020 & P600000 & 13.1 & 11.6 & 88.4 \\
\hline ETDC-8021 & P700000 & 14.9 & 13.0 & 87.0 \\
\hline ETDC-8022 & P800000 & 15.9 & 13.7 & 86.3 \\
\hline & & & & \\
\hline & & & & \\
\hline & & & & \\
\hline & & & & \\
\hline & & & & \\
\hline & & & & \\
\hline & & & & \\
\hline & & & & \\
\hline & & & & \\
\hline & & & & \\
\hline
\end{tabular}

ASTM D 2216 results are based on dry sample weight. SW846 results are based on wet sample weight.

Solids content is determined by subtracting the SW846 moisture $(\%)$ from 100 . 


\section{SPECIFIC GRAVITY ASTM D 854}

PROJECT NAME:

PROJECT NUMBER:

SAIC - Savannah River

777304.00010500

\begin{tabular}{l} 
IT LAB SAMPLE NO. \\
\begin{tabular}{|c|c|c|}
\hline ETDC-8015 & P100000 & 2.6407 \\
\hline ETDC-8016 & P200000 & 2.6446 \\
\hline ETDC-8017 & P300000 & 2.6429 \\
\hline ETDC-8018 & P400000 & 2.6374 \\
\hline ETDC-8019 & P500000 & 2.6428 \\
\hline ETDC-8020 & P600000 & 2.6408 \\
\hline ETDC-8021 & P700000 & 2.6435 \\
\hline ETDC-8022 & P800000 & 2.6428 \\
\hline & & \\
\hline & & \\
\hline & & \\
\hline & & \\
\hline & & \\
\hline & & \\
\hline & & \\
\hline & & \\
\hline & & \\
\hline
\end{tabular} \\
\hline
\end{tabular}




\section{ATTERBERG LIMITS \\ ASTM D 4318}

PROJECT NAME:

SAIC - Savannah River

PROJECT NO.

777304.00010500

\section{ATTERBERG LIMITS RESULTS}

\begin{tabular}{|c|c|c|c|c|c|c|}
\hline LAB SAMPLE & No. & FIELD $\mathbf{S}$ & LIQUID LIMIT & PLASTIC LIMIT & PLASTICITY INDEX & USCS SYMBOL \\
\hline ETDC-8015 & $\bullet$ & P100000 & 35 & 18 & 17 & $\mathrm{CL}$ \\
\hline ETDC-8016 & - & P200000 & 44 & 21 & 23 & $\mathrm{CL}$ \\
\hline ETDC-8017 & D & P300000 & 33 & 19 & 14 & $C L$ \\
\hline ETDC-8018 & 0 & P400Q00 & 38 & 20 & 18 & $\overline{C L}$ \\
\hline ETDC-8019 & + & P500000 & 39 & 19 & 20 & $C L$ \\
\hline ETDC-8020 & 0 & P600000 & 45 & 21 & 24 & $C L$ \\
\hline ETDC-8021 & E & P700000 & 46 & 20 & 26 & $\mathrm{CL}$ \\
\hline ETDC-8022 & $x$ & P800Q00 & 50 & 23 & 27 & $\mathrm{CH}$ \\
\hline & $\Delta$ & & & & & \\
\hline & * & & & & & \\
\hline
\end{tabular}

-NP = Nonplestic

\section{PLASTICITY CHART}

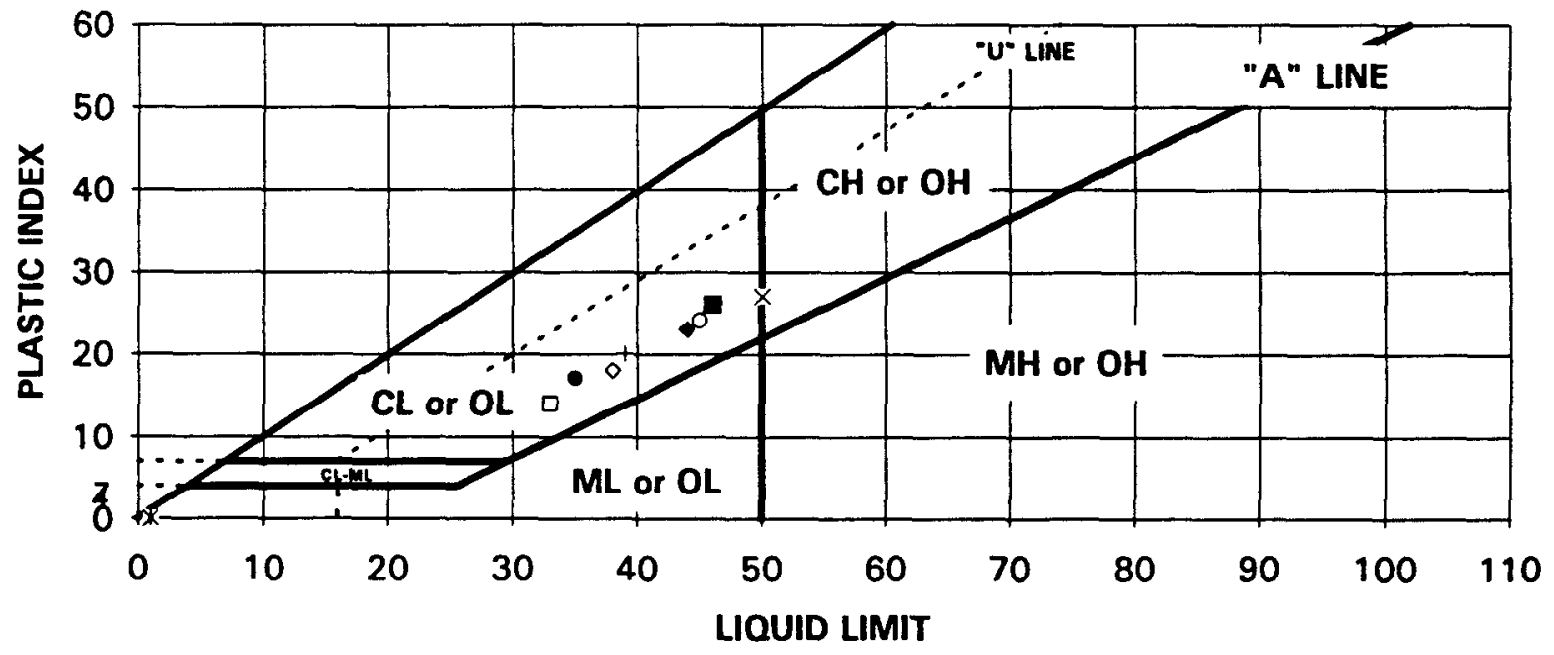




\section{SOIL CLASSIFICATION ASTM D 2487}

\begin{tabular}{|l|c|c|c|c|}
\hline & $\begin{array}{c}\text { ETDC } \\
\text { NUMBER: }\end{array}$ & $\begin{array}{c}\text { CLIENT } \\
\text { NUMBER: }\end{array}$ & $\begin{array}{c}\text { GROUP } \\
\text { SYMBOL: }\end{array}$ & $\begin{array}{c}\text { CLASSIFICATION- } \\
\text { GROUP NAME: }\end{array}$ \\
\hline SAMPLE 1: & ETDC-8015 & P100000 & SC & CLAYEY SAND \\
\hline SAMPLE 2: & ETDC-8016 & P200000 & SC & CLAYEY SAND \\
\hline SAMPLE 3: & ETDC-8017 & P300000 & SC & CLAYEY SAND \\
\hline SAMPLE 4: & ETDC-8018 & P400000 & SC & CLAYEY SAND \\
\hline SAMPLE 5: & ETDC-8019 & P500000 & SC & CLAYEY SAND \\
\hline SAMPLE 6: & ETDC-8020 & P600000 & SC & CLAYEY SAND \\
\hline SAMPLE 7: & ETDC-8021 & P700000 & SC & CLAYEY SAND \\
\hline SAMPLE 8: & ETDC-8022 & P800000 & SC & CLAYEY SAND \\
\hline SAMPLE 9: & & & & \\
\hline SAMPLE 10: & & & & \\
\hline
\end{tabular}




\section{SOIL CLASSIFICATION ASTM D 2487}

\begin{tabular}{|l|c|c|c|c|c|}
\hline & SAMPLE 1 & SAMPLE 2 & SAMPLE 3 & SAMPLE 4 & SAMPLE 5 \\
\hline ETDC NUMBER: & ETDC-8015 & ETDC-8016 & ETDC-8017 & ETDC-8018 & ETDC-8019 \\
\hline CLIENT NUMBER: & P100000 & P200000 & P300000 & P400000 & P500000 \\
\hline $\begin{array}{l}\text { (COARSE-GRAVELS, SANDS) } \\
\text { \% RETAINED ON \# 200 SIEVE: }\end{array}$ & 71.6 & & & & \\
\hline $\begin{array}{l}\text { (FINE-SILTS, CLAYS) } \\
\text { \% PASSING \# 200 SIEVE: }\end{array}$ & 28.4 & 30.0 & 69.5 & 65.2 & 68.3 \\
\hline$\%$ GRAVEL: & 3.8 & 30.0 & 30.5 & 34.8 & 31.7 \\
\hline \% SAND: & 67.7 & 1.2 & 0.9 & 1.6 & 1.4 \\
\hline I60 IN MM (DIAMETER AT 60\% PASSING): & 0.40685 & 0.41277 & 0.33018 & 0.29156 & 0.27654 \\
\hline J30 IN MM (DIAMETER AT 30\% PASSING): & 0.10171 & 0.07460 & 0.06911 & 0.00983 & 0.05882 \\
\hline D10 IN MM (DIAMETER AT 10\% PASSING): & 0.00078 & 0.00063 & 0.00068 & 0.00054 & 0.00060 \\
\hline CC: & 520.88 & 658.01 & 486.53 & 539.69 & 457.48 \\
\hline Cu: & 32.56 & 21.49 & 21.32 & 0.61 & 20.70 \\
\hline LIQUID LIMIT: & 35 & 44 & 33 & 38 & 39 \\
\hline PLASTIC INDEX: & 17 & 23 & 14 & 18 & 20 \\
\hline ATTERBERG CLASSIFICATION*: & $\mathrm{CL}$ & $\mathrm{CL}$ & $\mathrm{CL}$ & $\mathrm{CL}$ & $\mathrm{CL}$ \\
\hline
\end{tabular}

\begin{tabular}{|c|c|c|c|c|c|}
\hline & SAMPLE 6 & SAMPLE 7 & SAMPLE 8 & SAMPLE 9 & SAMPLE 10 \\
\hline ETDC NUMBER: & ETDC-8020 & ETDC-8021 & ETDC-8022 & & \\
\hline CLIENT NUMBER: & P600000 & P700000 & P800000 & & \\
\hline $\begin{array}{l}\text { (COARSE-GRAVELS, SANDS) } \\
\% \text { RETAINED ON \# } 200 \text { SIEVE: }\end{array}$ & 74.2 & 69.4 & 68.2 & & \\
\hline $\begin{array}{l}\text { (FINE-SILTS, CLAYS) } \\
\% \text { PASSING \# } 200 \text { SIEVE: }\end{array}$ & 25.8 & 30.6 & 31.8 & & \\
\hline \% GRAVEL: & 5.1 & 3.7 & 0.9 & & \\
\hline \% SAND: & 69.1 & 65.7 & 67.3 & & \\
\hline D60 IN MM (DIAMETER AT 60\% PASSING): & 0.57715 & 0.41961 & 0.44716 & & \\
\hline D30 IN MM (DIAMETER AT 30\% PASSING): & 0.15436 & 0.05435 & 0.05921 & & \\
\hline D10 IN MM (DIAMETER AT 10\% PASSING): & 0.00072 & 0.00063 & 0.00183 & & \\
\hline Cc: & 796.41 & 669.95 & 244.88 & & \\
\hline Cu: & 56.97 & 11.24 & 4.29 & & \\
\hline LIQUID LIMIT: & 45 & 46 & 50 & & \\
\hline JLASTIC INDEX: & 24 & 26 & 27 & & \\
\hline ATTERBERG CLASSIFICATION *: & $\mathrm{CL}$ & $\mathrm{CL}$ & $\mathrm{CH}$ & & \\
\hline
\end{tabular}




\section{PARTICLE-SIZE ANALYSIS \\ ASTM D 422}

$\begin{array}{llll}\text { Project Name } & \text { SAIC }- \text { Savannah River } & \text { Client Sample No. } & \text { P100000 } \\ \text { Project No. } & 777304.00010500 & \text { IT Lab Sample No. } & \text { ETDC-8015 } \\ \text { Specific Gravity }=2.6407 & \begin{array}{l}\text { Moisture Content }= \\ \text { besed on dry sample weight }\end{array}\end{array}$

SIEVE ANALYSIS

\begin{tabular}{|c|c|c|c|}
\hline & $\begin{array}{c}\text { Sieve } \\
\text { No. }\end{array}$ & $\begin{array}{c}\text { Diameter } \\
\mathrm{mm}\end{array}$ & $\begin{array}{c}\text { Percent } \\
\text { Finer }\end{array}$ \\
\cline { 2 - 4 } O & $3^{n}$ & 75.000 & $100.0 \%$ \\
\hline R & $1.5^{n}$ & 37.500 & $100.0 \%$ \\
\hline$S$ & $0.75^{n}$ & 19.000 & $100.0 \%$ \\
\hline E & $0.375^{n}$ & 9.500 & $97.1 \%$ \\
\cline { 2 - 4 } & $\# 4$ & 4.750 & $96.2 \%$ \\
\hline 410 & 2.000 & $94.4 \%$ \\
\hline
\end{tabular}

\begin{tabular}{|c|c|c|c|}
\hline \multirow{4}{*}{$F$} & $\begin{array}{c}\text { Sieve } \\
\text { No. }\end{array}$ & $\begin{array}{c}\text { Diameter } \\
\mathrm{mm}\end{array}$ & $\begin{array}{c}\text { Percent } \\
\text { Finer }\end{array}$ \\
\cline { 2 - 4 } $\mathrm{I}$ & $\# 20$ & 0.850 & $84.7 \%$ \\
\cline { 2 - 4 } & $\# 40$ & 0.425 & $61.8 \%$ \\
\hline & $\# 60$ & 0.250 & $44.0 \%$ \\
\cline { 2 - 4 } & $\# 100$ & 0.149 & $33.7 \%$ \\
\hline & $\# 140$ & 0.106 & $30.3 \%$ \\
\hline & $\# 200$ & 0.075 & $28.4 \%$ \\
\hline
\end{tabular}

HYDROMETER ANALYSIS

\begin{tabular}{|c|c|c|}
\hline \multirow{2}{*}{$\mathrm{H}$} & $\begin{array}{c}\text { Diameter } \\
\mathrm{mm}\end{array}$ & $\begin{array}{c}\text { Percent } \\
\text { Finer }\end{array}$ \\
\hline & & \\
\hline D & 0.04781 & $27.9 \%$ \\
\hline $\mathrm{R}$ & 0.03395 & $26.5 \%$ \\
\hline 0 & 0.02165 & $25.1 \%$ \\
\hline$M$ & 0.01260 & $23.8 \%$ \\
\hline $\begin{array}{l}E \\
T\end{array}$ & 0.00895 & $22.4 \%$ \\
\hline E & 0.00630 & $21.7 \%$ \\
\hline$R$ & 0.00453 & $19.7 \%$ \\
\hline & 0.00315 & $19.0 \%$ \\
\hline & 0.00133 & $17.0 \%$ \\
\hline
\end{tabular}


HYDROMETER

U.S. STANDARD SIEVE SIZES

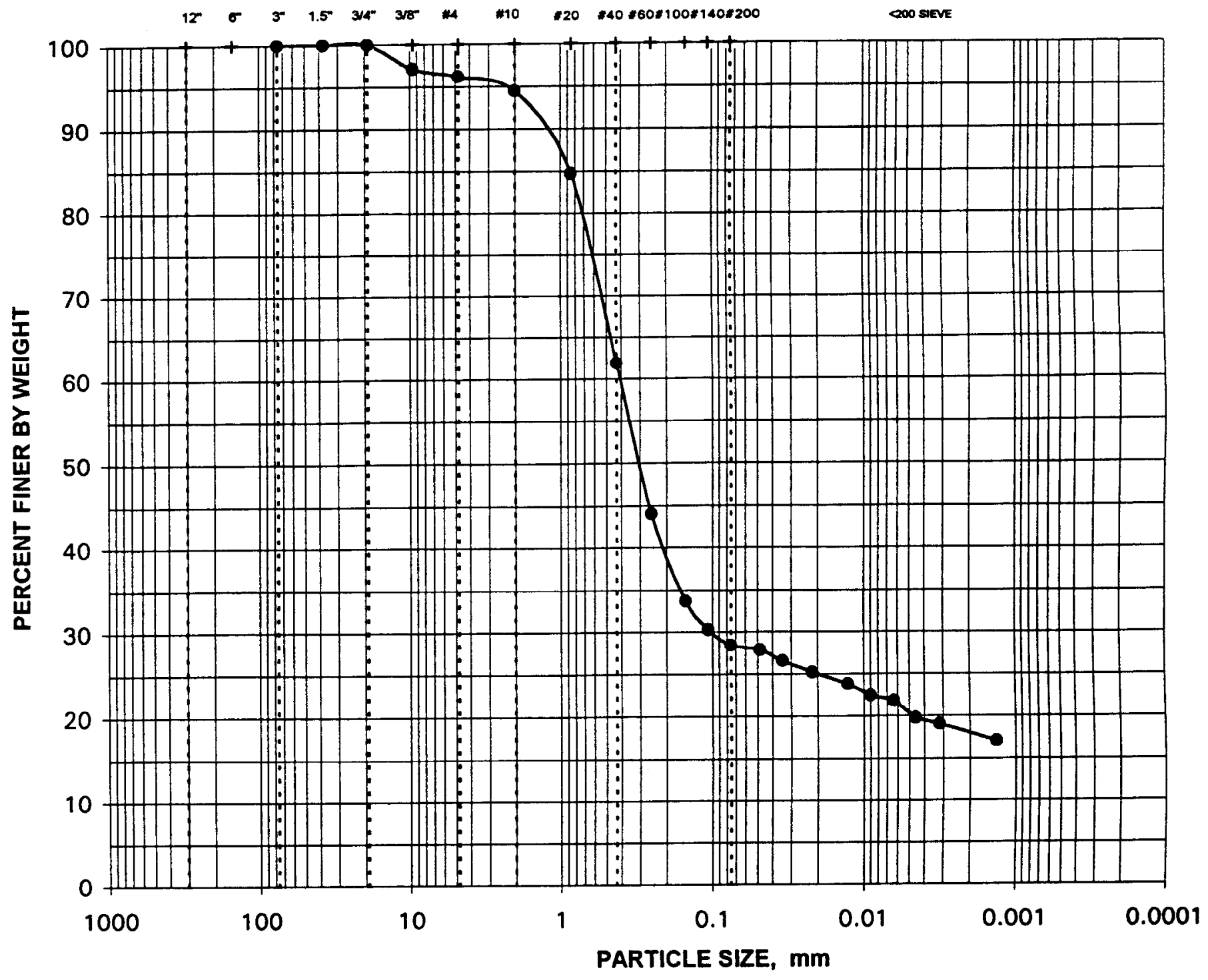

client sample no.: $\quad$ P100Q00

IT LAB SAMPLE NO:: $\quad$ ETDC-8015

\begin{tabular}{|c|c|c|c|c|c|c|c|}
\hline \multirow{2}{*}{$\begin{array}{l}B \\
O \\
U \\
L \\
D \\
E \\
\text { R } \\
\text { S }\end{array}$} & \multirow{2}{*}{$\begin{array}{l}C \\
0 \\
B \\
B \\
L \\
E \\
S\end{array}$} & \multicolumn{2}{|c|}{ GRAVEL } & \multicolumn{3}{|c|}{ SAND } & \multirow[b]{2}{*}{ CLAY $<2$ microns } \\
\hline & & $\begin{array}{l}\text { C } \\
0 \\
\hat{n} \\
\hat{k} \\
\text { E }\end{array}$ & $\begin{array}{l}F \\
1 \\
\text { N } \\
\text { E }\end{array}$ & $\begin{array}{l}\text { C } \\
0 \\
\hat{A} \\
\text { s } \\
\text { E }\end{array}$ & $\begin{array}{l}\text { M } \\
\text { E } \\
D \\
\vdots \\
U \\
\text { M }\end{array}$ & $\begin{array}{l}F \\
1 \\
N \\
E\end{array}$ & \\
\hline
\end{tabular}




\section{PARTICLE-SIZE ANALYSIS ASTM D 422}
Project Name SAIC - Savannah River
Client Sample No. P200000
Project No.
777304.00010500
IT Lab Sample No. ETDC-8016
Specific Gravity $=2.6446$
Moisture Content $=\quad \mathbf{1 4 . 9} \%$
based on dry sample woight

SIEVE ANALYSIS

\begin{tabular}{|c|c|c|c|}
\hline & $\begin{array}{c}\text { Sieve } \\
\text { No. }\end{array}$ & $\begin{array}{c}\text { Diameter } \\
\mathrm{mm}\end{array}$ & $\begin{array}{c}\text { Percent } \\
\text { Finer }\end{array}$ \\
\hline $\mathrm{O}$ & $3^{\prime \prime}$ & 75.000 & $100.0 \%$ \\
$\mathrm{~A}$ & $1.5^{\prime \prime}$ & 37.500 & $100.0 \%$ \\
$\mathrm{R}$ & $0.75^{\prime \prime}$ & 19.000 & $100.0 \%$ \\
$\mathrm{~S}$ & $0.375^{\prime \prime}$ & 9.500 & $100.0 \%$ \\
$\mathrm{E}$ & $\# 4$ & 4.750 & $98.8 \%$ \\
\cline { 2 - 4 } & $\# 10$ & 2.000 & $95.7 \%$ \\
\hline
\end{tabular}

\begin{tabular}{|c|c|c|c|}
\hline & $\begin{array}{c}\text { Sieve } \\
\text { No. }\end{array}$ & $\begin{array}{c}\text { Diameter } \\
\mathrm{mm}\end{array}$ & $\begin{array}{c}\text { Percent } \\
\text { Finer }\end{array}$ \\
\cline { 2 - 4 } $\mathrm{F}$ & $\# 20$ & 0.850 & $84.7 \%$ \\
\hline $\mathrm{N}$ & $\# 40$ & 0.425 & $61.0 \%$ \\
$\mathrm{E}$ & $\# 60$ & 0.250 & $46.1 \%$ \\
\cline { 2 - 4 } & $\# 100$ & 0.149 & $35.1 \%$ \\
\hline & $\# 140$ & 0.106 & $31.6 \%$ \\
\hline & $\# 200$ & 0.075 & $30.0 \%$ \\
\hline
\end{tabular}

HYDROMETER ANAL YSIS

\begin{tabular}{|c|c|c|}
\hline & $\begin{array}{c}\text { Diameter } \\
\mathrm{mm}\end{array}$ & $\begin{array}{c}\text { Percent } \\
\text { Finer }\end{array}$ \\
\hline$H$ & & \\
\cline { 2 - 3 } & & $29.1 \%$ \\
\hline$R$ & 0.04861 & $27.7 \%$ \\
\cline { 2 - 3 } & 0.03466 & $26.4 \%$ \\
\hline$M$ & 0.02201 & $25.7 \%$ \\
\hline & 0.01276 & $25.0 \%$ \\
\hline$T$ & 0.00906 & $24.3 \%$ \\
\hline & 0.00635 & $23.0 \%$ \\
\hline$R$ & 0.00452 & $22.3 \%$ \\
\cline { 2 - 3 } & 0.00310 & $20.9 \%$ \\
\hline
\end{tabular}




\section{SAIC - Savannah River}

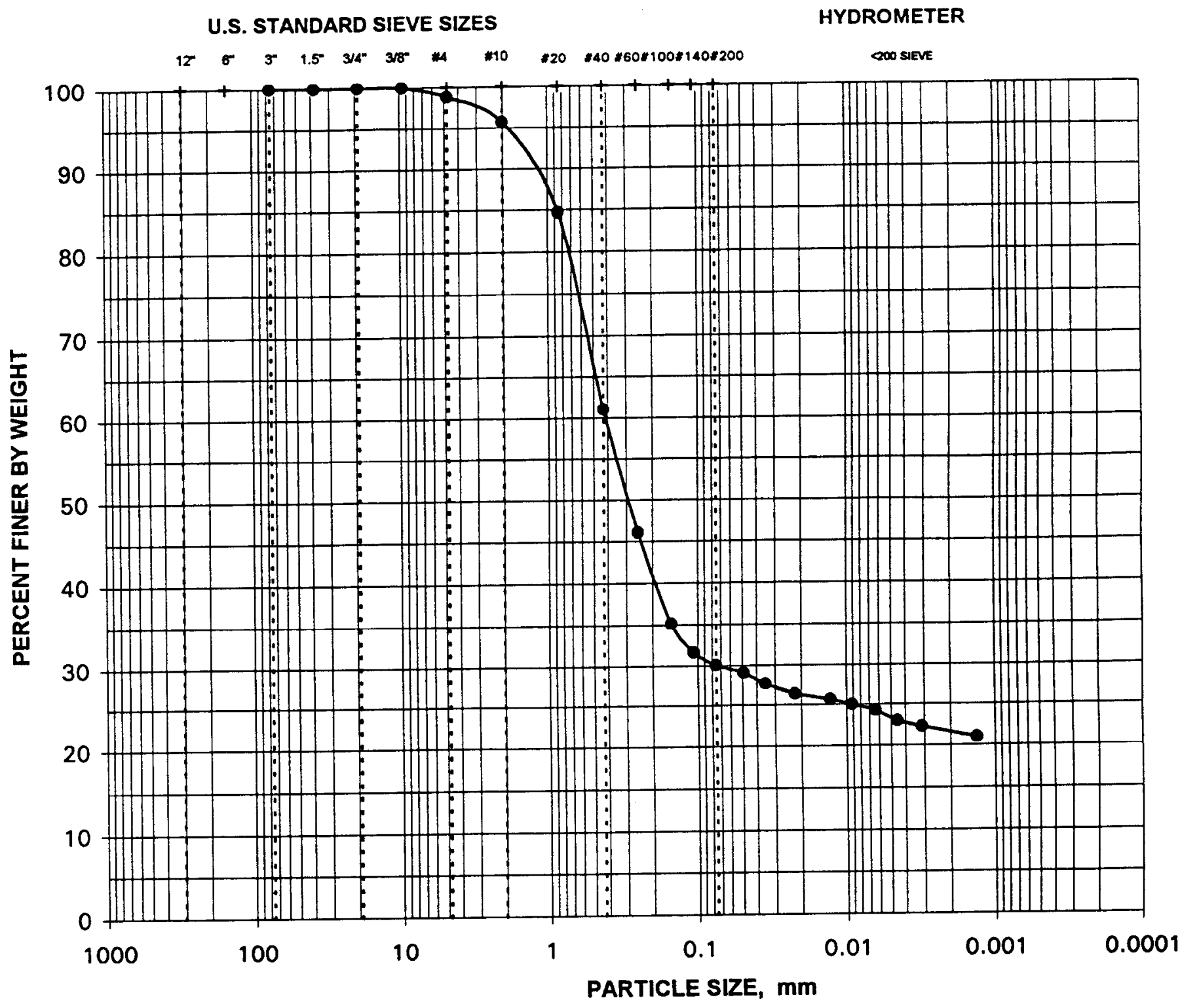

Client SAMPLE NO.: $\quad$ P200Q00

IT LAB SAMPLE NO.:

ETDC-8016

\begin{tabular}{|c|c|c|c|c|c|c|c|}
\hline \multirow{2}{*}{$\begin{array}{l}B \\
O \\
U \\
L \\
D \\
\text { E } \\
R \\
S\end{array}$} & \multirow{2}{*}{$\begin{array}{l}\text { C } \\
\text { O } \\
\text { B } \\
\text { B } \\
\text { L } \\
\text { E } \\
\text { S }\end{array}$} & \multicolumn{2}{|c|}{ GRAVEL } & \multicolumn{3}{|c|}{ SAND } & SILT 2 - 75 microns \\
\hline & & $\begin{array}{l}\mathrm{C} \\
\mathrm{a} \\
\hat{A} \\
\mathrm{R} \\
\mathrm{S} \\
\mathrm{E}\end{array}$ & $\begin{array}{l}F \\
1 \\
\text { N } \\
\text { E }\end{array}$ & $\begin{array}{l}\text { C } \\
0 \\
\hat{A} \\
\text { S } \\
\text { E }\end{array}$ & $\begin{array}{l}\text { M } \\
\mathbf{E} \\
\mathbf{D} \\
\mathbf{U} \\
\mathbf{U}\end{array}$ & $\begin{array}{l}F \\
\text { I } \\
\text { N } \\
\text { s }\end{array}$ & CLAY $<2$ microns \\
\hline
\end{tabular}




\section{PARTICLE-SIZE ANALYSIS \\ ASTM D 422}

$\begin{array}{llll}\text { Project Name } & \text { SAIC - Savannah River } & \text { Client Sample No. } & \text { P300000 } \\ \text { Project No. } & 777304.00010500 & \text { IT Lab Sample No. } & \text { ETDC-8017 } \\ \text { Specific Gravity }=2.6429 & \begin{array}{l}\text { Moisture Content }= \\ \text { based on dry sample weight }\end{array}\end{array}$

SIEVE ANALYSIS

\begin{tabular}{|c|c|c|c|}
\hline & $\begin{array}{c}\text { Sieve } \\
\text { No. }\end{array}$ & $\begin{array}{c}\text { Diameter } \\
\mathrm{mm}\end{array}$ & $\begin{array}{c}\text { Percent } \\
\text { Finer }\end{array}$ \\
\cline { 2 - 4 } $\mathrm{O}$ & $3^{\prime \prime}$ & 75.000 & $100.0 \%$ \\
$\mathrm{~A}$ & $1.5^{\prime \prime}$ & 37.500 & $100.0 \%$ \\
$\mathrm{R}$ & $0.75^{\prime \prime}$ & 19.000 & $100.0 \%$ \\
$\mathrm{~S}$ & $0.375^{\prime \prime}$ & 9.500 & $100.0 \%$ \\
$\mathrm{E}$ & $\# 4$ & 4.750 & $99.1 \%$ \\
\cline { 2 - 4 } & $\# 10$ & 2.000 & $98.2 \%$ \\
\hline
\end{tabular}

\begin{tabular}{|c|c|c|c|}
\hline & $\begin{array}{c}\text { Sieve } \\
\text { No. }\end{array}$ & $\begin{array}{c}\text { Diameter } \\
\mathrm{mm}\end{array}$ & $\begin{array}{c}\text { Percent } \\
\text { Finer }\end{array}$ \\
\cline { 2 - 4 } F & $\# 20$ & 0.850 & $91.9 \%$ \\
\hline N & $\# 40$ & 0.425 & $70.3 \%$ \\
\hline E & $\# 60$ & 0.250 & $51.3 \%$ \\
\cline { 2 - 4 } & $\# 100$ & 0.149 & $37.5 \%$ \\
\hline & $\# 140$ & 0.106 & $32.9 \%$ \\
\hline & $\# 200$ & 0.075 & $30.5 \%$ \\
\hline
\end{tabular}

HYDROMETER ANALYSIS

\begin{tabular}{|c|c|c|}
\hline \multirow{2}{*}{$H$} & $\begin{array}{c}\text { Diameter } \\
\mathrm{mm}\end{array}$ & $\begin{array}{c}\text { Percent } \\
\text { Finer }\end{array}$ \\
\hline & & \\
\hline D & 0.04922 & $28.3 \%$ \\
\hline $\mathrm{R}$ & 0.03495 & $27.6 \%$ \\
\hline 0 & 0.02220 & $26.8 \%$ \\
\hline$M$ & 0.01251 & $25.4 \%$ \\
\hline E & 0.00892 & $23.2 \%$ \\
\hline E & 0.00645 & $22.5 \%$ \\
\hline$R$ & 0.00458 & $21.8 \%$ \\
\hline & 0.00315 & $19.6 \%$ \\
\hline & 0.00133 & $19.6 \%$ \\
\hline
\end{tabular}




\section{SAIC - Savannah River}

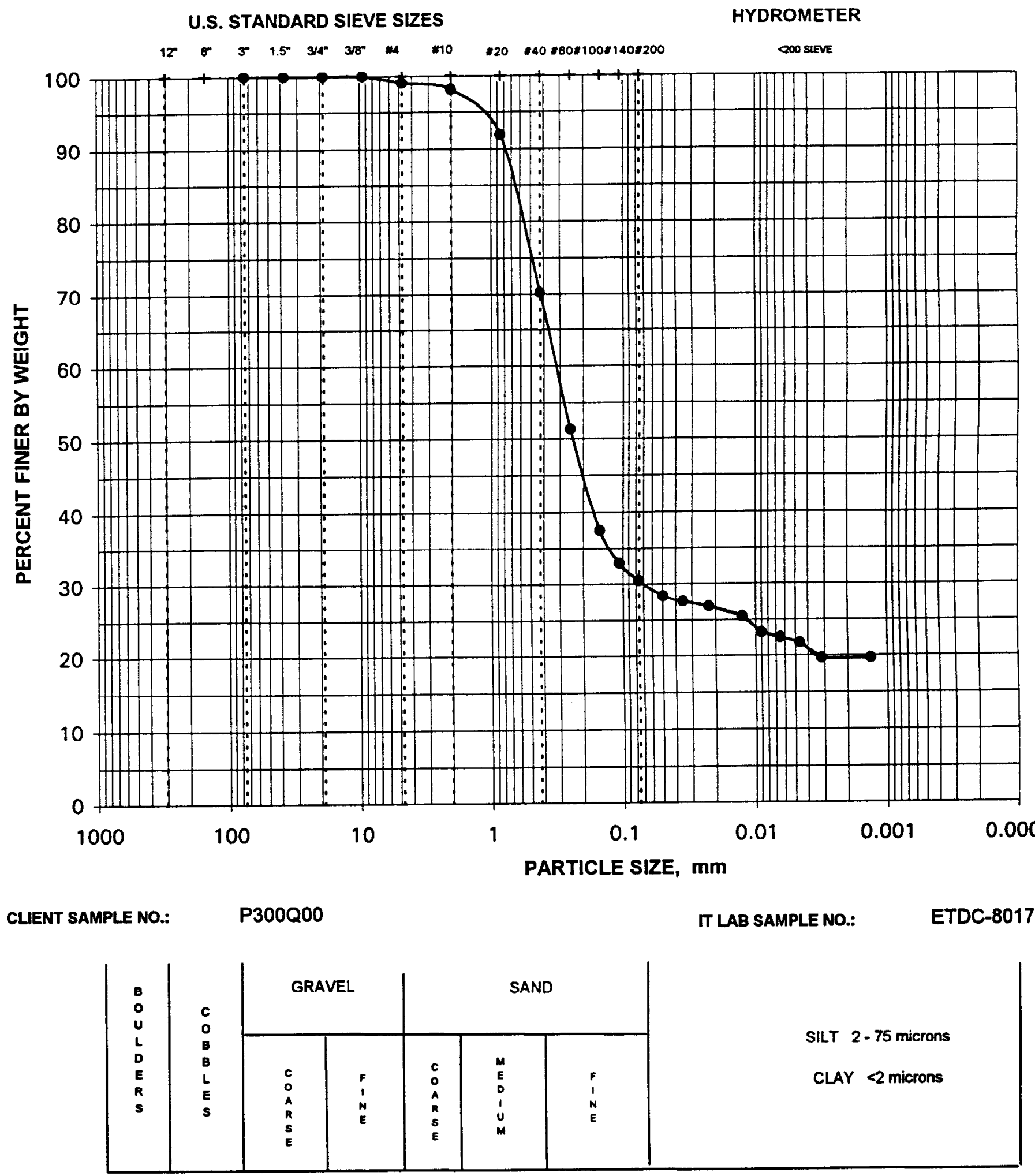




\section{PARTICLE-SIZE ANALYSIS \\ ASTM D 422}
Project Name
SAIC - Savannah River
Client Sample No.
P400000
Project No.
777304.00010500
IT Lab Sample No.
ETDC-8018
Specific Gravity $=2.6374$
Moisture Content $=19.6 \%$
based on dry sample woight

SIEVE ANALYSIS

\begin{tabular}{|c|c|c|c||}
\hline & $\begin{array}{c}\text { Sieve } \\
\text { No. }\end{array}$ & $\begin{array}{c}\text { Diameter } \\
\mathrm{mm}\end{array}$ & $\begin{array}{c}\text { Percent } \\
\text { Finer }\end{array}$ \\
\hline $\mathrm{O}$ & $3^{n}$ & 75.000 & $100.0 \%$ \\
\hline $\mathrm{A}$ & $1.5^{n}$ & 37.500 & $100.0 \%$ \\
\cline { 2 - 4 } $\mathrm{S}$ & $0.75^{n}$ & 19.000 & $100.0 \%$ \\
\cline { 2 - 4 } $\mathrm{E}$ & $0.375^{\prime \prime}$ & 9.500 & $99.2 \%$ \\
\cline { 2 - 4 } & $\# 4$ & 4.750 & $98.4 \%$ \\
\hline & $\# 10$ & 2.000 & $97.2 \%$ \\
\hline
\end{tabular}

\begin{tabular}{|c|c|c|c|}
\hline & $\begin{array}{c}\text { Sieve } \\
\text { No. }\end{array}$ & $\begin{array}{c}\text { Diameter } \\
\mathrm{mm}\end{array}$ & $\begin{array}{c}\text { Percent } \\
\text { Finer }\end{array}$ \\
\cline { 2 - 4 } $\mathrm{F}$ & $\# 20$ & 0.850 & $90.0 \%$ \\
\hline $\mathrm{N}$ & $\# 40$ & 0.425 & $71.4 \%$ \\
\hline $\mathrm{N}$ & $\# 60$ & 0.250 & $56.4 \%$ \\
\cline { 2 - 4 } & $\# 100$ & 0.149 & $41.6 \%$ \\
\cline { 2 - 4 } & $\# 140$ & 0.106 & $36.8 \%$ \\
\hline & $\# 200$ & 0.075 & $34.8 \%$ \\
\hline
\end{tabular}

HYDROMETER ANALYSIS

\begin{tabular}{|c|c|c|}
\hline \multirow{2}{*}{ H } & $\begin{array}{c}\text { Diameter } \\
\mathrm{mm}\end{array}$ & $\begin{array}{c}\text { Percent } \\
\text { Finer }\end{array}$ \\
\hline & & \\
\hline D & 0.04777 & $33.1 \%$ \\
\hline R & 0.03393 & $32.4 \%$ \\
\hline 0 & 0.02150 & $31.7 \%$ \\
\hline M & 0.01244 & $31.0 \%$ \\
\hline$E$ & 0.00887 & $29.6 \%$ \\
\hline E & 0.00625 & $28.3 \%$ \\
\hline$R$ & 0.00444 & $26.9 \%$ \\
\hline & 0.00309 & $24.1 \%$ \\
\hline & 0.00130 & $24.1 \%$ \\
\hline
\end{tabular}




\section{SAIC - Savannah River}

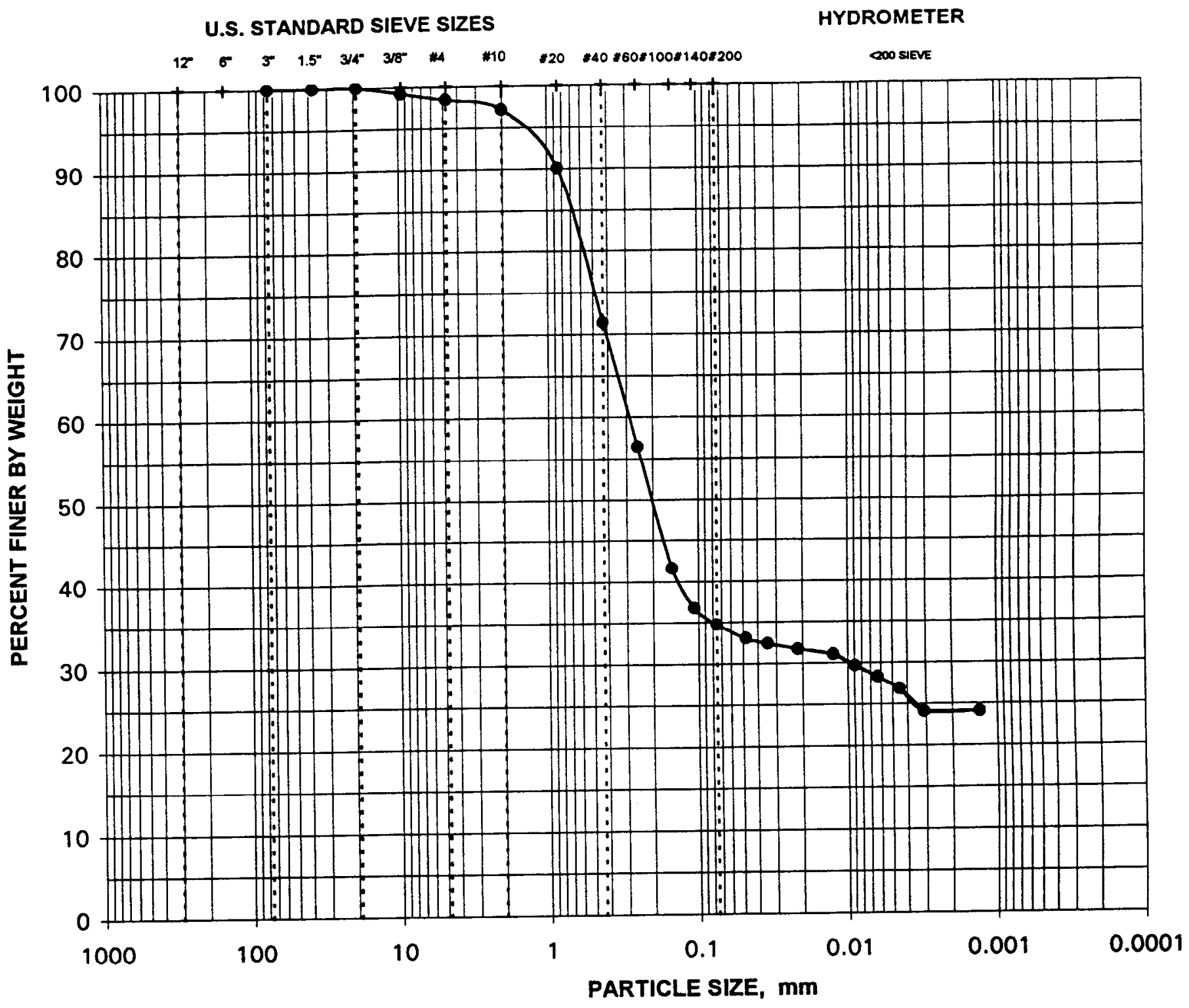

Client SAMPle No: $\quad$ P400Q00

IT LAB SAMPLE NO.:

ETDC-8018

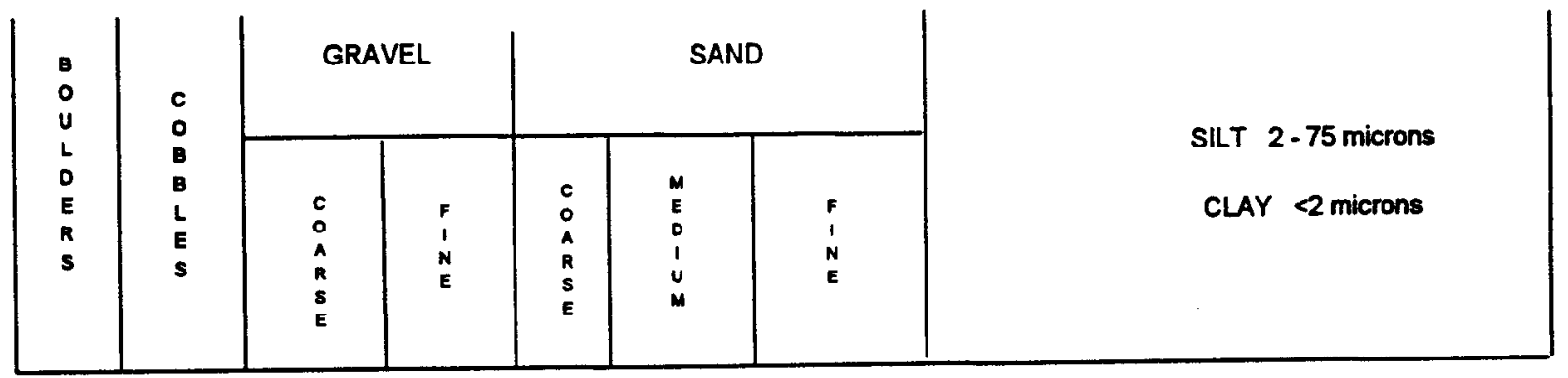




\section{PARTICLE-SIZE ANALYSIS \\ ASTM D 422}

Project Name SAIC - Savannah River

Project No.

Specific Gravity $=2.6428$
Client Sample No. P500000

IT Lab Sample No. ETDC-8019

Moisture Content $=14.9 \%$

based on dry sample weight

SIEVE ANALYSIS

\begin{tabular}{|c|c|c|c||}
\hline & $\begin{array}{c}\text { Sieve } \\
\text { No. }\end{array}$ & $\begin{array}{c}\text { Diameter } \\
\mathrm{mm}\end{array}$ & $\begin{array}{c}\text { Percent } \\
\text { Finer }\end{array}$ \\
\cline { 2 - 4 } $\mathrm{O}$ & $3^{n}$ & 75.000 & $100.0 \%$ \\
$\mathrm{~A}$ & $1.5^{n}$ & 37.500 & $100.0 \%$ \\
$\mathrm{R}$ & $0.75^{n}$ & 19.000 & $100.0 \%$ \\
S & $0.375^{n}$ & 9.500 & $99.2 \%$ \\
$\mathrm{E}$ & $\# 4$ & 4.750 & $98.6 \%$ \\
\cline { 2 - 4 } & $\# 10$ & 2.000 & $97.3 \%$ \\
\hline
\end{tabular}

\begin{tabular}{|c|c|c|c|}
\hline & $\begin{array}{c}\text { Sieve } \\
\text { No. }\end{array}$ & $\begin{array}{c}\text { Diameter } \\
\mathrm{mm}\end{array}$ & $\begin{array}{c}\text { Percent } \\
\text { Finer }\end{array}$ \\
\cline { 2 - 4 } $\mathrm{F}$ & $\# 20$ & 0.850 & $91.6 \%$ \\
\hline $\mathrm{N}$ & $\# 40$ & 0.425 & $75.0 \%$ \\
\cline { 2 - 4 } & $\# 60$ & 0.250 & $57.3 \%$ \\
\hline & $\# 100$ & 0.149 & $39.0 \%$ \\
\hline & $\# 140$ & 0.106 & $33.8 \%$ \\
\hline 200 & 0.075 & $31.7 \%$ \\
\hline
\end{tabular}

HYDROMETER ANALYSIS

\begin{tabular}{|c|c|c|}
\hline & $\begin{array}{c}\text { Diameter } \\
\mathrm{mm}\end{array}$ & $\begin{array}{c}\text { Percent } \\
\text { Finer }\end{array}$ \\
\hline H & & \\
\cline { 2 - 3 } Y & 0.04902 & $29.0 \%$ \\
\hline$R$ & 0.03473 & $28.3 \%$ \\
\hline & 0.02201 & $27.6 \%$ \\
M & 0.01276 & $26.8 \%$ \\
E & 0.00895 & $26.1 \%$ \\
\hline & 0.00635 & $25.4 \%$ \\
\hline$E$ & 0.00452 & $24.0 \%$ \\
\hline$R$ & 0.00311 & $22.6 \%$ \\
\hline & 0.00132 & $21.9 \%$ \\
\hline
\end{tabular}




\section{SAIC - Savannah River}

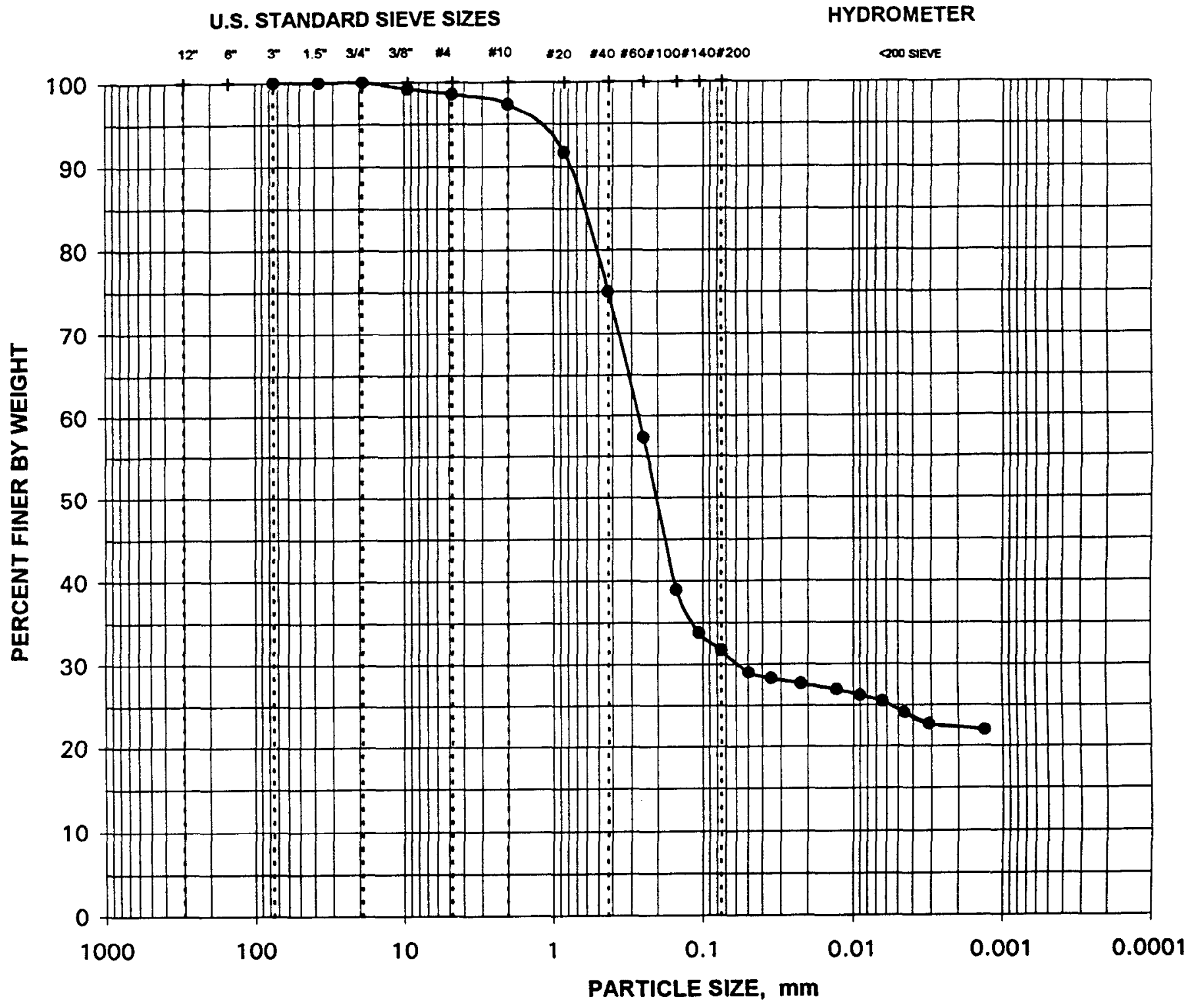

CLIENT SAMPLE NO.: $\quad$ P500Q00

IT LAB SAMPLE NO.:

ETDC-8019

\begin{tabular}{|c|c|c|c|c|c|c|c|}
\hline \multirow{2}{*}{$\begin{array}{l}B \\
D \\
U \\
L \\
D \\
E \\
R \\
\text { S }\end{array}$} & \multirow{2}{*}{$\begin{array}{l}C \\
0 \\
B \\
B \\
L \\
E \\
\text { S }\end{array}$} & \multicolumn{2}{|c|}{ GRAVEL } & \multicolumn{3}{|c|}{ SAND } & SILT 2 - 75 microns \\
\hline & & $\begin{array}{l}\mathbf{C} \\
0 \\
\hat{A} \\
R \\
\mathbf{S} \\
\mathbf{E}\end{array}$ & $\begin{array}{l}F \\
\text { F } \\
\text { E }\end{array}$ & $\begin{array}{l}c \\
0 \\
\hat{A} \\
\mathbf{R} \\
\mathbf{S} \\
\mathrm{E}\end{array}$ & $\begin{array}{l}M \\
\mathbf{E} \\
D \\
1 \\
U \\
M\end{array}$ & $\begin{array}{l}\mathbf{F} \\
\mathbf{N} \\
\mathbf{N}\end{array}$ & \\
\hline
\end{tabular}




\section{PARTICLE-SIZE ANALYSIS \\ ASTM D 422}

$\begin{array}{llll}\text { Project Name } & \text { SAIC - Savannah River } & \text { Client Sample No. } & \text { P600000 } \\ \text { Project No. } & 777304.00010500 & \text { IT Lab Sample No. } & \text { ETDC-8020 } \\ \text { Specific Gravity }=2.6408 & \begin{array}{l}\text { Moisture Content }= \\ \text { basod on dry samplo weight }\end{array}\end{array}$

SIEVE ANALYSIS

\begin{tabular}{|c|c|c|c|}
\hline & $\begin{array}{c}\text { Sieve } \\
\text { No. }\end{array}$ & $\begin{array}{c}\text { Diameter } \\
\mathrm{mm}\end{array}$ & $\begin{array}{c}\text { Percent } \\
\text { Finer }\end{array}$ \\
\cline { 2 - 4 } $\mathrm{O}$ & $3^{n}$ & 75.000 & $100.0 \%$ \\
$\mathrm{~A}$ & $1.5^{\prime \prime}$ & 37.500 & $100.0 \%$ \\
$\mathrm{R}$ & $0.75^{n}$ & 19.000 & $97.4 \%$ \\
S & $0.375^{n}$ & 9.500 & $96.4 \%$ \\
\hline & $\# 4$ & 4.750 & $94.9 \%$ \\
\cline { 2 - 4 } & $\# 4$ & 2.000 & $90.8 \%$ \\
\hline
\end{tabular}

\begin{tabular}{|c|c|c|c|}
\hline & $\begin{array}{c}\text { Sieve } \\
\text { No. }\end{array}$ & $\begin{array}{c}\text { Diameter } \\
\mathrm{mm}\end{array}$ & $\begin{array}{c}\text { Percent } \\
\text { Finer }\end{array}$ \\
\cline { 2 - 4 } F & $\# 20$ & 0.850 & $76.8 \%$ \\
\hline N & $\# 40$ & 0.425 & $50.6 \%$ \\
\hline E & $\# 60$ & 0.250 & $37.5 \%$ \\
\cline { 2 - 4 } & $\# 100$ & 0.149 & $29.6 \%$ \\
\hline & $\# 140$ & 0.106 & $27.1 \%$ \\
\hline & $\# 200$ & 0.075 & $25.8 \%$ \\
\hline
\end{tabular}

HYDROMETER ANALYSIS

\begin{tabular}{|c|c|c|}
\hline & $\begin{array}{c}\text { Diameter } \\
\mathrm{mm}\end{array}$ & $\begin{array}{c}\text { Percent } \\
\text { Finer }\end{array}$ \\
\cline { 2 - 3 } $\mathrm{H}$ & & \\
$\mathrm{Y}$ & & $24.6 \%$ \\
$\mathrm{D}$ & 0.04801 & $24.0 \%$ \\
\hline & 0.03409 & $23.4 \%$ \\
\hline $\mathrm{M}$ & 0.02165 & $22.7 \%$ \\
\cline { 2 - 3 } $\mathrm{E}$ & 0.01255 & $21.5 \%$ \\
\hline$T$ & 0.00839 & $20.9 \%$ \\
\hline E & 0.00633 & $19.0 \%$ \\
\hline & 0.00447 & $18.3 \%$ \\
\cline { 2 - 3 } & 0.00252 & $18.3 \%$ \\
\hline
\end{tabular}




\section{SAIC - Savannah River}

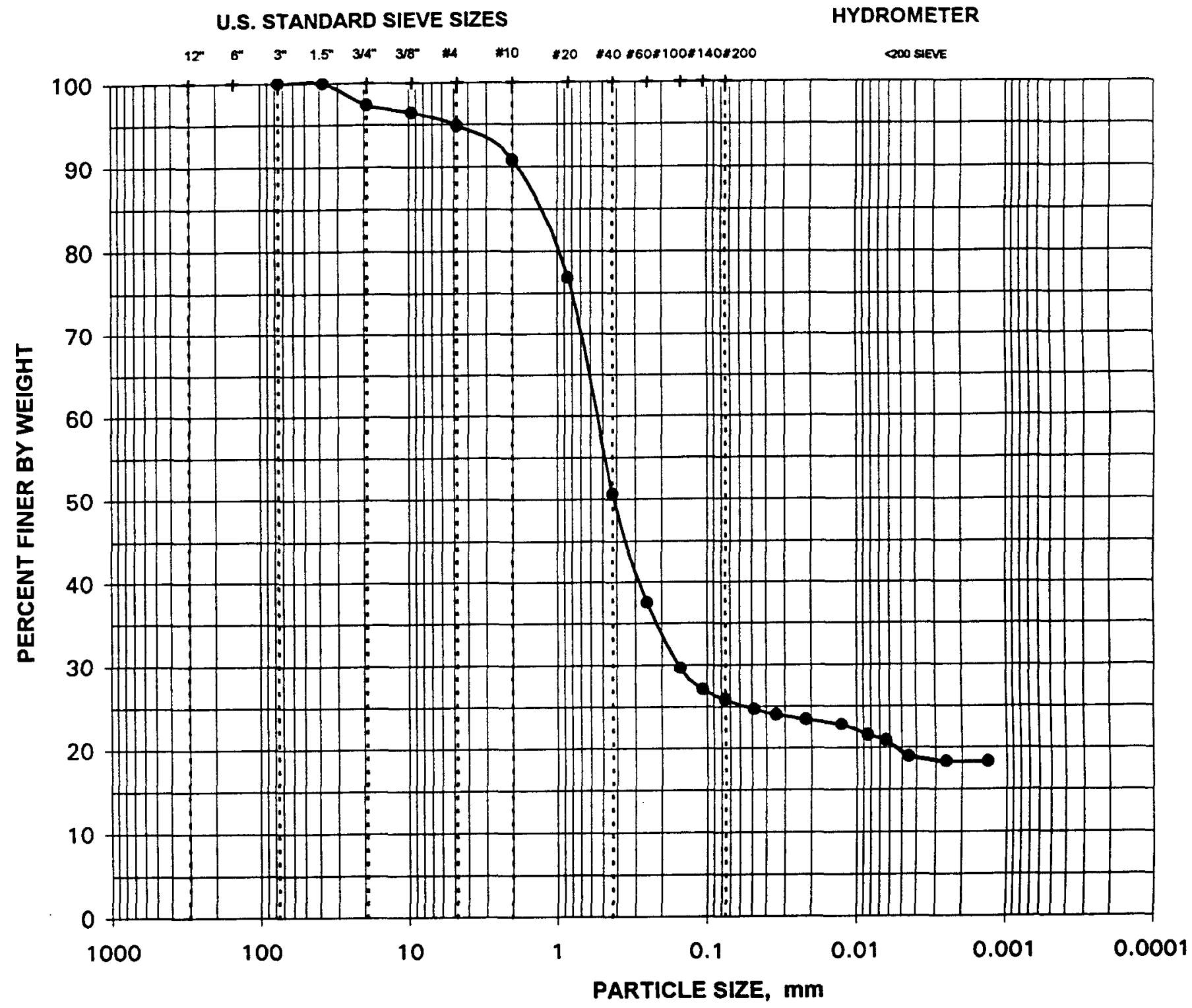

CLIENT SAMPLE NO:: P600Q00

IT LAB SAMPLE NO.:

ETDC-8020

\begin{tabular}{|c|c|c|c|c|c|c|c|}
\hline \multirow{2}{*}{$\begin{array}{l}B \\
O \\
U \\
L \\
D \\
E \\
R \\
S\end{array}$} & \multirow{2}{*}{$\begin{array}{l}\mathbf{C} \\
\mathbf{O} \\
\mathbf{B} \\
\mathbf{B} \\
\mathbf{L} \\
\mathbf{E} \\
\mathbf{S}\end{array}$} & \multicolumn{2}{|c|}{ GRAVEL } & \multicolumn{3}{|c|}{ SAND } & SILT 2 - 75 microns \\
\hline & & $\begin{array}{l}\mathrm{C} \\
0 \\
\hat{A} \\
\mathrm{R} \\
\mathrm{s} \\
\mathrm{E}\end{array}$ & $\begin{array}{l}F \\
\text { I } \\
\mathbf{N} \\
\mathbf{E}\end{array}$ & $\begin{array}{l}C \\
0 \\
\hat{R} \\
\hat{R} \\
\mathrm{E}\end{array}$ & $\begin{array}{l}M \\
E \\
D \\
1 \\
U \\
M\end{array}$ & $\begin{array}{l}\mathbf{F} \\
1 \\
\mathbf{N} \\
\mathbf{E}\end{array}$ & CLAY $<2$ microns \\
\hline
\end{tabular}




\section{PARTICLE-SIZE ANALYSIS ASTM D 422}

$\begin{array}{llll}\text { Project Name } & \text { SAIC }- \text { Savannah River } & \text { Client Sample No. } & \text { P700000 } \\ \text { Project No. } & 777304.00010500 & \text { IT Lab Sample No. } & \text { ETDC-8021 } \\ \text { Specific Gravity }=2.6435 & \begin{array}{l}\text { Moisture Content }= \\ \text { based on dry sample weight }\end{array}\end{array}$

SIEVE ANALYSIS

\begin{tabular}{|c|c|c|c||}
\hline & $\begin{array}{c}\text { Sieve } \\
\text { No. }\end{array}$ & $\begin{array}{c}\text { Diameter } \\
\mathrm{mm}\end{array}$ & $\begin{array}{c}\text { Percent } \\
\text { Finer }\end{array}$ \\
\cline { 2 - 4 } $\mathrm{O}$ & $3^{n}$ & 75.000 & $100.0 \%$ \\
$\mathrm{~A}$ & $1.5^{n}$ & 37.500 & $100.0 \%$ \\
$\mathrm{R}$ & $0.75^{n}$ & 19.000 & $100.0 \%$ \\
$\mathrm{~S}$ & $0.375^{n}$ & 9.500 & $97.3 \%$ \\
$\mathrm{E}$ & $\# 4$ & 4.750 & $96.3 \%$ \\
\cline { 2 - 4 } & $\# 10$ & 2.000 & $93.7 \%$ \\
\hline
\end{tabular}

\begin{tabular}{|c|c|c|c|}
\hline & $\begin{array}{c}\text { Sieve } \\
\text { No. }\end{array}$ & $\begin{array}{c}\text { Diameter } \\
\mathrm{mm}\end{array}$ & $\begin{array}{c}\text { Percent } \\
\text { Finer }\end{array}$ \\
\cline { 2 - 4 } $\mathrm{F}$ & $\# 20$ & 0.850 & $82.8 \%$ \\
\hline $\mathrm{N}$ & $\# 40$ & 0.425 & $60.5 \%$ \\
\hline $\mathrm{N}$ & $\# 60$ & 0.250 & $45.7 \%$ \\
\cline { 2 - 4 } & $\# 100$ & 0.149 & $35.5 \%$ \\
\hline & $\# 140$ & 0.106 & $32.2 \%$ \\
\hline & $\# 200$ & 0.075 & $30.6 \%$ \\
\hline
\end{tabular}

HYDROMETER ANALYSIS

\begin{tabular}{|c|c|c|}
\hline \multirow{2}{*}{$H$} & $\begin{array}{c}\text { Diameter } \\
\mathrm{mm}\end{array}$ & $\begin{array}{c}\text { Percent } \\
\text { Finer }\end{array}$ \\
\hline & & \\
\hline $\begin{array}{l}Y \\
D\end{array}$ & 0.04801 & $27.0 \%$ \\
\hline $\mathrm{R}$ & 0.03409 & $26.3 \%$ \\
\hline 0 & 0.02165 & $25.6 \%$ \\
\hline M & 0.01215 & $25.0 \%$ \\
\hline E & 0.00891 & $24.3 \%$ \\
\hline E & 0.00630 & $24.3 \%$ \\
\hline$R$ & 0.00444 & $22.2 \%$ \\
\hline & 0.00253 & $20.8 \%$ \\
\hline & 0.00130 & $20.8 \%$ \\
\hline
\end{tabular}




\section{SAIC - Savannah River}

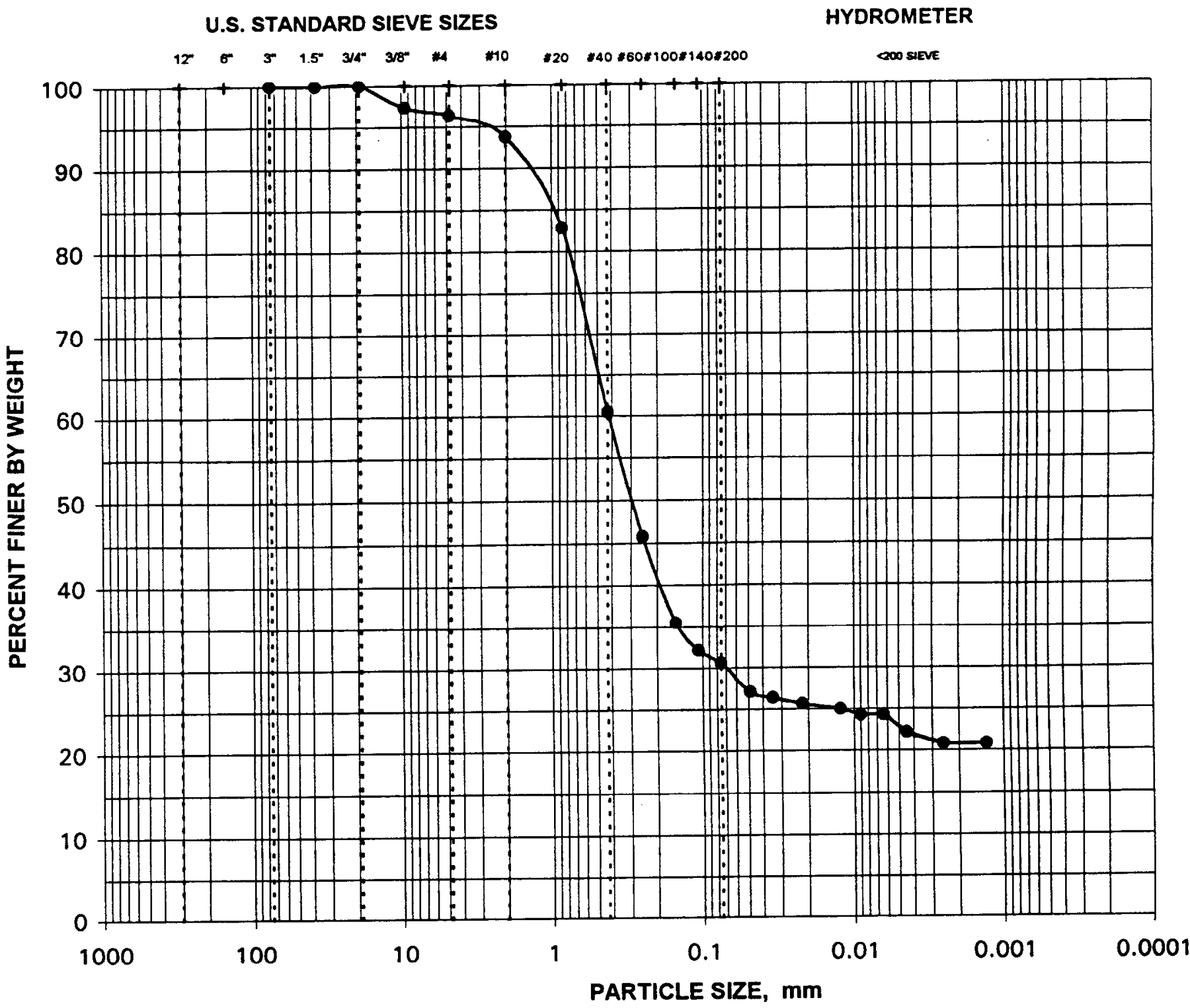

ClIENT SAMPLE NO: $\quad$ P700Q00

IT LAB SAMPLE NO.:

ETDC-8021

\begin{tabular}{|c|c|c|c|c|c|c|c|}
\hline \multirow{2}{*}{$\begin{array}{l}B \\
O \\
U \\
L \\
D \\
E \\
R \\
S\end{array}$} & \multirow{2}{*}{$\begin{array}{l}\mathbf{C} \\
\mathbf{D} \\
\mathbf{B} \\
\mathbf{B} \\
\mathbf{L} \\
\mathbf{E} \\
\mathbf{S}\end{array}$} & \multicolumn{2}{|c|}{ GRAVEL } & \multicolumn{3}{|c|}{ SAND } & SILT 2 - 75 microns \\
\hline & & $\begin{array}{l}\mathbf{C} \\
0 \\
\hat{A} \\
\mathbf{R} \\
\mathbf{S} \\
\mathrm{E}\end{array}$ & $\begin{array}{l}F \\
1 \\
N \\
E\end{array}$ & $\begin{array}{l}\text { O } \\
0 \\
\hat{R} \\
\text { s } \\
\text { E }\end{array}$ & $\begin{array}{l}M \\
\text { E } \\
\mathbf{D} \\
\mathbf{U} \\
\mathbf{M}\end{array}$ & $\begin{array}{l}\mathbf{F} \\
\mathbf{N} \\
\mathbf{N} \\
\mathbf{E}\end{array}$ & \\
\hline
\end{tabular}




\section{PARTICLE-SIZE ANALYSIS \\ ASTM D 422}

Project Name SAIC - Savannah River

Project No.

777304.00010500

Specific Gravity $=2.6428$
Client Sample No. $\quad$ P800000

IT Lab Sample No. ETDC-8022

Moisture Content $=15.9 \%$

besed on dry sample woight

SIEVE ANALYSIS

\begin{tabular}{|c|c|c|c|}
\hline & $\begin{array}{c}\text { Sieve } \\
\text { No. }\end{array}$ & $\begin{array}{c}\text { Diameter } \\
\mathrm{mm}\end{array}$ & $\begin{array}{c}\text { Percent } \\
\text { Finer }\end{array}$ \\
\cline { 2 - 4 } $\mathrm{O}$ & $3^{n}$ & 75.000 & $100.0 \%$ \\
$\mathrm{~A}$ & $1.5^{n}$ & 37.500 & $100.0 \%$ \\
$\mathrm{R}$ & $0.75^{n}$ & 19.000 & $100.0 \%$ \\
$\mathrm{~S}$ & $0.375^{n}$ & 9.500 & $99.7 \%$ \\
$\mathrm{E}$ & $\# 4$ & 4.750 & $99.1 \%$ \\
\cline { 2 - 4 } & $\# 10$ & 2.000 & $96.0 \%$ \\
\hline
\end{tabular}

\begin{tabular}{|c|c|c|c|}
\hline & $\begin{array}{c}\text { Sieve } \\
\text { No. }\end{array}$ & $\begin{array}{c}\text { Diameter } \\
\mathrm{mm}\end{array}$ & $\begin{array}{c}\text { Percent } \\
\text { Finer }\end{array}$ \\
\cline { 2 - 4 } $\mathrm{F}$ & $\# 20$ & 0.850 & $82.7 \%$ \\
\hline $\mathrm{N}$ & $\# 40$ & 0.425 & $58.8 \%$ \\
\hline \multirow{4}{*}{ E } & $\# 60$ & 0.250 & $45.2 \%$ \\
\cline { 2 - 4 } & $\# 100$ & 0.149 & $36.1 \%$ \\
\cline { 2 - 4 } & $\# 140$ & 0.106 & $33.2 \%$ \\
\hline & $\# 200$ & 0.075 & $31.8 \%$ \\
\hline
\end{tabular}

HYDROMETER ANALYSIS

\begin{tabular}{|c|c|c|}
\hline & $\begin{array}{c}\text { Diameter } \\
\mathrm{mm}\end{array}$ & $\begin{array}{c}\text { Percent } \\
\text { Finer }\end{array}$ \\
\hline H & 0.06647 & $30.0 \%$ \\
\cline { 2 - 3 } Y & 0.04741 & $28.7 \%$ \\
\cline { 2 - 3 } R & 0.03367 & $28.0 \%$ \\
\hline M & 0.02138 & $27.3 \%$ \\
\cline { 2 - 3 } E & 0.01234 & $27.3 \%$ \\
\hline$T$ & 0.00875 & $26.7 \%$ \\
\hline E & 0.00612 & $26.0 \%$ \\
\hline$R$ & 0.00438 & $24.0 \%$ \\
\hline & 0.00131 & $22.0 \%$ \\
\hline
\end{tabular}




\section{SAIC - Savannah River}

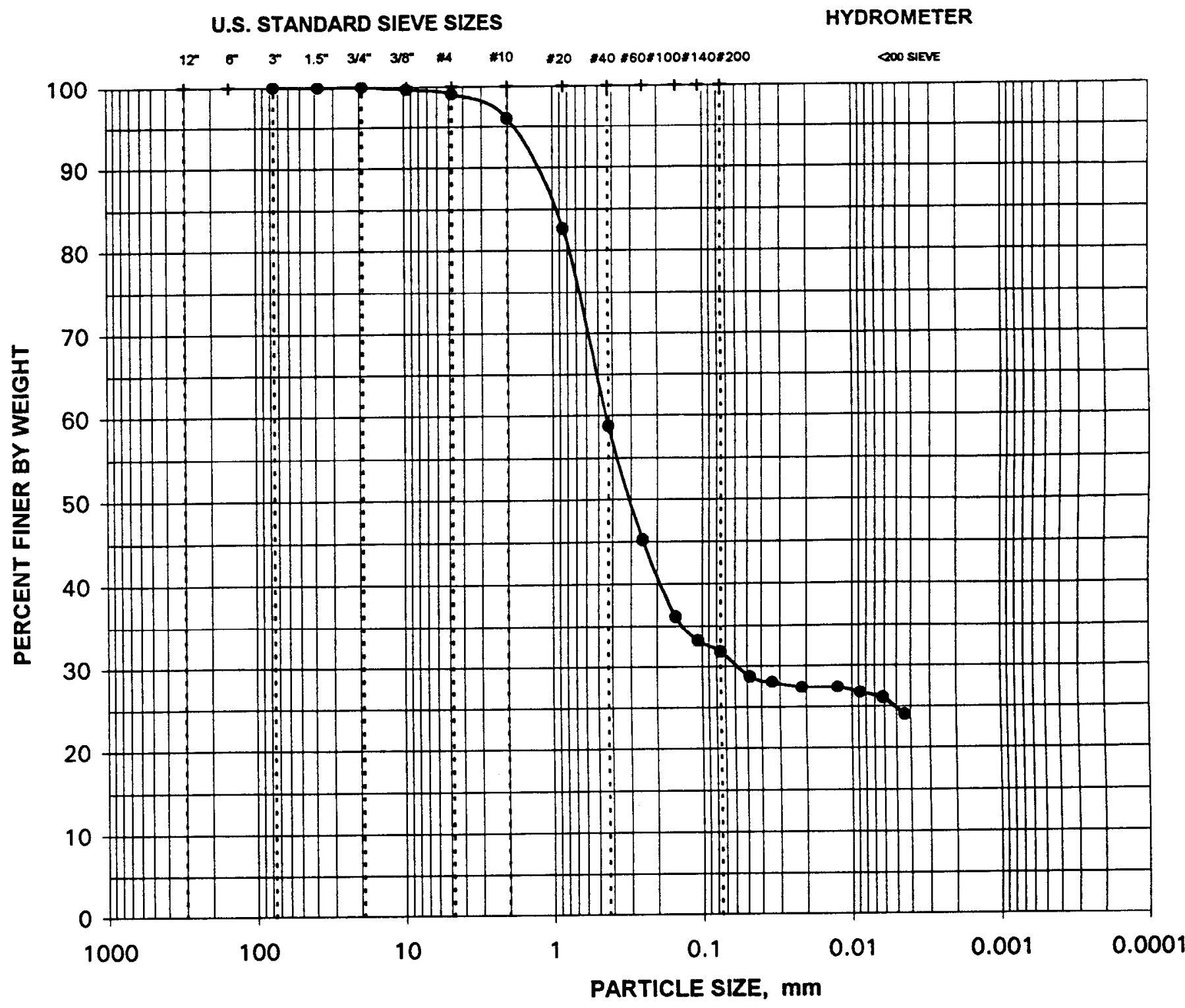

ClIENT SAMPLE NO: $\quad$ P800Q00

IT LAB SAMPLE NO.:

ETDC-8022

\begin{tabular}{|c|c|c|c|c|c|c|c|}
\hline \multirow{2}{*}{$\begin{array}{l}B \\
0 \\
U \\
L \\
D \\
E \\
R \\
S\end{array}$} & \multirow{2}{*}{$\begin{array}{l}C \\
0 \\
b \\
B \\
L \\
E \\
S\end{array}$} & \multicolumn{2}{|c|}{ GRAVEL } & \multicolumn{3}{|c|}{ SAND } & \multirow{2}{*}{$\begin{array}{l}\text { SILT } 2 \text { - } 75 \text { microns } \\
\text { CLAY }<2 \text { microns }\end{array}$} \\
\hline & & $\begin{array}{l}\mathbf{C} \\
\hat{0} \\
\hat{A} \\
\hat{R} \\
\mathbf{S} \\
\text { E }\end{array}$ & $\begin{array}{l}\text { F } \\
\text { E } \\
\text { E }\end{array}$ & $\begin{array}{l}c \\
0 \\
\hat{A} \\
\mathbf{S} \\
\mathrm{E}\end{array}$ & $\begin{array}{l}\text { M } \\
\mathbf{E} \\
\mathbf{D} \\
\mathbf{1} \\
\mathbf{M} \\
\mathbf{M}\end{array}$ & $\begin{array}{l}F \\
\text { F } \\
\text { E } \\
E\end{array}$ & \\
\hline
\end{tabular}




\section{P Series 28 Day UCS Results}




\section{UNCONFINED COMPRESSIVE STRENGTH - ASTM D 2166 28-DAY CURE}

Project Name SAIC - Savannah River

Project No. 777304.00010500

Specimen Mold Date

Specimen Test Date

STRESS AT FAILURE, psi

STRAIN AT FAILURE, \%

\begin{tabular}{ccc}
$\begin{array}{ccc}\text { AXIAL } \\
\text { STRAIN, \% }\end{array}$ & & $\begin{array}{c}\text { DEVIATOR } \\
\text { STRESS, psi }\end{array}$ \\
\cline { 1 - 1 } 0.00 & & 0.0 \\
0.10 & & 2.6 \\
0.20 & & 5.4 \\
0.30 & & 9.2 \\
0.40 & & 17.8 \\
0.50 & & 32.7 \\
0.75 & & 81.2 \\
1.00 & & 138.6 \\
1.25 & & 206.7 \\
1.51 & & 268.1 \\
1.76 & & 327.7 \\
2.01 & & 376.4 \\
2.26 & & 416.6 \\
2.51 & & 466.6 \\
2.76 & & 510.5 \\
3.01 & 544.5 \\
3.26 & 580.2 \\
3.51 & 592.3 \\
3.76 & 582.7 \\
4.02 & 580.6 \\
4.27 & 58.1
\end{tabular}

$12 / 22 / 98$

$1 / 19 / 99$

592.3

3.5
Client Sample No. P312F00

IT Lab Specimen No. ETDC-8047

Specimen Height, in.

3.9842

Specimen Diameter, in.

1.9962

Specimen Weight, $g$.

381.63

Moisture Content, \%

27.8

Wet Unit Weight, pcf.

116.6

Dry Unit Weight, pef.

91.2

Rate of Strain, in./min.

0.0400

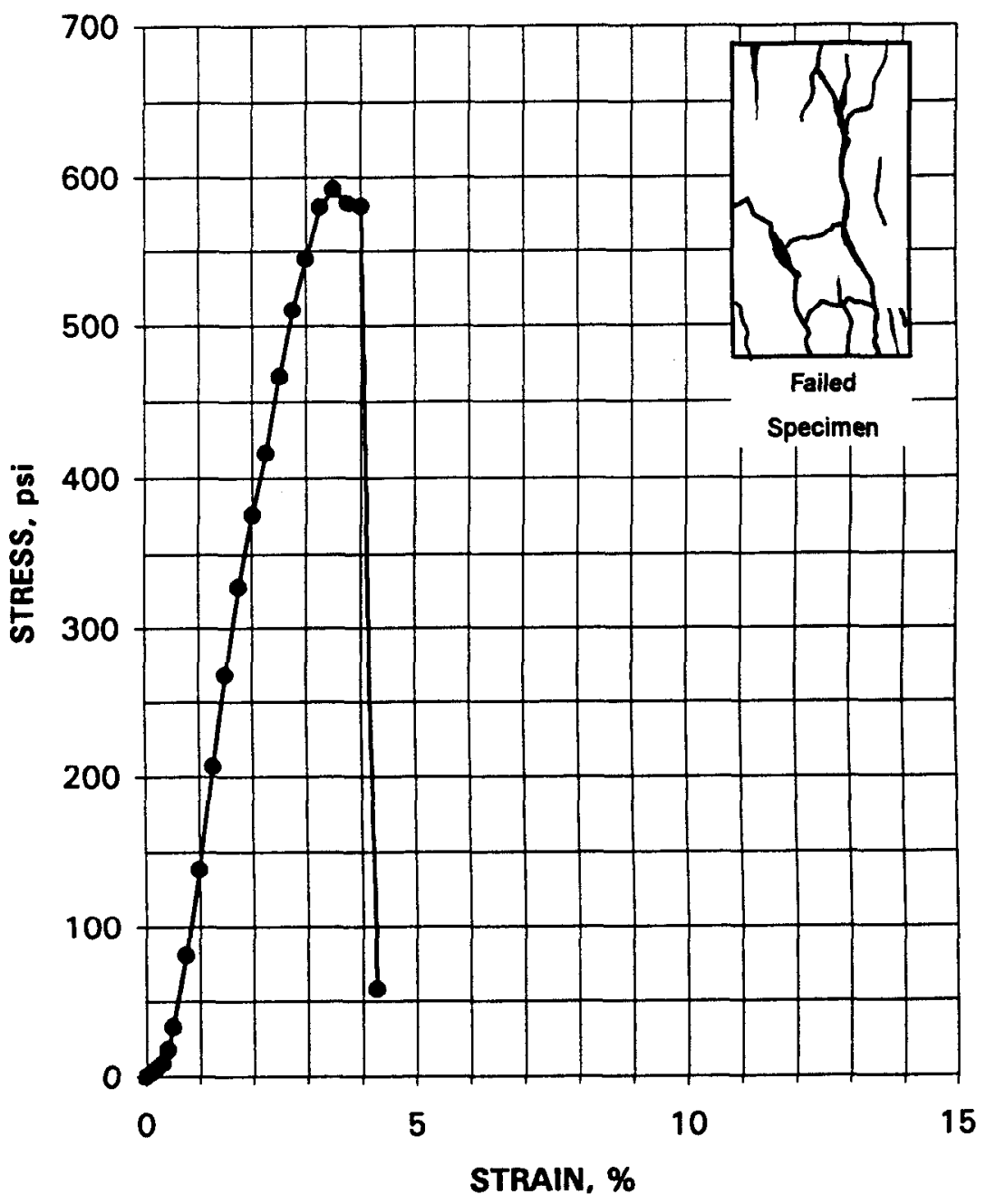




\section{UNCONFINED COMPRESSIVE STRENGTH - ASTM D 2166 27-DAY CURE}

$\begin{array}{ll}\text { Project Name } & \text { SAIC - Savannah River } \\ \text { Project No. } & 777304.00010500\end{array}$

Specimen Mold Date

Specimen Test Date

STRESS AT FAILURE, psi

STRAIN AT FAILURE, \%

AXIAL DEVIATOR

\begin{tabular}{ccc} 
STRAIN, $\%$ & STRESS, psi \\
\cline { 1 - 1 } 0.00 & & 0.0 \\
0.10 & & 2.5 \\
0.20 & & 5.1 \\
0.30 & & 7.6 \\
0.40 & & 11.4 \\
0.50 & & 17.7 \\
0.75 & & 46.2 \\
1.00 & & 99.1 \\
1.26 & & 171.8 \\
1.51 & & 253.1 \\
1.76 & & 319.0 \\
2.01 & & 389.9 \\
2.26 & & 452.1 \\
2.51 & & 500.7 \\
2.76 & & 531.4 \\
3.01 & & 535.3 \\
3.14 & & 537.3 \\
3.27 & & 520.4 \\
3.52 & 531.9 \\
3.77 & 522.9 \\
4.02 & 486.9 \\
4.27 & 376.4 \\
4.52 & 349.1 \\
4.77 & 327.4 \\
5.02 & & 309.6
\end{tabular}

$12 / 22 / 98$

$1 / 19 / 99$

537.3

3.1
Client Sample No. P322F00

IT Lab Specimen No. ETDC-8048

Specimen Height, in.

3.9803

Specimen Diameter, in.

2.0047

Specimen Weight, $\boldsymbol{g}$.

390.38

Moisture Content, \%

26.3

Wet Unit Weight, pcf.

118.4

Dry Unit Weight, pcf.

Rate of Strain, in./min.

0.0400

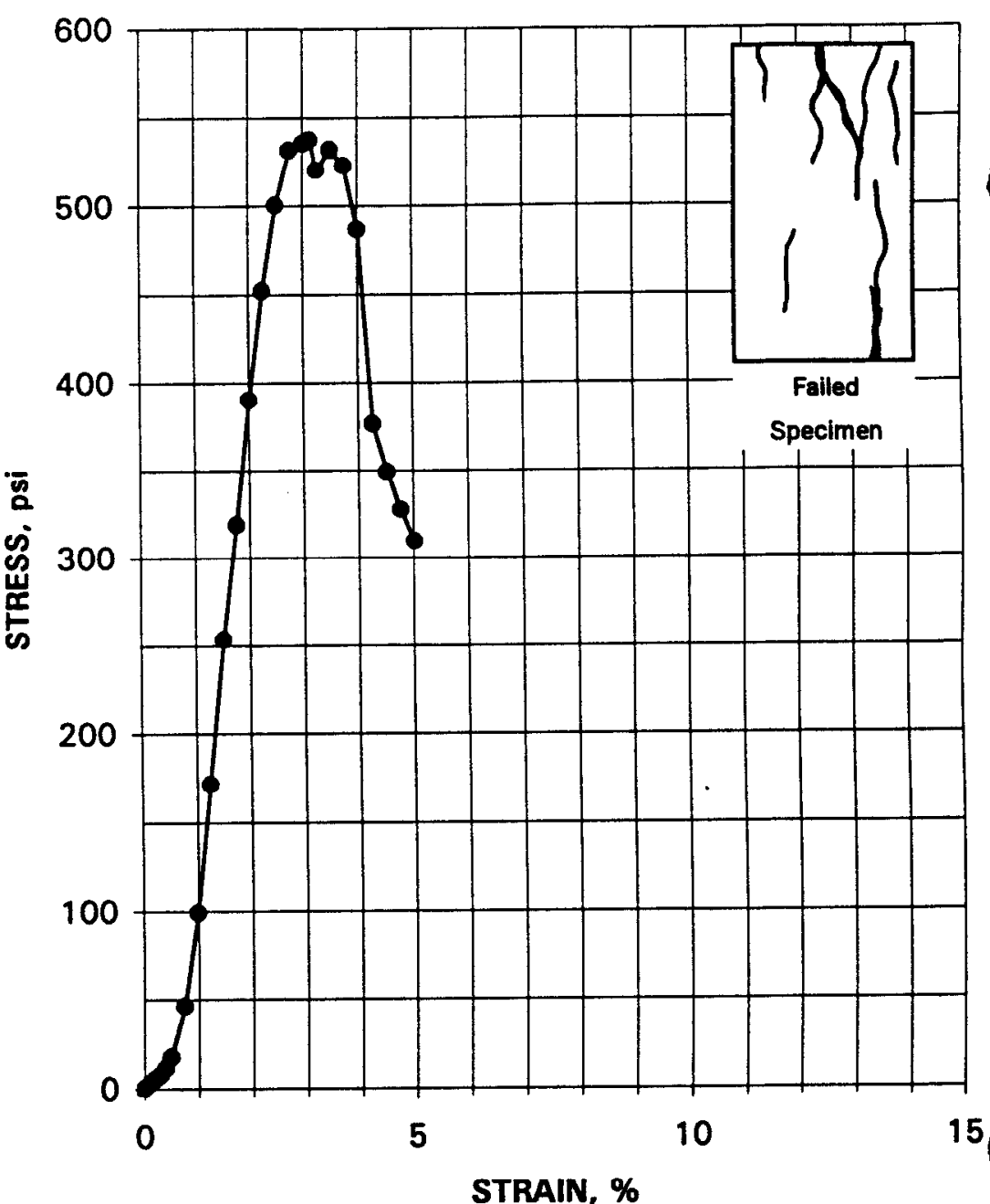




\section{UNCONFINED COMPRESSIVE STRENGTH - ASTM D 2166 28-DAY CURE}

Project Name SAIC - Savannah River

Project No. $\quad 777304.00010500$

Specimen Mold Date

Specimen Test Date

STRESS AT FAILURE, psi STRAIN AT FAILURE, \%

\begin{tabular}{ccc}
$\begin{array}{ccc}\text { AXIAL } \\
\text { STRAIN, \% }\end{array}$ & & $\begin{array}{c}\text { DEVIATOR } \\
\text { STRESS, psi }\end{array}$ \\
\cline { 1 - 1 } 0.00 & & 0.0 \\
0.10 & & 2.8 \\
0.20 & & 5.6 \\
0.30 & & 8.4 \\
0.40 & & 12.2 \\
0.50 & & 18.1 \\
0.75 & & 40.5 \\
1.00 & & 98.8 \\
1.25 & & 171.7 \\
1.51 & & 244.2 \\
1.76 & & 312.7 \\
2.01 & & 380.7 \\
2.26 & & 450.3 \\
2.51 & & 496.9 \\
2.76 & & 578.6 \\
3.01 & 629.2 \\
3.26 & 646.4 \\
3.39 & 646.8 \\
3.51 & & 7.6
\end{tabular}

Client Sample No. P332F00

IT Lab Specimen No. ETDC-8049

Specimen Height, in.

3.9855

Specimen Diameter, in.

2.0142

Specimen Weight, $g$. 380.08

Moisture Content, \%

28.5

Wet Unit Weight, pcf.

114.0

Dry Unit Weight, pcf.

88.7

Rate of Strain, in./min.

0.0400

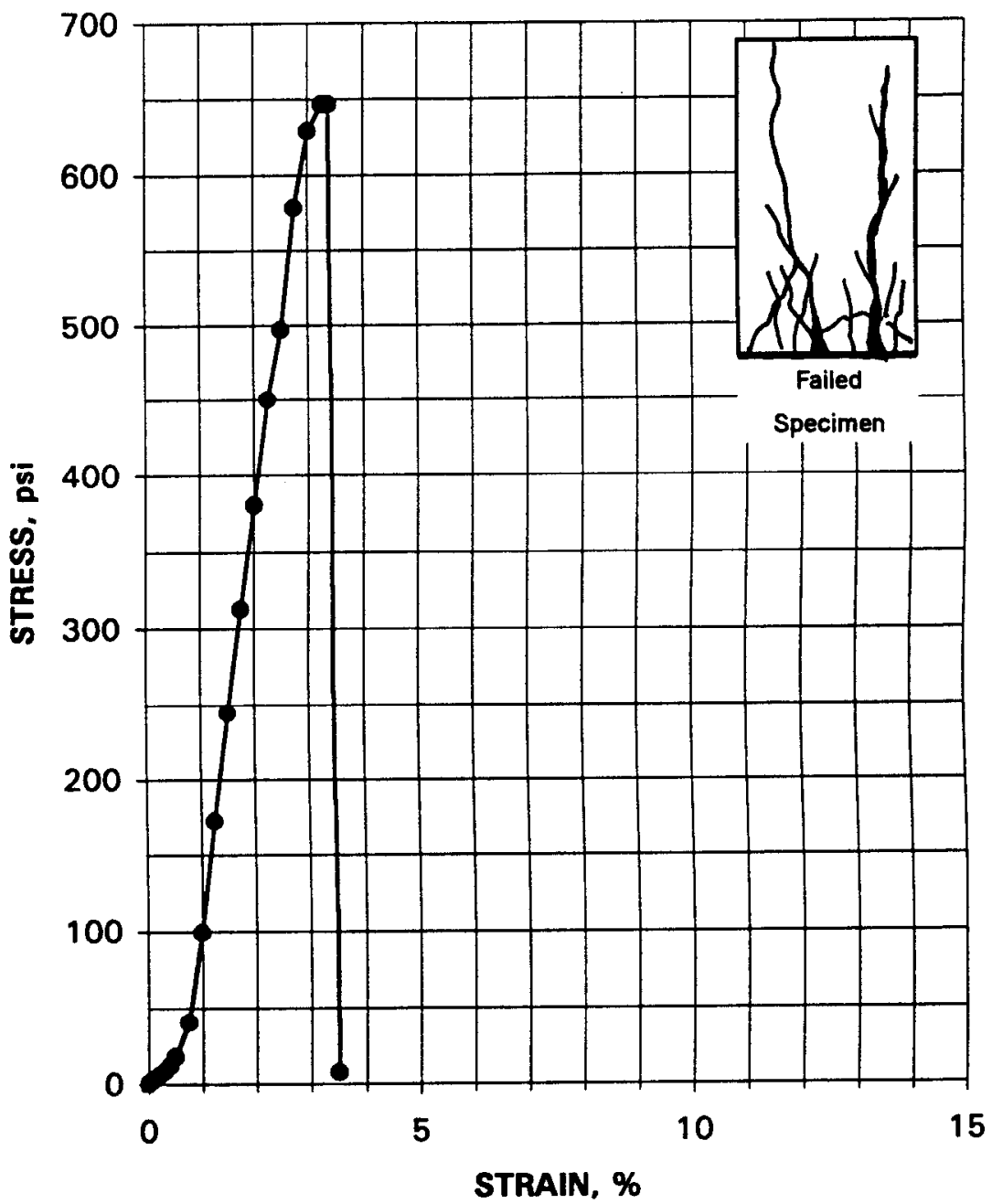




\section{UNCONFINED COMPRESSIVE STRENGTH - ASTM D 2166 28-DAY CURE}

Project Name SAIC - Savannah River

Project No. $\quad 777304.00010500$

Specimen Mold Date

Specimen Test Date

STRESS AT FAILURE, psi STRAIN AT FAILURE, \%

\begin{tabular}{|c|c|}
\hline AXIAL & DEVIATOR \\
\hline STRAIN, \% & STRESS, psi \\
\hline 0.00 & 0.0 \\
\hline 0.10 & 3.8 \\
\hline 0.20 & 7.3 \\
\hline 0.30 & 10.7 \\
\hline 0.40 & 17.7 \\
\hline 0.50 & 23.9 \\
\hline 0.75 & 48.1 \\
\hline 1.00 & 89.0 \\
\hline 1.25 & 154.2 \\
\hline 1.50 & 228.3 \\
\hline 1.75 & 314.2 \\
\hline 2.00 & 403.1 \\
\hline $\begin{array}{l}2.25 \\
2.50\end{array}$ & $\begin{array}{l}493.1 \\
555.1\end{array}$ \\
\hline 2.75 & 605.5 \\
\hline 3.00 & 637.1 \\
\hline 3.12 & 638.1 \\
\hline 3.25 & 37.7 \\
\hline
\end{tabular}

Client Sample No. P342F00

IT Lab Specimen No. ETDC-8050

Specimen Height, in.

4.0008

Specimen Diameter, in.

2.0052

Specimen Weight, $g$.

374.81

Moisture Content, \%

32.3

Wet Unit Weight, pcf.

113.0

Dry Unit Weight, pef.

85.5

Rate of Strain, in./min.

0.0400

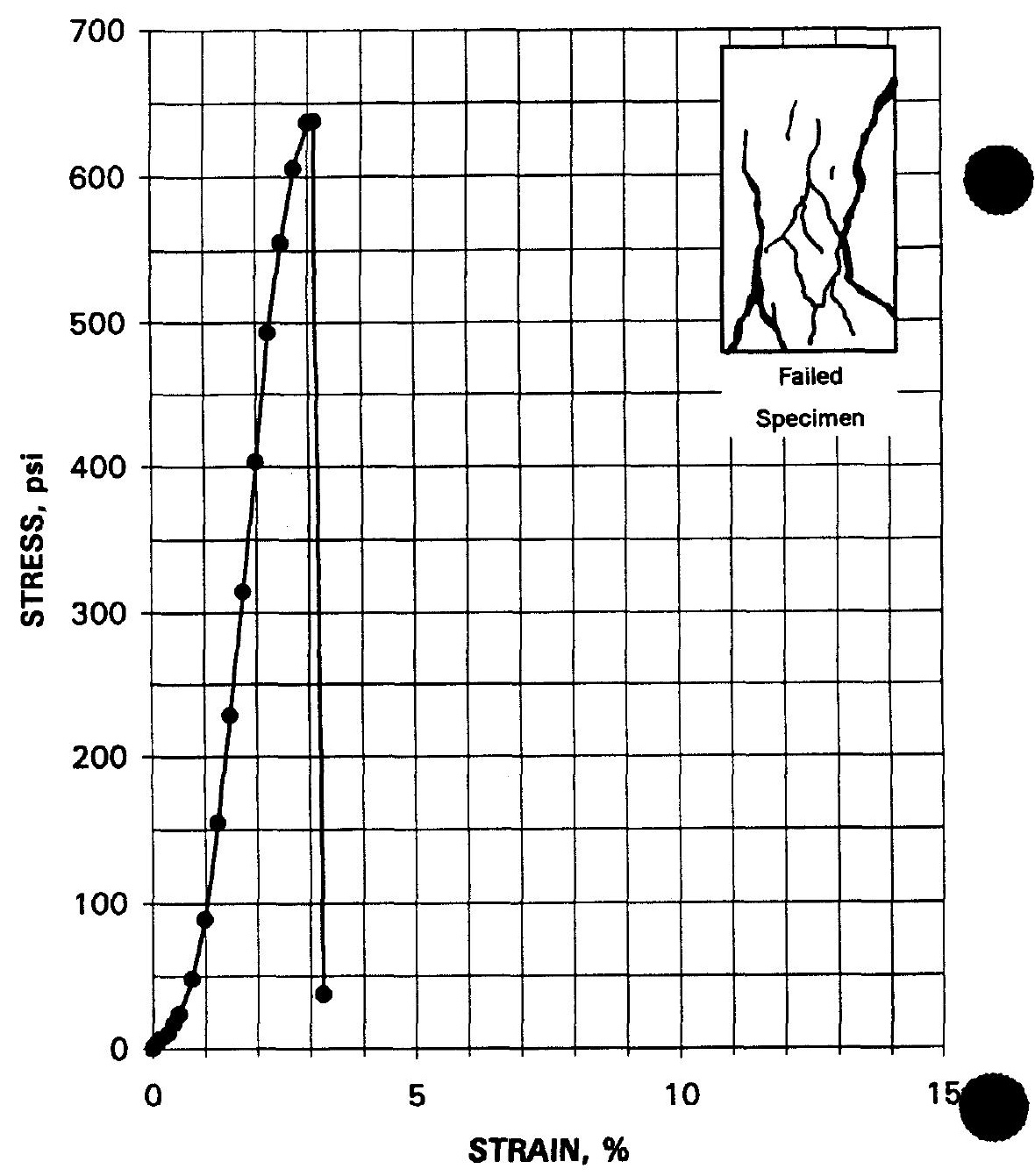




\section{UNCONFINED COMPRESSIVE STRENGTH - ASTM D 2166 28-DAY CURE}

Project Name SAIC - Savannah River

Project No. $\quad 777304.00010500$

Specimen Mold Date

Specimen Test Date

STRESS AT FAILURE, psi

STRAIN AT FAILURE, \%

\begin{tabular}{ccc}
$\begin{array}{c}\text { AXIAL } \\
\text { STRAIN, \% }\end{array}$ & & $\begin{array}{c}\text { DEVIATOR } \\
\text { STRESS, pSi }\end{array}$ \\
\cline { 1 - 1 } 0.00 & & 0.0 \\
0.10 & & 2.2 \\
0.20 & & 5.1 \\
0.30 & & 10.2 \\
0.40 & & 13.7 \\
0.50 & & 22.5 \\
0.75 & & 44.0 \\
1.01 & & 85.2 \\
1.26 & & 127.5 \\
1.51 & & 174.2 \\
1.76 & & 217.0 \\
2.01 & & 257.7 \\
2.26 & & 305.3 \\
2.51 & 345.2 \\
2.76 & 371.0 \\
3.02 & 389.5 \\
3.27 & 407.9 \\
3.39 & 411.1 \\
3.52 & 74.4
\end{tabular}

Client Sample No. P412F00

IT Lab Specimen No. ETDC-8051

Specimen Height, in.

3.9792

Specimen Diameter, in.

1.9987

Specimen Weight, $g$.

372.17

Moisture Content, \%

31.8

Wet Unit Weight, pef.

113.6

Dry Unit Weight, pcf.

86.2

Rate of Strain, in./min.

0.0400

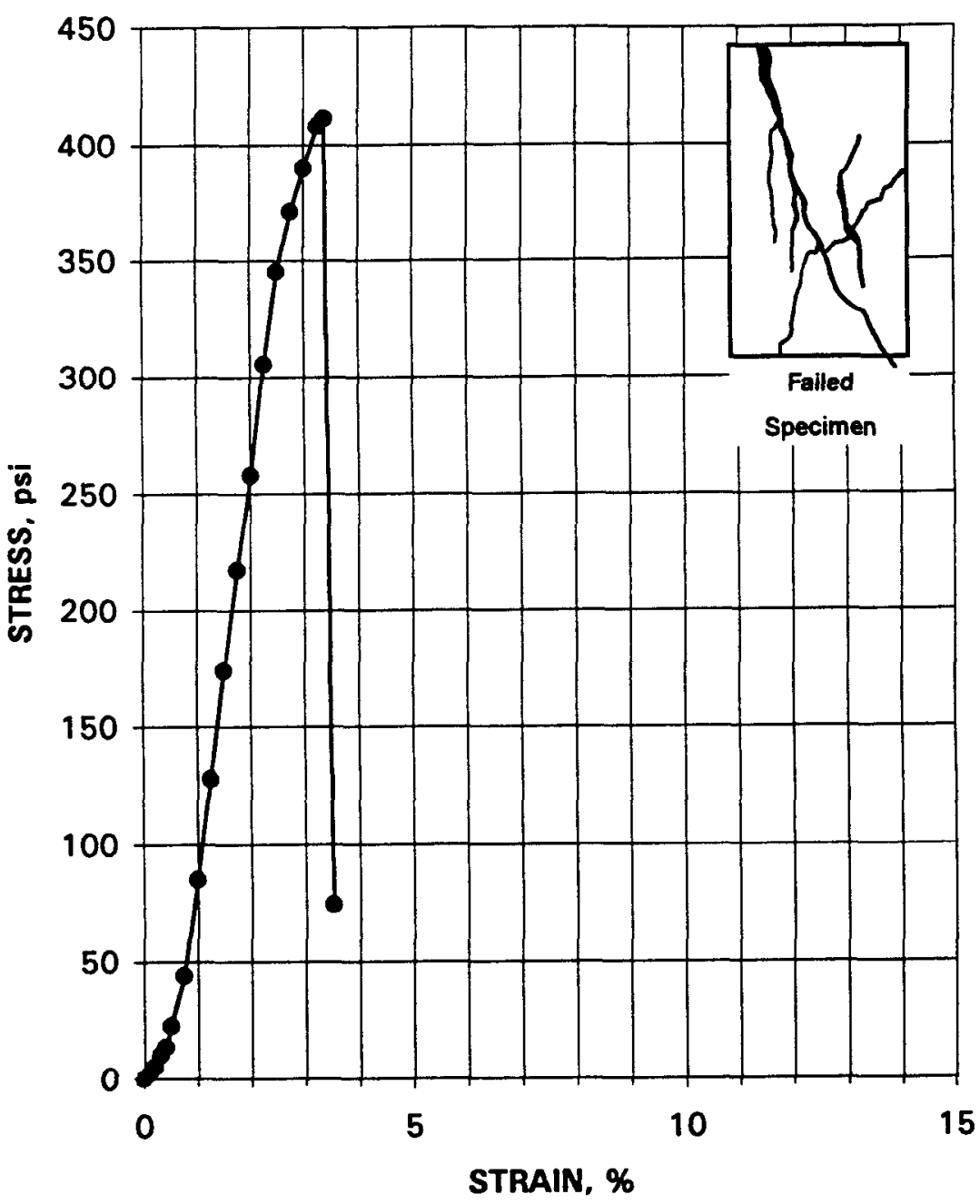




\section{UNCONFINED COMPRESSIVE STRENGTH - ASTM D 2166 28-DAY CURE}

Project Name SAIC - Savannah River

Project No. $\quad 777304.00010500$

Specimen Mold Date

Specimen Test Date

STRESS AT FAILURE, psi STRAIN AT FAILURE, \%

AXIAL DEVIATOR

\begin{tabular}{ccc} 
STRAIN, $\%$ & STRESS, psi \\
\cline { 1 - 1 } 0.00 & & 0.0 \\
0.10 & & 2.2 \\
0.20 & & 4.4 \\
0.30 & & 6.0 \\
0.40 & & 9.8 \\
0.50 & & 13.8 \\
0.75 & & 33.9 \\
1.00 & & 80.1 \\
1.25 & & 149.2 \\
1.50 & & 233.8 \\
1.75 & & 292.2 \\
2.00 & & 301.4 \\
2.25 & & 342.0 \\
2.30 & & 342.4 \\
2.50 & & 319.2 \\
2.75 & 284.9 \\
3.00 & 265.2 \\
3.25 & 239.4 \\
3.50 & 204.6 \\
3.75 & 184.3
\end{tabular}

Client Sample No. P422F00

IT Lab Specimen No. ETDC-8052

Specimen Height, in.

3.9953

Specimen Diameter, in.

2.0072

Specimen Weight, $g$.

382.76

Moisture Content, \%

30.4

Wet Unit Weight, pcf.

115.4

Dry Unit Weight, pcf.

88.5

Rate of Strain, in./min.

0.0400

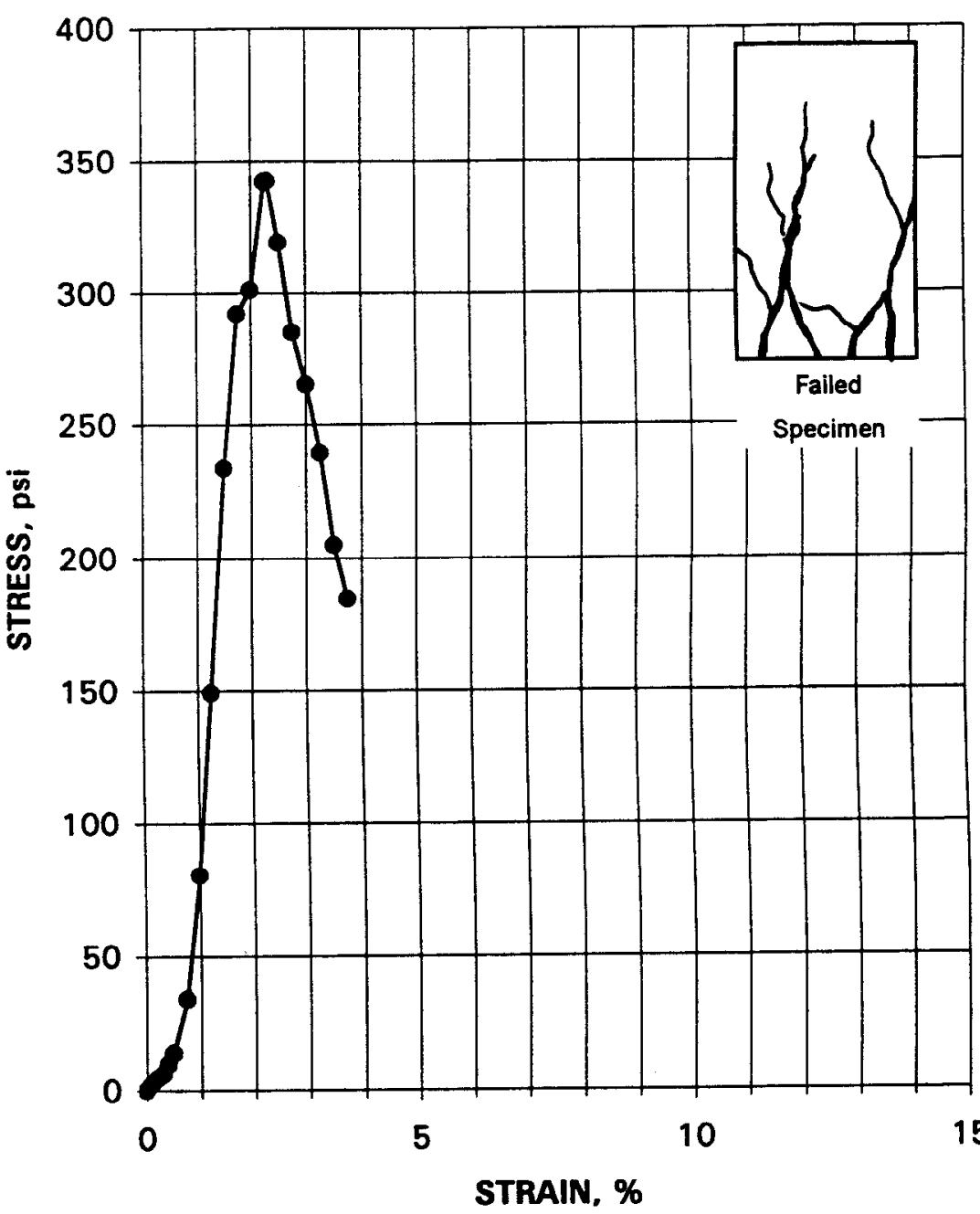




\section{UNCONFINED COMPRESSIVE STRENGTH - ASTM D 2166 28-DAY CURE}

Project Name SAIC - Savannah River

Project No. $\quad 777304.00010500$

Specimen Mold Date

Specimen Test Date

STRESS AT FAILURE, psi STRAIN AT FAILURE, \%

\begin{tabular}{|c|c|}
\hline $\begin{array}{c}\text { AXIAL } \\
\text { STRAIN, \% }\end{array}$ & $\begin{array}{l}\text { DEVIATOR } \\
\text { STRESS, psi }\end{array}$ \\
\hline 0.00 & 0.0 \\
\hline 0.10 & 2.9 \\
\hline 0.20 & 6.6 \\
\hline 0.30 & 9.2 \\
\hline 0.40 & 12.6 \\
\hline 0.50 & 18.6 \\
\hline 0.75 & 51.3 \\
\hline 1.00 & 92.3 \\
\hline 1.25 & 139.0 \\
\hline 1.51 & 175.8 \\
\hline 1.76 & 229.3 \\
\hline 2.01 & 272.5 \\
\hline $\begin{array}{l}2.26 \\
2.51\end{array}$ & $\begin{array}{l}308.4 \\
326.8\end{array}$ \\
\hline 2.76 & 335.8 \\
\hline 3.01 & 339.8 \\
\hline 3.26 & 339.6 \\
\hline 3.39 & 340.1 \\
\hline 3.51 & 307.8 \\
\hline 3.76 & 53.4 \\
\hline
\end{tabular}

Client Sample No. P432F00

IT Lab Specimen No. ETDC-8053

Specimen Height, in.

3.9855

Specimen Diameter, in.

2.0038

Specimen Weight, $g$.

361.96

Moisture Content, \%

37.5

Wet Unit Weight, pcf.

109.7

Dry Unit Weight, pcf.

79.8

Rate of Strain, in./min.

0.0400

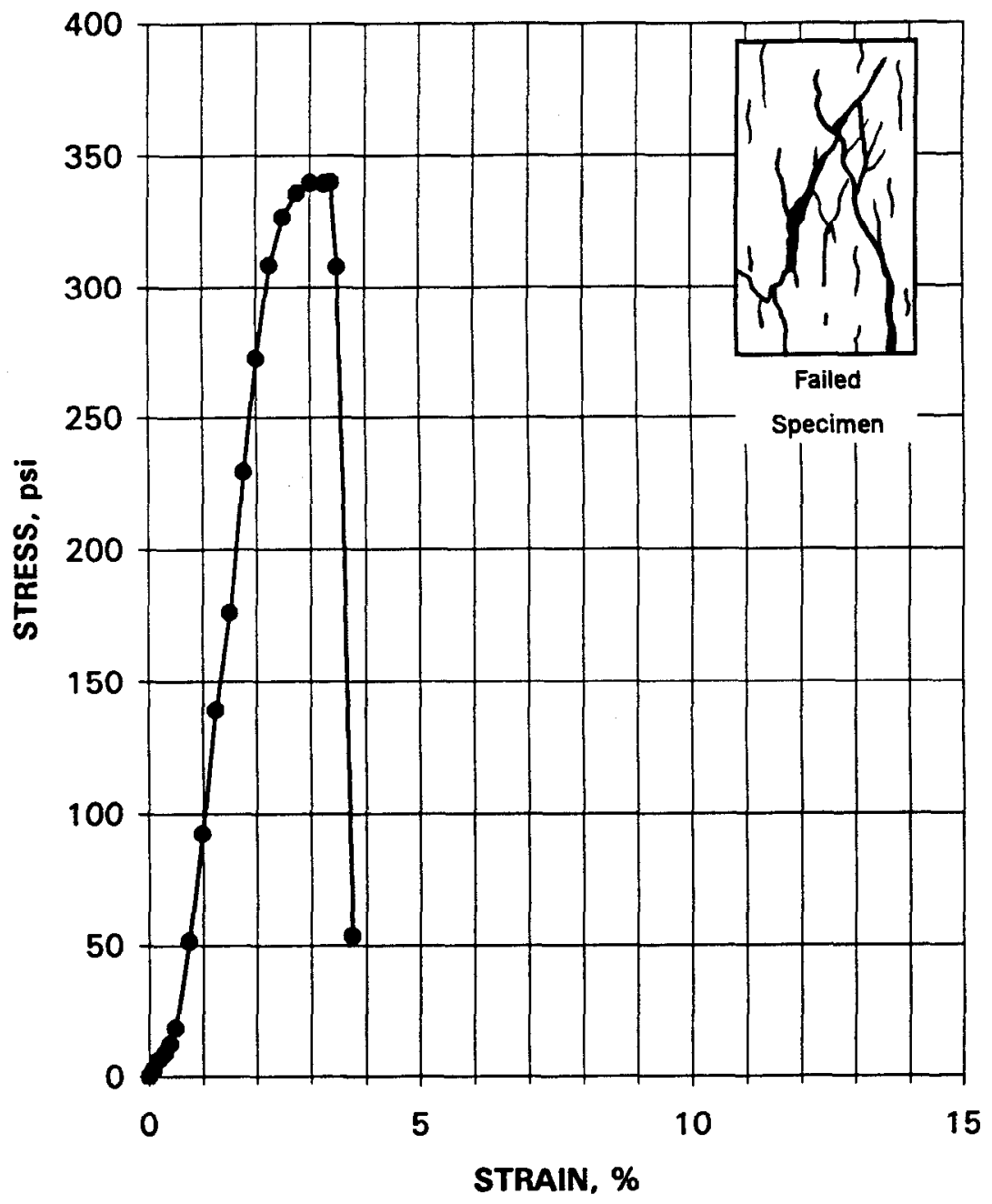




\section{UNCONFINED COMPRESSIVE STRENGTH - ASTM D 2166 28-DAY CURE}

Project Name SAIC - Savannah River

Project No. $\quad 777304.00010500$

Specimen Mold Date

Specimen Test Date

STRESS AT FAILURE, psi

STRAIN AT FAILURE, \%

AXIAL

STRAIN, \%

0.00

0.10

0.20

0.30

0.40

0.50

0.75

1.00

1.25

1.50

1.75

2.00

2.25

2.50

2.63

2.75

3.00

3.25
$1 / 19 / 99$

492.2

2.6
Client Sample No. P442F0O

IT Lab Specimen No. ETDC-8054

Specimen Height, in.

Specimen Diameter, in.

3.9987

Specimen Weight, $g$.

Moisture Content, \%

2.0082

364.40

36.0

Wet Unit Weight, pcf.

109.6

Dry Unit Weight, pcf.

80.6

Rate of Strain, in./min.

0.0400

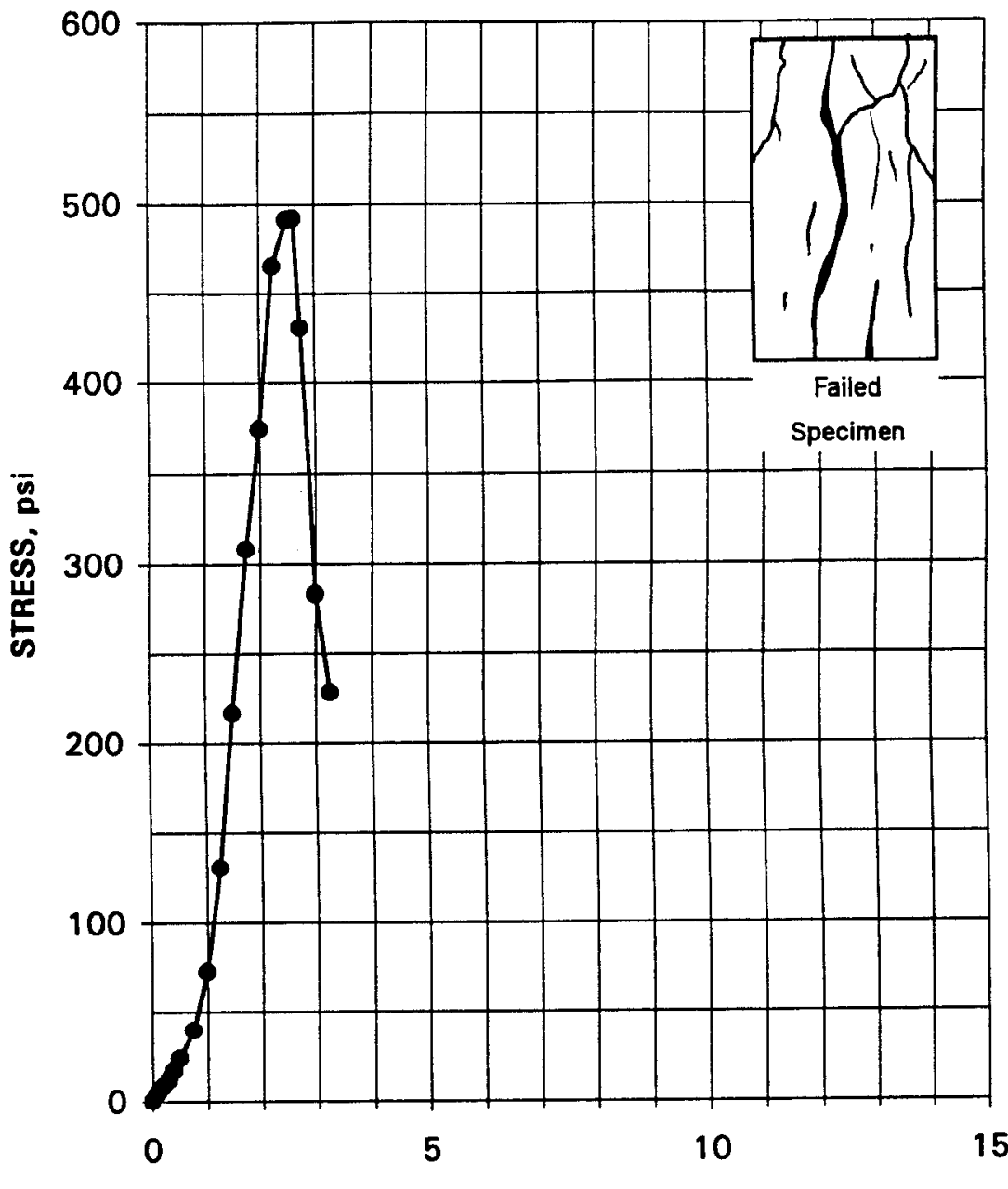

STRAIN, \% 


\section{UNCONFINED COMPRESSIVE STRENGTH - ASTM D 2166 28-DAY CURE}

Project Name SAIC - Savannah River

Project No. 777304.00010500

\section{Specimen Mold Date}

Specimen Test Date

STRESS AT FAILURE, psi

STRAIN AT FAILURE, \%

\begin{tabular}{ccc}
$\begin{array}{c}\text { AXIAL } \\
\text { STRAIN, \% }\end{array}$ & $\begin{array}{c}\text { DEVIATOR } \\
\text { STRESS, psi }\end{array}$ \\
\cline { 3 - 3 } 0.00 & 0.0 \\
0.10 & 4.1 \\
0.20 & 7.6 \\
0.30 & & 10.8 \\
0.40 & 16.5 \\
0.50 & 20.6 \\
0.75 & 54.9 \\
1.00 & 124.7 \\
1.26 & 216.7 \\
1.51 & 298.0 \\
1.76 & 365.6 \\
2.01 & 414.6 \\
2.26 & 488.8 \\
2.51 & 531.2 \\
2.76 & 541.9 \\
2.89 & 543.4 \\
3.01 & 513.7 \\
3.26 & 402.2 \\
3.51 & 344.1 \\
3.77 & 228.1
\end{tabular}

Client Sample No. P512F00

IT Lab Specimen No. ETDC-8055

Specimen Height, in.

3.9838

Specimen Diameter, in.

2.0007

Specimen Weight, $g$.

377.87

Moisture Content, \%

29.1

Wet Unit Weight, pcf.

115.0

Dry Unit Weight, pct.

89.1

Rate of Strain, in./min.

0.0400

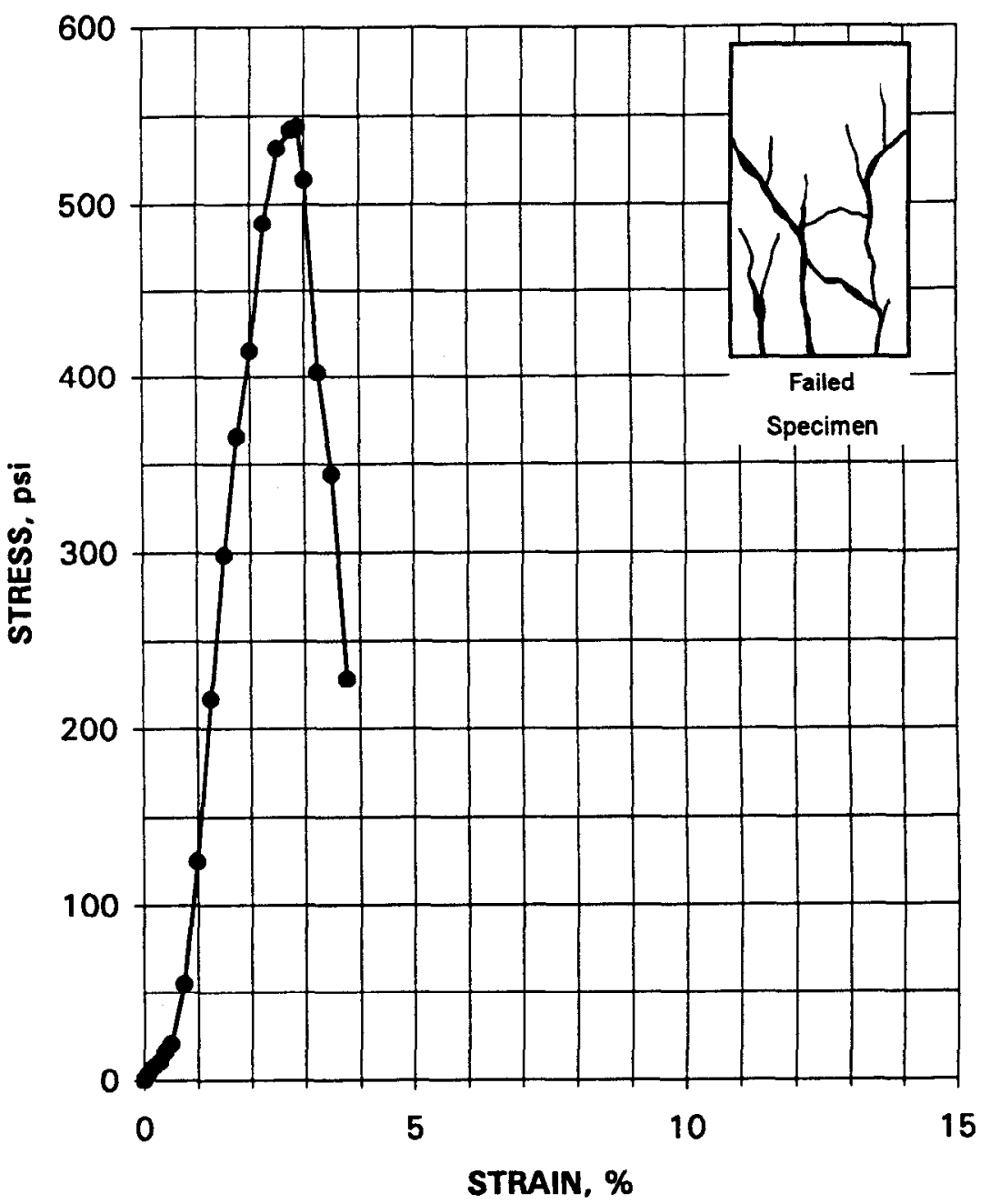




\section{UNCONFINED COMPRESSIVE STRENGTH - ASTM D 2166 28-DAY CURE}

Project Name SAIC - Savannah River

Project No. $\quad 777304.00010500$

Specimen Mold Date

Specimen Test Date

STRESS AT FAILURE, psi

STRAIN AT FAILURE, \%

\begin{tabular}{ccc}
$\begin{array}{ccc}\text { AXIAL } \\
\text { STRAIN, \% }\end{array}$ & & $\begin{array}{c}\text { DEVIATOR } \\
\text { STRESS, psi }\end{array}$ \\
\cline { 1 - 1 } 0.00 & & 0.0 \\
0.10 & & 3.8 \\
0.20 & & 11.8 \\
0.30 & & 26.7 \\
0.40 & & 48.9 \\
0.50 & & 62.8 \\
0.75 & & 114.3 \\
1.00 & & 179.0 \\
1.25 & & 264.2 \\
1.50 & & 353.0 \\
1.75 & & 437.1 \\
2.00 & & 499.4 \\
2.26 & & 598.8 \\
2.51 & 672.2 \\
2.76 & 714.8 \\
2.88 & 737.1 \\
3.01 & 734.6 \\
3.26 & 85.5
\end{tabular}

Client Sample No. P522F00

IT Lab Specimen No. ETDC-8056

Specimen Height, in.

3.9907

Specimen Diameter, in.

1.9982

Specimen Weight, $g$.

393.76

Moisture Content, \%

26.5

Wet Unit Weight, pcf.

119.9

Dry Unit Weight, pcf.

94.8

Rate of Strain, in./min.

0.0400

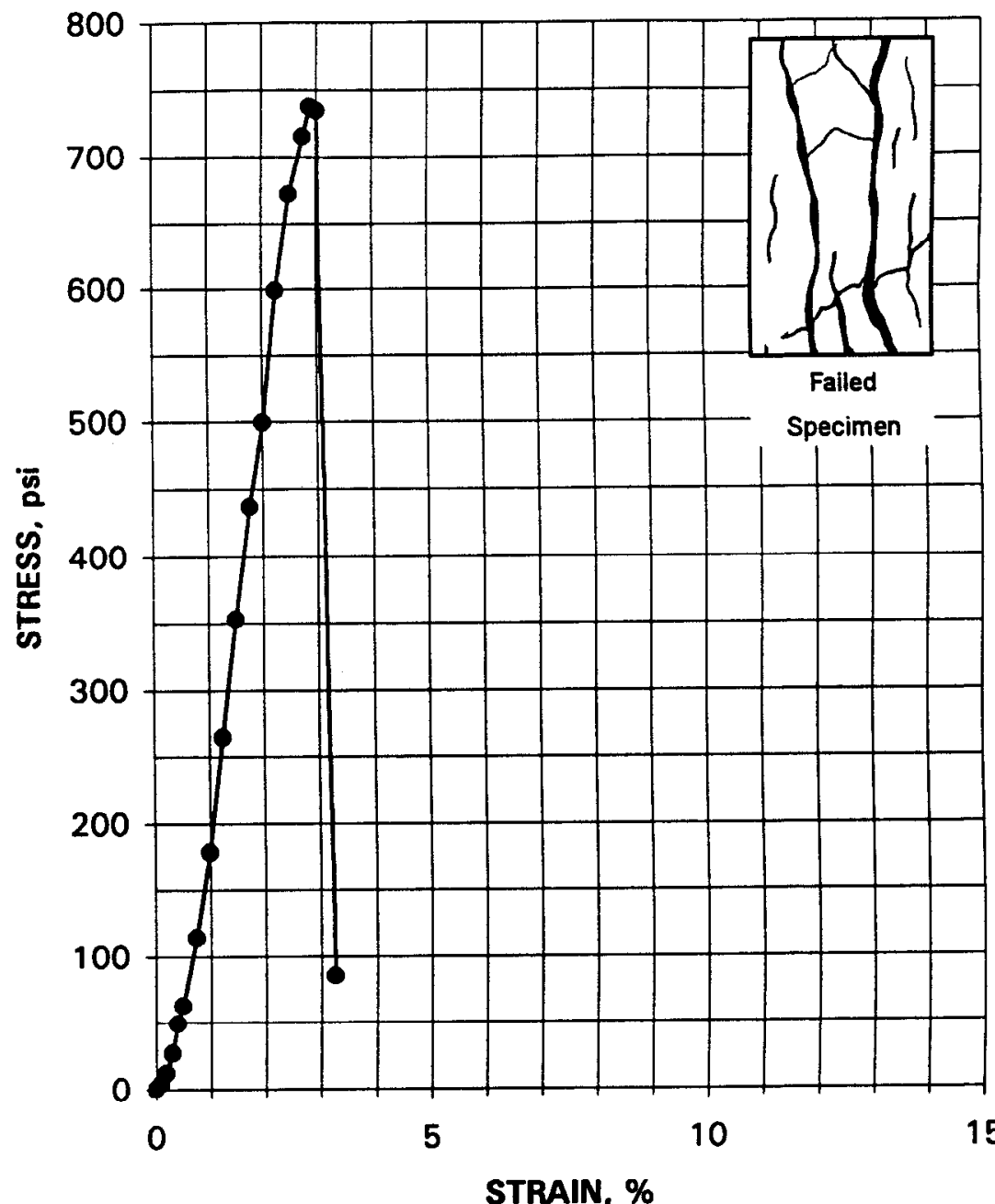




\section{UNCONFINED COMPRESSIVE STRENGTH - ASTM D 2166 28-DAY CURE}

\begin{tabular}{lc} 
Project Name & SAIC - Savan \\
Project No. & 777304.00010 \\
\multicolumn{3}{l}{ Nocimen Mold Date } \\
Specimen Test Date \\
STRESS AT FAILURE, psi \\
STRAIN AT FAILURE, \% \\
\multicolumn{2}{c}{} \\
AXIAL & DEVIATOR \\
STRAIN, \% & STRESS, psi \\
\cline { 2 - 2 } 0.00 & 0.0 \\
0.10 & 3.5 \\
0.20 & 10.2 \\
0.30 & 21.3 \\
0.40 & 43.1 \\
0.50 & 61.8 \\
0.75 & 139.4 \\
1.00 & 216.6 \\
1.25 & 277.3 \\
1.50 & 347.8 \\
1.75 & 403.6 \\
2.00 & 440.0 \\
2.25 & 475.0 \\
2.50 & 495.2 \\
2.75 & 142.1 \\
\end{tabular}

Client Sample No. P532F00

IT Lab Specimen No. ETDC-8057

Specimen Height, in.

$1 / 19 / 99$

Specimen Diameter, in.

1.9997

Specimen Weight, $g$.

Moisture Content, \%

Wet Unit Weight, pcf.

Dry Unit Weight, pcf.

90.5

Rate of Strain, in./min.

0.0400

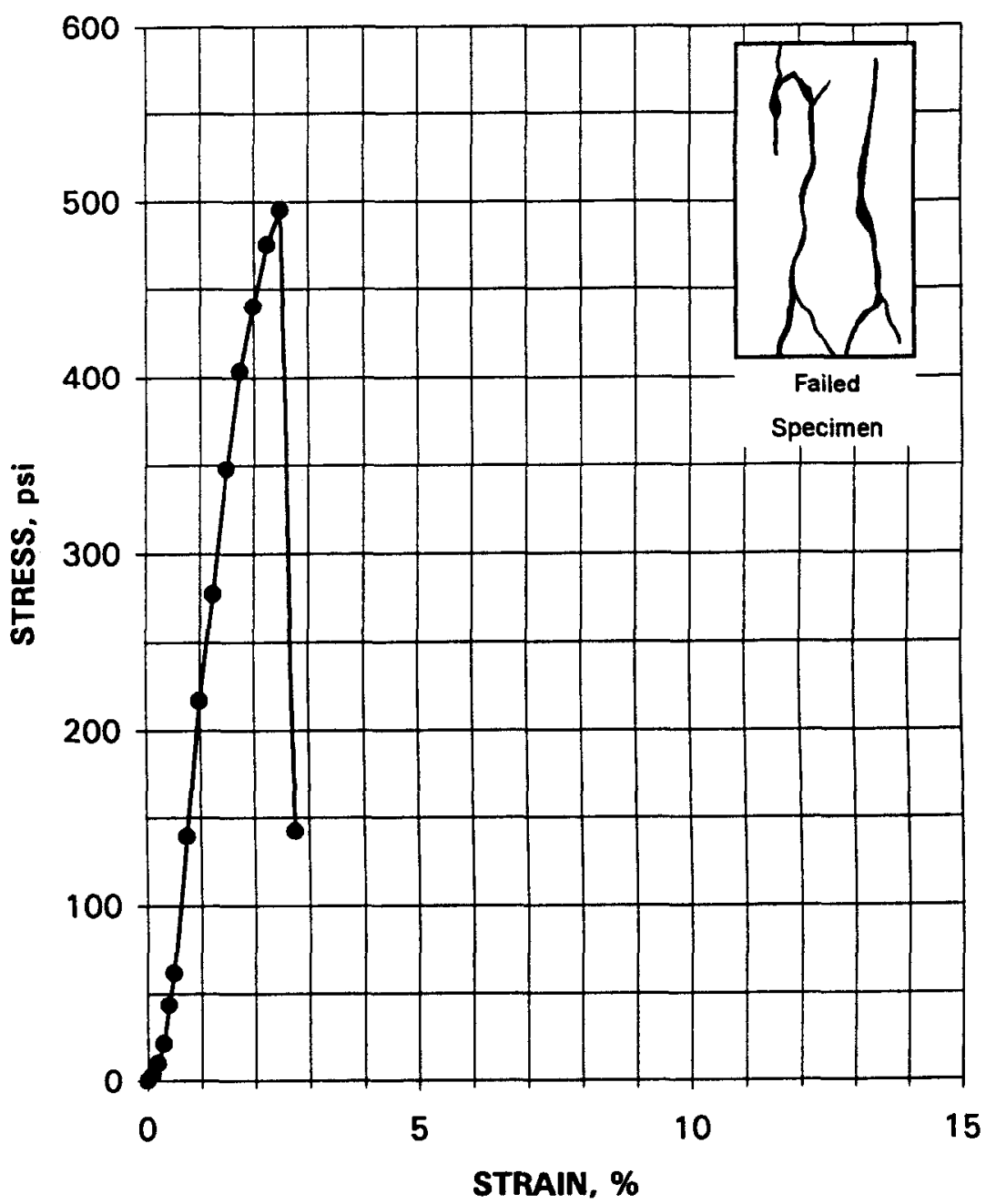




\section{UNCONFINED COMPRESSIVE STRENGTH - ASTM D 2166 28-DAY CURE}

Project Name SAIC - Savannah River

Project No. $\quad 777304.00010500$

Specimen Mold Date

Specimen Test Date

STRESS AT FAILURE, psi STRAIN AT FAILURE, \%

\begin{tabular}{ccc}
$\begin{array}{c}\text { AXIAL } \\
\text { STRAIN, \% }\end{array}$ & & $\begin{array}{c}\text { DEVIATOR } \\
\text { STRESS, psi }\end{array}$ \\
\cline { 1 - 1 } 0.00 & & 0.0 \\
0.10 & & 1.6 \\
0.20 & & 6.7 \\
0.30 & & 11.1 \\
0.40 & & 16.8 \\
0.50 & & 23.5 \\
0.75 & & 46.2 \\
1.00 & & 85.3 \\
1.25 & & 142.1 \\
1.49 & & 212.4 \\
1.74 & & 306.2 \\
1.99 & & 414.2 \\
2.24 & & 498.3 \\
2.49 & & 504.8 \\
2.74 & & 496.0 \\
2.99 & & 148.5
\end{tabular}

Client Sample No. P542F00

IT Lab Specimen No. ETDC-8058

Specimen Height, in.

4.0148

Specimen Diameter, in.

1.9980

Specimen Weight, $g$.

372.48

Moisture Content, \%

32.2

Wet Unit Weight, pcf.

112.7

Dry Unit Weight, pcf.

85.3

Rate of Strain, in./min.

0.0400

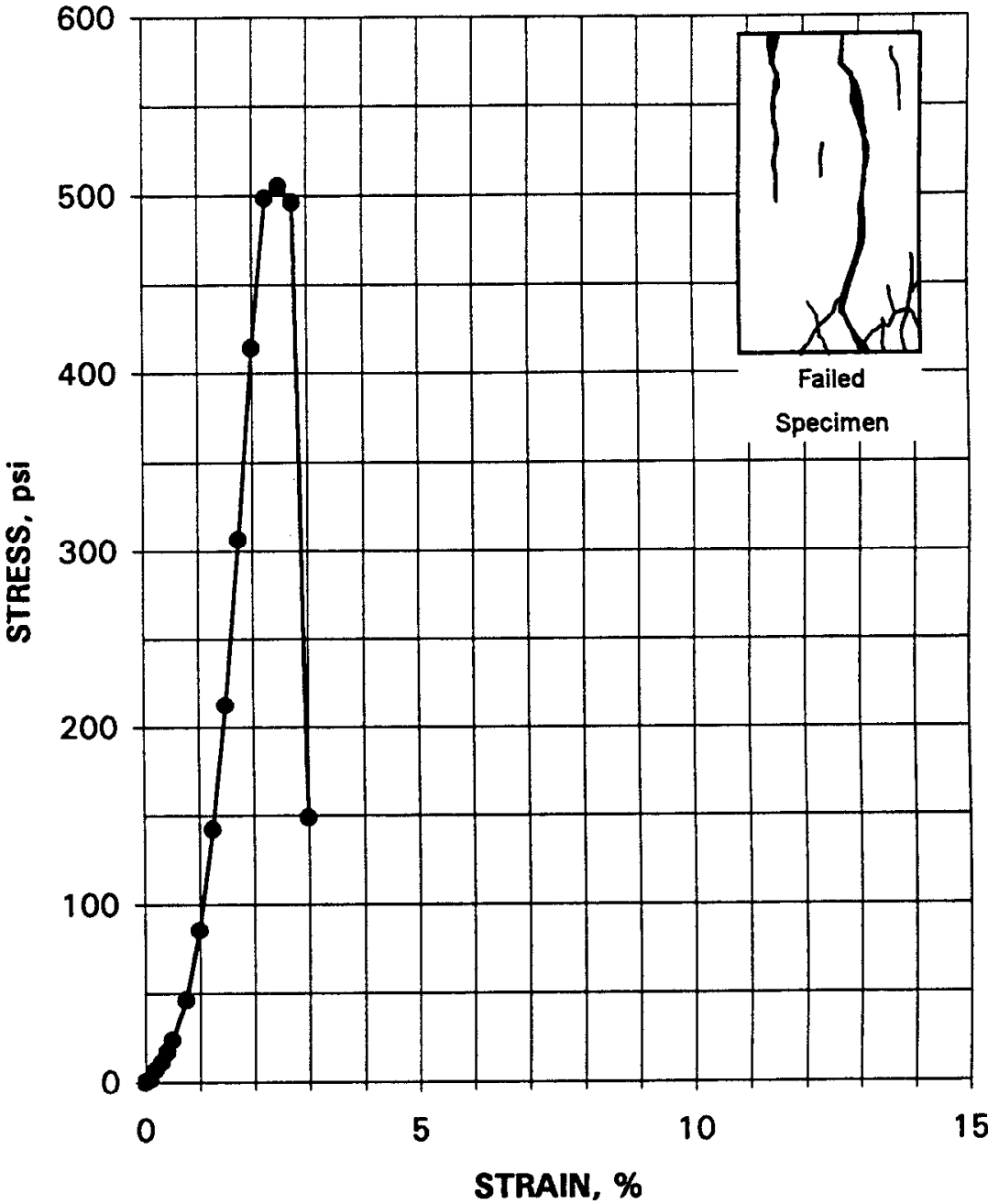




\section{UNCONFINED COMPRESSIVE STRENGTH - ASTM D 2166 28-DAY CURE}

Project Name SAIC - Savannah River

Project No. $\quad 777304.00010500$

Specimen Mold Date

Specimen Test Date

STRESS AT FAILURE, psi STRAIN AT FAILURE, \%

\begin{tabular}{ccc}
$\begin{array}{c}\text { AXIAL } \\
\text { STRAIN, \% }\end{array}$ & $\begin{array}{c}\text { DEVIATOR } \\
\text { STRESS, psi }\end{array}$ \\
\cline { 1 - 1 } 0.00 & & 0.0 \\
0.10 & & 3.8 \\
0.20 & & 7.6 \\
0.30 & & 12.1 \\
0.40 & & 18.1 \\
0.50 & & 26.7 \\
0.76 & & 56.3 \\
1.01 & & 124.1 \\
1.26 & & 193.4 \\
1.51 & & 267.9 \\
1.77 & & 325.8 \\
2.02 & & 393.7 \\
2.27 & & 451.0 \\
2.52 & & 505.5 \\
2.78 & & 539.2 \\
3.03 & 567.2 \\
3.28 & 584.9 \\
3.41 & 585.3 \\
3.53 & 577.5 \\
3.79 & 497.1 \\
4.04 & 371.9 \\
4.29 & 85.5
\end{tabular}

Client Sample No. P612F0O

IT Lab Specimen No. ETDC-8059

Specimen Height, in.

3.9608

Specimen Diameter, in.

1.9980

Specimen Weight, $g$.

377.70

Moisture Content, \%

28.9

Wet Unit Weight, pcf.

115.9

Dry Unit Weight, pcf.

89.9

Rate of Strain, in./min.

0.0400

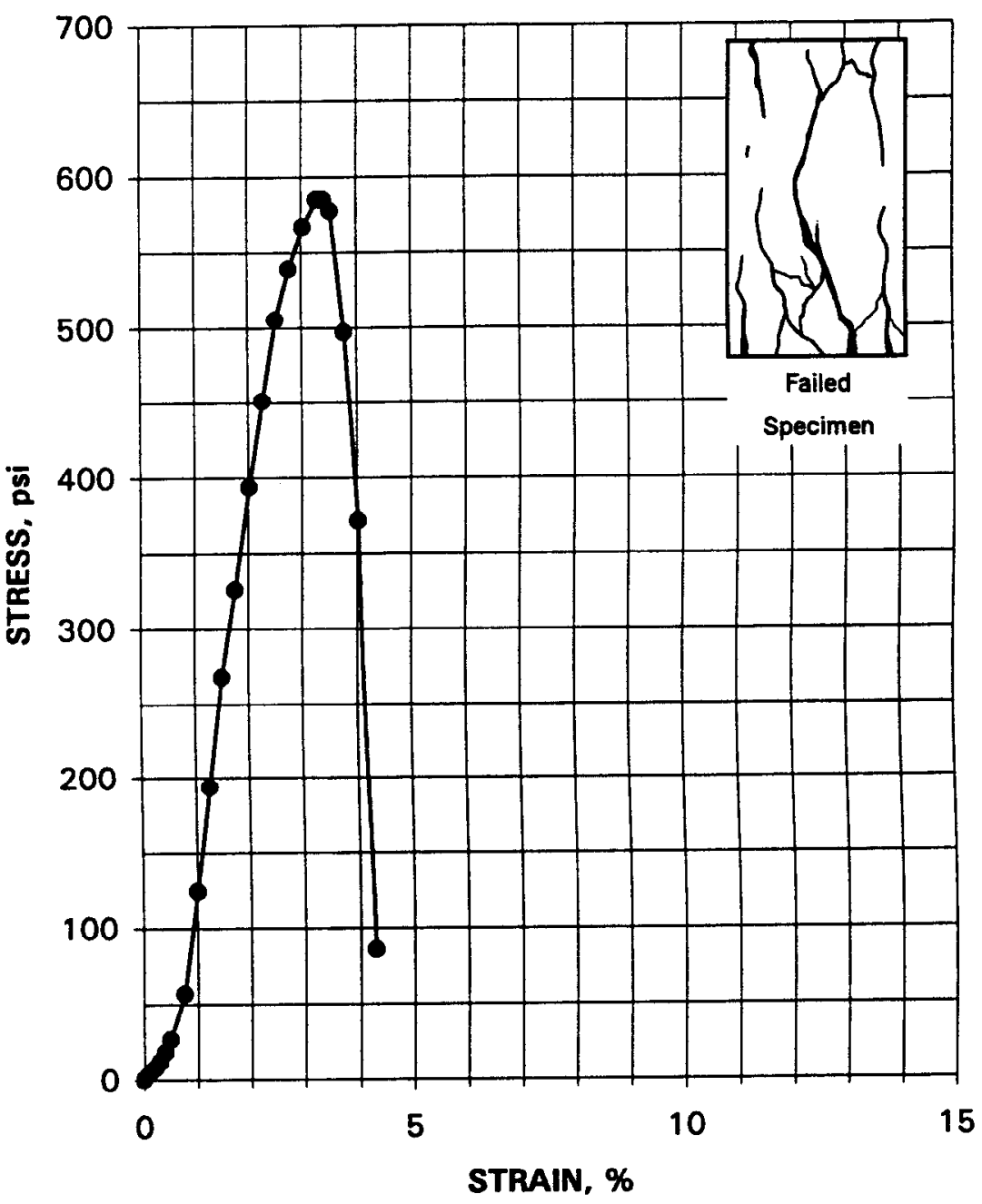




\section{UNCONFINED COMPRESSIVE STRENGTH - ASTM D 2166 28-DAY CURE}

Project Name SAIC - Savannah River

Project No. $\quad 777304.00010500$

Specimen Mold Date

Specimen Test Date

STRESS AT FAILURE, psi

$1 / 20 / 99$

STRAIN AT FAILURE, \%

\begin{tabular}{|c|c|}
\hline $\begin{array}{c}\text { AXIAL } \\
\text { STRAIN, \% }\end{array}$ & $\begin{array}{l}\text { DEVIATOR } \\
\text { STRESS, psi }\end{array}$ \\
\hline 0.00 & 0.0 \\
\hline 0.10 & 3.5 \\
\hline 0.20 & 7.6 \\
\hline 0.30 & 18.9 \\
\hline 0.40 & 41.6 \\
\hline 0.50 & 71.1 \\
\hline 0.75 & 161.6 \\
\hline 1.00 & 239.8 \\
\hline 1.25 & 309.5 \\
\hline 1.50 & 370.4 \\
\hline 1.75 & 408.0 \\
\hline 2.00 & 433.6 \\
\hline 2.25 & 460.0 \\
\hline 2.50 & 495.8 \\
\hline 2.75 & 531.1 \\
\hline 3.00 & 551.2 \\
\hline 3.25 & 563.6 \\
\hline 3.50 & 576.8 \\
\hline 3.76 & 587.7 \\
\hline 4.01 & 594.4 \\
\hline 4.26 & 585.0 \\
\hline 4.51 & 564.7 \\
\hline 4.76 & 465.1 \\
\hline 5.01 & 408.3 \\
\hline 5.26 & 361.6 \\
\hline
\end{tabular}

594.4

4.0
Client Sample No. P622F00

IT Lab Specimen No. ETDC-8060

Specimen Height, in.

3.9947

Specimen Diameter, in.

2.0065

Specimen Weight, $g$.

390.34

Moisture Content, \%

26.1

Wet Unit Weight, pcf.

117.7

Dry Unit Weight, pcf.

93.4

Rate of Strain, in./min.

0.0400

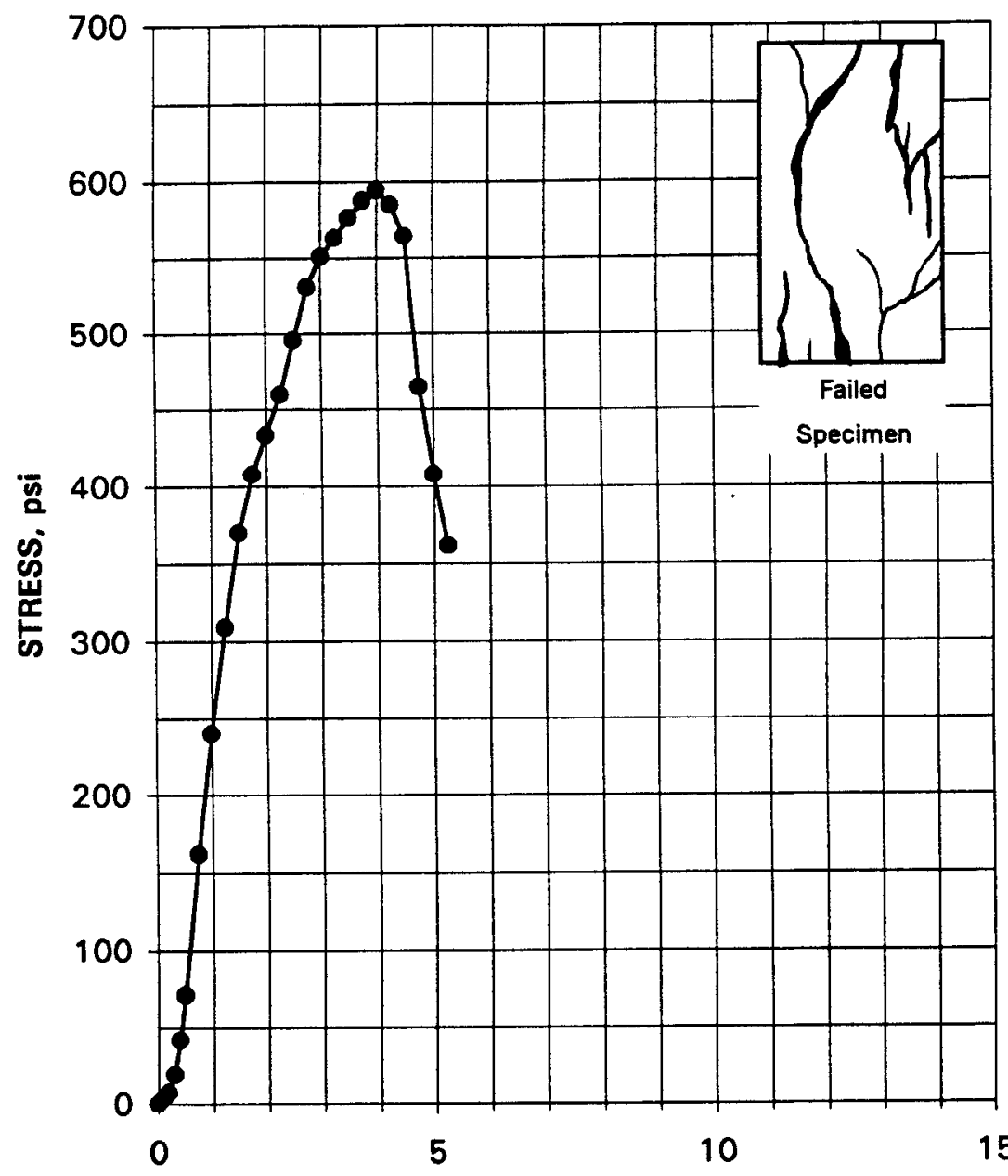

STRAIN, \% 


\section{UNCONFINED COMPRESSIVE STRENGTH - ASTM D 2166 28-DAY CURE}

$\begin{array}{ll}\text { Project Name } & \text { SAIC - Savannah River } \\ \text { Project No. } & 777304.00010500\end{array}$

Specimen Mold Date

Specimen Test Date

STRESS AT FAILURE, psi

STRAIN AT FAILURE, \%

\begin{tabular}{ccc}
$\begin{array}{c}\text { AXIAL } \\
\text { STRAIN, \% }\end{array}$ & & $\begin{array}{c}\text { DEVIATOR } \\
\text { STRESS, psi }\end{array}$ \\
\cline { 1 - 1 } 0.00 & & 0.0 \\
0.10 & & 3.5 \\
0.20 & & 7.6 \\
0.30 & & 15.2 \\
0.40 & & 21.2 \\
0.50 & & 32.3 \\
0.75 & & 82.8 \\
1.01 & & 154.7 \\
1.26 & & 223.8 \\
1.51 & & 297.5 \\
1.76 & & 363.9 \\
2.01 & 416.6 \\
2.26 & 436.4 \\
2.51 & 449.2 \\
2.77 & 478.1 \\
3.02 & 487.7 \\
3.27 & 105.6
\end{tabular}

Client Sample No. P632F00

IT Lab Specimen No. ETDC-8061

Specimen Height, in.

3.9770

Specimen Diameter, in.

2.0002

Specimen Weight, $\boldsymbol{g}$.

368.96

Moisture Content, \%

33.3

Wet Unit Weight, pcf.

112.5

Dry Unit Weight, pcf.

84.4

Rate of Strain, in./min.

0.0400

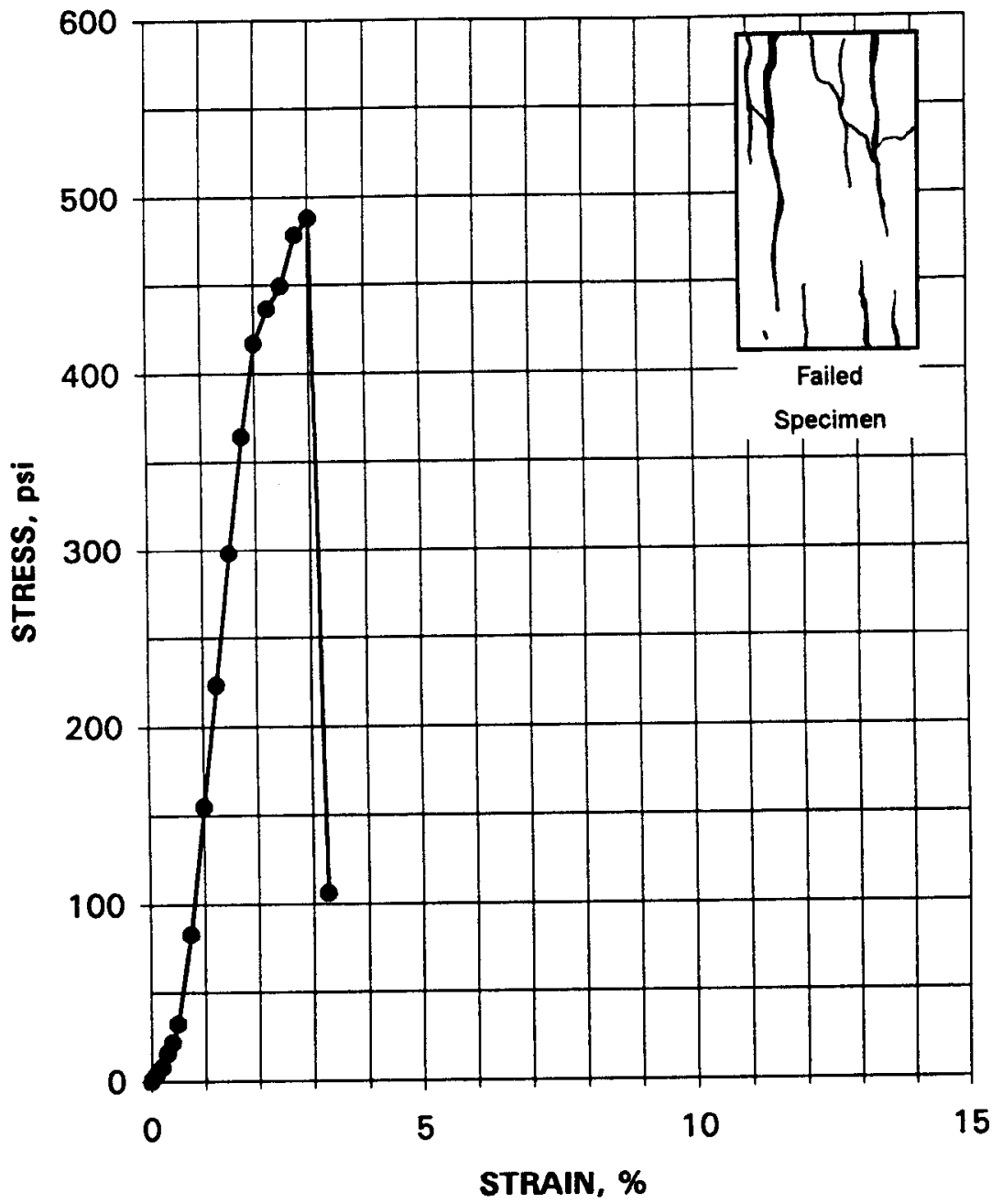




\section{UNCONFINED COMPRESSIVE STRENGTH - ASTM D 2166 28-DAY CURE}

Project Name

SAIC - Savannah River

Project No.

777304.00010500

Specimen Mold Date

Specimen Test Date

STRESS AT FAILURE, psi STRAIN AT FAILURE, \%

\begin{tabular}{|c|c|}
\hline $\begin{array}{c}\text { AXIAL } \\
\text { STRAIN, \% }\end{array}$ & $\begin{array}{l}\text { DEVIATOR } \\
\text { STRESS }\end{array}$ \\
\hline 0.00 & $\frac{0}{0.03}$ \\
\hline $\begin{array}{l}0.00 \\
0.10\end{array}$ & 38 \\
\hline $\begin{array}{l}0.10 \\
0.20\end{array}$ & 11.1 \\
\hline 0.20 & 23.1 \\
\hline 0.40 & 42.4 \\
\hline 0.50 & 64.8 \\
\hline 0.75 & 124.8 \\
\hline 1.00 & 184.0 \\
\hline 1.25 & 245.0 \\
\hline 1.50 & 309.4 \\
\hline 1.75 & 367.9 \\
\hline 2.00 & 434.2 \\
\hline 2.25 & 487.1 \\
\hline 2.50 & 512.8 \\
\hline 2.75 & $\begin{array}{l}529.7 \\
538.6\end{array}$ \\
\hline 3.25 & 537.8 \\
\hline 3.50 & 543.1 \\
\hline 3.75 & 538.1 \\
\hline 4.00 & 527.2 \\
\hline 4.25 & 511.2 \\
\hline 4.50 & 495.9 \\
\hline 4.75 & 478.0 \\
\hline 5.00 & 455.0 \\
\hline 5.25 & 413.5 \\
\hline 5.50 & 385.7 \\
\hline 5.75 & 362.2 \\
\hline
\end{tabular}

Client Sample No. P642F00

IT Lab Specimen No. ETDC-8062

Specimen Height, in.

4.0012

Specimen Diameter, in.

2.0022

Specimen Weight, $g$.

375.47

Moisture Content, \%

31.4

Wet Unit Weight, pcf.

113.6

Dry Unit Weight, pef.

86.4

Rate of Strain, in./min.

0.0400

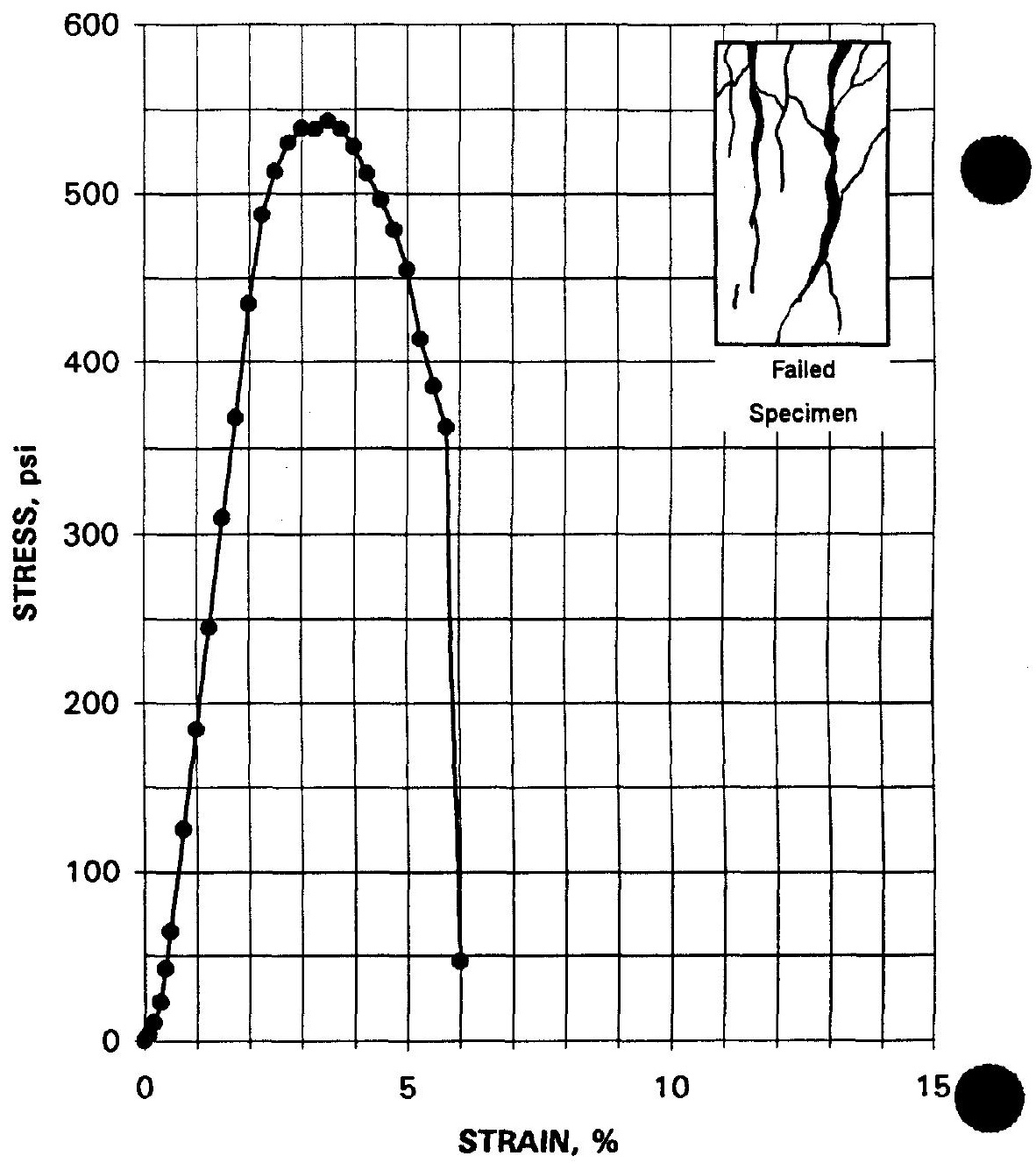




\section{P Series}

90 Day Immersion UCS Results 


\section{UNCONFINED COMPRESSIVE STRENGTH - ASTM D 2166 28-DAY CURE, 90-DAY SATURATION}

Project Name SAIC/SAVANNAH RIVER

Project No. $\quad 777304.00010500$

\section{Specimen Mold Date}

Specimen Test Date

STRESS AT FAILURE, psi

STRAIN AT FAILURE, \%

\begin{tabular}{ccc}
$\begin{array}{c}\text { AXIAL } \\
\text { STRAIN, \% }\end{array}$ & $\begin{array}{c}\text { DEVIATOR } \\
\text { STRESS, psi }\end{array}$ \\
\cline { 1 - 2 } \cline { 3 - 3 } 0.00 & 0.0 \\
0.10 & & 14.9 \\
0.20 & 37.0 \\
0.30 & & 71.1 \\
0.40 & & 104.9 \\
0.50 & & 138.8 \\
0.75 & & 218.4 \\
1.00 & & 289.7 \\
1.25 & 353.2 \\
1.50 & 403.5 \\
1.75 & 448.9 \\
2.00 & 494.7 \\
2.26 & 499.9 \\
2.51 & 506.1 \\
2.76 & 509.4 \\
2.88 & 509.7 \\
3.01 & 468.4 \\
3.26 & 448.2 \\
3.51 & 423.8 \\
3.76 & 131.8
\end{tabular}

$12 / 22 / 99$

$4 / 20 / 99$

509.7

2.9
Client Sample No. P313F00

IT Lab Specimen No. ETDC-8023

Specimen Height, in.

Specimen Diameter, in.

Specimen Weight, $\mathrm{g}$.

Moisture Content, \%

Wet Unit Weight, pcf.

Dry Unit Weight, pcf.

Rate of Strain, in./min.
3.9910

2.0038

388.04

30.8

117.5

89.8

0.0400

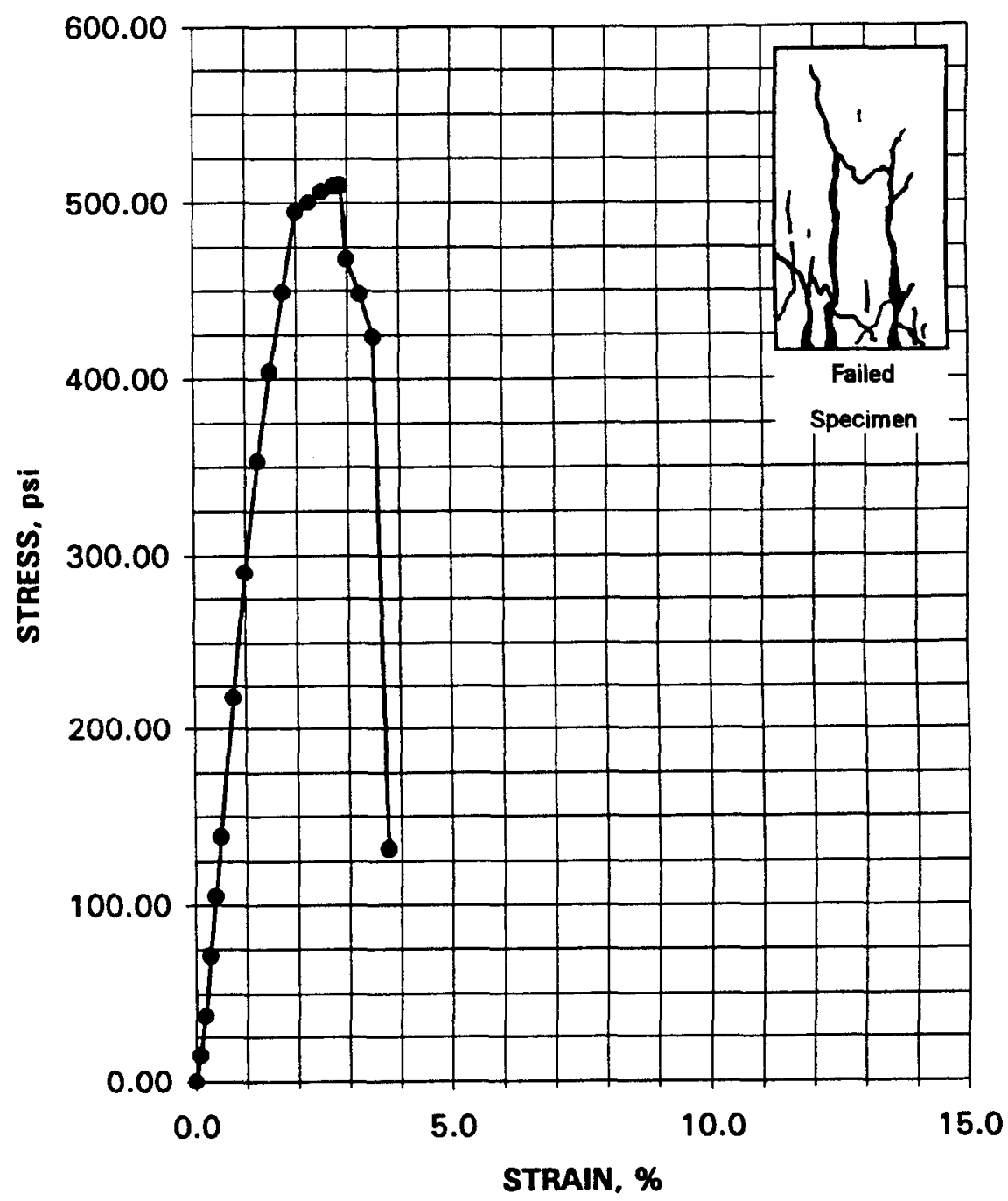




\section{UNCONFINED COMPRESSIVE STRENGTH - ASTM D 2166 28-DAY CURE, 90-DAY SATURATION}

Project Name SAIC/SAVANNAH RIVER

Project No. $\quad 777304.00010500$

Specimen Mold Date

Specimen Test Date

STRESS AT FAILURE, psi

STRAIN AT FAILURE, \%

\begin{tabular}{ccc}
$\begin{array}{c}\text { AXIAL } \\
\text { STRAIN, \% }\end{array}$ & $\begin{array}{c}\text { DEVIATOR } \\
\text { STRESS, psi }\end{array}$ \\
\cline { 1 - 3 } 0.00 & 0.0 \\
0.10 & 4.7 \\
0.20 & 16.4 \\
0.30 & 29.6 \\
0.40 & 48.5 \\
0.50 & 83.3 \\
0.75 & 168.0 \\
1.00 & 235.5 \\
1.25 & 294.5 \\
1.50 & 340.1 \\
1.75 & 373.0 \\
2.00 & 402.7 \\
2.25 & 434.1 \\
2.50 & 471.8 \\
2.75 & 504.1 \\
3.00 & 526.7 \\
3.25 & 546.1 \\
3.50 & 566.3 \\
3.63 & 571.4 \\
3.75 & 561.5 \\
4.00 & 531.0 \\
4.25 & 528.1 \\
4.51 & 307.7 \\
4.76 & 212.7
\end{tabular}

$12 / 22 / 99$

$4 / 19 / 99$

571.4

3.6
Client Sample No. P323F00

IT Lab Specimen No. ETDC-8024

Specimen Height, in.

3.9953

Specimen Diameter, in.

2.0077

Specimen Weight, $g$.

Moisture Content, \%

395.20

28.7

Wet Unit Weight, pcf.

119.1

Dry Unit Weight, pcf.

92.5

Rate of Strain, in./min.



STRAIN, \% 


\section{UNCONFINED COMPRESSIVE STRENGTH - ASTM D 2166 28-DAY CURE, 90-DAY SATURATION}

Project Name SAIC/SAVANNAH RIVER

Project No. $\quad 777304.00010500$

Specimen Mold Date

Specimen Test Date

STRESS AT FAILURE, psi

STRAIN AT FAILURE, \%

\begin{tabular}{ccc}
$\begin{array}{cc}\text { AXIAL } \\
\text { STRAIN, \% }\end{array}$ & $\begin{array}{c}\text { DEVIATOR } \\
\text { STRESS, pSi }\end{array}$ \\
\cline { 2 - 3 } 0.00 & 0.0 \\
0.10 & & 15.2 \\
0.20 & 53.3 \\
0.30 & & 91.5 \\
0.40 & 132.3 \\
0.50 & 161.2 \\
0.75 & 240.9 \\
1.00 & 311.7 \\
1.25 & 346.3 \\
1.50 & 401.8 \\
1.75 & 452.2 \\
2.00 & 504.6 \\
2.25 & 547.1 \\
2.50 & 577.0 \\
2.75 & 607.7 \\
3.00 & 621.5 \\
3.25 & 589.2 \\
3.50 & 491.7 \\
3.75 & 447.9 \\
4.00 & 434.9 \\
4.25 & 396.6 \\
4.50 & 370.7 \\
4.75 & 360.1 \\
5.00 & 344.0 \\
5.25 & 220.0
\end{tabular}

$12 / 22 / 99$

$4 / 20 / 99$

621.5

3.0
Client Sample No. P333F00

IT Lab Specimen No. ETDC-8025

Specimen Height, in.

3.9970

Specimen Diameter, in.

2.0020

Specimen Weight, $g$.

384.87

Moisture Content, \%

31.0

Wet Unit Weight, pcf.

116.6

Dry Unit Weight, pcf.

89.0

Rate of Strain, in./min.

0.0400

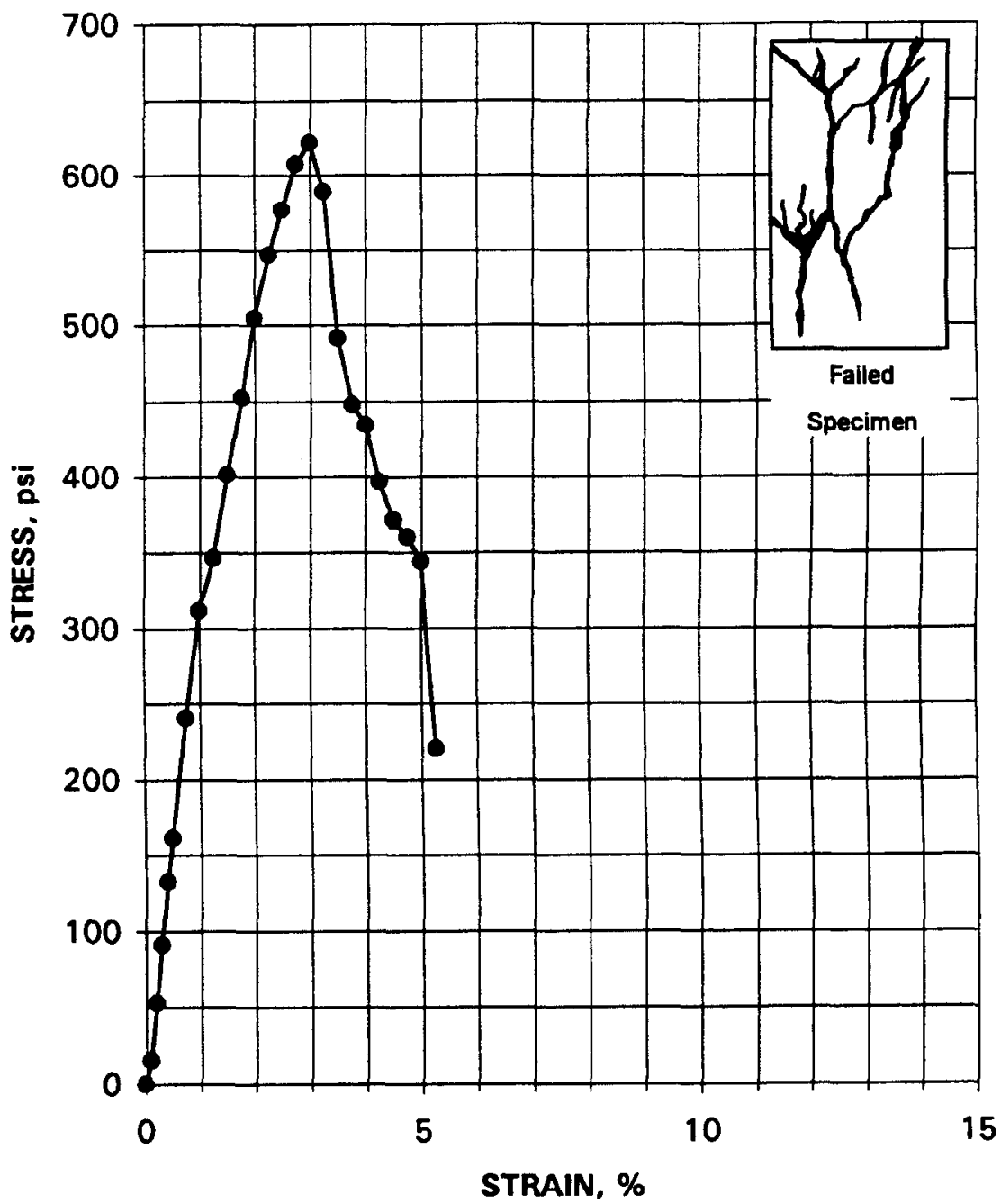




\section{UNCONFINED COMPRESSIVE STRENGTH - ASTM D 2166 28-DAY CURE, 90-DAY SATURATION}

Project Name SAIC/SAVANNAH RIVER

Project No. $\quad 777304.00010500$

Specimen Mold Date

Specimen Test Date

STRESS AT FAILURE, psi

STRAIN AT FAILURE, \%

\begin{tabular}{ccc}
$\begin{array}{c}\text { AXIAL } \\
\text { STRAIN, \% }\end{array}$ & $\begin{array}{c}\text { DEVIATOR } \\
\text { STRESS, psi }\end{array}$ \\
\cline { 1 - 1 } 0.00 & 0.0 \\
0.10 & 7.6 \\
0.20 & 23.1 \\
0.30 & & 46.4 \\
0.40 & 71.6 \\
0.50 & 101.1 \\
0.75 & 196.6 \\
1.00 & 298.3 \\
1.25 & 392.5 \\
1.50 & 485.4 \\
1.74 & 574.9 \\
1.99 & 630.5 \\
2.24 & 677.5 \\
2.49 & 706.9 \\
2.52 & 707.1 \\
2.74 & 119.4
\end{tabular}

Client Sample No. P343F00

IT Lab Specimen No. ETDC-8026

Specimen Height, in.

4.0115

Specimen Diameter, in.

2.0058

Specimen Weight, $g$.

379.20

Moisture Content, \%

33.4

Wet Unit Weight, pcf.

114.0

Dry Unit Weight, pcf.

85.4

Rate of Strain, in./min.

0.0400

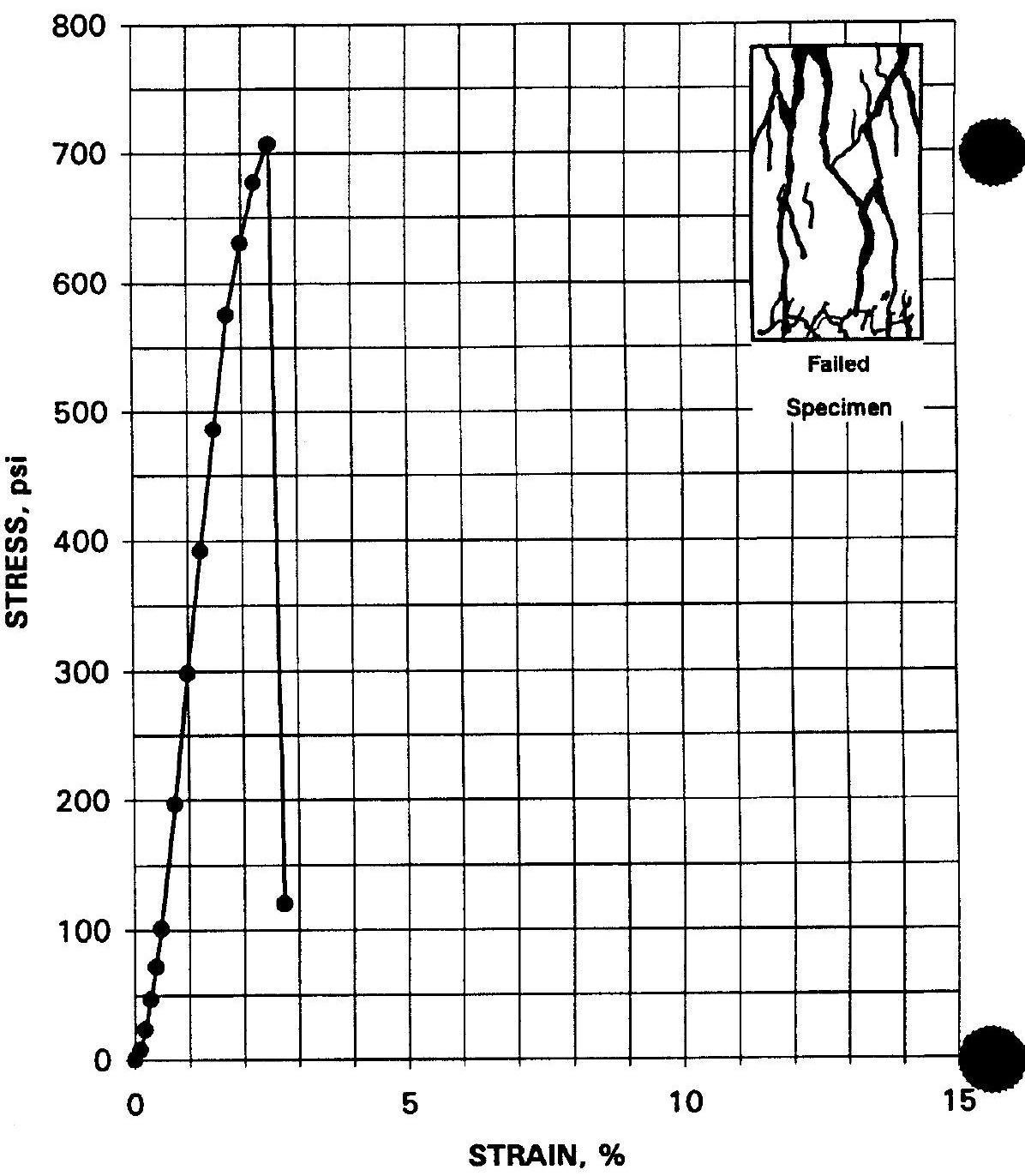




\section{UNCONFINED COMPRESSIVE STRENGTH - ASTM D 2166 28-DAY CURE, 90-DAY SATURATION}

Project Name SAIC/SAVANNAH RIVER

Project No. $\quad 777304.00010500$

Specimen Mold Date

Specimen Test Date

STRESS AT FAILURE, psi

STRAIN AT FAILURE, $\%$

\begin{tabular}{ccc}
$\begin{array}{cc}\text { AXIAL } \\
\text { STRAIN, \% }\end{array}$ & $\begin{array}{c}\text { DEVIATOR } \\
\text { STRESS, psi }\end{array}$ \\
\cline { 1 - 3 } 0.00 & 0.0 \\
0.10 & & 4.8 \\
0.20 & 10.5 \\
0.30 & & 22.8 \\
0.40 & 39.9 \\
0.50 & 51.9 \\
0.76 & 89.0 \\
1.01 & 157.8 \\
1.26 & 218.0 \\
1.51 & 267.2 \\
1.76 & 306.6 \\
2.01 & 314.2 \\
2.27 & 57.2 \\
2.52 & 34.7
\end{tabular}

$12 / 22 / 99$

$4 / 20 / 99$

314.2

2.0
Client Sample No.

P413F00

IT Lab Specimen No. ETDC-8027

Specimen Height, in.

3.9708

Specimen Diameter, in.

2.0007

Specimen Weight, $g$.

377.96

Moisture Content, \%

35.0

Wet Unit Weight, pct.

115.4

Dry Unit Weight, pcf.

85.5

Rate of Strain, in./min.

0.0400

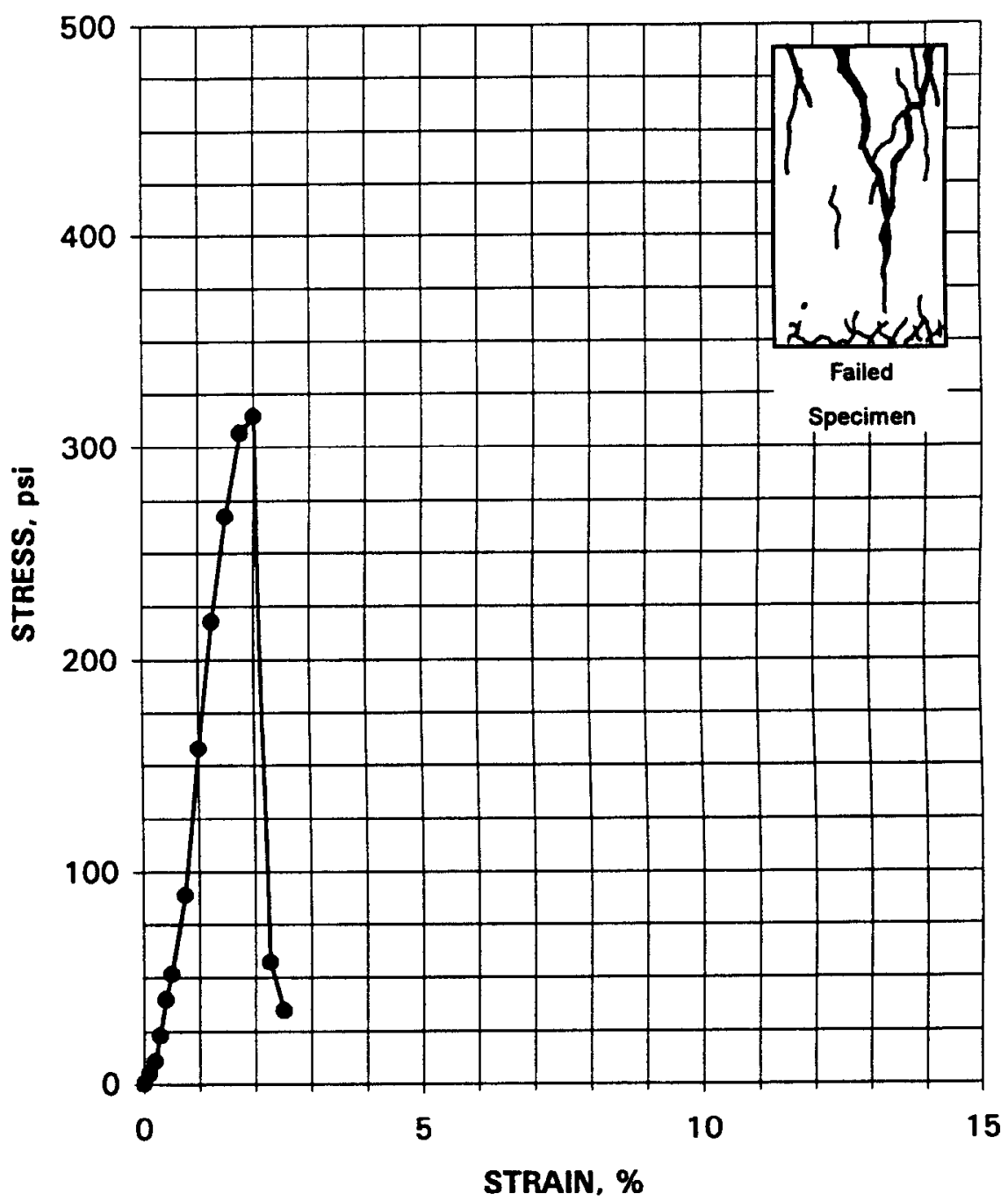




\section{UNCONFINED COMPRESSIVE STRENGTH - ASTM D 2166 28-DAY CURE, 90-DAY SATURATION}

Project Name SAIC/SAVANNAH RIVER

Project No. 777304.00010500

Specimen Mold Date

Specimen Test Date

STRESS AT FAILURE, psi STRAIN AT FAILURE, \%

\begin{tabular}{ccc}
$\begin{array}{c}\text { AXIAL } \\
\text { STRAIN, \% }\end{array}$ & & $\begin{array}{c}\text { DEVIATOR } \\
\text { STRESS, psi }\end{array}$ \\
\cline { 1 - 1 } \cline { 3 - 3 } 0.00 & & 0.0 \\
0.10 & & 2.8 \\
0.20 & & 16.1 \\
0.30 & & 49.7 \\
0.40 & & 108.7 \\
0.50 & & 150.0 \\
0.75 & & 185.9 \\
1.00 & & 198.6 \\
1.25 & & 246.7 \\
1.50 & & 297.0 \\
1.75 & 312.4 \\
2.00 & 313.4 \\
2.13 & 315.8 \\
2.25 & 243.3 \\
2.50 & 192.5
\end{tabular}

$4 / 20 / 99$
Client Sample No. P423F00

IT Lab Specimen No. ETDC-8028

Specimen Height, in.

Specimen Diameter, in.

3.9963

Specimen Weight, $g$.

2.0092

385.26

Moisture Content, \%

32.2

Wet Unit Weight, pcf.

115.9

Dry Unit Weight, pct.

87.6

Rate of Strain, in./min.

0.0400

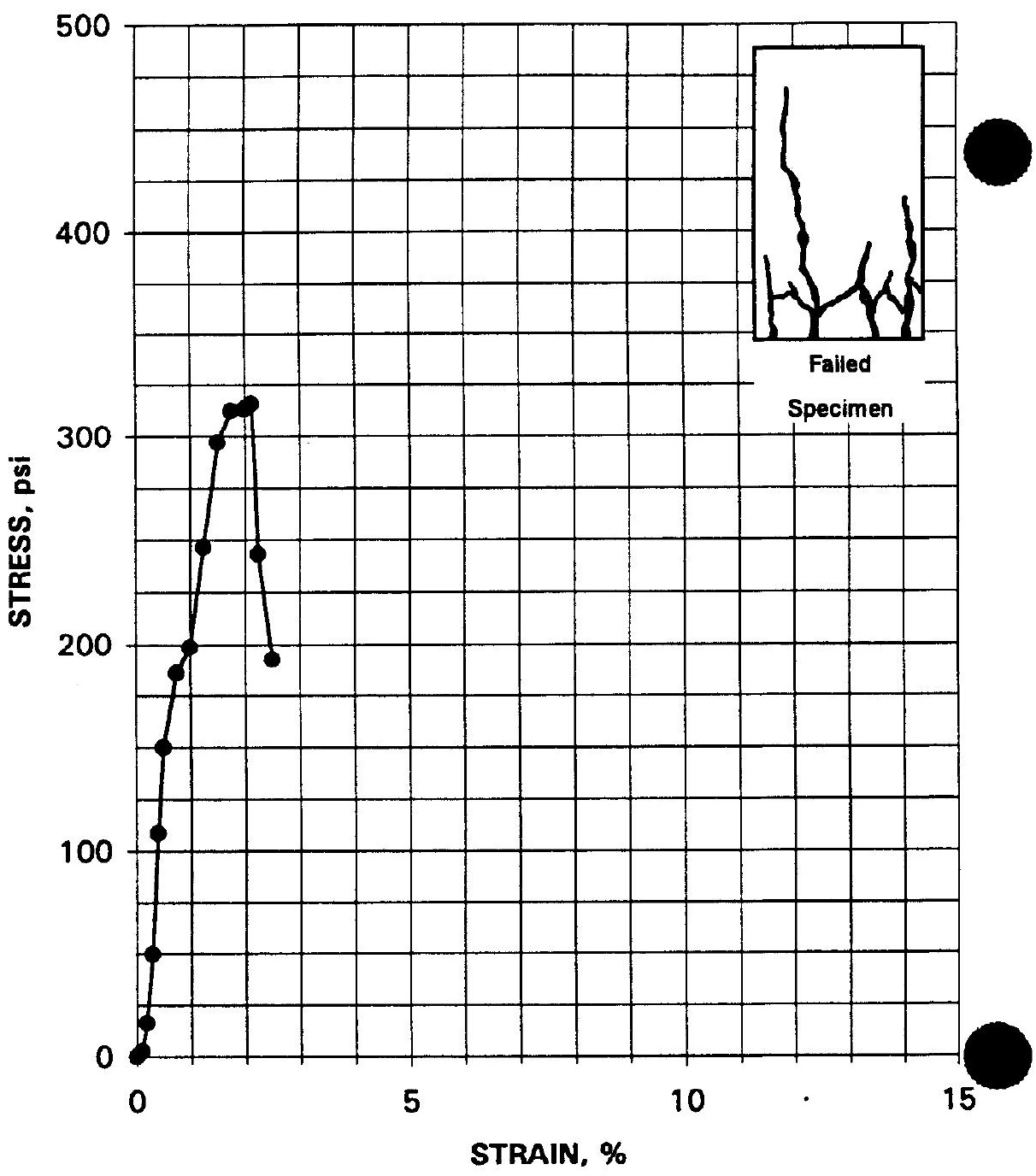




\section{UNCONFINED COMPRESSIVE STRENGTH - ASTM D 2166 28-DAY CURE, 90-DAY SATURATION}

Project Name

Project No.

SAIC/SAVANNAH RIVER

777304.00010500

Specimen Mold Date

Specimen Test Date

STRESS AT FAILURE, psi

STRAIN AT FAILURE, \%

\begin{tabular}{ccc}
$\begin{array}{c}\text { AXIAL } \\
\text { STRAIN, \% }\end{array}$ & $\begin{array}{c}\text { DEVIATOR } \\
\text { STRESS, psi }\end{array}$ \\
\cline { 1 - 1 } 0.00 & 0.0 \\
0.10 & & 5.1 \\
0.20 & 18.4 \\
0.30 & 39.6 \\
0.40 & 76.4 \\
0.50 & 119.3 \\
0.75 & 220.1 \\
1.00 & 312.1 \\
1.13 & 343.8 \\
1.25 & 35.5 \\
1.50 & 25.4
\end{tabular}

$12 / 22 / 99$

343.8

1.1
Client Sample No. P433FOO

IT Lab Specimen No. ETDC-8029

Specimen Height, in.

3.9997

Specimen Diameter, in.

2.0007

Specimen Weight, $g$.

368.01

Moisture Content, \%

38.8

Wet Unit Weight, pcf.

Dry Unit Weight, pcf.

111.5

80.4

Rate of Strain, in./min.

0.0400

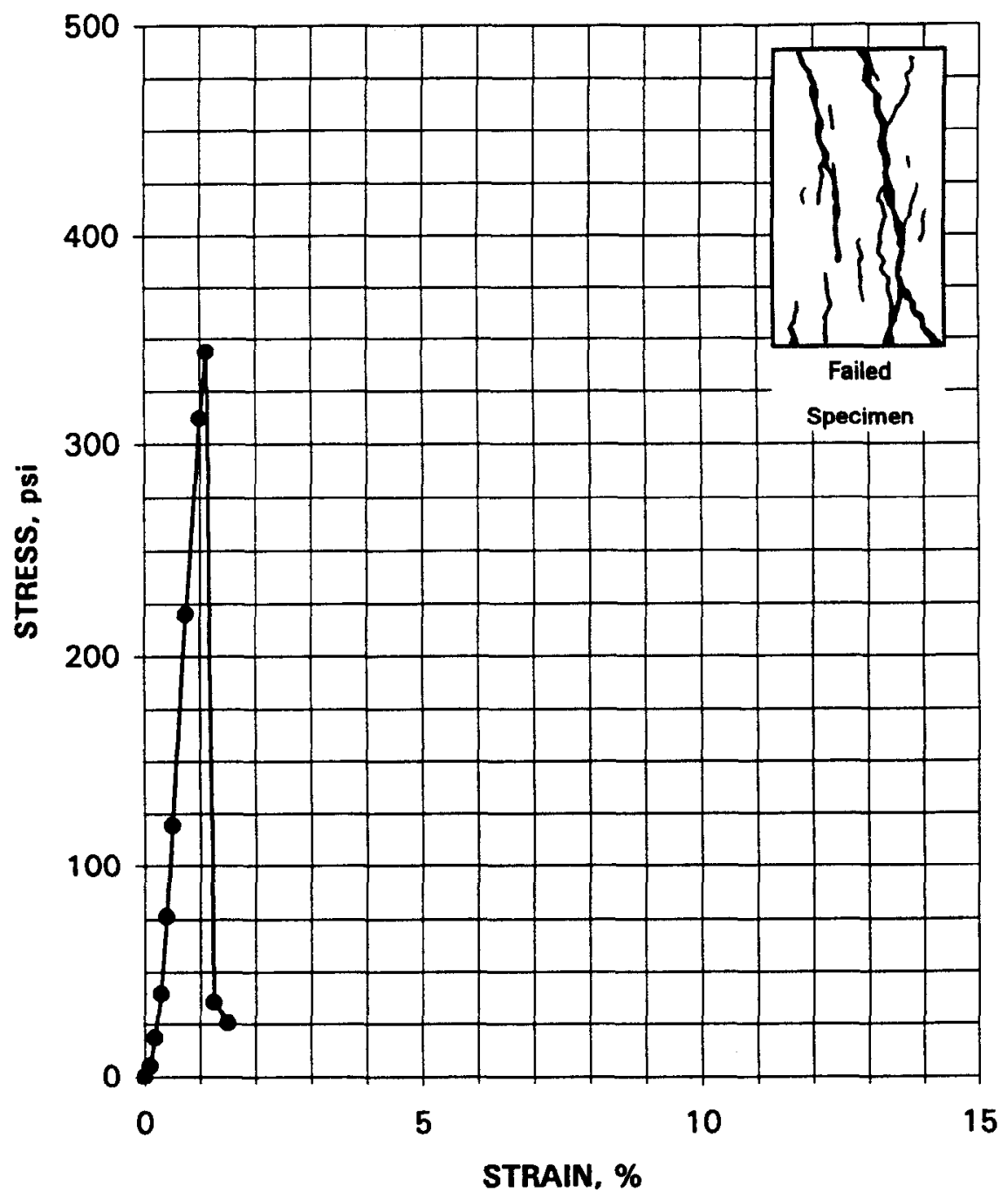




\section{UNCONFINED COMPRESSIVE STRENGTH - ASTM D 2166 28-DAY CURE, 90-DAY SATURATION}

Project Name SAIC/SAVANNAH RIVER

Project No. $\quad 777304.00010500$

Specimen Mold Date

Specimen Test Date

STRESS AT FAILURE, psi

STRAIN AT FAILURE, \%

AXIAL DEVIATOR

\begin{tabular}{ccc} 
STRAIN, \% & STRESS, psi \\
\cline { 1 - 3 } \cline { 3 - 3 } 0.00 & 0.0 \\
0.10 & & 3.1 \\
0.20 & & 7.2 \\
0.30 & & 17.0 \\
0.40 & & 27.0 \\
0.50 & & 33.6 \\
0.75 & & 46.6 \\
0.99 & & 79.3 \\
1.24 & & 140.7 \\
1.49 & & 223.5 \\
1.74 & & 298.8 \\
1.99 & 363.6 \\
2.24 & 428.6 \\
2.36 & 453.0 \\
2.48 & 407.5 \\
2.73 & 313.3 \\
2.98 & 123.8
\end{tabular}

Client Sample No. P443F00

IT Lab Specimen No. ETDC-8030

Specimen Height, in.

4.0257

Specimen Diameter, in.

2.0100

Specimen Weight, $g$.

Moisture Content, \%

371.64

36.9

Wet Unit Weight, pcf.

Dry Unit Weight, pcf.

110.9

Rate of Strain, in./min.

81.0

0.0400

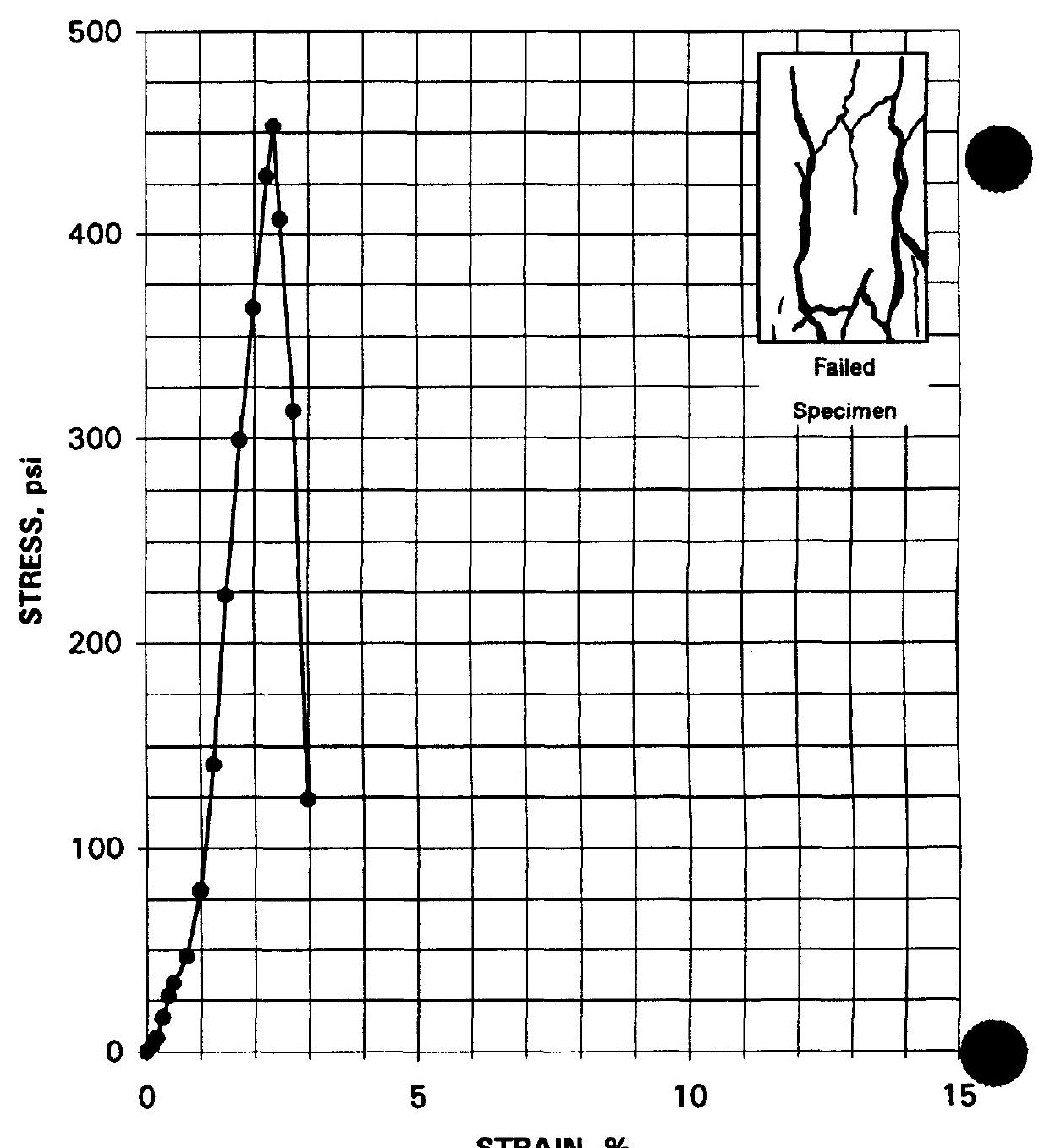

STRAIN, \% 


\section{UNCONFINED COMPRESSIVE STRENGTH - ASTM D 2166 28-DAY CURE, 90-DAY SATURATION}

\begin{tabular}{lc}
$\begin{array}{l}\text { Project Name } \\
\text { Sroject No. }\end{array}$ & 777304.00010 \\
Specimen Mold Date \\
Specimen Test Date \\
STRESS AT FAILURE, psi \\
STRAIN AT FAILURE, \% \\
\multicolumn{3}{|}{} \\
AXIAL & DEVIATOR \\
STRAIN, $\%$ & STRESS, psi \\
\hline 0.00 & 0.0 \\
0.10 & 6.0 \\
0.20 & 18.3 \\
0.30 & 29.0 \\
0.40 & 42.6 \\
0.50 & 60.5 \\
0.76 & 112.5 \\
1.01 & 184.6 \\
1.26 & 252.6 \\
1.51 & 334.6 \\
1.77 & 410.6 \\
2.02 & 488.7 \\
2.27 & 532.9 \\
2.52 & 432.8 \\
2.78 & 128.4
\end{tabular}

Client Sample No. P513F00

IT Lab Specimen No. ETDC-8031

Specimen Height, in.

Specimen Diameter, in.

Specimen Weight, $g$.

381.43

Moisture Content, \%

Wet Unit Weight, pcf.

116.1

Dry Unit Weight, pcf.

Rate of Strain, in./min.

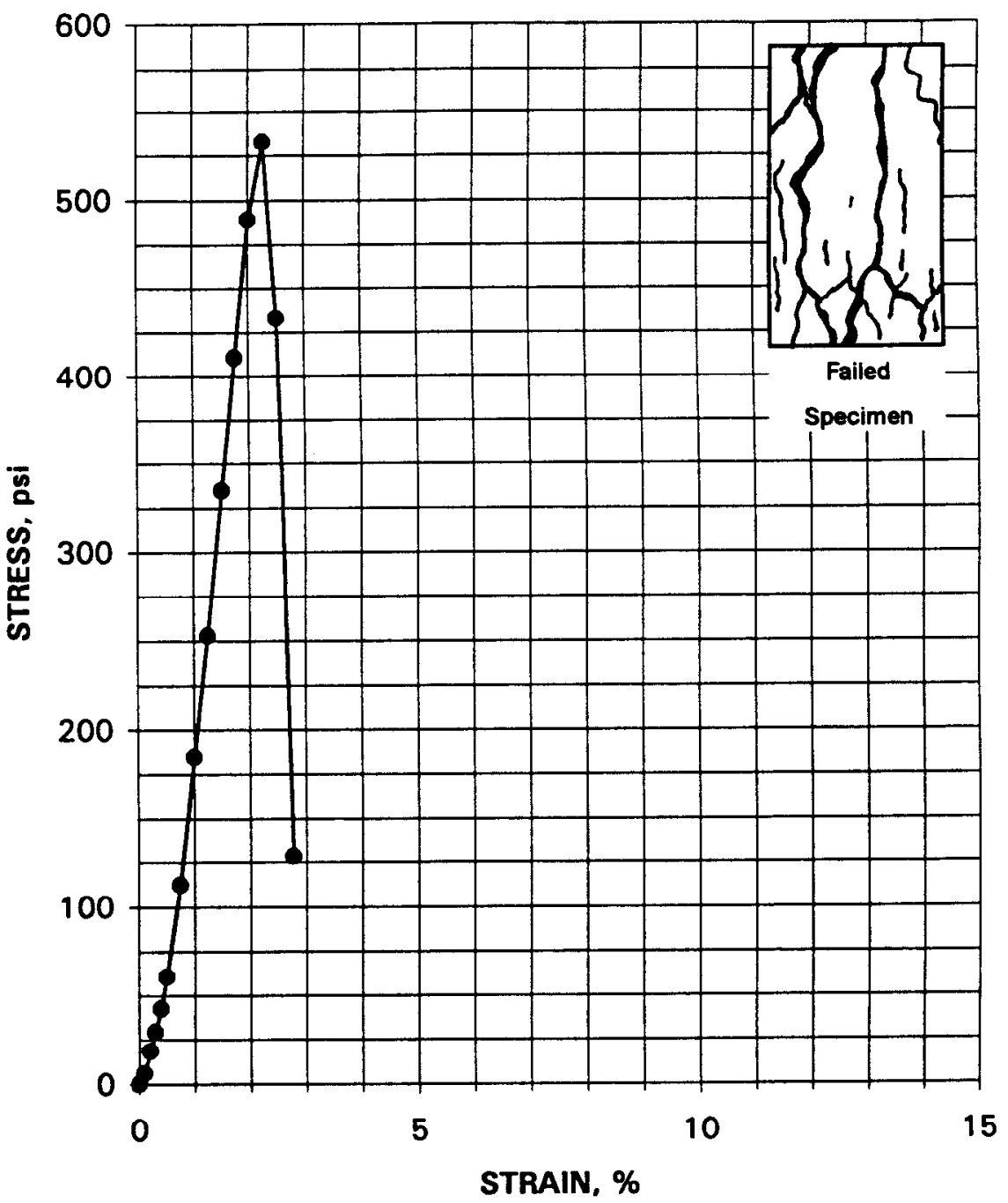




\section{UNCONFINED COMPRESSIVE STRENGTH - ASTM D 2166 28-DAY CURE, 90-DAY SATURATION}

Project Name SAIC/SAVANNAH RIVER

Project No. $\quad 777304.00010500$

Specimen Mold Date

Specimen Test Date

STRESS AT FAILURE, psi

STRAIN AT FAILURE, \%

AXIAL DEVIATOR

$\frac{\text { STRAIN, \% }}{0.00} \frac{\text { STRESS, psi }}{0.0}$

$0.10 \quad 9.2$

$0.20 \quad 22.1$

0.30

49.2

0.40

91.1

0.50

138.2

0.75

1.01

1.26

1.51

1.76

2.01

2.26

2.51

2.76

3.02

3.27

3.52

3.77

4.02

4.27

4.52

4.77

5.03

5.28

5.53

5.78
267.2

399.6

527.0

615.4

660.1

658.1

685.2

684.7

502.6

503.5

504.0

504.8

506.0

513.4

516.0

512.6

507.3

497.3

481.9

464.7

431.6
$12 / 22 / 99$

$4 / 20 / 99$

685.2

2.3
Client Sample No. P523F00

IT Lab Specimen No. ETDC-8032

Specimen Height, in.

3.9793

Specimen Diameter, in.

2.0062

Specimen Weight, $g$.

393.78

Moisture Content, \%

28.5

Wet Unit Weight, pcf.

119.3

Dry Unit Weight, pcf.

92.8

Rate of Strain, in./min.

0.0400

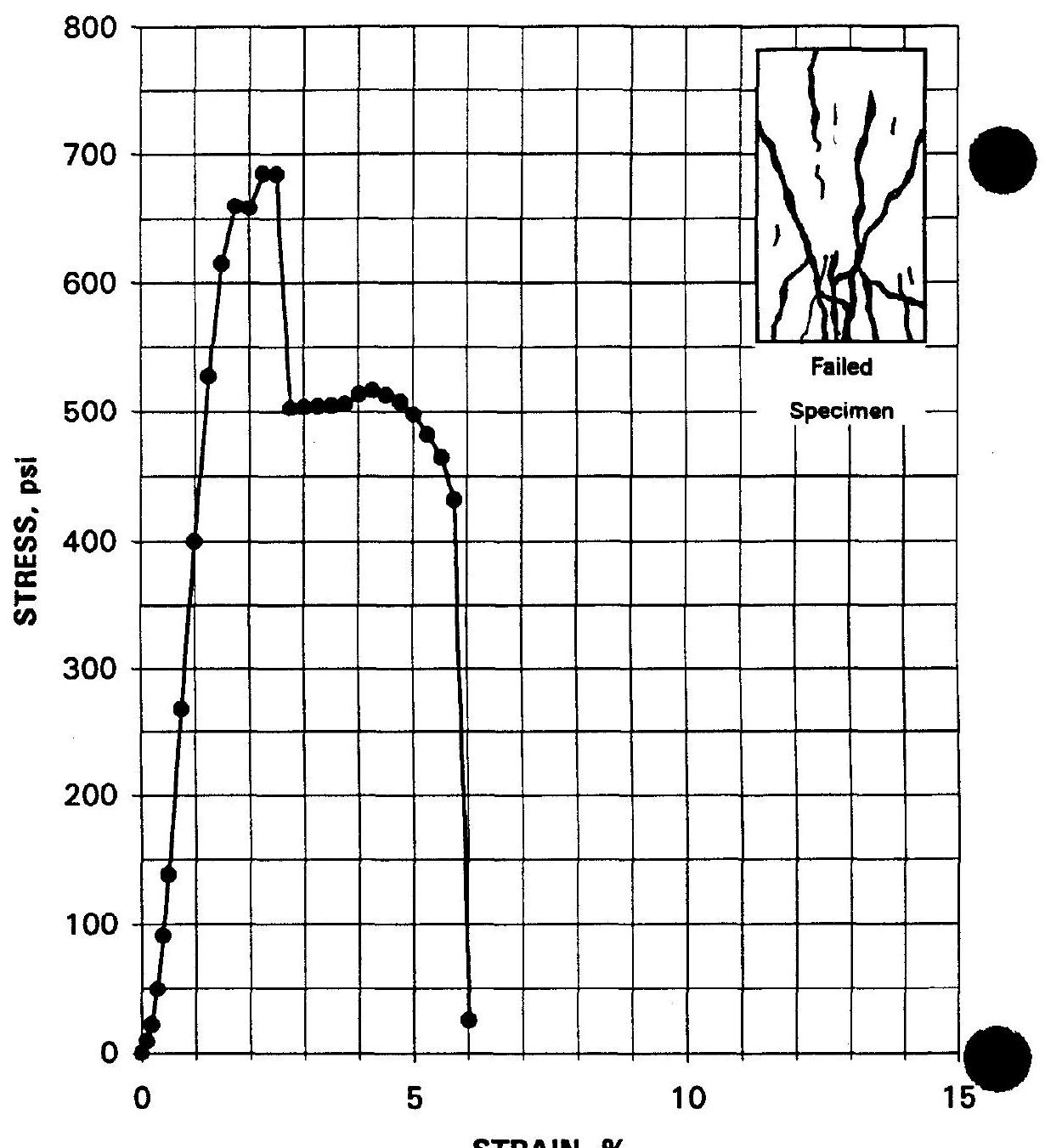

STRAIN, \% 


\section{UNCONFINED COMPRESSIVE STRENGTH - ASTM D 2166 28-DAY CURE, 90-DAY SATURATION}

Project Name SAIC/SAVANNAH RIVER

Project No. 777304.00010500

Specimen Mold Date

Specimen Test Date

STRESS AT FAILURE, psi

STRAIN AT FAILURE, \%

\begin{tabular}{cc}
$\begin{array}{c}\text { AXIAL } \\
\text { STRAIN, \% }\end{array}$ & $\begin{array}{c}\text { DEVIATOR } \\
\text { STRESS, psi }\end{array}$ \\
\cline { 2 - 3 } 0.00 & 0.0 \\
0.10 & 7.6 \\
0.20 & 16.2 \\
0.30 & 24.7 \\
0.40 & 35.1 \\
0.50 & 47.1 \\
0.75 & 91.4 \\
1.01 & 127.3 \\
1.26 & 173.4 \\
1.51 & 227.0 \\
1.76 & 265.4 \\
2.01 & 302.7 \\
2.26 & 339.4 \\
2.51 & 353.4 \\
2.76 & 342.6 \\
3.02 & 348.5 \\
3.27 & 353.5 \\
3.52 & 359.3 \\
3.64 & 359.7 \\
3.77 & 351.6 \\
4.02 & 338.5 \\
4.27 & 335.8 \\
4.52 & 333.1 \\
4.78 & 331.0 \\
5.03 & 331.4 \\
5.28 & 331.1 \\
5.53 & 325.4 \\
& \\
\hline
\end{tabular}

$12 / 22 / 99$

$4 / 20 / 99$

359.7

3.6
Client Sample No. P533F00

IT Lab Specimen No. ETDC-8033

Specimen Height, in.

Specimen Diameter, in.

3.9788

Specimen Weight, $\boldsymbol{o}$.

2.0027

371.50

Moisture Content, \%

36.0

Wet Unit Weight, pcf.

112.9

Dry Unit Weight, pcf.

83.1

Rate of Strain, in./min.

0.0400

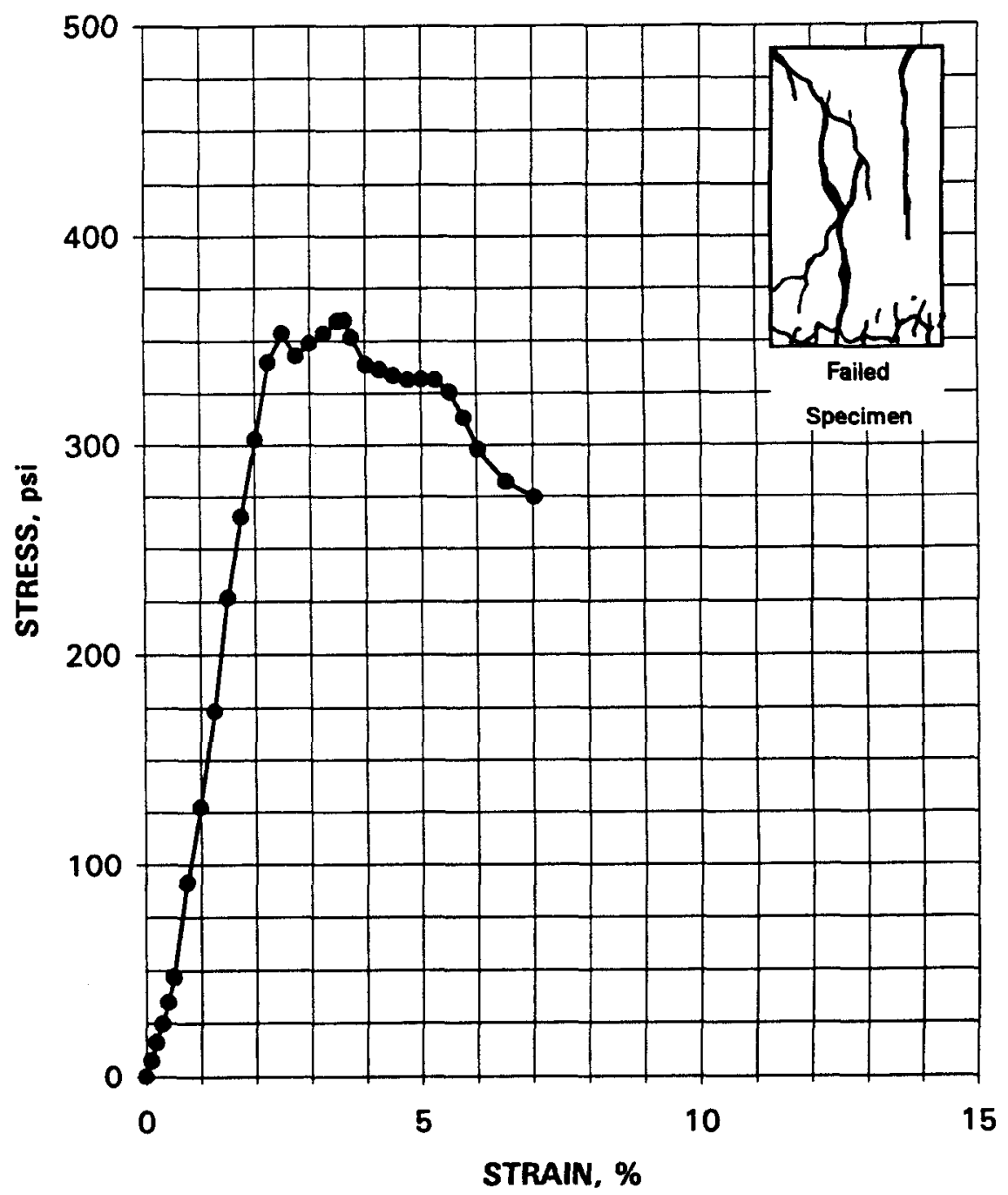




\section{UNCONFINED COMPRESSIVE STRENGTH - ASTM D 2166 28-DAY CURE, 90-DAY SATURATION}

Project Name SAIC/SAVANNAH RIVER

Project No. $\quad 777304.00010500$

Specimen Mold Date

Specimen Test Date

STRESS AT FAILURE, psi

STRAIN AT FAILURE, \%

\begin{tabular}{|c|c|}
\hline $\begin{array}{c}\text { AXIAL } \\
\text { STRAIN, \% }\end{array}$ & $\begin{array}{l}\text { DEVIATOR } \\
\text { STRESS, psi }\end{array}$ \\
\hline 0.00 & 0.0 \\
\hline 0.10 & 8.9 \\
\hline 0.20 & 21.5 \\
\hline 0.30 & 45.3 \\
\hline 0.40 & 81.0 \\
\hline 0.50 & 119.1 \\
\hline 0.75 & 214.9 \\
\hline 1.00 & 309.6 \\
\hline 1.25 & 391.0 \\
\hline 1.50 & 472.3 \\
\hline 1.75 & 566.9 \\
\hline 2.00 & 647.3 \\
\hline 2.25 & 687.8 \\
\hline 2.49 & 687.3 \\
\hline 2.74 & 250.7 \\
\hline 2.99 & 217.5 \\
\hline
\end{tabular}

$12 / 22 / 99$

$4 / 20 / 99$

687.8

2.2
Client Sample No. P543FOO

IT Lab Specimen No. ETDC-8034

Specimen Height, in.

4.0087

Specimen Diameter, in.

2.0025

Specimen Weight, $g$.

Moisture Content, \%

378.66

33.5

Wet Unit Weight, pcf.

Dry Unit Weight, pcf.

114.3

85.6

Rate of Strain, in./min.

0.0400

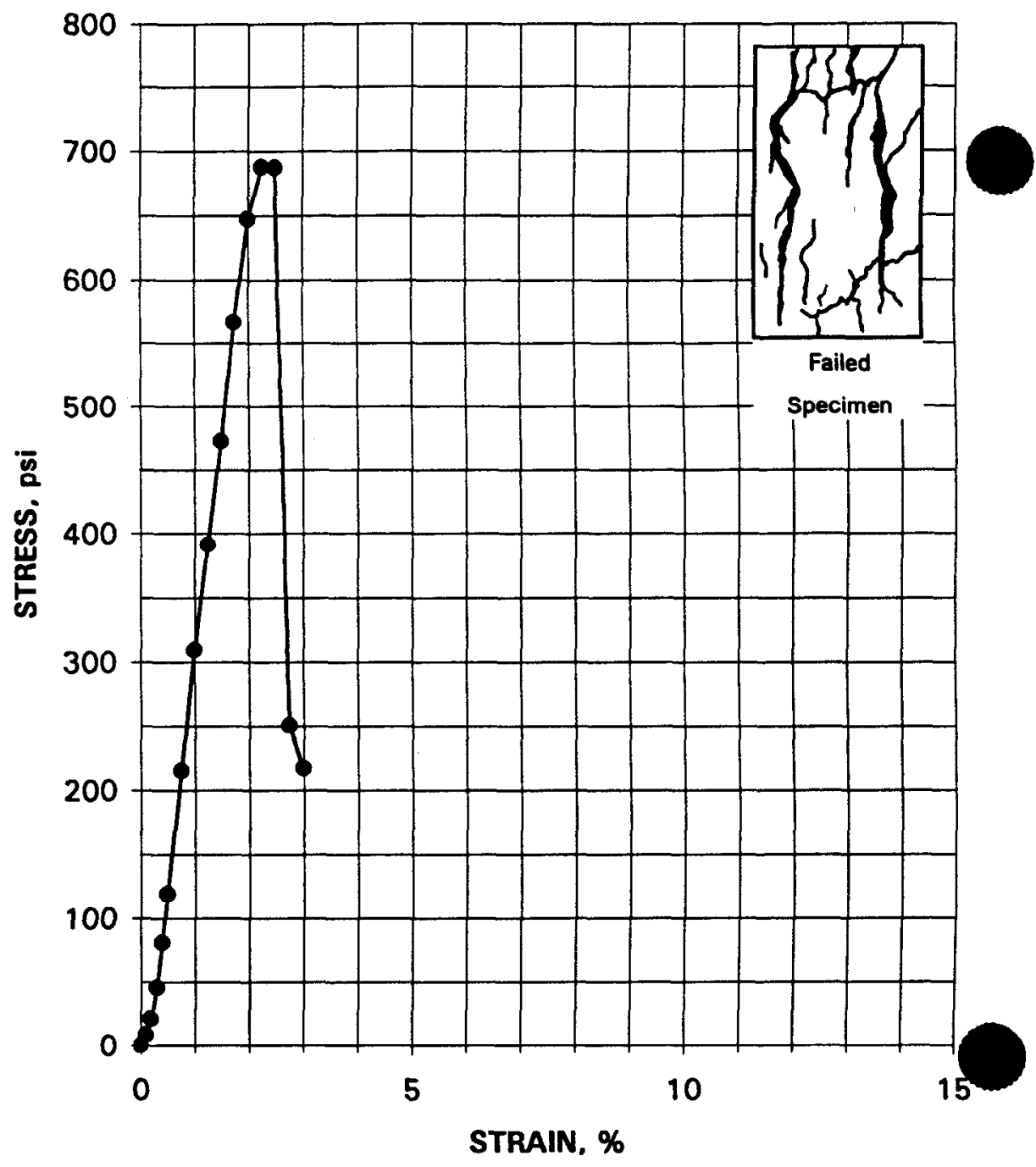




\section{UNCONFINED COMPRESSIVE STRENGTH - ASTM D 2166 28-DAY CURE, 90-DAY SATURATION}

Project Name SAIC/SAVANNAH RIVER

Project No. $\quad 777304.00010500$

\section{Specimen Mold Date}

Specimen Test Date

STRESS AT FAILURE, psi

STRAIN AT FAILURE, \%

AXIAL DEVIATOR

STRAIN, \% STRESS, pSi

$0.00-0.0$

0.10

10.5

0.20

0.30

0.40

0.50

0.75

1.00

1.25

1.51

1.76

2.01

2.26

2.51

2.76

2.88

3.01

3.26

3.51

3.76

4.01

4.26

4.52

4.77

5.02

5.27
$12 / 22 / 99$

$4 / 20 / 99$

392.6

2.9
Client Sample No.

P613F00

IT Lab Specimen No. ETDC-8035

Specimen Height, in.

3.9862

Specimen Diameter, in.

2.0008

Specimen Weight, $\mathbf{g}$.

388.93

Moisture Content, \%

Wet Unit Weight, pcf.

29.8

118.2

91.1

Rate of Strain, in./min.

0.0400

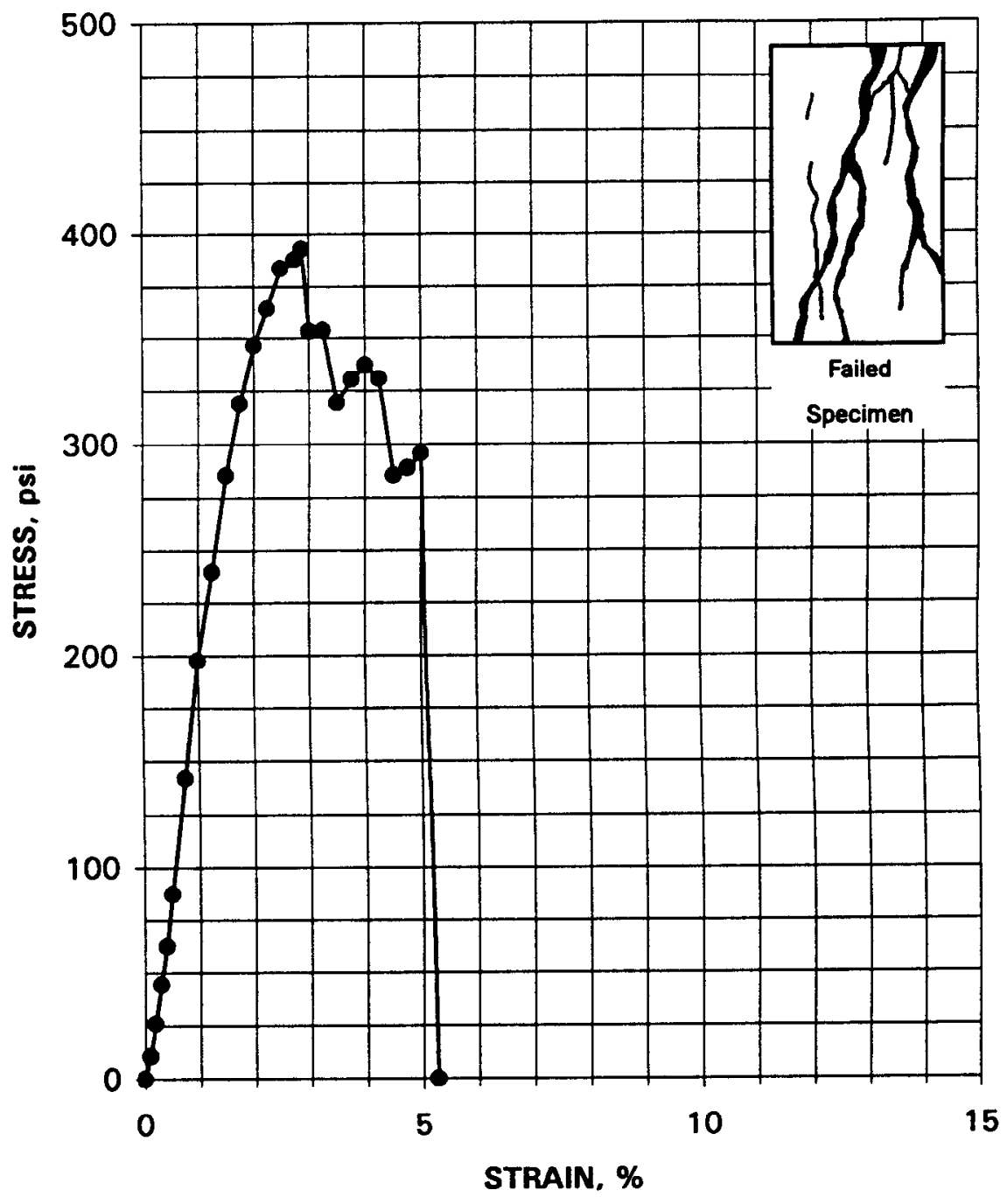




\section{UNCONFINED COMPRESSIVE STRENGTH - ASTM D 2166 28-DAY CURE, 90-DAY SATURATION}

$\begin{array}{ll}\text { Project Name } & \text { SAIC/SAVANNAH RIVER } \\ \text { Project No. } & 777304.00010500\end{array}$

Specimen Mold Date

Specimen Test Date

STRESS AT FAILURE, psi

STRAIN AT FAILURE, \%

\begin{tabular}{ccc}
$\begin{array}{c}\text { AXIAL } \\
\text { STRAIN, \% }\end{array}$ & $\begin{array}{c}\text { DEVIATOR } \\
\text { STRESS, psi }\end{array}$ \\
\cline { 1 - 3 } 0.00 & 0.0 \\
0.10 & 2.5 \\
0.20 & 6.6 \\
0.30 & 12.0 \\
0.40 & & 21.7 \\
0.50 & & 28.9 \\
0.75 & & 57.7 \\
1.00 & 131.7 \\
1.25 & 232.7 \\
1.50 & 315.3 \\
1.75 & 397.0 \\
2.00 & 454.2 \\
2.25 & 518.9 \\
2.50 & 573.6 \\
2.74 & 620.7 \\
2.99 & 649.4 \\
3.24 & 656.6 \\
3.49 & 658.9 \\
3.74 & 663.6 \\
3.87 & 663.6 \\
3.99 & 653.7 \\
4.24 & 428.1 \\
4.49 & 412.2 \\
4.74 & 361.1 \\
4.99 & 237.1
\end{tabular}

$12 / 22 / 99$

$4 / 20 / 99$

663.6

3.9
Client Sample No. P623FOO

IT Lab Specimen No. ETDC-8036

Specimen Height, in.

4.0077

Specimen Diameter, in.

2.0075

Specimen Weight, $g$.

Moisture Content, \%

395.09

27.9

Wet Unit Weight, pcf.

118.7

Dry Unit Weight, pcf.

92.8

Rate of Strain, in./min.

0.0400

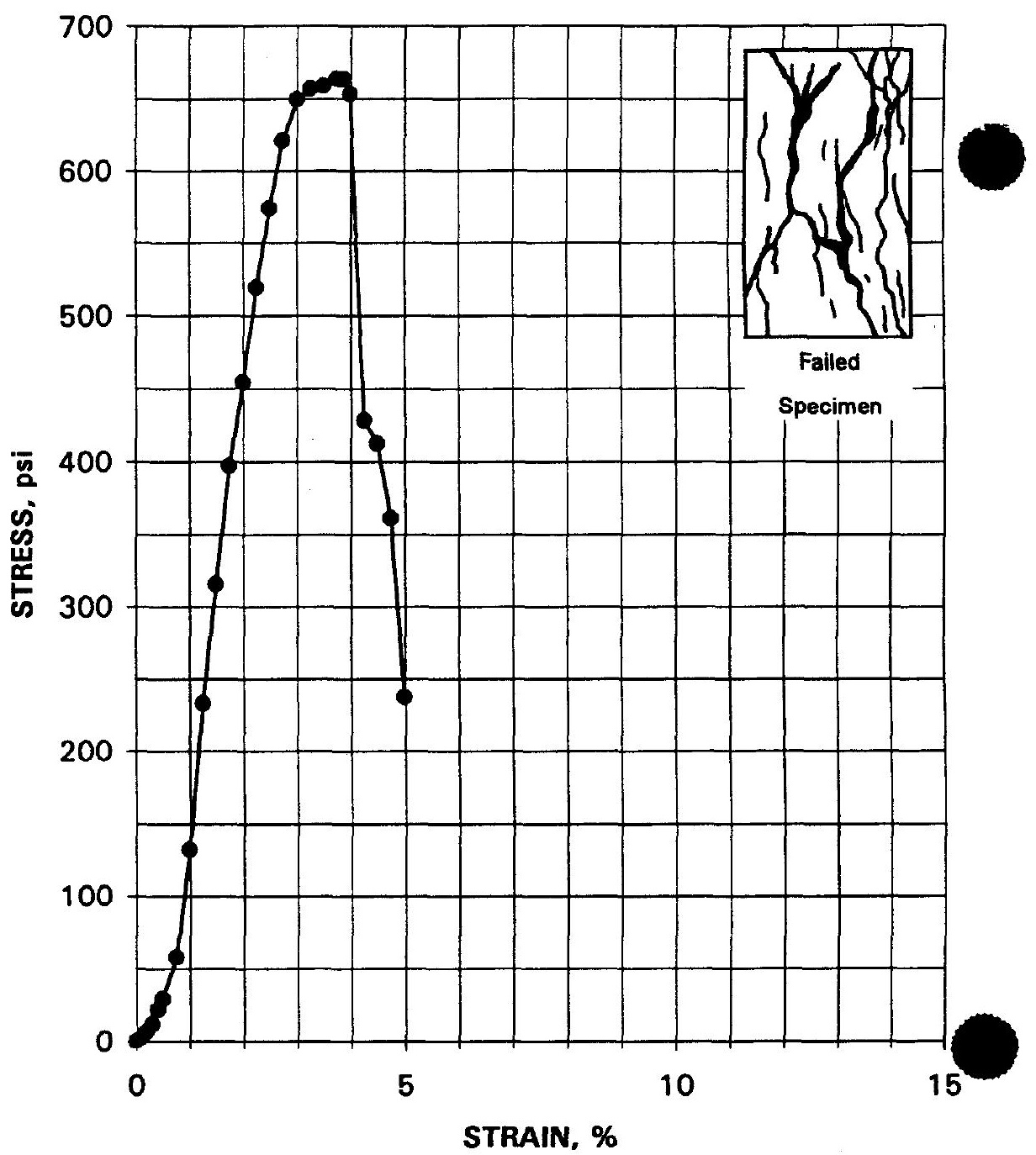




\section{UNCONFINED COMPRESSIVE STRENGTH - ASTM D 2166 28-DAY CURE, 90-DAY SATURATION}

Project Name SAIC/SAVANNAH RIVER

Project No. 777304.00010500

Specimen Mold Date

Specimen Test Date

STRESS AT FAILURE, psi

STRAIN AT FAILURE, \%

\begin{tabular}{ccc}
$\begin{array}{c}\text { AXIAL } \\
\text { STRAIN, \% }\end{array}$ & $\begin{array}{c}\text { DEVIATOR } \\
\text { STRESS, psi }\end{array}$ \\
\cline { 2 - 3 } 0.00 & 0.0 \\
0.10 & & 8.8 \\
0.20 & 16.4 \\
0.30 & & 43.5 \\
0.40 & & 80.9 \\
0.50 & & 125.7 \\
0.75 & & 216.0 \\
1.00 & & 294.3 \\
1.25 & 379.4 \\
1.50 & 473.0 \\
1.76 & 546.6 \\
2.01 & 574.6 \\
2.13 & 578.8 \\
2.26 & 531.5 \\
2.51 & 479.9 \\
2.76 & 459.6 \\
3.01 & 444.0 \\
3.26 & 413.8 \\
3.51 & 409.4 \\
3.76 & 403.5 \\
4.01 & 329.0
\end{tabular}

Client Sample No. P633F00

IT Lab Specimen No. ETDC-8037

Specimen Height, in.

3.9877

Specimen Diameter, in.

2.0075

Specimen Weight, $g$.

371.86

Moisture Content, \%

35.7

Wet Unit Weight, pcf.

112.3

Dry Unit Weight, pcf.

82.7

Rate of Strain, in./min.

0.0400

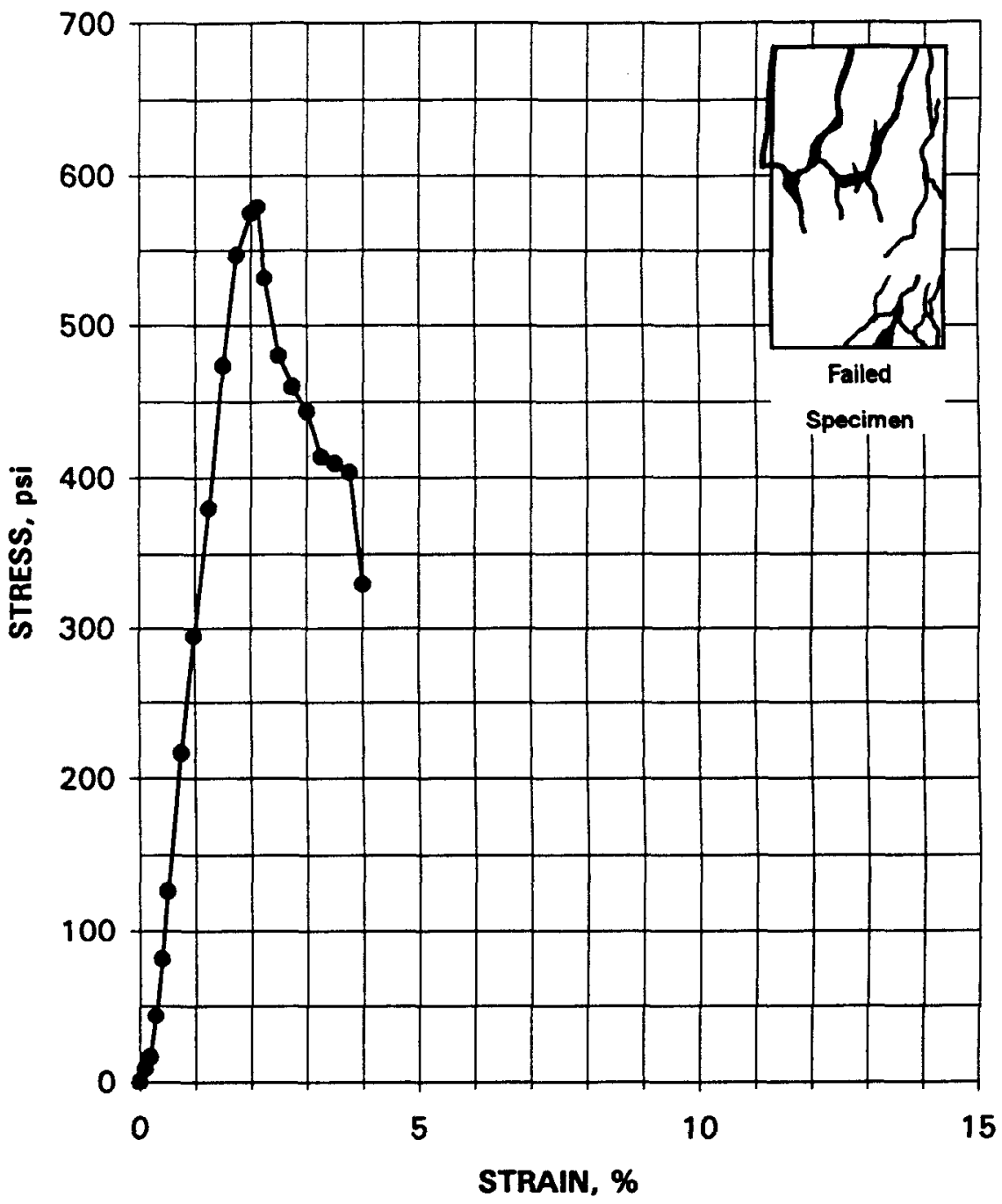




\section{UNCONFINED COMPRESSIVE STRENGTH - ASTM D 2166 28-DAY CURE, 90-DAY SATURATION}

Project Name

SAIC/SAVANNAH RIVER

Project No.

777304.00010500

Specimen Mold Date

Specimen Test Date

STRESS AT FAILURE, psi

STRAIN AT FAILURE, \%

\begin{tabular}{cc}
$\begin{array}{c}\text { AXIAL } \\
\text { STRAIN, \% }\end{array}$ & $\begin{array}{c}\text { DEVIATOR } \\
\text { STRESS, psi }\end{array}$ \\
\cline { 2 - 3 } 0.00 & 0.0 \\
0.10 & 6.0 \\
0.20 & 19.3 \\
0.30 & 37.5 \\
0.40 & 72.1 \\
0.50 & 115.5 \\
0.75 & 246.1 \\
1.00 & 375.7 \\
1.25 & 499.7 \\
1.50 & 608.4 \\
1.75 & 717.8 \\
2.00 & 769.3 \\
2.25 & 780.9 \\
2.50 & 681.8 \\
2.75 & 432.1 \\
3.00 & 259.8
\end{tabular}

Client Sample No. P634F00

IT Lab Specimen No. ETDC-8038

Specimen Height, in.

4.0042

Specimen Diameter, in.

2.0065

Specimen Weight, $g$.

378.35

Moisture Content, \%

33.1

Wet Unit Weight, pcf.

113.9

Dry Unit Weight, pcf.

85.5

Rate of Strain, in./min.

0.0400

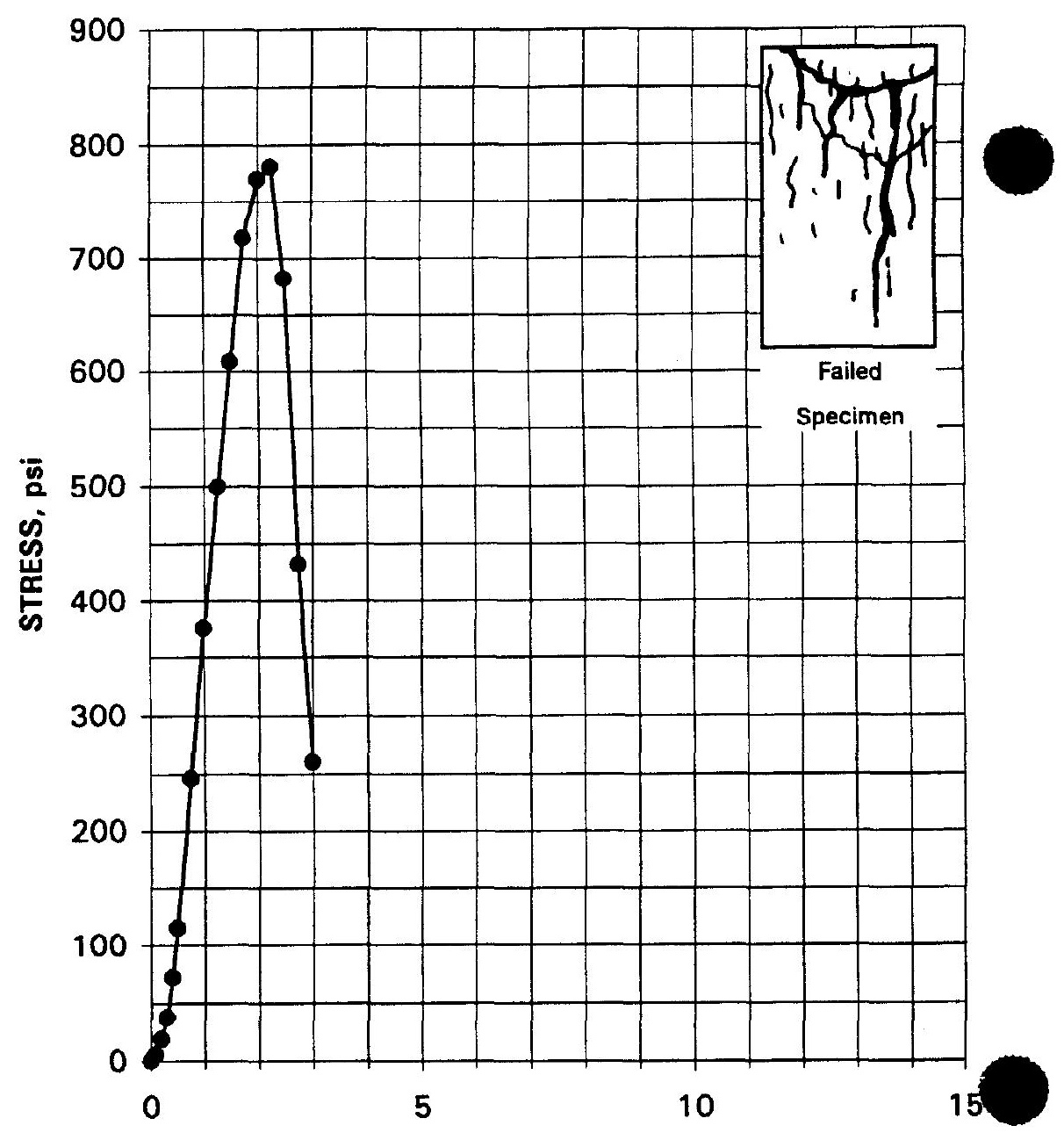

STRAIN, $\%$ 


\section{P Series}

28 Day Expansion / Shrinkage Results 


\section{One-Dimensional Expansion, Shrinkage, and Uplift Pressure of Soil-Lime Mixtures ASTM D 3877}

\section{8-Day Cure}

\begin{tabular}{ccccc}
$\begin{array}{c}\text { Geo Lab } \\
\text { Sample No. }\end{array}$ & $\begin{array}{c}\text { Knoxville Lab } \\
\text { Sample No. }\end{array}$ & Expansion, \% & Expansion Result & Shrinkage, $\%$ \\
\hline \hline ETDC-8071 & P312K00 & $-0.004 \%$ & No expansion & Insuff. Sample \\
ETDC-8072 & P322KO0 & $-0.308 \%$ & No expansion & $0.99 \%$ \\
ETDC-8073 & P332K00 & $-0.091 \%$ & No expansion & $2.72 \%$ \\
ETDC-8074 & P342KO0 & $-0.205 \%$ & No expansion & $0.55 \%$ \\
ETDC-8075 & P412K00 & $-0.212 \%$ & No expansion & $5.54 \%$ \\
ETDC-8076 & P422K00 & $-0.091 \%$ & No expansion & $0.53 \%$ \\
ETDC-8077 & P432K00 & $-0.192 \%$ & No expansion & $5.33 \%$ \\
ETDC-8078 & P442K00 & $-0.212 \%$ & No expansion & $1.14 \%$ \\
ETDC-8079 & P512K00 & $-0.175 \%$ & No expansion & $0.28 \%$ \\
ETDC-8080 & P522K00 & $-0.223 \%$ & No expansion & $1.38 \%$ \\
ETDC-8081 & P532K00 & $-0.025 \%$ & No expansion & $4.52 \%$ \\
ETDC-8082 & P542K00 & $-0.103 \%$ & No expansion & $0.81 \%$ \\
ETDC-8083 & P612K00 & $-0.089 \%$ & No expansion & $5.48 \%$ \\
ETDC-8084 & P622K00 & $-0.111 \%$ & No expansion & $0.73 \%$ \\
ETDC-8085 & P632K00 & $-0.081 \%$ & No expansion & $7.18 \%$ \\
ETDC-8086 & P642KO0 & $-0.040 \%$ & No expansion & $0.02 \%$ \\
& & & &
\end{tabular}




\section{P Series}

\section{Day Permeability Results}




\section{8-DAY HYDRAULIC CONDUCTIVITY / PERMEABILITY ASTM D 5084}

PROJECT NAME: SAIC / Savannah River PROJECT NO. 777304.00010500
CLIENT SAMPLE NO. P312G00

IT LAB SAMPLE NO. ETDC-8095

\begin{tabular}{lll|} 
& INITIAL & FINAL \\
\cline { 2 - 3 } & 5.09 & \\
Specimen diameter, $\mathrm{cm}$ & 10.07 & \\
Specimen length, cm & 378.31 & 386.16 \\
Wet weight of specimen, $\mathrm{g}$. & 20.3291 & \\
Specimen cross-sect. area, cm -2 & 29.9 & 32.6 \\
Water content, \% & 115.3 & \\
Wet unit weight, pcf & 88.8 & \\
Dry unit weight, pcf & 91.8 & \\
Estimated degree of saturation, \% & 9.65
\end{tabular}

Hydraulic gradient

27.9

Min. consolidation stress, psi

2.0

Max. consolidation stress, psi

8.0

Total backpressure, psi

Permeant Fluid

3.0

Sample molded

Deaired DI Water

Sample set up

$12 / 22 / 98$

Seven tests performed

\section{\begin{tabular}{|ll}
\hline Coefficient of Permeability, cm/s & 5.0E-09 \\
\hline
\end{tabular}}

\section{PERMEABILITY vS TIME}

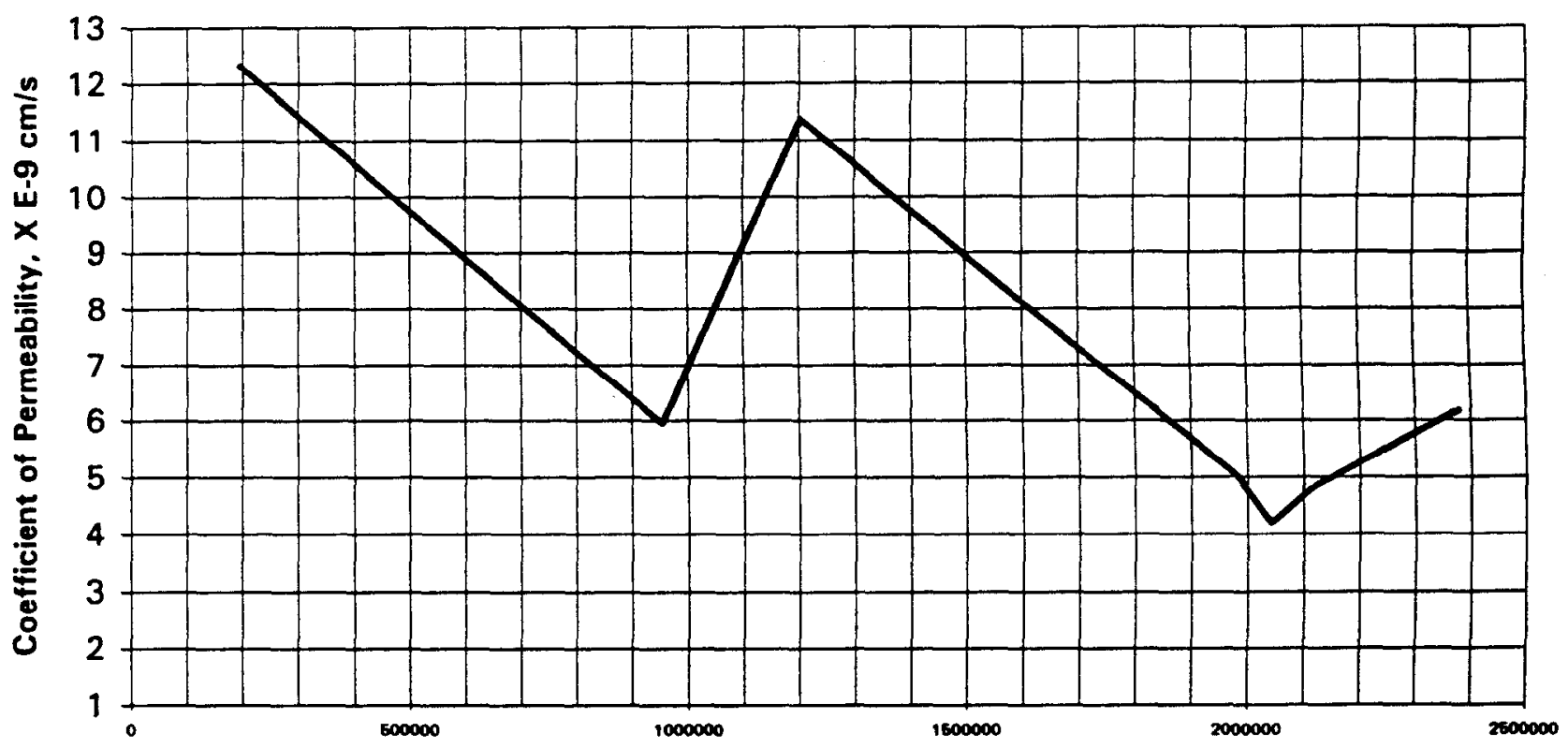

Cumulative Elapsed Time, seconds 


\section{8-DAY HYDRAULIC CONDUCTIVITY / PERMEABILITY ASTM D 5084}

PROJECT NAME: SAIC / Savannah River PROJECT NO. 777304.00010500
CLIENT SAMPLE NO. P322G00

IT LAB SAMPLE NO. ETDC-8096

\begin{tabular}{|c|c|c|c|c|}
\hline \multirow[b]{2}{*}{ Specimen diameter, $\mathrm{cm}$} & INITIAL & FINAL & & \\
\hline & \multicolumn{4}{|l|}{5.09} \\
\hline Specimen length, $\mathrm{cm}$ & 10.12 & & Hydraulic gradient & 27.8 \\
\hline Wet weight of specimen, $g$ & 391.92 & 396.32 & Min. consolidation stress, psi & 2.0 \\
\hline Specimen cross-sect. area, $\mathrm{cm}^{`} 2$ & 20.3630 & & Max. consolidation stress, psi & 8.0 \\
\hline Water content, $\%$ & 27.3 & 28.8 & Total backpressure, psi & 3.0 \\
\hline Wet unit weight, pcf & 118.7 & & Permeant Fluid Deaire & DI Water \\
\hline Dry unit weight, pcf & 93.2 & & Sample molded & $12 / 22 / 98$ \\
\hline Estimated degree of saturation, \% & 93.5 & & Sample set up & $1 / 20 / 99$ \\
\hline Estimated spec. gravity of solids & 2.6500 & & Six tests performed & \\
\hline
\end{tabular}

\section{PERMEABILITY vs TIME}

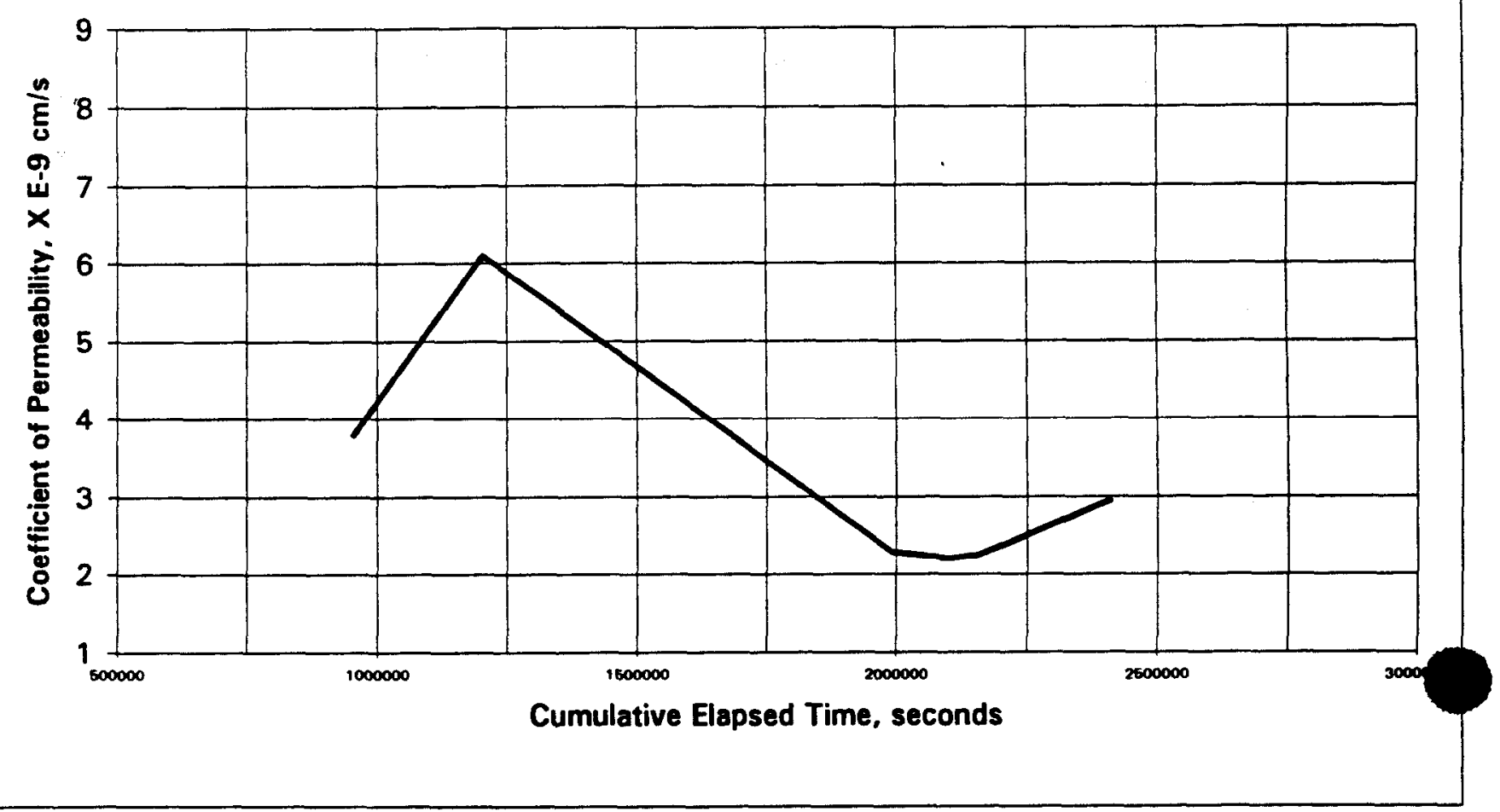




\section{8-DAY HYDRAULIC CONDUCTIVITY / PERMEABILITY ASTM D 5084}

PROJECT NAME:

PROJECT NO.

SAIC / Savannah River

777304.00010500

\section{CLIENT SAMPLE NO. \\ P332G00 \\ IT LAB SAMPLE NO. \\ ETDC.8097}

Specimen diameter, $\mathrm{cm}$

Specimen length, $\mathrm{cm}$

Wet weight of specimen, $\mathrm{g}$.

Specimen cross-sect. area, $\mathrm{cm}^{-} 2$

Water content, \%

Wet unit weight, pcf

Dry unit weight, pcf

Estimated degree of saturation, \%

Estimated spec. gravity of solids

\begin{tabular}{ll}
\hline INITIAL & FINAL \\
\hline 5.09 &
\end{tabular}

10.17

$382.45 \quad 388.8$

20.3867

$28.7 \quad 30.9$

115.2

89.5

89.7

2.65
Hydraulic gradient

27.7

Min. consolidation stress, psi

2.0

Max. consolidation stress, psi

9.0

3.0

Permeant Fluid

Sample molded

Deaired DI Water

Sample set up

12/22/98

$1 / 20 / 99$

Seven tests performed

\begin{tabular}{|ll|}
\hline Coefficient of Permeability, $\mathrm{cm} / \mathrm{s}$ & $3.5 \mathrm{E}-10$ \\
\hline
\end{tabular}

\section{PERMEABILITY vS TIME}

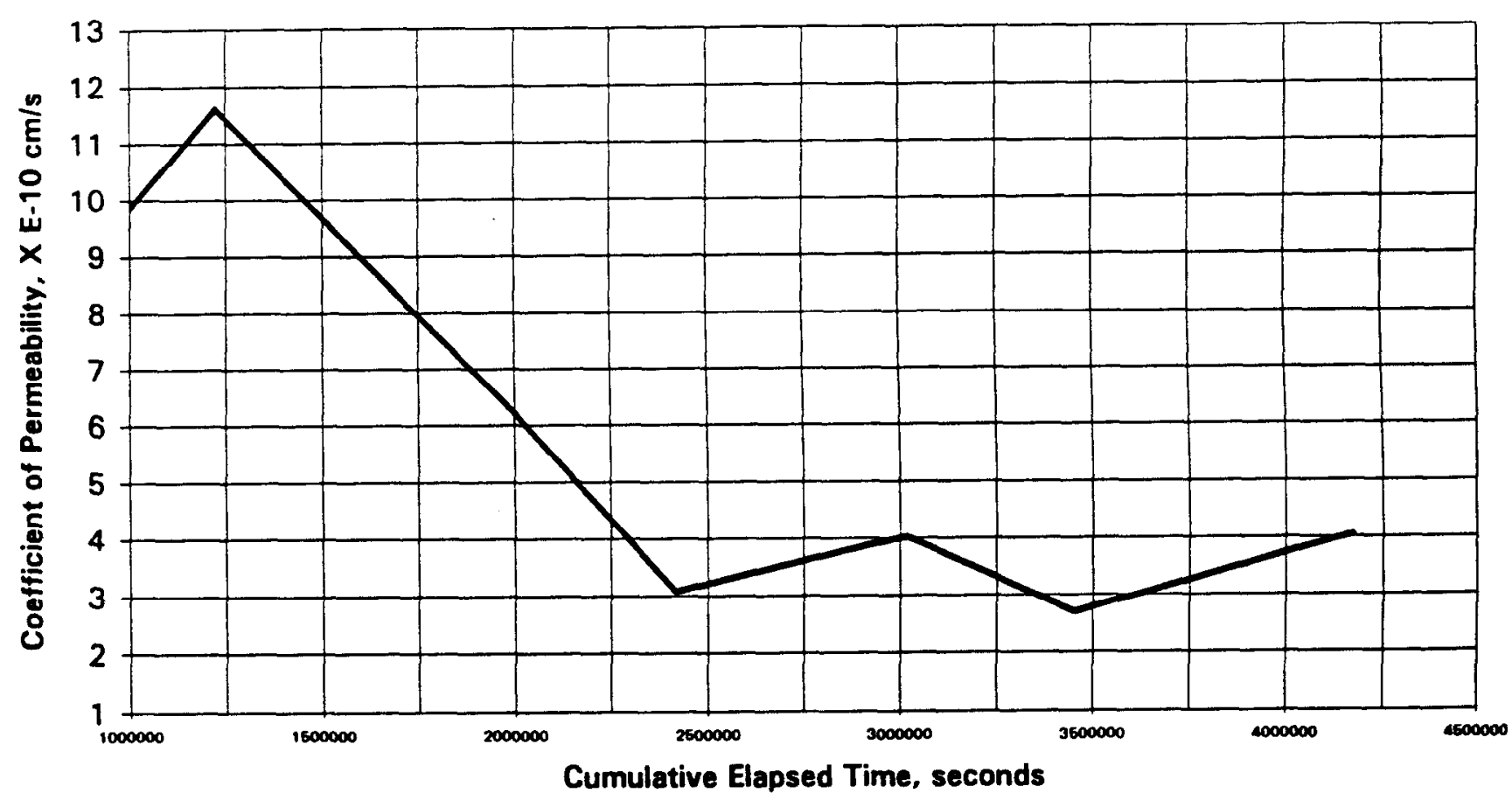




\section{8-DAY HYDRAULIC CONDUCTIVITY / PERMEABILITY ASTM D 5084}

PROJECT NAME: SAIC / Savannah River

PROJECT NO. $\quad 777304.00010500$
CLIENT SAMPLE NO. P342G00

IT LAB SAMPLE NO. ETDC-8098
Specimen diameter, $\mathrm{cm}$

Specimen length, $\mathrm{cm}$

Wet weight of specimen, $g$.

Specimen cross-sect. area, $\mathrm{cm}^{`} 2$

Water content, \%

Wet unit weight, pcf

Dry unit weight, pcf

Estimated degree of saturation, \%

Estimated spec. gravity of solids

INITIAL FINAL

5.10

10.23

$377.12 \quad 379.76$

20.3969

33.9

112.9

84.3

93.3

2.6500

34.9

Hydraulic gradient

27.5

Min. consolidation stress, psi

2.0

Max. consolidation stress, psi

6.0

2.0

Total backpressure, psi

Permeant Fluid

Sample molded

Deaired DI Water

Sample set up

$12 / 22 / 98$

$1 / 20 / 99$

Five tests performed

\begin{tabular}{|ll|}
\hline Coefficient of Permeability, $\mathrm{cm} / \mathrm{s}$ & $4.4 \mathrm{E}-08$ \\
\hline
\end{tabular}

\section{PERMEABILITY vS TIME}

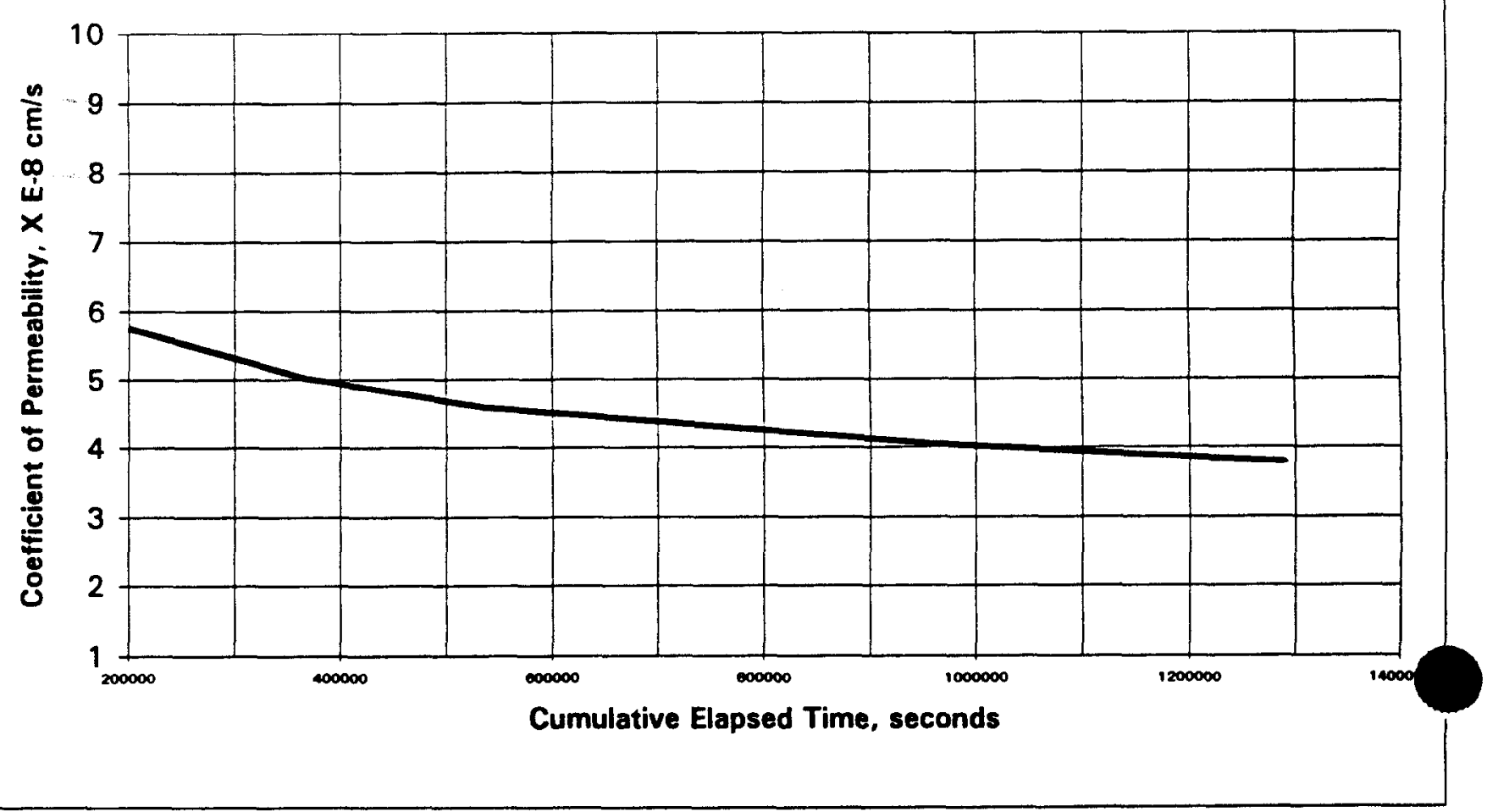




\section{8-DAY HYDRAULIC CONDUCTIVITY / PERMEABILITY ASTM D 5084}

PROJECT NAME: PROJECT NO.

SAIC / Savannah River 777304.00010500

\section{CLIENT SAMPLE NO. \\ P412G00 \\ IT LAB SAMPLE NO. \\ ETDC-8099}

Specimen diameter, $\mathrm{cm}$

Specimen length, $\mathrm{cm}$

Wet weight of specimen, $g$.

Specimen cross-sect. area, $\mathrm{cm}^{`} 2$

Water content, \%

Wet unit weight, pcf

Dry unit weight, pcf

Estimated degree of saturation, \%

Estimated spec. gravity of solids

\begin{tabular}{l}
\hline INITIAL FINAL \\
5.09
\end{tabular}

10.07

368.86

377.31

20.3698

33.2

112.2

84.2

91.3

2.6500

36.2
Hydraulic gradient

27.9

Min. consolidation stress, psi

2.0

Max. consolidation stress, psi

8.0

Total backpressure, psi

Permeant Fluid

3.0

Sample molded

Deaired DI Water

Sample set up

$12 / 22 / 98$

$1 / 20 / 99$

Four tests performed

\begin{tabular}{|ll|}
\hline Coefficient of Permeability, cm/s & $6.8 E-09$ \\
\hline
\end{tabular}

\section{PERMEABILITY vS TIME}

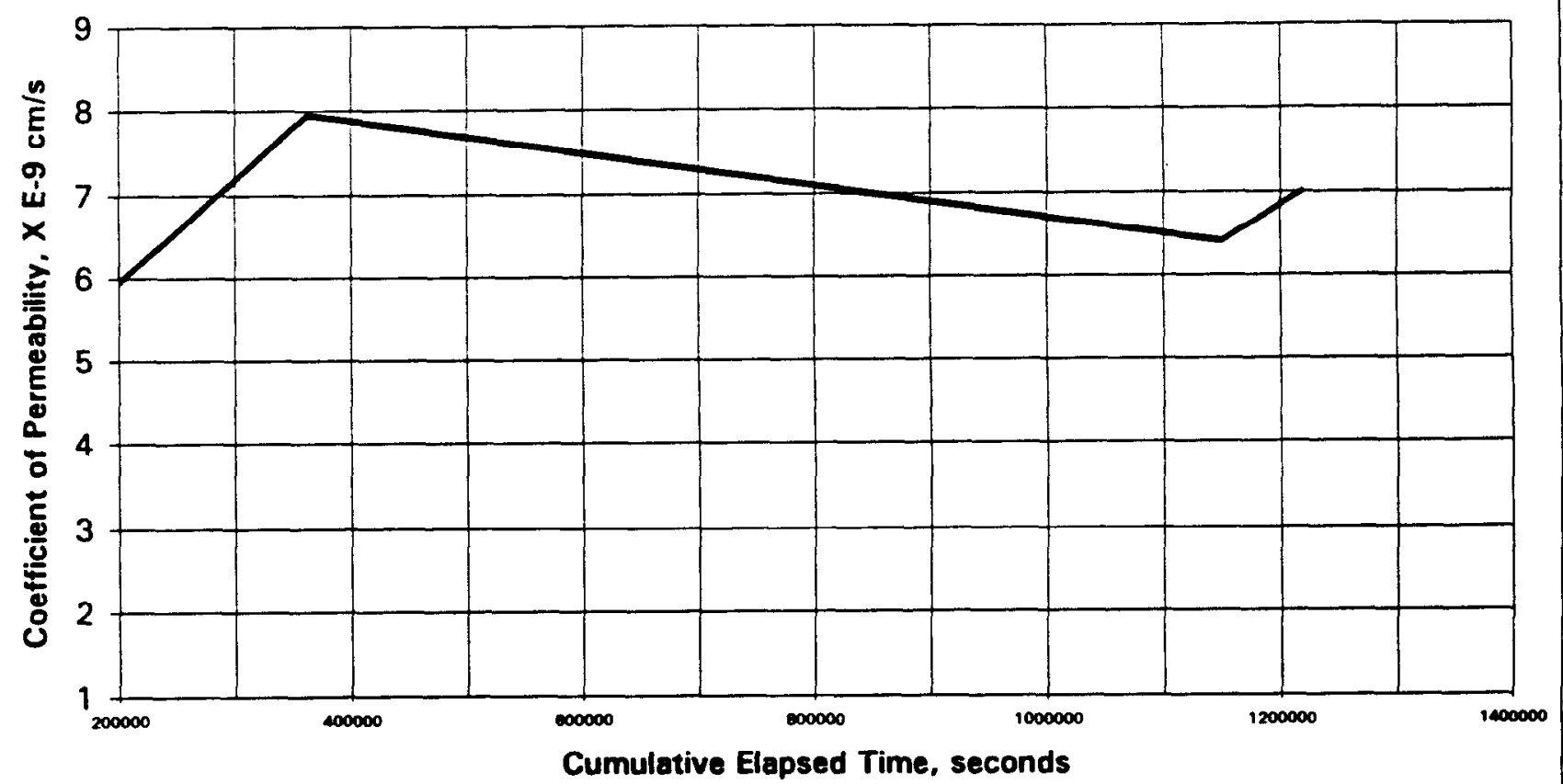




\section{8-DAY HYDRAULIC CONDUCTIVITY / PERMEABILITY ASTM D 5084}

PROJECT NAME: SAIC / Savannah River PROJECT NO.

777304.00010500
CLIENT SAMPLE NO. P422G00

IT LAB SAMPLE NO. ETDC-8100

\begin{tabular}{|c|c|c|c|c|}
\hline & & & & \\
\hline & INITIAL & FINAL & & \\
\hline Specimen diameter, $\mathrm{cm}$ & \multicolumn{4}{|l|}{5.09} \\
\hline Specimen length, $\mathrm{cm}$ & \multicolumn{2}{|l|}{10.12} & \multicolumn{2}{|l|}{ Hydraulic gradient } \\
\hline Wet weight of specimen, $g$. & 378.41 & 384.26 & \multicolumn{2}{|l|}{ Min. consolidation stress, psi } \\
\hline Specimen cross-sect. area, $\mathrm{cm} ` 2$ & \multicolumn{2}{|l|}{20.3596} & \multicolumn{2}{|l|}{ Max. consolidation stress, psi } \\
\hline Water content, $\%$ & 30.6 & 32.7 & Total backpressure, psi & 4.0 \\
\hline Wet unit weight, pcf & \multicolumn{2}{|l|}{114.6} & Permeant Fluid Deaire & Deaired DI Water \\
\hline Dry unit weight, pcf & \multicolumn{2}{|l|}{87.7} & Sample molded & $12 / 22 / 98$ \\
\hline Estimated degree of saturation, $\%$ & \multicolumn{2}{|l|}{91.7} & Sample set up & \multirow[t]{2}{*}{$1 / 20 / 99$} \\
\hline Estimated spec. gravity of solids & \multicolumn{2}{|l|}{2.6500} & Four tests performed & \\
\hline
\end{tabular}

\section{PERMEABILITY vs TIME}

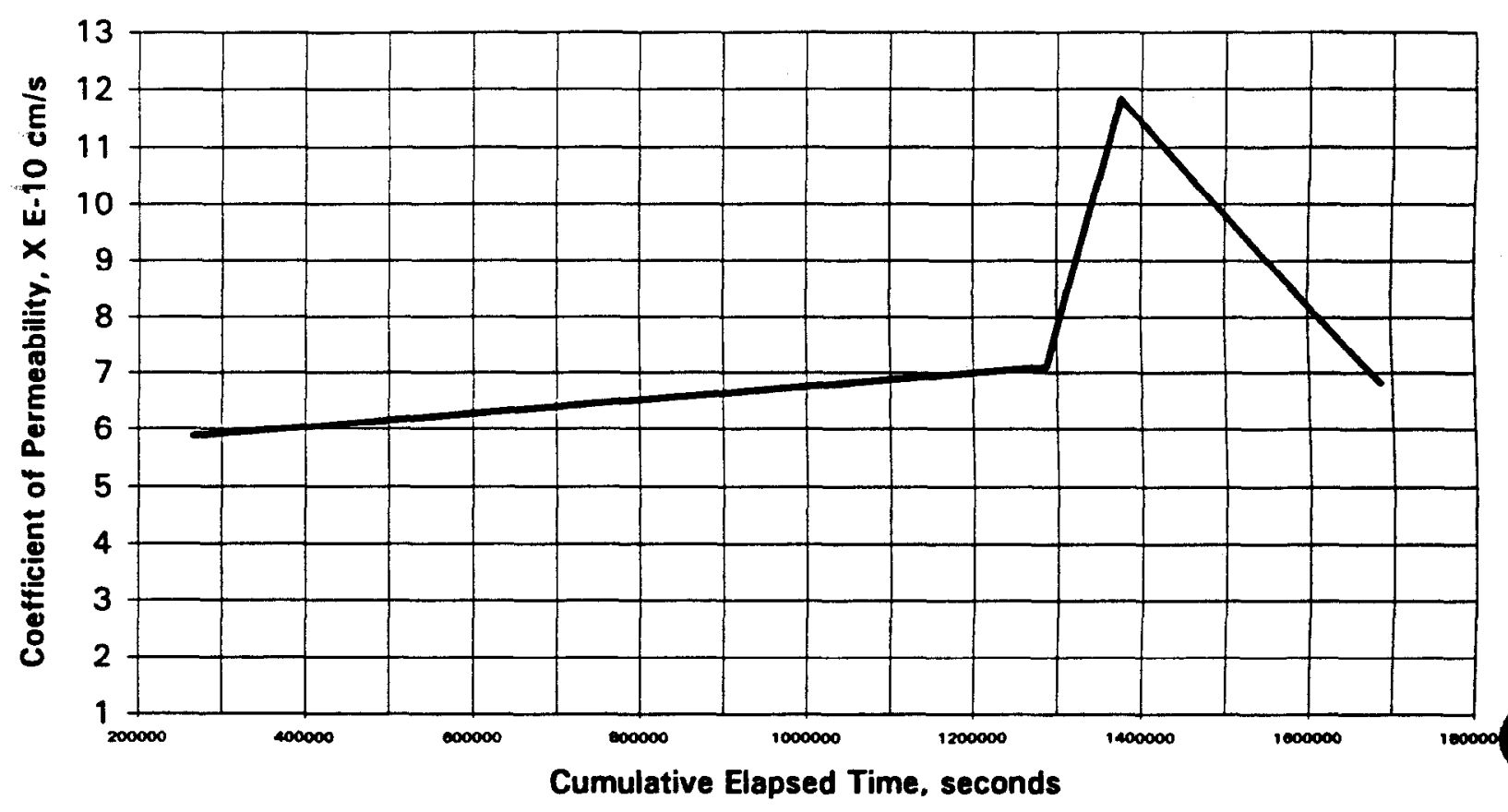




\section{8-DAY HYDRAULIC CONDUCTIVITY / PERMEABILITY ASTM D 5084}

PROJECT NAME:

PROJECT NO.

SAIC / Savannah River 777304.00010500
CLIENT SAMPLE NO.

P432G00

IT LAB SAMPLE NO.
Specimen diameter, $\mathrm{cm}$

Specimen length, cm

Wet weight of specimen, $g$.

Specimen cross-sect. area, $\mathrm{cm}^{\wedge} 2$

Water content, \%

Wet unit weight, pcf

Dry unit weight, pcf

Estimated degree of saturation, \%

Estimated spec. gravity of solids

\begin{tabular}{ll}
\hline INITIAL FINAL \\
5.08
\end{tabular}

10.13

$357.63 \quad 364.96$

20.3055

37.9

40.7

108.5

78.7

91.0

2.6500
Hydraulic gradient

27.8

Min. consolidation stress, psi

2.0

Max. consolidation stress, psi

10.0

4.0

Permeant Fluid

Deaired DI Water

Sample molded

$12 / 22 / 98$

Sample set up

$1 / 20 / 99$

\section{PERMEABILITY vS TIME}

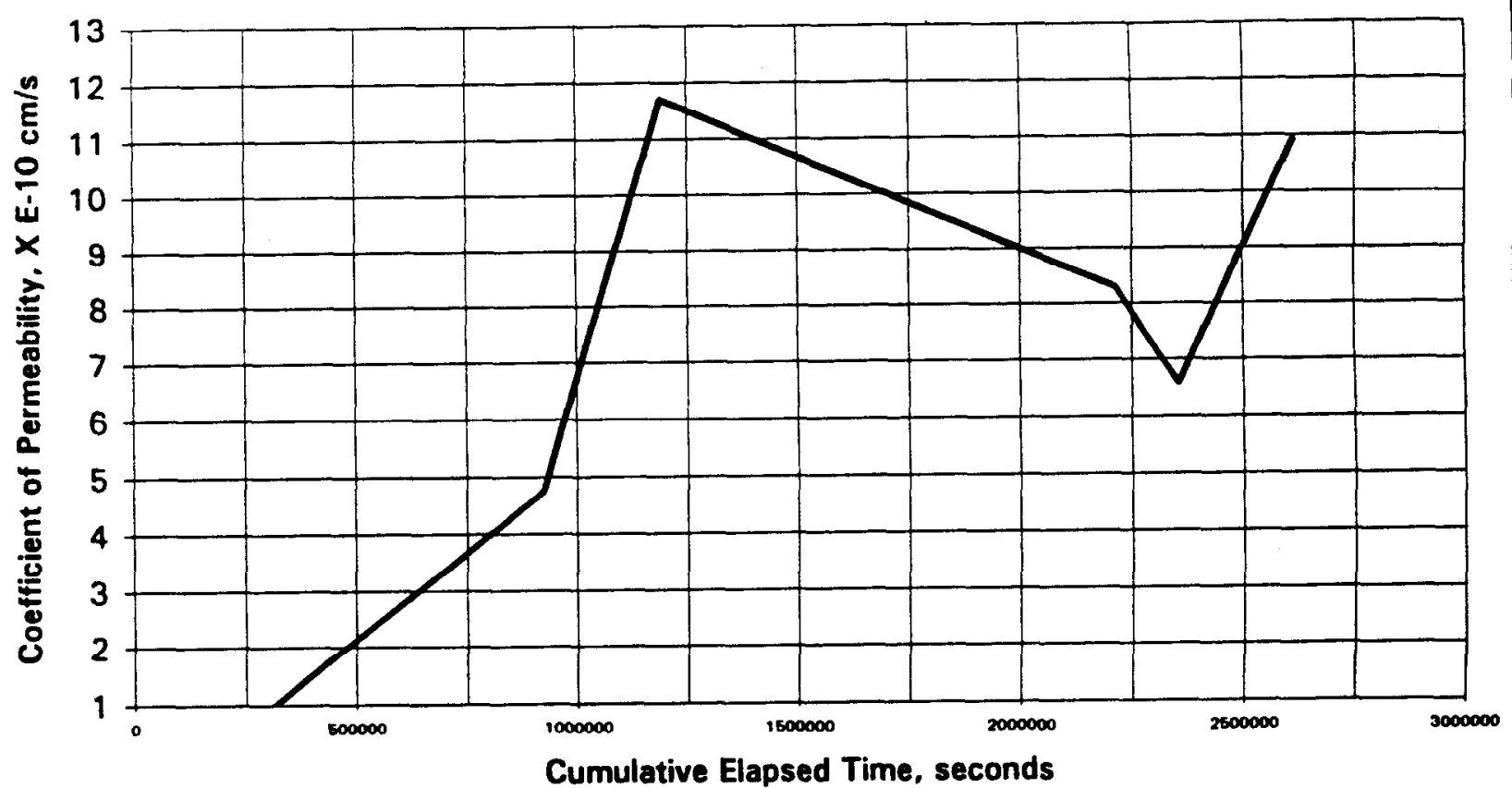




\section{8-DAY HYDRAULIC CONDUCTIVITY / PERMEABILITY ASTM D 5084}

PROJECT NAME:

PROJECT NO.

SAIC / Savannah River

777304.00010500
CLIENT SAMPLE NO. P442G00

IT LAB SAMPLE NO. ETDC-8102

INITIAL FINAL

Specimen diameter, $\mathrm{cm}$

Specimen length, $\mathrm{cm}$

Wet weight of specimen, $g$.

Specimen cross-sect. area, $\mathrm{cm}^{-2} 2$

Water content, \%

Wet unit weight, pcf

Dry unit weight, pcf

Estimated degree of saturation, \%

Estimated spec. gravity of solids

\subsection{0}

10.17

$373.54 \quad 373.54$

20.4138

38.0

112.4

2.65
Hydraulic gradient

20.8

Min. consolidation stress, psi

2.0

Max. consolidation stress, psi

9.0

5.0

Permeant Fluid

Deaired DI Water

Sample molded

$12 / 22 / 98$

Sample set up

$1 / 21 / 99$

Eight tests performed

\begin{tabular}{|ll|}
\hline Coefficient of Permeability, cm/s & 8.0E-10 \\
\hline
\end{tabular}

\section{PERMEABILITY VS TIME}

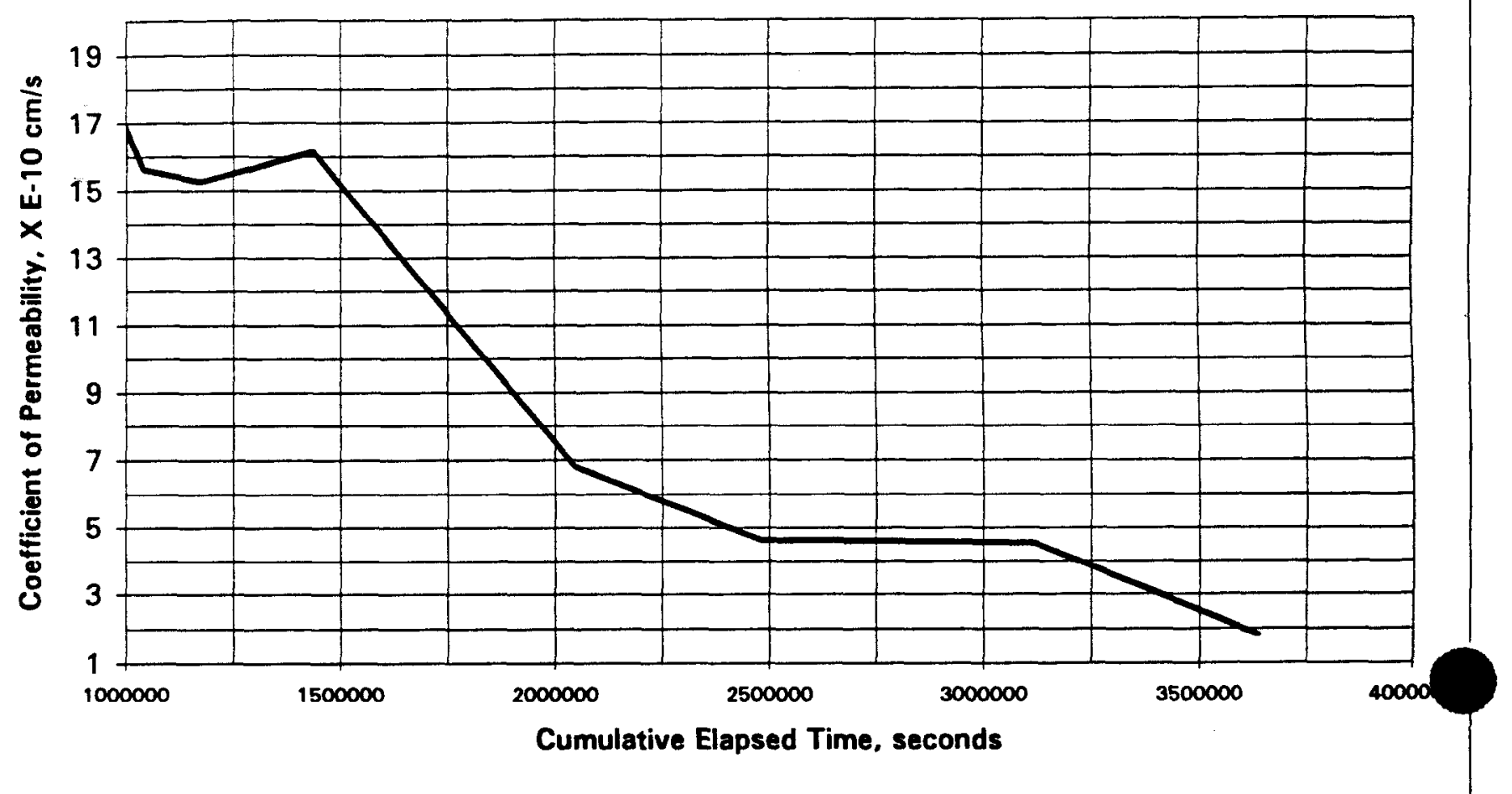




\section{8-DAY HYDRAULIC CONDUCTIVITY / PERMEABILITY ASTM D 5084}

PROJECT NAME: SAIC / Savannah River

PROJECT NO. $\quad 777304.00010500$
CLIENT SAMPLE NO. P512G00

IT LAB SAMPLE NO. ETDC-8103
Specimen diameter, $\mathrm{cm}$

Specimen length, $\mathrm{cm}$

Wet weight of specimen, $g$.

Specimen cross-sect. area, $\mathrm{cm}^{-} 2$

Water content, \%

Wet unit weight, pcf

Dry unit weight, pcf

Estimated degree of saturation, \%

Estimated spec. gravity of solids

\begin{tabular}{l}
\hline INITIAL FINAL \\
5.09 \\
10.18
\end{tabular}

Hydraulic gradient

$381.59 \quad 389.01$

20.3122

$29.1 \quad 31.6$

115.2

89.3

90.2

2.6500

Min. consolidation stress, psi

2.0

6.0

Max. consolidation stress, psi

4.0

Permeant Fluid

Deaired DI Water

Sample molded

$12 / 22 / 98$

Sample set up

$1 / 21 / 99$

Six tests performed

\section{PERMEABILITY vs TIME}

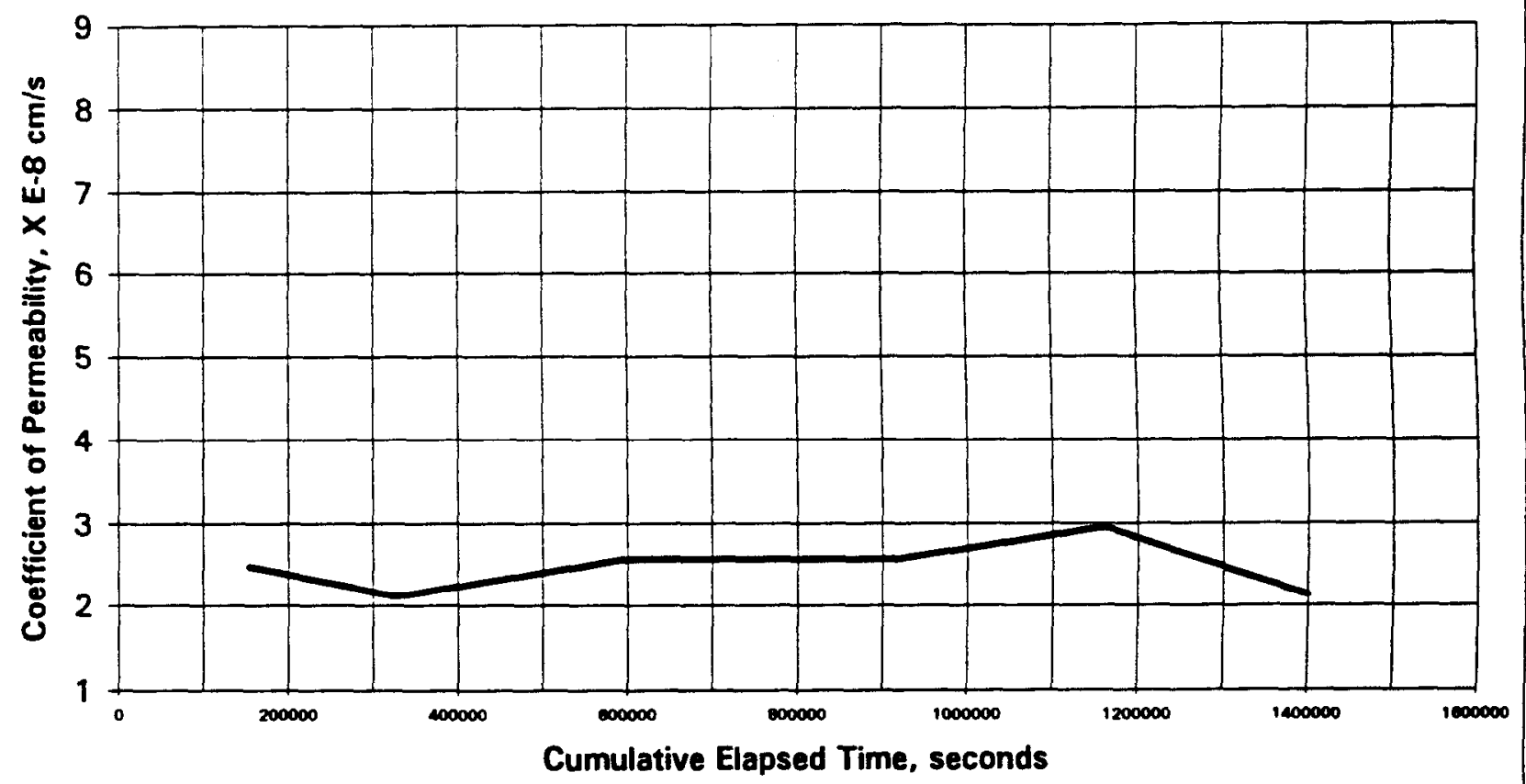




\section{8-DAY HYDRAULIC CONDUCTIVITY / PERMEABILITY ASTM D 5084}

PROJECT NAME: SAIC / Savannah River PROJECT NO. $\quad 777304.00010500$
CLIENT SAMPLE NO. P522G00

IT LAB SAMPLE NO. ETDC-8104

\begin{tabular}{|c|c|c|c|c|}
\hline \multirow[b]{2}{*}{ Cnpimp diameter $\mathrm{sm}$} & INITIAL & FINAL & & \\
\hline & \multicolumn{4}{|l|}{5.10} \\
\hline Specimen length, $\mathrm{cm}$ & \multicolumn{2}{|l|}{10.10} & Hydraulic gradient & 27.9 \\
\hline Wet weight of specimen, $g$ & \multicolumn{2}{|l|}{391.74} & Min. consolidation stress, psi & 2.0 \\
\hline Specimen cross-sect. area, $\mathrm{cm}^{\wedge} 2$ & \multicolumn{2}{|l|}{20.3935} & Max. consolidation stress, psi & 8.0 \\
\hline Water content, $\%$ & \multicolumn{2}{|l|}{26.6} & Total backpressure, psi & 4.0 \\
\hline Wet unit weight, pcf & \multicolumn{2}{|l|}{118.7} & Permeant Fluid Deaire & Deaired DI Water \\
\hline Dry unit weight, pcf & \multicolumn{2}{|l|}{93.8} & Sample molded & $12 / 22 / 98$ \\
\hline Estimated degree of saturation, $\%$ & \multicolumn{2}{|l|}{92.1} & Sample set up & $1 / 21 / 99$ \\
\hline Estimated spec. gravity of solids & \multicolumn{2}{|l|}{2.65} & \multicolumn{2}{|l|}{ Seven tests performed } \\
\hline
\end{tabular}

\section{PERMEABILITY vS TIME}

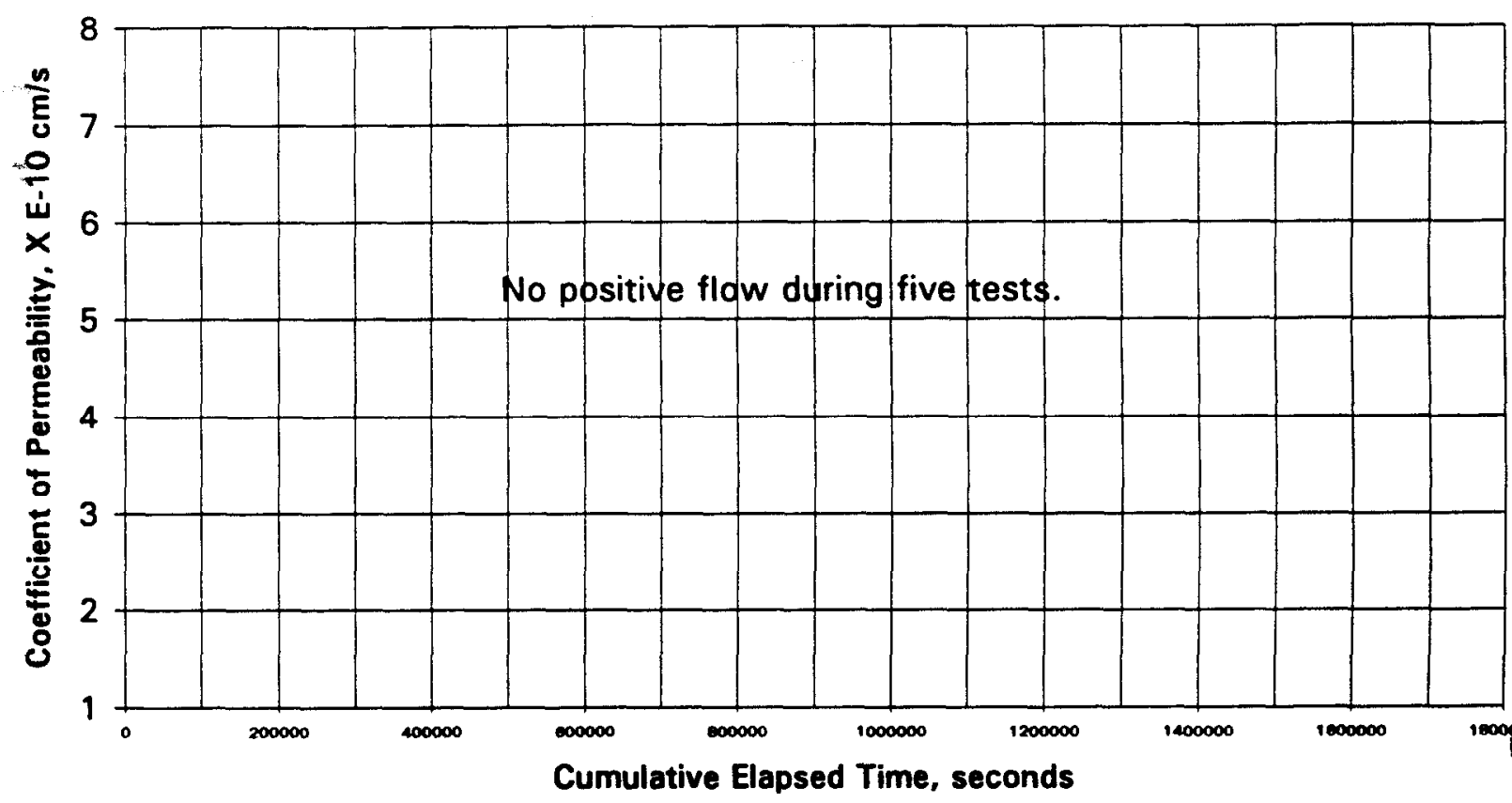




\section{8-DAY HYDRAULIC CONDUCTIVITY / PERMEABILITY ASTM D 5084}

PROJECT NAME:

SAIC / Savannah River

PROJECT NO.

777304.00010500

\section{CLIENT SAMPLE NO. P532G00 \\ IT LAB SAMPLE NO. ETDC-8105}

\begin{tabular}{|c|c|c|c|c|}
\hline \multirow[b]{2}{*}{ Specimen diameter, $\mathrm{cm}$} & INITIAL & FINAL & \multirow[b]{3}{*}{ Hydraulic gradient } & \multirow{3}{*}{28.0} \\
\hline & \multicolumn{2}{|l|}{5.08} & & \\
\hline Specimen length, $\mathrm{cm}$ & \multicolumn{2}{|l|}{10.05} & & \\
\hline Wet weight of specimen, $g$ & 364.11 & 370.7 & Min. consolidation stress, psi & 2.0 \\
\hline Specimen cross-sect. area, $\mathrm{cm}^{-2} 2$ & \multicolumn{2}{|l|}{20.2717} & Max. consolidation stress, psi & 9.0 \\
\hline Water content, $\%$ & 34.6 & 37.0 & Total backpressure, psi & 5.0 \\
\hline Wet unit weight, pcf & \multicolumn{2}{|l|}{111.6} & Permeant Fluid & Deaired DI Water \\
\hline Dry unit weight, pcf & \multicolumn{2}{|l|}{82.9} & Sample molded & $12 / 22 / 98$ \\
\hline Estimated degree of saturation, \% & \multicolumn{2}{|l|}{92.0} & Sample set up & \multirow[t]{2}{*}{$1 / 21 / 99$} \\
\hline Estimated spec. gravity of solids & 2.65 & & Five tests performed & \\
\hline
\end{tabular}

\section{Coefficient of Permeability, cm/s} 1.1E-09

Results based on influent volume. No effluent observed from sample.

\section{PERMEABILITY vs TIME}

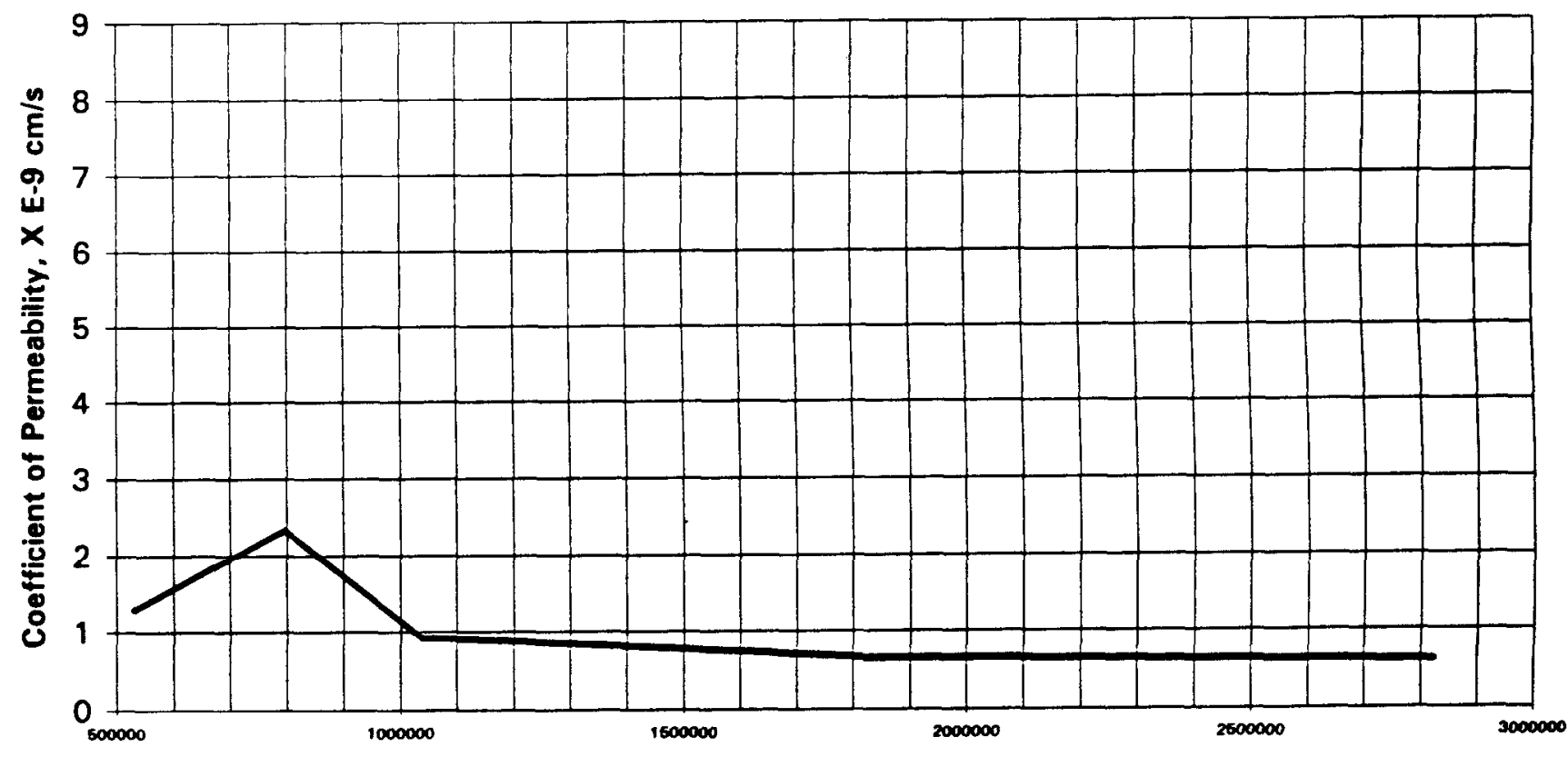

Cumulative Elapsed Time, seconds 


\section{8-DAY HYDRAULIC CONDUCTIVITY / PERMEABILITY ASTM D 5084}

PROJECT NAME: SAIC / Savannah River

PROJECT NO. $\quad 777304.00010500$
CLIENT SAMPLE NO. P542G00

IT LAB SAMPLE NO. ETDC-8106
Specimen diameter, $\mathrm{cm}$

Specimen length, $\mathrm{cm}$

Wet weight of specimen, $g$.

Specimen cross-sect. area, $\mathrm{cm}^{-2} \quad 20.3122$

Water content, \%

Wet unit weight, pcf

Dry unit weight, pcf

Estimated degree of saturation, \%

Estimated spec. gravity of solids

\begin{tabular}{|l|l|}
\hline INITIAL & FINAL \\
\hline 5.09 &
\end{tabular}

379.1

35.0

Hydraulic gradient

27.7

Min. consolidation stress, psi

2.0

Max. consolidation stress, psi

8.0

Total backpressure, psi

4.0

Permeant Fluid

Deaired DI Water

Sample molded

$12 / 22 / 98$

Sample set up

$1 / 21 / 99$

\begin{tabular}{|ll|}
\hline Coefficient of Permeability, $\mathrm{cm} / \mathrm{s}$ & $1.8 \mathrm{E}-08$ \\
\hline
\end{tabular}

\section{PERMEABILITY vs TIME}

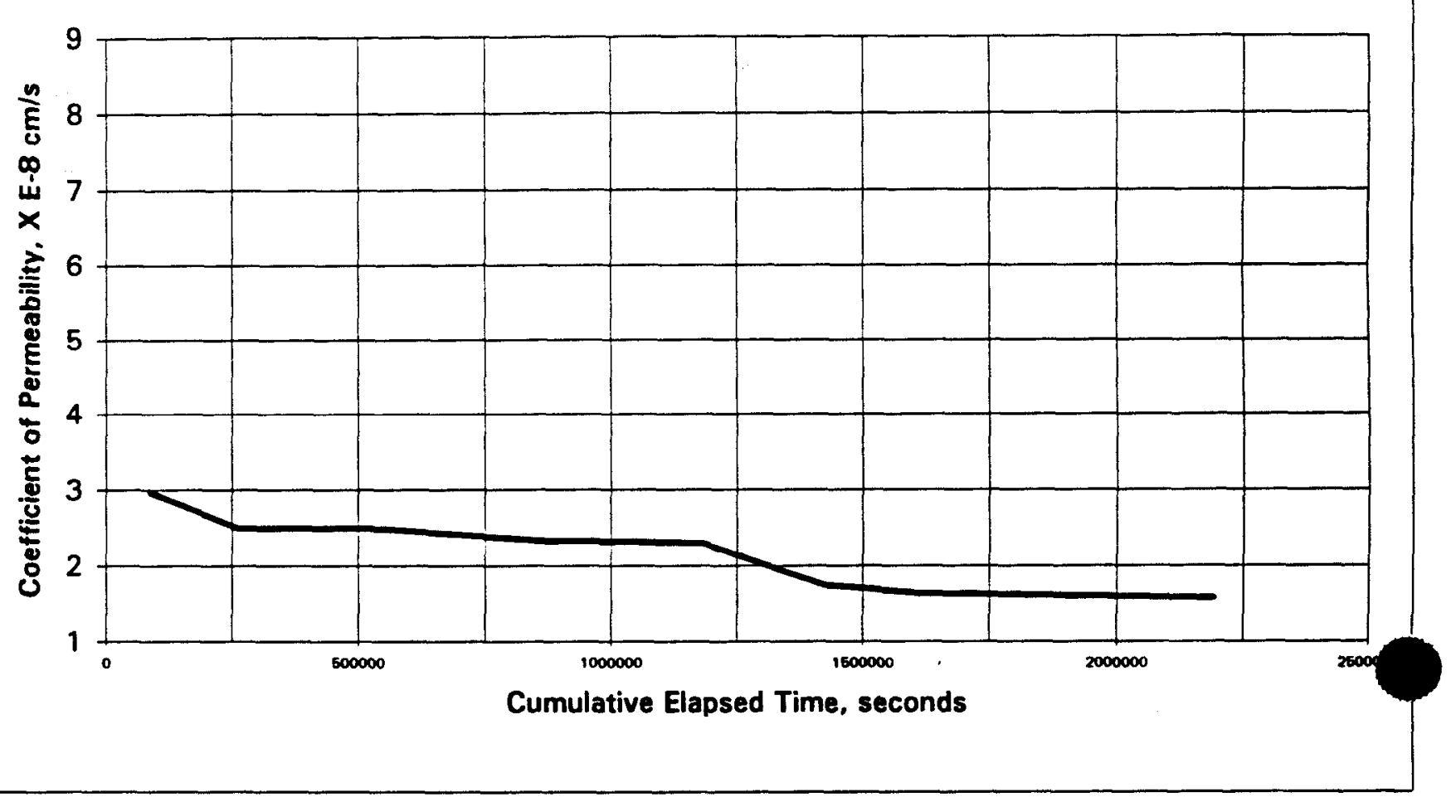




\section{8-DAY HYDRAULIC CONDUCTIVITY / PERMEABILITY ASTM D 5084}

PROJECT NAME: SAIC / Savannah River PROJECT NO. $\quad 777304.00010500$

\section{CLIENT SAMPLE NO. P612G00 \\ IT LAB SAMPLE NO. ETDC-8107}

\begin{tabular}{lll|}
\cline { 2 - 3 } & INITIAL & FINAL \\
\cline { 2 - 3 } Specimen diameter, $\mathrm{cm}$ & 5.09 & \\
Specimen length, cm & 10.08 & \\
Wet weight of specimen, g. & 373.1 & 380.15 \\
Specimen cross-sect. area, cm ${ }^{-2}$ & 20.3698 & \\
Water content, \% & 29.3 & 31.7 \\
Wet unit weight, pcf & 113.4 & \\
Dry unit weight, pcf & 87.7 & \\
Estimated degree of saturation, \% & 87.6 & \\
Estimated spec. gravity of solids & 2.65
\end{tabular}

Hydraulic gradient 34.9

Min. consolidation stress, psi

2.0

Max. consolidation stress, psi

9.0

Total backpressure, psi

Permeant Fluid

Deaired DI Water

Sample molded

$12 / 22 / 98$

Sample set up

$1 / 21 / 99$

Four tests performed

\section{Coefficient of Permeability, $\mathrm{cm} / \mathrm{s}$}

\subsection{E-09}

\section{PERMEABILITY vs TIME}

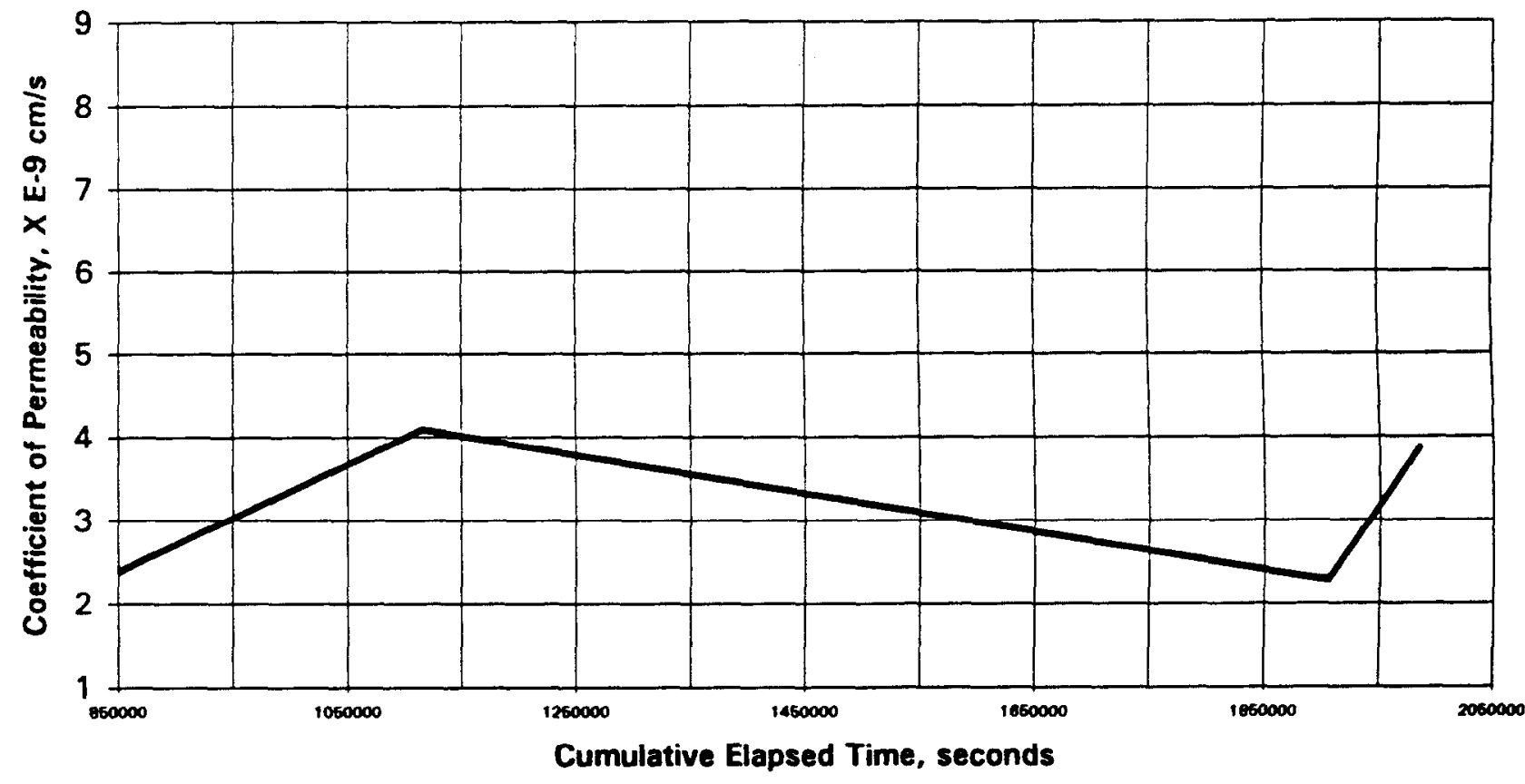




\section{8-DAY HYDRAULIC CONDUCTIVITY / PERMEABILITY ASTM D 5084}

PROJECT NAME: $\quad$ SAIC / Savannah River PROJECT NO.
CLIENT SAMPLE NO. P622G00

IT LAB SAMPLE NO. ETDC-8108

\begin{tabular}{|c|c|c|c|c|}
\hline \multirow[b]{2}{*}{ Specimen diameter, $\mathrm{cm}$} & & & & \multirow{3}{*}{27.8} \\
\hline & \multicolumn{2}{|l|}{5.08} & & \\
\hline Specimen length, cm & 10.13 & & Hydraulic gradient & \\
\hline Wet weight of specimen, $g$. & 388.27 & 391.91 & Min. consolidation stress, psi & 2.0 \\
\hline Specimen cross-sect. area, $\mathrm{cm}^{\wedge} 2$ & 20.3055 & & Max. consolidation stress, psi & 6.0 \\
\hline Water content, $\%$ & 27.1 & 28.3 & Total backpressure, psi & 4.0 \\
\hline Wet unit weight, pcf & 117.9 & & Permeant Fluid $\quad$ Deaire & DI Water \\
\hline Dry unit weight, pcf & 92.8 & & Sample molded & $12 / 22 / 98$ \\
\hline Estimated degree of saturation, $\%$ & 91.6 & & Sample set up & $1 / 21 / 99$ \\
\hline Estimated spec. gravity of solids & 2.65 & & Six tests performed & \\
\hline
\end{tabular}

\section{PERMEABILITY vS TIME}

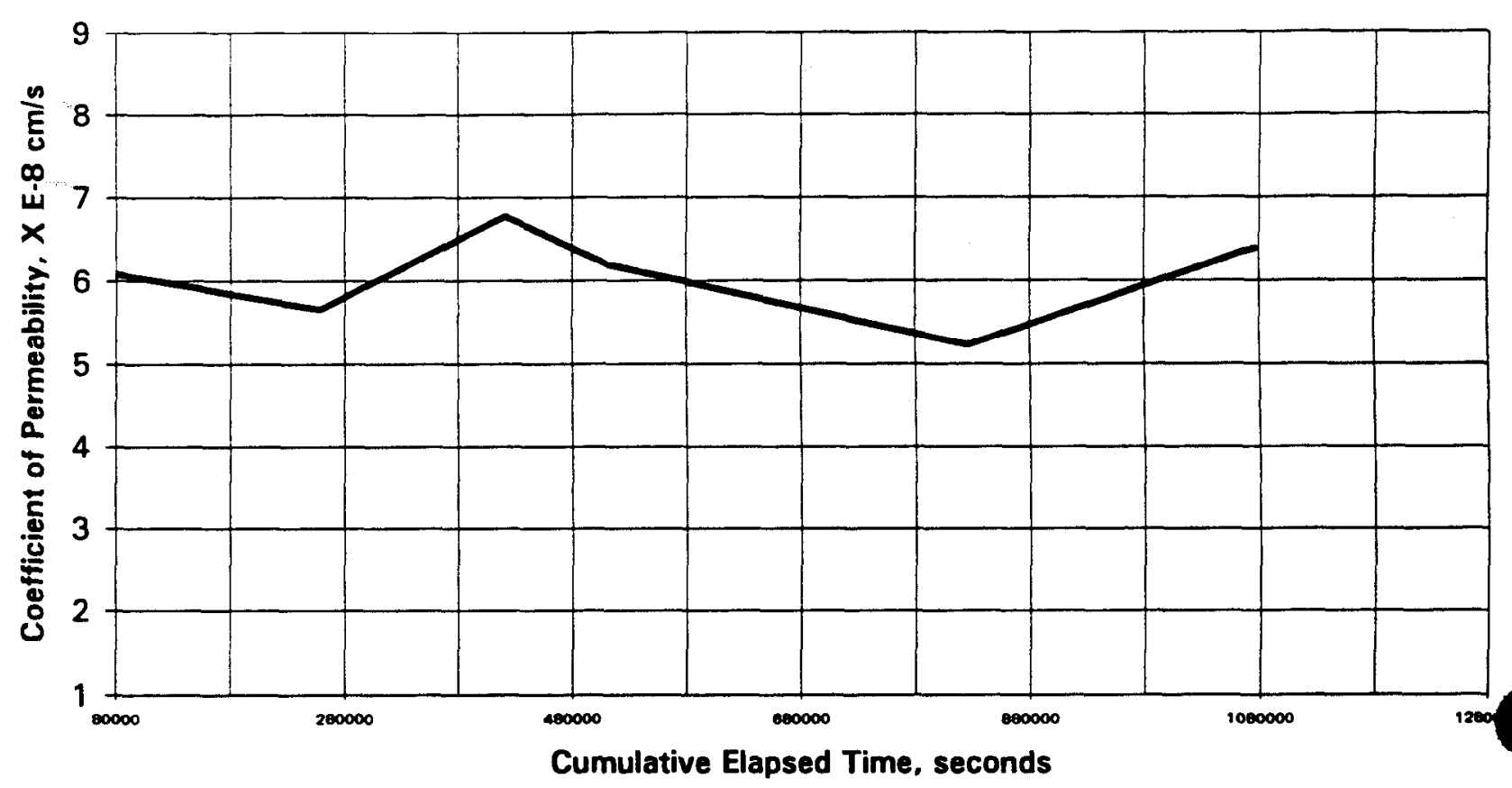




\section{8-DAY HYDRAULIC CONDUCTIVITY / PERMEABILITY ASTM D 5084}

PROJECT NAME: SAIC / Savannah River

PROJECT NO. 777304.00010500
CLIENT SAMPLE NO. P632G00

IT LAB SAMPLE NO. ETDC-8109

\begin{tabular}{lll|}
\cline { 2 - 3 } & INITIAL & FINAL \\
\cline { 2 - 3 } Specimen diameter, cm & 5.08 & \\
Specimen length, cm & 10.08 & \\
Wet weight of specimen, g. & 367.52 & 373.97 \\
Specimen cross-sect. area, cm `2 & 20.2987 & \\
Water content, \% & 34.0 & 36.3 \\
Wet unit weight, pcf & 112.1 & \\
Dry unit weight, pcf & 83.7 & \\
Estimated degree of saturation, \% & 92.2 & \\
Estimated spec. gravity of solids & 2.65 &
\end{tabular}

Hydraulic gradient 27.9

Min. consolidation stress, psi

2.0

Max. consolidation stress, psi

9.0

Total backpressure, psi

Permeant Fluid

4.0

Sample molded

Deaired DI Water

Sample set up

12/22/98

$1 / 21 / 99$

Six tests performed

\begin{tabular}{|ll}
\hline Coefficient of Permeability, $\mathrm{cm} / \mathrm{s}$ & $5.0 \mathrm{E}-10$ \\
\hline
\end{tabular}

\section{PERMEABILITY vS TIME}

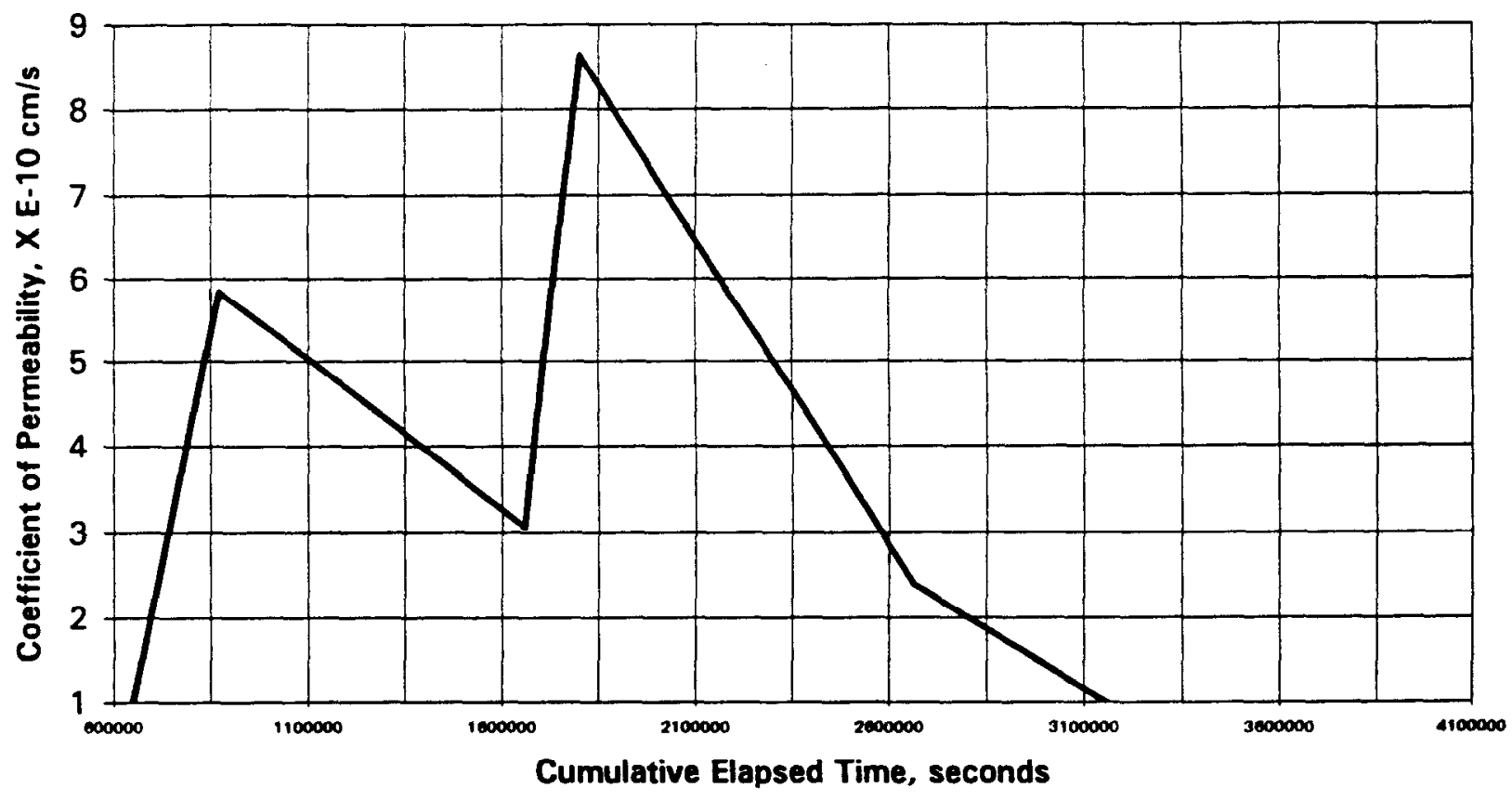




\section{8-DAY HYDRAULIC CONDUCTIVITY / PERMEABILITY ASTM D 5084}

PROJECT NAME: SAIC / Savannah River PROJECT NO. 777304.00010500
CLIENT SAMPLE NO. P642G00

IT LAB SAMPLE NO. ETDC-8110

\begin{tabular}{llll}
\cline { 2 - 2 } & INITIAL & FINAL \\
\cline { 2 - 2 } Specimen diameter, cm & 5.09 & \\
Specimen length, cm & 10.11 & \\
Wet weight of specimen, $g$. & 374.02 & 378.23 \\
Specimen cross-sect. area, $\mathrm{cm} \cdot 2$ & 20.3291 & \\
Water content, \% & 33.1 & 34.6 \\
Wet unit weight, pcf & 113.6 & \\
Dry unit weight, pcf & 85.3 & \\
Estimated degree of saturation, \% & 93.4 & \\
Estimated spec. gravity of solids & 2.65 &
\end{tabular}

Hydraulic gradient $\quad 27.8$

Min. consolidation stress, psi $\quad 2.0$

Max. consolidation stress, psi $\quad 6.0$

Total backpressure, psi $\quad 4.0$

Permeant Fluid Deaired DI Water

Sample molded $\quad 12 / 22 / 98$

Sample set up $\quad 1 / 20 / 99$

Seven tests performed

\begin{tabular}{|ll|}
\hline Coefficient of Permeability, $\mathrm{cm} / \mathrm{s}$ & $1.9 \mathrm{E}-08$ \\
\hline
\end{tabular}

\section{PERMEABILITY vs TIME}

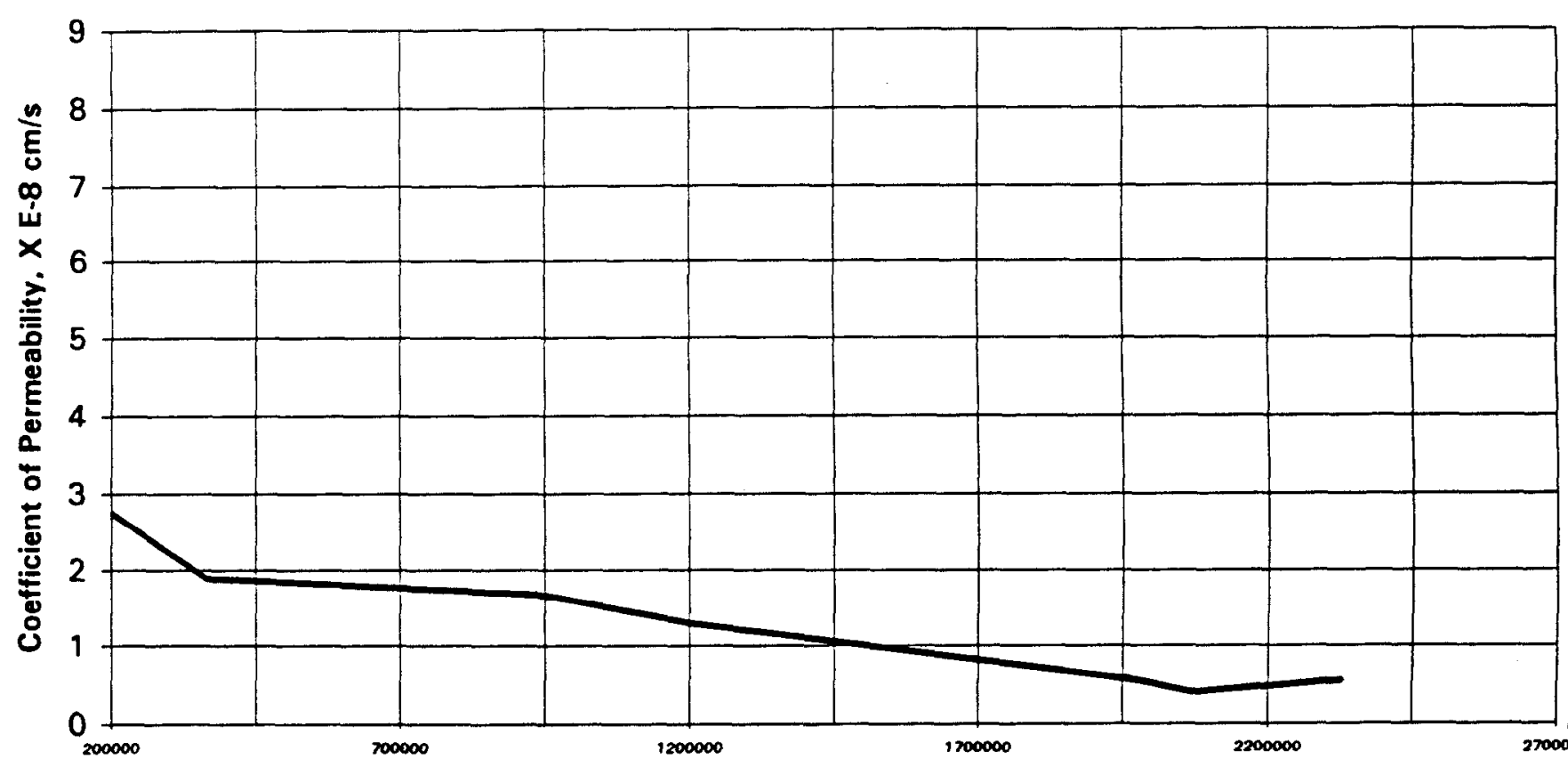

Cumulative Elapsed Time, seconds 


\section{P Series}

90 Immersion Permeability Results 


\section{0-DAY SATURATED HYDRAULIC CONDUCTIVITY / PERMEABILITY ASTM D 5084}

PROJECT NAME: SAIC / Savannah River PROJECT NO.
777304.00010500
CLIENT SAMPLE NO.

IT LAB SAMPLE NO.
P313G00

ETDC-8119

INITIAL FINAL

Specimen diameter, $\mathrm{cm}$

Specimen length, $\mathrm{cm}$

Wet weight of specimen, $g$.

Specimen cross-sect. area, $\mathrm{cm}^{\wedge} 2$ Water content, \%

Wet unit weight, pcf

Dry unit weight, pcf

Estimated degree of saturation, \% Estimated spec. gravity of solids Initial conditions are post-soak, pre-test

\subsection{9}

7.25

611.2

45.2093

30.2

116.4

89.4

94.2

2.65
Hydraulic gradient

38.8

615.03

Min. consolidation stress, psi

Max. consolidation stress, psi

2.0

4.0

31.0

Total backpressure, psi

Permeant Fluid

Sample molded

Sample set up

Five tests performed
Deaired DI Water

$12 / 22 / 98$

$4 / 18 / 99$

\section{\begin{tabular}{|ll|}
\hline Coefficient of Permeability, cm/s & $7.9 \mathrm{E}-07$ \\
\hline
\end{tabular}}

\section{PERMEABILITY VS TIME}

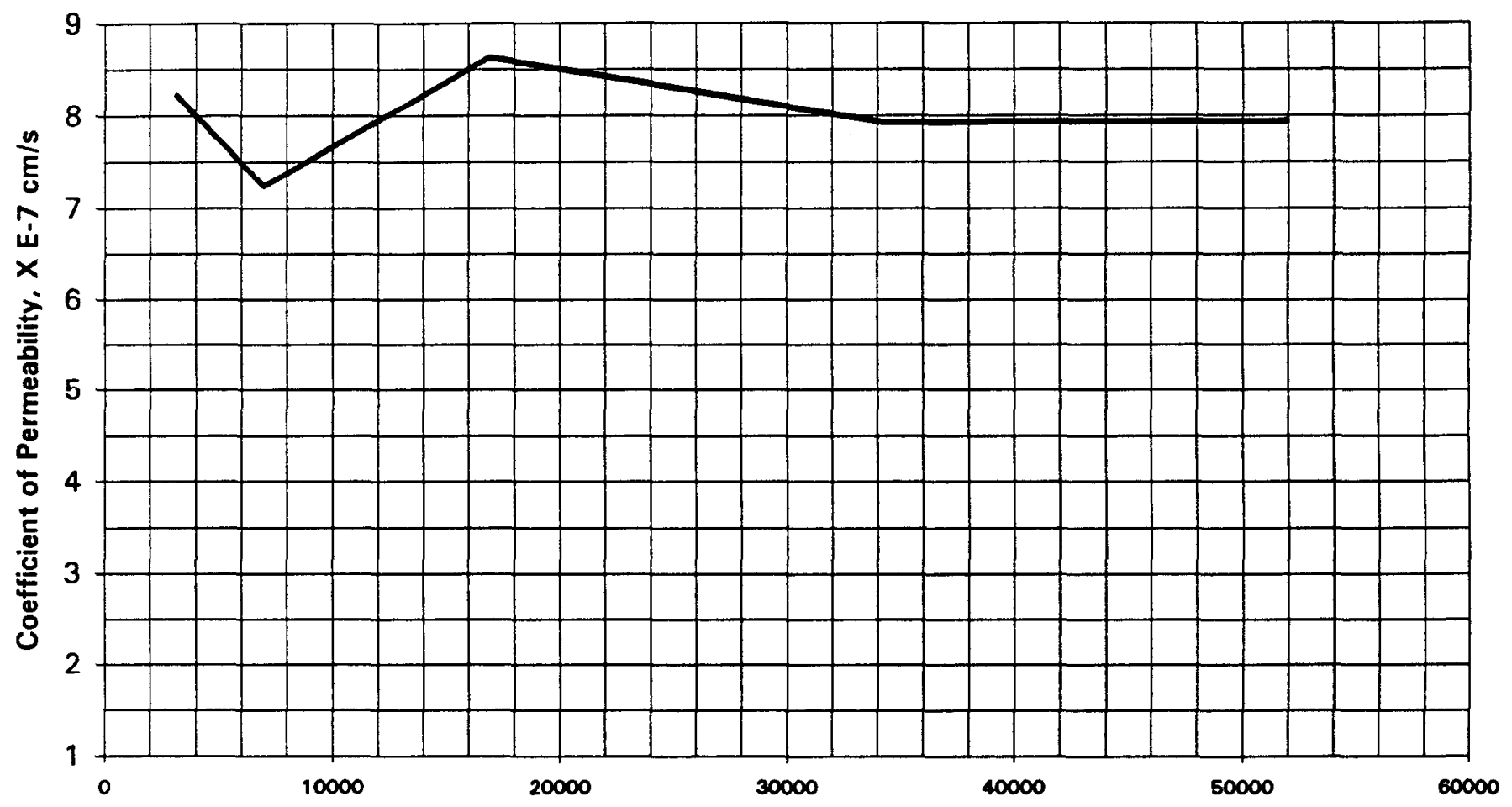

Cumulative Elapsed Time, seconds 


\section{0-DAY SATURATED HYDRAULIC CONDUCTIVITY / PERMEABILITY ASTM D 5084}

PROJECT NAME: SAIC / Savannah River

PROJECT NO. $\quad \mathbf{7 7 7 3 0 4 . 0 0 0 1 0 5 0 0}$
CLIENT SAMPLE NO. P323G00

IT LAB SAMPLE NO. ETDC-8120
Specimen diameter, $\mathrm{cm}$

Specimen length, $\mathrm{cm}$

Wet weight of specimen, $\mathbf{g}$.

Specimen cross-sect. area, $\mathrm{cm}^{\wedge} 2$ Water content, \%

Wet unit weight, pcf

Dry unit weight, pcf

Estimated degree of saturation, \% Estimated spec. gravity of solids Initial conditions are post-soak, pre-test

\begin{tabular}{|l|l|}
\hline INITIAL FINAL \\
\hline
\end{tabular}

\subsection{8}

6.32

538.06

538.02

45.1589

23.8

23.8

117.7

95.0

85.2

2.65
Hydraulic gradient

Min. consolidation stress, psi

44.5

2.0

Max. consolidation stress, psi $\quad 3.0$

Total backpressure, psi

7.0

Permeant Fluid

Deaired DI Water

Sample molded

Sample set up

Seven tests performed

\section{PERMEABILITY vs TIME}

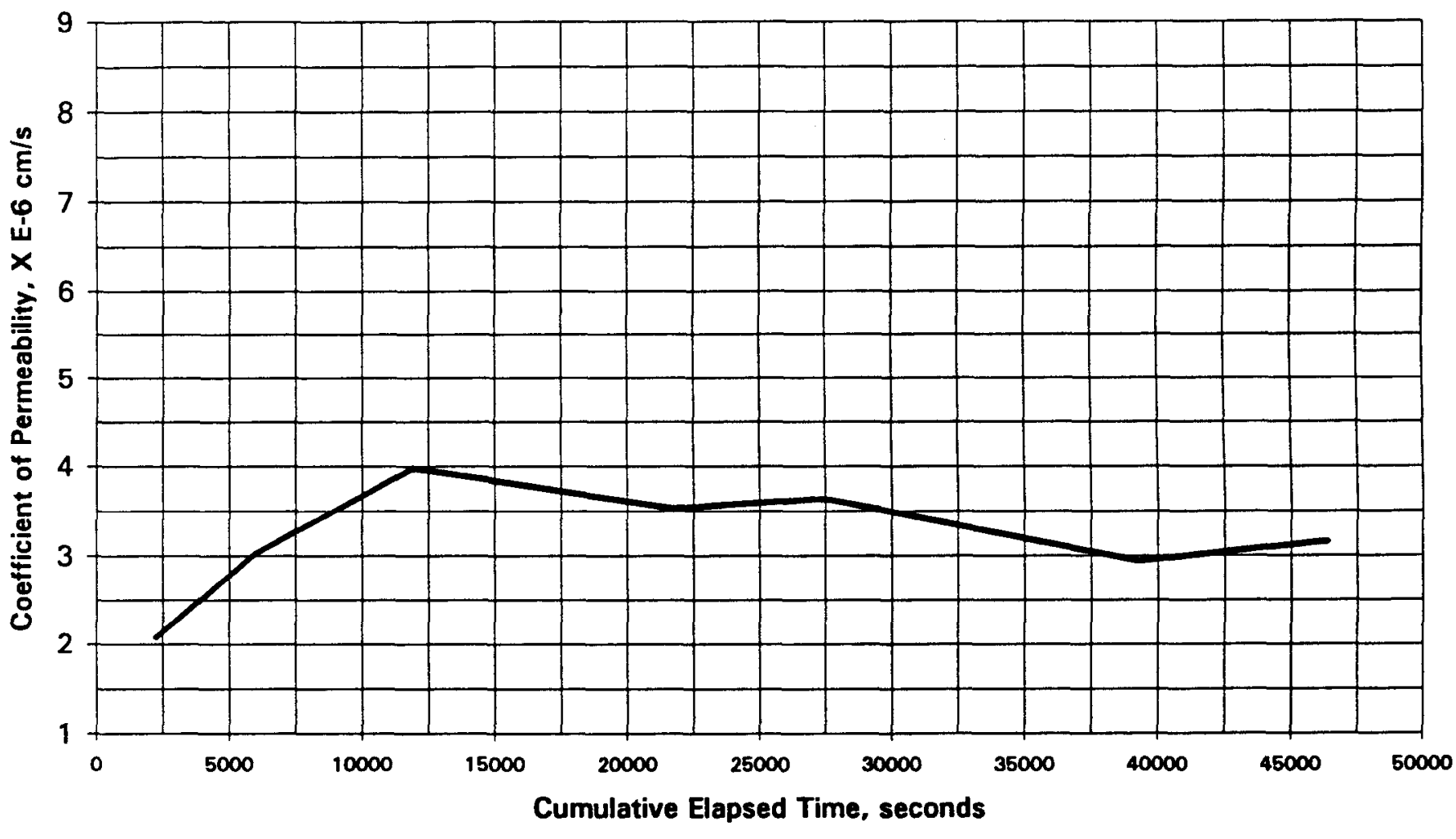




\section{0-DAY SATURATED HYDRAULIC CONDUCTIVITY / PERMEABILITY ASTM D 5084}

PROJECT NAME:

PROJECT NO.
SAIC / Savannah River

777304.00010500
CLIENT SAMPLE NO.

IT LAB SAMPLE NO.
P333G00

ETDC-8121
Specimen diameter, $\mathrm{cm}$

Specimen length, $\mathrm{cm}$

Wet weight of specimen, $g$.

Specimen cross-sect. area, $\mathrm{cm}^{\wedge} 2$

Water content, \%

Wet unit weight, pcf

Dry unit weight, pcf

Estimated degree of saturation, \%

Estimated spec. gravity of solids

Initial conditions are post-soak, pre-test

\section{INITIAL FINAL \\ 7.56}

7.88

$665.14 \quad 667.6$

44.8468

27.9

117.5

91.9

92.4

2.65
Hydraulic gradient

Min. consolidation stress, psi

35.7

2.0

Max. consolidation stress, psi

4.0

6.0

28.4 Total backpressure, psi

Permeant Fluid

Sample molded

Sampie set up

Five tests performed
Deaired DI Water

$12 / 22 / 98$

$4 / 19 / 99$

\section{Coefficient of Permeability, cm/s}

1.1E-06

\section{PERMEABILITY vs TIME}

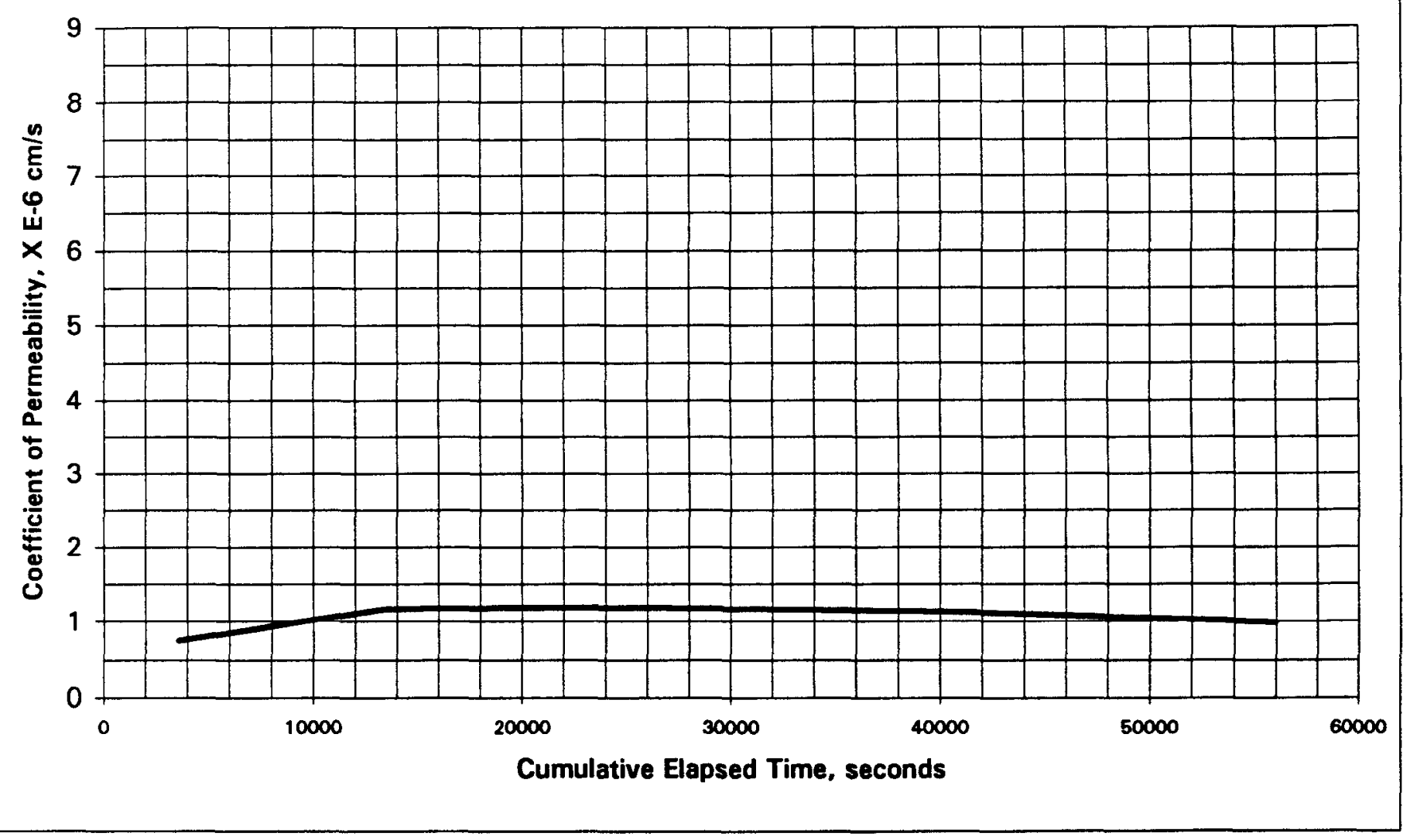




\section{0-DAY SATURATED HYDRAULIC CONDUCTIVITY / PERMEABILITY ASTM D 5084}

PROJECT NAME: SAIC / Savannah River PROJECT NO.
777304.00010500
CLIENT SAMPLE NO. P343G00

IT LAB SAMPLE NO. ETDC-8122

\begin{tabular}{|c|c|c|c|c|}
\hline \multirow[b]{2}{*}{ Specimen diameter, $\mathrm{cm}$} & INITIAL & \multirow[t]{2}{*}{ FINAL } & & \\
\hline & 7.60 & & & \\
\hline $\begin{array}{l}\text { Specimen length, cm } \\
\text { Wet weight of specimen, } \mathbf{g} \text {. }\end{array}$ & $\begin{array}{l}8.88 \\
729.9\end{array}$ & 731.47 & $\begin{array}{l}\text { Hydraulic gradient } \\
\text { Min. consolidation stress, psi }\end{array}$ & $\begin{array}{l}31.7 \\
2.0\end{array}$ \\
\hline Specimen cross-sect. area, $\mathrm{cm}^{\wedge} 2$ & 45.4113 & & Max. consolidation stress, psi & 4.0 \\
\hline Water content, \% & 30.4 & 30.7 & Total backpressure, psi & 6.0 \\
\hline Wet unit weight, pcf & 113.0 & & Permeant Fluid Deaire & DI Water \\
\hline Dry unit weight, pcf & 86.7 & & Sample molded & $12 / 22 / 98$ \\
\hline Estimated degree of saturation, $\%$ & 88.6 & & Sample set up & $4 / 19 / 99$ \\
\hline Estimated spec. gravity of solids & 2.65 & & Six tests performed & \\
\hline
\end{tabular}

Initial conditions are post-soak, pre-test

\section{PERMEABILITY vs TIME}

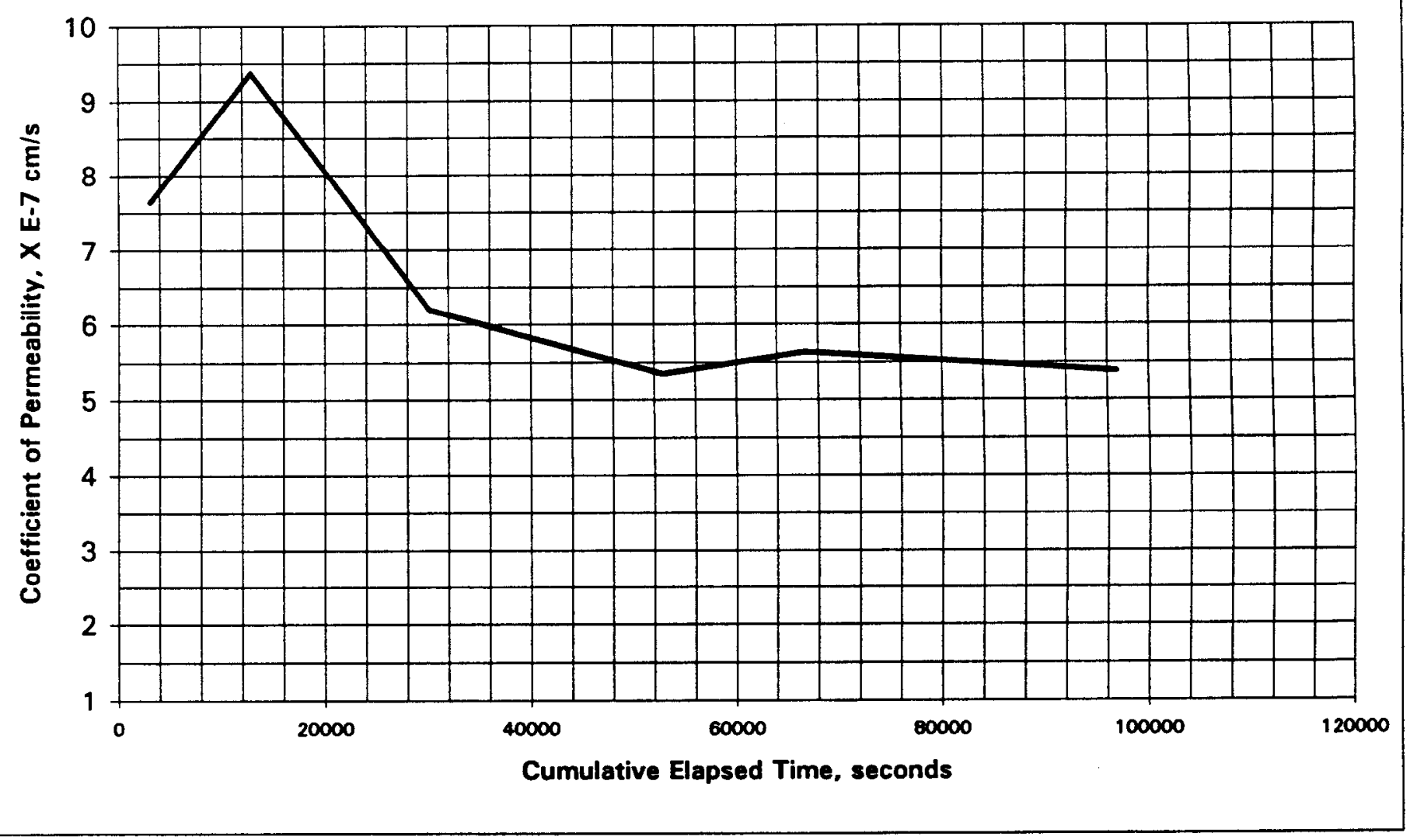




\section{0-DAY SATURATED HYDRAULIC CONDUCTIVITY / PERMEABILITY ASTM D 5084}

PROJECT NAME: SAIC / Savannah River PROJECT NO.
SAIC / Savannah Riv
777304.00010500
CLIENT SAMPLE NO.

IT LAB SAMPLE NO.
P413G00

ETDC-8123

\section{\begin{tabular}{|l|l|}
\hline INITIAL & FINAL \\
\hline
\end{tabular} \\ 7.59}

Specimen diameter, $\mathrm{cm}$

Specimen length, $\mathrm{cm}$

8.64

Wet weight of specimen, $g$.

754.34

45.3002

Water content, \%

30.8

120.4

92.0

Dry unit weight, pcf

102.3

2.65

31.2

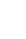

Estimated degree of saturation, \%

Estimated spec. gravity of solids

Initial conditions are post-soak, pre-test
Hydraulic gradient

Min. consolidation stress, psi

Max. consolidation stress, psi

32.6

2.0

4.0

6.0

Permeant Fluid

Deaired DI Water

Sample molded

$12 / 22 / 98$

Sample set up

$4 / 19 / 99$

\section{Eleven tests performed}

\section{\begin{tabular}{|ll|}
\hline Coefficient of Permeability, cm/s & $7.5 E-07$ \\
\hline
\end{tabular}}

\section{PERMEABILITY vs TIME}

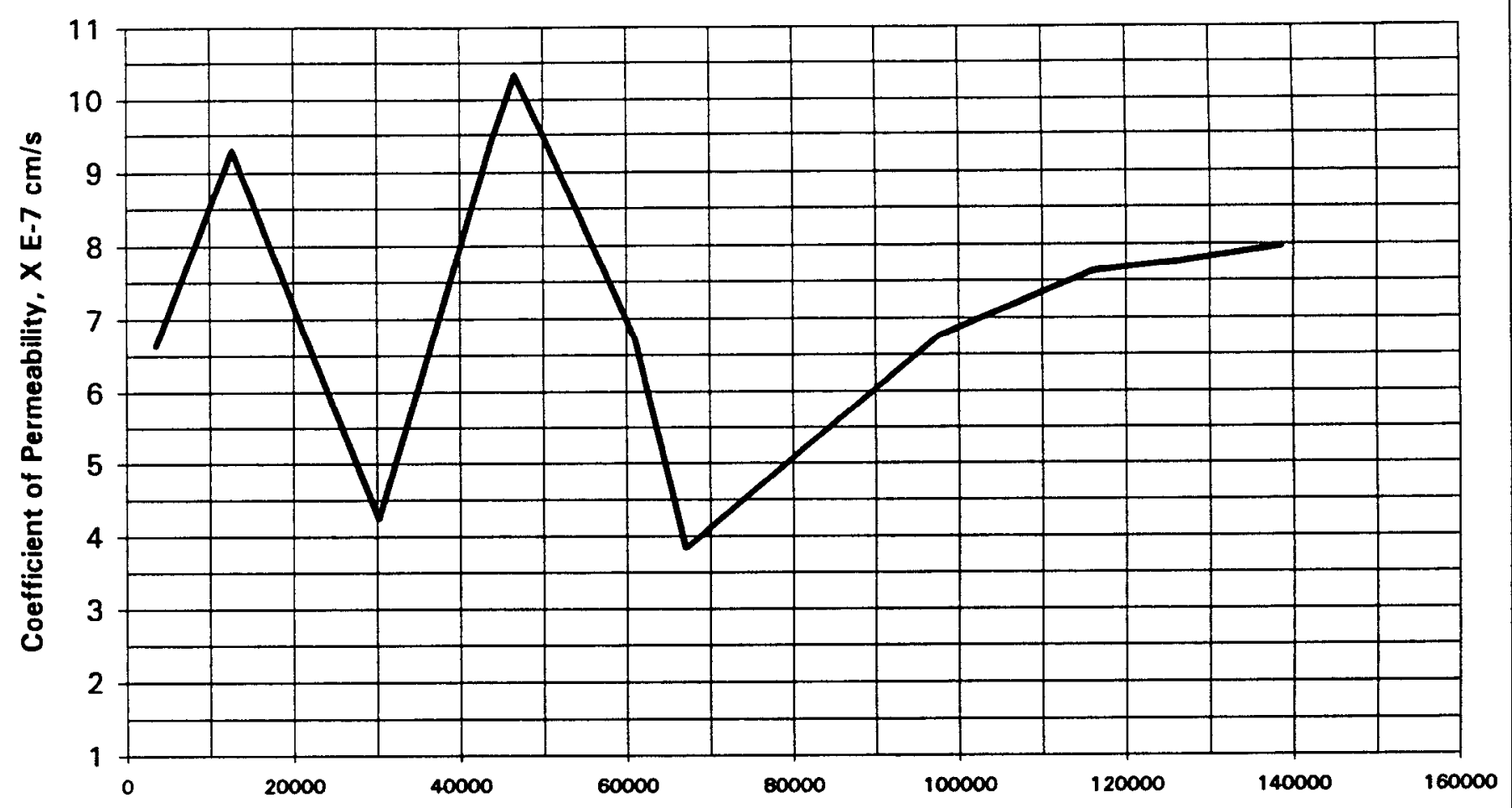

Cumulative Elapsed Time, seconds 


\section{0-DAY SATURATED HYDRAULIC CONDUCTIVITY / PERMEABILITY ASTM D 5084}

PROJECT NAME: SAIC / Savannah River PROJECT NO.

777304.00010500
CLIENT SAMPLE NO. P423G00

IT LAB SAMPLE NO. ETDC-8124
Specimen diameter, $\mathrm{cm}$

Specimen length, $\mathrm{cm}$

Wet weight of specimen, $g$.

Specimen cross-sect. area, $\mathrm{cm}^{\wedge} 2$

Water content, \%

Wet unit weight, pcf

Dry unit weight, pcf

Estimated degree of saturation, \%

Estimated spec. gravity of solids

Initial conditions are post-soak, pre-test

\begin{tabular}{|l|l|}
\hline INITIAL FINAL \\
\hline
\end{tabular}

\subsection{9}

7.69

642.14

643.28

45.2901

27.5

27.7

115.0

90.2

87.4

2.65
Hydraulic gradient 36.6

Min. consolidation stress, psi $\quad 2.0$

Max. consolidation stress, psi $\quad 4.0$

Total backpressure, psi

6.0

Permeant Fluid

Deaired DI Water

Sample molded

$12 / 22 / 98$

Sample set up

$4 / 19 / 99$ below of $\mathrm{OC}$ range of $+1-25 \%$.

\section{\begin{tabular}{ll}
\hline Coefficient of Permeability, cm/s & $1.2 E-06$ \\
\hline
\end{tabular}}

\section{PERMEABILITY vS TIME}

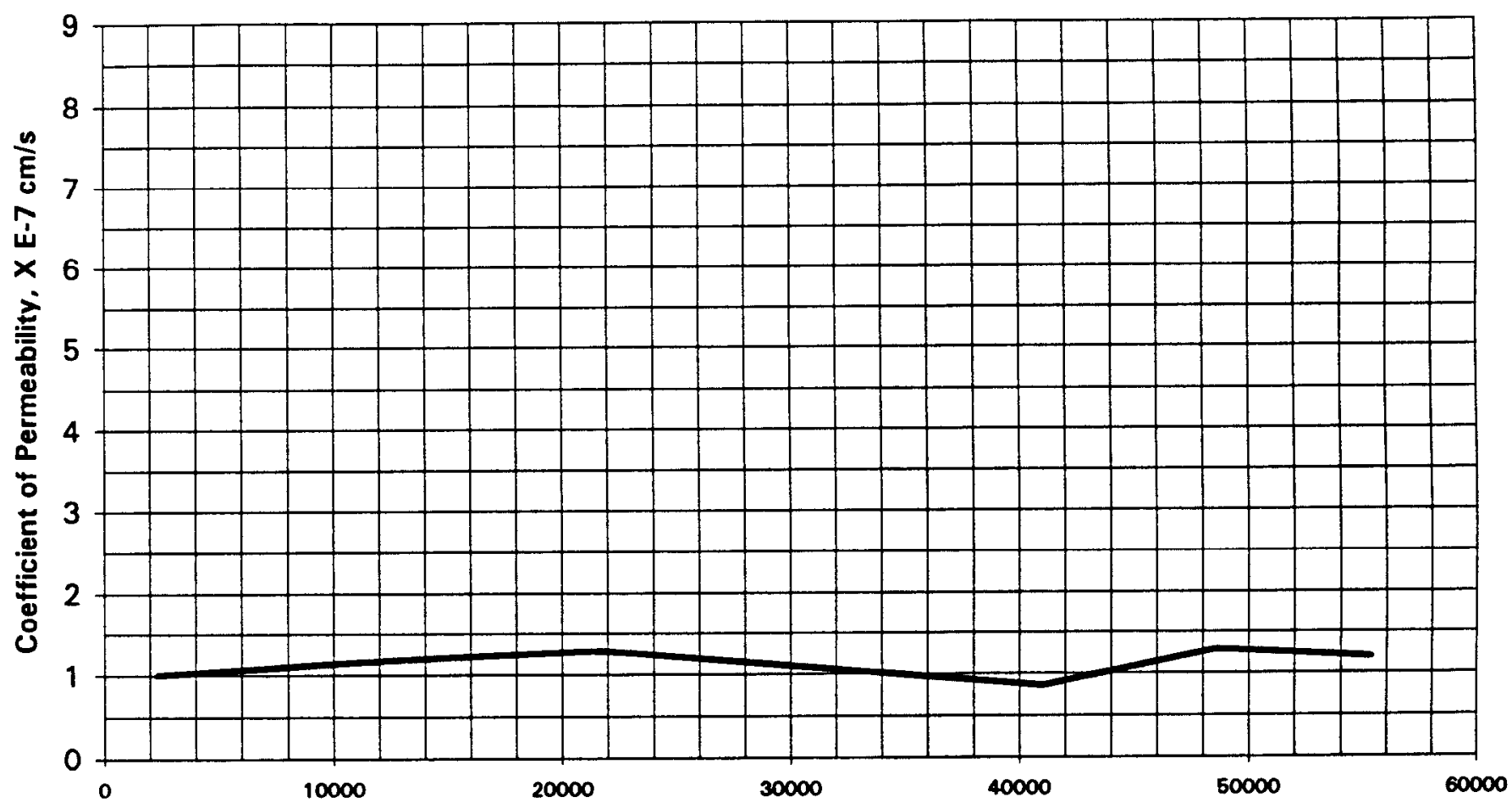

Cumulative Elapsed Time, seconds 


\section{0-DAY SATURATED HYDRAULIC CONDUCTIVITY / PERMEABILITY ASTM D 5084}

PROJECT NAME: SAIC / Savannah River PROJECT NO.
777304.00010500
CLIENT SAMPLE NO.

IT LAB SAMPLE NO.
P433G00

ETDC-8125

INITIAL FINAL

Specimen diameter, $\mathrm{cm}$

$$
7.60
$$

Specimen length, $\mathrm{cm}$

9.28

Wet weight of specimen, $\mathbf{g}$.

748.08

750.66

Specimen cross-sect. area, $\mathrm{cm}^{`} 2 \quad 45.3658$

Water content, \%

40.3

40.8

Wet unit weight, pcf

110.9

79.0

Estimated degree of saturation, \%

Estimated spec. gravity of solids

97.8

2.65

Initial conditions are post-soak, pre-test
Hydraulic gradient 30.3

Min. consolidation stress, psi

2.0

Max. consolidation stress, psi

4.0

6.0

Permeant Fluid

Deaired DI Water

Sample molded

$12 / 22 / 98$

Sample set up

$4 / 19 / 99$

\section{\begin{tabular}{|ll|}
\hline Coefficient of Permeability, cm/s & $4.5 E-07$ \\
\hline
\end{tabular}}

\section{PERMEABILITY vs TIME}

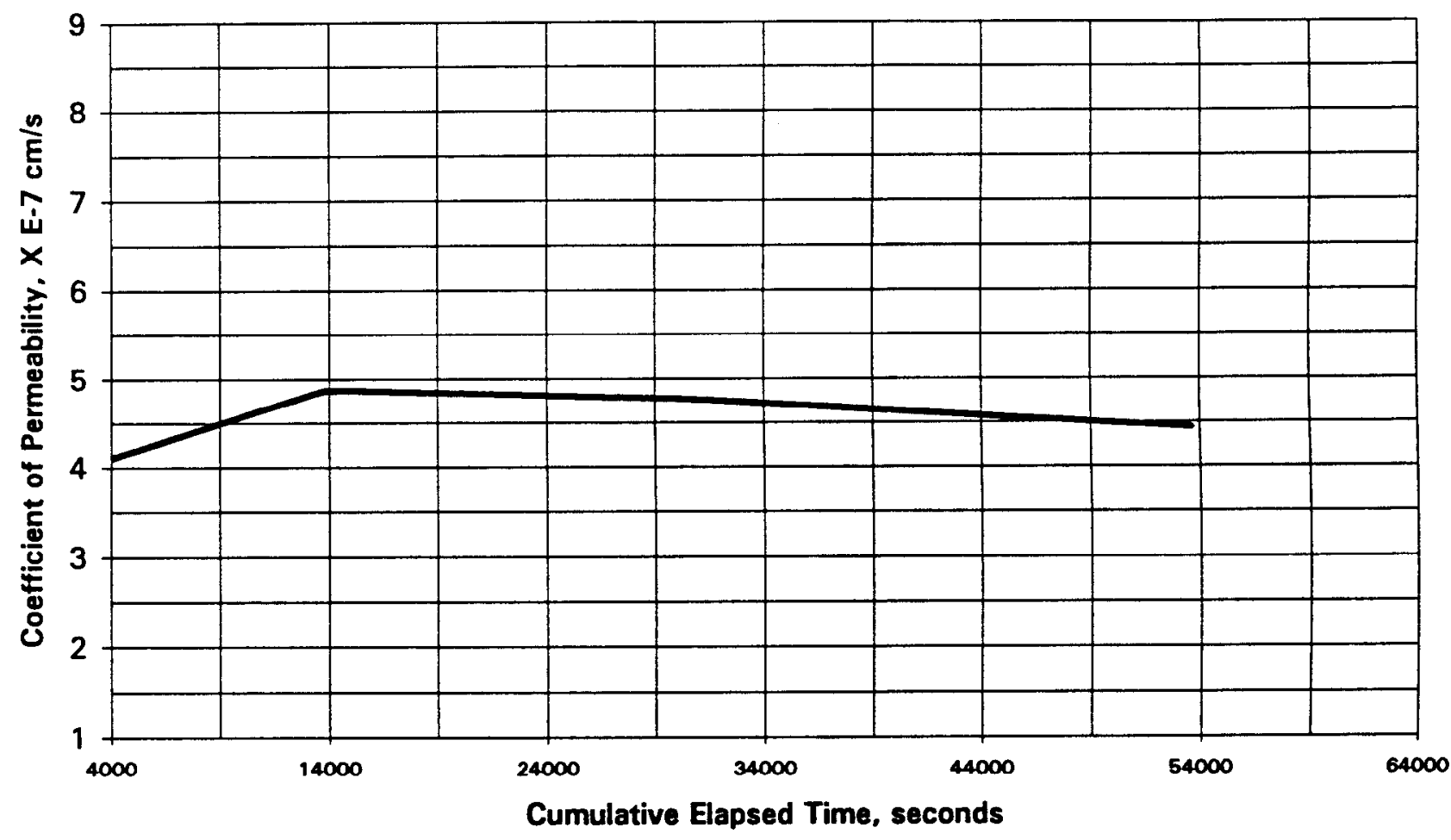




\section{0-DAY SATURATED HYDRAULIC CONDUCTIVITY / PERMEABILITY ASTM D 5084}

PROJECT NAME: SAIC / Savannah River PROJECT NO. $\quad \mathbf{7 7 7 3 0 4 . 0 0 0 1 0 5 0 0}$
CLIENT SAMPLE NO. P443G00

IT LAB SAMPLE NO. ETDC-8126

\begin{tabular}{|c|c|c|c|c|}
\hline \multirow[b]{2}{*}{ Specimen diameter, $\mathrm{cm}$} & INITIAL & \multirow[t]{2}{*}{ FINAL } & & \\
\hline & 7.60 & & & \\
\hline $\begin{array}{l}\text { Specimen length, } \mathrm{cm} \\
\text { Wet weight of specimen, } \mathrm{g} \text {. }\end{array}$ & $\begin{array}{l}9.89 \\
799.96\end{array}$ & 799.59 & $\begin{array}{l}\text { Hydraulic gradient } \\
\text { Min. consolidation stress, psi }\end{array}$ & $\begin{array}{l}14.2 \\
2.0\end{array}$ \\
\hline Specimen cross-sect. area, $\mathrm{cm}^{\wedge} 2$ & 45.3962 & & Max. consolidation stress, psi & 3.0 \\
\hline Water content, $\%$ & 38.3 & 38.2 & Total backpressure, psi & 7.0 \\
\hline Wet unit weight, pcf & 111.2 & & Permeant Fluid Deaire & DI Water \\
\hline Dry unit weight, pcf & 80.4 & & Sample molded & $12 / 22 / 98$ \\
\hline Estimated degree of saturation, \% & 96.0 & & Sample set up & $4 / 19 / 99$ \\
\hline Estimated spec. gravity of solids & 2.65 & & Six tests performed & \\
\hline
\end{tabular}

Initial conditions are post-soak, pre-test

\section{\begin{tabular}{|ll|}
\hline Coefficient of Permeability, $\mathrm{cm} / \mathrm{s}$ & $2.6 \mathrm{E}-06$ \\
\hline
\end{tabular}}

\section{PERMEABILITY vs TIME}

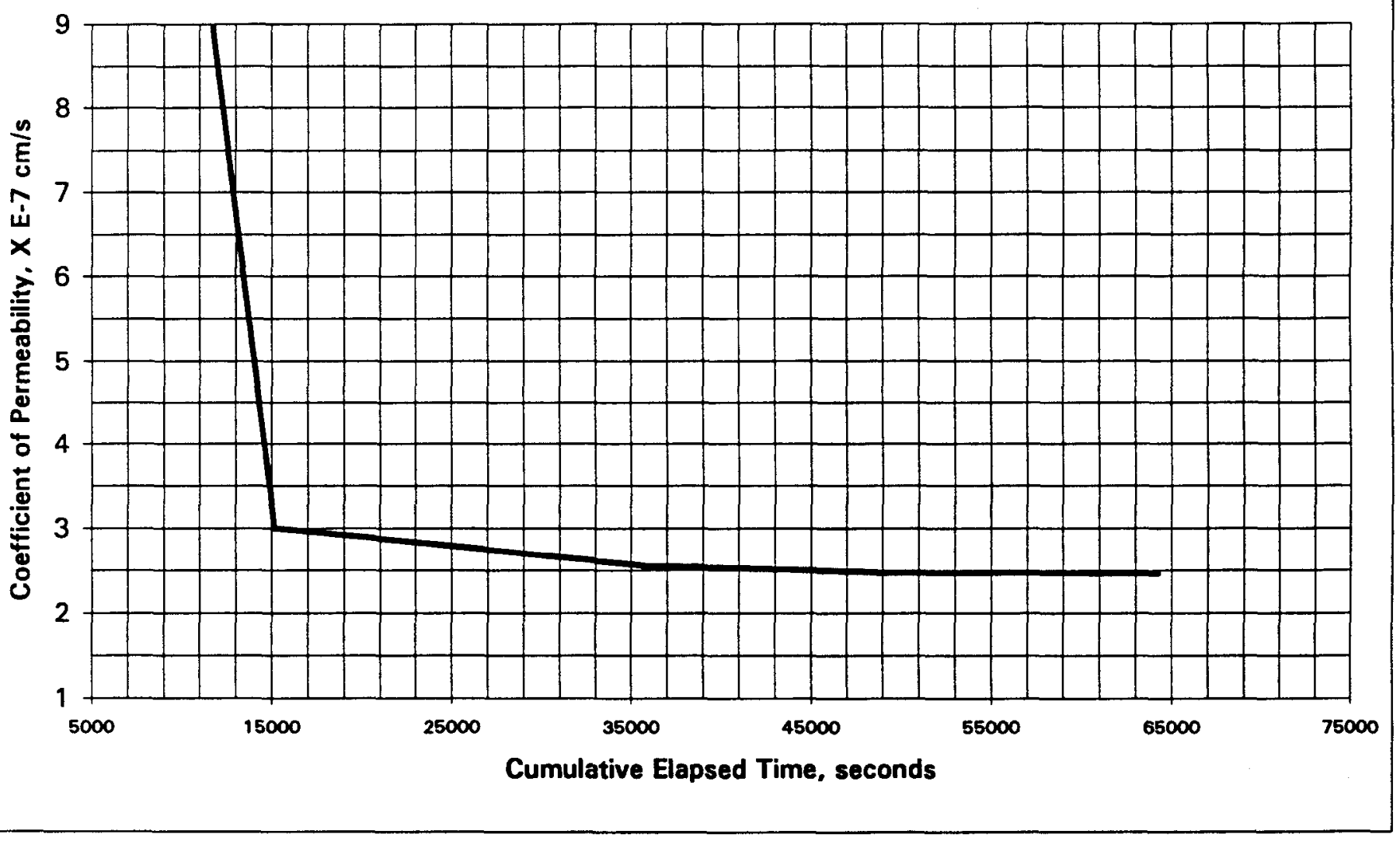




\section{0-DAY SATURATED HYDRAULIC CONDUCTIVITY / PERMEABILITY ASTM D 5084}

PROJECT NAME: SAIC / Savannah River PROJECT NO. 777304.00010500
CLIENT SAMPLE NO.

P513G00

IT LAB SAMPLE NO.

ETDC-8127

INITIAL FINAL

Specimen diameter, $\mathrm{cm}$

Specimen length, $\mathrm{cm}$

Wet weight of specimen, $g$.

Specimen cross-sect. area, $\mathrm{cm}^{\wedge} 2$ Water content, \%

Wet unit weight, pcf

Dry unit weight, pcf

Estimated degree of saturation, \% Estimated spec. gravity of solids Initial conditions are post-soak, pre-test

\subsection{8}

8.23

$697.28 \quad 700.48$

45.0933

29.1

117.2

90.9

93.8

2.65

29.6
Hydraulic gradient

17.1

Min. consolidation stress, psi

2.0

Max. consolidation stress, psi

4.0

Total backpressure, psi

Permeant Fluid

Sample molded

Deaired DI Water

Sample set up

Five tests performed

\section{\begin{tabular}{|ll}
\hline Coefficient of Permeability, $\mathrm{cm} / \mathrm{s}$ & $3.8 \mathrm{E}-07$ \\
\hline
\end{tabular}}

\section{PERMEABILITY vs TIME}

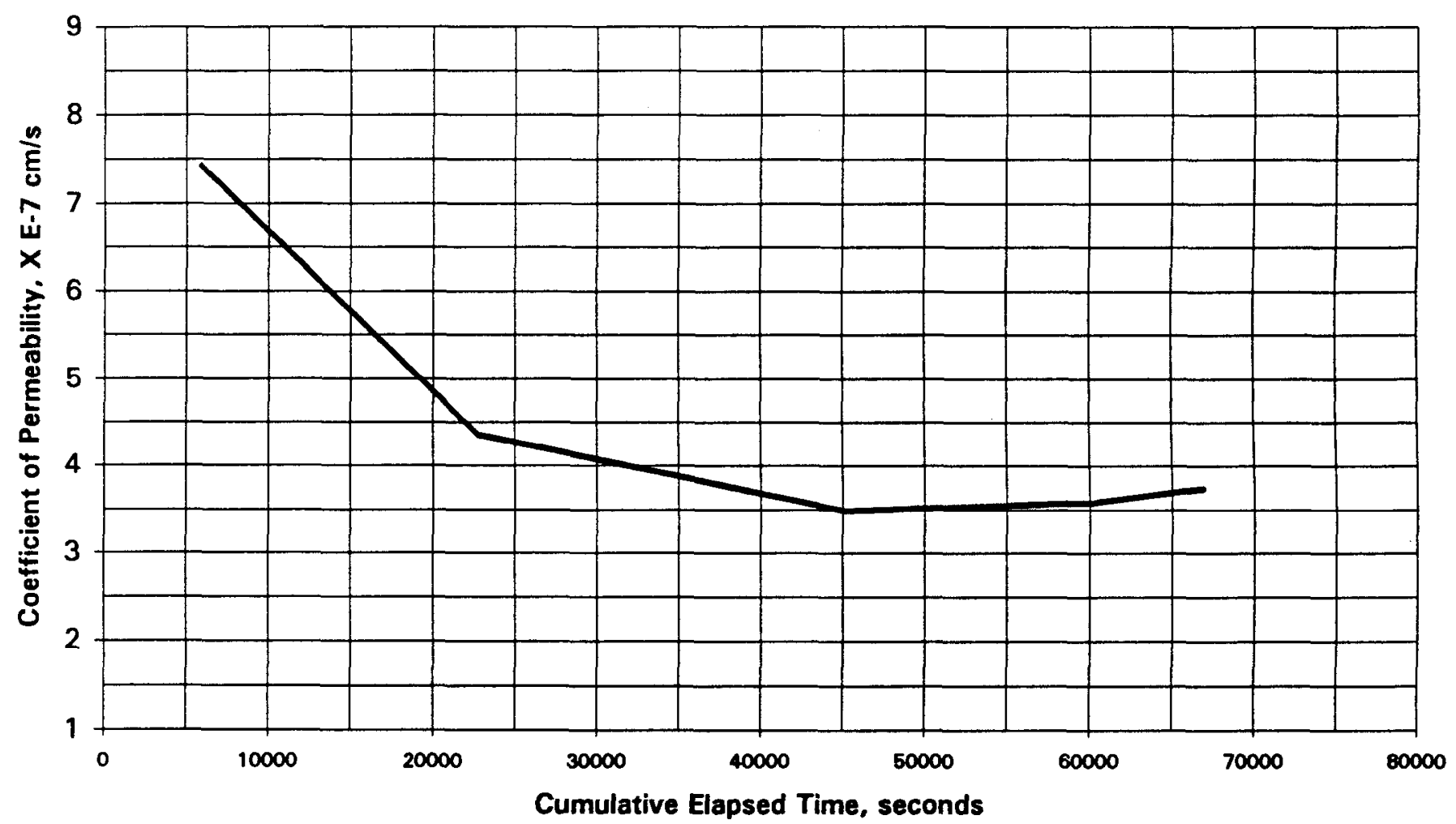




\section{0-DAY SATURATED HYDRAULIC CONDUCTIVITY / PERMEABILITY ASTM D 5084}

PROJECT NAME: SAIC / Savannah River PROJECT NO.

\section{CLIENT SAMPLE NO. P523G00}

IT LAB SAMPLE NO. ETDC-8128

\begin{tabular}{|c|c|c|c|c|}
\hline & INITIAL & FINAL & & \\
\hline Specimen diameter, $\mathrm{cm}$ & 7.58 & & & \\
\hline Specimen length, cm & 6.79 & & Hydraulic gradient & 41.5 \\
\hline Wet weight of specimen, $g$. & 566.64 & 567.81 & Min. consolidation stress, psi & 2.0 \\
\hline Specimen cross-sect. area, $\mathrm{cm}^{\wedge} 2$ & 45.1538 & & Max. consolidation stress, psi & 4.0 \\
\hline Water content, $\%$ & 23.3 & 23.6 & Total backpressure, psi & 6.0 \\
\hline Wet unit weight, pcf & 115.4 & & Permeant Fluid $\quad$ Deairec & DI Water \\
\hline Dry unit weight, pcf & 93.6 & & Sample molded & $12 / 22 / 98$ \\
\hline Estimated degree of saturation, $\%$ & 80.4 & & Sample set up & $4 / 18 / 99$ \\
\hline Estimated spec. gravity of solids & 2.65 & & Five tests performed & \\
\hline
\end{tabular}

Initial conditions are post-soak, pre-test

\section{PERMEABILITY vs TIME}

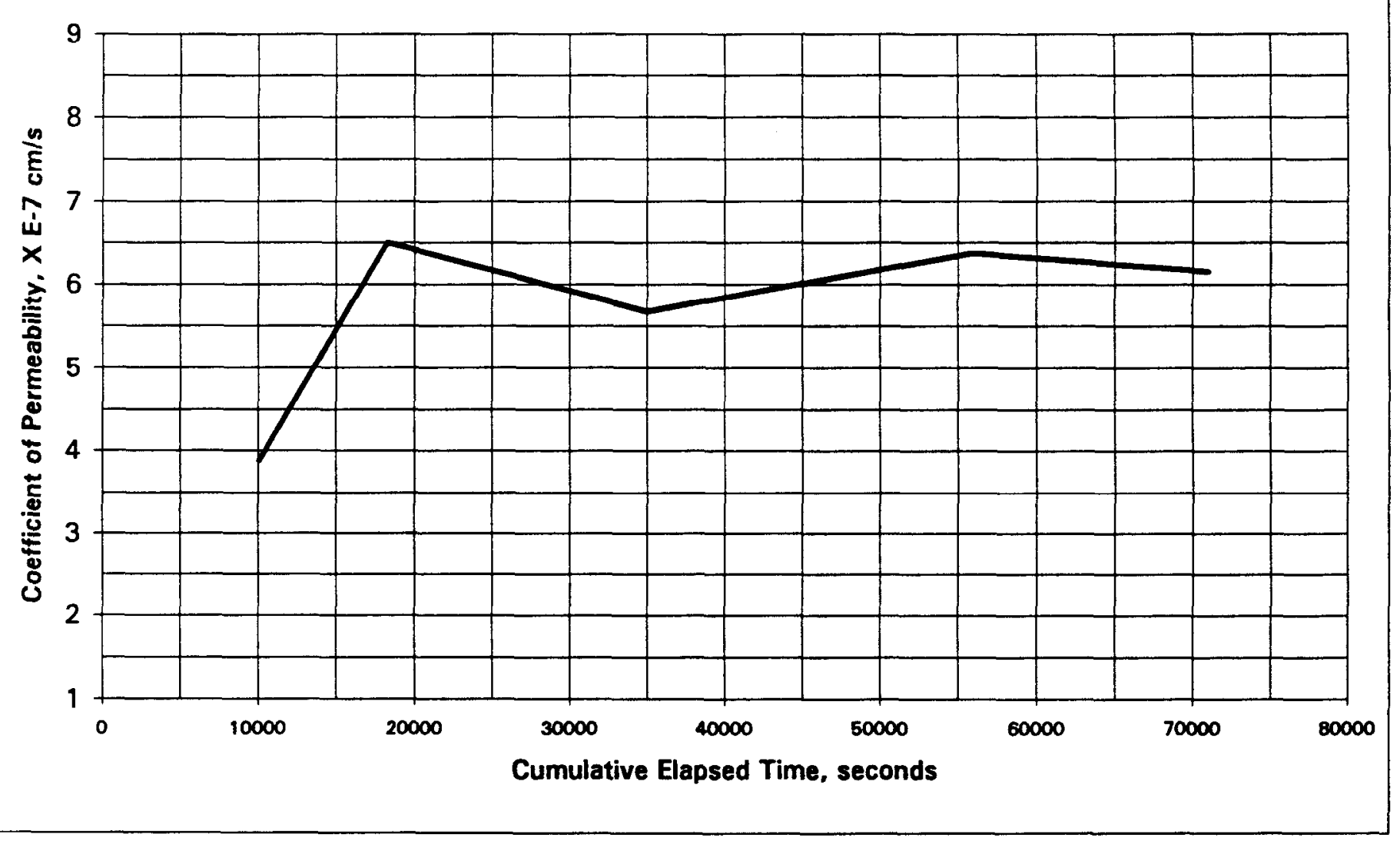




\section{0-DAY SATURATED HYDRAULIC CONDUCTIVITY / PERMEABILITY ASTM D 5084}

PROJECT NAME: SAIC / Savannah River PROJECT NO. $\quad 777304.00010500$
CLIENT SAMPLE NO. P533G00

IT LAB SAMPLE NO. ETDC-8129

\begin{tabular}{|c|c|c|c|c|}
\hline & INITIAL & FINAL & & \\
\hline Specimen diameter, $\mathrm{cm}$ & 7.58 & & & \\
\hline $\begin{array}{l}\text { Specimen length, } \mathrm{cm} \\
\text { Wet weight of specimen, } \mathrm{g} \text {. }\end{array}$ & $\begin{array}{l}9.25 \\
755.12\end{array}$ & 759.11 & $\begin{array}{l}\text { Hydraulic gradient } \\
\text { Min. consolidation stress, psi }\end{array}$ & $\begin{array}{l}30.4 \\
2.0\end{array}$ \\
\hline Specimen cross-sect. area, $\mathrm{cm}^{`} 2$ & 45.0782 & & Max. consolidation stress, psi & 4.0 \\
\hline Water content, $\%$ & 31.8 & 32.5 & Total backpressure, psi & 6.0 \\
\hline Wet unit weight, pcf & 113.0 & & Permeant Fluid Deaire & DI Water \\
\hline Dry unit weight, pcf & 85.8 & & Sample molded & $12 / 22 / 98$ \\
\hline Estimated degree of saturation, $\%$ & 90.7 & & Sample set up & $4 / 19 / 99$ \\
\hline Estimated spec. gravity of solids & 2.65 & & Ten tests performed & \\
\hline
\end{tabular}

Initial conditions are post-soak, pre-test

\begin{tabular}{|ll|}
\hline Coefficient of Permeability, cm/s & $8.6 E-07$ \\
\hline
\end{tabular}

\section{PERMEABILITY vS TIME}

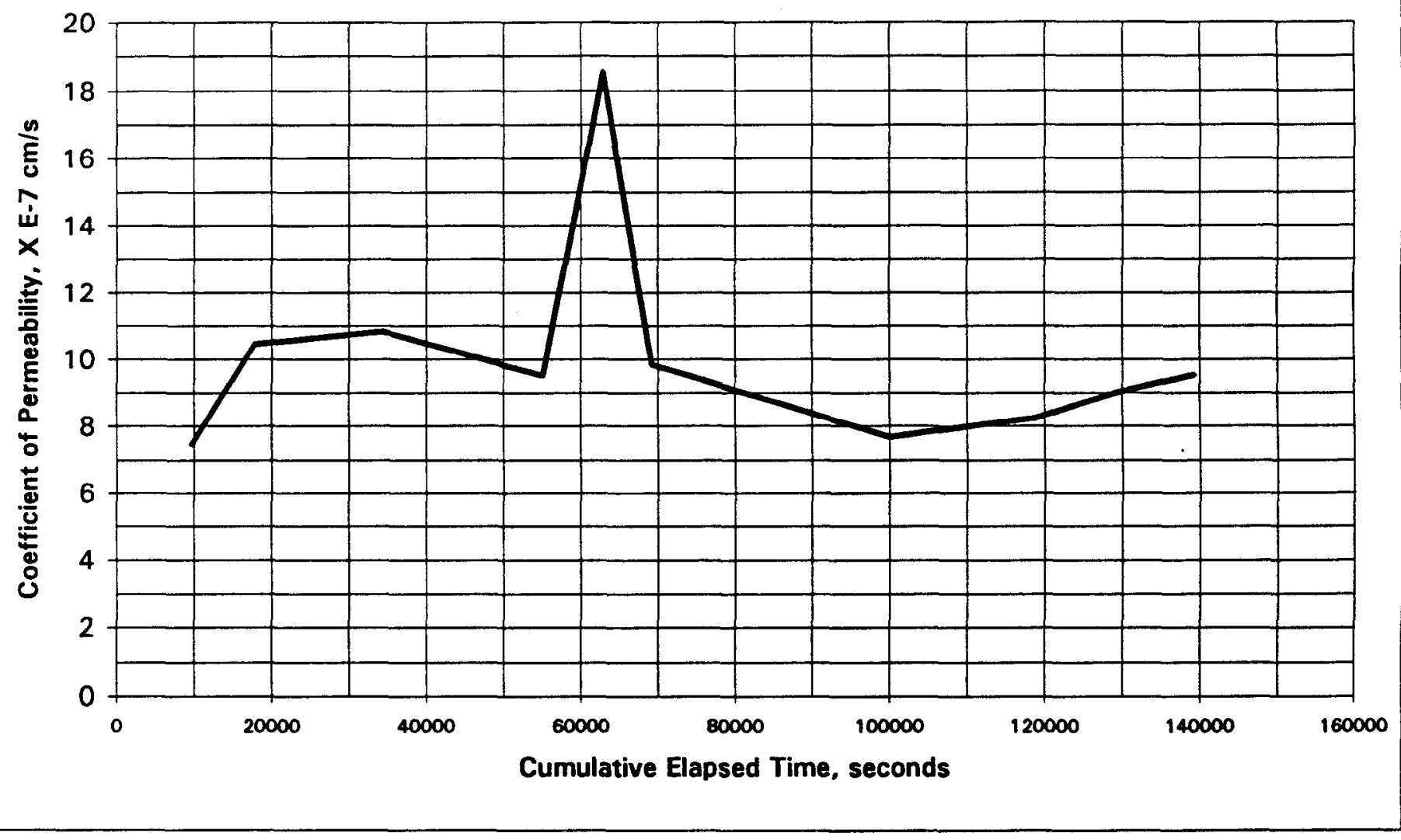




\section{0-DAY SATURATED HYDRAULIC CONDUCTIVITY / PERMEABILITY ASTM D 5084} $\begin{array}{ll}\text { PROJECT NAME: } & \text { SAIC / Savannah River } \\ \text { PROJECT NO. } & 777304.00010500\end{array}$

\section{CLIENT SAMPLE NO. P543G00}

IT LAB SAMPLE NO. ETDC-8130
Specimen diameter, $\mathrm{cm}$

INITIAL FINAL
Specimen length, $\mathrm{cm}$
7.58
Wet weight of specimen, $g$.
9.42
$777.52 \quad 779.15$
Specimen cross-sect. area, $\mathrm{cm}^{-} 2$
45.1286
Water content, \%
30.2

30.5
114.2
Wet unit weight, pcf
87.7
Dry unit weight, pcf
90.3
2.65
Hydraulic gradient
Min. consolidation stress, psi
2.0
Max. consolidation stress, psi
4.0
Total backpressure, psi
Permeant Fluid
6.0
Estimated degree of saturation, \%
Estimated spec. gravity of solids
Sample molded
Deaired DI Water
Sample set up
$12 / 22 / 98$
Six tests performed

Initial conditions are post-soak, pre-test

\section{\begin{tabular}{|ll|}
\hline Coefficient of Permeability, $\mathrm{cm} / \mathrm{s}$ & $1.4 \mathrm{E}-06$ \\
\hline
\end{tabular}}

\section{PERMEABILITY vS TIME}

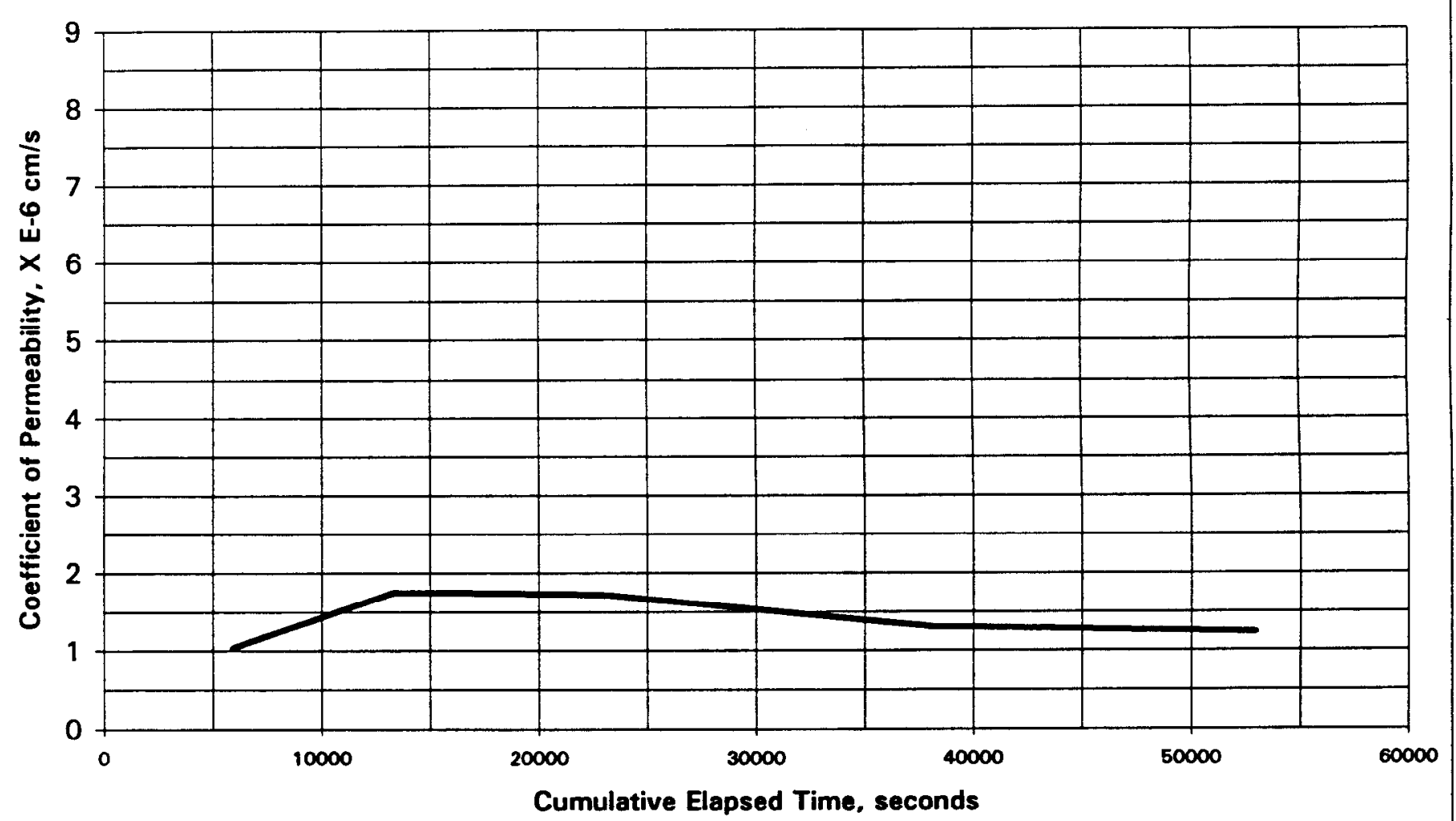




\section{0-DAY SATURATED HYDRAULIC CONDUCTIVITY / PERMEABILITY ASTM D 5084}

PROJECT NAME:

PROJECT NO.
SAIC / Savannah River

777304.00010500
CLIENT SAMPLE NO. P613G00

IT LAB SAMPLE NO. ETDC-8131

\section{INITIAL FINAL}

Specimen diameter, $\mathrm{cm}$

7.58

Specimen length, $\mathrm{cm}$

8.03

680.88

684.83

Hydraulic gradient

35.1

Wet weight of specimen, $\mathbf{g}$.

45.0681

Specimen cross-sect. area, $\mathrm{cm}^{`} 2$

27.3

28.1

117.5

92.3

91.4

2.65

Dry unit weight, pcf

Estimated degree of saturation, \%

Estimated spec. gravity of solids
Min. consolidation stress, psi

2.0

Max. consolidation stress, psi

4.0

Total backpressure, psi

Permeant Fluid

Deaired DI Water

Sample molded

$12 / 22 / 98$

Sample set up

$4 / 19 / 99$

Initial conditions are post-soak, pre-test

\section{PERMEABILITY vS TIME}

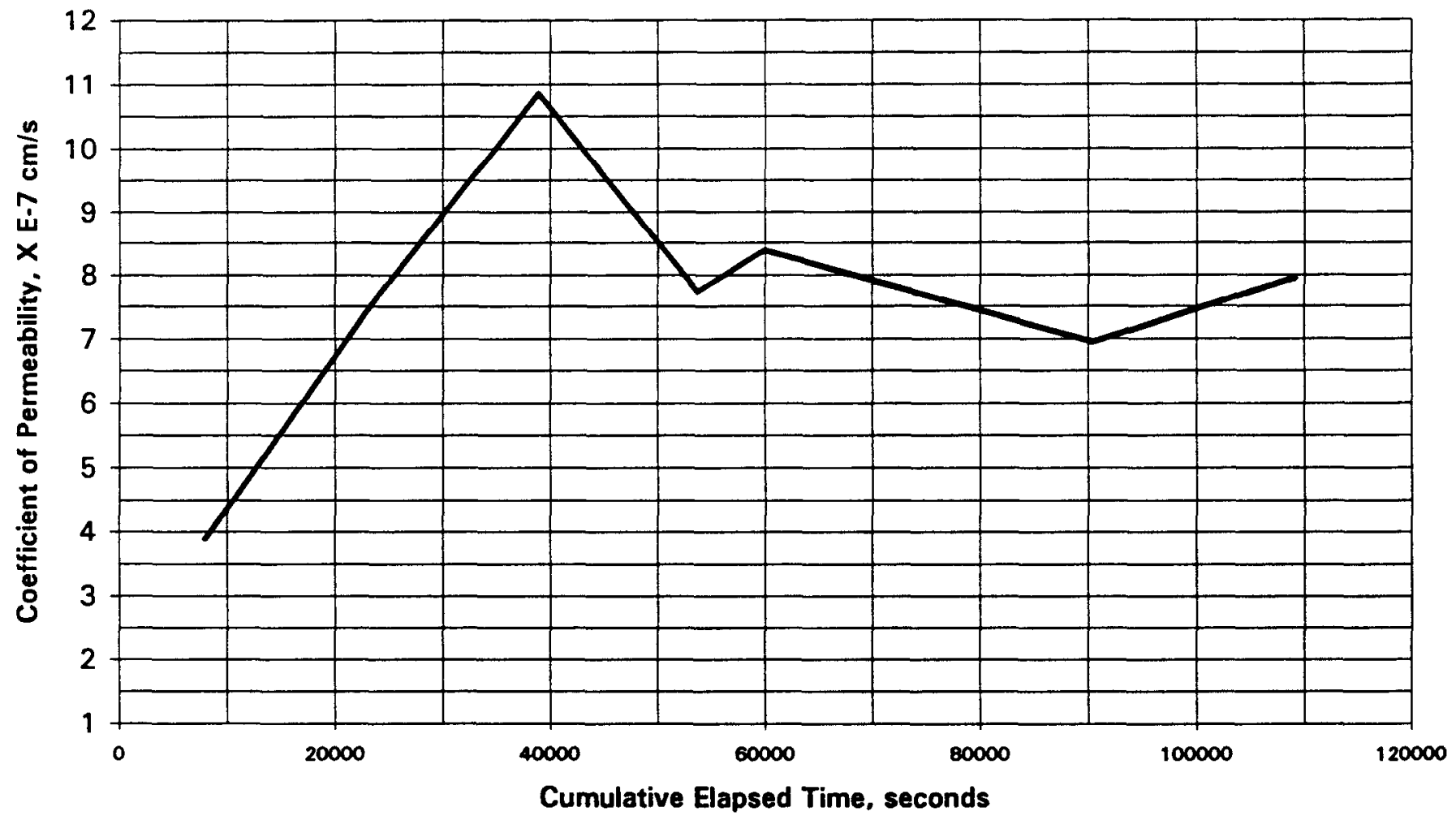

Cumulative Elapsed Time, seconds 


\section{0-DAY SATURATED HYDRAULIC CONDUCTIVITY / PERMEABILITY ASTM D 5084}

PROJECT NAME: SAIC / Savannah River

PROJECT NO.
777304.00010500
CLIENT SAMPLE NO.

IT LAB SAMPLE NO.
P623G00

ETDC-8132

\begin{tabular}{|c|c|c|}
\hline & INITIAL & FINAL \\
\hline Specimen diameter, $\mathrm{cm}$ & 7.57 & \\
\hline Specimen length, $\mathrm{cm}$ & 6.44 & \\
\hline Wet weight of specimen, $g$ & 549.91 & 551.55 \\
\hline Specimen cross-sect. area, $\mathrm{cm}^{`} 2$ & 44.9977 & \\
\hline Water content, $\%$ & 24.9 & 25.3 \\
\hline Wet unit weight, pcf & 118.5 & \\
\hline Dry unit weight, pcf & 94.8 & \\
\hline Estimated degree of saturation, \% & 88.7 & \\
\hline $\begin{array}{l}\text { Estimated spec. gravity of solids } \\
\text { Initial conditions are post-soak, pre-test }\end{array}$ & 2.65 & \\
\hline
\end{tabular}

Hydraulic gradient

21.9

Min. consolidation stress, psi

2.0

Max. consolidation stress, psi $\quad 3.0$

Total backpressure, psi

Permeant Fluid

7.0

Sample molded

Sample set up

Seven tests performed
Deaired DI Water

$12 / 22 / 98$

$4 / 19 / 99$

\section{Coefficient of Permeability, cm/s $2.3 E-06$}

\section{PERMEABILITY vs TIME}

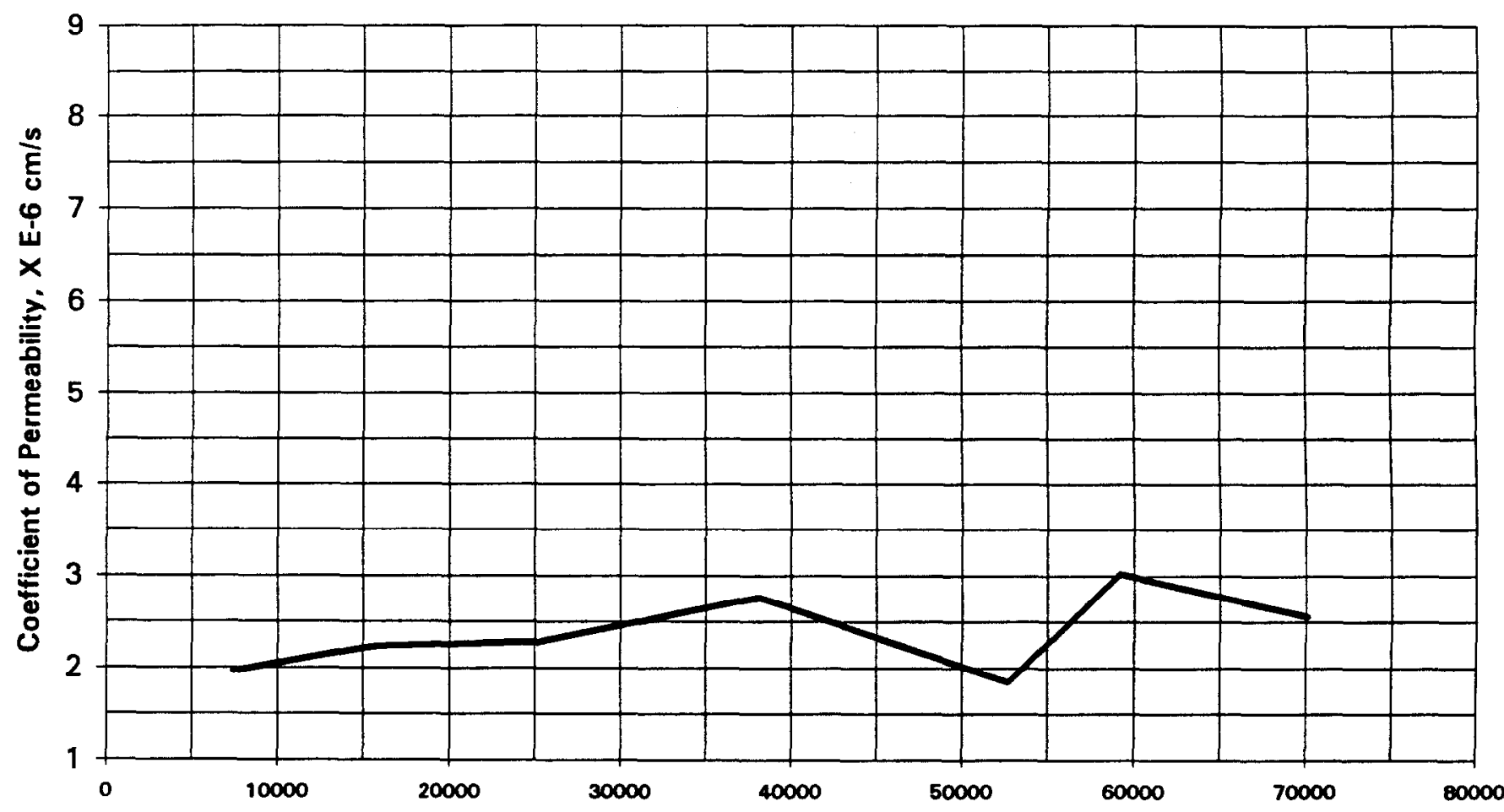

Cumulative Elapsed Time, seconds 


\section{0-DAY SATURATED HYDRAULIC CONDUCTIVITY / PERMEABILITY ASTM D 5084}

PROJECT NAME: SAIC / Savannah River PROJECT NO. $\quad 777304.00010500$
Client SAMPLE No. P633G00

IT LAB SAMPLE NO. ETDC-8133
Specimen diameter, cm

Specimen length, $\mathrm{cm}$

Wet weight of specimen, $g$.

Specimen cross-sect. area, $\mathrm{cm}^{`} 2$

Water content, \%

Wet unit weight, pcf

Dry unit weight, pcf

Estimated degree of saturation, \%

Estimated spec. gravity of solids

Initial conditions are post-soak, pre-test

\begin{tabular}{|l|l|}
\hline INITIAL FINAL \\
\hline 7.57
\end{tabular}

\subsection{7}

9.45

771.28

774.37

44.9624

33.3

113.3

85.0

93.3

2.65
Hydraulic gradient

Min. consolidation stress, psi

Max. consolidation stress, psi

Total backpressure, psi

Permeant Fluid

Sample molded

Sample set up

Five tests performed
29.8

2.0

4.0

6.0

Deaired DI Water

$12 / 22 / 98$

$4 / 19 / 99$

\section{\begin{tabular}{|ll|}
\hline Coefficient of Permeability, cm/s & $5.6 E-07$ \\
\hline
\end{tabular}}

\section{PERMEABILITY vS TIME}

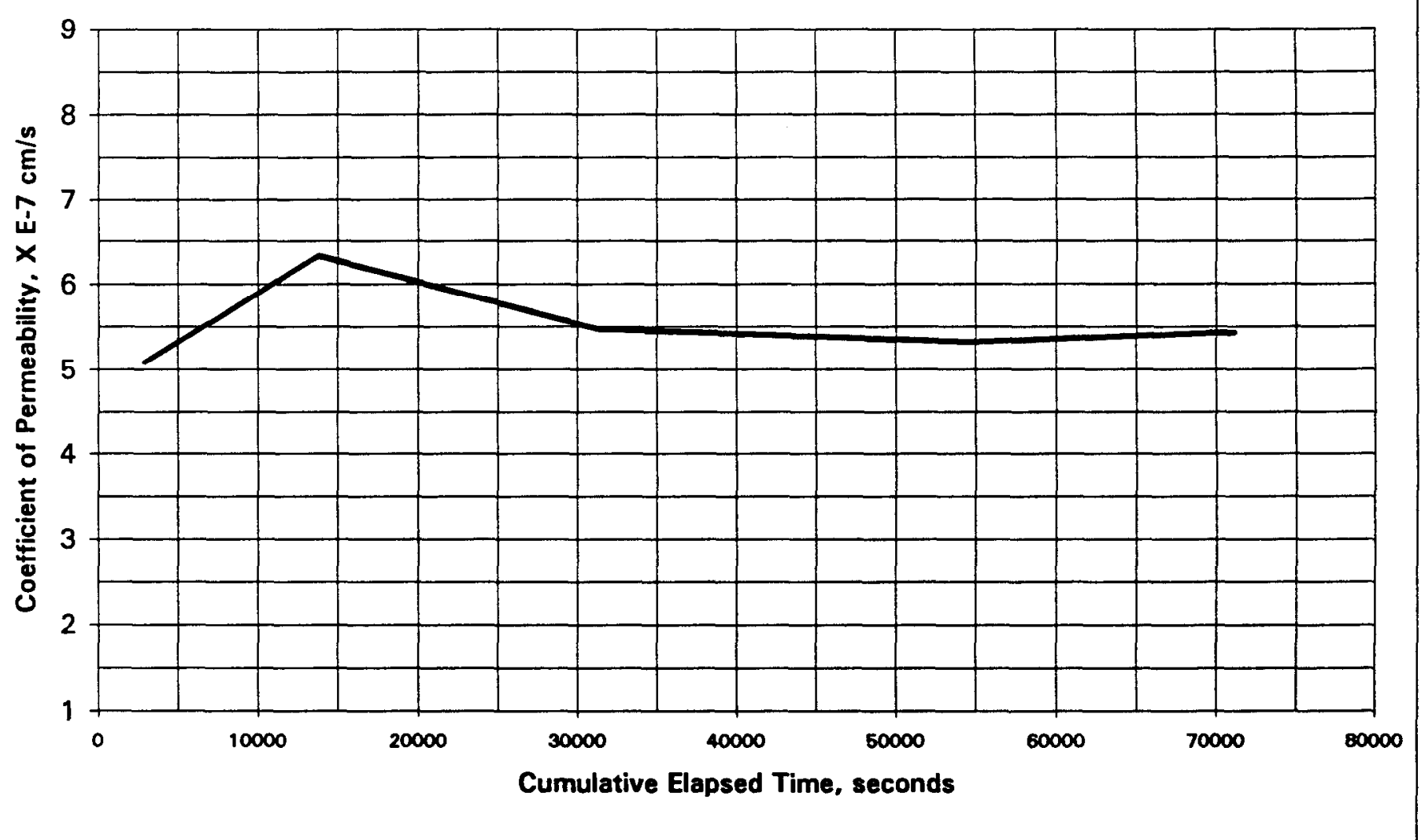




\section{0-DAY SATURATED HYDRAULIC CONDUCTIVITY / PERMEABILITY ASTM D 5084}

PROJECT NAME: SAIC / Savannah River PROJECT NO.
CLIENT SAMPLE NO. P643G00

IT LAB SAMPLE NO. ETDC-8134

\begin{tabular}{|c|c|c|c|c|}
\hline \multirow[b]{2}{*}{ Specimen diameter, cm } & INITIAL & FINAL & & \\
\hline & 7.60 & & & \\
\hline Specimen length, cm & 8.94 & & Hydraulic gradient & 31.5 \\
\hline Wet weight of specimen, $g$. & 737.2 & 739.25 & Min, consolidation stress, psi & 2.0 \\
\hline Specimen cross-sect. area, $\mathrm{cm}^{-} 2$ & 45.3103 & & Max. consolidation stress, psi & 4.0 \\
\hline Water content, $\%$ & 31.0 & 31.4 & Total backpressure, psi & 6.0 \\
\hline Wet unit weight, pcf & 113.6 & & Permeant Fluid Deaire & DI Water \\
\hline Dry unit weight, pcf & 86.7 & & Sample molded & $12 / 22 / 98$ \\
\hline Estimated degree of saturation, $\%$ & 90.5 & & Sample set up & $4 / 19 / 99$ \\
\hline Estimated spec. gravity of solids & 2.65 & & Eleven tests performed & \\
\hline
\end{tabular}

Initial conditions are post-soak, pre-test

\section{PERMEABILITY vS TIME}

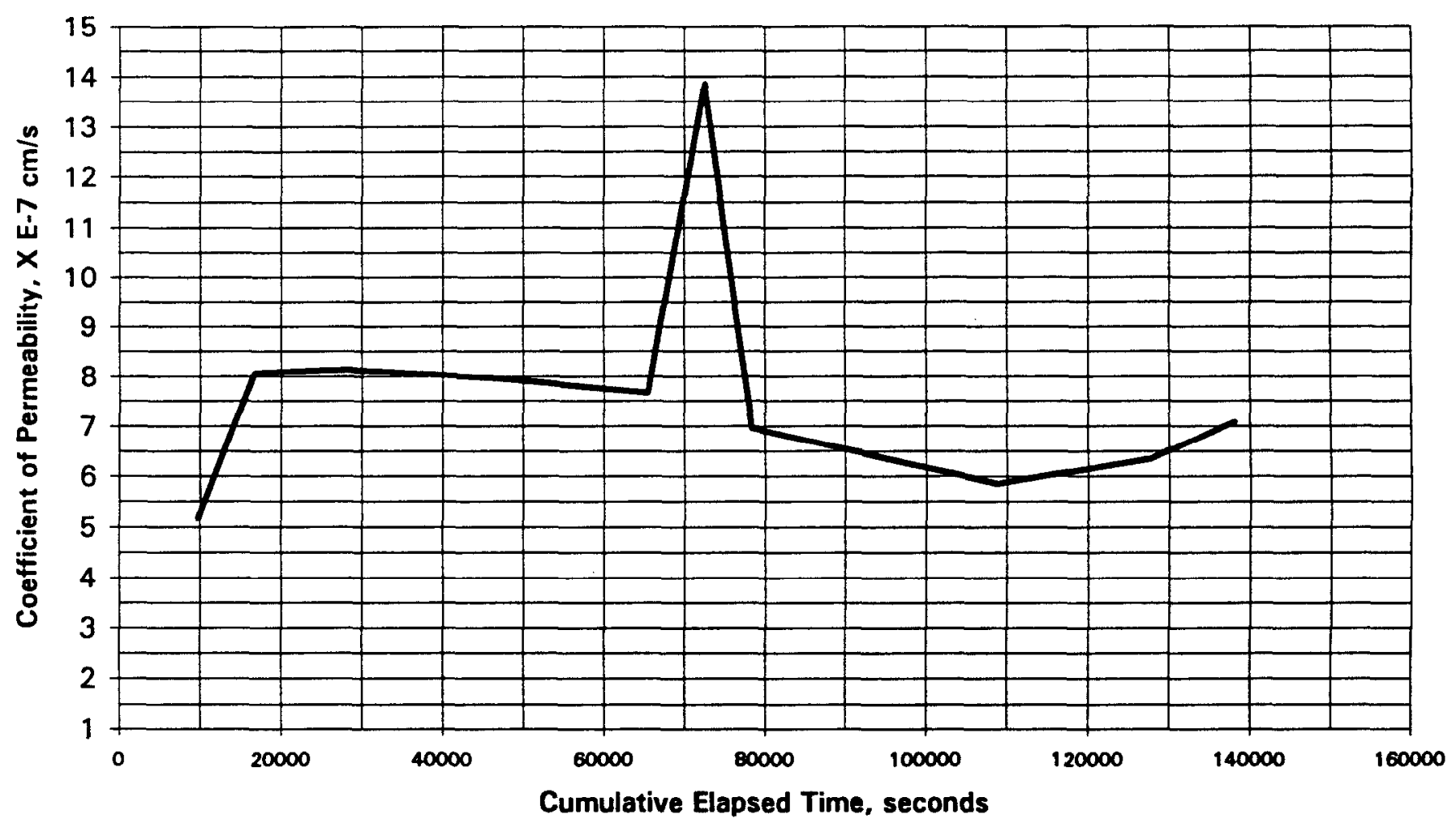

